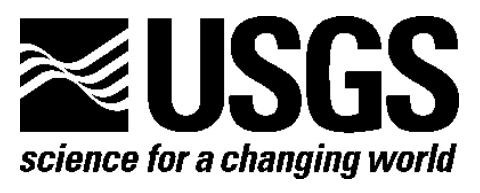

\title{
Exposure-Related Effects of Formulated Pseudomonas fluorescens Strain CL145A to Glochidia from Seven Unionid Mussel Species
}

By James A. Luoma, Kerry L. Weber, Todd J. Severson, Theresa M. Schreier, Denise A. Mayer, Douglas B. Aloisi and Nathan L. Eckert

Open-File Report 2015-1094

U.S. Department of the Interior

U.S. Geological Survey 


\section{U.S. Department of the Interior \\ SALLY JEWELL, Secretary}

\section{U.S. Geological Survey \\ Suzette M. Kimball, Acting Director}

U.S. Geological Survey, Reston, Virginia: 2015

For more information on the USGS-the Federal source for science about the Earth, its natural and living resources, natural hazards, and the environment-visit http://www.usgs.gov or call 1-888-ASK-USGS (1-888-275-8747)

For an overview of USGS information products, including maps, imagery, and publications, visit http://www.usgs.gov/pubprod

Any use of trade, firm, or product names is for descriptive purposes only and does not imply endorsement by the U.S. Government.

Although this information product, for the most part, is in the public domain, it also may contain copyrighted materials as noted in the text. Permission to reproduce copyrighted items must be secured from the copyright owner.

Suggested citation:

Luoma, J.A., Weber, K.L., Severson, T.J, Schreier ,T.M., Mayer, D.A., Aloisi, D.B, and Eckert N.L., 2015, Exposure-related effects of formulated Pseudomonas fluorescens strain CL145A to glochidia from seven unionid mussel species: U.S. Geological Survey Open-File Report 2015-1094, 474 p., http://dx.doi.org/10.3133/ofr20151094. 


\section{Acknowledgments}

This study was funded through a grant from the U.S. Environmental Protection Agency Great Lakes Restoration Initiative awarded to the New York State Education Department and U.S. Geological Survey appropriated funds. The authors thank Mark P. Gaikowski of the Upper Midwest Environmental Sciences Center for assisting with study design and statistical analysis as well as Jeremy K. Wise, Samuel M. Stafslien, Pajtshiab L. Moua, and Susan M. Schleis for technical assistance and data collection. 



\section{Contents}

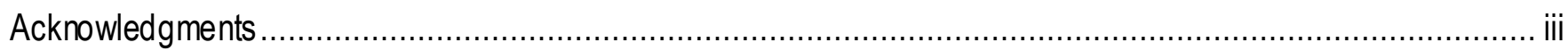

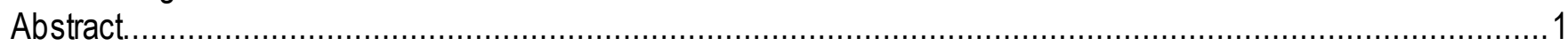

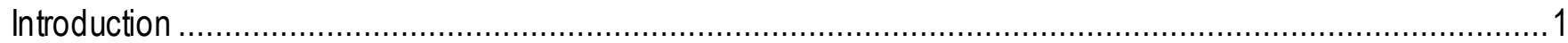

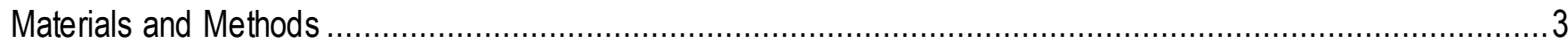

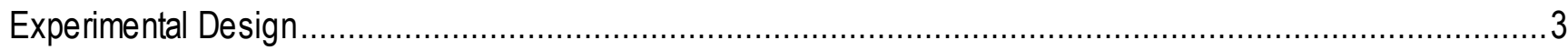

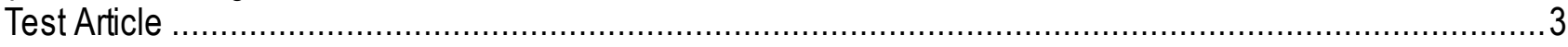

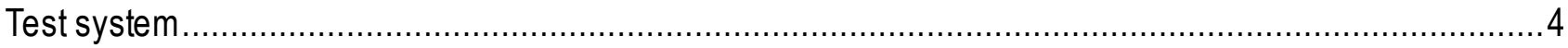

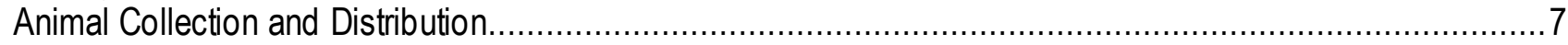

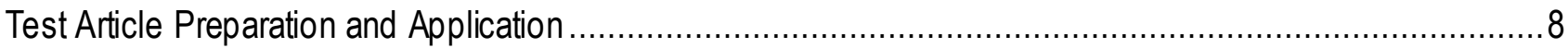

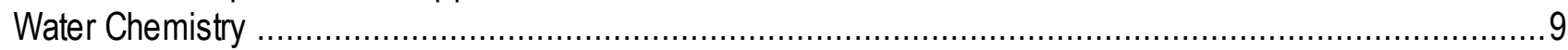

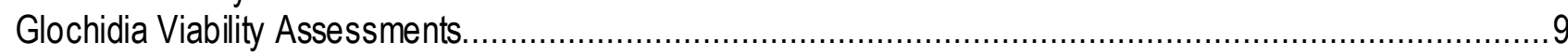

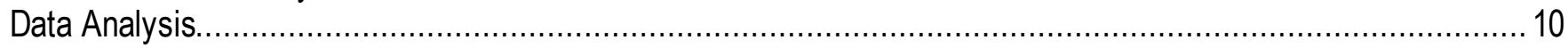

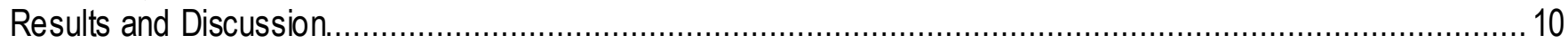

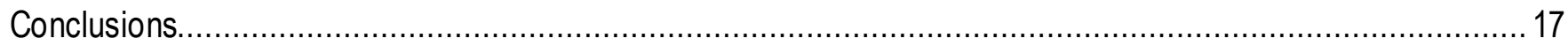

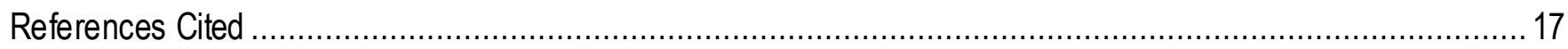

Appendix 1. Study Protocol, Amendments, and Datasheets ................................................................ 19

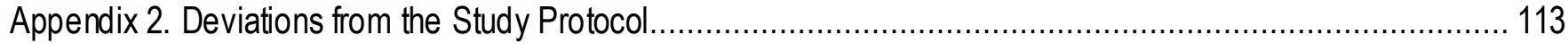

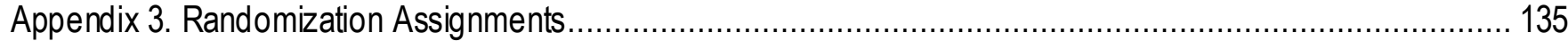

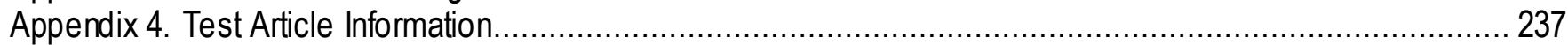

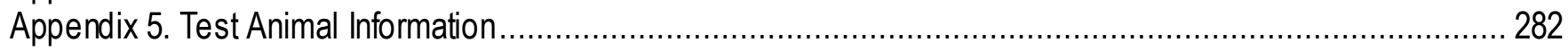

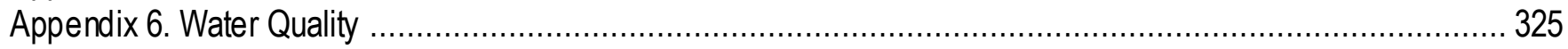

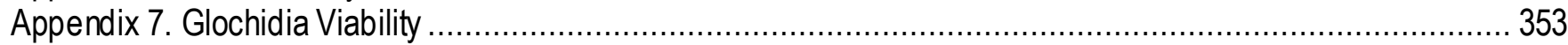




\section{Figures}

Figure 1. Photograph showing glochidia test system with individual test chambers................................... 6

Figure 2. Photographs showing $A$, the extraction of glochidia from a gravid female mussel and, $B$, extracted glochidia......

Figure 3. Photomicrographs showing $A$, glochidia before a drop of sodium chloride solution was added to induce valve closure in viable animals and, $B$, glochidia one minute after addition of the sodium chloride solution.

Figure 4. Photograph showing the distribution of glochidia to test chambers 8

\section{Tables}

Table 1. Data for test animals, test articles and, exposure dates for studies of selected native freshwater mussel glochidia exposed to two formulations of Pseudomonas fluorescens, strain CL145A. 4

Table 2. Data for initial glochidia viability by individual female mussel for each species..............................5

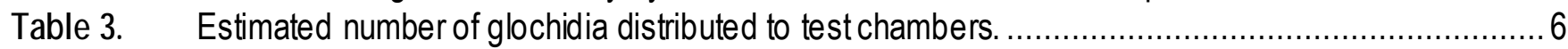

Table 4. Mean temperature, alkalinity, hardness, and conductivity of pre-exposure source water and pooledexposure termination-water samples 12

Table 5. Mean dissolved oxygen, mean temperature, and pH range of exposure water............................. 12

Table 6. Mean total ammonia nitrogen of exposure water by treatment group at 24 hours .......................... 14

Table 7. Mean un-adjusted glochidia viability from selected native freshwater mussels at 6, 12 and 24 hours

Table 8. Mean adjusted glochidia viability from selected native freshwater mussels at 6,12 and 24 hours.... 16 


\section{Conversion Factors}

International System of Units to Inch/Pound

\begin{tabular}{|c|c|c|}
\hline Multiply & By & To obtain \\
\hline \multicolumn{3}{|c|}{ Length } \\
\hline centimeter $(\mathrm{cm})$ & 0.3937 & inch (in.) \\
\hline micrometer $(\mu \mathrm{m})$ & $3.937 \times 10^{-5}$ & inch (in.) \\
\hline millimeter (mm) & 0.03937 & inch (in.) \\
\hline \multicolumn{3}{|c|}{ Volume } \\
\hline liter (L) & 1.057 & quart (qt) \\
\hline microliter $(\mu \mathrm{L})$ & 0.000033814 & ounce, fluid (fl. oz) \\
\hline milliliter $(\mathrm{mL})$ & 0.03382 & ounce, fluid (fl. oz) \\
\hline \multicolumn{3}{|c|}{ Flow rate } \\
\hline milliliter per minute $(\mathrm{mL} / \mathrm{min})$ & 0.0002642 & gallon per minute (gal/min) \\
\hline \multicolumn{3}{|c|}{ Mass } \\
\hline $\operatorname{gram}(\mathrm{g})$ & 0.03527 & ounce, avoirdupois (oz) \\
\hline milligram (mg) & $3.527 \times 10^{-5}$ & ounce, avoirdupois (oz) \\
\hline
\end{tabular}

\section{Abbreviations}

$\begin{array}{ll}\mathrm{Al} & \text { active ingredient } \\ \mathrm{a} & \text { alpha } \\ \mathrm{CaCO}_{3} & \text { calcium carbonate } \\ \mathrm{DO} & \text { dissolved oxygen } \\ \mathrm{FDP} & \text { freeze-dried powder } \\ \mathrm{HD} & \text { heat deactivated } \\ p & p \text {-value } \\ \mathrm{SDP} & \text { spray-dried powder } \\ \text { TAN } & \text { total ammonia nitrogen } \\ \text { USGS } & \text { U.S. Geological Survey }\end{array}$





\title{
Exposure-Related Effects of Formulated Pseudomonas fluorescens Strain CL145A to Glochidia from Seven Unionid Mussel Species
}

By James A. Luoma ${ }^{1}$, Kerry L. Weber ${ }^{1}$, Todd J. Severson ${ }^{1}$, Theresa M. Schreier ${ }^{1}$, Denise A. Mayer², Douglas B. Aloisi $^{3}$, and Nathan L. Eckert ${ }^{3}$

\begin{abstract}
The study was completed to evaluate the exposure-related effects of a biopesticide for dreissenid mussel (Dreissena polymorpha, zebra mussel and Dreissena rostriformis bugensis, quagga mussel) control on glochidia from unionid mussels endemic to the Great Lakes and Upper Mississippi River Basins. The commercially prepared biopesticide was either a spray-dried powder (SDP) or freeze-dried powder (FDP) formulation of Pseudomonas fluorescens, strain CL145A. Glochidia of the unionid mussel species Lampsilis cardium, Lampsilis siliquoidea, Lampsilis higginsii, Ligumia recta, Obovaria olivaria, and Actinonaias ligamentina were exposed to SDP-formulated P. fluorescens and Lampsilis cardium and Megalonaias nervosa were exposed to FDP-formulated P. fluorescens.

All exposures were static, 24 hours in duration, and included six treatment groups. The treatment groups included (1) an untreated control, (2) a positive control which received a nominal target active ingredient (AI) concentration of 300 milligrams per liter $(\mathrm{mg} / \mathrm{L})$ of heat-deactivated test article, and (3) treatments that received nominal target AI concentrations of 50,100, 200, and $300 \mathrm{mg} / \mathrm{L}$ of test article. All treatment concentrations are reported based on active ingredient.

Glochidia viability was reduced in two of the six species exposed to $50 \mathrm{mg} / \mathrm{L} \mathrm{SDP}$ and in four of the six species exposed to $100 \mathrm{mg} / \mathrm{L}$ SDP when compared to untreated control groups at 6,12 , and 24 hours. Regardless of sample time, concentrations of 200 and $300 \mathrm{mg} / \mathrm{L}$ of SDP and $300 \mathrm{mg} / \mathrm{L}$ of heat-deactivated SDP (positive control) substantially reduced glochidia viability in all species except, $L$. higginsii. Glochidia viability was only reduced for $L$. cardium exposed to FDP at concentrations $\geq$ $200 \mathrm{mg} / \mathrm{L}$. After 24 hours of FDP exposure, differences in glochidia viability were only detected in $M$. nervosa that were exposed to $300 \mathrm{mg} / \mathrm{L}$ of heat-deactivated SDP. However, given the low viability in the control group, the results for $M$. nervosa should be interpreted with caution.
\end{abstract}

\section{Introduction}

North America has the greatest diversity of freshwater mussels in the World with historical evidence of approximately 297 taxa consisting of 281 species and 16 subspecies, but because of their sedentary nature, freshwater mussels are particularly vulnerable to anthropogenic influences, such as

\footnotetext{
${ }^{1}$ U.S. Geological Survey

${ }^{2}$ New York State Education Department

${ }^{3}$ U.S. Fish and Wildlife Service
} 
habitat alteration; habitat degradation; pollution and overharvest; and more recently, to impacts from dreissenid mussels (Dreissena polymorpha, zebra mussel and Dreissena rostriformis bugensis, quagga mussel) (Williams and others, 1993, Neves and others, 1997, Strayer and Malcom, 2007). Master and others (1998) listed: 67 percent of freshwater mussels in the United States as extinct or vulnerable to extinction; 95 species of North American freshwater bivalves were listed as vulnerable, endangered, or critically endangered; and 29 were listed as extinct or possibly extinct on the International Union for Conservation of Nature's Red List (http://www.iucnredlist.org/, accessed March 6, 2015). Estimates indicate that 127 mussel species will become extinct in the next 100 years; however, this estimate may be low as it did not factor in impacts from dreissenid mussels (Ricciadi and Rasmussen, 1999). Native mussels in the southeastern United States are in particular danger with only 25 percent of the 269 historical species listed as stable, 13 percent presumed extinct, and 60 percent reported as either endangered, threatened or of special concern (Neves and others, 1997). The negative impact of dreissenid mussels on unionid mussels is well documented in the scientific literature with reports as early as 1937 of extirpation of unionid mussels from European waters due to dreissenid mussel colonization (Sebestyen, 1937; cited in Burlakova and others, 2000). Severe declines in unionid abundance linked to dreissenid colonization in North American waters were reported approximately one decade after dreissenids invaded the United States (Haag and others, 1993; Nalepa, 1994; Ricciardi and others, 1996).

The need to protect and recover imperiled species, such as freshwater mussels, was recognized in the 1973 Endangered Species Act (16 USC 1531-1544, 87 Stat. 884) and the U.S. Fish and Wildlife Service has developed recovery plans for many threatened and endangered unionid mussel species (U.S. Fish and Wildlife Service, 2013; http://www.fws.gov/endangered/species/recovery-plans.html, accessed April 1, 2015). Mitigating the severe impacts of dreissenid mussels on unionid species and the use of population enhancement and reintroduction into historical ranges with propagated animals are commonly cited tasks in unionid recovery plans (U.S. Fish and Wildlife Service, 2004). The U.S. Fish and Wildlife Service mussel propagation program uses wire-mesh rearing cages that are placed in natural waterways for approximately 18 months to rear unionid mussels (Brady and others, 2010). However, some areas previously used for rearing have been abandoned due to dreissenid mussel fouling of the rearing cages. A potential dreissenid mussel management tool for limited, high-value, open-water habitats, such as around unionid mussel rearing cages or mussel beds, is a commercially formulated biopesticide containing a specific strain (CL145A) of the common soil bacterium, Pseudomonas fluorescens as the active ingredient. Currently, the spray-dried powder (SDP) formulation of the biopesticide Zequanox ${ }^{\circledR}$ is manufactured by Marrone Bio Innovations, Inc. (Davis, California). It was registered by the U.S. Environmental Protection Agency (registration number 84059-15) for controlling dreissenid mussels in industrial water systems in 2012 and for open-water system use in 2014. The biopesticide containing $P$. fluorescens is readily ingested by dreissenid mussels, which causes necrosis of epithelial cells lining the digestive tract that results in the death of the dreissenid mussel (Molloy, Mayer, Gaylo, Morse, and others, 2013). The component of the P. fluorescens biopesticide that causes epithelial cell necrosis has been identified as a heat-liable natural metobilite associated with the bacterium's cell wall (Molloy, Mayer, Gaylo, Burlakova, and others 2013).

Freshwater mussels have a unique reproductive strategy that includes a short parasitic lifestage called glochidia. The ability of mature glochidia to attach to a suitable host (typically a fish) is a critical life-cycle step for most freshwater mussels (ASTM International, 2013). Therefore, evaluating the exposure-related effects of water-borne pesticides on freshwater mussel glochidia is prudent.

The objective of this study was to evaluate the exposure-related effects of a commercially formulated $P$. fluorescens, strain CL145A biopesticide on glochidia from select unionid mussel species 
present in the Great Lakes and Upper Mississippi River Basins. This report summarizes the methods and results of laboratory trials in which applications of SDP- or FDP-formulated P. fluorescens biopesticides were administered for 24 hours to static test chambers containing glochidia extracted from female unionid mussels. During the course of this study, the test article manufacturer, Marrone Bio Innovations, Inc., requested a change to a freeze-dried powder (FDP) formulation. However, the FDP formulation was quickly discontinued; the resulting data for the two species exposed to FDP (Lampsilis cardium and Megalonaias nervosa) are included within the report.

\section{Materials and Methods}

The protocol and amendments for this study are presented in appendix 1 (items 1-6). All methods and materials followed the written protocol and amendments except those instances that were identified as deviations (appendix 2, items 1-12). Notes to file provide further documentation on the procedures used during the study (appendix 1, items 7 and 8).

\section{Experimental Design}

The study was completed at the U.S. Geological Survey's Upper Midwest Environmental Sciences Center (La Crosse, Wisconsin). Seven species of unionid mussel glochidia (table 1) were exposed to either the SDP- (six species) or the FDP-formulation (two species) following procedures outlined in "Standard Guide for Conducting Laboratory Toxicity Tests with Freshwater Mussels" (Annex A1 of the ASTM International, 2013). The six species of unionid mussel glochidia exposed to the SDP-formulation were L. cardium, Lampsilis siliquoidea, Lampsilis higginsii, Ligumia recta, Obovaria olivaria, and Actinonaias ligamentina. The two species of unionid mussel glochidia exposed to the FDP-formulation were Lampsilis cardium and M. nervosa. For each species, glochidia were flushed from 3-4 gravid female mussels and viability was estimated to be over 80 percent before the glochidia were used in the study. Consistent with the Standard Guide for Conducting Laboratory Toxicity Tests with Freshwater Mussels, glochidia that closed their valves in response to sodium chloride exposure were considered viable (ASTM International, 2013). Photomicrographs were recorded before and after the addition of the sodium chloride solution and used for definitive determination of initial glochidia viability (table 2). Glochidia were pooled and distributed to test chambers according to a predetermined random distribution scheme (appendix 3, items 4-6). An estimated 843-4,602 glochidia were distributed to each test chamber (table 3).

Each species was assigned to one of three test block locations and treatments were assigned to test chambers using randomized block designs (appendix 3, items 1-3 and 7-9, respectively). Six treatments ( $n=3$ per treatment) were tested and included (1) an untreated control, (2) nominal target concentrations of 50, 100, 200, and 300 milligrams per liter $(\mathrm{mg} / \mathrm{L})$ of test article based on active ingredient (AI), and (3) a nominal target concentration of $300 \mathrm{mg} / \mathrm{L}$ AI of heat-deactivated test article group (300 HD, positive control). Exposures were static and lasted 24 hours. Glochidia from each test chamber were assessed for viability at 6,12 and 24 hours, with the exception of $M$. nervosa glochidia, which were only assessed at 24 hours.

\section{Test Article}

The test articles used in the study were commercially prepared SDP or FDP formulated $P$. fluorescens (strain CL145A) biopesticides containing 50 and 100 percent AI (weight-to-weight ratio $P$. fluorescens, strain CL145A cells), respectively. The test articles were produced by Marrone Bio 
Innovations, Inc. and test article use was documented in log books (appendix 4, items 4-6). All test article concentrations are reported as active ingredient. Verification of biological activity (the ability of the test article to induce dreissenid mussel mortality) was determined for each test article lot after use in the study by the New York State Museum Field Research Laboratory (Cambridge, New York). Biological activity for each lot of test article was confirmed as indicated by mean zebra mussel mortality ranging from 85.3 to 94.7 percent in the test article treated groups compared to 0.0 to 1.3 percent in the untreated control groups (table 1; appendix 4, items 7-9).

Table 1. Data for test animals, test articles and, exposure dates for studies of selected native freshwater mussel glochidia exposed to two formulations of Pseudomonas fluorescens, strain CL145A.

[PPB, plain pocketbook; WAS, washboard; BLS, black sandshell; FAM, fatmucket; HGE, Higgins eye; HIC, hickorynut; MUC, mucket; FDP, freeze-dried powder; SDP, spray-dried powder; MBI, Marrone Bio Innovations, Inc.]

\begin{tabular}{|c|c|c|c|c|c|c|}
\hline \multirow[b]{2}{*}{ Scientific name } & \multirow[b]{2}{*}{ Common name } & \multirow[b]{2}{*}{ Abbreviation } & \multicolumn{3}{|c|}{ Test article } & \multirow[b]{2}{*}{ Exposure date } \\
\hline & & & Formulation & Lot number & $\begin{array}{l}\text { Biological } \\
\text { activity }{ }^{1} \\
\text { (percent) }\end{array}$ & \\
\hline $\begin{array}{l}\text { Lampsilis } \\
\quad \text { cardium }\end{array}$ & Plain pocketbook & РPB & FDP & 110607WB-FD-E & $94.7 \pm 6.1$ & October 18, 2011 \\
\hline $\begin{array}{l}\text { Megalonaias } \\
\text { nervosa }\end{array}$ & Washboard & WAS & FDP & 110607WB-FD-E & $94.7 \pm 6.1$ & October 18, 2011 \\
\hline $\begin{array}{l}\text { Ligumia } \\
\text { recta }\end{array}$ & Black sandshell & BLS & SDP & MBI-401-SDP-4655-12-MIX & $85.3 \pm 11.5$ & January 17, 2012 \\
\hline $\begin{array}{l}\text { Lampsilis } \\
\quad \text { siliquoidea }\end{array}$ & Fatmucket & FAM & SDP & MBI-401 110308AI-BD-3 & $86.7 \pm 4.6$ & May 12, 2011 \\
\hline $\begin{array}{l}\text { Lampsilis } \\
\quad \text { higginsii }\end{array}$ & Higgins eye & HGE & SDP & MBI-401-110308AI-BD-3 & $86.7 \pm 4.6$ & May 12, 2011 \\
\hline $\begin{array}{l}\text { Obovaria } \\
\text { olivaria }\end{array}$ & Hickorynut & $\mathrm{HIC}$ & SDP & MBI-401-SDP-4655-12-MIX & $85.3 \pm 11.5$ & January 19, 2012 \\
\hline $\begin{array}{l}\text { Actinonaias } \\
\quad \text { ligamentina }\end{array}$ & Mucket & MUC & SDP & MBI-401-SDP-4655-12-MIX & $85.3 \pm 11.5$ & January 17, 2012 \\
\hline $\begin{array}{l}\text { Lampsilis } \\
\quad \text { cardium }\end{array}$ & Plain pocketbook & PPB & SDP & MBI-401 110308AI-BD-3 & $86.7 \pm 4.6$ & May 12, 2011 \\
\hline
\end{tabular}

${ }^{1}$ Biological activity is the mean percent zebra mussel mortality in test article treated groups during a bioassay performed by the New York State Museum Field Research Laboratory (Cambridge, New York).

\section{Test system}

The test system was a series of glass test chambers $(80 \times 40$ millimeters [mm], width $\times$ height; catalog number 89000-286; VWR International, West Chester, Pennsylvania) arranged in three blocks. Each block contained 3 rows with 6 test chambers, for a total of 18 test chambers per species (fig. 1). Test chambers were labelled to allow for identification of treatment type and replicate number. During the exposure, light aeration (1-2 bubbles per second) was provided to each test chamber through a disposable glass pipet. Test water was well water conditioned to ambient temperature $\left(\approx 20{ }^{\circ} \mathrm{C}\right)$ and dissolved-gas saturation by providing gentle aeration in 19-liter (L) glass jars for a minimum of 24 hours before use. Indirect fluorescent lighting (mean of 108 to 536 lux) was provided on an 18 hours light to 6 hours dark cycle in accordance with ASTM International guidelines (ASTM International, 2013). 
Table 2. Data for initial glochidia viability by individual female mussel for each species. [Viability of glochidia by individual female mussel and species during exposures of selected native freshwater mussel glochidia to two formulations of Pseudomonas fluorescens, strain CL145A. SD, standard deviation — number in parentheses; SDP, spray-dried powder; FDP, freeze-dried powder]

\begin{tabular}{|c|c|c|c|c|c|c|}
\hline Species & Formulation & $\begin{array}{l}\text { Mussel } \\
\text { number }\end{array}$ & $\begin{array}{c}\text { Total } \\
\text { glochidia }\end{array}$ & $\begin{array}{l}\text { Nonviable } \\
\text { glochidia }^{1}\end{array}$ & $\begin{array}{l}\text { Glochidia } \\
\text { viability } \\
\text { (percent) }\end{array}$ & $\begin{array}{l}\text { Mean percent } \\
\text { glocihidia } \\
\text { viability } \\
\text { (SD) }\end{array}$ \\
\hline \multirow[t]{3}{*}{ Lampsilis cardium } & FDP & 1 & 212 & 16 & 92.5 & $84.7(6.9)$ \\
\hline & & 2 & 222 & 39 & 82.4 & \\
\hline & & 3 & 150 & 31 & 79.3 & \\
\hline \multirow[t]{4}{*}{ Megalonaias nervosa } & FDP & 1 & 124 & 2 & 98.4 & $96.5(5.5)$ \\
\hline & & 2 & 155 & 1 & 99.4 & \\
\hline & & 3 & 51 & 5 & 90.2 & \\
\hline & & 4 & 104 & 0 & 100.0 & \\
\hline \multirow[t]{3}{*}{ Ligumia recta } & SDP & 1 & 312 & 32 & 89.7 & $84.5(6.7)$ \\
\hline & & 2 & 1,083 & 250 & 76.9 & \\
\hline & & 3 & 405 & 53 & 86.9 & \\
\hline \multirow[t]{3}{*}{ Lampsilis siliquoidea } & SDP & 1 & 548 & 70 & 87.2 & $80.3(7.1)$ \\
\hline & & 2 & 297 & 80 & 73.1 & \\
\hline & & 3 & 456 & 89 & 80.5 & \\
\hline \multirow[t]{3}{*}{ Lampsilis higginsii } & SDP & 1 & 129 & 49 & 62.0 & $80.8(16.5)$ \\
\hline & & 2 & 168 & 20 & 88.1 & \\
\hline & & 3 & 357 & 27 & 92.4 & \\
\hline \multirow[t]{3}{*}{ Obovaria olivaria } & SDP & 1 & 366 & 54 & 85.2 & $86.0(1.2)$ \\
\hline & & 2 & 352 & 51 & 85.5 & \\
\hline & & 3 & 309 & 39 & 87.4 & \\
\hline \multirow[t]{3}{*}{ Actinonaias ligamentina } & SDP & 1 & 161 & 14 & 91.3 & $92.7(1.4)$ \\
\hline & & 2 & 153 & 9 & 94.1 & \\
\hline & & 3 & 287 & 21 & 92.7 & \\
\hline \multirow[t]{3}{*}{ Lampsilis cardium } & SDP & 1 & 467 & 36 & 92.3 & $93.8(1.4)$ \\
\hline & & 2 & 537 & 27 & 95.0 & \\
\hline & & 3 & 927 & 55 & 94.1 & \\
\hline
\end{tabular}


Table 3. Estimated number of glochidia distributed to test chambers.

[Distributions to test chambers by species during exposures of selected native freshwater mussel glochidia to two formulations of Pseudomonas fluorescens, strain CL145A. SD, standard deviation - number in parentheses; SDP, spray-dried powder; FDP, freeze-dried powder]

\begin{tabular}{lcc}
\hline Species & Formulation & $\begin{array}{c}\text { Estimated } \\
\text { number (SD) } \\
\text { of glochidia per test chamber }\end{array}$ \\
\hline Lampsilis cardium & FDP & $4,602(1,225)$ \\
Megalonaias nervosa & FDP & $843(382)$ \\
Ligumia recta & SDP & $2,021(500)$ \\
Lampsilis siliquoidea & SDP & $2,702(1,094)$ \\
Lampsilis higginsii & SDP & $3,427(1,018)$ \\
Obovaria olivaria & SDP & $4,187(2,503)$ \\
Actinonaias ligamentina & SDP & $2,200(1,061)$ \\
Lampsilis cardium & SDP & $3,355(1,077)$ \\
\hline
\end{tabular}

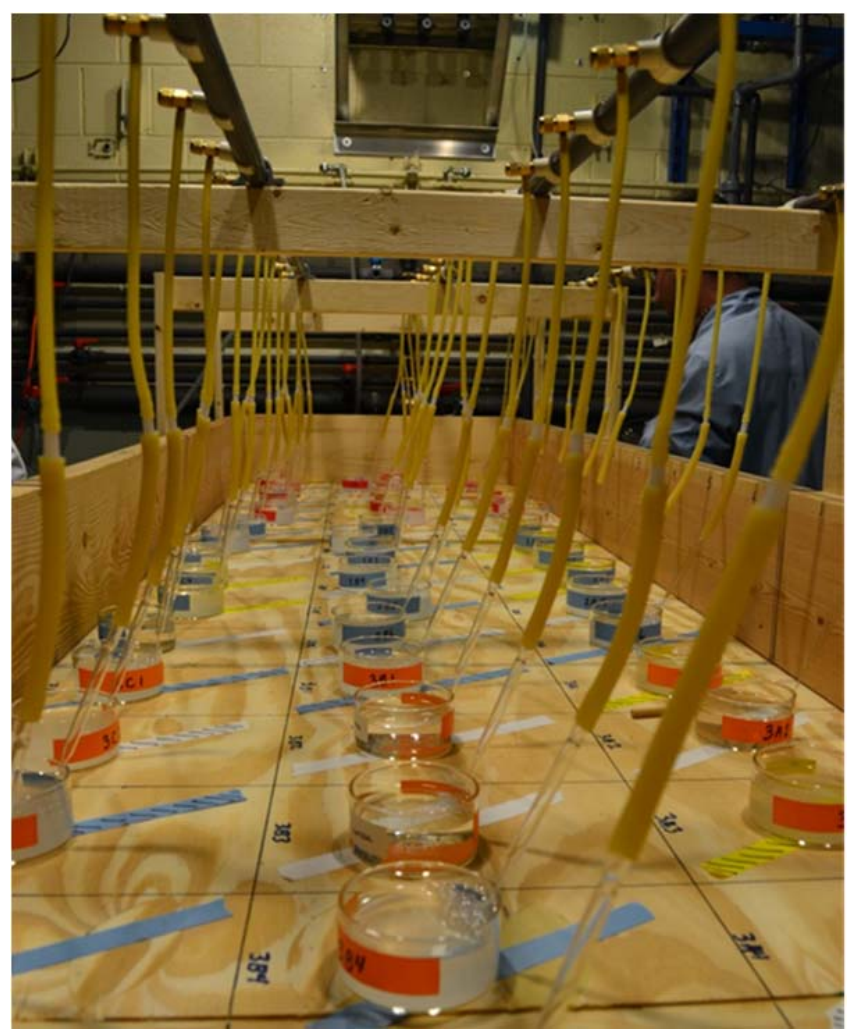

Figure 1. Photograph showing glochidia test system with individual test chambers. 


\section{Animal Collection and Distribution}

Test animal information is presented in appendix 5, items 1-8. Gravid female mussels were collected from the Upper Mississippi, Chippewa, and St. Croix Rivers and identified to species as described in Cummings and Mayer (1992) and Watters and others (2009) by biologists from the Genoa National Fish Hatchery (Genoa, Wis.). Gravid mussels were maintained at the Genoa National Fish Hatchery on flowing pond water for up to 120 days before being transported to the Upper Midwest Environmental Sciences Center for glochidia extraction. Glochidia were extracted from the gravid female mussels by gently opening the valves and using a syringe to flush the marsupium with water (fig. 2). Glochidia from 6-10 marsupial tubes were flushed into a 500-milliliter (mL) beaker and viability of the glochidia was estimated to be $>80$ percent by observation under a microscope before pooling. Glochidia viability was defined as valve closure in response to the addition of a drop of saturated sodium chloride solution (fig. 3). Glochidia from 3-4 mussels of each species were pooled in a 500-mL beaker containing water. Definitive initial viability of glochidia extracted from each female mussel was determined from photomicrographs after exposures were completed (table 2). The beaker containing the pooled glochidia was gently swirled to position the glochidia in a concentrated group at the bottom of the beaker. Glochidia were then indiscriminately collected by placing a 1,000-microliter $(\mu \mathrm{L})$ wide bore pipet tip (model T-1005-WB-C-R; Axygen Scientific, Union City, California) within the concentrated glochidia and withdrawing a $100-\mu \mathrm{L}$ sample with an adjustable pipette (model research plus 100-1,000 $\mu \mathrm{L}$; Eppendorf USA, Hauppauge, N.Y.). The glochidia were then placed into a randomly selected test chamber that contained $96.5 \mathrm{~mL}$ of water (fig. 4). After all test chambers had received glochidia, the distribution process was repeated until all test chambers received five independent glochidia distributions (appendix 3, items 4-6). Throughout the distribution process samples $(100 \mu \mathrm{L}, n=16$ per species) containing glochidia were placed into a $35-\mathrm{mm}$ petri dish and used to estimate the total number of glochidia distributed to the test chambers. Each treatment group contained three test chamber replicates with an estimated range of 843 to 4,602 glochidia per chamber (table 3). The volume of water within the test chambers after glochidia distribution and before test article administration was $97.0 \mathrm{~mL}$.

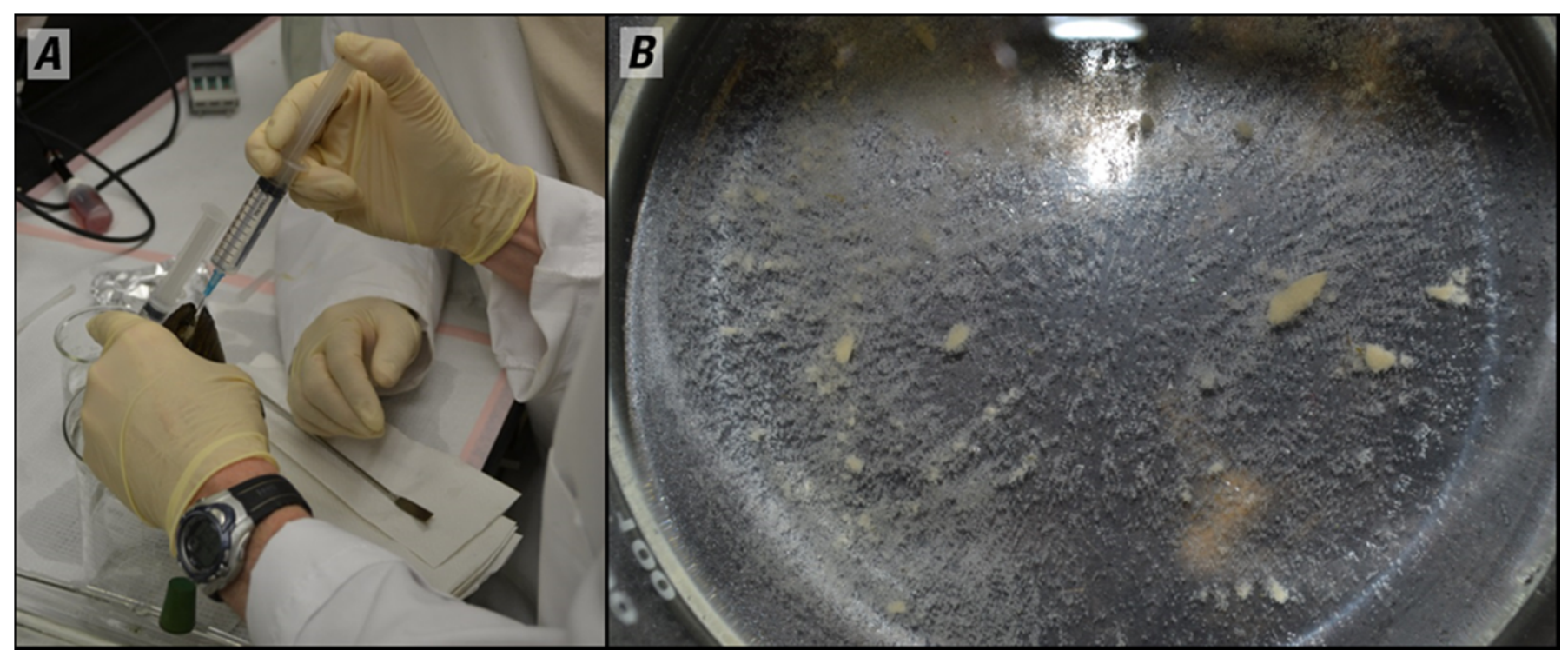

Figure 2. Photographs showing $A$, the extraction of glochidia from a gravid female mussel and, $B$, extracted glochidia. 


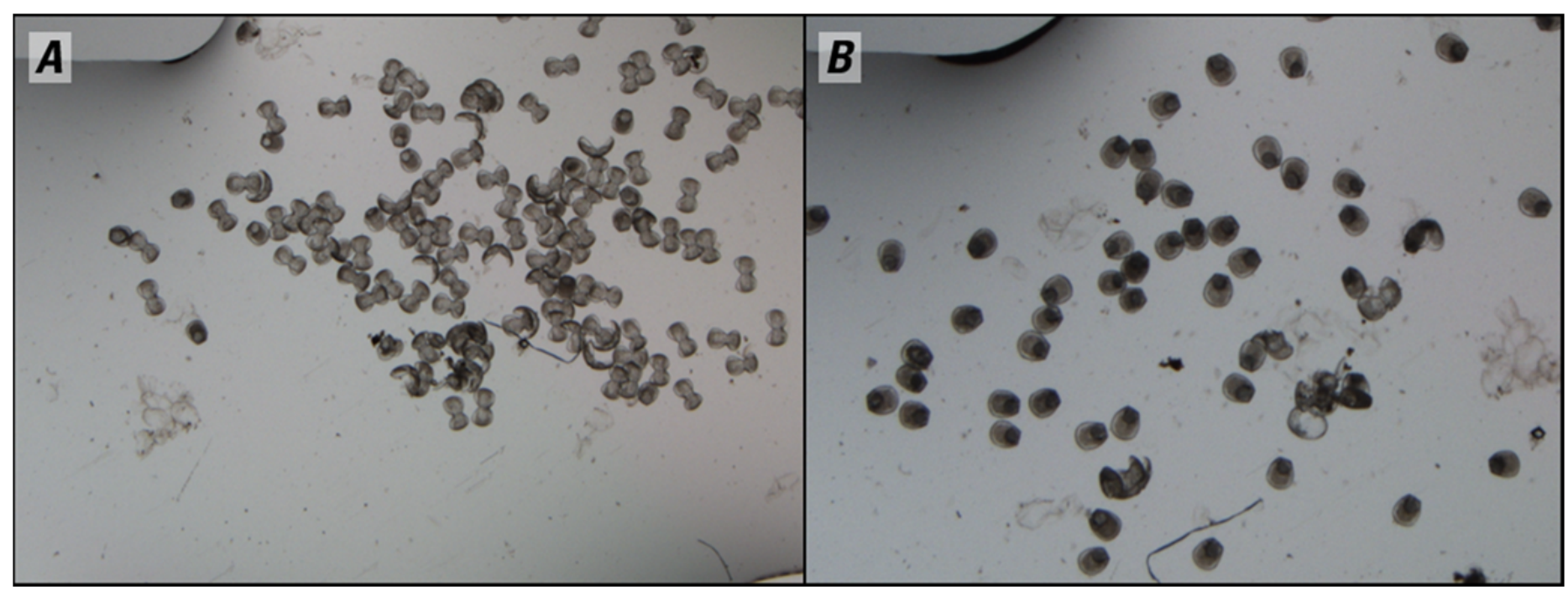

Figure 3. Photomicrographs showing $A$, glochidia before a drop of sodium chloride solution was added to induce valve closure in viable animals and, $B$, glochidia one minute after addition of the sodium chloride solution.

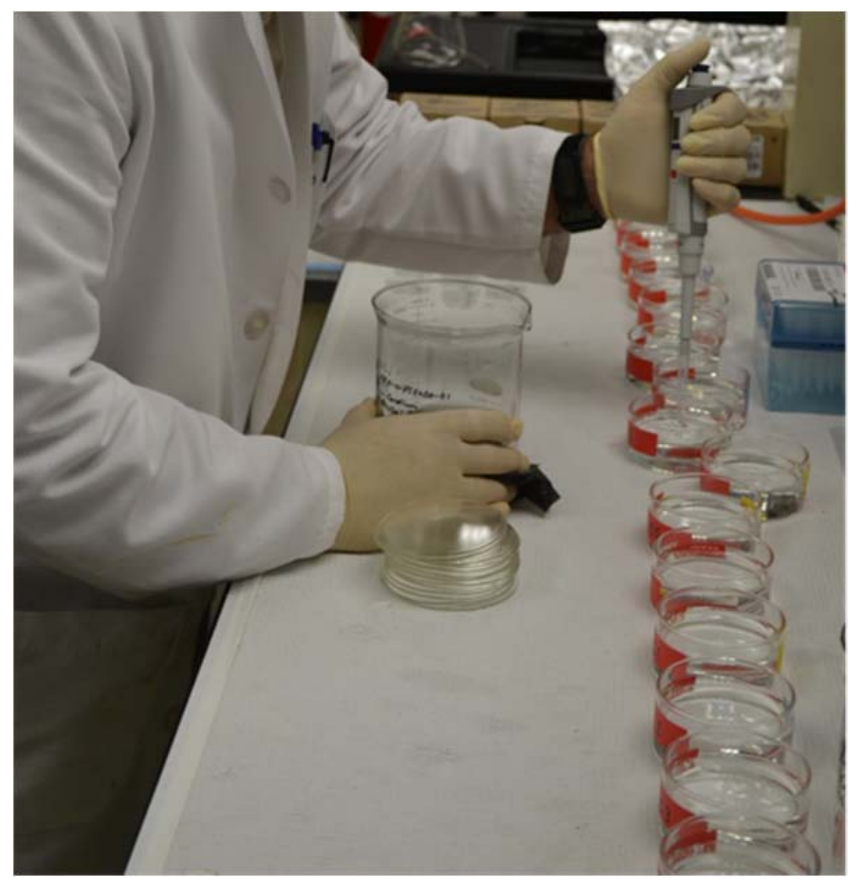

Figure 4. Photograph showing the distribution of glochidia to test chambers.

\section{Test Article Preparation and Application}

For each species, a separate test article stock solution was prepared for the 50, 100, 200 and $300 \mathrm{mg} / \mathrm{L}$ treatment groups immediately prior to application. A single heat-deactivated test article solution was prepared for each treatment day and used for all applications of heat-deactivated stock solution (positive control) that were completed on the same day. Untreated water was used as the test article stock solution for the untreated control treatment. The stock solutions were prepared by weighing a known amount of test article (1.0 grams FDP; 2.0 grams SDP) on an analytical balance and then 
placing the test article into a 100-mL flask with water, which resulted in an AI stock solution of $10,000 \mathrm{mg} / \mathrm{L}$. The heat-deactivated stock was prepared by placing the test article into a $100-\mathrm{mL}$ volumetric flask with approximately $50 \mathrm{~mL}$ of water, then the flask was placed into a 70 degrees Celsius $\left({ }^{\circ} \mathrm{C}\right)$ water bath for 45 minutes to degrade the activity of the P. fluorescens according to methods developed at the New York State Museum Field Research Laboratory (D. Mayer, Director of the New York State Museum Field Research Laboratory, oral commun., 2010). After cooling to ambient temperature, the heat-deactivated stock solution was brought to a final $100 \mathrm{~mL}$ volume with water.

Each test chamber received (1) $3.0 \mathrm{~mL}$ of untreated water (control), (2) $3.0 \mathrm{~mL}$ of heatdeactivated stock solution (positive control), (3) $3.0 \mathrm{~mL}$ of stock solution (300 mg/L treatment), or (4) $3.0 \mathrm{~mL}$ of the appropriate combination of untreated water and stock solution $(50,100$ and $200 \mathrm{mg} / \mathrm{L}$ treatments [appendix 1, items 20 and 24]). The final volume in each test chamber after application of the test article was $100 \mathrm{~mL}$.

\section{Water Chemistry}

Prior to exposure, temperature, hardness, and alkalinity were measured on the source water for all trials. Conductivity was measured on the source water for all trials, excluding those completed in May 2011 (L. cardium, L. siliquoidea, and L .higginsii SDP exposures; table 1). For the trials completed in May 2011 the water chemistry parameters measured during the exposure included duplicate measurements of dissolved oxygen (DO), $\mathrm{pH}$, and temperature on all control and high test article concentration $(300 \mathrm{mg} / \mathrm{L})$ test chambers and on one indiscriminately selected test chamber from the remaining treatment groups. For all other trials, the water chemistry parameters measured during the exposure included duplicate measurements of $\mathrm{DO}, \mathrm{pH}$, and temperature in all test chambers. At the termination of the exposure, the contents of each test chamber were pooled by treatment group and the hardness, alkalinity, conductivity, temperature, $\mathrm{pH}$, and total ammonia nitrogen (TAN) were measured on the pooled replicates. The TAN was measured using a YSI 9000 photometer (YSI, Inc., Yellow Springs, Ohio; L. cardium, L. siliquoidea, and L. higginsii SDP exposures) or using the automated phenate method (Standard Method 4500G in American Public Health Association and others, 2012; $L$. recta, O. olivaria, and A. ligamentina SDP exposures; L. cardium and M. nervosa FDP exposures).

\section{Glochidia Viability Assessments}

Definitive initial viability determinations for glochidia extracted from each female mussel and the viability of glochidia in samples collected during the exposures were completed from photomicrographs using equation 1:

$$
\begin{aligned}
& \text { Viability }(\text { percent })=\frac{\left(\mathrm{N}-\left[\mathrm{NC}_{\text {pre }}+\mathrm{NO}_{\text {post }}\right]\right)}{\mathrm{N}} \times 100 \\
& \text { where } \quad N \quad \text { is the total number of glochidia in the sample, } \\
& N C_{\text {pre }} \quad \text { is the number of glochidia closed before adding sodium chloride, and } \\
& N O_{\text {post }} \text { is the number of glochidia open after adding sodium chloride. }
\end{aligned}
$$

During the exposure, glochidia from each test chamber were assessed for viability at 6,12 , and 24 hours, with the exception of the M. nerovosa glochidia, which were assessed only at 24 hours. Water within each test chamber was gently swirled to position the glochidia in a concentrated group centered at the bottom exposure chamber. Then a 100- $\mu \mathrm{L}$ sample was withdrawn from each exposure chamber using a $1,000-\mu \mathrm{L}$ wide-bore pipet tip attached to an adjustable pipette. For each species, excluding $M$. 
nerovosa, the glochidia samples were placed into a 35- $\mathrm{mm}$ petri dish, viewed, and photomicrographed using a Nikon model SMZ 1500 compound stereo microscope fitted with a Nikon digital sight DS-Fi1 camera controlled by Nikon Imaging Systems Elements-BR ${ }^{\circledR}$ software, version 3.10 (Nikon Imaging systems, 1991-2010). Photomicrographs were recorded before and after a drop of saturated sodium chloride solution was added to induce valve closure in viable glochidia. The second photomicrograph was recorded one minute after sodium chloride addition to provide a standard valve closure response time. Glochidia on each photomicrograph were enumerated by valve position (either open or closed) and the percentage of viable glochidia was calculated by using equation 1. Megalonaias nervosa glochidia are released in a mucoidal matrix, which precluded the ability to obtain an image of the glochidia on a single focal plane. Therefore, $M$. nervosa glochidia were immediately assessed for viability by placing the glochidia under a dissecting microscope (model SMZ745; Nikon Instruments) and enumerating, by valve position, before and after a after a drop of saturated sodium chloride solution was added to induce valve closure in viable glochidia.

\section{Data Analysis}

Water chemistry (DO, $\mathrm{pH}$, temperature, hardness, alkalinity, conductivity, and TAN) data analyses were limited to simple descriptive statistics (appendix 6, items 1, 2, 4, and 7). In order to compensate for initial glochidia viabilities of less than 100 percent, the observed viabilities of glochidia during the exposure were adjusted with the initial viability of glochidia using the methods described by Wang and others (2007). Viability adjustments were completed using equation 2:

Adjusted viability $=\frac{\left(\mathrm{V}_{0}\right)}{\left(\mathrm{V}_{\mathrm{l}}\right)} \times 100$

where
$\mathrm{V}_{\mathrm{O}}$
is the observed (un-adjusted) viability of a treatment replicate (appendix 7, items 5-7), and
$\mathrm{V}_{\mathrm{I}}$
is the initial mean viability observed for the species, in percent (table 3).

For example, the mean initial viability of $A$. ligamentina glochidia was 92.7 percent. The observed (unadjusted) viability of $A$. ligamentina glochidia in a control group replicate, at 24 hours, was 90.0 percent, therefore, the adjusted viability of $A$. ligamentina glochidia in the control group replicate, at 24 hours, is $90 / 92.7 \times 100$ or 97.1 percent.

Statistical comparisons were completed with the observed (un-adjusted) glochidia viability of each treatment group replicate using SAS $^{\circledR}$ software version 9.3 (SAS, 2010). Significance was declared at $\alpha \leq 0.05$. A generalized linear mixed model was used to analyze the viability of glochidia by treatment group and species (appendix 7, item 2). The proportion of mortalities (number of nonviable glochidia compared to the total number of glochidia present) were modeled with a binomial distribution and a logit link function. A scale parameter was added to the model using the "random_residual_" statement. At each sampling time, glochidia viability in each treatment group was individually compared to the viability in the untreated control group using a two-sided means comparison test.

\section{Results and Discussion}

Pre-exposure water chemistry parameters (temperature, alkalinity, hardness, and conductivity) of the source water are summarized in table 4 and are presented in appendix 6 (item 1). Pre-exposure temperature ranged from 18.4 to $19.9^{\circ} \mathrm{C}$; hardness ranged from 171 to $175 \mathrm{mg} / \mathrm{L}$ as calcium carbonate $\left(\mathrm{CaCO}_{3}\right)$; alkalinity ranged from 122 to $130 \mathrm{mg} / \mathrm{L}$ as $\mathrm{CaCO}_{3}$; and conductivity ranged from 383 to 
422 microsiemens per centimeter $(\mu \mathrm{S} / \mathrm{cm})$. Water chemistry parameters ( $\mathrm{DO}, \mathrm{pH}$, and temperature) measured during the exposure are presented in table 5 and in appendix 6 (item 2). Dissolved oxygen remained well above the ASTM International criterion of $4 \mathrm{mg} / \mathrm{L}$ throughout the exposure period (ASTM International, 2013). The $\mathrm{pH}$ of exposure water ranged from 7.52 to 8.56 and the temperature ranged from 17.2 to $20.7^{\circ} \mathrm{C}$. The pooled replicate exposure water samples collected at exposure termination had mean temperatures from 18.2 to $20.3{ }^{\circ} \mathrm{C}$, hardness from 180 to $210 \mathrm{mg} / \mathrm{L}$ as $\mathrm{CaCO}_{3}$, alkalinity from 137 to $153 \mathrm{mg} / \mathrm{L}$ as $\mathrm{CaCO}_{3}$, and conductivity from 367 to $457 \mu \mathrm{S} / \mathrm{cm}$ (table 4; appendix 6, item 4). The mean TAN from samples collected at the termination of the exposure period are presented in table 6 and appendix 6 (items 5-7). The TAN remained below the 2013

U.S. Environmental Protection Agency criterion for acute exposure $\left(17 \mathrm{mg} / \mathrm{L}\right.$ at pH 7 and $\left.20{ }^{\circ} \mathrm{C}\right)$ throughout the entire exposure period for all species and treatment groups (U.S. Environmental Agency, 2013).

The initial viability of glochidia extracted for each female mussel ranged from 62 to 100 percent, with glochidia extracted from four individuals below the 80 percent viability recommended in the "Standard Guide for Conducting Laboratory Toxicity Tests with Freshwater Mussels" (ASTM International, 2013). However, the mean viability of glochidia extracted from the female mussels for each species ranged from 80.3 to 96.5 percent (table 2, appendix 7, item 1). Statistical analyses of the un-adjusted glochidia viability are presented in appendix 7 (item 4) and the un-adjusted-mean glochidia viability of each treatment group are presented by species in table 7 . The mean of the adjusted viability of each treatment group are presented by species in table 8 and in appendix 7 (item 5-7).

Regardless of species, the mean of the un-adjusted and adjusted control group viability during the SDP trials exceeded 66 and 82 percent, respectively, throughout the study. After 6 hours of SDP exposure at $50 \mathrm{mg} / \mathrm{L}$, the un-adjusted-mean viabilities of L. recta and A. ligamentina glochidia were significantly lower (11.7 percent $[p<0.01]$ and 71.7 percent $[p=0.04]$, respectively) than the untreated control groups (85.7 and 88.6 percent, respectively). Concentrations of SDP $\geq 100 \mathrm{mg} / \mathrm{L}$ for 6 hours significantly lowered glochidia viability for all species, except for L. cardium and L. higginsii, which were impacted at concentrations $\geq 200$ and $300 \mathrm{mg} / \mathrm{L}$, respectively. The observed lower viability for O. olivaria at 6 hours in the $100 \mathrm{mg} / \mathrm{L}$ SDP-treated group is likely an outlier, given no differences were detected at 12 and 24 hours in the viability of glochidia in the $100 \mathrm{mg} / \mathrm{L}$ SDP-treated group compared to the control group ( $p=0.16$ and 0.15 , respectively).

The mean for the un-adjusted and adjusted control group viability for L. cardium during the FDP trial exceeded 81 and 96 percent, respectively. The un-adjusted- and adjusted-mean control group glochidia viability for M. nervosa at 24 hours was 52.1 and 53.9 percent, respectively. During the L. cardium FDP-trial, glochidia exposed to FDP concentrations $\geq 200 \mathrm{mg} / \mathrm{L}$ were significantly less viable than those in the untreated control group, regardless of sample time. After 24 hours of exposure, no difference was detected between the viability of $M$. nervosa glochidia in the FDP-treated groups compared to the control group, with the exception of the positive control group. However, given the low viability observed in the $M$. nervosa untreated controls (un-adjusted viability of 52.1 percent) the results should be interpreted with caution. 
Table 4. Mean temperature, alkalinity, hardness, and conductivity of pre-exposure source water and pooledexposure termination-water samples.

[Samples were collected before and during exposures of selected native freshwater mussel glochidia to two formulations of Pseudomonas fluorescens, strain CL145A. ${ }^{\circ} \mathrm{C}$, degrees Celsius; mg/L, milligrams per liter; Cond., conductivity; $\mu \mathrm{S} / \mathrm{cm}$, microsiemens per centimeter at 25 degrees Celsius; SDP, spray-dried powder; FDP, freeze-dried powder; number in parentheses, standard deviation; --, no data collected or cannot calculate]

\begin{tabular}{|c|c|c|c|c|c|c|c|c|c|}
\hline \multirow[b]{2}{*}{ Species } & \multirow[b]{2}{*}{ Formulation } & \multicolumn{4}{|c|}{ Preexposure } & \multicolumn{4}{|c|}{ Exposure termination } \\
\hline & & $\begin{array}{c}\text { Temperature } \\
\left({ }^{\circ} \mathrm{C}\right)\end{array}$ & $\begin{array}{l}\text { Alkalinity } \\
(\mathrm{mg} / \mathrm{L})^{1}\end{array}$ & $\begin{array}{l}\text { Hardness } \\
(\mathrm{mg} / \mathrm{L})^{1}\end{array}$ & $\begin{array}{l}\text { Cond. } \\
(\mu \mathrm{S} / \mathrm{cm})\end{array}$ & $\begin{array}{c}\text { Temperature } \\
\left({ }^{\circ} \mathrm{C}\right)\end{array}$ & $\begin{array}{l}\text { Alkalinity } \\
(\mathrm{mg} / \mathrm{L})^{1}\end{array}$ & $\begin{array}{l}\text { Hardness } \\
(\mathrm{mg} / \mathrm{L})^{1}\end{array}$ & $\begin{array}{l}\text { Cond. } \\
(\mu \mathrm{S} / \mathrm{cm})\end{array}$ \\
\hline $\begin{array}{l}\text { Lampsilis } \\
\quad \text { cardium }\end{array}$ & FDP & $18.4(0.0)$ & $126(1)$ & $174(0)$ & 391 (--) & $18.2(0.2)$ & $144(3)$ & $196(4)$ & $431(12)$ \\
\hline $\begin{array}{l}\text { Megalonaias } \\
\text { nervosa }\end{array}$ & FDP & $18.4(0.0)$ & $126(1)$ & $174(0)$ & $391(--)$ & $18.7(0.3)$ & $148(5)$ & $201(2)$ & $440(18)$ \\
\hline $\begin{array}{l}\text { Lampsilis } \\
\quad \text { siliquoidea }\end{array}$ & SDP & $19.3(0.1)$ & $130(0)$ & $171(1)$ & -- & $19.9(0.1)$ & $137(2)$ & $181(2)$ & $367(13)$ \\
\hline $\begin{array}{l}\text { Lampsilis } \\
\quad \text { higginsii }\end{array}$ & SDP & $19.3(0.1)$ & $130(0)$ & $171(1)$ & -- & $19.5(0.1)$ & $142(4)$ & $185(3)$ & $387(17)$ \\
\hline $\begin{array}{l}\text { Obovaria } \\
\quad \text { olivaria }\end{array}$ & SDP & $19.9(0.2)$ & $122(1)$ & $175(1)$ & $383(2)$ & $19.7(0.7)$ & $147(7)$ & $210(4)$ & $457(27)$ \\
\hline
\end{tabular}

${ }^{1}$ Alkalinity and hardness reported as milligrams per liter of calcium carbonate.

Table 5. Mean dissolved oxygen, mean temperature, and $\mathrm{pH}$ range of exposure water. [Values are by treatment group, during exposures of selected native freshwater mussel glochidia to two formulations of Pseudomonas fluorescens, strain CL145A. mg/L, milligrams per liter; $\mathrm{pH}$, reported as standard units; ${ }^{\circ} \mathrm{C}$, degrees Celsius; SDP, spray-dried powder; number in parentheses, standard deviation; HD, heat-deactivated test article, FDP, freeze-dried powder]

\begin{tabular}{cccc}
\hline $\begin{array}{c}\text { Treatment } \\
\text { group }\end{array}$ & $\begin{array}{c}\text { Dissolved oxygen } \\
(\mathbf{m g} / \mathbf{L})\end{array}$ & $\begin{array}{c}\text { Temperature } \\
\left({ }^{\circ} \mathrm{C}\right)\end{array}$ & pH \\
\hline Lampsilis cardium, FDP formulation & \\
\hline 50 & $8.34(0.51)$ & $17.8(0.4)$ & $8.12-8.32$ \\
100 & $8.13(0.54)$ & $17.2(0.5)$ & $8.26-8.31$ \\
200 & $8.08(0.81)$ & $17.5(0.3)$ & $8.31-8.34$ \\
300 & $7.92(0.63)$ & $17.4(0.50$ & $8.31-8.34$ \\
$300 \mathrm{HD}$ & $7.81(0.62)$ & $17.6(0.5)$ & $8.29-8.33$ \\
& $7.19(0.89)$ & $17.7(0.4)$ & $8.31-8.36$ \\
\hline Control & Megalonaias nervosa, FDP formulation & $8.39-8.41$ \\
50 & $7.43(0.19)$ & $18.6(0.2)$ & $8.39-8.40$ \\
100 & $7.64(0.64)$ & $17.8(0.4)$ & $8.39-8.41$ \\
200 & $8.39(0.79)$ & $18.3(0.4)$ & $8.35-8.49$
\end{tabular}




\begin{tabular}{|c|c|c|c|}
\hline $\begin{array}{l}\text { Treatment } \\
\text { group }\end{array}$ & $\begin{array}{l}\text { Dissolved oxygen } \\
\quad(\mathrm{mg} / \mathrm{L})\end{array}$ & $\begin{array}{c}\text { Temperature } \\
\left({ }^{\circ} \mathrm{C}\right)\end{array}$ & pH \\
\hline 300 & $7.87(0.53)$ & $18.1(0.3)$ & $8.35-8.39$ \\
\hline $300 \mathrm{HD}$ & $7.84(0.68)$ & $18.4(0.2)$ & $8.37-8.40$ \\
\hline \multicolumn{4}{|c|}{ Ligumia recta, SDP formulation } \\
\hline Control & $9.17(0.31)$ & $20.4(0.4)$ & $8.27-8.56$ \\
\hline 50 & $9.36(0.48)$ & $20.7(0.7)$ & $8.34-8.44$ \\
\hline 100 & $9.59(0.94)$ & $20.5(0.4)$ & $8.32-8.38$ \\
\hline 200 & $8.72(0.57)$ & $20.2(0.3)$ & $8.21-8.37$ \\
\hline 300 & $8.75(1.09)$ & $20.6(0.4)$ & $8.15-8.31$ \\
\hline $300 \mathrm{HD}$ & $9.29(0.88)$ & $19.6(0.9)$ & $8.27-8.50$ \\
\hline \multicolumn{4}{|c|}{ Lampsilis siliquoidea, SDP formulation } \\
\hline Control & $8.59(0.28)$ & $19.4(0.4)$ & $8.39-8.48$ \\
\hline 50 & $7.71(0.57)$ & $19.3(0.4)$ & $8.05-8.36$ \\
\hline 100 & $7.58(0.81)$ & $19.5(0.8)$ & $8.09-8.35$ \\
\hline 200 & $7.31(1.82)$ & $19.5(0.6)$ & $7.73-8.26$ \\
\hline 300 & $6.92(2.09)$ & $19.7(0.7)$ & $7.52-8.37$ \\
\hline $300 \mathrm{HD}$ & $6.78(2.24)$ & $19.1(0.1)$ & $7.68-8.31$ \\
\hline \multicolumn{4}{|c|}{ Lampsilis higginsii, SDP formulation } \\
\hline Control & $8.05(0.20)$ & $19.3(0.6)$ & $8.38-8.46$ \\
\hline 50 & $7.59(0.26)$ & $19.3(0.2)$ & $8.08-8.38$ \\
\hline 100 & $8.02(0.76)$ & $19.0(1.1)$ & $8.00-8.29$ \\
\hline 200 & $7.49(0.68)$ & $18.7(0.9)$ & $8.03-8.30$ \\
\hline 300 & $7.21(1.54)$ & $19.3(0.8)$ & $7.58-8.41$ \\
\hline $300 \mathrm{HD}$ & $7.02(1.34)$ & $19.5(0.7)$ & $7.85-8.34$ \\
\hline \multicolumn{4}{|c|}{ Obovaria olivaria, SDP formulation } \\
\hline Control & $9.82(0.78)$ & $19.9(0.2)$ & $8.36-8.51$ \\
\hline 50 & $10.41(0.32)$ & $19.7(0.5)$ & $8.32-8.48$ \\
\hline 100 & $10.16(0.42)$ & $19.6(0.5)$ & $8.30-8.45$ \\
\hline 200 & $9.67(0.34)$ & $19.7(0.5)$ & $8.10-8.35$ \\
\hline 300 & $9.66(0.93)$ & $19.7(0.2)$ & $8.10-8.30$ \\
\hline $300 \mathrm{HD}$ & $10.57(0.30)$ & $19.3(1.0)$ & $8.11-8.36$ \\
\hline \multicolumn{4}{|c|}{ Actinonaias ligamentina, SDP formulation } \\
\hline Control & $8.40(0.63)$ & $20.1(0.1)$ & $8.34-8.53$ \\
\hline 50 & $9.20(0.47)$ & $20.1(0.1)$ & $8.33-8.43$ \\
\hline 100 & $8.65(0.18)$ & $19.8(0.5)$ & $8.31-8.43$ \\
\hline 200 & $8.96(0.50)$ & $19.7(0.2)$ & $8.26-8.40$ \\
\hline
\end{tabular}




\begin{tabular}{clcc}
\hline $\begin{array}{c}\text { Treatment } \\
\text { group }\end{array}$ & $\begin{array}{c}\text { Dissolved oxygen } \\
(\mathrm{mg} / \mathrm{L})\end{array}$ & $\begin{array}{c}\text { Temperature } \\
\left({ }^{\circ} \mathrm{C}\right)\end{array}$ & $\mathrm{pH}$ \\
\hline 300 & $8.74(0.25)$ & $19.8(0.3)$ & $8.29-8.38$ \\
$300 \mathrm{HD}$ & $8.63(0.31)$ & $19.7(0.6)$ & $8.23-8.42$ \\
\hline & Lampsilis cardium, SDP formulation & \\
\hline Control & $8.50(0.11)$ & $19.5(0.4)$ & $8.23-8.42$ \\
50 & $8.15(0.11)$ & $19.7(0.7)$ & $8.25-8.39$ \\
100 & $8.04(0.91)$ & $19.3(0.4)$ & $8.03-8.39$ \\
200 & $7.66(0.87)$ & $19.3(0.4)$ & $8.09-8.36$ \\
300 & $7.21(1.63)$ & $19.6(0.5)$ & $7.74-8.29$ \\
\hline $00 \mathrm{HD}$ & $7.46(1.17)$ & $19.6(0.6)$ & $8.07-8.37$ \\
\hline
\end{tabular}

Table 6. Mean total ammonia nitrogen of exposure water by treatment group at 24 hours.

[Total ammonia nitrogen in milligrams per liter measured at the termination of 24 hours exposures of selected native freshwater mussel glochidia to two formulations of Pseudomonas fluorescens, strain CL145A. FDP, freeze-dried powder; SDP, spray-dried powder; --, cannot calculate data; number in parentheses, standard deviation; HD, heat-deactivated product]

\begin{tabular}{|c|c|c|c|c|c|c|c|c|}
\hline \multirow[b]{2}{*}{$\begin{array}{l}\text { Treatment } \\
\text { group }\end{array}$} & \multicolumn{2}{|c|}{ FDP formulation } & \multicolumn{6}{|c|}{ SDP formulation } \\
\hline & $\begin{array}{l}\text { Lampsilis } \\
\text { cardium }\end{array}$ & $\begin{array}{c}\text { Megalonaias } \\
\text { nervosa }\end{array}$ & $\begin{array}{l}\text { Ligumia } \\
\text { recta }\end{array}$ & $\begin{array}{l}\text { Lampsilis } \\
\text { siliquoidea }\end{array}$ & $\begin{array}{c}\text { Lampsilis } \\
\text { higginsii }\end{array}$ & $\begin{array}{c}\text { Obovaria } \\
\text { olivaria }\end{array}$ & $\begin{array}{l}\text { Actinonaias } \\
\text { ligamentina }\end{array}$ & $\begin{array}{l}\text { Lampsilis } \\
\text { cardium }\end{array}$ \\
\hline Control & $\begin{array}{c}0.058 \\
(0.016)\end{array}$ & $\begin{array}{c}0.026 \\
(0.004)\end{array}$ & $\begin{array}{c}0.080 \\
(0.002)\end{array}$ & $\begin{array}{r}0.10 \\
(--)\end{array}$ & $\begin{array}{r}0.09 \\
(--)\end{array}$ & $\begin{array}{r}0.066 \\
(0.029)\end{array}$ & $\begin{array}{r}0.066 \\
(0.039)\end{array}$ & $\begin{array}{r}0.06 \\
(--)\end{array}$ \\
\hline 50 & $\begin{array}{c}0.107 \\
(0.013)\end{array}$ & $\begin{array}{c}0.050 \\
(0.010)\end{array}$ & $\begin{array}{r}0.297 \\
(0.121)\end{array}$ & $\begin{array}{r}0.25 \\
(--)\end{array}$ & $\begin{array}{r}0.17 \\
(--)\end{array}$ & $\begin{array}{c}0.210 \\
(0.097)\end{array}$ & $\begin{array}{r}0.268 \\
(0.234)\end{array}$ & $\begin{array}{r}0.08 \\
(--)\end{array}$ \\
\hline 100 & $\begin{array}{c}0.137 \\
(0.011)\end{array}$ & $\begin{array}{c}0.056 \\
(0.019)\end{array}$ & $\begin{array}{c}0.714 \\
(0.014)\end{array}$ & $\begin{array}{r}0.24 \\
(--)\end{array}$ & $\begin{array}{r}0.31 \\
(--)\end{array}$ & $\begin{array}{c}0.367 \\
(0.238)\end{array}$ & $\begin{array}{c}0.445 \\
(0.241)\end{array}$ & $\begin{array}{r}0.13 \\
(--)\end{array}$ \\
\hline 200 & $\begin{array}{c}0.225 \\
(0.014)\end{array}$ & $\begin{array}{c}0.047 \\
(0.013)\end{array}$ & $\begin{array}{c}1.054 \\
(0.127)\end{array}$ & $\begin{array}{r}0.44 \\
(--)\end{array}$ & $\begin{array}{r}0.53 \\
(--)\end{array}$ & $\begin{array}{c}0.243 \\
(0.027)\end{array}$ & $\begin{array}{c}0.853 \\
(0.072)\end{array}$ & $\begin{array}{r}0.24 \\
(--)\end{array}$ \\
\hline 300 & $\begin{array}{c}0.296 \\
(0.012)\end{array}$ & $\begin{array}{c}0.123 \\
(0.057)\end{array}$ & $\begin{array}{c}1.452 \\
(0.242)\end{array}$ & $\begin{array}{r}0.63 \\
(--)\end{array}$ & $\begin{array}{r}0.45 \\
(--)\end{array}$ & $\begin{array}{c}0.302 \\
(0.157)\end{array}$ & $\begin{array}{c}1.047 \\
(0.116)\end{array}$ & $\begin{array}{r}0.44 \\
(--)\end{array}$ \\
\hline $300 \mathrm{HD}$ & $\begin{array}{c}0.193 \\
(0.012)\end{array}$ & $\begin{array}{c}0.086 \\
(0.020)\end{array}$ & $\begin{array}{r}0.230 \\
(0.028)\end{array}$ & $\begin{array}{r}0.55 \\
(--)\end{array}$ & $\begin{array}{r}0.50 \\
(--)\end{array}$ & $\begin{array}{r}0.489 \\
(0.389)\end{array}$ & $\begin{array}{c}0.581 \\
(0.197)\end{array}$ & $\begin{array}{r}0.51 \\
(--)\end{array}$ \\
\hline
\end{tabular}


Table 7. Mean un-adjusted glochidia viability from selected native freshwater mussels at 6,12 and 24 hours.

[Selected native freshwater mussel glochidia exposed for 6, 12 and 24 hours to two formulations of Pseudomonas fluorescens, strain CL145A. FDP, freeze dried powder; SDP, spray dried powder; number in parentheses, standard deviation; --, no data collected; HD, heat-deactivated test article]

\begin{tabular}{|c|c|c|c|c|c|c|c|c|}
\hline \multirow[b]{2}{*}{$\begin{array}{l}\text { Treatment } \\
\text { group }\end{array}$} & \multicolumn{2}{|c|}{ FDP formulation } & \multicolumn{6}{|c|}{ SDP Formulation } \\
\hline & $\begin{array}{l}\text { Lampsilis } \\
\text { cardium }\end{array}$ & $\begin{array}{c}\text { Megalonaias } \\
\text { nervosa }\end{array}$ & $\begin{array}{c}\text { Ligumia } \\
\text { recta }\end{array}$ & $\begin{array}{l}\text { Lampsilis } \\
\text { siliquoidea }\end{array}$ & $\begin{array}{c}\text { Lampsilis } \\
\text { higginsii }\end{array}$ & $\begin{array}{c}\text { Obovaria } \\
\text { olivaria }\end{array}$ & $\begin{array}{l}\text { Actinonaias } \\
\text { ligamentina }\end{array}$ & $\begin{array}{c}\text { Lampsilis } \\
\text { cardium }\end{array}$ \\
\hline \multicolumn{9}{|c|}{6 hours } \\
\hline Control & $\begin{array}{l}82.3 \\
(4.7)\end{array}$ & -- & $\begin{array}{l}85.7 \\
(1.1)\end{array}$ & $\begin{array}{l}72.3 \\
(4.0)\end{array}$ & $\begin{array}{l}72.3 \\
(9.5)\end{array}$ & $\begin{array}{c}80.0 \\
(11.5)\end{array}$ & $\begin{array}{l}88.6 \\
(4.4)\end{array}$ & $\begin{array}{l}92.3 \\
(1.0)\end{array}$ \\
\hline 50 & $\begin{array}{c}69.6 \\
(11.5)\end{array}$ & -- & $\begin{array}{l}11.7^{\mathrm{a}} \\
(9.5)\end{array}$ & $\begin{array}{l}56.8 \\
(2.7)\end{array}$ & $\begin{array}{l}77.7 \\
(3.7)\end{array}$ & $\begin{array}{l}85.2 \\
(1.3)\end{array}$ & $\begin{array}{l}71.7^{\mathrm{a}} \\
(6.6)\end{array}$ & $\begin{array}{l}77.9 \\
(8.9)\end{array}$ \\
\hline 100 & $\begin{array}{l}65.7 \\
(3.4)\end{array}$ & -- & $\begin{array}{r}0.5^{\mathrm{a}} \\
(0.8)\end{array}$ & $\begin{array}{r}29.0^{\mathrm{a}} \\
(14.8)\end{array}$ & $\begin{array}{l}63.2 \\
(1.0)\end{array}$ & $\begin{array}{r}61.0^{\mathrm{a}} \\
(13.8)\end{array}$ & $\begin{array}{l}72.2^{\mathrm{a}} \\
(9.7)\end{array}$ & $\begin{array}{l}75.3 \\
(6.1)\end{array}$ \\
\hline 200 & $\begin{array}{r}48.7^{\mathrm{a}} \\
(11.4)\end{array}$ & -- & $\begin{array}{r}1.7^{\mathrm{a}} \\
(1.6)\end{array}$ & $\begin{array}{r}19.3^{\mathrm{a}} \\
(17.1)\end{array}$ & $\begin{array}{l}56.7 \\
(5.5)\end{array}$ & $\begin{array}{r}40.6^{\mathrm{a}} \\
(11.5)\end{array}$ & $\begin{array}{r}40.5^{\mathrm{a}} \\
(16.3)\end{array}$ & $\begin{array}{r}52.2^{\mathrm{a}} \\
(21.2)\end{array}$ \\
\hline 300 & $\begin{array}{r}40.7^{\mathrm{a}} \\
(14.7)\end{array}$ & -- & $\begin{array}{r}1.1^{\mathrm{a}} \\
(1.6)\end{array}$ & $\begin{array}{r}11.9^{\mathrm{a}} \\
(16.7)\end{array}$ & $\begin{array}{r}29.1^{\mathrm{a}} \\
(21.7)\end{array}$ & $\begin{array}{r}15.7^{\mathrm{a}} \\
(12.7)\end{array}$ & $\begin{array}{l}11.8^{\mathrm{a}} \\
(7.3)\end{array}$ & $\begin{array}{r}9.9^{\mathrm{a}} \\
(10.4)\end{array}$ \\
\hline $300 \mathrm{HD}$ & $\begin{array}{r}20.4^{\mathrm{a}} \\
(23.0)\end{array}$ & -- & $\begin{array}{r}0.8^{\mathrm{a}} \\
(1.5)\end{array}$ & $\begin{array}{r}2.3^{\mathrm{a}} \\
(2.4)\end{array}$ & $\begin{array}{r}13.3^{\mathrm{a}} \\
(12.3)\end{array}$ & $\begin{array}{l}13.8^{\mathrm{a}} \\
(6.3)\end{array}$ & $\begin{array}{r}3.6^{\mathrm{a}} \\
(2.1)\end{array}$ & $\begin{array}{r}3.4^{\mathrm{a}} \\
(3.1)\end{array}$ \\
\hline \multicolumn{9}{|c|}{12 hours } \\
\hline Control & $\begin{array}{l}81.8 \\
(3.4)\end{array}$ & -- & $\begin{array}{l}89.5 \\
(0.5)\end{array}$ & $\begin{array}{l}66.6 \\
(6.5)\end{array}$ & $\begin{array}{l}68.7 \\
(6.1)\end{array}$ & $\begin{array}{l}86.4 \\
(6.3)\end{array}$ & $\begin{array}{l}94.2 \\
(1.5)\end{array}$ & $\begin{array}{l}86.1 \\
(2.0)\end{array}$ \\
\hline 50 & $\begin{array}{l}76.7 \\
(6.2)\end{array}$ & -- & $\begin{array}{l}16.2^{\mathrm{a}} \\
(5.6)\end{array}$ & $\begin{array}{l}59.1 \\
(4.1)\end{array}$ & $\begin{array}{l}68.7 \\
(6.5)\end{array}$ & $\begin{array}{l}84.5 \\
(2.9)\end{array}$ & $\begin{array}{r}66.6^{\mathrm{a}} \\
(17.8)\end{array}$ & $\begin{array}{l}78.6 \\
(6.6)\end{array}$ \\
\hline 100 & $\begin{array}{l}70.6 \\
(8.4)\end{array}$ & -- & $\begin{array}{r}8.8^{\mathrm{a}} \\
(3.3)\end{array}$ & $\begin{array}{r}43.6^{\mathrm{a}} \\
(3.4)\end{array}$ & $\begin{array}{l}65.5 \\
(3.1)\end{array}$ & $\begin{array}{c}77.2 \\
(12.4)\end{array}$ & $\begin{array}{r}64.4^{\mathrm{a}} \\
(17.7)\end{array}$ & $\begin{array}{r}52.7^{\mathrm{a}} \\
(45.6)\end{array}$ \\
\hline 200 & $\begin{array}{r}34.9^{\mathrm{a}} \\
(30.6)\end{array}$ & -- & $\begin{array}{r}1.2^{\mathrm{a}} \\
(0.3)\end{array}$ & $\begin{array}{r}19.6^{\mathrm{a}} \\
(13.2)\end{array}$ & $\begin{array}{c}46.2 \\
(17.9)\end{array}$ & $\begin{array}{r}50.3^{\mathrm{a}} \\
(16.5)\end{array}$ & $\begin{array}{l}40.0^{\mathrm{a}} \\
(4.5)\end{array}$ & $\begin{array}{r}46.7^{\mathrm{a}} \\
(22.3)\end{array}$ \\
\hline 300 & $\begin{array}{r}9.1^{\mathrm{a}} \\
(8.8)\end{array}$ & -- & $\begin{array}{r}0.0^{\mathrm{a}} \\
(0.0)\end{array}$ & $\begin{array}{r}4.5^{\mathrm{a}} \\
(1.9)\end{array}$ & $\begin{array}{r}38.8^{\mathrm{a}} \\
(8.1)\end{array}$ & $\begin{array}{l}39.2^{\mathrm{a}} \\
(3.0)\end{array}$ & $\begin{array}{l}10.2^{\mathrm{a}} \\
(2.5)\end{array}$ & $\begin{array}{r}6.5^{\mathrm{a}} \\
(4.2)\end{array}$ \\
\hline $300 \mathrm{HD}$ & $\begin{array}{r}4.8^{\mathrm{a}} \\
(1.2)\end{array}$ & -- & $\begin{array}{r}0.9^{\mathrm{a}} \\
(0.8)\end{array}$ & $\begin{array}{l}2.1^{\mathrm{a}} \\
(2.8)\end{array}$ & $\begin{array}{r}9.5^{\mathrm{a}} \\
(0.9)\end{array}$ & $\begin{array}{r}1.2^{\mathrm{a}} \\
(0.4)\end{array}$ & $\begin{array}{r}8.2^{\mathrm{a}} \\
(5.0)\end{array}$ & $\begin{array}{r}5.8^{\mathrm{a}} \\
(2.6)\end{array}$ \\
\hline \multicolumn{9}{|c|}{24 hours } \\
\hline Control & $\begin{array}{l}81.9 \\
(1.4)\end{array}$ & $\begin{array}{c}52.1 \\
(11.3)\end{array}$ & $\begin{array}{l}84.5 \\
(1.9)\end{array}$ & $\begin{array}{l}69.4 \\
(8.2)\end{array}$ & $\begin{array}{l}69.3 \\
(7.3)\end{array}$ & $\begin{array}{l}87.0 \\
(1.8)\end{array}$ & $\begin{array}{l}92.6 \\
(2.2)\end{array}$ & $\begin{array}{l}92.1 \\
(0.9)\end{array}$ \\
\hline 50 & $\begin{array}{l}74.1 \\
(9.3)\end{array}$ & $\begin{array}{l}55.6 \\
(9.5)\end{array}$ & $\begin{array}{l}14.7^{\mathrm{a}} \\
(1.8)\end{array}$ & $\begin{array}{l}56.8 \\
(6.0)\end{array}$ & $\begin{array}{l}74.6 \\
(3.4)\end{array}$ & $\begin{array}{l}89.2 \\
(2.9)\end{array}$ & $\begin{array}{r}75.8^{\mathrm{a}} \\
(13.0)\end{array}$ & $\begin{array}{l}79.8 \\
(5.6)\end{array}$ \\
\hline 100 & $\begin{array}{l}67.7 \\
(3.8)\end{array}$ & $\begin{array}{c}48.1 \\
(17.0)\end{array}$ & $\begin{array}{r}7.0^{\mathrm{a}} \\
(4.9)\end{array}$ & $\begin{array}{r}41.6^{\mathrm{a}} \\
(6.5)\end{array}$ & $\begin{array}{l}65.5 \\
(4.9)\end{array}$ & $\begin{array}{l}78.3 \\
(6.8)\end{array}$ & $\begin{array}{r}63.5^{\mathrm{a}} \\
(11.2)\end{array}$ & $\begin{array}{r}66.9^{\mathrm{a}} \\
(15.8)\end{array}$ \\
\hline 200 & $\begin{array}{r}29.5^{\mathrm{a}} \\
(23.8)\end{array}$ & $\begin{array}{c}49.5 \\
(18.7)\end{array}$ & $\begin{array}{r}3.3^{\mathrm{a}} \\
(2.2)\end{array}$ & $\begin{array}{l}11.6^{\mathrm{a}} \\
(4.7)\end{array}$ & $\begin{array}{r}42.5^{\mathrm{a}} \\
(14.8)\end{array}$ & $\begin{array}{l}60.7^{\mathrm{a}} \\
(4.5)\end{array}$ & $\begin{array}{l}30.0^{\mathrm{a}} \\
(7.2)\end{array}$ & $\begin{array}{r}59.2^{\mathrm{a}} \\
(12.8)\end{array}$ \\
\hline 300 & $\begin{array}{l}12.0^{\mathrm{a}} \\
(1.8)\end{array}$ & $\begin{array}{c}40.1 \\
(20.8)\end{array}$ & $\begin{array}{r}0.7^{\mathrm{a}} \\
(0.6)\end{array}$ & $\begin{array}{r}6.0^{\mathrm{a}} \\
(1.7)\end{array}$ & $\begin{array}{r}22.3^{\mathrm{a}} \\
(26.6)\end{array}$ & $\begin{array}{l}26.5^{\mathrm{a}} \\
(3.2)\end{array}$ & $\begin{array}{r}6.0^{\mathrm{a}} \\
(3.5)\end{array}$ & $\begin{array}{r}6.1^{\mathrm{a}} \\
(7.2)\end{array}$ \\
\hline $300 \mathrm{HD}$ & $\begin{array}{r}4.9^{\mathrm{a}} \\
(2.3)\end{array}$ & $\begin{array}{c}26.8^{\mathrm{a}} \\
(10.6)\end{array}$ & $\begin{array}{r}0.7^{\mathrm{a}} \\
(0.8)\end{array}$ & $\begin{array}{r}1.7^{\mathrm{a}} \\
(0.9)\end{array}$ & $\begin{array}{c}13.9^{\mathrm{a}} \\
(10.4)\end{array}$ & $\begin{array}{r}0.5^{\mathrm{a}} \\
(0.3)\end{array}$ & $\begin{array}{r}5.2^{\mathrm{a}} \\
(3.4)\end{array}$ & $\begin{array}{r}4.7^{\mathrm{a}} \\
(1.3)\end{array}$ \\
\hline
\end{tabular}

${ }^{\mathrm{a}}$ At each sample time, the mean un-adjusted treatment group glochidia viability ( in percent) within the same column that have a letter superscript are significantly different $(\mathrm{p}>0.05)$ from the control group. 
Table 8. Mean adjusted glochidia viability from selected native freshwater mussels at 6, 12 and 24 hours. [Selected native freshwater mussel glochidia exposed for 6,12 and 24 hours to two formulations of Pseudomonas fluorescens, strain CL145A. FDP, freeze-dried powder; SDP, spray-dried powder; number in parentheses, standard deviation; --, no data collected; HD, heat-deactivated test article]

\begin{tabular}{|c|c|c|c|c|c|c|c|c|}
\hline \multirow[b]{2}{*}{$\begin{array}{l}\text { Treatment } \\
\text { group }\end{array}$} & \multicolumn{2}{|c|}{ FDP formulation } & \multicolumn{6}{|c|}{ SDP Formulation } \\
\hline & $\begin{array}{l}\text { Lampsilis } \\
\text { cardium }\end{array}$ & $\begin{array}{c}\text { Megalonaias } \\
\text { nervosa }\end{array}$ & $\begin{array}{l}\text { Ligumia } \\
\text { recta }\end{array}$ & $\begin{array}{l}\text { Lampsilis } \\
\text { siliquoidea }\end{array}$ & $\begin{array}{c}\text { Lampsilis } \\
\text { higginsii }\end{array}$ & $\begin{array}{c}\text { Obovaria } \\
\text { olivaria }\end{array}$ & $\begin{array}{l}\text { Actinonaias } \\
\text { ligamentina }\end{array}$ & $\begin{array}{c}\text { Lampsilis } \\
\text { cardium }\end{array}$ \\
\hline \multicolumn{9}{|c|}{6 hours } \\
\hline Control & $\begin{array}{l}97.1 \\
(5.6)\end{array}$ & -- & $\begin{array}{r}101.4 \\
(1.3)\end{array}$ & $\begin{array}{l}90.1 \\
(5.0)\end{array}$ & $\begin{array}{c}89.5 \\
(11.8)\end{array}$ & $\begin{array}{c}93.0 \\
(13.4)\end{array}$ & $\begin{array}{l}95.6 \\
(4.8)\end{array}$ & $\begin{array}{l}98.4 \\
(1.1)\end{array}$ \\
\hline 50 & $\begin{array}{c}82.1 \\
(13.6)\end{array}$ & -- & $\begin{array}{c}13.9 \\
(11.3)\end{array}$ & $\begin{array}{l}70.7 \\
(3.4)\end{array}$ & $\begin{array}{l}96.2 \\
(4.6)\end{array}$ & $\begin{array}{l}99.1 \\
(1.5)\end{array}$ & $\begin{array}{l}77.3 \\
(7.1)\end{array}$ & $\begin{array}{l}83.1 \\
(9.5)\end{array}$ \\
\hline 100 & $\begin{array}{l}77.6 \\
(4.1)\end{array}$ & -- & $\begin{array}{c}0.6 \\
(1.0)\end{array}$ & $\begin{array}{c}36.1 \\
(18.4)\end{array}$ & $\begin{array}{l}78.2 \\
(1.3)\end{array}$ & $\begin{array}{r}70.9 \\
(16.1)\end{array}$ & $\begin{array}{c}77.9 \\
(10.5)\end{array}$ & $\begin{array}{l}80.2 \\
(6.5)\end{array}$ \\
\hline 200 & $\begin{array}{c}57.5 \\
(13.5)\end{array}$ & -- & $\begin{array}{c}2.1 \\
(1.8)\end{array}$ & $\begin{array}{c}24.0 \\
(21.4)\end{array}$ & $\begin{array}{l}70.1 \\
(6.8)\end{array}$ & $\begin{array}{c}47.3 \\
(13.3)\end{array}$ & $\begin{array}{c}43.7 \\
(17.6)\end{array}$ & $\begin{array}{r}55.7 \\
(22.6)\end{array}$ \\
\hline 300 & $\begin{array}{r}48.0 \\
(17.3)\end{array}$ & -- & $\begin{array}{c}1.3 \\
(1.8)\end{array}$ & $\begin{array}{c}14.8 \\
(20.8)\end{array}$ & $\begin{array}{c}36.0 \\
(26.9)\end{array}$ & $\begin{array}{c}18.3 \\
(14.8)\end{array}$ & $\begin{array}{l}12.7 \\
(7.9)\end{array}$ & $\begin{array}{r}10.6 \\
(11.1)\end{array}$ \\
\hline $300 \mathrm{HD}$ & $\begin{array}{c}24.1 \\
(27.2)\end{array}$ & -- & $\begin{array}{r}1.0 \\
(1.7)\end{array}$ & $\begin{array}{r}2.9 \\
(2.9)\end{array}$ & $\begin{array}{r}16.4 \\
(15.2)\end{array}$ & $\begin{array}{l}16.1 \\
(7.3)\end{array}$ & $\begin{array}{r}3.9 \\
(2.2)\end{array}$ & $\begin{array}{r}3.7 \\
(3.3)\end{array}$ \\
\hline \multicolumn{9}{|c|}{12 hours } \\
\hline Control & $\begin{array}{l}96.6 \\
(4.0)\end{array}$ & -- & $\begin{array}{r}105.9 \\
(0.6)\end{array}$ & $\begin{array}{l}82.9 \\
(8.0)\end{array}$ & $\begin{array}{l}85.0 \\
(7.6)\end{array}$ & $\begin{array}{r}100.5 \\
(7.4)\end{array}$ & $\begin{array}{r}101.7 \\
(1.6)\end{array}$ & $\begin{array}{l}91.8 \\
(2.2)\end{array}$ \\
\hline 50 & $\begin{array}{l}90.5 \\
(7.4)\end{array}$ & -- & $\begin{array}{l}19.1 \\
(6.6)\end{array}$ & $\begin{array}{l}73.6 \\
(5.1)\end{array}$ & $\begin{array}{l}85.0 \\
(8.0)\end{array}$ & $\begin{array}{l}98.2 \\
(3.4)\end{array}$ & $\begin{array}{c}71.8 \\
(19.1)\end{array}$ & $\begin{array}{l}83.8 \\
(7.0)\end{array}$ \\
\hline 100 & $\begin{array}{c}83.3 \\
(10.0)\end{array}$ & -- & $\begin{array}{l}10.5 \\
(3.9)\end{array}$ & $\begin{array}{l}54.3 \\
(4.2)\end{array}$ & $\begin{array}{l}81.1 \\
(3.9)\end{array}$ & $\begin{array}{c}89.7 \\
(14.5)\end{array}$ & $\begin{array}{c}69.4 \\
(19.1)\end{array}$ & $\begin{array}{r}56.1 \\
(48.7)\end{array}$ \\
\hline 200 & $\begin{array}{c}41.2 \\
(36.1)\end{array}$ & -- & $\begin{array}{r}1.4 \\
(0.4)\end{array}$ & $\begin{array}{r}24.4 \\
(16.4)\end{array}$ & $\begin{array}{c}57.2 \\
(22.2)\end{array}$ & $\begin{array}{c}58.4 \\
(19.2)\end{array}$ & $\begin{array}{l}43.2 \\
(4.9)\end{array}$ & $\begin{array}{r}49.8 \\
(23.8)\end{array}$ \\
\hline 300 & $\begin{array}{c}10.8 \\
(10.4)\end{array}$ & -- & $\begin{array}{r}0.0 \\
(0.0)\end{array}$ & $\begin{array}{r}5.6 \\
(2.4)\end{array}$ & $\begin{array}{c}48.0 \\
(10.0)\end{array}$ & $\begin{array}{l}45.6 \\
(3.5)\end{array}$ & $\begin{array}{l}11.0 \\
(2.7)\end{array}$ & $\begin{array}{r}6.9 \\
(4.5)\end{array}$ \\
\hline $300 \mathrm{HD}$ & $\begin{array}{r}5.7 \\
(1.4)\end{array}$ & -- & $\begin{array}{c}1.1 \\
(1.0)\end{array}$ & $\begin{array}{c}2.7 \\
(3.5)\end{array}$ & $\begin{array}{l}11.8 \\
(1.1)\end{array}$ & $\begin{array}{r}1.4 \\
(0.4)\end{array}$ & $\begin{array}{r}8.8 \\
(5.4)\end{array}$ & $\begin{array}{r}6.2 \\
(2.8)\end{array}$ \\
\hline \multicolumn{9}{|c|}{24 hours } \\
\hline Control & $\begin{array}{l}96.7 \\
(1.7)\end{array}$ & $\begin{array}{r}53.9 \\
(11.7)\end{array}$ & $\begin{array}{r}100.0 \\
(2.3)\end{array}$ & $\begin{array}{r}86.5 \\
(10.2)\end{array}$ & $\begin{array}{l}85.8 \\
(9.0)\end{array}$ & $\begin{array}{r}101.2 \\
(2.0)\end{array}$ & $\begin{array}{l}99.9 \\
(2.4)\end{array}$ & $\begin{array}{l}98.2 \\
(1.0)\end{array}$ \\
\hline 50 & $\begin{array}{r}87.5 \\
(11.0)\end{array}$ & $\begin{array}{l}57.6 \\
(9.8)\end{array}$ & $\begin{array}{l}17.4 \\
(2.1)\end{array}$ & $\begin{array}{l}70.8 \\
(7.4)\end{array}$ & $\begin{array}{l}92.3 \\
(4.2)\end{array}$ & $\begin{array}{r}103.7 \\
(3.3)\end{array}$ & $\begin{array}{c}81.7 \\
(14.1)\end{array}$ & $\begin{array}{l}85.1 \\
(6.0)\end{array}$ \\
\hline 100 & $\begin{array}{l}80.0 \\
(4.5)\end{array}$ & $\begin{array}{c}49.8 \\
(17.6)\end{array}$ & $\begin{array}{c}8.2 \\
(5.8)\end{array}$ & $\begin{array}{l}51.9 \\
(8.0)\end{array}$ & $\begin{array}{l}81.0 \\
(6.0)\end{array}$ & $\begin{array}{l}91.0 \\
(8.0)\end{array}$ & $\begin{array}{c}68.5 \\
(12.1)\end{array}$ & $\begin{array}{r}71.3 \\
(16.8)\end{array}$ \\
\hline 200 & $\begin{array}{r}34.9 \\
(28.0)\end{array}$ & $\begin{array}{r}51.3 \\
(19.3)\end{array}$ & $\begin{array}{c}3.8 \\
(2.6)\end{array}$ & $\begin{array}{l}14.5 \\
(5.8)\end{array}$ & $\begin{array}{r}52.6 \\
(18.3)\end{array}$ & $\begin{array}{l}70.6 \\
(5.3)\end{array}$ & $\begin{array}{l}32.4 \\
(7.8)\end{array}$ & $\begin{array}{c}63.2 \\
(13.6)\end{array}$ \\
\hline 300 & $\begin{array}{l}14.2 \\
(2.2)\end{array}$ & $\begin{array}{r}41.6 \\
(21.5)\end{array}$ & $\begin{array}{c}0.9 \\
(0.8)\end{array}$ & $\begin{array}{c}7.5 \\
(2.2)\end{array}$ & $\begin{array}{c}27.6 \\
(32.9)\end{array}$ & $\begin{array}{l}30.9 \\
(3.8)\end{array}$ & $\begin{array}{c}6.5 \\
(3.8)\end{array}$ & $\begin{array}{r}6.5 \\
(7.7)\end{array}$ \\
\hline $300 \mathrm{HD}$ & $\begin{array}{c}5.8 \\
(2.7)\end{array}$ & $\begin{array}{c}27.7 \\
(11.0)\end{array}$ & $\begin{array}{c}0.9 \\
(0.9)\end{array}$ & $\begin{array}{c}2.1 \\
(1.1)\end{array}$ & $\begin{array}{c}17.2 \\
(12.9)\end{array}$ & $\begin{array}{c}0.6 \\
(0.4)\end{array}$ & $\begin{array}{r}5.6 \\
(3.7)\end{array}$ & $\begin{array}{r}5.1 \\
(3.3)\end{array}$ \\
\hline
\end{tabular}




\section{Conclusions}

The mean adjusted-viability of control group glochidia in trials with spray-dried powder (SDP) formulation of Pseudomonas fluorescens strain CL145A exceeded 82 percent. Differences in glochidia viability were detected in two of six species exposed to 50 milligrams per liter (mg/L) SDP and in four of six species exposed to $100 \mathrm{mg} / \mathrm{L}$ SDP at 6, 12, and 24 hours when compared to untreated controls. Regardless of sample time, viability of glochidia in the 200 and $300 \mathrm{mg} / \mathrm{L}$ SDP-treated groups and the $300 \mathrm{mg} / \mathrm{L}$ heat-deactivated SDP positive control groups were significantly lower than glochidia in the untreated control for all species except Lampsilis higginsii. The significant impact of the SDP positive control treatments on glochidia viability indicate that the decreases in glochidia viability may not be caused by the same mode of action that causes dreissenid mussel (zebra mussel, Dreissena polymorpha and quagga mussel, Dreissena rostriformis bugensis) mortality.

The mean adjusted-viability of control group glochidia in trials with freeze-dried powder (FDP) formulation of $P$. fluorescens (strain CL145A) exceeded 96 percent in the Lampsilis cardium trial and was 53.9 percent in the Megalonaias nervosa at 24 hours. Regardless of sample time, differences in glochidia viability were only detected in the L. cardium FDP-trial when the concentration was $\geq$ $200 \mathrm{mg} / \mathrm{L}$. In the M. nervosa FDP exposures, differences in glochidia viability were only detected between the $300 \mathrm{mg} / \mathrm{L}$ heat-deactivated FDP-treated group (positive control) and the untreated control group.

However, given the low viability observed in the $M$. nervosa control group (52.1 percent unadjusted viability), the results for $M$. nervosa should be interpreted with caution. Similar to the SDPtrials, the impact of the FDP positive control treatments indicate that the observed decreases in glochidia viability may not be caused from the same mode of action that causes dreissenid mussel mortality. The results of these exposures indicate that applications of a biopesticide formulated with $P$. fluorescens as the active ingredient may impact the viability of native freshwater mussel glochidia if they are present in the water column during an application. However, freshwater mussel glochidia are only present in the water column for a relatively short period which could be avoided by timing $P$. fluorescens biopesticide applications. Additionally, applications of a $P$. fluorescens biopesticide for dreissenid mussel control would be of short duration and the biological activity of $P$. fluorescens degrades rapidly; further reducing the risk of exposure to $P$. fluorescens to native mussel glochidia.

\section{References Cited}

American Public Health Association, American Water Works Association, and Water Environment Federation, 2012, Standard methods for examination of water and wastewater (22d ed): Washington, D.C., American Public Health Association, 1,360 p.

ASTM International, 2013, ASTM Standard E2455-06 (2013)—Standard guide for conducting laboratory toxicity tests with freshwater mussels: West Conshohocken, Pa., ASTM International, $52 \mathrm{p}$.

Brady, T.R., Aloisi, D., Gordon, D and Wege G., 2010, A method for culturing mussels using in-river cages: Journal of Fish and Wildlife Management, v. 2, no. 1, p.85-89.

Burlakova, L.E., Karatayev, A.Y., and Padilla, D.K., 2000, The impact of Dreissena polymorpha (PALLAS) invasion on unionid bivalves: International Review of Hydrobiology, v. 85, no. 5-6, p. 529-541.

Cummings S.K. and Mayer C.A., 1992, Field guide to freshwater mussels of the Midwest, Illinois Natural History Survey Manual 5, 194 pp. 
Haag, W.R., Berg, D.L., Garton, D.W., and Farris, J.L., 1993, Reduced survival and fitness in native bivalves in response to fouling by the introduced zebra mussel (Dreissena polymorpha) in western Lake Erie: Canadian Journal of Fisheries and Aquatic Sciences, v. 50, no. 1, p. 13-19.

Master, L.L., Flack, S.R. and Stein, B.A., eds., 1998, Rivers of Life: Critical Watersheds for Protecting Freshwater Biodiversity. The Nature Conservancy, Arlington, Virginia.

Molloy, D.P., Mayer, D.A., Gaylo, M.J., Burlakova, L.E., Karatayev, A.Y., Presti, K.T., Sawyko, P.M., and Morse, J.T., 2013, Non-target trials with Pseudomonas fluorescens strain CL145A, a lethal control agent of dreissenid mussels (Bivalvia: Dreissenidae): Management of Biological Invasions, v. 4, p. 71-79.

Molloy, D.P., Mayer, D.A., Gaylo, M.J., Morse, J.T., Presti, K.T., Sawyko, P.M., Karatayev, A.Y., Burlakova, L.E., Laruelle, F., Nishikawa, K.C., and Griffin, B.H., 2013, Pseudomonas fluorescens strain CL145A-A biopesticide for the control of zebra and quagga mussels (Bivalvia: Dreissenidae): Journal for Invertebrate Pathology, v. 113, p. 104-114.

Nalepa, T.F., 1994, Decline of native unionids in Lake St. Clair after infestation by the zebra mussel, Dreissena polymorpha: Canadian Journal of Fisheries and Aquatic Sciences, v. 51, p. 2227-2233.

Neves, R.J., Bogan, A.E., Williams, J.D., Ahlstedt, S.A., and Hartfield, P.W., 1997, Status of aquatic mollusks in the southeastern United States-A downward spiral of diversity, in Benz, G.W., and Collins, D.E., eds., Aquatic fauna in peril-The southeastern perspective: Decatur, Ga., Southeastern Aquatic Research Institute, Lenz Design and Communications, p. 43-85.

Nikon Imaging System Elements-BR, 1991-2010, Version 3.10, Nikon Instruments Inc., Melville, New York, USA.

Ricciardi, A., Whoriskey F.G., and Rasmussen J.B., 1996, Impact of the Dreissena invasion on native unionid bivalves in the upper St. Lawerence River, Canadian Journal of Fisheries and Aquatic Sciences, v. 53, p. 1434-1444.

Ricciardi, A., and Rasmussen, J.B., 1999, Extinction rates of North American freshwater fauna: Conservation Biology, v. 13, no. 5, p. 1220-1222.

SAS, 2010, Version 9.3, SAS Institute Inc., Cary, NC, USA.

Strayer, D.L., and Malcom, H.M., 2007, Effects of zebra mussels (Dreissena polymorpha) on native bivalves - The beginning of the end or the end of the beginning?: Journal of the North American Benthological Society, v. 26, no. 1, p. 111-122.

U.S. Environmental Protection Agency, 2013, Ambient water quality criteria for ammonia-Freshwater 2013: Washington, D.C., Office of Water, EPA 822-R-13-001, 242 p.

U.S. Fish and Wildlife Service, 2004, Higgins Eye Pearlymussel (Lampsilis higginsii) recovery plan: First Revision, Ft. Snelling, Minnesota, 126 p.

U.S. Fish and Wildlife Service, 2013, ESA Basics 40 Years of Conserving Endangered Species, 2 p., accessed April 1, 2015, at http://www.fws.gov/endangered/esa-library/pdf/ESA_basics.pdf/.

Wang, N., Augspurger, T., Barnhart, M.C., Bidwell, J. R., Cope, W. G., Dwyer, F. J., Geis S., Greer, E., Ingersoll, C.G., Kane, C.M, May, T.W., Neves, R.J., Newton T.J., Roberts, A.D., and Whites, D.W., 2007, Intra-and interlaboratory variability in acute toxicity tests with glochidia and juveniles of freshwater mussels (unionidae): Environmental Toxicology and Chemistry, v. 26, no. 10, 2029-2035.

Watters, G.T., M.A. Hoggarth and D.H. Stansbery, 2009, The freshwater mussels of Ohio: Columbus, Ohio, Ohio State University Press, 421 p.

Williams, J.D., Warren, M.L., Jr., Cummings, K.S., Harris, J.L., and Neves, R.J., 1993, Conservation status of freshwater mussels of the United States and Canada: Fisheries, v. 18, no. 9, p. 6-22. 


\section{Appendix 1. Study Protocol, Amendments, and Datasheets}

\begin{tabular}{|c|c|c|c|}
\hline $\begin{array}{l}\text { Item } \\
\text { number }\end{array}$ & Item description & $\begin{array}{c}\text { Number } \\
\text { of } \\
\text { pages }\end{array}$ & $\begin{array}{c}\text { Report } \\
\text { page } \\
\text { number }\end{array}$ \\
\hline 1 & $\begin{array}{l}\text { Prot ocol: "Effects of Pseudomonas fluorescens (Pf-CL145A) to glochidia from seven unionid mussel } \\
\text { species" }\end{array}$ & 21 & 20 \\
\hline 2 & Amendment 1: Revision of Study Protocol, Study \# AEH-11-PSEUDO-01 & 6 & 41 \\
\hline 3 & Amendment 2: Revision of Study Protocol, Study \# AEH-11-PSEUDO-01 & 8 & 47 \\
\hline 4 & Amendment 3: Revision of Study Protocol, Study \# AEH-11-PSEUDO-01 & 5 & 55 \\
\hline 5 & Amendment 4: Revision of Study Protocol, Study \# AEH-11-PSEUDO-01 & 7 & 60 \\
\hline 6 & Amendment 5: Revision of Study Protocol, Study \# AEH-11-PSEUDO-01 & 2 & 67 \\
\hline 7 & Note to File 1: Study \# AEH-11-PSEUDO-01 & 6 & 69 \\
\hline 8 & Note to File 2: Study \# AEH-11-PSEUDO-01 & 7 & 75 \\
\hline 9 & Version 1.1 "Donor Mussel UMESC Lot Number Assignment Form" Datasheet & 1 & 82 \\
\hline 10 & Version 1.1 "Daily Care Worksheet Donor Mussel Holding” Datasheet & 1 & 83 \\
\hline 11 & “Adult Mussel Holding Daily Algal Diet 1/13/2012” Datasheet & 1 & 84 \\
\hline 12 & Version 1.1 "Glochidia Test Organism UMESC Lot Number Assignment Form” Datasheet & 1 & 85 \\
\hline 13 & Version 1.1 "Glochidia Aliquot Distribution Form"Datasheet & 1 & 86 \\
\hline 14 & Version 1.1 "Glochidia Photomicrograph Counting Record" Datasheet & 1 & 87 \\
\hline 15 & Version 1.1 "Initial Viability and Concentration Determination of Glochidia" Datasheet & 1 & 88 \\
\hline 16 & $\begin{array}{l}\text { Version } 1.1 \text { “Water Quality - Form } 1 \text { Initial (Dilution water Hardness, Alkalinity, and Temperature)" } \\
\text { Datasheet }\end{array}$ & 1 & 89 \\
\hline 17 & Version 1.2 "WaterQuality- Form 2 During Exposure (Dissolved Oxygen, $\mathrm{pH}$, Temperature)" Datasheet & 1 & 90 \\
\hline 18 & $\begin{array}{l}\text { Version } 1.2 \text { "Water Quality-Form } 3 \text { Upon Termination (Hardness, Alkalinity, Conductivity, Temperature } \\
\text { and pH)" Datasheet }\end{array}$ & 1 & 91 \\
\hline 19 & Version 1.1 “Test Chemical Stock Preparation Data Form” Datasheet & 1 & 92 \\
\hline 20 & Version 1.2 "Chemical St ock Solut ion Determination and Preparation" Datasheet & 1 & 93 \\
\hline 21 & Version 1.1 "Pipette Calibration" Datasheet & 1 & 94 \\
\hline 22 & OLD Versions of Datasheets & 18 & 95 \\
\hline
\end{tabular}




\section{If}

\section{DRIGINAL}

Protocol Title:

Effects of Pseudomonas fluorescens (Pf-CL145A) to glochidia from seven unionid mussel species

Study Number: AEH-11-PSEUDO-01
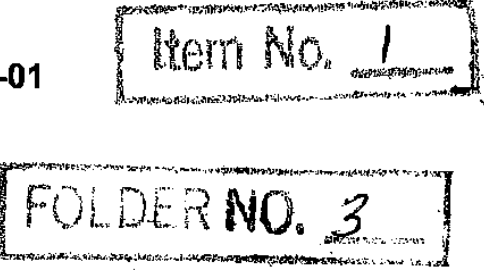

Test Facilities

Upper Midwest Environmental Sciences Center (UMESC)

US Geological Survey

2630 Fanta Reed Rd.

La Crosse, Wisconsin 54603

Proposed Experimental Start Date: May 2011

Proposed Experimental Termination Date: February 2012

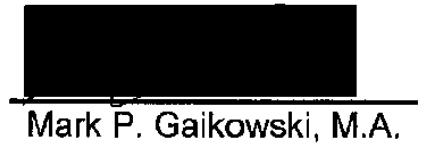

25 Apr2011

Protocol Approval

Reviewed by:

Supervisory Biologist
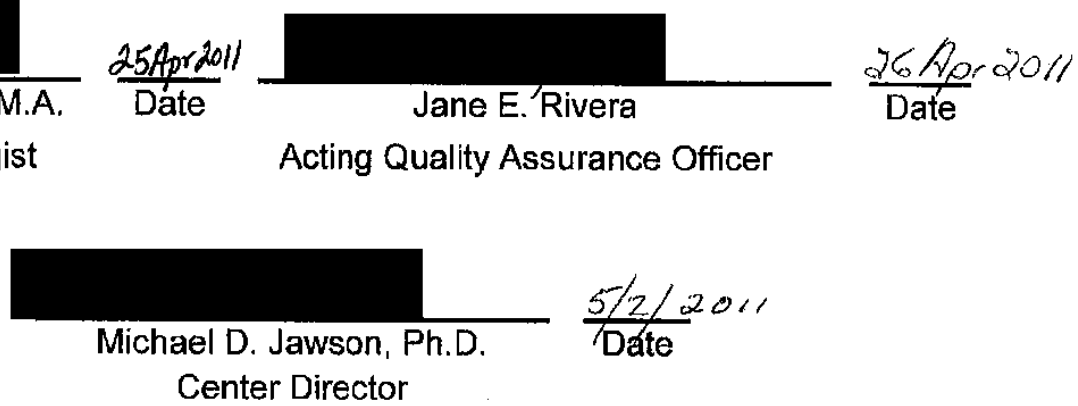

Center Director
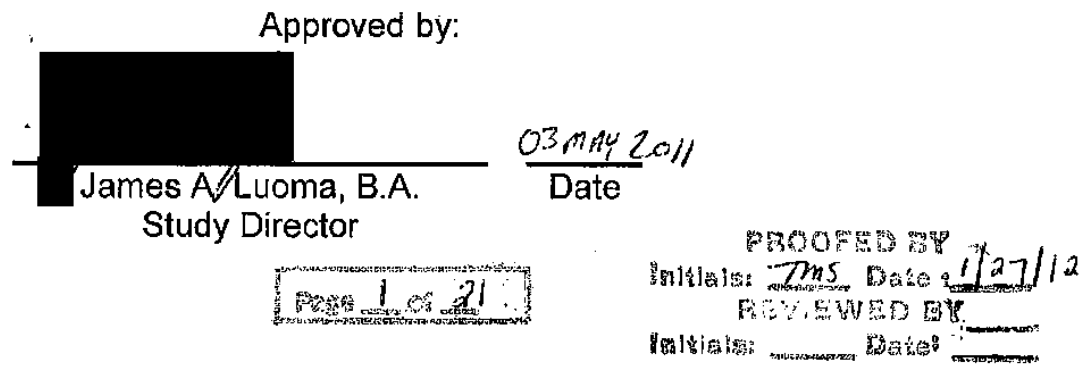


\section{TABLE OF CONTENTS}

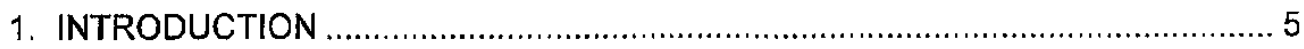

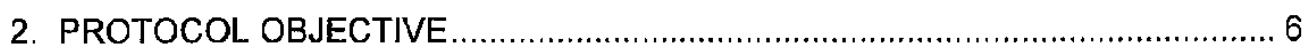

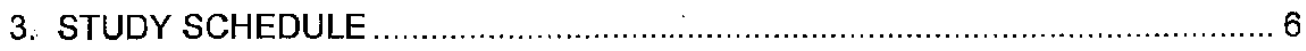

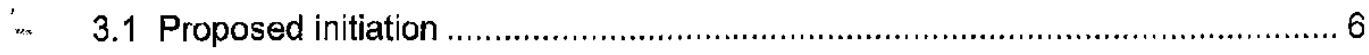

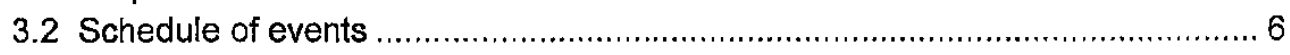

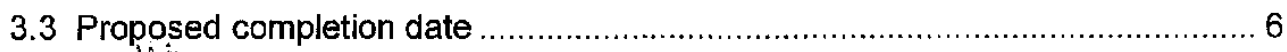

4. STUDY

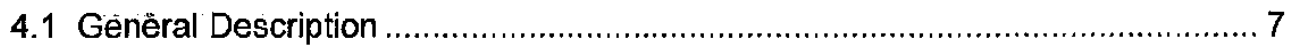

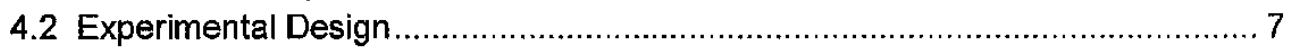

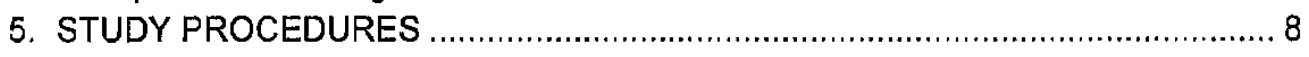

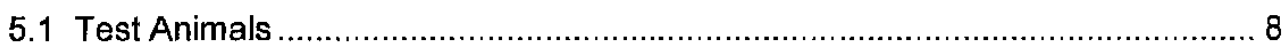

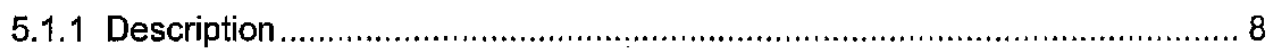

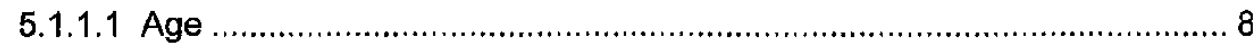

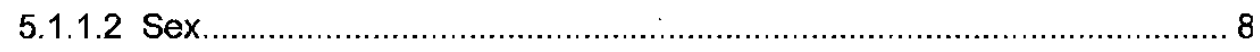

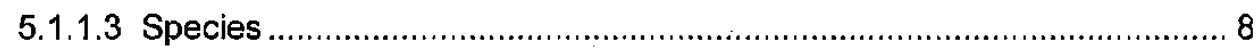

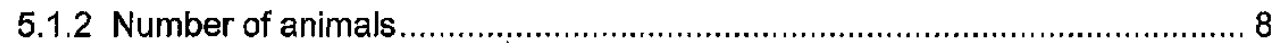

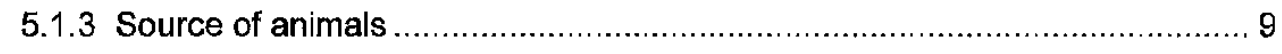

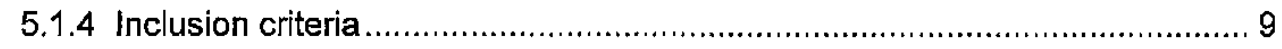

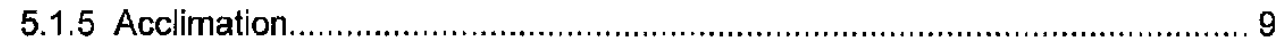

5.1 .6 Feeding

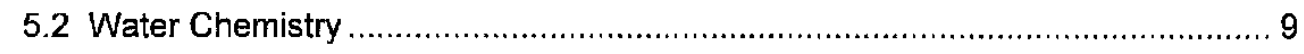

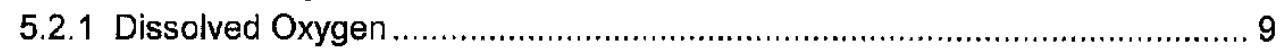

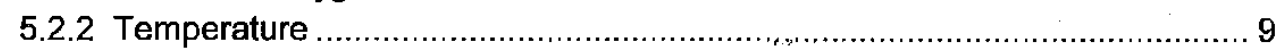

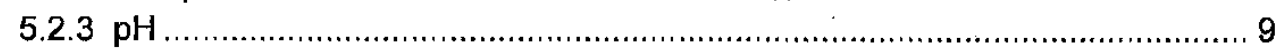

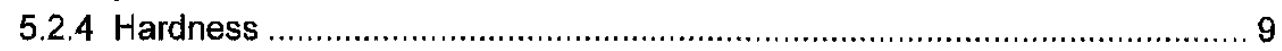

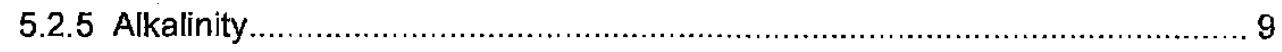

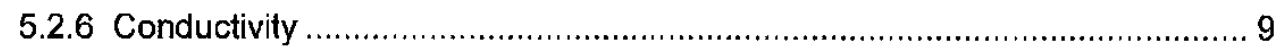

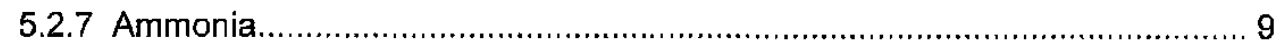

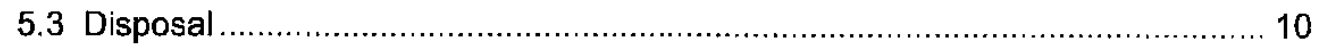

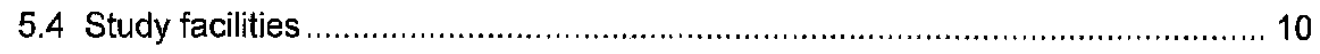

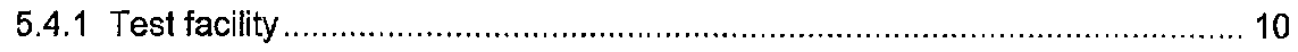

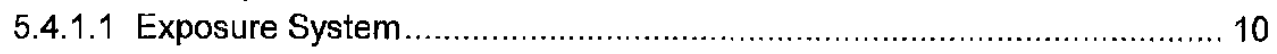

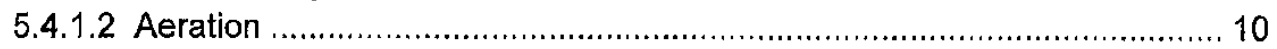

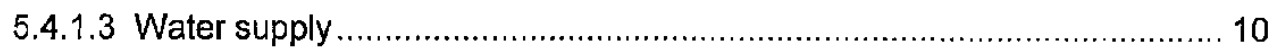

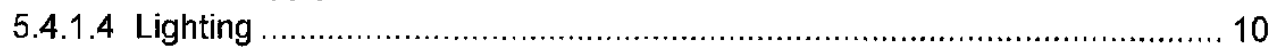

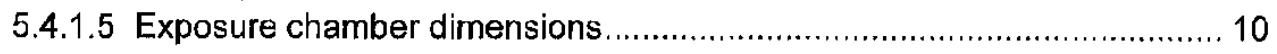

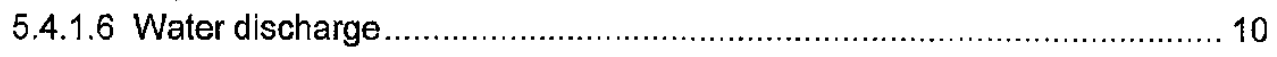

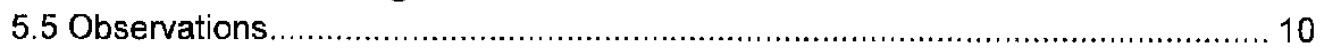




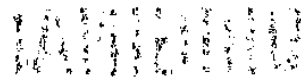

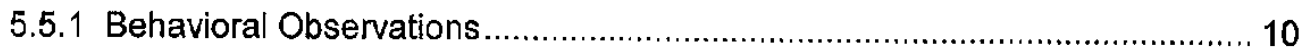

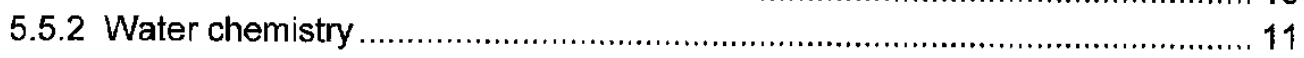

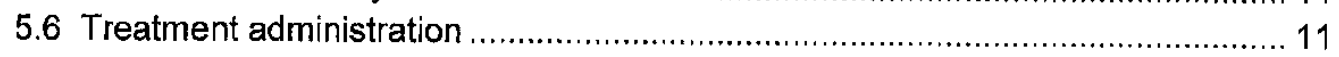

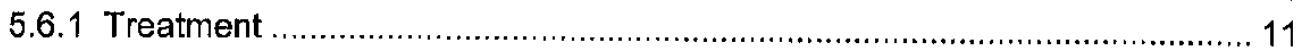

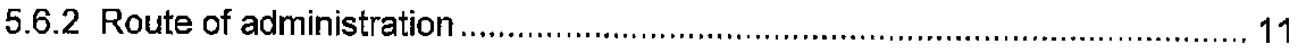

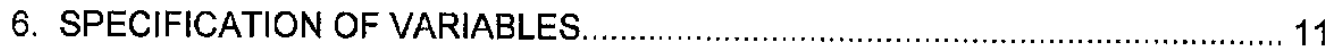

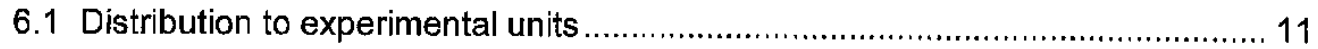

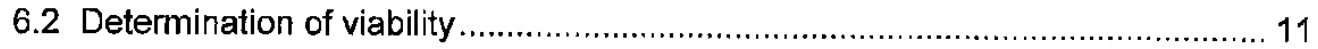

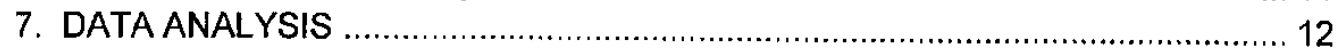

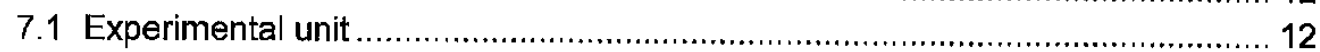

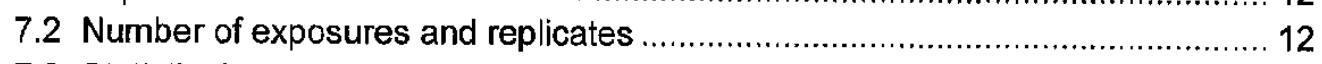

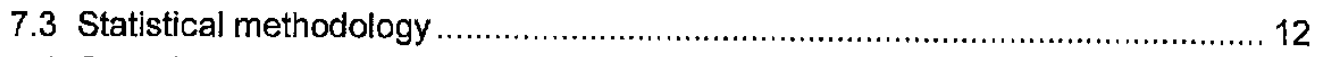

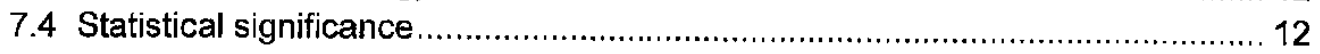

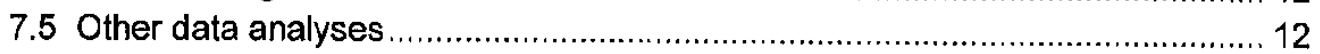

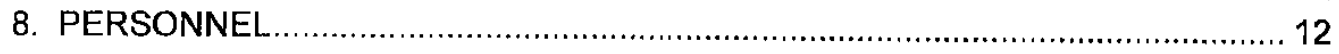

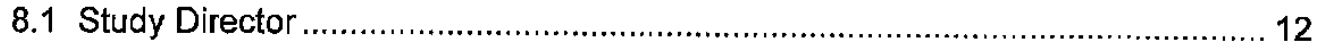

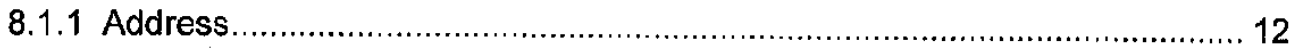

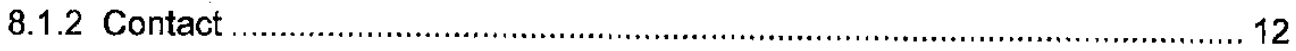

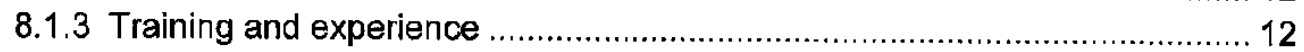

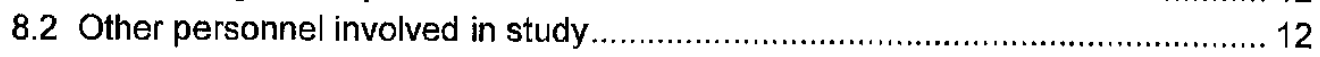

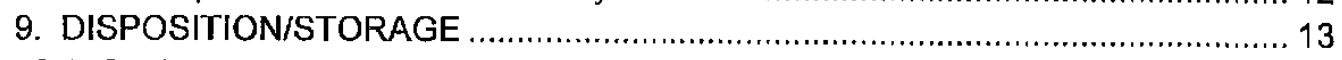

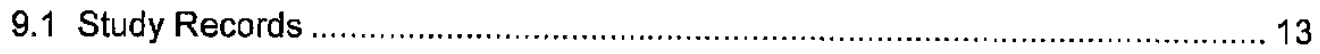

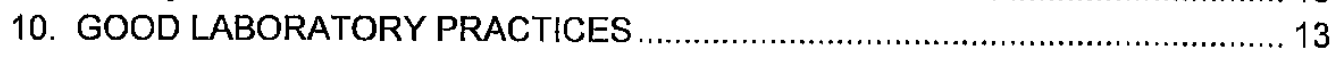

11. AMENDMENT/DEVIATIONS TO THE PROTOCOL ….................................... 13

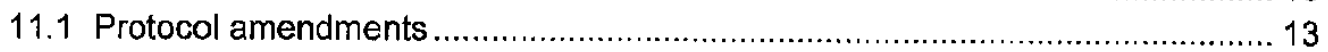

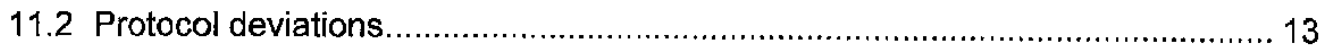

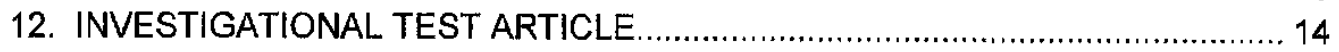

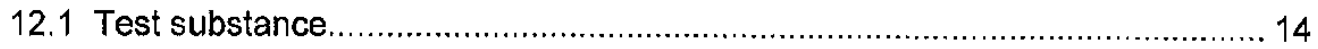

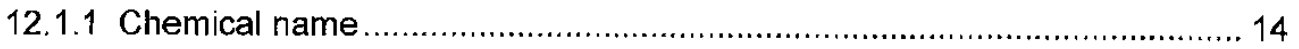

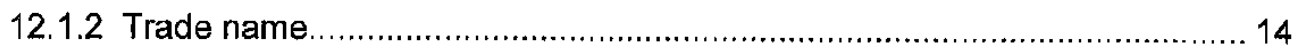

12.1.3 Active/inactive ingredients .......................................................... 14

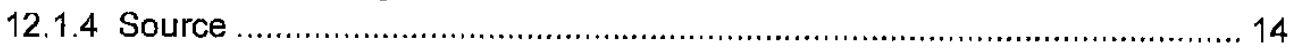

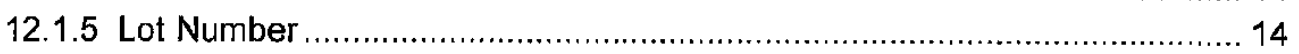

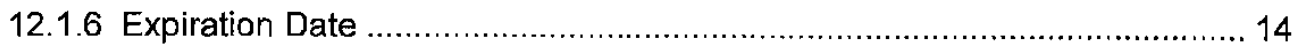

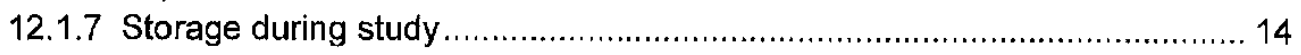

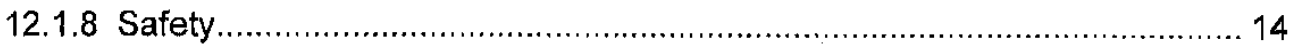

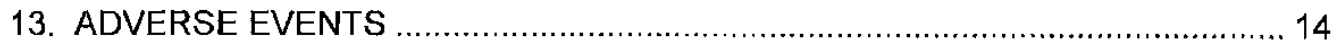

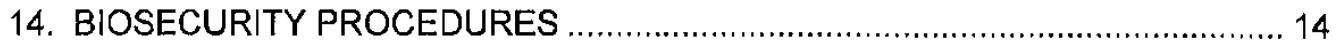

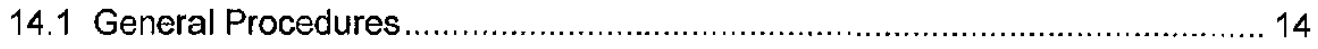

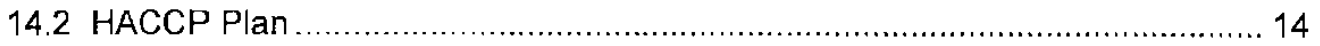




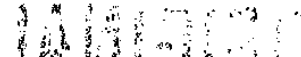

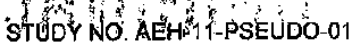

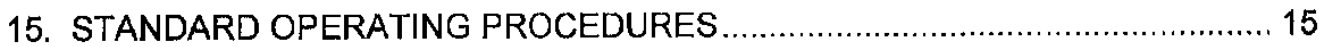

16. REFERENCES

17. APPENDIX

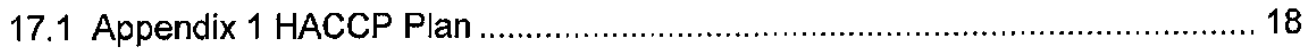


1. INTRODUCTION:

Historical native freshwater mussel populations of North America were considered the most diverse in the world with about 297 recognized taxa consisting of 281 species and 16 subspecies (Williams et al., 1993). Mussels are largely sedentary in nature, relying on movement of host fish during glochidial attachment as means of transport. Due to these facts, mussels are particularly vulnerable to a variety of anthropogenic influences including habitat degradation and alteration, pollution and over harvest. Master (1990) reported a survey conducted by the Nature Conservancy which showed $55 \%$ of North America's mussels as extinct or imperiled compared to $7 \%$ of terrestrial species which traditionally have received far greater attention. Ricciardi and Rasmussen (1999) projected that at least 127 imperiled mussel species will be lost in the next 100 years. The extinction rate was calculated to be $6.4 \%$ per decade and should be considered conservative because it did not take into account the extirpations caused by the invasive dreissenid mussels (Ricciardi and Rasmussen, 1999).

Neves et al (1997) reported the ominous status of native mussels in the Southeast with only 25 percent of the 269 species historically present reported as stable. Thirteen percent were reported as presumed extinct, 28 percent as endangered, followed by 14 percent as threatened and 18 percent listed as species of special concern. Many unionid mussel species in North America were imperiled prior to epizoic colonization by zebra mussels (Dreissena polymorpha), which has dramatically heightened concerns for their continued survival. Zebra mussels have been deemed responsible for the extirpation of unionids from waters in Europe as early as 1937 (Sebestyen, 1937). Severe declines in unionid abundance in Europe (Karatayev and Burlakova, 1995; Burlakova, 1998) and North America (Haag et al, 1993; Nalepa, 1994; Ricciardi et al., 1996) have since been well documented in the literature.

The 1973 Endangered Species Act (ESA) brought forth the need to recognize, protect and recover rare mussels in the United States. The United States Fish and Wildlife Service (USFWS) develops recovery plans for threatened and endangered species which utilize a range of tools to promote recovery of the species including restoring and acquiring critical habitat, removing introduced or invasive species and captive propagation and release into historic ranges.

As of 2004, mussel propagation work was being conducted in several different facilities in 7 states as well as in Ontario, Canada (Neves, 2004). The Genoa National Fish Hatchery (GNFH) in Wisconsin has been involved in mussel recovery since 2000, releasing tens of thousands of propagated subadult Higgins eye pearlymussel (Lampsilis higginsii) for recovery efforts. The GNFH produces subadult mussels using cage culture techniques. This technique involves placing glochidia laden host fish into 
submerged cages within natural water bodies such as the Upper Mississippi and St. Croix Rivers. The fish are released from the cages after mussel excystment and the mussels are allowed to grow on the cage bottom for an additional 6-18 months before being harvested. Areas that were previously successful in rearing mussels using this technique have been abandoned due to the proliferation and colonization by zebra mussels.

Biologists at the New York State Museum (NYSM) Field Research Laboratory have been researching dreissenid mussel control since 1991 and they discovered that a bacterium isolated from soils (Pseudomonas fluorescens [Pf-CL145A]) is efficacious for controlling zebra mussels. Marrone Bio Innovations (MB|; Davis, CA) is currently developing a commercial sprayed dried formulation of this bacterium called $\mathrm{MBI}-401$ SDP. The current commercial applications of this product are for use with closed systems such as power generating plant cooling systems. The NYSM has partnered with the USFWS (Genoa NFH) and United States Geological Survey's (USGS) Upper Midwest Environmental Sciences Center (UMESC) to determine the suitability of this product for open water zebra mussel control applications such as treatment of native mussel propagation cages or native mussel beds.

The Glochidia life stage of unionid mussels has been identified as a critical life stage and has been shown to be highly sensitive to various contaminants (ASTM E2455-06). This research will determine the animal effects of various concentrations of Pseudomonas fluorescens (Pf-CL145A) to glochidia from seven unionid mussels species present in the Great Lakes and Upper Mississippi River basins.

\section{PROTOCOL OBJECTIVE:}

This study will determine the animal effects of various concentrations of MBI-401 SDP (Pseudomonas fluorescens [Pf-CL145A]) to the glochidia lifestage of seven unionid mussels species present in the Great Lakes and Upper Mississippi River basins.

\section{STUDY SCHEDULE:}

3.1 Proposed initiation: May 2011

3.2 Schedule of events: A proposed schedule of events is provided in Table 1.

3.3 Proposed completion date: February 2012 
Table 1. Proposed Schedule of Events

\begin{tabular}{cc}
\hline Date & Activity \\
\hline May 2011-November 2011 & Glochidia exposures \\
November 2011-January 2012 & Data analysis \\
February 2012 & Final Report submission \\
\hline
\end{tabular}

\section{STUDY DESIGN:}

\subsection{General Description:}

Glochidia from 7 unionid mussel species (Table 2) endemic in the Great Lakes and Mississippi River basins will be evaluated for effects from exposure to varying concentrations of MBI-401 SDP (Pseudomonas fluorescens [Pf$\mathrm{CL} 145 \mathrm{~A}]$ ). Glochidia will be exposed in static exposures for $24 \mathrm{~h}$ as described in ASTM E-2455-06 and evaluated at 6, 12 and $24 \mathrm{~h}$ for viability as determined by valve closure response after addition of a saturated sodium chloride solution.

Table 2. Mussel species to be evaluated for MBI-401 SDP (Pseudomonas fluorescens [Pf-CL145A]) exposure effects.

\begin{tabular}{cc}
\hline Common name & Scientific name \\
\hline Black sandshell & Ligumia recta \\
Fatmucket & Lampsilis siliquoidea \\
Hickorynut & Obovaria olivaria \\
Higgins eye & Lampsilis higginsii \\
Mucket & Actinonaias ligamentina \\
Plain pocketbook & Lampsilis cardium \\
Washboard & Megalonaias nervosa \\
\hline
\end{tabular}

\subsection{Experimental Design:}

Gravid donor mussels will be collected by biologists from the Genoa National Fish Hatchery from the Upper lowa, Upper Mississippi or St. Croix Rivers. Mussels will be identified to species as described in Cummings and Mayer (1992) and Watters et al. (2009) and transported to the Genoa National Fish Hatchery. Glochidia from at least three gravid female mussels of each species will be extracted and evaluated for viability and if acceptable $(\geq 80 \%)$ glochidia groups will pooled for use by the Genoa NFH according to the procedures outlined in ASTM E2455-06 and immediately transferred to the UMESC in a 
cooler at $\sim 20^{\circ} \mathrm{C}$. Glochidia will be acclimated to test water by the addition of $50 \%$ UMESC well water at the Genoa NFH and upon arrival at UMESC. Prior to exposure initiation, viability will be assessed in a subsample of glochidia by the addition of a saturated sodium chloride solution $\left(12 \mathrm{~g} \mathrm{NaCl} / 50 \mathrm{~mL}\right.$ of D.l. $\mathrm{H}_{2} \mathrm{O}$ [ASTM E2455-06]). Viability will be assessed $\sim 1$ minute after addition of sodium chloride and must exhibit $>80 \%$ viability for use in exposures. An appropriate amount of glochidia suspension (ie: $2 \mathrm{~mL}$ ) will be randomly aliquoted to a exposure chamber ( $80 \mathrm{~mm} \times 40 \mathrm{~mm}$ crystallizing dish) containing acclimated well water to achieve a final volume of $100 \mathrm{~mL}$. Approximately 500 glochidia will be transferred to each exposure chamber in a minimum of two aliquots per chamber (ie: two $1 \mathrm{~mL}$ aliquots that contain $\sim 250$ glochidia each). Once all of the exposure chambers receive test animals the exposures will be initiated by the addition of the appropriate amount of MBI-401 SDP, deactivated MBI-401 SDP (positive control) or plain water (control) in a completely randomized design to achieve the desired test concentrations of 0 (control), $300 \mathrm{mg} / \mathrm{L}$ deactivated (positive control), or 50,100,200, and $300 \mathrm{mg} / \mathrm{L}$ of active MBI-401 SDP. The exposure chambers will be gently mixed with a glass rod or stainless steel spatula to ensure uniform distribution of the test chemical. All concentrations will be tested in triplicate for a total of 18 exposure chambers per species. exposure chambers will be held at $20^{\circ} \mathrm{C}$ in a water bath or controlled environment room and maintained on an 18:6 h light/dark cycle. Viability of a subsample of approximately 100 glochidia from each chamber will be assessed at 6,12 and $24 \mathrm{~h}$ post exposure by enumeration through a dissecting microscope.

Photomicrographs of subsamples may be recorded. The study will be terminated after $24 \mathrm{~h}$ post exposure evaluations are completed.

\section{STUDY PROCEDURES}

\subsection{Test Animals}

\subsubsection{Description:}

5.1.1.1 Age $-<24 \mathrm{~h}$ extracted glochidia.

5.1.1.2 Sex - glochidia are an immature lifestage and they cannot be identification sexually.

\subsubsection{Species - See Table 2}

5.1.2 Number of animals: Approximately 9,000 glochidia (500 per replicate $x$ 3 replicates $\times 6$ concentrations) of each species. This design uses the fewest number of glochidia possible, consistent with the objective of the study, contemporary scientific standards and ASTM guide E2455-06. 
5.1.3 Source of animals: All glochidia will be obtained from donor mussels located at the Genoa NFH.

5.1.4 Inclusion criterion: Glochidia will be used if they meet or exceed the following percentage of valve closure requirements after addition of a saturated sodium chloride solution ( $12 \mathrm{~g} \mathrm{NaCl} / 50 \mathrm{~mL}$ of D.I. $\mathrm{H}_{2} \mathrm{O}$ ). Glochidia must exhibit $\geq 80 \%$ valve closure to be pooled for use at the Genoa NFH and glochidia must exhibit $\geq 80 \%$ valve closure immediately prior study initiation at the UMESC.

5.1.5 Acclimation: Glochidia will be acclimated to UMESC well water and temperature $\left(20^{\circ} \mathrm{C}\right)$ by the addition of up to $50 \%$ UMESC well water at the Genoa NFH prior to transport to UMESC and again after arrival at the UMESC.

5.1.6 Feeding: In compliance with contemporary scientific standards, glochidia will not be fed during the exposures.

\subsection{Water Chemistry}

5.2.1 Dissolved oxygen: Dissolved oxygen will be measured and recorded at least twice during the exposure period in the control and high concentration. (UMESC SOP AEH 394 or equivalent).

5.2.2 Temperature: Temperature will be measured and recorded at least twice during the exposure period.

5.2.3 $\mathrm{pH}$ : The $\mathrm{pH}$ will be measured and recorded at least twice during the exposure period in the control and high concentration (UMESC SOP AEH 310,334 or equivalent).

5.2.4 Hardness: The hardness will be measured and recorded on dilution water prior to test initiation and upon test termination from pooled replicate samples from the control and high concentrations (UMESC SOP AEH 712 or equivalent).

5.2.5 Alkalinity: The alkalinity will be measured and recorded on dilution water prior to test initiation and upon test fermination from pooled replicate samples from the control and high concentrations (UMESC SOP AEH 706 or equivalent).

5.2.6 Conductivity: The conductivity will be measured and recorded upon test termination from pooled replicate samples of each test concentration (UMESC SOP AEH 188 or equivalent).

5.2.7 Ammonia: The ammonia will be measured and recorded at the termination of the exposure from pooled replicate samples from the controls and afl test concentrations (UMESC SOP AEH 301 or equivalent). 
5.3 Disposal: Upon study termination the glochidia in each test vessel will be euthanized by MS-222 overdose (UMESC SOP GEN 132) then disposed of by incineration.

\subsection{Study facilities:}

5.4.1Test Facility

U.S. Geological Survey, Upper Midwest Environmental Sciences Center 2630 Fanta Reed Rd

La Crosse, Wisconsin 54603

5.4.1.1 Exposure system: The test system is a series of eighteen static $80 \times 40$ $\mathrm{mm}$ crystallizing dishes ( $100 \mathrm{~mL}$ of test water) for each species, placed in a recirculating water bath system or on a bench top of a controlled environment room. Each replicate will be uniquely identified (eg: 1A2) to allow for identification of species treatment type and replicate number. Coding identification procedures will be documented in the laboratory notebook.

5.4.1.2 Aeration: Supplemental aeration will be supplied during exposures by gently bubbling in laboratory air through a pasteur pipet at a rate of approximately 1 bubble/second.

5.4.1.3 Water supply: UMESC well water will be temperature acclimated $\left(20^{\circ} \mathrm{C}\right)$ and aerated for at least $24 \mathrm{~h}$ prior to use for test water.

5.4.1.4 Lighting: Direct light may adversely affect test results, thus indirect lighting( 18 h L:6 h D; 100-1000 lux) will be provided; light intensity will be recorded at the initiation and completion of the exposure period (UMESC SOP AEH 308).

5.4.1.5 Exposure chamber dimensions: The exposure chambers are $80 \times 40 \mathrm{~mm}$ crystallizing dishes. Each exposure chamber will be filled with $100 \mathrm{~mL}$ of test water (ASTM E2455-06).

5.4.1.6 Water discharge: All water will be discharged into the UMESC invasive species isolation facility.

\subsection{Observations:}

5.5.1 Behavioral Observations: Behavioral observations will be limited to viability assessments. Viability is determined by the ability of the glochidia to constrict their adductor mussel thereby causing valve closure in response to saturated sodium chloride ( $12 \mathrm{~g} \mathrm{NaCl} 50 \mathrm{~mL}$ of D.I. $\mathrm{H}_{2} \mathrm{O}$ ) exposure. Viability will be determined on a subsample of approximately 100 glochidia at 6,12 and $24 \mathrm{~h}$ post exposure for each test chamber. 


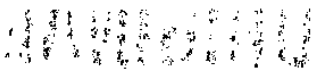

5.5.2 Water chemistry: Dissolved oxygen, $\mathrm{pH}_{1}$ and temperature will be monitored after the addition of the specified treatment. Alkalinity, hardness, conductivity and ammonia will be measured on a sample of dilution water at test initiation and on pooled replicate samples upon test termination. Alkalinity, hardness, conductivity and ammonia will not be measured during the exposures due to the quantity of test water required and probe submersion requirements to complete the analysis.

5.6 Treatment administration:

5.6.1 Treatment: Each species of mussel glochidia will be exposed to three replicates of either 0 (control), 50,100,200 or $300 \mathrm{mg} / \mathrm{L}$ (active ingredient) and a $300 \mathrm{mg} / \mathrm{L}$ heat deactivated $\left(70^{\circ} \mathrm{C} / 45\right.$ minutes) control as a one time single dose exposure.

5.6.2 Route of administration: To assure uniform exposure, an appropriate amount of freshly prepared stock solution will added to each test chamber from an agitated stock suspension with a pipet.

\section{SPECIFICATION OF VARIABLES}

6.1 Distribution to experimental units: The glochidia concentration (glochidia/mL) will be determined for each species prior to test initiation by enumeration of replicate subsamples of glochidial suspension through a dissecting microscope.

Appropriate volumes $(\mathrm{mL})$ will be determined that would contain approximately 500 glochidia. Test chambers will then be filled with appropriate amounts of dilution water to obtain a final volume of $100 \mathrm{~mL}$. Distribution to test chambers will then proceed by the addition of the calculated volume of suspension in at least two separate aliquots. Aliquots will be randomly assigned to a test chamber until all receive one aliquot. This procedure will then be repeated until all chambers receive approximately 500 glochidia in two or more aliquots. The test chambers will be randomly assigned treatment concentration.

6.2 Determination of viability: Viability will determined by the ability of the glochidia to constrict their adductor mussel thereby causing valve closure in response to saturated sodium chloride $\left(12 \mathrm{~g} \mathrm{NaCl} / 50 \mathrm{~mL}\right.$ of D.I. $\mathrm{H}_{2} \mathrm{O}$ ) exposure. Viability will be determined on a subsample of approximately 100 glochidia for each species prior to test initiation and at 6,12 and $24 \mathrm{~h}$ post exposure for each test replicate. A volume of water containing approximately 100 glochidia (eg: $1-2 \mathrm{~mL}$ ) will be placed on a $35 \mathrm{~mm}$ mini petri dish and examined under a dissecting scope to enumerate the percentage of glochidia with closed valves. Then approximately 1 drop of a saturated sodium chloride solution will be added to the sample and the glochidia will then be reassessed for valve closure after approximately one 
minute. Subsample photomicrographs of glochidia pre and post sodium chloride addition may be recorded and uniquely identified for documentation and optional later enumeration and/or validation of closure percentages. Only glochidia that are open and responsive to sodium chloride addition will be considered viable.

\section{DATA ANALYSIS}

7.1 Experimental unit: The experiment unit will be the exposure chamber.

7.2 Number of exposures and replicates: There will be a total of 6 treatment levels (control, positive control (heat treated Pf-CL145A) 50, 100, 200 and $300 \mathrm{mg} P f$ CL145A/L) for each replicate and three replicate exposure chambers per treatment level for a total of 18 exposure chambers for each mussel species.

7.3 Statistical methodology:

Viability data will be analyzed using a generalized linear mixed model (SAS PROC GLIMMIX). In every analysis, the exposure chamber will be treated as the experimental unit. The change in proportion of viability will be analyzed using a generalized linear mixed model where the distribution is binomial and the link used is the logit function.

If a significant effect of treatment is identified then pairwise comparison tests will be completed to compare each treatment group to the control group using unadjusted least squares means.

7.4 Statistical significance: Statistical significance will be declared at $p<0.05$.

7.5 Other data analyses: Statistical methods for other study data collected will include calculation of means, standard deviations and coefficients of variation.

\section{PERSONNEL}

8.1 Study Director: James A. Luoma, B.A.

8.1.1 Address: Upper Midwest Environmental Sciences Center, US Geological Survey, 2630 Fanta Reed Rd., La Crosse, Wisconsin 54603

8.1.2 Contact: Tel: (608) 781-6391, Fax: (608) 783-6066; jluoma@usgs.gov

8.1.3 Training and experience: CV on file at UMESC.

8.2 Other personnel involved in study: Technical staff involved in the study will be identified in the study raw data to include study function. UMESC technical staff training and experience will be documented in CVs included in the study raw data. 


\section{DISPOSITION/STORAGE}

9.1 Study Records: All data generated in the study at UMESC will be recorded in bound laboratory notebooks or kept in file folders (SOP No. GEN 008). All data sheets, file folders, laboratory notebooks and computer disks will be encoded with the study number when the data are generated and stored in secure files (SOP No. GEN 008). Raw data, laboratory notebooks and electronic files (including a CD-ROM containing the annotated SAS program used for the statistical analysis, the data files, SAS log and SAS output files) generated by UMESC and contract laboratory reports will be filed in the UMESC archives (SOP No. GEN 007) of the Upper Midwest Environmental Sciences Center, LaCrosse Wisconsin, before the final report is signed by the Study Director. The final report will then be signed and archived.

\section{GOOD LABORATORY PRACTICES}

Data collection, storage and retrieval procedures for the study will be conducted in compliance with FDA regulations for Good Laboratory Practices (GLP; 21 CFR, Part 58). The study protocol and progress of the study will be reviewed at the start of the study and periodically throughout the study by the Quality Assurance Unit (QAU). The Study Director has the responsibility of ensuring that all procedures used in conjunction with the study conform with Good Laboratory Practices.

\section{AMENDMENT/DEVIATIONS TO THE PROTOCOL}

11.1 Protocol amendments: A signed copy of the Study Protocol will be retained on-site. Proposed amendments to the protocol shall be brought to the attention of UMESC Management. When the Study Director and Management agree verbally, the study can proceed with the change. As soon as possible, the Study Director will then prepare a written protocol amendment that is signed by the Study Director, Branch Chief, UMESC Center Director, and UMESC-QA. The UMESC statistician or UMESC Animal Care and Use Chair may also sign as needed. The amendment then becomes an official part of the protocol.

11.2 Protocol deviations: All deviations from this approved protocol will be documented and reviewed by the Study Director. The Study Director will make a judgment on the impact of the deviations. The Study Director will notify Management, UMESC-QA, as soon as possible, in writing, of any deviations to the protocol, including their impact on the study. 
12. INVESTIGATIONAL TEST ARTICLE

12.1 Test Substance(s): MBI-401 SDP, Pseudomonas fluorescens (Pf-CL145A)

12.1.1 Chemical name: Pseudomonas fluorescens (Pf-CL145A)

12.1.2 Trade name: $\mathrm{MBI}-401$ SDP

12.1.3 Active ingredients: Pseudomonas fluorescens (Pf-CL145A) is the sole active ingredient, $50 \%$ active by weight.

12.1.4 Source: Marrone Bio Innovations (MBI); Davis, CA

12.1.5 Lot number: None established

12.1.6 Expiration date: None established

12.1.7 Storage during study: test chemical will be stored at refrigerated in a locked container within a restrictive entry laboratory. A subsample of the test chemical will be archived in the UMESC Chemical Archive (UMESC SOP GEN 011).

12.1.8 A NIOSH approved respirator will be used when preparing stock solutions to avoid inhalation. Protective eyewear, gloves and lab coats will be worn at all times when working with the test substance.

13. ADVERSE EVENTS: Any adverse event will be recorded in the study logbook and the Study Director will be notified.

\section{BIOSECURITY PROCEDURES}

14.1 General Procedures: All personnel involved in the study will review the UMESC biosecurity (UMESC SOP APP 075) and project HACCP plans. Testing will be conducted in a laboratory with controlled access. All effluent and sample water will be iodine or chlorine disinfected prior to discharge.

Biosecurity procedures outlined in UMESC SOP APP 075.0 will be followed for samples and equipment.

14.2 HACCP Plan: See Appendix 1 for the HACCP plan for this project. 


\section{STANDARD OPERATING PROCEDURES}

UMESC SOP GEN 007 - Archives Management for Regulated Studies UMESC SOP GEN 008 - Maintenance of Data Recording of Raw Data for Regulated Studies UMESC SOP GEN 012 - Receipt, Identification, Storage, Handling, Checkout and Documentation of Use of Test Chemicals

UMESC SOP APP 075 - Procedures to Minimize the Risk of Transfer of Pathogens and Invasive Species

UMESC SOP GEN 132 - Care, Maintenance \& Disposal of Aquatic Vertebrates UMESC SOP AEH 011 - Procedures for Labeling Chemicals and Specimens. UMESC SOP AEH 213 - Sartorius Balance, Model LC34000P, Serial 30303922 UMESC SOP AEH 188 - Accumet Portable Waterproof Conductivity meter Model \# AP75

UMESC SOP AEH 301 - Instrument Operating Procedure: YSI Photometer Model \# 9000 Serial \# 3638017

UMESC SOP AEH 308 - Instrument Operating Procedure: Milwaukee Light Meter Model \# SM7000 Serial \# 727298

UMESC SOP AEH 310 - Hanna pH Meter, Model HI991001, Serial Number 370973

UMESC SOP AEH 334 - Beckman Portable pH/mV Meter, Model F210, Serials 330167 \& 330168

UMESC SOP AEH 338 - Sartorius Model BP 3100S, Serial Number 12907582 UMESC SOP AEH 394 - YSI Handheld Dissolved Oxygen Meter, Model 55/12FT, Serials 94C17261 \& 97F0837AG

UMESC SOP AEH 606 - Methods Used to Weigh, Measure \& Mark Test Animals UMESC SOP AEH 706 - Determination of Total Alkalinity by the Titrimetric $(\mathrm{pH} \mathrm{4.5)}$ Method

UMESC SOP AEH 712 - Determination of Total Hardness UMESC SOP AEH 903 - Verification of Thermometer \& Temperature Recorder Calibration UMESC 


\section{REFERENCES.}

American Society for Testing and Materials. 2006. Standard guide for conducting laboratory toxicity tests with freshwater mussels. E2455-06. In Annual Book of ASTM Standards, Vol 11.06. Philidelphia, PA.

Burlakova, L.E., 1998. Ecology of Dreissena polymorpha (PALLAS) and its role in the structre and function of aquatic ecosystems. Candidate dissertation, Zoology Institute of the Academy of Science Republice Belarus, 168 p. (in Russian) in Burlakova L.E., A.Y. Karatayev and D. K. Padilla. 2000. The impact of Dreissena polymorpha (PALLAS) invasion on Unionid bivalves. Internat. Rev. hydrobiol. 85 (5-6):529-541.

Cummings S.K. and C.A. Mayer, 1992. Field guide to freshwater mussels of the Midwest. Illinois Natural History Survey Manual 5. 194 pp.

Haag, W.R., D.L. Berg, D.W. Garton, and J.L. Farris. 1993. Reduced survival and fitness in native bivalves in response to fouling by the introduced zebra mussel (Dreissena polymorpha) in western Lake Erie. Canadian Journal of Fisheries and Aquatic Sciences 50(1):13-19.

Karatayev, A.Y. and L.E. Burlakova, 1995. Present and further patterns in Dreissena population development in the Narochanskaya lakes system. Vestisi Akad. Navuk Belarusi. Ser. Biyol. Navuk 3: 95098 (in Belarussian) in Burlakova L.E., A.Y. Karatayev and D. K. Padilla. 2000. The impact of Dreissena polymorpha (PALLAS) invasion on Unionid bivalves. Internat. Rev. hydrobiol. 85 (5-6):529-541.

Master, L. 1990. The imperiled status of North American aquatic animals. Biodiversity Network News 3(3):5-8.

Napela, T.F., 1994. Decline of native unionid bivalves in Lake St. Clair after infestation by the zebra mussel, Dreissena polymorpha. Can. J. Fish. Aquat. Sci. 51:2227-2233.

Neves R. J. 2004. Propagation of endangered freshwater mussels in North America. Journal of Conchology, special publication 3:69-80.

Neves, R.J., A.E. Bogan, J.D. Williams, S.A. Ahlstedt, and P.W. Hartfield. 1997. Stafus of aquatic mollusks in the southeastern United States: a downward spiral of diversity. Pages 43-85 in G.W. Benz and D.E. Collins, editors. Aquatic fauna in peril: the southeastern perspective. Southeastern Aquatic Research Institute, Lenz Design and communications, Decatur, Georgia. 
Ricciardi, A. F.G. Whoriskey, and J.B. Rasamussen. 1996. Impact of the Dreissena invasion on native unionid bivalves in the upper St. Lawerance River. Can. J. Fish. Aquat. Sci. 53:1434-1444.

Ricciardi A., and J.B. Rasmussen. 1999. Extinction rates of North American freshwater fauna. Conservation Biology. Vol. 13 (5):1220-1222.

Sebestyn, O. 1937. Colonization of two new fauna-elements of Pontus-origin (Dreissena polymorpha Pall. And Corophuim curvispinum G.O. Sars forma devium Wundsch) in Lake Balaton, Verh. Int. Ver. Theor. Angew. Limnol. 8:169-182 in Burlakova L.E., A.Y. Karatayev and D. K. Padilla. 2000. The impact of Dreissena polymorpha (PALLAS) invasion on Unionid bivalves. Internat. Rev. hydrobiol. 85 (56):529-541.

Watters, G.T., M.A. Hoggarth and D.H. Stansbery. (2009) The Freshwater Mussels of Ohio. The Ohio State University Press, Columbus, OH. 421 pp.

Williams, J.D., M.L. Warren Jr., K.S. Cummings, J.L. Harris, and R.J. Neves. 1993. Conservation status of freshwater mussels of the United States and Canada. Fisheries 18(9):6-22. 


\section{APPENDIX.}

\subsection{Appendix 1. HACCP PLAN for the study Effects of Pseudomonas fluorescens (Pf-CL145A) to glochidia from seven unionid mussel species}

Step 1 - Activity Description

\begin{tabular}{|c|c|}
\hline $\begin{array}{l}\text { Facility: US Geo oglcal Survey-Upper Midwest Environmental Scłences } \\
\text { Center }\end{array}$ & Site: Lower B, rooms 15,16 and 17 \\
\hline Site Coordinator: Jim Luoma & \multirow{4}{*}{$\begin{array}{l}\text { Activlty: Determine the effects of various concentrations of } \\
\text { Pseudomonas fluorescens }(P f-C l .145 \mathrm{~A}) \text { to the glochidia lifestage of } \\
\text { seven unlonid mussels specles present in the Great Lakes and Upper } \\
\text { Mississippi River basins. }\end{array}$} \\
\hline Site Manager: Mark Galkowski & \\
\hline $\begin{array}{l}\text { Address: } 2630 \text { Fanta Reed Road } \\
\text { La Crosse WI, } 54601\end{array}$ & \\
\hline Phone: $608-781-6322$ & \\
\hline
\end{tabular}

\section{Project Description}

The objective of this study is to determine effects of various concentrations of Pseudomonas fivorescens (Pf-CL145A) to the glochidla lifestage of sever. unionld mussels species present In the Great Lakes and Upper Mississippi River basins.

Step 2 - Potentlal Hazards: Species whlch may potentially be moved/introduced

Vertebrates:

None

invertebrates

Zebra mussel (Dreissena polymorpha)

Plants: None

Other biologicals (dlsease, pathogen, parasite)

Largemouth Bass Virus

Spring Viremia of Carp Virus

B:uegill Virus

Infectious Pancreatic Necrosis Virus

Viral Hemorrhagic Septicemla

Furunculosis Aeromonas salmonicida

Enteric Redmouth Disease Yersinia ruckeri

Bacterial Kidney Dlsease Renibacterlum salmoninarum

Other Assorted parasites/pathogens commonly found in the upper Mississippi River Basin

Other (construction materials)

NA

Step 3-Flow Diagram

Flow dlagram outlining sequentlal tasks to complete activlty/project 


\begin{tabular}{|c|c|c|c|c|c|}
\hline Task 1 & \multicolumn{5}{|c|}{ Musse glochidia are obtalned from the Genoa NFH and transferred to UMESC. } \\
\hline Task 2 & \multicolumn{5}{|c|}{ Mussel glochidia distributed to test chambers in the invasive species laboratory } \\
\hline & & & & & \\
\hline Task 3 & \multicolumn{5}{|c|}{ Mussel g ochidia are removed for enumeration } \\
\hline Task 4 & \multicolumn{5}{|c|}{ Water samples collected and analyzed for chemlcal parameters } \\
\hline \multirow{2}{*}{$\frac{1}{\text { Task } 5}$} & \multirow{2}{*}{\multicolumn{5}{|c|}{$\begin{array}{l}\text { After exposure, the glochldia will be euthanlzed using a lethal dose of MS-2.22 and dlscarded according to UMESC SOPS } \\
\text { GEN 132. }\end{array}$}} \\
\hline & & & & & \\
\hline 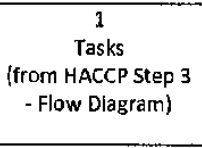 & $\begin{array}{l}2 \\
\text { Potential hazards } \\
\text { Identified in HACCP } \\
\text { Step } 2\end{array}$ & $\begin{array}{l}\text { Are any potentlal } \\
\text { hazards probable? } \\
\text { (yes/no) }\end{array}$ & $\begin{array}{l}\text { Justlfy evaluation for } \\
\text { column } 3\end{array}$ & $\begin{array}{c}5 \\
\text { What control } \\
\text { measures can be } \\
\text { app'jed to prevent } \\
\text { undesirable results? }\end{array}$ & $\begin{array}{l}\text { Is this task a critical } \\
\text { control point? } \\
\text { (yes/ro) }\end{array}$ \\
\hline
\end{tabular}

\begin{tabular}{|c|c|c|c|c|c|}
\hline \multirow{4}{*}{$\begin{array}{l}\text { Task } 1 \\
\text { Mussel glochidia are } \\
\text { obtained from the } \\
\text { Genoa NFH anc } \\
\text { transferred to } \\
\text { UMESC. }\end{array}$} & Vertebrates & No & $\begin{array}{l}\text { Mussel glochldia will } \\
\text { be transported in } \\
\text { clean well water and } \\
\text { the will not be } \\
\text { vertebrates present }\end{array}$ & $\mathrm{N} / \mathrm{A}$ & no \\
\hline & Invertebrates & yes & $\begin{array}{l}\text { Mussel glochidia will } \\
\text { be callected from } \\
\text { donor mussels } \\
\text { collected from wild } \\
\text { sources. }\end{array}$ & $\begin{array}{l}\text { Assure donor mussels } \\
\text { have been cleaned and } \\
\text { rinsed with well water } \\
\text { prior to use, Effluent } \\
\text { water Is treated and } \\
\text { equipment will be } \\
\text { disinfected. Equipment } \\
\text { remalns in the } \\
\text { laboratory. }\end{array}$ & ves \\
\hline & Plants & No & $\begin{array}{l}\text { The donor mussels } \\
\text { are cleaned upan } \\
\text { collection and held in } \\
\text { clean well water } \\
\text { prior to use. }\end{array}$ & $\begin{array}{l}\text { Any plant material will } \\
\text { be removed from } \\
\text { conor mussels and } \\
\text { equipment before } \\
\text { transporting Genoa } \\
\text { NFH. }\end{array}$ & no \\
\hline & Others & No & $\begin{array}{l}\text { The potential } \\
\text { transfer of flsh } \\
\text { diseases from } \\
\text { glochidla harvested } \\
\text { from donor mussels } \\
\text { Is extremely low. }\end{array}$ & $\begin{array}{l}\text { Effluent water is } \\
\text { treated and } \\
\text { equipment will be } \\
\text { disinfected. Equipment } \\
\text { remalns In the } \\
\text { laboratory. }\end{array}$ & no \\
\hline
\end{tabular}

\begin{tabular}{|c|c|c|c|c|c|}
\hline \multirow{4}{*}{$\begin{array}{l}\text { Task } 2 \\
\text { Mussel glochidia } \\
\text { distributed to test } \\
\text { chambers in the } \\
\text { invasive species } \\
\text { laboratory }\end{array}$} & Vertebrates & no & $\begin{array}{l}\text { Risk eliminated in } \\
\text { Task } 1\end{array}$ & $\mathrm{~N} / \mathrm{A}$ & no \\
\hline & invertebrates & no & $\begin{array}{l}\text { Risk eliminated in } \\
\text { Task } 1\end{array}$ & $\begin{array}{l}\text { Effluent water is } \\
\text { treated and } \\
\text { equipment will be } \\
\text { disinfected. Equipment } \\
\text { remalns in the } \\
\text { laboratary. }\end{array}$ & no \\
\hline & Piants & no & $\begin{array}{l}\text { Risk eliminated in } \\
\text { Task } 1\end{array}$ & $N / A$ & no \\
\hline & Others & no & $\begin{array}{l}\text { The potential } \\
\text { transfer of fish } \\
\text { diseases froru } \\
\text { glochidia has never } \\
\text { been documented. }\end{array}$ & $\begin{array}{l}\text { Effluent water is } \\
\text { treated and } \\
\text { equipment will be } \\
\text { disinfected. Equipment } \\
\text { remains in the } \\
\text { laboratory. }\end{array}$ & no \\
\hline
\end{tabular}




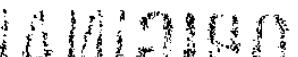

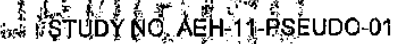

ORIGINAL

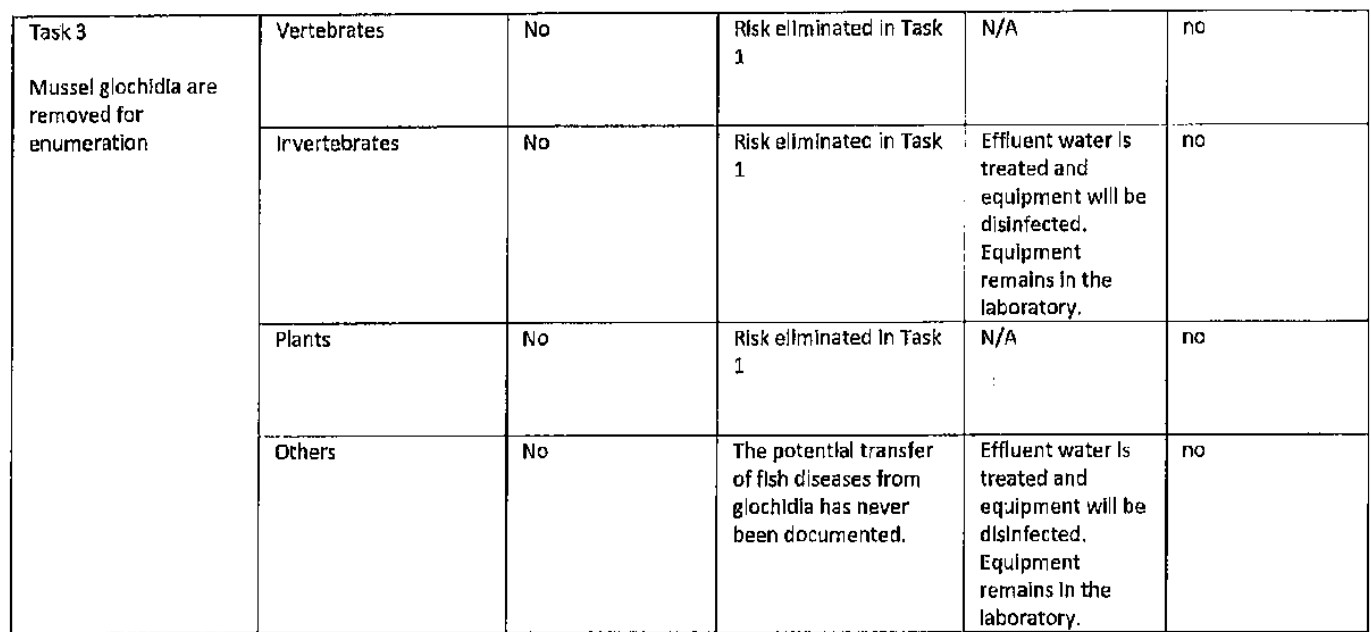

\begin{tabular}{|c|c|c|c|c|c|}
\hline \multirow{4}{*}{$\begin{array}{l}\text { Task 4 } \\
\text { Water samples } \\
\text { collected and analyzed } \\
\text { for chemlcal } \\
\text { parameters }\end{array}$} & Vertebrates & No & $\begin{array}{l}\text { Risk eliminated in Task } \\
1\end{array}$ & $N / A$ & no \\
\hline & Invertebrates & No & $\begin{array}{l}\text { Risk eliminated in Task } \\
1\end{array}$ & $\begin{array}{l}\text { Effluent water is } \\
\text { treated and } \\
\text { equipment will be } \\
\text { cisinfected. } \\
\text { Equipment } \\
\text { remains In the } \\
\text { laboratory. }\end{array}$ & no \\
\hline & Plants & No & $\begin{array}{l}\text { Rlsk eliminated in Task } \\
1\end{array}$ & $\mathrm{~N} / \mathrm{A}$ & no \\
\hline & Others & No & $\begin{array}{l}\text { The potential transfer } \\
\text { of fish diseases from } \\
\text { glochldla has never } \\
\text { been documented. }\end{array}$ & $\begin{array}{l}\text { Effluent water is } \\
\text { treated and } \\
\text { equipment w:l be } \\
\text { disinfected. } \\
\text { Equipment } \\
\text { remains in the } \\
\text { laboratory. }\end{array}$ & no \\
\hline
\end{tabular}

\begin{tabular}{|c|c|c|c|c|c|}
\hline $\begin{array}{l}\text { Task } 5 \\
\text { After exposure, the }\end{array}$ & Vertebrates & No & $\begin{array}{l}\text { Risk eliminated in Task } \\
1\end{array}$ & $N / A$ & no \\
\hline $\begin{array}{l}\text { euthanized usirg a } \\
\text { lethal dose of MS-222 } \\
\text { and discarded } \\
\text { according to UMESC }\end{array}$ & Invertebrates & No & $\begin{array}{l}\text { Risk eliminated in Task } \\
1\end{array}$ & $N / A$ & no \\
\hline 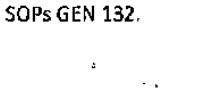 & Plarts & No & $\begin{array}{l}\text { Risk ellminated in Task } \\
1\end{array}$ & $\mathrm{~N} / \mathrm{A}$ & no \\
\hline
\end{tabular}




\begin{tabular}{|l|l|l|l|l|l|}
\hline & Others & No & $\begin{array}{l}\text { The potential transfer } \\
\text { of fish diseases from } \\
\text { glochldia has never } \\
\text { been documented. }\end{array}$ & $\begin{array}{l}\text { Effluent water is } \\
\text { treated and } \\
\text { equipment will be } \\
\text { disinfected. } \\
\text { Equipment } \\
\text { remains in the } \\
\text { laboratory. }\end{array}$ & \begin{tabular}{l} 
no \\
\hline
\end{tabular} \\
\hline
\end{tabular}

HACCP Plan Form

(all CCP's or "yes's" from column 6 of HACCP Step 4- Hazard Analysis Worksheet)

\begin{tabular}{|c|c|c|c|c|c|c|c|c|}
\hline & & & \multicolumn{4}{|c|}{ Monitaring } & \multirow[b]{2}{*}{$\begin{array}{c}\text { Evaluation \& } \\
\text { Correctlve } \\
\text { Action(s) } \\
\text { (If needed) }\end{array}$} & \multirow[b]{2}{*}{$\begin{array}{l}\text { Supporting } \\
\text { Documentation } \\
\text { (if any) }\end{array}$} \\
\hline $\begin{array}{l}\text { Critical } \\
\text { Control } \\
\text { Polnt } \\
\text { [CCP] } \\
\end{array}$ & $\begin{array}{c}\text { Signlficant } \\
\text { Haxard(s) }\end{array}$ & $\begin{array}{l}\text { Limits for each } \\
\text { Control Measure }\end{array}$ & What & How & Frequency & Who & & \\
\hline $\begin{array}{l}\text { Task } 1 \\
\text { Invertebrates }\end{array}$ & $\begin{array}{c}\text { Invertebrate } \\
\text { transfer }\end{array}$ & \begin{tabular}{|c|} 
Invertebrates \\
cannot be \\
transferred. \\
Donor missels \\
must be cleanec \\
and rinsed \\
Effluent water \\
must be captured \\
and treated.
\end{tabular} & $\begin{array}{c}\text { Macro/Micro } \\
\text { scopic } \\
\text { Invertebrates }\end{array}$ & $\begin{array}{l}\text { Mancally } \\
\text { remove } \\
\text { inverts } \\
\text { Clean and } \\
\text { rinse donor } \\
\text { mussels } \\
\text { effluent } \\
\text { water } \\
\text { disinfection } \\
\end{array}$ & $\begin{array}{c}\text { Da'ly } \\
\text { inspections } \\
\text { Immeciately } \\
\text { upan animal } \\
\text { or sample } \\
\text { transfer }\end{array}$ & All Staff & $\begin{array}{l}\text { Supervisor and staff } \\
\text { are responslble for } \\
\text { careful attention to } \\
\text { detail-Disinfection of } \\
\text { equipment }\end{array}$ & Records in log book \\
\hline \multicolumn{5}{|c|}{$\begin{array}{l}\text { Facility: } \\
\text { Upper Midwest Environmental Sciences Center }\end{array}$} & \multicolumn{4}{|c|}{$\begin{array}{l}\text { Activity: Determine the effects of various concentrations of } \\
\text { Pseudomonas fluarescens (Pf-CL14SA) to the glochidia llfestage of } \\
\text { seven unionid mussels. }\end{array}$} \\
\hline \multicolumn{9}{|c|}{$\begin{array}{l}\text { Address: } \\
2630 \text { Fanta Reed Road, La Crossc, WI } 54601\end{array}$} \\
\hline \multicolumn{5}{|c|}{$\begin{array}{l}\text { Signature: } \\
\text { HACCP Plan was followed. }\end{array}$} & \multicolumn{4}{|l|}{ Date: } \\
\hline
\end{tabular}

FOLDERNO. 3

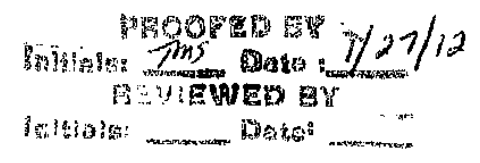




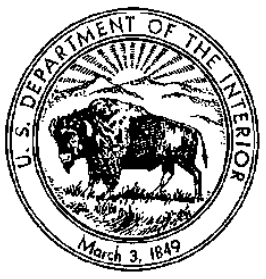

United States Department of the Interior

U.S. GEOLOGICAL SURVEY Biological Resources Division

Upper Midwest Environmental Sciences Center

2630 Fanta Reed Road

La Crosse, Wisconsin 54603

\section{OBIGINAL}

Date: May 9, 2011

To: The Record Study Number AEH-11PSEUDO-01

Subject: Amendment 1- Amendment to the study AEH-11-PSEUDO-01 "Effects of Pseudomonas fluorescens ( $P f$-CL145A) to glochidia from seven unionid mussel species"

Revision of Study Protocol, Study \# AEH-11PSEUDO-01 is proposed as detailed on pages 2-6 of this amendment. Revised text is indicated in bold.

This amendment 1) changes the physical location where glochidia are extracted from the gravid female mussels and adds a description of the acclimation procedures applied to the female mussels and glochidia; 2) adds the use of photomicrographs to enumerate glochidia; 3 ) changes the glochidia distribution and sampling methods; 4) adds the requirements to determine stock and test solution absorbance; and 5) includes the test material lot number and post-testing activity determination.

The amendment adjusts the glochidia collection methods to minimize the time period between collection and testing and maximizes acclimation to test conditions. The amendment adjusts the procedures used to enumerate, distribute and sample glochidia based on preliminary observations. The amendment also adds methods to assess the stock solution concentration. Last, the amendment adds the test material lot number and post-testing activity assessment.

Reviewed by:

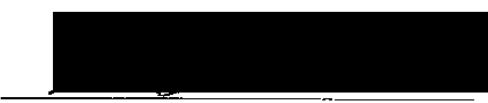

Mark P. Gaikowski, M.A.

Supervisory Biologist

Aquatic Ecosystem Health,

UMESC $^{1}$

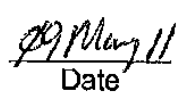

Date

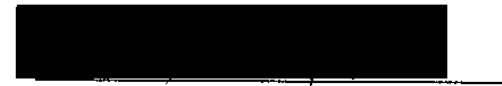

Jane E. Rivera, B.A?

Acting Quality Assurance Officer, UMESC
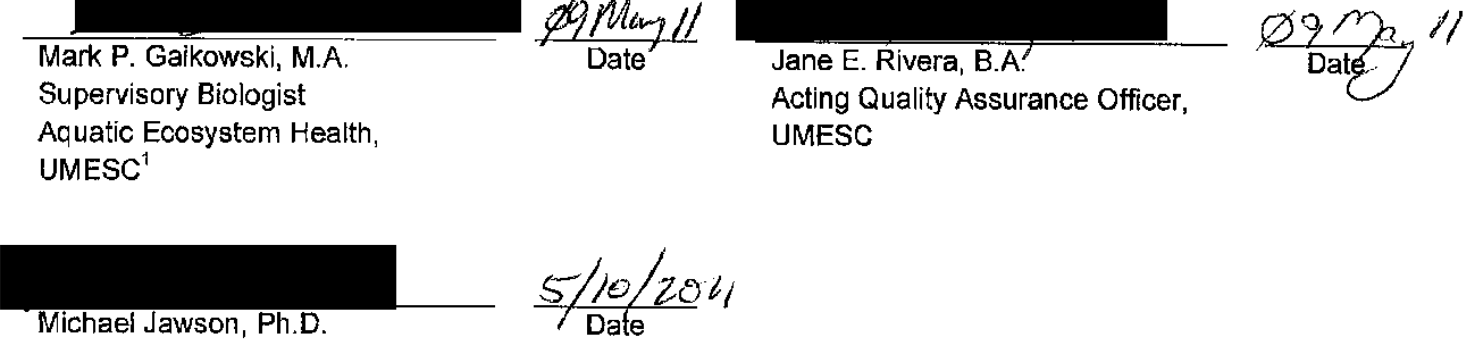

Center Director, UMESC

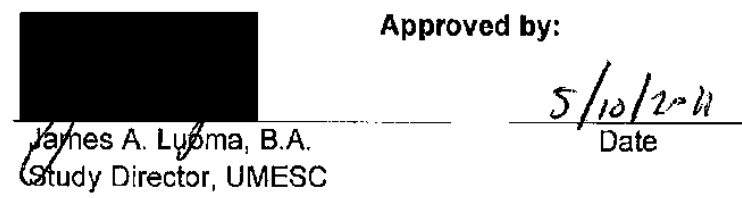

${ }^{1}$ UMESC: U.S. Geological Survey, Upper Midwest Environmental Sciences Center PQOOH

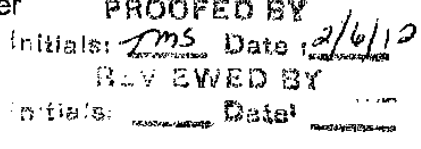

Study\# AEH-11-PSEUDO-01 Amendment \#1

Page 1 of 6 
Current text:

4.2 Experimental Design:

Gravid donor mussels will be collected by biologists from the Genoa National Fish Hatchery from the Upper lowa, Upper Mississippi or St. Croix Rivers. Mussels will be identified to species as described in Cummings and Mayer (1992) and Watters et al. (2009) and transported to the Genoa National Fish Hatchery. Glochidia from at least three gravid female mussels of each species will be extracted and evaluated for viability and if acceptable $(\geq 80 \%)$ glochidia groups will pooled for use by the Genoa NFH according to the procedures outlined in ASTM E2455-06 and immediately transferred to the UMESC in a cooler at $\sim 20^{\circ} \mathrm{C}$. Glochidia will be acclimated to test water by the addition of $50 \%$ UMESC well water at the Genoa NFH and upon arrival at UMESC. Prior to exposure initiation, viability will be assessed in a subsample of glochidia by the addition of a saturated sodium chloride solution (12 $\mathrm{g} \mathrm{NaCl} / 50 \mathrm{~mL}$ of D.I. $\mathrm{H}_{2} \mathrm{O}$ [ASTM E2455-06]). Viability will be assessed $\sim 1$ minute after addition of sodium chloride and must exhibit $>80 \%$ viability for use in exposures. An appropriate amount of glochidia suspension (ie: $2 \mathrm{~mL}$ ) will be randomly aliquoted to a exposure chamber ( $80 \mathrm{~mm} \times 40 \mathrm{~mm}$ crystallizing dish) containing acclimated well water to achieve a final volume of $100 \mathrm{~mL}$. Approximately 500 glochidia will be transferred to each exposure chamber in a minimum of two aliquots per chamber (ie: two $1 \mathrm{~mL}$ aliquots that contain $\sim 250$ glochidia each). Once all of the exposure chambers receive test animals the exposures will be initiated by the addition of the appropriate amount of MBI-401 SDP, deactivated $\mathrm{MBI}-401 \mathrm{SDP}$ (positive control) or plain water (control) in a completely randomized design to achieve the desired test concentrations of 0 (control), $300 \mathrm{mg} / \mathrm{L}$ deactivated (positive control), or $50,100,200$, and $300 \mathrm{mg} / \mathrm{L}$ of active MBI-401 SDP. The exposure chambers will be gently mixed with a glass rod or stainless steel spatula to ensure uniform distribution of the test chemical. All concentrations will be tested in triplicate for a total of 18 exposure chambers per species. exposure chambers will be held at $20^{\circ} \mathrm{C}$ in a water bath or controlled environment room and maintained on an 18:6 h light/dark cycle Viabillty of a subsample of approximately 100 glochidia from each chamber will be assessed at 6,12 and $24 \mathrm{~h}$ post exposure by enumeration through a dissecting microscope. Photomicrographs of subsamples may be recorded. The study will be terminated after $24 \mathrm{~h}$ post exposure evaluations are completed.

Revised text (in bold):

4.2 Experimental Design:

Gravid donor mussels will be collected by biologists from the Genoa National Fish Hatchery from the Upper lowa, Upper Mississippi or St. Croix Rivers. Mussels will be identified to species as described in Cummings and Mayer (1992) and Watters et al. (2009) and transported to the Genoa National Fish Hatchery. Approximately $72 \mathrm{~h}$ prior to test initiation the donor mussels will be transferred from the Genoa NFH to the UMESC and acclimated to test temperature. Glochidia from at least three gravid female mussels of each species will be extracted by personnel from the Genoa NFH. The viability of glochidia from each mussel will be assessed by drawing aliquots (e.g. $100 \mu \mathrm{L})$ of settled glochiclia from the stock container with an adjustable manual pipet fitted with a wide bore tip and placing it on a pre-labeled $35 \mathrm{~mm}$ petri dish. The sample will be diluted with a known and consistent volume $(e . g .200 \mu \mathrm{L})$ of acclimated well water to enhance dispersion of glochidla for more accurate enumeration. The sample will be viewed under a dissecting microscope, adjusted for maximum clarity and magnification, and a photomicrograph will be recorded. Approximately one drop of a saturated sodium chloride solution (12 $\mathrm{g} \mathrm{NaCl} / 50 \mathrm{~mL}$ of D.I. $\mathrm{H}_{2} \mathrm{O}$ [ASTM E2455-06]) will be added to the sample and after one minute a second photomicrograph will be recorded. Enumerations will be conducted for the total number of glochidla within the sample and the number of glochidla closed or open before and after sodium chloride addition. The percentage of viable glochidia (glochidia that respond to sodium chloride exposure by valve closure) will be calculated by the following method:

Percent viable $=([$ Total number of glochidia in sample - ftotal number of glochidia 


\section{OBIGINAL}

closed before addition of sodium chloride + number of glochidia open after sodium chloride addition] ]/Total number of glochidia in sample) * 100

If viability is acceptable ( $\geq 80 \%)$ for a mussel, the glochidia from that mussel will be pooled with glochidia from other mussels of that species. An appropriate amount of settled glochidla (e.g.: $500 \mu \mathrm{L}$ ) will be randomly drawn from the pooled glochidia stock and aliquoted to an exposure chamber $(80 \mathrm{~mm} \times 40 \mathrm{~mm}$ crystallizing dish) contalning exposure water to achieve a final volume of $100 \mathrm{~mL}$. Between $500-1,000$ glochidia will be transferred to each exposure chamber in a minimum of two aliquots (dependent on number of glochidia per aliquot and the estimated number of glochidia available) per chamber ( $\theta . \mathrm{g}$. two $100 \mu \mathrm{L}$, aliquots that contain $\sim 250$ glochidia each). Once all of the exposure chambers receive test animals the exposures will be initiated by the addition of the appropriate amount of MBI-401 SDP, deactivated MBI-401 SDP (positive control) or plain water (control) in a completely randomized design to achieve the desired test concentrations of $O$ (control), $300 \mathrm{mg} / \mathrm{L}$ deactivated (positive control), or 50,100,200, and $300 \mathrm{mg} / \mathrm{L}$ of active $\mathrm{MB} \mid-401 \mathrm{SDP}$. The exposure chambers will be gently mixed with a glass rod or stainless steel spatula to ensure uniform distribution of the test chemical. All concentrations will be tested in triplicate for a total of 18 exposure chambers per species. Exposure chambers will be held at $20^{\circ} \mathrm{C}$ in a water bath or controlled environment room and maintained on an 18:6 $\mathrm{h}$ llght/dark cycle. Viability of a subsample of approximately 100 glochidia from each chamber will be assessed at 6,12 and $24 \mathrm{~h}$ post exposure by enumeration from photomicrograph records pre- and post-sodium chloride addition. The study will be terminated after $24 \mathrm{~h}$ post exposure evaluations are completed.

Current text:

5. STUDY PROCEDURES

5.1 Test Animals

5.1.2 Number of animals: Approximately 9,000 glochidia (500 per replicate $\times 3$ replicates $\times 6$ concentrations) of each species. This design uses the fewest number of glochidia possible, consistent with the objective of the study, contemporary scientific standards and ASTM guide E2455-06.

5.1.3 Source of animals: All glochidia will be obtained from donor mussels located at the Genoa $\mathrm{NFH}$.

5.1.5 Acclimation: Glochidia will be acclimated to UMESC well water and temperature $\left(20^{\circ} \mathrm{C}\right)$ by the addition of up to $50 \%$ UMESC well water at the Genoa NFH prior to transport to UMESC and again after arrival at the UMESC

5.6 Treatment administration

5.6.1 Treatment: Each species of mussel glochidia will be exposed to three replicates of either 0 (control), 50,100, 200 or $300 \mathrm{mg} / \mathrm{L}$ (active ingredient) and a $300 \mathrm{mg} / \mathrm{L}$ heat deactivated $\left(70^{\circ} \mathrm{C} / 45\right.$ minutes) control as a one time single dose exposure.

5.6.2 Route of administration: To assure uniform exposure, an appropriate amount of freshly prepared stock solution will added to each test chamber from an agitated stock suspension with a pipet.

Revised text (in bold):

5. STUDY PROCEDURES

5.1 Test Animals

5.1.2 Number of animals: Approximately $9,000-18,000$ glochidia $(500-1,000$ per replicate $x$ 3 replicates $\times 6$ concentrations) of each species. This design uses the fewest number of glochidia possible, consistent with the objective of the study, contemporary scientific standards and ASTM guide E2455-06.

5.1.3 Source of animals: All glochidia will be obtained from donor mussels collected and temporarily held at the Genoa NFH. The donor mussels will be transported to the 
UMESC for acclimation to test conditions. Glochidia extraction will occur at UMESC; glochidia will be extracted by trained GNFH personnel.

5.1.5 Acclimation: Donor mussels will be acclimated from approximately $12{ }^{\circ} \mathrm{C}$ to the exposure temperature $\left(20^{\circ} \mathrm{C}\right)$ over a period of approximately $72 \mathrm{~h}$ upon arrival at UMESC.

5.6 Treatment administration:

5.6.1 Treatment: Each species of mussel glochidia will be exposed to three replicates of either 0 (control), 50,100, 200 or $300 \mathrm{mg} / \mathrm{L}$ (active ingredient) and a $300 \mathrm{mg} / \mathrm{L}$ heat deactivated ( $70^{\circ} \mathrm{C} / 45$ minutes) control as a one time single dose exposure,

5.6.2 Route of administration: To assure uniform exposure, an appropriate amount of freshly prepared stock solution will added to each test chamber from an agitated stock suspension with a pipet.

5.6.3 Dose verification: The activity of MBI-401 SDP (Pseudomonas fluorescens [PfCL145A]) cannot be assessed by current analytical techniques as the chemical nature of the active agent in the bacterium has not been determined or characterized. Dosage verification of MBI-401 SDP will be assessed by measuring and recording the absorbance of the prepared stock dosing solution and the high treatment concentrations. The collected absorbance information will be used to determine inter- and intra-test variability in the preparation of stock and test solutions.

Current text:

6.1 Distribution to experimental units:

The glochidia concentration ( $\mathrm{g} \mid$ ochidia/mL) will be determined for each species prior to test initiation by enumeration of replicate subsamples of glochidial suspension through a dissecting microscope. Appropriate volumes $(\mathrm{mL})$ will be determined that would contain approximately 500 glochidia. Test chambers will then be filled with appropriate amounts of dilution water to obtain a final volume of $100 \mathrm{~mL}$. Distribution to test chambers will then proceed by the addition of the calculated volume of suspension in at least two separate aliquots. Aliquots will be randomly assigned to a test chamber until all receive one aliquot. This procedure will then be repeated until all chambers receive approximately 500 glochidia in two or more aliquots. The test chambers will be randomly assigned treatment concentration.

6.2 Determination of viability:

Viability will determined by the abillty of the glochidia to constrict their adductor mussel thereby causing valve closure in response to saturated sodium chloride (12 $\mathrm{g} \mathrm{NaCl} / 50 \mathrm{~mL}$ of D.I. $\mathrm{H}_{2} \mathrm{O}$ ) exposure. Viability will be determined on a subsample of approximately 100 glochidia for each species prior to test initiation and at 6,12 and $24 \mathrm{~h}$ post exposure for each test replicate. A volume of water containing approximately 100 glochidia ( eg: 1-2 mL) will be placed on a $35 \mathrm{~mm}$ mini petri dish and examined under a dissecting scope to enumerate the percentage of glochidia with closed valves. Then approximately 1 drop of a saturated sodium chloride solution will be added to the sample and the glochidia will then be reassessed for valve closure after approximately one minute. Subsample photomicrographs of glochidia pre and post sodium chloride addition may be recorded and uniquely identified for documentation and optional later enumeration and/or validation of closure percentages. Only glochidia that are open and responsive to sodium chloride addition will be considered viable.

Revised text (in bold)

6.1 Distribution to experimental units:

The number of glochidia per $100 \mu \mathrm{L}$ aliquot will be estimated from samples taken to determine initial viability for each species prior to test initiation. Appropriate volumes $(\mu \mathrm{L})$ will be determined that would contain at least 500 glochidia. Test chambers will then be filled with appropriate amounts of dilution water to obtain a final volume of $100 \mathrm{~mL}$. Distribution to exposure chambers will then proceed by the addition of the calculated volume of settled glochidia in at least two separate aliquots. Aliquots will be randomly assigned to an exposure

Study\# AEH-11-PSEUDO-01 Amendment \#1

Page 4 of 6 
chamber until all receive one aliquot. This procedure will then be repeated until all chambers receive approximately 500 glochidia in two or more aliquots. The test chambers will be randomly assigned treatment concentration.

6.2 Determination of viability:

Vlability will determined by the ability of the glochidia to constrict their adductor mussel thereby causing valve closure in response to saturated sodium chloride (12 $\mathrm{g} \mathrm{NaCl} / 50 \mathrm{~mL}$ of D.I. $\mathrm{H}_{2} \mathrm{O}$ ) exposure. Viability will be determined on a subsample of approximately 100 glochidia from each mussel prior to test initiation and at 6,12 and $24 \mathrm{~h}$ post exposure for each test replicate. A subsample of at least 100 glochidia will be removed by drawing an aliquot (e.g. $100 \mu \mathrm{L}$ ) of settled glochidia from the exposure chamber with an adjustable manual plpet fitted with a wide bore tip and placing it on a pre-labeled $35 \mathrm{~mm}$ petri dish. The samples will be diluted with a known and consistent volume (e.g. $200 \mu \mathrm{L}$ ) of acclimated well water to enhance dlspersion of glochidia for more accurate enumeration. The sample will be viewed under a dissecting microscope, adjusted for maximum clarity and magnification, and a photomicrograph will be recorded. Approximately one drop of a saturated sodium chloride solution ( $12 \mathrm{~g} \mathrm{NaCl} / 50 \mathrm{~mL}$ of D.I. $\mathrm{H}_{2} \mathrm{O}$ [ASTM E2455-06]) will be added to the sample and after one minute a second photomicrograph will be recorded. Enumerations will be conducted for the total number of glochidia within the sample and the number of glochidia closed or open before and after sodium chloride addition. The percentage of viable glochidia (glochidia that respond to sodium chloride exposure by valve closure) will be calculated by the following method:

Percent viable $=$ ([Total number of glochidia in sample - total number of glochidia closed before addition of sodium chloride + number of glochidia open after sodium chloride addition\}]/Total number of glochidia in sample) ${ }^{*} 100$

Current text:

12. INVESTIGATIONAL TEST ARTICLE

12.1 Test Substance(s): MBI-401 SDP, Pseudomonas fluorescens (Pf-CL145A)

12.1.1 Chemical name: Pseudomonas fluorescens (Pf-CL145A)

12.1.2 Trade name: MBI-401 SDP

12.1.3 Active ingredients: Pseudomonas fluorescens (Pf-CL145A) is the sole active ingredient, $50 \%$ active by weight.

12.1.4 Source: Marrone Bio Innovations (MBI); Davis, CA

12.1.5 Lot number: None established

12.1.6 Expiration date: None established

12.1.7 Storage during study: test chemical will be stored at refrigerated in a locked container within a restrictive entry laboratory. A subsample of the test chemical will be archived in the UMESC Chemical Archive (UMESC SOP GEN 011).

12.1.8 A NIOSH approved respirator will be used when preparing stock solutions to avoid inhalation. Protective eyewear, gloves and lab coats will be worn at.all times when working with the test substance

Revised text (in bold)

12. INVESTIGATIONAL TEST ARTICLE

12.1 Test Substance(s): MB|-401 SDP, Pseudomonas fluorescens (Pf-CL145A)

12.1.1 Chemical name: Pseudomonas fluorescens (Pf-CL145A)

12.1.2 Trade name: MBI-401 SDP

12.1.3 Active ingredients: Pseudomonas fluorescens (Pf-CL145A) is the sole active ingredient $50 \%$ active by weight.

12.1.4 Source: Marrone Bio Innovations (MBI); Davis, CA 


\section{Whithas}

12.1.5 Lot number: MBI-401-110308AI-BD-3

12.1.6 Expiration date: None established, an aliquot of test material will be returned to the NYSM or MBI at the conclusion of exposures at UMESC for confirmatory posttest activity comparatlve zebra mussel bioassay tests (the standard testing

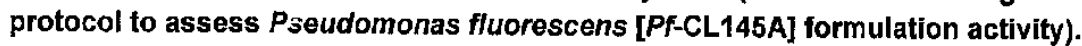
Results of these confirmation bioassays will be used to validate the activity retention of the MBI-401 SDP, Pseudomonas fluorescens (PF-CL145A).

12.1.7 Storage during study: test chemical will be stored refrigerated $\left(\leq 4^{\circ} \mathrm{C}\right)$ in a locked container within a restrictive entry iaboratory. A subsample of the test chemical will be archived in the UMESC Chemical Archive (UMESC SOP GEN 011)

12.1.8 A NIOSH approved respirator will be used when preparing stock solutions to avoid inhalation. Protective eyewear, gloves and lab coats will be worn at all times when working with the test substance.

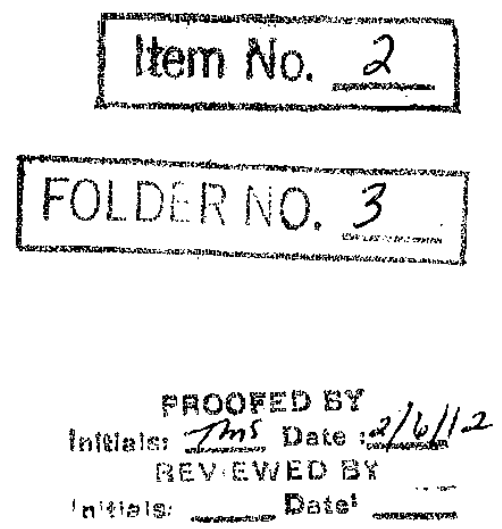




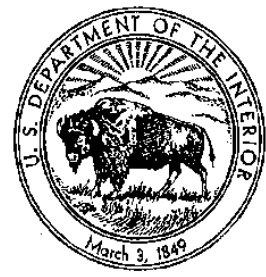

United States Department of the Interior

\section{U.S. GEOLOGICAL SURVEY Biological Resources Division Upper Midwest Environmental Sciences Center 2630 Fanta Reed Road La Crosse, Wisconsin 54603}

Date: October 19, 2011

To: The Record Study Number AEH-11-PSEUDO-01

Subject: Amendment 2 to the study AEH-11-PSEUDO-01 "Effects of Pseudomonas fluorescens (PfCL145A) to glochidia from seven unionid mussel species"

Revision of amended study protocol, study \# AEH-11-PSEUDO-01 is proposed as detailed on pages 2-8 of this amendment. Revised text is indicated in bold.

This amendment changes 1) the test material from a spray dried (SDP) to a freeze dried (FDP) formulation; 2) the procedures for acclimation of the donor mussels and 3) specifies the procedures for ammonia sample collection and analysis.

The FDP Pseudomonas fluorescens is a new manufacturer-preferred formulation that is considered to be $\sim 100 \%$ active compared to the spray-dried product (SDP) which was considered $\sim 50 \%$ active. The exposures will be conducted in an identical fashion except for the change in product type. Mussel species previously exposed to SDP will be exposed to the new FDP formulation; data collected during SDP exposures will be retained in the study data management system. Acclimation procedures previously outined may induce glochidial abortion in some species that are sensitive to handling (ie: washboard Megalonaias nervosa). The proposed accimation procedures provide flexibility to address acclimation at the direction of the Genoa NFH mussel biologist. The amendment specifies the procedures to determine ammonia concentration in exposure chamber water at the end of the 24-h exposure period.

Reviewed by:

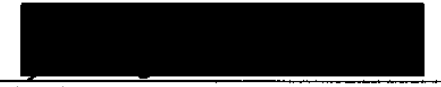

Mark P. Gaikowski, M.A. Supervisory Biologist Aquatic Ecosystem Health, UMESC $^{1}$

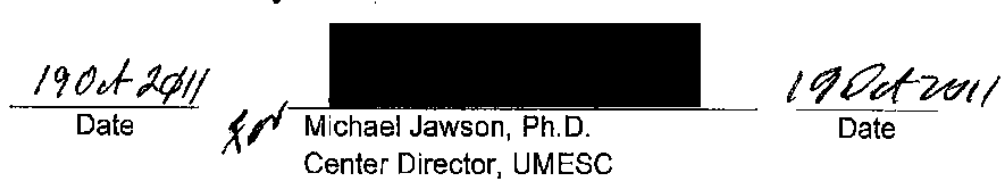

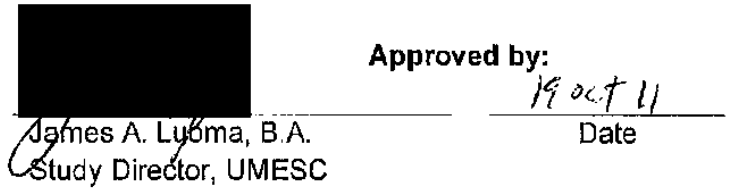

${ }^{1}$ UMESC: U.S. Geological Survey, Upper Midwest Environmental Sciences Center

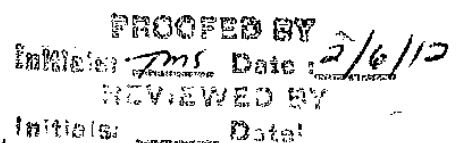

Study\# AEH-11-PSEUDO-01 Amendment \#2

Page 1 of 8 


\section{Current text:}

1. INTRODUCTION: (paragraph 4 of Section 1 is the only paragraph affected by this amendment)

Biologists at the New York State Museum (NYSM) Field Research Laboratory have been researching dreissenid mussel control since 1991 and they discovered that a bacterium isolated from soils (Pseudomonas fluorescens [Pf-CL145A]) is efficacious for controlling zebra mussels. Marrone Bio Innovations (MBl; Davis, $\mathrm{CA}$ ) is currently developing a commercial sprayed dried formulation of this bacterium called MBI-401 SDP. The current commercial applications of this product are for use with closed systems such as power generating plant cooling systems. The NYSM has partnered with the USFWS (Genca NFH) and United States Geological Survey's (USGS) Upper Midwest Environmental Sciences Center (UMESC) to determine the suitability of this product for open water zebra mussel control applications such as treatment of native mussel propagation cages or native mussel beds.

\section{PROTOCOL OBJECTIVE:}

This study will determine the animal effects of various concentrations of MBI-401 SDP (Pseudomonas fluorescens [Pf-CL145A]) to the glochidia lifestage of seven unionid mussels species present in the Great Lakes and Upper Mississippi River basins.

\subsection{General Description:}

Glochidia from 7 unionid mussel species (Table 2) endemic in the Great Lakes and Mississippi River basins will be evaluated for effects from exposure to varying concentrations of MB|-401 SDP (Pseudomonas fluorescens [Pf-CL145A]). Glochidia will be exposed in static exposures for $24 \mathrm{~h}$ as described in ASTM E-2455-06 and evaluated at 6,12 and $24 \mathrm{~h}$ for viability as determined by valve closure response after addition of a saturated sodium chloride solution.

\subsection{Experimental Design:}

Gravid donor mussels will be collected by biologists from the Genoa National Fish Hatchery from the Upper lowa, Upper Mississippi or St. Croix Rivers. Mussels will be identified to species as described in Cummings and Mayer (1992) and Watters et al. (2009) and transported to the Genoa National Fish Hatchery. Approximately $72 \mathrm{~h}$ prior to test initiation the donor mussels will be transferred from the Genoa NFH to the UMESC and acclimated to test temperature. Glochidia from at least three gravid female mussels of each species will be extracted by personnel from the Genoa NFH. The viability of glochidia from each mussel will be assessed by drawing aliquots (e.g. $100 \mu \mathrm{L}$ ) of settled glochidia from the stock container with an adjustable manual pipet fitted with a wide bore tip and placing it on a pre-labeled $35 \mathrm{~mm}$ petri dish. The sample will be diluted with a known and consistent volume (e.g. $200 \mu \mathrm{L}$ ) of acclimated well water to enhance dispersion of glochidia for more accurate enumeration. The sample will be viewed under a dissecting microscope, adjusted for maximum clarity and magnification, and a photomicrograph will be recorded. Approximately one drop of a saturated sodium chloride solution ( $12 \mathrm{~g} \mathrm{NaCl} / 50 \mathrm{~mL}$ of D.I. $\mathrm{H}_{2} \mathrm{O}$ [ASTM E2455-06]) will be added to the sample and after one minute a second photomicrograph will be recorded. Enumerations will be conducted for the total number of glochidia within the sample and the number of glochidia closed or open before and after sodium chloride addition. The percentage of 
viable glochidia (glochidia that respond to sodium chloride exposure by valve closure) will be calculated by the following method:

Percent viable $=([$ Total number of glochidia in sample $-\{$ total number of glochidia closed before addition of sodium chloride + number of glochidia open after sodium chloride addition\}]/Total number of glochidia in sample) * 100

If viability is acceptable ( $280 \%$ ) for a mussel, the glochidia from that mussel will be pooled with glochidia from other mussels of that species. An appropriate amount of settled glochidia (e.g.: $500 \mu \mathrm{L}$ ) will be randomly drawn from the pooled glochidia stock and aliquoted to an exposure chamber ( $80 \mathrm{~mm} \times 40 \mathrm{~mm}$ crystallizing dish) containing exposure water to achieve a final volume of $100 \mathrm{~mL}$. Between $500-1,000$ glochidia will be transferred to each exposure chamber in a minimum of two aliquots (dependent on number of glochidia per aliquot and the estimated number of glochidia available) per chamber (e.g. two $100 \mu \mathrm{L}$ aliquots that contain 250 glochidia each). Once all of the exposure chambers receive test animais the exposures will be initiated by the addition of the appropriate amount of MBI-401 SDP, deactivated MBI401 SDP (positive control) or plain water (control) in a completely randomized design to achieve the desired test concentrations of $O$ (control), $300 \mathrm{mg} / \mathrm{L}$ deactivated (positive control), or $50,100,200$, and $300 \mathrm{mg} / \mathrm{L}$ of active MB|-401 SDP. The exposure chambers will be gently mixed with a glass rod or stainless steel spatula to ensure uniform distribution of the test chemical. All concentrations will be tested in triplicate for a total of 18 exposure chambers per species. Exposure chambers will be held at $20^{\circ} \mathrm{C}$ in a water bath or controlled environment room and maintained on an 18:6 h light/dark cycle. Viability of a subsample of approximately 100 glochidia from each chamber will be assessed at 6,12 and $24 \mathrm{~h}$ post exposure by enumeration from photomicrograph records pre- and post-sodium chloride addition. The study will be terminated after $24 \mathrm{~h}$ post exposure evaluations are completed.

\section{STUDY PROCEDURES}

\subsection{Test Animals}

5.1.2 Number of animals: Approximately $9,000-18,000$ glochidia (500-1,000 per replicate $\times 3$ replicates $\times 6$ concentrations) of each species. This design uses the fewest number of glochidia possible, consistent with the objective of the study, contemporary scientific standards and ASTM guide E2455-06.

5.1.3 Source of animals: All glochidia will be obtained from donor mussels collected and temporarily held at the Genoa NFH. The donor mussels will be transported to the UMESC for acclimation to test conditions. Glochidia extraction will occur at UMESC; glochidia will be extracted by trained GNFH personnel.

5.1.4 Inclusion criterion: Glochidia will be used if they meet or exceed the following percentage of valve closure requirements after addition of a saturated sodium chloride solution ( $12 \mathrm{~g} \mathrm{NaCl} / 50 \mathrm{~mL}$ of D.I. $\mathrm{H}_{2} \mathrm{O}$ ). Glochidia must exhibit $\geq 80 \%$ valve closure to be pooled for use at the Genoa NFH and glochidia must exhibit $\geq 80 \%$ valve closure immediately prior study initiation at the UMESC.

5.1.5 Acclimation: Donor mussels will be acclimated from approximately $12{ }^{\circ} \mathrm{C}$ to the exposure temperature $\left(20^{\circ} \mathrm{C}\right)$ over a period of approximately $72 \mathrm{~h}$ upon arrival at UMESC.

\subsection{Water Chemistry}




\section{ORIGIMAL.}

5.2.7 Ammonia: The ammonia will be measured and recorded at the termination of the exposure from pooled replicate samples from the controls and ail test

5.5 Observations: concentrations (UMESC SOP AEH 301 or equivalent).

5.5.2 Water chemistry: Dissolved oxygen, $\mathrm{pH}_{\text {, }}$ and temperature will be monitored after the addition of the specified treatment. Alkalinity, hardness, conductivity and ammonia will be measured on a sample of dilution water at test initiation and on pooled replicate samples upon test termination. Alkalinity, hardness, conductivity and ammonia will not be measured during the exposures due to the quantity of test water required and probe submersion requirements to complete the analysis.

5.6 Treatment administration:

5.6.1 Treatment: Each species of mussel glochidia will be exposed to three replicates of either 0 (control), $50,100,200$ or $300 \mathrm{mg} / \mathrm{L}$ (active ingredient) and a $300 \mathrm{mg} / \mathrm{L}$ heat deactivated $\left(70^{\circ} \mathrm{C} / 45\right.$ minutes) control as a one time single dose exposure.

5.6.2 Route of administration: To assure uniform exposure, an appropriate amount of freshly prepared stock solution will added to each test chamber from an agitated stock suspension with a pipet.

5.6.3 Dose verification: The activity of MBI-401 SDP (Pseudomonas fluorescens [PfCL145A]) cannot be assessed by current analytical techniques as the chemical nature of the active agent in the bacterium has not been determined or characterized. Dosage verification of MBI-401 SDP will be assessed by measuring and recording the absorbance of the prepared stock dosing solution and the high treatment concentrations. The collected absorbance information will be used to determine inter- and intra-test variability in the preparation of stock and test solutions.

12. INVESTIGATIONAL TEST ARTICLE

12.1 Test Substance(s): MBI-401 SDP, Pseudomonas fluorescens (Pf-CL145A)

12.1.1 Chemical name: Pseudomonas fluorescens (PF-CL145A)

12.1.2 Trade name: MBI-401 SDP

12.1.3 Active ingredients: Pseudomonas fluorescens (Pf-CL145A) is the sole active ingredient, $50 \%$ active by weight.

12.1.4 Source: Marrone Bio Innovations (MBI); Davis, CA

12.1.5 Lot number: MBI-401-110308AI-BD-3

12.1.6 Expiration date: None established, an aliquot of test material will be returned to the NYSM or MBI at the conclusion of exposures at UMESC for confirmatory post-test activity comparative zebra mussel bioassay tests (the standard testing protocol to assess Pseudomonas fluorescens [Pf-CL145A] formulation activity). Results of these confirmation bioassays will be used to validate the activity retention of the MBI-401 SDP, Pseudomonas fluorescens (PfCL145A).

Revised text (in bold):

1. INTRODUCTION: (paragraph 4 of Section 1 is the only paragraph affected by this amendment) 


\section{ORIINAL}

Biologists at the New York State Museum (NYSM) Field Research Laboratory have been researching dreissenid mussel control since 1991 and they discovered that a bacterium isolated from soils (Pseudomonas fluorescens [Pf-CL145A]) is efficacious for controling zebra mussels. Marrone Bio Innovations (MBI; Davis, $\mathrm{CA}$ ) is currently developing a commercial freeze dried formulation of this bacterium referred to as MBI-401 FDP. The current commercial applications of this product are for use with closed systems such as power generating plant cooling systems. The NYSM has partnered with the USFWS (Genoa NFH) and United States Geological Survey's (USGS) Upper Midwest Environmental Sciences Center (UMESC) to determine the suitability of this product for open water zebra mussel control applications such as treatment of native mussel propagation cages or native mussel beds.

\section{PROTOCOL OBJECTIVE:}

This study will determine the animal effects of various concentrations of MBI-401 FDP (Pseudomonas fiuorescens [Pf-CL145A]) to the glochidia lifestage of seven unionid mussels species present in the Great Lakes and Upper Mississippi River basins.

\subsection{General Description:}

Glochidia from 7 unionid mussel species (Table 2) endemic in the Great Lakes and Mississippi River basins will be evaluated for effects from exposure to varying concentrations of MBI-401 FDP (Pseudomonas fluorescens [Pf-CL145A]). Glochidia will be exposed in static exposures for $24 \mathrm{~h}$ as described in ASTM E-2455-06 and evaluated at 6,12 and $24 \mathrm{~h}$ for viability as determined by valve closure response after addition of a saturated sodium chloride solution.

\subsection{Experimental Design:}

Gravid donor mussels will be collected by biologists from the Genoa National Fish Hatchery from the Upper lowa, Upper Mississippi or St. Croix Rivers. Mussels will be identified to species as described in Cummings and Mayer (1992) and Watters et al. (2009) and transported to the Genoa National Fish Hatchery. Prior to test initiation the donor mussels will be acclimated to the test temperature (see Section 5.1). Glochidia from at least three gravid female mussels of each species will be extracted by personnel from the Genoa NFH or UMESC. The viability of glochidia from each mussel will be assessed by drawing aliquots (e.g. $100 \mu \mathrm{L}$ ) of settled glochidia from the stock container with an adjustable manual pipet fitted with a wide bore tip and placing it on a pre-labeled $35 \mathrm{~mm}$ petri dish. The sample will be diluted with a known and consistent volume (e.g. $200 \mu \mathrm{L}$ ) of acclimated well water to enhance dispersion of glochidia for more accurate enumeration. The sample will be viewed under a dissecting microscope, adjusted for maximum clarity and magnification, and a photomicrograph will be recorded. Approximately one drop of a saturated sodium chloride solution (12 g NaCl/ $50 \mathrm{~mL}$ of D.I. $\mathrm{H}_{2} \mathrm{O}$ [ASTM E2455-06]) will be added to the sample and after one minute a second photomicrograph will be recorded. Enumerations will be conducted for the total number of glochidia within the sample and the number of glochidia closed or open before and after sodium chloride addition. The percentage of viable glochidia (glochidia that respond to sodium chloride exposure by valve closure) will be calculated by the following 
method:

Percent viable $=$ ([Total number of glochidia in sample - \{total number of glochidia closed before addition of sodium chloride + number of glochidia open after sodium chloride addition\}]/Total number of glochidia in sample) * 100

If viability is acceptable ( $\geq 80 \%$ ) for a mussel, the glochidia from that mussel will be pooled with glochidia from other mussels of that species. An appropriate amount of settled glochidia (e.g.: $500 \mu \mathrm{L}$ ) will be randomly drawn from the pooled glochidia stock and distributed to an exposure chamber ( $80 \mathrm{~mm} \times 40 \mathrm{~mm}$ crystallizing dish) containing exposure water to achieve a final volume of $100 \mathrm{~mL}$. Between $500-1,000$ glochidia will be transferred to each exposure chamber in a minimum of two distributions (dependent on number of glochidia per aliquot and the estimated number of glochidia available) per chamber (e.g. two $100 \mu \mathrm{L}$ aliquots that contain $\sim 250$ glochidia each). Once all of the exposure chambers receive test animals the exposures will be initiated by the addition of the appropriate amount of MBI-401 FDP, deactivated MBI-401 FDP (positive control) or plain water (control) in a completely randomized design to achieve the desired test concentrations of 0 (control), 300 $\mathrm{mg} / \mathrm{L}$ deactivated (positive control), or $50,100,200$, and $300 \mathrm{mg} / \mathrm{L}$ of active MBI-401 FDP. The exposure chambers will be gently mixed with a glass rod or stainless steel spatula to ensure uniform distribution of the test chemical. All concentrations will be tested in triplicate for a total of 18 exposure chambers per species. Exposure chambers will be held at $20^{\circ} \mathrm{C}$ in a water bath or controlled environment room and maintained on an 18:6 h light/dark cycle. Viability of a subsample of approximately 100 glochidia from each chamber will be assessed at 6,12 and $24 \mathrm{~h}$ post exposure by enumeration from photomicrograph records pre- and post-sodium chloride addition. The study will be terminated after $24 \mathrm{~h}$ post exposure evaluations are completed.

\section{STUDY PROCEDURES}

\subsection{Test Animals}

5.1.2 Number of animals: Approximately $9,000-18,000$ glochidia $(500-1,000$ per replicate $\times 3$ replicates $\times 6$ concentrations) of each species. This design uses the fewest number of glochidia possible, consistent with the objective of the study, contemporary scientific standards and ASTM guide E2455-06.

5.1.3 Source of animals: All glochidia will be obtained from donor mussels collected and temporarily held at the Genoa NFH. Donor mussel holding procedures (from collection to glochidia extraction) will be described in the study data management system. Glochidia extraction will occur at UMESC; glochidia will be extracted by trained GNFH or UMESC personnel.

5.1.5 Acclimation: Donor mussels will be acclimated from the collection/holding temperature to the exposure temperature $\left(20 \pm 2^{\circ} \mathrm{C}\right)$ before glochidia extraction at a rate specified by the Genoa NFH mussel biologist. The acclimation procedures will be described in the study data management system.

\subsection{Water Chemistry}

5.2.7 Ammonia: Total ammonia-nitrogen will be measured in a sample collected at the end of the 24-h exposure period from each exposure chamber. Samples $(\sim 3 \mathrm{~mL})$ will be filtered $(0.45 \mu \mathrm{m}$ syringe filter), acidified $(\mathrm{pH} \sim 2.5$ 
with $\mathrm{H}_{2} \mathrm{SO}_{4}$ ) and then stored at $\sim 4^{\circ} \mathrm{C}$. Total ammonia-nitrogen in samples will be determined by the UMESC Long Term Resources Monitoring Program (LTRMP) Water Quality Laboratory using the automated phenate method. Unionized ammonia will be calculated from the ammonia pKa (based on the $\mathrm{pH}$ and temperature recorded in the respective exposure chamber at the end of the 24-h exposure period) and the total ammonianitrogen concentration. The UMESC-LTRMP laboratory does not comply with Good Laboratory Practices (GLP) and thus data generated by the UMESC-LTRMP will be so annotated in the final report.

\subsection{Observations:}

5.5.2 Water chemistry: Dissolved oxygen, $\mathrm{pH}$, and temperature will be monitored after the addition of the specified treatment. Alkalinity, hardness, and conductivity will be measured using separate samples of dilution water collected before exposure initiation and from separate samples collected at the end of the 24-h exposure period. Water from replicate exposure chambers will be pooled to provide sufficient volume for these tests. Total ammonia-nitrogen will be measured in samples collected at the end of the 24-h exposure period (see Section 5.2.7). Alkalinity, hardness, conductivity and ammonia will not be measured during the exposures due to the quantity of test water required and probe submersion requirements to complete the analysis.

5.6 Treatment administration:

5.6.1 Treatment: Each species of mussel glochidia will be exposed to three replicates of either 0 (control), 50,100, 200 or $300 \mathrm{mg} / \mathrm{L}$ (active ingredient) and a $300 \mathrm{mg} / \mathrm{L}$ heat deactivated $\left(70^{\circ} \mathrm{C} / 45\right.$ minutes) control as a one time single dose exposure.

5.6.2 Route of administration: To assure uniform exposure, an appropriate amount of freshly prepared stock solution will added to each test chamber from an agitated stock suspension with a pipet

5.6.3 Dose verification: The activity of MBI-401 FDP (Pseudomonas fluorescens [PfCL145A]) cannot be assessed by current analytical techniques as the chemical nature of the active agent in the bacterium has not been determined or characterized. Dosage verification of MBI-401 FDP will be assessed by measuring and recording the absorbance of the prepared stock dosing solution and the high treatment concentrations. The collected absorbance information will be used to determine inter- and intra-test variability in the preparation of stock and test solutions.

\section{INVESTIGATIONAL TEST ARTICLE}

12.1 Test Substance(s): MBI-401 FDP, Pseudomonas fluorescens (Pf-CL145A)

12.1.1 Chemical name: Pseudomonas fluorescens (Pf-CL145A)

12.1.2 Trade name: $\mathrm{MBI}-401$ FDP

12.1.3 Active ingredients: Pseudomonas fluorescens (Pf-CL145A) is the sole active ingredient, $100 \%$ active by weight.

12.1.4 Source: Marrone Bio Innovations (MBI); Davis, CA

12.1.5 Lot number: More than one lot will be used. Test article lot numbers for each species will be recorded in the study files. 


\section{ORIGINAL}

12.1.6 Expiration date: Pseudomonas fluorescens (Pf-CL145A) FDP is susceptible to degradation if not properly handled. Therefore an aliquot of test material will be returned to the NYSM or MBI after each set of glochidia exposures at UMESC for post-exposure activity confirmation through completion of comparative zebra mussel bioassay tests (the standard testing protocol to assess Pseudomonas fluorescens [PfCL145A] formulation activity). Results of the zebra mussel bioassays will be used to validate the retention of the activity of the MBI-401 FDP lot used for a given mussel species.
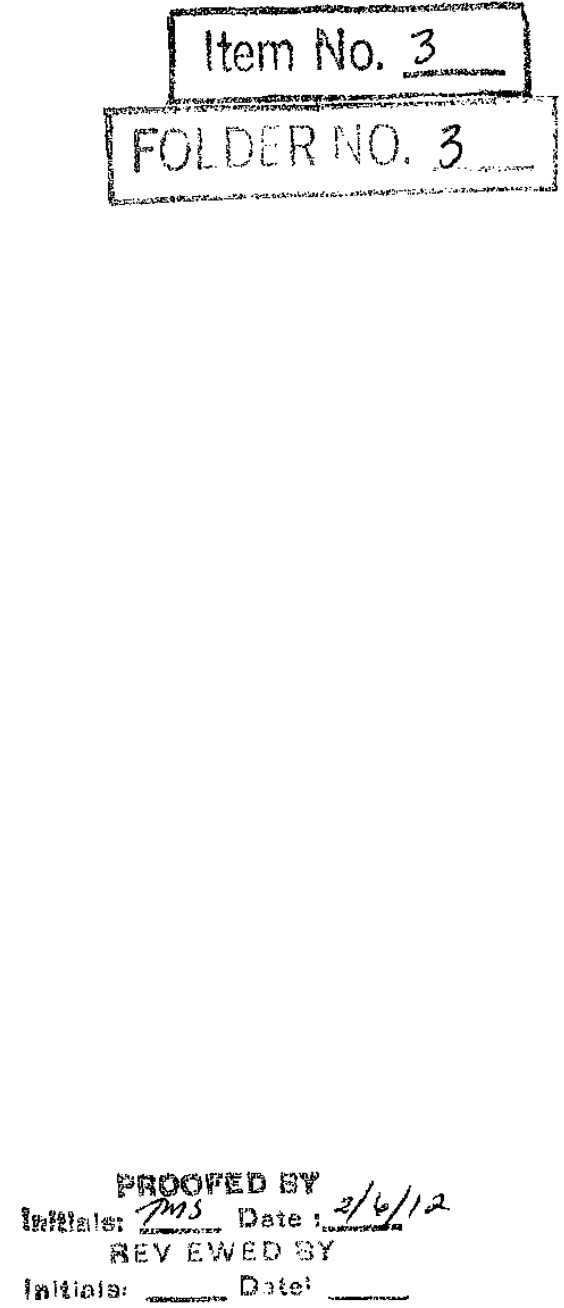


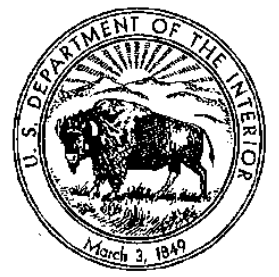

United States Department of the Interior

U.S. GEOLOGICAL SURVEY

Biological Resources Division

Upper Midwest Environmental Sciences Center

2630 Fanta Reed Road

La Crosse, Wisconsin 54603

\section{ORIGINAL}

Date: October 19, 2011

To: The Record Study Number AEH-11-PSEUDO-01

Subject: Amendment 3 to the study AEH-11-PSEUDO-01 "Effects of Pseudomonas fluorescens (PfCL.145A) to glochidia from seven unionid mussel species"

Revision of amended study protocol, study \# AEH-11-PSEUDO-01 is proposed as detailed on pages 2-5 of this amendment. Revised text is indicated in bold.

This amendment changes 1) the procedures for the viability assessment for mussels which cannot be reliably assessed from photomicrographic records (e.g. Megalonaias nervosa). M. nervosa glochidia are released from the donor mussel in a mucoidal matrix. Viability assessment from photomicrographic records is unreliable because glochidia within the mucus occupy several different focal planes, making it nearly impossible to bring all glochidia into focus. Therefore, this amendment specifies the procedures used to determine viability of species of donor mussels whose glochidia are released in a mucoidal matrix and when those assessments will be completed. The Study Director will determine the appropriate glochid la viability determination procedure to use for each mussel species after collecting glochidia from donor mussels. That decision will be recorded in the study data management system. This amendment serves as the record that the viability of glochidia of $M$. nervosa was determined using direct microscopic enumeration, not enumeration from photomicrographic recordings.

\section{Reviewed by:}

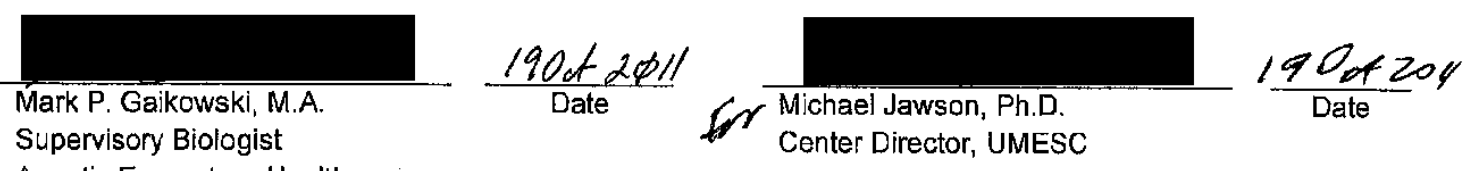
Aquatic Ecosystem Health, UMESC 1
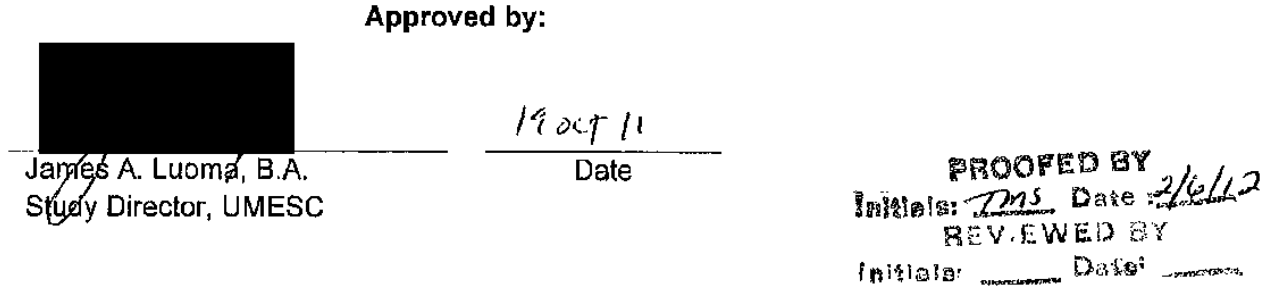

${ }^{1}$ UMESC: U.S. Geological Survey, Upper Midwest Environmental Sciences Center 


\section{Current text:}

\subsection{Experimental Design:}

Gravid donor mussels will be collected by biologists from the Genoa National Fish Hatchery from the Upper lowa, Upper Mississippi or St. Croix Rivers. Mussels will be identified to species as described in Cummings and Mayer (1992) and Watters et al. (2009) and transported to the Genoa National Fish Hatchery. Prior to test initiation the donor mussels will be acclimated to the test temperature (see Section 5.1). Glochidia from at least three gravid female mussels of each species will be extracted by personnel from the Genoa NFH or UMESC. The viability of glochidia from each mussel will be assessed by drawing aliquots (e.g. $100 \mu \mathrm{L}$ ) of settled glochidia from the stock container with an adjustable manual pipet fitted with a wide bore tip and placing it on a pre-labeled $35 \mathrm{~mm}$ petri dish. The sample will be diluted with a known and consistent volume (e.g. $200 \mu \mathrm{L}$ ) of acclimated well water to enhance dispersion of glochidia for more accurate enumeration. The sample will be viewed under a dissecting microscope, adjusted for maximum clarity and magnification, and a photomicrograph will be recorded. Approximately one drop of a saturated sodium chloride solution ( $12 \mathrm{~g} \mathrm{NaCl} / 50 \mathrm{~mL}$ of D.I. $\mathrm{H}_{2} \mathrm{O}$ [ASTM E2455-06]) will be added to the sample and after one minute a second photomicrograph will be recorded. Enumerations will be conducted for the total number of glochidia within the sample and the number of glochidia closed or open before and after sodium chloride addition. The percentage of viable glochidia (glochidia that respond to sodium chloride exposure by valve closure) will be calculated by the following method:

Percent viable $=([$ Total number of glochidia in sample $-\{$ total number of glochidia closed before addition of sodium chloride + number of glochidia open after sodium chloride addition] $] /$ Total number of glochidia in sample) * 100

If viability is acceptable ( $280 \%$ ) for a mussel, the glochidia from that mussel will be pooled with glochidia from other mussels of that species. An appropriate amount of settled glochidia (e.g.: $500 \mu \mathrm{L}$ ) will be randomly drawn from the pooled glochidia stock and distributed to an exposure chamber ( $80 \mathrm{~mm} \times 40 \mathrm{~mm}$ crystallizing dish) containing exposure water to achieve a final volume of $100 \mathrm{~mL}$. Between $500-1,000$ glochidia will be transferred to each exposure chamber in a minimum of two distributions (dependent on number of glochidia per aliquot and the estimated number of glochidia available) per chamber (e.g. two $100 \mu \mathrm{L}$ aliquots that contain $\sim 250$ glochidia each). Once all of the exposure chambers receive test animals the exposures will be initiated by the addition of the appropriate amount of MBI-401 FDP, deactivated MBI-401 FDP (positive control) or plain water (control) in a completely randomized design to achieve the desired test concentrations of 0 (control), 300 $\mathrm{mg} / \mathrm{L}$ deactivated (positive control), or 50,100,200, and $300 \mathrm{mg} / \mathrm{L}$ of active $\mathrm{MBI}-401$ FDP. The exposure chambers will be gently mixed with a glass rod or stainless steel spatula to ensure uniform distribution of the test chemical. All concentrations will be tested in triplicate for a total of 18 exposure chambers per species. Exposure chambers will be held at $20^{\circ} \mathrm{C}$ in a water bath or controlled environment room and maintained on an 18:6 h light/dark cycle. Viability of a subsample of approximately 100 glochidia from each chamber will be assessed at 6,12 and $24 \mathrm{~h}$ post exposure by enumeration from photomicrograph records pre- and post-sodium chloride 
addition. The study will be terminated after $24 \mathrm{~h}$ post exposure evaluations are completed.

5.5 Observations:

5.5.1 Behavioral Observations: Behavioral observations will be limited to viability assessments. Viability is determined by the ability of the glochidia to constrict their adductor mussel thereby causing valve closure in response to saturated sodium chloride ( $12 \mathrm{~g} \mathrm{NaCl} / 50 \mathrm{~mL}$ of D.I. $\mathrm{H}_{2} \mathrm{O}$ ) exposure. Viability will be determined on a subsample of approximately 100 glochidia at 6,12 and $24 \mathrm{~h}$ post exposure for each test chamber.

Revised text (in bold):

4.2 Experimental Design:

Gravid donor mussels will be collected by biologists from the Genoa National Fish Hatchery from the Upper lowa, Upper Mississippi or St. Croix Rivers. Mussels will be identified to species as described in Cummings and Mayer (1992) and Watters et al. (2009) and transported to the Genoa National Fish Hatchery. Prior to test initiation the donor mussels will be acclimated to the test temperature (see Section 5.1). Glochidia from at least three gravid female mussels of each species will be extracted by personnel from the Genoa NFH or UMESC. The viability of glochidia from each mussel will be assessed by drawing aliquots (e.g. $100 \mu \mathrm{L}$ ) of settled glochidia from the stock container with an adjustable manual pipet fitted with a wide bore tip and placing it on a pre-labeled $35 \mathrm{~mm}$ petri dish. The sample will be diluted with a known and consistent volume (e.g. $200 \mu \mathrm{L}$ ) of acclimated well water to enhance dispersion of glochidia for more accurate enumeration.

The Study Director will determine which method of glochidia viability assessment will be used (enumeration from photomicrographic recording or direct enumeration) after glochidia are collected from donor mussels. That decision will be recorded in the study data management system. If glochidia can be enumerated and viability status (i.e. open or closed) reliably determined through photomlcrographic records (i.e. the glochidia remain on a single focal plane) then the following procedures will be used for viability assessment. The sample will be viewed under a dissecting microscope, adjusted for maximum clarity and magnification, and a photomicrograph will be recorded. Approximately one drop of a saturated sodium chloride solution $(12 \mathrm{~g} \mathrm{NaCl} / 50$ $\mathrm{mL}$ of D.I. $\mathrm{H}_{2} \mathrm{O}$ [ASTM E2455-06]) will be added to the sample and after one minute a second photomicrograph will be recorded. The total number of glochidia within the sample and the number of glochidia closed or open before and after sodium chloride addition will be determined. The percentage of viable glochidia (glochidia that respond to sodium chloride exposure by valve closure) will be calculated by the following method:

Percent viable $=([$ Total number of glochidia in sample - \{total number of glochidia closed before addition of sodium chloride + number of glochidia open after sodium chloride addition\}]/Total number of glochidia in sample) * 100

If glochidia enumeration and vlability cannot be reliably determined through 
photomicrographic records because the glochidia do not occupy a single focal plane (e.g. Megalonaias nervosa and other mussels that release glochidia in a mucoidal matrix) the following procedures will be used to assess viability.

The sample will be viewed under a dissecting microscope, adjusted for maximum clarity and magnification, and the total number of glochidia and the number of closed or open glochidia (discretion of the individual performing the count) will be directly enumerated. Approximately one drop of a saturated sodium chloride solution (12 g NaCl/ $50 \mathrm{~mL}$ of D.I. $\mathrm{H}_{2} \mathrm{O}$ [ASTM E2455-06]) will be added to the sample. After one minute, the number of glochidia that remain open after sodium chloride addition will be directly enumerated. The percentage of viable glochidia (glochidia that respond to sodium chloride exposure by valve closure) will be calculated by the following method:

Percent viable $=([$ Total number of glochidia in sample - \{total number of glochidia closed before addition of sodium chloride + number of glochidia open after sodium chloride addition\}]/Total number of glochidia in sample) * 100

If glochidia viability is acceptable $(\geq 80 \%)$ for a donor mussel, the glochidia from that donor mussel will be pooled with glochidia from other donor mussels of that species. An appropriate amount of settled glochidia (e.g.: $500 \mu \mathrm{L}$ ) will be randomly drawn from the pooled glochidia stock and distributed to an exposure chamber ( $80 \mathrm{~mm} \times 40 \mathrm{~mm}$ crystallizing dish) containing exposure water to achieve a final volume of $100 \mathrm{~mL}$. Between 500-1,000 glochidia will be transferred to each exposure chamber in a minimum of two distributions (dependent on number of glochidia per aliquot and the estimated number of glochidia available) per chamber (e.g. two $100 \mu \mathrm{L}$ aliquots that contain $\sim 250$ glochidia each). Once all of the exposure chambers receive test animals the exposures will be initiated by the addition of the appropriate amount of MBI-401 FDP, deactivated MBI-401 FDP (positive control) or plain water (control) in a completely randomized design to achieve the desired test concentrations of 0 (control), $300 \mathrm{mg} / \mathrm{L}$ deactivated (positive control), or 50,100 , 200 , and $300 \mathrm{mg} / \mathrm{L}$ of active MBI-401 FDP. The exposure chambers will be gently mixed with a glass rod or stainless steel spatula to ensure uniform distribution of the test chemical. All concentrations will be tested in triplicate for a total of 18 exposure chambers per species. Exposure chambers will be held at $20^{\circ} \mathrm{C}$ in a water bath or controlled environment room and maintained on an 18:6 h light/dark cycle. For mussel species for which the viability of glochidia can be assessed from photomicrographic records, the viability of a subsample of approximately 100 glochidia from each chamber will be assessed at 6, 12 and $24 \mathrm{~h}$ post exposure by enumeration from photomicrograph records pre- and post-sodium chloride addition. For mussel species for which the viability of glochidia cannot be assessed from photomicrographic records (e.g. M. nervosa), the viability of a subsample of approximately 100 glochidia from each chamber will be assessed only at $24 \mathrm{~h}$ post exposure using the methods described for initial glochidia viability assessment.

The study will be terminated after $24 \mathrm{~h}$ post exposure evaluations are completed.

\subsection{Observations:}

\subsubsection{Behavioral Observations: Behavioral observations will be limited to}




\section{ORIGMAL}

viability assessments. Viability is determined by the ability of the glochidia to constrict their adductor mussel thereby causing valve closure in response to saturated sodium chloride $\left(12 \mathrm{~g} \mathrm{NaCl} / 50 \mathrm{~mL}\right.$ of D.I. $\mathrm{H}_{2} \mathrm{O}$ ) exposure. For mussel species for which the viability of glochidia can be assessed from photomicrographlc records, the viability of a subsample of approximately 100 glochidia from each chamber will be assessed at 6,12 and $24 \mathrm{~h}$ post exposure by enumeration from photomicrograph records pre- and post-sodium chloride addition. For mussel species for which the viability of glochidia cannot be assessed from photomicrographic records (e.g. M. nervosa), the viability of a subsample of approximately 100 glochidia from each chamber wlll be assessed only at $24 \mathrm{~h}$ post exposure using the methods described for initial glochidia viability assessment.

The study will be terminated after $24 \mathrm{~h}$ post exposure evaluations are completed.

Item No. 4

FOLDER NO. 3

解OOFEDY

Evilielss: $7 m$ s Date: $2 / 4 / 10$

REYVIEWED EY

Pitials Datat 


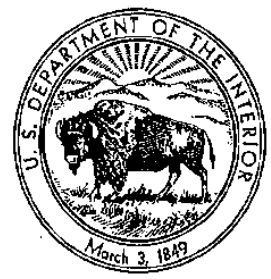

United States Department of the Interior
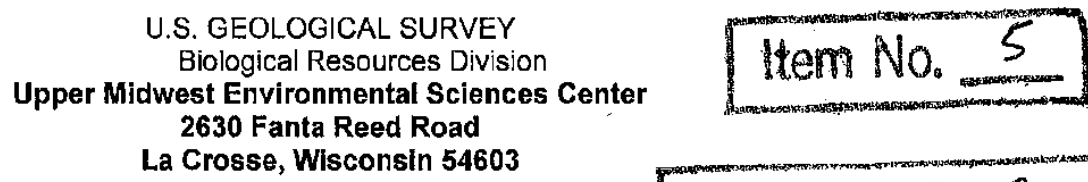

FOLDERNO. 3

Date: December 20, 2011

To: $\quad$ The Record Study Number AEH-11-PSEUDO-01

Subject: Amendment 4 to the study AEH-11-PSEUDO-01 "Effects of Pseudomonas fluorescens (Pf-CL145A) to glochidia from seven unionid mussel species"

Revision of amended study protocol, study \# AEH-11-PSEUDO-01 is proposed as detailed on pages 2-7 of this amendment. Revised text is indicated in bold.

This amendment changes the test material from a Freeze dried (FDP) to a spray dried (SDP) formulation.

The initially manufacturer-preferred freeze dried formulation (FDP) of Pseudomonas fluorescens has recently been discontinued and the manufacturer has indicated support for a spray-dried (SDP) formulation.

The exposures will be conducted in an identical fashion except for the change in product formulation. Mussel species previously exposed to FDP will not be re-evaluated with the SDP formulation; data collected during FDP exposures will be retained in the study data management system.

Reviewed by:

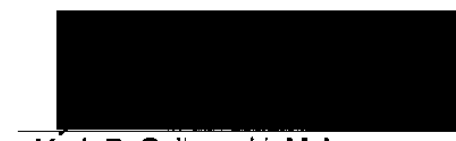

Mark P. Gaikowski, M.A.

Supervisory Biologist

Aquatic Ecosystem Health,

UMESC $^{1}$

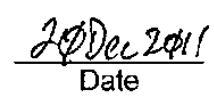

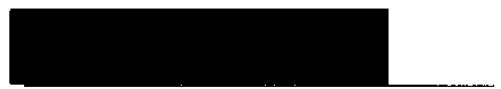

Michael Jawson, Ph.D. Center Director, UMESC

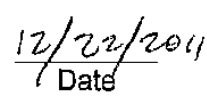

Approved by:

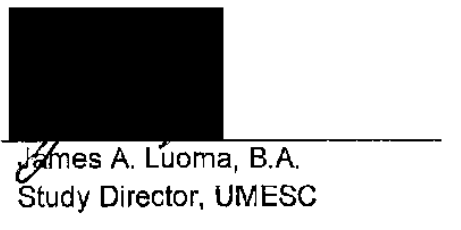

$\frac{12 / 22 / 11}{\text { Date }}$

${ }^{1}$ UMESC: U.S. Geological Survey, Upper Midwest Environmental Sciences Center thatsiaim

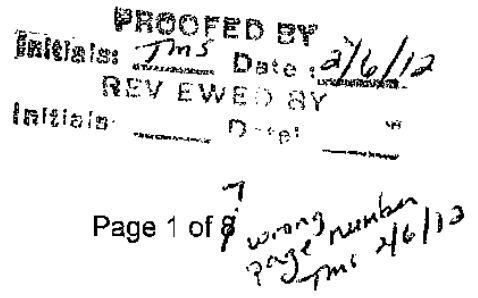

Study\# AEH-11-PSEUDO-01 Amendment \#4 $p^{e} \sum_{-1}^{2} m^{6}$ 
Current text:

\section{ORIGINAL}

1. INTRODUCTION: (paragraph 4 of Section 1 is the only paragraph affected by this amendment)

Biologists at the New York State Museum (NYSM) Field Research Laboratory have been researching dreissenid mussel control since 1991 and they discovered that a bacterium isolated from soils (Pseudomonas fluorescens [Pf-CL145A]) is efficacious for controlling zebra mussels. Marrone Bio Innovations (MBl; Davis, CA) is currently developing a commercial freeze dried formulation of this bacterium referred to as MB|-401 FDP. The current commercial applications of this product are for use with closed systems such as power generating plant cooling systems. The NYSM has partnered with the USFWS (Genoa NFH) and United States Geological Survey's (USGS) Upper Midwest Environmental Sciences Center (UMESC) to determine the suitability of this product for open water zebra mussel control applications such as treatment of native mussel propagation cages or native mussel beds.

\section{PROTOCOL OBJECTIVE:}

This study will determine the animal effects of various concentrations of MBI-401 FDP (Pseudomonas fluorescens [Pf-CL145A]) to the glochidia lifestage of seven unionid mussels species present in the Great Lakes and Upper Mississippi River basins.

\subsection{General Description:}

Glochidia from 7 unionid mussel species (Table 2) endemic in the Great Lakes and Mississippi River basins will be evaluated for effects from exposure to varying concentrations of MB|-401 FDP (Pseudomonas fluorescens [Pf-CL145A]). Glochidia will be exposed in static exposures for $24 \mathrm{~h}$ as described in ASTM E-2455-06 and evaluated at 6,12 and $24 \mathrm{~h}$ for viability as determined by valve closure response after addition of a saturated sodium chloride solution.

\subsection{Experimental Design:}

4.2 Experimental Design:

Gravid donor mussels will be collected by biologists from the Genoa National Fish Hatchery from the Upper lowa, Upper Mississippi or St. Croix Rivers. Mussels will be identified to species as described in Cummings and Mayer (1992) and Watters et al. (2009) and transported to the Genoa National Fish Hatchery. Prior to test initiation the donor mussels will be acclimated to the test temperature (see Section 5.1). Glochidia from at least three gravid female mussels of each species will be extracted by personmel from the Genoa NFH or UMESC. The viability of glochidia from each mussel will be assessed by drawing aliquots (e.g. $100 \mu \mathrm{L}$ ) of settled glochidia from the stock container with an adjustable manual pipet fitted with a wide bore tip and placing it on a pre-labeled $35 \mathrm{~mm}$ petri dish. The sample will be diluted with a known and consistent volume (e.g. $200 \mu \mathrm{L}$ ) of acclimated well water to enhance dispersion of glochidia for more accurate enumeration.

The Study Director will determine which method of glochidia viability assessment will be used (enumeration from photomicrographic recording or direct enumeration) after glochidia are collected from donor mussels. That decision will be recorded in the study data management system. If glochidia can be enumerated and viability status (i.e. open or closed) reliably determined through photomicrographic records (i.e. the 


\section{DRIUIVAL}

glochidia remain on a single focal plane) then the following procedures will be used for viability assessment. The sample will be viewed under a dissecting microscope, adjusted for maximum clarity and magnification, and a photomicrograph will be recorded. Approximately one drop of a saturated sodium chloride solution (12 g $\mathrm{NaCl} / 50 \mathrm{~mL}$ of D.I. $\mathrm{H}_{2} \mathrm{O}$ [ASTM E2455-06]) will be added to the sample and after one minute a second photomicrograph will be recorded. The total number of glochidia within the sample and the number of glochidia closed or open before and after sodium chloride addition will be determined. The percentage of viable glochidia (glochidia that respond to sodium chloride exposure by valve closure) will be calculated by the following method:

Percent viable $=([$ Total number of glochidia in sample $-\{$ total number of glochidia closed before addition of sodium chloride + number of glochidia open after sodium chloride addition\}]/Total number of glochidia in sample) ${ }^{*} 100$

If glochidia enumeration and viability cannot be reliably determined through photomicrographic records because the glochidia do not occupy a single focal plane (e.g. Megalonaias nervosa and other mussels that release glochidia in a mucoidal matrix) the following procedures will be used to assess viability.

The sample will be viewed under a dissecting microscope, adjusted for maximum clarity and magnification, and the total number of glochidia and the number of closed or open glochidia (discretion of the individual performing the count) will be directly enumerated. Approximately one drop of a saturated sodium chloride solution $(12 \mathrm{~g}$ $\mathrm{NaCl} / 50 \mathrm{~mL}$ of D.I. $\mathrm{H}_{2} \mathrm{O}$ [ASTM E2455-06]) will be added to the sample. After one minute, the number of glochidia that remain open after sodium chloride addition will be directly enumerated. The percentage of viable glochidia (glochidia that respond to sodium chloride exposure by valve closure) will be calculated by the following method:

Percent viable $=([T$ tal number of glochidia in sample $-\{$ total number of glochidia closed before addition of sodium chloride + number of glochidia open after sodium chloride addition\}]/Total number of glochidia in sample) * 100

If glochidia viability is acceptable ( $280 \%$ ) for a donor mussel, the glochidia from that donor mussel will be pooled with glochidia from other donor mussels of that species. An appropriate amount of settled glochidia (e.g.: $500 \mu \mathrm{L}$ ) will be randomly drawn from the pooled glochidia stock and distributed to an exposure chamber $(80 \mathrm{~mm} x$ $40 \mathrm{~mm}$ crystallizing dish) containing exposure water to achieve a final volume of $100 \mathrm{~mL}$. Between 500-1,000 glochidia will be transferred to each exposure chamber in a minimum of two distributions (dependent on number of glochidia per aliquot and the estimated number of glochidia available) per chamber (e.g. two $100 \mu \mathrm{L}$ aliquots that contain $\sim 250$ glochidia each). Once all of the exposure chambers receive test animals the exposures will be initiated by the addition of the appropriate amount of $\mathrm{MBI}-401$ FDP, deactivated MBI-401 FDP (positive control) or plain water (control) in a completely randomized design to achieve the desired test concentrations of 0 (control), $300 \mathrm{mg} / \mathrm{L}$ deactivated (positive control), or $50,100,200$, and $300 \mathrm{mg} / \mathrm{L}$ of active MB|-401 FDP. The exposure chambers will be gently mixed with a glass rod or stainless steel spatula to ensure uniform distribution of the test chemical. All concentrations will be tested in triplicate for a total of 18 exposure chambers per species. Exposure chambers will be held at $20^{\circ} \mathrm{C}$ in a water bath or controlled 


\section{ORIGINAI}

environment room and maintained on an 18:6 h light/dark cycle. For mussel species for which the viability of glochidia can be assessed from photomicrographic records, the viability of a subsample of approximately 100 glochidia from each chamber will be assessed at 6,12 and $24 \mathrm{~h}$ post exposure by enumeration from photomicrograph records pre- and post-sodium chloride addition. For mussel species for which the viability of glochidia cannot be assessed from photomicrographic records (e.g. M. nervosa), the viability of a subsample of approximately 100 glochidia from each chamber will be assessed only at $24 \mathrm{~h}$ post exposure using the methods described for initial glochidia viability assessment.

The study will be terminated after $24 \mathrm{~h}$ post exposure evaluations are completed.

\section{STUDY PROCEDURES}

5.6 Treatment administration:

5.6.1 Treatment: Each species of mussel glochidia will be exposed to three replicates of either 0 (control), 50,100,200 or $300 \mathrm{mg} / \mathrm{L}$ (active ingredient) and a $300 \mathrm{mg} / \mathrm{L}$ heat deactivated $\left(70^{\circ} \mathrm{C} / 45\right.$ minutes) control as a one time single dose exposure.

5.6.2 Route of administration: To assure uniform exposure, an appropriate amount of freshly prepared stock solution will added to each test chamber from an agitated stock suspension with a pipet.

5.6.3 Dose verification: The activity of MBI-401 FDP (Pseudomonas fluorescens [PFCL145AJ) cannot be assessed by current analytical techniques as the chemical nature of the active agent in the bacterium has not been determined or characterized. Dosage verification of MBI-401 FDP will be assessed by measuring and recording the absorbance of the prepared stock dosing solution and the high treatment concentrations. The collected absorbance information will be used to determine inter- and intra-test variability in the preparation of stock and test solutions.

\section{INVESTIGATIONAL TEST ARTICLE}

12.1 Test Substance(s): MBl-401 FDP, Pseudomonas fluorescens (Pf-CL145A)

12.1.1 Chemical name: Pseudomonas fluorescens (Pf-CL145A)

12.1.2 Trade name: $\mathrm{MBI}-401 \mathrm{FDP}$

12.1.3 Active ingredients: Pseudomonas fluorescens (Pf-CL145A) is the sole active ingredient, $100 \%$ active by weight.

12.1.4 Source: Marrone Bio Innovations (MBI); Davis, CA

12.1.5 Lot number: More than one lot will be used. Test article lot numbers for each species will be recorded in the study files.

Revised text:

1. INTRODUCTION: (paragraph 4 of Section 1 is the only paragraph affected by this amendment)

Biologists at the New York State Museum (NYSM) Field Research Laboratory have been researching dreissenid mussel control since 1991 and they discovered that a bacterium isolated from soils (Pseudomonas fluorescens [Pf-CL145A]) is efficacious for controlling zebra mussels. Marrone Bio Innovations (MBl; Davis, $\mathrm{CA}$ ) is currently developing a commercial spray dried formulation of this bacterium referred to as MB|-401 SDP. The current commercial applications 
of this product are for use with closed systems such as power generating plant cooling systems. The NYSM has partnered with the USFWS (Genoa NFH) and United States Geological Survey's (USGS) Upper Midwest Environmental Sciences Center (UMESC) to determine the suitability of this product for open water zebra mussel control applications such as treatment of native mussel propagation cages or native mussel beds.

\section{PROTOCOL OBJECTIVE:}

This study will determine the animal effects of various concentrations of MBI-401 SDP (Pseudomonas fluorescens [Pf-CL145A]) to the glochidia lifestage of seven unionid mussels species present in the Great Lakes and Upper Mississippi River basins.

\subsection{General Description:}

Glochidia from 7 unionid mussel species (Table 2) endemic in the Great Lakes and Mississippi River basins will be evaluated for effects from exposure to varying concentrations of MBI-401 SDP (Pseudomonas fluorescens [Pf-CL145A]). Glochidia will be exposed in static exposures for $24 \mathrm{~h}$ as described in ASTM E-2455-06 and evaluated at 6,12 and $24 \mathrm{~h}$ for viability as determined by valve closure response after addition of a saturated sodium chloride solution.

\subsection{Experimental Design:}

4.2 Experimental Design:

Gravid donor mussels will be collected by biologists from the Genoa National Fish Hatchery from the Upper lowa, Upper Mississippi or St. Croix Rivers. Mussels will be identified to species as described in Cummings and Mayer (1992) and Watters et al. (2009) and transported to the Genoa National Fish Hatchery. Prior to test initiation the donor mussels will be acclimated to the test temperature (see Section 5.1). Glochidia from at least three gravid female mussels of each species will be extracted by personnel from the Genoa NFH or UMESC. The viability of glochidia from each mussel will be assessed by drawing aliquots (e.g. $100 \mu \mathrm{L}$ ) of settled glochidia from the stock container with an adjustable manual pipet fitted with a wide bore tip and placing it on a pre-labeled $35 \mathrm{~mm}$ petri dish. The sample will be diluted with a known and consistent volume (e.g. $200 \mu \mathrm{L}$ ) of acclimated well water to enhance dispersion of glochidia for more accurate enumeration.

The Study Director will determine which method of glochidia viability assessment will be used (enumeration from photomicrographic recording or direct enumeration) after glochidia are collected from donor mussels. That decision will be recorded in the study data management system. If glochidia can be enumerated and viability status (i.e. open or closed) reliably determined through photomicrographic records (i.e. the glochidia remain on a single focal plane) then the following procedures will be used for viability assessment. The sample will be viewed under a dissecting microscope, adjusted for maximum clarity and magnification, and a photomicrograph will be recorded. Approximately one drop of a saturated sodium chloride solution $12 \mathrm{~g}$ $\mathrm{NaCl} / 50 \mathrm{~mL}$ of D.I. $\mathrm{H}_{2} \mathrm{O}$ [ASTM E2455-06]) will be added to the sample and after one minute a second photomicrograph will be recorded. The total number of glochidia within the sample and the number of glochidia closed or open before and after sodium chloride addition will be determined. The percentage of viable glochidia (glochidia that respond to sodium chloride exposure by valve closure) will be calculated by the following method: 


\section{ORIGINAL}

Percent viable $=([$ Total number of glochidia in sample $-\{$ total number of glochidia closed before addition of sodium chloride + number of glochidia open after sodium chloride addition] ]/Total number of glochidia in sample) * 100

If glochidia enumeration and viability cannot be reliably determined through photomicrographic records because the glochidia do not occupy a single focal plane (e.g. Megalonaias nervosa and other mussels that release glochidia in a mucoidal matrix) the following procedures will be used to assess viability.

The sample will be viewed under a dissecting microscope, adjusted for maximum clarity and magnification, and the total number of glochidia and the number of closed or open glochidia (discretion of the individual performing the count) will be directly enumerated. Approximately one drop of a saturated sodium chloride solution $(12 \mathrm{~g}$ $\mathrm{NaCl} / 50 \mathrm{~mL}$ of D.I. $\mathrm{H}_{2} \mathrm{O}$ [ASTM E2455-06]) will be added to the sample. After one minute, the number of glochidia that remain open after sodium chloride addition will be directly enumerated. The percentage of viable glochidia (glochidia that respond to sodium chloride exposure by valve closure) will be calculated by the following method:

Percent viable $=\{[$ Total number of glochidia in sample $-\{$ total number of glochidia closed before addition of sodium chloride + number of glochidia open after sodium chloride addition\}]/Total number of glochidia in sample) * 100

If glochidia viability is acceptable $(\geq 80 \%)$ for a donor mussel, the glochidia from that donor mussel will be pooled with glochidia from other donor mussels of that species. An appropriate amount of settled glochidia (e.g.: $500 \mu \mathrm{L}$ ) will be randomly drawn from the pooled glochidia stock and distributed to an exposure chamber $(80 \mathrm{~mm} \times 40$ $\mathrm{mm}$ crystallizing dish) containing exposure water to achieve a final volume of 100 $\mathrm{mL}$. Between 500-1,000 glochidia will be transferred to each exposure chamber in a minimum of two distributions (dependent on number of glochidia per aliquot and the estimated number of glochidia available) per chamber (e.g. two $100 \mu \mathrm{L}$ aliquots that contain $\sim 250$ glochidia each). Once all of the exposure chambers receive test animals the exposures will be initiated by the addition of the appropriate amount of MBI-401 SDP, deactivated MB|-401 SDP (positive control) or plain water (control) in a completely randomized design to achieve the desired test concentrations of 0 (control), $300 \mathrm{mg} / \mathrm{L}$ deactivated (positive control), or $50,100,200$, and $300 \mathrm{mg} / \mathrm{L}$ of active MBI-401 SDP. The exposure chambers will be gently mixed with a glass rod or stainless steel spatula to ensure uniform distribution of the test chemical. All concentrations will be tested in triplicate for a total of 18 exposure chambers per species. Exposure chambers will be held at $20^{\circ} \mathrm{C}$ in a water bath or controlied environment room and maintained on an $18: 6 \mathrm{~h}$ light/dark cycle. For mussel species for which the viability of glochidia can be assessed from photomicrographic records, the viability of a subsample of approximately 100 glochidia from each chamber will be assessed at 6,12 and $24 \mathrm{~h}$ post exposure by enumeration from photomicrograph records pre- and post-sodium chloride addition. For mussel species for which the viability of glochidia cannot be assessed from photomicrographic records (e.g. $M$. nervosa), the viability of a subsample of approximately 100 glochidia from each chamber will be assessed only at $24 \mathrm{~h}$ post exposure using the methods described for initial glochidia viability assessment.

The study will be terminated after $24 \mathrm{~h}$ post exposure evaluations are completed. 


\section{STUDY PROCEDURES}

5.6 Treatment administration:

5.6.1 Treatment: Each species of mussel glochidia will be exposed to three replicates of either 0 (control), 50,100,200 or $300 \mathrm{mg} / \mathrm{L}$ (active ingredient) and a $300 \mathrm{mg} / \mathrm{L}$ heat deactivated $\left(70^{\circ} \mathrm{C} / 45\right.$ minutes) control as a one time single dose exposure.

5.6.2 Route of administration: To assure uniform exposure, an appropriate amount of freshly prepared stock solution will added to each test chamber from an agitated stock suspension with a pipet.

5.6.3 Dose verification: The activity of MBI-401 SDP (Pseudomonas fluorescens [PFCL145A]) cannot be assessed by current analytical techniques as the chemical nature of the active agent in the bacterium has not been determined or characterized. Dosage verification of MBI-401 SDP will be assessed by measuring and recording the absorbance of the prepared stock dosing solution and the high treatment concentrations. The collected absorbance information will be used to determine inter- and intra-test variability in the preparation of stock and test solutions.

\section{INVESTIGATIONAL TEST ARTICLE}

12.1 Test Substance(s): MBI-401 SDP, Pseudomonas fluorescens (Pf-CL145A)

12.1.1 Chemical name: Pseudomonas fluorescens (Pf-CL145A)

12.1.2 Trade name: MBI-401 SDP

12.1.3 Active ingredients: Pseudomonas fluorescens (Pf-CL145A) is the sole active ingredient, $\mathbf{5 0} \%$ active by weight.

12.1.4 Source: Marrone Bio Innovations (MBI); Davis, CA

12.1.5 Lot number: More than one lot will be used. Test article lot numbers for each species will be recorded in the study files.
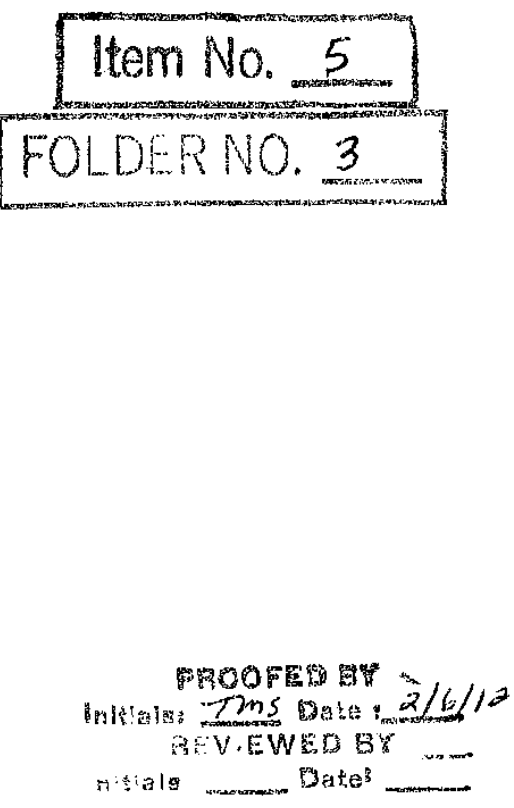


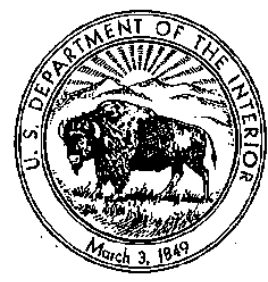

United States Department of the Interior

U.S. GEOLOGICAL SURVEY Biological Resources Division

Upper Midwest Environmental Sciences Center

2630 Fanta Reed Road

La Crosse, Wisconsin 54603

Date: May 12, 2014

To: The Record Study Number AEH-11-PSEUDO-01

5 tops $5 /, i / 1, i j i$

Subject: Amendment 7 - Amendment to the study AEH-11-PSEUDO-01 "Effects of Pseudomonas fluorescens (Pf-CL145A) to glochidia from seven unionid mussel species"

Revision of Study Protocol, Study \# AEH-11PSEUDO-01 as detailed on page 2 of this amendment. Revised text is in bold and underlined, deleted text has a strike hrough.

This amendment documents the change of status of study number AEH-11-PSEUDO-01 titled "Effects of Pseudomonas fluorescens (Pf-CL145A) to glochidia from seven unionid mussel species" from a Good Laboratory Practices (GLP) regulated study to a non-GLP regulated study. The data resulting from this study will not be used to support a product registration, therefore, a verbal decision with UMESC management was agreed to on April 22, 2014 to change the status of the study to non-regulated.

Reviewed by:

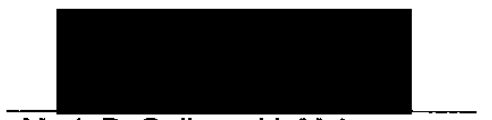

Mark P. Gaikowski, M.A.

Supervisory Biologist

Aquatic Ecosystem Health,

UMESC $^{1}$

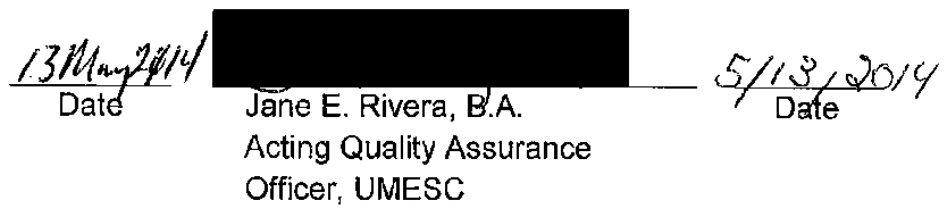

Kevin D. Richards, Ph.D.
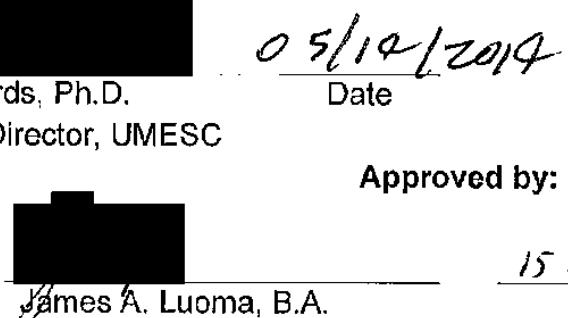

Approved by:

James A. Luoma, B.A.

$\frac{15 \text { nat } 2: 14}{\text { Date }}$

Study Director, UMESC

${ }^{1}$ UMESC: U.S. Geological Survey, Upper Midwest Environmental Sciences Center 
Current text:

10. GOOD LABORATORY PRACTICES

Data collection, storage and retrieval procedures for the study will be conducted in compliance with FDA regulations for Good Laboratory Practices (GLP; 21 CFR, Part 58). The study protocol and progress of the study will be reviewed at the start of the study and periodically throughout the study by the Quality Assurance Unit (QAU). The Study Director has the responsibility of ensuring that all procedures used in conjunction with the study conform with Good Laboratory Practices.

\section{Revised text (in bold and underlined):}

\section{GOOD LABORATORY PRACTICES}

Data collection, storage and retrieval procedures for the study will be not be conducted in compliance with FDA regulations for Good Laboratory Practices (GLP; 21 CFR, Part 58). The study protocol and progress of the study may will be reviewed at the start of the study and periodically throughout the study by the Quality Assurance Unit (QAU). The Study Director has the responsibility of ensuring that all procedures used in conjunction with the study conforms to with Good Scientific taboratory Practices. 


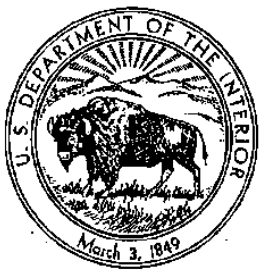

United States Department of the Interior

U.S. GEOLOGICAL SURVEY

Biological Resources Division

Upper Midwest Environmental Sciences Center

2630 Fanta Reed Road

La Crosse, Wisconsin 54603

MEMORANDUM

Date: February 4, 2013

To: The Record study Number AEH-11-PSEUDO-01

Subject: Note To File \#1 to the study AEH-11-PSEUDO-01 "Effects of Pseudomonas fluorescens (PfCL145A) to glochidia from seven unionid mussel species"

This note to file is to document and clarify the lab notebook entries for the study events and procedures used in the Lampsilis cardium, L. Higginsii and L. Siliquoidea glochidia exposures conducted in May of 2011. This document was prepared on May 31, 2011 and signed on February 4, 2013.

\section{Pre-exposure period:}

Test Chemical: (Lab notebook pages $2,3 \& 7$ )

Approximately $96 \mathrm{~g}$ of Pseudomonas fluorescens (PF-CL 145A) test material (lot\# MBI-401-110308A1-BD3; SDP formulation) was received on 28 April, 2011 from the New York State Museum Field Research Laboratory (NYSM-FRL) located in Cambridge, NY. The test material was shipped from Denise Mayer (NYSM-FRL) on 27 April, 2011. The test material was received with a thermometer inside the cooler which indicated a temperature of $9.3^{\circ} \mathrm{C}$ above the ice packs in the cooler. A clean glass thermometer inserted into the test chemical indicated a temperature of $8.5^{\circ} \mathrm{C}$. The temperature observed was greater than the recommend storage temperature of $4^{\circ} \mathrm{C}$. Therefore, an aliquot of test material was returned to the NYSM-FRL after the conclusion of testing at UMESC (shipped May 17, 2011) to confirm the activity of the product. A chemical lab notebook was prepared for the test material upon arrival and the material was placed in a lock box located in a refrigerator in Room 122. On 29 April, 2011, $1.00147 \mathrm{~g}$ of test material was placed into a pre-cleaned glass archive vial for storage in the UMESC ultra-cold freezer chemical archive. All weights and documentation were recorded in the chemical lab notebook.

Donor Mussels: (Lab notebook pages 3-6)

On 09 May 2011, four gravid adult mussels of 3 species [Lampsilis cardium (lot \# 111100), L. higginsii (lot \# 111300) and L. siliquoidea (lot\# 111200)] were received from the Genoa NFH at approximately $1130 \mathrm{~h}$. The mussels were received at approximately $11.7^{\circ} \mathrm{C}$ and they were placed directly into holding tank 1 (compartments $1 \mathrm{~A}, 1 \mathrm{~B}$ and $1 \mathrm{C})$ in room 11 which contained cold $\left(\sim 13^{\circ} \mathrm{C}\right)$ UMESC well water flowing at approximately $1.1 \mathrm{LPM} \mathrm{(1} \mathrm{tank} \mathrm{exchange/hr).} \mathrm{A} \mathrm{temperature} \mathrm{ramp} \mathrm{was} \mathrm{set} \mathrm{to} \mathrm{acclimate} \mathrm{the} \mathrm{donor}$ mussels to test temperature $\left(20^{\circ} \mathrm{C}\right)$ over a period of $60 \mathrm{~h}$. The donor mussels were feed by mixing the following Reed Mariculture (Campbell, CA) instant algae with $14,400 \mathrm{~mL}$ of UMESC well water: $44.0 \mathrm{~g}$ of shellfish diet, $35.3 \mathrm{~g}$ of tetraselmis and $92.4 \mathrm{~g}$ of Thalassiorsira weissflogii. This recipe approximates a $1: 1: 1$ dry weight ratio of $\sim 10 \mathrm{mg} / L$ total dry weight of food in the culture system when delivered to the holding tank at $\sim 10.0 \mathrm{~mL} / \mathrm{min}$. The donor mussels were feed this diet on a continuous basis throughout the entire holding period.

Note: Separate Daily Care Worksheets were prepared for each section of the tank that held mussels, however, water chemistry analysis were only completed on a single compartment, farthest from the incoming water. The measurements were recorded for all 3 sections as the water is not separated between compartments. The data sheets were labeled (5/12/11) with a note (dated 5/12/11) detailing this procedure (File Folder 7A).

cc: UMESC QAU

Page 1 of 6 
UMESC lot numbers were obtained from the UMESC fish culture for the donor mussels. The information regarding the assignment of lot numbers was placed into File Folder $7 \mathrm{~B}$. The following lot numbers were assigned: $L$. Cardium (plain pocketbook; PPB) $=$ Lot \# 11110; $L$. Siliquoidea $($ fat mucket; FAM) $=$ Lo\# $111200 ;$ L. Higginsii (Higgins eye; HGE) $=$ Lot\# 111300.

On May 10, 2011 the temperature acclimation was found to have failed after running 660 minutes. The temperature then increased to the $20^{\circ} \mathrm{C}$ study temperature. A screen capture of the acclimation ramp setting and curve were recorded in the electronic study record (I:LEH-11-PSEUDO-01LDonor mussels) and was saved to disk. The temperatures were then set to remain at the test temperature until the use. The temperature increase would likely have no impacts to the study as the temperature increase was buffered from the existing tank water and it likely took several hours to raise the temperature to $20^{\circ} \mathrm{C}$ in the holding tank.

Daily observations for donor mussels included pH, temperature and dissolved oxygen (D.O.) in addition to daily preparation of food.

Equipment calibration: (Lab notebook pages 4-5)

Adjustable pipettors were calibrated on 09 and 10 May, 2011 for the tips and volumes to be used in the study. Results from the calibrations were placed into File Folder $\uparrow 3$.

Test Water preparation: (Lab notebook pages 6-7)

Three $20 \mathrm{~L}$ glass jars were filled with UMESC well water on 10 May 2011 and supplied with air from an air pump and the water was allowed to equilibrate to room temperature for use in exposures of glochidia. The test water prepared on 10 May 2011 was discarded and replaced with fresh well water on 11 May 2011 due to over aeration of the water. A smaller air pump was used to lightly bubble air into the water acclimation jars. Two jars were placed on bench top in room 15 for use in all aspects of the study (glochidia extraction, stock preparation and dilution water). Siphons were placed into the jars for easy removal of water as required.

Randomization Procedures: (Lab notebook pages 4)

Randomizations were prepared by Mark Gaikowski on 10 May 2011 to perform the following:

1) Assignment of each mussel species to a treatment block $(1,2$, or 3$)$

2) The distribution order of glochidia to exposure chambers for each species

a. Ten separate rounds prepared for each species by exposure chamber

b. Only 5 rounds of prepared distribution rounds were used during the distribution

3) The assignment of treatment to each exposure vessel

a. One of 6 treatment types to each vessel

i. $0,50,100,200$ or $300 \mathrm{mg} / \mathrm{L}$ active ingredient

ii. $300 \mathrm{mg} / \mathrm{L}$. heat deactivated product

b. Three replicates per species

\section{Exposure Period: (initiated 12 May 2011)}

Pipette calibration verification: (Lab notebook pages 7-8)

Pipette calibrations were verified for pipettes $1-4$ by weighing $20^{\circ} \mathrm{C}$ distilled water using a calibrated analytical balance (BAL.1NTS2). The pipette setting and target volume $(\mu \mathrm{l})$ were recorded along with the verification reading $(\mathrm{g})$. Additionally, a summary table was prepared for each pipette indicating the delivery volume, delivery setting and tip type to use for each pipette assigned to the study. The pipette verifications were performed by Todd Severson. The data was placed into File Folder 13.

Initial water Chemistry: (Lab notebook pages 7,13$)$

The water quality parameters were measured on the initial water dilution water (from the $320 \mathrm{~L}$ glass jars) and the donor mussel culture water between 0645 and 0730 by Jeremy Wise on 12 May 2011. The initial parameters included hardness, alkalinity and temperature. The measurements were recorded on Water Quality Form 1 and were filed in File Folder 10a. The initial measurements conducted by Jeremy Wise CC: UMESC QAU

Page 2 of 6 
were found to be conducted in error as he mixed water from the dilution vessels with the donor mussel culture tanks and then performed the water chemistry analysis. Once the mistake was realized, new water chemistry parameters were conducted on remaining dilution water and donor mussel culture tank water (separately) at $\sim 1900 \mathrm{~h}$ by Todd Severson on 12 May 2011 . The water chemistry values were found to be nearly identical to the earlier mixed samples. This was explained on the lab notebook page 13 and the original data sheets that contained the values from the mixed water were noted as such and retained. All of the data sheets were placed into File Folder 10a.

Test Chemical Stock Preparations: (Lab notebook pages 8-10)

In order to preserve efficacy and to maintain similar times between stock preparation and dosing, one stock solution was prepared for each species active dosing (i.e., 50, 100, 200 and $300 \mathrm{mg} / \mathrm{L}$ ). A common stock solution was prepared for dosing the heat deactivated controls for use in all 3 species tested on 12 May 2011. Individual $50 \mathrm{~mL}$ beakers were labeled and tared on analytical balance BAL1. Then $(\sim 0645 \mathrm{~h})$ approximately $2.0 \mathrm{~g}$ of test material was weighed into each beaker. The exact weight of test material was recorded on each beaker and the beaker was then covered with parafilm and refrigerated until used in making a test material solution. The exact weights for each stock solution were as follows: $1.99990 \mathrm{~g}$ used for heat deactivated stock; $2.00062 \mathrm{~g}$ used to make $\mathrm{L}$. cardium active stock; $2.00008 \mathrm{~g}$ used to make $L$. higginsii active stock and $2.00062 \mathrm{~g}$ used to make $L$, siliquoidea active stock. Each stock solution was prepared in the following fashion: the test material was poured through a funnel into a $100 \mathrm{~mL}$ volumetric flask. The $50 \mathrm{~mL}$ beaker and funnel were rinsed with well water and the flask filled approximately $25 \%$ with well water and shaken to suspend the test material. The flask was then filled to $75 \%$ and was shaken for $\sim 15$ minutes to suspend the test material. The resulting foam was allowed to dissipate and the volumetric flask was brought to volume with well water. The heat deactivated stock was placed in a $70^{\circ} \mathrm{C}$ water bath for 45 minutes for deactivation at approximately $0725 \mathrm{~h}$. The heat deactivated stock was allowed to cool to room temperature prior to use in deactivated control dosing. Other stocks were prepared $\sim 30$ minutes prior to use. Test material was weighed by Kerry Weber and the information regarding the stock preparation was recorded on page 8 of the laboratory notebook, File Folder 8 and in the test material lab notebook.

Glochidia extraction: (Lab notebook pages 8-10)

Glochidia were extracted in the order of block assignment (Block $1=L$. cardium, Block $2=L$. Higginsii and Block $3=L$. siliquoidea). The glochidia were all extracted by Nathan Eckert (Genoa NFH) following the same procedures. The glochidia were extracted by irrigating donor gills with well water from a $10 \mathrm{cc}$ syringe fitted with a 1.5 inch 25 gauge needle. The gill water tubes containing glochidia were pierced with the needle and the gill was gently irrigated to express the glochidia. The glochidia were captured in a pre-wetted $500 \mathrm{~mL}$ beaker. From 6-10 water tubes from each mussel were extracted. For more detailed information regarding glochidia extraction and the donor mussels see the laboratory notebook pages 8 10. Glochidia from three mussels of each species were extracted for use in the exposures. The glochidia from each mussel were assessed visually for viability prior to acceptance for use. After acceptable viability was determined $(>80 \%)$, the glochidia were pooled into a common $500 \mathrm{~mL}$ beaker for distribution. Glochidia of each species were extracted at the following times: $L$. cardium $=0900,0910$ and $0922 \mathrm{~h}$; $L$. higginsii $=1033,1041$ and $1045 \mathrm{~h} ; L$. siliquoidea $=1215,1220$ and $1225 \mathrm{~h}$.

Glochidia viability assessment and pooling: (Lab notebook pages 9-10) Glochidia from each mussel were assessed for viability immediately after extraction to determine acceptable viability $(>80 \%)$ for use in exposure trials. Assessments were conducted by Kerry Weber and Theresa Schreier for all the mussels. The procedures used for all mussels were as follows: A $100 \mu \mathrm{l}$ sample of settle glochidia was placed on a $35 \mathrm{~mm}$ petri dish. $200 \mu \mathrm{l}$ of well water was added to the sample and the sample was placed under a dissecting scope (SCO 1). The scope was adjusted for maximum clarity and a photomicrograph was recorded using Image-Pro Express software (SOFT1) and stored in the AEH-11-PSEUDO-01 electronic study folder at the following path I:VEH-11-PSEUDO01YPictures/12May11 1/nitial Viability. A drop of saturated sodium chloride solution $(12 \mathrm{~g} \mathrm{NaCl} / 50 \mathrm{~mL}$ well water, page 7 of lab notebook) was then added to the sample and allowed to sit for 1 minute. A second photomicrograph was recorded for each sample and stored in the same location and path as the previous. The samples were coded for species, mussel number, and before or after salt addition. The samples were visually assessed for viability and if they appeared to have greater than $80 \%$ viability then cC: UMESC QAU

Page 3 of 6 
the mussels were used for the study. No mussels that were assessed were rejected due to initial glochidia viability. Two of the HGE mussels visually appeared to have near $80 \%$ viability so a preliminary hand count was done to determine that they were indeed greater than $80 \%$ viable. The method used was: (total number of glochidia in the sample - [number of glochidia closed prior to addition of the salt solution + number of glochidia open after the addition of the salt solution])/total number of glochidia in the sample *100. The initial counts revealed that the glochidia viability exceeded the minimum threshold of $80 \%$ viability so they were used in the study. The photomicrograph records will be used to determine and document the exact initial viability for each mussel used in the study.

After the glochidia were verified to be of acceptable viability they were pooled into a common $500 \mathrm{~mL}$ beaker and then distributed to the exposure chambers.

Glochidla Lot number assignments: (Lab notebook page 11)

The glochidia from each species were assigned a UMESC lot number by Steve Redman after they were pooled and an estimate of the number of glochidia was available. Glochidia lot number assignment data sheets were completed and filed in File Folder 7C. The lot numbers assigned were as follows: $L$. cardium $\# 111400$, L. higginsii \#111500, and L. siliquoidea \#111600.

Glochidia distribution to the exposure chambers: (Lab notebook pages 9-10,13-14)

The glochidia from all three species were distributed to the exposure chambers using identical procedures. The glochidia were distributed in 5 separate randomized rounds for each species with each exposure chamber receiving a $100 \mu \mathrm{L}$ aliquot of settled glochidia during each round. The order of the aliquots to each exposure chambered followed the randomization order produced by SAS and prepared by Mark Gaikowski (File Folder 14b). Prior to the start of each distribution round and every sixth aliquot, a separate $100 \mu \mathrm{L}$ aliquot was placed on a petri dish for enumeration. The enumeration aliquots will be used to estimate the total number of glochidla that were placed in each exposure chamber. The distribution to the exposure chambers followed these procedures:

1) The exposure chambers were filled with $97 \mathrm{~mL}$ of acclimated test water using a $100 \mathrm{~mL}$ graduated cylinder. Then $500 \mu \mathrm{L}$ was removed from each chamber using a Biohit pipette (PIP3) fitted with a $5000 \mu \mathrm{L}$ tip. The starting volume was then $96.5 \mathrm{~mL}$; which when added to the $500 \mu \mathrm{L}$ of glochidia aliquots and the $3.0 \mathrm{~mL}$ of dosing aliquots the resulting final volume will be $100 \mathrm{~mL}$.

2) The exposure chambers were placed in order in the bench top in room 15.

3) The distributions were performed by James Luoma and were conducted by drawing $100 \mu$ of settled glochidia from the source $1000 \mathrm{~mL}$ beaker of pooled glochidia using an Eppendorf Research Plus pipette (PIP1) fitted with $1000 \mu \mathrm{L}$ wide bore pipette tip. The glochidla were then released below the surface of the water in the exposure chamber identified in the randomization order or placed on a petri dish for distribution enumeration. A watch glass was then placed over the exposure chamber to denote that the chamber had received an aliquot of glochidia for that distribution round. After completion of the distribution round the watch glasses were removed and the procedure was repeated until a total of 5 rounds were completed.

\section{Exposure chamber Dosing: (Lab notebook Pages 9-11)}

After the glochidia were distributed to the exposure chambers the chambers were dosed with the appropriate amount of stock solutions to attain the desired concentration of test chemical. The exposure chambers were previously labeled with color coded tape to denote the assigned test concentration that was determined in the dosing randomization procedures (File Folder 14c). The color coding was as follows: white $=$ control; white with stripes $=300 \mathrm{mg} / \mathrm{L}$ heat deactivated control; yellow $=50 \mathrm{mg} / \mathrm{L}$ active ingredient (A.I.); yellow with stripes $=100 \mathrm{mg} / \mathrm{L} \mathrm{A.I.;} \mathrm{blue}=200 \mathrm{mg} / \mathrm{L} \mathrm{A.I.}$; and blue with stripes $=$ $300 \mathrm{mg} / \mathrm{L} \mathrm{A.I}$. The exposure chambers for each species were arranged on the bench top in room 15 by concentration for the dosing.

All exposure chambers received $3 \mathrm{~mL}$ of the appropriate amount of stock solution(s) required to attain the desired concentration. Chambers that received a combination of control (well water) stock and active ingredient stock to attain the desired concentration received the control water stock first followed by the active ingredient stock. This procedure allowed the final volume of all chambers to be $100 \mathrm{~mL}$ and all

cC: UMESC QAU

Page 4 of 6 
chambers received $3 \mathrm{~mL}$ of stock(s). The amount of each stock that each chamber received was documented on the "Chemical Stock Solution Determination" sheet and the sheets were filed in File Folder 16 along with the "Glochidia Exposure Dosing Form" that was prepared for each species. Dosing for each species was initiated and completed at the following times (File Folder 16): L. cardium: 1036$1040 \mathrm{~h}$; L. higginsii: $1155-1204 \mathrm{~h}$ and $L$, siliquoidea: 1317-1325.

After the dosing was completed for each species the exposure chambers were placed into their appropriate position within the test system (room 16) as determined by the randomization procedures (File Folder 14a). The exposure chambers were then provided light aeration via a disposable glass pipet that was suspended from a PVC air supply line with a length of latex tubing. Air was bubbled into the chambers at a rate to provide approximate 1-5 bubbles/sec.

\section{Water Chemistry - Exposure Period Measurements: (Lab notebook Pages 11 \&15)}

Water chemistry parameters including dissolved oxygen, $\mathrm{pH}$ and temperature were measured and recorded twice during the exposure period, after all dosings were completed on May 12, 2011 and prior to completion of the exposure period on May 13,2011. Measurements were recorded for all test block replicates for the control and high $(300 \mathrm{mg} / \mathrm{L}$ active ingredient) concentrations and one replicate of each of the other concentrations $(50,100,200 \mathrm{mg} / \mathrm{L}$ active ingredient and the $300 \mathrm{mg} / \mathrm{L}$ heat deactivated). Data for the water quality measurements including dissolved oxygen, $\mathrm{pH}$ and temperature were recorded on Water Quality - Form 2 and placed into study File Folder 10b. Additionally, light intensity was measured at the center of each species block and recorded on the bottom of Form 2 . The measurements were conducted by Jeremy Wise and Pajtshiab Moua.

\section{Glochidia Viability Assessments: (Lab notebook pages 14 \& 15)}

Samples of glochidia from each exposure replicate from all species were removed at 6,12 and $24 \mathrm{~h}$ post exposure to determine the viability. The exposure chambers were slightly stirred to bring the glochidia to the center of the chamber. A $100 \mu \mathrm{l}$ sample of settled glochidia was removed from each exposure chamber using an adjustable $1000 \mu /$ pipette (PIP1) fitted with a wide bore pipet tip. The sample was placed onto a pre-labeled (chamber code + sample time) $35 \mathrm{~mm}$ petri dish. $200 \mu \mathrm{ll}$ of $20^{\circ} \mathrm{C}$ well water was added to each sample to increase dispersion for easier enumeration from photomicrograph records. A photomicrograph was recorded for each sample and then 1 drop of saturated sodium chloride was added to each petri dish to induce closure of viable glochidia. A second photomicrograph was recorded after allowing the sample to stand for 1 minute. During the 1 minute closure time the sample was removed from the stage and a new sample petri dish was placed on the stage and a pre-sodium chloride photomicrograph was recorded. Immediately after the 1 minute had elapsed for valve closure the sample was returned to the stage and the after sodium chloride addition photomicrograph was recorded. All photomicrograph records were recorded in the studies electronic file log and were coded for exposure chamber, sampling time, and before (bs) or after (as) sodium chloride addition. The photomicrograph records were recorded by Kerry Weber and Theresa Schreier;', the samples were collected by Jim Luoma and Todd Severson.

Note: No 24h data was collected from exposure chamber $2 \mathrm{B1}$ (HGE control) as it was inadvertently pooled with the control replicates for Block 1 during the pooling for final water quality analysis. When this was discovered, additional $24 \mathrm{~h}$ viability samples were removed and processed as described above for the 2 remaining HGE control chambers, $2 \mathrm{C} 3$ and $2 \mathrm{C} 6$. This is further explained in study deviations \# 1 and 2 , dated $6 / 3 / 2011$. The study deviation is located in the electronic study file as well as File Folder 3.

Water Chemistry - Experimental Termination Measurements: (Lab notebook Page16)

After completion of the $24 \mathrm{~h}$ viability sampling and the second exposure period water quality measurements (DO, $\mathrm{pH}$, temp) the replicate concentrations from each species block were pooled for final hardness, alkalinity, conductivity and ammonia analysis.

cC: UMESC QAU 
Initially, hardness and alkalinity data analysis were performed on the control and high concentrations as described in the study protocol. Due to a slight variance in the observed measurements, hardness and alkalinity measurements were performed for all pooled concentrations

Conductivity and total ammonia were recorded for all pooled replicate samples. $\mathrm{pH}$ and temperature were not initially determined for the pooled replicate samples (Block 1, PPB) however, due to what appeared to be high ammonia concentrations, the termination water quality (Form 3 ) was modified and $\mathrm{pH}$ and temperature data were recorded for the pooled replicate samples for Blocks 2 and 3 (HGE and FAM).

Un-ionized ammonia concentrations for the pooled replicate samples from Blocks 2 and 3 can be determined using the collected $\mathrm{pH}$ and temperature data. Un-ionized ammonia concentrations for Block 1 will have to be estimated using the second exposure period temperature and $\mathrm{pH}$ measurements collected prior to termination. The meters used to collect the terminal water quality data were calibrated according to their respective SOP's and are denoted in the study as pH2, Therm 3, CON1 and PHO1. All data sheets for the terminal water quality were placed into study File Folder $10 \mathrm{C}$.

\section{Final Termination: (Lab notebook Page17)}

The glochidla were euthanized by MS-222 overdose and discarded. All sample analysis was completed by $1530 \mathrm{~h}$ on $05 / 13 / 2011$.

Prepared by:

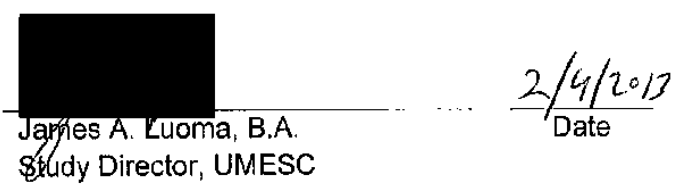

cC: UMESC QAU

Page 6 of 6 


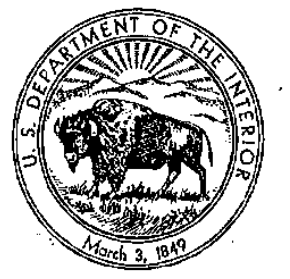

United States Department of the Interior

U.S. GEOLOGICAL SURVEY

Biological Resources Division

Upper Midwest Environmental Sciences Center

2630 Fanta Reed Road

La Crosse, Wisconsin 54603

\section{MEMORANDUM}

Date: October 31,2012

To: The Record study Number AEH-11-PSEUDO-01

Subject: Note To File \#2 to the study AEH-11-PSEUDO-01 "Effects of Pseudomonas fluorescens (PfCL145A) to glochidia from seven unionid mussel species"

This note to file is to document and clarify the path, folder and file names for glochidia photomicrographs for study number AEH-11-PSEUDO-01.

Pictures of glochidia (photomicrographs) were used to: 1) determine the viability of extracted glochidia that were used in the exposures; 2 ) estimate the number of glochidia distributed to each exposure replicate and; 3$)$ to assess the treatment effects on the viability of glochidia at three time points $(6,12$ and 24 hour) during the exposure period.

The original and enumerated photomicrograph files are saved as described below. Additionally, how the files were enumerated is also described.

\section{ORIGINAL PHOTOMICROGRAPHS}

Initial Viability

Photomicrographs were recorded to document the viability of glochidia extracted from each donor mussel. The glochidia were photographed before and after a concentrated salt solution was added to the sample to induce valve closure as a valve closure response indicates viability. Glochidia that visually appeared to have suitable viability $(>80 \%)$ were pooled for use prior to determining the initial viability. Examples of path and filenames for the original initial viability photomicrographs are shown in Table 1.

Table 1. Initial Viability Path and Filenames

Path ||$_{\substack{\text { Study Folder } \\ \text { (Location of all study specific data) }}}^{\text {(Location of all study photomicrographs) }}$

Page 1 of 7 
Table 1 (con.) Initial Viability Path and Filenames

Filename $\left.\right|_{\substack{\text { Mussel } \#(1,2 \text { or } 3) \\ \text { Formulation Type (FDP or SDP) } \\ \text { Species } 3 \text { Letter Code }}} ^{\text {FAM-SDP-2-I-bS }}$

\section{Distribution}

Photomicrographs were recorded on $100 \mu \mathrm{L}$ glochidia samples collected during each round of glochidia distribution to the exposure chambers. Glochidia distribution was completed in 5 randomized rounds with $18100 \mu \mathrm{L}$ glochidia aliquots (1 aliquot per exposure chamber) per round. Photomicrographs were recorded on the initial aliquot and then after every 6 aliquots for a total of 16 distribution samples per species. Distribution samples that had very dense quantities of glochidia were subdivided into more manageable counts before being photomicrographed. Plain pocketbook (PPB) was the only species exposed to both SDP and FDP formulations. Therefore, only the distribution file names for PPB were modified to include the formulation type. Examples of path and filenames for the original distribution photomicrographs are shown in Table 2.

Table 2. Distribution Path and Filenames

\begin{tabular}{|c|c|c|}
\hline Path & $\begin{array}{l}\text { I://AEH-11-PSEUDO-01/Pictures/1800 } \\
\text { (Location of all study specific data) } \\
\text { (Location of all st } \\
\text { (Location of all study data) }\end{array}$ & $\begin{array}{l}\text { IDistribution/PPB-R2-2.1 } \\
\text { Pample Type Folder } \\
\text { (Initial Viability, Distribution, } 6 \mathrm{~h}, 12 \mathrm{~h} \text { or } 24 \mathrm{~h} \text { ) } \\
\text { late Folder } \\
180 \mathrm{C} T 11,17 \mathrm{JAN} 12 \text { or 19JAN12) } \\
\text { hotomicrographs) }\end{array}$ \\
\hline Filename & 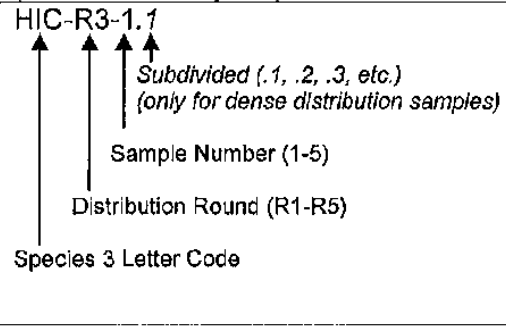 & $\left.\right|_{\text {Formulation Type (FDP or SDP) }} ^{\text {Distribution Round (R1-R5) }}$ \\
\hline
\end{tabular}

Viability Samples $(6,12$ and 24 hour)

Two photomicrographs were recorded for each sample collected for exposure period viability assessment. The first photomicrograph was recorded prior to the addition of a salt solution to induce valve closure and the second after induced closure. Photomicrographs were recorded on $100 \mu \mathrm{L}$ glochidia samples collected from each exposure chamber at 6,12 and 24 hours post-dose initiation. 
Examples of path and filenames for the original viability samples $(6,12$ and 24 hour $)$ photomicrographs are shown in Table 3.

Table 3. Viability Samples $(6,12$ and 24 hour) Path and Filenames

Path ||$_{\text {Filename }}^{\text {B. }}$

\section{ENUMERATED PHOTOMICROGRAPHS}

Enumerated data files are saved in different electronic folders, depending on when they were enumerated. Some photomicrograph files were inadvertently over-written during file conversion and during the enumeration process (see Deviations 8 and 9 ). To prevent reoccurrence, the folders containing the raw data files were locked to prevent additional file overwrites. All of the initial viability photomicrographs were enumerated prior to the locking of the electronic file folders. All of distribution photomicrographs were enumerated after the locking of the electronic file folders. Some of the viability samples were enumerated prior to the locking of the electronic file folders (FAM, PPB and HGE from 12 May 11 at 12 and 24 hour; PPB from 180 CT11 at 6,12 and 24 hour) while other viability samples were enumerated after the locking of the electronic file folders (FAM, PPB and HGE from 12May11 at 6h; BLS and MUC from 17JAN12 at 6, 12 and 24 hour; HIC from 19JAN12 at 6, 12, and 24 hour). Those files that were enumerated prior to the locking of the electronic file folders were saved in the raw data file folders in 
a separate electronic folder entitled "Enumerated" (e.g., l://AEH-11-PSEUDO-

01/Pictures/180CT11/6h/Enumerated/PPB-FDP-ctrl-3B6-6h-bs-C). Those files that were enumerated after the locking of the electronic file folders were saved in a separate enumerated file folder within the study folder (e.g., l://AEH-11-PSEUDO-01/Pictures/Enumerated/180CT11/6h/PPB-FDP-ctrl-3B6-6h-bsC).

\section{Initial Viability}

All of the initial viability photomicrographs were enumerated prior to the locking of the raw data electronic file folders. The photomicrographs were enumerated for total number of glochidia within the sample and the number of glochidia open or closed before and after salt addition. The percentage of viable glochidia (i.e., glochidia that respond to salt exposure by valve closure) was calculated by the following method

Percent Viability $=$ $\left(\frac{\text { [Total number of glochidla in sample-(Number of glochldia closed before salt }+ \text { Number of glochldia open after salt) })}{\text { Total number of glochldia in sample }}\right) * 100$

For more specific details regarding the enumeration process, refer to Amendment 1 of the protocol. Examples of path and filenames for the enumerated initial viability photomicrographs are shown in Table 4.

Table 4. Enumerated Initial Viability Path and Filename

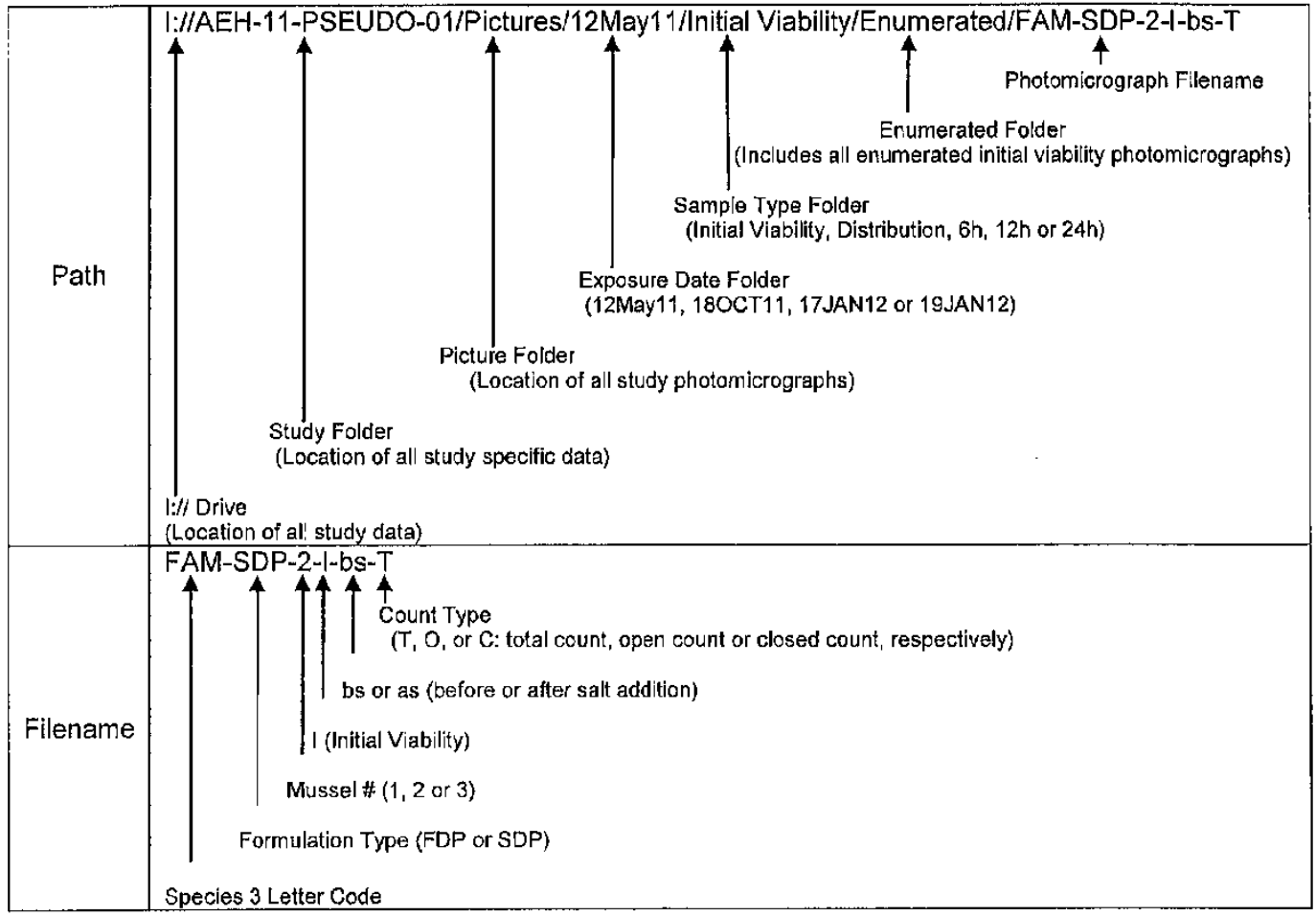

Page 4 of 7 


\section{Distribution}

All of the distribution photomicrographs were enumerated after the locking of the raw data electronic file folders containing the original photomicrograph files. The photomicrographs were recorded and enumerated to estimate the number of animals distributed to each chamber. Distribution samples in which the glochidia were too dense to enumerate from a single photomicrograph were subdivided and multiple photomicrographs were recorded for each sample. Plain pocketbook (PPB) was the only species exposed to both SDP and FDP formulations, therefore, the distribution file names for PPB were the only files renamed to include the formulation type used. Distribution photomicrographs were enumerated for total counts only. All glochidia, regardless of shell position (open or closed) were counted for each sample. Enumerated files were saved with a " $T$ " to designate that they had been counted. Examples of path and filenames for the enumerated distribution photomicrographs are shown in Table 5.

Table 5. Enumerated Distribution Path and Filename

\begin{tabular}{|c|c|c|}
\hline Path & \multicolumn{2}{|c|}{ 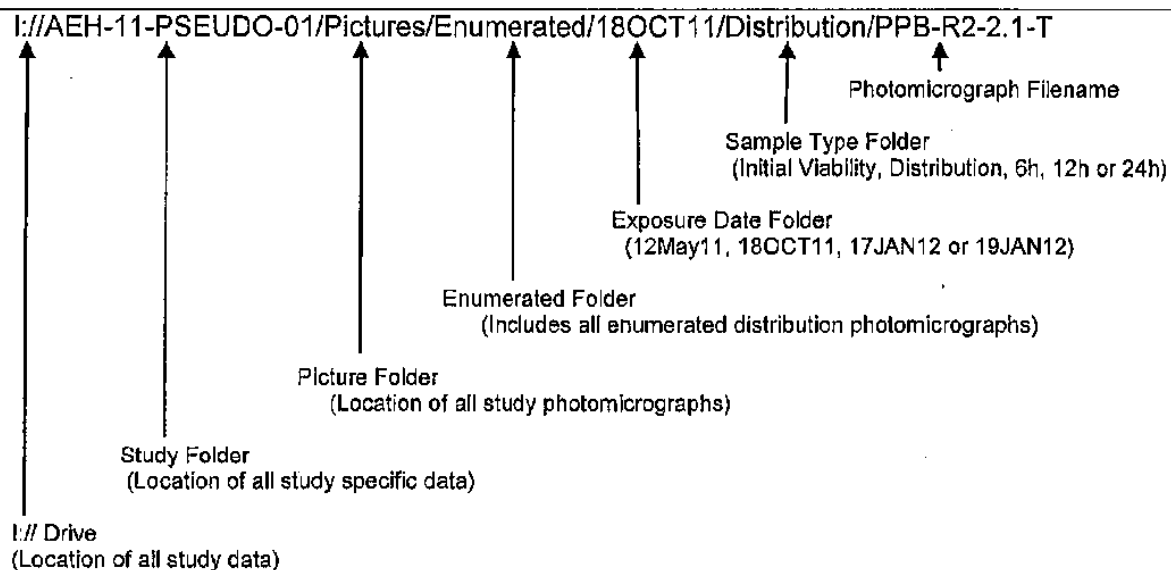 } \\
\hline Filename & $\begin{array}{l}\text { Count Type ( } T=\text { total count) } \\
\text { Subdivided }(1, .2, .3 \text {, etc.) } \\
\text { (only for dense distribution samples) }\end{array}$ & 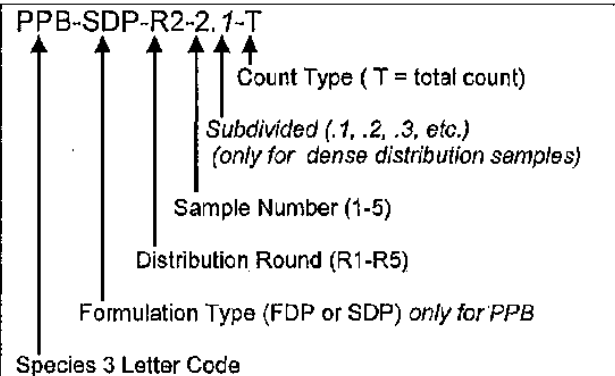 \\
\hline
\end{tabular}

Viability Samples $(6,12$ and 24 hour $)$

Photomicrographs were recorded on glochidia samples collected from each exposure chamber at 6,12 and 24 hours post-dose initiation for viability assessment. Two photomicrographs were recorded for each sample. The first photomicrograph was recorded prior to the addition of a salt solution to induce valve closure (a closure response indicates viability) and the second after induced closure. To determine viability the photomicrographs were examined to 1) determine the total number of glochidia with the sample, 2) to determine the number of glochidia in an open state prior to induced valve closure and 3 ) the number of open glochidia (prior to the addition of salt solution ) that responded to the addition of the salt solution by valve closure. The photomicrographs were enumerated for total number of glochidia within the sample $(T)$ and the number of glochidia open $(O)$ or closed $(C)$ before and after salt addition. The percentage of viable glochidia (i.e., glochidia that respond to salt exposure by valve closure) was 
calculated by the following method:

Percent Viability $=$

$\left(\frac{\text { Trotal number of glochidia in sample }-(\text { Number of glochidia closed before salt }+ \text { Number of glochidla open after salt })}{\text { Total number of glochidia in sample }}\right) * 100$

For more specific details regarding the enumeration process, refer to Amendment 1 of the protocol.

Enumerated photomicrograph files were saved in one of two locations. Viability samples enumerated prior to the locking of the electronic file folders (FAM, PPB and HGE from 12May 11 at 12 and 24 hour; PPB from $180 C T 11$ at 6,12 and 24 hour) are saved within the raw data file folders in a separate electronic folder entitled enumerated. Viability samples enumerated after the locking of the electronic file folders (FAM, PPB and HGE from 12May11 at 6h; BLS and MUC from 17JAN12 at 6, 12 and 24 hour; HIC from 19JAN12 at 6,12, and 24 hour) are saved in a separated enumerated file folder within the study folder. Examples of path and filenames for the enumerated viability samples $(6,12$ and 24 hour) photomicrographs are shown in Table 6.

Table 6. Enumerated 6, 12 and 24 hour Viability Samples Path and Filenames

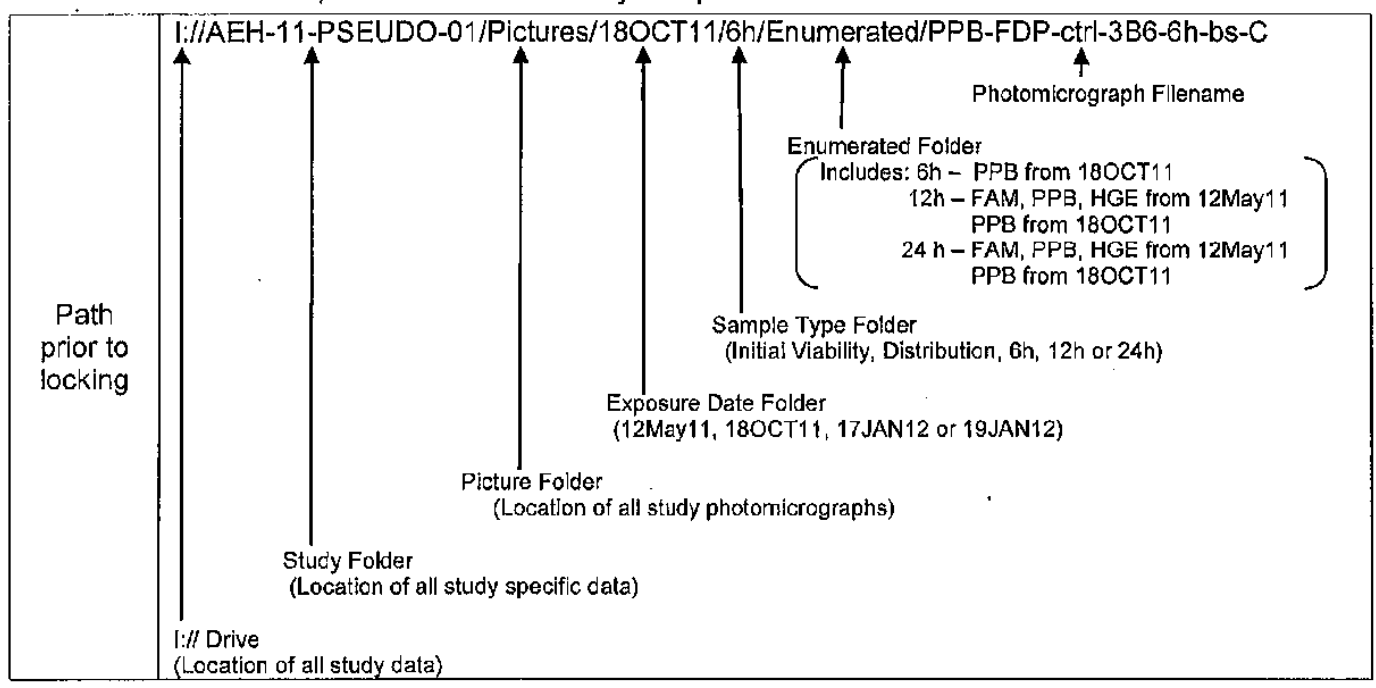


Table 6 (con.) Enumerated 6, 12 and 24 hour Viability Samples Path and Filenames

\begin{tabular}{|c|c|}
\hline $\begin{array}{l}\text { Path } \\
\text { after } \\
\text { locking }\end{array}$ & 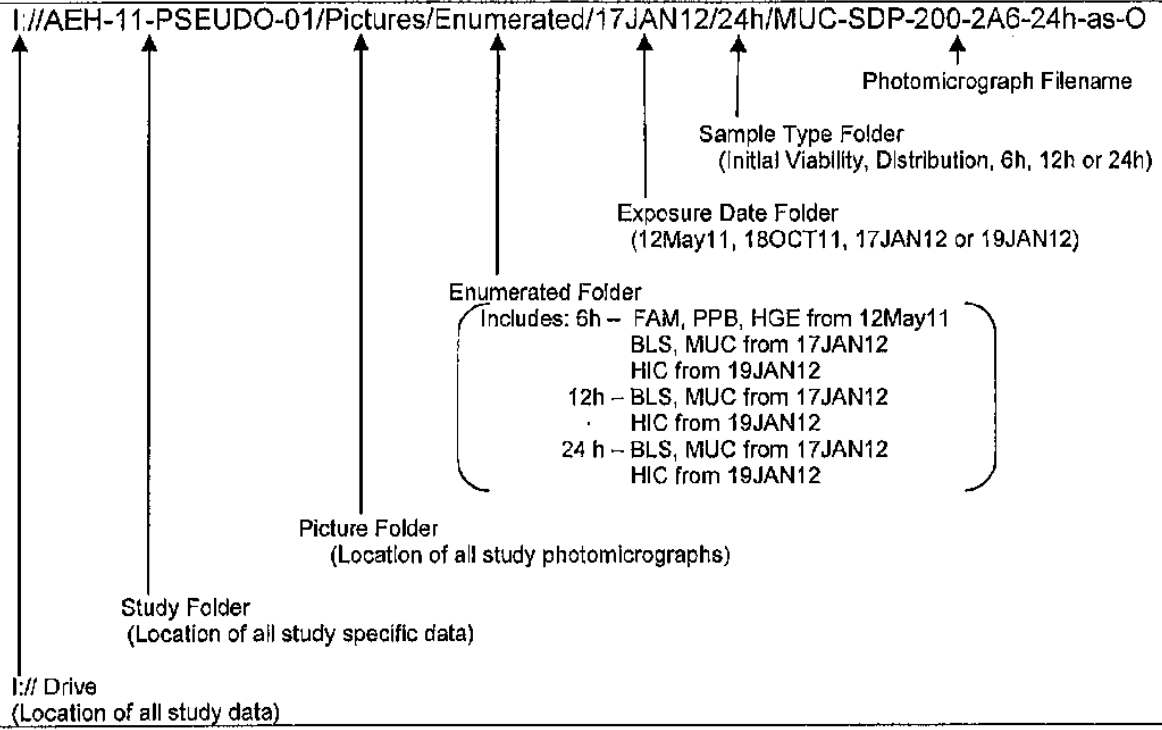 \\
\hline Filename & 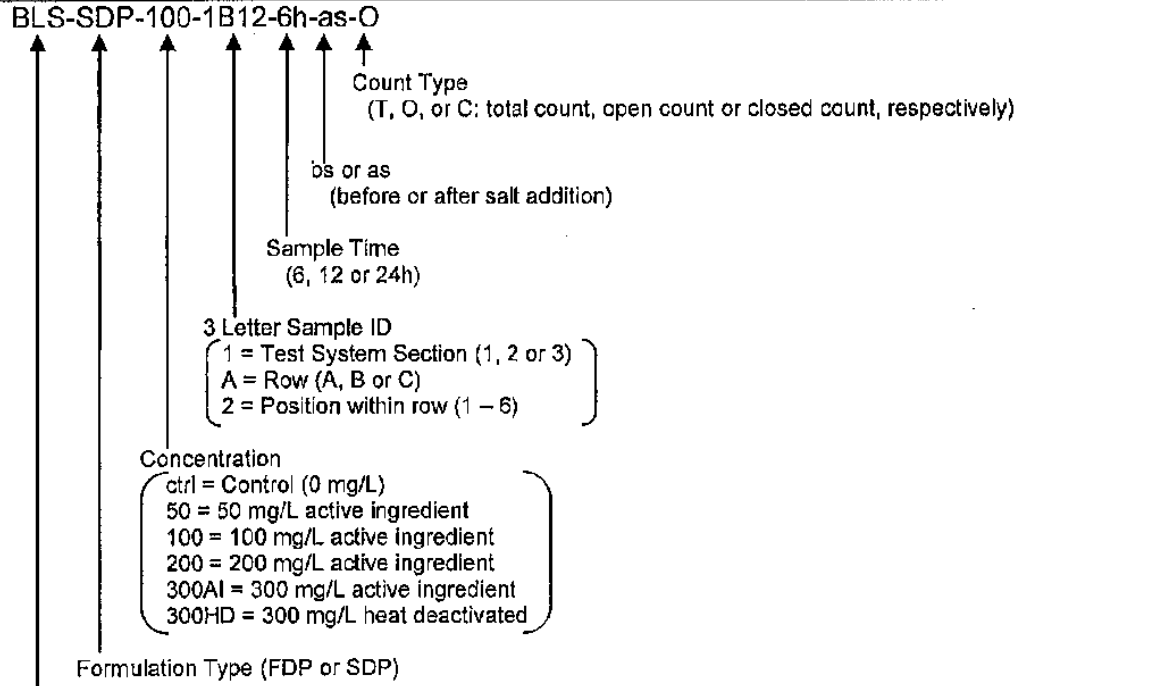 \\
\hline
\end{tabular}

The electronic log can be used to also determine where the raw and enumerated files are located in the electronic study folder.
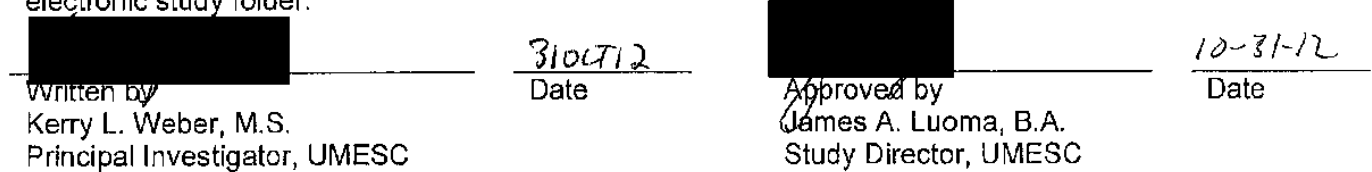

C: UMESC QAU

Page 7 of 7 
Study Title: "Effects of Pseudomonas fluorescens (Pf-CL145A) to glochidia from seven unionid mussel species"

Study number: AEH-11-PSEUDO-01

File Folder:

Lab book/pgs:

Reviewed: Verified:

\section{Donor Mussel UMESC Lot Number Assignment Form}

DONOR MUSSEL INFORMATION:

Species:

Number of Donor Mussels: Collection Date:

Collection Location:

Species Identification performed by:

Title/affiliation of identifier:

UMESC Arrival Date

Receiving UMESC tank/room \#

Additional information:

UMESC LOT NUMBER DESIGNATION:

Witness and form recorded by:

UMESC

Printed Name

Signature

Affiliation

Date

This datasheet was approved by

11JAN2012/Version 1.1

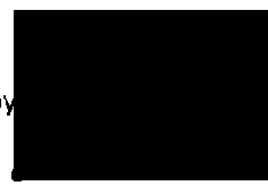

on $125 \mathrm{sin} 2012$ 
Study Number AEH-11-PSEUDO-01

File Folder:

Lab book/pgs:

Reviewed:

Verified:

Daily Care Worksheet

Donor Mussel Holding

\begin{tabular}{|c|c|c|c|c|c|c|c|}
\hline \multirow{3}{*}{$\begin{array}{l}\text { Tank \#: } \\
\text { Species: } \\
\text { Instrume } \\
\text { Day }\end{array}$} & \multicolumn{2}{|c|}{ Section \#: } & \multicolumn{2}{|c|}{$\begin{array}{l}\text { Room \#: } \\
\text { Lot Number: }\end{array}$} & \multicolumn{2}{|c|}{ Month/Year: } & \multirow[b]{3}{*}{ Initials } \\
\hline & \multirow{2}{*}{$\begin{array}{c}\text { Feed } \\
\text { Time } \\
\text { (military) } \\
\end{array}$} & \multicolumn{2}{|c|}{$\begin{array}{l}\text { Flow Rate } \\
\text { (mL/min) }\end{array}$} & \multirow[b]{2}{*}{$\begin{array}{c}\text { Temperature } \\
\left({ }^{\circ} \mathrm{C}\right)\end{array}$} & \multirow[b]{2}{*}{$\begin{array}{c}\text { Dissolved } \\
\text { Oxygen (mg/L) } \\
\end{array}$} & \multirow[b]{2}{*}{$\mathrm{pH}$} & \\
\hline & & Inflow & Pump & & & & \\
\hline 1 & & & & & & & \\
\hline 2 & & & & & & & \\
\hline 3 & & & & & & & \\
\hline 4 & & & & & & & \\
\hline 5 & & & & & & & \\
\hline 6 & & & & & & & \\
\hline 7 & & & & & & & \\
\hline 8 & & & & & & & \\
\hline 9 & & & & & & & \\
\hline 10 & & & & & & & \\
\hline 11. & & & & & & & \\
\hline 12 & & & & & & & \\
\hline 13 & & & & & & & \\
\hline 14 & & & & & & & \\
\hline 15 & & & & & & & \\
\hline 16 & & & & & & & \\
\hline 17 & & & & & & & \\
\hline 18 & & & & & & & \\
\hline 19 & & & & & & & \\
\hline 20 & & & & & & & \\
\hline 21 & & & & & & & \\
\hline 22 & & & & & & & \\
\hline 23 & & & & & & & \\
\hline 24 & & & & & & & \\
\hline 25 & & & & & & & \\
\hline 26 & & & & & & & \\
\hline 27 & & & & & & & \\
\hline 28 & & & & & & & \\
\hline 29 & & & & & & & \\
\hline 30 & & & & & & & \\
\hline 31 & & & & & & & \\
\hline
\end{tabular}

This datasheet was approved $b$ 11JAN2012/version1.1

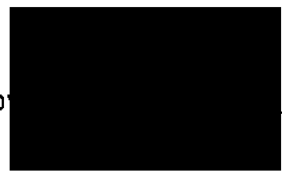

on $12 \sqrt{\pi} \times 2 / 2$. 
AEH-11-PSEUDO-01

Adult Mussel Holding Daily Algal Diet

1/13/2012

Tank Dimensions and volume

$48^{\prime \prime} \times 14^{\prime \prime} \times 6^{\prime \prime}(121.92 \mathrm{~cm} \times 35.56 \mathrm{~cm} \times 15.24 \mathrm{~cm})=66,073 \mathrm{~cm}^{3}$

$6^{\prime \prime}(15.24 \mathrm{~cm})$ standpipe: $66.073 \mathrm{~L}$

Flow rate (1 turnover per hour): $1.1 \mathrm{~L} / \mathrm{m}$ )

Daily water volume: $1.1 \mathrm{~L} / \mathrm{m} \times 60 \mathrm{~m} / \mathrm{h} \times 24 \mathrm{~h} / \mathrm{d}=1584 \mathrm{~L} / \mathrm{d}$

Diet weights ( $50 \%$ Tetraselmis and 50\% Nannochloropsis)

to achieve $10 \mathrm{mg} / \mathrm{L}$ by dry weight:

Tetrase/mis: Target 5.0 mg/L (17.93\% Dry Wt); Nannochloropsis: Target $5.0 \mathrm{mg} / \mathrm{L}$ (20.48\% Dry Wt)

Tetrase/mis: $27.89 \mathrm{mg} / \mathrm{L}$; Nannochloropsis: $24.41 \mathrm{mg} / \mathrm{L}$

$\times 1584 \mathrm{~L} / \mathrm{d}$

Tetraselmis: $44.2 \mathrm{~g} / \mathrm{d}$; Nannochloropsis: $38.7 \mathrm{~g} / \mathrm{d}$

Diet Inflow Rate: $\quad 10 \mathrm{~mL} / \mathrm{min} \times 60 \mathrm{~m} / \mathrm{h} \times 24 \mathrm{~h} / \mathrm{d}=14400 \mathrm{~mL} / \mathrm{d}$

Daily Care:

Mix 44.2g Tetraselmis and $38.7 \mathrm{~g}$ Nannochloropsis with $14,400 \mathrm{~mL}$ well water each day, to be delivered by peristaltic pump at $10 \mathrm{~mL}$ per minute. Prepare fresh diet daily.

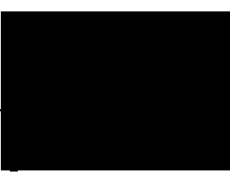

Date: $13 \operatorname{sen} 20 / 2$ 
Study Title: "Effects of Pseudomonas fluorescens (Pf-CL145A) to glochidia from seven unionid mussel species"

Study number: AEH-11-PSEUDO-01

File Folder: Lab book/pgs:

Reviewed: Verified:

\section{Glochidia Test Organism UMESC Lot Number Assignment Form}

Species:

Number of Donor Mussels: Donor Mussel Lot \#:

Extraction Date: $\quad / \quad / \quad$ Extraction Time (military)

Extraction Location: Container ID:

Approximate Number of glochidia:

Glochidia extracted by: Affiliation:

Additional information:

\section{UMESC GLOCHIDIA LO'T NUMBER DESIGNATION:}

Witness and form recorded by:

$\frac{\text { Printed Name }}{\frac{\text { Signature }}{\text { UMESC }}} \frac{\text { Affiliation }}{\text { Date }}$

This datasheet was approved by

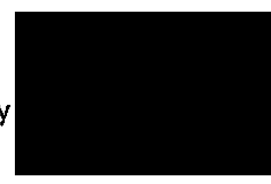

on $12 . \sqrt{P A} \mathrm{Z}$. 
Study Títle: "Effects of Pseudomonas fluorescens (Pf-CL145A) to glochidia from seven unionid mussel species"

Study number: AEH-11-PSEUDO-01

File Folder: Lab book/pgs: Reviewed: Verified:

\section{Glochidia Aliquot Distribution Form}

Species: UMESC LOT NUMBER:

Test Block Assignment (circle one): \begin{tabular}{lll}
$1 \quad 2 \quad 3$ \\
\hline
\end{tabular}

Aliquot Volume ( $\mu$ ): Estimated \# glochidia/aliquot

Initial Distribution aliquot date/time (military):

Final Distribution Aliquot date/time (military):

Number of Aliquot rounds: Estimated \# glochidia/chamber

Additional information:

Witness and form recorded by:

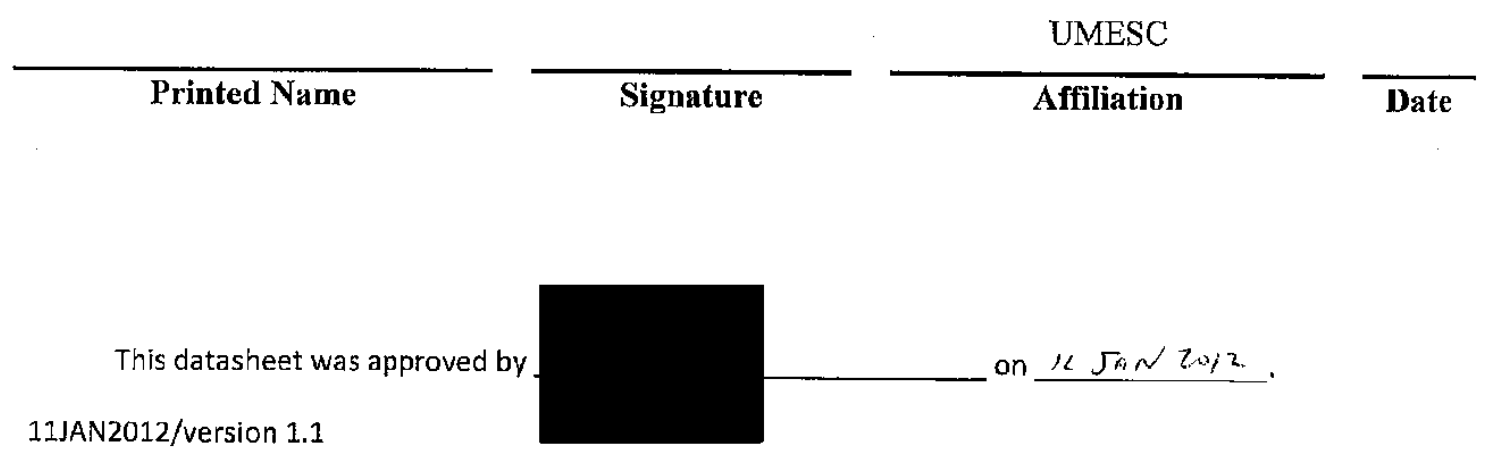




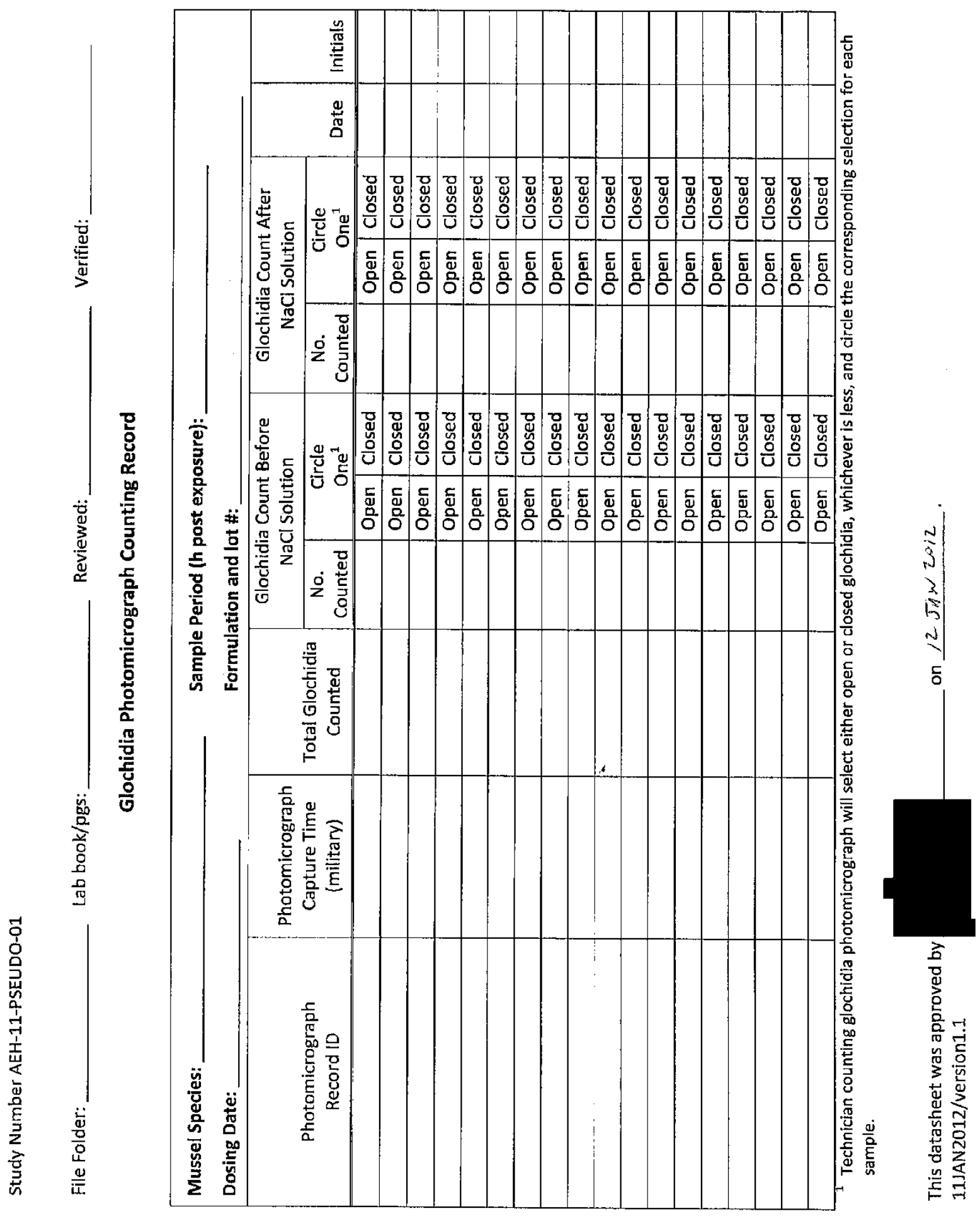



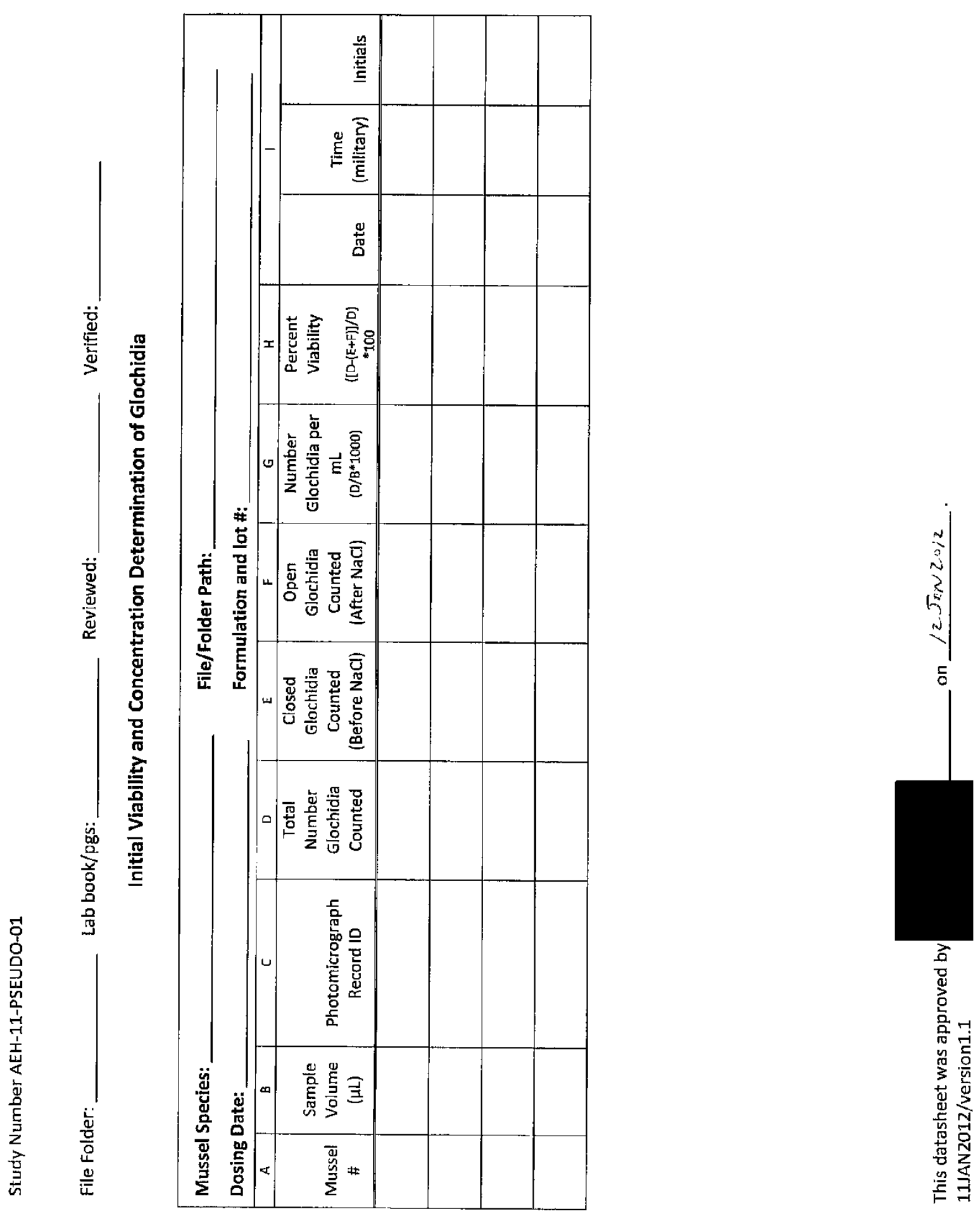
Study Number AEH-11-PSEUDO-01

File Folder: Lab book/pgs:

Reviewed: Verified:

\section{Water Quality - Form 1}

Initial (Dilution Water Hardness, Alkalinity, and Temperature)

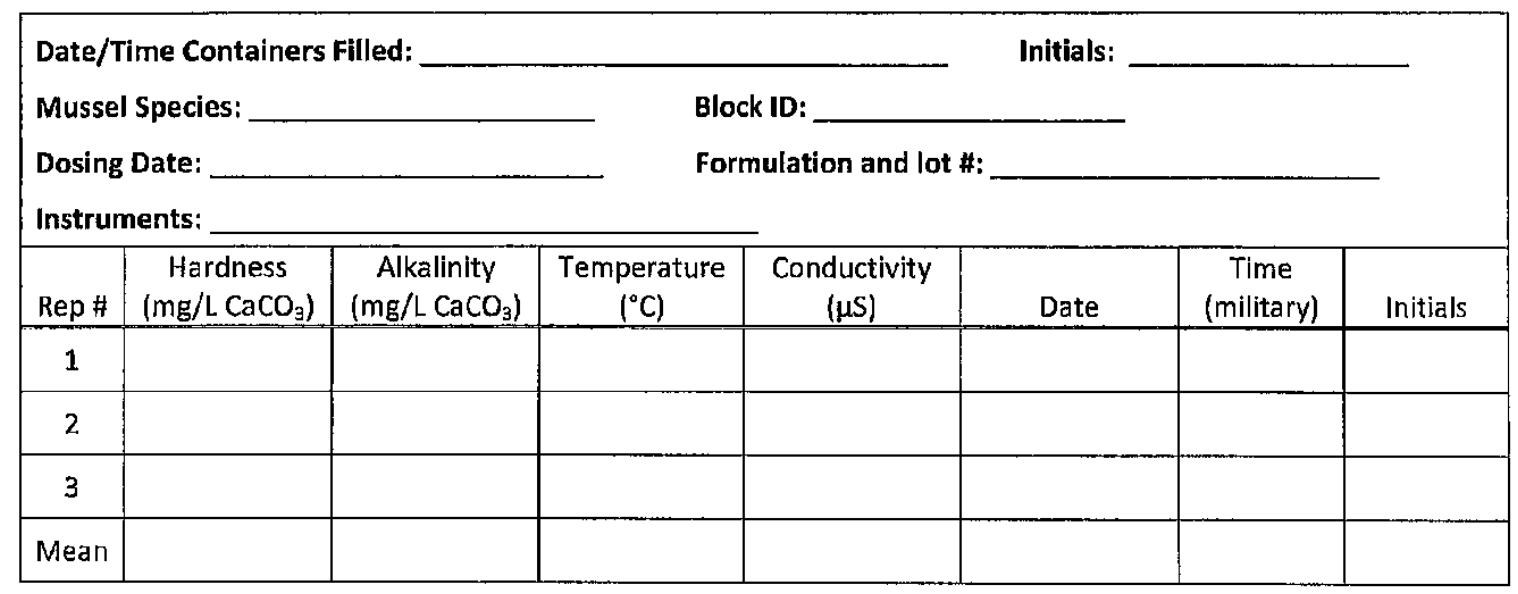

This datasheet was approved by 11JAN2012/Version 1.1

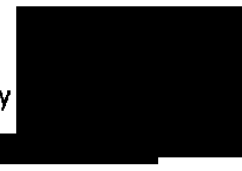

on $17 \sqrt{11} \times 2,2$ 
Study Number AEH-11-PSEUDO-01

File Folder: Lab book/pgs:

Reviewed: Verified:

Water Quality - Form 2

During Exposure (Dissolved Oxygen, pH, Temperature)

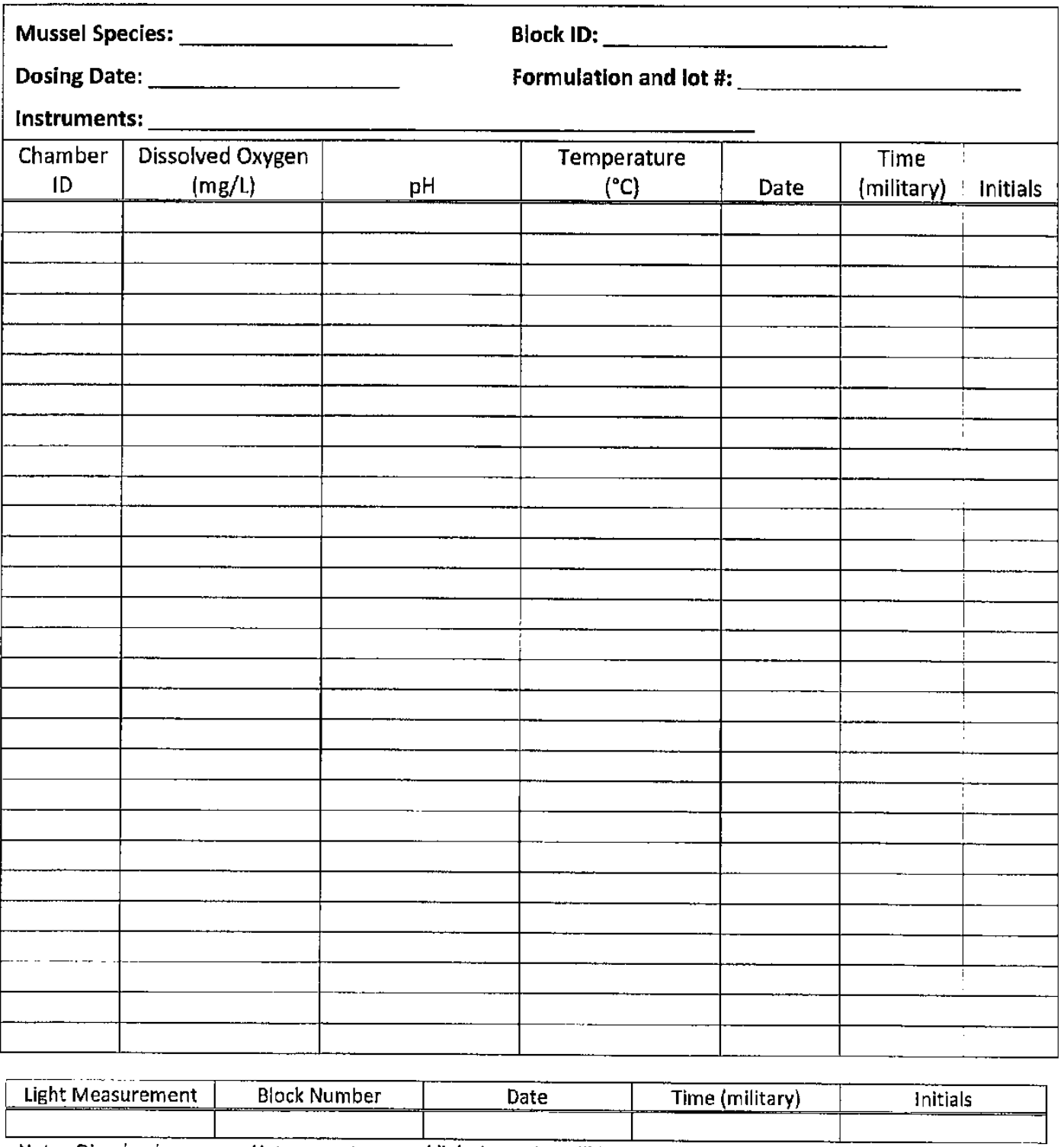

Note: Dissolved oxygen, $\mathrm{pH}$, temperature, and light intensity will be measured at least twice during the exposure period in the controls and high concentrations.

11JAN2012/vs1.2

This datasheet was approved by

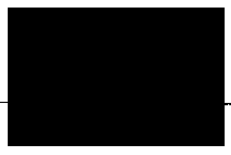
on $12.0720 \% 2$ 
Study Number AEH-11-PSEUDO-01

File Folder:

Lab book/pgs:

Reviewed:

Verified:

Water Quality - Form 3

Upon Termination (Hardness, Alkalinity, Conductivity, Temperature, and pH)

Mussel Species:

Dosing Date:

Instruments:
Block ID:

Formulation and lot \#:

\begin{tabular}{|c|c|c|c|c|c|c|c|}
\hline $\begin{array}{l}\text { Pooled Sample } \\
\text { Concentration }\end{array}$ & Chamber IDs & $\begin{array}{l}\text { Hardness } \\
\left(\mathrm{mg} / \mathrm{LCaCO}_{3}\right)\end{array}$ & $\begin{array}{c}\text { Alkalinity } \\
\left(\mathrm{mg} / \mathrm{LCaCO}_{3}\right)\end{array}$ & $\begin{array}{l}\text { Conductivlty } \\
(\mu \mathrm{S} / \mathrm{cm})\end{array}$ & Date & $\begin{array}{c}\text { Time } \\
\text { (military) }\end{array}$ & Initials \\
\hline & & & & & & & \\
\hline & & & & & & & \\
\hline & & & & & & . & \\
\hline & & & & & & & \\
\hline & & & & & & & \\
\hline & & & & & & & \\
\hline
\end{tabular}

Note: Hardness, alkalinity, and conductivity will be measured upon termination of trial from pooled replicate samples.

\begin{tabular}{|c|c|c|c|c|c|}
\hline Chamber ID & Temperature $\left({ }^{\circ} \mathrm{C}\right)$ & $\mathrm{pH}$ & Date & Time (military) & Initials \\
\hline & & & & & \\
\hline & & & & & \\
\hline & & & & & \\
\hline & & & & & \\
\hline & & & & & \\
\hline & & & & & \\
\hline & & & & & \\
\hline & & & & & \\
\hline & & & & & \\
\hline & & & & & \\
\hline & & & & & \\
\hline & & & & & \\
\hline & & & & & \\
\hline & & & & & \\
\hline & & & & & \\
\hline & & & & & \\
\hline & & & & & \\
\hline & & & & & \\
\hline
\end{tabular}

Note: Temperature and $\mathrm{pH}$ will be measured upon termination of trial from each indlvidual test chamber. These values will be used for ammonia analysis.

13JAN2012/V51.2

This datasheet was approved by

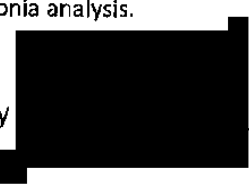

on $17 \sin 2002$. 


\section{Study Title: "Effects of Pseudomonas fluorescens (Pf-CL145A) to glochidia from seven different unionid species"}

Study number: AEH-11-PSEUDO-01

File Folder: Lab book/pgs:

Reviewed: Verified;

\section{Test Chemical Stock Preparation Data Form}

Test Chemical: Pseudomonas fluorescens strain 145A

Test Chemical Lot \# Date Rec'd Exp. Date

Mussel Species Block ID

Instruments:

\section{Chemical Weighing:}

\begin{tabular}{|c|c|c|c|}
\hline Sample I.D. & Sample wt. (g) & Date/Time & Initials \\
\hline & & & \\
\hline & & & \\
\hline & & & \\
\hline & & & \\
\hline & & & \\
\hline
\end{tabular}

*Chemical samples to be stored refrigerated until used for stock preparation.

\section{Stock Preparation:}

\begin{tabular}{|c|c|c|c|c|c|}
\hline Sample I.D. & $\begin{array}{l}\text { Dilution Vol. } \\
\text { (ml) }\end{array}$ & $\begin{array}{l}\text { Dilution } \\
\text { time }\end{array}$ & $\begin{array}{c}\text { Use (ie: Active stock for } \\
\text { HGE) }\end{array}$ & $\begin{array}{l}\text { Date/ } \\
\text { Time }\end{array}$ & Initials \\
\hline & & & & & \\
\hline & & & & & \\
\hline & & & & & \\
\hline & & & & & \\
\hline & & & & & \\
\hline
\end{tabular}

* Stocks to be prepared immediately before use, except for heat deactivated stock which will be prepared prior to use to allow for deactivation and cooling.

This datasheet was approved by 11JAN2012/version 1.1

$$
\text { on } 12 \sqrt{10} \times 20,2 .
$$




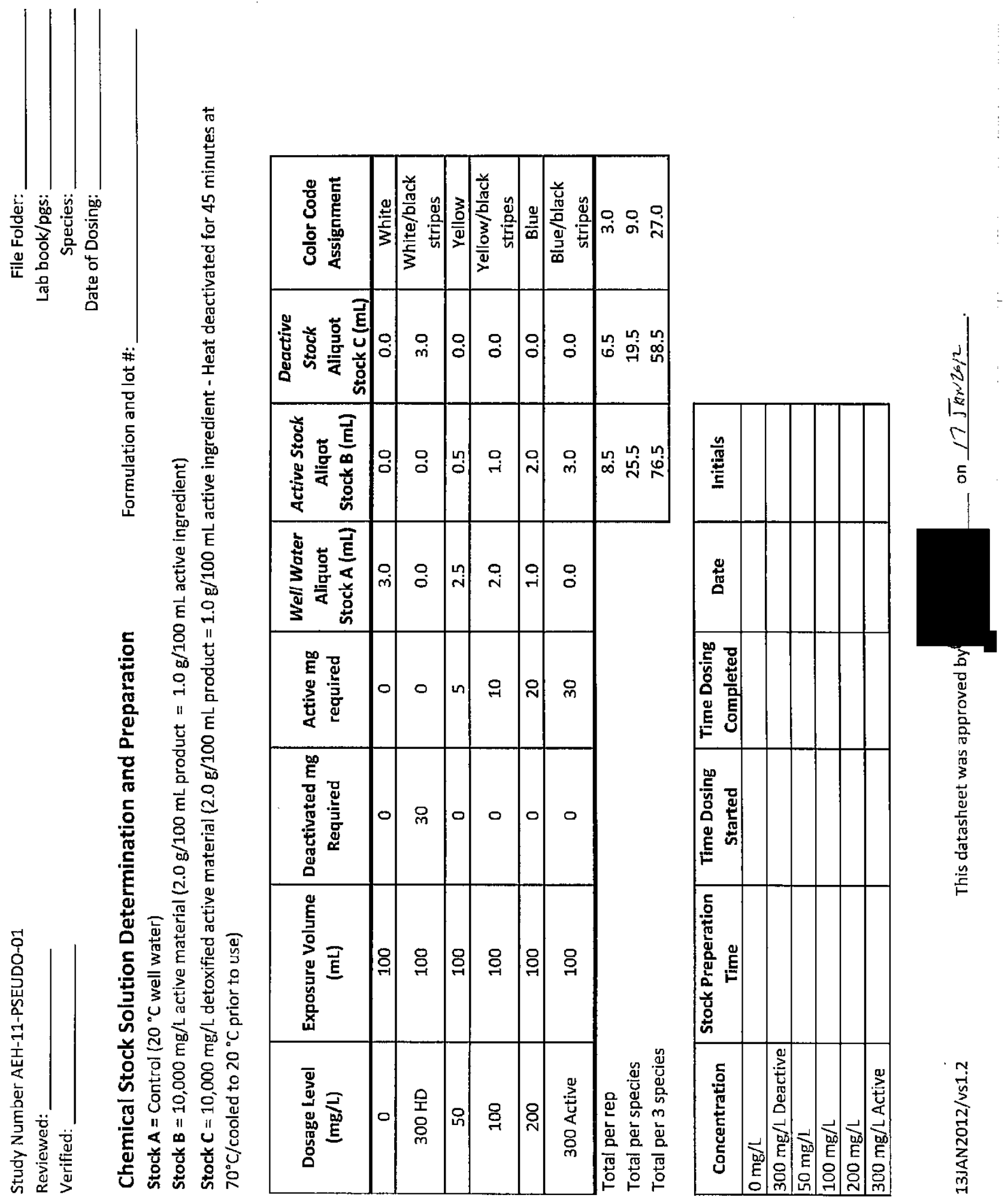


Study Number AEH-11-PSEUDO-01

File Folder:

Lab book/pgs:

Reviewed:

Verified:

\section{Pipette Calibration}

Pipette Brand/Model/SN

Tip Type:

Balance:

Thermometer:

Temperature: Water Density ${ }^{1}(\mathrm{~g} / \mathrm{mL})$ :

\begin{tabular}{|c|c|c|c|c|c|}
\hline $\begin{array}{c}\text { Pipette Setting } \\
(\mu \mathrm{L})\end{array}$ & Rep\# & $\begin{array}{c}\text { Water Mass } \\
(\mathrm{g})\end{array}$ & Date & $\begin{array}{c}\text { Time } \\
\text { (military) }\end{array}$ & Initials \\
\hline \hline & 1 & & & & \\
\hline & 2 & & & & \\
\hline & 3 & & & & \\
\hline & 4 & & & & \\
\hline & 5 & & & & \\
\hline & 7 & & & & \\
\hline & 9 & & & & \\
\hline & 10 & & & & \\
\hline & Standard Deviation & & & & \\
\hline
\end{tabular}

Relative Standard Deviation (Standard Deviation / Mean *100):

Pass or Fail (Pass = Relative Standard Deviation $\leq 1 \%$ ):

Pipette Volume (Mean mass / Water Density ${ }^{1}$ ):

Note: Pipette callbration must be performed with deionized water left to adjust to ambient room temperature for at least 24 hours prior to calibration.

1 Water density $(\mathrm{g} / \mathrm{mL})$ at varying temperatures: $18^{\circ} \mathrm{C}: 0.9985976 ; 19^{\circ} \mathrm{C}: 0.9984073 ; 20^{\circ} \mathrm{C}: 0.9982063 ; 21^{\circ} \mathrm{C}$ : $0.9979948 ; 22^{\circ} \mathrm{C} ; 0.9977730$.

This datasheet was approved by

11JAN2012/version 1.1

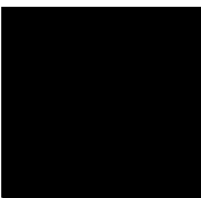

on 1250.0212. 
Study Title: "Effects of Pseudomonas fluorescens (Pf-CL145A) to glochidia from seven different unionid species"

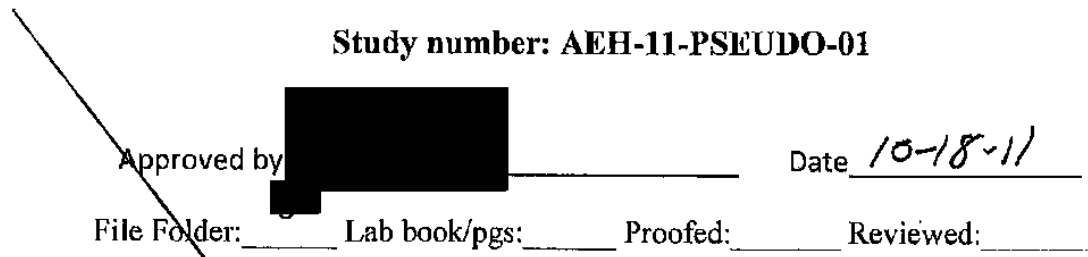

\section{Test Chemical Stock Preparation Data Form}

Test Chemical Lot \#

Test Chemical: Pseudomonas fluorescens strain 145A

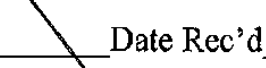

Exp. Date

\section{Chemical Weighing:}

\begin{tabular}{|l|l|l|l|}
\hline Sample I.D. & Sample wt. (g) & Date/Time & Initials \\
\hline & & & \\
\hline & & & \\
\hline & & & \\
\hline & & & \\
\hline
\end{tabular}

"Chemical samples to be stored refrigerated until used for stock preparation.

$$
\text { These forms were }
$$

Stock Preparation: revised from 12JAW 2012

$$
\text { revised from } 18 \mathrm{JAT} \text { Jol } 2012 \text { and will }
$$

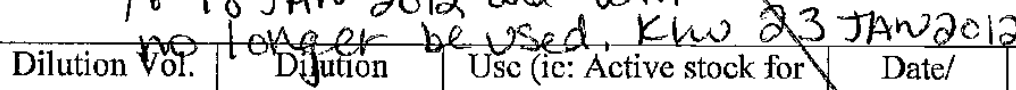

\begin{tabular}{|c|c|c|c|c|c|}
\hline Sample I.D. & $\begin{array}{c}\text { Dilution Vol. } \\
(\mathrm{ml})\end{array}$ & $\begin{array}{c}\text { Dijution } \\
\text { time }\end{array}$ & $\begin{array}{c}\text { Use (ic: Active stock for } \\
\text { HGE) }\end{array}$ & $\begin{array}{c}\text { Date/ } \\
\text { Time }\end{array}$ & Initials \\
\hline & & & & & \\
\hline & & & & & \\
\hline & & & & & \\
\hline
\end{tabular}

${ }^{*}$ Stocks to be prepared immediately before use, except for heat deactivated stock which will be prepared at least $1 \mathrm{hr}$ prior to use to allow for deactivation and cooling.

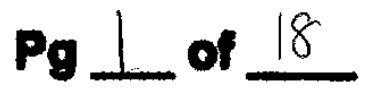


Study Number AEH-11-PSEUDO-01

File Folder: Lab book/pgs:

Proofed: Reviewed:

Water Quality - Form 2

During Exposure (Dissolved Oxygen, pH, Temperature)

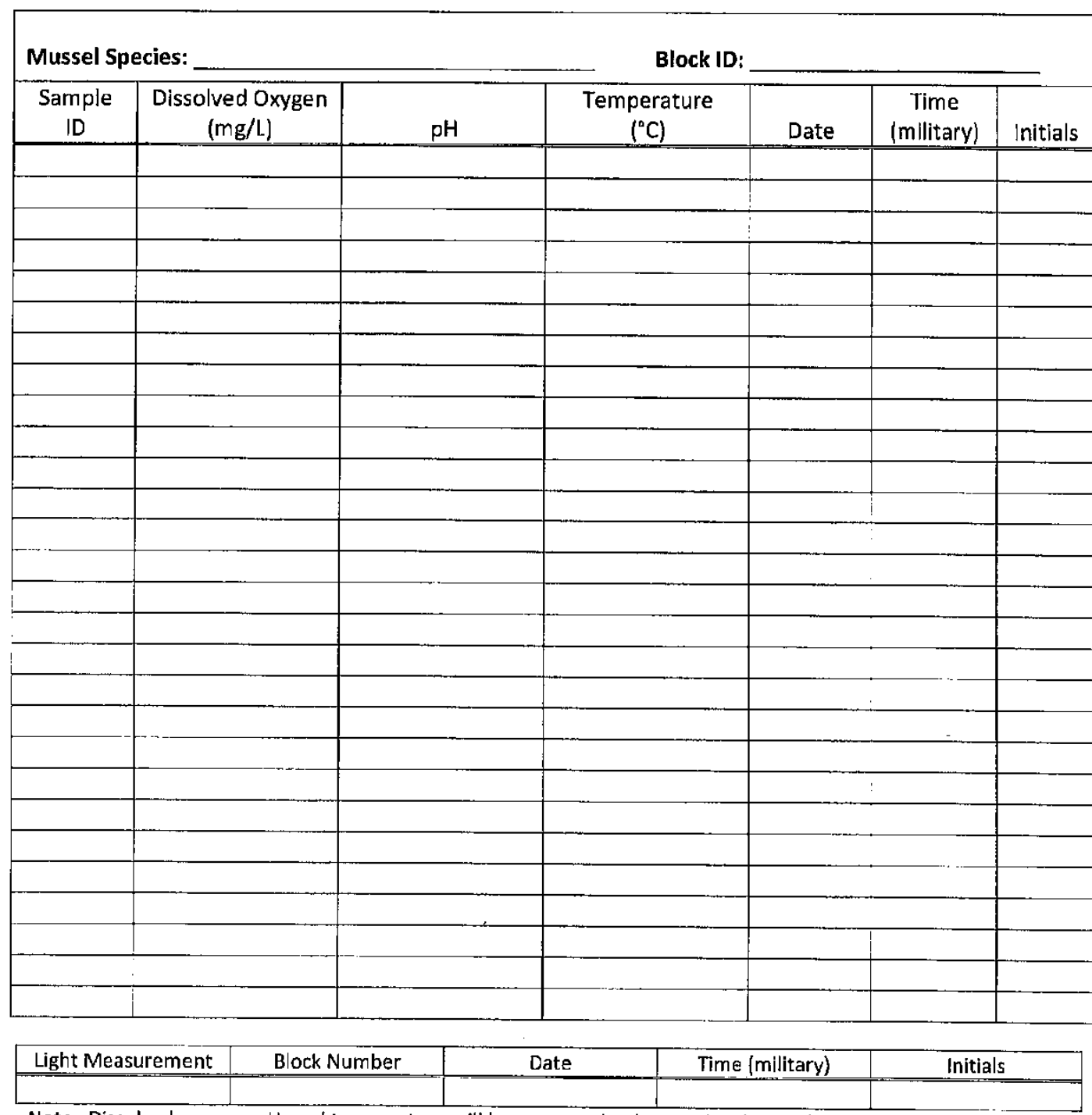

Note: Dissolved oxygen, $\mathrm{pH}$, and temperature will be measured at least twice during the exposure period in the controls and high concentrations.

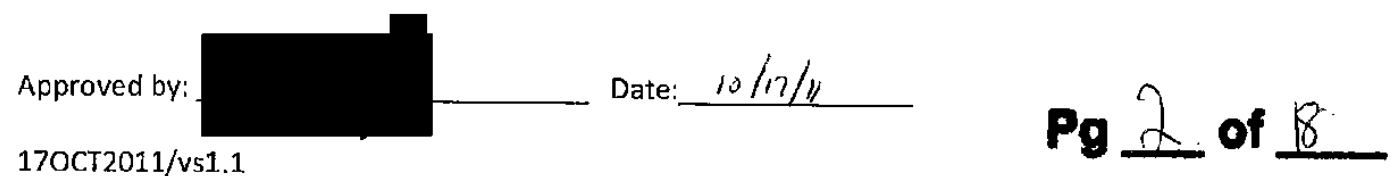



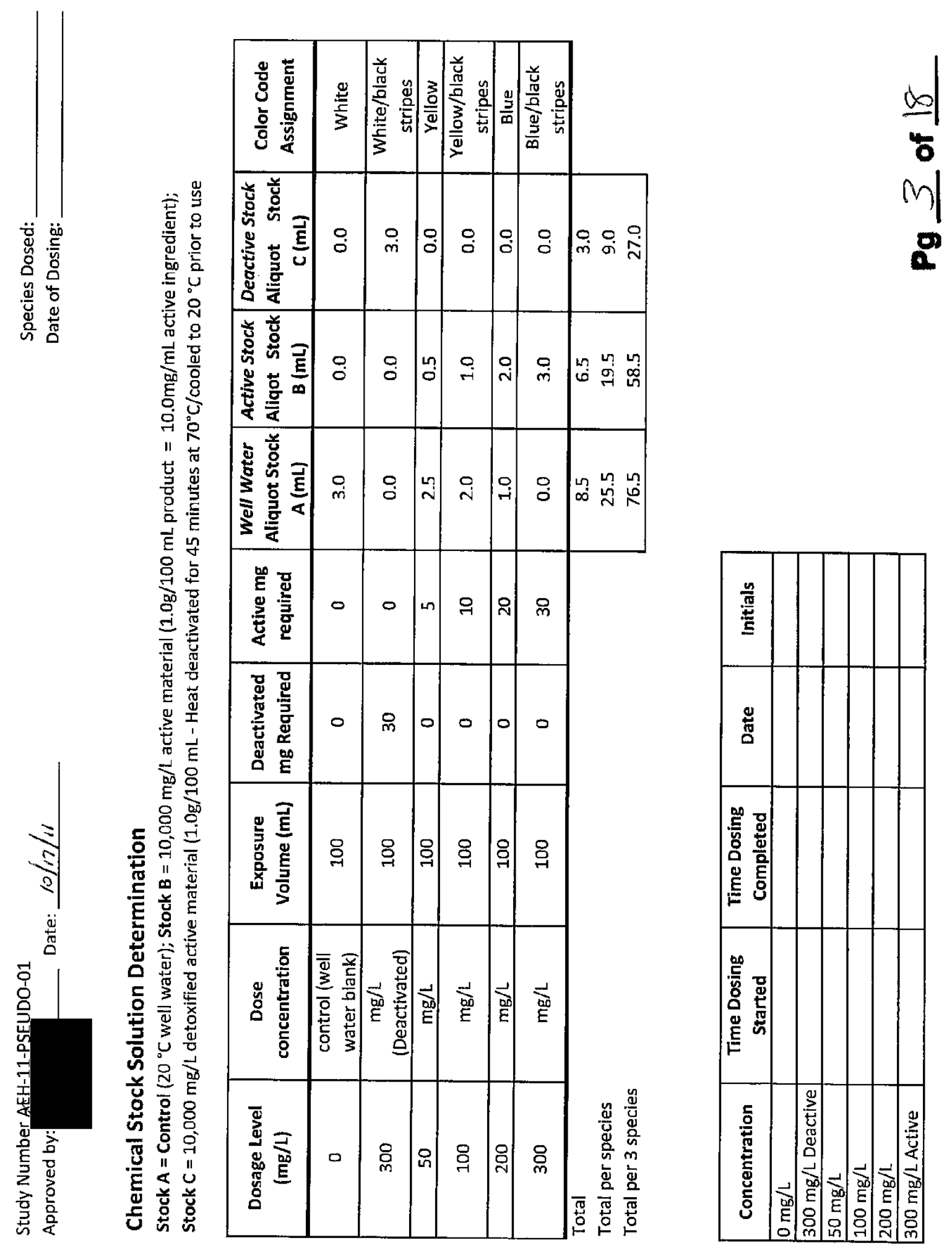

ت্. 
Study Number AEH-11-PSEUDO-01

File Folder:

Lab book/pgs:

Proofed:

Reviewed:

Water Quality - Form 1

Initial (Dilution Water Hardness, Alkalinity, and Temperature)

\begin{tabular}{|c|c|c|c|c|c|c|}
\hline \multicolumn{5}{|c|}{ Date/Time Containers Filled: } & \multicolumn{2}{|c|}{ Initials: } \\
\hline Rep\# & $\begin{array}{c}\text { Hardness } \\
\left(\mathrm{mg} / \mathrm{LCaCO}_{3}\right)\end{array}$ & $\begin{array}{c}\text { Alkalinity } \\
\left(\mathrm{mg} / \mathrm{L} \mathrm{CaCO}_{3}\right)\end{array}$ & $\begin{array}{c}\text { Temperature } \\
\left({ }^{\circ} \mathrm{C}\right) \\
\end{array}$ & Date & $\begin{array}{c}\text { Time } \\
\text { (military) } \\
\end{array}$ & Initials \\
\hline 1 & & & & & & \\
\hline 2 & & & & & & \\
\hline 3 & & & & & & \\
\hline & & & & & & \\
\hline Mean & & & & & & \\
\hline & & & & & & \\
\hline & & & & & & \\
\hline & & & & & & \\
\hline & & & & & & \\
\hline & & & & & & \\
\hline
\end{tabular}

Note: Hardness, alkalinity, and temperature will be measured prior to test initiation from well water aerated and allowed to acclimate to room temperature for at least 24 hours prior to use.

Approved by:

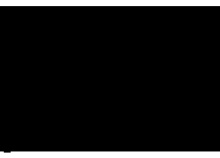

Date: $5-1 /-11$

$\operatorname{Pg} \underline{4}$ of 18 
Study Number AEH-11-PSEUDO-01

File Folder: Lab book/pgs:

Proofed:

Reviewed:

Water Quality - Form 3

Upon Termination (Hardness, Alkalinity, Conductivity, Temperature, and pH)

\section{Mussel Species:}

\section{Block ID:}

\begin{tabular}{|c|c|c|c|c|c|c|c|}
\hline $\begin{array}{l}\text { Pooled Sample } \\
\text { Concentration }\end{array}$ & Sample IDs & $\begin{array}{l}\text { Hardness } \\
\left(\mathrm{mg} / \mathrm{LCaCO}_{3}\right)\end{array}$ & $\begin{array}{c}\text { Alkalinity } \\
\left(\mathrm{mg} / \mathrm{LCaCO}_{3}\right)\end{array}$ & $\begin{array}{c}\text { Conductivity } \\
(\mu \mathrm{S} / \mathrm{cm})\end{array}$ & Date & $\underset{\text { (military) }}{\text { Time }}$ & Initials \\
\hline & & & & & & & \\
\hline & & & & & & & \\
\hline & & & & & & & \\
\hline & & & & & & & \\
\hline & & & & & & & \\
\hline & & & & & & & \\
\hline
\end{tabular}

Note: Hardness, alkalinity, and conductivity will be measured upon termination of trial from pooled replicate samples.

\begin{tabular}{|c|c|c|c|c|c|c|}
\hline Sample ID & $\begin{array}{c}\text { Dissolved Oxygen } \\
(\mathrm{mg} / \mathrm{L})\end{array}$ & Temperature $\left({ }^{\circ} \mathrm{C}\right)$ & $\mathrm{pH}$ & Date & Time (military) & Initials \\
\hline & & & & & & \\
\hline & & & & & & \\
\hline & & & & & & \\
\hline & & & & & & \\
\hline & & & & & & \\
\hline & & & & & & \\
\hline & & & & & & \\
\hline & & & & & & \\
\hline & & & & & & \\
\hline & & & & & & \\
\hline & & & & & & \\
\hline & & & & & & \\
\hline & & & & & & \\
\hline & & & & & & \\
\hline & & & & & & \\
\hline & & & & & & \\
\hline & & & & & & \\
\hline & & & & & & \\
\hline
\end{tabular}

Note: D.O., temperature and $\mathrm{nH}$ will be measured upon termination of trial from each individual test chamber for ammonia analysis.

Approved by:

Date: $10 / 17 / 11$

$170 \mathrm{CT} 2011 / \mathrm{vs1.1}$

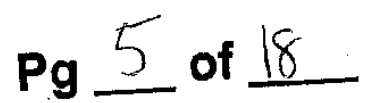


File Folder: Lab book/pgs:

Proofed Reviewed:

Pipette Calibration

Pipette Brand/Model/SN:

Balance:

Temperature: Water Density ${ }^{1}(\mathrm{~g} / \mathrm{mL})$ :

\begin{tabular}{|c|c|c|c|c|c|}
\hline $\begin{array}{c}\text { Pipette Setting } \\
(\mu \mathrm{L}) \\
\end{array}$ & Rep \# & $\begin{array}{c}\text { Water Mass } \\
\text { (g) }\end{array}$ & Date & $\begin{array}{c}\text { Time } \\
\text { (military) }\end{array}$ & Initials \\
\hline & 1 & & & & \\
\hline & 2 & & & & \\
\hline & 3 & & & & \\
\hline & 4 & & & & \\
\hline & 5 & & & & \\
\hline & 6 & & & & \\
\hline & 7 & & & & \\
\hline & 8 & & & & \\
\hline & 9 & & & & \\
\hline & 10 & & & & \\
\hline & & & & & \\
\hline & Mean & & & & \\
\hline & Standard Deviation & & & & \\
\hline
\end{tabular}

Relative Standard Deviation (Standard Deviation / Mean *100):

Pass or Fail (Pass $=$ Relative Standard Deviation $\leq 1 \%$ ):

Pipette Volume (Mean mass / Water Density ${ }^{1}$ ):

Note: Pipette calibration must be performed with deionized water left to adjust to ambient room temperature for at least 24 hours prior to calibration.

${ }^{1}$ Water density $(\mathrm{g} / \mathrm{mL})$ at varying temperatures: $18^{\circ} \mathrm{C}: 0.9985976 ; 19^{\circ} \mathrm{C}: 0.9984073 ; 20^{\circ} \mathrm{C}: 0.9982063 ; 21^{\circ} \mathrm{C}$ : $0.9979948 ; 22^{\circ} \mathrm{C}: 0.9977730$.

Date: $59-1$ 
Study Title: "Effects of Pseudomonas fluorescens (Pf-CL145A) to glochidia from seven unionid mussel species"

Study number: AEH-11-PSEUDO-01

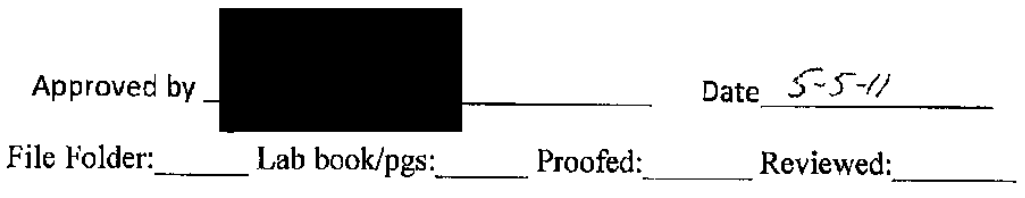

\section{Donor Mussel UMESC Lot Number Assignment Form} DONOR MUSSEL INFORMATION:

Species:

Number of Donor Mussels: Collection Date:

Collection Location:

Species Identification performed by:

Title/affiliation of identifier:

UMESC Arrival Date:

Receiving UMESC tank/room \#

Additional information:

\section{UMESC LOT NUMBER DESIGNATION:}

Witness and form recorded by:

Printed Name

Signature

UMESC

Affiliation

Datc

$\operatorname{Pg} 7$ of 18 
Study Title: "Effects of Pseudomonas fluorescens (Pf-CL145A) to glochidia from seven unionid mussel species"

Study number: AEH-11-PSEUDO-01

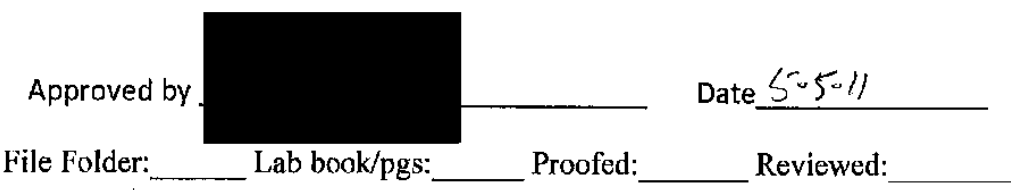

\section{Glochidia Test Organism UMESC Lot Number Assignment Form}

Species:

Number of Donor Mussels: Donor Mussel Lot H:

Extraction Date: 1201 Extraction Time (military)

Extraction Location: UMESC rm 15 Container ID:

Approximate Number of glochidia:

Glochidia extracted by: Affiliation:

Additional information:

UMESC GLOCHIDIA LOT NUMBER DESIGNATION:

Witness and form recorded by:

UMESC

Affiliation

Date

Pg 8 of 18 
Study Title: "Effects of Pseudomonas fluorescens (Pf-CL145A) to glochidia from seven unionid mussel species"

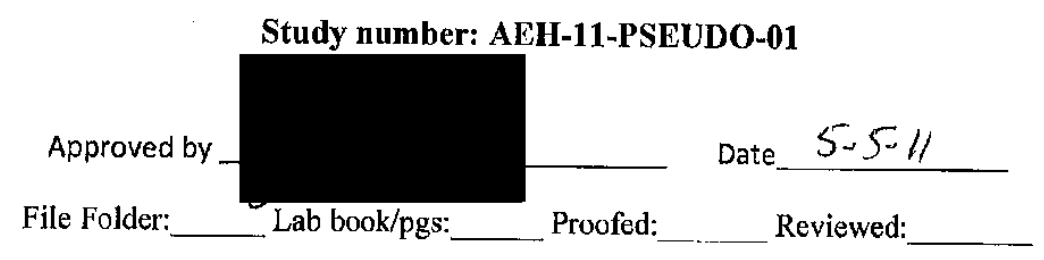

\section{Glochidia Exposure Dosing Form}

Species: UMESC lot number:

Test Block Assignment (circle one):

Estimated \# glochidia/Chamber

Date/time (military) of dosing initiation:

Date/time (military) of dosing completion:

Additional information:

Witness and form recorded by:

Printed Name

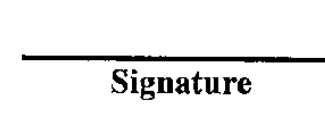

JMMESC

Affiliation

Date

$\operatorname{Pg} 9$ of 18 
Study Title: "Effects of Pseudomonas fluorescens (Pf-CL145A) to glochidia from seven unionid mussel species"

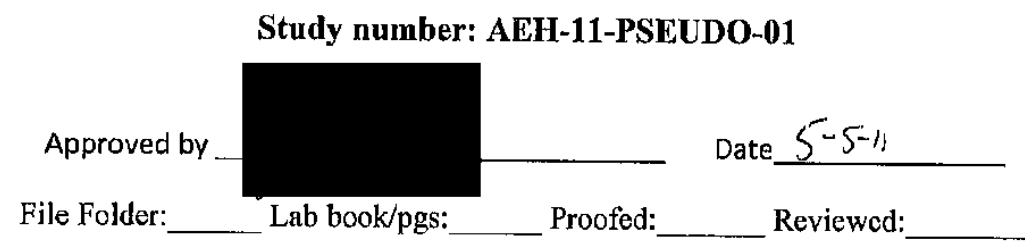

\section{Glochidia Aliquot Distribution Form}

Species: UMESC LOT NUMBER:

Test Block Assignment (circle one):_1 1

Aliquot Volume $(\mu \mathrm{l})$ :

Estimated \# glochidia/aliquot

Initial Distribution aliquot date/time (military):

Final Distribution Aliquot date/time (military):

Number of Aliquot rounds: Estimated \# glochidia/chamber

Additional information:

Witness and form recorded by:

UMESC

$\begin{array}{ll}\text { Printed Name } & \text { Affiliation } \\ & \text { Pg } 10 \text { of } 18\end{array}$


File Folder: Lab book/pgs:

Proofed:

Reviewed:

\section{Daily Care Worksheet}

Tank \#:

Room \#:

Month/Year:

Species:

Lot Number:

\begin{tabular}{|c|c|c|c|c|c|c|c|}
\hline \multirow[b]{2}{*}{ Day } & \multirow{2}{*}{$\begin{array}{c}\text { Feed } \\
\text { Time } \\
\text { (military) } \\
\end{array}$} & \multicolumn{2}{|c|}{$\begin{array}{l}\text { Flow Rate } \\
\text { (mL/min) }\end{array}$} & \multirow[b]{2}{*}{$\begin{array}{c}\text { Temperature } \\
\left({ }^{\circ} \mathrm{C}\right)\end{array}$} & \multirow[b]{2}{*}{$\begin{array}{c}\text { Dissolved } \\
\text { Oxygen (mg/L) }\end{array}$} & \multirow[b]{2}{*}{$\mathrm{pH}$} & \multirow[b]{2}{*}{ Initials } \\
\hline & & Inflow & Pump & & & & \\
\hline \multicolumn{8}{|l|}{1} \\
\hline \multicolumn{8}{|l|}{2} \\
\hline \multicolumn{8}{|l|}{3} \\
\hline \multicolumn{8}{|l|}{4} \\
\hline \multicolumn{8}{|l|}{5} \\
\hline \multicolumn{8}{|l|}{6} \\
\hline \multicolumn{8}{|l|}{7} \\
\hline \multicolumn{8}{|l|}{8} \\
\hline \multicolumn{8}{|l|}{9} \\
\hline \multicolumn{8}{|l|}{10} \\
\hline \multicolumn{8}{|l|}{11} \\
\hline \multicolumn{8}{|l|}{12} \\
\hline \multicolumn{8}{|l|}{13} \\
\hline \multicolumn{8}{|l|}{14} \\
\hline \multicolumn{8}{|l|}{15} \\
\hline \multicolumn{8}{|l|}{16} \\
\hline \multicolumn{8}{|l|}{17} \\
\hline \multicolumn{8}{|l|}{18} \\
\hline \multicolumn{8}{|l|}{19} \\
\hline \multicolumn{8}{|l|}{20} \\
\hline \multicolumn{8}{|l|}{21} \\
\hline \multicolumn{8}{|l|}{22} \\
\hline 23 & & & & & & & \\
\hline 24 & & & & & & & \\
\hline 25 & & & & & & & \\
\hline 26 & & & & & & & \\
\hline 27 & & & & & & & \\
\hline 28 & & & & & & & \\
\hline 29 & & & & & & & \\
\hline 30 & & & & & & & \\
\hline 31 & & & & & & & \\
\hline
\end{tabular}

Daily Food Prep: Mix 44.0g Shellfish diet, $35.3 \mathrm{~g}$ Tetraselmis, and $92.4 \mathrm{~g}$ Thalassiosira weissflogii with $14,400 \mathrm{~mL}$ well water each day, to be delivered by peristaltic pump at $10 \mathrm{~mL}$ per minute. Target water inflow rate is $1,100 \mathrm{~mL} / \mathrm{min}$. Prepare fresh diet daily.

Approved by

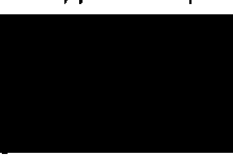

Date: $5-9-11$

$\mathrm{Pg} \Perp$ of 18 
Study Number AEH-11-PSEUDO-01

File Folder: Lab book/pgs:

Proofed: Reviewed:

Water Quality - Form 2

During Exposure (Dissolved Oxygen, pH, Temperature)

Mussel Species:

Block ID:

\begin{tabular}{|c|c|c|c|c|c|c|}
\hline $\begin{array}{l}\text { Sample } \\
\text { ID }\end{array}$ & $\begin{array}{c}\text { Dissolved Oxygen } \\
(\mathrm{mg} / \mathrm{L})\end{array}$ & $\mathrm{pH}$ & $\begin{array}{c}\text { Temperature } \\
\left({ }^{\circ} \mathrm{C}\right)\end{array}$ & Date & $\begin{array}{c}\text { Time } \\
\text { (military) } \\
\end{array}$ & Initials \\
\hline & & & & & & \\
\hline & & & & & & \\
\hline & & & & & & \\
\hline & & & & & & \\
\hline & & & & & & \\
\hline & & & & & & \\
\hline & & & & & 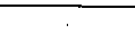 & \\
\hline & & & & & & \\
\hline & & & & & & \\
\hline & & & & & & \\
\hline & & & & & & \\
\hline & & & & & & \\
\hline & & & & & & \\
\hline & & & & & & \\
\hline & & & & & & \\
\hline & & & & & & \\
\hline & & & & & & \\
\hline & & & & & & \\
\hline & & & & & & \\
\hline & & & & & & \\
\hline & & & & & & \\
\hline & & & & & & \\
\hline & & & & & & \\
\hline & & & & & & \\
\hline & & & & & & \\
\hline & & & & & & \\
\hline & & & & & & \\
\hline & & & & & & \\
\hline
\end{tabular}

Note: Dissolved oxygen, $\mathrm{pH}$, and temperature will be measured at least twice during the exposure period in the controls and high concentrations.

Approved by:

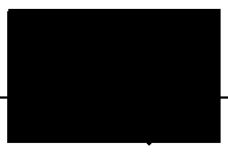

Date: $5-11-11$

$\operatorname{Pg} 12$ of 18 


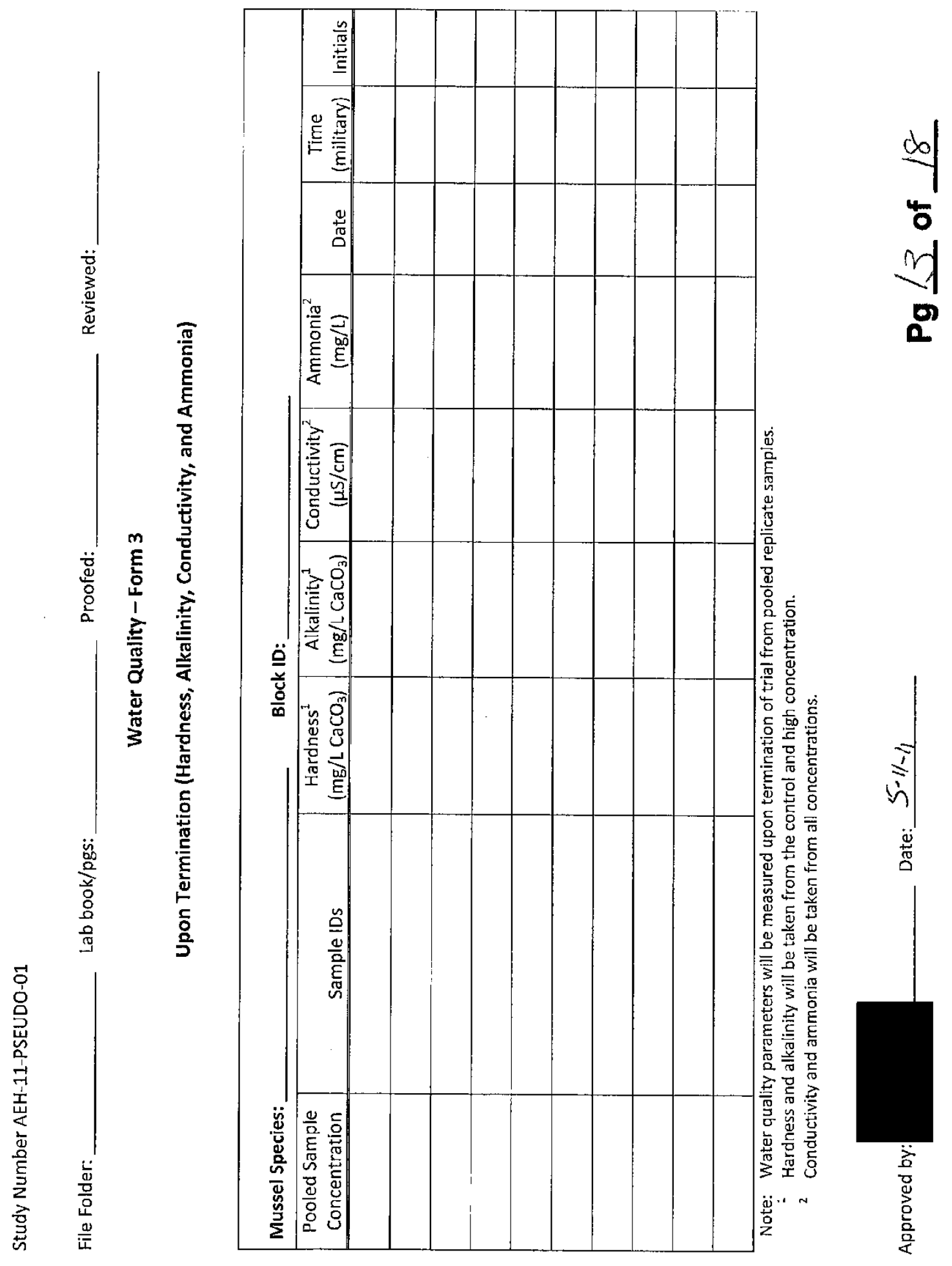




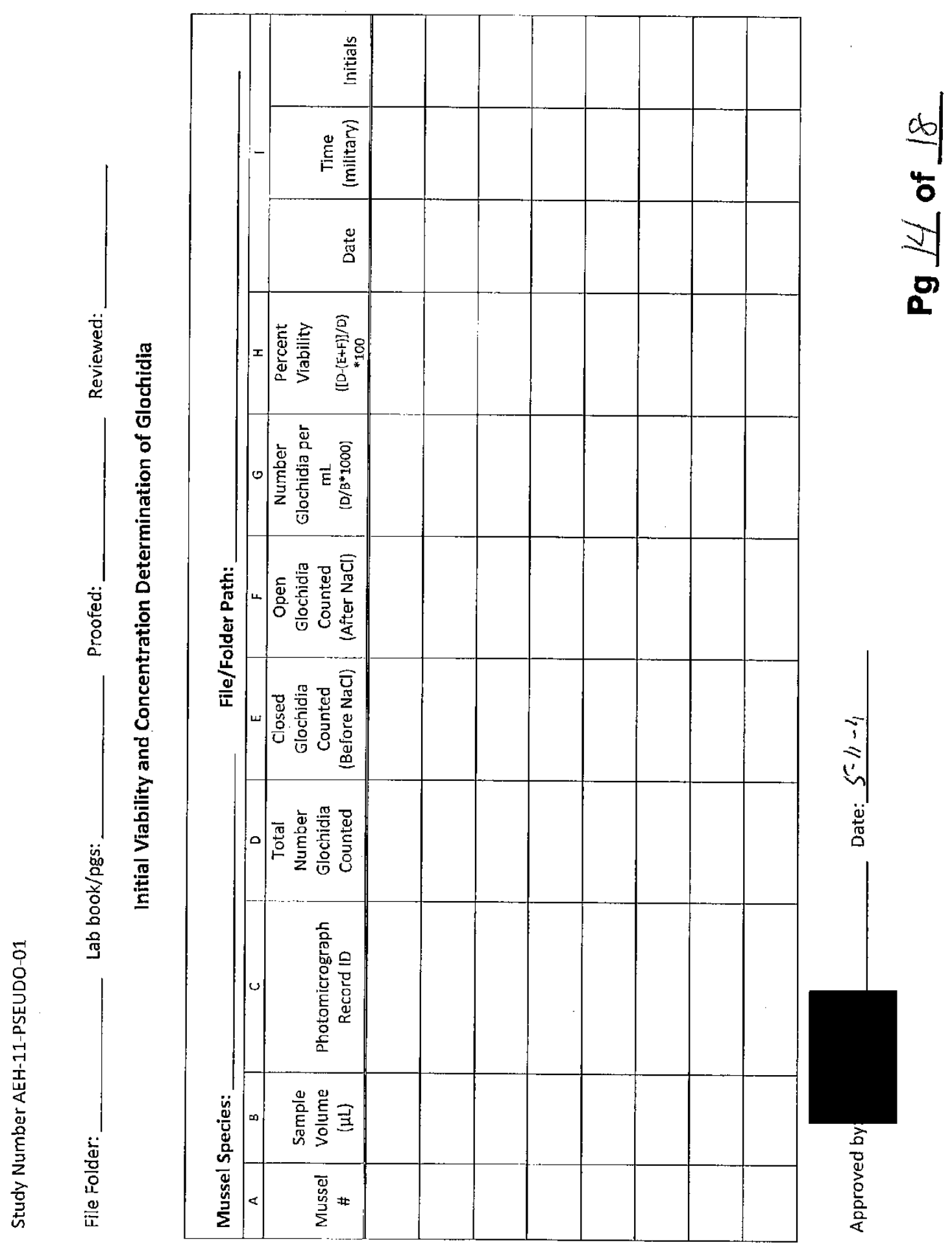




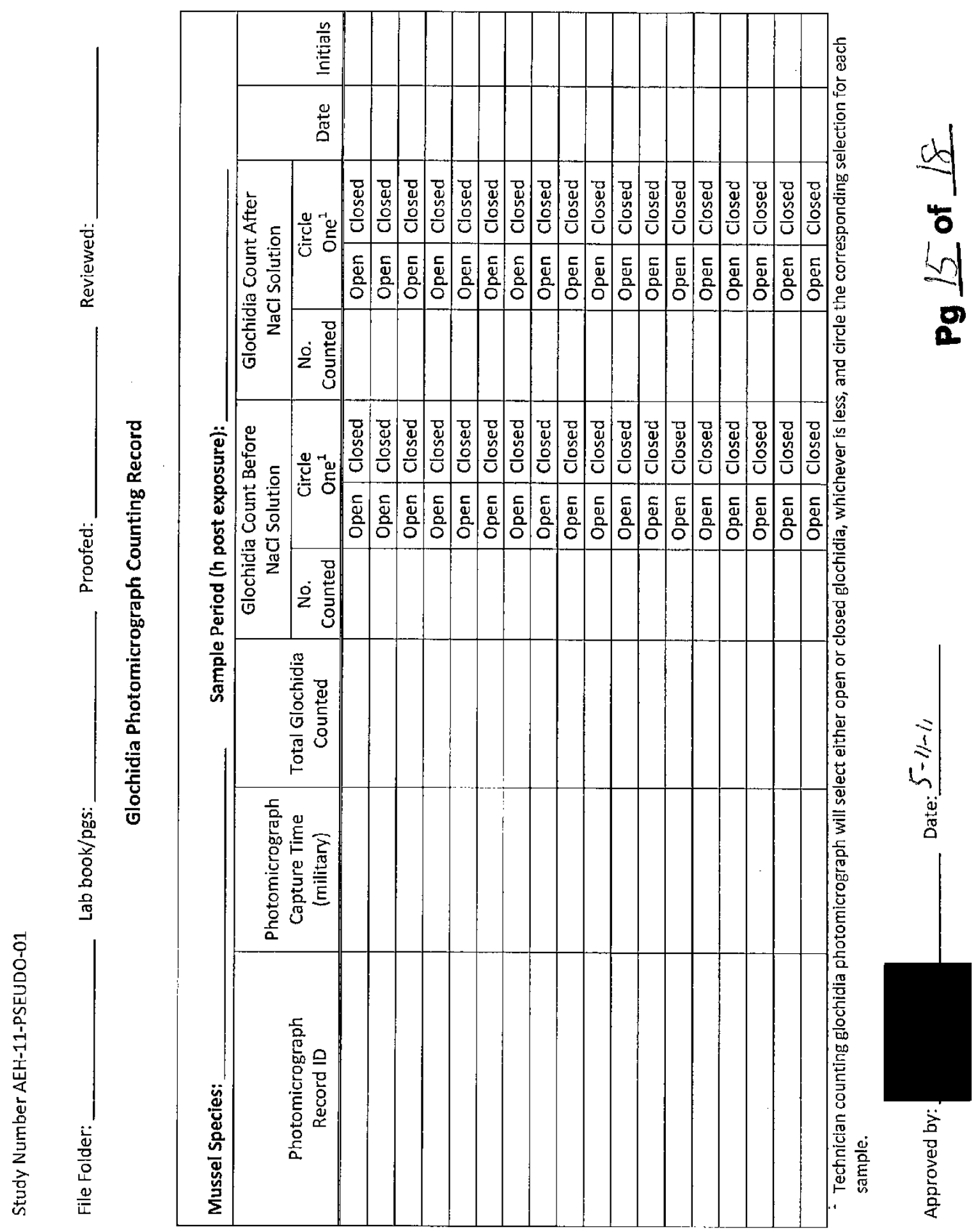




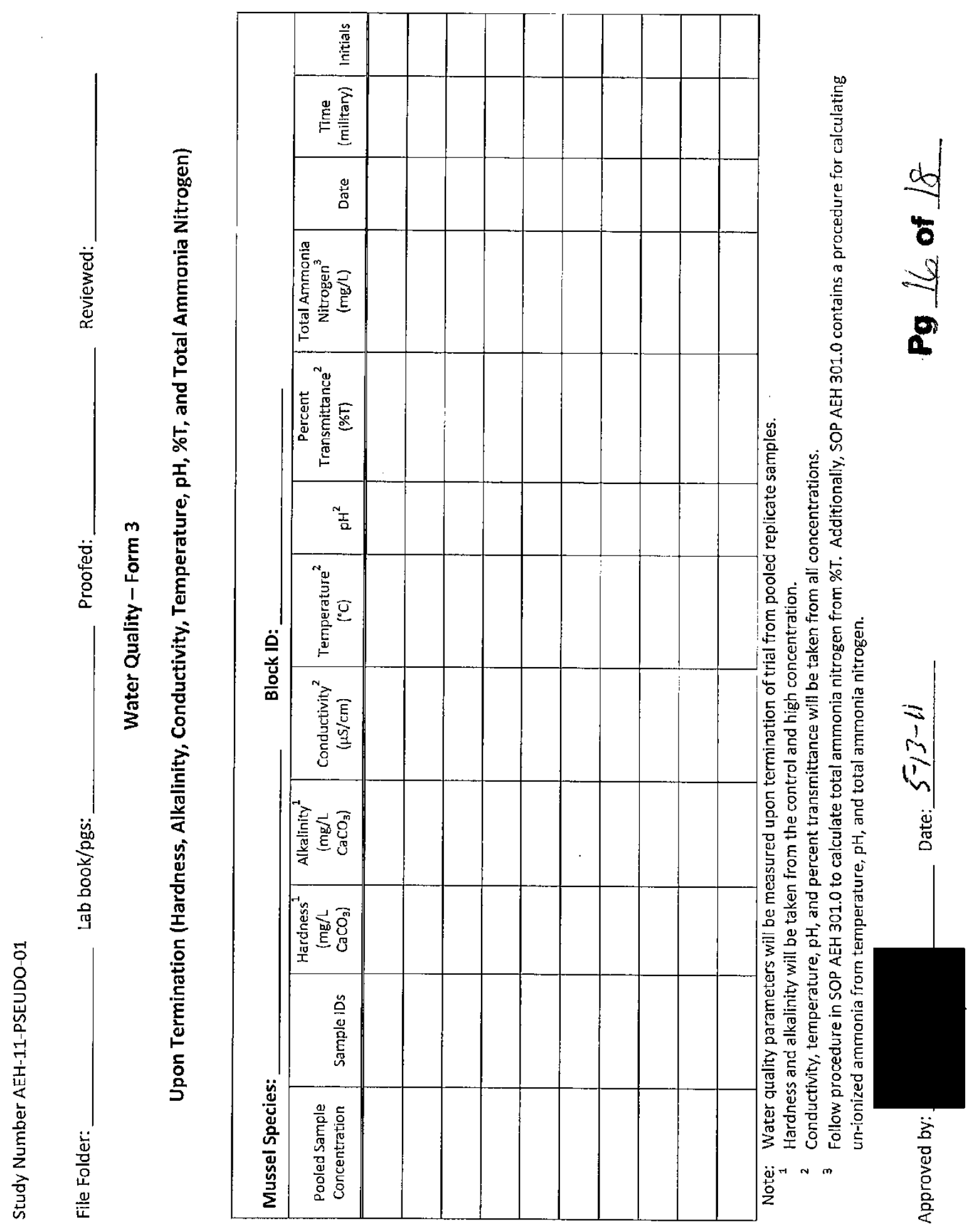


Study Title: "Effects of Pseudomonas fluorescens (Pf-CL145A) to glochidia from seven unionid mussel species"

Study number: AEH-11-PSEUDO-01

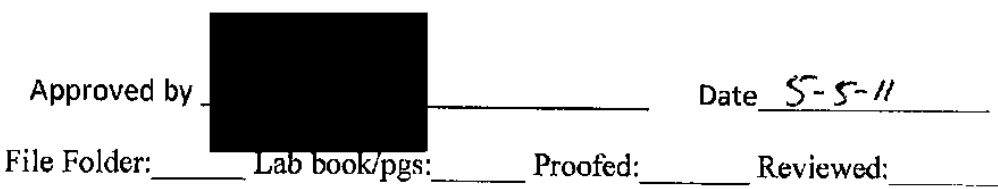

\section{Donor Mussel UMESC Lot Number Assignment Form}

DONOR MUSSEL INFORMATION:

Species:

Number of Donor Mussels:

Collection Date:

Collection Location:

Species Identification performed by:

Title/affiliation of identifier:

UMESC Arrival Date:

Receiving UMESC tank/room \#

Additional information:

UMESC LOT NUMBER DESIGNATION:

Witness and form recorded by:

Printed Name
Signature
UMESC

Affiliation $\overline{\text { Date }}$

$\operatorname{Pg} 17$ of 18 


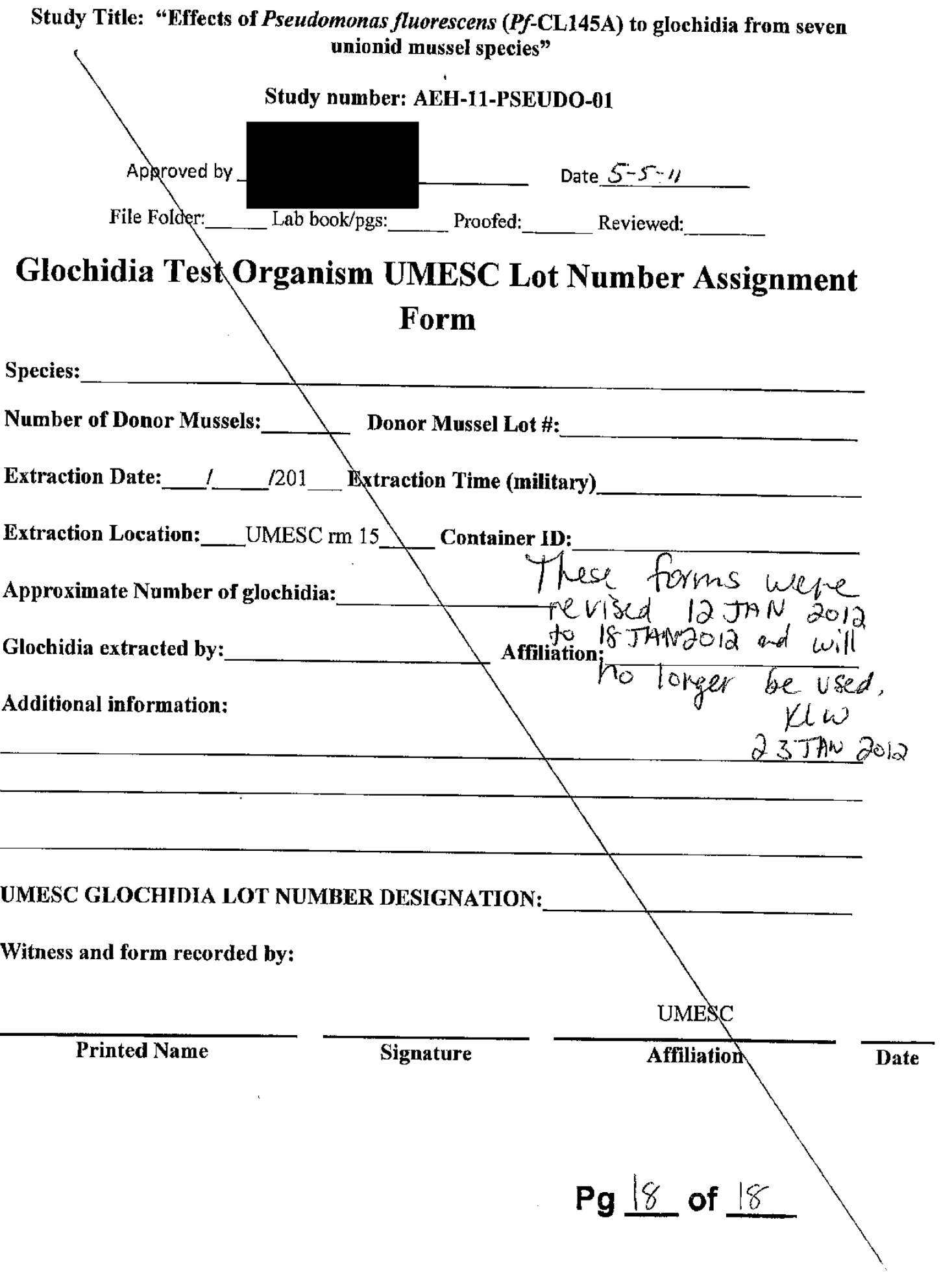




\section{Appendix 2. Deviations from the Study Protocol}

\begin{tabular}{clcc}
\hline $\begin{array}{c}\text { Item } \\
\text { num ber }\end{array}$ & Item description & $\begin{array}{c}\text { Number } \\
\text { of } \\
\text { pages }\end{array}$ & $\begin{array}{c}\text { Report } \\
\text { page } \\
\text { number }\end{array}$ \\
\hline 1 & Deviations \#1 and \#2 from Study Protocol, Study \# AEH-11-PSEUDO-01 & 1 & 114 \\
2 & Deviation \#3 from Study Protocol, Study \# AEH-11-PSEUDO-01 & 1 & 115 \\
3 & Deviation\#4 from Study Protocol, Study \# AEH-11-PSEUDO-01 & 1 \\
4 & Deviation\#5 from Study Protocol, Study \# AEH-11-PSEUDO-01 & 116 \\
5 & Deviation\#6 from Study Protocol, Study \# AEH-11-PSEUDO-01 & 117 \\
6 & Deviation\#7 from Study Protocol, Study \# AEH-11-PSEUDO-01 & 1 & 118 \\
7 & Deviation\#8 from Study Protocol, Study \# AEH-11-PSEUDO-01 & 2 & 119 \\
8 & Deviation\#9 from Study Protocol, Study \# AEH-11-PSEUDO-01 & 2 & 121 \\
9 & Deviation\#10 from Study Protocol, Study \# AEH-11-PSEUDO-01 & 1 & 123 \\
10 & Deviation\#11 from Study Protocol, Study \# AEH-11-PSEUDO-01 & 12 \\
11 & Deviation\#12 from Study Protocol, Study \# AEH-11-PSEUDO-01 & 124 \\
12 & Deviation\#13 from Study Protocol, Study \# AEH-11-PSEUDO-01 & 129 \\
\hline
\end{tabular}




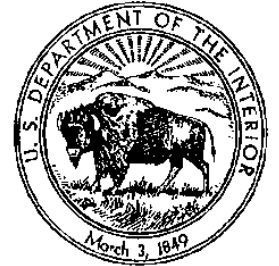

\author{
United States Department of the Interior \\ U.S. GEOLOGICAL SURVEY \\ Biological Resources Division
}

Upper Midwest Environmental Sciences Center

2630 Fanta Reed Road

La Crosse, Wisconsin 54603

\section{MEMORANDUM}

Date: June 3, 2011

To: The Record Study Number AEH-11-PSEUDO-01

\section{ORIGINAL}

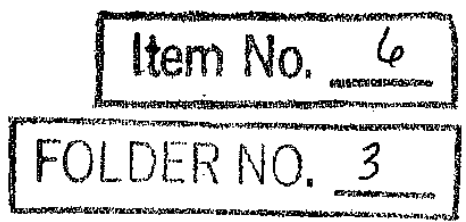

Subject: Deviations 1 and 2 to study AEH-11-PSEUDO-01 "Effects of Pseudomonas fluorescens (Pf$C L 145 A)$ to glochidia from seven unionid mussel species"

Deviation \#1 - Sections 4.2 and 6.2 (Experimental Design and Determination of viability) state that the viability will determined on subsamples of approximately 100 glochidia taken from each exposure chamber at 6,12 and $24 \mathrm{~h}$ post exposure. After completing the $24 \mathrm{~h}$ viability assessments for Block 1 (Lampsilis cardium) the test replicates for each concentration were pooled for the final water quality measurements. A control chamber (2B1) from Block 2 (L. higginsii) was inadvertently pooled with the control chambers from Block 1. Because chamber $2 \mathrm{~B} 1$ was pooled with the control chambers from Block 1, a glochida sample was not taken for this one control replicate at $24-\mathrm{h}$ post exposure to assess $L$. higginsii viability in that one control replicate. The remaining two L. higginsii controls (chambers $2 \mathrm{C} 3$ and $2 \mathrm{C} 6$ ) were sampled to assess glochida viability at 24-h post exposure according to the study protocol except that two $\sim 100-\mu \mathrm{L}$ aliquots were collected per replicate and the glochida viability recorded in each aliquot (two separate glochida viability determinations per control replicate).

Deviation \#2 - Section 5.2 (Water Chemistry) of the study protocol specifies that water remaining in the replicates will be pooled after glochidia aliquots are taken to determine glochidia viability at 24 -h post exposure. Lampsilis higginsii control replicate chamber, 38 P was inadvertently combined with the $L$. cardium control chambers. Therefore, the water quality measurements at $24-\mathrm{h}$ post exposure for $L$. cardium were determined in a water sample of which a portion was derived from the $L$. higginsii control. The water quality measurements for the L. higginsii controls were made in a water sample which only contained water from control replicate chambers $2 \mathrm{C} 3$ and $2 \mathrm{C} 6$.

The impact of these deviations to the outcome of the study will not be fully known until the glochidia viability data are analyzed. Therefore, an assessment of the impact of this deviation will be rendered in the final study report.

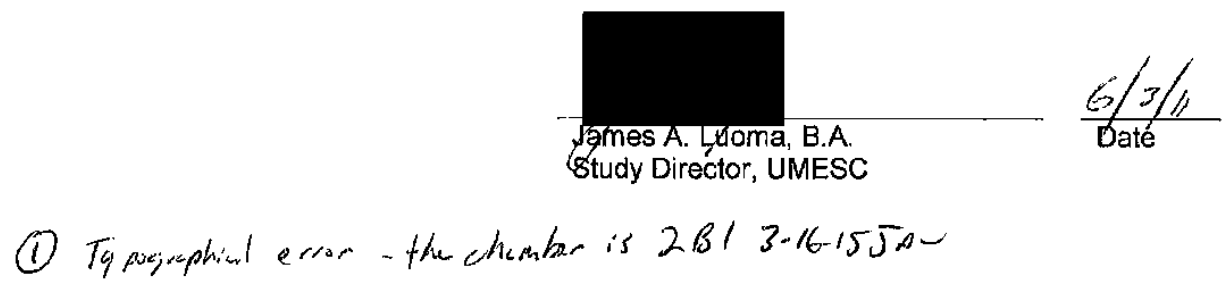

cC: UMESC QAU

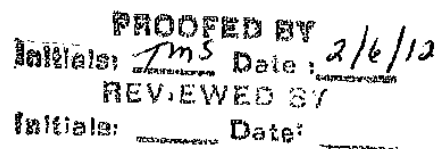

Page 1 of 1 


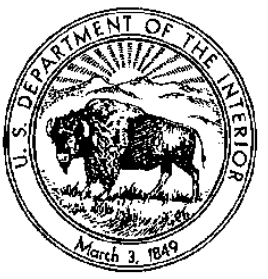

MEMORANDUM

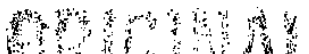

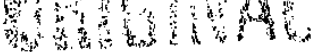

United States Department of the Interior
\end{abstract}

U.S. GEOLOGICAL SURVEY

Biological Resources Division

Upper Midwest Environmental Sclences Center

2630 Fanta Reed Road

La Crosse, Wisconsin 54603

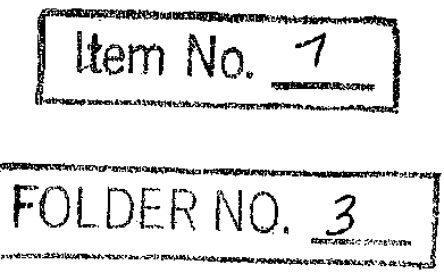

Date: June 3, 2011

To: The Record Study Number AEH-11-PSEUDO-01

Subject: Deviation 3 to study AEH-11-PSEUDO-01 "Effects of Pseudomonas fluorescens (Pf-CL145A) to glochidia from seven unionid mussel species"

Deviation \#3 - Sections 4.2 and 6.2 (Experimental Design and Determination of Viability) of the amended protocol states that the viability of glochidia from each mussel will be assessed by "drawing aliquots (e.g. $100 \mu \mathrm{L}$ ) of settled glochidia from the stock container with an adjustable manual pipet fitted with a wide bore tip and placing it on a pre-labeled $35 \mathrm{~mm}$ petri dish. The sample will be diluted with a known and consistent volume (e.g. $200 \mu \mathrm{L}$ ) of acclimated well water to enhance dispersion of glochidia for more accurate enumeration. The sample will be viewed under a dissecting microscope, adjusted for maximum clarity and magnification, and a photom/crograph will be recorded. Approximately one drop of a saturated sodium chloride solution ( $12 \mathrm{~g} \mathrm{NaCl} / 50 \mathrm{~mL}$ of D.f. $\mathrm{H}_{2} \mathrm{O}$ [ASTM E2455-06]) will be added to the sample and after one minute a second photomicrograph will be recorded. Enumerations will be conducted for the total number of glochidia within the sample and the number of glochidia closed or open before and after sodium chloride addition. The percentage of viable glochidia (glochidia that respond to sodium chloride exposure by valve closure) will be calculated by the following method:

Percent viable $=$ ([Total number of glochidia in sample - [total number of glochidia closed before addition of sodium chloride + number of glochidia open after sodium chloride addition]]/Total number of glochidia in sample) * 100

If viability is acceptable ( $280 \%$ ) for a mussel, the glochidia from that mussel will be pooled with glochidia from other mussels of that species."

The procedures as described in the protocol were followed except mussels that were observed to have high viability, the exact viability percentage was not determined prior to allowing the glochidia into the study. For these mussels a preliminary visual observation was used to determine acceptable viability. Only two $\mathrm{L}$. higginsii mussels appeared to have viability near the threshold limit of $80 \%$ vlability. For these mussels a preliminary estimation of the viability using the formula in the protocol showed that the viability was greater than the minimum $80 \%$ for acceptance.

The methods described were used to substantially reduce the period from glochidia extraction until use in the study. No negative impacts to the study are anticipated as the exact viability of the glochidia will be determined from the photomicrograph records, viability $\geq 80 \%$ for all but two mussels was easily identified by visual observation and the two higginsii mussels with somewhat reduce viability were determined to have acceptable viability by using the formula described in the protocol. The calculations were preliminary and not recorded, however they did indicate greater than $80 \%$ viability.

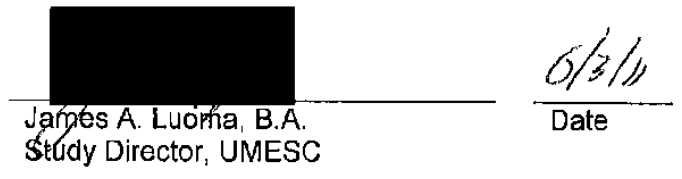

CC: UMESC QAU

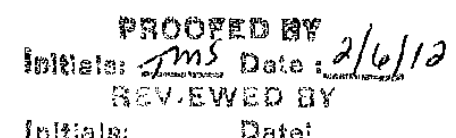

Page 1 of 1

ให pata? 


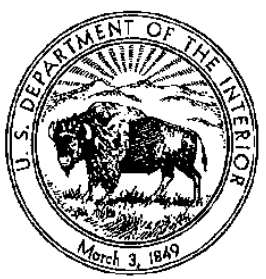

MEMORANDUM
United States Department of the Interior

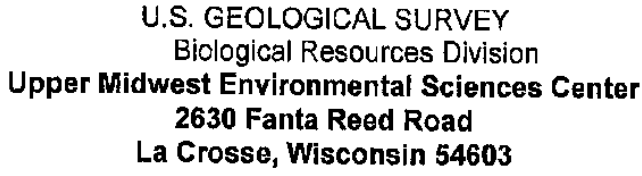

U.S. GEOLOGICAL SURVEY Biological Resources Division Upper Midwest Environmental Sciences Center 2630 Fanta Reed Road La Crosse, Wisconsin 54603

\section{ORIGLïAL}

Date: June 7, 2011

To: The Record Study Number AEH-11-PSEUDO-01

Subject: Deviation 4 to study AEH-11-PSEUDO-01 "Effects of Pseudomonas fluorescens (PF-CL145A) to glochidia from seven unionid mussel species"

Deviation \#4 - UMESC SOP GEN 012.2 section $C_{1} 3 \& 4$, specify that " the chemical removed from the test chemical container will be weighed into a tared vessel and the weight of the chemical mass recorded in the Test Chemical Use Log (Form GEN 012.2b)." and "After the chemical has been removed from the test chemical container (including the cap or lid) re-weigh the container and its contents and record the weight in the use log".

When the test chemical was removed from the container, four separate aliquots of $\sim 2.0$ grams each were weighed into separate tared $50-\mathrm{mL}$ beakers. The exact weight of test material weighed into each beaker was recorded on a piece of laboratory tape fixed to the beaker. At the conclusion of the all samples being removed the container was re-weighed and a single entry was placed into the chemical use logbook. The original tapes (containing the exact chemical weights) affixed to the beakers were retained and were filed in file folder 8 of the study records along with a description and time of their use. Additionally, an entry explaining the procedures used were recorded in the chemical logbook and laboratory notebook. No adverse impacts to the study are anticipated as a result of this deviation.

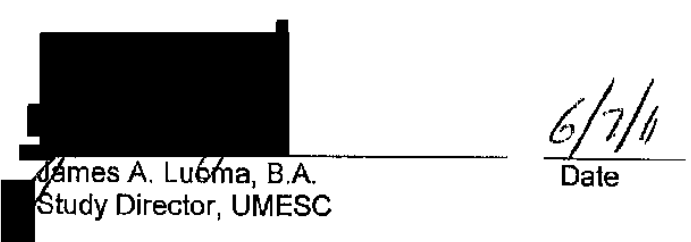




\section{U.S. GEOLOGICAL SURVEY \\ Upper Midwest Environmental Sciences Center 2630 Fanta Reed Road \\ La Crosse, Wisconsin 54603}

Ltem No. 9

MEMORANDUM

FOLDER NO. 3

Date: January 11, 2012

To: The Record Study Number AEH-11-PSEUDO-01

Subject: Deviation 5 to study AEH-11-PSEUDO-01 "Effects of Pseudomonas fluorescens (PfCL145A) to glochidia from seven unionid mussel species"

Deviation \#5 - Sections 4.2 and 6.2 (Experimental Design and Determination of viability) of the amended protocol (see Amendment 3) state that "approximately one drop of saturated sodium chloride solution (12 $\mathrm{g} \mathrm{NaCl} / 50 \mathrm{~mL}$ of D.I. $\mathrm{H}_{2} \mathrm{O}$ [ASTM E2455-06]) will be added to the sample and after one minute a second photomicrograph will be recorded. The total number of glochidia within the sample and the number of glochidia closed or open before and after sodium chloride addition will be determined". Due to the large quantity of samples to be processed, samples were placed on the microscope stage and photographed to enumerate for viable glochidia (i.e., those with open valves). One drop of a saturated sodium chloride solution was added to the sample and a timer was started. The sample was then removed from the microscope stage and replaced with the next sample. Once the minute was over, the original sample exposed to the saturated sodium chloride solution was again placed on the microscope staged and photographed to enumerate for viable glochidia (i.e., those with closed valves). This process was used for the initial viability, distribution, 6,12 , and 24 hour viability determinations.

The method described was only used for samples processed on May 12-13, 2011. No negative impacts to the study are anticipated as each sample was re-photographed at the one minute postexposure to saturated sodium chloride. The photographs are not aligned in the same way from before salt to after salt, but will have no impact on enumeration.

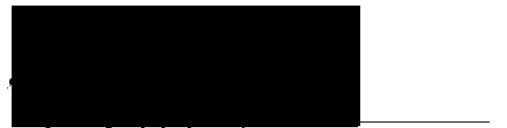

Writen by

Kerry L. Weber, M.S.

Principal Investigator, UMESC

cc: UMESC QAU

\section{JANADHR Date} Date

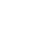

.


United States Department of the Interior

U.S. GEOLOGICAL SURVEY

Upper Midwest Environmental Sciences Center 2630 Fanta Reed Road

La Crosse, Wisconsin 54603
OBCans

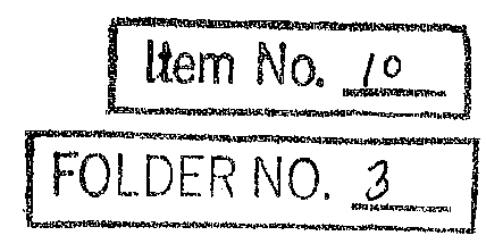

Date: January 11, 2012

To: The Record Study Number AEH-11-PSEUDO-01

Subject: Deviation 6 to study AEH-11-PSEUDO-01 "Effects of Pseudomonas fluorescens ( $P f$ CL145A) to glochidia from seven unionid mussel species"

Deviation $\# 6$ - Sections 4.2 and 6.2 (Experimental Design and Determination of viability) of the amended protocol (see Amendment 3) state that "if glochidia can be enumerated and viability statues (i.e. open or closed) reliably determined through photomicrograph records (i.e. the glochidia remain on a single focal plane)" then the viability of the subsample of approximately 100 glochidia from each chamber will be assessed at 6,12 , and $24 \mathrm{~h}$ post exposure by enumeration through a dissection microscope. The $24 \mathrm{~h}$ control dosing $(0 \mathrm{mg} / \mathrm{L})$ photographs taken on May 12, 2011 for Plain pocketbook (Lampsilis cardium), Higgins eye (Lampsilis higginsii) and Fatmucket (Lampsilis siliquoidea) were enumerated on May 16, 2011 using Image Pro Software. The ncw Nikon NIS-Elements BR imaging software was received and installed after this enumeration occurred. Therefore, the samples were recounted on August 10-16, 2011 using the new software. The original enumcrations from Image Pro were retained as .jpg images. The enumerations using the Nikon NIS-Elements BR software were saved as tif files. All count data from both enumerations were recorded on the Glochidia Photomicrograph Counting Record datasheet (File Folder 12C) and within the electronic log (pages 1 to 24 of 1://AEH-1 1-PSEUDO$01 /$ Pictures/12May 11/24h). The count data from the Nikon NIS-Elements BR software were used during analysis.

Samples that were re-counted using the Nikon NIS-Elements BR software include:

\begin{tabular}{|c|c|c|}
\hline Plain Pocketbook & Higgins Eye & Fatmucket \\
\hline IVI & & 34224 \\
\hline $1 \mathrm{~A} 3-24 h-2$ & $2 \mathrm{C} 3-24 \mathrm{~h}-\mathrm{R} 2$ & $3 \mathrm{~B} 2-24 \mathrm{~h}$ \\
\hline 20 & & \\
\hline $1 \mathrm{C} 3-24 \mathrm{~h}$ & 2C6-24-R2 & \\
\hline
\end{tabular}

No negative impacts to the study are anticipated. The images analyzed with Nikon BR Elements

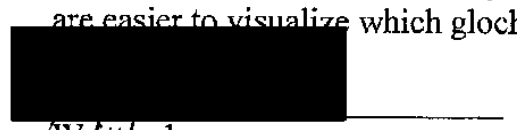

Writyen by

Kerry L. Weber, M.S.

Principal Investigator, UMESC

cc: UMESC QAU

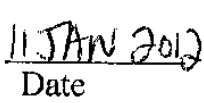

$\frac{11.5}{\text { Date }}$

in the

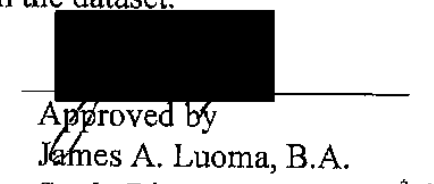

Study Director, UMESC $\frac{1 / 5 \text { No } 2012}{\text { Date }}$ Ims 00 se $2 / 6 / 12$ BEV: EWED RY Page 1 of 1 
United States Department of the Interior

U.S. GEOLOGICAL SURVEY

Upper Midwest Environmental Sciences Center

2630 Fanta Reed Road

La Crosse, Wisconsin 54603

MEMORANDUM

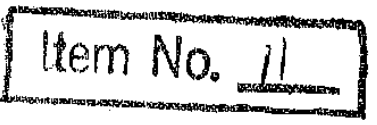

FOLDER NO. 3

Date: January 11, 2012

To: The Record Study Number AEH-11-PSEUDO-01

Subject: Deviation 7 to study AEH-11-PSEUDO-01 "Effects of Pseudomonas fluorescens (PfCL145A) to glochidia from seven unionid mussel species"

Deviation \#7 - Section 6.2 (Determination of viability) of the amended protocol (see Amendment 3) states that "the total number of glochidia within the sample and the number of glochidia closed or open bcfore and after sodium chloride addition will be determined." Samples taken on May 12, 2011 for Plain pocketbook (Lampsilis cardium), Higgins eye (Lampsilis

higginsii) and Fatmucket (Lampsilis siliquoidea) were enumerated from August 10 to 22,2011 in this manner, which is prior to the written amendment. The samples analyzed following this procedure can be found in Table 1 on page 2 of this deviation.

No negative impacts to the study are anticipated as an official amendment was approved for this procedure after the fact.

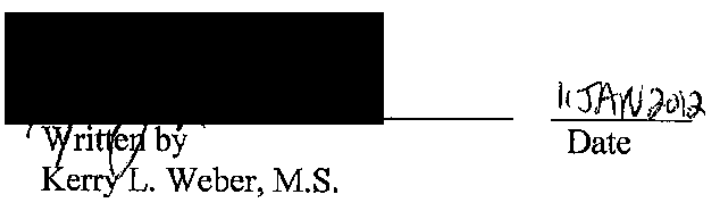

Principal Investigator, UMESC
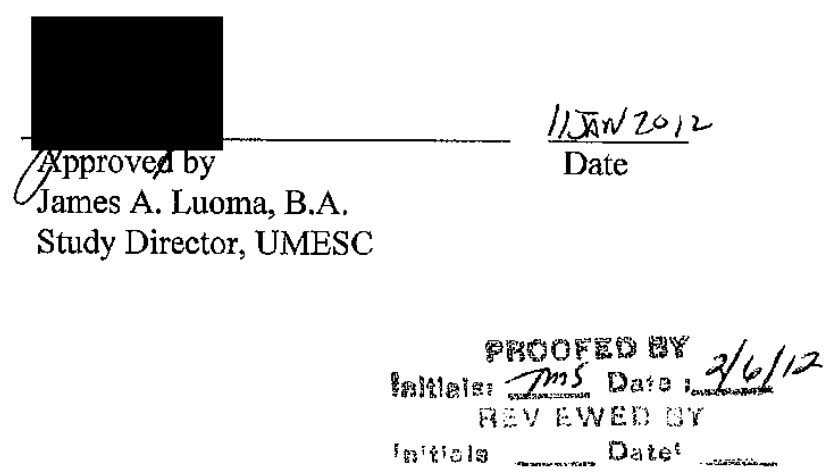

cc: UMESC QAU

Page 1 of 2 
AEH-II-PSEUDO-OI

Table 1. Samples analyzed using modified enumeration procedure outlined in amended protocol (see Amendment 3)

\begin{tabular}{|c|c|c|c|}
\hline & $\begin{array}{c}\text { Plain } \\
\text { Pocketbook }\end{array}$ & Higgins Eye & Fatmucket \\
\hline & 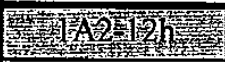 & 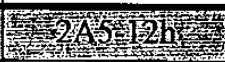 & $4 y^{4} y_{2}$ \\
\hline 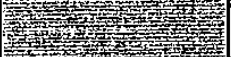 & $1 \mathrm{~A} 5-12 \mathrm{~h}$ & $2 \mathrm{~A} 6-12 \mathrm{~h}$ & $3 \mathrm{~A} 6-12 \mathrm{~h}$ \\
\hline (1) & 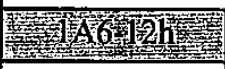 & 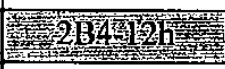 & 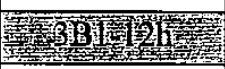 \\
\hline Shoul & $1 \mathrm{~B} 3-12 \mathrm{~h}$ & $2 \mathrm{~B} 6-12 \mathrm{~h}$ & 3B4-12h \\
\hline 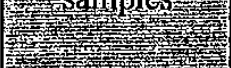 & 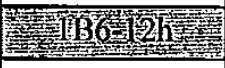 & 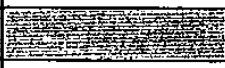 & 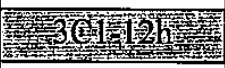 \\
\hline 7y & $1 \mathrm{C} 2-12 \mathrm{~h}$ & & $3 \mathrm{C} 2-12 \mathrm{~h}$ \\
\hline 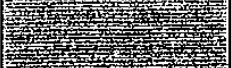 & 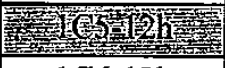 & W I & 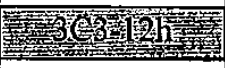 \\
\hline 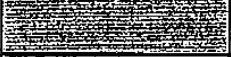 & $1 \mathrm{C} 6-12 \mathrm{~h}$ & & $3 \mathrm{C} 4-12 \mathrm{~h}$ \\
\hline & 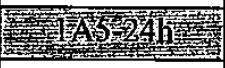 & 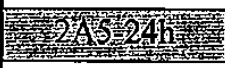 & 154 \\
\hline 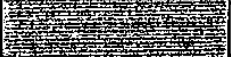 & $1 \mathrm{~A} 6-24 \mathrm{~h}$ & $2 \mathrm{~B} 3-24 \mathrm{~h}$ & $3 \mathrm{~A} 5-24 \mathrm{~h}$ \\
\hline 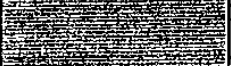 & 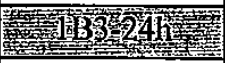 & 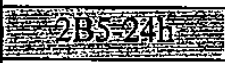 & 5ing \\
\hline (1) & $1 \mathrm{~B} 6-24 \mathrm{~h}$ & $2 \mathrm{~B} 6-24 \mathrm{~h}$ & $3 B 1-24 h$ \\
\hline samples & 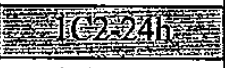 & 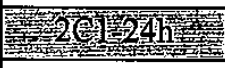 & 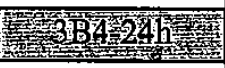 \\
\hline ע th & $1 \mathrm{C} 5-24 \mathrm{~h}$ & & $3 \mathrm{~B} 6-24 \mathrm{~h}$ \\
\hline L & 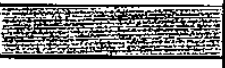 & 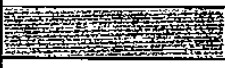 & 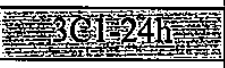 \\
\hline 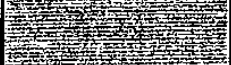 & & & $3 \mathrm{C} 2-24 \mathrm{~h}$ \\
\hline & & & 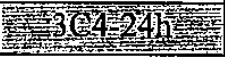 \\
\hline
\end{tabular}

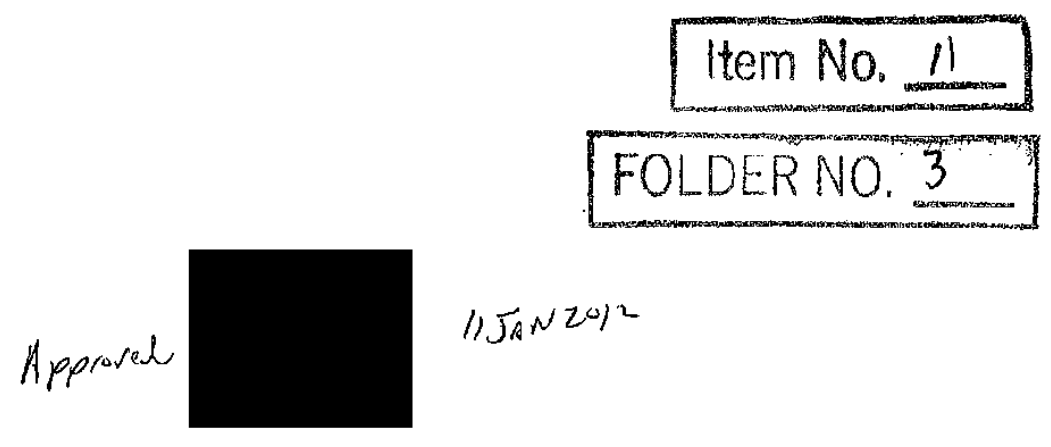

MOOFE

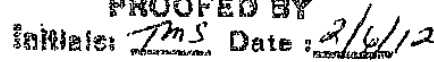
GEV EWED增 inisis Date:

cc: UMESC QAU

Page 2 of 2 


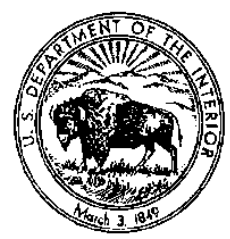

MEMORANDUM

Date: January 11, 2012

\section{U.S. GEOLOGICAL SURVEY \\ Upper Midwest Environmental Sciences Center 2630 Fanta Reed Road \\ La Crosse, Wisconsin 54603}

Itern No. 12

To: The Record Study Number AEH-11-PSEUDO-01

Subject: Deviation 8 to study AEH-11-PSEUDO-01 "Effects of Pseudomonas fluorescens (PfCL145A) to glochidia from seven unionid mussel species"

Deviation \#8-Sections 4.2 and 6.2 (Experimental Design and Determination of viability) of the amended protocol (see Amendments 2 and 3) state that "if glochidia can be enumerated and viability status (i.e. open or closed) reliably determined through photomicrographic records (i.e. the glochidia remain on a single focal plane" then "the sample will be viewed under a dissecting microscope, adjusted for maximum clarity and magnification, and a photomicrograph will be recorded". Photographs taken on May 13, 2011 were saved as .jpg files. During a training session, it was brought to our attention that compression inherent to .jpg files actually discards information associated with images. Over time, the loading and saving of .jpg images can cause enough information to be lost as to actually degrade the image. Therefore, the .jpg photographs taken on May 13, 2011 were converted to a tif file on August 3, 2011, which were then enumerated from August 9-22, 2011. During the conversion process and/or enumeration, 5 of the photographs were inadvertently over-written. Two of the over-written photographs were restored by Martin Tagesen using the network back-up while three of the over-written photographs were converted back to a tif file from the original .jpg file. See the Table 1 on page 2 of this deviation for detailed information.

No negative impacts to the study are anticipated as the tif files are have greater integrity and each photograph was restored to its original file before analysis was conducted.

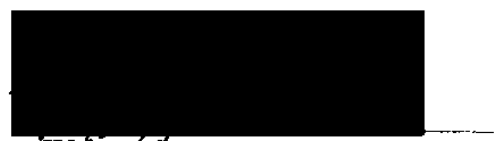

W/itten by

Kerrylu. Weber, M.S.

Principal Investigator, UMESC

\section{SAN DOID Date}

cc: UMESC QAU
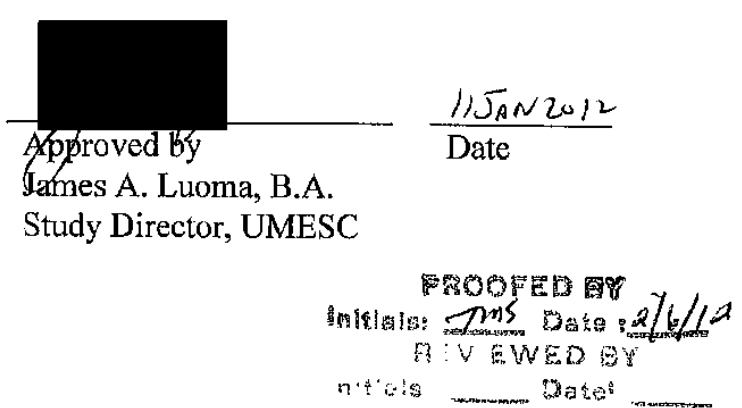

Page 1 of 2 
AEH-11-PSEUDO- 01

\section{OBJGiWAL}

Table 1. Detailed information regarding restoration for over-written files:

\begin{tabular}{|c|c|}
\hline & \\
\hline \multicolumn{2}{|c|}{ 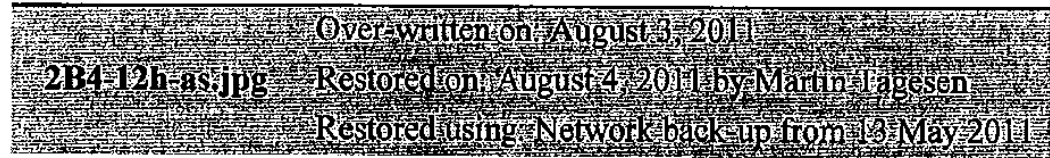 } \\
\hline \multirow{3}{*}{ 3B1-12h-as.jpg } & Over-written on: August 3, 2011 \\
\hline & Restored o \\
\hline & Restored \\
\hline \multicolumn{2}{|c|}{ 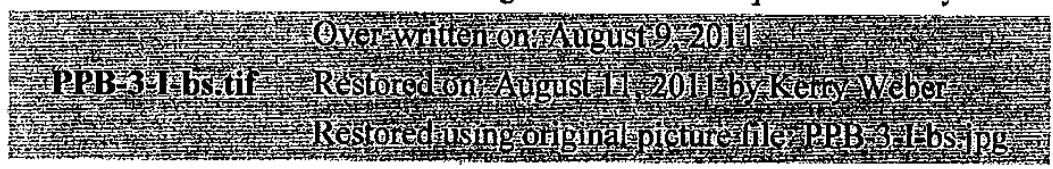 } \\
\hline \multirow{3}{*}{ 2A5-24h-bs.tif } & Over-written on: August 12,2011 \\
\hline & Restored on: August 12, 2011 by Kerry W \\
\hline & Restored using original picture file: 2 A5-24h-bs.jpg \\
\hline \multicolumn{2}{|c|}{ 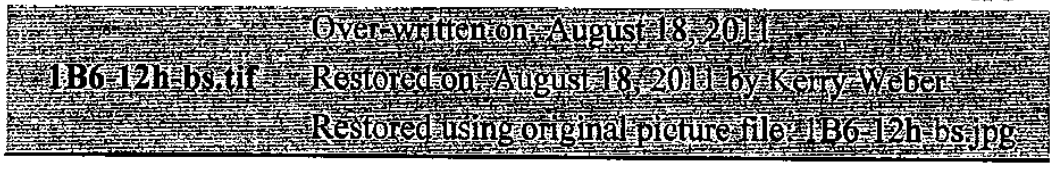 } \\
\hline
\end{tabular}

Item No. 12

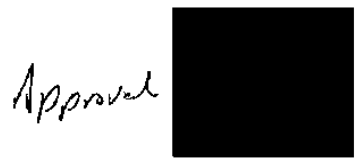

$115 \mathrm{NaN} 20 / 2$

FOLDER NO. 3

cc: UMESC QAU

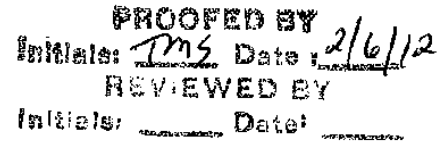

Page 2 of 2 


\section{United States Department of the Interior \\ U.S. GEOLOGICAL SURVEY \\ Upper Midwest Environmental Sciences Center 2630 Fanta Reed Road \\ La Crossc, Wisconsin 54603}

MEMORANDUM

Item No. 13

File Folden: 3

Date: February 7, 2012

To: The Record Study Number AEH-11-PSEUDO-01

Subject: Deviation 9 to study AEH-11-PSEUDO-01 "Effects of Pseudomonas fluorescens (PfCL145A) to glochidia from seven unionid mussel species"

Deviation \#9 - Sections 4.2 and 6.2 (Expcrimental Design and Determination of viability) of the amended protocol (see Amendments 2 and 3) state that "if glochidia can be enumerated and viability status (i.e. open or closed) reliably determined through photomicrographic records (i.e. the glochidia remain on a single focal plane" then "the sample will be viewed under a dissecting microscope, adjusted for maximum clarity and magnification, and a photomicrograph will be recorded". During enumeration of Hickorynut glochidia (dosed on January 19, 2012) for initial viability, the following photograph was inadvertently over-written: HIC-3-I-bs.tif. The original file was restored by Martin Tagesen using the network back-up from January 19, 2012.

No adverse impacts to the study will result from this error as the over-written file was restored from a backup of the original file.

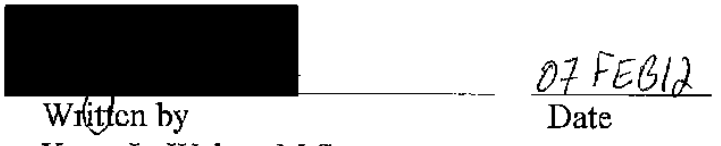

Kerry L. Wcber, M.S.

Principal Investigator, UMESC

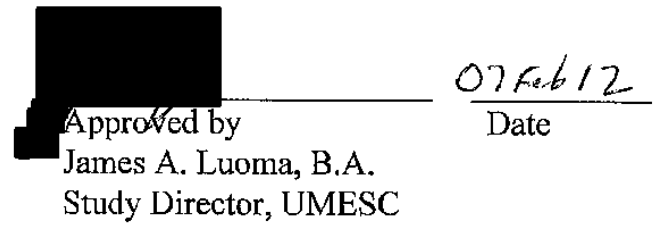

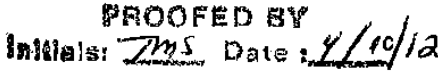

REV EWED BY

Inilialo: Date:

cc: UMESC QAU 


\section{United States Department of the Interior \\ U.S. GEOLOGICAL SURVEY \\ Upper Midwest Environmental Sciences Center \\ 2630 Fanta Reed Road \\ La Crosse, Wisconsin 54603}

MEMORANDUM

Date: October 31, 2012 ,

To: The Record Study Number AEH-11-PSEUDO-01

Subjcct: Deviation 10 to study AFH-11-PSEUDO-01 "Iffects of Pseudomonas fluorescens (PfCL145A) to glochidia from seven unionid mussel species"

Deviation \#10 - Filcnames for the photomicrographs of glochidia samples were changed to allow for unique file identification. The original photomicrograph codes used were not unique as the test system was used for multiple species and formulations and the original photomicrograph codes did not identify the species or product formulation. The filenames for each photomicrograph were renamed to include unique identifiers for each time point at which photomicrographs were recorded. Codes were used within the filenames to make the file identifiable to species, formulation, concentration, sample, exposure time and before or after salt addition, as applicable. The resulting codes allow for unambiguous photomicrograph tracking.

\section{INITIAL VIABILITY}

Photomicrographs were used to determine the suitability of glochidia for inclusion in the exposurcs. Photomicrographs were recorded for samples of glochidia flushed from each gravid mussel. The glochidia were photographed before and after a concentrated salt solution was added to the sample to induce valve closure. The photomicrographs were enumerated according to the procedures described in Amendment 1 of the protocol.

The original codes used in the original filenames were not unique as two formulations were used in exposures. The formulation type was incorporated into the filcname. Additionally, once files were enumerated, they were then saved with a new filename that had the addition of $\mathrm{T}, \mathrm{O}$ or $\mathrm{C}$ (Total count, Open count or Closed count) at the end of the filename which designate the type of count performed. See Table 1 for the original filenames, the new filenames and enumerated filenames for the initial viability samples. 
Table 1. Initial Viability Filenames

\begin{tabular}{|c|c|}
\hline Original Filename & $\left.\right|_{\text {Mussel } \#(1,2 \text { or } 3)} ^{\text {PPB-1 I Initial Viability) }}$ \\
\hline New Filename & $\left.\right|_{\text {Formulation Type (FDP or SDP) }} ^{\text {PPB-SDP-1-I-bs }}$ \\
\hline $\begin{array}{l}\text { Enumcrated } \\
\text { Filename }\end{array}$ & ||$_{\text {Formulation Type (FDP or SDP) }}^{\text {PPB-SDP-1 -I-bs-T }}$ \\
\hline
\end{tabular}

\section{DISTRIBUTION}

Photomicrographs were recorded on samples collected during each round of distribution to the exposure chambers. Distributions were completed in 5 rounds with 18 aliquots per round.

Photomicrographs were recorded on the initial aliquot and then after every 6 aliquots for a total of 16 distribution samples for each species. The photomicrographs were recorded and cnumerated to estimate the number of animals distributed to each chamber. Distribution samples that had very dense quantities of glochidia were subdivided into more manageable counts bcfore being photomicrographed. The photomicrographs were enumerated according to the procedures described in Amendment 1 of the protocol. 
The codes used in the original filenames were unique and were retained with the exception of the plain pocketbook (PPB) photomicrograph files. Plain pocketbook was the only species that was exposed to both formulations. Therefore, the formulation type was added only to the PPB distribution photomicrographs. Additionally, once files were enumerated, they were then saved with a new filename that had the addition of $T$ (Total count) at the end of the filename which designate the type of count performed. See Table 2 for the original filenames, the new filenames and enumerated filenames for the distribution samples.

Table 2. Distribution Filenames

\begin{tabular}{|c|c|}
\hline Original Filename & ||$_{\text {Distribution Round (R1-R5) }}^{\text {PPB-R2-2.1 }}$ \\
\hline New Filename & 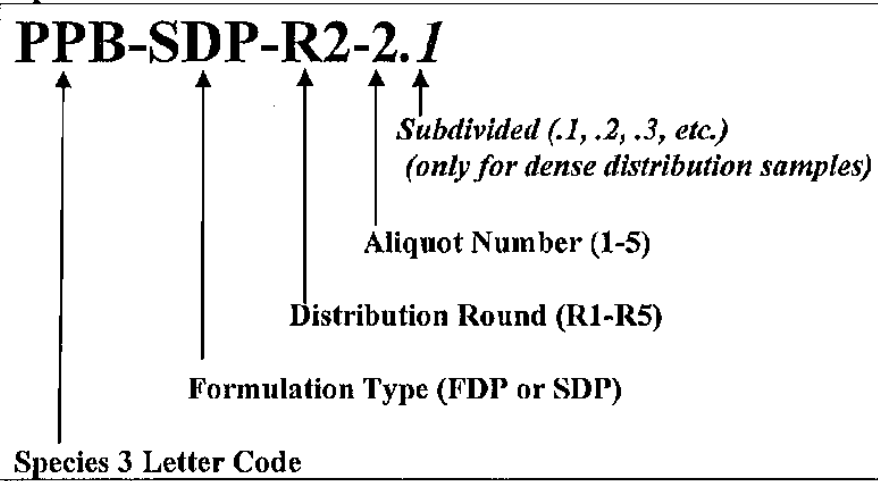 \\
\hline $\begin{array}{c}\text { Enumerated } \\
\text { Filename }\end{array}$ & PPB-SDP-R2-2.1-T \\
\hline
\end{tabular}

cc: UMESC QAU

Page 3 of 5 


\section{VIABILITY SAMPLES (6, 12 AND 24 HOUR)}

Photomicrographs were recorded on glochidia samples collected from each exposure chamber at 6,12 and 24 hours post-dose initiation to determine viability. Two photomicrographs were recorded for each sample collected for viability assessment. The first photomicrograph was recorded prior to the addition of a salt solution to induce valve closure and the second after induced closure. The photomicrographs were enumerated according to the procedures described in Amendment 1 of the protocol.

The codes used in the original filenames were not unique as multiple species were exposed using the same test system and two formulations were used in the exposures. Therefore, the species $\mathrm{ID}$, formulation type and concentration were added to the filenames. Additionally, once files were enumcrated, they were then saved with a new filename that had the addition of $\mathrm{T}, \mathrm{O}$ or $\mathrm{C}$ (Total count, Open count or Closed count) at the end of the filename which designate the type of count performed. See Table 3 for the original filenames, the new filenames and enumerated filenames for the viability samples at 6,12 and 24 hours.

Table 3. Viability Samples $(6,12$ and 24 hour $)$

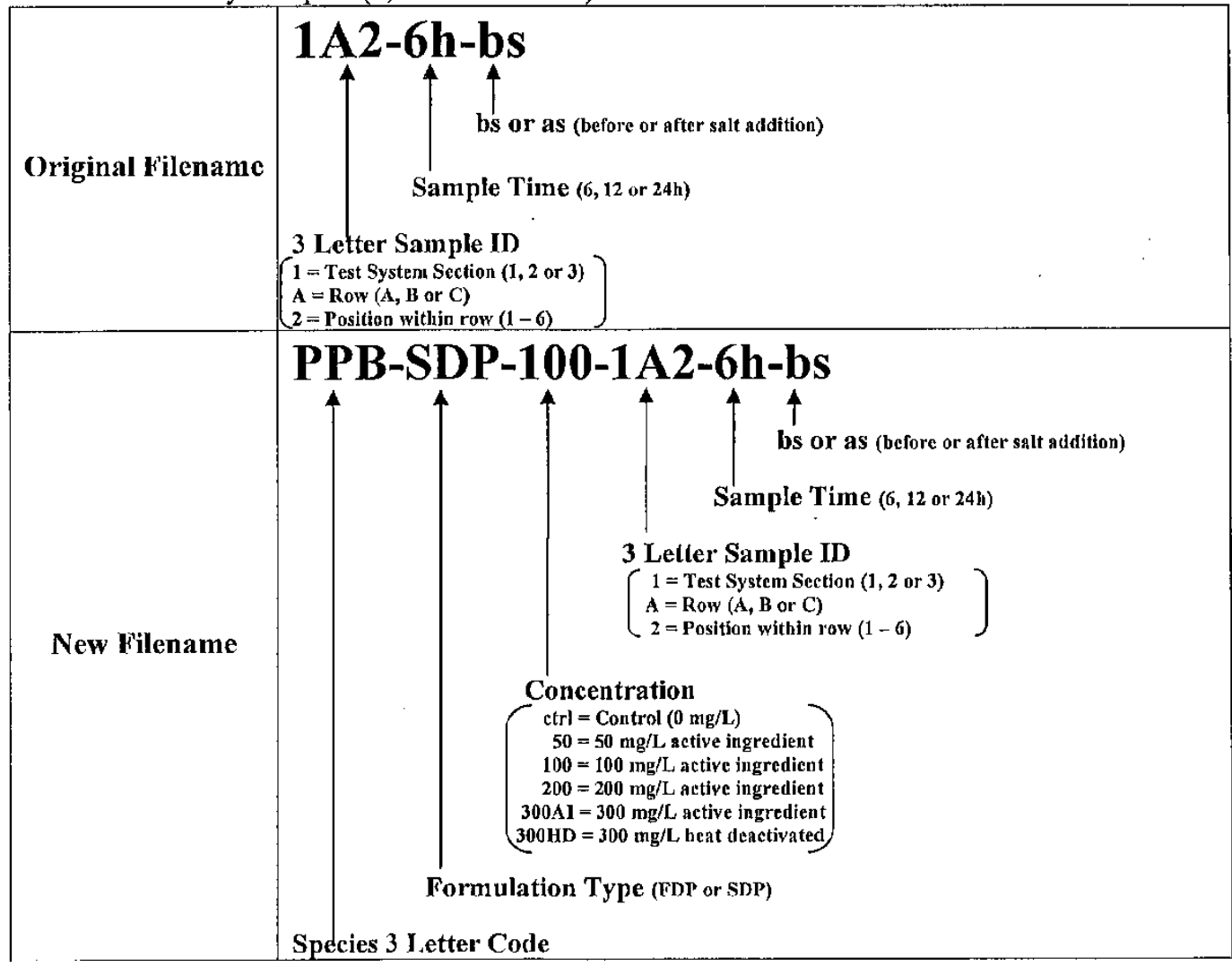

cc: UMESC QAU 
Table 3 (con.) 6, 12 and 24 Hour Viability Samples

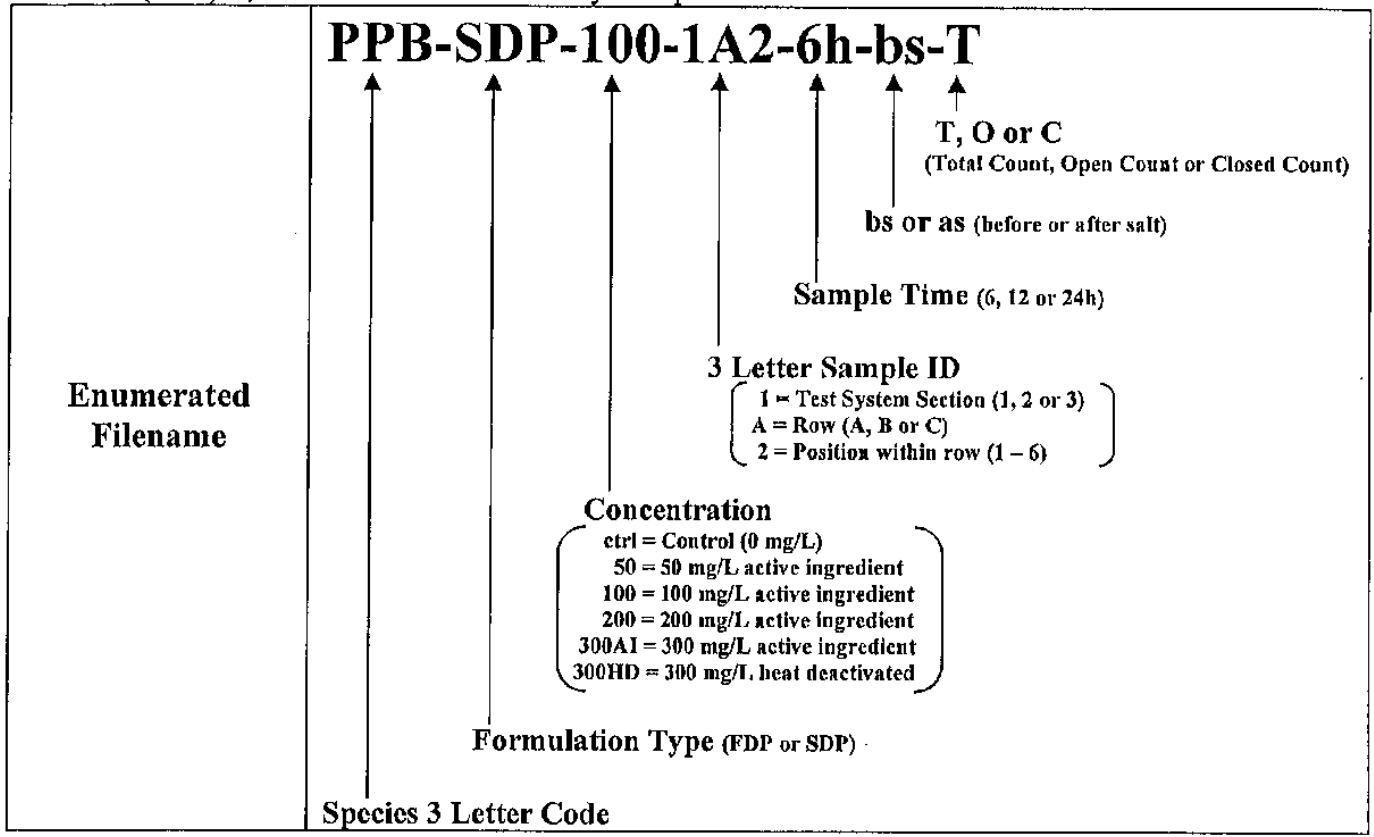

No adverse impacts to the study will result from this change.

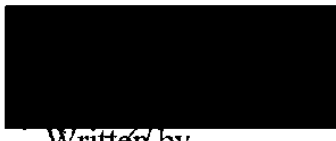

Writter by

Kerry L. Weber, M.S.

Principal Investigator, UMESC

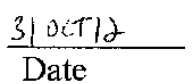

Date

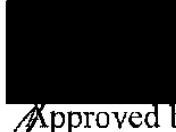

Approved by

James A. Luoma, B.A.

Study Director, UMESC
$10-31-12$

Date 


\section{United States Department of the Interior \\ U.S. GEOLOGICAL SURVEY \\ Upper Midwest Environmental Sciences Center \\ 2630 Fanta Reed Road \\ Ia Crosse, Wisconsin 54603}

MEMORANDUM

Date: November 27, 2012

To: The Record Study Number AEH-11-PSEUDO-01

Subject: Deviation 11 to study AEH-11-PSEUDO-01 "Effects of Pseudomonas fluorescens (PfCL145A) to glochidia from seven unionid musscl species"

Deviation \#11 - Sections 4.2 (Experimental Design) and 5.1.4 (Test Animal: Inclusion criterion) of the amendment 1 of the protocol entitled "Effects of Pseudomonas fluorescens (Pf-CL145A) to glochidia from seven unionid mussel species" states that "if viability [of glochidia] is acceptable ( $\geq 80 \%$ ) for a mussel, the glochidia from that mussel will be pooled with glochidia from other mussels of that species". Deviation 3 further states that "mussels that were observed to have high viability, the exact viability percentage was not determined prior to allowing the glochidia into the study. For these mussels a preliminary visual observation was used to determine acceptable viability... exact viability of the glochidia will be determined from the photomicrograph records..."

The initial viability of three individual mussels [Fatmucket (FAM 2), Higgins eye (HGE 1) and Plain pocketbook (PPB 3)] appeared (by visual observation) to be greater than $80 \%$. Upon conducting the final counts of the photomicrograph records, it was discovered that the viability of the glochidia from these individual mussels were slightly below $80 \%$. See Table 1 for the mussel ID and the percent viability.

The initial viability of one individual mussel [Black sandshell (BLS 2)] did not appear (by visual observation) to be grcater than $80 \%$ (photomicrograph enumeration $=54.4 \%$ viable). Two additional glochidia samples (BLS 2.2 and BLS 2.3) from this mussel were observed for initial viability and they appeared to have near or greater than $80 \%$ viability (photomicrograph enumeration $=79.4 \%$ and $85.4 \%$, respectively). Upon visual observation of the two subsequent samples, the glochidia from this mussel were pooled for use in the study.

The results obtained from the three viability samples (BLS 2, BLS 2.2 and BLS 2.3) photomicrograph record counts were averaged to determine the viability of glochidia cxtracted from BLS 2. Additionally, the mean initial viability from BLS 2 was used to determine the pooled viability of all glochidia extracted from the BLS donor mussels. See Table 1 for the 
mussel ID and the percent viabilities.

No negative impacts to the study are anticipated as the mean glochidia viability for all pooled samples were greater than $80 \%$ criteria identified in the study protocol.

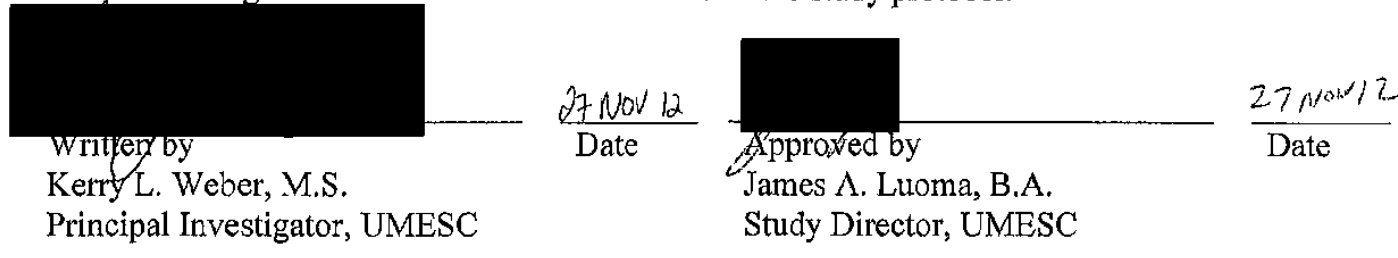

Table 1. Photomicrograph record determined glochidia viability from individual mussels.

\begin{tabular}{|c|c|c|}
\hline Mussel ID & $\begin{array}{c}\text { Individual Percent Viability } \\
\qquad(\%)\end{array}$ & Pooled Viability (\%) \\
\hline \multicolumn{3}{|c|}{ 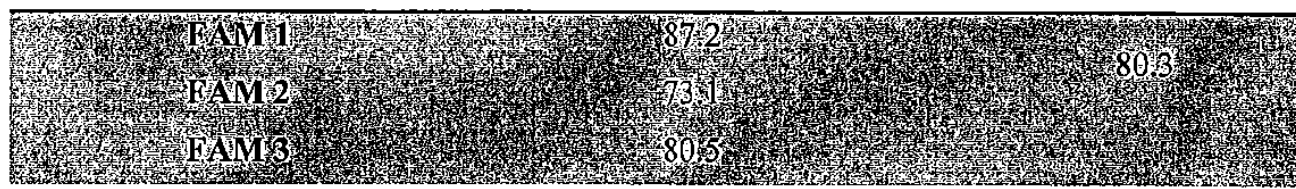 } \\
\hline HGE 1 & 62.0 & \\
\hline HGE 2 & 88.1 & 80.8 \\
\hline HGE 3 & 92.4 & \\
\hline 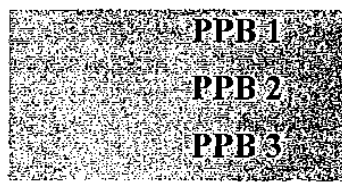 & 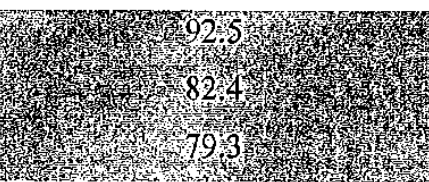 & Mry \\
\hline BLS 1 & 89.7 & \\
\hline BLS 2 & $73.1^{\mathrm{a}}$ & 83.2 \\
\hline BLS 3 & 86.9 & \\
\hline
\end{tabular}

${ }^{n}$ This value is the mean percent initial viability (\%) of the 3 glochidia samples (BLS 2, BLS 2.2, BLS 2.3) from BLS mussel 2. This value was used to determine the pooled initial viability (\%) from the BLS donor mussels.

cc: UMESC QAU

Page 2 of 2 


\section{United States Department of the Interior \\ U.S. GEOLOGICAL SURVEY \\ Upper Midwest Environmental Sciences Center \\ 2630 Fanta Reed Road \\ La Crosse, Wisconsin 54603}

\section{MEMORANDUM}

Date: December 17, 2012

To: The Record Study Number AEH-11-PSEUDO-01

Subject: Deviation 12 to study AEH-11-PSEUDO-01 "Effects of Pseudomonas fiuorescens ( $P f$ CL145A) to glochidia from seven unionid mussel species"

Deviation \#1.2 - Sections 4.2 (Experimental Design) and 6.2 (Determination of viability) of the amendment 3 of the protocol entitled "Effects of Pseudomonas fluorescens (Pf-CL145A) to glochidia from seven unionid mussel species" states that "if glochidia can be cnumerated and viability status (i.c. open or closed) reliably determined through photomicrograph records (i.e. the glochidia remain on a single focal plane)" then the viability of the subsample of approximately 100 glochidia from each chamber will be assessed at 6,12 , and $24 \mathrm{~h}$ post exposure by enumeration through a dissection microscope. As outlined in Note to File \#2, the glochidia photomicrograph records were used to: 1) determine the viability of extracted glochidia that were used in the exposures; 2) estimate the number of glochidia distributed to each exposure replicate and; 3) assess the treatment effects on the viability of glochidia at three time points $(6,12$ and $24 \mathrm{~h}$ ) during the exposure period. Note to File $\# 2$ provides clarification to the path and unique folder and filenames for the photomicrograph records. Enumerated photomicrographs each contain a text box legend containing the study number, species, formulation type (SDP vs. FDP), concentration, filename and the glochidia count (i.e. Total Number Glochidia $=$ the total number of glochidia present before salt; Glochidia Open or Glochidia Closed $=$ the number of glochidia open or closed before or after sait). The legend allows the photomicrograph to be uniquely identified. Legend data were typed in by the analyst upon completion of the enumerations and the legend was then burned as an overlay onto the image.

During data proofing, the hard copy data was verified against the legend information on the enumerated photomicrographs. Typographical errors were noted and corrected on 6 photomicrographs. The individual images were opened in the Nikon NIS-Flements BR software. A new legend was created with the correct information. The new legend was burned as an overlay on the image before it was saved. See Table 1 for the photomicrograph filename, the typographical error and the correction.

No negative impacts to the study are anticipated as the data in the legend reflects the original cc: UMESC QAU 
hard copy data. Enumeration data was immediately recorded on the "Glochidia Photomicrograph Counting Record" datasheet (File Folders 11, 12A, 12B, 12C and 12D).

Table 1. Typographical errors in AEH-11-PSEUDO-01 enumerated photomicrograph records.

\begin{tabular}{|l|l|}
\hline \multicolumn{1}{|c|}{ Photomicrograph Filename } & \multicolumn{1}{c|}{ Typographical error and correction } \\
\hline HGE-SDP-300HD-2A5-6h-bs-T.tif & $\begin{array}{l}\text { Error: HGE-SDP-300HD-2A506h-bs-T.tif } \\
\text { Correction: HGE-SDP-300HD-2A5-6h-bs-T.tif }\end{array}$ \\
\hline HIC-SDP-100-3C5-6h-bs-T.tif & $\begin{array}{l}\text { Error: HIC-SDP-100-3C6-6h-bs-T.tif } \\
\text { Correction: HIC-SDP-100-3C5-6h-bs-T.tif }\end{array}$ \\
\hline BLS-SDP-50-1A1-6h-bs-T.tif & $\begin{array}{l}\text { Error: BLS-SDP-50-1A1-6h-as-T.tif } \\
\text { Correction: BLS-SDP-50-1A1-6h-bs-T.tif }\end{array}$ \\
\hline MUC-SDP-100-2A2-12h-bs-C.tif & $\begin{array}{l}\text { Error: Glochidia Closed }=\mathbf{0} \\
\text { Correction: Glochidia Closed }=\mathbf{9 1}\end{array}$ \\
\hline HIC-SDP-300AI-3B5-12h-bs-T.tif & $\begin{array}{l}\text { Error: HIC-SDP-300Al-3B5012h-bs-T.tif } \\
\text { Correction: HIC-SDP-300AI-3B5-12h-bs-T.tif }\end{array}$ \\
\hline $\begin{array}{l}\text { H(iE-SDP-300HD-2A5-24h-as-O.tif } \\
\text { Error: Glochidia Open }=\mathbf{1 3 7} \\
\text { Correction: Glochidia Open }=\mathbf{4 2}\end{array}$ \\
\hline
\end{tabular}

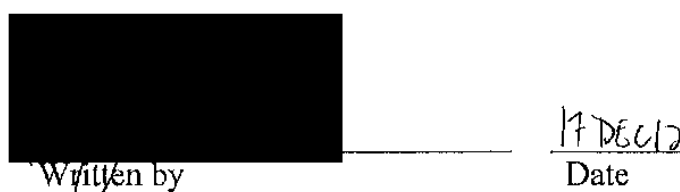

Kerry L. Weber, M.S.

Principal Investigator, UMESC

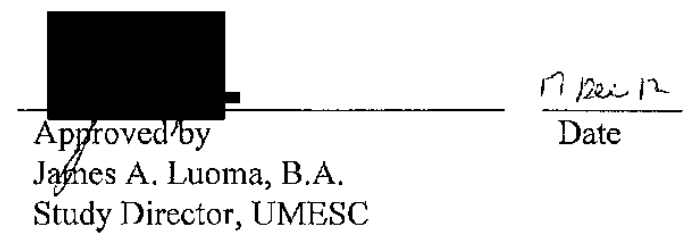

Study Director, UMESC

cc: UMESC QAU

Page 2 of 2 


\title{
United States Department of the Interior
}

\author{
U.S. GEOLOGICAL SURVEY \\ Upper Midwest Environmental Sciences Center \\ 2630 Fanta Reed Road \\ La Crosse, Wisconsin 54603
}

MEMORANDUM

Date: March 16, 2015

To: The Record Study Number AEH-11-PSEUDO-01

Subject: Deviation 13 to study AEH-11-PSEUDO-01 "Effects of Pseudomonas fluorescens (PfCL145A) to glochidia from seven unionid mussel species"

Deviation \#13 - Sections 4.2 (Experimental Design) and 5.1.4 (Test Animal: Inclusion criterion) of the amendment 1 of the protocol entitled "Effects of Pseudomonas fluorescens (Pf-CL145A) to glochidia from seven unionid mussel species" states that "if viability [of glochidia] is acceptable ( $\geq 80 \%$ ) for a mussel, the glochidia from that mussel will be pooled with glochidia from other mussels of that species".

Deviation 11 calculated black sandshell donor mussel 2 (BLS 2) percent viability by taking the mean of all three BLS 2 sample percent viabilities (BLS 2, BLS 2.2, and BLS 2.3). See deviation 11 for clarification. Deviation 13 calculates percent viability of BLS 2 using the sum total of all glochidia and non-viable glochidia (those closed before the addition, and open after the addition of salt) from all three BLS 2 samples.

See Table 1 for viable and non-viable glochidia counts and percent viability calculations.

The mean percent viability of the glochidia from all black sandshell donor mussels was 84.5 percent. No negative impacts to the study were observed from the viability of glochidia from donor mussel BLS 2 being 76.9 percent.

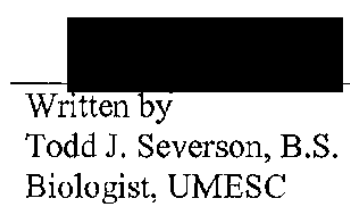

Biologist, UMESC

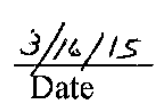

Date
Approved by

Janes A. Luoma, B.A.

Study Director, UMESC

cc: UMESC QAU

Page 1 of 2 
Table 1. Black sandshell donor mussel 2 initial glochidia viability samples and calculations.

\begin{tabular}{cccc}
\hline Sample ID & Total glochidia & $\begin{array}{c}\text { Non-viable } \\
\text { glochidia' }^{1}\end{array}$ & Percent viability \\
\hline BLS 2 & 237 & 108 & $54.4^{2}$ \\
BLS 2.2 & 310 & 64 & $79.4^{2}$ \\
BLS 2.3 & 536 & 78 & $85.4^{2}$ \\
\hline BLS 2 sum total & 1083 & 250 & $76.9^{3}$ \\
\hline
\end{tabular}

'Non-viable glochidia are glochidia closed prior to the addition of, and open after the addition of salt.

2Percent viability calculation: (total glochidia - non-viable glochidia) / total glochidia * 100 .

${ }^{3}$ Percent viability calculation: (sum total glochidia - sum total non-viable glochidia) / sum total glochidia * 100 . 


\section{Appendix 3. Randomization Assignments}

\begin{tabular}{|c|c|c|c|}
\hline $\begin{array}{c}\text { Item } \\
\text { num ber }\end{array}$ & Item description & $\begin{array}{c}\text { Number } \\
\text { of } \\
\text { pages }\end{array}$ & $\begin{array}{c}\text { Report } \\
\text { page } \\
\text { number }\end{array}$ \\
\hline 1 & $\begin{array}{l}\text { SAS random assignment of mussel species to block for plain pocketbook, Higgins eye, and fatmucket } \\
(10 \text {-May-11) }\end{array}$ & 4 & 136 \\
\hline 2 & SAS random assignment of mussel species to block for washboard and plain pocketbook (14-Oct-1 1) & 4 & 140 \\
\hline 3 & $\begin{array}{l}\text { SAS random assignment of mussel species to block for black sandshell, mucket, and hickory nut } \\
\text { (09-Jan-12) }\end{array}$ & 5 & 144 \\
\hline 4 & SAS random assignment of glochidia to tank for plain pocketbook, Higgins eye, and fatmucket (12-May-11) & 20 & 149 \\
\hline 5 & SAS random assignment of glochidia to tank for washboard and plain pocketbook (14-Oct-1 1) & 13 & 169 \\
\hline 6 & SAS random assignment of glochidia to tank for black sandshell, mucket, and hickorynut (09-Jan-12) & 20 & 182 \\
\hline 7 & SAS random assignment of treatment to tank for plain pocketbook, Higgins eye, and fatmucket (12-May-11) & 13 & 202 \\
\hline 8 & SAS random assignment of treatment to tank for washboard and plain pocketbook (14-Oct-11) & 9 & 215 \\
\hline 9 & SAS random assignment of treatment to tank for black sandshell, mucket, and hickorynut (09-Jan-12) & 13 & 224 \\
\hline
\end{tabular}




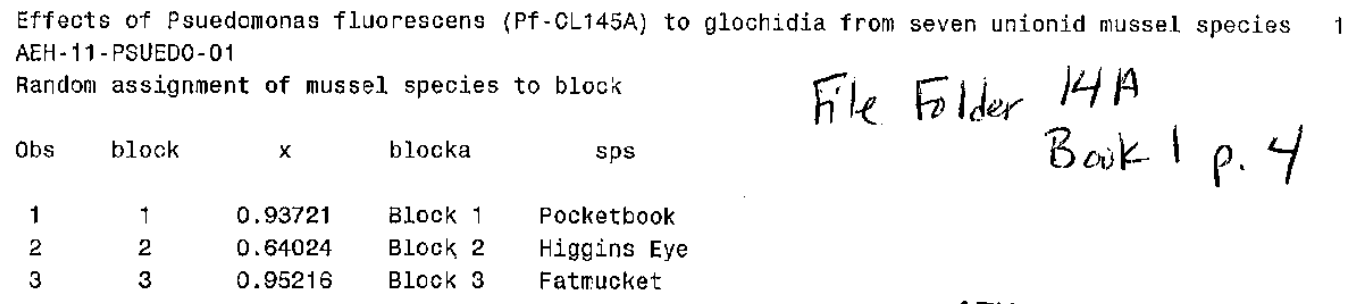

File Folder B 14 A

AEH-11-PSEUDO-01 Hem No. 1 


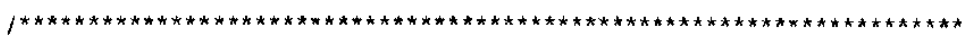

* Study Number : AEH 11 .PSUED0-01

* Study Director: Jim Luoma

* date created : 22Apr11. MPG MPZ

* Verified by: (Date:_)

* Random allocation of mussel species to block. sas

page of

**********************************************************************)

DM 'LOG; Clear; OUtPUT; CleAR;'; * CleAR LOG AND OUTPUT;

FOOTNOTE1 'Analysis performed by M. Gaikowski, SAS version ' \&SYSVER \&SYSTIME \&SYSDATE;

options $/{ }^{*} \mathrm{ls}=85 \mathrm{ps}=40$ formdlim=' $-1 * /$ pageno $=1$ nocenter nodate nosource2;

$l$ *Random assignment of mussel species to block* $f$

data sps;

do block $=1$ to 3 by 1 ;

$x=\operatorname{ranuni}(-1)$;

output;

end;

run;

proc sort data $=$ sps;

by $x$;

run;

data block; set sps;

if block $=1$ then blocka =' Block 1 ';

if block $=2$ then blocka $=$ 'Block $2 '$;

if block $=3$ then blocka $={ }^{\prime B l o c k} 3 '$; run;

data assign_sps; set block;

if $n_{1}=1$ then sps = 'Higgins Eye';

if ${ }_{-}{ }_{-}=2$ then sps $=$ 'Pocketbook';

if $n_{-}=3$ then sps = 'Fatmucket';

run;

proc sort data=assign_sps;

by block;

run;

proc print data $=$ assign.sps;

title1 $h=2$ 'Effects of Psuedomonas fluorescens (Pf-CL145A) to glochidia from seven unionid mussel title2 $h=1.5$ 'AEH-11-PSUEDO-O1';

title3 $h=1$ 'Randon assignment of mussel species to block';

run;

Pas 20 


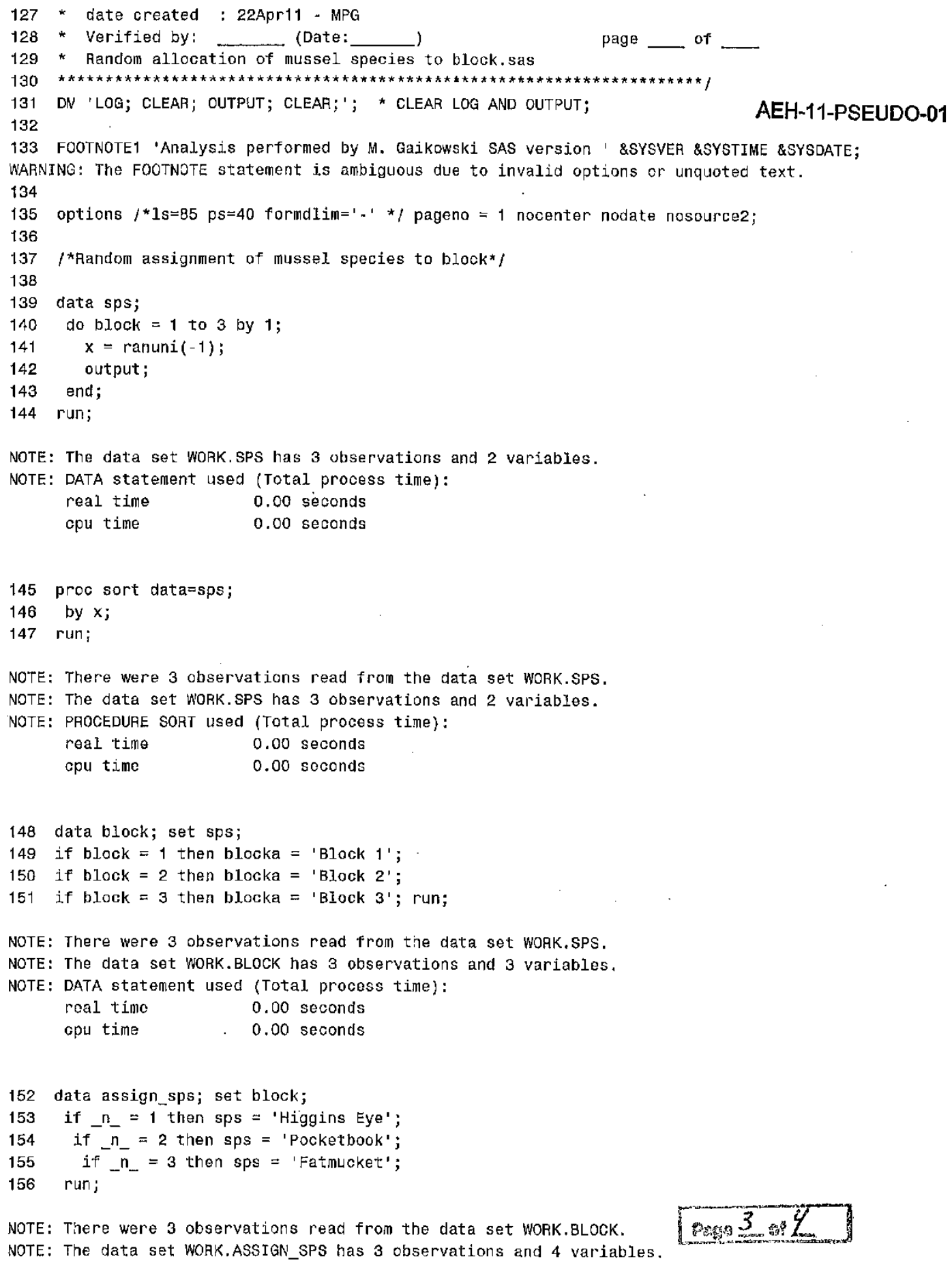

NOTE: There were 3 observations read from the data set wORK.SPS. NOTE: The data set WORK.BLOCK has 3 observations and 3 variables. NOTE: DATA statement used (Total process time):

NOTE: There were 3 observations read from the data set WORK. BLOCK. NOTE: The data set WORK.ASSIGN_SPS has 3 observations and 4 variables. 
NOTE: DATA statement used (Total process time):

real time $\quad 0.00$ seconds

AEH-11-PSEUDO-01

cpu time

0.00 seconds

157 proc sort data=assign_sps;

158 by block;

159 run;

NOTE: There were 3 observations read from the data set WORK.ASSIGN_SPS.

NOTE: The data set WORK.ASSIGN_SPS has 3 observations and 4 variables.

NOTE: PPOCEDURE SORT used (Total process time):
real time
0.00 seconds

cpu tine

0.00 seconds

160 proc print data= assign_sps;

161 title1 $h=2$ 'Effects of Psuedomonas fluorescens (Pf-CL145A) to giochidia from seven unionid

161 ! mussol species';

162 title2 $h=1.5$ 'AEH-11-PSUEDO-01';

163 title3 $h=1$ 'Random assignment of mussel species to block';

164 run;

NOTE: There were 3 observations read from the data set WORK.ASSIGN_SPS.

NOTE: PROCEDURE PRINT used (Total process time):

$\begin{array}{ll}\text { real time } & 0.00 \text { seconds } \\ \text { opu time } & 0.00 \text { seconds }\end{array}$ 


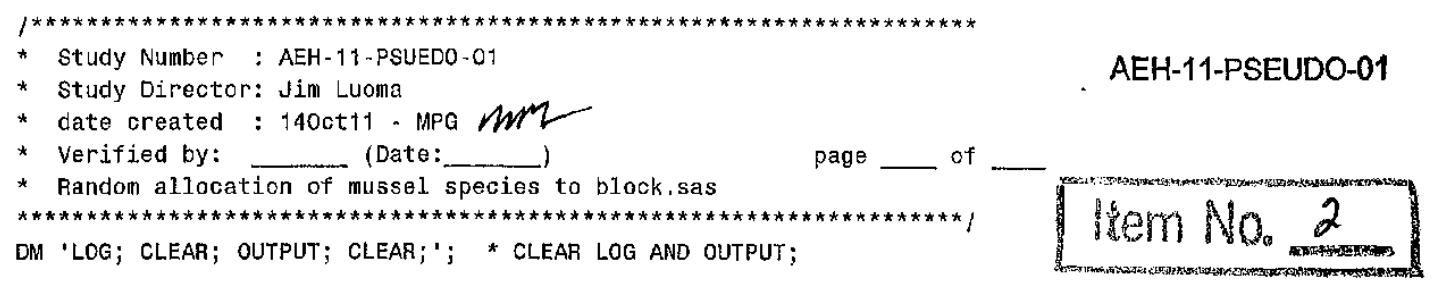

FOOTNOTE1 'Analysis perforned by M. GaikowSki SAS version ' \&SYSVER \&SYSTIME \&SYSDATE;

options $/{ }^{*} \mathrm{ls}=85 \mathrm{ps}=40$ formdlim=1.1*/ pageno $=1$ nocenter nodate nosource 2 ;

$f$ Random assignment of mussel species to block*

data sps;

do block $=1$ to 2 by 1 ;

$x=\operatorname{ranuni}(-1)$;

output;

end;

run;

proc sort data=sps;

by $x$;

run;

data block; set sps;

if block $=1$ then blocka $=$ 'Block 1 ';

if block $=2$ then blocka $=$ 'Block 3'; run; $/{ }^{*}$ Note: only two mussel species tested therefore Block

data assign_sps; set block;

if $n_{-}=1$ then sps = 'Washboard";

if $\bar{n}_{-}=2$ then $\operatorname{sps}=$ 'Fatmucket';

run;

proc sort data=assign_.sps;

by block;

run;

proc print data= assign_sps;

title1 $h=2$ 'Effects of Psuedomonas fluarescens (Pf-CL145A) to glochidia from seven unionid mussel

title2 $h=1.5$ 'AEH-11-PSUEDO-01';

title3 $h=1$ 'Random assignment of mussel species to block';

run;

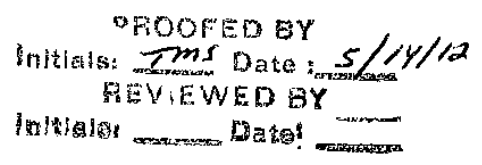

Pas 1 


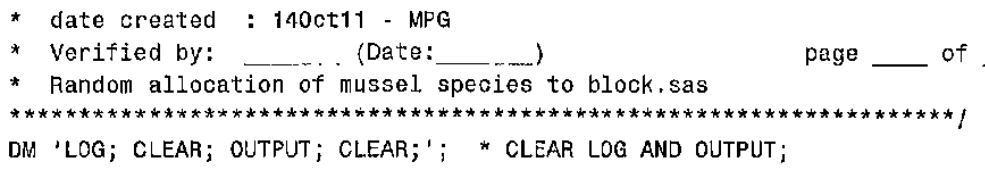

FOOTNOTE1 'Analysis performed by M. Gaikowski SAS version ' \&SYSVER \&SYSTIME \&SYSDATE; WARNING: The FOOTNOTE statement is ambiguous due to invalid options or unquoted text.

options $/ * 1 s=85$ ps=40 formdlim=' - * $/$ pageno $=1$ nocenter nodate nosourco2;

$l *$ Randiom assignment of mussel species to block* $t$

data sps;

do block $=1$ to 2 by 1 ;

NOTE: The data set WORK.SPS has 2 observations and 2 variables.

NOTE: DATA statement used (Total, process time):

real time $\quad 0.36$ seconds

cpu time $\quad 0.09$ seconds

22 proc sort data=sps;

by $x$;

run;

NOTE: There were 2 observations read from the data set WOAK.SPS.

NOTE: The data set WORK.SPS has 2 observations and 2 variables.

NOTE: PROCEDURE SORT used (Total process time):
real time
0.05 seconds

cpu time

0.03 seconds

25 data block; set sps;

26 if block $=1$ then blocka $=$ 'Block 1 ';

27 if block $=2$ then blocka $=$ 'Block $3^{\prime}$; run;

NOTE: There were 2 observations read from the data set WOAK.SPS.

NOTE: The data set WORK.BLOCK has 2 observations and 3 variables.

NOTE: DATA statement used (Total process time):
rea.1 time
0.01 seconds
cpu time
0.01 seconds

$27 !$

27 ! Block 2 was not used. MPG Oct 14, 2011*/

data assign_sps; set block;

if $n_{-}=1$ then $\operatorname{sps}^{-}=$'Washboard';

if $\vec{n}_{-}=2$ then sps = 'Fatmucket';

run;

NOTE: There were 2 observations road from the data set wORK.BLOCK.

NOTE: The data set WORK.ASSIGN SPS has 2 observations and 4 variables.

NOTE: DATA statement used (Total process time):

real time $0.0 t$ seconds

cpu time $\quad 0.03$ seconds

/*Note: only two mussel species tested therefore 
32 proc sort data=assign_sps;
33 by block;

34 run;

AEH-11-PSEUD0.01

NOTE: There were 2 observations read from the data set WORK.ASSIGN_SPS.

NOTE: The data set WORK.ASSIGN_SPS has 2 observations and 4 variables.

NOTE: PROCEDURE SORT used (Total process time):
real time
0.01 seconds
cpu time
0.01 seconds

35 proc print data= assign_sps:

36 title1 $h=2$ 'Effects of Psuedomonas fluorescens (Pf-CL145A) to glochidia from seven unionid

36 "I mussel species';

37 title2 $h=1.5$ 'AEH-11-PSUEDO-01';

38 title3 $\mathrm{h}=1$ 'Random assignment of mussel species to block';

39 run;

NOTE: There were 2 observations read from the data set WORK.ASSIGN_SPS.

NOTE: PROCEDURE PRINT used (Total process time):

$\begin{array}{ll}\text { real time } & 0.33 \text { seconds } \\ \text { cpu time } & 0.06 \text { seconds }\end{array}$

Fasp 3 a 


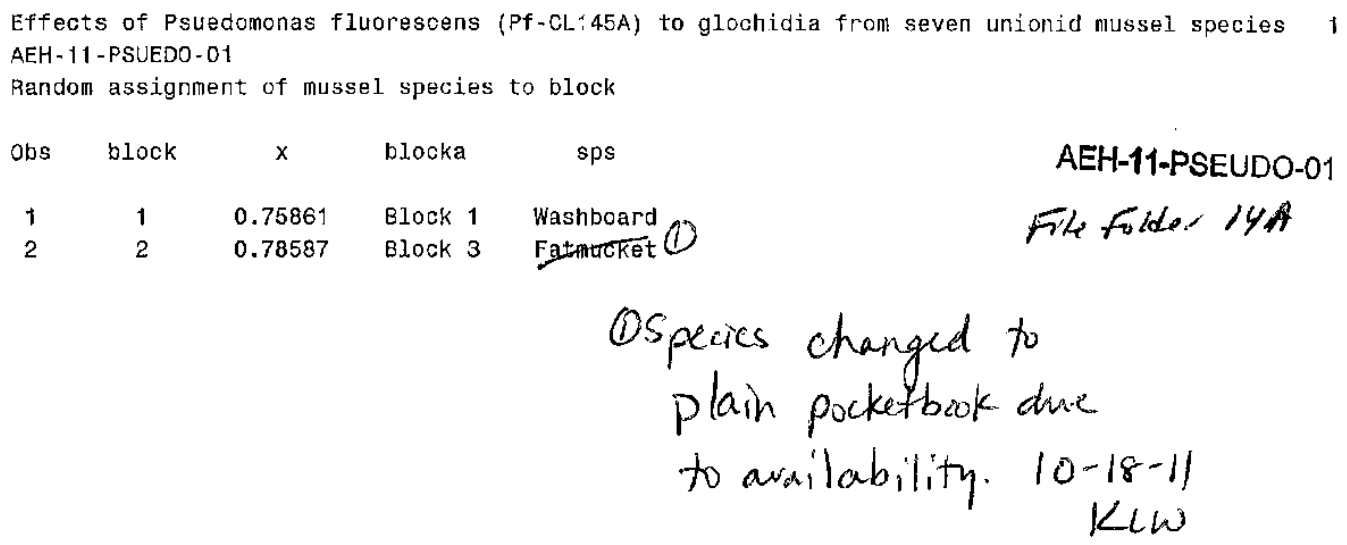

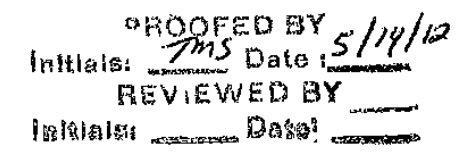

Pags 94

Analysis performed by $M$. Gaikowski SAS version $0.211: 03$ 1400T11 $\mathrm{m} / \mathrm{L}$ 


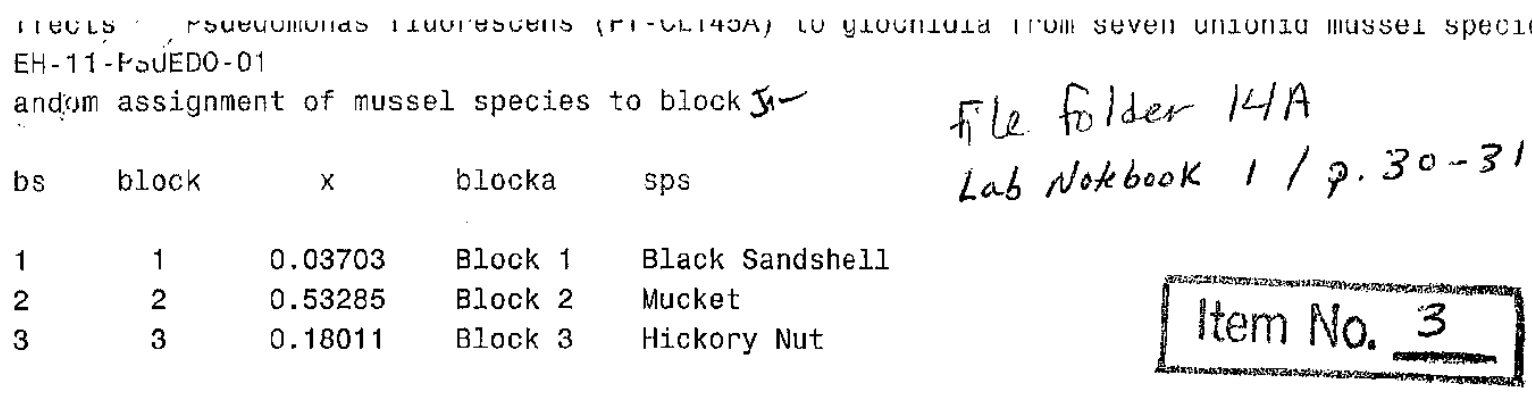

\section{AEH-11-PSEUDO-01}

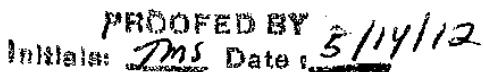
REVIEWED BY Inthlatio: Datet

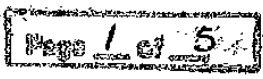

nalysis performed by $J$. Luoma SAS version $9.2 \quad 10: 50$ 09JAN12 
Study Number : AEH-11-PSUEDO-01

Study Director: Jim Luona

date created : 09Jan12 - JÁ

Verified by: _........ (Date:

page of

Random aljocation of mussel species to block.sas

$* * * * * * * * * * * * * * * * * * * * * * * * * * * * * * * * * * * * * * * * * * * * * * * * * * * * * * * * * * * * * * * * * * * * /$

M 'LOG; ClEAR; OUTPUT; CLEAR;'; * CleAR LOG AND OUTPUT;

AEH-11-PSEUDO-01

OOTNOTE1 'Analysis performed by $J$. Luoma SAS version ' \&SYSVER \&SYSTIME \&SYSDATE;

ptions $/{ }^{*} \mathrm{~s}=85 \mathrm{ps}=40$ formdlim= $-1 * /$ pageno $=1$ nocenter nodate nosource $;$

*Random assignment of mussel species to block*/

ata sps;

do block $=1$ to 3 by 1 ;

$x=\operatorname{ranuni}(-1)$;

output;

end;

un;

roc sort data=sps;

by $x$;

un;

ata block; set sps;

$f$ block $=1$ then blocka $={ }^{\prime}$ Block $1^{\prime}$;

$f$ block $=2$ then blocka $=$ Block 2 ;

$f$ block $=3$ then blocka $={ }^{\prime B l o c k ~} 3^{\prime}$; run;

ata assign_sps; set block;

if $n_{-}=1$ then sps = 'Black Sandshell';

if ${ }_{n}{ }_{-}=2$ then sps $=$ 'Hickory Nut';

if $n_{\ldots}=3$ then sps = 'Mucket';

run;

proc sort data-assign_sps;

by block;

un;

roc print data= assign_sps;

itle1 h=2 'Effects of Psuedomonas fluorescens (Pf-CL145A) to glochidia from seven unionid mussel sper itle2 h=1.5 'AEH-11-PSUEDO-01';

itle3 $h=1$ 'Random assignment of mussel species to block';

un;

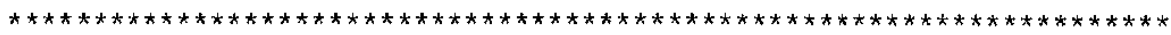

Study Number : AEH-11-PSUEDO-01

Study Director: Jim Luoma

date created : 09Jan12 - JAL

Verified by: (Date:

Random allocation of mussel species to block. sas

page of

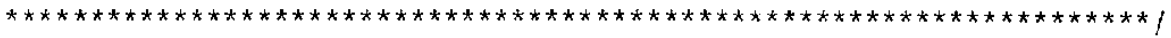

M 'LOG; ClEAR; OUTPUT; ClEAR;'; * CLEAR LOG AND OUTPUT;

OOTNOTE1 'Analysis performed by $J$. Luoma SAS version ' \&SYSVER \&SYSTIME \&SYSDATE;

ptions $/ * 1 s=85$ ps=40 formdlim=' - * * pageno = 1 nocenter nodate nosource2;

*Random assignment of mussel species to block*1 
ata sps;

do block $=1$ to 3 by 1 ;

$x=\operatorname{ranuni}(-1)$;

output;

end;

un;

AEH-11-PSEUDO-01

roc sort data $=s p s$;

by $x$;

un;

ata block; set sps;

$f$ block $=1$ then blocka $=$ 'Block 1 ';

$f$ block $=2$ then blocka $=$ 'Block 2';

$f$ block $=3$ then blocka $={ }^{\prime}$ Block $3^{\prime}$; run;

ata assign_sps; set block;

if ${ }_{n}=1$ then sps $=$ ' Bl-ack -Sandshel:1';

if $\bar{n}_{-}=2$ then sps $=$ 'Hickory Nut';

if $\bar{n}_{-}=3$ then sps = 'Mucket';

run;

proc sort data=assign_sps;

by block;

un;

roc print data= assign_sps;

itle1 $h=2$ 'Effects of Psuedomonas fluorescens (Pf-CL145A) to glochidia from seven unionid mussel sper itle2 $h=1.5$ 'AEH-11-PSUEDO-01';

itle3 $h=1$ 'Random assignment of mussel species to block';

un;

Lage 35 


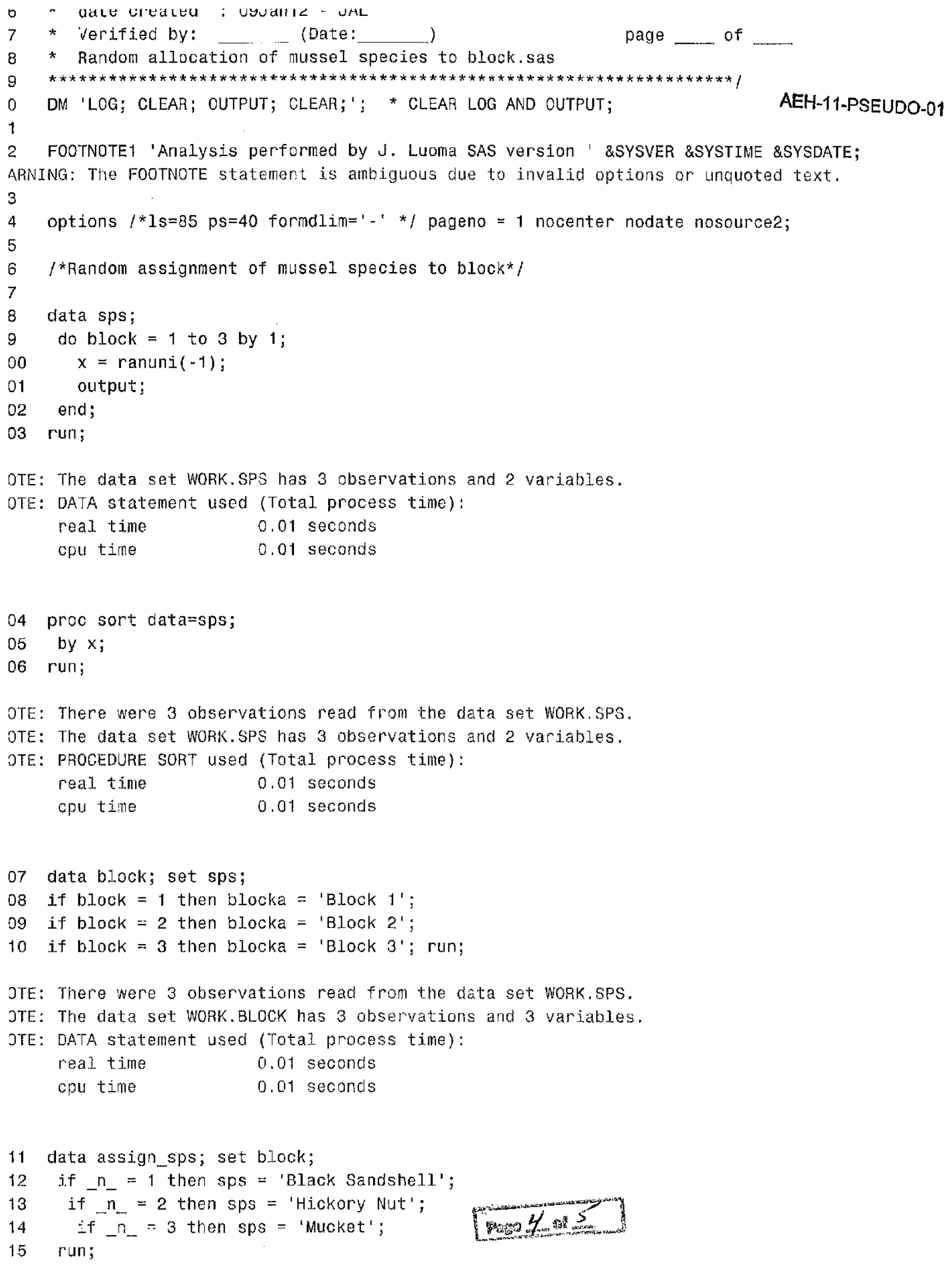


OTE: The data set WORK.ASSIGN SPS has 3 observations and 4 variables.

OTE: DATA statement used (Total process time):

real time $\quad 0.01$ seconds

cpu time $\quad 0.01$ seconds

16 proc sort data=assign_sps;

17 by block;

18 run;

OTE: There were 3 observations read from the data set WORK.ASSIGN_SPS.

OTE: The data set WORK.ASSIGN_SPS has 3 observations and 4 variables.

OTE: PROCEDURE SORT used (Total process time):

real time

0.01 seconds

cpu time ............... 0.01 seconds

19 proc print data= assign_sps;

20 titlel $h=2$ 'Effects of Psuedomonas fluorescens (Pf-CL145A) to glochidia from seven unionid

20! mussel species';

21 title2 $h=1.5$ 'AEH-11-PSUEDO-01';

22 title3 $h=1$ 'Random assignment of mussel species to block';

23 run;

OTE: There were 3 observations read from the data set WORK.ASSIGN SPS.

OTE: PROCEDURE PRINT used (Total process time):

real time 0.00 seconds

cpu time $\quad 0.00$ seconds

\section{AEH-11-PSEUDO-01 \\ File folder $14 \mathrm{~A}$}




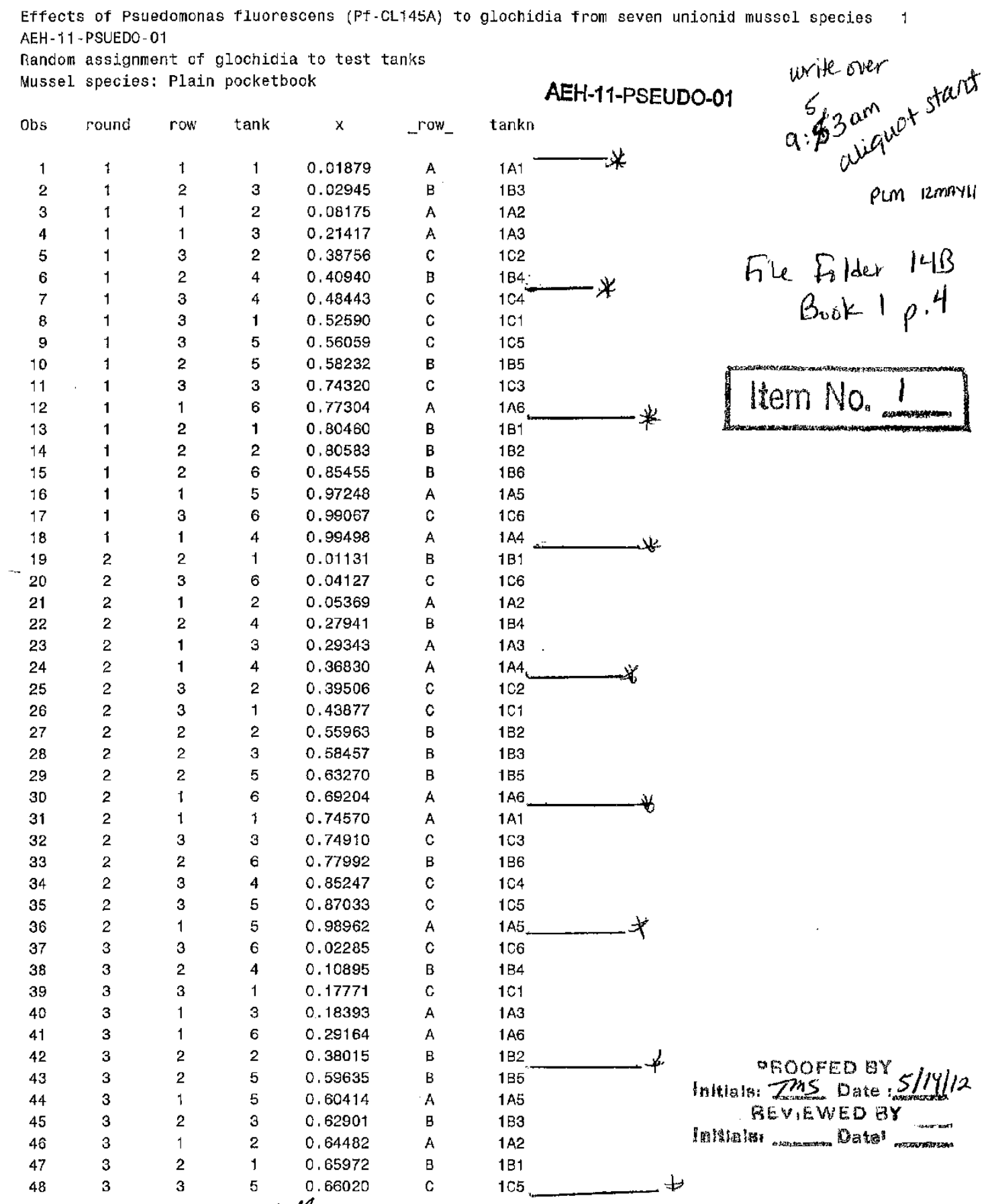

Analysis performed by M. Gaikowski SAS version $9.2 \quad 07: 41$ 12MAY11

$$
\begin{aligned}
& \text { * Scroplia fur } \\
& \text { distributow alrgat } \\
& 5-12-11 \\
& 5
\end{aligned}
$$


Effects of Psuedomonas fluorescens ( $P f-C L 145 A)$ to glochidia from seven unionid mussel species 2 AEH - 11 - PSUED0-01

Aandom assignment of glochidia to test tanks

Mussel species: Plain pocketbook

obs round row tank $x$ row tankn AEH-11-PSEUDO-01

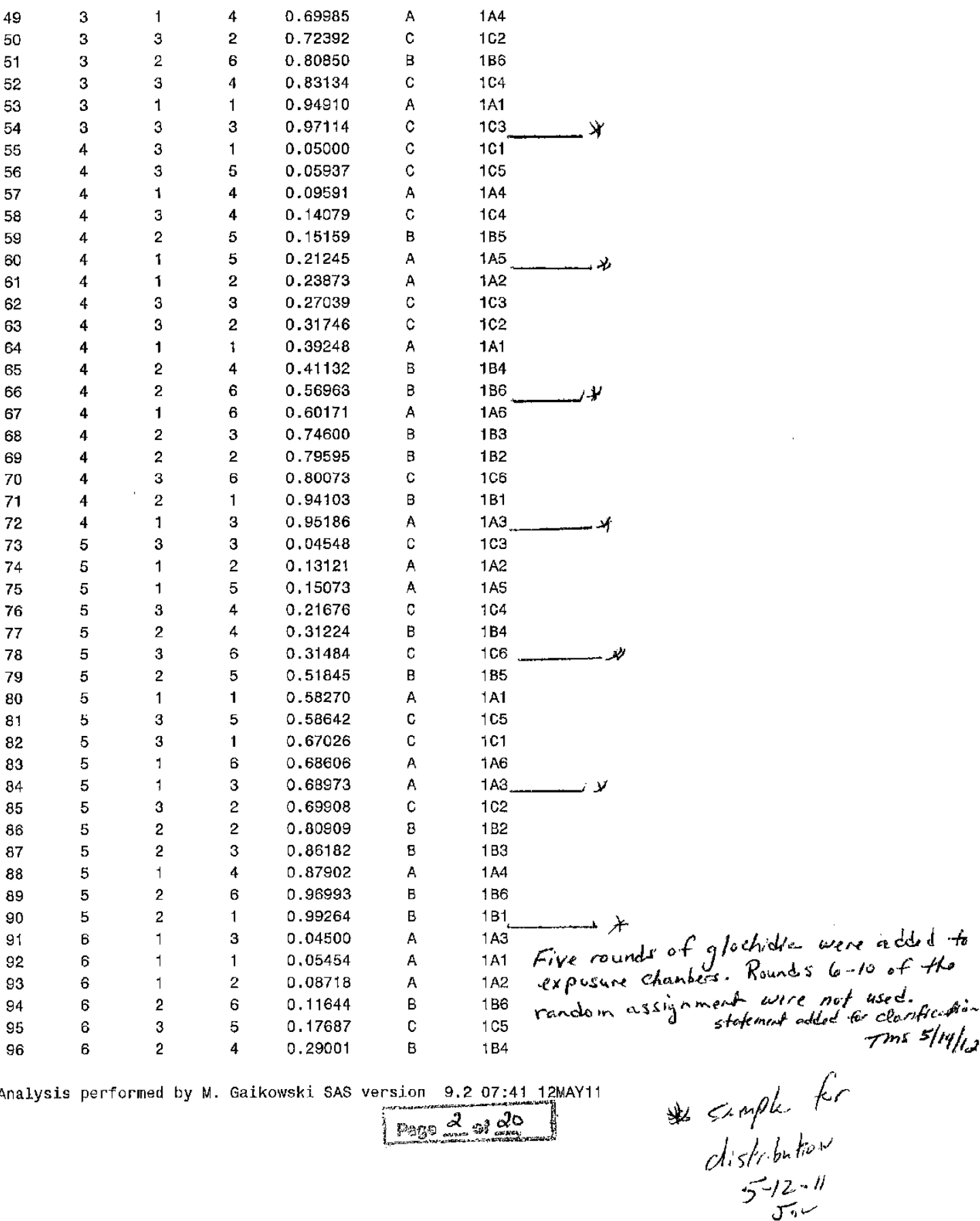


Effects of Psuedomonas fluorescens (Pf-CL145A) to glochidia from seven unionid mussel species 3 AEH-11-PSUEDO-01

Random assignment of glochidia to test tanks

Mussel species: Plain pocketbook

\begin{tabular}{|c|c|c|c|c|c|c|}
\hline Obs & round & row & tank & $x$ & _row_ & tankn \\
\hline 97 & 6 & 3 & 2 & 0.29738 & C & $1 \mathrm{C} 2$ \\
\hline 98 & 6 & 1 & 5 & 0.40275 & A & $1 \mathrm{A5}$ \\
\hline 99 & $\hat{o}$ & 1 & 6 & 0.43727 & $A$ & $1 A 6$ \\
\hline 100 & 6 & 3 & 3 & 0.48861 & $\mathrm{C}$ & $1 \mathrm{C3}$ \\
\hline 101 & 6 & 2 & 2 & 0.50353 & $\mathrm{~B}$ & 182 \\
\hline 102 & 6 & 3 & 6 & 0.56958 & $c$ & 106 \\
\hline 103 & 6 & 2 & 1 & 0.62141 & $\mathrm{~B}$ & $1 \mathrm{~B} 1$ \\
\hline 104 & 6 & 2 & 3 & 0.76177 & $B$ & 183 \\
\hline 105 & 6 & 1 & 4 & 0.89170 & A & $1 \mathrm{~A} 4$ \\
\hline 106 & 6 & 3 & 1 & 0.89421 & c & $1 \mathrm{C} 1$ \\
\hline 107 & 6 & 2 & 5 & 0.94943 & B & iB5 \\
\hline 108 & 6 & 3 & 4 & 0.95587 & $c$ & $1 \mathrm{C} 4$ \\
\hline 109 & 7 & 1 & 6 & 0.01753 & A & $1 A 6$ \\
\hline 110 & 7 & 1 & 3 & 0.05265 & A & $1 \mathrm{A3}$ \\
\hline 111 & 7 & 3 & 1 & 0.06021 & $c$ & $1 \mathrm{C} 1$ \\
\hline 112 & 7 & 2 & 3 & 0.13135 & B & $1 B 3$ \\
\hline 113 & 7 & 1 & 4 & 0.15031 & A & $1 \mathrm{A4}$ \\
\hline 114 & 7 & 3 & 2 & 0.16166 & $\mathrm{C}$ & $1 \mathrm{C} 2$ \\
\hline 115 & 7 & 1 & 2 & 0.17852 & $A$ & $1 \mathrm{~A} 2$ \\
\hline 116 & 7 & 1 & 1 & 0.18945 & A & $1 \mathrm{~A} 1$ \\
\hline 117 & 7 & 1 & 5 & 0.21711 & $A$ & $1 \mathrm{~A} 5$ \\
\hline 118 & 7 & 2 & 2 & 0.30167 & B & $1 \mathrm{~B} 2$ \\
\hline 119 & 7 & 3 & 6 & 0.34314 & c & $1 \mathrm{C6}$ \\
\hline 120 & 7 & 2 & 6 & 0.48469 & B & $1 \mathrm{~B} 6$ \\
\hline 121 & 7 & 3 & 4 & 0.75785 & c & $1 \mathrm{C4}$ \\
\hline 122 & 7 & 3 & 3 & 0.76057 & $c$ & $1 \mathrm{C} 3$ \\
\hline$\uparrow 23$ & 7 & 3 & 5 & 0.79406 & C & 105 \\
\hline 124 & 7 & 2 & 4 & 0.81814 & B & $1 \mathrm{~B} 4$ \\
\hline 125 & 7 & 2 & 1 & 0.84270 & B & 181 \\
\hline 126 & 7 & 2 & 5 & 0.97628 & B & 185 \\
\hline 127 & 8 & 3 & 4 & 0.01800 & C & $1 \mathrm{C4}$ \\
\hline 128 & 8 & 1 & 3 & 0.05110 & A & $1 \mathrm{A3}$ \\
\hline 129 & 8 & 1 & 4 & 0.06923 & A & $1 \mathrm{~A} 4$ \\
\hline 130 & 8 & 1 & 2 & 0.08503 & A & $1 \mathrm{~A} 2$ \\
\hline 131 & 8 & 3 & 5 & 0.14368 & $\mathrm{c}$ & 105 \\
\hline 132 & 8 & 1 & 6 & 0.14720 & A & $1 A 6$ \\
\hline 133 & 8 & 1 & 5 & 0.18610 & A & TA5 \\
\hline 134 & 8 & 3 & 6 & 0.31005 & c & $1 \mathrm{C} 6$ \\
\hline 135 & 8 & 3 & 2 & 0.47913 & C & $1 C 2$ \\
\hline 136 & 8 & 2 & 1 & 0.55774 & $B$ & $1 \mathrm{~B} 1$ \\
\hline 137 & 8 & 2 & 6 & 0.61696 & $\mathrm{~B}$ & $1 \mathrm{~B} 6$ \\
\hline 138 & 8 & 2 & 5 & 0.63578 & $B$ & $1 \mathrm{~B} 5$ \\
\hline 139 & 8 & 3 & 1 & 0.75157 & $\mathrm{C}$ & 101 \\
\hline 140 & 8 & 3 & 3 & 0.76715 & $c$ & 103 \\
\hline 141 & 8 & 2 & 2 & 0.77851 & $B$ & $1 \mathrm{~B} 2$ \\
\hline 142 & 8 & 2 & 4 & 0.78177 & 8 & $1 \mathrm{~B} 4$ \\
\hline 143 & 8 & 2 & 3 & 0.80188 & $B$ & $1 \mathrm{~B} 3$ \\
\hline 144 & 8 & 1 & 1 & 0.83057 & A & $1 \mathrm{~A} 1$ \\
\hline
\end{tabular}

Analysis performed by M. Gaikowski SAS version $9.207: 41$ 12NAY11

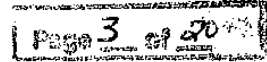


Effects of Psuedomonas fIuorescens (Pf-CL145A) to glochidia from seven unionid mussel species 4 AEH - 11-PSUEDO-01

Random assignment of glochidia to test tanks

Mussel species: Plain pocketbook

\begin{tabular}{|c|c|c|c|c|c|c|}
\hline Obs & round & row & tank & $x$ & row_ & $\operatorname{tankn}$ \\
\hline$\{45$ & 9 & 3 & 1 & 0.00567 & $c$ & $1 \mathrm{C} 1$ \\
\hline 146 & 9 & 1 & 4 & 0.04799 & A & $1 \mathrm{~A} 4$ \\
\hline 147 & 9 & 3 & 4 & 0.09070 & $c$ & $1 C 4$ \\
\hline 148 & 9 & 1 & 2 & 0.13436 & A & $1 \mathrm{~A} 2$ \\
\hline 149 & 9 & 1 & 5 & 0.17031 & A & tA5 \\
\hline 150 & 9 & 2 & 2 & 0.18916 & $B$ & $1 B 2$ \\
\hline 151 & 9 & 1 & 3 & 0.28699 & A & $1 \mathrm{AB}$ \\
\hline 152 & 9 & 3 & 5 & 0.41896 & $c$ & 105 \\
\hline 153 & 9 & 2 & 6 & 0.45724 & $B$ & 186 \\
\hline 154 & 9 & 1 & 1 & 0.47143 & $A$ & $1 \mathrm{~A} 1$ \\
\hline 155 & 9 & 3 & 3 & 0.50109 & $c$ & $1 \mathrm{C3}$ \\
\hline 156 & 9 & 1 & 6 & 0.51486 & $A$ & $1 \mathrm{A6}$ \\
\hline 157 & 9 & 2 & 3 & 0.53944 & 8 & $1 \mathrm{~B} 3$ \\
\hline 158 & 9 & 2 & 5 & 0.62377 & $B$ & $1 \mathrm{~B} 5$ \\
\hline 159 & 9 & 3 & 2 & 0.63384 & $c$ & $1 \mathrm{C} 2$ \\
\hline 160 & 9 & 3 & 6 & 0.91619 & $\mathrm{C}$ & $1 \mathrm{C6}$ \\
\hline 161 & 9 & 2 & 4 & 0.97574 & $B$ & $1 \mathrm{~B} 4$ \\
\hline 162 & 9 & 2 & 1 & 0.98193 & $B$ & $1 \mathrm{~B} 1$ \\
\hline 163 & 10 & 1 & 5 & 0.13894 & A & $1 \mathrm{~A} 5$ \\
\hline 164 & 10 & 3 & 1 & 0.16112 & $\mathrm{c}$ & $1 \mathrm{Ct}$ \\
\hline 185 & 10 & 3 & 5 & 0.18016 & $c$ & 105 \\
\hline 166 & 10 & 2 & 1 & 0.21049 & $B$ & tB1 \\
\hline 167 & 10 & 1 & 4 & 0.28226 & A & IA4 \\
\hline 168 & 10 & 3 & 4 & 0.28484 & $c$ & 104 \\
\hline 169 & 10 & 2 & 5 & 0.39719 & $B$ & 1B5 \\
\hline 170 & 10 & 2 & 4 & 0.45883 & $B$ & 1B4 \\
\hline 171 & 10 & 1 & 2 & 0.47575 & A & $1 \mathrm{~A} 2$ \\
\hline 172 & 10 & 2 & 6 & 0.55730 & B & $1 \mathrm{~B} 6$ \\
\hline 173 & 10 & 1 & 1 & 0.71916 & A & $1 \mathrm{~A} 1$ \\
\hline 174 & 10 & 2 & 3 & 0.75737 & $B$ & $1 \mathrm{~B} 3$ \\
\hline 175 & 10 & 3 & 3 & 0.80742 & $c$ & 103 \\
\hline 176 & 10 & 3 & 6 & 0.84725 & $\mathrm{C}$ & $1 \mathrm{c} 6$ \\
\hline 177 & 10 & 1 & 6 & 0.86038 & $A$ & $1 \mathrm{~A} G$ \\
\hline 178 & 10 & 2 & 2 & 0.86791 & B & $1 \mathrm{~B} 2$ \\
\hline 179 & 10 & 3 & 2 & 0.95113 & $c$ & $1 \mathrm{ce}$ \\
\hline 180 & 10 & 1 & 3 & 0.96901 & A & $1 \mathrm{AB}$ \\
\hline
\end{tabular}

AEH-11-PSEUDO-01 
Effects of Psuedomonas fluorescens (Pf-CL145A) to glochidia from seven unionid mussel species 5 AEH-11-PSUEDO- 01

Randon assignment of glochidia to test tank

Mussel species: Higgins eye

Obs round row tank

$x \quad$ row_ tankn

AEH-11-PSEUDO-01 Start: 11:32 Am

$\begin{array}{lllllll}1 & 1 & 2 & 5 & 0.00353 & \mathrm{~B} & 2 \mathrm{~B} 5\end{array}$

$\begin{array}{lllllll}2 & 1 & 3 & 1 & 0.01828 & \mathrm{C} & 2 \mathrm{C} 1 \\ 3 & 1 & 3 & 3 & 0.06547 & \mathrm{C} & 2 \mathrm{C} 3\end{array}$

$0.09979-A$

$\begin{array}{lllllll}5 & 1 & 2 & 2 & 0.11238 & B & 282\end{array}$

$\begin{array}{lllllll}6 & 1 & 1 & 6 & 0.27072 & A & 2 A 6\end{array}$

$\begin{array}{lllllll}7 & 1 & 2 & 1 & 0.28549 & B & 2 B 1\end{array}$

$\begin{array}{lllllll}8 & 1 & 3 & 2 & 0.30041 & G & 2 \mathrm{C} 2\end{array}$

$\begin{array}{lllllll}9 & 1 & 3 & 6 & 0.34286 & \mathrm{C} & 2 \mathrm{C} 6\end{array}$

$\begin{array}{lllllll}10 & 1 & 2 & 3 & 0.44640 & B & 2 B 3\end{array}$

$\begin{array}{lllllll}11 & 1 & 2 & 6 & 0.54274 & \text { B } & 2 B 6\end{array}$

$\begin{array}{lllll}12 & 1 & 2 & 4 & 0.54557\end{array}$

$\begin{array}{lllll}13 & 1 & 1 & 2 & 0.59140\end{array}$

$\begin{array}{lllll}14 & 1 & 1 & 4 & 0.63600\end{array}$

0.65528

0.65562

0.68469

0.88493

0.09555

0.10853

0.17714

0.25215

0.32275

0.33037

0.35151

0,43424

0.47595

0.47888

0.54611

0.66566

0.73720

0.79288

0.82948

0.85988

0.91676

0.97926

0.00275

0.02144

0.08661

0.11286

0.19395

0.19567

0.36903

0.37635

0.39921

0.41669

0.44630

0.44748

2B4

$2 A 2$

$2 \mathrm{~A} 4$

$2 A 3$

2A5

$2 \mathrm{C5}$

$\frac{2 \mathrm{C} 4}{2 \mathrm{C} 6}$

2B2

2B1

$2 \mathrm{C} 1$

2B6

$2 \mathrm{C} 2$

$2 \mathrm{C3}$

2A1

$2 \mathrm{~B} 3$

$2 A 6$

$2 A 2$

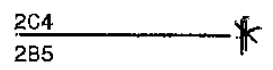

2A4

$2 A 5$

$2 \mathrm{C5}$

2B4

$2 A 3$

2C3

2A5

$2 A 6$

2A4

$2 \mathrm{~A}$

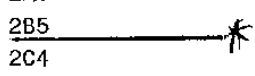

$2 \mathrm{~A} 2$

2B3

2C1

206

2B2

Analysis performed by M. Gaikowski SAS version 9.2 07:41 12MAY11

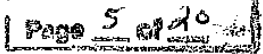

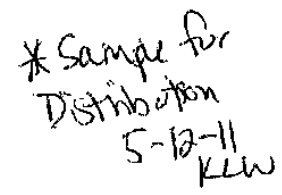


Effects of Psuedomonas fluorescens (Pf-CL145A) to glochidia from seven unionid mussel species 6 AEH-11-PSUEDO-01

Random assignment of glochidia to test tank

Mussel species: Higgins eye

obs round row tank $x$ _row_ tankn AEH-11-PSEUDO-01

\begin{tabular}{|c|c|c|c|c|c|c|}
\hline 49 & 3 & 1 & 3 & 0.50721 & A & $2 \mathrm{AB}$ \\
\hline 50 & 3 & 3 & 5 & 0.60580 & $c$ & $2 \mathrm{C5}$ \\
\hline 51 & 3 & 2 & 6 & 0.69776 & 3 & 2B6 \\
\hline 52 & 3 & 2 & 4 & 0.72794 & B & 2B4 \\
\hline 53 & 3 & 2 & 1 & 0.88609 & $B$ & $2 \mathrm{B1}$ \\
\hline 54 & 3 & 3 & 2 & 0.93325 & 0 & $2 C 2$ \\
\hline 55 & 4 & 3 & 5 & 0.11301 & C & $2 \mathrm{C5}$ \\
\hline 56 & 4 & 2 & 1 & 0.21527 & $B$ & $2 \mathrm{~B} 1$ \\
\hline 57 & 4 & 2 & 5 & 0.27124 & $\mathrm{~B}$ & $2 \mathrm{~B} 5$ \\
\hline 58 & 4 & 1 & 1 & 0.38224 & A & $2 \mathrm{~A} 1$ \\
\hline 59 & 4 & 3 & 1 & 0.41408 & $c$ & $2 \mathrm{C} 1$ \\
\hline 60 & 4 & 1 & 5 & 0.44653 & A & $2 A 5 ـ$ \\
\hline 61 & 4 & 3 & 2 & 0.46886 & $\mathrm{C}$ & $2 \mathrm{C} 2$ \\
\hline 62 & 4 & 2 & 3 & 0.51095 & $\mathrm{~B}$ & $2 B 3$ \\
\hline 63 & 4 & 3 & 3 & 0.54719 & C & $2 \mathrm{C3}$ \\
\hline 64 & 4 & 2 & 2 & 0.60320 & $B$ & $2 \mathrm{~B} 2$ \\
\hline 65 & 4 & 1 & 4 & 0.69545 & A & $2 \mathrm{~A} 4$ \\
\hline 66 & 4 & 2 & 4 & 0.69847 & $\mathrm{~B}$ & $2 \mathrm{~B} 4 \ldots$ \\
\hline 67 & 4 & 2 & 6 & 0.73361 & B & $2 \mathrm{~B} 6$ \\
\hline 68 & 4 & 3 & 4 & 0.73394 & $\mathrm{C}$ & $2 \mathrm{C} 4$ \\
\hline 69 & 4 & 3 & 6 & 0.77816 & $\mathrm{C}$ & $2 \mathrm{C6}$ \\
\hline 70 & 4 & 1 & 3 & 0.80287 & A & $2 A 3$ \\
\hline 71 & 4 & 1 & 2 & 0.82110 & A & $2 \mathrm{~A} 2$ \\
\hline 72 & 4 & 1 & 6 & 0.86940 & A & $2 A 6 \ldots K$ \\
\hline 73 & 5 & 2 & 5 & 0.04537 & B & $2 \mathrm{~B} 5$ \\
\hline 74 & 5 & 1 & 1 & 0.09335 & A & $2 A 1$ \\
\hline 75 & 5 & 1 & 4 & 0.12238 & A & $2 A 4$ \\
\hline 76 & 5 & 3 & 6 & 0.22538 & $\mathrm{C}$ & $2 \mathrm{C6}$ \\
\hline 77 & 5 & 3 & 3 & 0.27257 & $\mathrm{C}$ & $2 \mathrm{C} 3$ \\
\hline 78 & 5 & 1 & 5 & 0.37465 & A & $2 A 5$ _ $*$ \\
\hline 79 & 5 & 2 & 1 & 0.40649 & 8 & $2 \mathrm{~B} 1$ \\
\hline 80 & 5 & 3 & 4 & 0.46054 & C & $2 \mathrm{C} 4$ \\
\hline 81 & 5 & 1 & 2 & 0.47441 & A & $2 A 2$ \\
\hline 82 & 5 & 1 & 3 & 0.55586 & A & $2 A 3$ \\
\hline 83 & 5 & 2 & 4 & 0.57846 & $B$ & 284 \\
\hline 84 & 5 & 3 & 5 & 0.58119 & $\mathrm{c}$ & 205 - $x$ \\
\hline 85 & 5 & 2 & 2 & 0.61383 & B & $2 \mathrm{~B} 2$ \\
\hline 86 & 5 & 2 & 3 & 0.62711 & B & 2B3 \\
\hline 87 & 5 & 1 & 6 & 0.76919 & A & $2 A 6$ \\
\hline 88 & 5 & 2 & 6 & 0.77658 & $\mathrm{~B}$ & 286 \\
\hline 89 & 5 & 3 & 2 & 0.78874 & $\mathrm{C}$ & $2 \mathrm{C} 2$ \\
\hline 90 & 5 & 3 & 1 & 0.98232 & $\mathrm{C}$ & $2 \mathrm{C} 1$ מ \\
\hline 91 & 6 & 3 & 3 & 0.01723 & $\mathrm{c}$ & $2 \mathrm{C} 3$ \\
\hline 92 & 6 & 3 & 1 & 0.06903 & $\mathrm{C}$ & PLM 12 MALII \\
\hline 93 & 6 & 2 & 6 & 0.19358 & B & 2B6 Five counds of glochidic were added to \\
\hline 94 & 6 & 2 & 5 & 0.26894 & B & 2B5 Five rounds at gos exposure chambers. Rounds 6 -to of the \\
\hline $\begin{array}{l}95 \\
96\end{array}$ & 6 & $\begin{array}{l}1 \\
3\end{array}$ & $\begin{array}{l}3 \\
6\end{array}$ & $\begin{array}{l}0.27596 \\
0.29174\end{array}$ & A & 2A3 ratidam assignment were not used if \\
\hline 96 & 0 & 3 & 0 & 0.29174 & 6 & 0 solutementadded tor crar ol \\
\hline
\end{tabular}

Analysis performed by M. Gaikowski SAS version $9.207: 41$ 12MAY11

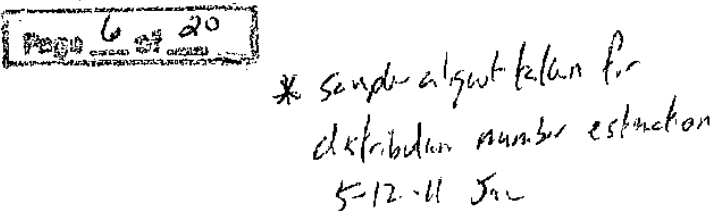


Effects of Psuedomonas fluorescens (Pf-CL145A) to glochidia from seven unionid mussel species 7 AEH- 11 -PSUEDO- 01

Random assignment of glochidia to tost tank

Mussel species: Higgins eye

Obs round row tank $x \quad$ row_ tankn

\begin{tabular}{|c|c|c|c|c|c|c|}
\hline 97 & 6 & $t$ & 4 & 0.33462 & A & $2 \mathrm{~A} 4$ \\
\hline 98 & 6 & 2 & 1 & 0.34254 & B & $2 \mathrm{~B} 1$ \\
\hline 99 & 6 & 2 & 3 & 0.42686 & B & $2 \mathrm{~B} 3$ \\
\hline 100 & 6 & $t$ & 1 & 0.43456 & A & $2 A 1$ \\
\hline 101 & 6 & 2 & 2 & 0.59493 & B & $2 B 2$ \\
\hline 102 & 6 & 3 & 2 & 0.59626 & $C$ & $2 \mathrm{C} 2$ \\
\hline 103 & 6 & $t$ & 6 & 0.59777 & A & $2 A B$ \\
\hline 104 & 6 & 1 & 2 & 0.63322 & A & $2 \mathrm{~A} 2$ \\
\hline 105 & 6 & 3 & 4 & 0.76409 & $c$ & $2 \mathrm{C} 4$ \\
\hline 106 & 6 & 2 & 4 & 0.77924 & B & 2B4 \\
\hline 107 & 6 & 1 & 5 & 0.82305 & A & $2 \mathrm{~A} 5$ \\
\hline 108 & 6 & 3 & 5 & 0.91307 & $\mathrm{C}$ & $2 \mathrm{C5}$ \\
\hline 109 & 7 & 3 & 3 & 0.01123 & C & $2 \mathrm{C} 3$ \\
\hline 110 & 7 & 1 & 2 & 0.13433 & A & $2 A 2$ \\
\hline 111 & 7 & 2 & 4 & 0.15831 & B & $2 B 4$ \\
\hline 112 & 7 & 2 & 6 & 0.28654 & B & $2 \mathrm{~B} 6$ \\
\hline 113 & 7 & 3 & 2 & 0.29506 & C & $2 \mathrm{c} 2$ \\
\hline 114 & 7 & 2 & 3 & 0.33579 & B & $2 \mathrm{~B} 3$ \\
\hline 115 & 7 & 1 & 6 & 0.43376 & A & $2 A 6$ \\
\hline 116 & 7 & 3 & 6 & 0.48583 & C & $2 \mathrm{C} 6$ \\
\hline 117 & 7 & 3 & 1 & 0.52022 & $\mathrm{C}$ & $2 \mathrm{C} 1$ \\
\hline 118 & 7 & 1 & 5 & 0.57020 & A & $2 A 5$ \\
\hline 119 & 7 & 1 & 1 & 0,57770 & A & $2 A 1$ \\
\hline 120 & 7 & 2 & 2 & 0.62832 & $B$ & 282 \\
\hline 121 & 7 & 2 & 5 & 0.73838 & 8 & $2 \mathrm{~B} 5$ \\
\hline 122 & 7 & 2 & 1 & 0.79867 & B & $2 \mathrm{~B} 1$ \\
\hline 123 & 7 & 3 & 4 & 0.81943 & C & 204 \\
\hline 124 & 7 & 1 & 4 & 0.84167 & A & $2 \mathrm{~A} 4$ \\
\hline 125 & 7 & 3 & 5 & $0.92596^{\circ}$ & $\mathrm{C}$ & $2 \mathrm{C5}$ \\
\hline 126 & 7 & 1 & 3 & 0.99065 & A & $2 A 3$ \\
\hline 127 & 8 & 3 & 3 & 0.06761 & C & $2 \mathrm{C} 3$ \\
\hline 128 & 8 & 2 & 3 & 0.16831 & B & $2 \mathrm{~B} 3$ \\
\hline 129 & 8 & 2 & 2 & 0.23416 & B & $2 \mathrm{~B} 2$ \\
\hline 130 & 8 & 2 & 5 & 0.29976 & B & $2 B 5$ \\
\hline 131 & 8 & 1 & 1 & 0.30323 & A & $2 \mathrm{~A} 1$ \\
\hline 132 & 8 & 3 & 4 & 0.31483 & C & $2 \mathrm{C} 4$ \\
\hline 133 & 8 & 1 & 4 & 0.34192 & A & $2 \mathrm{~A} 4$ \\
\hline 134 & 8 & 3 & 1 & 0.35878 & C & 201 \\
\hline 135 & 8 & 3 & 5 & 0.38611 & C & $2 \mathrm{C5}$ \\
\hline 136 & 8 & 1 & 2 & 0.39165 & A & $2 \mathrm{~A} 2$ \\
\hline 137 & 8 & 2 & 1 & 0.42688 & B & $2 \mathrm{~B} 1$ \\
\hline 138 & 8 & 3 & 2 & 0.43867 & $c$ & $2 \mathrm{C} 2$ \\
\hline 139 & 8 & 2 & 4 & 0.47883 & B & $2 \mathrm{~B} 4$ \\
\hline 140 & 8 & 1 & 3 & 0.58091 & A & $2 \mathrm{~A} 3$ \\
\hline 141 & 8 & 1 & 6 & 0.67692 & A & $2 A 6$ \\
\hline 142 & 8 & 2 & 6 & 0.74573 & $B$ & $2 \mathrm{~B} 6$ \\
\hline 143 & 8 & 3 & 6 & 0.89282 & $\mathrm{C}$ & $2 \mathrm{c} 6$ \\
\hline 144 & 8 & 1 & 5 & 0.91038 & A & $2 \mathrm{~A} 5$ \\
\hline
\end{tabular}

Analysis performed by M. Gaikowski SAS version 9.2 07:41 12MAY11

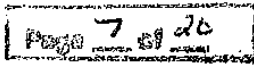


Effects of Psuedomonas fluorescens (Pf-CL145A) to glochidia from seven unionid mLssez species 8 AEH-11-FSUEDO-01

Randon assignment of glochidia to test tank

Mussel species: Higgins eye

$\begin{array}{lrrrccc}\text { Obs } & \text { round } & \text { row } & \text { tank } & \text { x } & \text { row_ } & \text { tankn } \\ 145 & 9 & 1 & 1 & 0.06975 & \text { A } & 2 \mathrm{~A} 1 \\ 146 & 9 & 2 & 6 & 0.08304 & \mathrm{~B} & 2 \mathrm{~B} 6 \\ 147 & 9 & 3 & 6 & 0.14826 & \mathrm{C} & 2 \mathrm{C} 6 \\ 148 & 9 & 2 & 4 & 0.17937 & \mathrm{~B} & 2 \mathrm{~B} 4 \\ 149 & 9 & 2 & 5 & 0.19933 & \mathrm{~B} & 2 \mathrm{~B} 5 \\ 150 & 9 & 1 & 3 & 0.36557 & \mathrm{~A} & 2 \mathrm{~A} 3 \\ 151 & 9 & 3 & 2 & 0.38197 & \mathrm{C} & 2 \mathrm{C} 2 \\ 152 & 9 & 1 & 4 & 0.44056 & \mathrm{~A} & 2 \mathrm{A4} \\ 153 & 9 & 2 & 3 & 0.53384 & \mathrm{~B} & 2 \mathrm{~B} 3 \\ 154 & 9 & 2 & 1 & 0.55441 & \mathrm{~B} & 2 \mathrm{~B} 1 \\ 155 & 9 & 3 & 1 & 0.60235 & \mathrm{C} & 2 \mathrm{C} 1 \\ 156 & 9 & 2 & 2 & 0.60503 & \mathrm{~B} & 2 \mathrm{~B} 2 \\ 157 & 9 & 1 & 6 & 0.62606 & \mathrm{~A} & 2 \mathrm{~A} 6 \\ 158 & 9 & 3 & 4 & 0.85074 & \mathrm{C} & 2 \mathrm{C} 4 \\ 159 & 9 & 3 & 5 & 0.85759 & \mathrm{C} & 2 \mathrm{C} 5 \\ 160 & 9 & 3 & 3 & 0.86157 & \mathrm{C} & 2 \mathrm{C} 3 \\ 161 & 9 & 1 & 2 & 0.93100 & \mathrm{~A} & 2 \mathrm{~A} 2 \\ 162 & 9 & 1 & 5 & 0.96584 & \mathrm{~A} & 2 \mathrm{~A} 5 \\ 163 & 10 & 1 & 4 & 0.01688 & \mathrm{~A} & 2 \mathrm{~A} 4 \\ 164 & 10 & 3 & 5 & 0.05395 & \mathrm{C} & 2 \mathrm{C} 5 \\ 165 & 10 & 2 & 1 & 0.06399 & \mathrm{~B} & 2 \mathrm{~B} 1 \\ 166 & 10 & 3 & 1 & 0.08176 & \mathrm{C} & 2 \mathrm{C} 1 \\ 167 & 10 & 3 & 6 & 0.08998 & \mathrm{C} & 2 \mathrm{C} 6 \\ 168 & 10 & 2 & 2 & 0.19534 & \mathrm{~B} & 2 \mathrm{~B} 2 \\ 169 & 10 & 2 & 4 & 0.32319 & \mathrm{~B} & 2 \mathrm{~B} 4 \\ 170 & 10 & 3 & 4 & 0.37064 & \mathrm{C} & 2 \mathrm{C} 4 \\ 171 & 10 & 1 & 5 & 0.47450 & \mathrm{~A} & 2 \mathrm{~A} 5 \\ 172 & 10 & 2 & 6 & 0.52635 & \mathrm{~B} & 2 \mathrm{~B} 6 \\ 173 & 10 & 1 & 2 & 0.59619 & \mathrm{~A} & 2 \mathrm{~A} 2 \\ 174 & 10 & 1 & 3 & 0.61180 & \mathrm{~A} & 2 \mathrm{~A} 3 \\ 175 & 10 & 1 & 6 & 0.63640 & \mathrm{~A} & 2 \mathrm{~A} 6 \\ 176 & 10 & 1 & 1 & 0.70002 & \mathrm{~A} & 2 \mathrm{~A} 1 \\ 177 & 10 & 2 & 3 & 0.76863 & \mathrm{~B} & 2 \mathrm{~B} 3 \\ 178 & 10 & 3 & 3 & 0.89173 & \mathrm{C} & 2 \mathrm{CB} \\ 179 & 10 & 2 & 5 & 0.94232 & \mathrm{~B} & 2 \mathrm{~B} 5 \\ 180 & 10 & 3 & 2 & 0.97204 & \mathrm{C} & 2 \mathrm{C} 2\end{array}$

AEH-11-PSEUDO-01

Analysis performed by M. Gaikowski. SAS version $9.207: 41$ 12MAY1

$12 x=8$ 
Effects of Psuedomonas fluorescons ( $P f-C L 145 A$ ) to glochicia from seven unionid mussel species $\theta$ AEH-11-PSUED0-01

Random assignment of glochidia to test tank

Mussel species: Fatmucket

\begin{tabular}{|c|c|c|c|c|c|c|}
\hline obs & round & row & tank & $x$ & row_ & $\operatorname{tankn}$ \\
\hline 1 & 1 & 1 & 6 & 0.06425 & $A$ & $3 A 6$ \\
\hline 2 & 1 & 2 & 4 & 0.23487 & B & 3B4 \\
\hline 3 & 1 & 3 & 3 & 0.28423 & $c$ & 303 \\
\hline 4 & 1 & 2 & 3 & 0.29083 & B & $3 B 3$ \\
\hline 5 & 1 & 1 & 3 & 0.31575 & A & $3 A 3$ \\
\hline 6 & 1 & 2 & 1 & 0.34859 & B & 3B1 \\
\hline 7 & 1 & 2 & 2 & 0.44260 & B & 382 \\
\hline 8 & 1 & 3 & 2 & 0.46605 & c & $3 C 2$ \\
\hline 9 & 1 & 2 & 6 & 0.50123 & B & $3 B 6$ \\
\hline 10 & 1 & 3 & 5 & $0.58+07$ & $c$ & $3 c 5$ \\
\hline 11 & 1 & 1 & 1 & 0.61879 & A & $3 A 1$ \\
\hline 12 & 1 & 2 & 5 & 0.63059 & B & 385 \\
\hline 13 & 1 & 3 & 1 & 0.68919 & $\mathrm{C}$ & $3 \mathrm{C} 1$ \\
\hline 14 & 1 & 1 & 5 & 0.69536 & A & $3 A 5$ \\
\hline 15 & 1 & 1 & 4 & 0.71002 & A & $3 A 4$ \\
\hline 16 & 1 & 3 & 6 & 0.79854 & C & $3 C 6$ \\
\hline 17 & 1 & 1 & 2 & 0.84196 & A & $3 A 2$ \\
\hline 18 & 1 & 3 & 4 & 0.92360 & c & $3 \mathrm{C} 4$ \\
\hline 19 & 2 & 3 & 3 & 0.21575 & $\mathrm{C}$ & $3 \mathrm{cs}$ \\
\hline 20 & 2 & 2 & 3 & 0.21897 & B & $3 \mathrm{B3}$ \\
\hline 21 & 2 & 3 & 5 & 0.27131 & $c$ & 305 \\
\hline 22 & 2 & 1 & 5 & 0.36197 & A & $3 \mathrm{~A} 5$ \\
\hline 23 & 2 & 3 & 2 & 0.45861 & $\mathrm{c}$ & $3 \mathrm{C} 2$ \\
\hline 24 & 2 & 1 & 6 & 0.46067 & $A$ & $3 A 6$ \\
\hline 25 & 2 & 2 & 6 & 0.56608 & B & $3 B 6$ \\
\hline 26 & 2 & 1 & 1 & 0.56645 & A & $3 A 1$ \\
\hline 27 & 2 & 2 & 1 & 0.58251 & $B$ & $3 B 1$ \\
\hline 28 & 2 & 1 & 2 & 0.74533 & A & $3 \mathrm{~A} 2$ \\
\hline 29 & 2 & 2 & 5 & 0.81915 & B & $3 B 5$ \\
\hline 30 & 2 & 3 & 6 & 0.82026 & $\mathrm{c}$ & $3 c 6$ \\
\hline 31 & 2 & 3 & 4 & 0.83029 & $\mathrm{c}$ & $3 \mathrm{C} 4$ \\
\hline 32 & 2 & 2 & 4 & 0.83100 & B & 384 \\
\hline 33 & 2 & 1 & 4 & 0.83244 & A & $3 \mathrm{A4}$ \\
\hline 34 & 2 & 3 & 1 & 0.87722 & $\mathrm{C}$ & $3 \mathrm{C} 1$ \\
\hline 35 & 2 & 2 & 2 & 0.89739 & $\mathrm{~B}$ & 382 \\
\hline 36 & 2 & 1 & 3 & 0.97844 & A & $3 A 3$ \\
\hline 37 & 3 & 2 & 2 & 0.13149 & B & $3 \mathrm{~B} 2$ \\
\hline 38 & 3 & 1 & 1 & 0.14025 & A & $3 A 1$ \\
\hline 39 & 3 & 2 & 3 & 0.18648 & $B$ & $3 B 3$ \\
\hline 40 & 3 & 1 & 2 & 0.28969 & A & $3 \mathrm{~A} 2$ \\
\hline 41 & 3 & 2 & 1 & 0.31270 & 8 & $3 \mathrm{~B} 1$ \\
\hline 42 & 3 & 1 & 4 & 0.32951 & A & $3 A 4$ \\
\hline 43 & 3 & 1 & 3 & 0.40179 & $A$ & 3A3 \\
\hline 44 & 3 & 2 & 6 & 0.40607 & $B$ & $3 \mathrm{~B} 6$ \\
\hline 45 & 3 & 3 & 6 & 0.48170 & $c$ & 306 \\
\hline 46 & 3 & 2 & 4 & 0.59046 & $B$ & $3 B 4$ \\
\hline 47 & 3 & 3 & 4 & 0.71623 & C & $3 \mathrm{C} 4$ \\
\hline 48 & 3 & 3 & 5 & 0.74142 & $c$ & 365. \\
\hline
\end{tabular}

Analysis performed by M. Gaikowski. SAS version 9.2 07:41 12MAY11

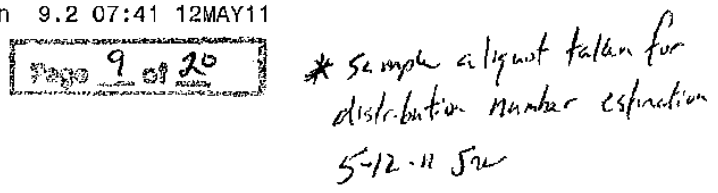


Effects of Psuedomonas fluorescens (Pf-CL145A) to glochidia from seven unionid mussel species 10 AEH - 11-PSUEDO-01

Random assignment of glochidia to test tank

Mussel species: Fatmucket

AEH-11-PSEUDO-01

\begin{tabular}{|c|c|c|c|c|c|c|c|}
\hline Obs & round & row & tank & $x$ & row & tankn & \\
\hline 49 & 3 & 3 & 3 & 0.84026 & $\mathrm{C}$ & 303 & \\
\hline 50 & 3 & $\dagger$ & 6 & 0.84708 & A & $3 A 6$ & \\
\hline 51 & 3 & 2 & 5 & 0.94185 & $B$ & 3B5 & \\
\hline 52 & 3 & 1 & 5 & 0.94902 & A & $3 A 5$ & \\
\hline 53 & 3 & 3 & 2 & 0.95766 & C & $3 \mathrm{C2}$ & \\
\hline 54 & 3 & 3 & 1 & 0.97980 & c & $3 \mathrm{C} 1$ & X \\
\hline 55 & 4 & 1 & 6 & 0.00485 & A & $3 A 6$ & \\
\hline 56 & 4 & 3 & 2 & 0.04653 & C & $3 \mathrm{C} 2$ & \\
\hline 57 & 4 & 2 & 6 & 0.08927 & 8 & $3 \mathrm{~B} 6$ & \\
\hline 58 & 4 & 1 & 2 & 0.26321 & A & $3 A 2$ & \\
\hline 59 & 4 & 2 & 4 & 0.27095 & $B$ & 384 & \\
\hline 60 & 4 & 2 & 5 & 0.27927 & B & $3 B 5$ & $t$ \\
\hline 61 & 4 & 3 & 5 & 0.35826 & $c$ & 305 & 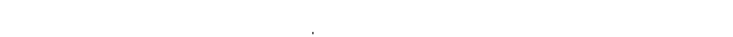 \\
\hline 62 & 4 & 3 & 6 & 0.43440 & c & $3 \mathrm{C} 6$ & \\
\hline 63 & 4 & 3 & 1 & 0.57582 & $c$ & $3 \mathrm{C} 1$ & \\
\hline 64 & 4 & 2 & 2 & 0.57707 & B & $3 \mathrm{~B} 2$ & \\
\hline 65 & 4 & 1 & 1 & 0.65035 & A & 3A1 & \\
\hline 66 & 4 & 1 & 3 & 0.66001 & A & $3 \mathrm{~A}_{3}$ & 每 \\
\hline 67 & 4 & 3 & 4 & 0.73456 & $\mathrm{c}$ & $3 \mathrm{C4}$ & \\
\hline 68 & 4 & 2 & 3 & 0.75676 & B & 383 & \\
\hline 69 & 4 & 2 & 1 & 0.75680 & $B$ & 381 & \\
\hline 70 & 4 & 1 & 4 & 0.77378 & A & $3 A 4$ & \\
\hline 71 & 4 & 3 & 3 & 0.91226 & C & $3 \mathrm{C3}$ & \\
\hline 72 & 4 & 1 & 5 & 0.98150 & A & $3 A 5$ & $\ldots$ \\
\hline 73 & 5 & 1 & 2 & 0.02882 & A & $3 \mathrm{~A} 2$ & \\
\hline 74 & 5 & 3 & 2 & 0.08059 & $c$ & 362 & \\
\hline 75 & 5 & 2 & 1 & 0.11022 & B & 3B1 & \\
\hline 76 & 5 & 2 & 3 & 0.14474 & $B$ & $3 B 3$ & \\
\hline 77 & 5 & 3 & 3 & 0.15604 & C & $3 \mathrm{C3}$ & \\
\hline 78 & 5 & 2 & 4 & 0.16623 & B & $3 \mathrm{~B} 4$ & $-x$ \\
\hline 79 & 5 & 3 & 4 & 0.25175 & $\mathrm{C}$ & $3 \mathrm{C} 4$ & \\
\hline 80 & 5 & 2 & 5 & 0.32841 & B & $3 B 5$ & \\
\hline 81 & 5 & 2 & 6 & 0.36155 & $B$ & 386 & \\
\hline 82 & 5 & 1 & 5 & 0.41550 & A & 3A5 & \\
\hline 83 & 5 & 3 & 1 & 0.45032 & $\mathrm{c}$ & $3 c 1$ & \\
\hline 84 & 5 & 1 & 3 & 0.48246 & A & $3 \mathrm{~A} 3$ & $-t$ \\
\hline 85 & 5 & $t$ & 4 & 0.56627 & A & $3 A 4$ & \\
\hline 86 & 5 & 1 & 6 & 0.63987 & A & 3A6 & \\
\hline 87 & 5 & 2 & 2 & 0.72266 & B & 382 & \\
\hline 88 & 5 & 1 & 1 & 0.89897 & A & $3 A 1$ & \\
\hline 89 & 5 & 3 & 6 & 0.92983 & $\mathrm{c}$ & $3 \mathrm{C} 6$ & \\
\hline 90 & 5 & 3 & 5 & 0.93030 & C & $3 \mathrm{C5}$ & - \\
\hline 91 & 6 & 2 & 4 & 0.03396 & B & 384 & Five rounds of glochidice ware added to \\
\hline 92 & 6 & 3 & 2 & 0.05586 & C & $3 \mathrm{C} 2$ & Five rounds of $J_{\text {Rourds }} 6$ m 10 of the random \\
\hline 93 & 6 & 3 & 6 & 0,22820 & $c$ & 306 & exposure chambers. Rourds \\
\hline 94 & 6 & 1 & 4 & 0.25609 & A & $3 A 4$ & 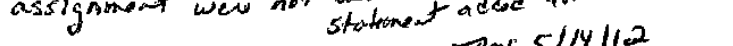 \\
\hline 95 & 6 & 3 & 4 & 0.25905 & C & $3 \mathrm{C} 4$ & Mns $5 / 1411$ ad \\
\hline 96 & 6 & 1 & 5 & 0.27999 & A & $3 \mathrm{A5}$ & \\
\hline
\end{tabular}

Analysis performed by $M$. Gaikowski SAS version 9.2 07:41 12MAY11

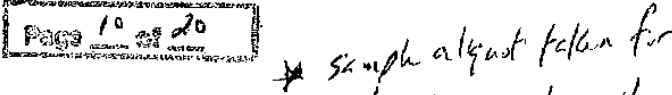

$$
\begin{aligned}
& \text { distribulion nunber estineton } \\
& 5-12 \cdot 1150
\end{aligned}
$$


Effects of Psuedomonas fluorescens (Pf-CL145A) to glochidia from seven unionid mussel species AEH-11-PSUEDO-01

Random assignment of glochidia to test tank

Mussel species: Fatmucket

AEH-11-PSEUDO-01

\begin{tabular}{|c|c|c|c|c|c|c|}
\hline Obs & round & row & tank & $x$ & row_ & $\operatorname{tankn}$ \\
\hline 97 & 6 & 1 & 6 & 0.30692 & A & $3 A 6$ \\
\hline 98 & 6 & 3 & 3 & 0.30840 & $c$ & 303 \\
\hline 99 & 6 & 2 & 6 & 0.33386 & $B$ & $3 B 6$ \\
\hline 100 & 6 & 2 & 3 & 0.41121 & $\mathrm{~B}$ & $3 \mathrm{~B} 3$ \\
\hline 101 & 6 & 2 & 2 & 0.52134 & $\mathrm{~B}$ & $3 \mathrm{~B} 2$ \\
\hline 102 & 6 & 1 & 3 & 0.64758 & A & 3A3 \\
\hline 103 & 6 & 3 & 1 & 0.70774 & C & $3 \mathrm{C1}$ \\
\hline 104 & 6 & 3 & 5 & 0.73370 & $\mathrm{C}$ & $3 \mathrm{C5}$ \\
\hline 105 & 6 & 1 & 1 & 0.74906 & A & $3 \mathrm{~A} 1$ \\
\hline 106 & 6 & 2 & 5 & 0.85655 & $B$ & 3B5 \\
\hline 107 & 6 & 1 & 2 & 0.89278 & A & $3 \mathrm{~A} 2$ \\
\hline 108 & 6 & 2 & 1 & 0.98807 & $B$ & $3 B 1$ \\
\hline 109 & 7 & 3 & 6 & 0.01515 & C & $3 \mathrm{C} 6$ \\
\hline 110 & 7 & 2 & 1 & 0.27756 & B & 3B1 \\
\hline 111 & 7 & 3 & 5 & 0.28546 & $\mathrm{C}$ & $3 C 5$ \\
\hline 112 & 7 & 1 & 4 & 0.31072 & A & $3 A 4$ \\
\hline 113 & 7 & 3 & 4 & 0.33916 & $C$ & $3 C 4$ \\
\hline 114 & 7 & 1 & 5 & 0.35827 & A & $3 A 5$ \\
\hline 115 & 7 & 1 & 2 & 0.46221 & $A$ & $3 \mathrm{~A} 2$ \\
\hline 116 & 7 & 2 & 3 & 0.51697 & $B$ & 383 \\
\hline 117 & 7 & 1 & 6 & 0.59115 & A & 3A6 \\
\hline 118 & 7 & 2 & 2 & 0,60333 & B & 382 \\
\hline 119 & 7 & 2 & 5 & 0.66243 & B & 385 \\
\hline 120 & 7 & 3 & 1 & 0.68544 & $\mathrm{C}$ & $3 \mathrm{Cl}$ \\
\hline 121 & 7 & 3 & 2 & 0.69659 & C & $3 \mathrm{C} 2$ \\
\hline 122 & 7 & 2 & 4 & 0.87014 & B & $3 B 4$ \\
\hline 123 & 7 & 1 & 3 & 0.89719 & A & $3 A 3$ \\
\hline 124 & 7 & 1 & 1 & 0.91395 & A & 3A1 \\
\hline 125 & 7 & 2 & 6 & 0.92644 & $\mathrm{~B}$ & $3 \mathrm{~B} 6$ \\
\hline 126 & 7 & 3 & 3 & 0.96712 & $c$ & $3 \mathrm{C3}$ \\
\hline 127 & 8 & 3 & 3 & 0.03720 & $c$ & $3 \subset 3$ \\
\hline 128 & 8 & 2 & 3 & 0.07049 & $B$ & 383 \\
\hline 12.9 & 8 & 3 & 4 & 0.17372 & C & 304 \\
\hline 130 & 8 & 2 & 6 & 0.23243 & $B$ & $3 \mathrm{~B} 6$ \\
\hline 131 & 8 & 3 & 6 & 0.26338 & $c$ & $3 \mathrm{C6}$ \\
\hline 132 & 8 & 1 & 4 & 0.27158 & A & $3 A 4$ \\
\hline 133 & 8 & 1 & 3 & 0.29892 & A & $3 A 3$ \\
\hline 134 & 8 & 2 & 2 & 0.42426 & B & 382 \\
\hline 135 & $B$ & 2 & 1 & 0.53568 & B & 381 \\
\hline 136 & 8 & 3 & 2 & 0,53767 & $\mathrm{c}$ & $3 \mathrm{C} 2$ \\
\hline 137 & 8 & 3 & 1 & 0.59074 & C & $3 \mathrm{C} 1$ \\
\hline 138 & 8 & 1 & 1 & 0.65401 & A & 3A1 \\
\hline 139 & 8 & 2 & 4 & 0.77251 & $B$ & $3 B 4$ \\
\hline 140 & 8 & 1 & 5 & 0.81738 & $A$ & $3 A 5$ \\
\hline 141 & 8 & 1 & 6 & 0.87037 & $A$ & $3 A 6$ \\
\hline 142 & 8 & 1 & 2 & 0.88416 & A & $3 A 2$ \\
\hline 143 & 8 & 2 & 5 & 0.94410 & 8 & 385 \\
\hline 144 & 8 & 3 & 5 & 0.98817 & $\mathrm{C}$ & $3 C 5$ \\
\hline
\end{tabular}

Analysis performed by M. Gaikowski SAS version 9.2 07:41 12MAY11

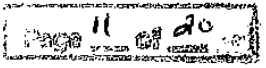


Effects of Psuedomonas fluorescens (Pf,CL145A) to glochidia from soven unionid mussel species 12 AEH - 11 - PSUEDO-01

Random assignment of glochidia to test tank

Mussel species: Fatmucket

\begin{tabular}{|c|c|c|c|c|c|c|}
\hline Obs & round & row & $\operatorname{tank}$ & $x$ & _row_ & tankn \\
\hline 145 & 9 & 1 & 1 & 0.10991 & A & $3 \mathrm{~A} 1$ \\
\hline 146 & 9 & 2 & 4 & 0.13007 & $B$ & $3 B 4$ \\
\hline 147 & 9 & 2 & 3 & 0.18093 & $B$ & $3 \mathrm{~B} 3$ \\
\hline 148 & 9 & 2 & 6 & 0.18945 & $B$ & 386 \\
\hline 149 & 9 & 1 & 3 & 0.19692 & A & $3 A 3$ \\
\hline 150 & 9 & 1 & 2 & 0.21057 & A & $3 A 2$ \\
\hline 151 & 9 & 3 & 3 & 0.27487 & $c$ & $3 \mathrm{CO}$ \\
\hline 152 & 9 & 3 & 5 & 0.32850 & c & $3 C 5$ \\
\hline 153 & $\theta$ & 3 & 1 & 0.39289 & $\mathrm{C}$ & $3 c 1$ \\
\hline 154 & $\theta$ & 3 & 2 & 0.40678 & $\mathrm{C}$ & $3 \mathrm{C} 2$ \\
\hline 155 & 9 & 3 & 4 & 0.45710 & $C$ & $3 C 4$ \\
\hline 156 & 9 & 3 & 6 & 0.54291 & $c$ & $3 C 6$ \\
\hline 157 & 9 & 1 & 6 & 0.61225 & A & $3 A 6$ \\
\hline 158 & 9 & 1 & 5 & 0.73560 & A & $3 \mathrm{A5}$ \\
\hline 159 & $\mathbf{g}$ & 2 & 5 & 0.75981 & B & 385 \\
\hline 160 & 9 & 2 & 1 & 0.90249 & B & 381 \\
\hline 161 & 9 & 2 & 2 & 0.91701 & B & $3 \mathrm{~B} 2$ \\
\hline 162 & 9 & 1 & 4 & 0.93682 & A & $3 A 4$ \\
\hline 163 & 10 & 3 & 6 & 0.03932 & $\mathrm{c}$ & 306 \\
\hline 164 & 10 & 1 & 1 & 0.05556 & A & $3 A 1$ \\
\hline 165 & 10 & 2 & 5 & 0.08212 & B & $3 B 5$ \\
\hline 166 & 10 & 2 & 4 & 0.16615 & B & 3B4 \\
\hline 167 & 10 & 1 & 2 & 0.43469 & A & $3 \mathrm{~A} 2$ \\
\hline 168 & 10 & 1 & 4 & 0.45582 & A & $3 \mathrm{A4}$ \\
\hline 169 & 10 & 1 & 3 & 0.46757 & $A$ & $3 A 3$ \\
\hline 170 & 10 & 2 & 6 & 0.56078 & B & $3 \mathrm{~B} 6$ \\
\hline 171 & 10 & 1 & 5 & 0.61491 & A & $3 A 5$ \\
\hline 172 & 10 & 1 & 6 & 0.61818 & A & $3 A 6$ \\
\hline 173 & 10 & 3 & 5 & 0.67004 & c & $3 \mathrm{C5}$ \\
\hline 174 & 10 & 2 & 2 & 0.69138 & B & $3 B 2$ \\
\hline 175 & 10 & 2 & 1 & 0.76353 & B & 3B1 \\
\hline 176 & 10 & 3 & 3 & 0.78170 & $c$ & 303 \\
\hline 177 & 10 & 3 & 1 & 0.90674 & $c$ & $3 \mathrm{Cl}$ \\
\hline 178 & 10 & 3 & 2 & 0.93409 & C & $3 c 2$ \\
\hline 179 & 10 & 3 & 4 & 0.94792 & C & 364 \\
\hline 180 & 10 & 2 & 3 & 0.99146 & B & 3B3 \\
\hline
\end{tabular}

AEH-11-PSEUDO-01

$$
\text { mit }
$$

Analysis performed by $v$. Gaikowski sAs version 9.2 07:41 12MAY11

20120 


\section{AEH-11-PSEUDO-01}

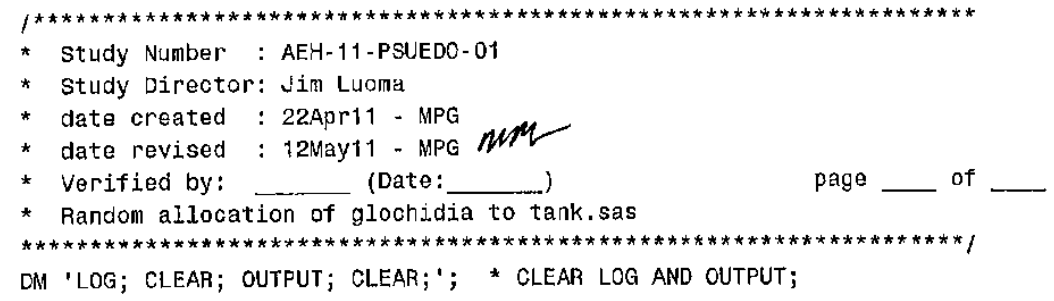

FOOTNOTE1 'Analysis performed by M. Gaikowski SAS version ' \&SYSVER \&SYSTIME \&SYSDATE;

options $/{ }^{*} 1 s=85 \mathrm{ps}=40$ formdlim=' $-1 * /$ pageno $=1$ nocenter nodate nosource 2

$1 *$ Random distribution of glochidia to experinental tanks*/

$1^{*} \operatorname{tank} 1$ to $18=\operatorname{tank} A 1$ to $A 6(1-6)$, tank B1 to B6 (7-12), tank C1 to C6 (13-18)

round = distribution rounds 1 to 2 , place one aliquot of glochidia per tank per round */

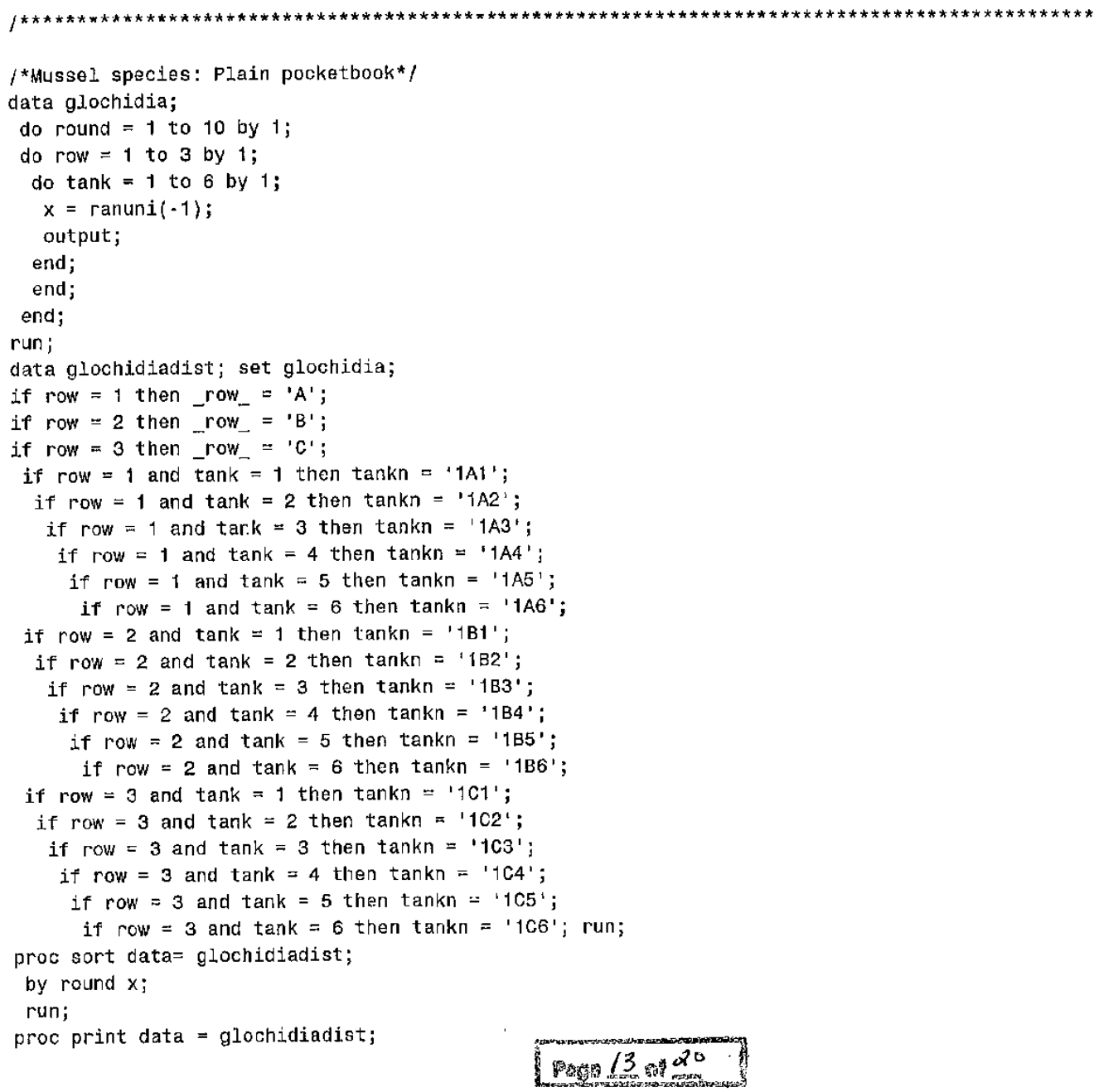




\section{AEH-11-PSEUDO-01}

titlef $h=2$ 'Effects of Psuedomonas fluorescens ( $\mathrm{ff}-\mathrm{CL}$ 145A) to glochidia from seven unionid mussel title2 $h=1.5$ 'AEH-11-PSUEDO-01';

title $h=1$ 'Random assignment of glochidia to test tanks';

title4 $h=1$ 'Mussel species: Plain pocketbook';

run;

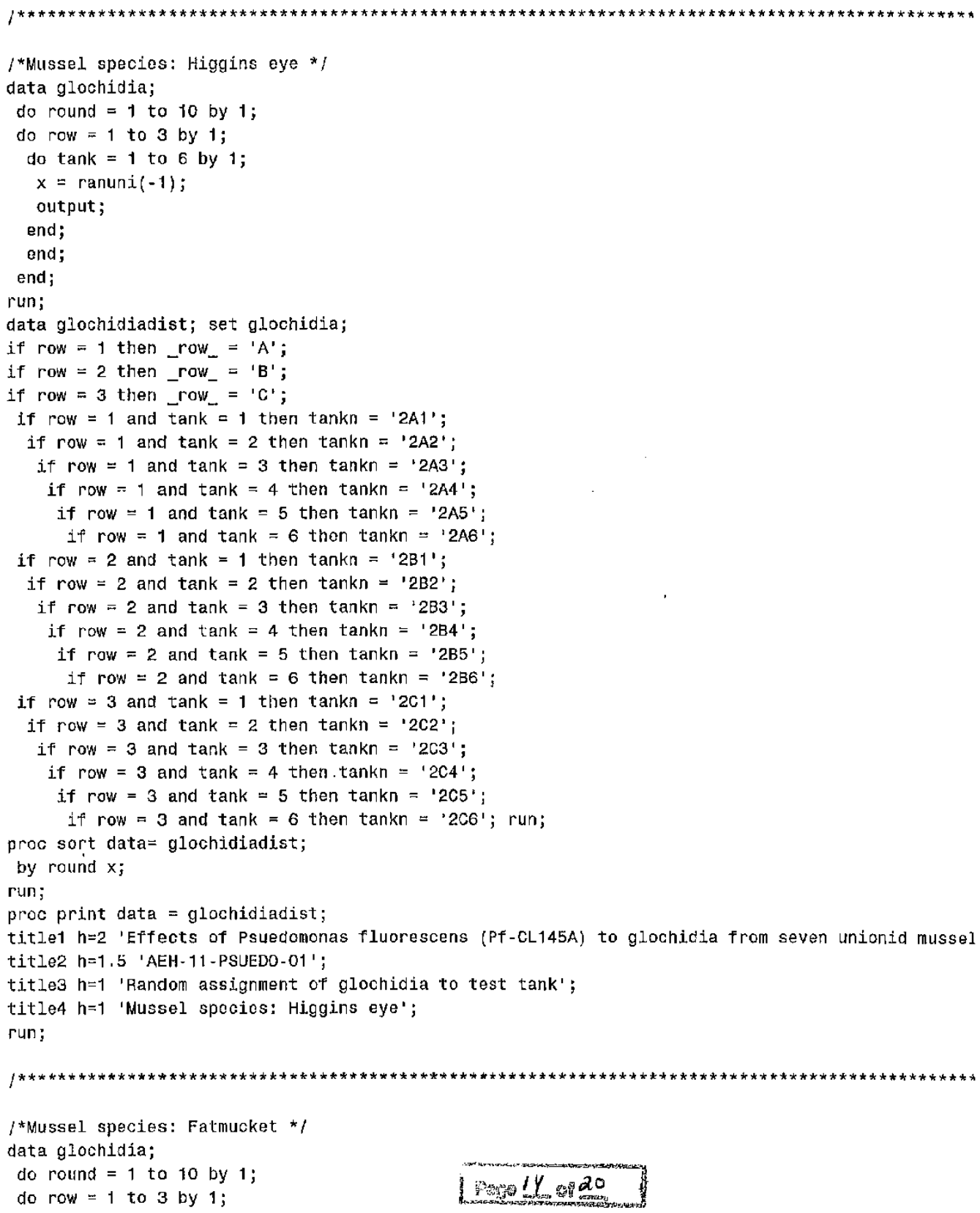




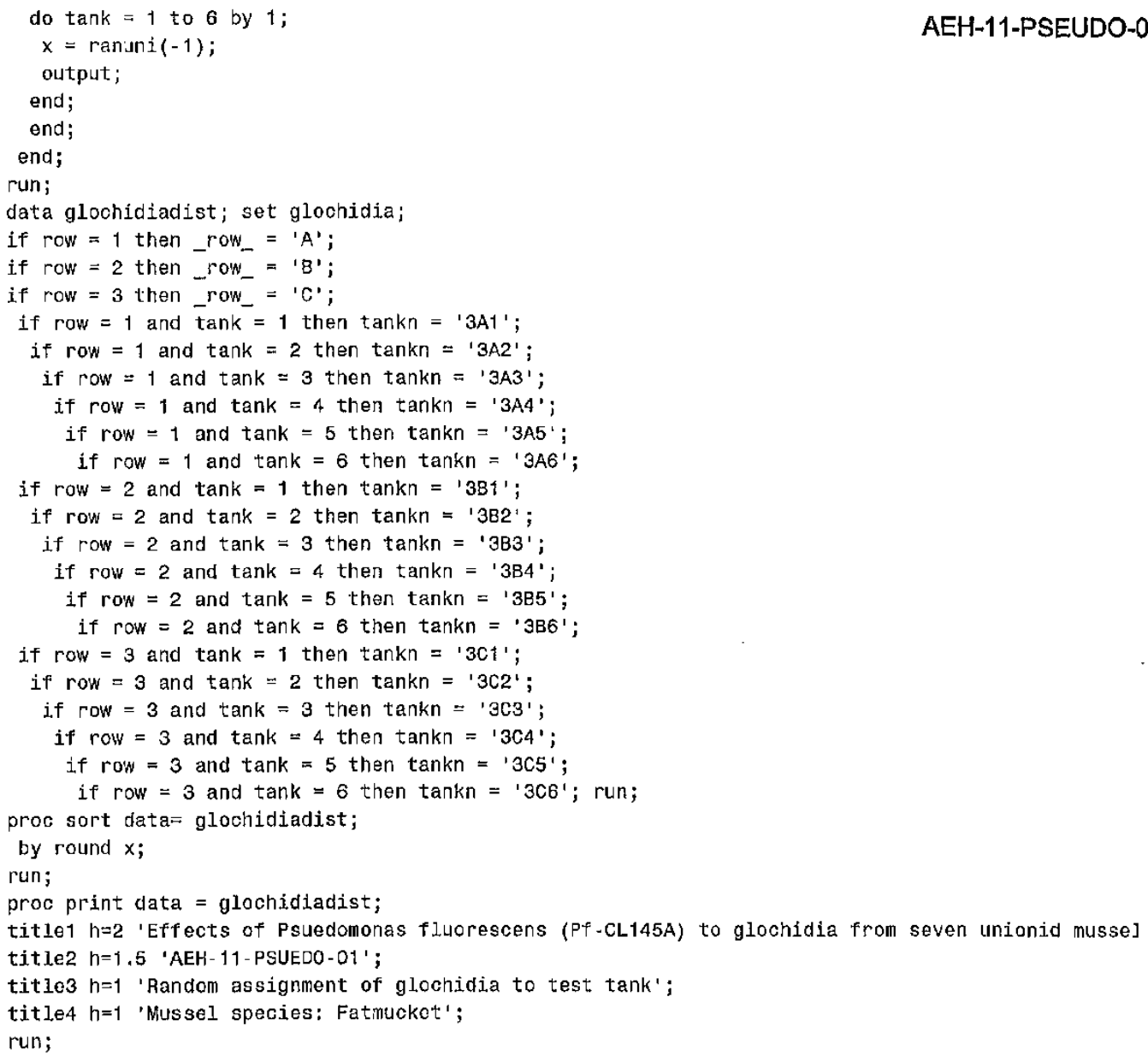




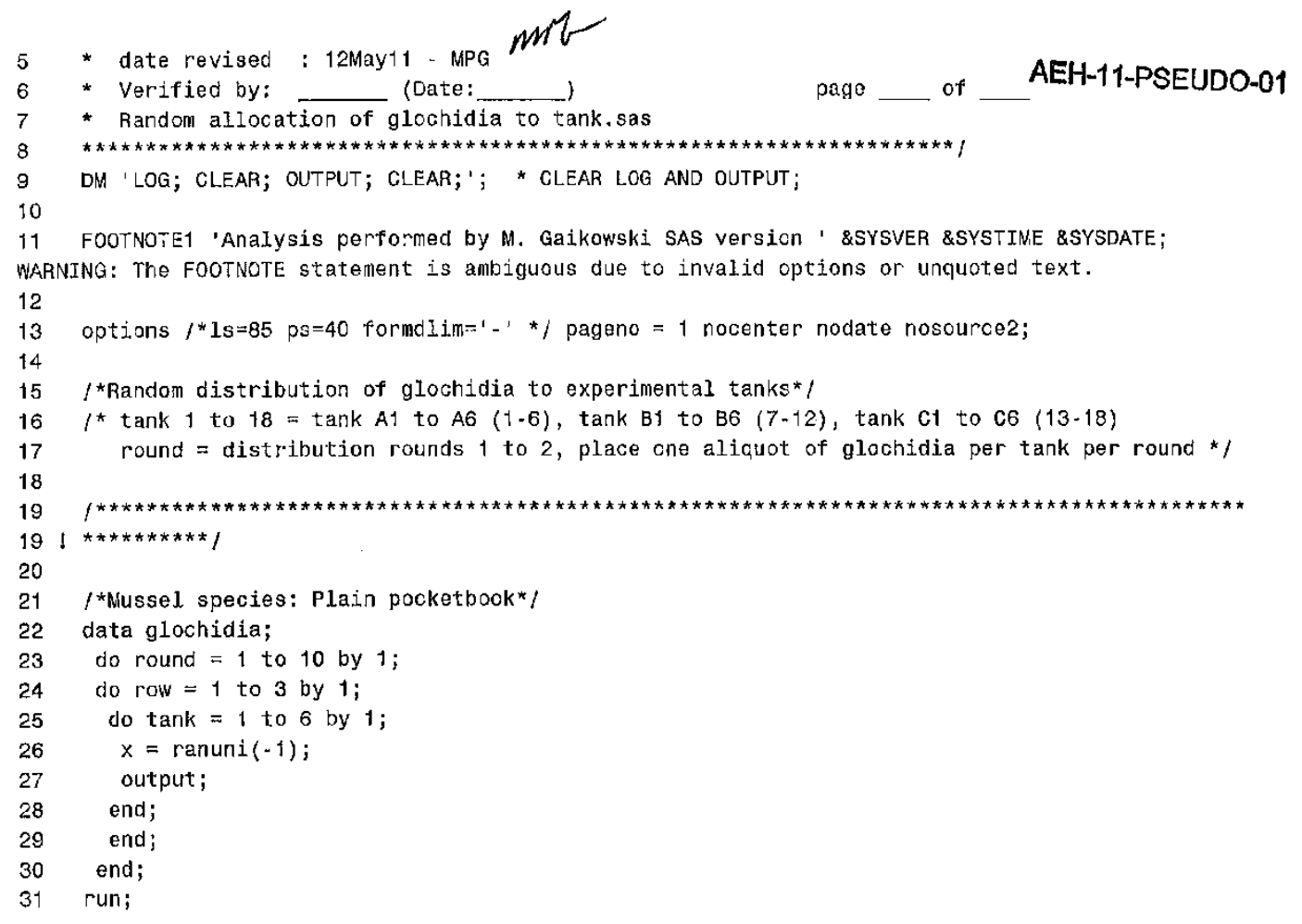

NOTE: The data set WORK.GLOCHIDIA has 180 observations and 4 variables.

NOTE: DATA statement used (Total process time):
real time
0.10 seconds
cpu time
0.07 seconds

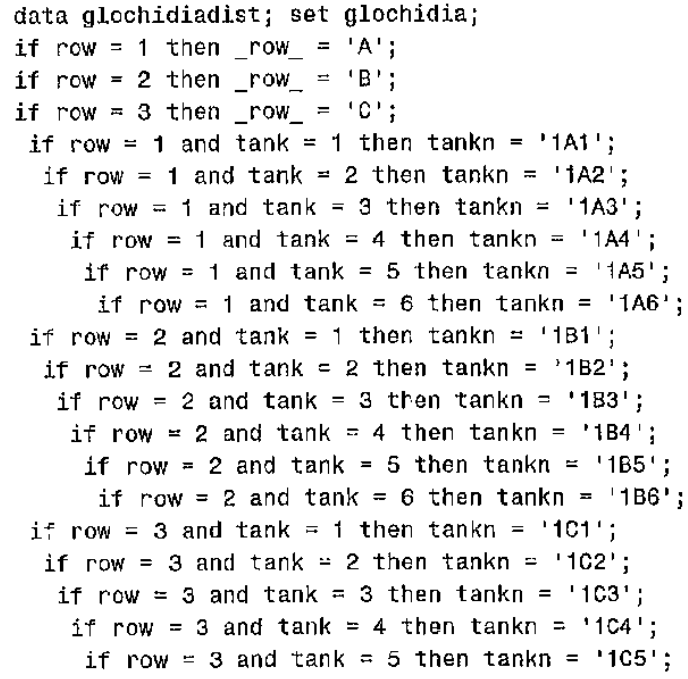


NOTE: There were 180 observations read from the data set WORK.GLOCHIDIA.

NOTE: The data set WORK.GLOCHIDIADIST has 180 observations and 6 variables.

NOTE: DATA statement used (Total process time):
real timo
0,03 seconds
cpu time
0.01 seconds
54 proo sort data $=$ glochidiadist
55 by round $x$;
56 run;

NOTE: There were 180 observations read from the data set WORK.GLOCHIDIADIST.

NOTE: The data set WORK.GLOCHIDIADIST has 180 observations and 6 variables.

NOTE: PROCEDURE SORT used (Total process time):
real time
0.04 seconds
cpu time
0.03 seconds

57 proc print data $=$ glochidiadist;

58 title1 h=2 'Effects of Psuedomonas fluorescens (Pf-CL145A) to giochidia from seven unionid

58 ! mussol species';

59 title2 $\mathrm{h}=1.5$ 'AEH-11-PSUEDO-01';

60 title3 $\mathrm{h}=1$ 'Random assignment of glochicia to test tanks';

61 title4 $\mathrm{h}=1$ 'Mussel species: Plain pocketbook';

62 run;

NOTE: There were 180 observations read from the data set WORK.GLOCHIDIADIST,

NOTE: PROCEDURE PRINT used (Total process time):

real time $\quad 0.06$ seconds

cpu time $\quad 0.04$ seconds

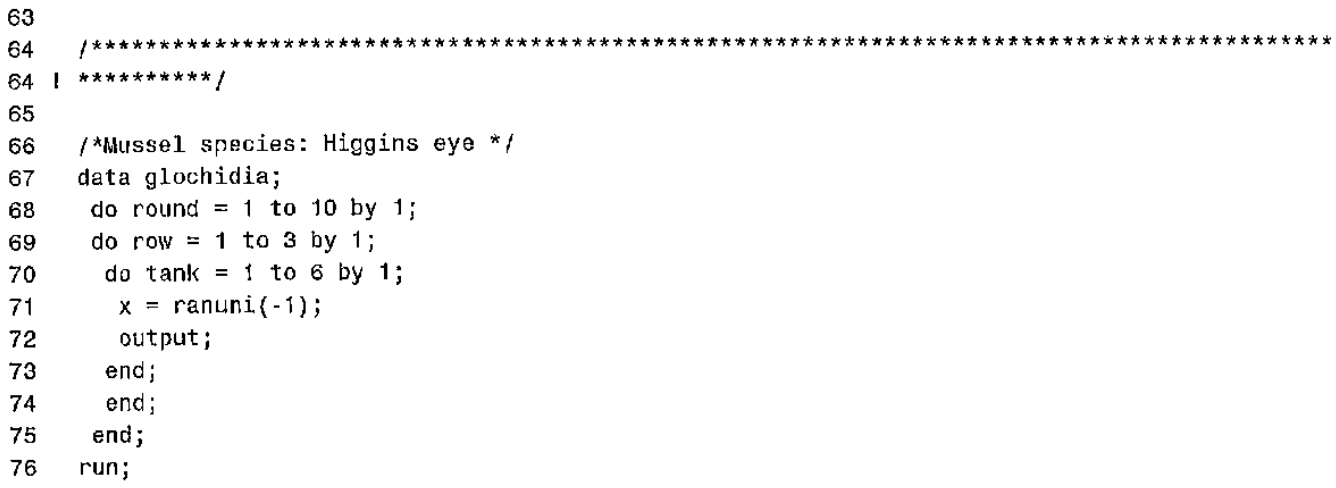

NOTE: The data set WORK.GLOCHIDIA has 180 observations and 4 variables.

NOTE: DATA statement used (Total process time):
real time
0.00 seconds
cp's time
0.00 seconds

77 data glochidiadist; set glochidia;

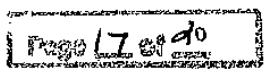




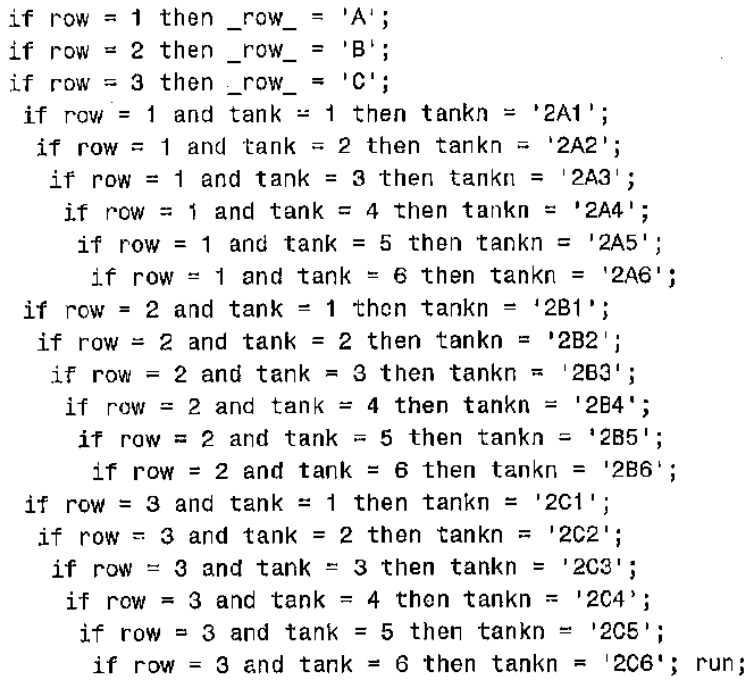

NOTE: There were 180 observations read from the data set WOAK.GLOCHIDIA.

NOTE: The data set WORK.GLOCHIDIADIST has 180 observations and 6 variables.

NOTE: DATA statement used (Total process time):
real time
0.03 seconds

cpu time

0.03 seconds

99 proc sort data= glochidiadist;
100 by round $x_{;}$
101 run;

NOTE: There were 180 observations read from the data set WOFK.GLOCHIDIADIST. NOTE: The data set WORK.GLOCHIDIADIST has 180 observations and 6 variables.

NOTE: PROCEDURE SORT used (Total process time):
real time
0.01 seconds
cpu time
0.01 seconds

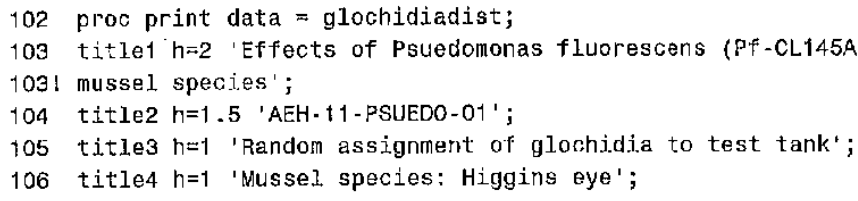

NOTE: There were 180 observations read from the data set WORK.GLOCHIDIADIST.

NOTE: PRCCECURE PRINT used (Total process time):
real time
0.01 seconds
cpu time
0.01 seconds

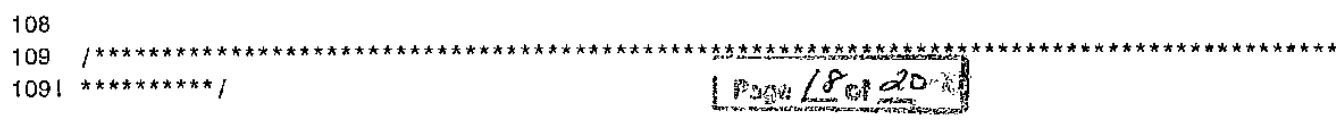




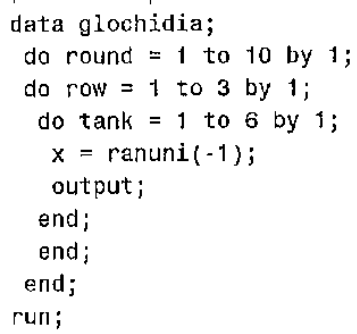

NOTE: The data set WOHK.GLOCHIDIA has 180 observations and 4 variables.

NOTE: DATA statement used (Total process time):
real time
0.01 seconds
cpu time
0.01 seconds

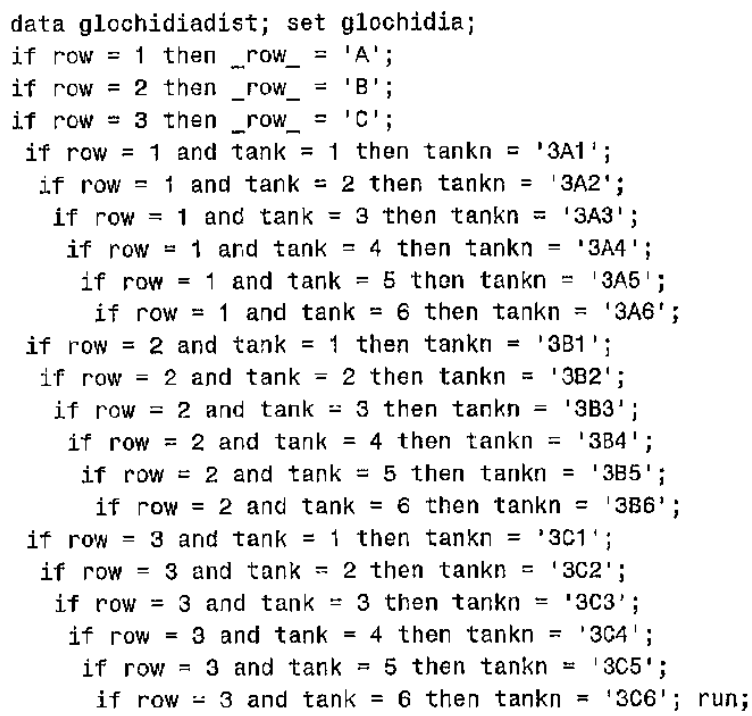

NOTE: There were 180 observatjons read from the data set WOAK.GLOCHIDIA.

NOTE: The data set WORK.GLOCHIDIADIST has 180 observations and 6 variables.

NOTE: DATA statement used (Total process tine):

$\begin{array}{ll}\text { real time } & 0.01 \text { seconds } \\ \text { cpu time } & 0.01 \text { seconds }\end{array}$

144 proc sort data $=$ glochidiadist;

145 by round $x$;

146 run;

NOTE: There were 180 observations read from the data set WORK.GLOCHIDIADIST. NOTE: The data set WORK.GLOCHIDIADIST has 180 observations and 6 variables.

NOTE: PROCEDURE SORT used (Total process time):
reai time
0.00 seconds

19720 and 
147 proc print data $=$ glochidiadist;

148 title1 $h=2$ 'Effects of Psuedomonas fluorescens (Pf-CL.145A) to glochidia from seven unionid

148 ! mussel species';

149 title2 $h=1.5$ 'AEH-11-PSUEDO-01';

150 title3 $h=1$ 'Random assignment of glochidia to test tank';

151 title4 h=1 'Mussel species: Fatmucket';

152 run;

NOTE: There were 180 cbservations read from the data set WORK.GLOCHIDIADIST.

NOTE: PROCEDURE PAINT used (Total process time):
real time
0.01 seconds
cpu time
0.01 seconds

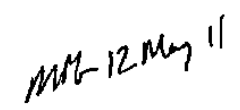

File foldan 148

WROOFEO BY

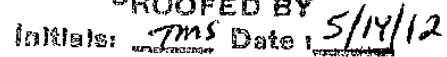

FVIEWED WY

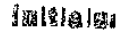

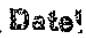

170020 


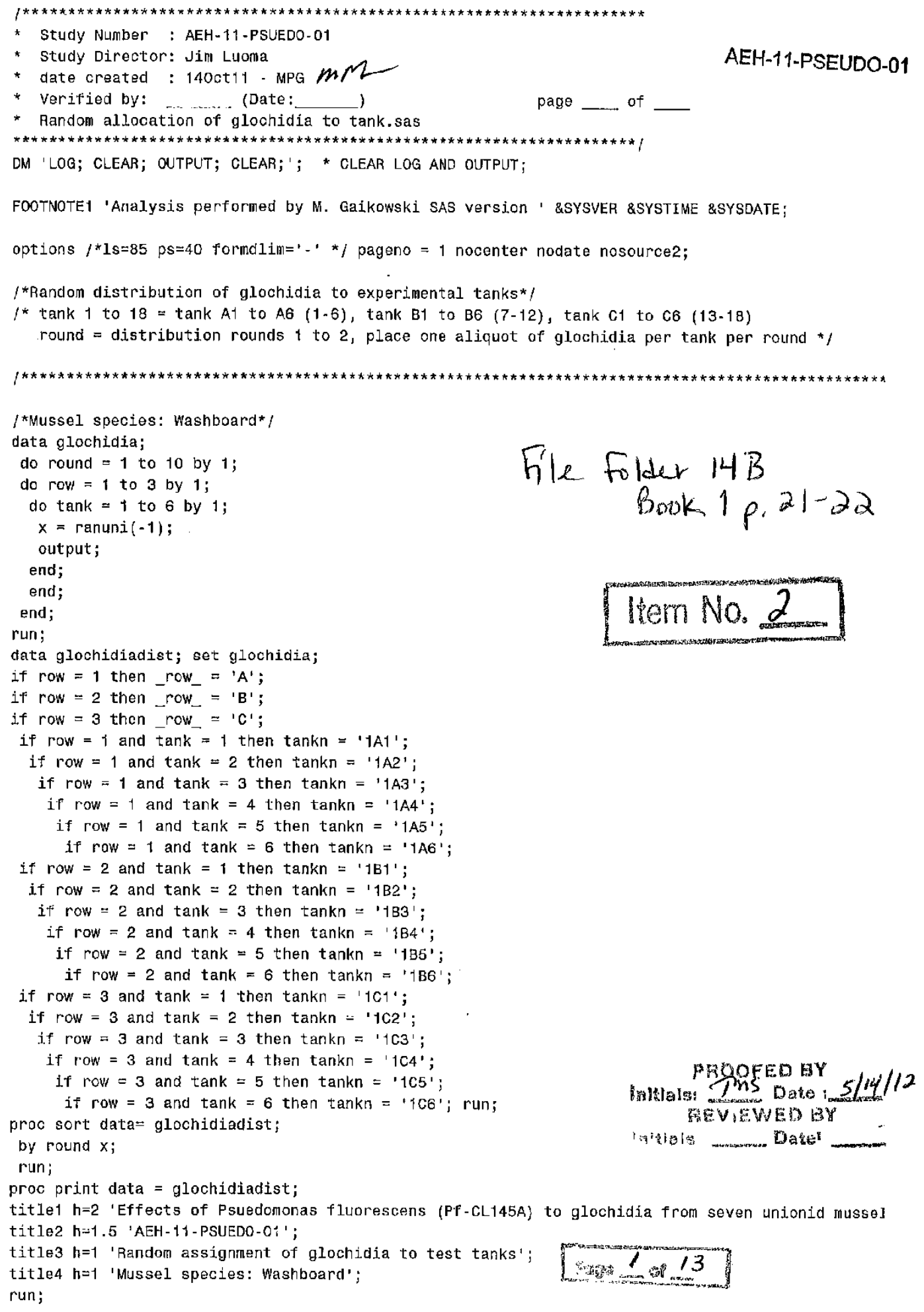

File folder $14 B$ Book 1 p. 21-22 


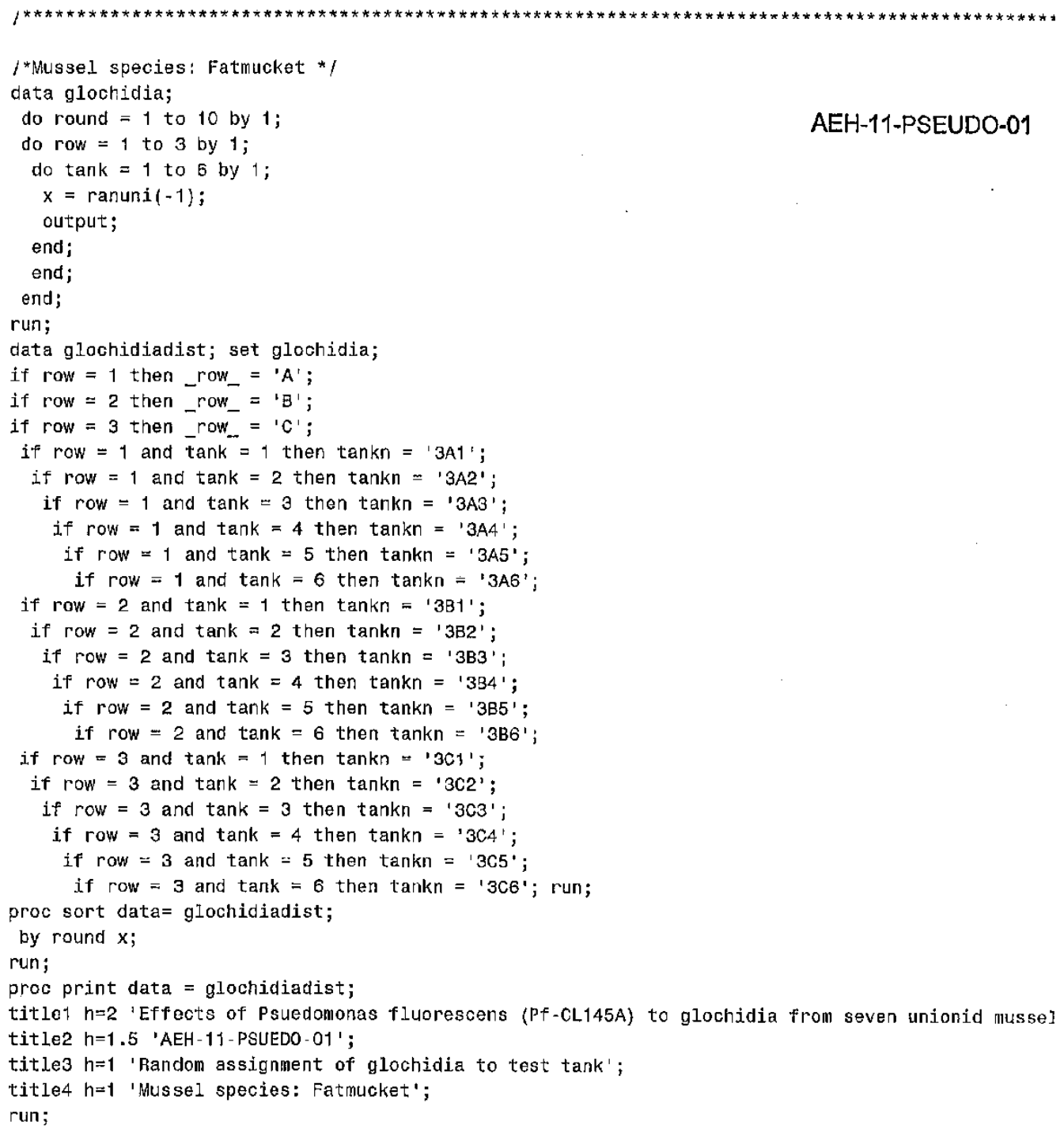




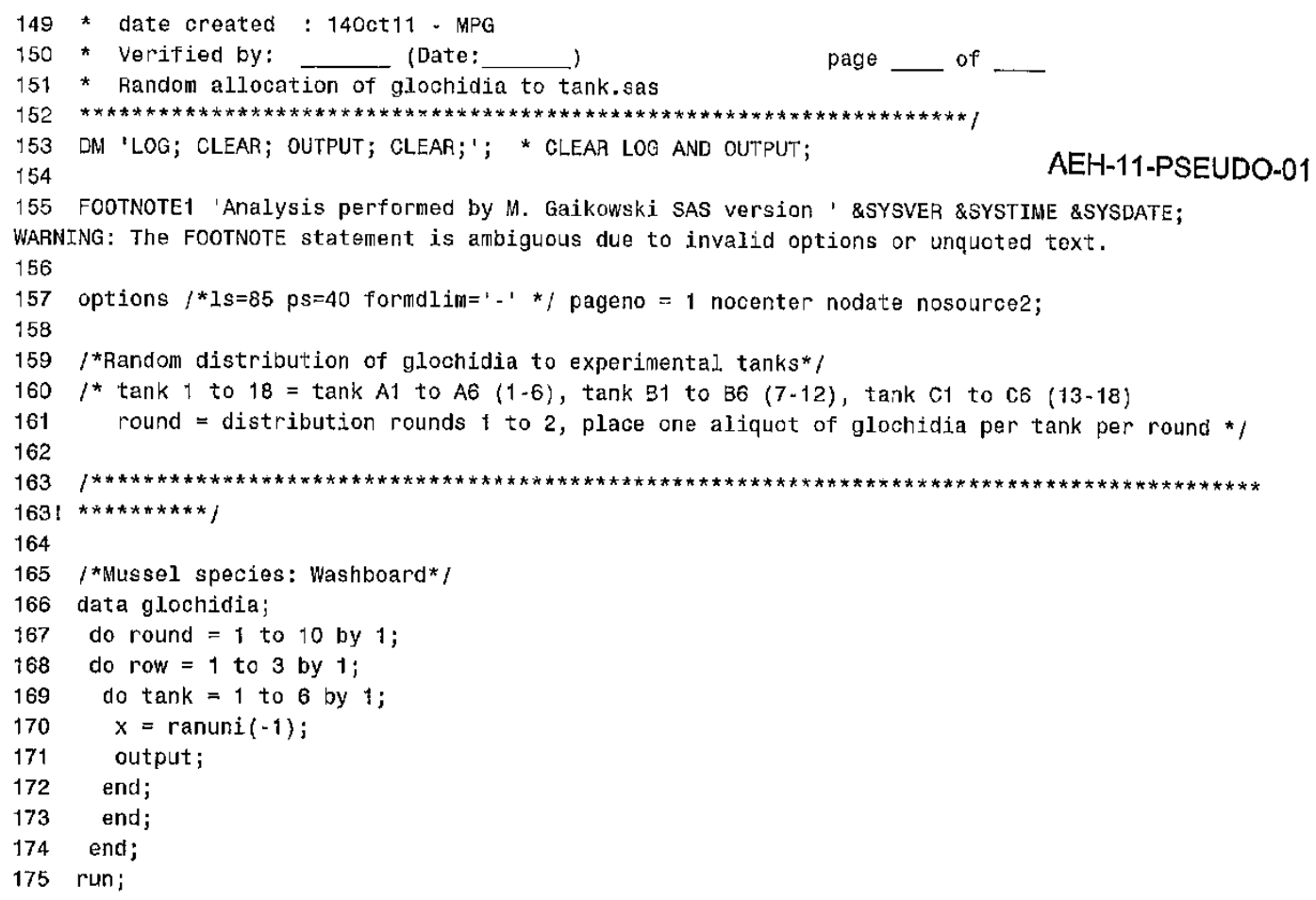

NOTE: The data set WORK.GLOCHIDIA has 180 observations and 4 variables.

NOTE: DATA statement used (Total process time):

real time $\quad 0.00$ seconds

cpu time $\quad 0.01$ seconds

176 data glochidiadist; set glochidia;

177 if row $=1$ then raw $_{-}=\mathrm{A}^{\prime} \mathrm{A}$;

178 if row $=2$ then row $_{-}=\mathrm{B}^{\prime}$ ';

179 if row $=3$ then row $=' \mathrm{C}$ ';

180 if row $=1$ and $\operatorname{tank}=1$ then $\operatorname{tankn}=$ 'tA' ';

181 if row $=1$ and tank $=2$ then $\operatorname{tankn}=1$ ' $1 \mathrm{~A} 2$ ',

182 if row $=1$ and tank $=3$ then tankn $=11 \mathrm{~A}^{\prime}$;

183 if row $=1$ and $\tan k=4$ then $\tan k n=1$ ' 1 A4'

if row $=1$ and $\operatorname{tank}=5$ then $\operatorname{tankn}=1 \mathrm{~A} 5$ ';

if row $=1$ and $\operatorname{tank}=6$ then $\operatorname{tankn}=' 1 \mathrm{~A} \mathrm{C}^{\prime}$;

if row $\bar{m} 2$ and $\operatorname{tank}=1$ then tankn $=11 \mathrm{~B} 1 '$;

if row $=2$ and tank $=2$ then $\operatorname{tankn}=11 \mathrm{~B} 2$ ';

if row $=2$ and $\operatorname{tank}=3$ then tankn $=' 1 B 3 '$;

if row $=2$ and tank $=4$ then tankn $=11 \mathrm{~B} 4$ ';

if row $=2$ and tank $=5$ then tankn $=1185$ ';

if row $=2$ and $\operatorname{tank}=6$ then $\operatorname{tankn}=$ ' $1 \mathrm{~B} \mathrm{C}^{\prime}$;

if row $=3$ and $\tan k=1$ then $\operatorname{tankn}={ }^{\prime} 1 \mathrm{C} 1^{\prime}$ ';

if row $=3$ and tank $=2$ then $\operatorname{tankn}=' 1 \mathrm{c} 2$ ';

if row $=3$ and tank $=3$ then tankn $=11 \mathrm{c} 3$ '

if row $=3$ and $\operatorname{tank}=4$ then $\operatorname{tankn}=11 \mathrm{C} 4$ ';

if row $=3$ and $\operatorname{tank}=5$ then $\operatorname{tankn}=11 \mathrm{c} 5$ ';

if row $=3$ and tank $=6$ then tankn $=$ ' 1 C6'; run;

NOTE: There were 180 observations read from the data set WORK. GLOCHIDIA.

NOTE: The data set WORK.GLOCHIDIADIST has 180 observations and 6 variables. 3 
NOTE: DATA statement used (Total process time):

real time $\quad 0.01$ seconds

AEH-11-PSEUDO-01

cpu time $\quad 0.03$ seconds

198 proc sort data= glochidiadist;

499 by round $x$;

200 run;

NOTE: There were 180 observations read from the data set WORK.GLOCHIDIADIST.

NOTE: The data set WORK.GLOCHIOIADIST has 180 observations and 6 variables.

NOTE: PROCEDURE SORT used (Total process time) :
real time
0.00 seconds
cpu time
0.01 seconds

201 proc print data $=$ glochidiadist;

202 title1 $h=2$ 'Effects of Psuedomonas fluorescens (Pf-CLf45A) to glochidia from seven unionid

202 ! mussel species';

203 title2 $h=1.5$ 'AEH-11-PSUEDO-01';

204 title3 $h=1$ 'Random assignment of glochidia to test tanks';

205 title4 $h=1$ 'Mussel species: Washboard';

206 run;

NOTE: There were 180 observations read from the data sot VORK.GLOCHIDIADIST.

NOTE: PROCEDURE PRINT used (Total process time):
real time
0.00 seconds
cpu time
0.01 seconds

207

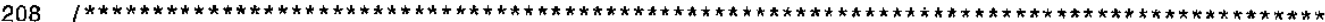

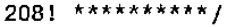

209

210 /*Mussel species: Fatmucket */

211 data glochidia;

212 do round $=1$ to 10 by 1 ;

213 do row $=1$ to 3 by 1

214 do tank $=1$ to 6 by 1 ;

$215 \quad x=\operatorname{ranuni}(-1)$;

216 output;

217 end

218 end;

219 end;

220 run;

NOTE: The data set WORK,GLOCHIDIA has 180 observations and 4 variables

NOTE: DATA statoment used (Total process time):
real time
0.01 seconds
cpu time
0.00 seconds 


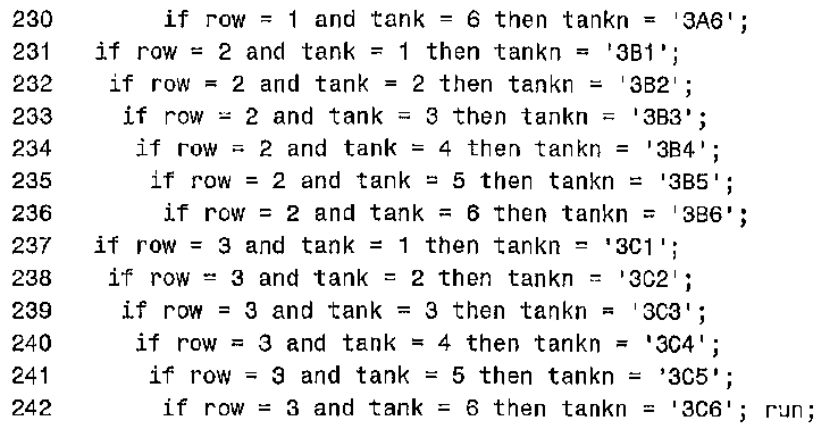

NTE: There were 180 observations read from the data set WORK.GLOCHIDIA.

NOTE: The data set WORK. GLOCHIDIADIST has 180 observations and 6 variables.

NOTE: DATA statement used (Total process time):

real time $\quad 0.01$ seconds

cpu time $\quad 0.01$ seconds

243 proc sort data $=$ glochidiadist;

244 by round $x$;

245 run;

NOTE: There were 180 observations read from the data set WORK.GLOCHIDIADIST.

NOTE: The data set WORK.GLOCHIDIADIST has 180 observations and 6 variables,

NOTE: PROCEDURE SORT used (Total process time):
real time
0.00 seconds
cpu time
0.01 seconds

246 proc print data = glochidiadist;

247 title1 $h=2$ 'Effects of Psuedomonas fluorescens (Pf-CL-145A) to glochidia from seven unionid

247 ! mussel species';

248 title2 $\mathrm{h}=1.5$ 'AEH-11-PSUEDO-01';

249 titles $h=1$ 'Random assignment of glochidia to tost tank';

250 title4 $\mathrm{h}=1$ 'Mussel species: Fatmucket'.

251 run;

NOTE: There were 180 observations read from the data set WORK.GLOCHIDIADIST.

NOTE: PROCEDURE PAINT used (Total process time):
real time
0.00 seconds
epu time
0.00 seconds 
Effects of Psuedomonas fluorescens (Pf-CL145A) to glochidia from seven unionid mussel species 1 AEH- 11 -PSUEDO-01

Random assignment of glochidia to test tanks

Nussel species: Washboard

\begin{tabular}{|c|c|c|c|c|c|c|c|}
\hline Obs & round & row & $\operatorname{tank}$ & $x$ & row $_{\text {_ }}$ & tankn & AEH-11-PSEUDO-01 \\
\hline 1 & 1 & 2 & 3 & $0.0483 \mathrm{~B}$ & $B$ & $1 \mathrm{~B} 3 \checkmark$ & \\
\hline 2 & 1 & 2 & 1 & 0.15057 & B & $1 \mathrm{~B} 1 \checkmark$ & \\
\hline 3 & 1 & 2 & 5 & 0.16278 & $B$ & $1 \mathrm{~B} 5 /$ & \\
\hline 4 & 1 & 3 & 6 & 0.18466 & $c$ & $106 \checkmark$ & \\
\hline 5 & $\uparrow$ & 2 & 4 & 0,36690 & $B$ & $1 B 4 /$ & \\
\hline 6 & 1 & 3 & 5 & 0.37006 & $\mathrm{C}$ & $105 V$ & \\
\hline 7 & 1 & 1 & 1 & 0.44366 & $\bar{A}$ & IA1 $\checkmark$ & \\
\hline 8 & 1 & 3 & 2 & 0.44674 & $C$ & $102 \checkmark$ & \\
\hline 9 & 1 & 1 & 5 & 0.45064 & A & $1 \mathrm{~A} 5 \checkmark$ & \\
\hline 10 & 1 & 1 & 2 & 0.49027 & A & $1 \mathrm{~A} 2 \checkmark$ & \\
\hline 11 & 1 & 1 & 6 & 0.56970 & A & $1 \mathrm{~A} 6 \checkmark$ & \\
\hline 12 & 1 & 2 & 6 & 0.63330 & $B$ & $186 \sqrt{2}$ & \\
\hline 13 & 1 & 3 & 1 & 0.70052 & $c$ & 1048 & \\
\hline 14 & 1 & 3 & 1 & 0.77579 & $c$ & $101 \checkmark$ & \\
\hline 15 & 1 & 3 & 3 & 0.77615 & $c$ & $1 \mathrm{c3} \checkmark$ & \\
\hline 16 & 1 & 1 & 3 & 0.77930 & A & tA3 & \\
\hline 17 & 1 & 1 & 4 & 0.82716 & A & $1 \mathrm{~A} 4 \checkmark$ & \\
\hline 18 & 1 & 2 & 2 & 0.86307 & B & $1 \mathrm{~B} 2 \mathrm{~V}$ & \\
\hline 19 & 2 & 2 & 6 & 0.05096 & $\vec{B}$ & $1 \mathrm{~B} 6 \mathrm{~V}$ & \\
\hline 20 & 2 & 1 & 3 & 0.13832 & A & $13^{\prime}$ & \\
\hline 21 & 2 & 3 & 3 & 0.21191 & $c$ & $103 v$ & \\
\hline 22 & 2 & 3 & 2 & 0.36940 & $c$ & 1021 & \\
\hline 23 & 2 & 1 & 2 & 0.38742 & A & 1A2 $\checkmark$ & \\
\hline 24 & 2 & 2 & 3 & 0.41658 & $\mathrm{~B}$ & $183 \sqrt{ }$ & \\
\hline 25 & 2 & 1 & 4 & 0.54504 & $\bar{A}$ & $1 \mathrm{A4V}$ & \\
\hline 26 & 2 & 3 & 5 & 0.58445 & $\mathrm{c}$ & $1 \mathrm{C5}$ & \\
\hline 27 & 2 & 2 & 1 & 0.61804 & B & $1 \mathrm{Bi} \sqrt{ }$ & \\
\hline 28 & 2 & 1 & 6 & 0.66695 & A & $1 \mathrm{~A} 6$ & \\
\hline 29 & 2 & 2 & 2 & 0.77401 & B & $1 \mathrm{~B} 2 \mathrm{~V}$ & \\
\hline 30 & 2 & 3 & 1 & 0.84946 & $\frac{c}{B}$ & $\frac{101 \sqrt{184}}{18}$ & \\
\hline $\begin{array}{l}31 \\
32\end{array}$ & $\begin{array}{l}2 \\
2\end{array}$ & $\begin{array}{l}2 \\
3\end{array}$ & $\begin{array}{l}4 \\
6\end{array}$ & $\begin{array}{l}0.85021 \\
0.88869\end{array}$ & $\begin{array}{l}B \\
C\end{array}$ & $106 \checkmark$ & \\
\hline 33 & 2 & 1 & i & $0.907 \div 6$ & A & $1 \mathrm{~A} 1$ & \\
\hline 34 & 2 & 2 & 5 & 0.96065 & $B$ & $185 \checkmark$ & \\
\hline 35 & 2 & 1 & 5 & 0.98905 & A & $1 A 5$ & \\
\hline 36 & 2 & 3 & 4 & 0.99356 & $\mathrm{c}$ & 104 & \\
\hline 37 & 3 & 3 & 3 & 0.00319 & C & $103 \sqrt{ }$ & \\
\hline 38 & 3 & 2 & 1 & 0.00885 & B & $1 \mathrm{B1}$ & $\cdot$ \\
\hline 39 & 3 & 3 & 6 & 0.06275 & $c$ & $106 /$ & \\
\hline 40 & 3 & 1 & 4 & 0.15052 & A & $1 \mathrm{~A} 4$ & \\
\hline 41 & 3 & 2 & 3 & 0.20804 & B & $13^{2}$ & \\
\hline 42 & 3 & 3 & 4 & 0.46083 & $\mathrm{c}$ & 104 & \\
\hline 43 & 3 & 2 & 2 & 0.47385 & B & $1 B 2 /$ & \\
\hline 44 & 3 & 2 & 6 & 0.56072 & $B$ & $1 \mathrm{~B} 6 \mathrm{~J}$ & \\
\hline 45 & 3 & 2 & 4 & 0.56497 & B & $1 \mathrm{~B} 4 r$ & \\
\hline 46 & 3 & 3 & 2 & 0.57337 & $c$ & $1 \mathrm{C} 2 \mathrm{~J}$ & \\
\hline 47 & 3 & 3 & 1 & 0.63977 & $c$ & $101 /$ & \\
\hline 48 & 3 & 1 & 2 & 0.65438 & A & $1 \mathrm{~A} 2 \mathrm{~V}$ & \\
\hline 49 & 3 & 2 & 5 & 0.73127 & $B$ & $185 \checkmark$ & \\
\hline 50 & 3 & 3 & 5 & 0.82134 & c & 105 & \\
\hline 51 & 3 & $t$ & 6 & 0.82425 & $\begin{array}{l}A \\
A\end{array}$ & $\begin{array}{l}1 \mathrm{~A} 6 \\
1 \mathrm{~A} 5\end{array}$ & \\
\hline 52 & 3 & 1 & 5 & 0.92219 & A & & \\
\hline
\end{tabular}


Effects of Psuedomonas fluorescons (Pf-CL145A) to glochidia from seven unionid mussel species 2 AEH - 11 - PSUEDO-01

Random assignment of glochidia to test tanks

Mussel species: Washboard

Obs round row tank $x$ row_ tankn AEH-11-PSEUDO-01

$\begin{array}{lllll}53 & 3 & 1 & 1 & 0.96150 \\ 54 & 3 & 1 & 3 & 0.99602\end{array}$

54

$\begin{array}{lllll}56 & 4 & 2 & 3 & 0.03225\end{array}$

0.03225
0.07077

0.07077

0.10623

0.27354

0.29696

0.29785

0.31372

0.43548

0.49463

0.57987

0.72352

0.91329

0.81675

0.92533

0.96086

0.97561

0.01106

0.18516

0.21654

0.26751

0.30069

0.43225

0.48596

0.48743

0.54357

0.63776

0.73840

0.75303

0.77589

0.80186

0.80385

0.88062

0.91017

0.93914

0.10293

0.12505

0.13401

0.17936

0.27550

0.31435

0.35475

0.37608

0.38250

0.45321

0.48700

0.49334

0.57207

0.64539

\begin{tabular}{cl}
$A$ & $1 A 1 \sqrt{A}$ \\
\hline$A$ & $1 A 3 \sqrt{A}$ \\
\hline$A$ & $N A C$ \\
$A$ & NAB
\end{tabular}

C $\mathrm{NCB}$

C $+\mathrm{C2}$

$\frac{B}{B} \quad$ LAB

B $-1 B 1$

C $\because C 6$

A

$A \quad A A 4$

$\frac{A}{C}=1 \mathrm{~A} 5$

B 대 34

C $\quad * C 4$

B

$A$ HAS

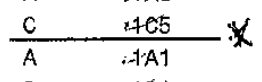

B $\angle B 1$

$B \quad 4 B 6$

C $A C 1$

$A$ HA2

$\frac{B}{A}-\frac{1 B 2}{A A 6} *$

$B \quad 2-135$

$C$ 대

C $\quad 703$

B, 484

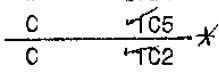

B HE3

C TC6

A WA3

A ITA5

$\frac{A}{A} \frac{\text { TA } 4}{1 A 3} *$ rounds of glochidic were addod

A $1 \mathrm{A2}$ Five rounds of glochidia were acod $6-10$ of

C 101 to exporare chambers. Rounds bere not used.

B 185 the randorn assignoment were neted for clarificotion

A IA4 5 toms $5 / 14 / 10$

A. $\quad 1 \mathrm{AG}$

C $\quad 1 C 4$

A $1 \mathrm{~A} 1$

B $1 B 4$

C $1 \mathrm{Ce}$

B $1 \mathrm{~B} 3$

A

Analysis performed by $M$. Gaikowski SAS version $9.211: 03$ 140GT11

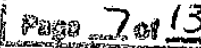

(1) Distribution aliquot afiter IA3 not 111 KKW $B$ BOCTII 
Effects of Psuedomonas fluorescens (Pf-CL145A) to glochidia from seven unionid mussel. species 3 AEH- $1 \uparrow$-PSUED0-01

Pandom assignment of glochidia to test tanks

Nussel species: Wastboard

\begin{tabular}{|c|c|c|c|c|c|c|}
\hline Obs & round & row & tank & $x$ & row & tankn \\
\hline 105 & 6 & 2 & 1 & 0.65028 & B & $1 B 1$ \\
\hline 106 & 6 & 3 & 3 & 0.69455 & $c$ & 103 \\
\hline 107 & 6 & 3 & 2 & 0.74112 & $c$ & $1 \mathrm{C} 2$ \\
\hline 108 & 6 & 3 & 5 & 0.91209 & $c$ & 105 \\
\hline 109 & 7 & 2 & 2 & 0.07653 & B & $1 \mathrm{~B} 2$ \\
\hline 110 & 7 & 3 & 4 & 0.12108 & $\mathrm{C}$ & $1 \mathrm{C4}$ \\
\hline 111 & 7 & 1 & 4 & 0.12753 & $A$ & $1 \mathrm{A4}$ \\
\hline 112 & 7 & 2 & 3 & 0.14355 & B & $1 \mathrm{B3}$ \\
\hline 113 & 7 & 1 & 3 & 0.21451 & A & $1 \mathrm{AB}$ \\
\hline 114 & 7 & 3 & 1 & 0.21495 & 0 & 101 \\
\hline 115 & 7 & 3 & 6 & 0.24565 & $c$ & 106 \\
\hline 116 & 7 & 3 & 2 & 0.35784 & $c$ & $1 \mathrm{C2}$ \\
\hline 117 & 7 & 2 & 6 & 0.37076 & $B$ & $1 \mathrm{~B} 6$ \\
\hline 118 & 7 & 1 & 5 & 0.48846 & $A$ & $1 \mathrm{~A} 5$ \\
\hline 119 & 7 & 1 & 6 & 0.49531 & $A$ & $1 A 6$ \\
\hline 120 & 7 & 3 & 5 & 0.62835 & $c$ & $1 \mathrm{C5}$ \\
\hline 121 & 7 & 1 & 1 & 0.66040 & A & $1 \mathrm{~A} 1$ \\
\hline 122 & 7 & 2 & 1 & 0.78412 & B & $1 \mathrm{B1}$ \\
\hline 123 & 7 & 3 & 3 & 0.81819 & C & 103 \\
\hline 124 & 7 & 2 & 5 & 0.84879 & $B$ & $1 \mathrm{BS}$ \\
\hline 125 & 7 & 2 & 4 & 0.91208 & $B$ & 184 \\
\hline 126 & 7 & 1 & 2 & 0.93405 & A & $1 \mathrm{~A} 2$ \\
\hline 127 & 8 & 1 & 4 & 0.07285 & A & $1 \mathrm{A4}$ \\
\hline 128 & 8 & 2 & 2 & 0.13071 & $\mathrm{~B}$ & $1 \mathbf{B 2}$ \\
\hline 129 & 8 & 3 & 3 & 0.17105 & C & $1 \mathrm{C3}$ \\
\hline 130 & 8 & 1 & 6 & 0.17484 & A & $1 \mathrm{~A} 6$ \\
\hline 131 & 8 & 1 & 1 & 0.17628 & A & $1 \mathrm{~A} 1$ \\
\hline 132 & 8 & 3 & 4 & 0.21731 & $\mathrm{C}$ & $1 \mathrm{C} 4$ \\
\hline 133 & 8 & 3 & 1 & 0.29783 & $\mathrm{C}$ & $1 \mathrm{C1}$ \\
\hline 134 & 8 & 2 & 3 & 0.39927 & $B$ & $1 \mathrm{~B} 3$ \\
\hline 135 & 8 & 3 & 2 & 0.44313 & $c$ & $1 \mathrm{C2}$ \\
\hline 136 & 8 & 3 & 5 & 0.46488 & $c$ & 105 \\
\hline 137 & 8 & 2 & 5 & 0.48661 & $\mathrm{~B}$ & $1 \mathrm{BS}$ \\
\hline 138 & 8 & 2 & 1 & 0.65784 & $B$ & $1 \mathrm{~B} 1$ \\
\hline 139 & 8 & 3 & 6 & 0.74051 & $\mathrm{C}$ & $1 \mathrm{C6}$ \\
\hline 140 & 8 & 1 & 5 & 0.74383 & A & $1 \mathrm{~A} 5$ \\
\hline 141 & 8 & 1 & 2 & 0.75499 & A & $1 \mathrm{~A} 2$ \\
\hline 142 & 8 & 2 & 4 & 0.77273 & B & $1 \mathrm{BA}$ \\
\hline 143 & 8 & 2 & 6 & 0.85171 & $B$ & $1 \mathrm{~B} 6$ \\
\hline 144 & 8 & 1 & 3 & 0.98418 & A & $1 \mathrm{~A} 3$ \\
\hline 145 & 9 & 2 & 5 & 0.00420 & $B$ & $1 \mathrm{BS}$ \\
\hline 146 & 9 & 1 & 5 & 0.07321 & A & $1 \mathrm{AS}$ \\
\hline 147 & 9 & 2 & 4 & 0.10943 & $B$ & $1 \mathrm{~B} 4$ \\
\hline 148 & 9 & 1 & 1 & 0.12746 & A & $1 \mathrm{~A} 1$ \\
\hline 149 & 9 & 1 & 3 & 0.12940 & A & $1 \mathrm{AB}$ \\
\hline 150 & 9 & 2 & 3 & 0.21011 & $B$ & $1 \mathrm{~B} 3$ \\
\hline 151 & 9 & 3 & 2 & 0.22581 & $c$ & $1 \mathrm{C2}$ \\
\hline 152 & 9 & 3 & 4 & 0.23737 & $c$ & $1 \mathrm{C} 4$ \\
\hline 153 & 9 & 3 & 3 & 0.24321 & $c$ & $1 \mathrm{C3}$ \\
\hline 154 & $\theta$ & 3 & 6 & 0.56414 & 0 & $1 \mathrm{C6}$ \\
\hline 155 & 9 & 1 & 4 & 0.63829 & A & $1 \mathrm{A4}$ \\
\hline 156 & $\theta$ & 2 & 6 & 0.63940 & $B$ & $1 \mathrm{~B} 6$ \\
\hline
\end{tabular}

Analysis performed by 14 . Gaikowski SAS version 9.2 11:03 1400T11

AEH-11-PSEUDO-01

8 ast 13 
Effects of Psuedomonas fluorescens ( $\mathrm{Pf}-\mathrm{CL} 145 \mathrm{~A}$ ) to glochidia from seven unionid mussel species 4 AEH - 11-PSUEDO- 01

Randon assignment of glochidia to test tanks

Mussel species: Washboard

\begin{tabular}{|c|c|c|c|c|c|c|}
\hline Obs & round & row & tank & $x$ & row & tankn \\
\hline 157 & $\theta$ & 3 & 1 & 0.78984 & $c$ & $1 \mathrm{Ct}$ \\
\hline$\uparrow 58$ & 9 & 1 & 2 & 0.83409 & A & $1 \mathrm{AZ}$ \\
\hline 159 & $\theta$ & 2 & 2 & 0.83827 & B & $1 \mathrm{~B} 2$ \\
\hline 160 & 9 & 2 & 1 & 0.86606 & B & 1B1 \\
\hline 161 & 9 & 1 & $B$ & 0.93247 & A & $1 \mathrm{~A} 6$ \\
\hline 162 & 9 & 3 & 5 & 0.97047 & $c$ & 105 \\
\hline 163 & 10 & 1 & 5 & 0.13868 & A & $1 \mathrm{A5}$ \\
\hline 164 & 10 & 2 & 3 & 0.19793 & $B$ & $1 \mathrm{BO}$ \\
\hline 165 & 10 & 2 & 1 & 0.22524 & B & $1 \mathrm{~B} 1$ \\
\hline 166 & 10 & 2 & 2 & 0.26319 & $B$ & $1 \mathrm{~B} 2$ \\
\hline 167 & 10 & 1 & 1 & 0.33809 & A & $1 \mathrm{~A} 1$ \\
\hline 168 & 10 & 1 & 2 & 0.35208 & A & $1 \mathrm{~A} 2$ \\
\hline 169 & 10 & 2 & 6 & 0.40875 & B & $1 \mathrm{~B} 6$ \\
\hline 170 & 10 & 2 & 5 & 0.49523 & $B$ & $1 \mathrm{~B} 5$ \\
\hline 171 & 10 & 3 & 2 & 0.54407 & $c$ & $1 \mathrm{C} 2$ \\
\hline 172 & 10 & 3 & 6 & 0.62890 & $c$ & $1 \mathrm{Cb}$ \\
\hline$\$ 73$ & 10 & 3 & 4 & 0.66996 & $c$ & $1 \mathrm{C4}$ \\
\hline 174 & 10 & 1 & 3 & 0.69539 & A & $1 \mathrm{AB}$ \\
\hline 175 & 10 & 1 & 6 & 0.73423 & A & $1 A 6$ \\
\hline 176 & 10 & 3 & 1 & 0.77376 & $c$ & 101 \\
\hline 177 & 10 & 2 & 4 & 0.81274 & $B$ & $1 \mathrm{~B} 4$ \\
\hline 178 & 10 & 3 & 5 & 0.83455 & $c$ & 105 \\
\hline 179 & 10 & 3 & 3 & 0.98332 & $c$ & $1 \mathrm{C3}$ \\
\hline 180 & 10 & 1 & 4 & 0.99509 & $A$ & $1 \mathrm{~A} 4$ \\
\hline
\end{tabular}

AEH-11-PSEUDO-01 
Effects of Psuedomonas fluorescens (Pf.CL145A) to glochidia from seven unionid mussel species 5 AEH-11-PSUEDO-01

Random assignment of glochidia to test tank

Mussel species: Fatmueket p/ain Poila $/$ bosk

obs round row tank

\subsection{4}

0.02206

0.03535

0.06789

0. 09694

0.14775

0.16191

0.20688

0.20991

0.30468

0.36183

0.45100

0.54897

0.68545

0.69170

0.70064

0.70843

0.90442

0.00545

0.11329

0.13927

0.17332

0.22802

0.31714

o. 38788

0.39340

0.40032

0.47171

0.54855

0.57131

0.61824

0.72234

0.79335

0.82843

0.93168

0.99853

0.02972

0.05996

0.20484

0.29113

0.29706

0.31291

0.38686

0.42806

0.53249

0.57085

0.57194

0.58116

0.65021

0.68760

0.91176

0.93952 _row_ tankn

B
B
B
C
C

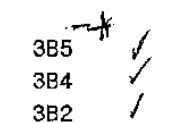

3B2 $f$

3B1 $\checkmark$

3C6 6

3034

$3 \mathrm{C} 1$

3 A3

उA6 $I$

3A2

$3 \mathrm{~B} 3 \quad \sqrt{3}-\mathrm{d}$

3C5 $\frac{d}{3 \mathrm{~B} 6}$

$3 A 4 \checkmark$

$3 A 1 \checkmark$

3A5 $f$

$302 \sqrt{ }+1$

301

385

3A2 $\checkmark$

$3 \mathrm{C6} \checkmark$

$3 \mathrm{~A} 6 \checkmark$

$384 \checkmark-\approx$

$3 B 6 \sqrt{ }$

3A1 $\checkmark$

3A5 $\checkmark$

$381 /$

$3 \mathrm{C} 57$

$3 \mathrm{C} 2 \checkmark$

$3 \mathrm{A3} \gamma$

3 B3

3A4 $\sqrt{ }$

$304 \mathrm{f}$

302

$3 \mathrm{C} 4$

3A1 $f$

$3 \mathrm{~B} 31$

385

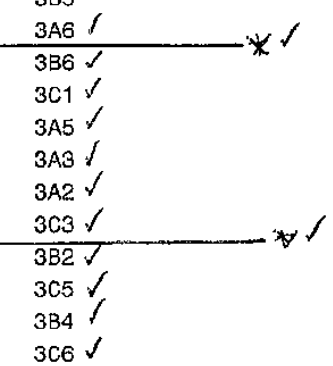

(1) Species chengel to

plain pockest toosk

dow to avarkletile

$10-18 \cdot 4$

sits

\section{AEH-11-PSEUDO-01}

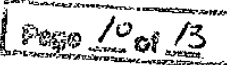

Analysis performed by M. Gaikowski SAS version $9.211: 03$ 1400T11

$$
\begin{aligned}
& \text { * sopel fire andind adstritulor } \\
& \text { conet. } 10 \times 451 \mathrm{~J} \text {. }
\end{aligned}
$$


Effects of Psuedomonas fluorescens (Pf-CL145A) to glochidia from seven unionid mussel species 6 AEH-11-PSUEDO-01 Randon assignment of glochidia to test tank

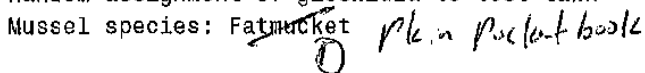

\begin{tabular}{|c|c|c|}
\hline Obs & round & row \\
\hline 53 & 3 & 2 \\
\hline 54 & 3 & 1 \\
\hline 55 & 4 & 1 \\
\hline 56 & 4 & 3 \\
\hline 57 & 4 & 2 \\
\hline 58 & 4 & 1 \\
\hline 59 & 4 & 3 \\
\hline 60 & 4 & 3 \\
\hline 61 & 4 & 2 \\
\hline 62 & 4 & 2 \\
\hline 63 & 4 & 3 \\
\hline 64 & 4 & 2 \\
\hline 65 & 4 & 1 \\
\hline 66 & 4 & 2 \\
\hline 67 & 4 & 2 \\
\hline 68 & 4 & 1 \\
\hline 69 & 4 & 1 \\
\hline 70 & 4 & 1 \\
\hline 71 & 4 & 3 \\
\hline 72 & 4 & 3 \\
\hline 73 & 5 & 1 \\
\hline 74 & 5 & 3 \\
\hline 75 & 5 & 1 \\
\hline 76 & 5 & 3 \\
\hline 77 & 5 & 3 \\
\hline 78 & 5 & 1 \\
\hline 79 & 5 & 2 \\
\hline 80 & 5 & 2 \\
\hline 81 & 5 & 1 \\
\hline 82 & 5 & 2 \\
\hline 83 & 5 & 2 \\
\hline 84 & 5 & 2 \\
\hline 85 & 5 & 1 \\
\hline 86 & 5 & 3 \\
\hline 87 & 5 & 3 \\
\hline 88 & 5 & 1 \\
\hline 89 & 5 & 2 \\
\hline 90 & 5 & 3 \\
\hline 91 & 6 & 3 \\
\hline 92 & 6 & 2 \\
\hline 93 & 6 & 3 \\
\hline 94 & 6 & 3 \\
\hline 95 & 6 & 3 \\
\hline 96 & 6 & 1 \\
\hline 97 & 6 & 1 \\
\hline 98 & 6 & 1 \\
\hline 99 & 6 & 1 \\
\hline 100 & 6 & 2 \\
\hline 101 & 6 & 3 \\
\hline 102 & 6 & 2 \\
\hline 103 & 6 & 2 \\
\hline 104 & 6 & 1 \\
\hline
\end{tabular}

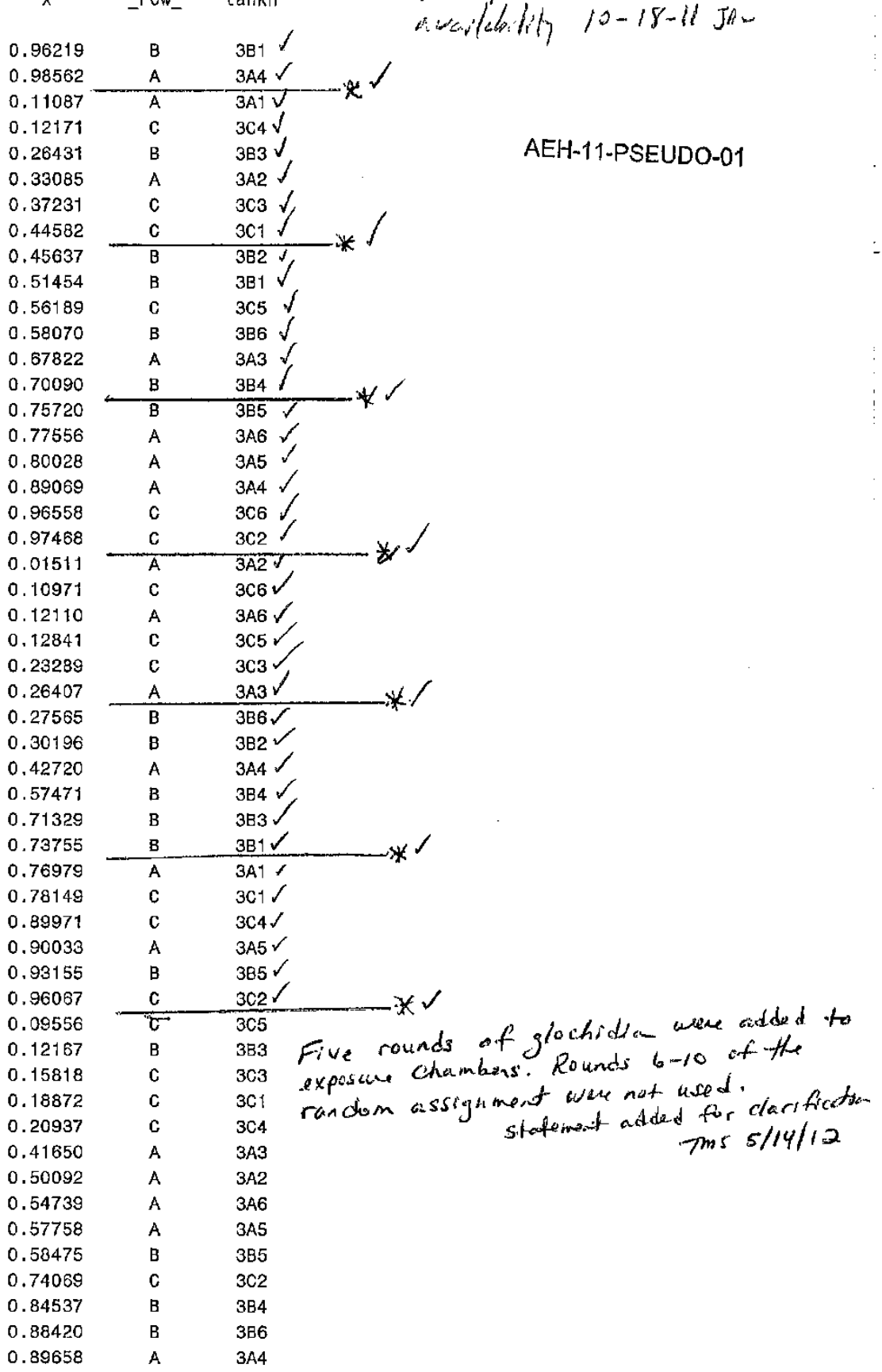

Analysis performed by $M$. Gaikowski SAS version $9.211: 03$ 140CT11

D speces chingel to

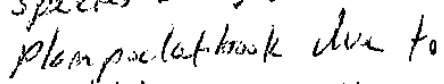
riwailcbility 10-18-11 JAw

AEH-11-PSEUDO-01 
Effects of Psuedomonas fluorescens (Pf-CL145A) to glochidia from seven unionid mussel species 7 AEH-11 - PSUEDO-01

Random assignment of glochidia to test tank

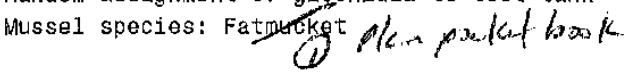

Obs round row tank $x \quad$ row_ tankn

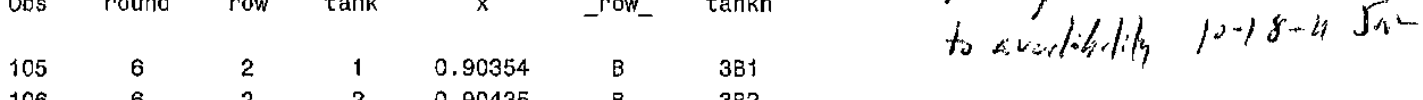

\begin{tabular}{|c|c|c|c|c|c|c|}
\hline & & & & & & \\
\hline 106 & 6 & 2 & 2 & 0.90435 & $B$ & $3 B 2$ \\
\hline 107 & 6 & 3 & 6 & 0.96706 & $c$ & $3 C 6$ \\
\hline 108 & 6 & 1 & 1 & 0.97874 & A & $3 A 1$ \\
\hline 109 & 7 & 2 & 4 & 0.02158 & $B$ & $3 B 4$ \\
\hline 110 & 7 & 2 & 6 & 0.06957 & B & $3 B 6$ \\
\hline 111 & 7 & 3 & 3 & 0.11259 & c & 303 \\
\hline 112 & 7 & 1 & 6 & 0.18067 & A & $3 A 6$ \\
\hline 113 & 7 & 1 & 2 & 0.27586 & A & $3 A 2$ \\
\hline 114 & 7 & 1 & 5 & 0.28208 & A & 3A5 \\
\hline 115 & 7 & 3 & 2 & 0.29497 & $c$ & $3 \mathrm{C} 2$ \\
\hline 116 & 7 & 2 & 2 & 0.35074 & $B$ & Зв2 \\
\hline 117 & 7 & 3 & 4 & 0.38960 & $c$ & $3 \mathrm{C} 4$ \\
\hline 118 & 7 & 2 & 1 & 0.50335 & $B$ & 3日1 \\
\hline 119 & 7 & 1 & 3 & 0.51187 & A & $3 A 3$ \\
\hline 120 & 7 & 2 & 5 & 0.77789 & $B$ & 385 \\
\hline 121 & 7 & 1 & 1 & 0.80690 & A & $3 A 1$ \\
\hline 122 & 7 & 3 & 1 & 0.89798 & C & $3 \mathrm{CI}$ \\
\hline 123 & 7 & 1 & 4 & 0.95446 & A & 3A4 \\
\hline 124 & 7 & 3 & 5 & 0.96114 & c & $3 \mathrm{C5}$ \\
\hline 125 & 7 & 2 & 3 & 0.99428 & B & $3 \mathrm{~B} 3$ \\
\hline 126 & 7 & 3 & 6 & 0.99761 & C & $3 \mathrm{C6}$ \\
\hline 127 & 8 & 1 & 3 & 0.01429 & A & $3 A 3$ \\
\hline 128 & 8 & 3 & 1 & 0.07414 & $c$ & $3 \mathrm{C} 1$ \\
\hline 129 & 8 & 1 & 5 & 0.14921 & A & 3A5 \\
\hline 130 & 8 & 3 & 4 & 0.21611 & 0 & $3 C 4$ \\
\hline 131 & 8 & 2 & 5 & 0.26547 & $B$ & 3B5 \\
\hline 132 & 8 & 1 & 1 & 0.31691 & A & $3 A 1$ \\
\hline 133 & 8 & 3 & 5 & 0.34881 & C & $3 \mathrm{C5}$ \\
\hline 134 & 8 & 3 & 6 & 0.37552 & c & $3 \mathrm{C} 6$ \\
\hline 135 & 8 & 2 & 2 & 0.37813 & B & $3 \mathrm{~B} 2$ \\
\hline 136 & 8 & 1 & 6 & 0.39360 & A & $3 A C$ \\
\hline 137 & 8 & 2 & 1 & 0.49526 & $B$ & $3 \mathrm{~B} 1$ \\
\hline 138 & 8 & 2 & 6 & 0.50407 & B & $3 \mathrm{~B} 6$ \\
\hline 139 & 8 & 2 & 4 & 0.65842 & $B$ & $3 B 4$ \\
\hline$\{40$ & 8 & 3 & 2 & 0.71088 & C & $3 \mathrm{C} 2$ \\
\hline 141 & 8 & 1 & 4 & 0.76065 & A & $3 A 4$ \\
\hline 142 & 8 & 1 & 2 & 0.83296 & A & $3 \mathrm{~A} 2$ \\
\hline$\{43$ & 8 & 3 & 3 & 0.94481 & 0 & 363 \\
\hline 144 & 8 & 2 & 3 & 0.97151 & B & 3B3 \\
\hline 145 & 9 & 3 & 3 & 0.09622 & c & $3 \mathrm{CO}$ \\
\hline 146 & 9 & 1 & 2 & 0.13471 & A & $3 A 2$ \\
\hline 147 & 9 & 1 & 4 & 0.18266 & A & $3 A 4$ \\
\hline 148 & 9 & 3 & 4 & 0.37323 & $c$ & $3 C 4$ \\
\hline 149 & 9 & 2 & 2 & 0.41030 & B & $3 B 2$ \\
\hline 150 & 9 & 3 & 2 & 0.41378 & 0 & $3 \mathrm{C2}$ \\
\hline 151 & 9 & 1 & 1 & 0.42871 & A & $3 A 1$ \\
\hline 152 & $\theta$ & 3 & 1 & 0.46783 & c & 301 \\
\hline 153 & 9 & 2 & 6 & 0.59253 & B & 3B6 \\
\hline 154 & 9 & 1 & 5 & 0.59983 & A & 3A5 \\
\hline 155 & 9 & 2 & 5 & 0.61762 & B & $3 B 5$ \\
\hline 156 & 9 & 1 & 3 & 0.66848 & A & $3 A 3$ \\
\hline
\end{tabular}

AEH-11-PSEUDO-01

Analysis performed by $M$. Gajkowski SAS version $9.211: 03$ 140CT11

1213 


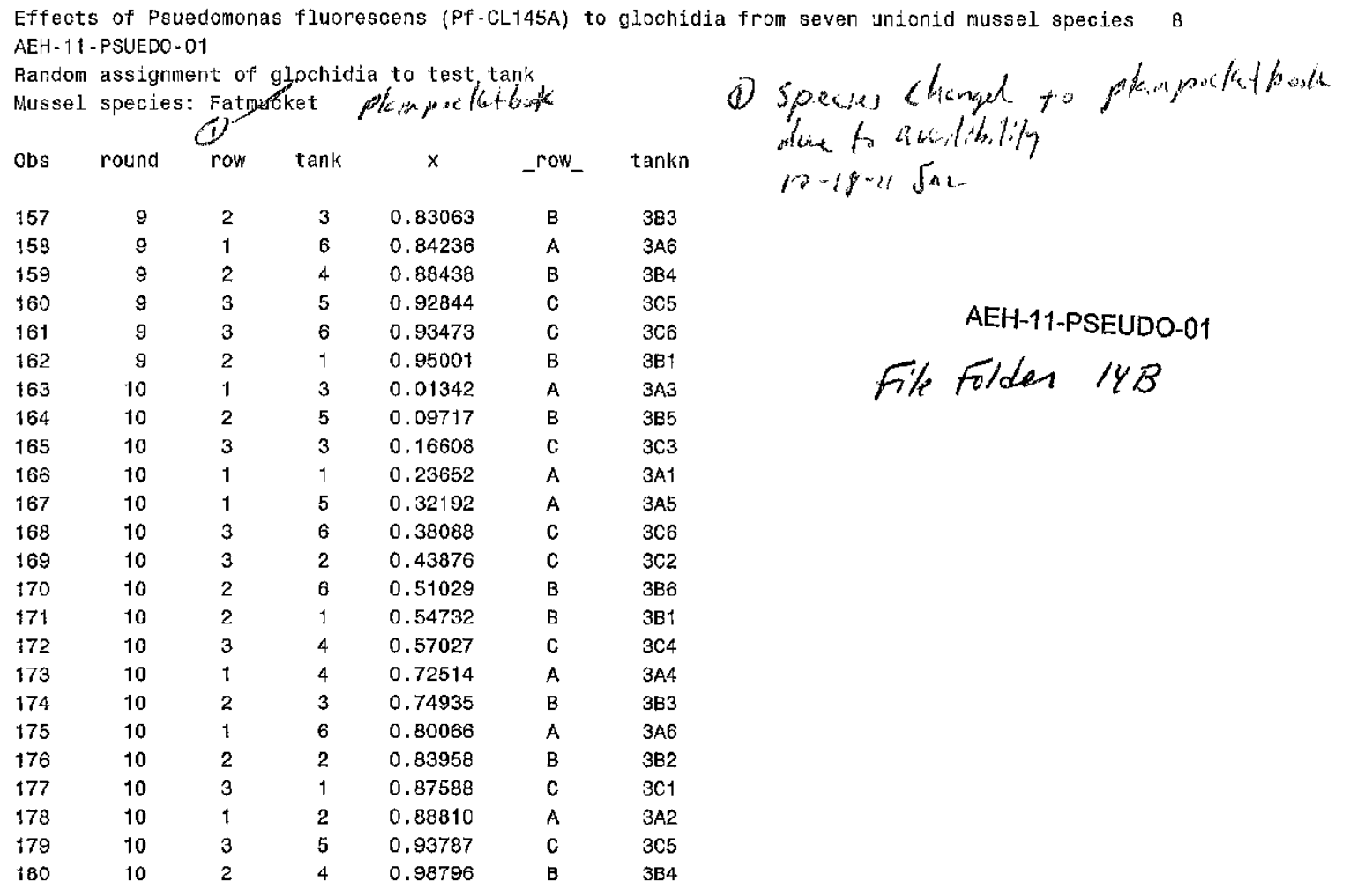

WOOED

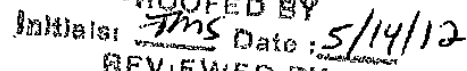

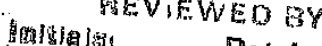

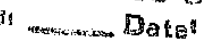

Analysis performed by M. Gaikowski SAS version $9.211: 03$ 1400711 AMPL 


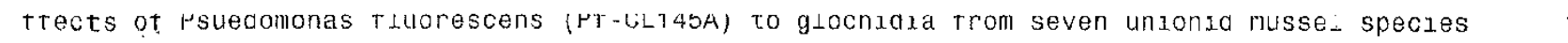
EH-11-PS JEDO-01 andom assignment of glochidia to test tanks Jiv ussel species: Black sandshell.

$L \cdot g$ buik 1,8$) 30-31$

fie foldew ${ }^{A E H} 11$-PSEUDO-01

\begin{tabular}{|c|c|c|c|c|c|c|c|}
\hline bs & round & row & $\tan k$ & $x$ & row $_{-}$ & $\operatorname{tankn}$ & $\begin{array}{c}\text { Initiatez at } 1026 \text { h } 5 K W \\
172 A N 11\end{array}$ \\
\hline 1 & 1 & 1 & 1 & 0.03540 & A & $1 \mathrm{~A} 1 \mathrm{~J}$ & \\
\hline 2 & 1 & 2 & 5 & 0.15720 & $\mathrm{~B}$ & $1 \mathrm{~B} 5$ & 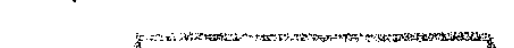 \\
\hline 3 & 1 & 1 & 2 & 0.16134 & $A$ & $1 \mathrm{~A} 2 /$ & 7 \\
\hline 4 & 1 & 2 & 3 & 0.36077 & $\mathrm{~B}$ & 1 B3 $\checkmark$ & 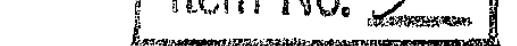 \\
\hline 5 & 1 & 1 & 5 & 0.39275 & A & $1 \mathrm{~A} 5 \checkmark$ & \\
\hline 6 & 1 & 3 & 6 & 0.39514 & C & $1 \mathrm{C6} \checkmark$ & \\
\hline 7 & 1 & 2 & 2 & 0.42251 & $\mathrm{~B}$ & $1 \mathrm{~B} 2 \sqrt{2}$ & \\
\hline 8 & 1 & 1 & 6 & 0.43934 & A & $1 \mathrm{~A} 6^{\prime}$ & \\
\hline 9 & 1 & 3 & 4 & 0.45629 & $\mathrm{C}$ & 104 & \\
\hline 10 & 1 & 3 & 1 & 0.46298 & C & $1 \mathrm{C1}$ & \\
\hline 11 & 1 & 1 & 4 & 0.46826 & $A$ & $1 \mathrm{~A} 4^{\sqrt{ }}$ & \\
\hline 12 & 1 & 3 & 3 & 0.49509 & C & $1 \mathrm{C} 3 \checkmark$ & \\
\hline 13 & 1 & 3 & 2 & 0.53590 & $\mathrm{C}$ & $\overline{1 C 2 ~} \sqrt{2}$ & \\
\hline 14 & 1 & 2 & 4 & 0.63459 & $B$ & $1 \mathrm{~B} 4 \checkmark$ & \\
\hline 15 & 1 & 1 & 3 & 0.72859 & A & 1 A3 $\checkmark$ & \\
\hline 16 & 1 & 3 & 5 & 0.73196 & $c$ & $1 \mathrm{c} 5$ & \\
\hline 17 & 1 & 2 & 6 & 0.85674 & B & 1B6 & \\
\hline 18 & 1 & 2 & 1 & 0.86215 & $B$ & $1 B 1 \checkmark$ & 032 \\
\hline 19 & 2 & 1 & 1 & 0.00152 & A & $1 \mathrm{~A} 1 \checkmark$ & \\
\hline 20 & 2 & 2 & 6 & 0.01044 & $B$ & $1 \mathrm{~B} 6 /$ & \\
\hline 21 & 2 & 2 & 1 & 0.01183 & $B$ & iB1 $\checkmark$ & \\
\hline 22 & 2 & 1 & 6 & 0.09963 & A & $1 A 6$ & \\
\hline 23 & 2 & 3 & 6 & 0.16381 & C & 106 & \\
\hline 24 & 2 & 3 & 1 & 0.37238 & $C$ & $101 \checkmark$ & \\
\hline 25 & 2 & 3 & 2 & 0.39250 & $c$ & $1 \mathrm{c2} \checkmark$ & \\
\hline 26 & 2 & 1 & 5 & 0.39924 & A & 1A5 $\checkmark$ & \\
\hline 27 & 2 & 1 & 3 & 0.48046 & A & $1 A 3 \checkmark$ & \\
\hline 28 & 2 & 2 & 3 & 0.57825 & $B$ & $1 \mathrm{~B} 3 /$ & \\
\hline 29 & 2 & 3 & 4 & 0.65481 & $C$ & $1 \mathrm{C} 4 \checkmark$ & \\
\hline 30 & 2 & 2 & 2 & 0.71580 & $B$ & $1 \mathrm{~B} 2 \sqrt{ }$ & \\
\hline 31 & 2 & 3 & 3 & 0.73748 & $c$ & $103 \checkmark$ & \\
\hline 32 & 2 & 3 & 5 & 0.76035 & $c$ & $105 \checkmark$ & \\
\hline 33 & 2 & 1 & 4 & 0.79744 & A & $1 \mathrm{~A} 4 \checkmark$ & \\
\hline 34 & 2 & 1 & 2 & 0.87184 & $A$ & $1 \mathrm{~A} 2 \mathrm{~V}$ & \\
\hline 35 & 2 & 2 & 4 & 0.93146 & $\mathrm{~B}$ & $1 \mathrm{~B} 4 /$ & \\
\hline 36 & 2 & 2 & 5 & 0.94375 & $B$ & $185 \sqrt{ }$ & \\
\hline 37 & 3 & 1 & 3 & 0.08401 & A & $\overline{\mathrm{A} 3} \sqrt{ }$ & 1037 \\
\hline 38 & 3 & 3 & 5 & 0.11492 & C & $105 \checkmark$ & $90 \mathrm{~Eb}$ \\
\hline 39 & 3 & 1 & 4 & 0.12051 & A & $1 \mathrm{~A} 4 \checkmark$ & 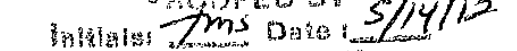 \\
\hline 40 & 3 & 1 & 1 & 0.17095 & A & $1 \mathrm{~A} 1 \checkmark$ & 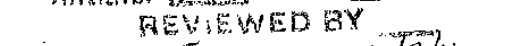 \\
\hline 41 & 3 & 2 & 6 & 0.25392 & $B$ & $1 \mathrm{~B} 6 \sqrt{ }$ & 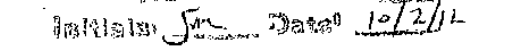 \\
\hline 42 & 3 & 2 & 3 & 0.26144 & $B$ & $1 \mathrm{~B} 3 \checkmark$ & \\
\hline 43 & 3 & 3 & 4 & 0.40117 & $\mathrm{C}$ & $\overline{104 /}$ & \\
\hline 44 & 3 & 1 & 6 & 0.41257 & A & $1 A 6 \checkmark$ & 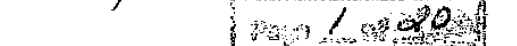 \\
\hline 45 & 3 & 1 & 2 & 0.45539 & A & $1 \mathrm{~A} 2 \checkmark$ & \\
\hline 46 & 3 & 2 & 5 & 0.51031 & $\mathrm{~B}$ & $1 \mathrm{~B} 5 \mathrm{~J}$ & \\
\hline 47 & 3 & 1 & 5 & 0.55130 & A & $1 \mathrm{~A} 5 \mathrm{~V}$ & * Sample for \\
\hline
\end{tabular}



ffects of Psuedomonas fluorescens (Pf-CL145A)
EH-11-PSUEDO-01
andom assignment of glochidia to test tanks $\mathrm{J}$,
ussel species: Black sandshell

bs round row tank $x$ row_ tankn AEH-11-PSEUDO-D1

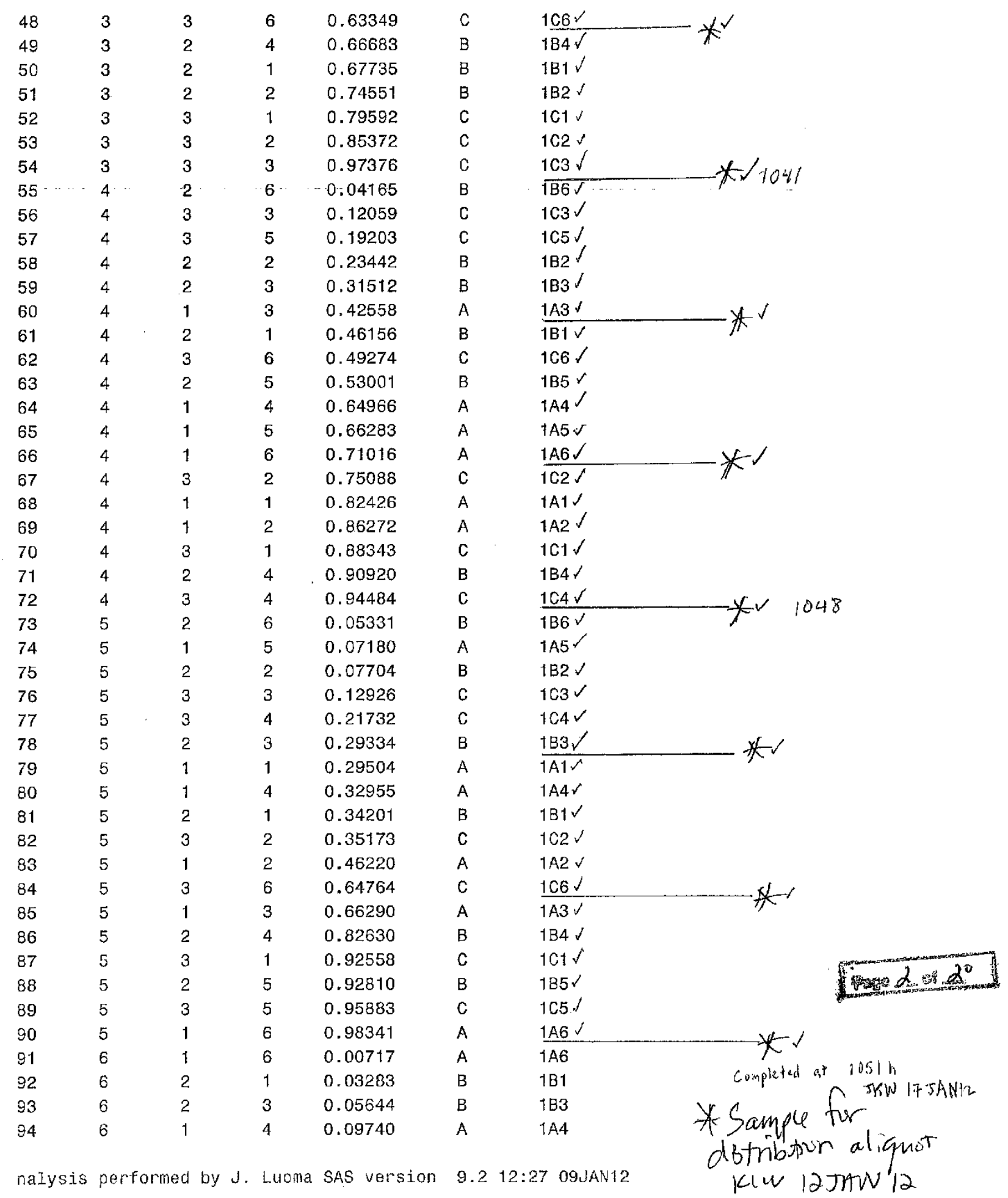


ffects of Psuedomonas thorescens (Pt-CL145A) to glochidia trom seven unionid mussed species EH-11-PSUEDO-01

andom assignment of glochidia to test tanks $J_{4}$ ussel species: Black sandshell

AEH-11-PSEUDO-01

bs round row tank $x \quad$ row_ tankn

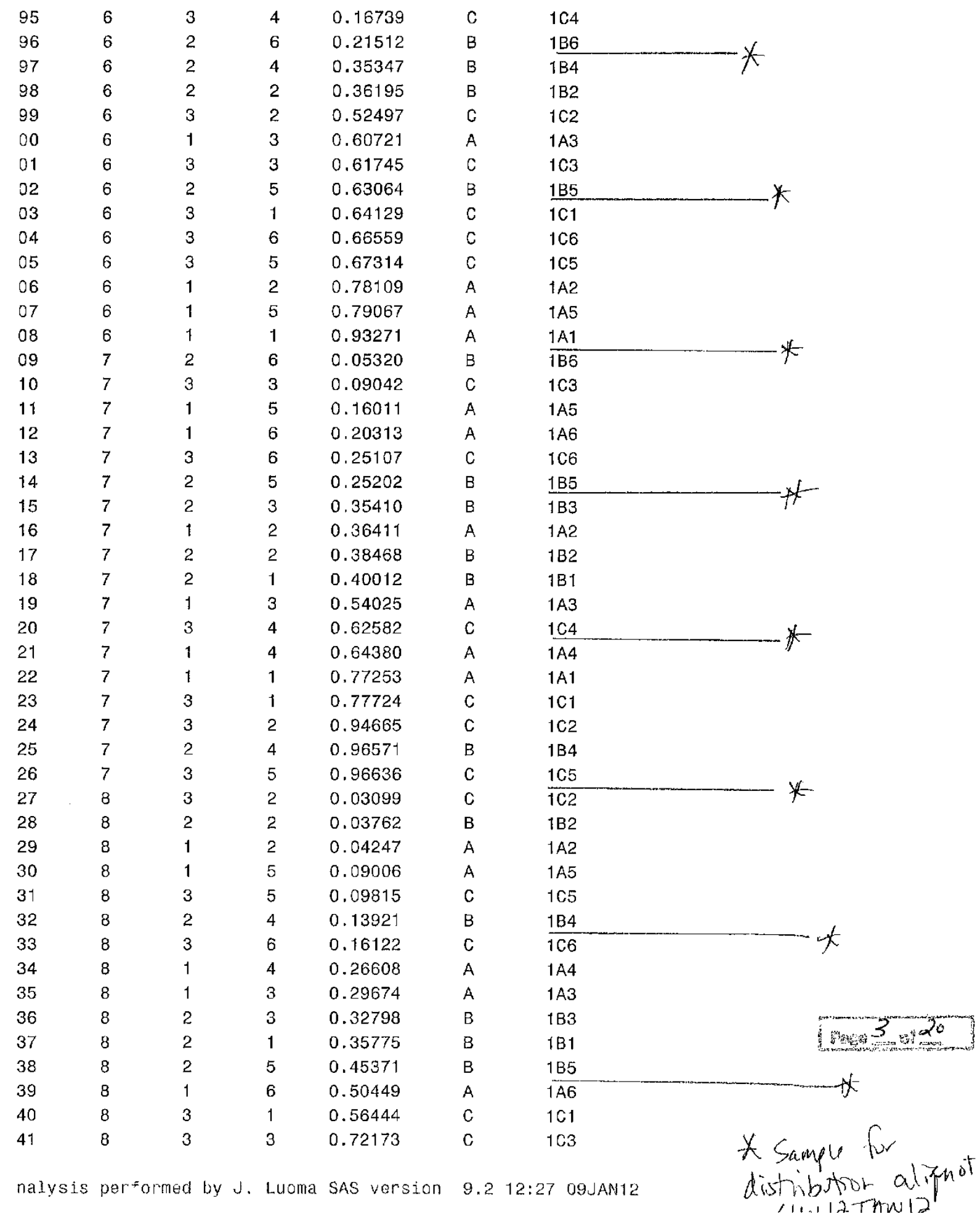


ffects of Psuedomonas fluorescens (Pf.CL145A) to glochidia from seven unionid mussel species 4 EH-11-PSUEDO - 01

andom assignment of glochidia to test tanks 5 -

ussel species: Black sandshell

bs round row tank $x$ row_ tankn AEH-11-PSEUDO-01

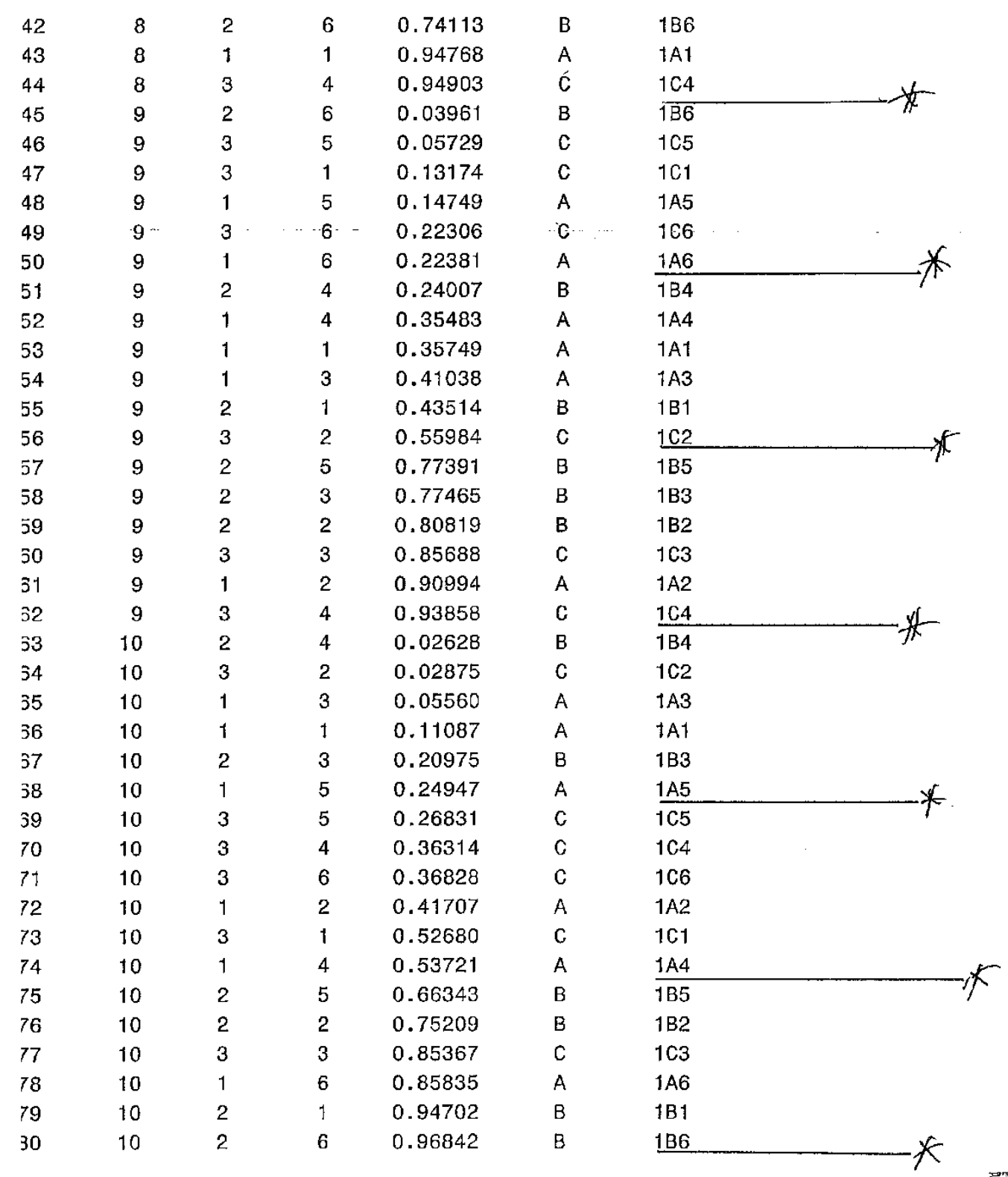

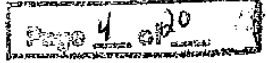

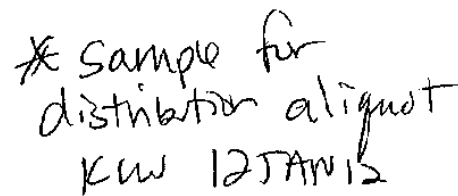

lalysis performed by $J$. Luoma SAS version $9.2 \quad 12: 27 \quad 090$ AN12 
trects ot, Hsuedomonas thuorescens (w-LL.145A) to glocnidia trom seven unionza mussel species b EH - 11 - PSUEDO - 01

andom assignment of glochidia to test tank $J_{-}$ ussel species: Mucket

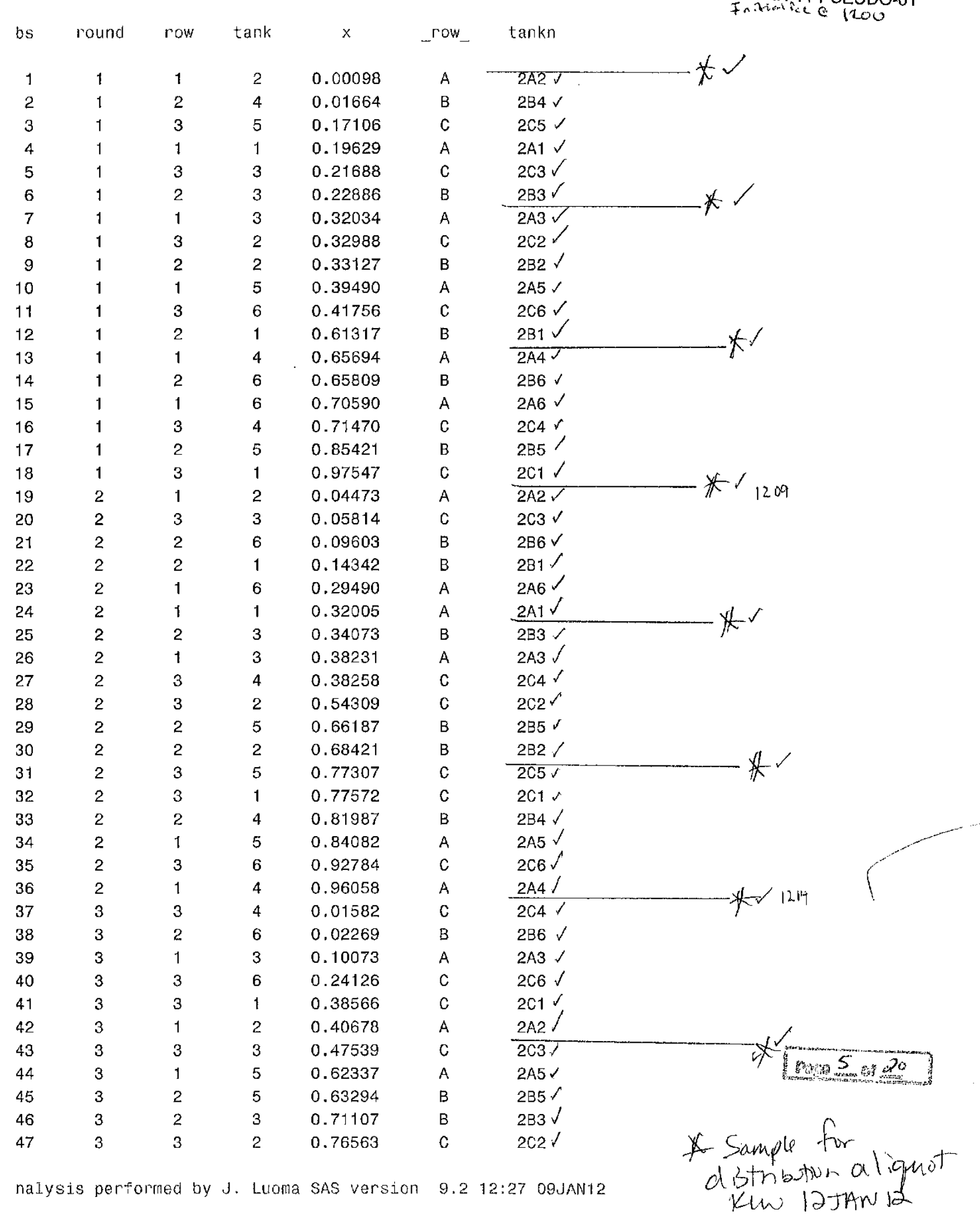


ffects of Psuedomonas fluorescens (Pf-CL145A) to glochidia from seven unionid mussel species 6 EH-11-PSUEDO-01 andom assignment of glochidia to test tanks

ussel species: Mucket

AEH-11-PSEUDO-01

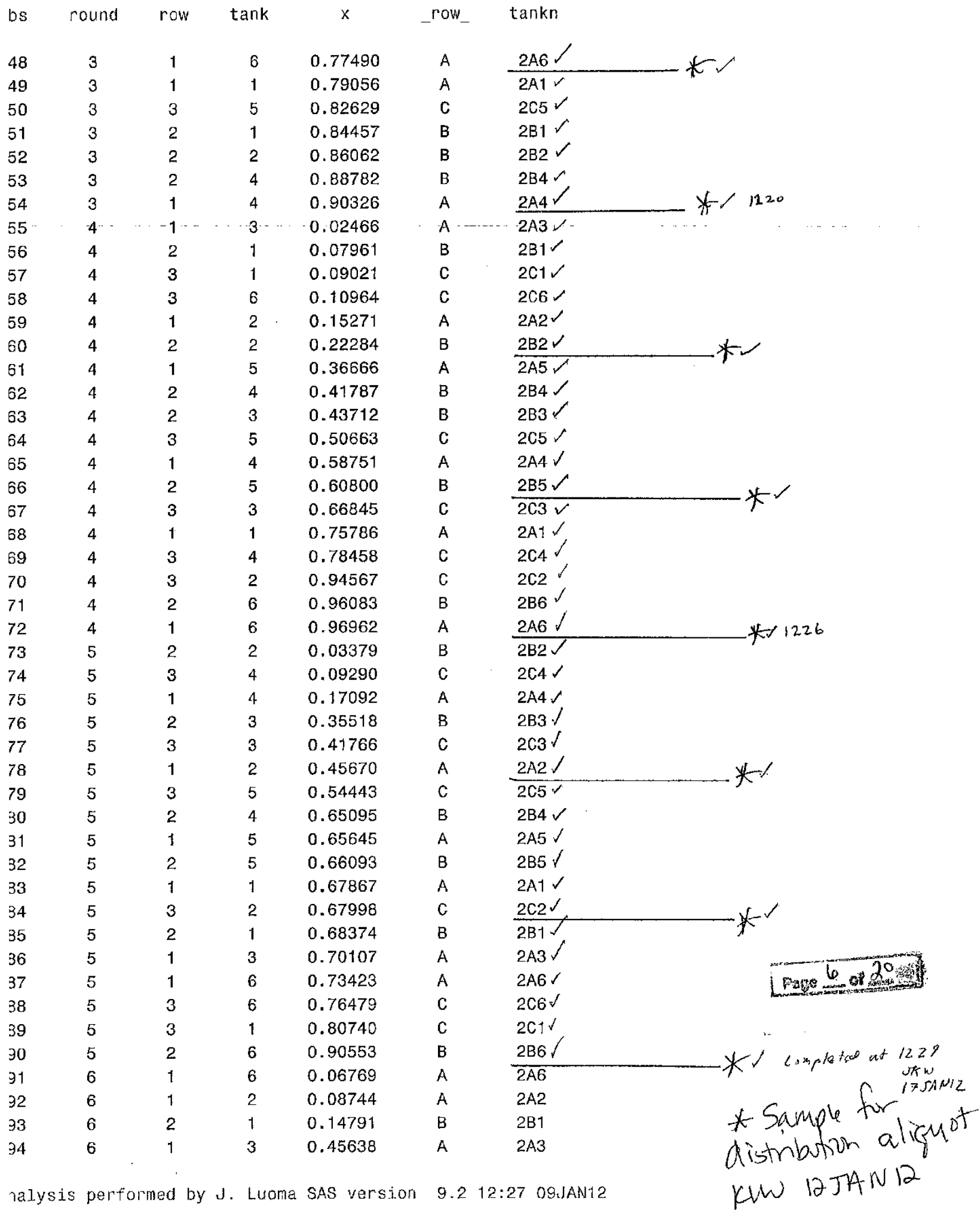


rtects or rsuedomonas rluorescens (HT-LL 145A) to glocniala trom seven unionid mussed species EH-11-PSUEDO-01

zndom assignment of glochidia to test tank Siw usse 1 species: Mucket

js round row tank $x$ row_ tankn. AEH-11-PSEUDO-01

\begin{tabular}{|c|c|c|c|c|}
\hline 35 & 6 & 3 & 3 & 0.46071 \\
\hline 36 & 6 & 3 & 6 & 0.49449 \\
\hline 37 & 6 & 1 & 5 & 0.52489 \\
\hline$\exists 8$ & 6 & 3 & 1 & 0.61590 \\
\hline 39 & 6 & 3 & 4 & 0.70732 \\
\hline 30 & 6 & 2 & 5 & 0.71307 \\
\hline 31 & 6 & 3 & 5 & 0.75936 \\
\hline 32 & 6 & 1 & 1 & 0.78284 \\
\hline 33 & 6 & 2 & 3 & 0.83665 \\
\hline 34 & 6 & 3 & 2 & 0.84431 \\
\hline 35 & 6 & 2 & 4 & 0.84525 \\
\hline 36 & 6 & 2 & 2 & 0.88368 \\
\hline 37 & 6 & 2 & 6 & 0.90205 \\
\hline 38 & 6 & 1 & 4 & 0.96568 \\
\hline 39 & 7 & 2 & 1 & 0.04213 \\
\hline 10 & 7 & 2 & 2 & 0.05244 \\
\hline 11 & 7 & 3 & 5 & 0.08552 \\
\hline 12 & 7 & 1 & 6 & 0.11230 \\
\hline 13 & 7 & 3 & 2 & 0.16857 \\
\hline 14 & 7 & 2 & 5 & 0.22141 \\
\hline 15 & 7 & 2 & 6 & 0.22335 \\
\hline 16 & 7 & 2 & 4 & 0.25653 \\
\hline 17 & 7 & 1 & 4 & 0.33381 \\
\hline 18 & 7 & 1 & 1 & 0.40108 \\
\hline 19 & 7 & 2 & 3 & 0.56124 \\
\hline 20 & 7 & 3 & 1 & 0.60276 \\
\hline 21 & 7 & 3 & 6 & 0.78412 \\
\hline 22 & 7 & 1 & 2 & 0.81734 \\
\hline 23 & 7 & 3 & 3 & 0.82288 \\
\hline 24 & 7 & 3 & 4 & 0.88472 \\
\hline 25 & 7 & 1 & 3 & 0.96413 \\
\hline 26 & 7 & 1 & 5 & 0.99816 \\
\hline 27 & 8 & 1 & 4 & 0.01906 \\
\hline 28 & 8 & 2 & 1 & 0.02411 \\
\hline 29 & 8 & 3 & 4 & 0.06716 \\
\hline 30 & 8 & 3 & 5 & 0.23709 \\
\hline 31 & 8 & 2 & 3 & 0.26067 \\
\hline 32 & 8 & 2 & 5 & 0.30873 \\
\hline 33 & 8 & 1 & 1 & 0.40705 \\
\hline 34 & 8 & 1 & 5 & 0.48246 \\
\hline 35 & 8 & 3 & 2 & 0.64868 \\
\hline 36 & 8 & 3 & 1 & 0.69547 \\
\hline 37 & 8 & 2 & 6 & 0.71882 \\
\hline 38 & 8 & 2 & 2 & 0.74359 \\
\hline 39 & 8 & 2 & 4 & 0.76087 \\
\hline 40 & 8 & 1 & 2 & 0.80410 \\
\hline 41 & 8 & 1 & 3 & 0.82372 \\
\hline
\end{tabular}

$2 \mathrm{C} 3$

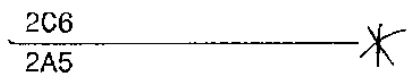

$2 \mathrm{C} 1$

$2 \mathrm{C} 4$

$2 \mathrm{~B} 5$

$2 \mathrm{C} 5$

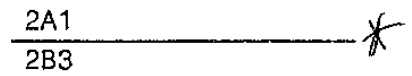

$2 \mathrm{C} 2$

2B4

2B2

2B6

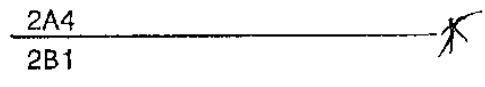

2B2

$2 \mathrm{C} 5$

2A6

$2 \mathrm{C} 2$

$\frac{2 \mathrm{~B} 5}{2 \mathrm{~B} 6} \mathrm{~K}$

2B4

2A4

2A1

2B3

$2 \mathrm{C} 1$

2A2

$2 \mathrm{C} 3$

$2 \mathrm{C} 4$

$2 A 3$

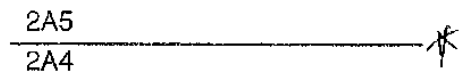

2B1

$2 \mathrm{C} 4$

$2 \mathrm{C5}$

2B3

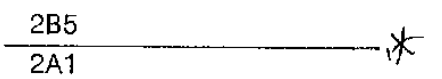

$2 A 5$

$2 \mathrm{C} 2$

$2 \mathrm{C} 1$

2B6

2B2

$2 \mathrm{~B} 4$

$2 \mathrm{~A} 2$

$2 \mathrm{A3}$

7alysis performed by $J$. Luoma SAS varsion $9.2 \quad 12: 27 \quad 09 J A N i z$

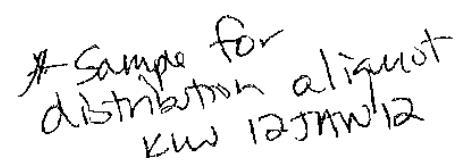


ffects of Psuedomonas fluorescens (Pf-CL145A) to glochidia from seven unionid mussel. species 8 EH - 11 - PSUEDO-01 andom assignment of glochidia to test tank for $^{\mathrm{J}}$ ussel species: Mucket

bs round row tank $x$ row tankn AEH-11-PSEUDO-01

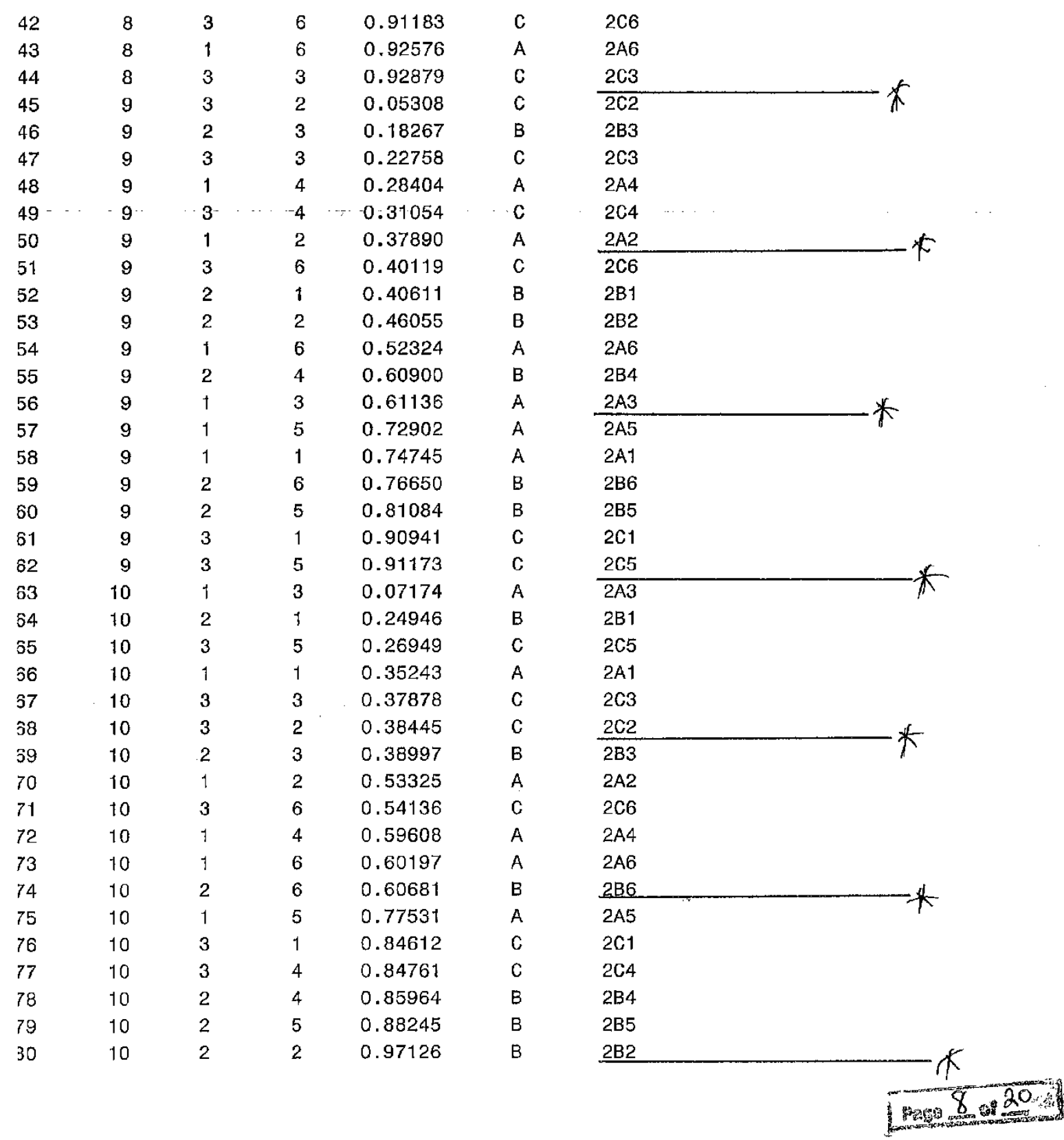




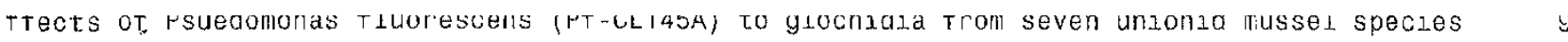
EH-11-PSUEOO-01

andom assignment of glochidia to test tank Jn-

ussel species: Hickorynut

AEH-11-PSEUDO-01

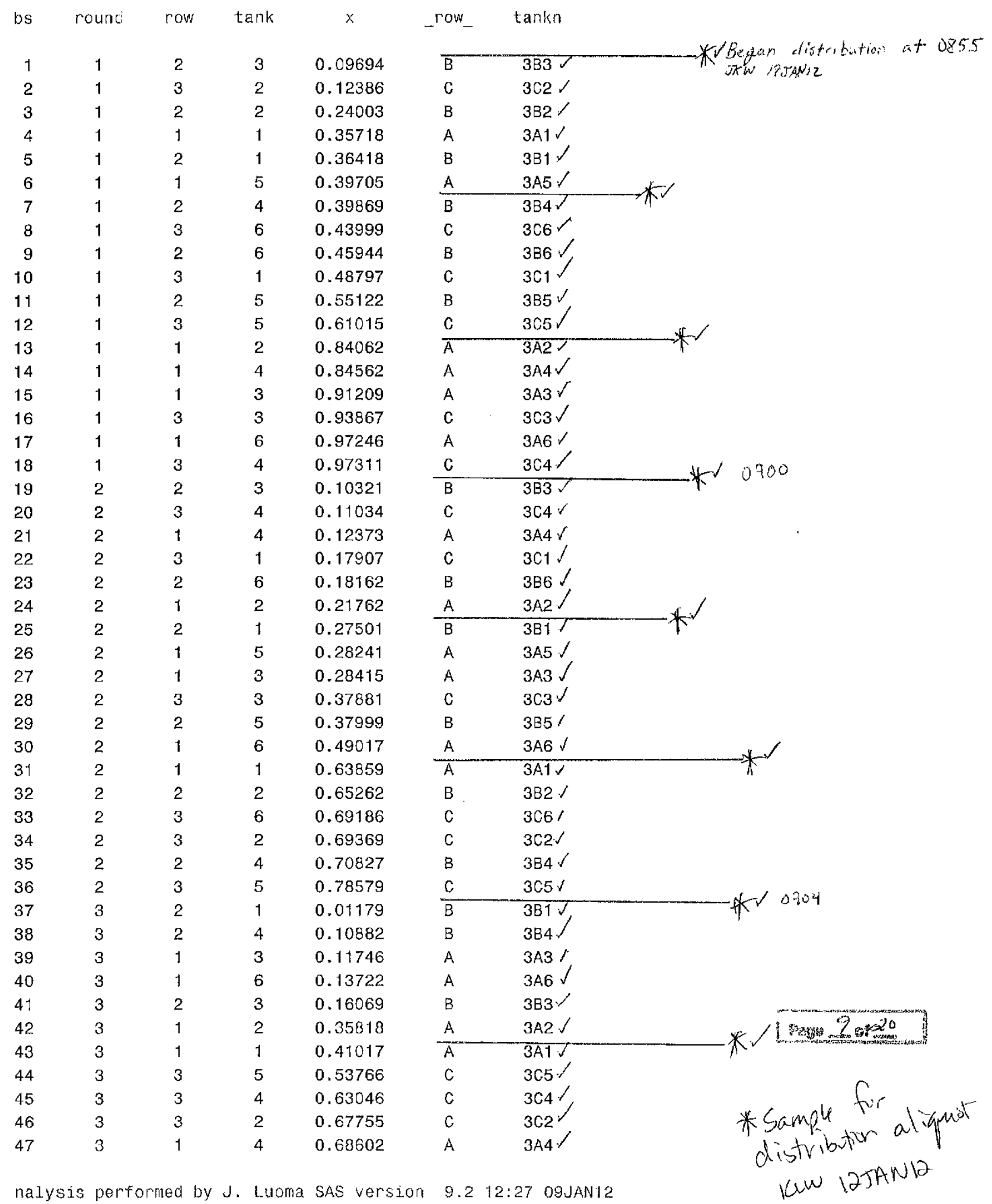


ffects of Psuedomonas fluorescens (Pf-CL145A) to glochidia from seven unionid musse1 species 10 EH-11-PSUEDO - 01 andom assignment of glochidia to test tank for ussel species: Hickorynut

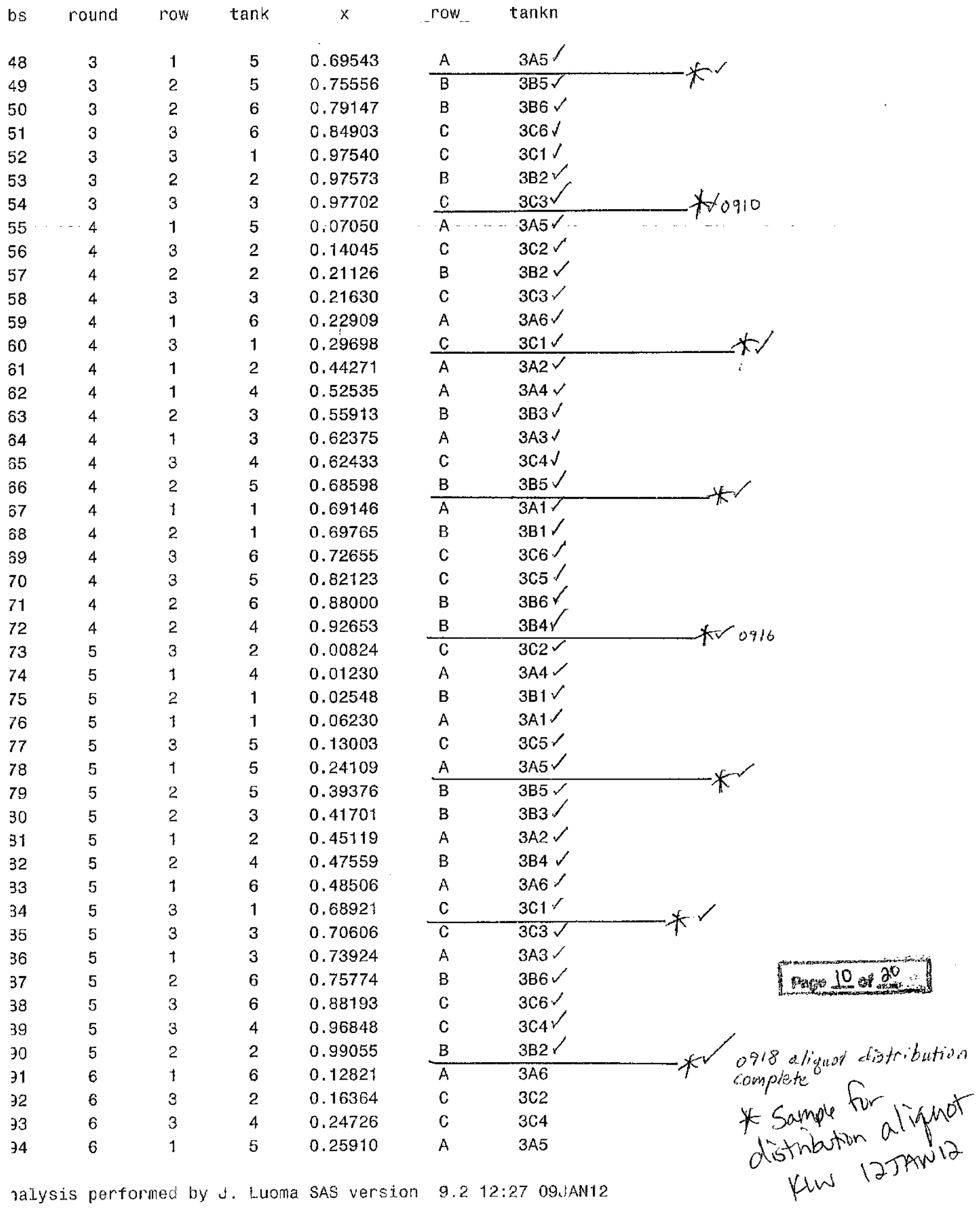




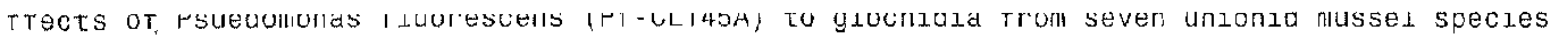
EH-11-PSUEDO-01

andom assignment of glochidia to test tank tiv

usse1 species: Hickorynut

AEH-11-PSEUDO-01

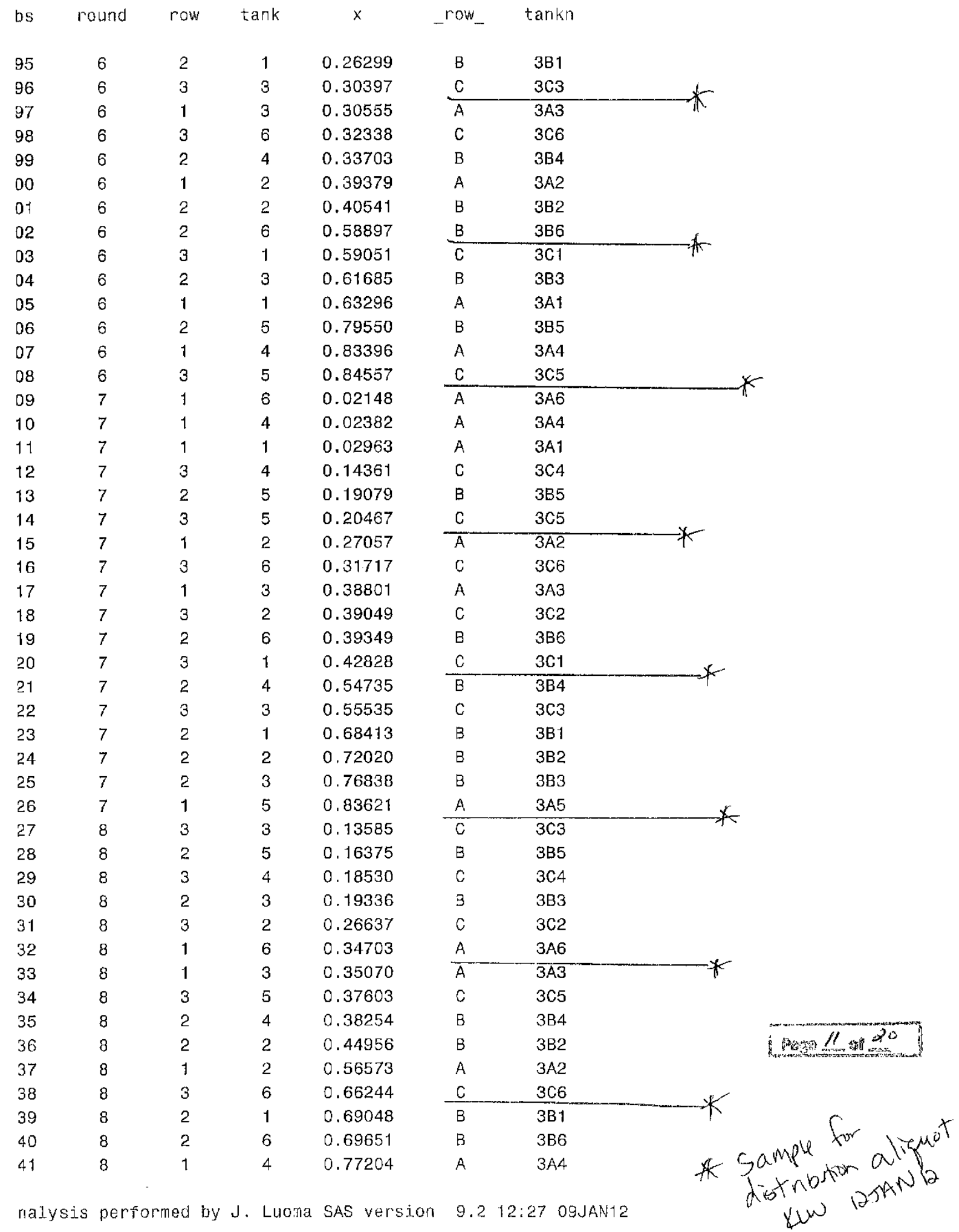


ffects of Psuedomonas fluorescens (Pf-CL145A) to glochidia from seven unionid mussel species 12 EH-11-PSUEDO-01

andom assignment of glochidia to test tank $x^{2}$

ussel species: Hickorynut

bs round row tank $x$ row_ tankn AEH-11-PSEUDO-01

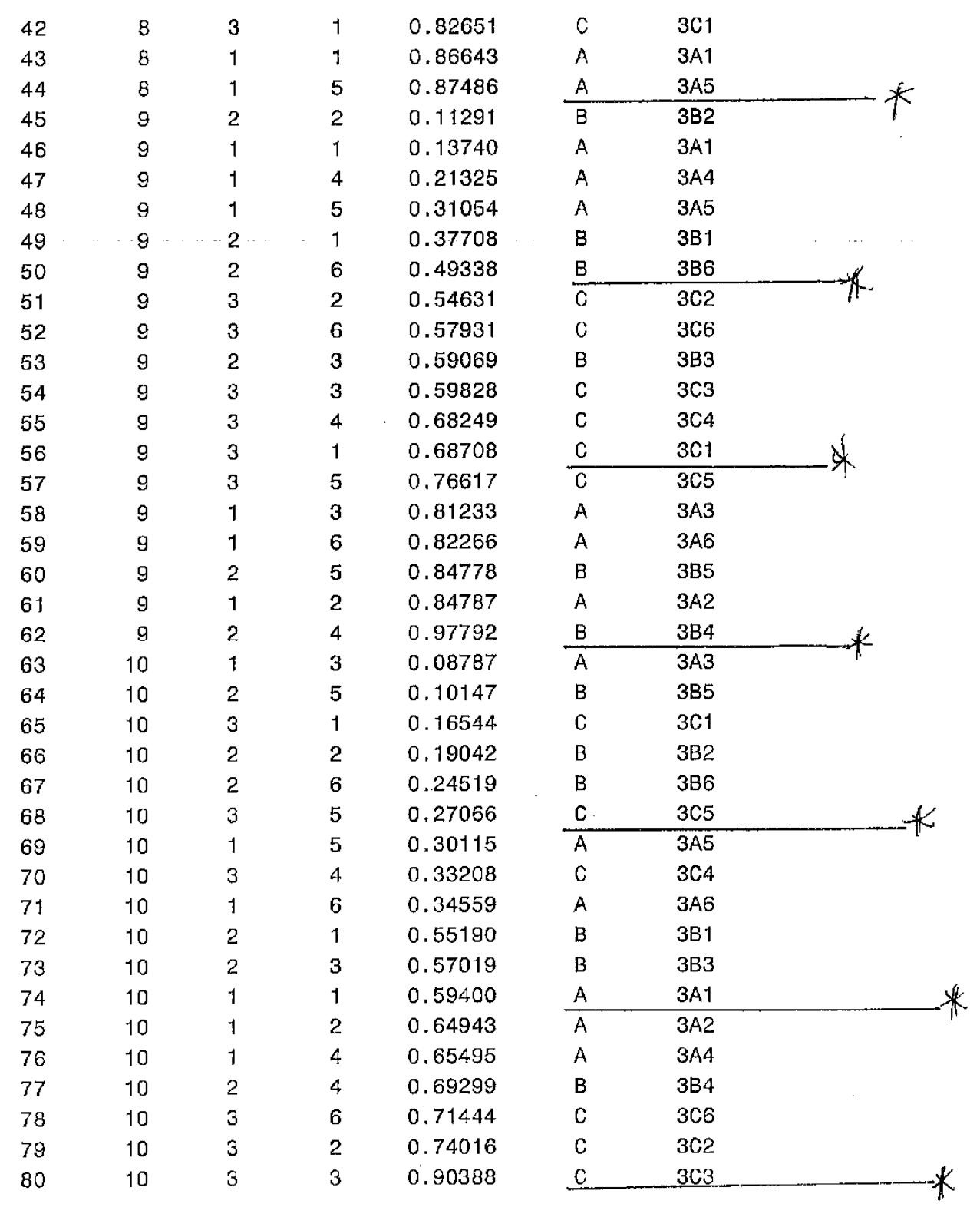

$12+2$

nalysis performed by J. Luoma SAS version 9.2 12:27 09JAN12 


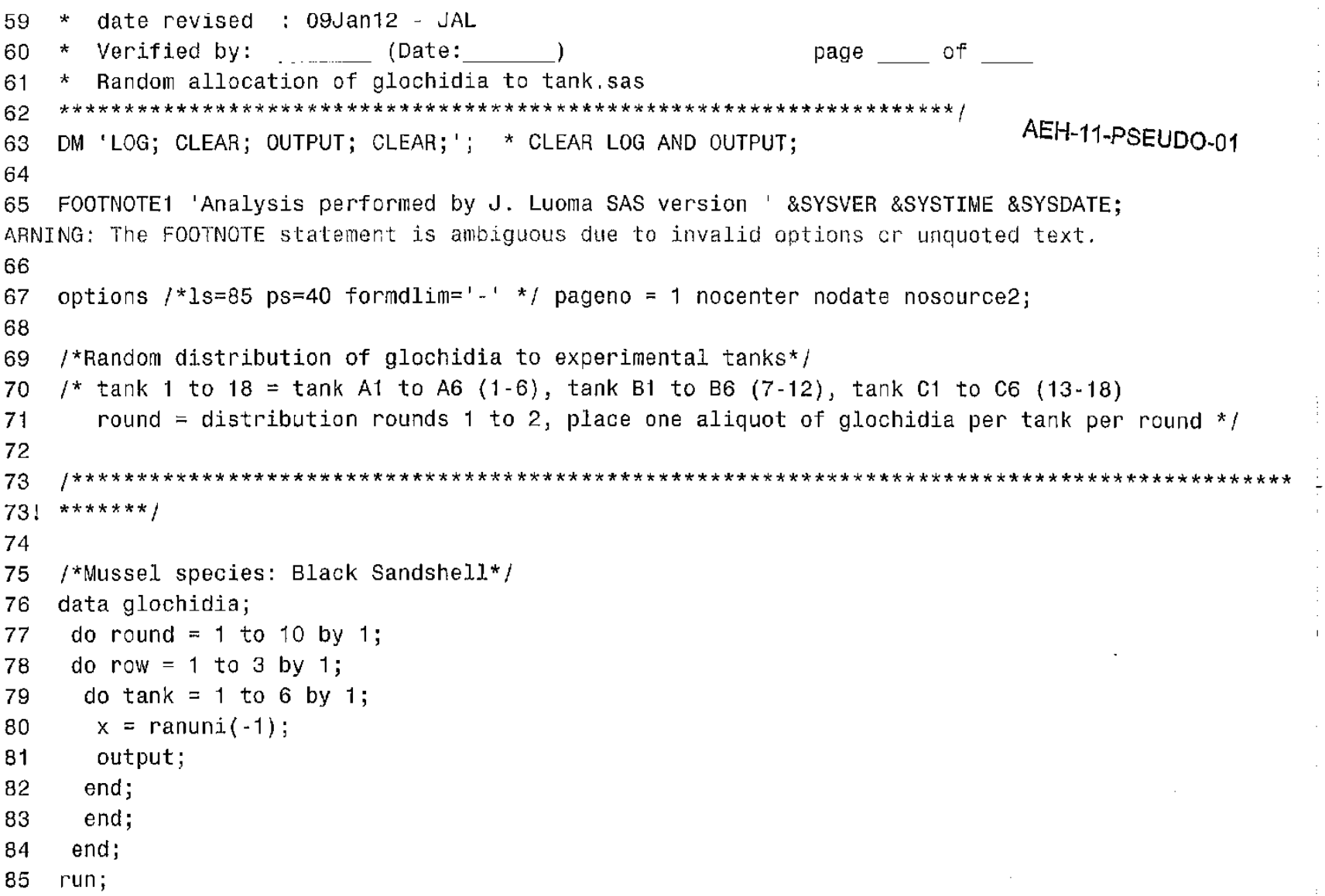

OTE: The data set WORK.GLOCHIDIA has 180 observations and 4 variabies.

OTE: DATA statement used (Total process time):
real time
0.01 seconds
cpu time
0.01 seconds

data glochidiadist; set glochidia;

if row $=1$ then_row_ $=$ ' $A$ ';

if row $=2$ then_row_ $=$ ' $B$ ';

if row $=3$ then row $={ }^{\prime} \mathrm{C}$ ';

if row $=1$ and tank $=1$ then tankn $=11 \mathrm{Al}$ ';

if row $=1$ and $\operatorname{tank}=2$ then $\operatorname{tankn}=$ ' $1 \mathrm{~A} 2$ ';

if row $=1$ and tank $=3$ then tankn $=11 \mathrm{~A} 3^{\prime}$;

if row $=1$ and tank $=4$ then tankn = ' $1 \mathrm{~A} 4$ ';

if row $=1$ and tank $=5$ then tankn $=11 \mathrm{~A} 5^{\prime}$;

if row $=1$ and $\operatorname{tank}=6$ then $\operatorname{tankn}={ }^{\prime} 1 \mathrm{~A}^{\prime}{ }^{\prime}$;

if row $=2$ and $\operatorname{tank}=1$ then $\operatorname{tankn}=' 1 \mathrm{~B} 1$ ';

if row $=2$ and tank $=2$ then tankn $=$ ' $1 \mathrm{~B} 2$ ';

if row $=2$ and tank $=3$ then $\operatorname{tankn}=11 \mathrm{~B} 3 '$;

if row $=2$ and tank $=4$ then tankn $=' 1 \mathrm{~B} 4$ ';

if row $=2$ and tank $=5$ then tankn $=' 1 B 5 '$;

if row $=2$ and tank $=6$ then tankn $=1186 '$;

if row $=3$ and $\tan k=1$ then $\operatorname{tankn}=101 '$;

if row $=3$ and tank $=2$ then tankn $={ }^{\prime} 1 \mathrm{C} 2{ }^{\prime}$;

if row $=3$ and $\operatorname{tank}=3$ then $\tan k n=' 1 \mathrm{c} 3$ ';

if row $=3$ and tank $=4$ then tankn $=' 1 \mathrm{C} 4$ '; 
OTE: There were 180 observations read from the data set WORK.GLOCHIDIA.

OTE: The data set WORK.GLOCHIDIADIST has 180 observations and 6 variables.

OTE: DATA statement used (Total process time):
real time
0.04 seconds

cpu time

0.04 seconds

AEH-11-PSEUDO-01

08 proc sort data $=$ glochidiadist;

09 by round $x$;

10 run;

OTE: There were -180 observations read-from the data set WORK. GLOCHIDIADIST,

OTE: The data set WORK.GLOCHIDIADIST has 180 observations and 6 variables.

OTE: PROCEDURE SORT used (Total process time):
real time
0.01 seconds

cpu time

0.01 seconds

11 proc print data = glochidiadist;

12 title1 $h=2$ 'Effects of Psuedomonas fluorescens (Pf-CL.145A) to glochidia from seven unionid

12 ! mussel species';

13 title2 $h=1.5$ 'AEH-11-PSUEDO-01';

14 title3 $h=1$ 'Random assignment of glochidia to test tanks';

15 title4 h=1 'Mussel species: Black sandshell';

16 run;

OTE: There were 180 observations read from the data set WORK.GLOCHIDIADIST.

OTE: PROCEDURE PRINT used (Total process time):
real time
0.00 seconds

cpu time

0.00 seconds

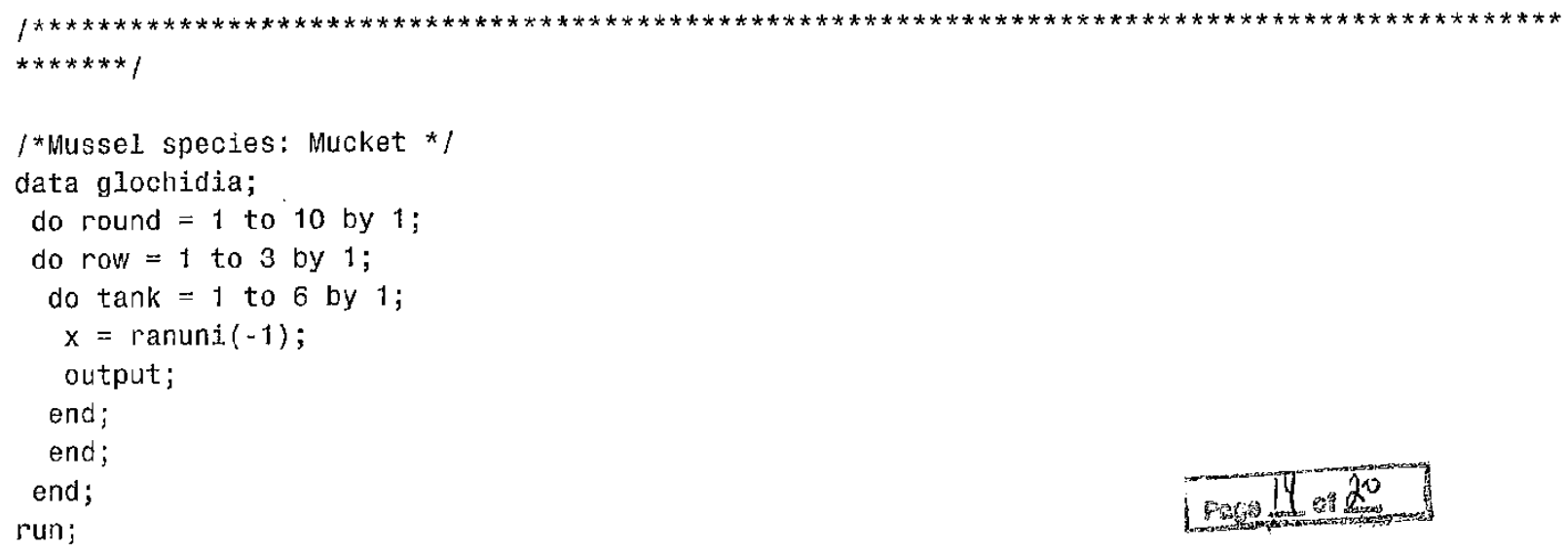

TTE: The data set WORK.GLOCHIDIA has 180 observations and 4 variables.

OTE: DATA statement used (Total process time):
real time
0.01 seconds

cpu time

0.01 seconds 


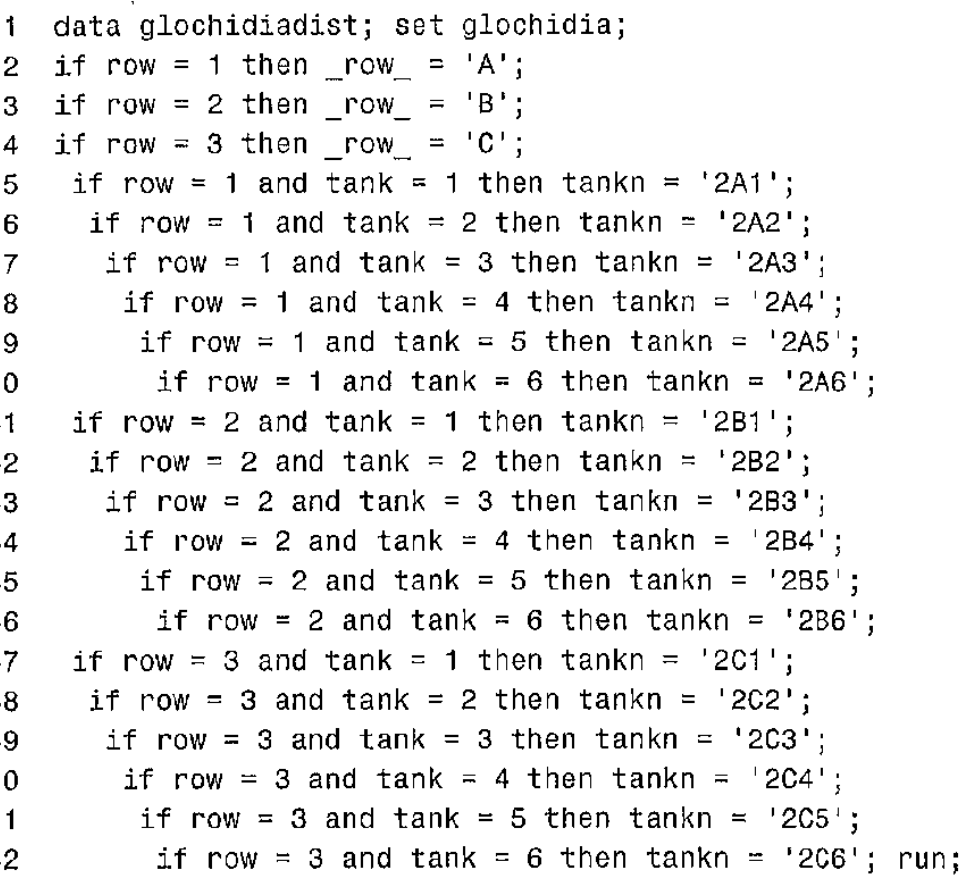

AEH-11-PSEUDO-01

OTE: There were 180 observations read from the data set WORK.GLOCHIDIA.

OTE: The data set WORK.GLOCHIDIADTST has 180 observations and 6 variables.

OTE: DATA statement used (Total process time):
real time
0.03 seconds

cpu time

0.03 seconds

53 proc sort data $=$ glochidiadist;

54 by round $x$;

55 run;

OTE: There were 180 observations read from the data set WORK.GLOCHIDIADIST.

OTE: The data set WORK.GLOCHIDIADIST has 180 observations and 6 variables.

OTE: PROCEDURE SORT Used (Total process time):
real time
0.01 seconds
cpu time
0.01 seconds

56 proc print data $=$ glochidiadist;

57 title1 $h=2$ 'Effects of Psuedomonas fluorescens (Pf-CL145A) to glochidia from seven unionid

57 ! mussel species';

58 title2 $h=1.5$ 'AEH-11-PSUED0-01';

59 title3 $h=1$ 'Random assignment of glochidja to test tank';

60 title4 $h=1$ 'Mussel species: Mucket';

61 run;

OTE: There were 180 observations read from the data set WORK.GLOCHIDIADIST

OTF: PROCEDURE PRINT used (Total process time):
real time
0.01 seconds

cpu time

0.01 seconds

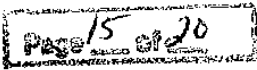


62

63

63 !

64

65

66

67

68

69

70

71

72

73

74

75
AEH-11-PSEUDO-01

/*Mussel species: Hickorynut */

data glochidia;

do round $=1$ to 10 by 1 ;

do row $=1$ to 3 by 1 ;

do $\operatorname{tank}=1$ to 6 by $t$;

$x=\operatorname{ranuni}(-1)$;

output;

end;

end;

end;

run;-

OTE: The data set WORK.GLOCHIDIA has 180 observations and 4 variables.

OTE: DATA statement used (Total process time):

$\begin{array}{ll}\text { real time } & 0.01 \text { seconds } \\ \text { cpu time } & 0.01 \text { seconds }\end{array}$

76 data glochidiadist; set glochidia;

77 if row $=1$ then row $_{-}={ }^{\prime} \mathrm{A}^{\prime}$;

78 if row $=2$ then row $_{-}={ }^{\prime} \mathrm{B}$ ';

79 if row $=3$ then_row_ $=$ ' $\mathrm{C}$ ';

80 if row $=1$ and tank $=1$ then tankn = ' 3 A 1 ';

81 if row $=1$ and $\operatorname{tank}=2$ then $\operatorname{tankn}={ }^{\prime}$ ' $3 \mathrm{~A} 2$ ';

if row $=1$ and tank $=3$ then $\operatorname{tankn}={ }^{\prime} 3 A 3^{\prime}$;

if row $=1$ and $\operatorname{tank}=4$ then tankn $={ }^{\prime} 3{ }^{\prime} 4^{\prime}$;

if row $=1$ and $\operatorname{tank}=5$ then $\operatorname{tankn}={ }^{\prime} 3 \mathrm{~A} 5^{\prime}$;

if row $=1$ and $\operatorname{tank}=6$ then tankn $={ }^{\prime} 3 A 6^{\prime}$;

if row $=2$ and tank $=1$ then $\operatorname{tankn}=$ ' $3 \mathrm{~B} 1$ ';

if row $=2$ and tank $=2$ then $\operatorname{tankn}=$ ' $3 \mathrm{~B} 2$ ';

if row $=2$ and $\operatorname{tank}=3$ then tankn $=$ ' $3 \mathrm{~B} 3{ }^{\prime}$;

if row $=2$ and tank $=4$ then tankn $=$ ' $3 \mathrm{~B} 4^{\prime}$;

if row $=2$ and tank $=5$ then tankn $=$ ' $3 \mathrm{~B} 5$ ';

if row $=2$ and $\operatorname{tank}=6$ then tankn $=$ ' $3 \mathrm{~B} 6$ ';

if row $=3$ and tank $=1$ then $\operatorname{tankn}=' 3 \mathrm{C} 1$ ';

if row $=3$ and tank $=2$ then $\operatorname{tankn}={ }^{\prime} 3 \mathrm{C} 2{ }^{\prime}$;

if row $=3$ and tank $=3$ then $\operatorname{tankn}={ }^{\prime} 3 \mathrm{C} 3^{\prime}$;

if $r o w=3$ and $\operatorname{tank}=4$ then tankn $=$ ' $3 \mathrm{C} 4$ ';

if row $=3$ and tank $=5$ then $\operatorname{tankn}=$ ' $3 \mathrm{C5}$ ';

if row $=3$ and $\operatorname{tank}=6$ then tankn $=$ ' $3 \mathrm{C6} 6^{\prime}$; run;

OTE: There were 180 observations read from the data set WORK. GLOCHIDIA,

OTE: The data set WORK.GLOCHIDIADIST has 180 observations and 6 variables.

OTE: DATA statement used (Total process time):

real time $\quad 0.01$ seconds

cpu time $\quad 0.01$ seconds

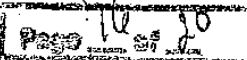

98 proc sort data $=$ glochidiadist;

99 by round $x$;

00 run; 
JTE: There were 180 observations read trom the data set WUKK. GLUCHIULALISI.

ITE: The data set WORK.GLOCHIDIADIST has 180 observations and 6 variables.

JTE: PROCEDURE SORT used (Total process time):

real time $\quad 0.01$ seconds

cpu time $\quad 0.01$ seconds

AEH-11-PSEUDO-01

1 proc print data = glochidiadist;

)2 title1 $h=2$ 'Effects of Psuedomonas fluorescens (Pf-CL145A) to glochidia from seven unionid

J2! mussel species';

33 title2 $\mathrm{h}=1.5$ 'AEH-11-PSUEDO-01';

34 title3 $h=1$ 'Random assignment of glochidia to test tank';

5 title4 $\mathrm{h}=1$ 'Mussel species: Hickorynut';

36 run;

ITE: There were 180 observations read from the data set WORK.GLOCHIDIADIST.

JTE: PROCEDURE PRINT used (Total process time):

real time $\quad 0.00$ seconds

cpu time $\quad 0.00$ seconds

1. 


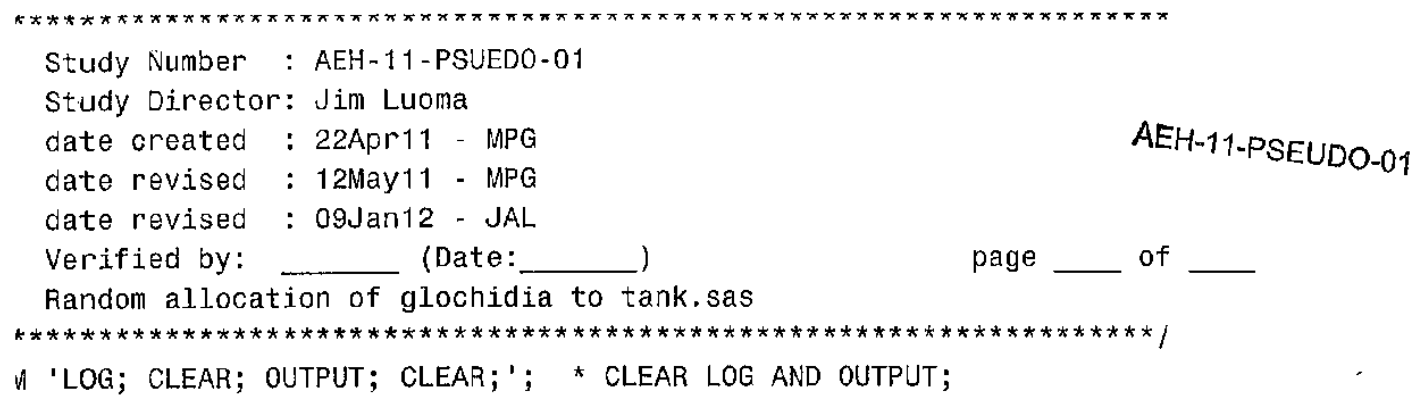

JOTNOTE1 'Analysis performed by $J$. Luoma SAS version ' \&SYSVER \&SYSTIME \&SYSDATE;

otions $/{ }^{*} \mathrm{ls}=85 \mathrm{ps}=40$ formdlim=' ${ }^{\prime} * /$ pageno $=1$ nocenter nodate nosource2;

*Random distribution of glochidia to experimental tanks* /

* tank 1 to $18=\operatorname{tank} A 1$ to $A 6(1-6)$, tank B1 to B6 $(7-12)$, tank $\mathrm{C} 1$ to $\mathrm{C6}(13-18)$

round $=$ distribution rounds 1 to 2 , place one aliquot of glochidia per tank per round *

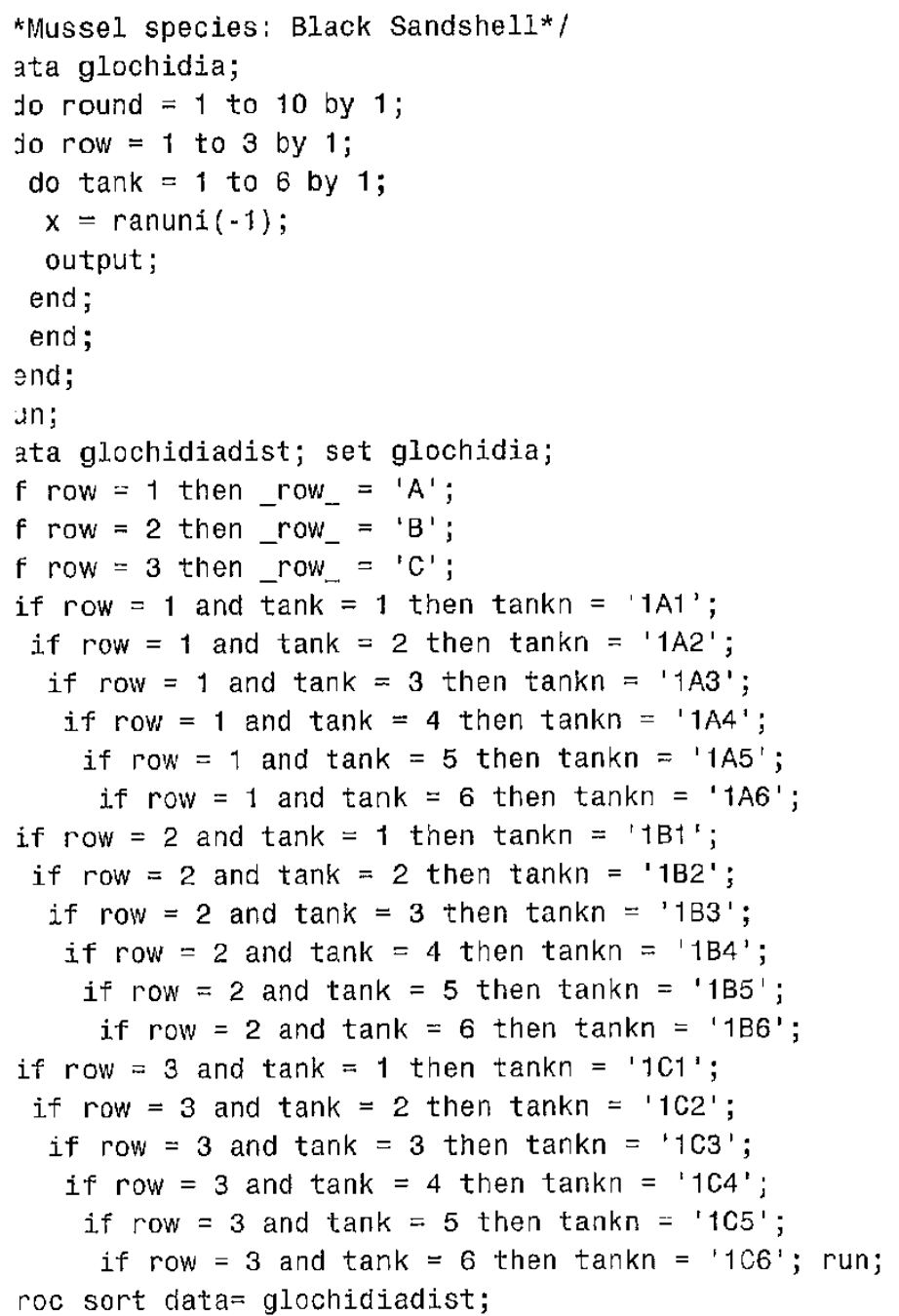

oy round $x$; 


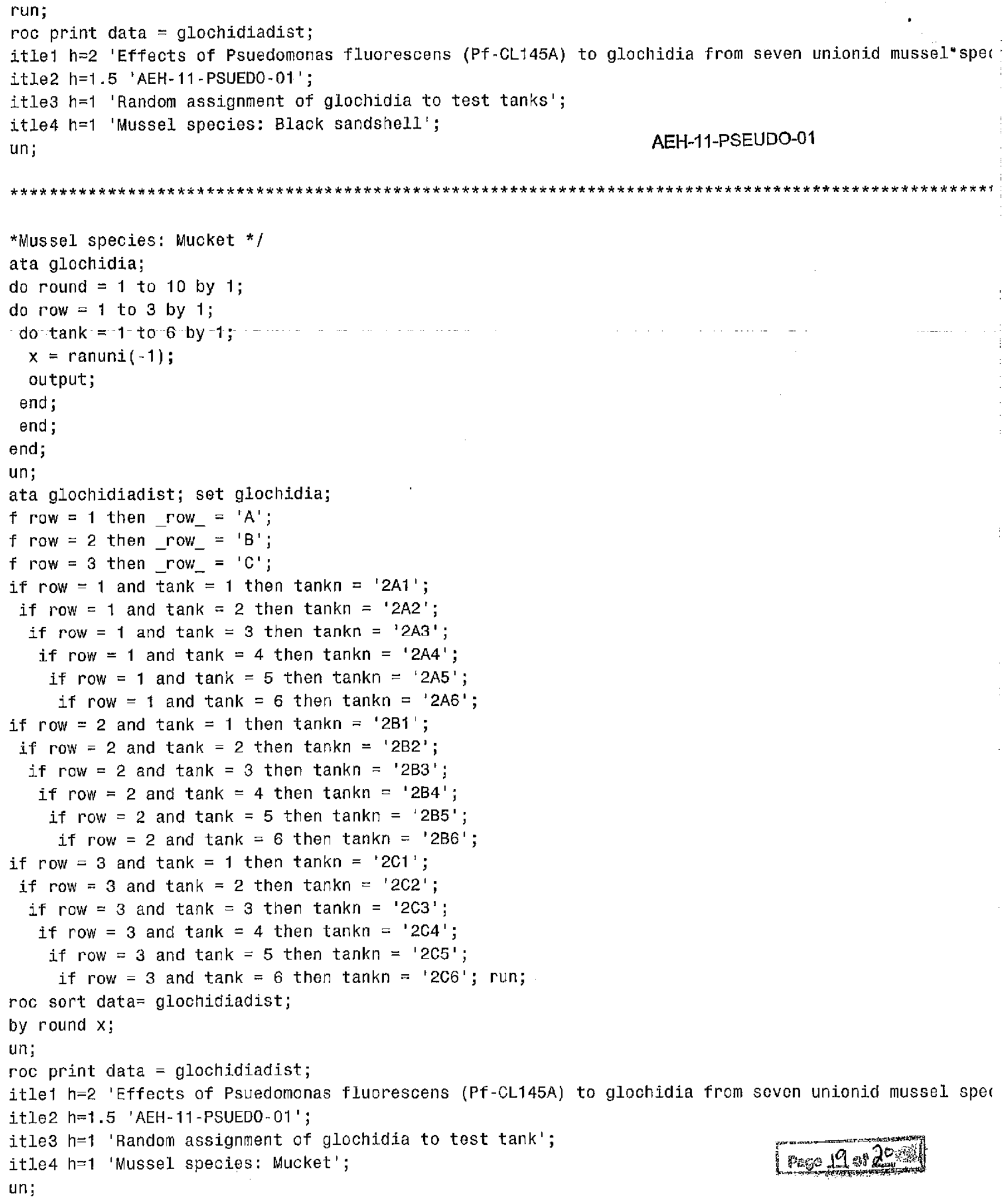




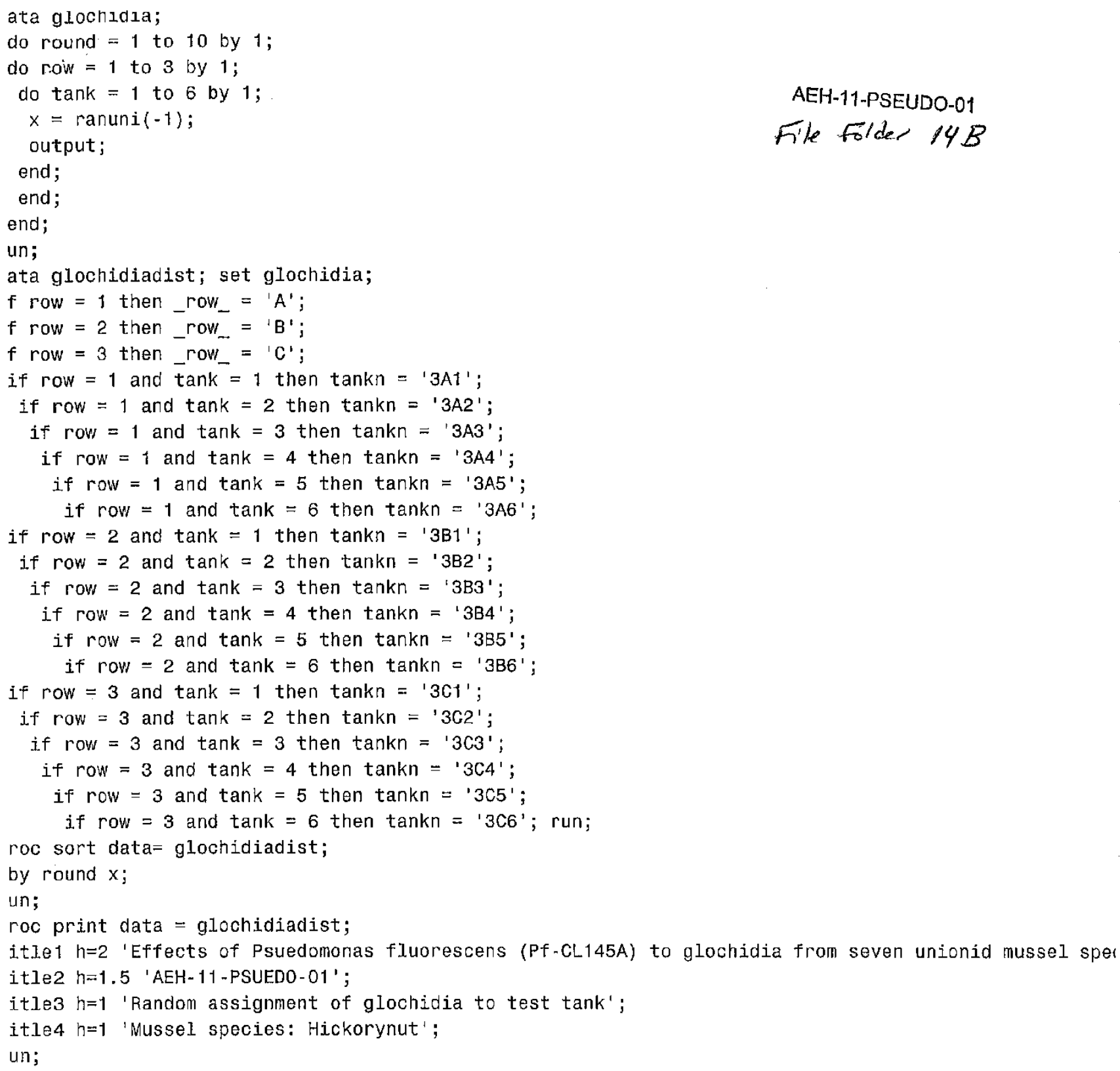

20 


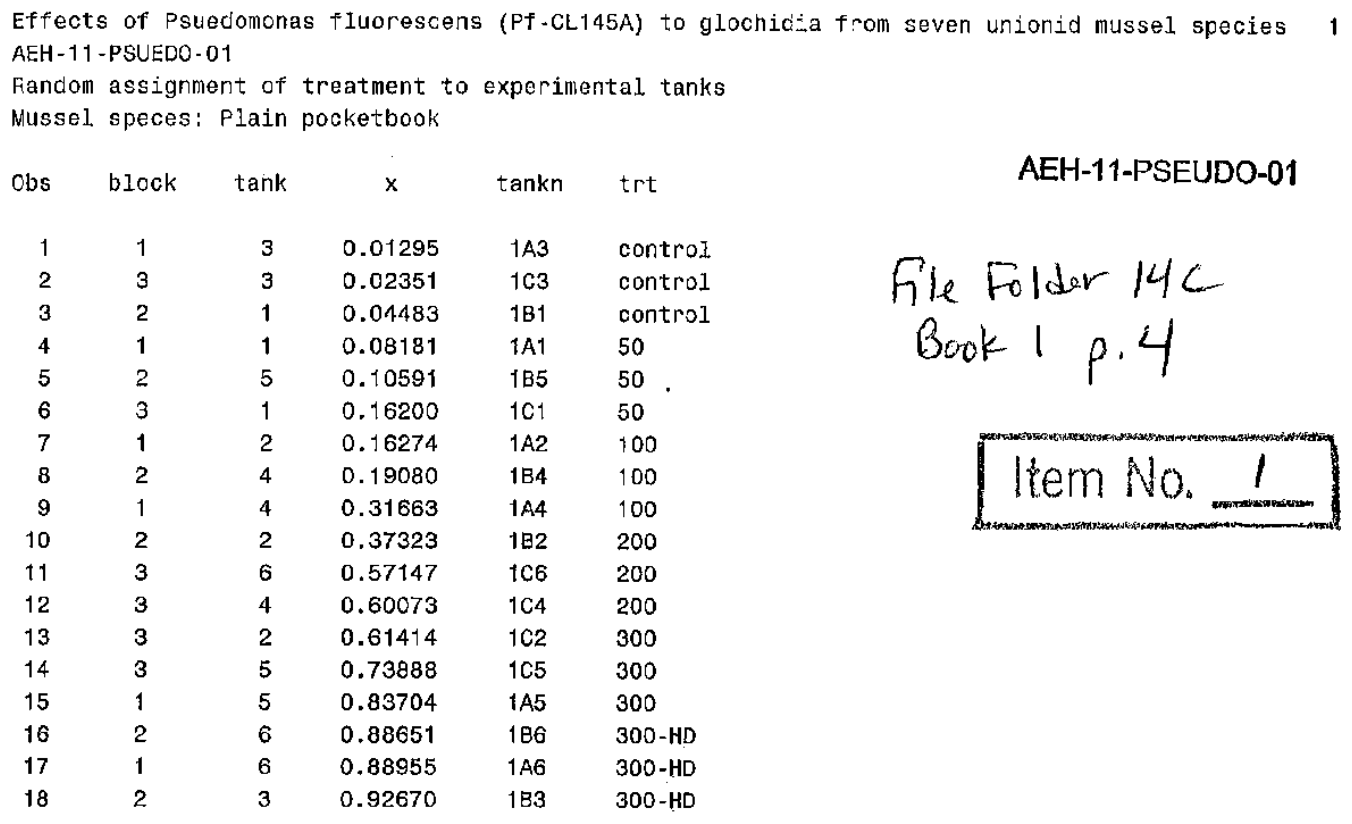

\footnotetext{
MOOFEDEY $5 / 15 / 12$ Initlals: $7 m s$ Date : REVIEWED BY

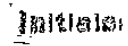
Batat
}

\footnotetext{
MrL
}

Analysis performed by M. Gaikowski SAS version 0.2 08:56 10MAY11

por 13 


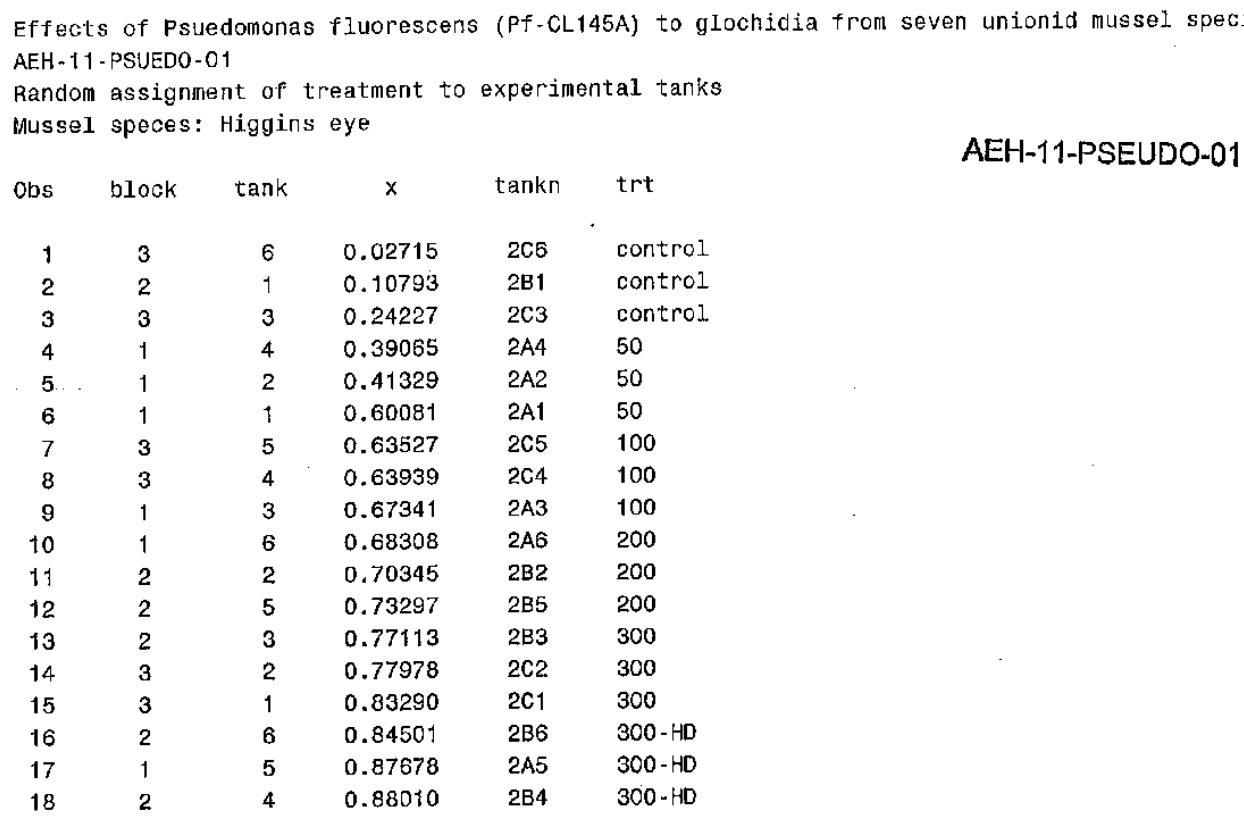

Analysis performed by M. Gaikowski. SAS version $9.208: 56$ 10MAY11 
Effects of Psuedomonas fluorescens (Pf-CL145A) to glochidia from seven unionid mussel species 3 AEH-11-PSUEDO-01

Random assignment of treatment to experimental tanks

Mussel speces: Fatmucket

$\begin{array}{rrrcrl}\text { Obs } & \text { block } & \text { tank } & x & \text { tankn } & \text { trt } \\ 1 & 1 & 2 & 0.08330 & 3 \mathrm{~A} 2 & \text { control } \\ 2 & 2 & 2 & 0.10124 & 3 \mathrm{~B} 2 & \text { control } \\ 3 & 2 & 3 & 0.31999 & 3 \mathrm{~B} 3 & \text { control } \\ 4 & 3 & 5 & 0.32027 & 3 \mathrm{C} 5 & 50 \\ 5 & 3 & 6 & 0.35692 & 3 \mathrm{C} 6 & 50 \\ 6 & 2 & 5 & 0.45716 & 3 \mathrm{~B} 5 & 50 \\ 7 & 1 & 1 & 0.52092 & 3 \mathrm{~A} 1 & 100 \\ 8 & 1 & 3 & 0.52278 & 3 \mathrm{~A} 3 & 100 \\ 9 & 1 & 5 & 0.55777 & 3 \mathrm{~A} 5 & 100 \\ 10 & 2 & 4 & 0.63422 & 3 \mathrm{~B} 4 & 200 \\ 11 & 2 & 6 & 0.73185 & 3 \mathrm{~B} 6 & 200 \\ 12 & 3 & 4 & 0.78148 & 3 \mathrm{C} 4 & 200 \\ 13 & 3 & 1 & 0.81279 & 3 \mathrm{C} 1 & 300 \\ 14 & 2 & 1 & 0.83781 & 3 \mathrm{~B} 1 & 300 \\ 15 & 3 & 3 & 0.84275 & 3 \mathrm{C3} & 300 \\ 16 & 1 & 4 & 0.84300 & 3 \mathrm{~A} 4 & 300-\mathrm{HD} \\ 17 & 3 & 2 & 0.89865 & 3 \mathrm{C} 2 & 300 \cdot \mathrm{HD} \\ 18 & 1 & 6 & 0.91420 & 3 \mathrm{AG} & 300-\mathrm{HD}\end{array}$

\section{AEH-11-PSEUDO-01}

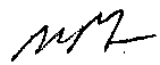

Analysis performed by $M$. Gaikowski. SAS version 9.2 08:56 10MAY11 


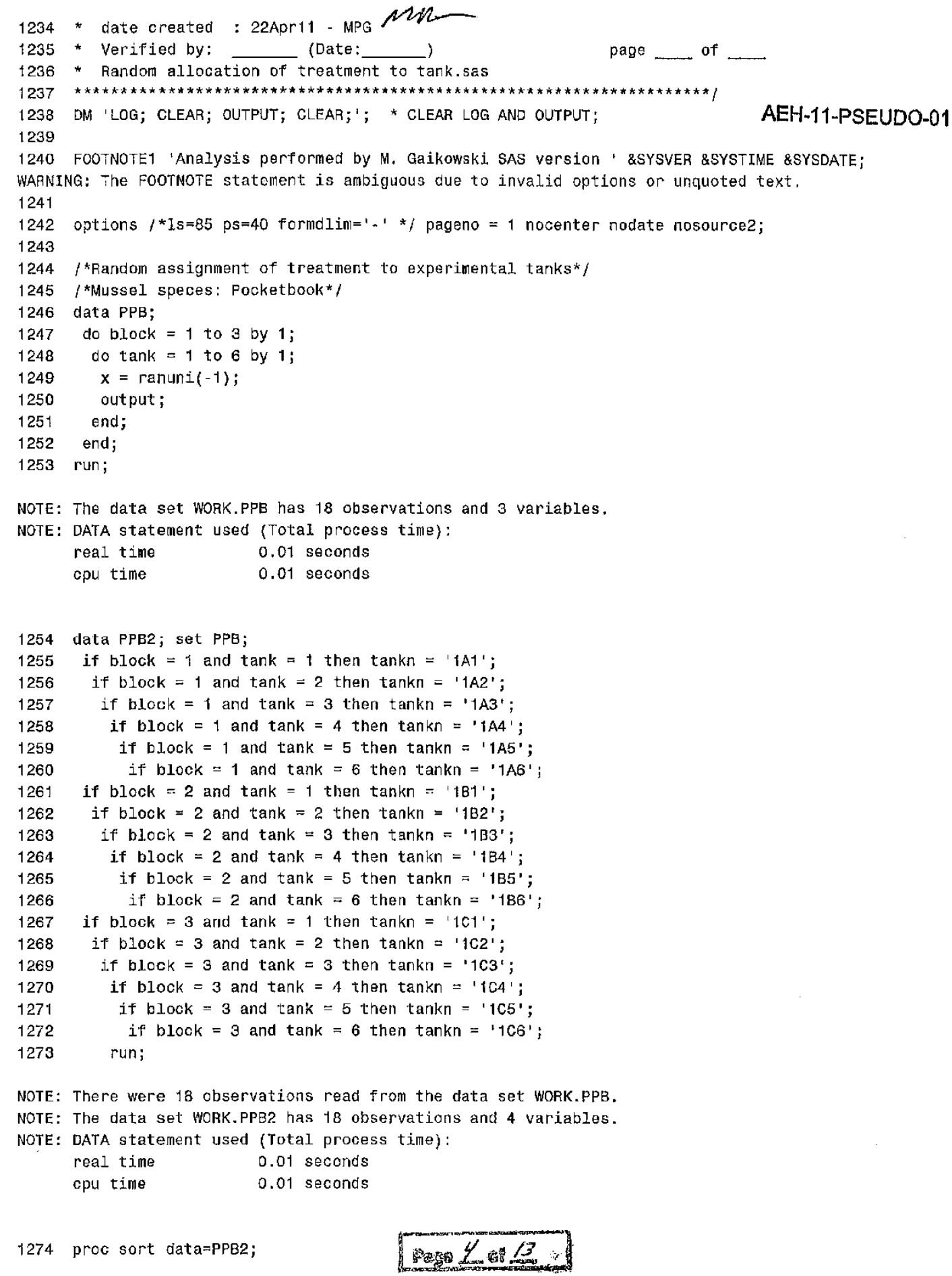


1275 by $x$;

1276 run;

AEH-11-PSEUDO-01

NOTE: There were 18 observations read from the data set wORK.PPB2.

NOTE: The data sot WORK.PPB2 has 18 observations and 4 variables.

NOTE: PROCEDURE SORT used (Total process time):

real time $\quad 0.01$ seconds

cpu time $\quad 0.01$ seconds

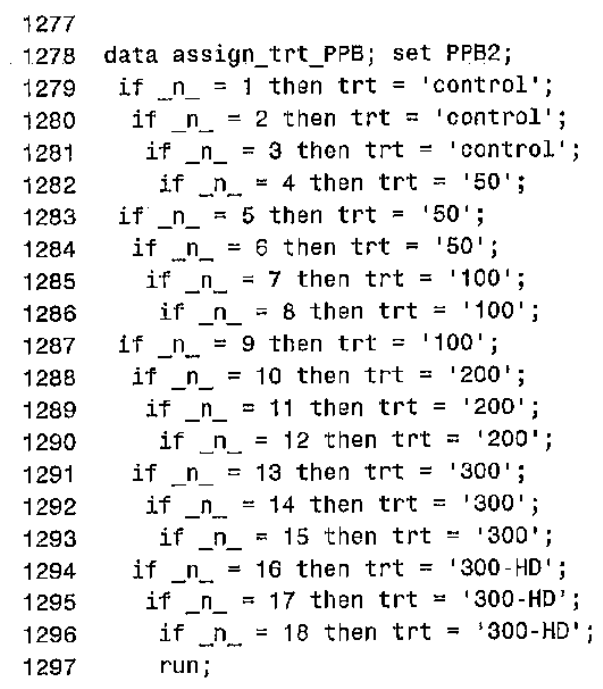

NOTE: There were 18 observations read from the data set WORK.PPB2.

NOTE: The data set WORK.ASSIGN_TRT_PPB has 18 observations and 5 variables.

NOTE: DATA statement used (Total process time):

rcal time $\quad 0.01$ seconds

cpu time $\quad 0.01$ seconds

1298 proc print data= assign_trt_PPB;

1299 title1 $h=2$ 'Effects of Psuedomonas fluorescens (Pf-CL145A) to glochidia from seven unionid

1299 ! mussel species';

1300 title2 $h=1.5$ 'AEH-11-PSUED0-01'

1301 titie3 $h=1$ 'Random assignment of treatment to experimental tanks';

1302 title4 $h=1$ 'Mussel speces: Plain pocketbook';

1303 run;

NOTE: There were 18 observations read from the data set WORK.ASSIGN_TRT_PPB.

NOTE: PROCEDURE PRINT used (Total process time):

real time $\quad 0.01$ soconds

cpu time $\quad 0.01$ seconds

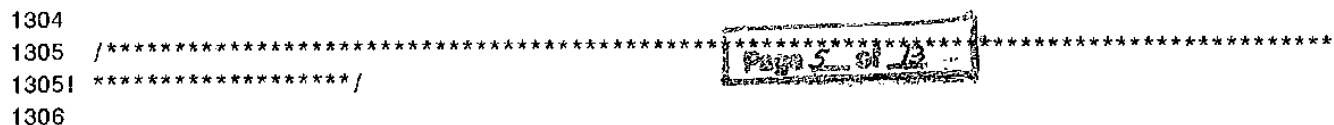


1307 /*Mussel, speces: Higgins eye*/

1308 data HGE;

1309 do block $=1$ to 3 by $1 ;$

1310 do $\operatorname{tank}=1$ to 6 by 1 ;

$1311 \quad x=\operatorname{ranuni}(-1)$;

1312 output;

1313 end;

1314 end;

1315 run;

NOTE: The data set WORK. MGE has 18 observations and 3 variables.

NOTE: DATA statement used (TotaI process time):
real time
0.00 seconds
cpu time
0.00 seconds

1316 data HGE2; set HGE

1317 if block $=1$ and $\operatorname{tank}=1$ then $\operatorname{tankn}=$ ' $2 \mathrm{~A} 1$ ';

1318 if block $=1$ and tank $=2$ then tankn $=' 2 \mathrm{~A} 2^{\prime}$;

if block $=1$ and tank $=3$ then tankn = ' $2 \mathrm{~A} 3^{\prime}$;

if block $=1$ and tank $=4$ then $\operatorname{tankn}={ }^{\prime} 2 \mathrm{A4}{ }^{\prime}$;

if block $=1$ and tank $=5$ then tankn $=$ ' $2 \mathrm{~A} 55^{\prime}$;

if block $=1$ and tank $=6$ then $\operatorname{tankn}={ }^{\prime} 2 A 6^{\prime}$;

if block $=2$ and tank $=1$ then $\operatorname{tankn}=' 2 \mathrm{~B} 1$ ' ';

if block $=2$ and $\operatorname{tank}=2$ then $\operatorname{tankn}={ }^{\prime} 2 \mathrm{~B} 2$ ';

if block $=2$ and tank $=3$ then tankn $={ }^{\prime} 2 \mathrm{~B}^{\prime}$ ';

if block $=2$ and tank $=4$ then $\operatorname{tankn}={ }^{\prime} 2 \mathrm{~B} 4$ ';

if block $=2$ and tank $=5$ then tankn $=$ ' $2 B 5$ ';

if b.lock $=2$ and $\operatorname{tank}=6$ then $\operatorname{tankn}={ }^{\prime} 2 \mathrm{~B}^{\prime}{ }^{\prime}$;

if block $=3$ and tank $=1$ then $\operatorname{tankn}=$ ' $2 \mathrm{C} 1$ ';

if block $=3$ and $\operatorname{tank}=2$ then $\tan k n={ }^{\prime} 2 \mathrm{C} 2{ }^{\prime}$;

if block $=3$ and tank $=3$ then tankn $={ }^{\prime} 2 \mathrm{cs}{ }^{\prime}$;

if block $=3$ and tank $=4$ then $\operatorname{tankn}=$ ' $2 \mathrm{C} 4$ ';

if block $=3$ and tank $=5$ then tankn $={ }^{\prime} 2 \mathrm{C} 5^{\prime}$;

if block $=3$ and tank $=6$ then tankn $=$ ' 2 c 6 ' ;

run;

NOTE: There were 18 observations read from the data set WORK. HGE.

NOTE: The data set WORK.HGE2 has 18 observations and 4 variables.

NOTE: DATA statement used (Total process time):
real time
0.01 seconds

cpu time

0.01 seconds

1336 proc sort data=rige2;

1337 by $x$;

1338 run;

NOTE: There were 18 observations read from the data set WORK. HGE2.

NOTE: The data set WORK. HGE2 has 18 observations and 4 variables.

NOTE: PROCEDURE SORT used (Total process time):
real time
0.00 seconds
cpu time
0.00 seconds

1339

1340 data assign_treat_HGE; set HGE2;

Fus 


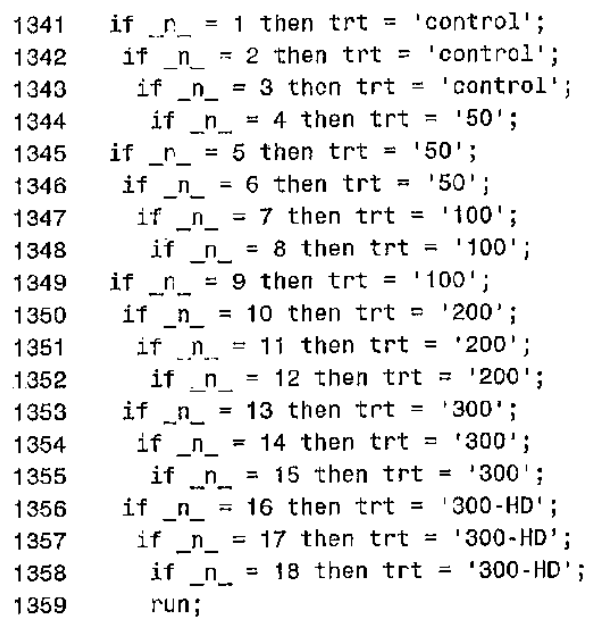

NOTE: There were 18 observations read from the data set WORK. HGE2.

NOTE: The data set WOHK.ASSIGN_TREAT_HGE has 18 observations and 5 variables.

NOTE: DATA statement used (Total process time):
real time
0.01 seconds
cpu time
0.01 seconds

1360 proc print data= assign_treat HGE;

1361 title1 $h=2$ 'Effects of Psuedomonas fluorescens (Pf-CL145A) to glochidia from seven unionid

1361: mussel species' :

1362 ti.tle2 $h=1.5$ 'AEH-11-PSUEDO-01';

1363 title3 $h=1$ 'Handom assignment of treatment to experimental tanks';

1364 ti.te4 $h=1$ 'Mussel speces: Higgins eye';

1365 run;

NOTE: There were 18 observations read from the data set WORK.ASSIGN_TREAT_HGE.

NOTE: PROCEDURE PRINT used (Total process time):
real time
0.00 seconds

cpu time

0,00 seconds

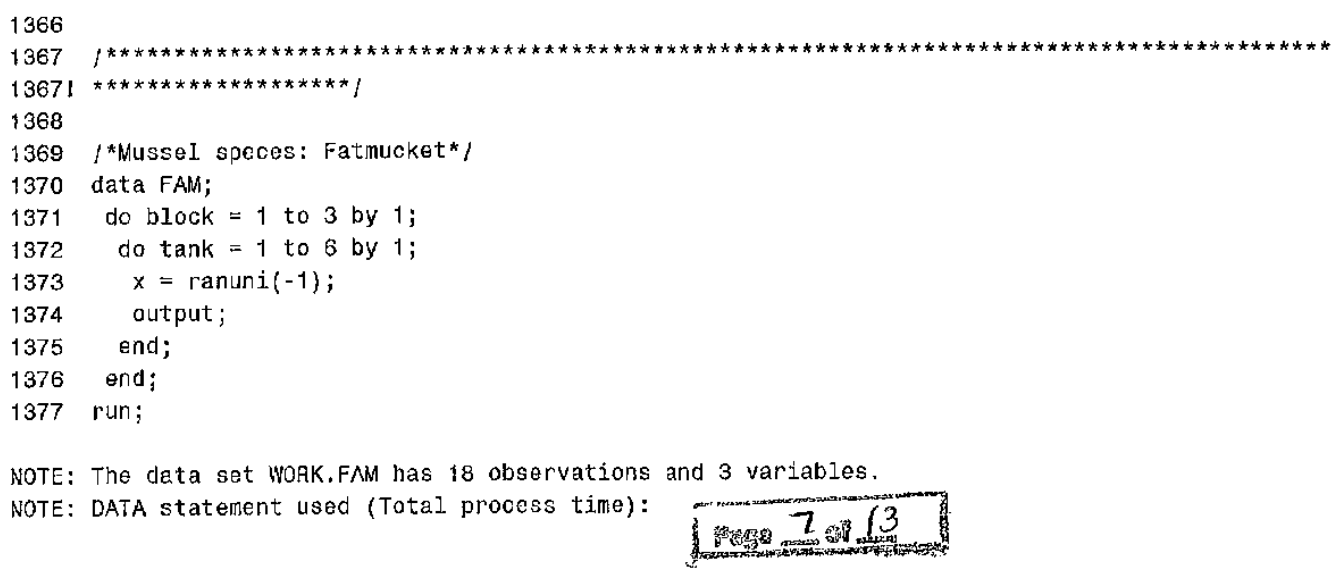




$$
\begin{array}{ll}
\text { real time } & 0.01 \text { seconds } \\
\text { cpu time } & 0.01 \text { seconds }
\end{array}
$$

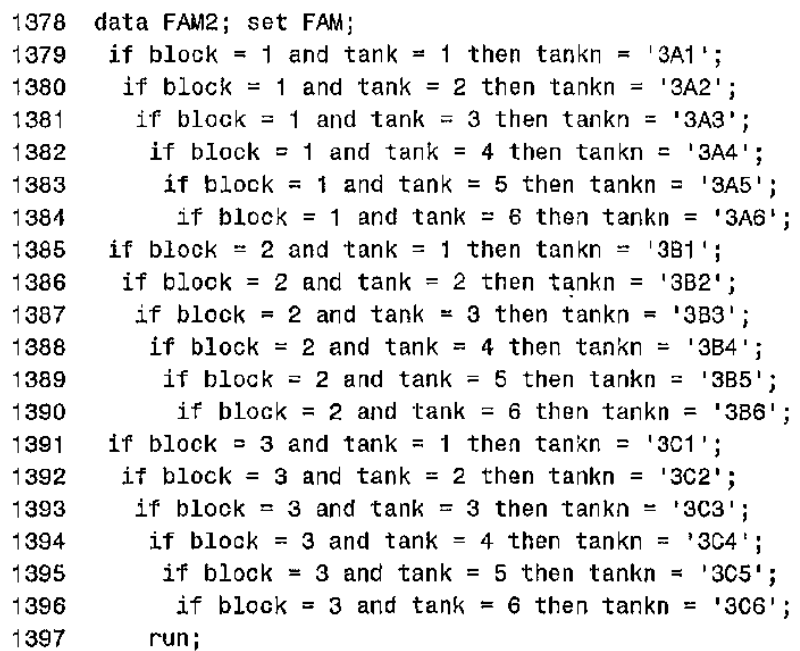

NOTE: There were 18 observations read from the data set WORK.FAM. NOTE: The data set WORK.FAM2 has 18 observations and 4 variables. NOTE: DATA statement used (Total process time):
real time
0.01 seconds

cpu time

0.01 seconds

NOTE: There were 18 observations read from the data set WORK.FAM2.

NOTE: The data set WORK.FAM2 has 18 observations and 4 variables.

NOTE: PROCEDURE SORT used (Total process time):
real time
0.01 seconds
cpu timo
0.01 seconds

1401

1402 data assign_treat_FAM; set FAM2;

1403 if $n_{-}=1$ then trt $\simeq$ 'control';

1404 if $n_{-}=2$ then trt = 'control';

1405 if $n_{-}=3$ then trt $=$ 'control',

1406 if $n_{-}=4$ then trt $={ }^{\prime} 50$ ';

1407 if $n_{-}=5$ then trt $=' 50^{\prime}$;

1408 if $n_{-}=6$ then trt $=150$;

1409 if $\bar{n}_{-}=7$ then trt $=11001$;

1410 if $n_{-}=8$ then trt $=1100^{\prime}$;

1411 if $n_{-}=9$ then trt $=1100 '$;

1412 if ${ }_{n}{ }_{-}=10$ then trt $=1200 '$;

1413 if $\bar{n}_{-}=11$ then trt $=' 200 '$;

1414 if ${ }_{n} n_{-}=12$ then trt $=' 200 '$;

8013 


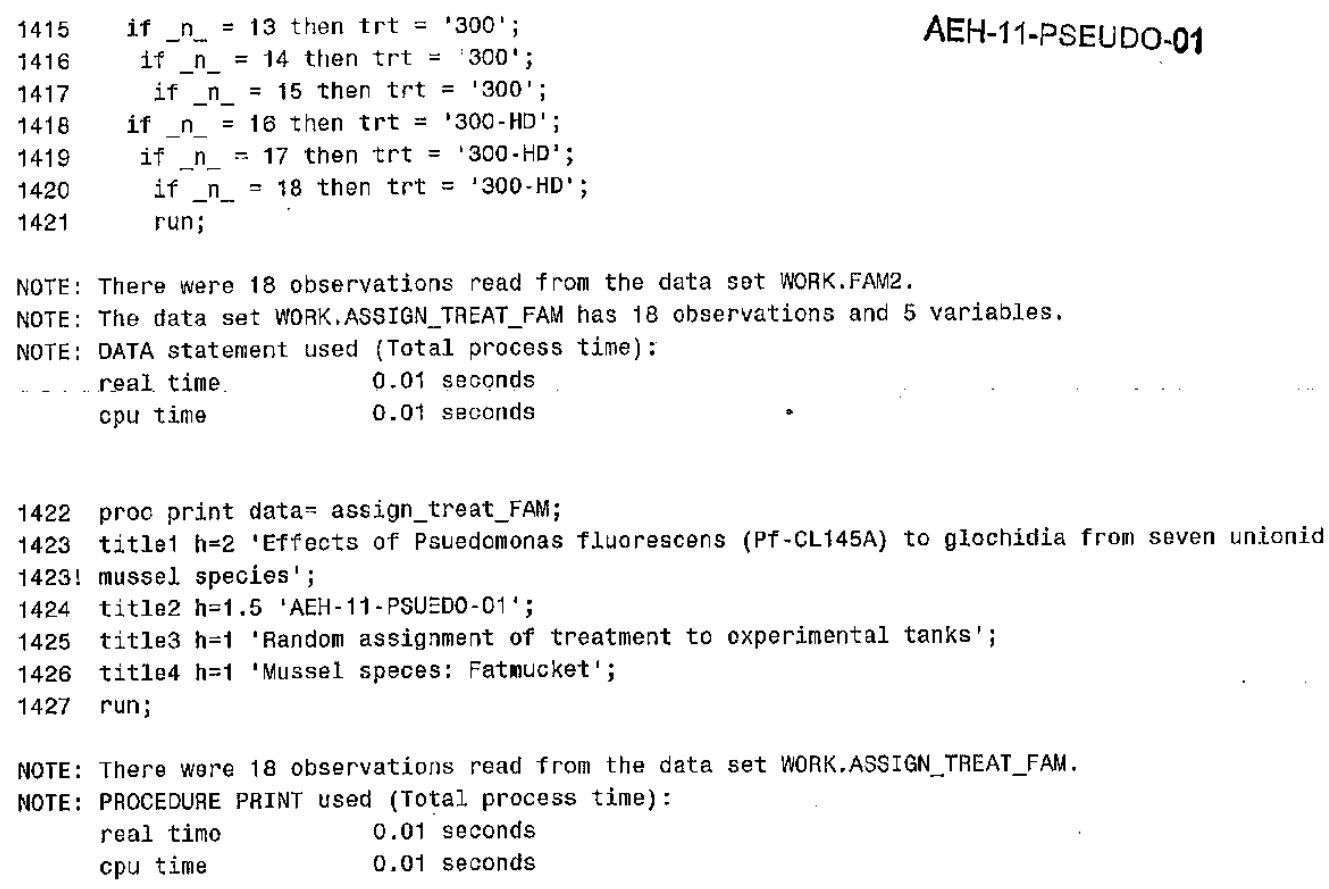

NOTE: There were 18 observations read from the data sot WORK.FAM2

NOTE: The data set WORK.ASSIGN_TREAT_FAM has 18 observations and 5 variables.

NOTE: DATA statement used (Total process time):

real time. $\quad 0.01$ seconds

cpu time $\quad 0.01$ seconds

\section{AEH-11-PSEUDO-01}

1422 proo print data= assign_treat_FAM

1423 title1 $h=2$ 'Effects of Psuedomonas fluorescens (Pf-CL145A) to glochidia from seven unionid

1423 ! mussel species';

1424 title2 $h=1.5$ 'AEH-11-PSUEDO-0.1';

1425 title3 $h=1$ 'Random assignment of treatment to oxperimental tanks';

1426 title4 $\mathrm{h}=1$ 'Mussel speces: Fatmucket';

1427 run;

NOTE: There wore 18 observations read from the data set WORK.ASSIGN_TREAT_FAM.

NOTE: PROCEDURE PRINT used (Total process time):
real time
0.01 seconds
cpu time
0.01 seconds 


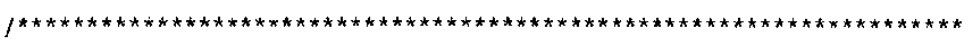

* Study Number : AEH-11-PSUEDO-01

AEH-11-PSEUDO-01

* Study Director: Jim Luoma

* date created : 22Apr11 - MPG MML-

* Aandom allocation of treatment to tank, sas

$\star * * * * * * * * * * * * * * * * * * * * * * * * * * * * * * * * * * * * * * * * * * * * * * * * * * * * * * * * * * * * * * * * * * * *)$

DM 'LOG; CleAR; OUTPUT; ClEAR;'; * CLEAR LOG AND OUTPUT;

FOOTNOTE1 'Analysis performed by M. Gaikowski. SAS version ' \&SYSVER \&SYSTIME \&SYSDATE;

options $/{ }^{*} \mathrm{ls}=85 \mathrm{ps}=40$ formdlim=' - ' */ pageno $=1$ nocenter nodate nosource 2 ;

1 *Random assignment of treatment to experimental tanks*/

$I^{*}$ Musse.I speces: Pocketbook*/

data PPB;

do block $=1$ to 3 by 1 ;

do $\operatorname{tank}=1$ to 6 by 1 ;

$x=\operatorname{ranuni}(-1)$;

output;

end;

end;

run;

data PPB2; set PPB;

if block $=1$ and $\operatorname{tank}=1$ then tankn = ' $1 \mathrm{A1}$ ';

if block $=1$ and tank $=2$ then tankn $=11 \mathrm{~A}^{\prime}$;

if block $=1$ and tank $=3$ then tankn $=$ ' $1 \mathrm{~A} 33^{\prime}$;

if block $=1$ and tank $=4$ then $\operatorname{tankn}=11 \mathrm{~A} 4$ ';

If block $=1$ and tank $=5$ then $\operatorname{tankn}=11 \mathrm{~A} 5$ ';

if block $=1$ and tank $=6$ then tankn $=1 \mathrm{~A} 6^{\prime}$;

if block $=2$ and tank $=1$ then $\operatorname{tankn}=11 \mathrm{B1}$ ';

if block $=2$ and tank $=2$ then tankn $=11 \mathrm{~B} 2^{\prime}$;

if block $=2$ and tank $=3$ then $\operatorname{tankn}=$ '1B3';

if block $=2$ and tank $=4$ then tankn $=11 \mathrm{~B} 4$ ';

if block $=2$ and tank $=5$ then $\operatorname{tankn}=185 '$;

if block $=2$ and tank $=6$ then tankn $=' 186$ ';

if block $=3$ and $\operatorname{tank}=1$ then $\operatorname{tankn}=$ ' 101 ';

if block $=3$ and tank $=2$ then tankn $=11 \mathrm{c} 2$ ';

if block $=3$ and tank $=3$ then tankn $=' 1 \mathrm{C3}$ ';

if block $=3$ and tank $=4$ then $\operatorname{tankn}=1 \mathrm{C} 4$ ';

if $b$ lock $=3$ and tank $=5$ then tankn $=105 '$;

if block $=3$ and $\operatorname{tank}=6$ then $\operatorname{tankn}=' 1 \mathrm{C} 6 '$;

run;

proc sort data=PPB2;

by $x$;

run;

data assign_trt_PPB; set PPB2;

if $n_{-}=1$ then trt = 'control';

if $\bar{n}_{-}=2$ then trt $=$ 'control';

if $n_{-}=3$ then trt $=$ 'control';

if $\bar{n}=4$ then trt $=$ ' $50^{\prime}$ ';

if $n_{-}=5$ then trt $={ }^{\prime} 50^{\prime}$;

if $n_{-}=6$ then trt $=' 50^{\prime}$;

if $n_{-}=7$ then trt $=1100 '$;

if $\vec{n}_{-}=8$ then trt $=1100^{\prime}$;

if $n_{-}=9$ then trt $=100^{\prime}$;

10.10 


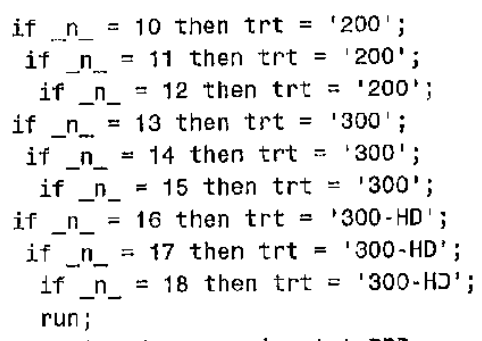

\section{AEH-11-PSEUDO-01}




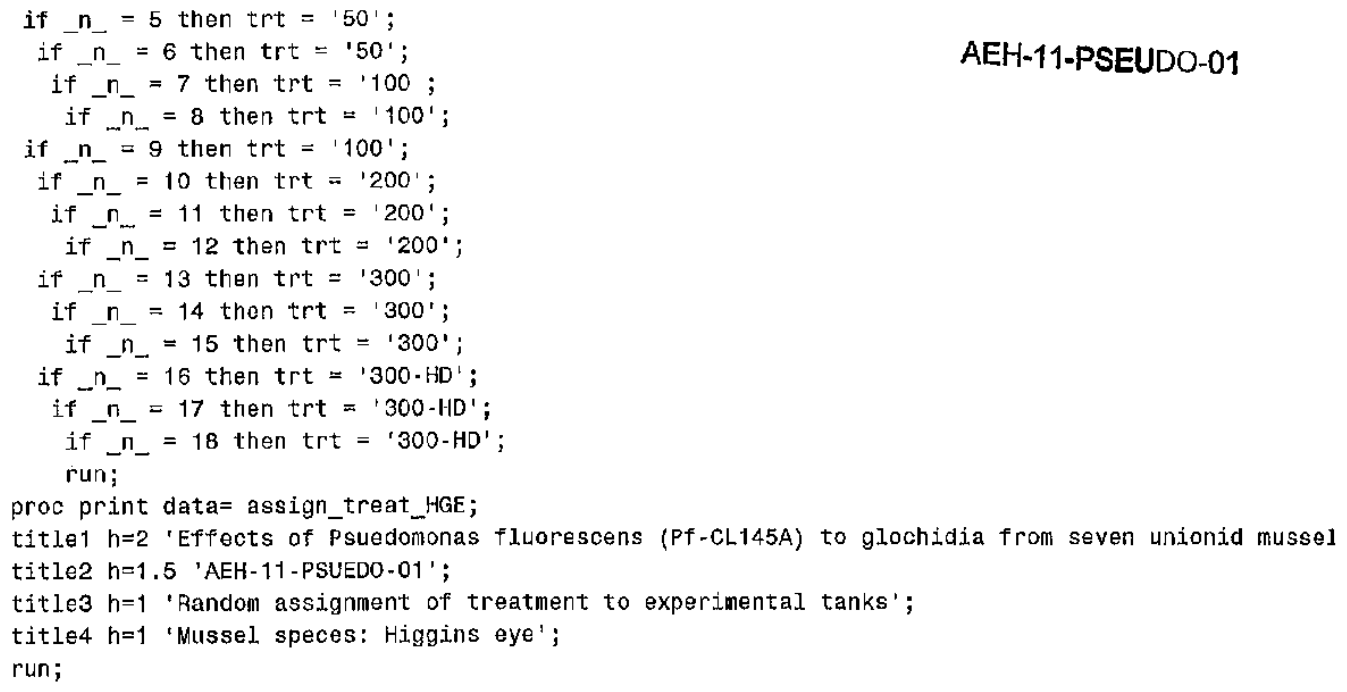


data assign_treat_FAM; set FAM2;

if $n_{-}=1$ then trt = 'control.';

if $\vec{n}_{-}=2$ then trt $=$ 'control';

if $n=3$ then trt $=$ 'control';

if $\vec{n}_{-}=4$ then trt $={ }^{\prime} 50^{\prime}$;

if $n=5$ then trt $=150 '$;

if $\bar{n}_{-}=6$ then trt $=' 50^{\prime}$;

if $n=7$ then trt $=1100 '$;

if $\bar{n}_{-}=8$ then trt $=1100 '$;

if $n=9$ then trt $=1100 '$;

if $\bar{n}_{-}=10$ then $\operatorname{trt}={ }^{\prime} 200^{\prime}$;

if $\vec{n}=11$ then $t r t=' 200$ ';

if $\vec{n}_{-}=12$ then trt $=1200^{\prime}$;

if $n=13$ then $\operatorname{trt}=1300^{\prime}$;

if $\bar{n}_{-}=14$ then trt $=' 300 '$;

if $n=15$ then trt $=1300^{\prime}$;

if $n_{-}=16$ then trt $=300-\mathrm{HO}^{\prime}$;

if $\bar{n}_{-}=17$ then trt $=1300-H^{\prime}$ ';

if ${ }_{-}{ }_{-}=18$ then trt $=300-\mathrm{HD}^{\prime}$; run;

proc print data= assign_treat_FAM;

title1 $h=2$ 'Effects of Psuedomonas fluorescens (Pf-CL145A) to glochidia from seven unionid mussel title2 $h=1.5$ 'AEH-11-PSUEDO-01';

title3 $h=1$ 'Random assignment of treatment to experimental tanks';

ti.tle4 $h=1$ 'Mussel speces: Fatmucket';

run;

\section{AEH-11-PSEUDO-01 \\ File folder $14 \mathrm{C}$}

$m i z$

loinly 4

Mmals: 1 TMS Date $5 / 15 / 12$

REVIEWED BY

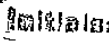
的的?

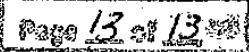




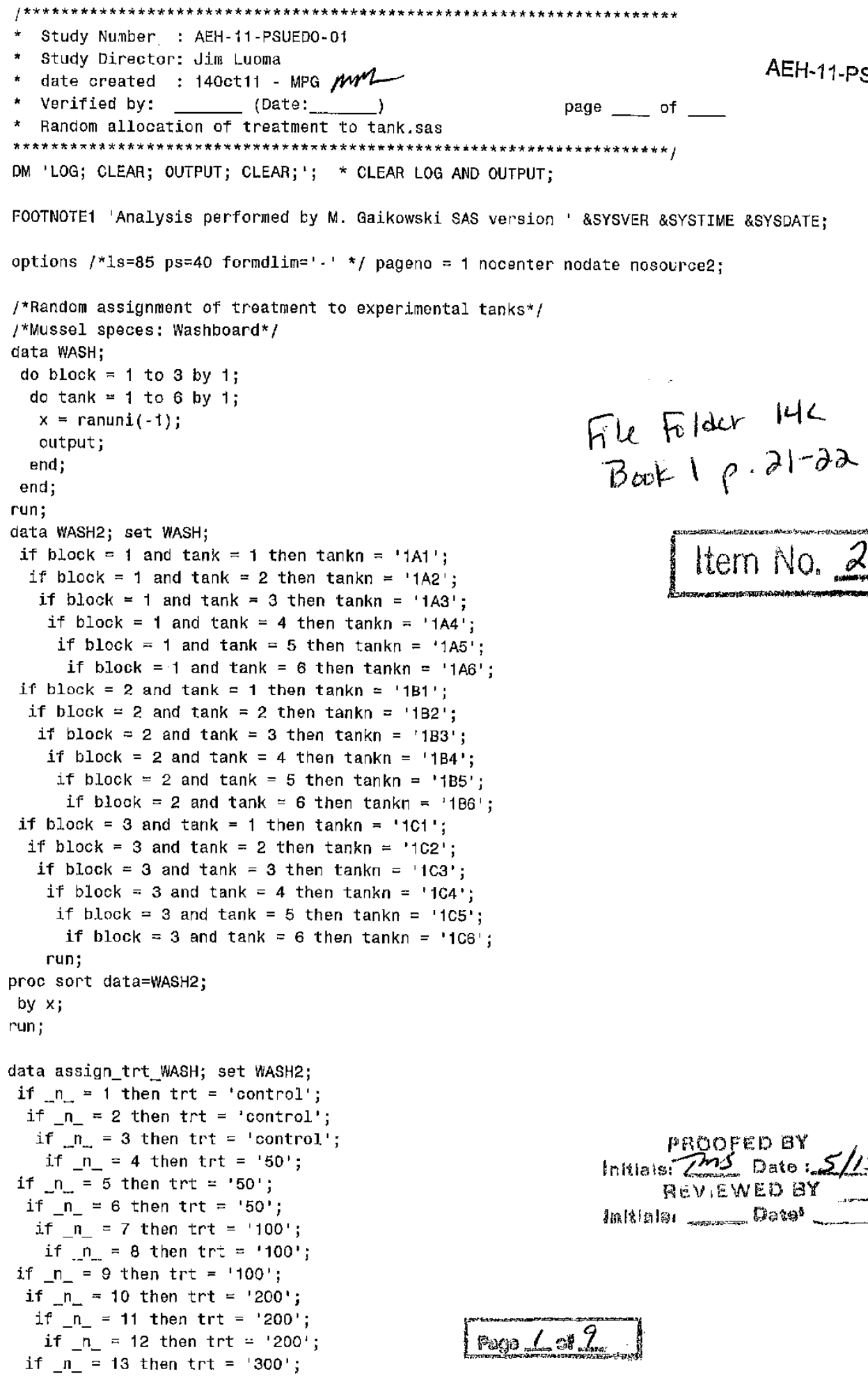

Item No. 2 


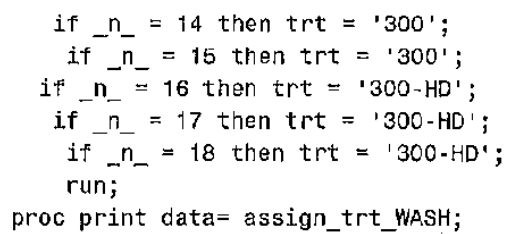




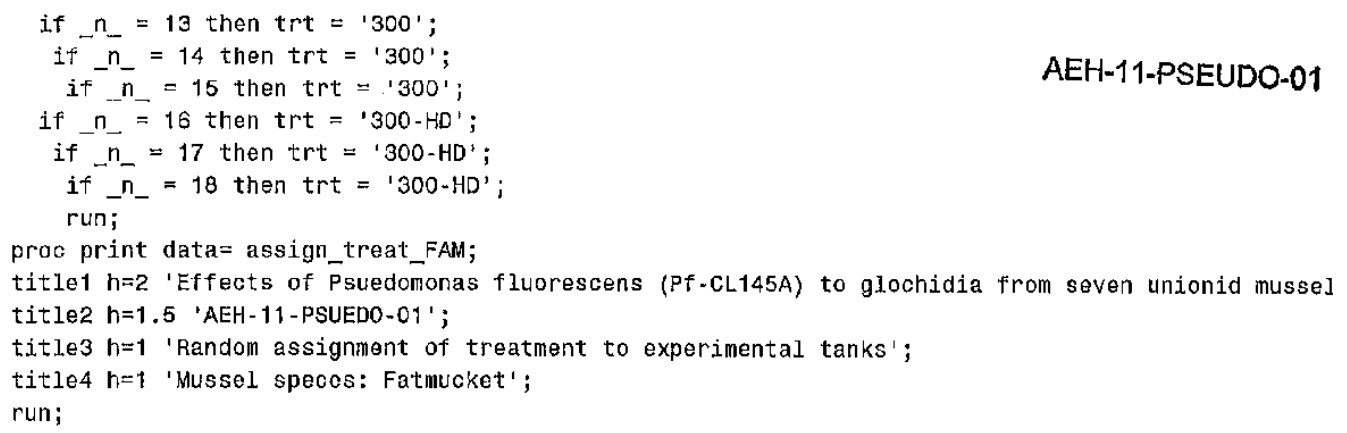

\section{AEH-11-PSEUDO-01}

pogs 3.9 


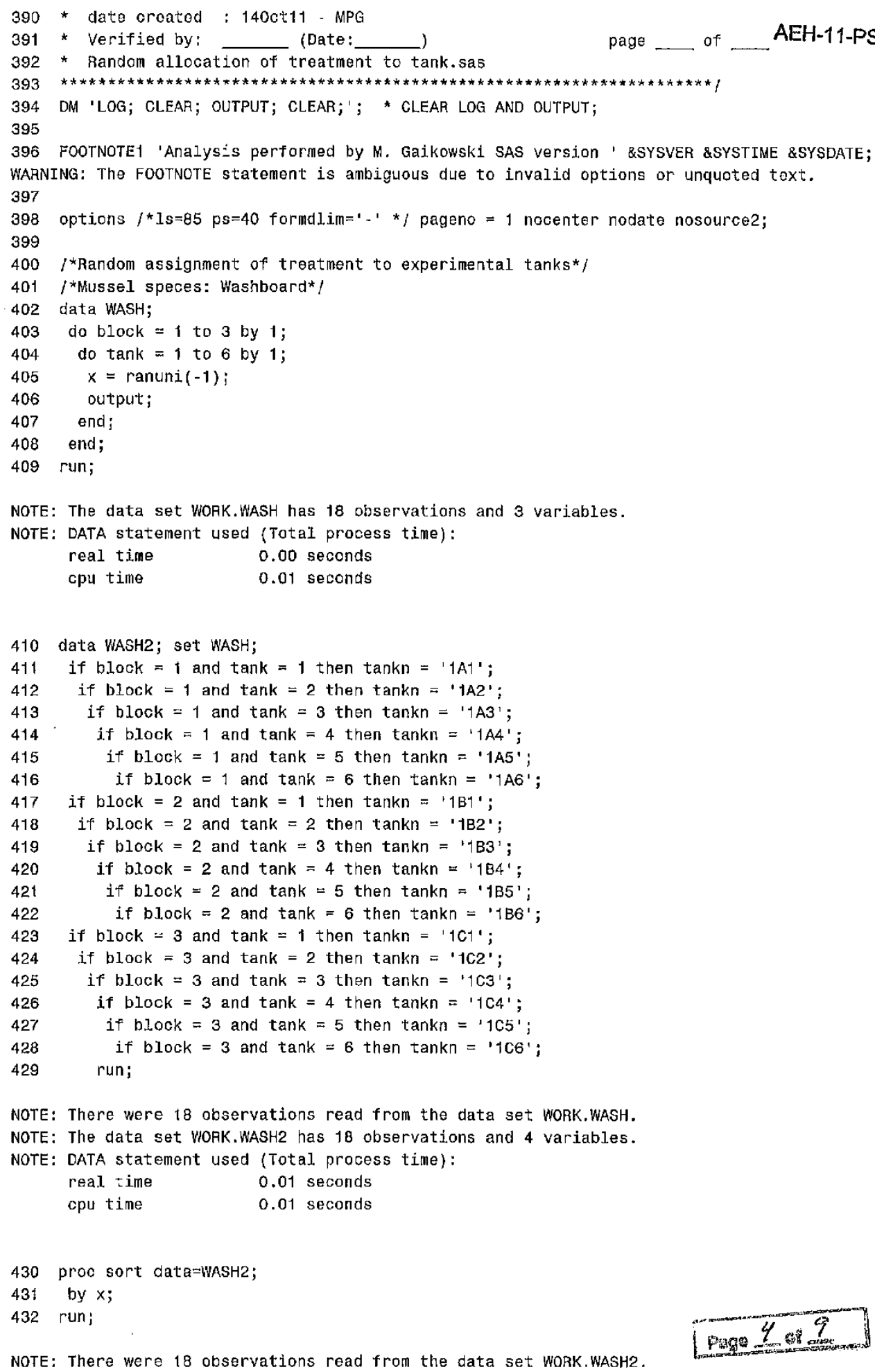

NOTE: There were 18 observations read from the data set. WORK. WASH. NOTE: The data set WORK.WASH2 has 18 observations and 4 variables. NOTE: DATA statement used (Total. process time):
real time
0.01 seconds
cpu time
0.01 seconds

430 proc sort data=WASH2;

43 by $x$;

432 run;

NOTE: There were 18 observations read from the data set WORK. WASH2. 
NOTE: The data set WORK.WASH2 has 18 observations and 4 variables,

NOTE: PROCEDURE SORT used (Total process time):
real time
0.00 seconds
cpu time
0.01 seconds

AEH-11-PSEUDO-01

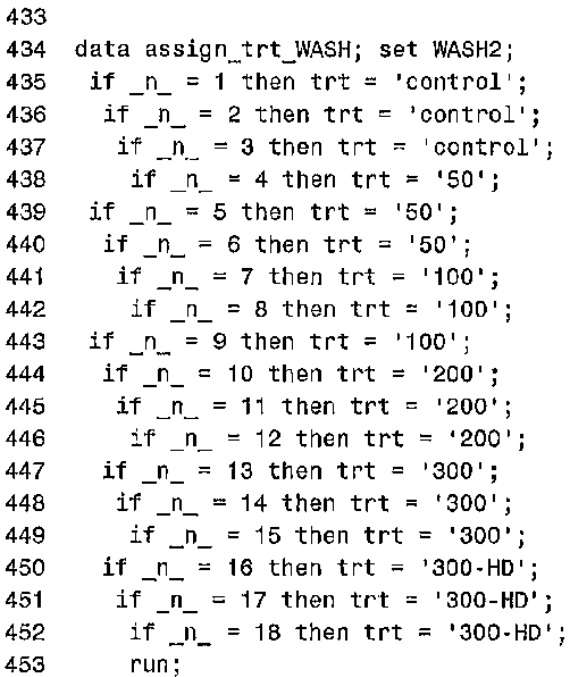

NOTE: There were 18 observations read from the dat a set WOAK. WASH2,

NOTE: The data set WORK.ASSIGN_TRT WASH has 18 observations and 5 variables.

NOTE: DATA statement used (Total process time):
real time
0.01 seconds
cpu time
0.01 seconds

454 proc print data= assign_trt_WASH

455 titlet $h=2$ 'Effects of Psuedomonas fluorescens (Pf-CL145A) to glochidia from seven unionid

455 ! mussel species',

456 tit.le2 $h=1.5$ 'AEH-11-PSUEDO-01';

457 title3 $\mathrm{h}=1$ 'Random assignment of treatment to experimental tanks';

458 title4 $h=1$ 'Mussel speces: Washboard';

459 run;

NOTE: There were 18 observations read from the data set WORK.ASSIGN TRT WASH.

NOTE: PROCEDURE PRINT used (Tota.L process time)
real time
0.00 seconds
cpu time
0.00 seconds

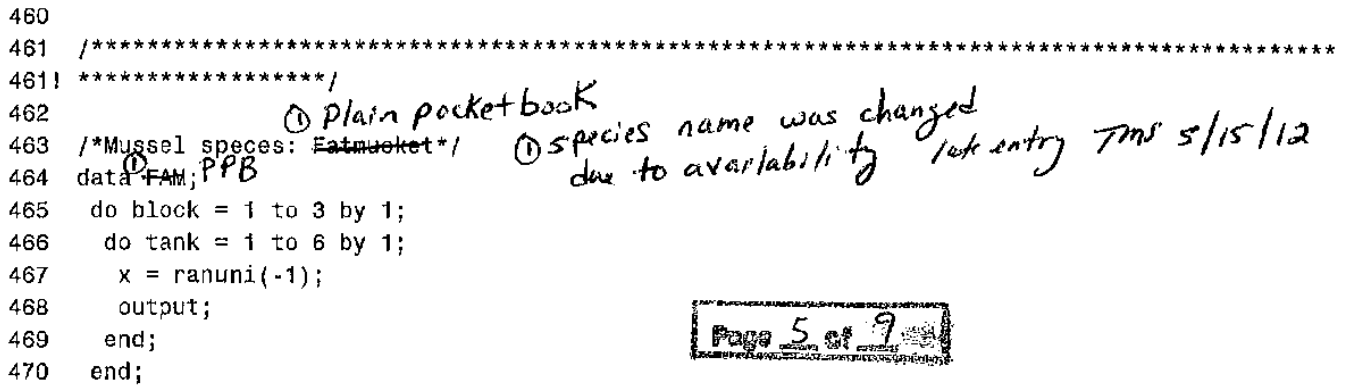


471 run; $\quad 0_{P P B}$

NOTE: The data set WORK. FAl has 18 observations and 3 variables.

NOTE: DATA statement usod (Total process time):

AEH-11-PSEUDO-01

$\begin{array}{ll}\text { real time } & 0.01 \text { seconds } \\ \text { cpu time } & 0.01 \text { seconds }\end{array}$

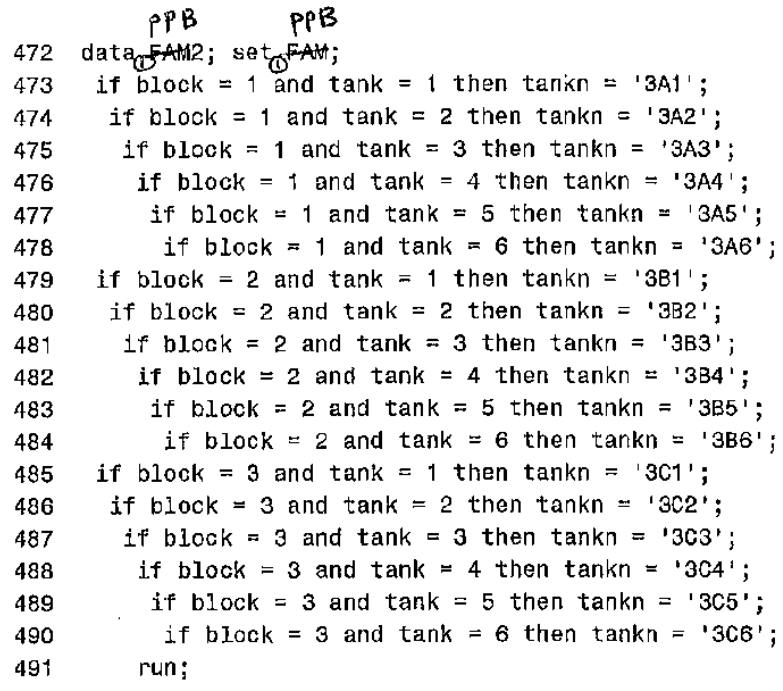

NOTE: There were 18 observations read from the data set WORK. FAM?

NOTE: The data set WORK. FAffy has 18 observations and 4 variables.

NOTE: DATA statement used (Total process time):

real time $\quad 0.01$ seconds

cpu time $\quad 0.01$ seconds

$P P B$

492 proc sort data=FAM2;

493 by $x$;

494 run;

$P P B$

NOTE: There were 18 observations read from the data set WORK. FAM2.

NOTE: The data set WORK. FAM2 has 18 observations and 4 variables.

NOTE: PROCEDURE SORT used (Total process timo):
real time
0.00 seconds
cpu time
0.00 seconds

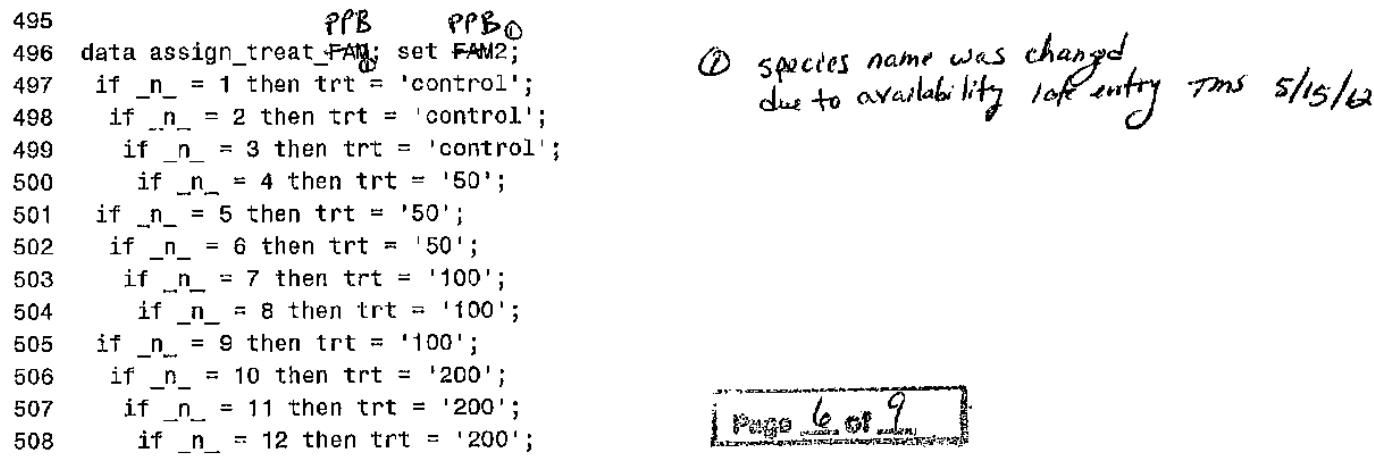




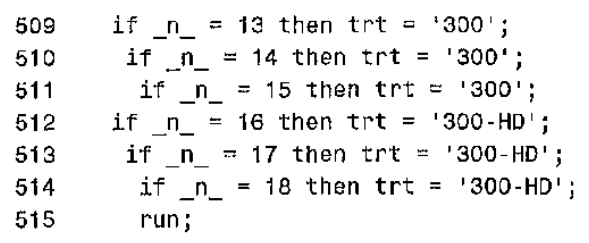

NOTE: There were 18 observations read from the data set WORK. EAM2.

NOTE: The data set WORK.ASSIGN_TREAT_FAd has 18 observations and 5 variables.

NOTE: DATA statement used (Total procesS time):
real time
0.01 seconds
cpu time
0.00 seconds

516 proc print data= assign_treat_PAd

517 title1 h=2 'Effects of Psuedomonas fluorescens (Pf-CL145A) to glochidia from seven unionid 517 ! mussel species';

518 title2 $h=1.5$ 'AEH-11.PSUEDO-01';

519 title3 $h=1$ 'Random assignment of treatment to experimental tanks';

520 title4 $h=1$ 'Mussel speces i to

521 run; Oplain Pocket book

NOTE: There were 18 observations read from the data set WOAK.ASSIGN_TREAT_FATh.

NOTE: PROCEDURE PRINT used (Total process time):
real time
0.00 seconds
cpu time
0.00 seconds

O species name changed des to
avarlability lake entry Toms $5 / 1$ ster 


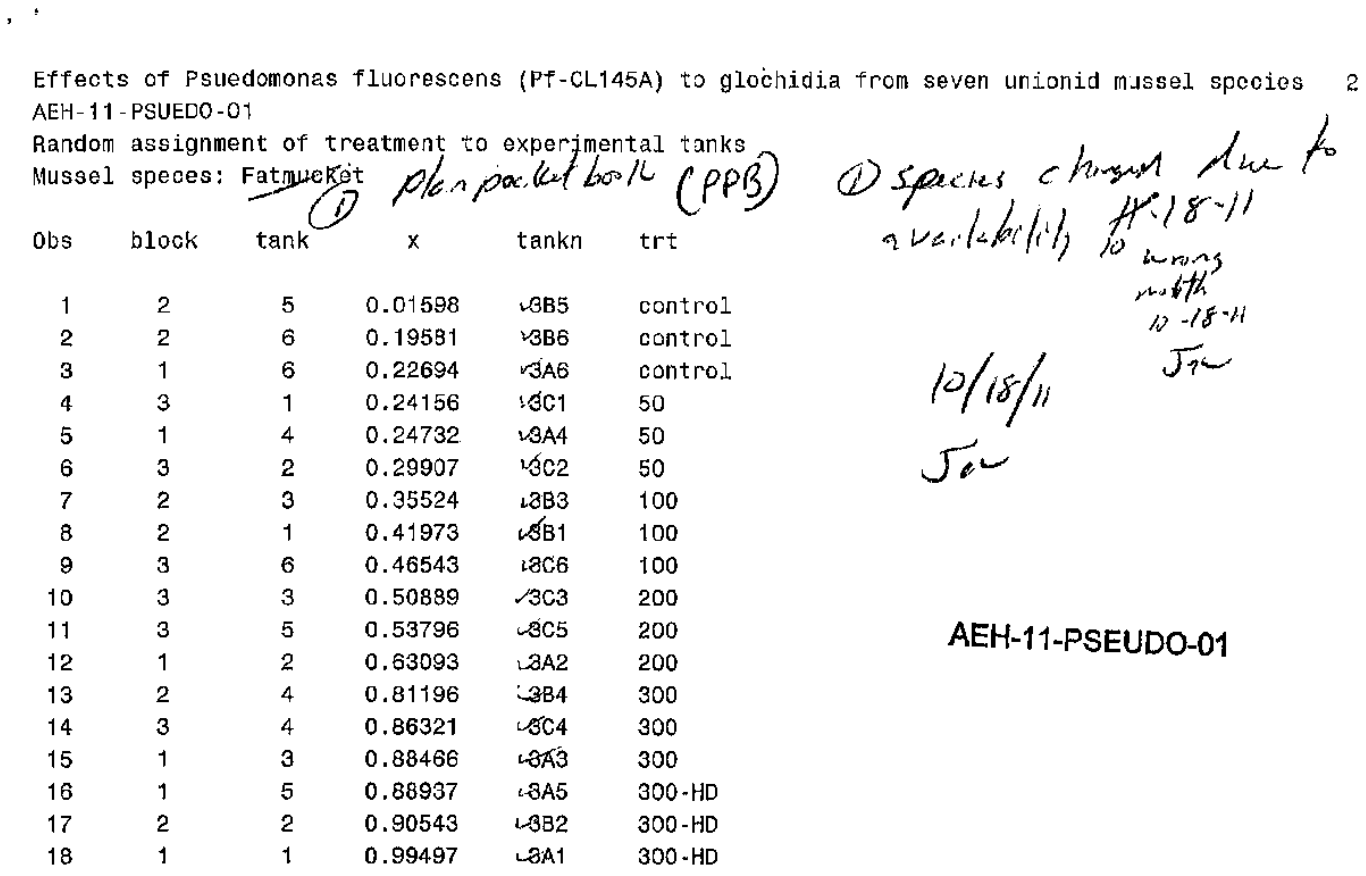

Analysis performed by M. Gaikowski SAS version $9.211: 03$ 1400711 M/P 
Effects of Psuedomonas fluorescens (Pf-CL145A) to glochidia from seven unionid mussel species 1 AEH - 11 - PSUEDO-01

Random assignment of treatment to experimental tanks Mussel speces: Washboard

AEH-11-PSEUDO-01 File folden $14 \mathrm{c}$

\begin{tabular}{|c|c|c|c|c|c|}
\hline Obs & block & tank & $x$ & $\operatorname{tankn}$ & trt \\
\hline 1 & 2 & 2 & 0.04902 & 482 & control \\
\hline 2 & 1 & 5 & 0.19550 & LA5 & control \\
\hline 3 & 2 & 4 & 0.22508 & 너B4 & control \\
\hline 4 & 3 & 2 & 0.25731 & ᄂ1C2 & 50 \\
\hline 5 & 1 & 1 & 0.26201 & WA1 & 50 \\
\hline 6 & 3 & 1 & 0.29013 & Uc1 & 50 \\
\hline 7 & 3 & 6 & 0.48750 & $+c_{6}$ & 100 \\
\hline 8 & 1 & 2 & 0.51734 & TA2 & 100 \\
\hline 9 & 1 & 6 & 0.60907 & VAG & 100 \\
\hline 10 & 2 & 5 & 0.69893 & $m+m_{5}$ & 200 \\
\hline 11 & 3 & 4 & 0.75130 & $+c_{4}$ & 200 \\
\hline 12 & 3 & 3 & 0.78912 & 463 & 200 \\
\hline 13 & 2 & 1 & 0.79399 & TB1 & 300 \\
\hline 14 & 3 & 5 & 0.79401 & - 4'C5 & 300 \\
\hline 15 & 1 & 4 & 0.80175 & WA4 & 300 \\
\hline 16 & 2 & 3 & 0.83625 & -183 & $300-H D$ \\
\hline 17 & 1 & 3 & 0.91376 & UAB & $300-H D$ \\
\hline 18 & 2 & 6 & 0.95637 & $4 B 6$ & $300-H D$ \\
\hline
\end{tabular}

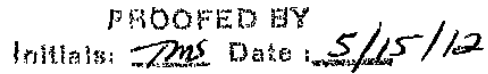
REVIEWED BY Initale:

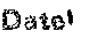

Analysis performed by M. Gaikowski SAS version $9.211: 03140 \mathrm{CT11}$ 


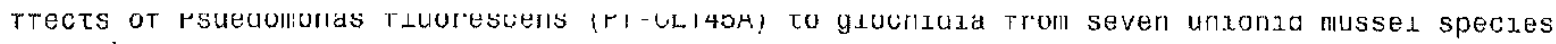
EH-11-PSUEDO-01

andom assignment of treatment to experimental tanks $\zeta_{n}$ ussel speces: Black Sandshell

AEH-11-PSEUDO-01

\begin{tabular}{|c|c|c|c|c|c|c|}
\hline bs & block & $\operatorname{tank}$ & $x$ & $\operatorname{tankn}$ & trt & File folder ILC \\
\hline 1 & 3 & 6 & 0.04349 & $1 \mathrm{C} 6$ & control & Log6ook 1,133 \\
\hline 2 & 1 & 5 & 0.05493 & $1 \mathrm{~A} 5$ & control & \\
\hline 3 & 2 & $t$ & 0.06777 & 1B1 & control & \\
\hline 4 & 1 & 1 & 0.06965 & $1 \mathrm{~A} 1$ & 50 & \\
\hline 5 & 3 & 1 & 0.07510 & $1 \mathrm{C} 1$ & 50 & 3 \\
\hline 6 & 1 & 3 & 0.07952 & $1 \mathrm{A3}$ & 50 & 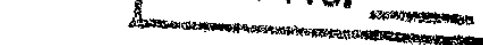 \\
\hline 7 & 2 & 2 & 0.09559 & $1 \mathrm{~B} 2$ & 100 & \\
\hline 8 & 2 & 3 & 0.21685 & $1 \mathrm{B3}$ & 100 & \\
\hline 9 & 3 & 5 & 0.29355 & $1 \mathrm{C5}$ & 100 & \\
\hline 10 & 1 & 6 & 0.32497 & $1 \mathrm{~A} 6$ & 200 & \\
\hline 11 & 2 & 6 & 0.36986 & $1 \mathrm{~B} 6$ & 200 & \\
\hline 12 & 2 & 5 & 0.41768 & 185 & 200 & \\
\hline 13 & 3 & 2 & 0.43417 & $1 \mathrm{C} 2$ & 300 & \\
\hline 14 & 2 & 4 & 0.48474 & $1 B 4$ & 300 & \\
\hline 15 & 1 & 2 & 0.70423 & $1 \mathrm{~A} 2$ & 300 & \\
\hline 16 & 3 & 4 & 0.72592 & $1 \mathrm{C} 4$ & $300-H D$ & \\
\hline 17 & 3 & 3 & 0.74208 & $1 \mathrm{C} 3$ & $300-H D$ & \\
\hline 18 & 1 & 4 & 0.80908 & $1 \mathrm{~A} 4$ & $300-H D$ & \\
\hline
\end{tabular}

PR incials: 27 S Daze $5 / 15 / 12$

PEVIEWED BY

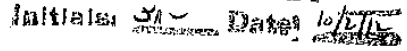

10

nalysis performed by $J$. Luoma SAS version 9.2 11:32 09JAN12 


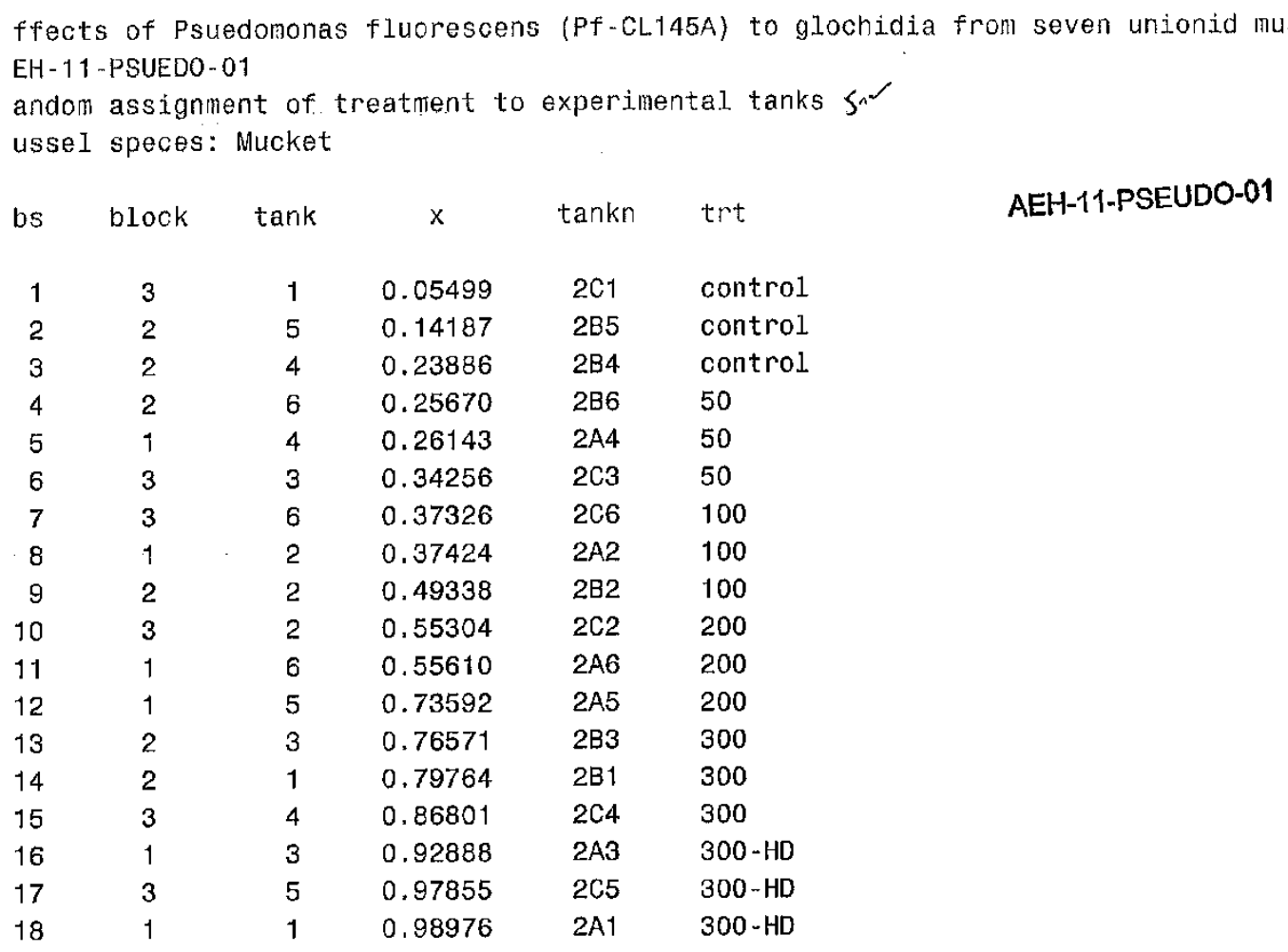

2013 


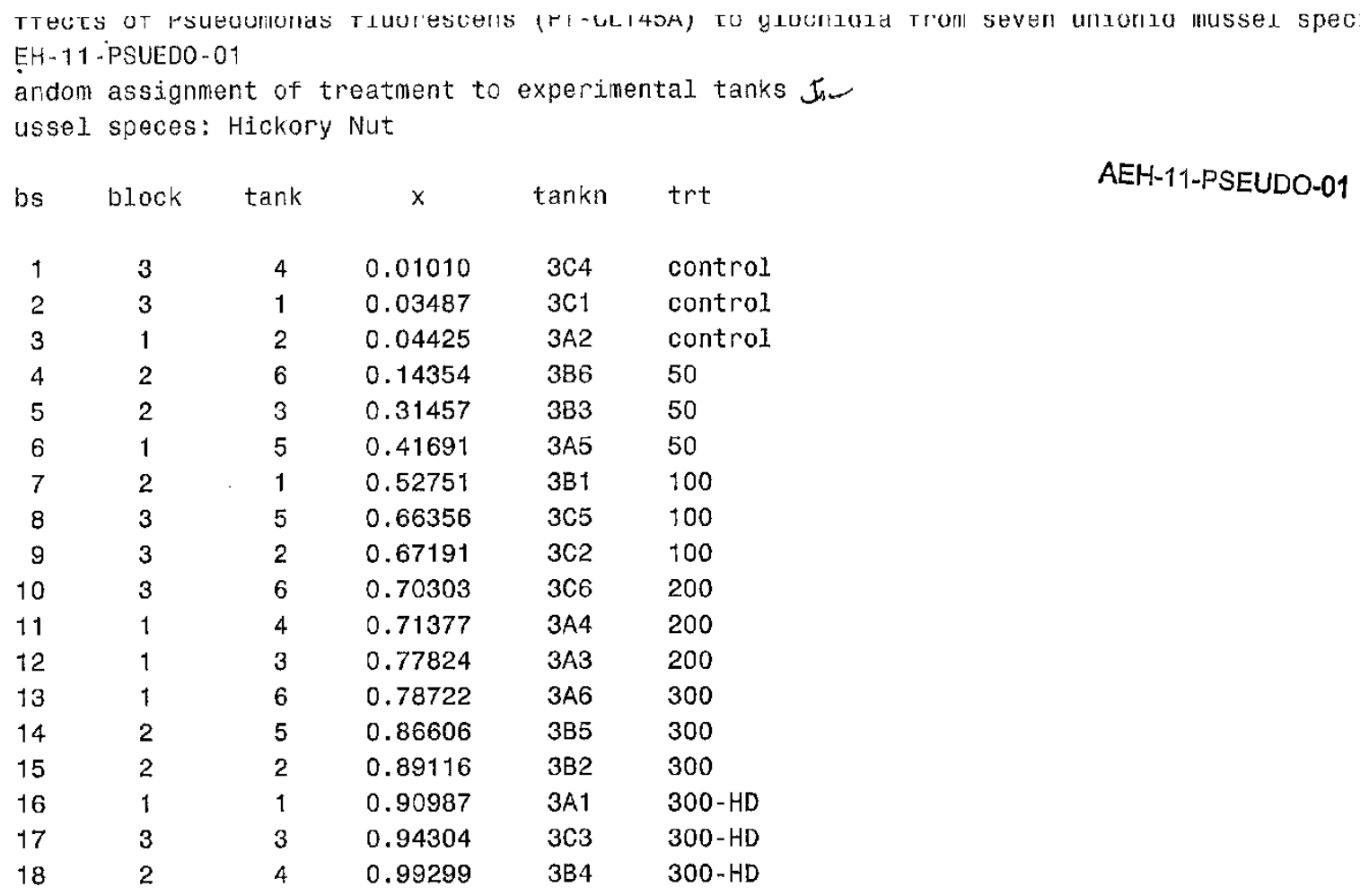

130.3

nalysis performed by $J$. Luoma SAS version 9.2 11:32 09JAN12 


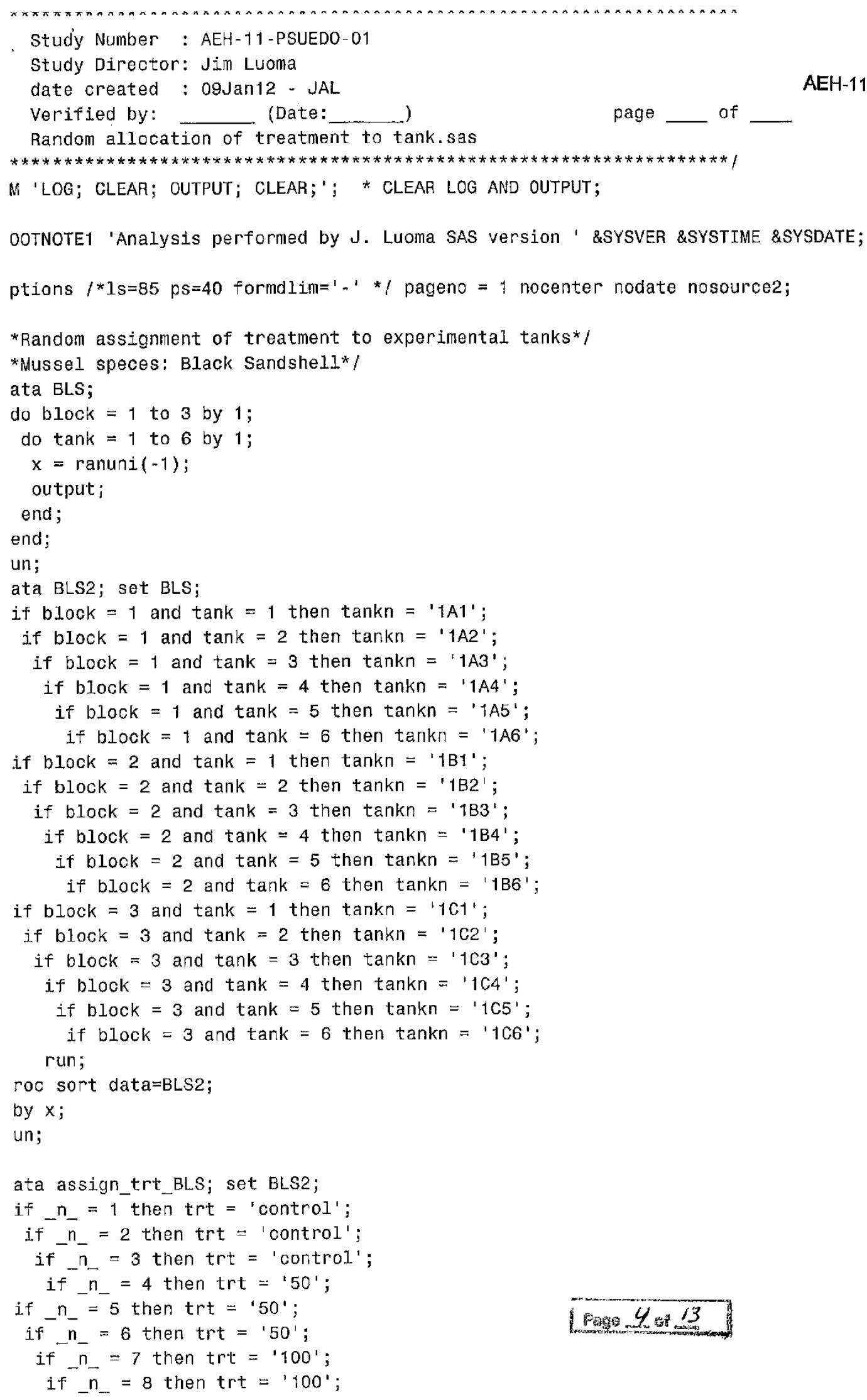




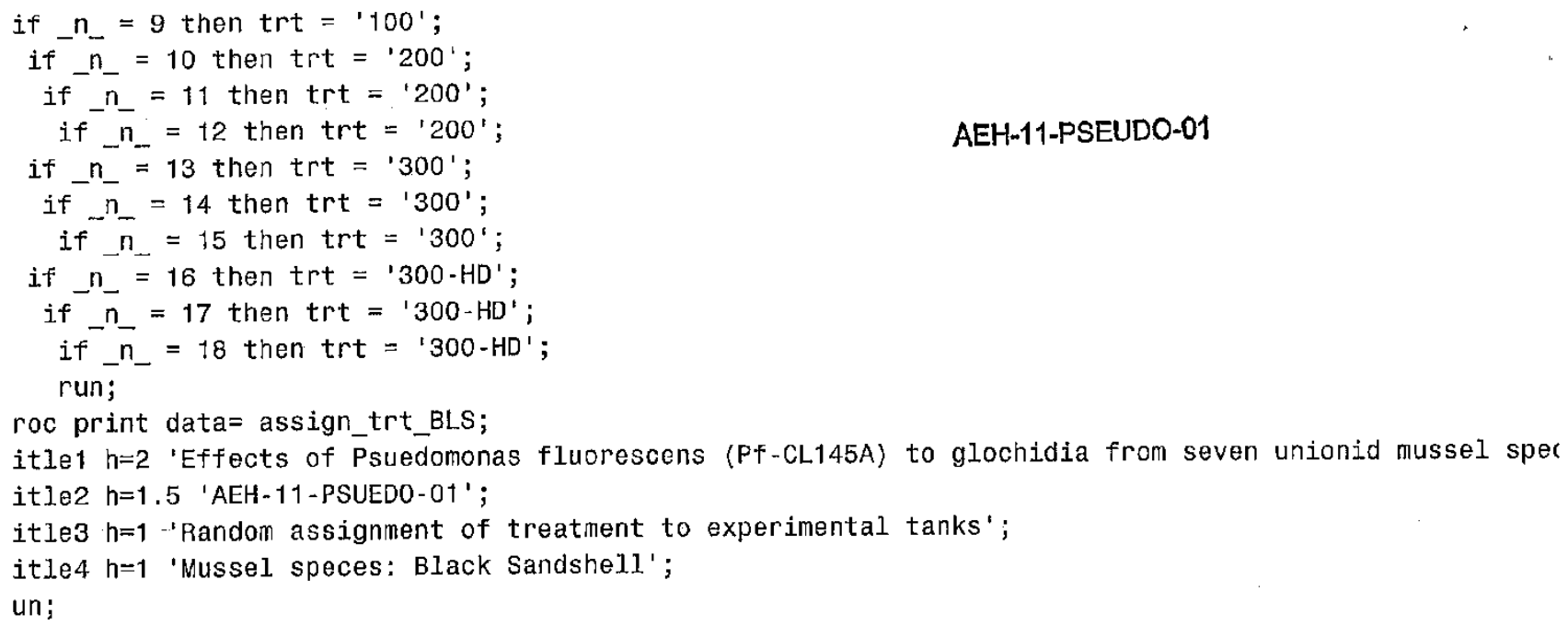

\section{AEH-11-PSEUDO-01}




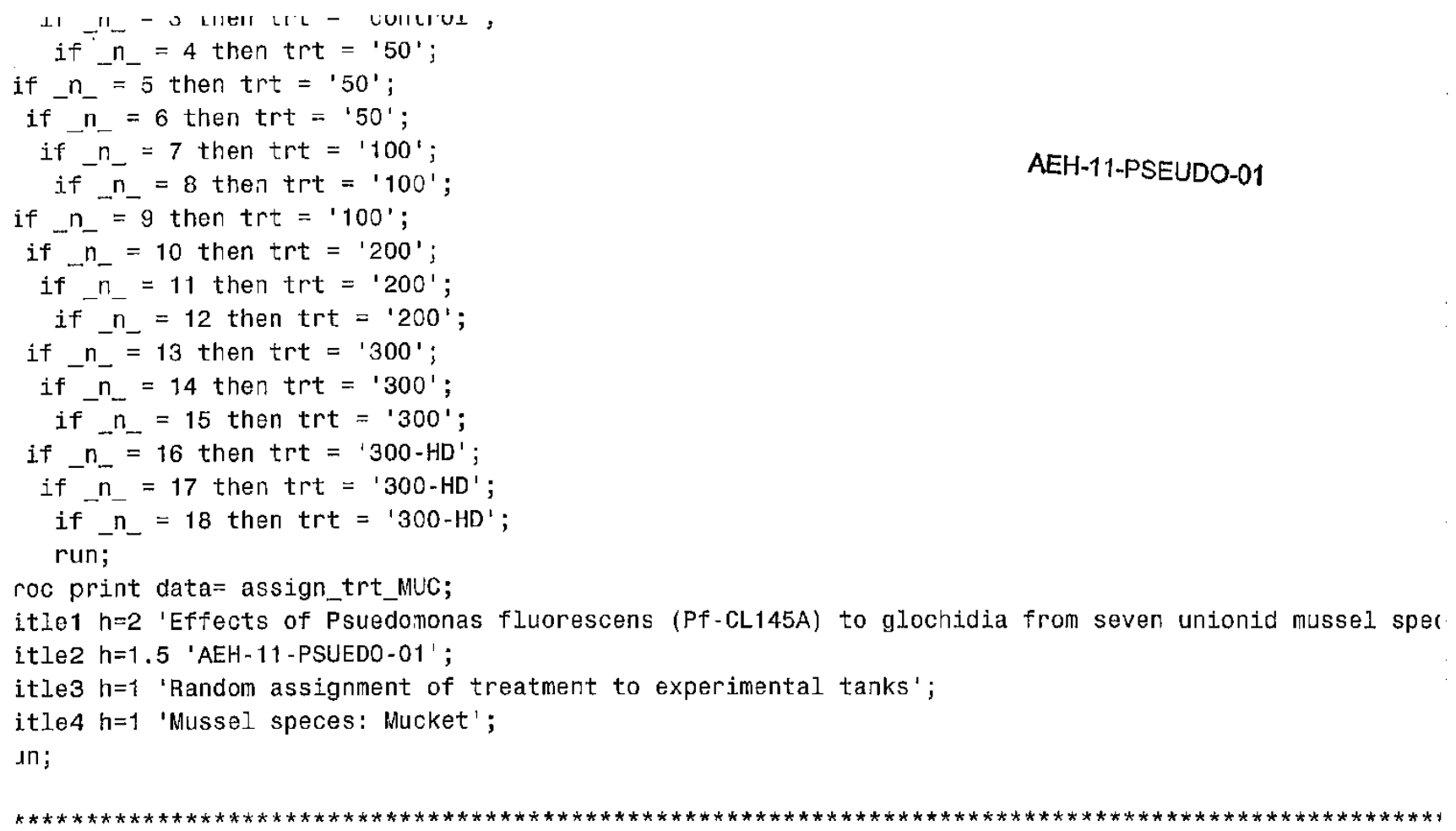

*Mussel speces: Hickory Nut*/

zta HIC;

to block $=1$ to 3 by 1 ;

do $\operatorname{tank}=1$ to 6 by 1 ;

$x=\operatorname{ranuni}(-1)$;

output;

end;

and;

in;

ata $\mathrm{HIC2}$; set $\mathrm{HIC}$;

if block $=1$ and tank $=1$ then tankn = ' $3 \mathrm{~A} 1$ ';

if block $=1$ and tank $=2$ then tankn $=$ ' $3 \mathrm{~A} 2^{\prime}$ ';

if block $=1$ and tank $=3$ then tankn $=$ ' $3 \mathrm{~A} 33^{\prime}$;

if block $=1$ and tank $=4$ then $\operatorname{tankn}={ }^{\prime} 3 A 4 '$;

if block $=1$ and $\operatorname{tank}=5$ then $\operatorname{tankn}={ }^{\prime} 3 \mathrm{~A} 5^{\prime}$;

if block $=1$ and tank $=6$ then tankn $={ }^{\prime} 3 \mathrm{~A} 6^{\prime}$;

if $\mathrm{block}=2$ and tank $=1$ then tankn $=$ ' $3 \mathrm{~B} 1$ ';

if block $=2$ and tank $=2$ then tankn $=$ ' $3 \mathrm{~B} 22^{\prime}$;

if block $=2$ and tank $=3$ then tankn $={ }^{\prime} 3 \mathrm{~B} 3^{\prime}$;

if block $=2$ and $\operatorname{tank}=4$ then $\operatorname{tankn}=$ ' $3 \mathrm{~B} 44^{\prime}$;

if b.lock $=2$ and $\operatorname{tank}=5$ then $\operatorname{tankn}={ }^{\prime} 3 B 5$ ';

if block $=2$ and tank $=6$ then tankn $=$ ' $3 \mathrm{~B} 6$ ';

i.f block $=3$ and tank $=1$ then tankn $={ }^{\prime} 3 \mathrm{C} 11^{\prime}$;

if block $=3$ and tank $=2$ then tankn $={ }^{\prime} 3 \mathrm{C} 2 '$;

if block $=3$ and tank $=3$ then tankn $={ }^{\prime} 3 \mathrm{C} 3^{\prime}$;

if block $=3$ and $\operatorname{tank}=4$ then $\operatorname{tankn}={ }^{\prime} 3 \mathrm{C} 4{ }^{\prime}$;

if block $=3$ and tank $=5$ then tankn $=$ ' $305^{\prime}$;

if block $=3$ and tank $=6$ then $\operatorname{tankn}={ }^{\prime} 3 \mathrm{C} 6^{\prime}$;

13060 run;

roc sort data=HIC2; 
by $x$;

un;

ata assign_trt_HIC; set HIC2;

if $n_{-}=1$ then $\operatorname{trt}=$ 'control';

if $\bar{n}_{-}=2$ then trt $=$ 'control';

if ${ }_{-}{ }_{-}=3$ then trt $=$ 'control';

if $\bar{n}_{-}=4$ then trt $=$ ' $50^{\prime}$;

if $n_{-}=5$ then $\operatorname{trt}={ }^{\prime} 50^{\prime}$;

if $n_{-}=6$ then trt $={ }^{\prime} 50^{\prime}$;

if $n_{-}=7$ then $\operatorname{trt}=100^{\prime}$;

if $n_{-}=8$ then trt $=' 100^{\prime}$;

if $n=9$ then trt $=1100^{\prime}$;

if $\bar{n}_{-}=10$ then trt $={ }^{\prime} 200^{\prime}$;

if $-n=11$ - then $t r t=' 200^{\prime}$;

if $n_{-}=12$ then trt $=' 200^{\prime}$;

if $n_{-}=13$ then $\operatorname{trt}={ }^{-} 300^{\prime}$;

if $\bar{n}_{-}=14$ then trt $={ }^{\prime} 300^{\prime}$;

if $\bar{n}_{-}=15$ then trt $=1300^{\prime}$;

if $\mathrm{n}=16$ then trt $=300-\mathrm{HD}^{\prime}$;

if $n_{-}=17$ then trt $=$ ' $300-\mathrm{HD}^{\prime}$;

if ${ }_{-} n_{-}=18$ then $\operatorname{trt}={ }^{\prime} 300-\mathrm{HD}^{\prime}$;

run;

roc print data= assign_trt_HIC;

itle1 h=2 'Effects of Psuedomonas fluorescens (Pf-CL145A) to glochidia from seven unionid mussel sper itle2 $h=1.5$ 'AEH-11-PSUEDO-01';

itle3 $h=1$ 'Random assignment of treatment to experimental tanks';

itle4 $h=1$ 'Mussel speces: Hickory Nut';

un;

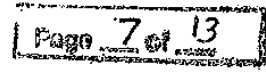




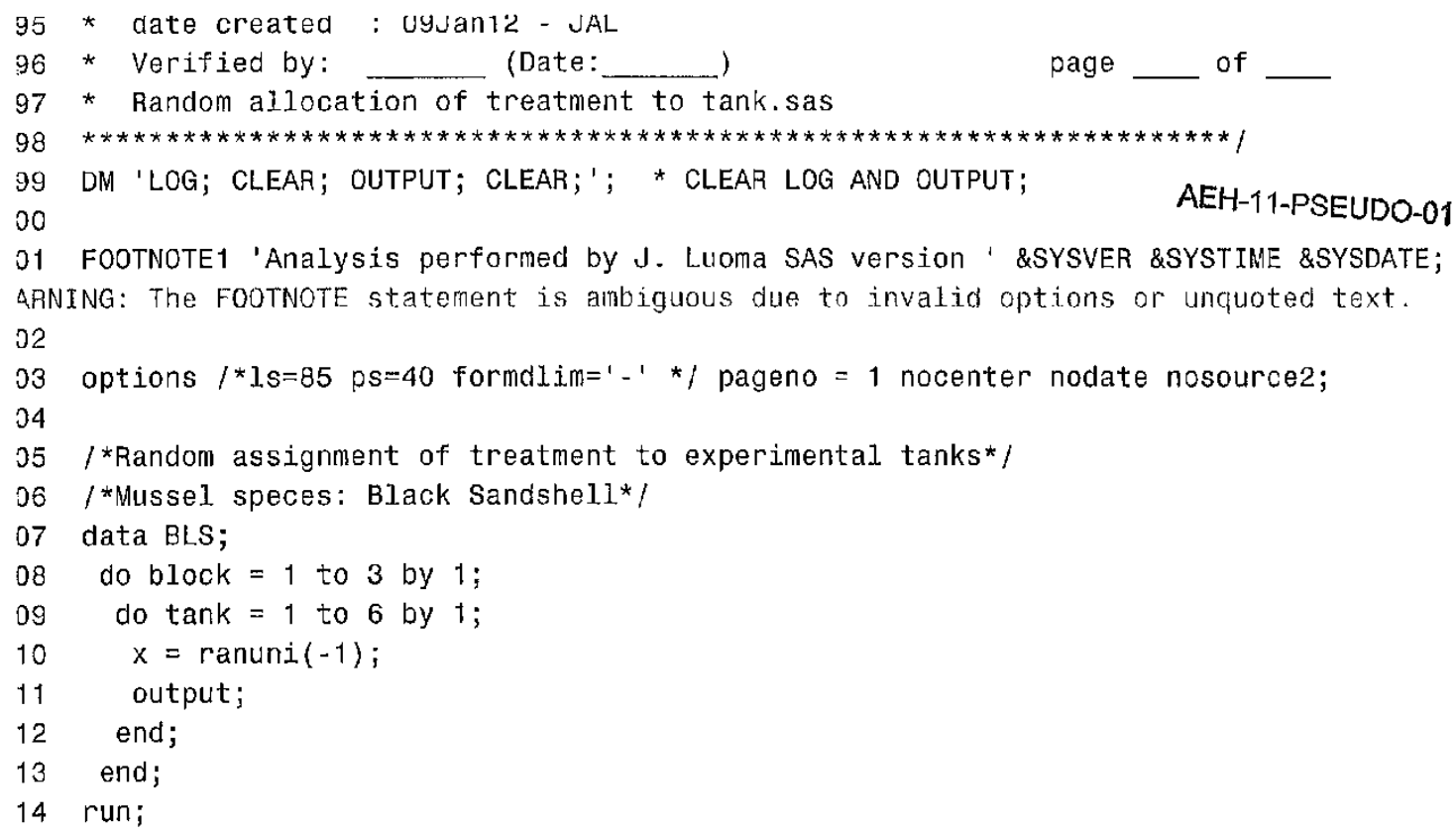

OTE: The data set WORK.BLS has 18 observations and 3 variables.

OTE: DATA statement used (Total process time):

real time $\quad 0.01$ seconds

cpu time $\quad 0.01$ seconds

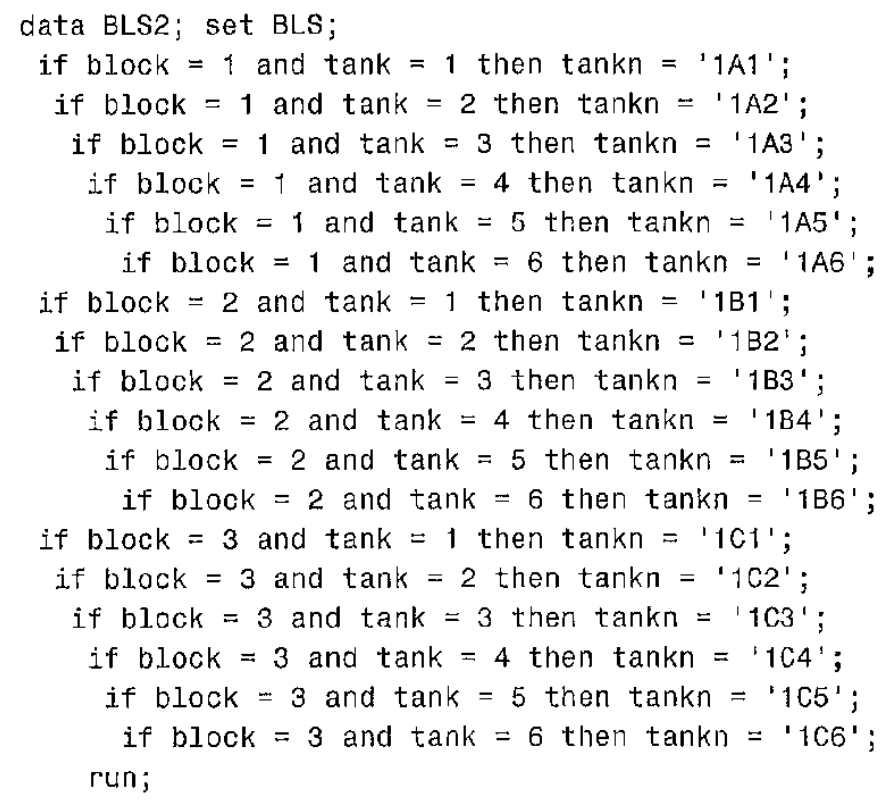

DTE: There were 18 observations read from the data set wORK.BLS.

OTE: The data set WORK.BLS2 has 18 observations and 4 variables.

OTE: DATA statement used (Total process time):

real time $\quad 0.01$ seconds

cpu time $\quad 0.01$ seconds

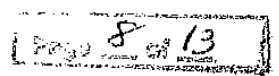


35 proc sort data=BLS2;

36 by $x$;

37 run;

OTE: There were 18 observations read from the data set WORK.BLS2.

OTE: The data set WORK.BL.S2 has 18 observations and 4 variables.

OTE: PROCEDURE SORT used (Total process time):

\section{AEH-11-PSEUDO-01}

real time $\quad 0.01$ seconds

cpu time $\quad 0.01$ seconds

38

39 data assign_trt_BLS; set BLS2;

40 if $n_{-}=1$ then trt = 'control';

41 if $\bar{n}_{-}=2$ then trt $=$ 'control';

42 if $n_{-}=3$ then trt $=$ 'control';

43 if $n_{-}=4$ then trt $=' 50^{\prime}$;

44 if $n_{-}=5$ then trt $={ }^{\prime} 50^{\prime}$;

45 if $\bar{n}_{-}=6$ then trt $=$ ' 50 ';

46 if $n_{-}=7$ then trt $=1100$ ';

47 if $n_{-}=8$ then trt $=1100^{\prime}$;

48 if $n_{-}=9$ then trt $={ }^{\prime} 100^{\prime}$;

49 i.f $\bar{n}_{-}=10$ then trt $={ }^{\prime} 200^{\prime}$;

50 if $\bar{n}_{-}=11$ then trt $=' 200^{\prime}$;

51 if $\bar{n}_{-}=12$ then trt $=1200^{\prime}$;

52 if $n_{-}=13$ then $\operatorname{trt}={ }^{\prime} 300^{\prime}$;

53 if $n_{-}=14$ then trt $=' 300^{\prime}$;

54 if $n_{-}=15$ then trt $=1300^{\prime}$;

55 if $n=16$ then trt $=$ ' $300-\mathrm{HD}^{\prime}$;

56 if $\bar{n}_{-}=17$ then trt $=$ ' $300-\mathrm{HD}^{\prime}$;

57 if $\vec{n}_{-}=18$ then trt $=1300-\mathrm{HD}^{\prime}$;

58 run;

JTE: There were 18 observations read from the data set WORK.BLS2.

TTE: The data set WORK. ASSIGN TRT_BLS has 18 observations and 5 variables.

JTE: DATA statement used (Total process time):
real time
0.01 seconds

cpu time

0.01 seconds

9 proc print data= assign_trt_BLS;

50 titlet $h=2$ 'Effects of Psuedomonas fluorescens (Pf-CL145A) to glochidia from seven unionid

30 ! mussel species';

o1 title2 $h=1.5$ 'AEH-11-PSUEDO-01';

32 title3 $h=1$ 'Random assignment of treatment to experimental tanks';

33 title4 $h=1$ 'Mussel. speces: Black Sandshell';

34 run;

JTE: There were 18 observations read from the data set WORK.ASSIGN_TRT_BLS.

JTE: PROCEDURE PRINT used (Total process time):
real time
0.00 seconds
cpu time
0.00 seconds
1 Page 9013

35

36

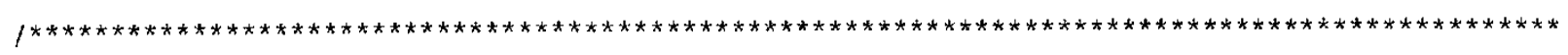




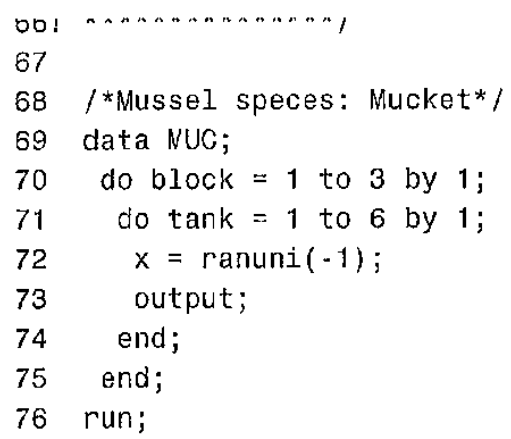

OTE: The data set WORK.MUC has 18 observations and 3 variables.

OTE: DATA statement used (Total process time):

real time $\quad 0.00$ seconds

cpu time $\quad 0.00$ seconds

77 data MUC2; set MUC;

78 if block $=1$ and $\operatorname{tank}=1$ then $\operatorname{tankn}=$ ' $2 \mathrm{~A} 1$ ';

79 if block $=1$ and $\operatorname{tank}=2$ then $\operatorname{tankn}={ }^{\prime} 2 \mathrm{~A} 2$ ';

80 if block $=1$ and tank $=3$ then tankn = ' $2 \mathrm{~A} 3^{\prime}$;

81 if block $=1$ and $\operatorname{tank}=4$ then $\operatorname{tankn}={ }^{\prime} 2 \mathrm{~A} 4^{\prime}$;

82 if block $=1$ and tank $=5$ then tankn $={ }^{\prime} 2 \mathrm{~A}^{\prime}{ }^{\prime}$;

83 if block $=t$ and $\operatorname{tank}=6$ then tankn $={ }^{\prime} 2 \mathrm{~A} 6^{\prime}$;

84 if block $=2$ and $\operatorname{tank}=1$ then $\operatorname{tankn}={ }^{\prime} 2 \mathrm{~B} 11^{\prime}$;

85 if block $=2$ and tank $=2$ then tankn $={ }^{\prime} 2 \mathrm{~B} 2{ }^{\prime}$;

86 if block $=2$ and tank $=3$ then tankn $={ }^{\prime} 2 \mathrm{~B} 3^{\prime}$;

87 if block $=2$ and tank $=4$ then $\operatorname{tankn}={ }^{\prime} 284^{\prime}$;

88 if block $=2$ and tank $=5$ then tankn $={ }^{\prime} 2 B 5^{\prime}$;

89 if block $=2$ and $\operatorname{tank}=6$ then $\operatorname{tankn}={ }^{\prime} 2 \mathrm{~B} 6^{\prime}$;

90 if block $=3$ and $\operatorname{tank}=1$ then $\operatorname{tankn}={ }^{\prime} 2 \mathrm{C} 1$ ';

91 if block $=3$ and $\operatorname{tank}=2$ then $\operatorname{tankn}={ }^{\prime} 2 \mathrm{C} 2^{\prime}$;

if block $=3$ and tank $=3$ then tankn $={ }^{\prime} 2 \mathrm{C} 3^{\prime}$;

if block $=3$ and tank $=4$ then $\operatorname{tankn}={ }^{\prime} 2 \mathrm{C} 4$ ';

if block $=3$ and tank $=5$ then $\operatorname{tankn}={ }^{\prime} 205^{\prime}$;

if block $=3$ and tank $=6$ then tankn $={ }^{\prime} 2 \mathrm{C} 6^{\prime}$;

run;

OTE: There were 18 observations read from the data set WOAK.MUC.

OTE: The data set WORK. MUC2 has 18 observations and 4 variables.

OTE: DATA statement used (Total process time):

real time $\quad 0.00$ seconds

cpu time $\quad 0.00$ seconds

97 proc sort data=MUC2;

98 by $x$;

99 run;

OTE: There were 18 observations read from the data set wORK. UUC2.

OTE: The data set WORK. MUC2 has 18 obscrvations and 4 variables.

OTE: PROCEDURE SORT used (Total process time):
real time
0.01 seconds
cpu time
0.01 seconds
10013 


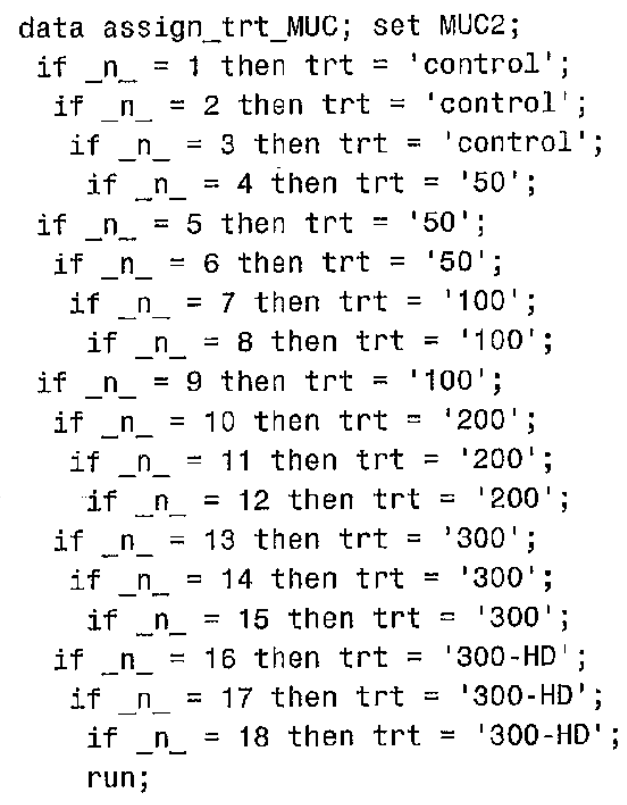

JTE: There were 18 observations read from the data set WORK. MUC2.

TTE: The data set WORK.ASSIGN TRT_MUC has 18 observations and 5 variables.

JTE: DATA statement used (Total process time):
real time
0.01 seconds
cpu time
0.01 seconds

21 proc print data= assign_trt_MUC;

22 title1 $n=2$ 'Effects of Psuedomonas fluorescens (Pf-CL145A) to glochidia from seven unionid

22! mussel species';

23 title2 $h=1.5$ 'AEH-11-PSUEDO-01';

24 title $3 h=1$ 'Random assignment of treatment to experimontal tanks';

25 title4 h=1 'Mussel speces: Mucket';

26 run;

JTE: There were 18 observations read from the data set WORK.ASSIGN_TRT MUC.

TTE: PROCEDURE PRINT used (Total process time):
real time
0.00 seconds
cpu time
0.00 seconds

27

28

28 !

20

30

31

32

33

34

35

36

37

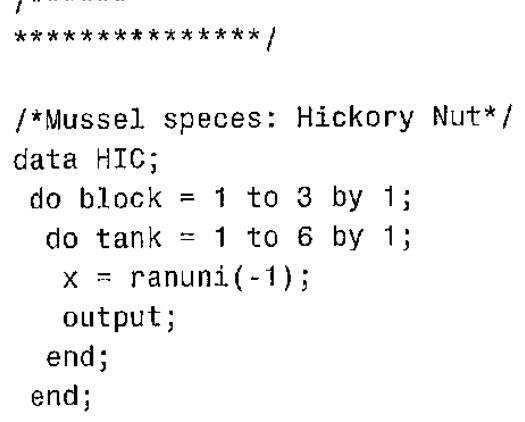


OTE: The data set WORK.HTC has 18 observations and 3 variables.

OTE: DATA statement used (Total process time):

real time $\quad 0.00$ seconds

cpu time $\quad 0.00$ seconds

AEH-11-PSEUDO-01

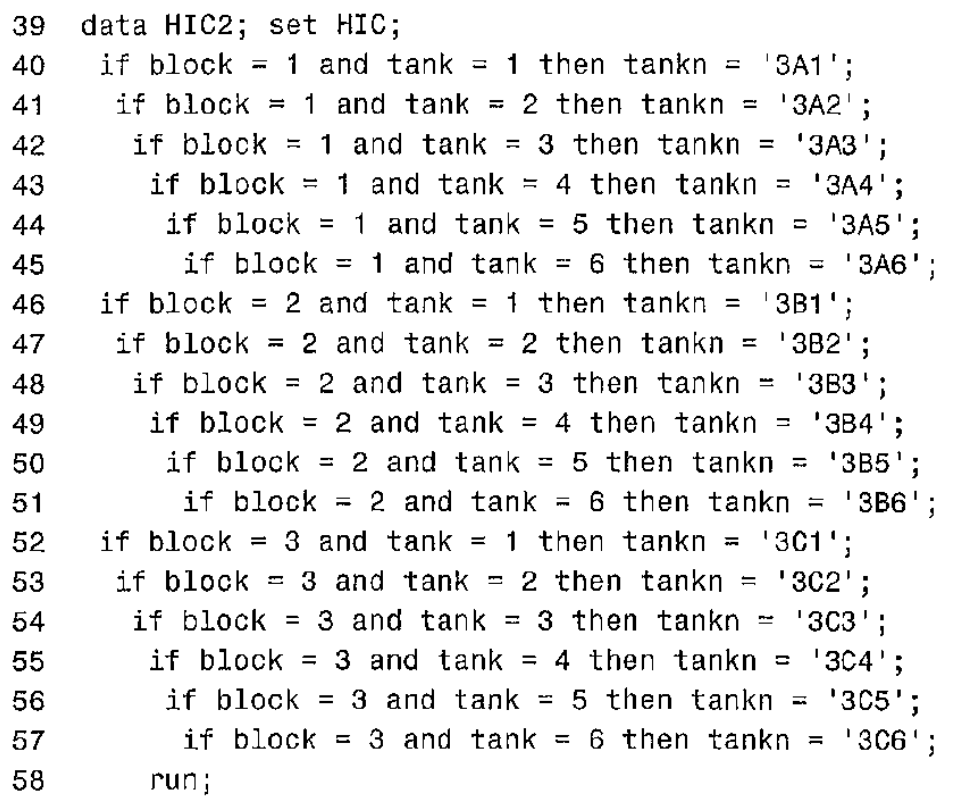

OTE: There were 18 observations read from the data set WORK. HIC.

OTE: The data set WORK. HIC2 has 18 observations and 4 variables.

OTE: DATA statement used (Total process time):

real time $\quad 0.01$ seconds

cpu time $\quad 0.01$ seconds

59 proc sort data=HIC2;

60 by $x$;

61 run;

OTE: There were 18 observations read from the data set WORK.HTC2.

OTE: The data set WORK.HIC? has 18 observations and 4 variables.

OTE: PROCEDURE SORT uscd (Total procoss time):

real time $\quad 0.01$ seconds

cpu time $\quad 0.01$ seconds

62

63 data assign_trt_HIC; set HIC2;

64 if $n=1$ then trt $n_{-}=$'contral' $^{\prime}$;

65 if $n_{-}=2$ then trt = 'control';

66 if $\bar{n}_{-}=3$ then trt $=$ 'control';

67 if $n_{-}=4$ then trt $={ }^{\prime} 50^{\prime}$;

68 if $n_{-}=5$ then trt $=' 50$ ';

69 if $n_{-}=6$ then trt $=' 50^{\prime}$;

70 if $\bar{n}_{-}=7$ then trt $=1100^{\prime}$; 


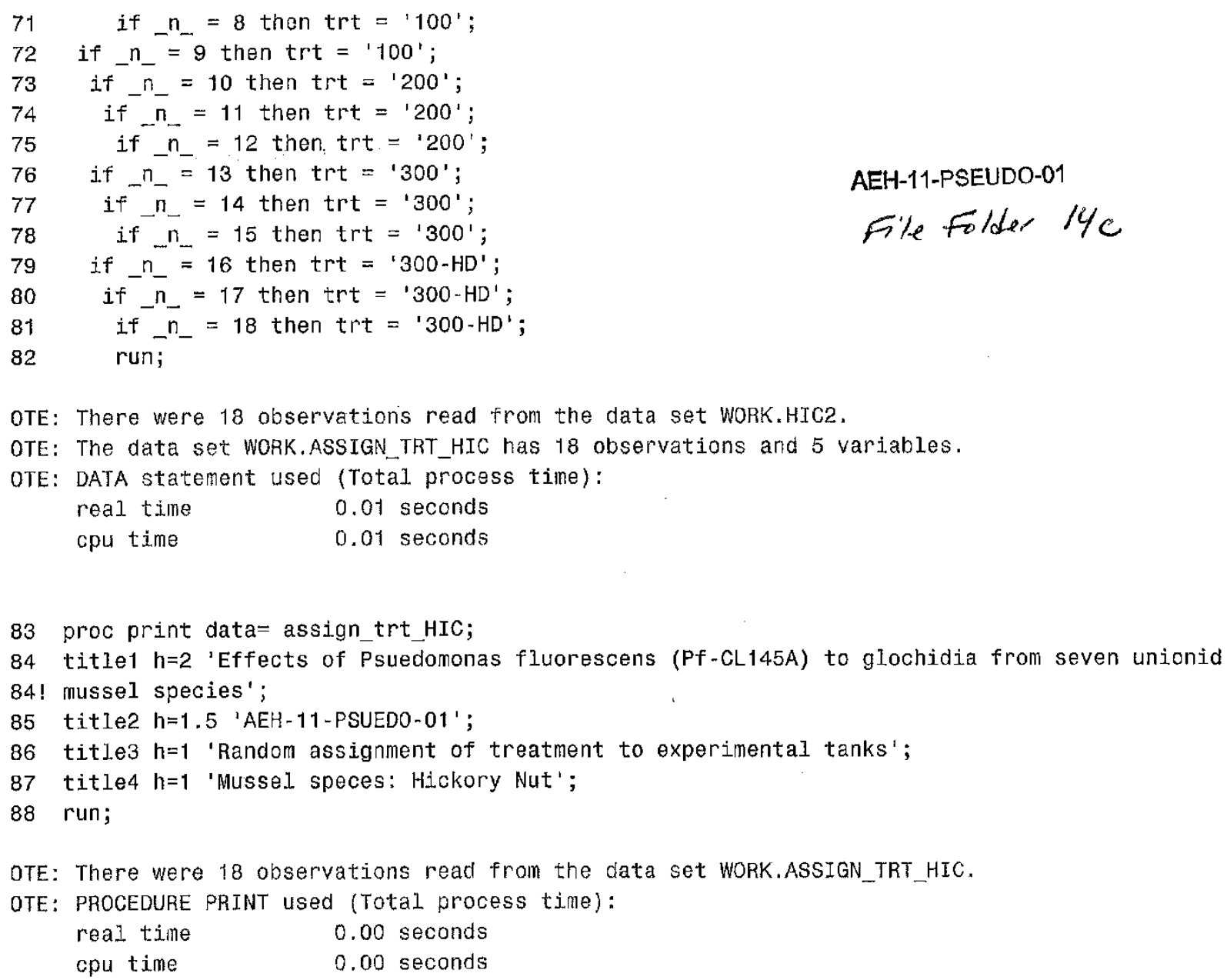

OTE: There were 18 observations read from the data set WORK.ASSIGN_TRT_HIC.

OTE: PROCEDURE PRINT used (Total process time):

real time $\quad 0.00$ seconds

cpu time $\quad 0.00$ seconds

\section{AEH-11-PSEUDO-01




\section{Appendix 4. Test Article Information}

\begin{tabular}{|c|c|c|c|}
\hline $\begin{array}{c}\text { Item } \\
\text { number }\end{array}$ & Item description & $\begin{array}{c}\text { Number } \\
\text { of } \\
\text { pages }\end{array}$ & $\begin{array}{c}\text { Report } \\
\text { page } \\
\text { number }\end{array}$ \\
\hline 1 & Material Safety Data Sheet: MBI-401 Spray Dried Powder & 2 & 238 \\
\hline 2 & Material Safety Data Sheet: MBI-401 Freeze Dried Powder & 2 & 240 \\
\hline 3 & MBI-401 FDP (lot \# 1 10607WB-FD-E) Test Article: Certificate of Analysis & 1 & 242 \\
\hline 4 & $\begin{array}{l}\text { Copy of test article information from test article log book for MBI-401 SDP; lot number MBI-401- } \\
110308 \text { AI-BD-3 }\end{array}$ & 5 & 243 \\
\hline 5 & Copy of test article information from test article log book for MBI-401 FDP; lot number $110607 \mathrm{WB}-\mathrm{FD}-\mathrm{E}$ & 4 & 248 \\
\hline 6 & $\begin{array}{l}\text { Copy of test article information from test article log book for MBI-401 SDP; lot number MBI-401 SDP } \\
\text { 4655-12-Mix }\end{array}$ & 4 & 252 \\
\hline 7 & NYSM Post-Treatment Product Validation Assay (lot number MBI-401 SDP 1 10308AI-BD-3) & 2 & 256 \\
\hline 8 & NYSM Post-Treatment Product Validation Assay (lot number MBI-401 FDP 110607 WB-FD-E) & 2 & 258 \\
\hline 9 & NYSM Post-Treatment Product Validation Assay (lot number MBI-401 SDP 4655-12-Mix) & 2 & 260 \\
\hline 10 & Test Chemical Weights (12-May-11) & 1 & 262 \\
\hline 11 & Glochidia Exposure Dosing Form and Chemical Stock Solution Determination(PPB SDP) & 2 & 263 \\
\hline 12 & Glochidia Exposure Dosing Form and Chemical St ock Solution Determination(HGE SDP) & 2 & 265 \\
\hline 13 & Glochidia Exposure Dosing Form and Chemical Stock Solution Determination(FAM SDP) & 2 & 267 \\
\hline 14 & Test Chemical Stock Preparation Data Form (18-Oct-11) & 1 & 269 \\
\hline 15 & Glochidia Exposure Dosing Form and Chemical St ock Solution Determination (PPB FDP) & 2 & 270 \\
\hline 16 & Glochidia Exposure Dosing Form and Chemical St ock Solut ion Determination(WASFDP) & 2 & 272 \\
\hline 17 & Test Chemical Stock Preparation Data Form(17-Jan-12) & 1 & 274 \\
\hline 18 & Glochidia Exposure Dosing Form and Chemical Stock Solution Determination (BLSSDP) & 2 & 275 \\
\hline 19 & Glochidia Exposure Dosing Form and Chemical St ock Solut ion Determination(MUC SDP) & 2 & 277 \\
\hline 20 & Test Chemical Stock Preparation Data Form(19-Jan-12) & 1 & 279 \\
\hline 21 & Glochidia Exposure Dosing Form and Chemical Stock Solution Determination (HIC SDP) & 2 & 280 \\
\hline
\end{tabular}



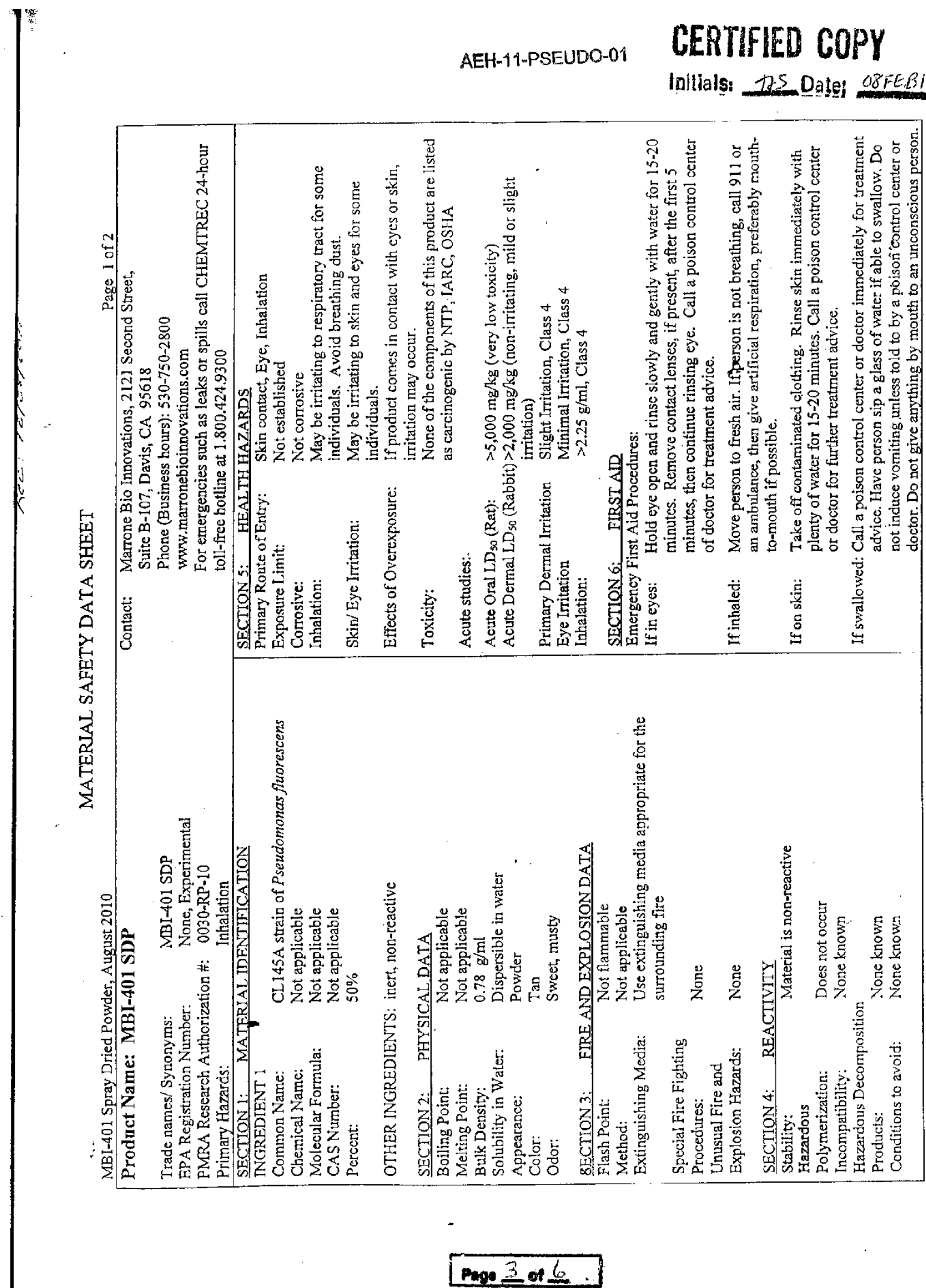


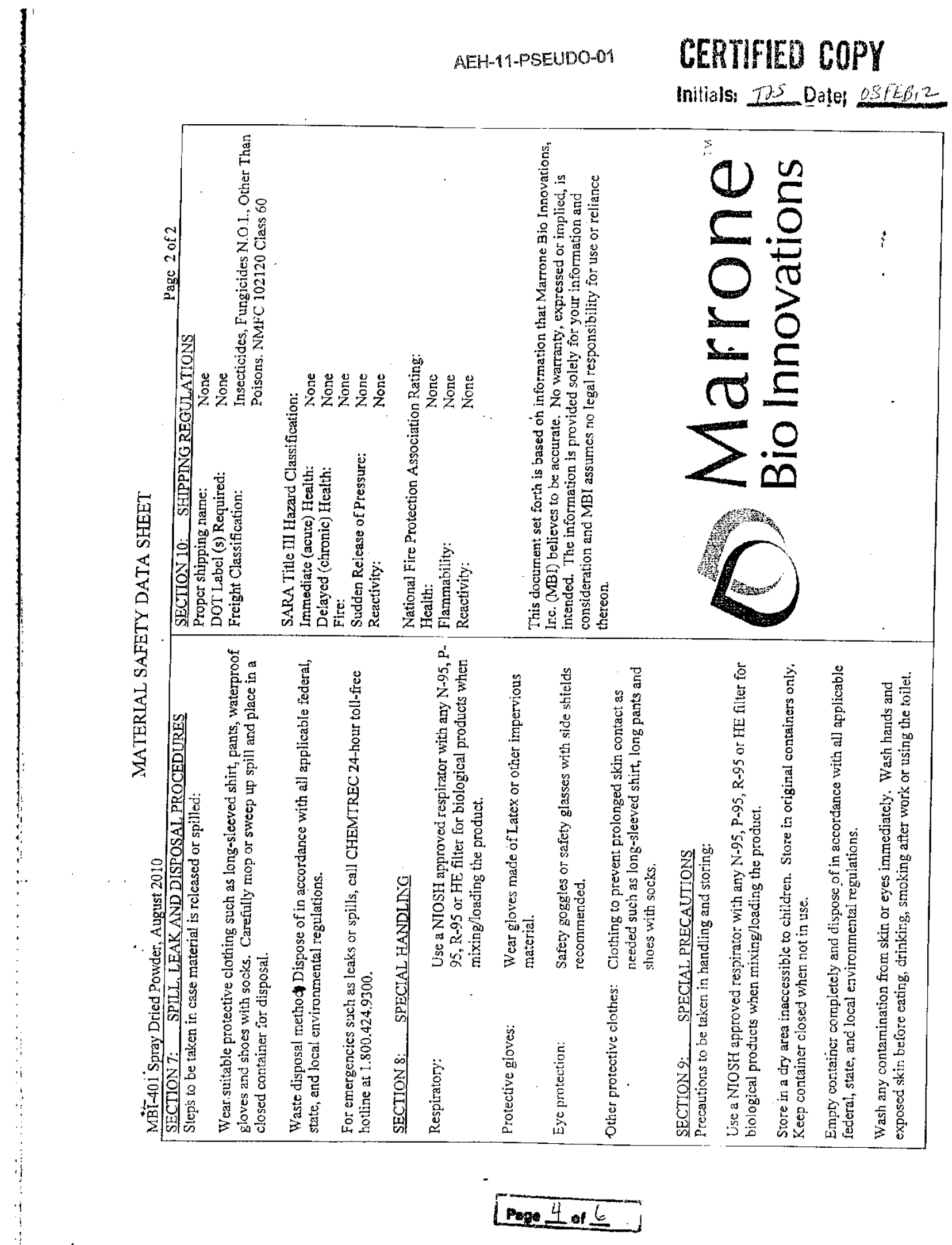




\section{AEH-11-PSEUDO-01 CERTIFIED COPY \\ Inittalsi t7SDate O8FEBiz}

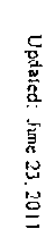
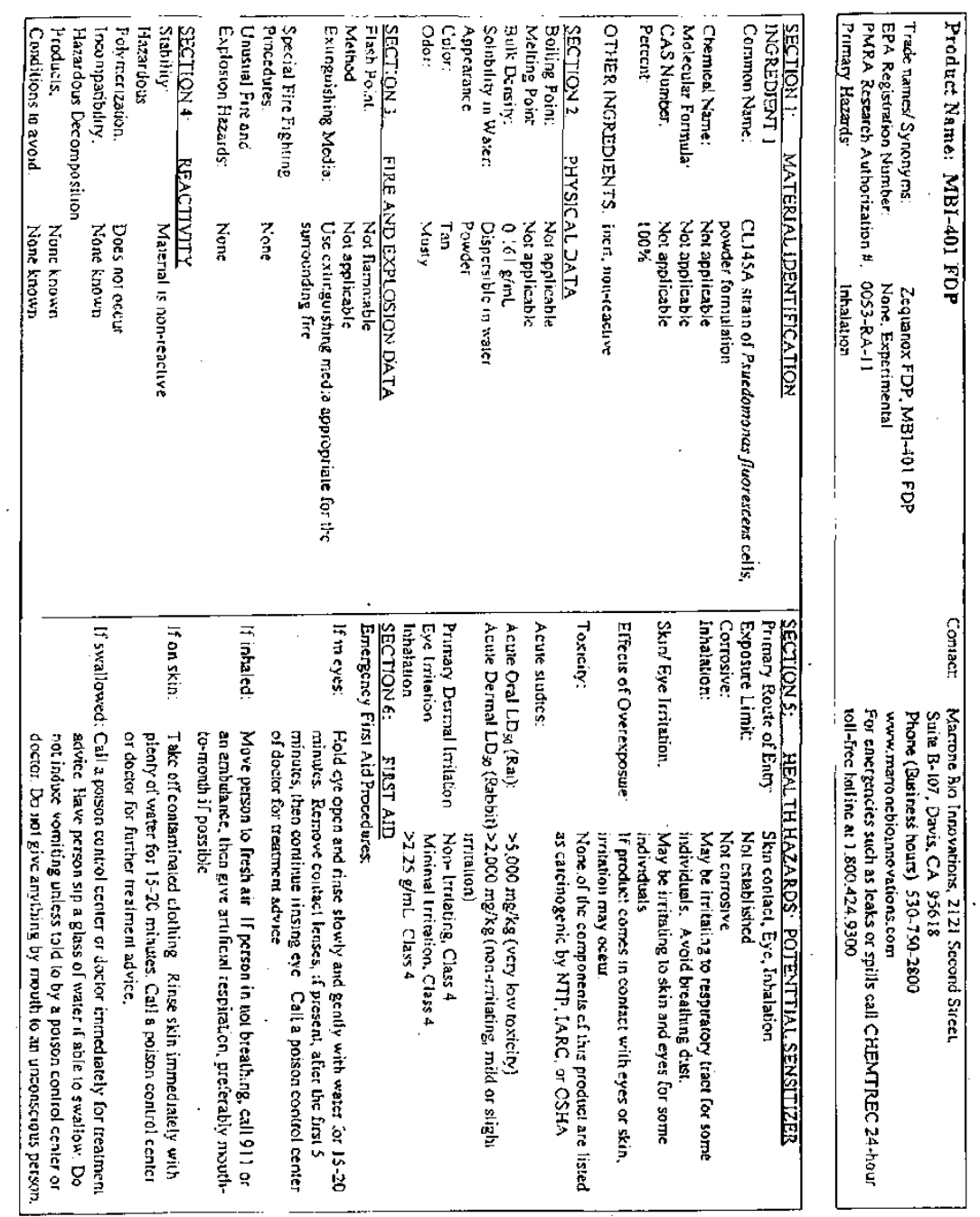

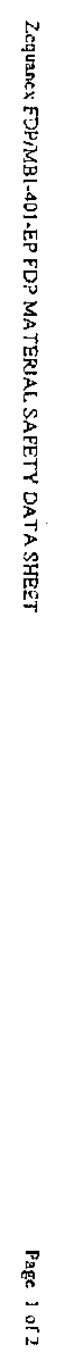

$\because$

and 3 


\section{AEH-11-PSEUDO-01 CERTIFIED COPY}

Intials: DOSDate; OSFEB 12

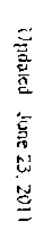

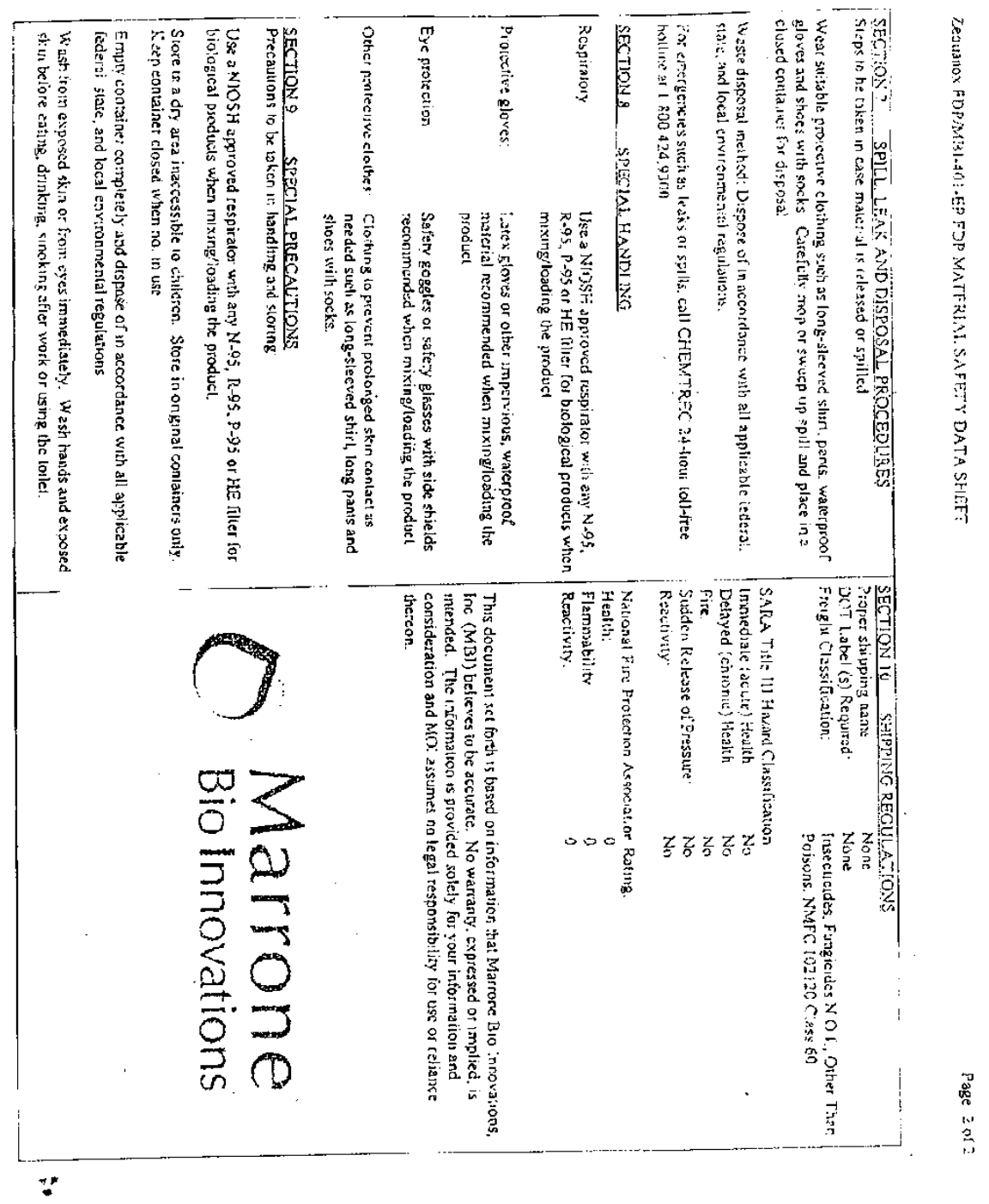

Page 4 of 7 . 


\section{AEH-11-PSEUDO-01 CERTIFIEU COPY}

Initials: 725 Datei $08 E E B$ I2

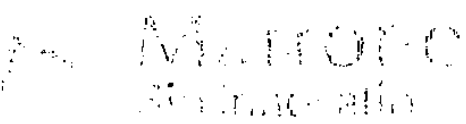

\section{CERTIFICATE OF ANALYSIS}

\begin{tabular}{|c|c|}
\hline Name of Produci: & MBI-AOI PDP \\
\hline Aclive lngredient: & 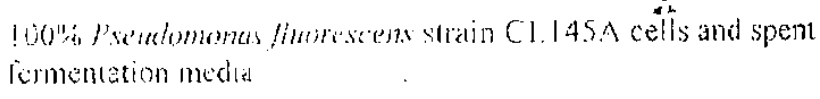 \\
\hline Chilg: & 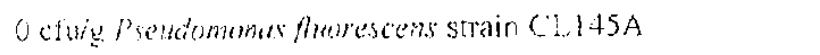 \\
\hline Lot Nurnbes: & i $10607 W+3-1.1)-[$ \\
\hline Mussel Bioassay: & Pass \\
\hline Appearance: & Tan powalo \\
\hline Storage Conditions: & $4^{\circ} \mathrm{C}:$ in the darl \\
\hline Date of Manufacture: & $s^{\prime}:=1: 11$ \\
\hline Expiration Dille: & $\begin{array}{l}\text { Ths prothe: is siable for } 6 \text { months liom date of manufacture } \\
\text { provided the sample is stored under whe recommended storage } \\
\text { conditionls. }\end{array}$ \\
\hline
\end{tabular}

J hereby certify that the above mormation is the and contect.

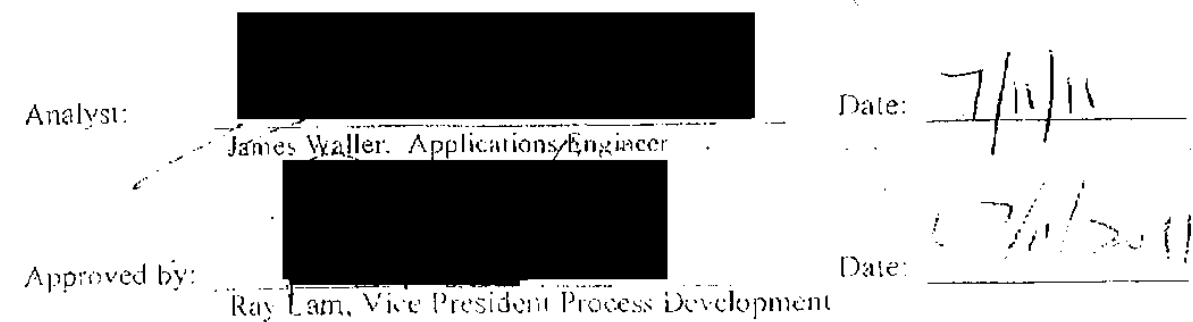

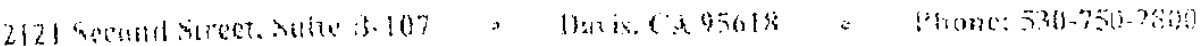

\section{Pase 5 of 7}


CEmited oth

AEH-11-PSEUDO-01

Initials: DTS Date: OBFEAIZ

\title{
CHEMICAL LOG BOOK
}

\author{
MBI-401 SDP
}

(CL145A strain of Pseudomonas fluorescens)

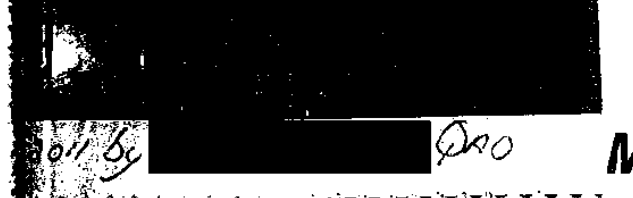

Manufacturer:

Marrone Bio Innovations (MBI)

Davis, California

\section{Lot Number:}

MBI-401-110308AI-BD-3

FILE FOLDER $\rightarrow 8$

ITEM $M$ H

Pape 19
PROOFED BY

Initlala: $\frac{\text { T2S DaLe : }}{\text { REV E }}$

Initlalm: Date' 
SIGNATURE PAGE

All personnel making an entry in this log must fill out the form below in accordance with SOP GEN 009.

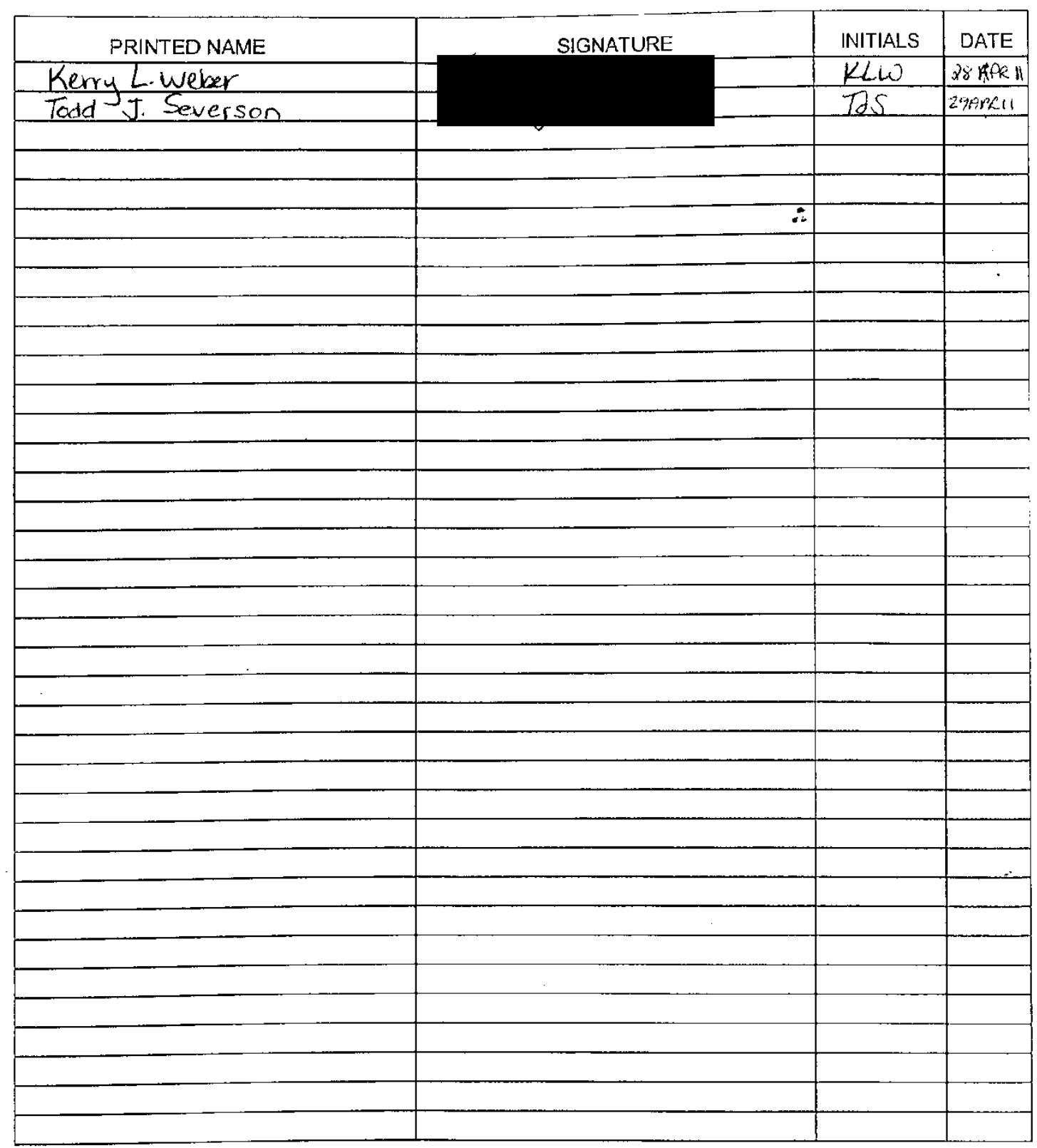

"This Use and Maintenance Log Book has been inspected and found to be in compliance with SOP GEN 009.

inspected and sealed on $/ m_{y} / 1,2011$
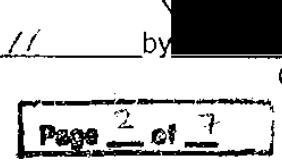
Prepared By:

Denise Mayer

New York State Museum

Field Research Laboratory

51 Fish Hatchery Road

Cambridge, NY 12816

(518) 677-8245

Shipped to:

Attn: Jim Luoma

Upper Midwest Environmental Sciences Center 2630 Fanta Reed Rd.

LaCrosse, WI 54603

(608)783-6451

Shipment Date:

$4 / 27 / 11$

Storage Information:

Store below $4 \mathrm{C}$ at all times.

Material Specs:

Material Lot\#: MBI-401-110308AI-BD-3

Formulation: SDP

Dry Cell Weight: $50 \%$ (by weight of powder)

Volume per Aliquot: $96 \mathrm{~g}$ ( $=48 \mathrm{~g}$ active ingredient)

Number of Aliquots: 1 


\section{OPdow}

TEST CHEMICAL DATA I ORM

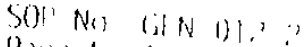

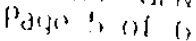

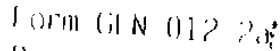 \\ listol of a
}

Test Material (Chemical Name) CLIHSA strain of Psendomonas flucrescens

Trade Name of:Chemical (Synonyms) MBI-4\&1 SDP $\therefore$

Source of Chemical (Manufacturer) Marrone Bio Inrovations (MBI)

Storage Location Room 122 Hotpoint Refrigerator in lock box

Date Received 28 APR 2 al Date Opened 28 APR 2 all Expiration 0ate N/A

(5 years unless otherwise stated)

Test Chemical Lot Number MBI-4\%1-1103\%4r-Purity of Chemical $50 \%$

Amount of Test Chemical Available or Received (if known) $96 \mathrm{Gg}$

Initial weight (with cover on) of Test Chemical and Container $130.678 \mathrm{~g}$

Characterization of Test Chemical: Color Tan

Physical State: Tiquid $\mathrm{s}$ solid $X$

Solid Form: powder $\mathbf{X}$ crystal a pellet

Chemical Abstract Service Number $N / A$

Manufacturer Certificale of Analysis Yes $\square$ No $\mathbb{X}$

Additional Comments About Test Chemical:

This lot of Pf-CL145A was used for L. Cardium, L. siliqueidea
and L. higginsii glochidia (dosing date: 12 MAY 11). TOS O8FEB

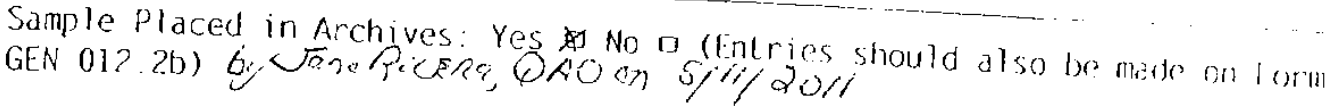

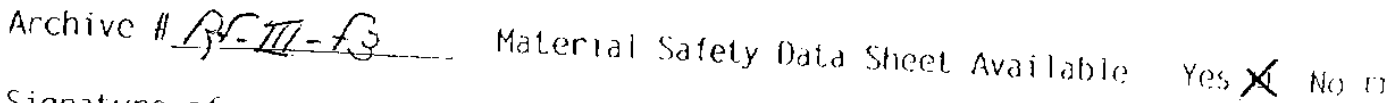

Signature of person (Study Director or designated representative) matading
Test Chemical Use log and date:

Signature

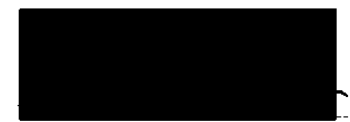

Pape bef 7

$$
\text { n. OS- AOP Ini }
$$




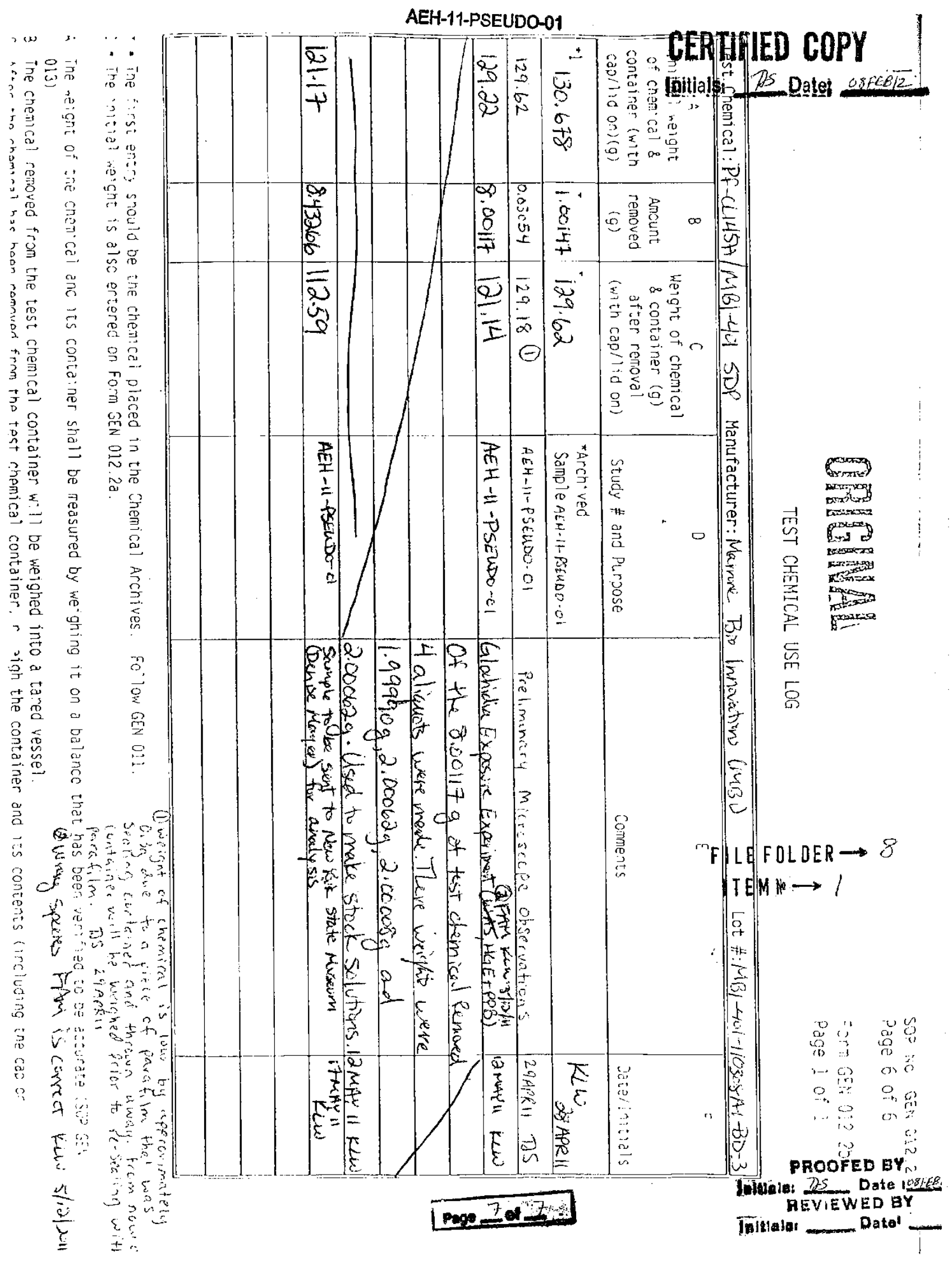




\section{CHEMICAL LOG BOOK}

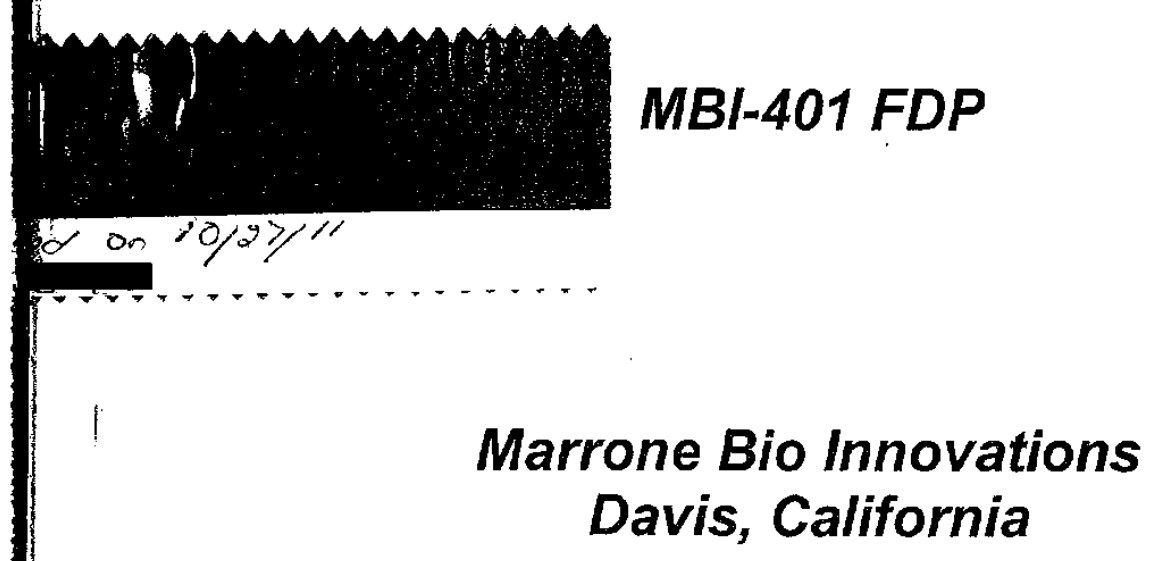

\section{LOT NUMBER: 110607WB-FD-E}

FILE FOLDER $\rightarrow 8$

ITEM $\rightarrow 2$
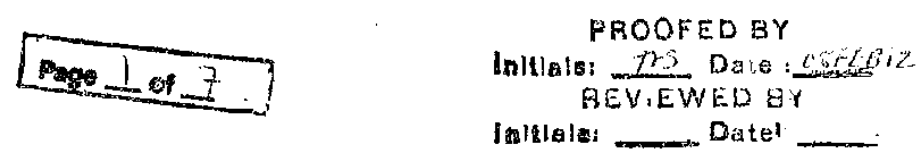
AEH-11-PSEUDO-01

CEDTIFIED COPY

Inilials: $2 \mathrm{~s}$ Date 03168,2

SIGNATURE PAGE

All personnel making an entry in this log must fill out the form below in accordance with SOP GEN 009.

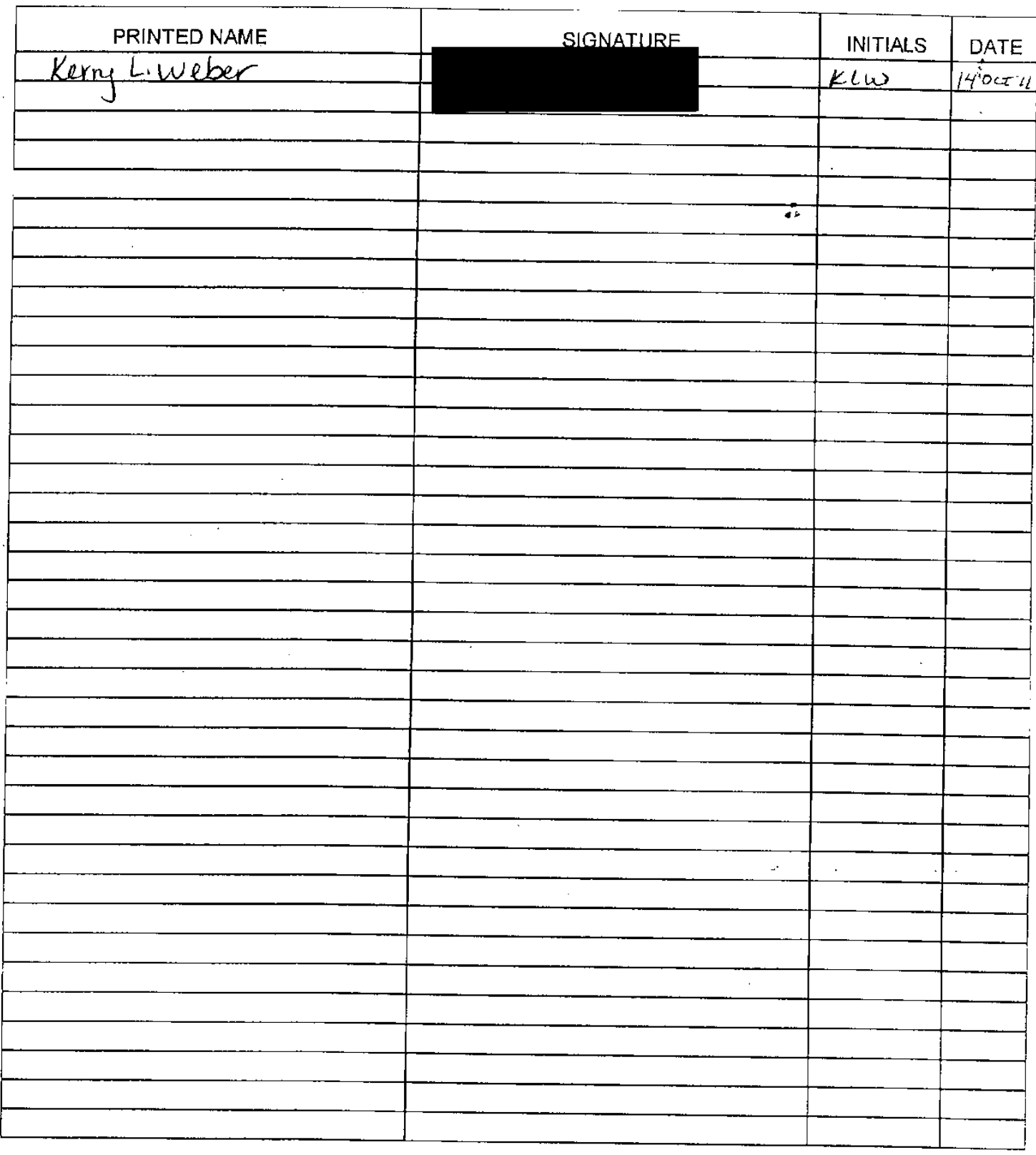

"This Use and Maintenance Log Book has been inspected and found to be incomnliance with SOP GEN 009. Inspected and sealed on
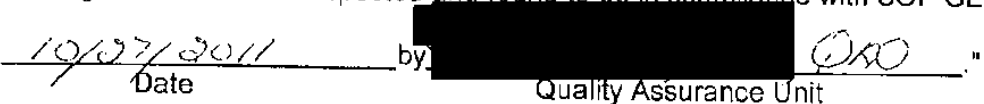

Quality Assurance Unit

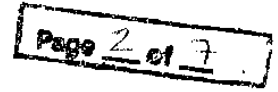




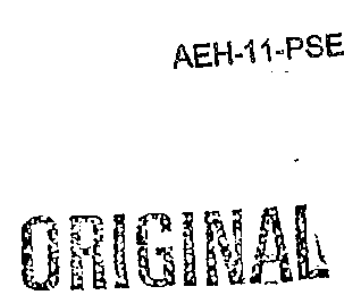

TEST CHEMICAL DATA FORM

\section{CERTIFIED COPY}

Inilials: DSDatei $\triangle 8 F E B$ iz

SOP NO. GEN 012.2

Page 5 of 6

Form GEN 012.2a.

Page 3 of 1 ;

Test Material (Chemical Name) MBI-401 FDP

Trade Name of: Chemical (Synonyms) Pf - CL 145A; Zequknex.

Source of Chemical (Manufacturer) Marrove Bio Innorations

storage Location Refrigerator in $\mathrm{Rm} 122$ in $10 \mathrm{cklax}(4 \%)$

Date Received 140 CT II Date Opened $140 \mathrm{CT}$ II Expiration Date 31 Nov 2011

(5 years unless otherwise stated)

Test Chemical Lot Number 110607 WB-FD-E Purity of Chemical $100 \%$

Amount of Test Chemical Available or Received (if known) $100 \mathrm{~g}$

Initial weight (with cover on) of Test Chemical and Container $161.68 \mathrm{~g}$

Characterization of Test Chemical: color Tan

Physical State: Tiquid a solid $\&$

Solid Form: powder $X$ crystal a pellet $\square$

Chemical Abstract Service Number N/A

Manufacturer Certificate of Analysis Yes $X$ No $\square$

Additional Comments About Test Chemical:

Temperature in cocler was $4.4^{\circ} \mathrm{C}$ upon veceipt.

This lot of Pf-CLI4SA was used for L. cardium and M. nervasa

glochidia (dosing date: $1800 T 11$ ). TOS OBFEB 2

Sample Placed in Archives: Yes $X$ No a (Entries should also be made on form GLN 012.2b)

Archive \#ff IIf -9 Material Safety Data Sheet Available: Yes o No D Signature of person (Study Director or designated representative) initiating Test Chenical Use $\log$ and date:

'Tind.ut?

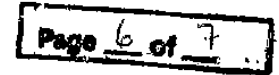




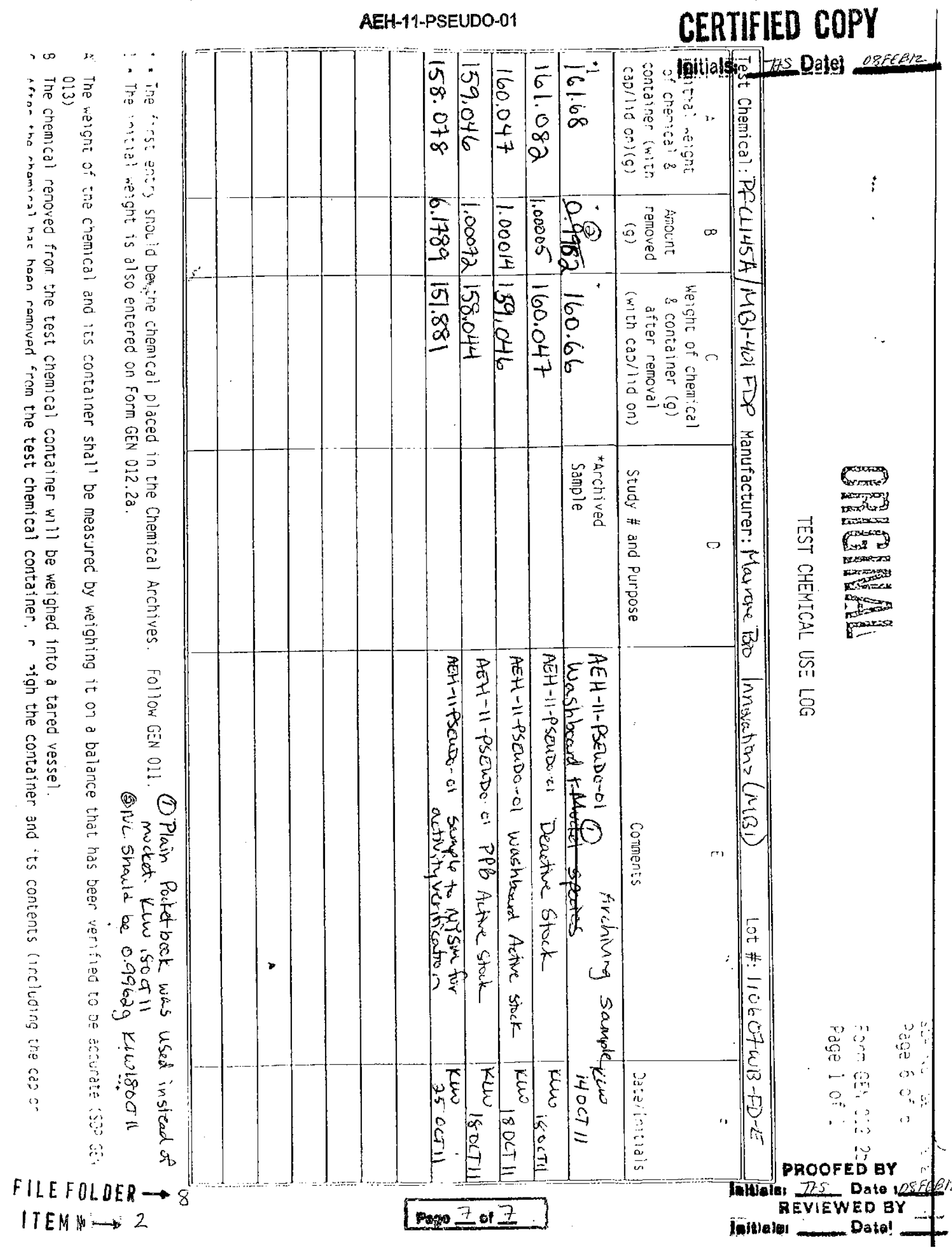




\title{
CHEMICAL LOG BOOK
}

\author{
MBI-401 SDP
}

\author{
Marrone Bio Innovations \\ Davis, California
}

\section{LOT NUMBER: MBI-401 SDP 4655-12-Mix}

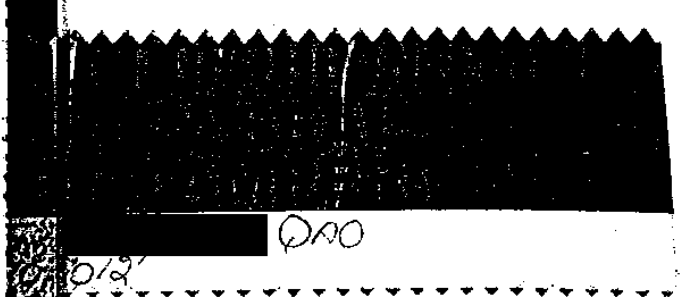

FILE FOLDER $\rightarrow 8$

ITEMH $\rightarrow 3$
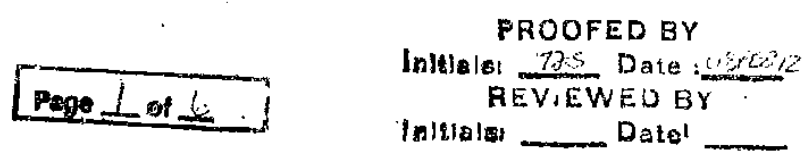


\section{SIGNATURE PAGE}

All personnel making an entry in this log must fill out the form below in accordance with SOP GEN 009.

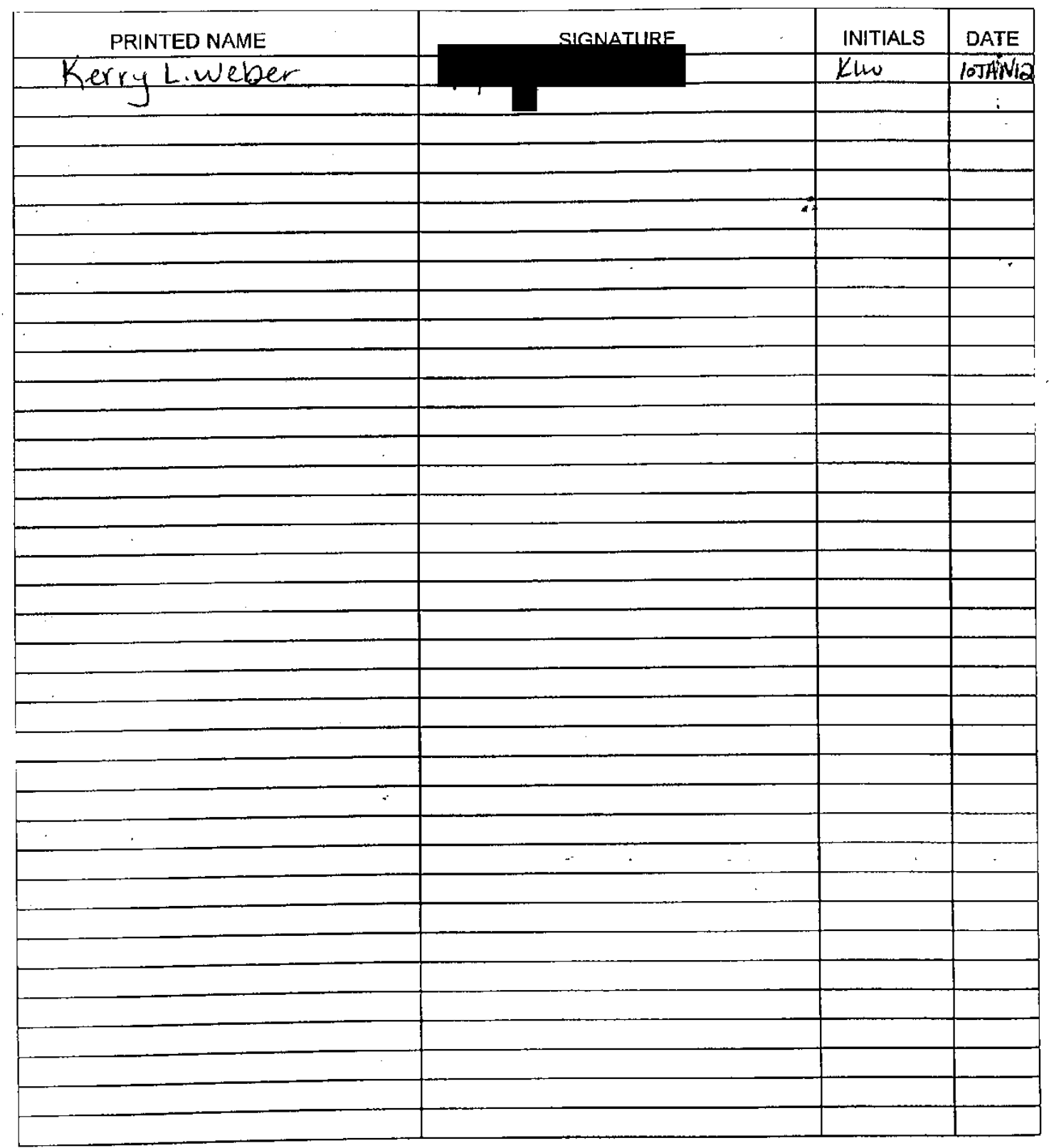

"This Use and Maintenance Log Book has been inspected and found to be in compliance with SOP GEN 009.

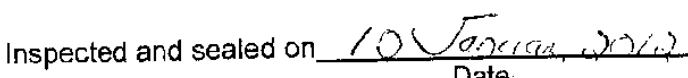
Date 
AEH-11-PSEUDO-01

ORIGINAL

TEST CHEMICAL DATA FORM

\section{CERTIFIED COPY}

Initialsi TfS Datei OSFEBiz

SOP No. GEN 012.3

Page 5 of 7

Form GEN 012.3a

Page 1 of 1

Test Chemical (Chemical Name) MBI -401 SDP

Circle one: Test Article Control Article

Trade Name of Test Chemical (Synonyms) Pf - CL 145A; Zequanox

Source of Test Chemical (Manufacturer) Marrone Bib Innovations

Storage Location Refrigerator in $\operatorname{Rm} 122$ in lakbox $\left(0-5^{\circ} \mathrm{C}\right)$

Date Received Io JAN 2012 Date Opened 10 JAR2012 Expiration Date 10 JAN 2017

(5 years unless otherwise stated) (Nove provida

Test Chemical Lot Number MBI-401 SDP

$4655-12-M i x$

Amount of Test Chemical Available or Received (if known) $100 \mathrm{q}$

Initial Mass (with cover on) of Test Chemical and Container $162.057 \mathrm{~g}$

Characterization of Test Chemical: Color Tan

Physical State: liquid solidX

Chemical Abstract Service Number N/A

Solid Form: powder X crystal _ pellet

Manufacturer Certificate of Analysis Yes _ No $\underline{X}$

Additional Comments about the Test Chemical:

Temperature in coder ypon receipt was $3.1 \%$

This lot of Pf-cliusA was used for Liecta, A. ligamentina, and

o. olivaria glochidia (dosing dates: $17 J A N 12$ and 19 JANi2). TIS OBFEB/2.

Sample Placed in Archives: Yes $X$ No ___ (Entries should also be made on Form GEN 012.b)

Archive Location $\underline{R}$ III- $F \|$ Material Safety Data Sheet Available: Yes $X$ No

Signature of Study Director or designee initiating Tost Chemical Use Log and date:

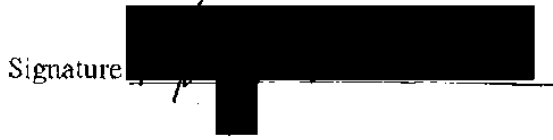

Date 10 TAN 2012

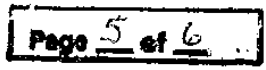




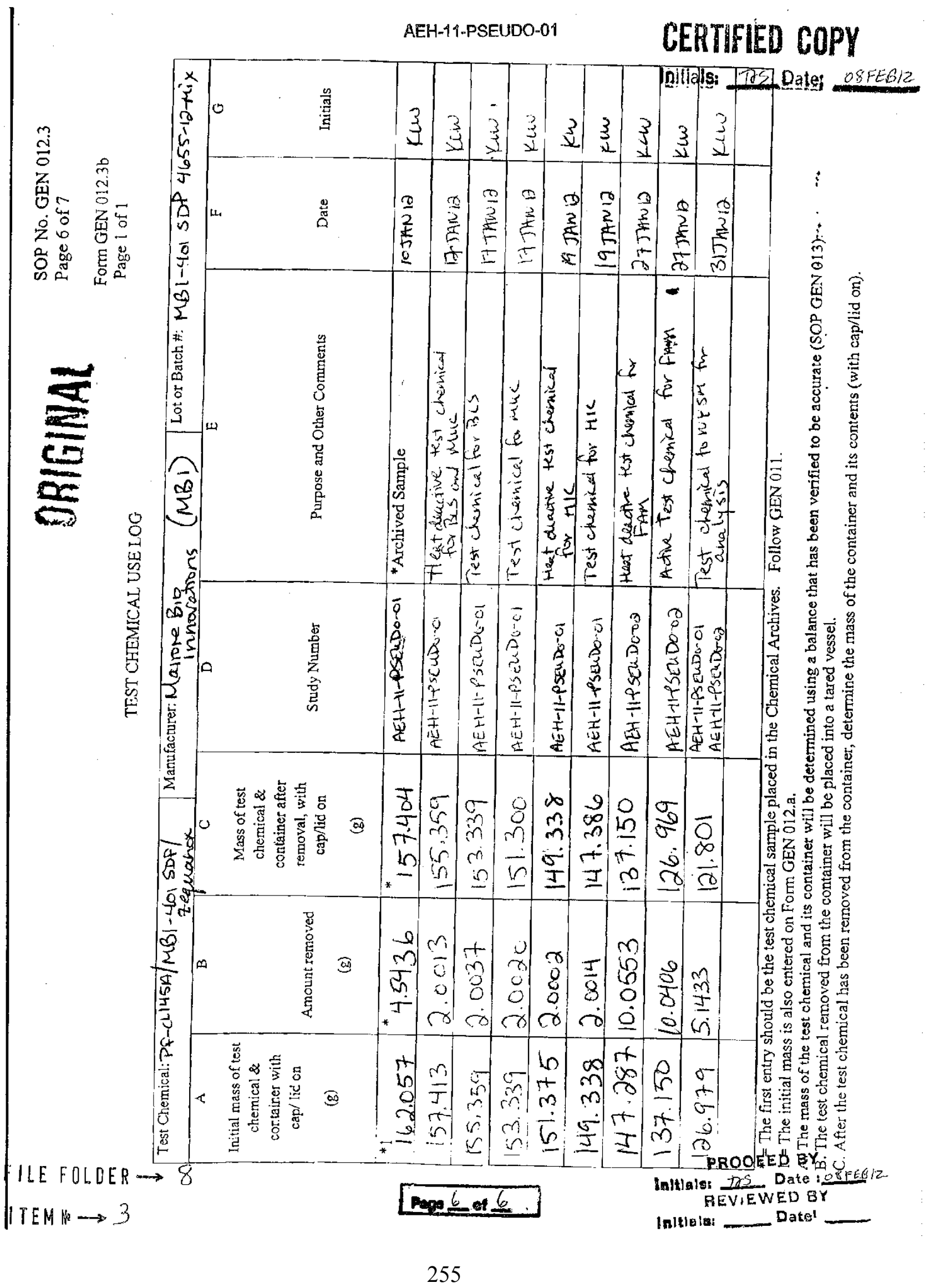




\section{NYSM Post-Treatment Product Validation Assay MBI-401 SDP 110308AI-BD-3 (USGS Study \#AEH-1 1-PSEUDO-01)}

Date product received from USGS: 2011/05/18

Date of start of test: 09/13/2011

BACKGROUND: As standard protocol for the USEPA project, each time a batch of Zequanox product is used in a test a UMESC, a portion of the product is bioassayed by the NYSM to validate toxicity post-treatment.

- MBI-401 SDP 110308AI-BD-3

○ USGS Study \#AEH-11-PSEUDO-01: Glochidia (FAM, HGE, PPB)

PURPOSE: Post-test product validation of MBI-401 SDP 110308AI-BD-3 from USGS-UMESC.

\section{MATERIALS AND METHODS:}

Preparation of product for testing:

Product was shipped under cold conditions and held in the laboratory refrigerator at $4^{\circ} \mathrm{C}$ until use. Within $30 \mathrm{~min}$ of treatment application, prepare each at treatment stock of each MBI-401 formulated product:

MBI 401-110308AI-BD-3 (SDP $-50 \%$ active ingredient): Weigh out $1.0 \mathrm{~g}$ of the powder into a labeled $50 \mathrm{ml}$ centrifuge tube and bring volume up to $20 \mathrm{ml}$ with dilution water. Vortex until evenly dispersed $=50 \mathrm{mg}$ product $/ \mathrm{ml}$ or $25 \mathrm{mg} \mathrm{ai} / \mathrm{ml}$. For $200 \mathrm{ppm}$ ai treatments in testing jars, add $4 \mathrm{ml}$ to each jar $(500 \mathrm{ml})$.

Cambridge CF (Standard for Positive Controls):

As an efficacy standard, use Pf-CL145A killed CF that was maintained at $-80^{\circ} \mathrm{C}$ (Cambridge CF). Since its production in 2005 , this material has been valuable as a reference standard. The Cambridge CF was produced in 2005 (2005-0027) in 100-L, batches 10,11 and 12 and E-beamed to kill the cells. The solution, at $110 \mathrm{mg} / \mathrm{ml}$ dry weight, is stored in $1 \mathrm{~cm}$ thick sheets in the Cambridge ultrafreezer at $80^{\circ} \mathrm{C}$. A scction of the sheet was broken off and weighed to determine volume (ca. $1 \mathrm{~g}=1 \mathrm{ml}$ ).

For this bioassay, the positive control suspension was produced on 5-2-2011 by weighing out $33.84 \mathrm{~g}$ of frozen Batch 10-12 block (killed cell suspension from test 2005-0027) and diluting with ca. $35 \mathrm{ml}$ dilution water. $7 \mathrm{ml}$ of this suspension were dispersed into 50 - $\mathrm{ml}$ centrifuge tubes and placed in the ultrafreezer (11 tubes). The dry weight of the material was $43.75 \mathrm{mg} / \mathrm{ml}$; therefore $2.3 \mathrm{ml}$ were added to each testing jar to treat at $200 \mathrm{ppm}$.

Mussel collections:

Mussels attached to small stones were collected from Hedges Lake (Washington County, NY) and brought back to the Cambridge Lab in coolers. Mussels were scraped from substrates at the lab and placed in pint-sized canning jars, covered with mesh, and placed in aquaria containing unchlorinated tap water with circulation (1 Whisper filter) and aeration at ambient laboratory temperature $\left(20-23^{\circ} \mathrm{C}\right)$.

FILE FOLOER $\rightarrow$ 8a<smiles>I[13IH][13IH]</smiles>

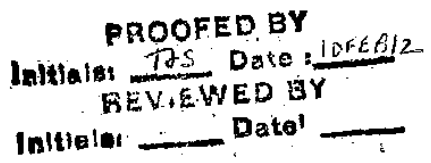

Pag 12 


\section{AEH-11-PSEUDO-01}

MBI-401 SDP 110308AI-BD-3 Post-test - 2 -

Mussel collection and handling:

\begin{tabular}{|c|c|c|c|c|}
\hline \hline Species & Collection site & $\begin{array}{c}\text { Collection } \\
\text { date }\end{array}$ & $\begin{array}{c}\text { Date in lab } \\
\left(20^{\circ} \mathrm{C}\right)\end{array}$ & Picked for test \\
\hline Zebra mussels & $\begin{array}{c}\text { Hedges Lake } \\
\text { (Washington County) }\end{array}$ & $09 / 09 / 2011$ & $09 / 09 / 2011$ & $\begin{array}{c}09 / 13 / 2011 \\
\mathrm{KP}\end{array}$ \\
\hline
\end{tabular}

Experimental design:

For validation of efficacy the following treatments will be set up:

Zebra mussels (25 mussels/jar):

$$
\begin{aligned}
& 3 \text { - Untreated Control } \\
& 3 \text { - } 200 \text { ppm (ai) Positive Control - Cambridge CF (A, B, C) } \\
& 3-200 \text { ppm (ai) SDP MBI 401-110308AI-BD-3 (A, B, C) }
\end{aligned}
$$

Testing jar bioassay protocol:

On the day prior to treatmont (09/13/2011) mussels were carefully examined and 25 mussels placed into each testing jar containing ca. $100 \mathrm{ml}$ aerated hard water and allowed to attach overnight. The next morning (09/14/2011), unattached mussels were removed and replaced with attached mussels from an extra glass Petri dish. Water was replaced with $500 \mathrm{ml}$ fresh aerated hard water.

After at least one hour, the treatment was applied. The optical density of each jar was measured in duplicate ( $A_{660 \mathrm{~mm}}$ Genesys Spectrophotometer).

After 24 hr of treatment, mussel mortality was checked and mussels were transferred to square plastic dishes with fresh aerated hard water. Mortality was checked and recorded each day with water replacements, for an additional 22 days. On the final day of mortality checks, 20 mussels were measured from the untreated controls using a caliper.

\section{Results:}

Mussel length: Zebra mussels $12.35 \pm 2.33 \mathrm{~mm}$.

Optical density of troatments:

\begin{tabular}{|c|c|}
\hline Treatment & Mcan $( \pm \mathrm{SD}) \mathrm{OD}\left(\mathrm{A}_{660 \mathrm{~nm}}\right)$ \\
\hline \hline Untreated Control & $-0.006 \pm 0.001$ \\
\hline Positive Control - Cambridge CF & $0.175 \pm 0.009$ \\
\hline SDP MBI-401 110308AI-BD-3 & $0.246 \pm 0.002$ \\
\hline
\end{tabular}

Zebra mussel mortality: Mussels were treated in triplicate testing jars $(500 \mathrm{ml})$ at $20^{\circ} \mathrm{C}$ for 24 hr and mortality was recorded for a total of 23 days.

\begin{tabular}{|c|c|}
\hline Treatment & Mean \% mortality (+SD) \\
\hline \hline Untreated Control & $1.3 \pm 2.3 \%$ \\
\hline Positive Control - Cambridge CF & $80.0 \pm 6.9 \%$ \\
\hline SDP MBI-401 110308AI-BD-3 & $86.7 \pm 4.6 \%$ Pass \\
\hline
\end{tabular}

\section{Activity of MBI-401 SDP 110308AI-BD-3 was acceptable for this study.}

\section{FILE FOLOER $\rightarrow 8 a$ ITEM $\rightarrow$}

PROOFED EY

Iamialai THS Date LUFER/2. REVIEWED BY

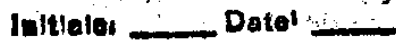

Page 2 of 2 


\section{NYSM Post-Treatment Product Validation Assay MBI-401 FDP 110607/6B-FD-E (USGS Study \#AEH-11-PSEUDO-01) *W \\ Date product arrived from USGS: $2011 / 10 / 22$}

Date of start of test: 11/15/2011

BACKGROUND: As standard protocol for the USEPA project, each time a batch of Zequanox product is used in a test a UMESC, a portion of the product is bioassayed by the NYSM to validate toxicity post-treatment. W/ $1 / 23 / \mathrm{r}^{2} \mathrm{~mm}$

- MBI-401 FDP 11060710B-FD-E

- USGS Study \#AEH-1 1-PSEUDO-01: Glochidia (WAS, PPB)

PURPOSE: Post-test product validation of MBI-401 FDP 110607 $16 \mathrm{~W}$ B-FD-E from USGS-UMESC.

\section{MATERIALS AND METHODS:}

Preparation of product for testing:

Product was shipped under cold conditions and held in the laboratory refrigerator at $4^{\circ} \mathrm{C}$ until use. Within $30 \mathrm{~min}$ of treatment application, prepare treatment stock of MBI 401 formulated product:

$W$ incing lot number trons a/8/1a

MBI 11060710B-FD-E (FDP - 100\% active ingredient): Weigh out $1.0 \mathrm{~g}$ of the powder into a labeled $50 \mathrm{ml}$ centrifuge tube and bring volume up to $20 \mathrm{ml}$ with dilution water. Vortex until evenly dispersed $=50 \mathrm{mg}$ product $/ \mathrm{ml}$ or $50 \mathrm{mg} \mathrm{ai} / \mathrm{ml}$. For $200 \mathrm{ppm}$ ai treatments in testing jars, add $2 \mathrm{ml}$ to each jar $(500 \mathrm{ml})$.

Cambridge CF (Standard):

As an efficacy standard, use $P f$-CL145A killed $\mathrm{CF}$ that was maintained at $-80^{\circ} \mathrm{C}$ (Cambridge $\mathrm{CF}$ ). Since its production in 2005 , this material has been valuable as a reference standard. The Cambridge CF was produced in 2005 (2005-0027) in 100-L batches 10, 11 and 12 and E-beamed to kill the cells. The solution, at $110 \mathrm{mg} / \mathrm{ml}$ dry weight, is stored in $1 \mathrm{~cm}$ thick sheets in the Cambridge ultrafreezer at $80^{\circ} \mathrm{C}$. A section of the sheet was broken off and weighed to determine volume (ca. $1 \mathrm{~g}=1 \mathrm{ml}$ ).

For this bioassay, the positive control suspension was produced on 5-2-2011 by weighing out $33.84 \mathrm{~g}$ of frozen Batch 10-12 block (killed cell suspension from test 2005-0027) and diluting with ca. $35 \mathrm{ml}$ dilution water. $7 \mathrm{ml}$ of this suspension were dispersed into $50-\mathrm{ml}$ centrifuge tubes and placed in the ultrafreezcr (11 tubes). The dry weight of the material was $43.75 \mathrm{mg} / \mathrm{ml}$; therefore $2.3 \mathrm{ml}$ were added to each testing jar to treat at $200 \mathrm{ppm}$.

In this test verified the activity of a new batch of positive control suspension produced on 11-14-2011; produced as described above and having a dry weight of $65.65 \mathrm{mg} / \mathrm{ml} .1 .52 \mathrm{ml}$ were added to each testing jar $(500 \mathrm{ml})$ to treat at $200 \mathrm{ppm}$.

Musscl collections:

Mussels were scraped from substrates (rocks) in the field and placed in plastic bins. Bins containing mussels were set in a cooler with towels and frozen ice packs to maintain lemperature to be transported back to the laboratory in Cambridge. In the laboratory, mussels were placed in pint-sized canning jars, covered with mesh, and placed in aquaria containing unchlorinated tap water with circulation (1 Whisper filter) and aeration at ambient laboratory temperature $\left(20^{\circ} \mathrm{C}\right)$.

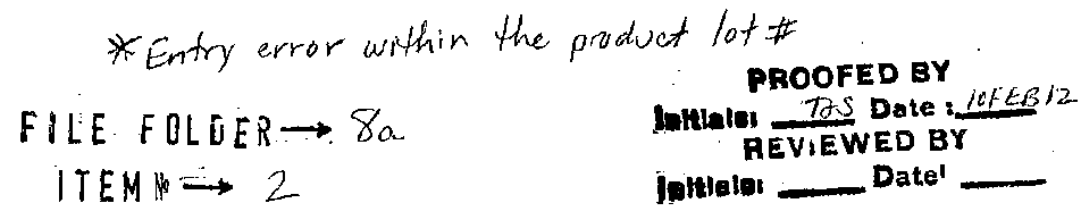


Mussel collection and handling:

\begin{tabular}{|c|c|c|c|c|}
\hline Species & Collection site & $\begin{array}{c}\text { Collection } \\
\text { date }\end{array}$ & $\begin{array}{c}\text { Date in lab } \\
\left(20^{\circ} \mathrm{C}\right)\end{array}$ & Picked for test \\
\hline Zebra mussels & $\begin{array}{c}\text { Hedges Lake } \\
\text { (Washington County) }\end{array}$ & $10 / 18 / 2011$ & $10 / 18 / 2011$ & $\begin{array}{c}11 / 14 / 2011 \\
\text { PS }\end{array}$ \\
\hline
\end{tabular}

Experineental design:

For validation of efficacy the following treatments will be set up:

Zebra mussels (25 mussels/jar):

\section{3 - Untreated Control}

3-200 ppm (ai) Cambridge CF Positive Control A (A, B, C) (produced on 5-2-201 I)

3-200 ppm (ai) Cambridge CF Positive Control B (A, B, C) (produced on 11-14-2011)

$3-200 \mathrm{ppm}$ (ai) MBI $110607 \not 0 \mathrm{~B}-\mathrm{FD}-\mathrm{E}$ (A, B, C

Testing jar bioassay protocol:

$$
\text { W } 123 / /^{2} A^{m}
$$

On the day prior to treatment $(11 / 14 / 2011)$ mussels were carcfully cxamined and 25 mussels placed into each testing jar containing ca. $100 \mathrm{ml}$ aerated hard water and allowed to attach overnight.' The next morning (11/15/2011), unattached mussels were removed and replaced with attached mussels from an extra glass Petri dish. Water was replaced with $500 \mathrm{ml}$ fresh aerated hard water.

After at least one hour, the treatment was applicd. The optical density of each jar was measured in duplicate ( $\mathrm{A}_{660 \mathrm{~nm}}$ Genesys Spectrophotometer).

After 24 hr of treatment, mussel mortality was checked and mussels were transferred to square plastic dishes with fresh aerated hard water. Mortality was checked and recorded each day with water replacements, for an additional 22 days. On the finat day of mortality checks, 20 mussels were measured from the untreated controls using a caliper.

\section{Results:}

Mussel length: Zebra mussels $12.46 \pm 2.29 \mathrm{~mm}$.

Optical density of treatments:

\begin{tabular}{|c|c|}
\hline Treatment & Mean $( \pm \mathrm{SD})$ OD $\left(\mathrm{A}_{660 \mathrm{~nm}}\right)$ \\
\hline Untreated Control & $-0.005 \pm 0.001$ \\
\hline Cambridge CF A (Positive Control) & $0.171 \pm 0.013$ \\
\hline Cambridge CF B (Positive Control) & $0.176 \pm 0.002$ \\
\hline MBI-401 FDP-11060710B-FD-E & $0.184 \pm 0.003$ \\
\hline
\end{tabular}

W $1 / 23 / 1^{2}$ mam

Zebra mussel mortality: Mussels were treated in triplicate lesting jars $(500 \mathrm{ml})$ at $20^{\circ} \mathrm{C}$ for $24 \mathrm{hr}$ and mortality was recorded for a total of 10 days.

\begin{tabular}{|c|c|}
\hline Treatment & Mean \% mortality ( \pm SD) \\
\hline Untreated Control & $0.0 \pm 0.0 \%$ \\
\hline Cambridgc CF A (Positive Control) & $88.0 \pm 4.0 \%$ \\
\hline Cambridge CF B (Positive Control) & $79.6 \pm 11.0 \%$ \\
\hline MBI-401 FDP-11060710B-FD-E & $94.7 \pm 6.1 \%$ Pass \\
\hline
\end{tabular}

Activity of MBI-401 FDP-11060710 1 B-FD-E was acceptable for this study.

\footnotetext{
FILE FOLEER $\rightarrow 8 a$ ITEMH $\rightarrow 2$
}

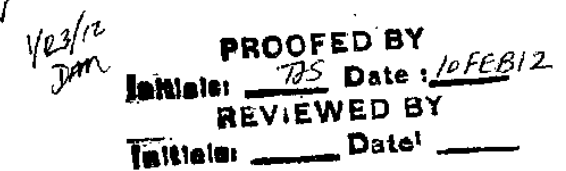

Tinitialar

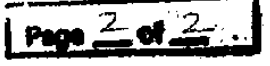




\section{NYSM Post-Treatment Product Validation Assay MBI-401 SDP 4655-12-Mix (USGS Study \#AEH-11-PSEUDO-01) FiLEFOLDER $\rightarrow 8$ a \\ ITEM $\rightarrow$ \& Date product received from USGS: 2012/02/01 \\ Date of start of test: 02/09/2012}

BACKGROUND: As standard protocol for the USLPA project, each time a batch of Zequanox product is used in a test a UMESC, a portion of the product is bioassayed by the NYSM to validate toxicity post-treatment.

- MBI-401 SDP 4655-12-Mix

- USGS Study \#AEH-11-PSEUDO-01: Glochidia (BLS, MUC, HIC)

0 USGS Study \#AEH-11-PSEUDO-02: Juvenile (FAM)

PURPOSE: Post-test product validation of MBI-401 SDP 4655-12-Mix from USGS-UMESC.

\section{MATERIALS AND METHODS:}

Preparation of product for testing:

Product was shipped under cold conditions and held in the laboratory refrigerator at $4{ }^{\circ} \mathrm{C}$ until use. Within 30 min of treatment application, prepare each at treatment stock of each MBI-401 formulated product:

MBI-401 SDP 4655-12-Mix (SDP - 50\% active ingredient): Weigh out $2 \mathrm{~g}$ of the powder and add slowly to a beaker with water stirring for even suspension. Total volume should be $20 \mathrm{ml}$ in dilution water. Transfer to a $50 \mathrm{ml}$ centrifuge and store in refrigerator until ready to use. Mix until evenly dispersed $=100 \mathrm{mg}$ product $/ \mathrm{ml}$ or $50 \mathrm{mg}$ a.i. $/ \mathrm{ml}$. For $200 \mathrm{ppm}$ ai treatments in testing jars, add $2 \mathrm{ml}$ to cach jar $(500 \mathrm{ml})$.

Cambridge CF (Standard for Positive Controls):

As an efficacy standard, use $P f$-CL145A killed CF that was maintained at $-80^{\circ} \mathrm{C}$ (Cambridge $\mathrm{CF}$ ). Sincc its production in 2005 , this material has been valuable as a reference standard. The Cambridge CF was produced in 2005 (2005-0027) in 100-L batches 10,11 and 12 and E-beamed to kill the cells. The solution, at $110 \mathrm{mg} / \mathrm{ml}$ dry weight, is stored in $1 \mathrm{~cm}$ thick sheets in the Cambridge ultrafreezer at $80^{\circ} \mathrm{C}$. A section of the sheet was broken off and weighed to determine volume (ca. $1 \mathrm{~g}=1 \mathrm{ml}$ ).

For this bioassay, the positive control suspension was produced on 11-14-2011 and then stored at $80^{\circ} \mathrm{C}$ in 50 -ml centrifuge tubes. The dry weight of the material is $65.65 \mathrm{mg} / \mathrm{ml}$; therefore $1.5 \mathrm{ml}$ was added to each testing jar to treat at $200 \mathrm{ppm}$.

Mussel collections:

Mussels were scraped from substrates (rocks) in the field and placed in plastic bins. Bins containing mussels were set in a cooler with towels and frozen ice packs to maintain temperature to be transported back to the laboratory in Cambridge. In the laboratory, mussels were placed in pint-sized canning jars, covered with mesh, and placed in aquaria containing unchlorinated tap water with circulation (1 Whisper filter) and aeration at ambient laboratory temperature $\left(20^{\circ} \mathrm{C}\right)$.

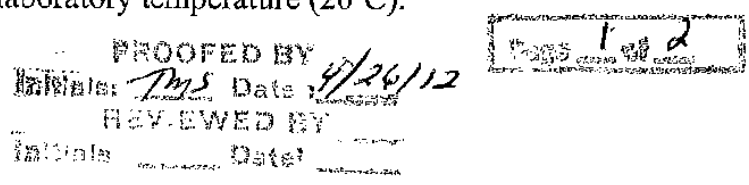


MBI-401 SDP 4655-12-Mix Post-test.02 - 2-

Mussel collection and handling:

\begin{tabular}{|c|c|c|c|c|}
\hline Species & Collection site & Collection date & Date in lab $\left(20^{\circ} \mathrm{C}\right)$ & Picked for test \\
\hline $\begin{array}{l}\text { Zebra } \\
\text { mussels }\end{array}$ & $\begin{array}{c}\text { Hedges Lake } \\
\text { (Washington County) }\end{array}$ & $11 / 30 / 2011$ & $01 / 31 / 2012$ & $02 / 08 / 2012$ \\
\hline
\end{tabular}

Experimental design:

For validation of efficacy the following treatments will be set up:

AEH-11-PSEUDO-01

Zebra mussels (25 mussels/jar):

3 - Untreated Control

3- 200 ppm (a.i.) Cambridge CF Positive Control B (A, B, C) (produced on 11-14-2011)

3-200 ppm (a.i.) MBI-401 SDP 4655-12-Mix (A, B, C)

Testing jar bioassay protocol:

On the day prior to treatment $(02 / 08 / 2012)$ mussels were carefully examined and 25 mussels placed into each testing jar containing ca. $100 \mathrm{ml}$ aerated hard water and allowed to attach overnight. The next morning (02/09/2012), unattached musscls werc removed and replaced with attached mussels from an extra glass Petri dish. Water was replaced with $500 \mathrm{ml}$ fresh aerated hard water.

After at least one hour, the treatment was applied. The optical density of each jar was measured in duplicate $\left(\mathrm{A}_{660 \mathrm{~nm}}\right.$ Genesys Spectrophotometer).

After $24 \mathrm{hr}$ of treatment, mussel mortality was checked and mussels were transferred to square plastic dishes with fresh aerated hard water. Mortality was checked and recorded each day with water replacements, for an additional 13 days. On the final day of mortality checks, 20 mussels were measured from the untreated controls using a caliper.

\section{Results:}

Mussel length: Zebra mussels $12.28 \pm 2.89 \mathrm{~mm}$.

Optical density of treatments:

\begin{tabular}{|c|c|}
\hline Treatment & Mean $( \pm \mathrm{SD})$ OD $\left(\mathrm{A}_{660 \mathrm{~nm}}\right)$ \\
\hline \hline Cambridge CF (Positive Control) & $0.191 \pm 0.008$ \\
\hline MBI-401 SDP 4655-12-Mix & $0.283+0.007$ \\
\hline
\end{tabular}

Zebra mussel mortality: Mussels were treated in triplicate testing jars $(500 \mathrm{ml})$ at $20^{\circ} \mathrm{C}$ for $24 \mathrm{hr}$ and mortality was recorded for a total of 14 days.

\begin{tabular}{|c|c|}
\hline Treatment & Mean \% mortality $( \pm \mathrm{SD})$ \\
\hline \hline Untrcatcd Control & $0.0 \pm 0.0 \%$ \\
\hline Cambridge CF (Positive Control) & $78.4 \pm 6.0 \%$ \\
\hline MBI-401 SDP 4655-12-Mix & $85.3 \pm 11.5 \%$ \\
\hline
\end{tabular}

MBI-401 SDP 4655-12-Mix PASSED the post-test bioassay validation ( $85 \%$ mortality). Untreated control mortality was $0 \%$.
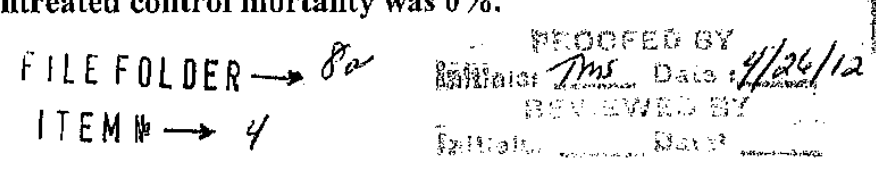

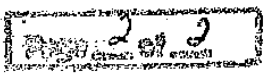


Stindy Number: AEH-11-rStusion

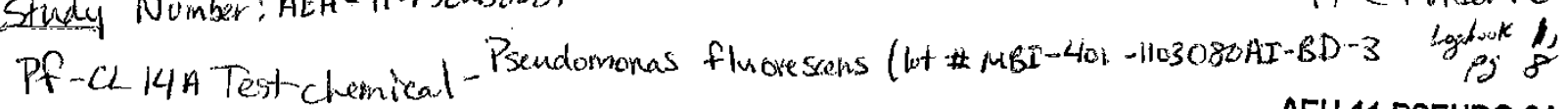

Test Chemical Wights (12 MAY $2011 \mathrm{KLW}$ ) Proofed: Z7S OZFER12 Reviewed:
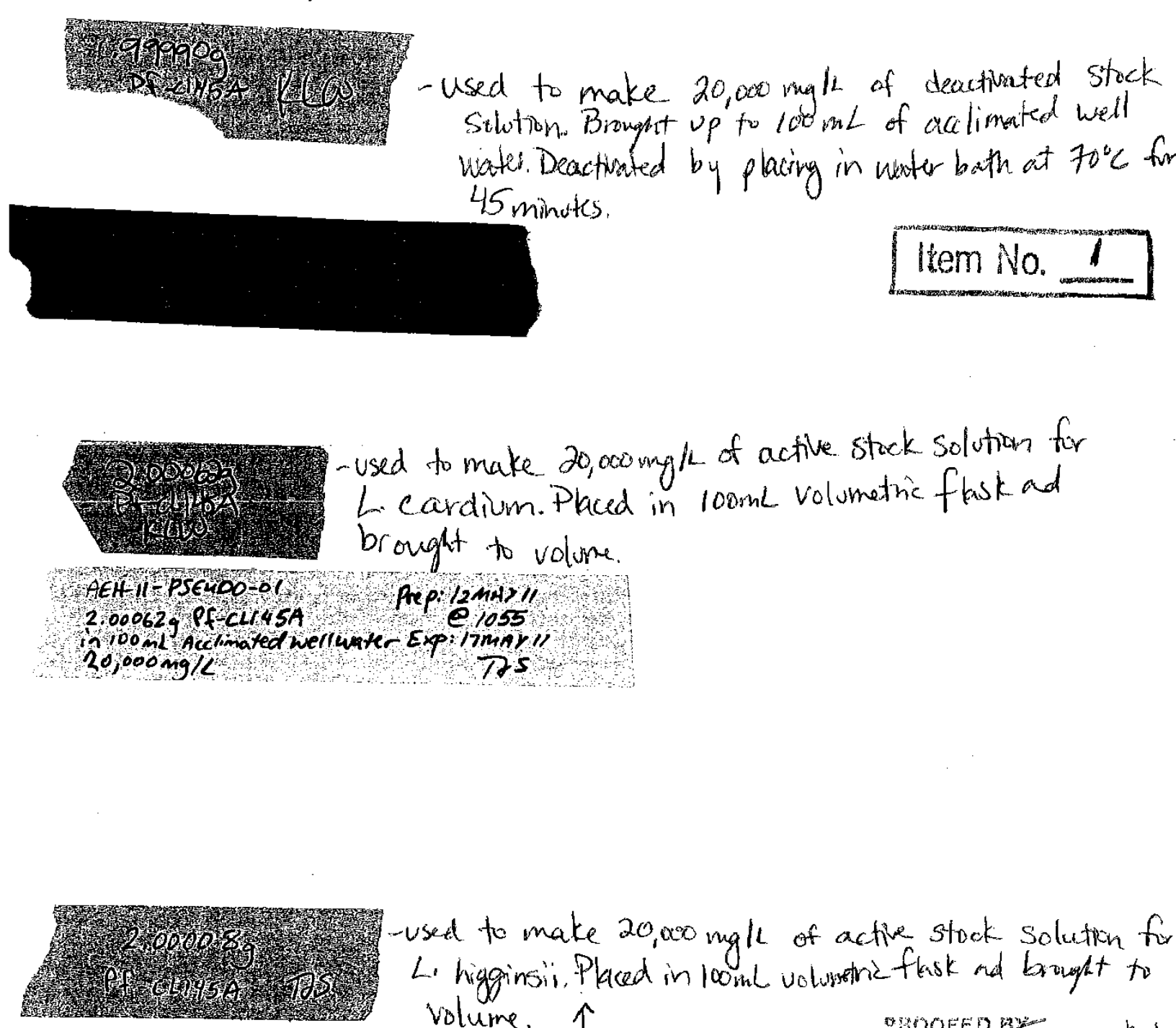

-used to make $20,000 \mathrm{mg} / \mathrm{c}$ of active stock solution for L. higginsii. Placed in $100 \mathrm{~mL}$ volumbric flask and baught to
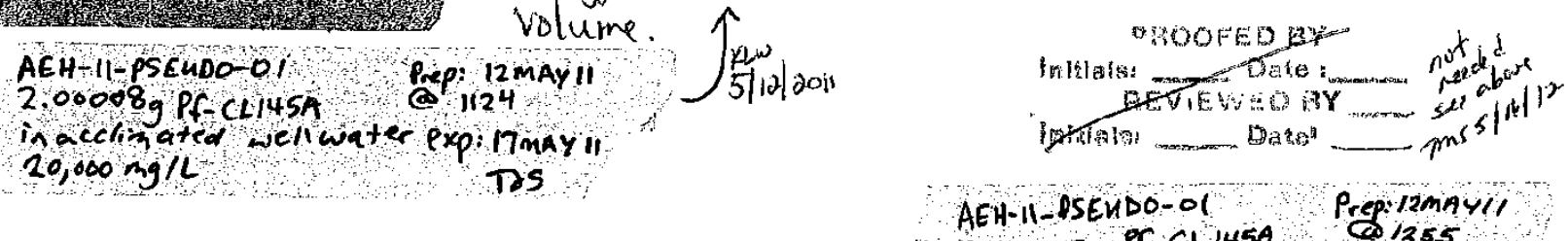

$2,00062 \mathrm{~g} \mathrm{PF} C \mathrm{Cl} 145 \mathrm{~A}$ well water $125 \mathrm{~g}$

Sh $100 \mathrm{~mL}$ accliniated well water. 70

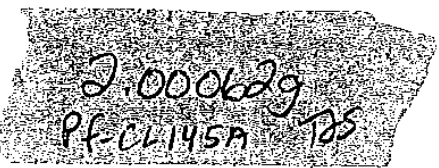

-used to make $20,000 \mathrm{mg} / \mathrm{L}$

$20,000 \mathrm{mg} / \mathrm{L}$

exporsmaris

kew

L. siliguoidea. Placed in $100 \mathrm{~mL}$ olumethe flask in brovolht to voluse.

12 MAY 2011 KLW 
Study Title: "Lffects of $P$ seudomonas fluorescens ( $P f$-CL145A) to glochidia from seven unionid mussel species"

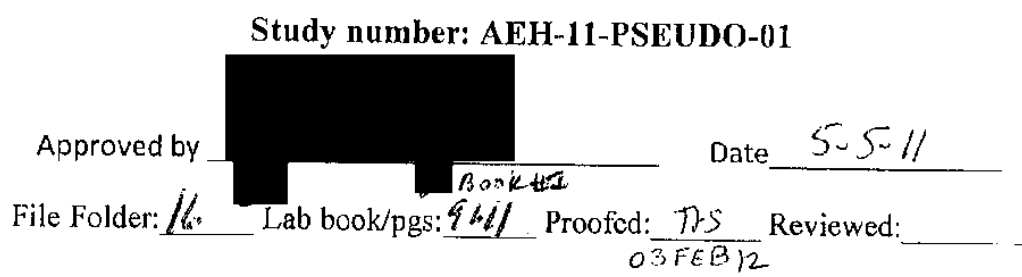

\section{Glochidia Exposure Dosing Form}

Item No. 2

Species: L. CLidhm UMESC lot number: $/ / 1400$

Test Block Assignment (circle one): 12

Estimated \# glochidia/Chamber $>1,000$

Date/time (military) of dosing initiation: 1036

Date/time (military) of dosing completion: 1040

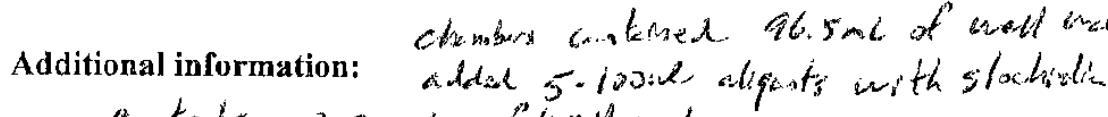

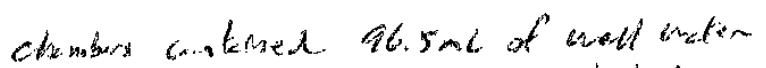

controls $=3,0 \mathrm{mi}$ of iwell water

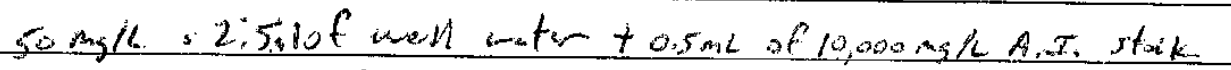

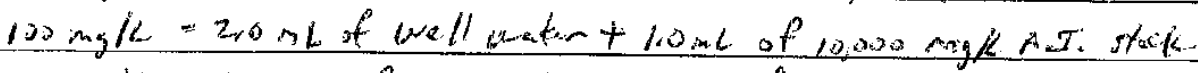

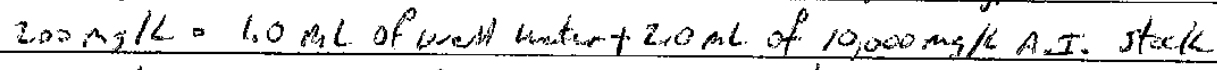

$300 \mathrm{Ag} / \mathrm{L}=3.0 \mathrm{~mL}$ of $10,000 \mathrm{mg} / \mathrm{L}$ A I $\mathcal{T}_{\mathrm{u}}$ stock

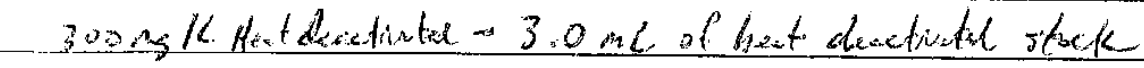

Witness and form recorded by:

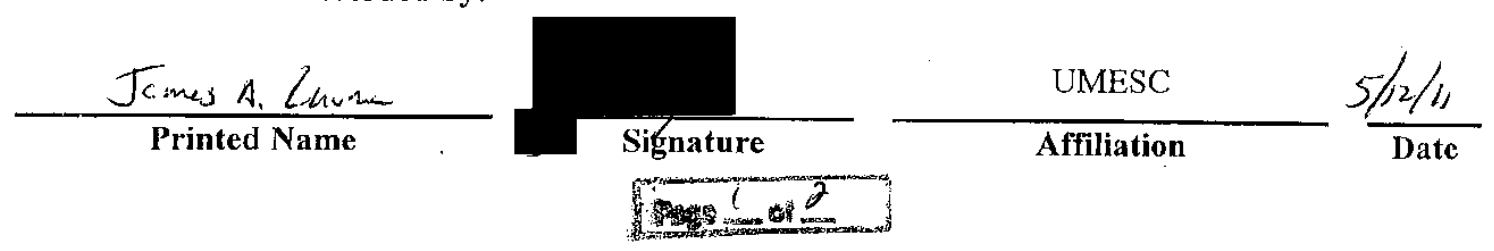




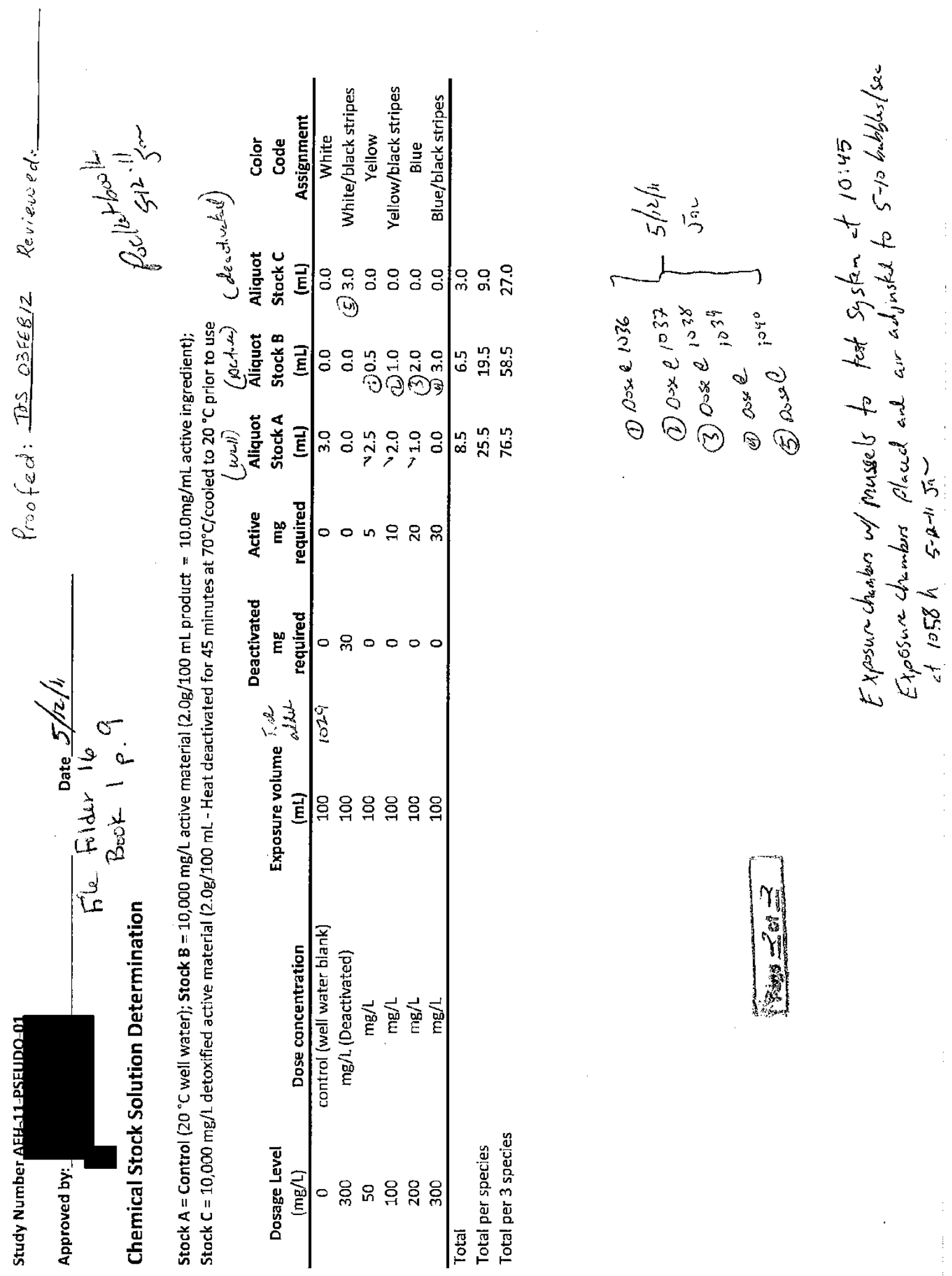


Study Title: "Effects of Psesdomonas fluorescens (Pf-CL145A) to glochidia from seven unionid mussel species"

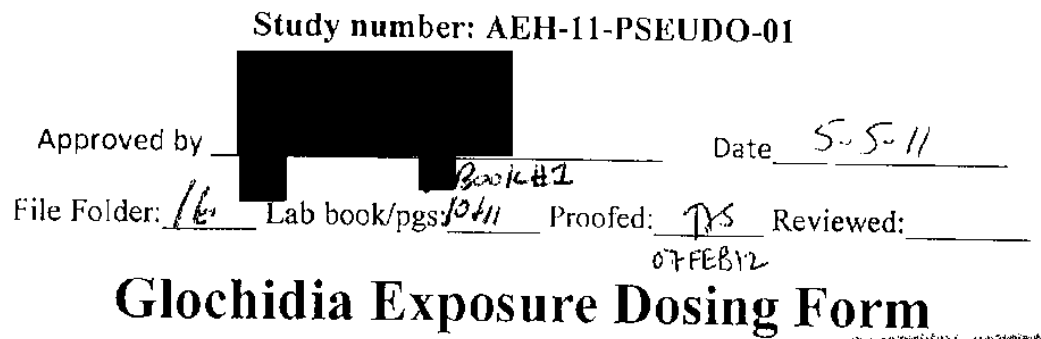

$\tan N \mathrm{No}, 3$

Species: H.spins Ege UMESC lot number: $/ 11500$

Test Block Assignment (circle one): 1

Estimated \# glochidia/Chamber $>/ 0 \infty$

Date/time (military) of dosing initiation: $\quad 1 / 55$

Datc/time (military) of dosing completion: / $/ 204$

Additional information:

Chambers cantured $46.5 \mathrm{~m}$ 's $\mathrm{H}_{2} \mathrm{O}$

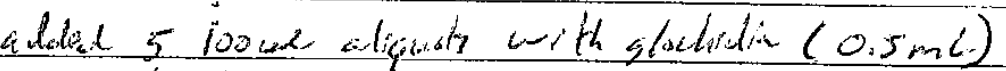

Each chambio recerval $3 \mathrm{~mL}$ of a stock on coimbinction of stock.

Ciantes $1 \leq 3 \mathrm{ml}$ of well butem

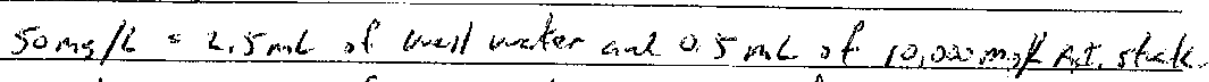

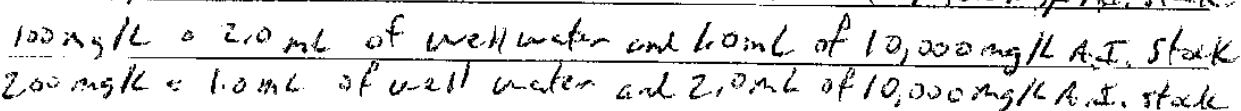

Witness and form recorded by:

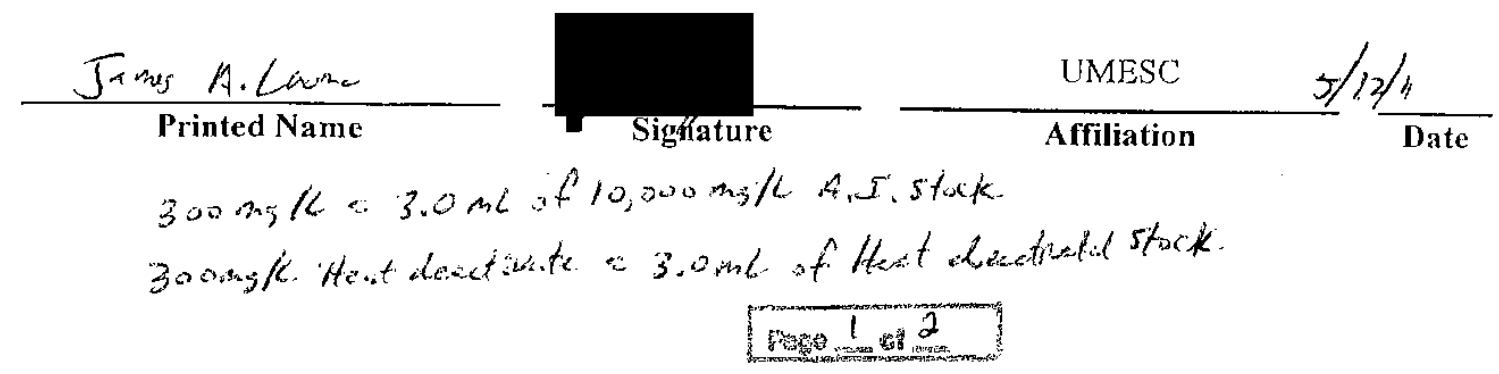




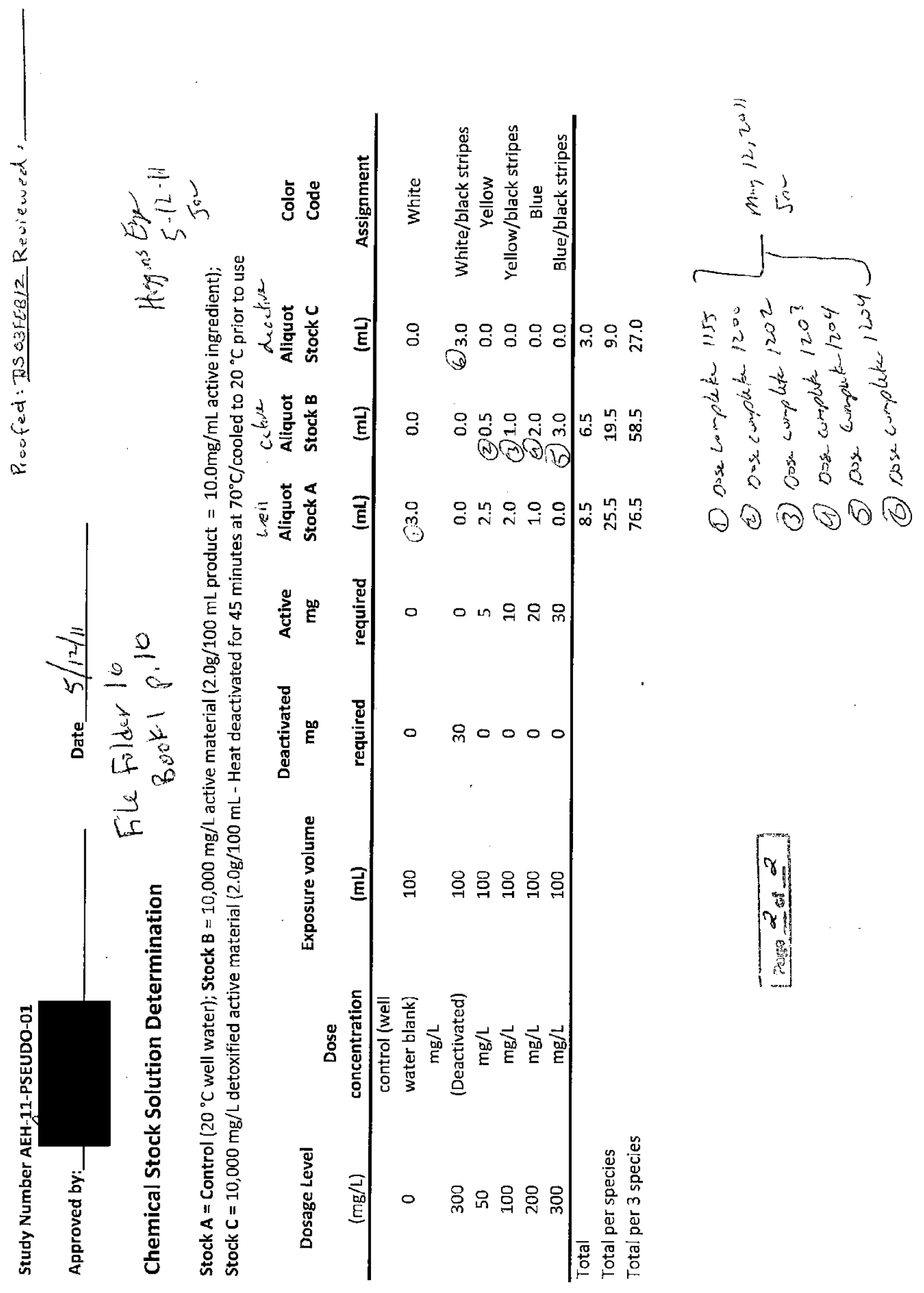


Study Title: "Effects of Pseudomonas fluorescens (Pf-CL145A) to glochidia from seven unionid mussel species"

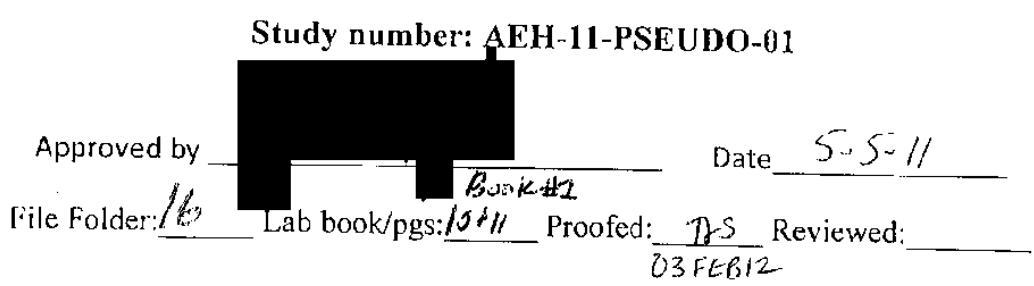

Glochidia Exposure Dosing Form

Species: Fat Murlat lem No. 4

Test Block Assignment (circle one): 1

Estimated \# glochidia/Chamber $2 / 000$

Date/time (military) of dosing initiation:

$13 / 7$

Date/time (military) of dosing completion: 132.5

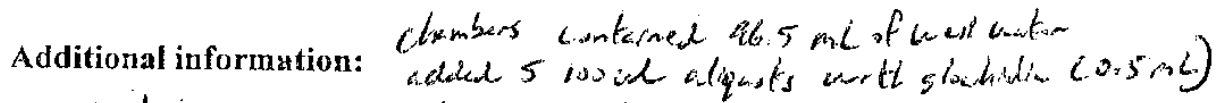

contrats $=3.0 \mathrm{~mL}$ of went water

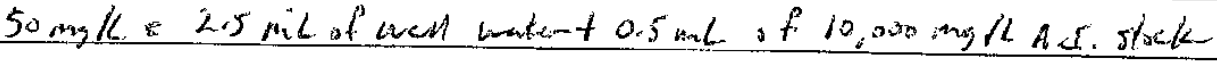

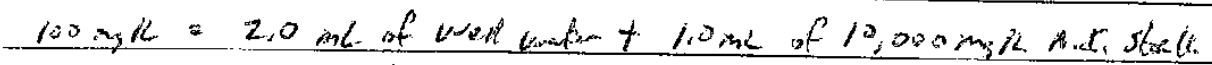

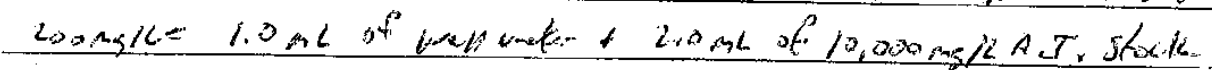

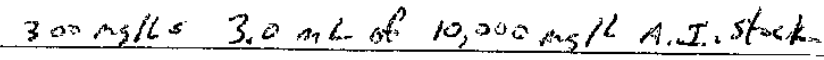

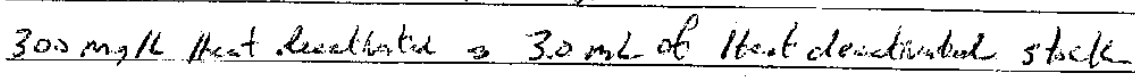

Witness and form recorded by:

$\frac{\text { Joms A. Lum }}{\text { Printed Name }} \frac{\text { UMESC }}{\text { Signature }} \frac{5 / 12 / 1}{\text { Date }}$ 


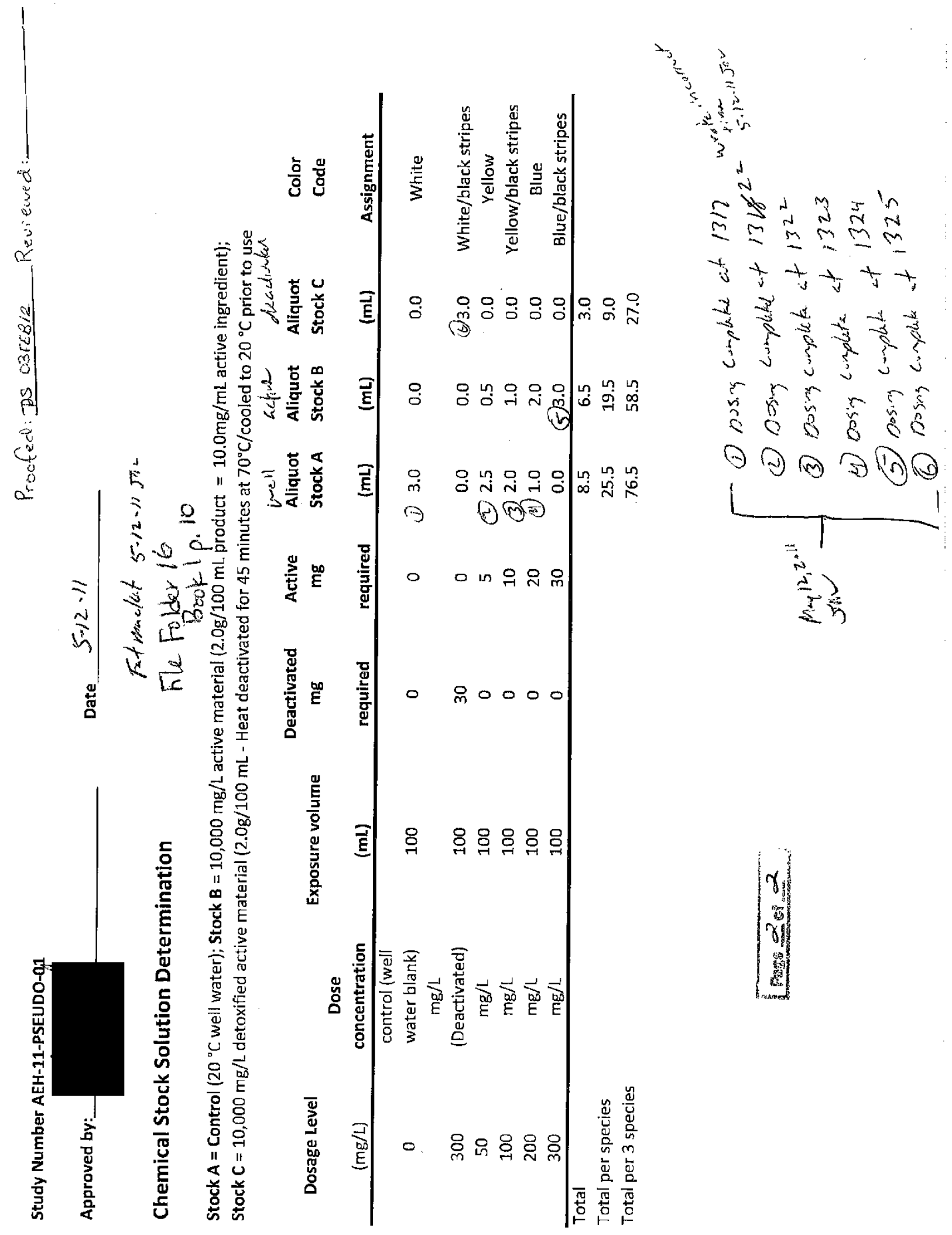


Study Title: "Effects of Pseudomonas fluorescens (Pf-CL145A) to glochidia from seven different unionid species"

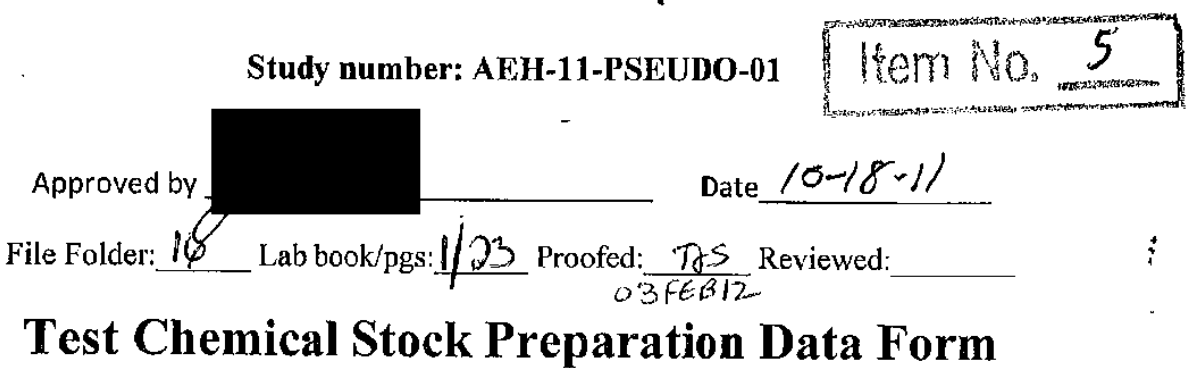

Test Chemical: Pseudomonas fluorescens strain 145A

Test Chemical Lot \# $\frac{110607 \text { WB }}{E}$-Do-Date Rec'd 14 act 2011 Exp. Date $11 / 31 / 11$

Chemical Weighing:

\begin{tabular}{|c|c|c|c|}
\hline Sample I.D. & Sample wt. (g) & Date/Time & Initials \\
\hline Deactive & 1.00005 & 18 OCT $11 / 0.725$ & KLW \\
\hline WAS Active & 1.00014 & $1800+11 / 0735$ & kew \\
\hline PPB Active & 1.00072 & $1800 \mathrm{~T} 11 / 0740$ & kLw \\
\hline & & $(2)$ & \\
\hline & & & \\
\hline
\end{tabular}

${ }^{\star}$ Chemical samples to be stored refrigerated until used for stock preparation.

\section{Stock Preparation:}

\begin{tabular}{|c|c|c|c|c|c|}
\hline Sample I.D. & $\begin{array}{l}\text { Dilution Vol. } \\
\qquad(\mathrm{ml})\end{array}$ & $\begin{array}{l}\text { Dilution } \\
\text { time }\end{array}$ & $\begin{array}{l}\text { Use (ie: Active stock for } \\
\text { IIGE) }\end{array}$ & $\begin{array}{l}\text { Date/ } \\
\text { Time }\end{array}$ & Initials \\
\hline Deactive & $100 \mathrm{~mL}$ & 0855 & Deactive ant both os 35 & $\begin{array}{r}\text { isoutll } \\
0900\end{array}$ & KLW \\
\hline WAS Active & $100 \mathrm{~mL}$ & 12.50 & 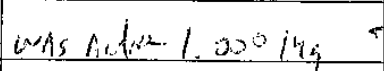 & $15 / 0+10$ & sin \\
\hline PPB Active & $100 \mathrm{~mL}$ & $1 / 2-0$ & PPB cution $1.0007^{2}$ & $180+14 / 1123$ & $\sqrt{n}-$ \\
\hline & & & (2) & & \\
\hline & & & & & \\
\hline
\end{tabular}

*Stocks to be prepared immediately before use, except for heat deactivated stock which will be prepared at least $1 \mathrm{hr}$ prior to use to allow for deactivation and cooling.
(2) No data recorded. TOS OBFEBZ2.

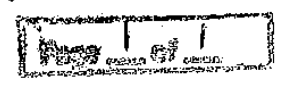

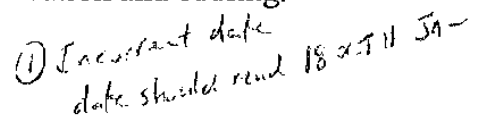


Study Title: "Lffects of Pseudomonas fluorescens (Pf-CL145A) to glochidia from seven unionid mussel species"

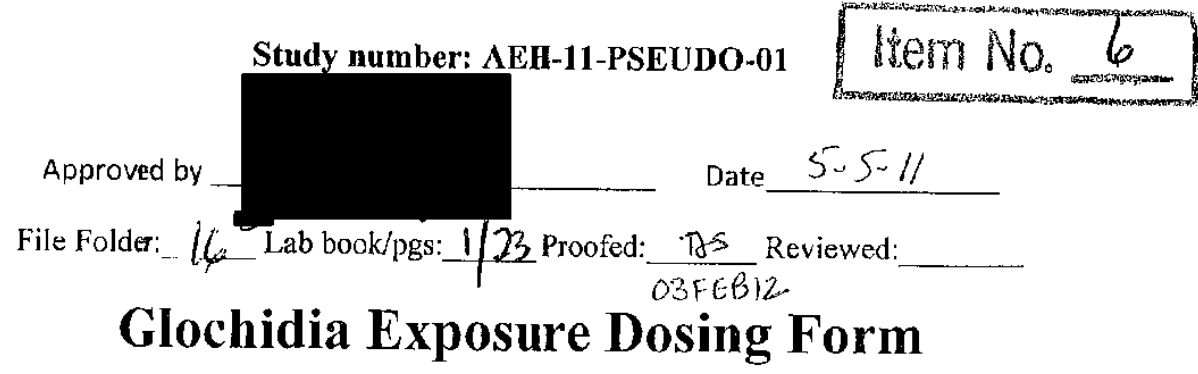

Species:_Licalium UMESC lot number: $1 / 540^{\circ}$

Test Block Assignment (circle one): 1

Estimated \# glochidia/Chamber 1000

Date/time (military) of dosing initiation: 1137

Date/time (military) of dosing completion: $1 / 46$

Additional information:

Witness and form necorded by:

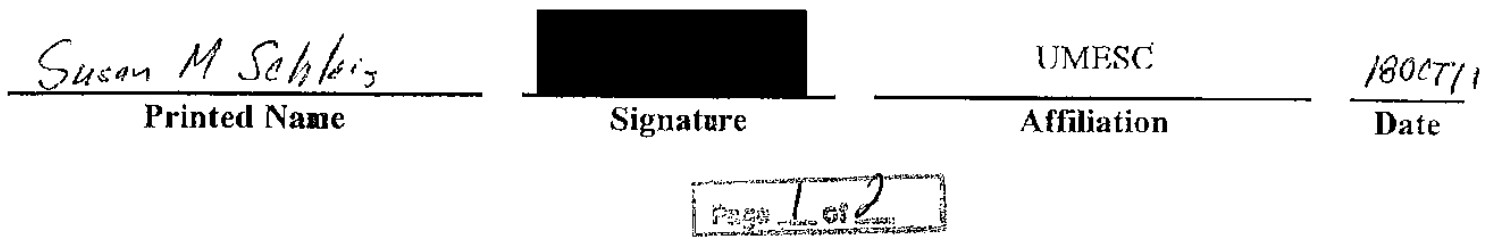




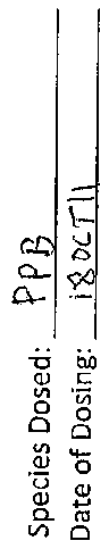

\begin{tabular}{|c|c|c|c|c|c|c|c|}
\hline 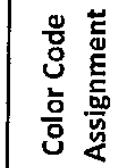 & 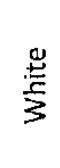 & 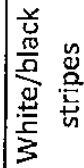 & $\frac{3}{0}$ & 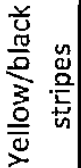 & $\frac{2}{3}$ & 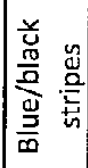 & \\
\hline 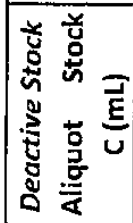 & $\stackrel{\circ}{\circ}$ & $\stackrel{\circ}{m}$ & 잉 & $\stackrel{\circ}{\circ}$ & : & $\stackrel{\circ}{\circ}$ & 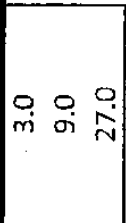 \\
\hline 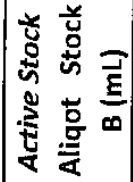 & $\stackrel{\circ}{\circ}$ & $\ddot{\circ}$ & : & 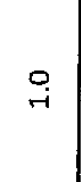 & & $\stackrel{\circ}{\dot{m}}$ & 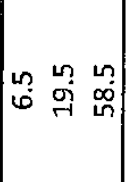 \\
\hline 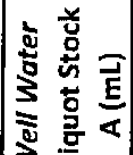 & $\stackrel{\circ}{\text { mi }}$ & 웅 & $\stackrel{n}{\sqrt[n]{2}}$ & $\stackrel{\circ}{\mathrm{N}}$ & تُ & $\stackrel{\circ}{\circ}$ & 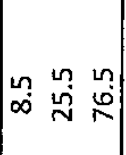 \\
\hline
\end{tabular}

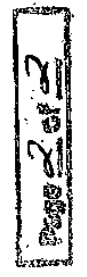

2. $\vec{E} \frac{\vec{E}}{\mathscr{G}}$

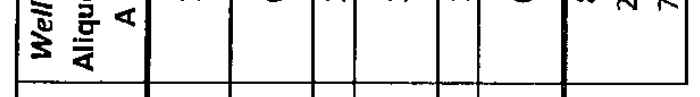
i $2 \ln$ of

$>\infty \frac{\pi}{\frac{\pi}{2}}$

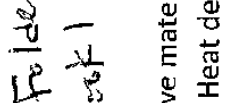

Q 0 离 ir क्षे

\begin{tabular}{|c|c|c|c|c|c|}
\hline 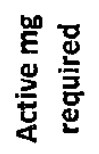 & 0 & 0 & 10 & 요 & ID \\
\hline 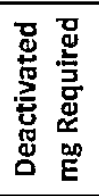 & 0 & $\stackrel{\circ}{\circ}$ & 10 & 0 & 0 \\
\hline 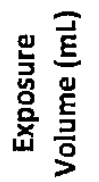 & ర్తి & 욤 & 암 & 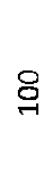 & $\stackrel{-1}{9}$ \\
\hline
\end{tabular}

$\therefore \quad$ \&

촌

究究

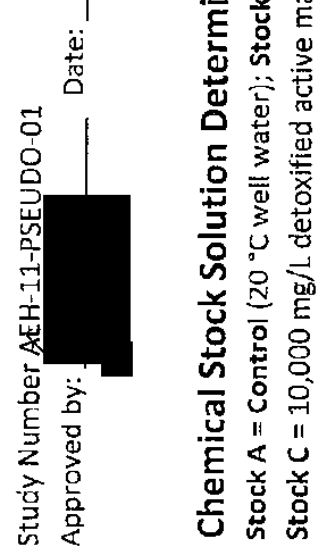

\begin{tabular}{|c|c|c|c|c|c|c|}
\hline & 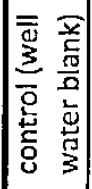 & 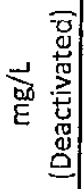 & है & के & & $\overrightarrow{\mathrm{a}}$ \\
\hline 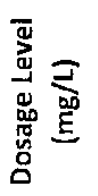 & 0 & 总 & 용 & 윰 & 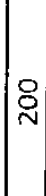 & 总 \\
\hline
\end{tabular}

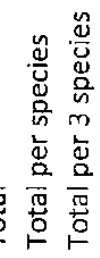

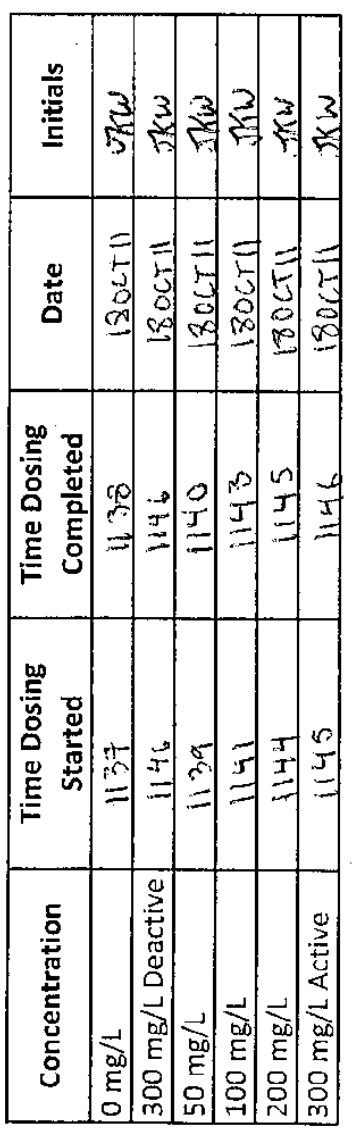

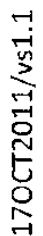


Study Title: "Effects of Pseudomonas fluorescens ( $P f$-CL145A) to glochidia from seven unionid mussel species"

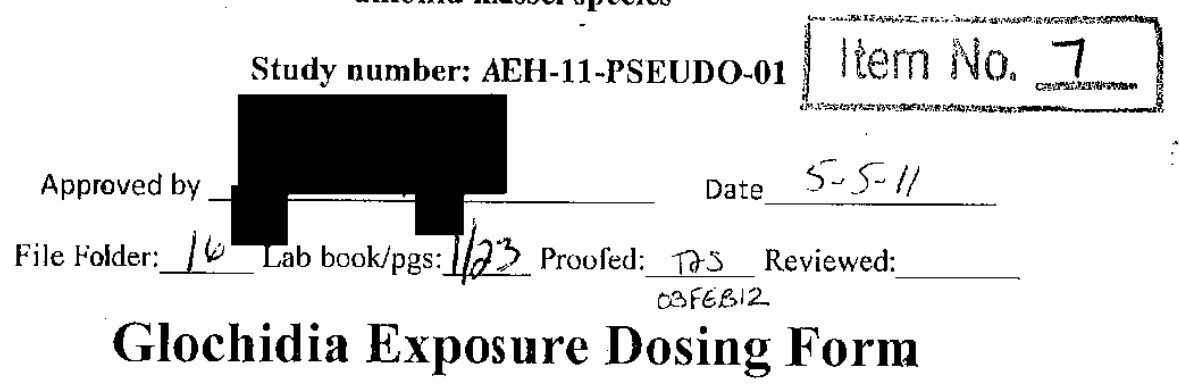

Species: Washboard UMESC lot number: $1 / 5500$

Tcst Block Assignment (circle one): 12

Estimated \# glochidia/Chamber 500

Date/time (military) of dosing initiation: $\quad 1305$

Date/time (military) of dosing completion: 1313

Additional information:

Witness and form recorded by:

$\frac{\text { Susan M Schleis }}{\text { Printed Name }} \frac{\text { UMESC }}{\text { Signature }} \frac{\text { Affiliation }}{\text { Date }}$




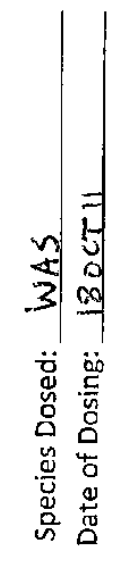

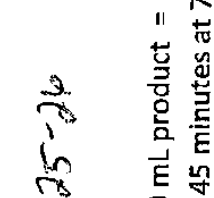

क

$\pm-\quad$ 总

है (5)

प⿺⿻一⿰冫⿰亅⿱丿丶丶⿱⿰㇒一乂

की

$15 \quad 50$

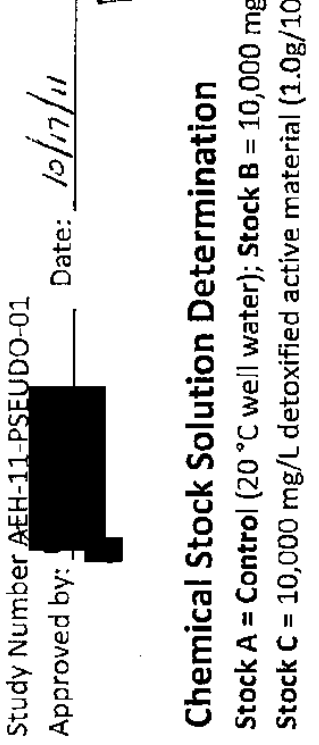

\begin{tabular}{|c|c|c|c|c|c|c|c|}
\hline 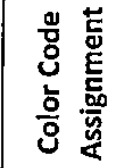 & $\begin{array}{l}\frac{0}{5} \\
\frac{5}{3}\end{array}$ & 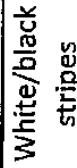 & $\mid \frac{3}{2}$ & 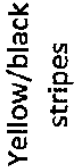 & $\frac{\mathrm{g}}{\mathrm{a}}$ & 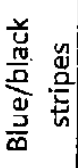 & \\
\hline 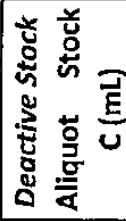 & $\stackrel{\circ}{\circ}$ & $\stackrel{\circ}{\text { if }}$ & 잉 & : & $\stackrel{\circ}{0}$ & : & \begin{tabular}{lll}
0 & 0 & 0 \\
$\dot{m}$ & $\sigma$ & \multirow{N}{*}{}
\end{tabular} \\
\hline 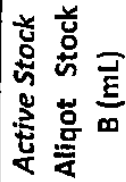 & $\stackrel{\circ}{\circ}$ & $\stackrel{\circ}{\circ}$ & $\stackrel{4}{3}$ & $\stackrel{\circ}{\rightarrow}$ & $\stackrel{\leftrightarrow}{\mathrm{i}}$ & $\stackrel{\text { in }}{\text {. }}$ & 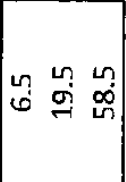 \\
\hline 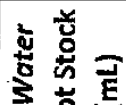 & 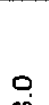 & & in & 0 & & 0 & ก กุ กุ \\
\hline
\end{tabular}

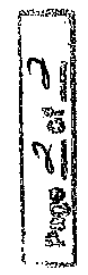

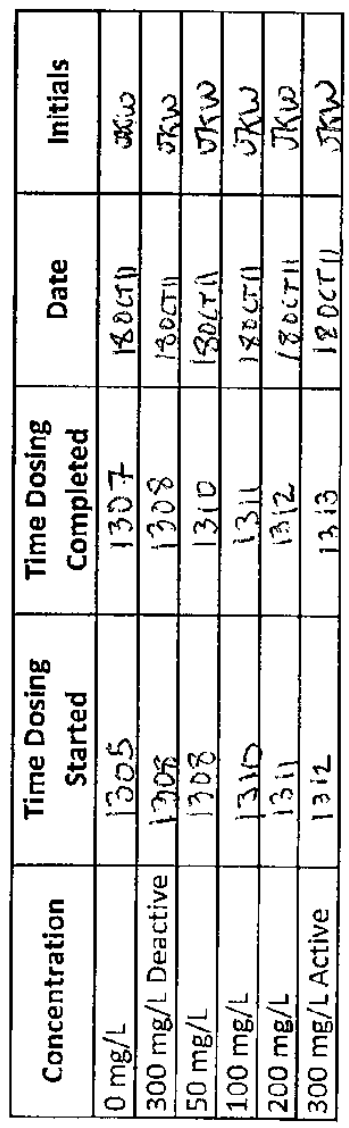

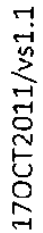


Study Title: “Effects of Pseudomonas fluorescens (Pf-CL145A) to glochidia from seven different unionid species"

Study number: AEH-11-PSEUDO-01

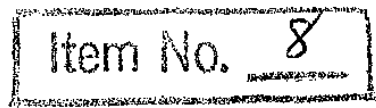

File Folder: 16 Lab book/pgs: 1/31 Reviewed: ThS Verified:

\section{Test Chemical Stock Preparation Data Form}

Test Chemical: Pseudomonas fluorescens strain 145A -

Test Chemical Lot \# $4 \mathrm{MBI}_{55-40) \text { SDP }}$

Test Chemical Lot \#4655-12-Mix Date Rec'd 10 JAN 2012 Exp. Date 10 JAN 2017

Mussel Species BLS+MUC Block ID BLS=1;MUC=2 (none providel)

Instruments: $B A L$ 1, BAL4 and WTS 2

\section{Chemical Weighing:}

\begin{tabular}{|c|c|c|c|}
\hline Sample I.D. & Sample wt. (g) & Date/Time & Initials \\
\hline Deadive & 2.0013 & $17 \pi 4 \omega i 2 / 8000$ & klow \\
\hline Active BLS & 2,0037 & 17 JAn a/ $/ 0807$ & kw \\
\hline Active MUC & 2,0020 & 17 TAN 1210815 & kw \\
\hline & & (1) $\mathrm{Ku}_{\mathrm{W}}$ & \\
\hline & & (I) & 10 \\
\hline
\end{tabular}

*Chemical samples to be stored refrigerated until used for stock preparation.

\section{Stock Preparation:}

\begin{tabular}{|c|c|c|c|c|c|}
\hline Sample I.D. & $\begin{array}{l}\text { Dilution Vol. } \\
\qquad(\mathrm{ml})\end{array}$ & $\begin{array}{l}\text { Dilution } \\
\text { time }\end{array}$ & $\begin{array}{c}\text { Use (ie: Active stock for } \\
\text { HGE) }\end{array}$ & $\begin{array}{l}\text { Date/ } \\
\text { Time }\end{array}$ & Initials \\
\hline Deactive & $100 \mathrm{~mL}$ & 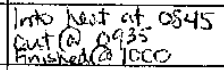 & Deactive stock for & 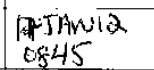 & kus \\
\hline Active BLS & $100 \mathrm{~mL}$ & 1035 & Active stack for BLS & 19 TAMONO & Rino \\
\hline \multirow[t]{3}{*}{ Active muc } & $100 \mathrm{~mL}$ & 1205 & Active stock formuc & $\begin{array}{c}175 A N 12 \\
1205\end{array}$ & Tars \\
\hline & & & (1) Kin 17thon 3012 & & \\
\hline & & & & & \\
\hline
\end{tabular}

*Stocks to be prepared immediately before use, except for heat deactivated stock which will be prepared prior to use to allow for deactivation and cooling.

This datasheet was approved by 11.JAN2012/version 1.1

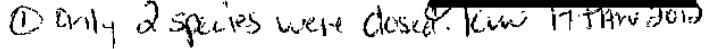
on $12 \sin 2 \cdot 2$ 
Study Title: "Effects of Pseudomonas fluorescens (Pf-CL145A) to glochidia from seven unionid species"

Study number: AEH-11-PSEUDO-01

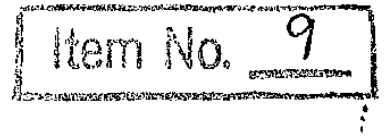

\section{File Folder: 16 Lab book/pgs: $1 / 32$ Reviewed: $\frac{175}{07 F E B 12}$ Verified:
Glochidia Exposure Dosing Form}

Species: $\quad$ BLS UMLSC Iot number: 120800

Test System Assignment (circle one): (1) 2

Estimated \# glochidia/chamber $\leq 500$

Date/time (military) of dosing initiation: 1100

Date/time (military) of dosing completion: $\leq-50$ illl

Additional information:

Chambers cortained $96.5 \mathrm{~mL} H 30$. Added $5100 \mathrm{~mL}$ aliquot with glochidia $(0.5 \mathrm{~mL})$. Each chamber received $3 \mathrm{~mL}$ of a stock or combination of stocks. Control $=3 \mathrm{~mL}$ of well water; $50 \mathrm{rrg} / \mathrm{L}=2.5 \mathrm{ml}$ of wedl water and $0.5 \mathrm{~mL}$ of $10,000 \mathrm{mg} / \mathrm{LA} . I_{\text {. }} 5 \mathrm{tack}$; $100 \mathrm{mg} / \mathrm{h}=2.0 \mathrm{~mL}$ of well water and $1.0 \mathrm{~mL}$ of $10,000 \mathrm{mg} / \mathrm{L}$. A. . . Stack; $200 \mathrm{mgl} /=$ $1.0 \mathrm{~mL}$ of wed water and $2.0 \mathrm{~mL}$ of $10,000 \mathrm{mg} / \mathrm{L}$ A.T. staki $300 \mathrm{mg} / \mathrm{L}=$ $3 \mathrm{~mL}$ of $A \cdot T \cdot$ Stock; $300 \mathrm{mg} / \mathrm{L}$ Deactivated $=3 \mathrm{~mL}$ of $10,000 \mathrm{ng} / \mathrm{L}$ deactivated
Stack. Kno 17 JAh 2012

Witness and form recorded by:

Jeremy K. Wise Printed Name

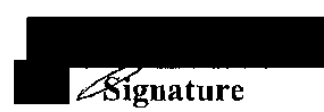

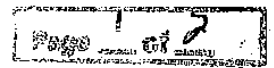

This datasheet was approved by 13JAN2012/version 1.1

(i) Misentry, SKW BJTANL 


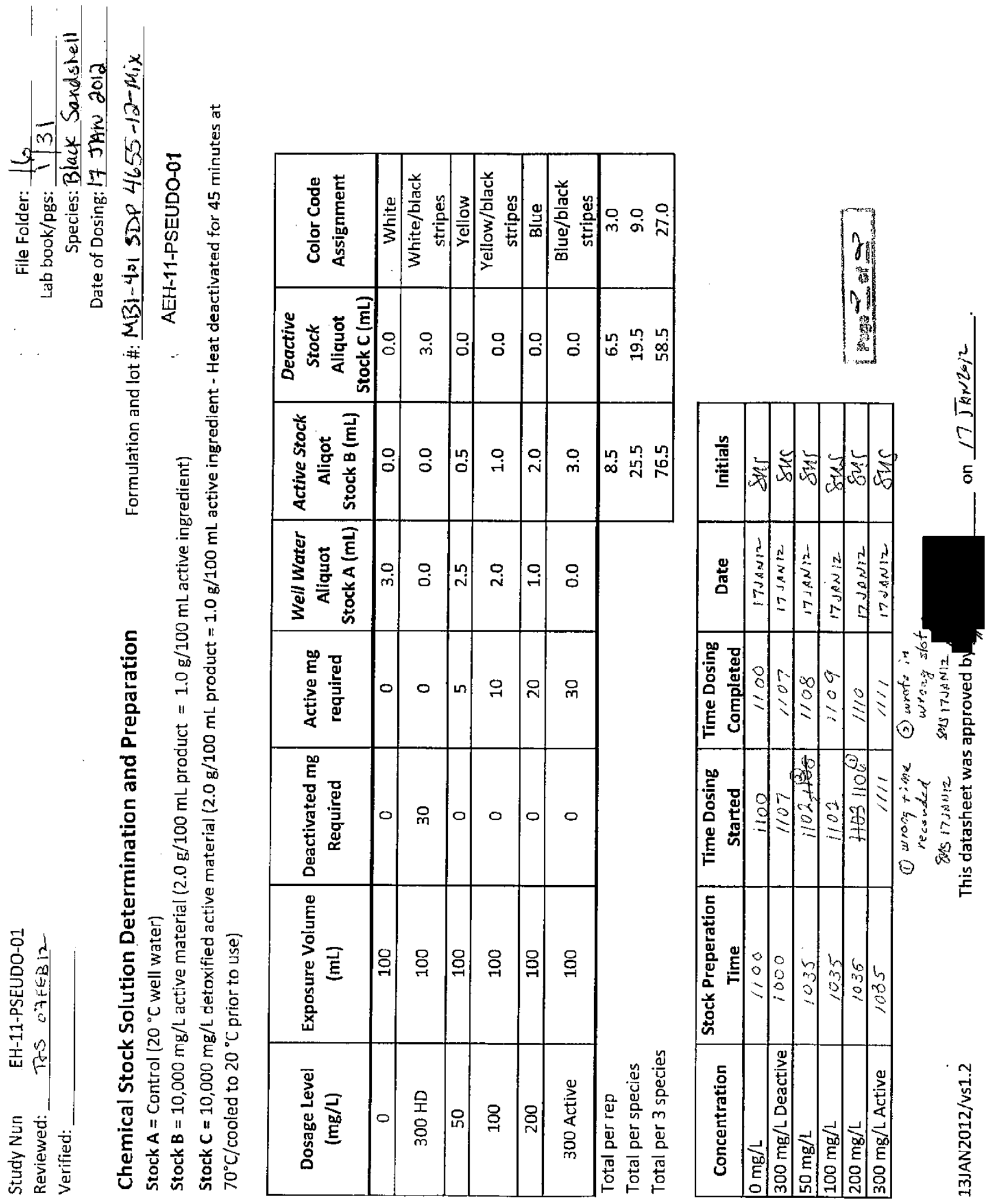


Study Title: "Effects of Pseudomonas fluorescens (Pf-CL145A) to glochidia from seven unionid species"

Study number: AEH-11-PSEUDO-01

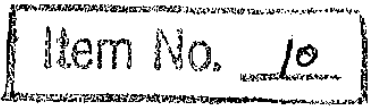

File Folder: 16 Lab book/pgs: 1132 Revicwed: $\frac{75}{67 F G B, 2}$ Verified:

\section{Glochidia Exposure Dosing Form}

Species: MUL UMESC lot number: 120700

Test System Assignment (circle one):

(2) 3

Estimated \# glochidia/chamber $\leq 500$

Date/time (military) of dosing initiation: $1232 / 17 \mathrm{JAN} / 2$

Date/time (military) of dosing completion: $1238 /$ 17 JAN 12

Additional information:

Chambers contaited $96.5 \mathrm{~mL}$ t. 0 . Added 5100 ut aliquots with glochidia (0.5mL). Each chamber received $3 \mathrm{~mL}$ of a stock or conbination of stocks. Cantrol = $3 \mathrm{~mL}$ of weil water. $50 \mathrm{mg} / \mathrm{L}=2.5 \mathrm{~mL}$ of weil watior and $0.5 \mathrm{~mL}$ of $100 \mathrm{n}$ A.I. Stock; $100 \mathrm{mg} / \mathrm{L}=2.0 \mathrm{~mL}$ of well water and $1.0 \mathrm{~mL}$ of $10,000 \mathrm{ng} / \mathrm{L}$ A.T. Stock; $200 \mathrm{mg} / \mathrm{L}=1.0 \mathrm{~mL}$ of $v e+1 /$ water ond $2.0 \mathrm{mb}$ of $10,000 \mathrm{mg} / \mathrm{h}$ A.T stock; $300 \mathrm{mg} / \mathrm{L}$ Active $=3 \mathrm{~mL}$ of $10,000 \mathrm{mg} / \mathrm{L}$ A.t stock; $300 \mathrm{mg} / \mathrm{l}$ Deactivated $=3 \mathrm{~mL}$ of $10,000 \mathrm{mg} / \mathrm{L}$ deactivated Stack. KCw 17 tato 2010

Witness and form recorded by:

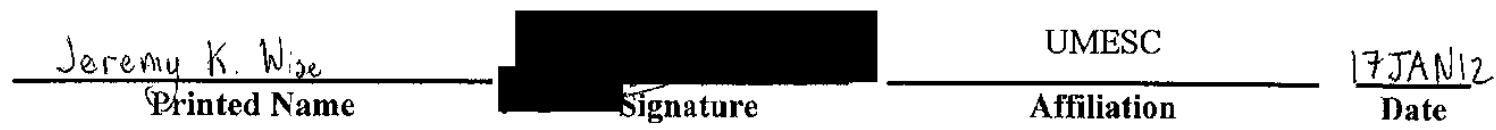

(1)ER. Should be 10,000mg/h Kin 177 W 2012

This datasheet was approved by

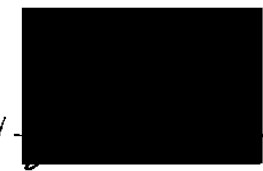
on $17 \sqrt{4} \times 120 \% 2$ 


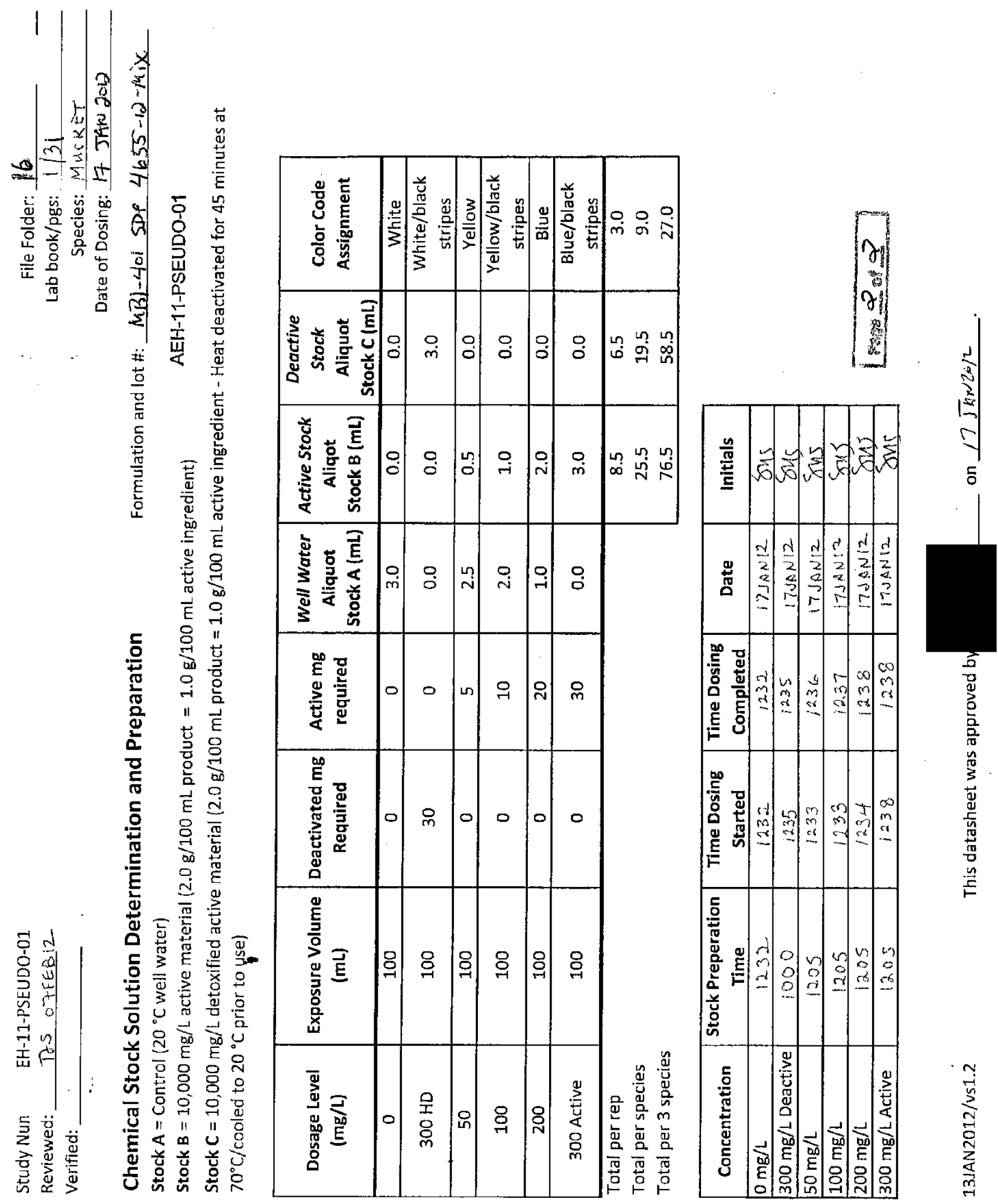


Study Title: "Effects of Pseudomonas fluorescens (Pf-CL145A) to glochidia from seven different unionid species"

Study number: AEH-11-PSEUDO-01

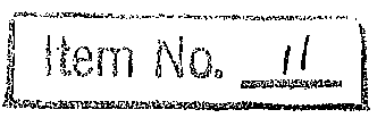

File Folder: 16 Lab book/pgs: 133,34 Reviewed: Ths Verifjed:

\section{Test Chemical Stock Preparation Data Form}

Test Chemical: Pseudomonas fluorescens strain 145A

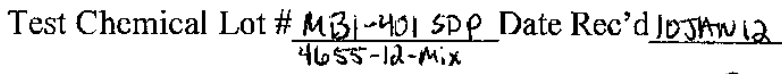

Mussel Species HKC Block ID 3

Exp. Date 10 JAN 2017

Instruments: BAL I BAL 4, WTS

(nive provideds)

Chemical Weighing:

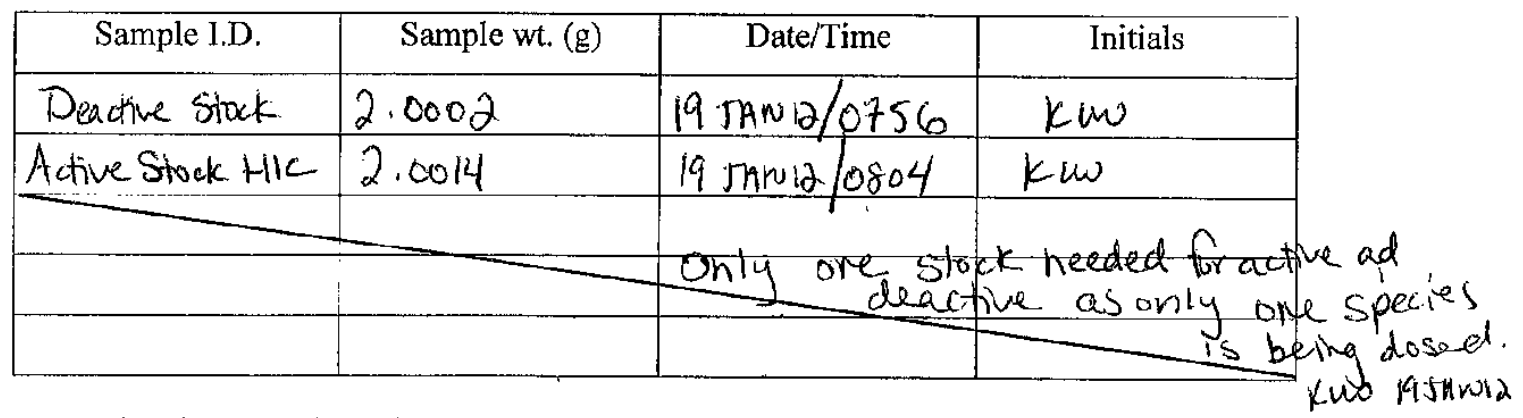

*Chemical samples to be stored refrigerated until used for stock preparation.

\section{Stock Preparation:}

\begin{tabular}{|c|c|c|c|c|c|}
\hline Sample I.D. & $\begin{array}{l}\text { Dilution Vol. } \\
\text { (ml) }\end{array}$ & $\begin{array}{l}\text { Dilution } \\
\text { time }\end{array}$ & $\begin{array}{c}\text { Usc (ic: Active stock for } \\
\text { HGE) }\end{array}$ & $\begin{array}{l}\text { Date/ } \\
\text { Time }\end{array}$ & Initials \\
\hline Deactive sta.k & 100 & into heat eosis & Af Ru RE & $195 \mathrm{AN} Z \mathrm{Z} / 8808$ & ThS \\
\hline \multirow[t]{4}{*}{ Active Stock } & 100 & 0900 & Active Strek for Hic & $195 \mathrm{JAN} / 2 / 10900$ & DS \\
\hline & 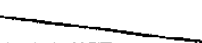 & & & & \\
\hline & & & $y$ & km & $19.5 A N 12$ \\
\hline & & & & & \\
\hline
\end{tabular}

* Stocks to be prepared immediately before use, except for heat deactivated stock which will be prepared prior to use to allow for deactivation and cooling.

$$
1 x^{2}
$$

This datasheet was approved by 11JAN2012/version 1.1

$$
\text { on } 12 \sin 2 \cdots 2 .
$$


Study Title: "Effects of Pseudomonas fluorescens (Pf-CL145A) to glochidia from seven unionid species"

Study number: AEH-11-PSEUDO-01

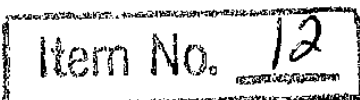

File Folder: 16 Lab book/pgs: $1 / 34$ Reviewed: $\frac{75}{0.7 F B / 2}$ Verified:

\section{Glochidia Exposure Dosing Form}

Species: HIC UMESC lot number: 120400

Test System Assignment (circle one): 1 \begin{tabular}{lll}
1 & 2 & 3 \\
\hline
\end{tabular}

Estimated \# glochidia/chamber $\leq 1000$

Date/time (military) of dosing initiation: 0925

Date/time (military) of dosing completion:

0929

Additional information:

Chaimbers contained $96.5 \mathrm{ml}$ Ho. Added $5100 \mathrm{ul}$ a liquots woth glochidia $(0.5 \mathrm{~mL})$. Each chamber received $3 \mathrm{~mL}$ of a stock or concentration of stocks. Control $=3 \mathrm{~mL}$ of well water; $50 \mathrm{mg} / \mathrm{L}=2.5 \mathrm{~mL}$ of well water and $0.5 \mathrm{~mL}$

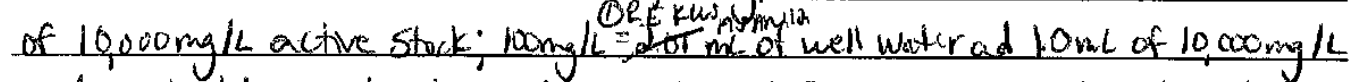
active streck; $200 \mathrm{mg} / \mathrm{l}=1.0 \mathrm{~mL}$ of well water and $20 \mathrm{~min}$ of $10,000 \mathrm{mg} / \mathrm{c}$ active stock; $300 \mathrm{rg} / \mathrm{c}$ active $=3 \mathrm{~mL}$ of $10,000 \mathrm{mg} / \mathrm{L}$ active stuck $300 \mathrm{mg} / \mathrm{L}$ Deactivated $=3 \mathrm{~mL}$ of $10,000 \mathrm{ng} / \mathrm{L}$ deactivated stock. KWW 19 ThW 12

Witness and form recorded by:

$\frac{\text { Jeremy } x \text {. Wire }}{\text { Printed Nam }}$

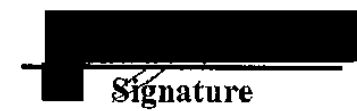

UMESC

Affiliation

19 FAN12

(1) Should be $2.0 \mathrm{mg} / \mathrm{L}$ kWw $95 \mathrm{tan} / 2$

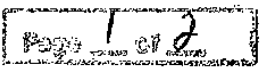

This datasheet was approved by

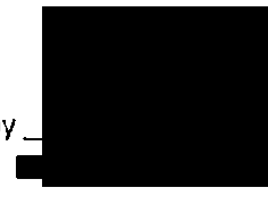

on $17 \sqrt{\mathrm{A}} \mathrm{N} / 2 \mathrm{x} / 2$ 


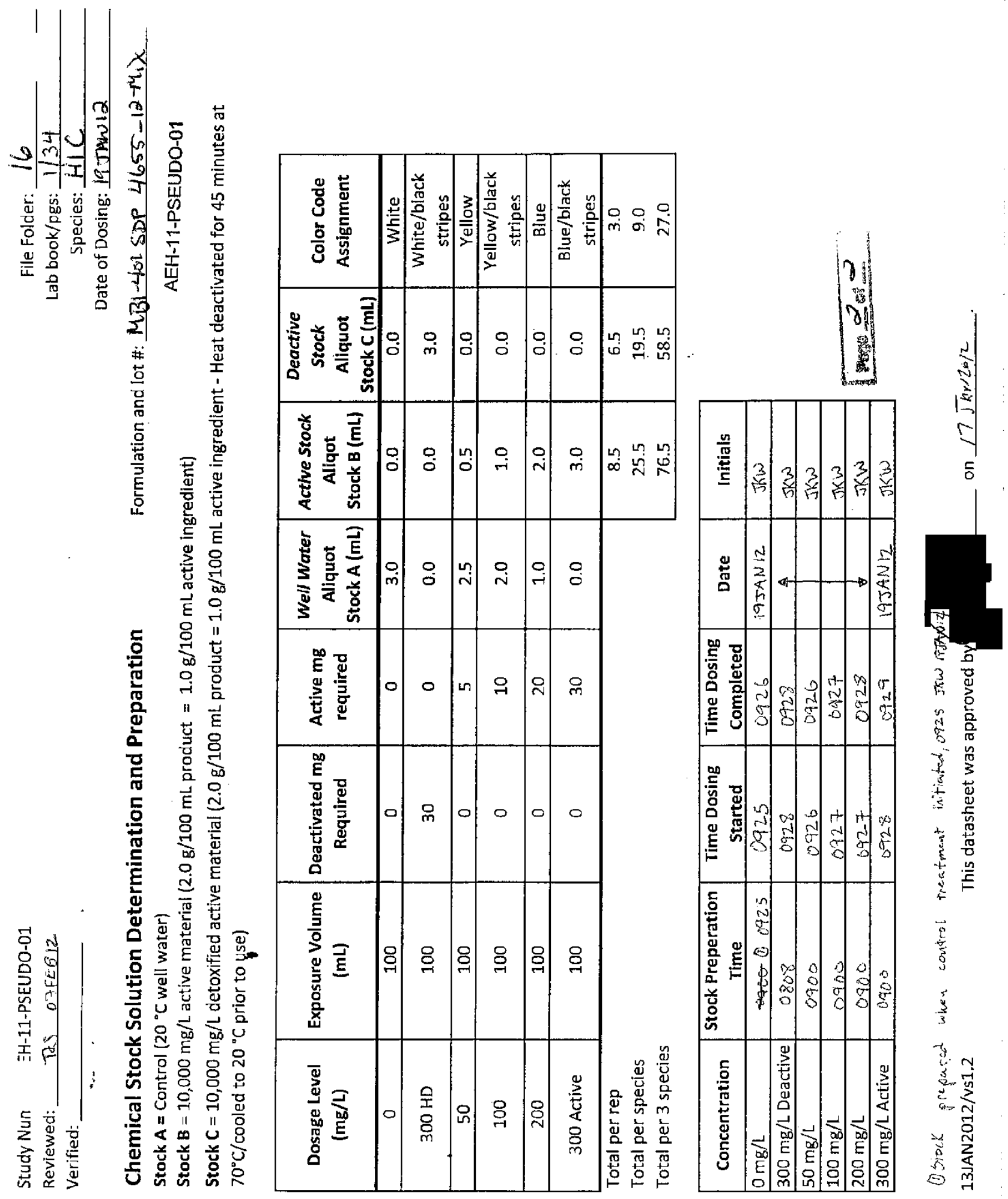




\section{Appendix 5. Test Animal Information}

\begin{tabular}{clcc}
\hline $\begin{array}{c}\text { Item } \\
\text { number }\end{array}$ & \multicolumn{1}{c}{ Item description } & $\begin{array}{c}\text { Number } \\
\text { of } \\
\text { pages }\end{array}$ & $\begin{array}{c}\text { Report } \\
\text { number } \\
\text { number }\end{array}$ \\
\hline 1 & Test Organism Species List, Collection and Inclusion Criteria & 1 & 283 \\
2 & Amended T est Organism Species List, Collection and Inclusion Criteria & 2 & 284 \\
3 & Daily Care Worksheets (PPB, HGE, FAM, HIC, BLS, MUC) & 6 & 286 \\
4 & Adult Mussel Holding Daily Algal Diet (1/13/2012) & 1 & 292 \\
5 & Donor Mussel UMESC Lot Number Assignment Forms & 8 & 293 \\
6 & USGS UMESC Fish/Animal History Forms (Donor Mussels) & 8 & 301 \\
7 & Glochidia T est Organism UMESC Lot Number Assignment Forms & 309 \\
8 & USGS UMESC Fish/Animal History Forms (Glochidia) & 317 \\
\hline
\end{tabular}


Study Title: "Effects of Pseudomonas fluorescens (Pf-CL145A) to glochidia from seven unionid mussel species"

\begin{tabular}{cc} 
Study number: AEH-11-PSEUDO-01 \\
Test Organism Species List, Collection and Inclusion Crit \\
\hline Common name & Satentific name \\
\hline Black sandshell & Ligumia recta \\
Fatmucket & Lampsilis siliquoidea \\
Hickorynut & Obovaria olivaria \\
Higgins eye & Lampsilis higginsii \\
Mucket & Actinonaias ligamentina \\
Plain pocketbook & Lampsilis cardium \\
Washboard & Megalonaias nervosa \\
\hline
\end{tabular}

\section{Collection and inclusion criteria (see protocol sections 4.1-4.2):}

Gravid donor mussels will be collected by biologists from the Genoa National Fish Hatchery from the Upper Iowa, Upper Mississippi or St. Croix Rivers. Mussels will be identified to species as described in Cummings and Mayer (1992) and Watters et al. (2009) and transported to the Genoa National Fish Hatchery. Glochidia from at least three gravid female mussels of each species will be extracted and evaluated for viability and if acceptable $(\geq 80 \%)$ glochidia groups will pooled for use by the Genoa NFH according to the procedures outlined in ASTM E2455-06 and immediately transferred to the UMESC in a cooler at $\sim 20^{\circ} \mathrm{C}$. Glochidia will be acclimated to test water by the addition of $50 \%$ UMESC well water at the Genoa NFI and upon arrival at UMESC. Prior to exposure initiation, viability will be assessed in a subsample of glochidia by the addition of a saturated sodium chloride solution ( $12 \mathrm{~g} \mathrm{NaCl} / 50 \mathrm{ml}_{\text {s }}$ of D.I. $\mathrm{H}_{2} \mathrm{O}$ [ASTM E2455-06]). Viability will be assessed $\sim 1$ minute after addition of sodium chloride and must exhibit $>80 \%$ viability for use in exposures.

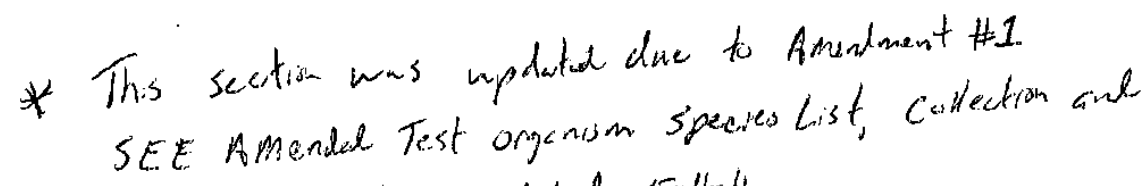

$$
\begin{aligned}
& \text { Inclusion critere dated 5-11-11 } \\
& \text { Man } 11.201 \\
& \text { Jal } \\
& \text { 1) }
\end{aligned}
$$


Study Title: "Effects of Pseudomonas fluorescens (Pf-CL145A) to glochidia from seven unionid mussel species"

\begin{tabular}{cc} 
Study number: AEH-11-PSEUDO-01 \\
Amended Test Organism Species List, Collection and Inclu \\
Criteria \\
\hline Common name & Scientific name \\
\hline Black sandshell $50 / /-20 / /$ \\
Fatmucket & Ligumia recta \\
Hickorynut & Lampsilis siliquoidea \\
Higgins eye & Obovaria olivaria \\
Mucket & Lampsilis higginsii \\
Plain pocketbook & Actinonaias ligamentina \\
Washboard & Lampsilis cardium \\
& Megalonaias nervosa \\
\hline
\end{tabular}

\section{Collection and inclusion criteria (see protocol sections 4.1- 4.2):}

Gravid donor mussels will be collected by biologists from the Genoa National Fish Hatchery from the Upper lowa, Upper Mississippi or St. Croix Rivers. Mussels will be identified to species as described in Cummings and Mayer (1992) and Watters et al. (2009) and transported to the Genoa National Fish Hatchery. Approximately $72 \mathrm{~h}$ prior to test initiation the donor mussels will be transferred from the Genoa NFH to the UMESC and acclimated to test temperature. Glochidia from at least three gravid female mussels of each species will be extracted by personnel from the Genoa NFH. The viability of glochidia from each mussel will be assessed by drawing aliquots (e.g. $100 \mu \mathrm{L}$ ) of settled glochidia from the stock container with an adjustable manual pipet fitted with a wide bore tip and placing it on a pre-labeled $35 \mathrm{~mm}$ petri dish. The sample will be diluted with a known and consistent volume (e.g. $200 \mu \mathrm{L}$ ) of acclimated well water to enhance dispersion of glochidia for more accurate enumeration. The sample will be viewed under a dissecting microscope, adjusted for maximum clarity and magnification, and a photomicrograph will be recorded. Approximately one drop of a saturated sodium chloride solution (12 $\mathrm{g} \mathrm{NaCl} / 50 \mathrm{~mL}$ of D.I. $\mathrm{H}_{2} \mathrm{O}$ [ASTM E2455-06]) will be added to the sample and after one minute a second photomicrograph will be recorded. Enumerations will be conducted for the total number of glochidia within the sample and the number of glochidia closed or open before and after sodium chloride addition. The percentage of viable glochidia (glochidia that respond to sodium chloride exposure by valve closure) will be calculated by the following method:

Percent viable $=([T$ tatal number of glochidia in sample $-\{$ total number of glochidia closed before addition of sodium chloride + number of glochidia open after sodium chloride addition\}]/Total number of glochidia in sample) * 100

If viability is acceptable ( $\geq 80 \%$ ) for a mussel, the glochidia from that mussel will be pooled with glochidia from other mussels of that species. An appropriate amount of settled glochidia (e.g.: $500 \mu \mathrm{L}$ ) will be randomly drawn from the pooled glochidia stock and aliquoted to an exposure chamber ( $80 \mathrm{~mm} \times 40 \mathrm{~mm}$ crystallizing dish) containing exposure water to achieve

Page 1 of $\mathbf{2}$

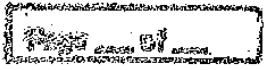




\section{Study Title: "Effects of Pseudomonas fluorescens (Pf-CL145A) to glochidia from seven unionid mussel species"}

\section{Study number: AEH-11-PSEUDO-01}

a final volume of $100 \mathrm{~mL}$. Between 500-1,000 glochidia will be transferred to each exposure chamber in a minimum of two aliquots (dependent on number of glochidia per aliquot and the estimated number of glochidia available) per chamber (e.g. two $100 \mu \mathrm{L}$ aliquots that contain $\sim 250$ glochidia each). Once all of the exposure chambers receive test animals the exposures will be initiated by the addition of the appropriate amount of MBI-401 SDP, deactivated MBI401 SDP (positive control) or plain water (control) in a completely randomized design to achieve the desired test concentrations of 0 (control), $300 \mathrm{mg} / \mathrm{L}$ deactivated (positive control), or $50,100,200$, and $300 \mathrm{mg} / \mathrm{L}$ of active MBI-401 SDP. The exposure chambers will be gently mixed with a glass rod or stainless steel spatula to ensure uniform distribution of the test chemical. All concentrations will be tested in triplicate for a total of 18 exposure chambers per species. Exposure chambers will be held at $20^{\circ} \mathrm{C}$ in a water bath or controlled environment room and maintained on an 18:6 h light/dark cycle. Viability of a subsample of approximately 100 glochidia from each chamber will be assessed at 6, 12 and $24 \mathrm{~h}$ post exposure by enumeration from photomicrograph records pre- and post-sodium chloride addition. The study will be terminated after $24 \mathrm{~h}$ post exposure evaluations are completed.

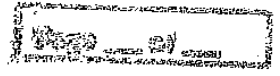

Page 2 of 2 
Study Number AEH-11.-PSEUDO-01

Book-1

File Folder: 79

Lab book/pgs: 4.8

Proofed: To $\angle 2 F E B 12$ Reviewed:

Daily Care Worksheet

Item No.

/

Tank\#: IA

Species: L. Cardium

Room \#:

11

Month/Year: 05/2011

Lot Number: 111100

\begin{tabular}{|c|c|c|c|c|c|c|c|}
\hline \multirow[b]{2}{*}{ Day } & \multirow{2}{*}{$\begin{array}{c}\text { Feed } \\
\text { Time } \\
\text { (military) } \\
\end{array}$} & \multicolumn{2}{|c|}{$\begin{array}{l}\text { Flow Rate } \\
(\mathrm{mL} / \mathrm{min})\end{array}$} & \multirow[b]{2}{*}{$\begin{array}{c}\text { Temperature } \\
\left({ }^{\circ} \mathrm{C}\right) \\
\end{array}$} & \multirow[b]{2}{*}{$\begin{array}{c}\text { Dissolved } \\
\text { Oxygen (mg } / \mathrm{L})\end{array}$} & \multirow[b]{2}{*}{$\mathrm{pH}$} & \multirow[b]{2}{*}{ Initials } \\
\hline & & Infiow & Pump & & & & \\
\hline 1 & & \multicolumn{2}{|c|}{1} & & & & \\
\hline \multicolumn{8}{|l|}{2} \\
\hline \multicolumn{8}{|l|}{3} \\
\hline \multicolumn{8}{|l|}{$\frac{4}{5}$} \\
\hline \multicolumn{8}{|l|}{5} \\
\hline \multicolumn{8}{|l|}{6} \\
\hline \multicolumn{8}{|l|}{7} \\
\hline \multicolumn{8}{|l|}{8} \\
\hline 9 & 1330 & 1100 & 9.8 & 13.0 & 10.18 & 8.19 & KLW \\
\hline 10 & 1335 & 1100 & 9.7 & 19.5 & 7.97 & 8.07 & 125 \\
\hline 11 & 1240 & 1100 & 9.5 & 19.4 & 7.81 & 7.90 & D. \\
\hline 12 & 2810 & 1100 & 9.3 & 21.0 & 8.15 & 7.98 & Kew \\
\hline \multicolumn{8}{|l|}{13} \\
\hline \multicolumn{8}{|l|}{14} \\
\hline 15 & & & & & & & $=$ \\
\hline 16 & \multicolumn{4}{|c|}{ Note: Mussel hplding tanks $|A|$,$B ,$} & and 12 ar & \multicolumn{2}{|c|}{ e three sedtions } \\
\hline 17 & \multirow{2}{*}{\multicolumn{2}{|c|}{ of one }} & \multirow{2}{*}{\multicolumn{2}{|c|}{$\begin{array}{l}\operatorname{tank} \text { divided by } \\
\text { grameftes were }\end{array}$}} & \multicolumn{3}{|c|}{ mesh Scraens. These water } \\
\hline 18 & & & & & measured & \multicolumn{2}{|c|}{ only in sektion } \\
\hline 19 & \multicolumn{2}{|c|}{$1 C, y e t$} & \multicolumn{2}{|c|}{ recorded on sheet } & \multicolumn{3}{|c|}{ S for $I A$ and IB as well. } \\
\hline \multicolumn{8}{|c|}{ - The inm 1211} \\
\hline \multicolumn{8}{|l|}{21} \\
\hline \multicolumn{8}{|l|}{22} \\
\hline 23 & & & & & & & \\
\hline 24 & & & & (i) & & Fisen & 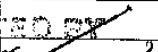 \\
\hline 25 & & & & & & 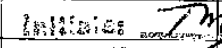 & Dew?: \\
\hline 26 & & & & & & do $m y$ & 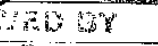 \\
\hline 27 & & & & & & 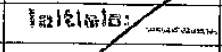 & Dent \\
\hline 28 & & & & & & 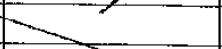 & \\
\hline 29 & & & & & & & \\
\hline 30 & & & & & & & \\
\hline 31 & & & & & & & \\
\hline
\end{tabular}

Daily Food Prep: Mix 44.0g Shellfish diet, 35.3g Tetraselmis, and $92.4 \mathrm{~g}$ Thalassiosira weissflogii with $14,400 \mathrm{~mL}$ well water each day, to be delivered by peristaltic pump at $10 \mathrm{~mL}$ per minute. Target water inflow rate is $1,100 \mathrm{~mL} / \mathrm{min}$. Prepare fresh diet

daily.

Approved by:
Page 1 of 1

(1) No data recorded.
Th 12 M 11 
Study Number AEH-11-PSEUDO-01

Buok-HI

File Folder: $-7 q$

Lab book/pgs: if 8

Proofed: DSS OLFEB/2 Reviewed:

\section{Daily Care Worksheet}

Item No.

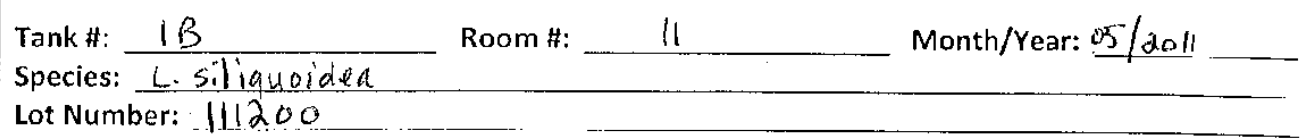

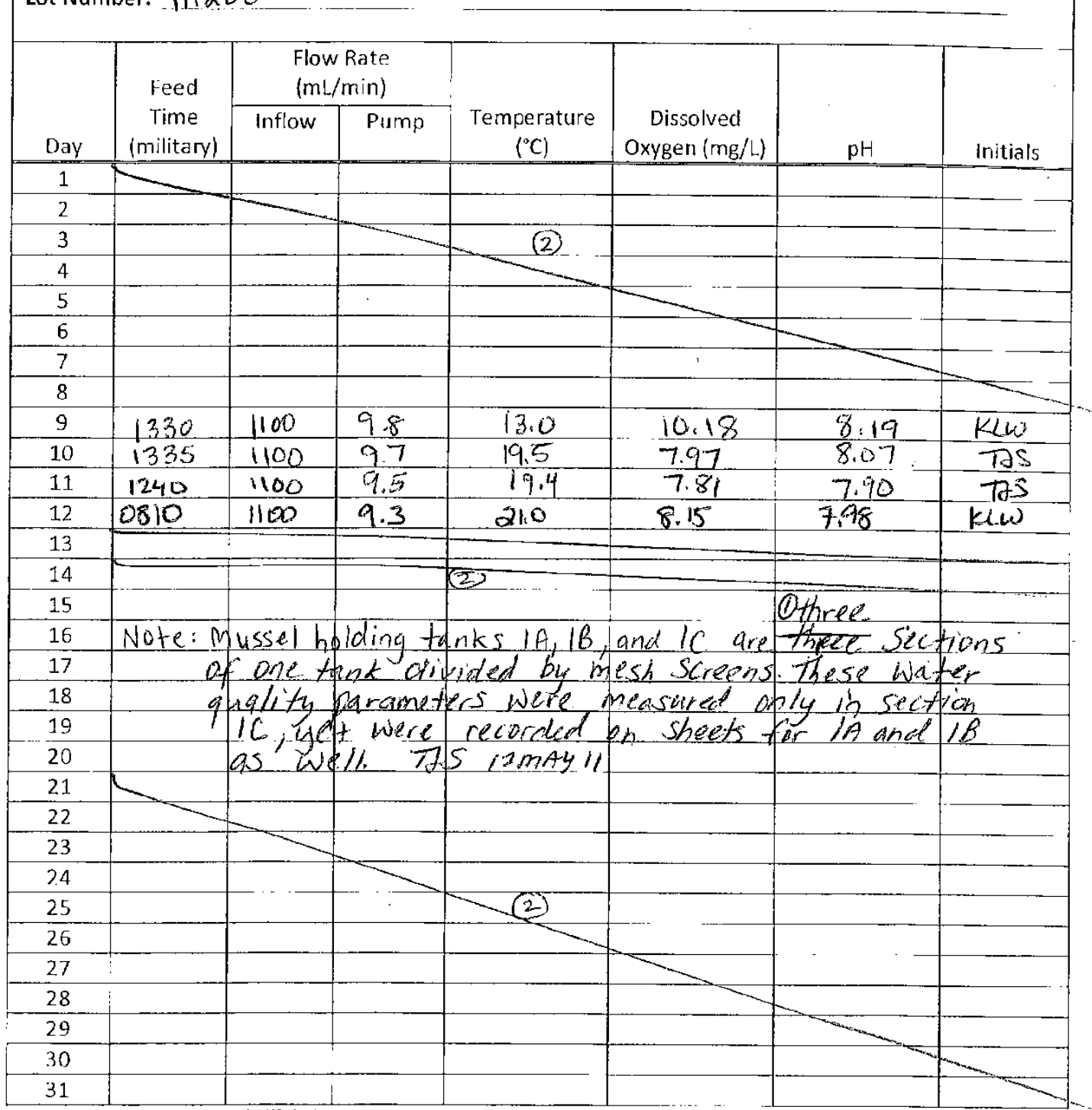

Daily Food Prep: Mix $44.0 \mathrm{~g}$ Sheilfish diet, $35.3 \mathrm{~g}$ Tetraselmis, and $92.4 \mathrm{~g}$ Thatassiosira weissflogï with $14,400 \mathrm{~mL}$. well water each day, to be delivered by peristaltic pump at $10 \mathrm{~mL}$ per minute. Target water inflow rate is $1,100 \mathrm{~mL} / \mathrm{min}$. Prepare fresh diet
daily.
pige $|\mathrm{g}|$
Approved by
Date: 5-9-11
(1) Spelling error.
TJS IIMAYII
(2) No data recorded.
t5. 12 MAY"1 
Study Number AEH-11-PSEUDO-01

Book. \#1

File Folder: $7 a$ Lab book/pgs: $4-8$ Proofed: DS O2FEB 12 Reviewed

Daily Care Worksheet

Item No. 3 :

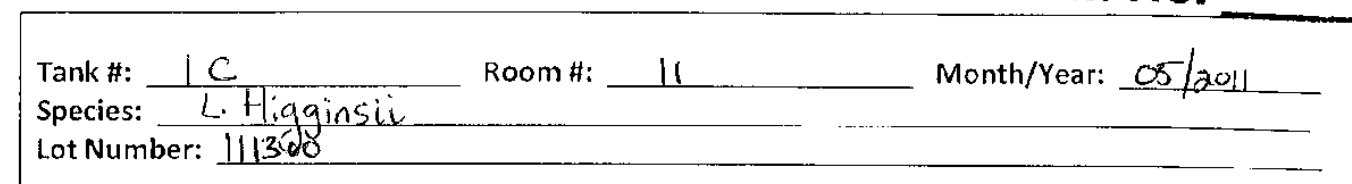

\begin{tabular}{|c|c|c|c|c|c|c|c|}
\hline \multirow[b]{2}{*}{ Day } & \multirow{2}{*}{$\begin{array}{c}\text { Feed } \\
\text { Time } \\
\text { (military) }\end{array}$} & \multicolumn{2}{|c|}{$\begin{array}{l}\text { Flow Rate } \\
(\mathrm{mL} / \mathrm{min})\end{array}$} & \multirow[b]{2}{*}{$\begin{array}{c}\text { Temperature } \\
\left({ }^{\circ} \mathrm{C}\right)\end{array}$} & \multirow[b]{2}{*}{$\begin{array}{c}\text { Dissolved } \\
\text { Oxygen }(\mathrm{mg} / \mathrm{L}) \\
\end{array}$} & \multirow[b]{2}{*}{$\mathrm{pH}$} & \multirow[b]{2}{*}{ Initials } \\
\hline & & Inflow & Pump & & & & \\
\hline 1 & 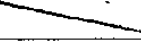 & & & & & & \\
\hline 2 & & & & & & & \\
\hline
\end{tabular}

\begin{tabular}{|c|c|c|c|c|c|c|c|}
\hline & & & & $=$ (1) & & & \\
\hline 4 & & & & & & & \\
\hline 5 & & & & & & & \\
\hline 6 & & & & & & 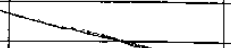 & \\
\hline 7 & & & & & & & \\
\hline 8 & & & & & & & \\
\hline 9 & 1330 & 1100 & 9.8 & 13.0 & 10.18 & 8.19 & KLW \\
\hline 10 & 1335 & 1100 & 9.7 & 19.5 & 7.97 & 8.07 & BSS \\
\hline 11 & 1240 & 1100 & 9.5 & 19.4 & 7.81 & 7.90 & 725 \\
\hline 12 & $0 \$ 10$ & 1100 & 9.3 & 21.0 & 8.15 & 7.98 & $k(w)$ \\
\hline 13 & & & & & & & \\
\hline 14 & & & & (1) & & & \\
\hline 15 & & & & & & 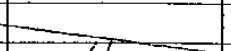 & \\
\hline 16 & Note: $n$ & Dussel & bolding & tanks 19,18 & $B$ and $/ C$ & re three 8 & Ctions \\
\hline 17 & 0 & fone $\rightarrow$ & $\ln k d$ & ivided by & mesh scree & hs. These in & Water \\
\hline 18 & & kality & paramed & ers idene A & beasured 0 & phy in sec & tion \\
\hline 19 & & 1c.yes & Were & recorded \& & Pn sheets & (a) $A$ and & $\beta$ \\
\hline 20 & & as we & 11.725 & $12 \mathrm{may} 11$ & & & \\
\hline 21 & & & & & & & \\
\hline 22 & & & & & & & \\
\hline 23 & & & & & & & \\
\hline 24. & & & & & & & \\
\hline 25 & & & & (i) & & & \\
\hline 26 & & & & & & & \\
\hline 27 & & & & & & & \\
\hline 28 & & & & & & & \\
\hline 29 & . & & & & & & \\
\hline 30 & & & & & & & \\
\hline 31 & & & & & & & \\
\hline
\end{tabular}

Oaily Food Prep: Mix 44.0g Shellfish diet, 35.3g Tetraseimis, and 92.4g Tholossiosira weissfiogi with $14.400 \mathrm{~mL}$ well water each day, to be delivered by peristaltic pump at $10 \mathrm{~mL}$ per minute. Target water inflow rate is $1,100 \mathrm{~mL} / \mathrm{min}$. Prepare fresh dict
daily.
pinge $\log ^{\prime}$
(1) No deata recordea?
12 MAY 11795
Approved by:
Date: 5-9-11 
Study Number AEH-11-PSEUDO-01

File Folder: $7 a$ Lab book/pgs: $1 / 31,33,34$ Reviewed: IAS O2FEB 12 Verified:

Daily Care Worksheet

Donor Mussel Holding

Item No. 5

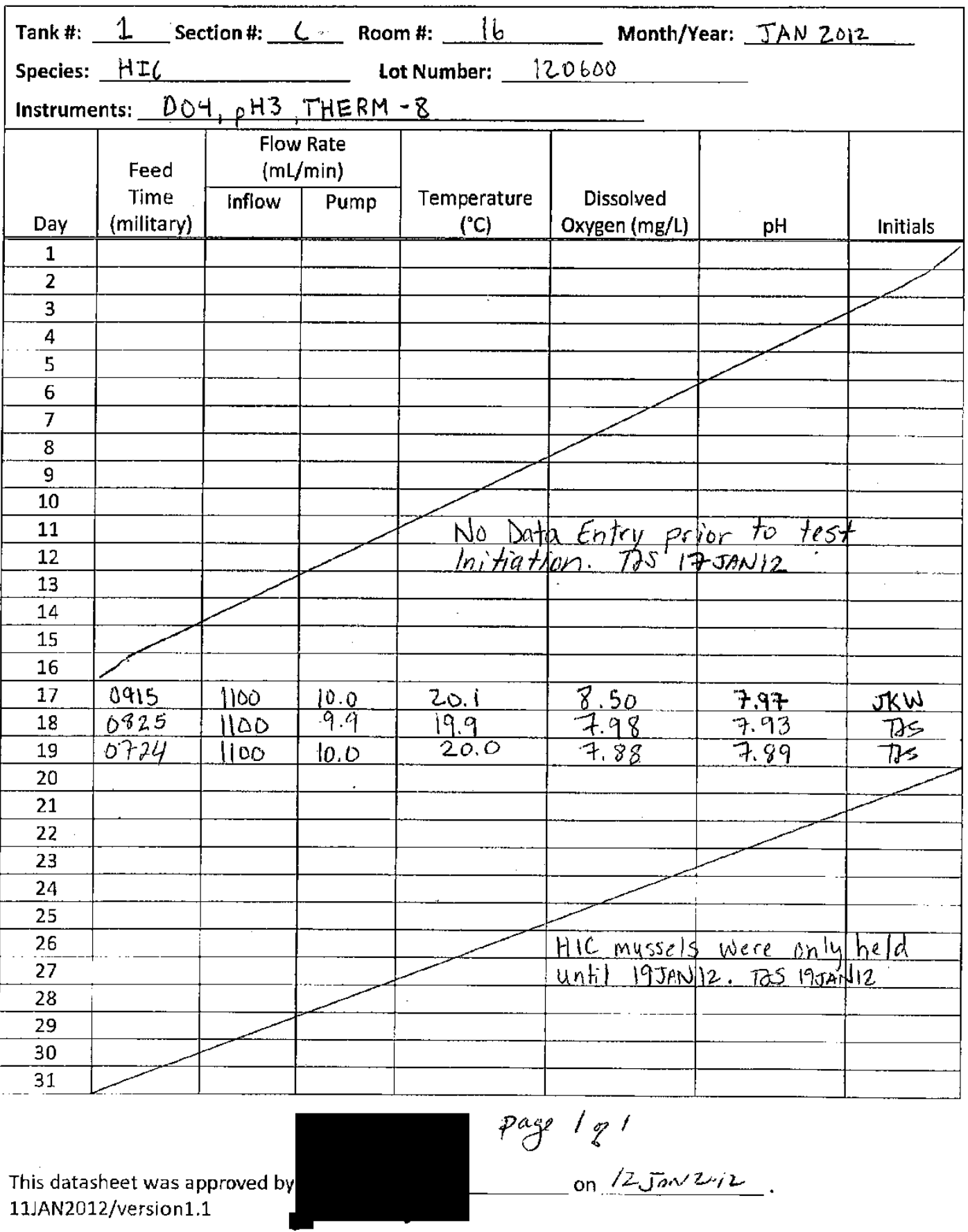


Study Number AEH-11-PSEUDO-01

File Folder: 7a Lab book/pgs: $1 / 31$ Reviewed: T2S O2FEB/2 Verified:

Daily Care Worksheet

Donor Mussel Holding Item MO. 6

\begin{tabular}{|c|c|c|c|c|}
\hline Tank \#: & 1. Section \#: A & Room \#: 16 & Month/Year: & 2012 \\
\hline Species: & BLS & Lot Number: & 120400 & \\
\hline
\end{tabular}

Instruments: $\mathrm{DO} 4, \mathrm{pH}$, THERM

\begin{tabular}{|c|c|c|c|c|c|c|c|}
\hline \multirow[b]{2}{*}{ Day } & \multirow{2}{*}{$\begin{array}{c}\text { Feed } \\
\text { Time } \\
\text { (military) }\end{array}$} & \multicolumn{2}{|c|}{$\begin{array}{l}\text { Flow Rate } \\
\text { (mL/min) }\end{array}$} & \multirow[b]{2}{*}{$\begin{array}{c}\text { Temperature } \\
\left({ }^{\circ} \mathrm{C}\right)\end{array}$} & \multirow[b]{2}{*}{$\begin{array}{c}\text { Dissolved } \\
\text { Oxygen (mg/L) }\end{array}$} & \multirow[b]{2}{*}{$\mathrm{pH}$} & \multirow[b]{2}{*}{ Initials } \\
\hline & & Inflow & Pump & & & & \\
\hline
\end{tabular}

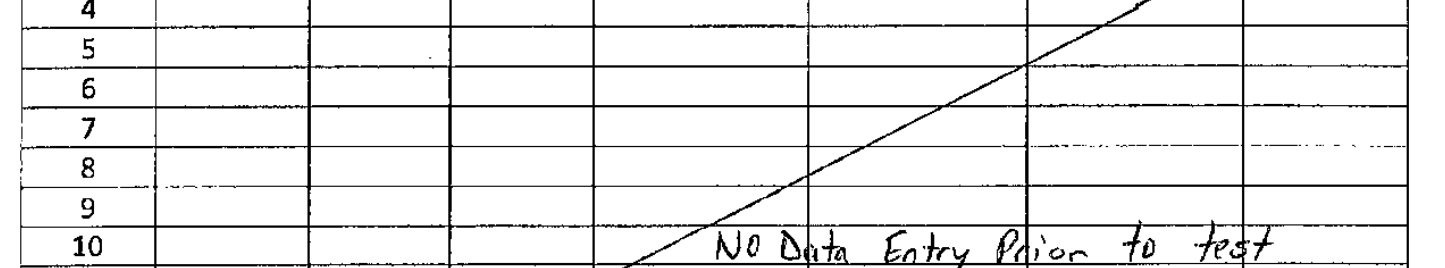

\begin{tabular}{|c|c|c|c|c|c|c|c|}
\hline 10 & & & & \multicolumn{4}{|c|}{ No Odita Entry prion to } \\
\hline 11 & & & & \multicolumn{3}{|c|}{ lnitidion. TOS/TJAN/2 } & \\
\hline \multicolumn{8}{|c|}{ 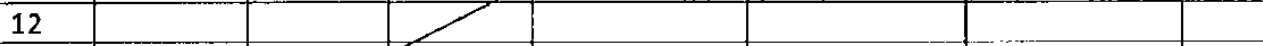 } \\
\hline \multicolumn{8}{|l|}{13} \\
\hline \multicolumn{8}{|l|}{14} \\
\hline \multicolumn{8}{|l|}{15} \\
\hline \multicolumn{8}{|l|}{16} \\
\hline 17 & 0915 & 1100 & 10,0 & 20.1 & 8.50 & 7.97 & JKW \\
\hline \multicolumn{8}{|l|}{18} \\
\hline 19 & & & & & & & \\
\hline
\end{tabular}

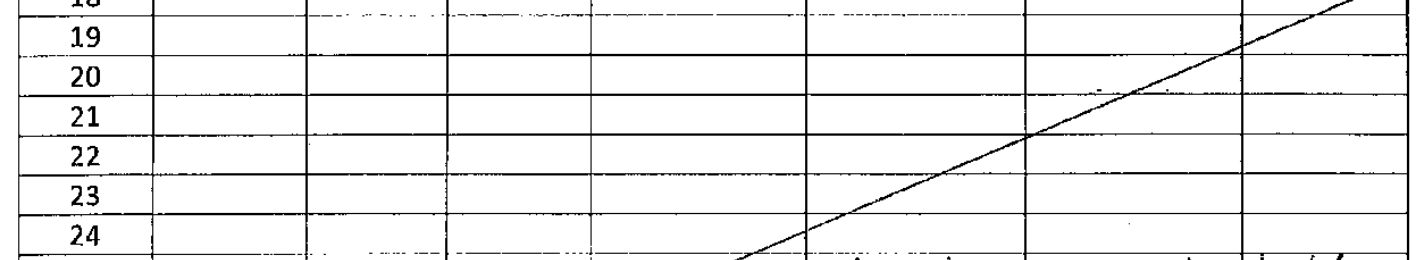

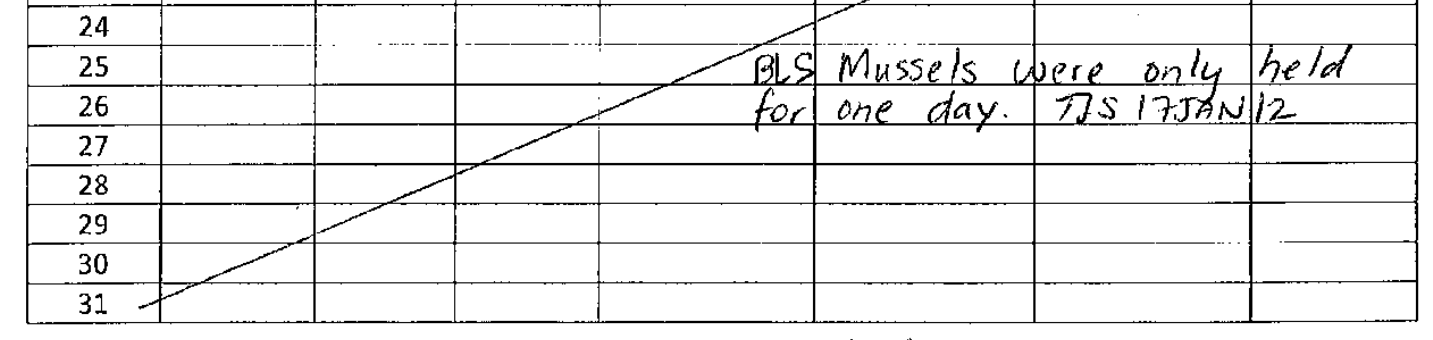

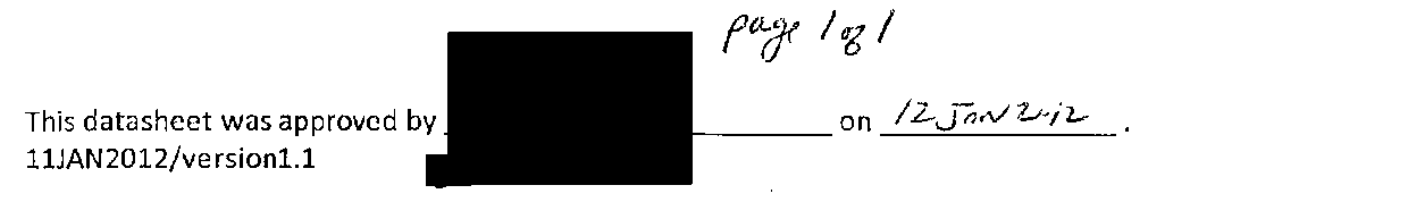


Study Number AEH-11-PSEUDO-01

File Folder: $-7 a$

Lab book/pgs: $1 / 31$

Reviewed: $D_{5}$ OZFEB 12 Verified:

\section{Daily Care Worksheet}

Donor Mussel Holding

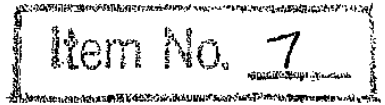

\begin{tabular}{|c|c|c|c|c|c|c|c|}
\hline \multirow[b]{2}{*}{ Day } & \multirow{2}{*}{$\begin{array}{c}\text { Feed } \\
\text { Time } \\
\text { (military) } \\
\end{array}$} & \multicolumn{2}{|c|}{$\begin{array}{l}\text { Flow Rate } \\
\text { (mL/min) }\end{array}$} & \multirow[b]{2}{*}{$\begin{array}{c}\text { Temperature } \\
\left({ }^{\circ} \mathrm{C}\right) \\
\end{array}$} & \multirow[b]{2}{*}{$\begin{array}{c}\text { Dissolved } \\
\text { Oxygen (mg/L) } \\
\end{array}$} & \multirow[b]{2}{*}{$\mathrm{pH}$} & \multirow[b]{2}{*}{ Initlals } \\
\hline & & Inflow & Pump & & & & \\
\hline \multicolumn{8}{|l|}{1} \\
\hline \multicolumn{8}{|l|}{2} \\
\hline \multicolumn{8}{|l|}{3} \\
\hline \multicolumn{8}{|l|}{4.} \\
\hline \multicolumn{8}{|l|}{5} \\
\hline \multicolumn{8}{|l|}{6} \\
\hline \multicolumn{8}{|l|}{7} \\
\hline \multicolumn{8}{|c|}{ 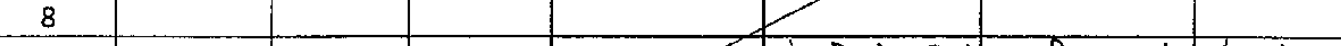 } \\
\hline 9 & & & & & No Data Ent & y Prior to & test \\
\hline 10 & & & & & Initiation. & TDS 17 JANIL & \\
\hline \multicolumn{8}{|c|}{ 管 } \\
\hline \multicolumn{8}{|l|}{12} \\
\hline \multicolumn{8}{|l|}{13} \\
\hline \multicolumn{8}{|l|}{14} \\
\hline \multicolumn{8}{|l|}{15} \\
\hline \multicolumn{8}{|l|}{16} \\
\hline 17 & 0715 & 1100 & 10.0 & 20.1 & 8.50 & 7.97 & JKW \\
\hline \multicolumn{8}{|l|}{18} \\
\hline \multicolumn{8}{|l|}{19} \\
\hline \multirow{2}{*}{\multicolumn{8}{|c|}{$\begin{array}{l}20 \\
21\end{array}$}} \\
\hline & & & & & & & \\
\hline \multicolumn{8}{|l|}{22} \\
\hline \multicolumn{8}{|l|}{23} \\
\hline \multicolumn{8}{|l|}{24} \\
\hline 25 & & & & & & Is were on & ly held \\
\hline 26 & & & & & \multicolumn{3}{|c|}{ for one day. TS 1704 N 12} \\
\hline \multicolumn{8}{|c|}{ 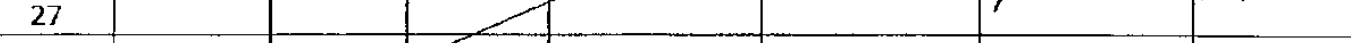 } \\
\hline 28 & & & & & & & \\
\hline 29 & & & & & & & \\
\hline 30 & & & & & & & \\
\hline 31 & & & & & & & \\
\hline
\end{tabular}

$$
\text { page } 181
$$

This datasheet was approved by on $12 \operatorname{san} 2, i 2$ 
Adult Mussel Holding Daily Algal Diet $1 / 13 / 2012$

\section{Item No.}

Tank Dimensions and volume

$48^{\prime \prime} \times 14^{\prime \prime} \times 6^{\prime \prime}(121.92 \mathrm{~cm} \times 35.56 \mathrm{~cm} \times 15.24 \mathrm{~cm})=66,073 \mathrm{~cm}^{3}$

$6^{\prime \prime}(15.24 \mathrm{~cm})$ standpipe: $66.073 \mathrm{~L}$

Flow rate (1 turnover per hour): $1.1 \mathrm{~L} / \mathrm{m}$ )

Daily water volume: $1.1 \mathrm{~L} / \mathrm{m} \times 60 \mathrm{~m} / \mathrm{h} \times 24 \mathrm{~h} / \mathrm{d}=1584 \mathrm{~L} / \mathrm{d}$

Diet weights (50\% Tetraselmis and 50\% Nannochloropsis)

to achieve $10 \mathrm{mg} / \mathrm{L}$ by dry weight:

Tetraselmis: Target $5.0 \mathrm{mg} / \mathrm{L}$ (17.93\% Dry Wt); Nannochloropsis: Target $5.0 \mathrm{mg} / \mathrm{L}(20.48 \%$ Dry Wt)

Tetraselmis: $27.89 \mathrm{mg} / \mathrm{L}$; Nannochloropsis: $24.41 \mathrm{mg} / \mathrm{L}$

$\times 1584 \mathrm{~L} / \mathrm{d}$

Tetraselmis: $44.2 \mathrm{~g} / \mathrm{d}$; Nannochloropsis: $38.7 \mathrm{~g} / \mathrm{d}$

Diet Inflow Rate: $\quad 10 \mathrm{~mL} / \mathrm{min} \times 60 \mathrm{~m} / \mathrm{h} \times 24 \mathrm{~h} / \mathrm{d}=14400 \mathrm{~mL} / \mathrm{d}$

Daily Care:

Mix 44.2g Tetraselmis and $38.7 \mathrm{~g}$ Nannochloropsis with $14,400 \mathrm{~mL}$ well water each day, to be delivered by peristaltic pump at $10 \mathrm{~mL}$ per minute. Prepare fresh diet daily.

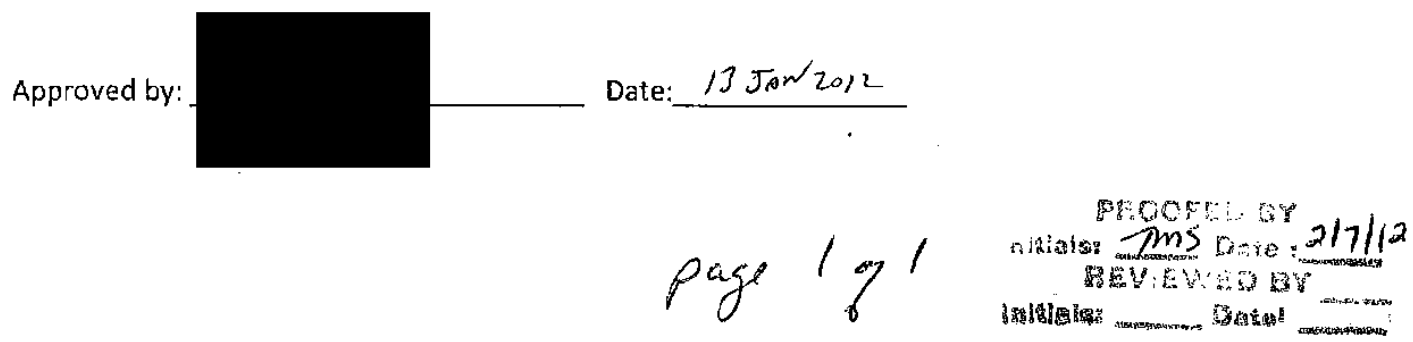


Study Title: "Effects of Pseudomonas fluorescens (Pf-CL145A) to glochidia from seven unionid mussel species"

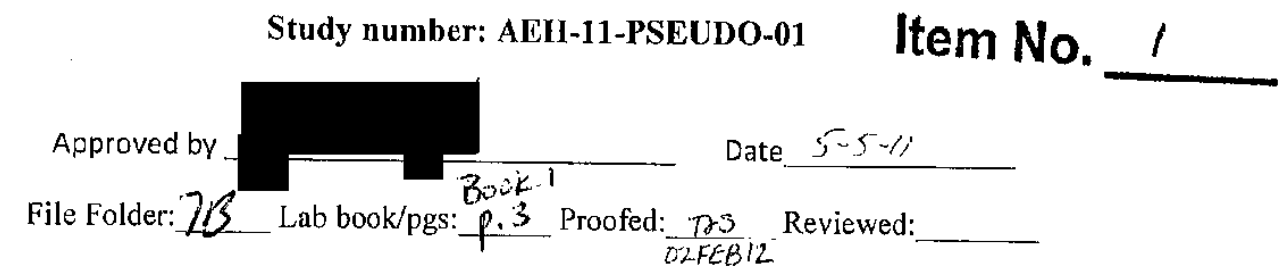

\section{Donor Mussel UMESC Lot Number Assignment Form} DONOR MUSSEL INHORMATION:

species: Plain poeketbook (Lampsilis canlum)

Number of Donor Mussels: Collection Date: $4 / 2 g / 11$

Collection Location: st. Craix River o Hadsun Narrows

Species Identification performed by: Nathan Eackent

Title/affiliation of identifier: Minsse' Bsoloy,st / Genda NFA

UMESC Arrival Date: $5-9-11 \quad 1130 \mathrm{~h}$

Receiving UMESC tank/room \#_IA Roon /I

Additional information:

Racievel e $53^{\circ} \mathrm{F}\left(11.7^{\circ} \mathrm{C}\right)$ Ramp stated from $12^{\circ} \mathrm{C}$ to $20^{\circ} \mathrm{C}$
e 1230 set to man for $60 \mathrm{~h}$

UMESC LOT NUMBER DESIGNATION: $\quad 1 / / 100$

Witness and form recorded by:

James 4. Luome.
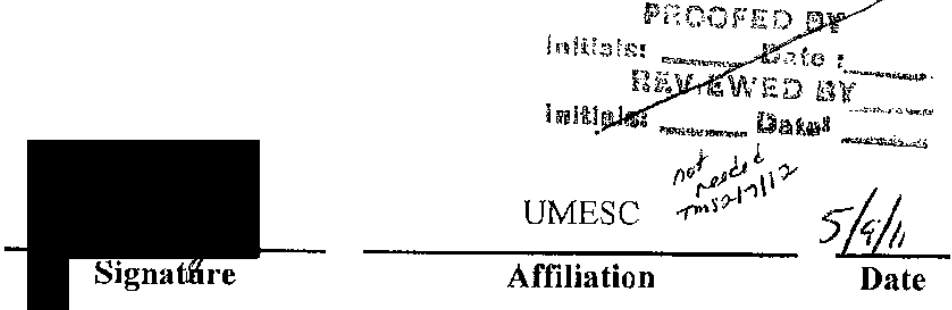

Printed Name

$$
\text { page } 1 \text { of } 1
$$


Study Title: "Effects of Pseudomonas fluorescens (Pf-CL145A) to glochidia from seven unionid mussel species"

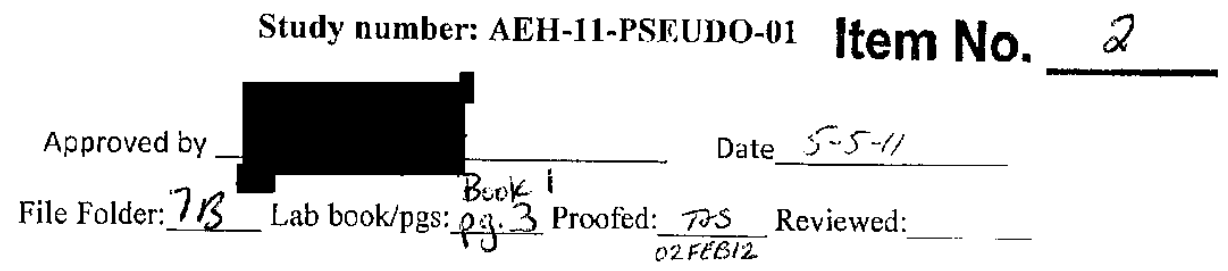

\section{Donor Mussel UMESC Lot Number Assignment Form}

\section{DONOR MUSSEL INFORMATION:}

Species: Fat thatlat (L. Siliquodec)

Number of Donor Mussels: 4 Collection Date: $4 / 2.9 / 4$

Collection Location: st. Croix River e Hadson Warrangs

Species Identification performed by: Nathen Eckent

Title/affiliation of identifier: Mussel Biologiof / GeNOW WPH

UMESC Arrival Date: $.5-9 \cdot 11 \quad 1130 \mathrm{~h}$

Receiving UMESC tank/room \#/B R.00 m II

Additional information:

Recerved e $5 g^{\circ} \times\left(11.7^{\circ} \mathrm{C}\right)$ Romp statiel frim $12^{\circ} \mathrm{C}$ to $20^{\circ} \mathrm{CO} / 230 \mathrm{~h}$ sit to run for $60 \mathrm{~h}$

UMESC LOT NUMBER DESIGNATION: $1 / 12.00$

Witness and form recorded by:

James A. Linume

Printed Name

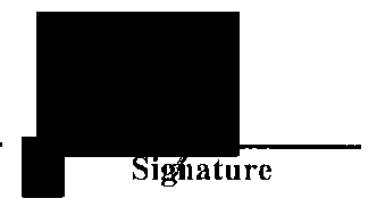

$$
\text { pagiof } 1
$$

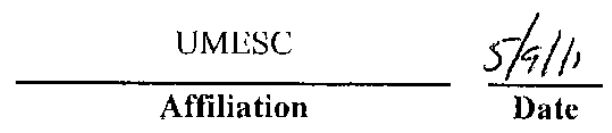

Affiliation Date 
Study Title: "Effects of Pseudomonas fluorescens (Pf-CL145A) to glochidia from seven unionid mussel species"

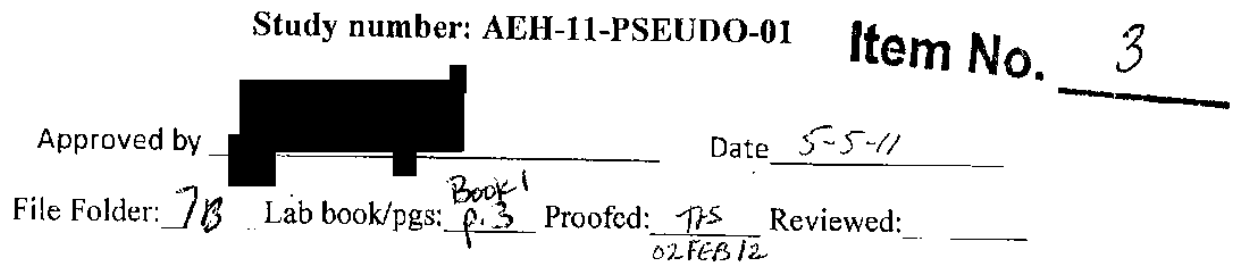

\section{Donor Mussel UMESC Lot Number Assignment Form}

DONOR MUSSEL INFORMATION:

Species:_Higgins Eque (L. higginsti)

Number of Donor Mussels: 4 Collection Date: $4 / 24 / 4$

Collection Location:st. Croix River Q Hadsow Marrows

Species Identification performed by: Wathen Eck kr t

Title/affiliation of identifier: Mussel Broloy, / Gevora NFH

UMESC Arrival Date: $5-9-11$ e $1130 \mathrm{hr}$

Receiving UMESC tank/room \#_/C KNom II

Additional information:

Hocerved (e) $53^{\circ}$ ( $\left.11.7^{\circ} \mathrm{C}\right)$ hemp stated e $1230 \mathrm{~h}$ to remp from $12^{\circ} \mathrm{C}$ to zoc aner boh.

UMESC LOT NUMBER DESIGNATION: $1 / 1300$

Witness and form recorded by:

Jabus h. Litume
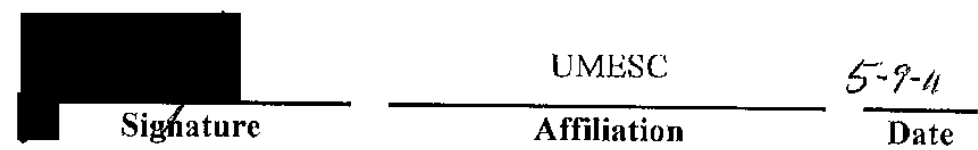

Printed Name

$$
\text { page } 191
$$


Study Title: "Effects of Pseudomonas fluorescens (Pf-CL145A) to glochidia from seven unionid mussel species"

\section{Study number: AEH-11-PSEUDO-01 Item No. 4}

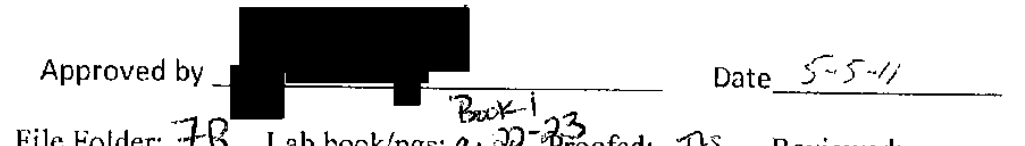

File Folder: $7 B$ Lab book/pgs: $9.22-33$ Proofed: $\frac{T S}{2 F E B 12}$ Reviewed:

\section{Donor Mussel UMESC Lot Number Assignment Form}

DONOR MUSSEL INFORMATION:

Species:_Lampgilis ciodinm (Plen peluthouk)

Number of Donor Mussels: 3 Collection Date: $10 / 8 / 11$

Collection Location: $\quad u m / p_{0} / 9$

Species Identification performed by: Genow NFA Nathon Eckert

Title/affiliation of identifier: Minsse. B Brologiot

UMESC Arrival Date: $10-18-1 /$

Receiving UMESC tank/room \# 15

Additional information:

UMESC LOT NUMBER DESIGNATION: 115200

Witness and form recorded by:

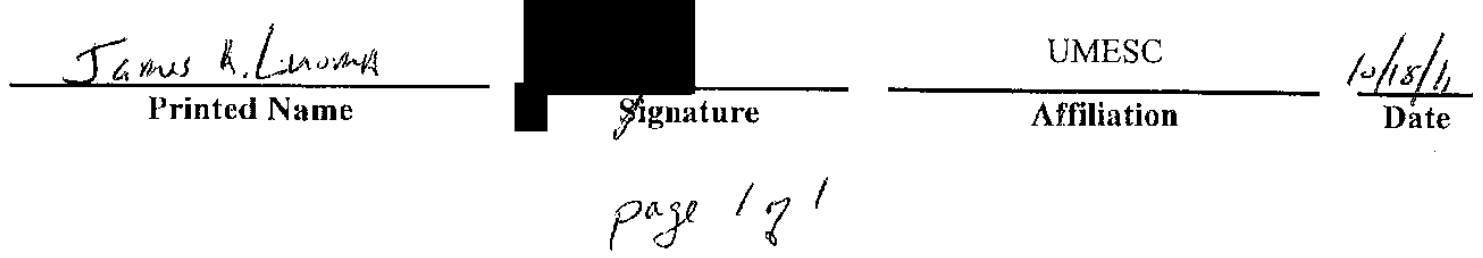


Study Title: "Effects of Pseudomonas fluorescens (Pf-CL,145A) to glochidia from seven unionid mussel species"

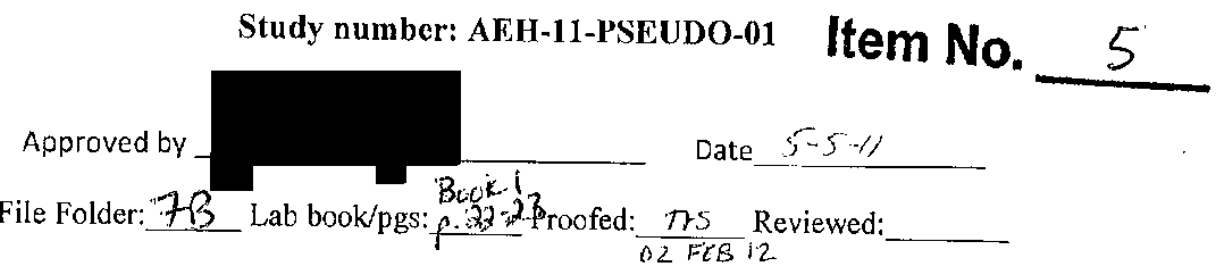

\section{Donor Mussel UMESC Lot Number Assignment Form}

DONOR MUSSEL INFORMA'TION:

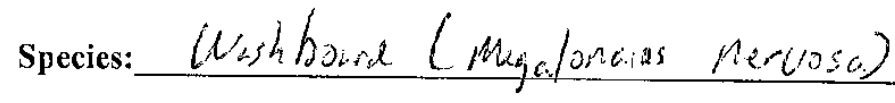

Number of Donor Mussels: $4 \quad$ Collection Date: $|0-17-1|$

Collection Location: LMR Pool 9

Species Identification performed by: Wathen Eskint

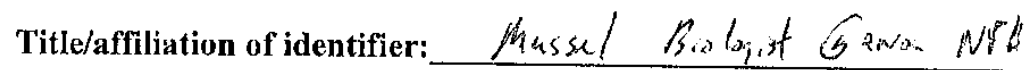

UMESC Arrival Date: $\quad|3-18-1|$

Receiving UMESC tank/room \#_R P

Additional information:

UMESC LOT NUMBER DESIGNATION: 115300

Witness and form recorded by:

$\frac{\text { James } L_{\text {mums }}}{\text { Printed Name }}-\frac{\text { UMESC }}{\text { Signature }}-\frac{10 / 18 / 11}{\text { Date }}$


Study Title: "Effects of Pseudomonas fluorescens (Pf-CL145A) to glochidia from seven unionid mussel species"

Study number: AEH-11-PSEUDO-01 Item No. 6

File Folder: $7 \mathrm{~h}$ Lab book/pgs: $1 / 31$ Reviewed: $\frac{\pi 5}{02 F E B / 2}$ Verified:

\section{Donor Mussel UMESC Lot Number Assignment Form}

\section{DONOR MUSSEL INFORMATION:}

species: Black Sandshell

Number of Donor Mussels: 4 Collection Date: 25 ort 2011

Collection Location:Chippewa River

Species Identification performed by: Nathan Eckert

Title/affiliation of identifier: MUssel Bologist $/ G N F+1$

UMESC Arrival Date: 17 JAN 2012

Receiving UMESC tank/room \# UMESC Rm 15

Additional information:

Arrived at 0915@18\%. Placed in 50\% UMEs water followed by $100 \%$ umesc water $@ 20 \% \mathrm{C}$.

UMESC LOT NUMBER DESIGNATION:

120400

Witness and form recorded by:

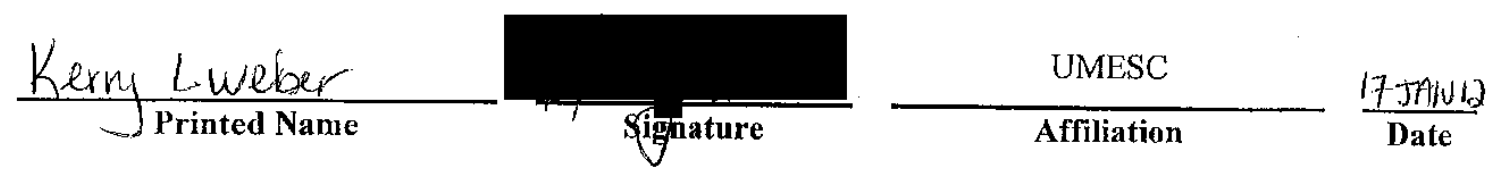

$$
\text { page } 1 \% 1
$$

This datasheet was approved by

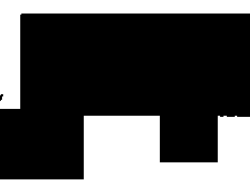


Study Title: "Effects of Pseudomonas fluorescens (Pf-CL145A) to glochidia from seven unionid mussel species"

Study number: AEH-11-PSEUDO-01 Item No.

File Folder: $7 b$ Lab book/pgs: $1 / 31$ Reviewed: $\frac{\text { tos }}{02 F E 612}$ Verified:

\section{Donor Mussel UMESC Lot Number Assignment Form}

DONOR MUSSEL INFORMATION:

Species: Mucket

Number of Donor Mussels: Collection Date: 20 SeP 2011

Collection Location:St. Croix River

Species Identification performed by: Nathan Eckert

Title/affiliation of identifier:Mvssel Biplogist /GWFH

UMESC Arrival Date: 17 JAN 2012

Receiving UMESC tank/room \# UMESC Rm 15

Additional information:

Arrived at 0915@18\%. Placed in 50\%unesc werter followed by $100 \%$ umesc water e $20 \% \mathrm{C}$.

UMESC LOT NUMBER DESIGNATION: $\quad \mid 20500$

Witness and form recorded by:

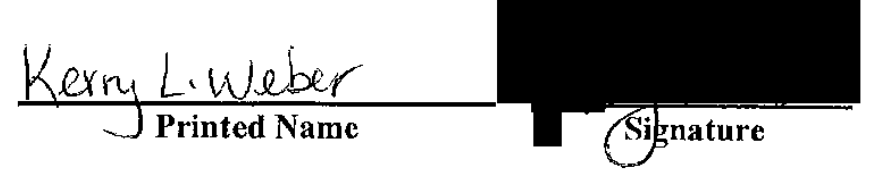

UMESC

Affiliation

$\frac{\text { FJAL12 }}{\text { Date }}$

$$
\text { page } 1 \text { o } 1
$$

This datasheet was approved by

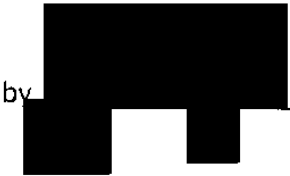

on 1257.212. 
Study Title: "Effects of Pseudomonas fluorescens (Pf-CL145A) to glochidia from seven unionid mussel species"

Study number: AEH-11-PSEUDO-01 Item No.

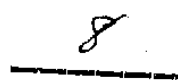

File Folder: th Lab book/pgs: $1 / 31$ Reviewed: $\frac{7 S}{O L E B / 2}$ Verified:

\section{Donor Mussel UMESC Lot Number Assignment Form}

DONOR MUSSEL INFORMATION:

Species: Hickorynut

Number of Donor Mussels: 4 Collection Date: 25 oct 2 al

Collection Location: Chippewa River

Species Identification performed by: Nathan Ecket

Title/affiliation of identifier:Mvssel Bologist/GMFH

UMESC Arrival Date: 17 TAN 201

Recciving UMESC tank/room \# UMES _ em 15

Additional information:

Arrived at 0915@ $18 \%$. Placed in $50 \%$ untesc water followed by
$100 \%$ uMesc water a $20 \%$.

UMESC LOT NUMBLR DESIGNATION: $\quad 120600$

Witness and form recorded by:
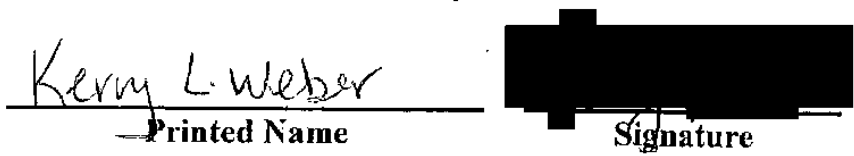

$\frac{\text { UMESC }}{\text { Affiliation }} \frac{17 J 74 N 12}{\text { Date }}$

$$
\text { page log } 1
$$

This datasheet was approved by

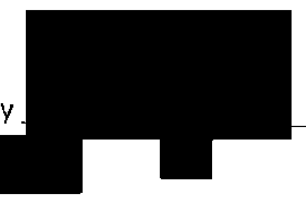


AEH-11-PSEUDO-01

USGS Upper Midwest Environmental Sciences Center

Page

of

Fish/Anima! History Form

CERTIFIED COPY

NA = not applicable

IN = inside $\quad$ OUT = outside

Initials: Jos Dates OTFEB $\overline{2}$ -

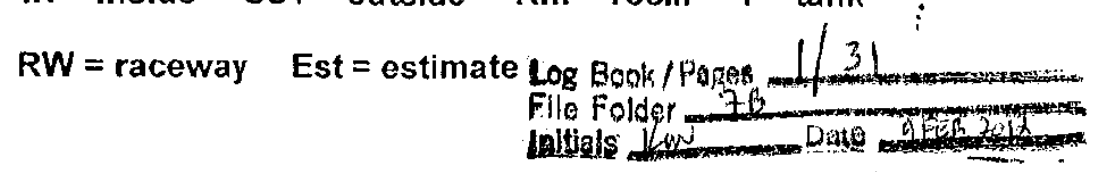

UMESC Lot Number:

120600

Source Facility Lot \#:

Origin/Source: Gen NFH

Species/Strain:__ Hickory Nut

Species Verified:

Number Received:

$\longleftarrow$

Date received/lot \# assigned:

$1 / 17 / 12$

Life stage at receipt/lot \#:

egg larval juvenile adult

Health certificate:

Yes (No)

If egg, disinfected:

Date spawn: Actual/Estimate

Date hatch: Actual/Estimate

First UMESC rearing unit: $\frac{R m 15}{\text { (egg. room, tank, raceway, pond and rearing unit number) }}$

comments: Collected) from Chifpiowa River $10125 / 11$ by Gena NFH

Date completed:

$-117 / 2$

Completed by:

FILE FOLDER $\rightarrow 7 b$

ITEM $\rightarrow 9$

PROOFED BY

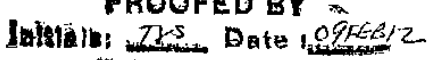

REVIEWED RY

Initialed Dato

301 


\section{AEH-11-PSEUDO-01 \\ USGS Upper Midwest Environmental Sciences Center Page $\angle$ of 2 Fish/Animal History Form

$$
\begin{aligned}
& \text { NA = not applicable } \quad I N=\text { inside } \quad \text { OUT }=\text { outside } \quad R m=r o o m \quad T=\operatorname{tank}
\end{aligned}
$$

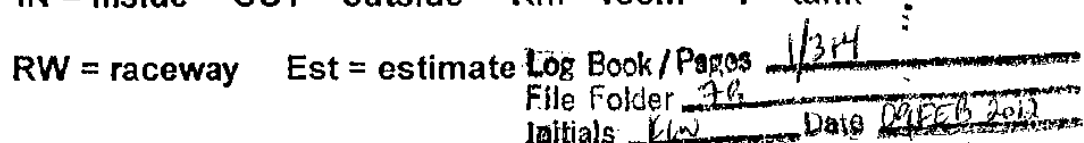

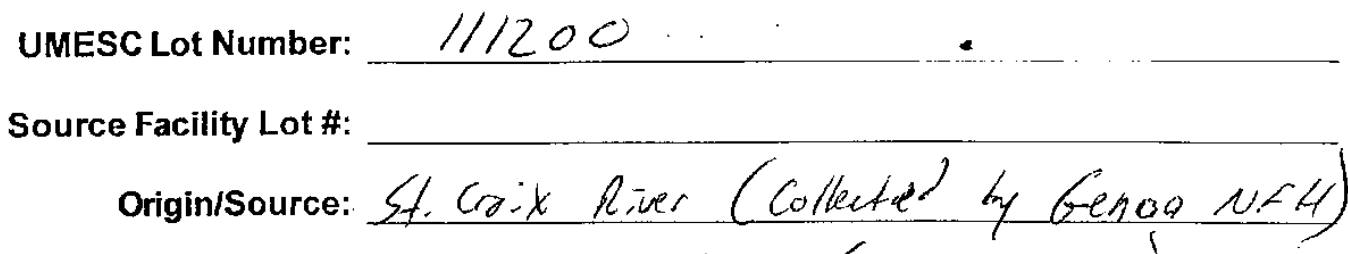

species/strain: Fat mockett (L. Siliquidie) baural

Species Verified:

$$
\text { 工 }
$$

Number Received:

$$
4
$$

$\begin{array}{rrr}\text { Date received/lot \# assigned: } & 5 / 9 / 1 / & \\ & \text { egg larval juvenile adult } \\ \text { Health certificate: } & \text { Yes } \mathrm{No}\end{array}$

If egg, disinfected:

Date spawn: Actual/Estimate

Date hatch: Actual/Estimate

First UMESC rearing unit: $\frac{\operatorname{Room} / / \quad \operatorname{taN} / \mathrm{B}}{\text { (e.g. room, tank, raceway, pond and rearing unit number) }}$

$$
\begin{aligned}
& \text { comments: Collewted 4/29/11 by fenera wett } \\
& \text { trassend to ones } 2 \quad 5 / 9 / 11
\end{aligned}
$$

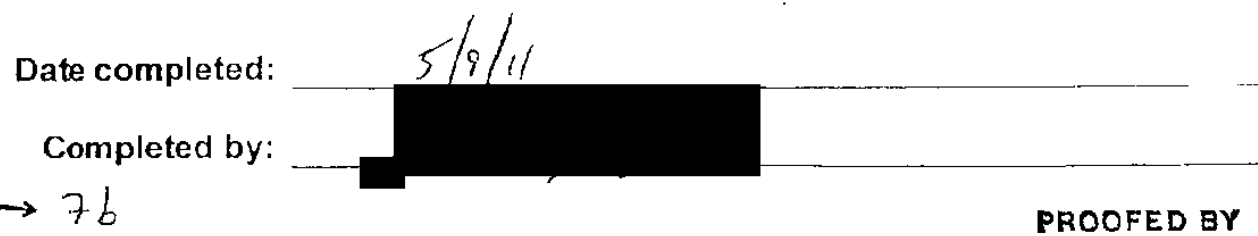

$I T E M \rightarrow 710$ wrote incorrect item number. TSSOTFEBIz Dater 


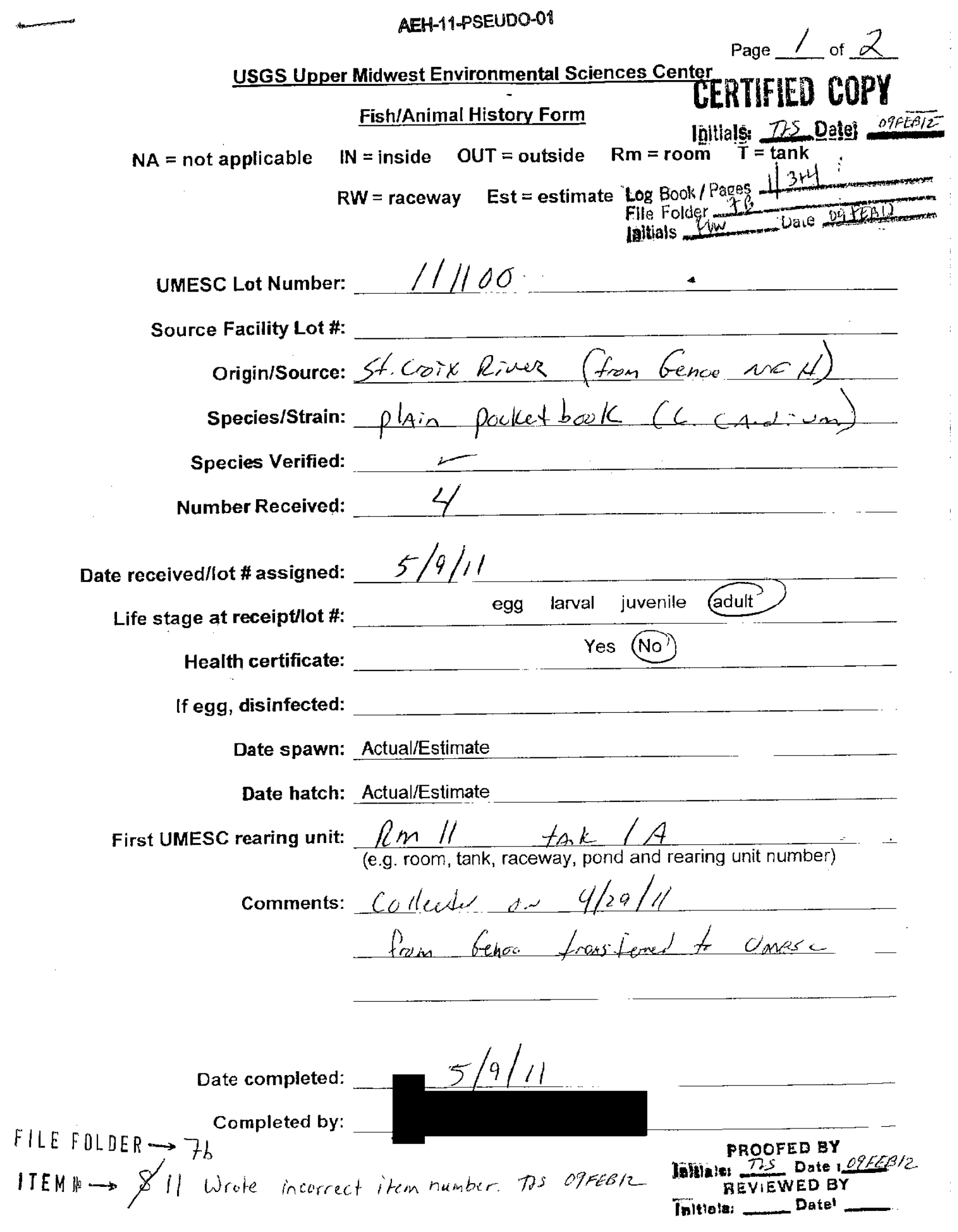




\section{AEH-11-PSEUDO-01 \\ USGS Upper Midwest Environmental Sciences Center Page 1 of 2 \\ Fish/Animal History Form}

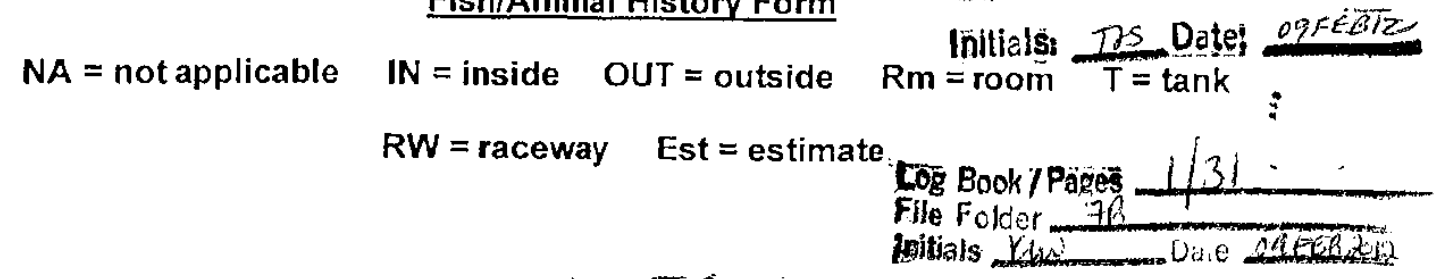

UMESC Lot Number: 120500

Source Facility Lot \#:

Origin/Source: Gen kno tt

Species/Strain: Muckett musjeL

Species Verified:

Number Received:

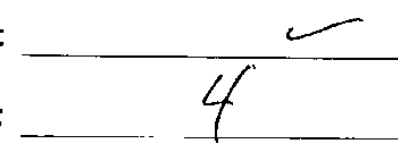

Date received/lot \# assigned:

Life stage at receipt/lot \#:

Health certificate:

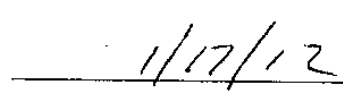

Yes (arval juvenile (adult)

If egg, disinfected:

Date spawn: Actual/Estimate

Date hatch: Actual/Estimate

First UMESC rearing unit: $\Omega_{m} 15$

(e.g. room, tank, raceway, pond and rearing unit number)

comments: collewer from ST. Cro.x Kiver

$$
9 / 20 / 11 \quad V_{y} \text { Gewein NFH }
$$

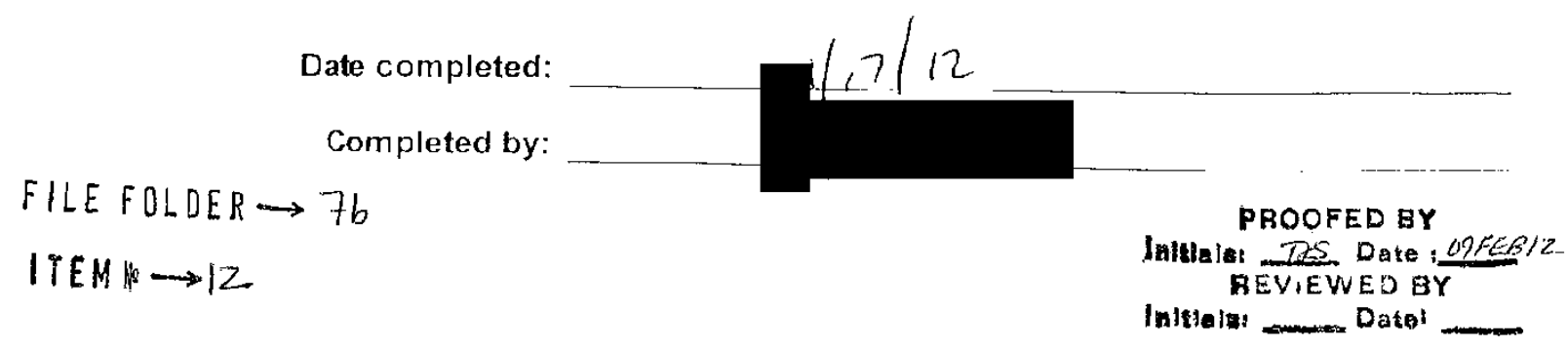


AEH-11-PSEUDO-01 Page 1 of 2

USGS Upper Midwest Environmental Sciences Center

Fish/Animal History Form

NA = not applicable

IN = inside

$\mathrm{Rm}=\underset{\text { room }}{\mathrm{rathials}} \mathrm{T}=\operatorname{tank}$

RW $=$ raceway $\quad$ Est $=$ estimate

Log Book/Pakges _.

UMESC Lot Number: $\quad 12040^{\circ}$

Fille Folder

I pitials Uin

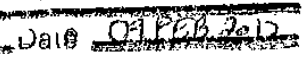

Source Facility Lot \#:

Origin/Source: GeN NFH

Species/Strain: Black Sandestell mussec

Species Verified:

Number Received:

Date received/lot \# assigned: $1 / 17 / 12$

Life stage at receipt/lot \#: _____ egg larval juvenile adult

Health certificate:

Yes (No)

If egg, disinfected:

Date spawn: Actual/Estimate

Date hatch: Actual/Estimate

First UMESC rearing unit: .... $2 \mathrm{~m} / 5$

(e.g. room, tank, raceway, pond and rearing unit number)

Comments:

Collecteron $10 / 25 / 11$

$\begin{aligned} & \text { Date completed: } 1 / 17 / 12 \\ & \text { FILE FOLOER } \rightarrow 7 b \text { Completed by: }\end{aligned}$

ITEM $\rightarrow 3$

PROOFED BY .

Dowala 725 Date :02Eg/2 REVIEWED BY

Initialos Datol 


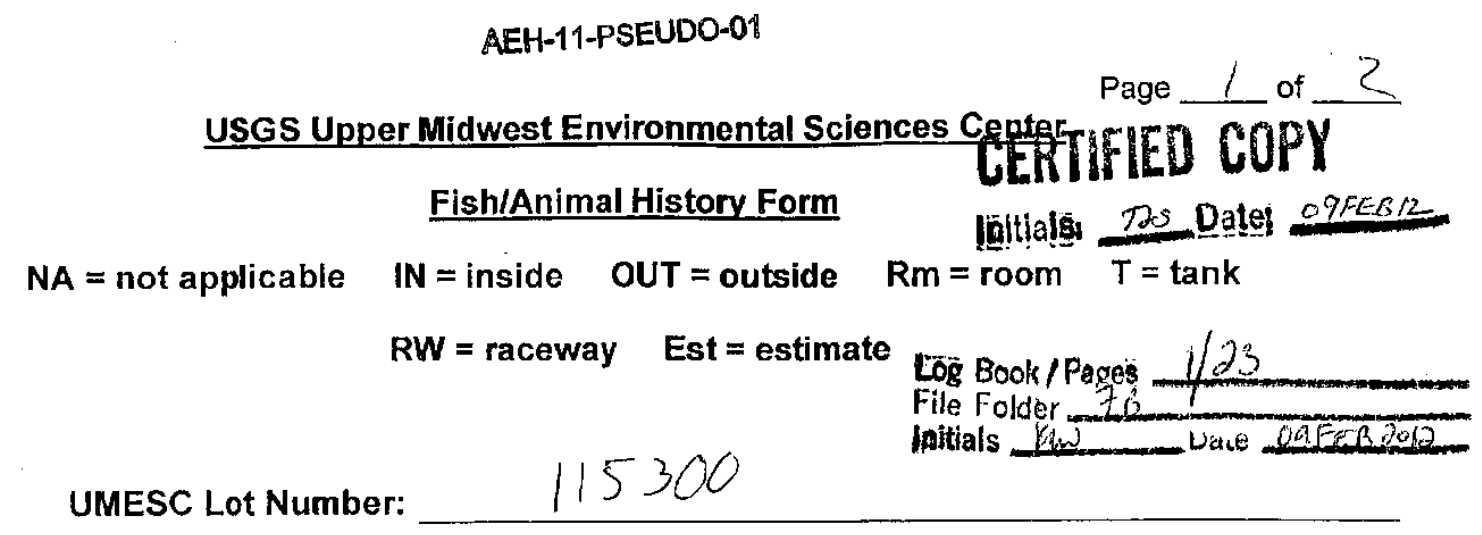

Source Facility Lot \#:

Origin/Source: poro 9 Upar niss: Reres

Species/Strain: Washboard (megaloraras Nervosa)

Species Verified: —u (Gencea of t Nathav Eckent)

Number Received:

4

Date received/lot \# assigned:

Life stage at receipt/lot \#:

$\frac{10 / 18 / 11}{\text { egg larval juvenile dult }}$

Health certificate:

Yes

If egg, disinfected:

Date spawn: Actual/Estimate

Date hatch: Actual/Estimate

First UMESC rearing unit: $\ln / 5$

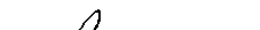

(e.g. room, tank, raceway, pond and rearing unit number)

comments: Collecta) on 10/.7/4

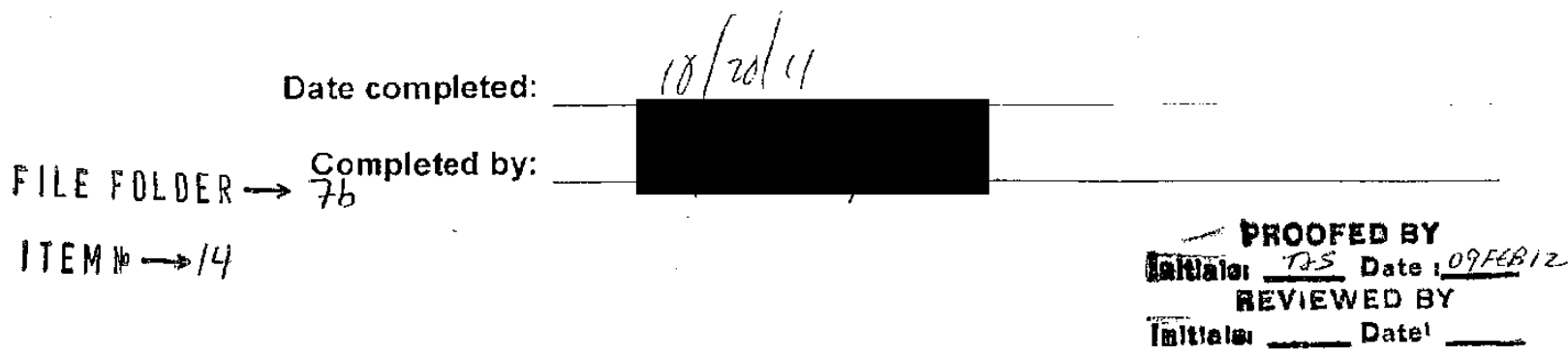




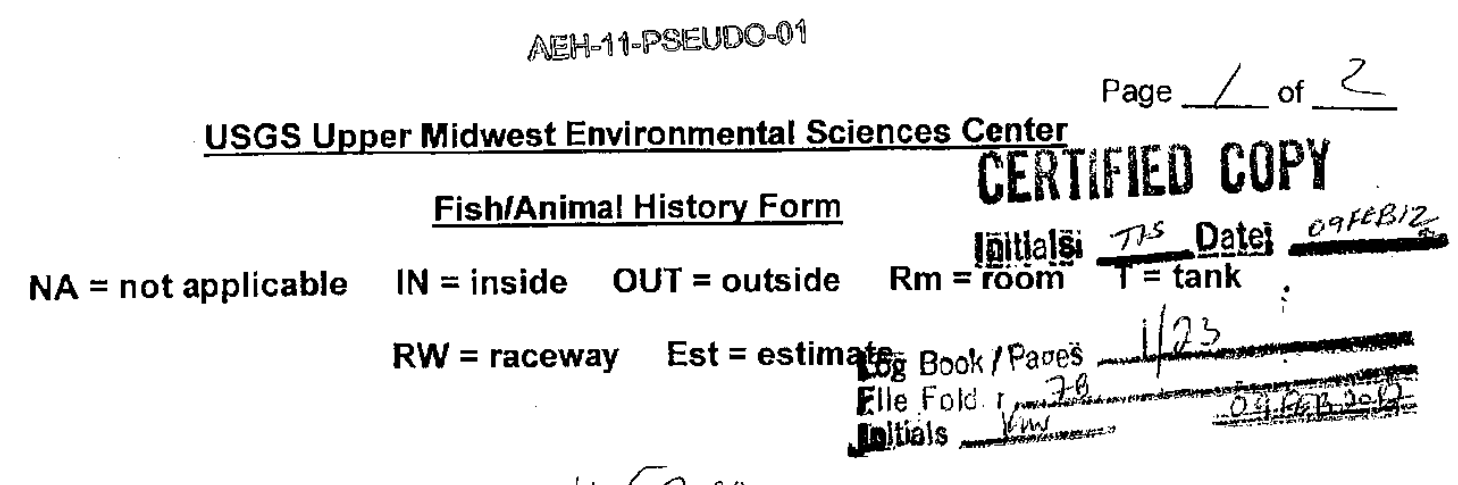

UMESC Lot Number:

115200

Source Facility Lot \#:

Origin/Source:

Pool 9 um $R$

Species/Strain: Plain Pocketbook

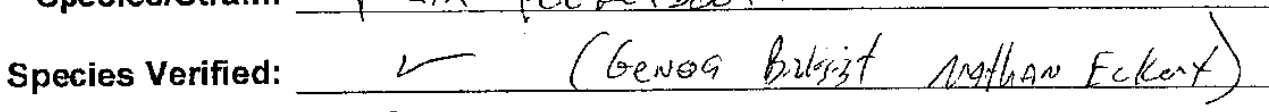

Number Received: 3

Date received/lot \# assigned:

Life stage at receipt/lot \#: $16 / 18 / 4$

$\frac{10 / 18 / 4}{\text { egg larval juvenile adult }}$

Health certificate:

Yes (No)

If egg, disinfected:

Date spawn: Actual/Estimate

Date hatch: Actual/Estimate

First UMESC rearing unit: $R 00 \mathrm{~m} 15$

(e.g. room, tank, raceway, pond and rearing unit number)

Comments: Collecte ov $10 / 8 / 14$

Date completed: $10 / 20 / 4$
FILE FOLDER $\rightarrow$ Completed by:
76

ITEM $\rightarrow 15$

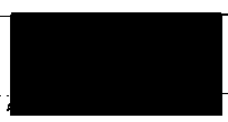

PRODFED BY

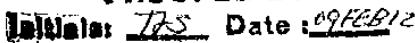

REVIEWED BY

Tinitialas Dater 


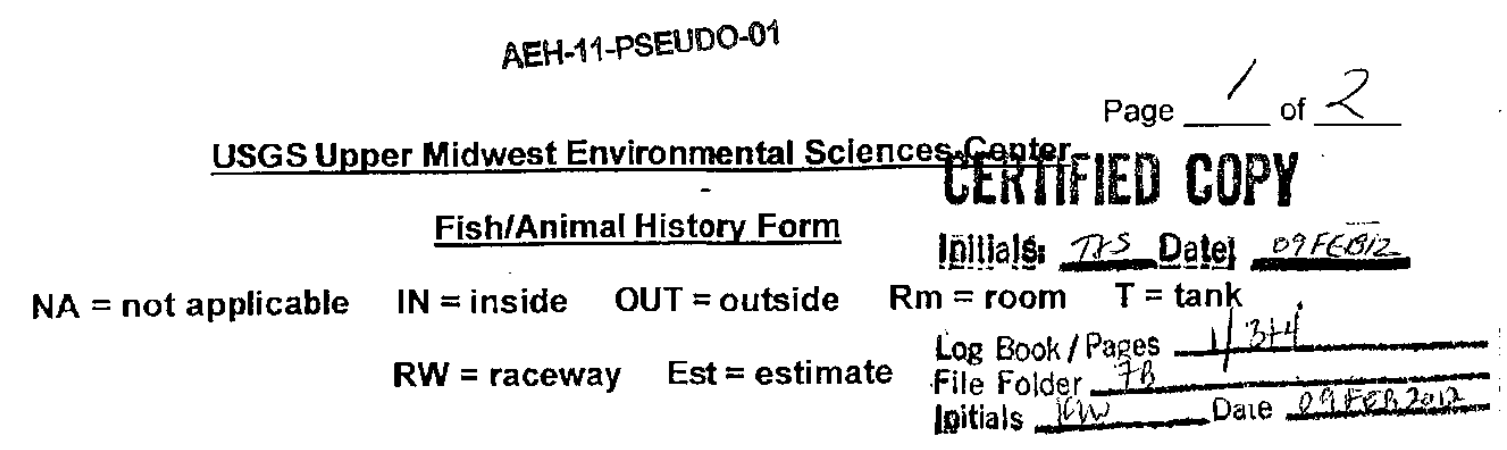

UMESC Lot Number: $1 / 1300$

Source Facility Lot \#:

Origin/Source: St croix River (colleted by GenOa wAlt)

species/Strain: Higgins eye (L.biggiasir)

Species Verified:

Number Received:

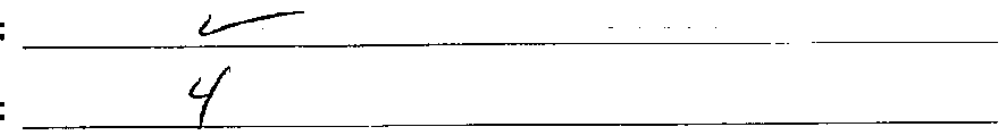

Date received/lot \# assigned:

Life stage at receipt/lot \#:

$5 / 9 / 11$

Health certificate:

If egg, disinfected:

Date spawn: Actual/Estimate

Date hatch: Actual/Estimate

First UMESC rearing unit: $\ln \|$ tank $/ C$

(e.g. room, tank, raceway, pond and rearing unit number)

comments: collectet on $4 / x^{2} / 11$ by Geneq wEAt trasterrel to Umesc an $5 / 0 / 11$

FILE FOLDER $\rightarrow 76$

Date completed:

ITEM $\rightarrow 16$ Date' 
Study Title: "Effects of Pseudomonas fluorescens (Pf-CL145A) to glochidia from seven unionid mussel species"

\section{Study number: AEH-11-PSEUDO-01}

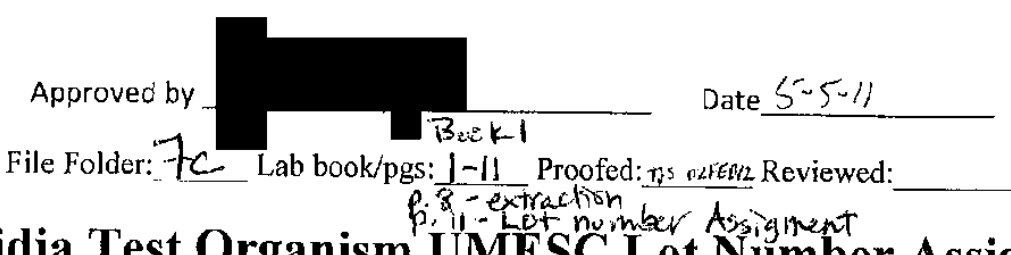

\section{Glochidia Test Organism UMESC L Lot Number Assignment Form}

Species: Lampsilis caydium

Number of Donor Mussels: 3 Donor Mussel Lot \#: 111100

Extraction Date: $0.5 / 12 / 201 \_$Extraction Time (military) 0900 to 0922

Extraction Location:__LMESC rm 15___ Container ID: Pool Block 1

Approximate Number of glochidia: 40,000

Glochidia extracted by: Nathan Eckert__ Affiliation:Genoo NFH

Additional information:

UMESC GLOCHIDIA LOT NUMBER DESIGNATIION: $\| 1400$

Witness and form recorded by:

$\frac{\text { Kerry L. Weber }}{\text { Printed Name }}$

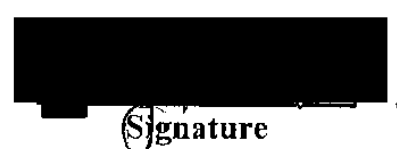

UMESC

Affiliation

$\frac{05 / 121201}{\text { Date }}$

ITEMH $\rightarrow 9$

Page $\perp \perp$ 
Study Title: "Effects of Pseudomonas fluorescens (Pf-CL145A) to glochidia from seven unionid mussel species"

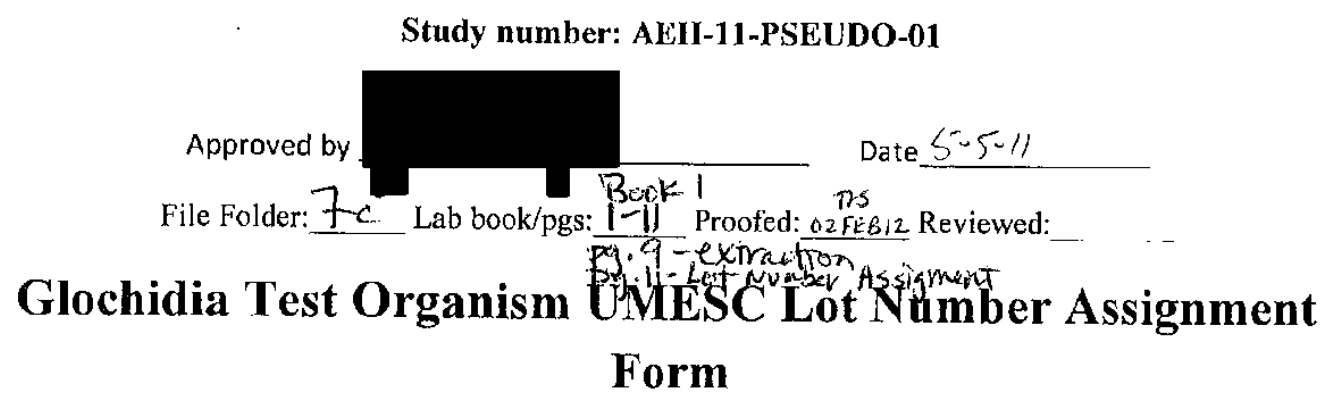

species: L. higginsii

Number of Donor Mussels: 3 Donor Mussel Lot \#: 111300

Extraction Date: $05 / 12$ /2011 Extraction Time (military) 1033 to 1045

Extraction Location:___UMESC $\mathrm{rm} 15 \_$Container ID: Poo _ Block 2

Approximate Number of glochidia: $40,00025,000$

Glochidia extracted by: Nathan Eckert

Affiliation: Gena NFH

Additional information:

UMESC GLOCHIDIA LOT NUMBER DESIGNATION: 111500

Witness and form recorded by:

$\frac{\text { Kevry L. Weber }}{\text { JPrinted Name }}$

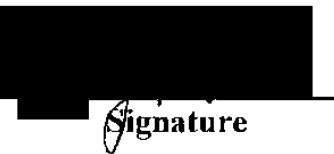

$\frac{\text { UMESC }}{\text { Affiliation }} \frac{05(12) \text { don }}{\text { Date }}$

O) wring Number Recouled i2 MAY zon kW
ITEM $\rightarrow 10$

Page 1 of 1


Study Title: "Effects of Pseudomonas fluorescens (Pf-CL145A) to glochidia from seven unionid mussel species"

Study number: AEH-11-PSEUDO-01

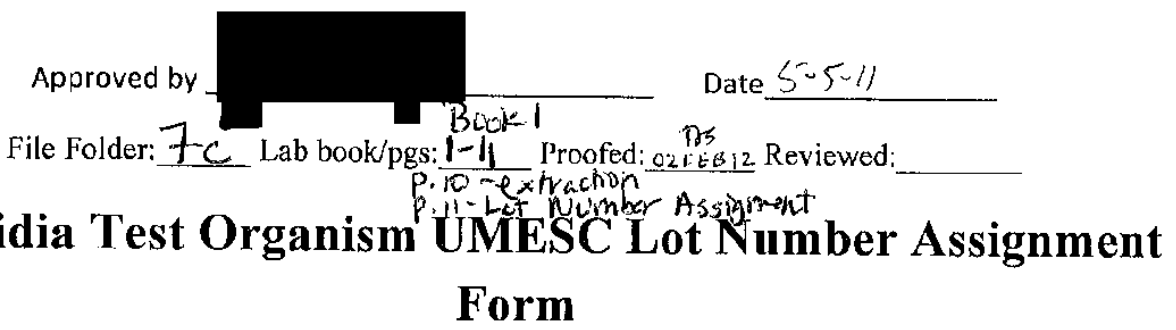

Species: L. siliguaidea

Number of Donor Mussels:

3

Donor Mussel Lot \#: 111200

Extraction Date: $05 / 12 / 2011$ Extraction Time (military) 1215 to 1229

Extraction Location:___ UMESC rm 15___ Container ID: Pool Block 3

Approximate Number of glochidia: 40,000

Glochidia extracted by: Wathon Eckert Affiliation: Genoa NFH

Additional information:

\section{UMESC GLOCHIDIA LOT NUMBER IDESIGNATION: 11600}

Witness and form recorded by:

$\frac{\text { Kerry L. Neber }}{\text { Printed Name }}$
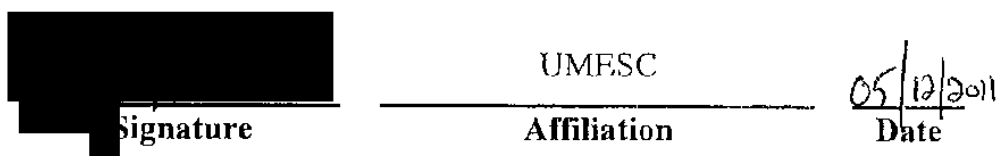

ITEM $\rightarrow \|$

Page I of I 
Study Title: "Effects of Pseudomonas fluorescens (Pf-CL145A) to glochidia from seven unionid mussel species"

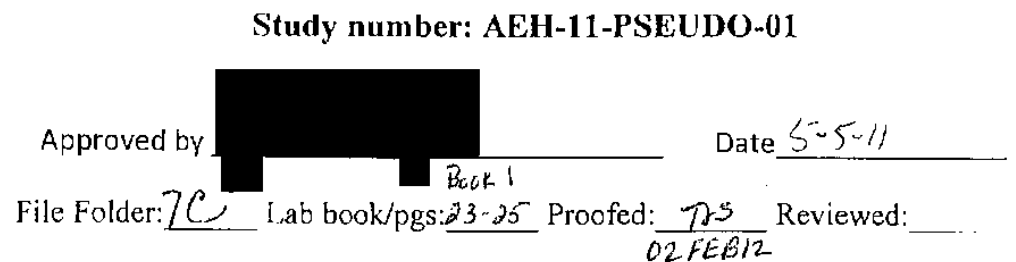

\section{Glochidia Test Organism UMESC Lot Number Assignment Form}

Species: L. Cerdinn

Number of Donor Mussels: Donor Mussel Lot \#: $\quad 1 / 5200$

Extraction Date: $10 / 18 / 201 /$ Extraction Time (military) 0945.

Extraction Location: UMESC rm 15 Container ID: $\quad 1,2+3$

Approximate Number of glochidia: $\pm 5,000$ from each

Glochidia extracted by: $\operatorname{Tim} \operatorname{Lim} A$ Affiliation: 1 M MESL

Additional information:

UMESC GLOCHIDIA LOT NUMBER DESIGNATION:_ $/ 15 \% 00$

Witness and form recorded by:

TAmes A. LlinomA Printed Name

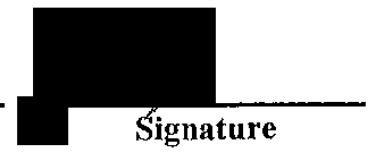

Signature

$\frac{\text { UMESC }}{\text { Affiliation }} \frac{18 x \tau / 1}{\text { Date }}$

ITEM $\rightarrow 12$ 
Study Title: "Effects of Pseudomonas fluorescens (Pf-CL145A) to glochidia from seven unionid mussel species"

\section{Study number: AEH-11-PSEUDO-01}

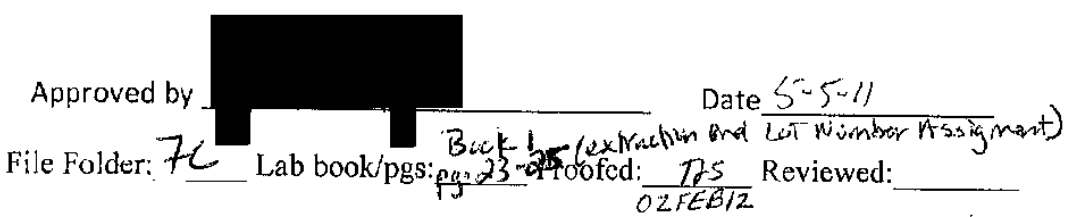

\section{Glochidia Test Organism UMESC Lot Number Assignment} Form

Species: Wash baund (Whegalonacs newasc)

Number of Donor Mussels: 4 Donor Mussel Lot \#: H5500 115300

Extraction Date: $10 / 18 / 201 /$ Extraction Time (military) 1015

Extraction Location: UMESC $\operatorname{rm} 15$ Container ID:

$1,2,3,4$

Approximate Number of glochidia: $3-4,000$ tiach

Glochidia extracted by: Jorge Buxing Affiliation: Gevar WFH

Additional information:

\section{UMESC GLOCHIDIA LOT NUMBER DESIGNA'IION: $\| 5500$}

Witness and form recorded by:

Jantes M. Linm
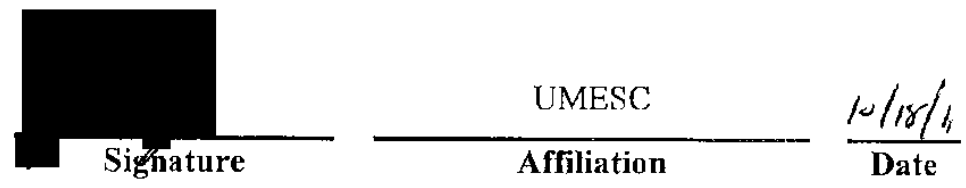

ITEM $\rightarrow 13$ 
Study Title: "Effects of $P_{\text {seudomonas fluorescens }}(P f$-CL145A) to glochidia from seven unionid mussel species"

Study number: AEH-11-PSEUDO-01

\section{Filc Folder: $7 c$ Lab book/pgs: $1 / 31$ Reviewed: $\frac{175}{0,2 F E B i 2}$ Verified: \\ Glochidia Test Organism UMESC Lot Number Assignment Form}

species: Black Sandstell

Number of Donor Mussels: 4 Donor Mussel Lot \#:_120400

Extraction Date: $01 \quad 17 / 2012$ Extraction Time (military) 0945

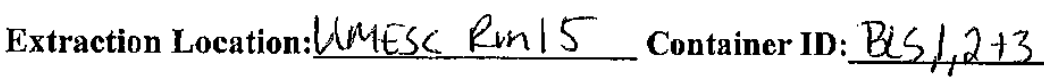

Approximate Number of glochidia: $\quad 20,000$

Glochidia extracted by: _ affiliation: fiecsa NFH

Additional information;

UMESC GLOCHIDIA LOT NUMBER DESIGNATION: 120800

Witness and form recorded by:

$\frac{\text { Keri L. Welour }}{\text { Srinted Name }}$

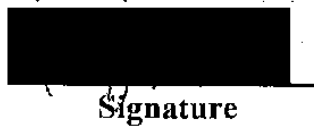

UMESC

Affiliation

$\frac{17571212}{\text { Date }}$

$$
\text { ITEM } \rightarrow 14
$$

This datasheet was approved by

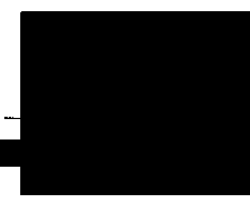

on:2. Shin 2,12

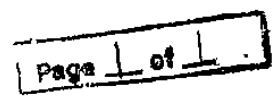


Study Title: "Effects of Pseudomonas fluorescens (Pf-CL145A) to glochidia from seven unionid mussel species"

Study number: AEH-11-PSEUDO-01

File Folder: $7_{C}$ Lab book/pgs: $1 / 31$ Reviewed: $\frac{175}{02 F E B / 2}$ Verified:

\section{Glochidia Test Organism UMESC Lot Number Assignment Form}

Species: Mucket

Number of Donor Mussels: Donor Mussel Lot \#:

120500

Extraction Date: $01 / 17 / 2012$ Extraction Time (military) 1100

Extraction Location: UMESC Rm 15 container ID: MUC 1,2,3 Approximate Number of glochidia: 20,000

Glochidia extracted by

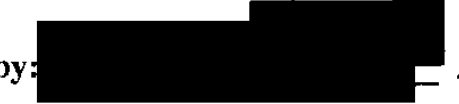
Affiliation: Genox Nfft

Additional information:

\section{UMESC GLOCHIDIA LOT NUMBER DESIGNATION:}

120700

Witness and form recorded by:

$\frac{\text { Kexn L Webar }}{\text { J Printed Name }}$

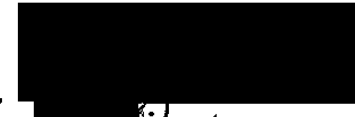

Signature
UMESC

Affiliation
17 JAN12

Date
This datasheet was approved by

11JAN2012/Version 1.1

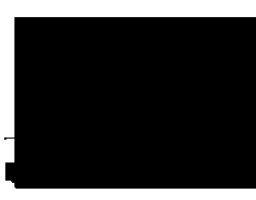

ITEM $\rightarrow 15$ on:2. Shiv? $2,12$.
Page I of I. 
Study Title: "Effects of Pseudomonas fluorescens ( $P$ f-CL145A) to glochidia from seven unionid mussel species"

Study number: AEH-11-PSEUDO-01

File Folder: $7 \mathrm{C}$ Lab book/pgs: $1 / 34$ Reviewed: $\frac{\nabla s}{02 t \in G / 2}$ Verified:

\section{Glochidia Test Organism UMESC Lot Number Assignment Form}

Species: $\frac{\text { Hickorynut }}{\text { Hon }}$

Number of Donor Mussels: 4 Donor Mussel Lot \#: 120600

Extraction Date: $01 / 19 / 2012$ Extraction Time (military) 0832

Extraction Location: UMESC Rm. 15 Container ID: HIC I, $2+3$

Approximate Number of glochidia: 20,000

Glochidia extracted by: Jim Luoma Affiliation: WMESC

Additional information:

UMESC GLOCHIDIA LOT NUMBER DESIGNATION: 120900

Witness and form recorded by:

Kery LiWeber

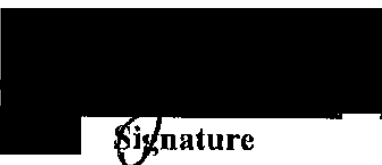

UMESC

Affiliation

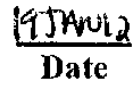

ITEM $\rightarrow 16$

This datasheet was approved by
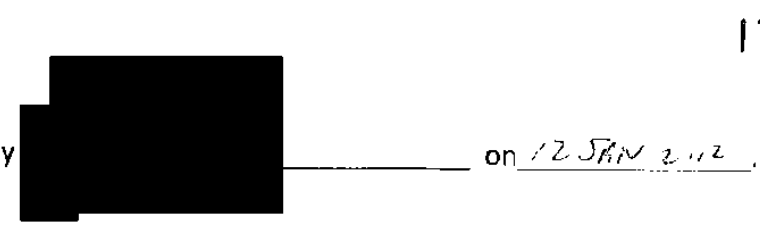

Page I ot L 


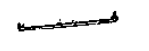

\section{AEH-11-PSEUDO-01 \\ USGS Upper Midwest Environmental Sciences Center \\ Page 1 of 2}

Fish/Animal History Form

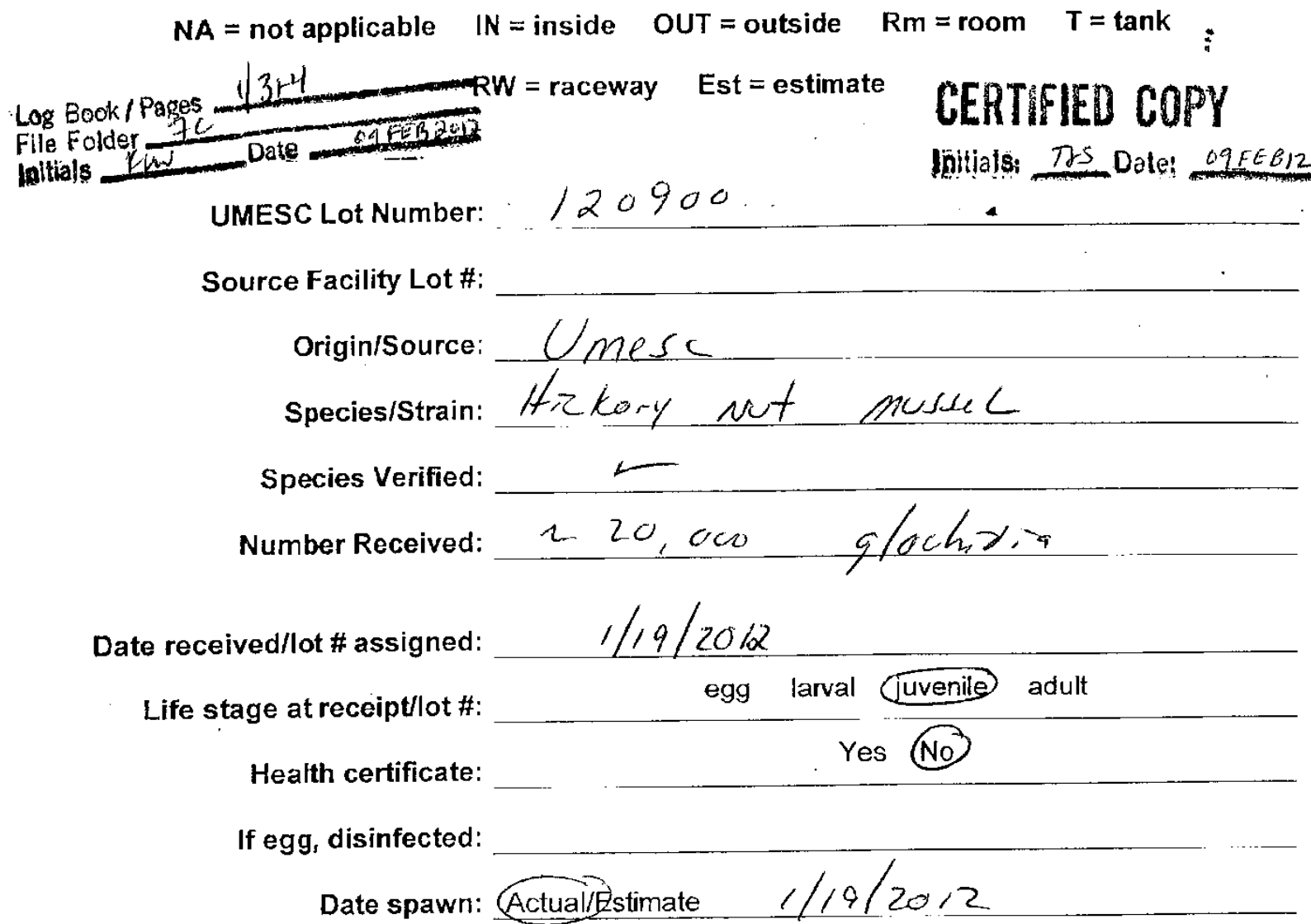

Date hatch: Actual/Estimate

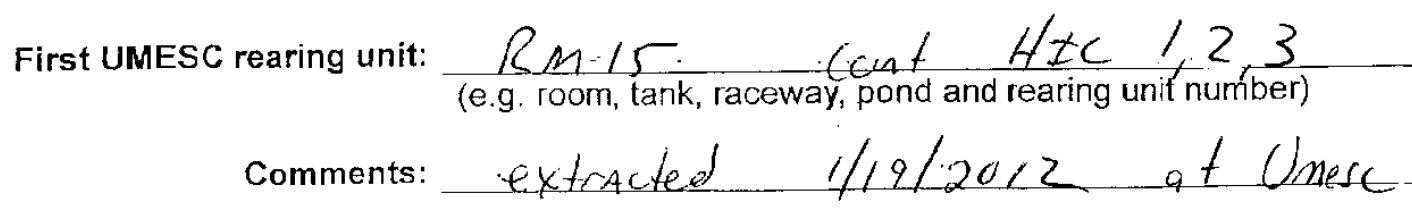

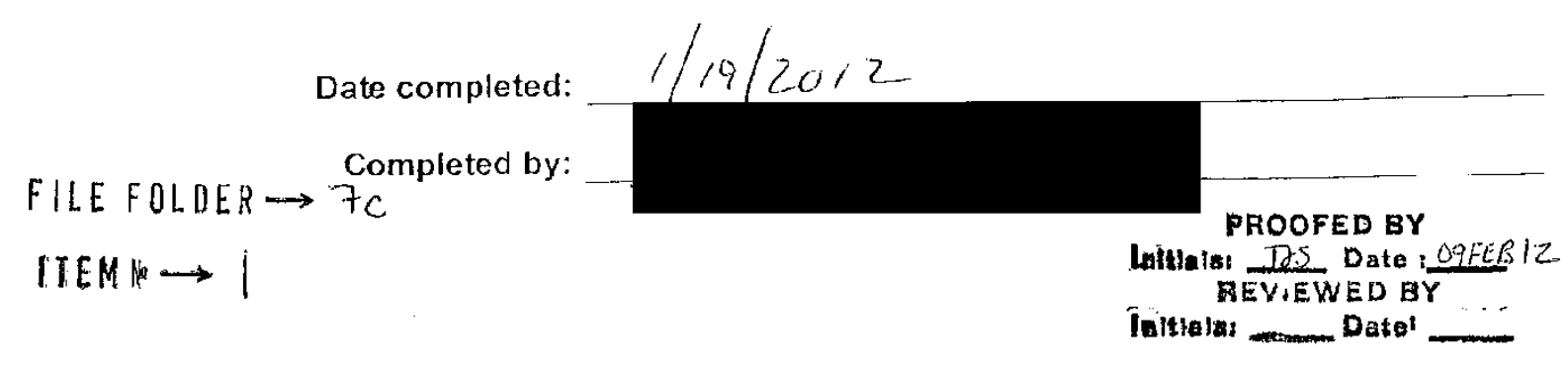




\section{AEH-11-PSEUDO-01}

\section{USGS Upper Midwest Environmental Sciences Center Page 1 of 2 \\ Fish/Animal History Form}

$$
\text { NA = not applicable } \quad \text { IN }=\text { inside } \quad \text { OUT }=\text { outside } \quad R m=\text { room } \quad T=\operatorname{tank}
$$

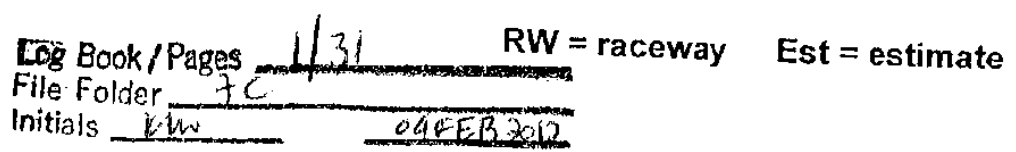

Initials vinw

UMESC Lot Number:

120800

\section{CERTIEA COPY}

IGitials: TSS Dote: ogFEB/Z

Source Facility Lot \#:

Origin/Source: Umesc

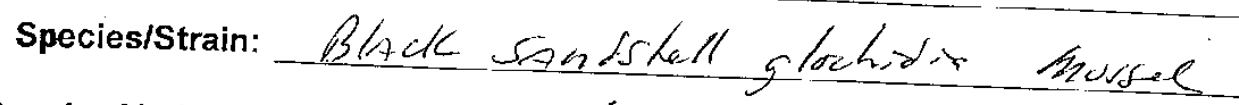
Species Verified:

Number Received: 4

N. ore $=20$, ous

Date received/lot \# assigned:

Life stage at receipt/lot \#: $1 / 17 / 12$

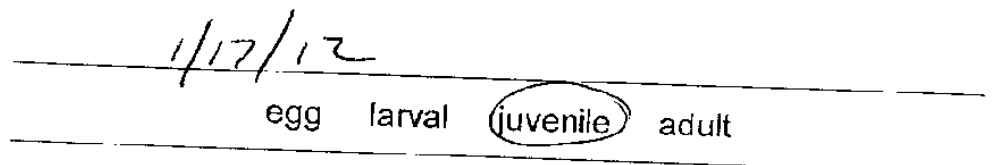

Health certificate: Yes (No)

If egg, disinfected:

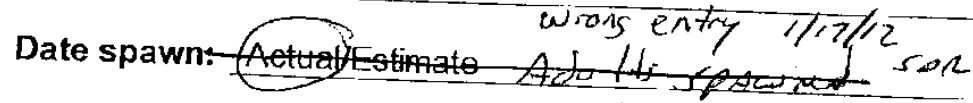

Date hatch: Actual/Estimate

First UMESC rearing unit: $R m \frac{15}{2}$ Cont $20 B C S 1,2,3$

(e. . room, tank, raceway, pond and rearing unit number)

Comments:

$$
\begin{aligned}
& \text { glochine extracted } 1 / 17 / 12 \\
& \text { at umesc }
\end{aligned}
$$

Date completed:

Completed by:

FILE FOLOER $\rightarrow 7 C$

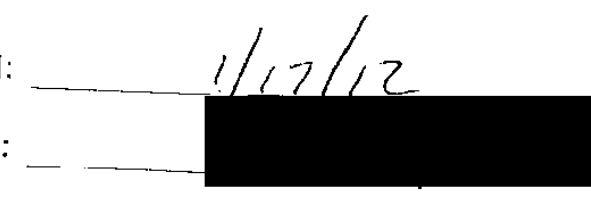

ITEM $\rightarrow 2$

PROOFED GY

laikina: 725 Date:DqESE/2

REVIEWEO BX

Toitiatai Date: 


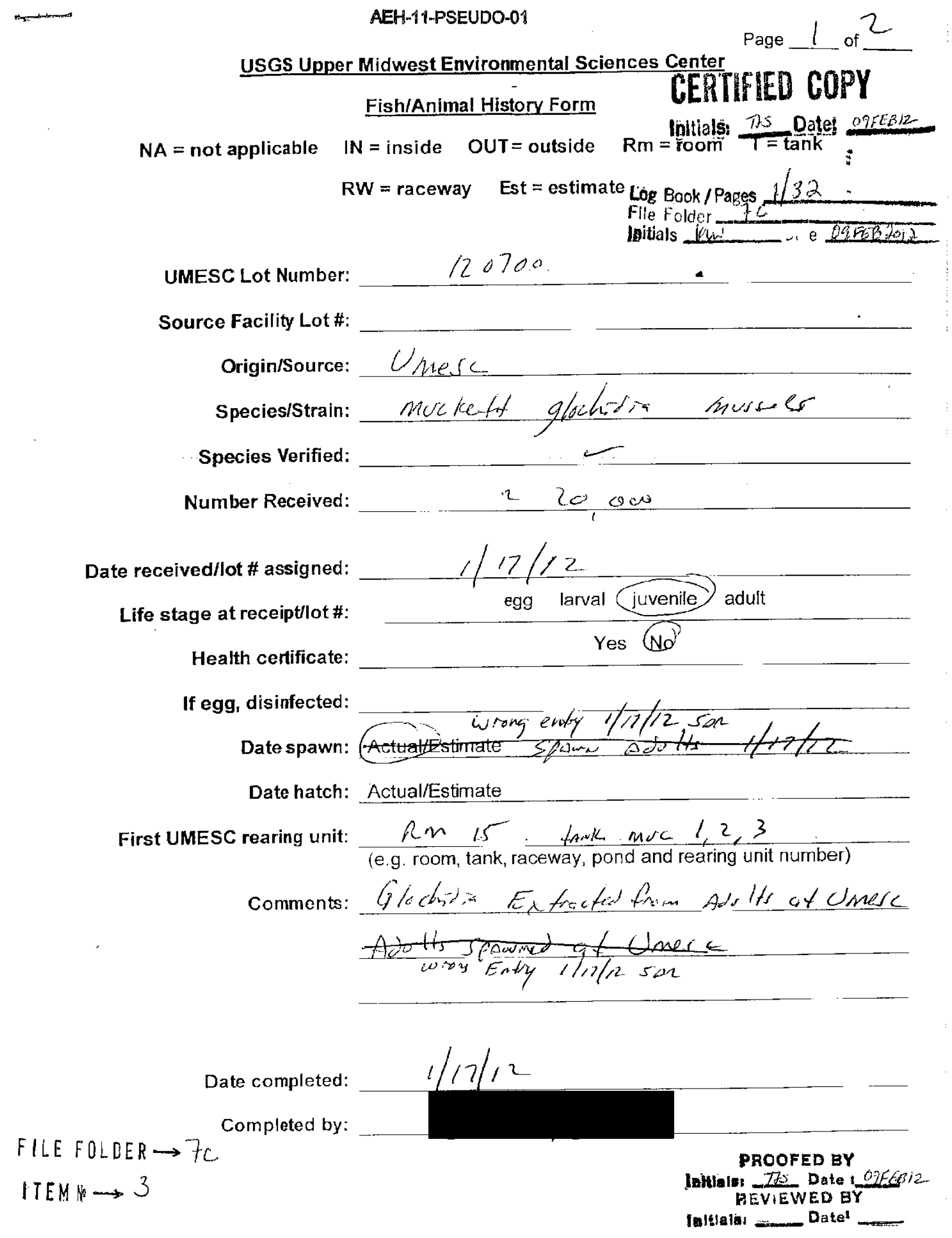




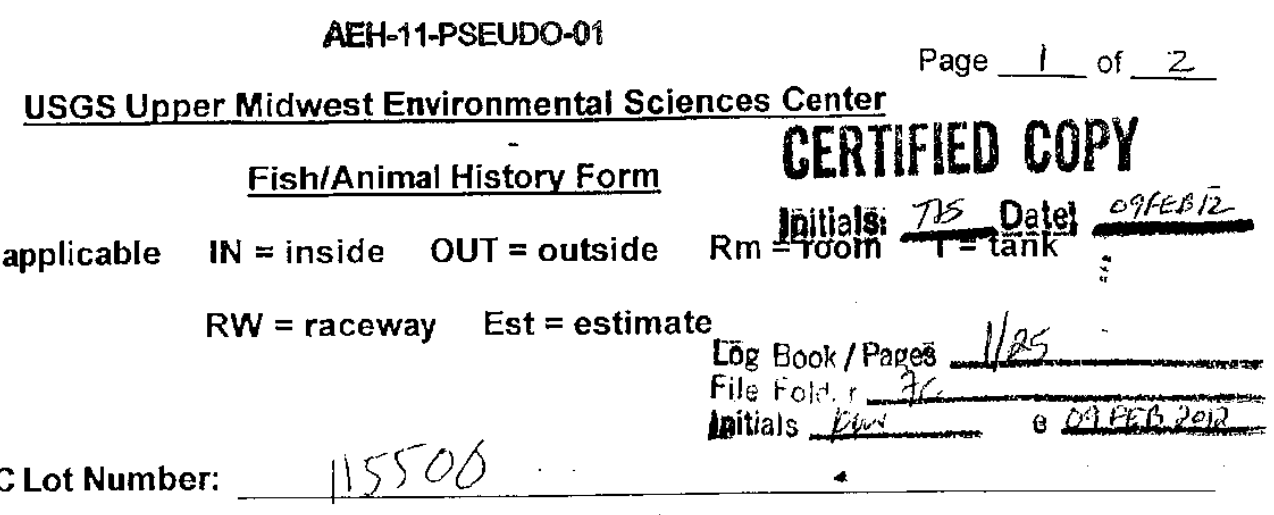

Source Facility Lot \#:

Origin/Source: Umesc

Species/Strain: Washboan mursel ( $M$-Nevosa)

Species Verified: L ( Tin Luoina)

Number Received: $16,000 \quad 3,000-4000 /$ gra in female

Date received/lot \# assigned:

Life stage at receipt/lot \#:

Health certificate:

$\frac{10 / 18 / 1 /}{\text { Yes } \sqrt{10}}$

If egg, disinfected:

Date spawn: Actual/Estimate

Date hatch: Actudil/Estimate $10 / 18 / 11$

First UMESC rearing unit:

$\operatorname{lin} 15$ Gantain (e.g. room, tank, raceway, pond and rearing unit number)

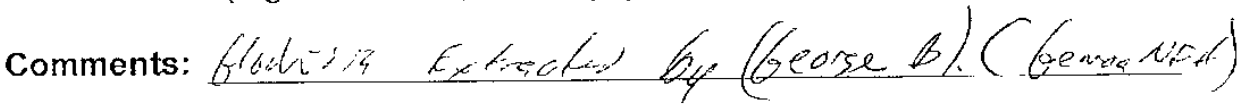

FILE FOLOER $\rightarrow 7 \mathrm{C}$

Date completed: $\quad$ lo/ $/ 20 / 4$

Completed by:

ITEMH $\rightarrow 4$

PROOFED BY

Tillaioi 275 Date 09 FEB/2

REVIEWED BY

Taiziolar 


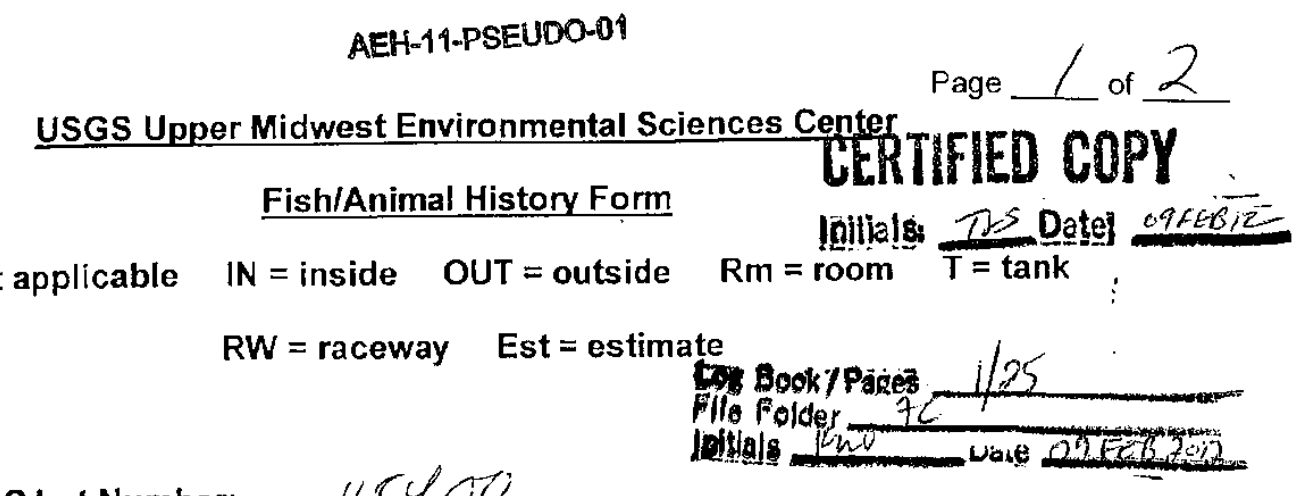

UMESC Lot Number: 115400

Source Facility Lot \#:

Origin/Source:

species/Strain: flaiv paktedtrok CL (Andivu)

Species Verified: $\sim$ ( Tim cuoms)

Number Received: \pm 15 , oue

Date received/lot \# assigned: $\quad 10 / 18 / 11$

Life stage at receipt/iot \#:

Health certificate:

$\frac{10 / 18 / 11}{\text { egg larval juvenile adult }}$

If egg, disinfected:

Date spawn: Actual/Estimate $10 / 18 / 4$

Date hatch: Actual/Estimate

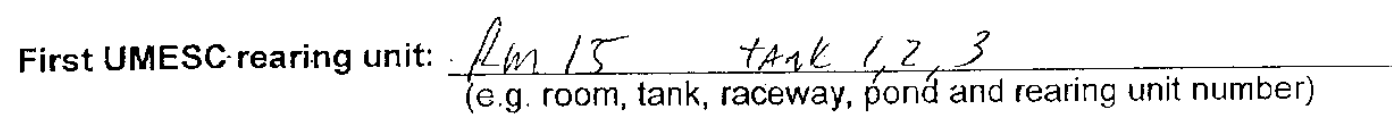

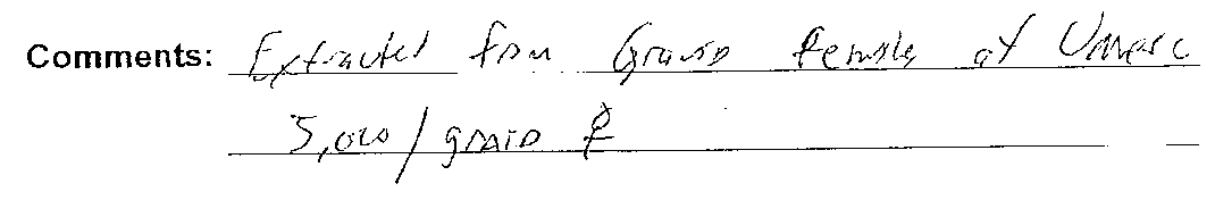

\section{FILE FOLDER $\rightarrow 7 c$}

Date completed:

Completed by:

ITEM $\rightarrow 5$

PROOFED BY

Dithla DS Date: 09ELE/2. REVIEWED BY

Timilia _Datel 


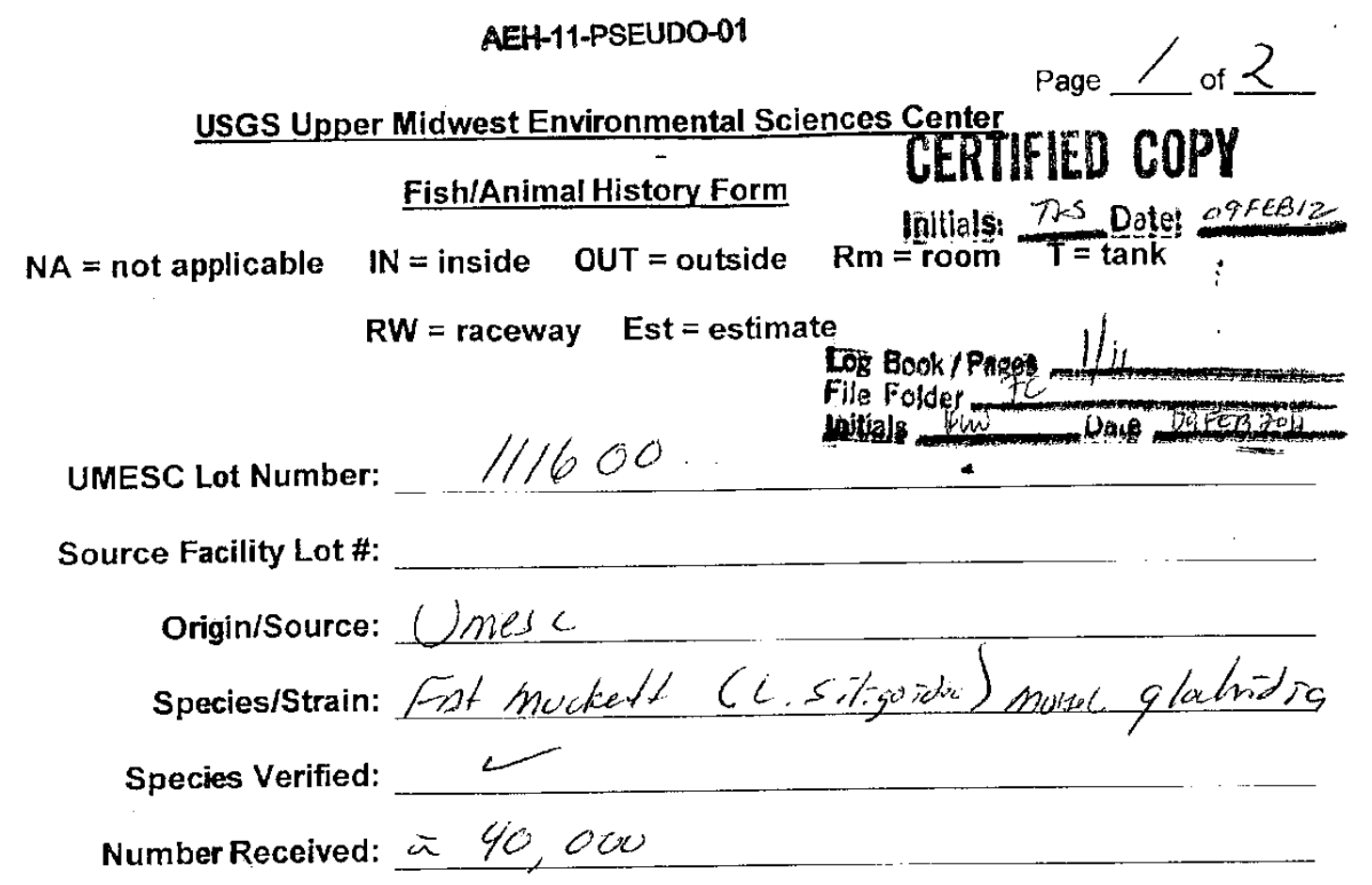

$\begin{array}{rr}\text { Date received/lot \# assigned: } & 5 / 12 / 11 \\ \text { Life stage at receipt/lot \#: } & \text { egg larval uvenile adult } \\ \text { Health certificate: } & \text { Yes }\end{array}$

If egg, disinfected:

Date spawn: Actual/Estimate $5 /, 2 / 1$

Date hatch: Actual/Estimate

First UMESC rearing unit: $\mathrm{Rm} / 5$ Com to B Blok 43

(e.g. room, tank, raceway, pond and rearing unit number)

comments: Dewar muitel tot "ll $11 / 2$ co qlahidg extrained by Genac wet

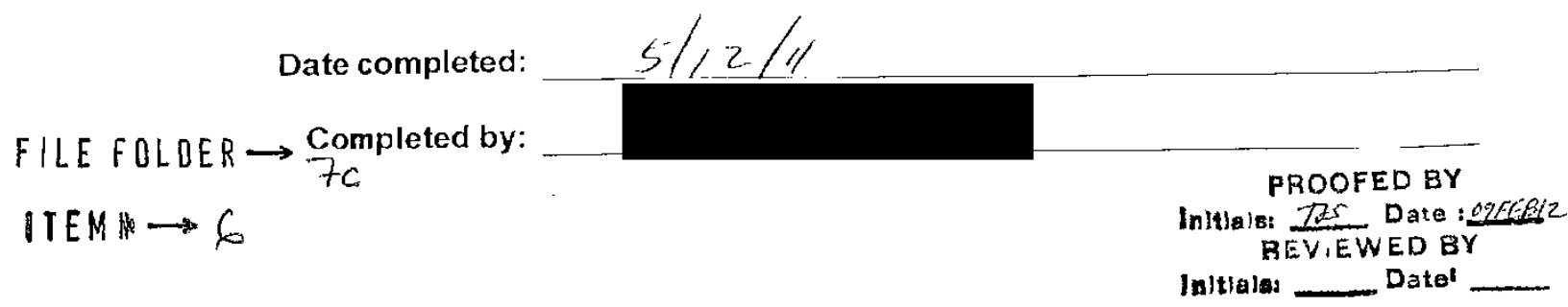


AEH-11-PSEUDO-01

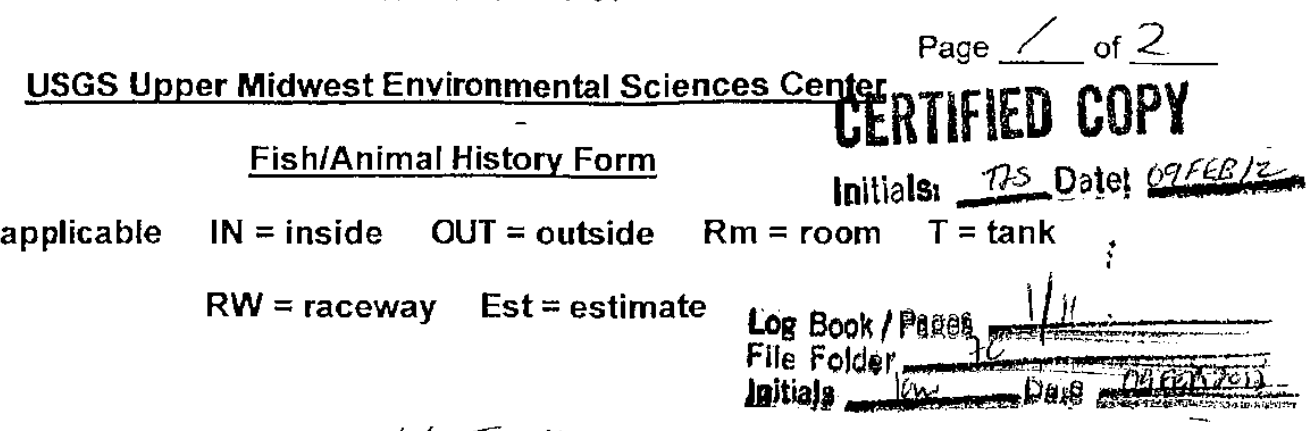

UMESC Lot Number:

$1 / 1500$

Source Facility Lot \#:

Origin/Source: Umesc

species/Strain: hisgins eyes. mosel glockidia

Species Verified:

Number Received: $\simeq 25$, eso

Date received/lot \# assigned:

Life stage at receiptrlot \#:

Health certificate:

$5 / 12 / 11$

egg larval juvenile adult

If egg, disinfected:

Date spawn: Actuati Estimate $5 / 2 / 11$

Date hatch: Actual/Estimate

First UMESC rearing unit: $2 \mathrm{~m} / \mathrm{s}$ cont $t a \beta$ lack 42

(e.g, room, tank, raceway, pond and rearing unit number)

$$
\begin{aligned}
& \text { comments: Dowor mostic lad th } 1 / 1300 \\
& \text { glochies extracted } \therefore \text { Rm } 15 \text { Blok } 2 \\
& \text { by genwe wo } 4
\end{aligned}
$$

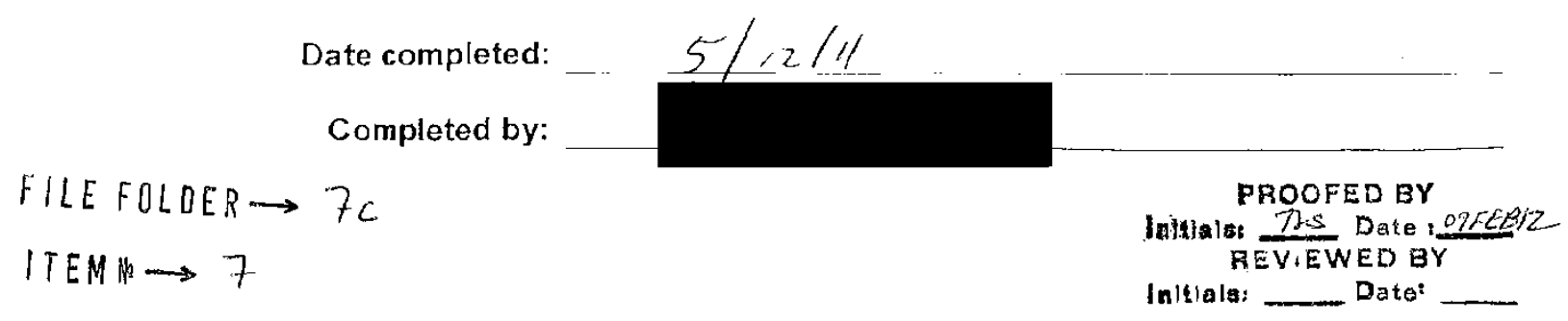




\section{AEH-11-PSEUDO-01 \\ USGS Upper Midwest Environmental Sciences Cogen Page $\frac{1}{2}$ of $\frac{2}{\mathrm{C}}$}

Fish/Animal History Form

NA $=$ not applicable

IN = inside $\quad$ OUT $=$ outside

Initials: DSS Datel

$\mathrm{RW}=$ raceway $\quad$ Est $=$ estimate

$R m=$ room $T=$ tank wrong date.

UMESC Lot Number: $\quad$ _ $/ / 1400$

Log Book / Pages

File Folder $7 c$

Intivals kañ

1

Source Facility Lot \#:

Origin/Source: $\frac{\text { Umesc }}{\text { Plain }}$

Species/Strain: pecketbook (l. (andium)

Species Verified:

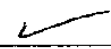

Number Received: ¿t 40 , oce

Date received/lot \# assigned:

Life stage at receiptllot \#:

$5 / 2 / 11$

Health certificate:

egg larval juvenile adult

If egg, disinfected:

Date spawn: Actual/Estimate $5 / 12 / 11$

Date hatch: Actual/Estimate

First UMESC rearing unit:

(e.g. room, tank, raceway, pond and rearing unit number)

Comments: Doper musse lat lo $1 / 1100$

glochide extracted in fem /5 cout pori Black 41 of Genee Not

Date completed:

$5 / 12 / 11$

Completed by:

ILE FOLDER $\rightarrow 7 \mathrm{C}$

$T E M H \rightarrow 8$

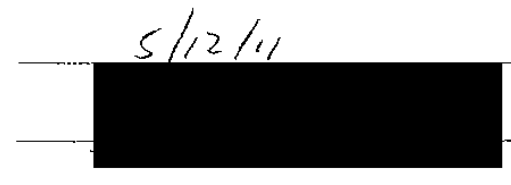




\section{Appendix 6. Water Quality}

\begin{tabular}{|c|c|c|c|}
\hline $\begin{array}{c}\text { Item } \\
\text { number }\end{array}$ & Item description & $\begin{array}{c}\text { Number } \\
\text { of } \\
\text { pages }\end{array}$ & $\begin{array}{c}\text { Report } \\
\text { page } \\
\text { number }\end{array}$ \\
\hline 1 & Initial (Pre Exposure) Water Chemistry - Data Summary & 2 & 326 \\
\hline 2 & Exposure Water Chemistry-Data Summary & 9 & 328 \\
\hline 3 & Exposure Light Intensity - Data Summary & 2 & 337 \\
\hline 4 & Termination Water Chemistry - Data Summary & 2 & 339 \\
\hline 5 & $\begin{array}{l}\text { Report of Analysis - Ammonia Report from Water Quality Laboratory at UMESC - Report Dated } \\
\text { November 9,2011 }\end{array}$ & 1 & 341 \\
\hline 6 & $\begin{array}{l}\text { Report of Analysis - Ammonia Report from Water Quality Laboratory at UMESC - Report Dated February } \\
\text { 3,2012 }\end{array}$ & 1 & 342 \\
\hline 7 & Total Ammonia Nitrogen - Data Summary & 10 & 343 \\
\hline
\end{tabular}




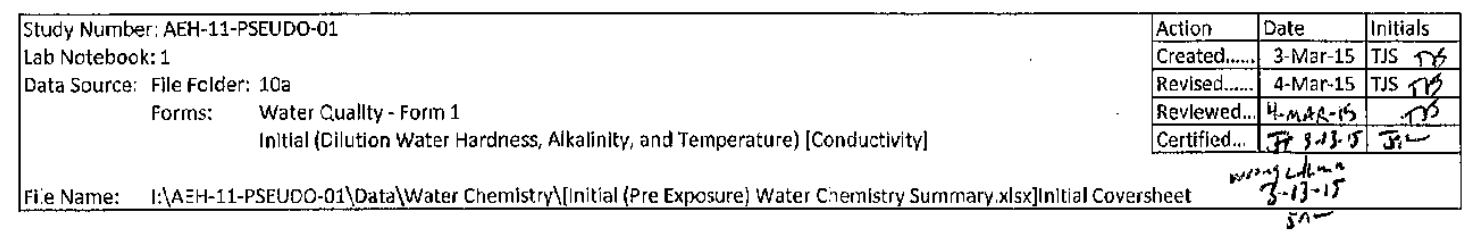

Initial (Pre Exposure) Water Chemistry

Test Article: Pseudomonas fluorescens ( $P f-C L 145 A)$ spray dried powder (SDP) and freeze drled powder (FDP)

Article Lot \#s: SDP = MBI-401-110308Al-BD-3 and $\mathrm{MB|}-401$ SDP 4655-12-MlX; FDP = 110607WB-FD-E

Exposure Dates: 12-May-11, 18-Oct-11, 17-Jan-12, and 19-Jan-12

Species Tested: PPB, FAM, HGE, WAS, BLS, MUC, and HIC

Data Explanation:

Specles $A$ bbreviations

PP3 - plain pocketbook (Lampsilis cardtum)

FAM - fatmucket (Lampsills siliquoldeo)

HGE - Higgins eye (Lampsills higginsii)

WAS - washboard (Megalonaias rervosa)

$B L S$ - black sandshell (Ligumia recta)

MUC - mucket (Actinonalos ligamentina)

HIC - hickorynut [Obovaria olivaria\}

Data anomalies and deviations:

1) Initial conductivity reading was not measured on 12-MAY-11.

2) Only one replicate sample of conductivity was measured on 18-Oct-11.

3) Initial water chemistry parameters were measured in triplicate for each exposure day (12-May-11, 18-Oct-11, 17-Jan-12, and 19-Jan-12) but not separately for each specics, therefore, for species that were tested on the same day the data is transcribed for clarity.

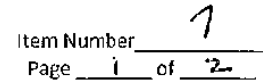


SiddY Number: AEH-11.PSEUDO-01

Lab Notebook 1

Ja:a Source: 'File folder: 10

Forms: Water Qualry - Form 1

Initlal (Dllutlon Water Hardness, Alkall Ilky, and Temperature) [Conductivity]
Test Article: MBI 401 (Pf-CL 145N [SDP] and [FDP)]

Artcle Lot H: $\quad$ MBl-401-110308Al-ED-3, MBI-401 SDP 4655-12-Mix, 110607WB-FD-

Exposure Dates: 12-May-11, 18-Oct-11, 17-Jan-12, and 19-Jan-12

Species Tested: PPB, FAM, HGE, WAAS, BLS, MUC, and HIC

Initial (Pre Exposure) Water Chemistry

\begin{tabular}{|c|c|c|c|c|c|c|c|}
\hline Species & Formulation & Test Date & Replicate & $\begin{array}{l}\text { Hardness } \\
\left(\mathrm{rug} / \mathrm{L} \mathrm{CaCO}_{3}\right)\end{array}$ & $\begin{array}{c}\text { Alkalinity } \\
\left(\mathrm{ng} / \mathrm{LCaCO}_{3}\right\}\end{array}$ & $\begin{array}{c}\text { Conductivity } \\
(\mu \mathrm{s} / \mathrm{cm})\end{array}$ & $\begin{array}{c}\text { Temperature } \\
\left({ }^{\circ} \mathrm{C}\right)\end{array}$ \\
\hline \multirow[t]{7}{*}{ PPB } & SDP & 12 -Мву 11 & 1 & 170 & 130 & $M D$ & 19.4 \\
\hline & & & $?$ & 172 & 130 & ND & 19.3 \\
\hline & & & 3 & 170 & 130 & $A D$ & 19.3 \\
\hline & & & Mean & 171 & 130 & $A D$ & 19.3 \\
\hline & & & SD & 1 & 0 & $\Lambda D$ & 0.1 \\
\hline & & & $\operatorname{Min}$ & 170 & 130 & NO & 19.3 \\
\hline & & & Max & 172 & 130 & ND & 19.4 \\
\hline \multirow[t]{7}{*}{ FAM } & SDP & $\mid 12+M a y-11$ & 1 & 170 & 130 & AD & 19.4 \\
\hline & & & 2 & 172 & 130 & $A D$ & 19.3 \\
\hline & & & 3 & 170 & 130 & ND & 19.3 \\
\hline & & & Mean & 171 & 130 & $A D$ & 19.3 \\
\hline & & & SD & 1 & 0 & ND & 0.1 \\
\hline & & & Min & 170 & 130 & ND & 19.3 \\
\hline & & & Max & 172 & 130 & $N D$ & 19.4 \\
\hline \multirow[t]{7}{*}{ HEE } & SDP & 12-May-11! & 1 & 170 & 130 & $A D$ & 19,4 \\
\hline & & & 2 & 172. & 130 & DO & 19.3 \\
\hline & & & 3 & 170 & 130 & $A D$ & 19.3 \\
\hline & & & Mean & 171 & 130 & ND & 19.3 \\
\hline & & & SD & 1 & 0 & ND & 0.1 \\
\hline & & & Min & 170 & 130 & ND & 19.3 \\
\hline & & & $\operatorname{Max}$ & 172 & 130 & ND & 19.4 \\
\hline \multirow[t]{7}{*}{ PPB } & FDP & 18-Oct-11 & 1 & 174 & 126 & 391 & 18.4 \\
\hline & & & 2 & 174 & 126 & ND & 18.4 \\
\hline & & & 3 & 174 & 127 & ND & 18.4 \\
\hline & & & Mean & 174 & 126 & 391 & 18.4 \\
\hline & & & SD & 0 & 1 & ND & 0.0 \\
\hline & & & Min & 174 & 126 & 391 & 18.4 \\
\hline & & & Max & 174 & 1.27 & 391 & 18.4 \\
\hline \multirow[t]{7}{*}{ WAS } & FDP & 18-0ct-11 & 1 & 174 & 126 & 391 & 18.4 \\
\hline & & & 2 & 174 & 126 & $A D$ & 18.4 \\
\hline & & & 3 & 174 & 127 & $A D$ & 18.4 \\
\hline & & & Mean & 174 & 126 & 391 & 18.4 \\
\hline & & & SD & 0 & 1 & $A D$ & 0.0 \\
\hline & & & Min & 174 & 126 & 391 & 18.4 \\
\hline & & & $\operatorname{Max}$ & 174 & 127 & 391 & 18.4 \\
\hline \multirow[t]{7}{*}{ BLS } & SDP & 17- $\tan -12$ & 1 & 172 & 125 & 412 & 19.9 \\
\hline & & & 2 & 171 & 124 & 425 & 19.9 \\
\hline & & & 3 & 170 & 124 & 429 & 19.9 \\
\hline & & & Mean & 171 & 124 & 422 & 19.9 \\
\hline & & & SD & 1 & 1 & 9 & 0.0 \\
\hline & & & Mln & 170 & 124 & 412 & 19.9 \\
\hline & & & $\operatorname{Max}$ & 172 & 125 & 429 & 19.9 \\
\hline \multirow[t]{7}{*}{ MUC } & SDP & 17-1an-12 & 1 & 172 & 125 & 412 & 19.9 \\
\hline & & & 2 & 171 & 124 & 425 & 19.9 \\
\hline & & & 3 & 170 & 124 & 429 & 19.9 \\
\hline & & & Mean & 171 & 124 & 422 & 19.9 \\
\hline & & & SD & 1 & 1 & 9 & 0.0 \\
\hline & & & Min & 170 & 124 & 412 & 19.9 \\
\hline & & & Max & 172 & 125 & 429 & 19.9 \\
\hline \multirow[t]{7}{*}{ HIC } & SDP & $19-\tan -12$ & 1 & 174 & 121 & 382 & 20.1 \\
\hline & & & 2 & 174 & 122 & 331 & 19.9 \\
\hline & & & 3 & 176 & 122 & 385 & 19.8 \\
\hline & & & Mean & 175 & 122 & 383. & 19,9 \\
\hline & & & SD & 1 & 1 & 2 & 0.2 \\
\hline & & & Mln & 174 & 121 & 381 & 19.8 \\
\hline & & & Max & 176 & 122 & 335 & 20.1 \\
\hline
\end{tabular}




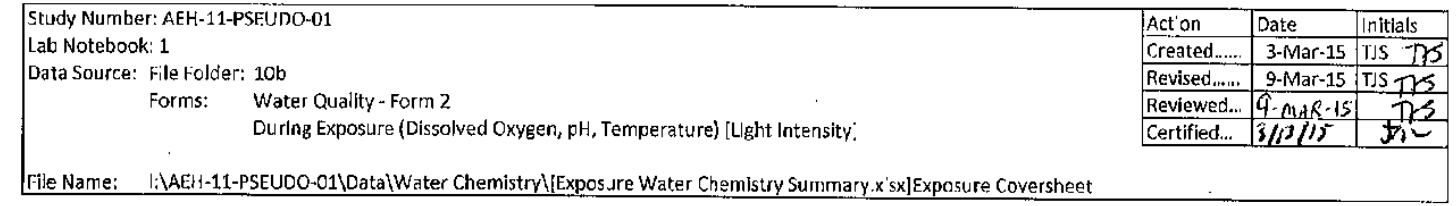

\section{Exposure Water Chemistry}

Test Article: Pseudomonas fluorescens (Pf-CL145A) spray dried powder (SDP) and freeze dried powder (FDP)

Article Lot Hs: SDP = MBI-401-1103JSAl-BD-3 and MBI-401 SDP 4655-12-Mix; FDP = 110607WB-FD-E

Exposure Dates: 12-May-11, 18-Oct-11, 17-Jan-12, and 19-Jan-12.

Species Tested: PPB, FAM, HGE, WAS, B:S, MUC, and HIC

Data Explanation:

$300 \mathrm{HD}=300 \mathrm{mg} / \mathrm{L}$ Heat Deactivated

Specles Abbrevlations

PPB - plain pocketbook (Lampsilis cardium)

FAM - fatmucket (Lampsilts siliquoidea)

HGE - Higgins eye (Lampsilis higginsii)

WAS - washboard (Megalonalas nervosa)

BLS - black sandshell (Ligumla recta)

MUC - mucket (Actinonaias ligamentina)

IIIC - hickorynut (Obovoria olivaria)

Water chemistry was measured twice during exposure:

1) Upon exposure initiation

2) Prior to exposure termination

For PPB, FAM, and HGE (SDP), water chemlstry parameters were measured in all replicates ( 3 ) for controls $(0 \mathrm{mg} / \mathrm{L}$ ) and 300 mg $/$ t teatments, however, only one replicate was measured for $50,100,200 \mathrm{mg} / \mathrm{L}$, and $300 \mathrm{mg} / \mathrm{L}$ heat deactivated (HD). All other species had all replicates measured for all treatment concentrations

$\mathrm{pH}$ means calculated on hydrogen ion concentration; $\mathrm{pH}$ standard devlations calculated on $\mathrm{pH}$ values.

Data anomalies and deviations:

None 


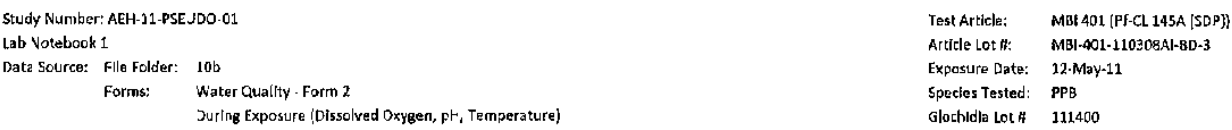

Exposure Water Chemistry - Plain Pocketbook SDP

\begin{tabular}{|c|c|c|c|c|c|}
\hline Test & Treatment & Sample & Dissolved & & Temperature \\
\hline Chamber & $(\mathrm{mg} / \mathrm{L})$ & Time & Oxpen $(\mathrm{mg} / \mathrm{L})$ & $\mathrm{pH}$ & $\left({ }^{\circ} \mathrm{C}\right)$ \\
\hline \multirow[t]{2}{*}{$1 \mathrm{AB}$} & 0 & 1 & 8.57 & 8.42 & 99.3 \\
\hline & & 2 & 8.39 & 8.23 & $: 9.1$ \\
\hline \multirow[t]{2}{*}{$1 \mathrm{B1}$} & & 1 & 8.43 & 8.30 & 20,0 \\
\hline & & 2 & 8.55 & 8,33 & 9.5 \\
\hline \multirow[t]{6}{*}{$1 \mathrm{C3}$} & & 1 & 8.66 & 8.39 & 20.0 \\
\hline & & 2 & 8.39 & 8.41 & 19.0 \\
\hline & & Mear. & 8.50 & 8,35 & 19.5 \\
\hline & & SD & 0.21 & 0.07 & 0.4 \\
\hline & & $\mathrm{V} \ln$ & 8.39 & 8.23 & 19.0 \\
\hline & & Max & 8.66 & 8.42 & 20.0 \\
\hline 185 & 50 & 1 & 8.23 & 8.39 & 20.2 \\
\hline \multirow[t]{5}{*}{$1 \mathrm{~A} 1$} & & 2 & 8.07 & 8.25 & 19.2 \\
\hline & & Mean & 8.25 & 8.33 & 19.7 \\
\hline & & so & 0.11 & 0.10 & 0.7 \\
\hline & & Mln & 8.07 & 8.25 & 19.2 \\
\hline & & Max & 8.23 & 8,39 & 20.2 \\
\hline 184 & 100 & 1 & 8.68 & 8.39 & 19.6 \\
\hline \multirow[t]{5}{*}{$1 \mathrm{AA}$} & & 2 & 7.40 & 8.03 & 19.0 \\
\hline & & Mean & 8.04 & 8.25 & 19,3 \\
\hline & & SD & 0.91 & 0.25 & 0.4 \\
\hline & & $\operatorname{Min}$ & 7.40 & 8.03 & 19.0 \\
\hline & & Max & 8.68 & 8.39 & 19.6 \\
\hline $1 \mathrm{C6}$ & 200 & 1 & 8.27 & 8.36 & 19.5 \\
\hline \multirow[t]{5}{*}{$1 C 4$} & & 2 & 7.04 & 8.09 & 19.0 \\
\hline & & Mean & 7.66 & 8.25 & 19,3 \\
\hline & & SD & 0.87 & 0.19 & 0.4 \\
\hline & & Min & 7.04 & 8.09 & 19.0 \\
\hline & & $\operatorname{Max}$ & 8.27 & 8.36 & 19.5 \\
\hline \multirow[t]{2}{*}{$1 \mathrm{AS}$} & 300 & 1 & 8.80 & 8.27 & 20.0 \\
\hline & & 2 & 6.20 & 7.85 & 19.0 \\
\hline \multirow[t]{2}{*}{$1 \mathrm{C} 2$} & & 1 & 8.54 & 8.29 & 20.0 \\
\hline & & 2 & 5.83 & 7.87 & 19.5 \\
\hline \multirow[t]{6}{*}{$1 C 5$} & & 1 & 8.68 & 0.27 & 20.0 \\
\hline & & 2 & 5.22 & 7.74 & 19.1 \\
\hline & & Mean & 7.21 & 8.11 & 19.6 \\
\hline & & SD & 1.63 & 0.25 & 0.5 \\
\hline & & MIn & 5.27 & 7.74 & 19.0 \\
\hline & & Max & 8.80 & 8.29 & 20.0 \\
\hline & $300 \mathrm{HD}$ & 1 & 8.29 & 8.37 & 20.0 \\
\hline \multirow[t]{5}{*}{$1 \mathrm{AB}$} & & 2 & 6.63 & $8.0 t$ & 19.1 \\
\hline & & Mean & 7.46 & 8.25 & 19.6 \\
\hline & & 50 & 1.17 & 0.21 & 0.6 \\
\hline & & MIn & 6.63 & 8.07 & 19.1 \\
\hline & & $\operatorname{Max}$ & 8.29 & 8.37 & 20.0 \\
\hline
\end{tabular}

Sample Time 1 was measured upo

$$
\text { Item Number-197}
$$




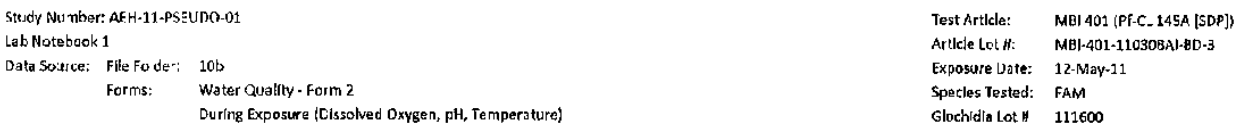

Test Artcle: ME1 401 (PFC 145A [SDP])

Artcle Lot H: MEl-401-110308Al-8D-3

Exposure Date: 12-May-11

Spetles Tested: FAM

Glochidia Lot : 111600

Exposure Water Chemistry - Fatmucket SDP

\begin{tabular}{|c|c|c|c|c|c|}
\hline Test & Treatment & Sample & Dissolved & & Ternperature \\
\hline Chamber & (mg/L) & Time & Oxygen (mg/L) & $\mathrm{PH}$ & $\left|{ }^{\circ} \mathrm{C}\right\rangle$ \\
\hline \multirow{2}{*}{$3 A 2$} & 0 & 1 & 8.65 & 8,39 & 19.7 \\
\hline & & 2 & 8.85 & 8.47 & 19.0 \\
\hline \multirow[t]{2}{*}{$3 \mathrm{~B} 2$} & & 1 & 8.56 & 8.48 & 19.4 \\
\hline & & 2 & 8.05 & 8.43 & 19,0 \\
\hline \multirow[t]{6}{*}{383} & & 1 & 8.67 & 8.44 & 20.0 \\
\hline & & 2 & 8.75 & 8.42 & 19.1 \\
\hline & & Mean & 8.59 & 8.44 & 19.4 \\
\hline & & SD & 0.28 & c.03 & 0.4 \\
\hline & & Min & $8 . c 5$ & 8.39 & 19.0 \\
\hline & & $\operatorname{Max}$ & 8.96 & 8.48 & 20.0 \\
\hline $3 C 5$ & 50 & 1 & 8.11 & 8.36 & 19.5 \\
\hline \multirow[t]{5}{*}{385} & & 2 & 7.91 & 8.05 & 19.0 \\
\hline & & Mean & 7.71 & 8.23 & 19.3 \\
\hline & & so & 0.57 & 0.22 & 0.4 \\
\hline & & $M^{\prime} n$ & 7.31 & 8.05 & 19.0 \\
\hline & & Max & 8.11 & 8.36 & 19.5 \\
\hline $3 A 5$ & 100 & 1 & 8.15 & 8.35 & 20.0 \\
\hline \multirow[t]{5}{*}{$3 A 3$} & & 2 & 7.01 & 8.09 & 18.9 \\
\hline & & Mear & 7.58 & 8.24 & 19.5 \\
\hline & & SD & 0.81 & 0.18 & 0.8 \\
\hline & & Min & 7.01 & 8.09 & 18.9 \\
\hline & & $\max$ & 8.15 & 8.35 & 20.0 \\
\hline $3 \mathrm{CA}$ & 200 & 1 & 8.60 & 8.20 & 19.9 \\
\hline \multirow[t]{5}{*}{384} & & 2 & 6.02 & 7.73 & 19.1 \\
\hline & & Mean & 7.31 & 8.07 & 19.5 \\
\hline & & SD & 1.82 & 0.37 & 0.6 \\
\hline & & Mln & 6.02 & 7.73 & 19.1 \\
\hline & & Max & 8.60 & 8.25 & 19.9 \\
\hline \multirow[t]{2}{*}{381} & 300 & 1 & 8.52 & 8.24 & 20.3 \\
\hline & & 2 & 4.97 & 7.57 & 19.0 \\
\hline \multirow[t]{2}{*}{$3 \mathrm{Cl}$} & & 1 & 8.50 & 8.37 & 20.3 \\
\hline & & 2 & 7.31 & 7.92 & 19.0 \\
\hline \multirow[t]{3}{*}{ 3C3 } & & 1 & 8.50 & 8.27 & 20.2 \\
\hline & & 2 & 3.71 & 7.52 & 19.1 \\
\hline & & Mean & 6.92 & 8,10 & 19.7 \\
\hline \multirow[t]{3}{*}{. } & & SD & 2.09 & 0.37 & 0.7 \\
\hline & & Min & 3.71 & 7.52 & 19.0 \\
\hline & & Max & 8.52 & 8.37 & 20.3 \\
\hline $3 \mathrm{C2}^{2}$ & $300 \mathrm{HD}$ & 1 & 8.36 & 8.31 & 19.2 \\
\hline \multirow[t]{5}{*}{$3 A 4$} & & 2 & 5.19 & 7.68 & 19.0 \\
\hline & & Mean & 6.78 & 8.10 & 19.1 \\
\hline & & SD & 2.21 & 0.45 & 0.1 \\
\hline & & Min & 5.19 & 7.68 & 19.0 \\
\hline & & Max & 8.36 & 8.31 & 19.2 \\
\hline
\end{tabular}

ust prlor to cxposure termination.

$$
\text { tom Number } \frac{1}{3}
$$


Study Number: AFH-X1-PS:IJDO-01

Lat Natebook 1

Tost Articla: MBt 401 (PF-CL 14EA [SOP])

Data Source: File Folder: 10

Forms: Water Quality - Form 2

Arricle Lot $H$ MBS-A01-110308Al-BD-3

Exposure Date: 12-May-11

Species Tested: HGE

During Exposure (DIssolved Dxygen, pH, Teriperature)

Glochidla Lot a 111500

Exposure Water Chemistry - Higgins Eye SDP

\begin{tabular}{|c|c|c|c|c|c|}
\hline Test & Treatment & Samcle & Dissolved & & Temperature \\
\hline Chamber & $(\mathrm{mg} / \mathrm{L})$ & Tlme & Oxygen $\{\mathrm{mg} /$ L & $p-1$ & $\left({ }^{\circ} \mathrm{C}\right)$ \\
\hline \multirow[t]{2}{*}{$2 \mathrm{B1}$} & 0 & 1 & 7.82 & 8.41 & 19.9 \\
\hline & & 2 & 828 & 8.39 & 19.0 \\
\hline \multirow[t]{2}{*}{$2 \mathrm{C} 3$} & & 1 & 7.85 & 8.38 & 19,0 \\
\hline & & 2 & 7.97 & 8,44 & 18.5 \\
\hline \multirow[t]{6}{*}{206} & & 1 & 8.23 & 8.46 & 20.1 \\
\hline & & 2 & B.15 & 8.44 & 19.0 \\
\hline & & Mean & 8.05 & 8,42 & 19.3 \\
\hline & & SD & 0.20 & 0.03 & 0.6 \\
\hline & & Min & 7.82 & 8,38 & 18.5 \\
\hline & & Max & 8.28 & 8.46 & 20.1 \\
\hline $2 \mathrm{AA}$ & 50 & 1 & 7.77 & 8.38 & 19.4 \\
\hline \multirow[t]{5}{*}{$2 \mathrm{A2}$} & & 2 & 7.40 & 8.08 & 19.1 \\
\hline & & Mean & 7.59 & 8.26 & 19.3 \\
\hline & & 50 & 0.26 & 0.21 & 0.2 \\
\hline & & Min & 7.40 & 8.08 & 19.1 \\
\hline & & Max & 7.77 & 8.38 & 19.4 \\
\hline $2 \mathrm{CS}$ & 100 & 1 & 8.55 & 8.29 & 19.3 \\
\hline \multirow[t]{5}{*}{204} & & 2 & 7.48 & 8.00 & 18.2 \\
\hline & & Mea, & 8.02 & 8,17 & 19.0 \\
\hline & & SD & 0.76 & 0.21 & 1.1 \\
\hline & & Min & 7.48 & 8.00 & 18.2 \\
\hline & & Max & 8.55 & 8.29 & 19,7 \\
\hline 282 & 200 & 1 & 7.97 & 8.30 & 19.3 \\
\hline \multirow[t]{5}{*}{282} & & 2 & 7.01 & 8.03 & 18.0 \\
\hline & & Mean & 7.49 & 8.19 & 18.7 \\
\hline & & SD & 0.68 & 0.19 & 0.9 \\
\hline & & Mln & 7.01 & 8.03 & 18.0 \\
\hline & & Max & 7.97 & 8.30 & 19.3 \\
\hline \multirow[t]{2}{*}{$2 \mathrm{B3}$} & 300 & 1 & 7.70 & 8.26 & 20.2 \\
\hline & & 2. & 4.08 & 7.58 & 18.5 \\
\hline \multirow[t]{2}{*}{$2 \mathrm{C1}$} & & 1 & 7.77 & 8.41 & 19.8 \\
\hline & & 2 & 7.78 & 7.99 & 18.5 \\
\hline \multirow[t]{6}{*}{$2 \mathrm{C} 2$} & & 1 & 7.81 & 8.39 & 20.0 \\
\hline & & 2 & 8.11 & 8.05 & 18.5 \\
\hline & & Mean & 7.21 & 8.19 & 19.3 \\
\hline & & SD & 1.54 & 0.31 & 0.8 \\
\hline & & MIn & 4.08 & 7.58 & 18.5 \\
\hline & & Max & 8.11 & 8.41 & 20.2 \\
\hline & $300 \mathrm{HD}$ & 1 & 7.97 & 8.34 & 20.0 \\
\hline \multirow[t]{5}{*}{284} & & 2 & 6.07 & 7.85 & 19.0 \\
\hline & & Mean & 7.02 & $8: 26$ & 19.5 \\
\hline & & SD & 1.34 & 0.35 & 0.7 \\
\hline & & MIn & 6.07 & 7.85 & 19,0 \\
\hline & & Max & 7.97 & 8.34 & 20.0 \\
\hline
\end{tabular}

Sample Thme 1 was measured upon

Page 4 of ${ }^{\circ}-9$ 
Study Vumber: AEH-11.PSEUDO-01

Lab Notebook 1

Data Sauree: Fle Folder:

Forms: Water Quakty - Form 2

Iest Art cle: MBDI 401 (PF-CL 145A [HUP])

Artic e Lot \#: 110607 WB-FD.E

Exposure Date: $18-0 \mathrm{ct}-1$

Ourling Exposure (Dissolved Oxyger, pH, Tamperature)

Specles rested: Was

Glochidla Lot \# 115500

Exposure Water Chemistry - Washboard FDP

\begin{tabular}{|c|c|c|c|c|c|}
\hline $\begin{array}{c}\text { Test } \\
\text { chamber }\end{array}$ & Treatment & $\begin{array}{c}\text { Sample } \\
\text { T/me }\end{array}$ & $\begin{array}{c}\text { Olssolved } \\
\text { Oxygen (mg/L) }\end{array}$ & $\mathrm{pH}$ & $\begin{array}{c}\text { Tempe'cture } \\
\left({ }^{\circ} \mathrm{C}\right)\end{array}$ \\
\hline \multirow[t]{2}{*}{$1 \mathrm{~A} 5$} & 0 & 1 & 7.58 & 8.41 & 18.6 \\
\hline & & 2 & 7.36 & 8.41 & 18.3 \\
\hline \multirow[t]{2}{*}{182} & & 1 & 7.66 & 8.39 & 18.7 \\
\hline & & 2 & 7.37 & 8.39 & 18.6 \\
\hline \multirow[t]{6}{*}{184} & & 1 & 7.45 & 8,40 & 18.7 \\
\hline & & 2 & 7.13 & 8.40 & 18.7 \\
\hline & & Mear & 7.43 & 8.40 & 18,6 \\
\hline & & SD & 0.19 & 0.01 & 0.2 \\
\hline & & Mln & 7.13 & $8.3 \mathrm{~s}$ & 18,3 \\
\hline & & Max & 7.66 & 8.41 & 18.7 \\
\hline \multirow[t]{2}{*}{$1 \mathrm{~A} 1$} & 50 & 1 & 7.75 & 8.40 & 18.1 \\
\hline & & 2 & 6.80 & $8.3 \mathrm{~s}$ & 17.7 \\
\hline \multirow[t]{2}{*}{$1 \mathrm{CI}$} & & 1 & 8.11 & 8.40 & 17.4 \\
\hline & & 2 & 7.11 & 8.40 & 17.3 \\
\hline \multirow[t]{6}{*}{$1 C 2$} & & 1 & 8.55 & 8.39 & 18.3 \\
\hline & & 2 & 7.54 & 8.40 & 18.1 \\
\hline & & Mean & 7.64 & 8.40 & 17,9 \\
\hline & & SD & 0.64 & 0.01 & 0.4 \\
\hline & & Mln & 6.80 & 8.39 & 17.3 \\
\hline & & $\operatorname{Max}$ & 8.55 & 8.40 & 18,3 \\
\hline \multirow[t]{2}{*}{$1 \mathrm{~A} 2$} & 100 & 1 & 9.25 & 8.41 & 18.8 \\
\hline & & 2 & 8.00 & $8.3 \mathrm{c}$ & 18.6 \\
\hline \multirow[t]{2}{*}{$1 \mathrm{~A} 6$} & & 1 & 9.11 & 8.41 & 18,6 \\
\hline & & 2 & 7.68 & 8.39 & 18.1 \\
\hline \multirow[t]{6}{*}{106} & & 1 & 8.90 & 8.41 & 18.2 \\
\hline & & 2 & 7.40 & 8.39 & 17.7 \\
\hline & & Mean & 8.39 & 8.40 & 18.9 \\
\hline & & SD & 0.79 & 0.01 & 0.4 \\
\hline & & Mln & 7.40 & 8.39 & 17.7 \\
\hline & & Max & 9.25 & 8.41 & 18.8 \\
\hline \multirow[t]{2}{*}{105} & 200 & 1 & 9.09 & 8.40 & 18.7 \\
\hline & & 2 & 7.44 & 8.49 & 18.5 \\
\hline \multirow[t]{2}{*}{$1 C_{3}$} & & 1 & 8.30 & 8.37 & 18.0 \\
\hline & & 2 & 7.60 & 8.37 & 18.2 \\
\hline \multirow[t]{6}{*}{164} & & 1 & 8.72 & 8.35 & 18.8 \\
\hline & & 2 & 7.59 & 8.35 & 18.7 \\
\hline & & Mean & 8.12 & 8.39 & 18.5 \\
\hline & & SD & 0.69 & 0.05 & 0.3 \\
\hline & & Min & 7.44 & 8.35 & 28.0 \\
\hline & & Max & 9.09 & 8.49 & 18.8 \\
\hline \multirow[t]{2}{*}{$1 \mathrm{~A} 4$} & 300 & 1 & 8.64 & 8,38 & 18.5 \\
\hline & & 2 & 7.37 & 8.37 & 18.2 \\
\hline \multirow[t]{2}{*}{$1 B 1$} & & 1 & 8.09 & 8.39 & 17.9 \\
\hline & & 2 & 7.36 & 8.36 & 17.6 \\
\hline \multirow[t]{6}{*}{$1 C 5$} & & 1 & 8.23 & 8.37 & 18.3 \\
\hline & & 2 & 7.55 & 8.35 & 18.3 \\
\hline & & Mean & 7.87 & 8,37 & 18.1 \\
\hline & & $5 \mathrm{D}$ & 0.53 & 0.01 & 0.3 \\
\hline & & Min & 7.36 & 8.35 & 17.6 \\
\hline & & $\operatorname{Max}$ & 8.64 & 8.39 & 18.5 \\
\hline \multirow[t]{2}{*}{$\overline{1 A 3}$} & $300 \mathrm{HO}$ & 1 & 8.44 & 8.40 & 18.6 \\
\hline & & 2 & 7.42 & 8.37 & 18.3 \\
\hline $1 \mathrm{~B} 3$ & & 1 & 8.09 & 8.39 & 18.3 \\
\hline & & 2 & 7.27 & 8,37 & 18.0 \\
\hline 186 & & 1 & 8.74 & 8.37 & 18.6 \\
\hline & & 2 & 7,07 & 8,37 & 18.3 \\
\hline & & Mean & 7.84 & 8.38 & 18.4 \\
\hline & & SD & 0.68 & $0.0=$ & 0.2 \\
\hline & & $\mathrm{M} \mid \mathrm{n}$ & 7.07 & 8.37 & 18.0 \\
\hline & & $\operatorname{Max}$ & 8.74 & 8.40 & 18.6 \\
\hline
\end{tabular}

just prior to exposure termination.

$$
\begin{aligned}
& 17 \\
& \text { Page } 5 \text { or } 9 \\
& \hline
\end{aligned}
$$


Study Number: AEH-11-P5EUDO-01

Lab ^otebook 1

Data Source: Fille =older: $10 \mathrm{~b}$

Forms: Water Quality - Form 2

During Exposure (Dlssclved Oxygen, 3H, Temperature!
Test Arti:le: $\quad$ MBI 401 (Pr. CL 1A5A [FDP])

Attcle Lot 1: $\quad 110607 \mathrm{WB}_{\mathrm{B}} \mathrm{FD} \cdot \mathrm{E}$

Exposure Date: $\quad 18-$ Oct-11

Specles Tested: PPB

Gloctidis 115400

Exposure Water Chemistry - Plain Pocketbook FDP

\begin{tabular}{|c|c|c|c|c|c|}
\hline Tes: & Treatment & Sample & Dlssolved & & Teriperature \\
\hline Chamber & $(\mathrm{mg} / \mathrm{L})$ & Time & Oxygen $(\mathrm{mg} / \mathrm{L})$ & $\mathrm{pH}$ & ("C) \\
\hline \multirow[t]{2}{*}{$3 A 6$} & 0 & 1 & 8.43 & 8.32 & 18.3 \\
\hline & & 2 & 8.00 & 8.29 & 17.7 \\
\hline \multirow[t]{2}{*}{$3 \mathrm{~B} .5$} & & 1 & 9.10 & 8.12 & 17.7 \\
\hline & & 2 & 7.74 & 8.22 & 17.2 \\
\hline \multirow[t]{6}{*}{$3 B 6$} & & 1 & 8.72 & 8.19 & 18.1 \\
\hline & & 2 & 8.04 & 8.25 & 17.6 \\
\hline & & Mean & 8.34 & 8.24 & 17.8 \\
\hline & & SD & 0.51 & 0.07 & 0.4 \\
\hline & & Min & 7.74 & 8.12 & 17.2 \\
\hline & & Max & 9.10 & 8.32 & 18.3 \\
\hline \multirow[t]{2}{*}{ 3A4 } & 50 & 1 & 8.70 & 8.26 & 18.1 \\
\hline & & 2 & 7.81 & 8.27 & 17.4 \\
\hline \multirow[t]{2}{*}{$3 C_{1}$} & & 1 & 8.64 & 8.28 & 17,3 \\
\hline & & 2 & 7.54 & 3.31 & 16.8 \\
\hline \multirow[t]{6}{*}{$3 \mathrm{C} 2$} & & 1 & 8.50 & 8.26 & 17.2 \\
\hline & & 2 & 7.59 & 8.31 & 16.6 \\
\hline & & Mean & 8.13 & 8.28 & 17.2 \\
\hline & & SD & 0.54 & 0.02 & 0.5 \\
\hline & & Min & 7.54 & 8.26 & 16.6 \\
\hline & & Max & 8.70 & 8.31 & 18.1 \\
\hline \multirow[t]{2}{*}{$3 \mathrm{~B} 1$} & 100 & 2 & 8.90 & 8.34 & 17.9 \\
\hline & & 2 & 7.46 & B.31 & 17.4 \\
\hline \multirow[t]{2}{*}{383} & & 1 & 8.88 & B.33 & 17.8 \\
\hline & & 2 & 7.25 & B.31 & 17.3 \\
\hline \multirow[t]{6}{*}{$3 C 6$} & & 1 & 8.65 & 8,34 & 17.7 \\
\hline & & 2 & 7.31 & 8.31 & 17.1 \\
\hline & & Mean & 8.08 & 8.32 & 17.5 \\
\hline & & SD & 0.81 & 0.02 & 0.3 \\
\hline & & Min & 7.25 & 8.31 & 17.1 \\
\hline & & $\operatorname{Max}$ & 8.90 & 8.34 & 17.9 \\
\hline \multirow[t]{2}{*}{$3 A 2$. } & 200 & 1 & 8.50 & 8,31 & 17.7 \\
\hline & & 2 & 7.55 & 8.31 & 17.1 \\
\hline \multirow[t]{2}{*}{$3 \mathrm{C} 3$} & & 1 & 8.41 & 8.34 & 17.3 \\
\hline & & 2 & 7.21 & 8.31 & 16.8 \\
\hline \multirow[t]{6}{*}{$3 \mathrm{C5}$} & & 1 & 8.55 & 8.31 & 18.1 \\
\hline & & 2 & 7.29 & 8.31 & 17,4 \\
\hline & & Mean & 7.92 & 8,32 & 17,4 \\
\hline & & SD & 0.63 & 0.01 & 0.5 \\
\hline & & Mln & 7.21 & 8.31 & 16.8 \\
\hline & & Max & 8.55 & 8.34 & 18.1 \\
\hline \multirow[t]{2}{*}{$3 A 3$} & 300 & 1 & 8.47 & 8.29 & 18.2 \\
\hline & & 2 & 7.71 & 8.29 & 17,6 \\
\hline \multirow[t]{2}{*}{$3 B 4$} & & 1 & 8.45 & 8.31 & 18.0 \\
\hline & & 2 & 7,54 & 8.30 & 17.3 \\
\hline \multirow[t]{6}{*}{$3 \mathrm{C4}$} & & 1 & 7.88 & 8.33 & 17.5 \\
\hline & & 2 & 6.81 & 8.51 & 15.8 \\
\hline & & Mean & 7.81 & 8.31 & 17.6 \\
\hline & & so & 0.62 & 0.02 & 0.5 \\
\hline & & Min & 6.81 & 8.29 & 16.8 \\
\hline & & $\operatorname{Max}$ & 8.47 & 8.33 & 18.2 \\
\hline $3 \mathrm{~A} 1$ & $300 \mathrm{HO}$ & 1 & 8.04 & 8.34 & 18.2 \\
\hline & & 2 & 6.04 & 8.31 & 17.7 \\
\hline $3 A 5$ & & 1 & 7.85 & $8.3 E$ & 18. C \\
\hline & & 2 & 6.90 & 8.32 & 17.5 \\
\hline 382 & & 1 & 7.97 & 8.34 & 17.7 \\
\hline & & 2 & 6.32 & 8.34 & 17.2 \\
\hline & & Mean & 7.19 & 8.34 & 17.7 \\
\hline & & so & 0.89 & 0.02 & 0.4 \\
\hline & & $\mathrm{M}$ ' & 6.04 & 8.31 & 17.2 \\
\hline & & $\operatorname{Max}$ & 8.04 & 8.36 & 18.2 \\
\hline
\end{tabular}

Sample Time 1 was measured upon exposure initiation and sample Time 2 was measured just prior to exposure termination.

$$
\text { Item Number } \frac{17}{6 \text { of } \frac{17}{6}}
$$


Study Number: AEH-11-PSELDDO-01

Lat Notebook 1

Data Source: Flle Folder; $10 \mathrm{~b}$

Forms Water Quality - Form

During Exposure (DIssolved Oxygen, pH, Temperature\}
Test Article: $\quad$ MEl 401 \{PF-CL 145A [SDP]]

Artcle Lot H. MB1-401 5DP 4655-12-MIX

Exposure Date: 17-Jan-1?

Exposure Water Chemistry - Black Sandshell SDP

\begin{tabular}{|c|c|c|c|c|c|}
\hline Test & Treatment & Sample & Dlssolved & & Tenperature \\
\hline Chamber & $(\mathrm{mg} / \mathrm{L})$ & Time & Oxygen $(\mathrm{mg} / \mathrm{L})$ & $\mathrm{pH}$ & $\left({ }^{\circ} \mathrm{C}\right)$ \\
\hline \multirow[t]{2}{*}{$1 \mathrm{~A} 5$} & 0 & 1 & 8.69 & 8.27 & 20.2 \\
\hline & & 2 & $B, 90$ & 8.55 & 20.0 \\
\hline \multirow[t]{2}{*}{181} & & 1 & 9.39 & 8.40 & 21.2 \\
\hline & & 2 & 9.27 & 8.56 & 20.1 \\
\hline \multirow[t]{6}{*}{106} & & 1 & 9.50 & 8.42 & 20.6 \\
\hline & & 2 & 9.24 & 8.55 & 20.4 \\
\hline & & Yean & 9.17 & 8.47 & 20.4 \\
\hline & & so & 0.31 & 0.12 & 0.4 \\
\hline & & Min & 8.69 & 8.27 & 20.0 \\
\hline & & Max & 9.50 & 8.56 & 21.2 \\
\hline \multirow[t]{2}{*}{$1 \mathrm{~A} 1$} & 50 & 1 & 9.47 & 8.41 & 21.0 \\
\hline & & 2 & 9.67 & $8.4 \bar{\varepsilon}$ & 20.4 \\
\hline \multirow[t]{2}{*}{$1 \mathrm{A3}$} & & 1 & 9.68 & 8.41 & 20.6 \\
\hline & & 2 & 9.68 & 8.44 & 20.0 \\
\hline \multirow[t]{6}{*}{$1 \subset 1$} & & 1 & 8.45 & 8.39 & 21,9 \\
\hline & & 2 & 9.18 & 8.34 & 20.4 \\
\hline & & Mean & 9,36 & 8.40 & 20.7 \\
\hline & & SD & 0.48 & 0.03 & 0.7 \\
\hline & & $\mathrm{Mn}$ & 8.45 & 8.34 & 20.0 \\
\hline & & $\operatorname{Max}$ & 9.68 & 8.44 & 21.9 \\
\hline \multirow[t]{2}{*}{$18 ?$} & 100 & 1 & 10.68 & 8.36 & 21.3 \\
\hline & & 2 & 8.97 & 8.33 & 20.5 \\
\hline \multirow[t]{2}{*}{183} & & 1 & 10.49 & 8.37 & 20.1 \\
\hline & & 2 & 8.90 & 8.38 & 20.3 \\
\hline \multirow[t]{6}{*}{105} & & 1 & 10.03 & 8.37 & 20.4 \\
\hline & & 2 & 8.44 & 8.32 & 20.3 \\
\hline & & Mear & 9.59 & 8.36 & 20.5 \\
\hline & & SD & 0.94 & 0.02 & 0.4 \\
\hline & & Min & 8.44 & 8.32 & 20.1 \\
\hline & & $\operatorname{Max}$ & 10.68 & 8.38 & 21.3 \\
\hline \multirow[t]{2}{*}{$1 \mathrm{AG}$} & 200 & 1 & 9.15 & 8.31 & 20.4 \\
\hline & & 2 & 8.46 & 8.30 & 20.2 \\
\hline \multirow[t]{2}{*}{185} & & 1 & 9.10 & 8.36 & 19.8 \\
\hline & & 2 & 8.34 & 8.37 & 19.8 \\
\hline \multirow[t]{6}{*}{$1 B 6$} & & 1 & 9.37 & 8.31 & 20.3 \\
\hline & & 2 & 7.90 & 8.21 & 20.5 \\
\hline & & Mean & 8.72 & 8.31 & 20.2 \\
\hline & & So & 0.57 & 0.06 & 0.3 \\
\hline & & Min & 7.90 & 8.21 & 19.8 \\
\hline & & $\operatorname{Max}$ & 9.37 & 8.37 & 20.5 \\
\hline \multirow[t]{2}{*}{$1 \mathrm{~A} 2$} & 300 & 1 & 10.23 & 8.26 & 20.8 \\
\hline & & 2 & 7,86 & 8.15 & 20.5 \\
\hline \multirow[t]{2}{*}{184} & & 1 & 8.11 & 8.31 & 20.2 \\
\hline & & 2 & 8.44 & 8.30 & 20.3 \\
\hline \multirow[t]{6}{*}{$1 \mathrm{C2}$} & & 1 & 10.02 & 8.28 & 21.2 \\
\hline & & 2 & 7.85 & 8.15 & 20.5 \\
\hline & & Mean & 8.75 & 8.25 & 20.6 \\
\hline & & SD & 1.09 & 0.07 & 0.4 \\
\hline & & Mln & 7.85 & 8.15 & 20.2 \\
\hline & & Max & 10.23 & 8.31 & 21.2 \\
\hline $1 \mathrm{AM}$ & $300 \mathrm{HD}$ & 1 & 8.34 & 8.33 & 18.3 \\
\hline & & 2 & 8.65 & 8.50 & 18.5 \\
\hline $1 C 3$ & & 1 & 10.30 & 8.37 & 20.4 \\
\hline & & 2 & 9.11 & $8.3 \mathrm{~S}$ & 20.0 \\
\hline $1 C 4$ & & 1 & 10.45 & 8.37 & 20.3 \\
\hline & & 2 & 8.88 & 8.27 & 20.1 \\
\hline & & Mean & 9.29 & 8.38 & 19.6 \\
\hline & & SD & 0,88 & 0.08 & 0.9 \\
\hline & & Mln & 8.34 & 8.27 & 18.3 \\
\hline & & $\operatorname{Max}$ & 10,45 & 8.50 & 20.4 \\
\hline
\end{tabular}




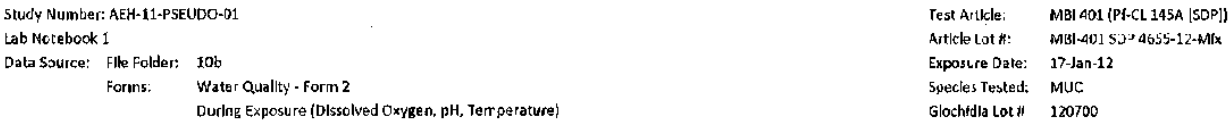

Exposure Water Chemistry - Mucket SDP

\begin{tabular}{|c|c|c|c|c|c|}
\hline Test & Treatment & Sample & Dissolved & & Termperature \\
\hline Chamoer & $(m g / 2)$ & rime & Oxysen (mig/L) & $\mathrm{pH}$ & ('C) \\
\hline \multirow[t]{2}{*}{ 2BA } & 0 & 1 & 8.64 & 8.34 & 20.1 \\
\hline & & 2 & 7.44 & 8.52 & 20.1 \\
\hline \multirow[t]{2}{*}{$2 B 5$} & & 1 & 8.02 & 8.37 & 20,0 \\
\hline & & 2 & 8.32 & 8.53 & 20.0 \\
\hline \multirow[t]{6}{*}{$2 \mathrm{C1}$} & & 1 & 9.30 & 8,28 & 20.2 \\
\hline & & 2 & 8.66 & 8.52 & 20.3 \\
\hline & & Mean & 8.40 & 8,45 & 20.1 \\
\hline & & SD & 0.63 & 0.09 & $c .1$ \\
\hline & & Min & 7.44 & 8.34 & 20.0 \\
\hline & & $\operatorname{Max}$ & 9.30 & 8.53 & 20,3 \\
\hline \multirow[t]{2}{*}{$2 A L$} & 50 & 1 & 9.85 & 8.93 & 20.0 \\
\hline & & 2 & 8.89 & 8,38 & 20.3 \\
\hline \multirow[t]{2}{*}{286} & & 1 & 9.55 & 8.36 & 20.1 \\
\hline & & 2 & 8.80 & 8.98 & 20.3 \\
\hline \multirow[t]{6}{*}{$2 \mathrm{Ca}$} & & 1 & 9.43 & 8.36 & 20.0 \\
\hline & & 2 & 8.70 & 8.43 & 20.0 \\
\hline & & Mean & 9.20 & 8.37 & 20.1 \\
\hline & & SD & 0.47 & 0.03 & 0.1 \\
\hline & & Min & 8.70 & 8.93 & 20,0 \\
\hline & & Max & 9.85 & 8.43 & 20.3 \\
\hline \multirow[t]{2}{*}{$2 \mathrm{~A} 2$} & 100 & 1 & 8.88 & 8,32 & 20.1 \\
\hline & & 2 & 8.72 & 8.37 & 20.3 \\
\hline \multirow[t]{2}{*}{282} & & 1 & 8.72 & 8.34 & 19.7 \\
\hline & & 2 & 8.44 & 8,31 & 20.2 \\
\hline \multirow[t]{6}{*}{$2 \mathrm{Cb}$} & & 1 & 8.72 & 8,35 & 19.3 \\
\hline & & 2 & 8.43 & 8.43 & 19.2 \\
\hline & & Mean & 8.65 & 8.36 & 19.8 \\
\hline & & SD & 0.18 & 0.64 & 0.5 \\
\hline & & Mln & 8.43 & 8.31 & 19.2 \\
\hline & & Vax & 8.88 & 8.43 & 20.3 \\
\hline \multirow[t]{2}{*}{$2 \mathrm{~A} 5$} & 200 & 1 & 9.44 & 8.32 & 19,9 \\
\hline & & 2 & 8.46 & 8.35 & 19.8 \\
\hline \multirow[t]{2}{*}{$2 A G$} & & 1 & 9.14 & 8.30 & 19.9 \\
\hline & & 2 & 8.56 & 8.35 & 19.5 \\
\hline \multirow[t]{6}{*}{$2 \mathrm{C} 2$} & & 1 & 9.60 & 8.26 & 19.6 \\
\hline & & 2 & 8.57 & 8.40 & 19.7 \\
\hline & & Mean & 8.96 & 8.33 & 19.7 \\
\hline & & SD & 0.50 & 0.05 & 0.2 \\
\hline & & Mln & 8.46 & 8.25 & 19.5 \\
\hline & & Vax & 9.60 & 8.40 & 19.9 \\
\hline \multirow[t]{2}{*}{281} & 300 & 1 & 8,33 & 8.29 & 20.1 \\
\hline & & 2 & 8.78 & 8.34 & 20.3 \\
\hline \multirow[t]{2}{*}{283} & & 1 & 8.8 .5 & 8.29 & 19.6 \\
\hline & & 2 & 8.83 & 8.32 & 19.6 \\
\hline \multirow[t]{6}{*}{$2 \mathrm{C4}$} & & 1 & 9.07 & $8.3 \mathrm{C}$ & 19,8 \\
\hline & & 2 & 8.60 & 8.38 & 19.6 \\
\hline & & Mean & 8.74 & 8.32 & 19,8 \\
\hline & & SD & 0.25 & 0.04 & 0.3 \\
\hline & & Min & 8.33 & 8.29 & 19.6 \\
\hline & & Vax & 9.07 & 8.38 & 20.3 \\
\hline $2 A 1$ & $300 \mathrm{HD}$ & 1 & 8.93 & 8.30 & 20.0 \\
\hline & & 2 & 8.33 & 8.54 & 19.9 \\
\hline $2 A ?$ & & 1 & 8.71 & 8.32 & 18.8 \\
\hline & & 2 & 8.26 & 8.23 & 20.5 \\
\hline $2 \mathrm{CS}$ & & 1 & 9.00 & $8.3 \pm$ & 19.7 \\
\hline & & 2 & 8.55 & 8.47 & 19.4 \\
\hline & & Mean & 8.63 & 8.32 & 19.7 \\
\hline & & SD & 0.31 & 0.06 & $0 . \tilde{6}$ \\
\hline & & Mln & 8.26 & 8.23 & 18.8 \\
\hline & & Max & 9.00 & 8.42 & 20.5 \\
\hline
\end{tabular}

Sample Time 1 was measured uson exposurc nittiatlon and Sample Tline 2 was més just prou to exposure lerninatlon.

$$
\text { Item Number } \frac{19}{8}
$$


StuJy Number: AEH-11-PSEUDO-01

Lab Notebook 1

Data Source: File Folder: 10 forms:
Waler Quallty - Farm 2

During Exposare (Dissolved Oxygen, pH, Temperature)
Test Artec: MBl 401 (Pf-Cl 145A (SDP])

Arlicle Lot H: MB1-401 SDP A655-12-Mix

Exposure Date: 19-an-12

Specles Testec: HIC

Gloclidlatule 120000

Exposure Water Chemistry - Hickorynut SDP

\begin{tabular}{|c|c|c|c|c|c|}
\hline Test & Treatment & Sarple & Dissolved & & Temperature \\
\hline Chamber & $(\mathrm{mg} / L)$ & Tlme & Oxygen (mg/L) & $\mathrm{pH}$ & $\left({ }^{\circ} \mathrm{C}\right)$ \\
\hline \multirow{2}{*}{$3 A 2$} & 0 & 1 & 10.32 & 8.36 & 20.1 \\
\hline & & 2 & 10.73 & 8.51 & 19,9 \\
\hline \multirow[t]{2}{*}{$3 \mathrm{Cl}$} & & 1 & 8.46 & $8.3 \epsilon$ & 20.1 \\
\hline & & 2 & 9.55 & 8.43 & 19.8 \\
\hline \multirow[t]{6}{*}{$3 C 4$} & & 1 & 9.88 & 8.36 & 20.0 \\
\hline & & 2 & 9.95 & 8.50 & 19,5 \\
\hline & & Mean & 9.82 & 8.42 & 19.9 \\
\hline & & 50 & 0.78 & 0.07 & 0.2 \\
\hline & & $\mathrm{Mn}$ & 8.46 & 8.36 & 19.5 \\
\hline & & $\operatorname{Max}$ & 10.73 & 8.51 & 20.1 \\
\hline \multirow[t]{2}{*}{ 3A5 } & 50 & 1 & 10.37 & 8.39 & 20.0 \\
\hline & & 2 & 10.06 & 8.42 & 19.5 \\
\hline \multirow[t]{2}{*}{383} & & 1 & 11.00 & 8,37 & 19.7 \\
\hline & & 2 & 10.43 & 8.48 & 18.8 \\
\hline \multirow[t]{6}{*}{$3 B 6$} & & 1 & 10.36 & 8.32 & 20.0 \\
\hline & & 2 & 10.23 & 8.34 & 19.9 \\
\hline & & Mean & 10.41 & 8.38 & 19.7 \\
\hline & & SD & 0.32 & 0.06 & 0.5 \\
\hline & & Min & 10.06 & 8.32 & 18.8 \\
\hline & & $\operatorname{Max}$ & 11.00 & 8.48 & 20.0 \\
\hline \multirow[t]{2}{*}{$3 B 1$} & 100 & 1 & 10.91 & 8.34 & 20.1 \\
\hline & & 2 & 10.09 & 8.38 & 19.7 \\
\hline \multirow[t]{2}{*}{$3 \mathrm{C} 2$} & & 1 & 10.00 & 8.36 & 19.6 \\
\hline & & 2 & 9.91 & 8.45 & 18,6 \\
\hline \multirow[t]{6}{*}{$3 \mathrm{C5}$} & & 1 & 10.35 & 8.32 & 20.0 \\
\hline & & 2 & 9,74 & $8.3 c$ & 19.6 \\
\hline & & Mean & 10.16 & $8.3 E$ & 19.6 \\
\hline & & so & 0.42 & 0.05 & 0.5 \\
\hline & & Min & 9.74 & $8,5 \mathrm{C}$ & 18.6 \\
\hline & & $\max$ & 10.91 & 8.65 & 20.1 \\
\hline \multirow[t]{2}{*}{$3 A 3$} & 200 & 1 & 9.95 & 8.24 & 20.0 \\
\hline & & 2 & 9.08 & $8.1 \mathrm{C}$ & 19.9 \\
\hline \multirow[t]{2}{*}{$3 A 4$} & & 1 & 9.87 & 8.27 & 19,9 \\
\hline & & 2 & 9.49 & 8.24 & 19.7 \\
\hline \multirow[t]{6}{*}{$3 C 6$} & & 1 & 9.96 & 8.32 & 19,9 \\
\hline & & 2 & 9.66 & 8.35 & 18.6 \\
\hline & & Mean & 9.67 & 8.26 & 13.7 \\
\hline & & SD & 0,34 & 0.05 & 0.5 \\
\hline & & Min & 9.08 & 8.10 & 18.6 \\
\hline & & Max & 9.96 & 8.35 & 23,0 \\
\hline \multirow[t]{2}{*}{$3 A 6$} & 300 & 1 & 10.70 & 8.18 & 19.9 \\
\hline & & 2 & 8.58 & 8.10 & 19.8 \\
\hline \multirow[t]{2}{*}{382} & & 1 & 10.45 & 8.21 & 19.8 \\
\hline & & 2 & 8.96 & 8.15 & 19.7 \\
\hline \multirow[t]{2}{*}{385} & & 1 & 10.31 & 8.30 & 19.7 \\
\hline & & 2 & 8.95 & 8.26 & 19.4 \\
\hline & & Mean & 9.66 & 8.21 & 19.7 \\
\hline & & so & 0.93 & 0.07 & 0.2 \\
\hline & & Min & 8.58 & 8.10 & 19.4 \\
\hline & & $\operatorname{Max}$ & 10,70 & 8.30 & 19.9 \\
\hline $3 A 1$ & $300 \mathrm{HD}$ & 1 & 10.75 & 8.11 & 20.1 \\
\hline & & 2 & 9.97 & 8.14 & 19.7 \\
\hline $3 B 4$ & & 1 & 10.64 & 8.13 & $20 . c$ \\
\hline & & 2 & 10.68 & 8.36 & 17.4 \\
\hline $3 C 3$ & & 1 & 10.76 & 8.15 & 19.8 \\
\hline & & 2 & 10.61 & 8.32 & 19.0 \\
\hline & & Mear & 10.57 & 8.21 & 19.3 \\
\hline & & SD & 0.30 & 0.11 & $\therefore 0$ \\
\hline & & Min & 9.97 & 8..1 & 17,4 \\
\hline & & Max & 10.76 & 8.36 & 20.1 \\
\hline
\end{tabular}




\begin{tabular}{|c|c|c|c|c|}
\hline \multirow{2}{*}{\multicolumn{2}{|c|}{$\begin{array}{l}\text { Study Number: AEH-11-PSEUDO-01 } \\
\text { Lab Notebook: } 1\end{array}$}} & Actlon & Date & Injtials \\
\hline & & Created....... & 9-Mar-15 & TJS Th \\
\hline \multirow{2}{*}{ Data Source: } & File Fo der: $10 \mathrm{~b}$ & Revised...... & 9-Mar-15 & TIS tos \\
\hline & Fcrms: Water Quality - Form 2 & \begin{tabular}{|l|} 
Reviewed... \\
\end{tabular} & $9-M A R \cdot 15$ & 78 \\
\hline
\end{tabular}

\section{Exposure Light Intensity}

Test Article: Fseudomonas fluorescens (Pf-CL145A) spray dried powder (SDP) and freeze dried powder (FOP) Article Lot \#s: SDP = MBI-401-110308AJ-BD-3 and MBI-401 SDP 46S5-12-Mix; FDP = 110607WB-F D-E

Exposure Dates: 12-MaY-11, 18-Oct-11, 17-Jan-12, and 19-Jan-12

Specles Tested: PPB, FAM, HGE, WAS, BLS, MUC, and HIC

Data Explanation:

Light intensity was measured twice during exposure:

1) Upon exposure initiation

2) Prlor to exposure terminatlon

Species Abbreviations

PPB - plain pocketbook (Lampslis cardium)

FAM - fatmucket (Lampsilis siliquoidea)

HGE - Higgins eye (Lompsilss higginsll)

WAS - washboard (Megalonaias nervosa)

BLS - black sandshell (Ligumia recto)

MUC - mucket (Actinonaias Iigamentina)

HiC - hickorynut (Obovaria olivaria)

Data anomalles and deviations:

none

I:em Number 18

Page 1 of 2 
STudy Number: AEH-11-PSEUDO-01

Lab Notcbook 1

Data Source: Fille folder: 10

Forms: Water Quality - Form 2

Burlng Exposure (Dissolved Oxygen, pH, Temperature) [Llght Intenstiy]
Test Article: NE1 401 (Pf-CL 145A [SDP] and [FDP])

Article Lot \#: MBI-401-110308Al-BD-3, MB1-401 5DP 4655-12-MIX, 110607WB-FI-E

Exposure Jates: 12-May-11, 18-Oct-11, 17-Jan-12, and 19-tan-12

Specles Tested; PPB, FAM, HGE, WAS, BLS, MUC, and HIC

Exposure Light Intensity

\begin{tabular}{|c|c|c|c|c|}
\hline Spccies & Formulation & Test Date & $\begin{array}{c}\text { Sample } \\
\text { Time } \\
\end{array}$ & $\begin{array}{c}\text { Light } \\
\text { Intensity (lux) }\end{array}$ \\
\hline \multirow[t]{2}{*}{ PPB } & SDP & 12-May-11 & $\begin{array}{l}1 \\
2\end{array}$ & $\begin{array}{l}204 \\
242\end{array}$ \\
\hline & & & $\begin{array}{c}\text { Mean } \\
\text { SD }\end{array}$ & $\begin{array}{l}223 \\
27 \\
\end{array}$ \\
\hline \multirow[t]{2}{*}{ FAM } & SDP & 12-May-11 & $\begin{array}{l}1 \\
2\end{array}$ & $\begin{array}{l}239 \\
211\end{array}$ \\
\hline & & & $\begin{array}{c}\text { Mean } \\
\text { SD }\end{array}$ & $\begin{array}{l}225 \\
20 \\
\end{array}$ \\
\hline \multirow[t]{2}{*}{$\mathrm{HGE}$} & SDP & $12-$ - $a y-11$ & $\begin{array}{l}1 \\
2 \\
2\end{array}$ & $\begin{array}{l}218 \\
220\end{array}$ \\
\hline & & & $\begin{array}{c}\text { Mean } \\
\text { SD }\end{array}$ & $\begin{array}{c}219 \\
1 \\
\end{array}$ \\
\hline \multirow[t]{2}{*}{ PPB } & FDP & $18-0 \mathrm{ct}-11$ & $\begin{array}{l}1 \\
2\end{array}$ & $\begin{array}{c}120 \\
96\end{array}$ \\
\hline & & & $\begin{array}{c}\text { Mean } \\
\text { SD }\end{array}$ & $\begin{array}{l}108 \\
17\end{array}$ \\
\hline \multirow[t]{2}{*}{ WAS } & FDP & $18-0 \mathrm{ct}-11$ & $\begin{array}{l}1 \\
2\end{array}$ & $\begin{array}{l}500 \\
571\end{array}$ \\
\hline & & & $\begin{array}{c}\text { Mean } \\
\text { SD }\end{array}$ & $\begin{array}{c}536 \\
50 \\
\end{array}$ \\
\hline & SDP & $17-\operatorname{Jan}-12$ & $\begin{array}{l}1 \\
2 \\
\end{array}$ & $\begin{array}{l}283 \\
465\end{array}$ \\
\hline & & & $\begin{array}{c}\text { Mean } \\
\text { SD }\end{array}$ & $\begin{array}{l}374 \\
129\end{array}$ \\
\hline \multirow[t]{2}{*}{$M U C$} & SDP & 17-Јaๆ-12 & $\begin{array}{l}1 \\
2\end{array}$ & $\begin{array}{l}176 \\
277\end{array}$ \\
\hline & & & $\begin{array}{c}\text { Mean } \\
\text { SD }\end{array}$ & $\begin{array}{r}227 \\
71 \\
\end{array}$ \\
\hline \multirow[t]{3}{*}{ HIC } & SOP & 19-Jan-12 & $\begin{array}{l}1 \\
2\end{array}$ & $\begin{array}{l}228 \\
295\end{array}$ \\
\hline & & & $\begin{array}{c}\text { Mean } \\
\text { SD }\end{array}$ & $\begin{array}{c}262 \\
47 \\
\end{array}$ \\
\hline & & & $\begin{array}{c}\text { Grand Mean } \\
\text { SD } \\
\text { Min } \\
\text { Max }\end{array}$ & $\begin{array}{c}272 \\
132 \\
96 \\
571 \\
\end{array}$ \\
\hline
\end{tabular}

Time 2 was measured just prior to exposure termination.

\begin{tabular}{l} 
Item Number $\frac{18}{2}$ \\
Page of 2 \\
\hline
\end{tabular} 


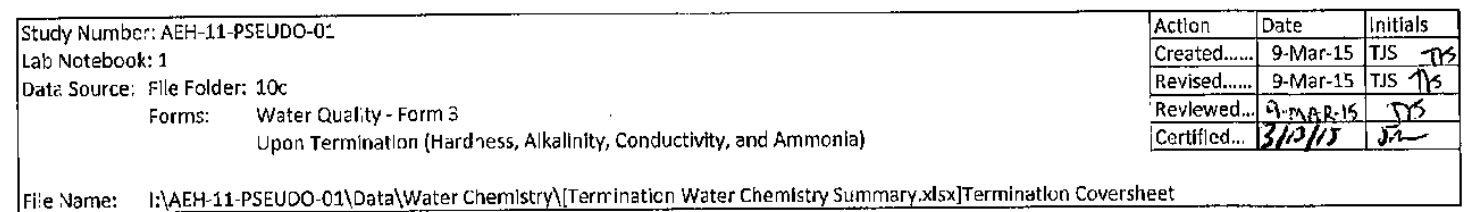

\section{Terminatlon Water Chemistry}

Test Article: Pseudomonas fluorescens (Pf-CL145A) spray dried powder (SDP) and freeze dried powder (FDP)

Article Lot \#5: SD? = MBi-401-110308Al-BD-3 ard MBI-401 SOP 4655-12-Mix; FDP = 1.10607WB-FD-E

Exposure Dates: 12-May-11, 18 Cct-11, 17-Jan-12, and 19-Jan-12

Species Tested: PPB, FAM, HGE, WAS, BLS, MUC, and HIC

Data Explanation:

$300 \mathrm{mg} / \mathrm{LHD}=$ Heat Deactivated

Species Abbreviations

$\mathrm{PPB}$ - plain pocketbook (Lampsilis cardium)

FAM - fatmucket (Lampsills siliquoldea)

HGE - Higgins eve (Lampsills higginsil\}

WAS - wash board (Megatonalas nervosa)

BLS - black sandshell (Ligumla recta)

MUC- mucket (Actinonalas ligamentina)

HIC- hickorynut (Obovoria olivaria)

Upon termination, all replicate treatment chambers (3) were pooled and water chemistry was measured from pooled sample.

Data anomalles and deviations:

Upon termination of 12-May-11 SDP exposure, one control replicate from chamber 281 (HGE) was accldentally pooled wlth control replicates from block 1 (PPB); this resulted in controls for PPB having 4 pooled control replicates and cuntrols for HGE having only 2 pooled replicates.

Item Number $\frac{9}{1}$ 


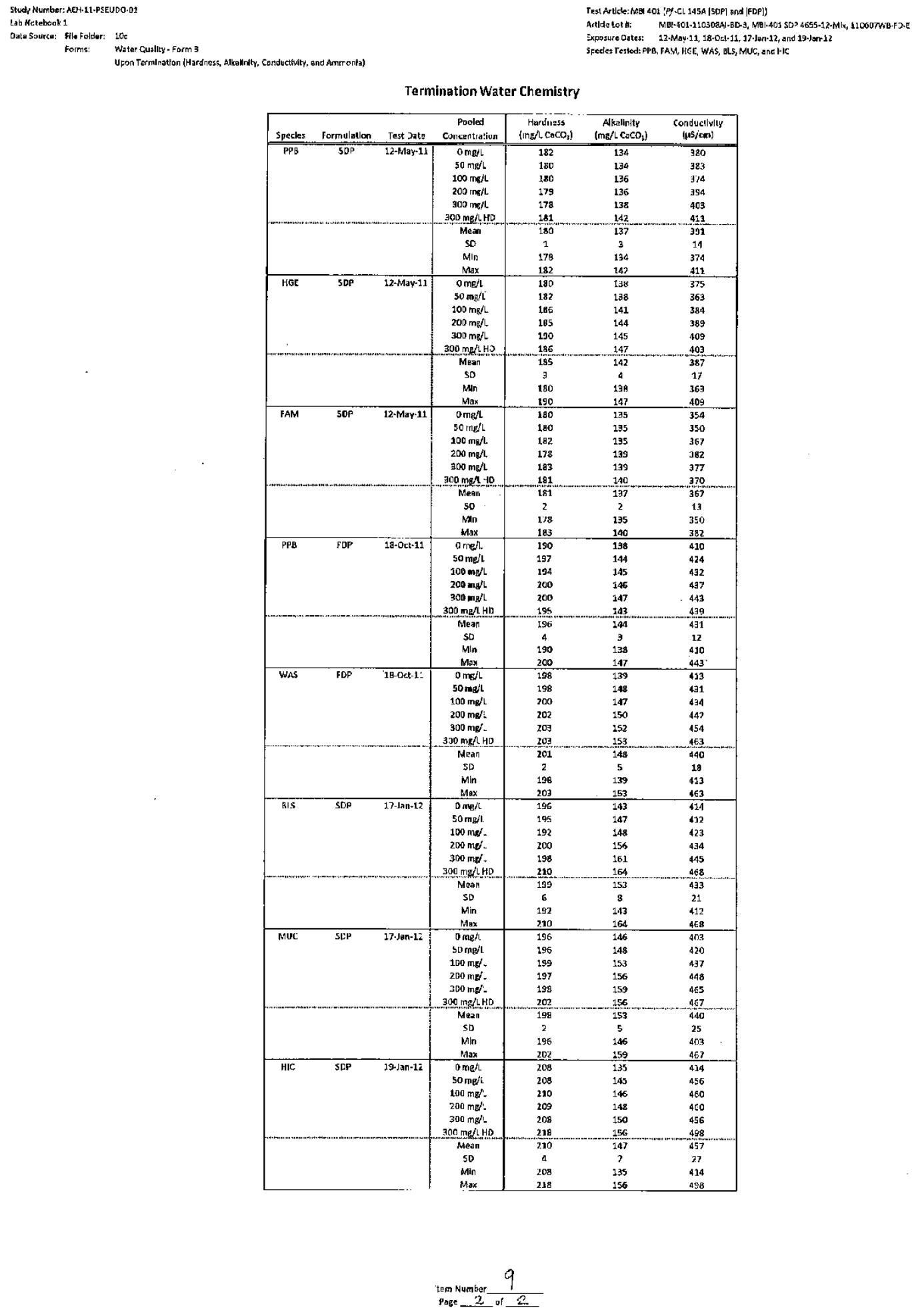


File Folder: $10 d$

Sample Description: $3 \mathrm{~mL}$ acidlfied exposure water $(0.45 \mu \mathrm{m}$ filtrated $)$ Study \#: AEH-11-PSEUDO-D1

Upper Midwest Environmental Sciences Center attn: James L.uoma

2630 Fanta Reed Road

La Crosse, WI54603

REPORT OF ANALYSIS

Sample Code

PPB3A1

PPB3A2

PPB3A3

PPB3A4

PPB3AS

PPB3A6

PPB3B1

PPB3B2

PPB3B3

PPB384

PPB3B5

РPB3B5

РPB3C1

PPB3C2.

PPB3C3

PPB3C4

PPB3C5

PPB3C6

WAS1A1

WAS1A2

WAS1A3

WAS1A4

WASIA5

WAS1A6

WASIB1

WAS1B2

WAS1B3

WAS1B4

WAS1B5

WAS1B6

WAS1C1

WAS1C2

WAS1C3

WAS1C4

WAS1C5

WA51C6

USGS

Water Quality Laboratory

Laboratory Director

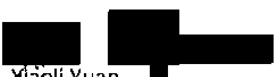

Yiàoli Yuan
Water Quality Laboratory

Upper Midwest Environmental Sciences Center

USGS

2630 Fanta Reed Road

La Crosse, W/ 54603

Analyzing Date: $11 / 07 / 2011$

Report Date: 11/09/2011

Results (Total Ammonia Nitrogen, mg $\mathrm{NH}_{3}-\mathrm{N} / \mathrm{L}$ ) 
File folder: $10 d$

Sarple Description: $3 \mathrm{~mL}$ acidiffed exposure vater $(0.45 \mu \mathrm{m}$ filtrated $)$ Study \#: AEH-11-PSEUDO-01

Upper Midwest Environmental Sciences Center

attn: James Luoina

2630 Fanta Reed Road

La Crosse, W154603

REPORT OF ANALYS|S

Sample Code

BL51A1:24

B151A2T24

BL1A3T24

BLS1A4T24

BLSIAST24

BLSIAG'24

BL51B1T24

BLS1B2T24

BLS1B3T24

BLS1B4T24

BLS165T24

BLS186T2A

BLS1C:T24

BLS1C2T24

BL51C3124

BLS1C4T24

BLS1C5T24

BLS1C6T24

HIC3A:T24

HIC3A2T24

HIC $3 \wedge 3 T 2 A$

HIC3A4T24

HIC3A5T24

HIC3A6T24

HIC3日1T2A

HIC. $3 \mathrm{H} 2 \mathrm{T2A}$

НIC3B 3 T2A

HIC3B4T24

HIC3B5T2A

HIC3B6T24

HC3C1T24

HIC3C2T2A

AIC3C3T24

HIC3C4T24

HIC3C5T24

НIC3С6T24

MUC2A1T24

MUC2A2T24

MUC2A3T24

MUC2A4T24

MUC2A5T24

MUCZAGT24

MUC2B1T24

MUC2B2T24

MUC2B3 I2.4

MUC2B4T24

MUC2B5T24

MUC2B6T24

MUC2C1T24

MUC2C2T24

MUC2C3T24

MUC2C4T24

Muc2C5T24

MUC2C6T24

USGS

Waler Quality Laborator

Laboratory Directo

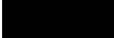

Xifali Yvan
Water Quallty Laboratory

Upper Midwest Enviranmental Sciences Center

USGS

2630 fanta Reed Road

La Crosse, WI54603

Asalyzing Date: 01/25/2012

Aeport Date: 02/03/2012

Results (Total Ammonia Nitrogen, me NH $-\mathrm{N} / \mathrm{L}$ )

0.215

1.661

0.241

0.247
0.082

1.078

0.079
0.698

0.719

1.187

0.916

1.167
0.436

1.509

0.245

0.724

0.08

0.885

0.082

0.263

0.263
0.265

0.482

0.533

0.231

0.107

0.192

0.266
0.084

0.094

0.474

0.032

0.473
0.213

0.537

0.691

0.795

0.088

0.861

0.777

1.136
0.43

0.916

0.052

0.052

0.533

0.921

0.183

1.088

0.212

$\mathrm{Pg} \perp$ of 1 


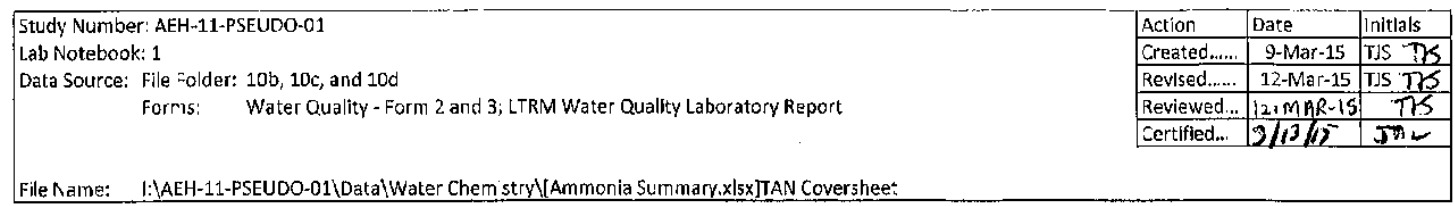

\section{Total Ammonia Nitrogen Coversheet}

Test Article: Pseudomonos fluorescens ( $P f$-Cl.145A) spray drled powder (SDP) and freeze drled powder (FDP) Article Lot H5: SDP $=$ MBl-401-110308Al-BD-3 and MBI-401 SDP 4655-12-V.ix; FDP = 110607WD-FD-E Exposure Dates: 12-May-11, 18-Oct-11, 17-Jan-12, and :9-Jan-12 Species Tested: PPB, FAM, HGE, WAS, BLS, MUC, and HIC

Data Explanation:

Specles Abbreviations

$$
\begin{aligned}
& \text { PPB - plaln pocketbook (Lampsillis cardium) } \\
& \text { FAM - fatmucket (Lampsilis sillquoidea) } \\
& \text { HGE - Hlggins eve (Lompsills hlgginsil) } \\
& \text { WAS - washboard (Megalonalas nervosa) } \\
& \text { BLS - black sandshell (Ugumia recto) } \\
& \text { MUC - mucket (Actinonaias igamentina) } \\
& \text { HIC - hIckorynut (Obovaria ollvaria) }
\end{aligned}
$$

For $5: P$ treatrrents inltiated on 12-May-11. (FAM, PPB, and HGE), total ammonla רitrogen was measured using a YSI 9000 photometer. For, all ather treatments (SDP and FDP), total ammonla nitrogen analyses were conducted by the UMESC LTRM Water Quality Laboratory using the authomated phenate method.

$\mathrm{pH}-\mathrm{H}$ means were calculated on hydrogen ion concentration; $\mathrm{pH}$ standarc deviations were calculated on $\mathrm{pH}$ values.

TAN toxlcity is determined according to U.S. Env|ronmental Protection Agency, 2013, Ambient Water Quality Criteria for Ammonia -Freshwater 2013. Washington, D.C., Office of Water, EPA 822-R-13-001, $242 \mathrm{p}$. The EPA document sets the acute criterlon magnitude at $17 \mathrm{mg} \mathrm{TAN} / \mathrm{L}\left(1\right.$ hour average, $\left.\mathrm{pH} 7,20^{\circ} \mathrm{C}\right)$ and a chronic criterion magnitude at $1.9 \mathrm{mg}$ TAN/L $\left(30\right.$-day rolling averagc, $\left.\mathrm{pH} 7,20^{\circ} \mathrm{C}\right)$ with the stipulation that the chronlc criterion connot exceed a 4 -day average of $4.8 \mathrm{mg} / \mathrm{L}$.

Data anomalles and deviations:

For the PPB (SDP) exposures on 12-May-11, $\mathrm{pH}$ and temperature data were not measured on pooled samples used to measure total armmonia nitrogen. Therefore, the mean $\mathrm{pH}$ and temperature of each treatment group measured at the end of exposure period (24 hour) were used to calculate the TAN toxicity.

$$
\text { Item Vumber } \frac{3}{10}
$$


Plain Pocketbook (SDP) Total Ammonia Nitrogen

\begin{tabular}{|c|cccc|}
\hline $\begin{array}{c}\text { Treatment } \\
(\mathrm{mg} / \mathrm{L})\end{array}$ & $\begin{array}{c}\text { Temperature } \\
\left.\left({ }^{\circ} \mathrm{C}\right)\right)^{\prime}\end{array}$ & $\mathrm{pH}^{\prime}$ & $\begin{array}{c}\text { Total Ammonia } \\
\text { Nitrogen }\left(\mathrm{mg} \mathrm{NH} \mathrm{H}_{3} \mathrm{~N} / \mathrm{L}\right)\end{array}$ & $\begin{array}{c}\text { Ammonia } \\
\text { Toxicity }\end{array}$ \\
\hline \hline 0 & 19.2 & 8.34 & 0.06 & $\mathrm{NT}$ \\
50 & 19.2 & 8.25 & 0.08 & $\mathrm{NT}$ \\
100 & 19 & 8.03 & 0.13 & $\mathrm{NT}$ \\
200 & 19 & 8.09 & 0.24 & $\mathrm{NT}$ \\
300 & 19.2 & 7.82 & 0.44 & $\mathrm{NT}$ \\
$300 \mathrm{HD}$ & 19.1 & 8.07 & 0.51 & $\mathrm{NT}$ \\
\hline Grand Mean & 19.1 & 8.10 & 0.24 & $\mathrm{NT}$ \\
SD & 0.1 & 0.18 & 0.19 & $\mathrm{NA}$ \\
\hline
\end{tabular}

$N T^{\top}=$ non-toxic levels of total ammonia nitrogen (below chronic and acute levels).

Note: Control water sample from $2 B 1$ (HGE) was accidentally pooled with the 3lock 1 (PPB)

Control group, therefore four control samples were pooled for determination of PPB control TAN.

Plain Pocketbook (SDP) End of Exposure $\mathrm{pH}$ and Temperature

\begin{tabular}{|cc|cc|}
\hline $\begin{array}{c}\text { Test } \\
\text { Chamber }\end{array}$ & $\begin{array}{c}\text { Treatment } \\
(\mathrm{mg} / \mathrm{L})\end{array}$ & $\mathrm{pH}$ & $\begin{array}{c}\text { Temperature } \\
\left({ }^{\circ} \mathrm{C}\right)\end{array}$ \\
\hline $1 \mathrm{~A} 3$ & 0 & 8.23 & 19.1 \\
$1 \mathrm{~B} 1$ & & 8.35 & 19.5 \\
$1 \mathrm{C} 3$ & & 8.41 & 19.0 \\
\hline & Mean & 8.34 & 19.2 \\
\hline $1 \mathrm{~A} 1$ & 50 & 8.25 & 19.2 \\
\hline & Mean & 8.25 & 19.2 \\
\hline $1 \mathrm{~A} 4$ & 100 & 8.03 & 19.0 \\
\hline & Mean & 8.03 & 19.0 \\
\hline $1 \mathrm{CC}$ & 200 & 8.09 & 19.0 \\
\hline & Mean & 8.09 & 19.0 \\
\hline $1 \mathrm{~A} 5$ & 300 & 7.85 & 19.0 \\
$1 \mathrm{C} 2$ & & 7.87 & 19.5 \\
$1 \mathrm{C5}$ & & 7.74 & 19.1 \\
\hline & Mean & 7.82 & 19.2 \\
\hline $1 \mathrm{~A} 6$ & $300 \mathrm{HD}$ & 8.07 & 19.1 \\
\hdashline Mean & 8.07 & 19.1 \\
\hline
\end{tabular}

' $\mathrm{pH}$ and temperature data measured at the end of exposure pertod (24 hour)

are used for maklng total ammonia nitrogen toxiclty determinations since

there was no termination $\mathrm{pH}$ and temperdure data taken for PPB (SDP)

exposure. 
Study Number: AEH-11-PSEUDO-01

Lab Notelook 1

Ja:a Source: Fite Folder: $10 \mathrm{~d}$

Forms: Water Quallty - Form 3

Upon Termination (Hardness, Alkalinity, Conductivity, and Ammonia)
Test Article: $\quad M B \mid 401$ (PF-CL 145A [SDP])

Article Lot \#: MB-401-110308AL-BD-3

Exposure Date: 12-May-11

Species Tested: HGE

Glochldia Lo: \# 111500

Higgins Eye (SDP) Total Ammonia Nitrogen

\begin{tabular}{|c|cccc|}
\hline $\begin{array}{c}\text { Treatment } \\
(\mathrm{mB} / \mathrm{L})\end{array}$ & $\begin{array}{c}\text { Temperature } \\
\left({ }^{\circ} \mathrm{C}\right)\end{array}$ & $\mathrm{pH}$ & $\begin{array}{c}\text { Total Ammonia } \\
\text { Nitrogen }\left(\mathrm{mg} \mathrm{NH}_{3}-\mathrm{N} / \mathrm{L}\right)\end{array}$ & $\begin{array}{c}\text { Ammonla } \\
\text { Toxicity }\end{array}$ \\
\hline \hline 0 & 19.5 & 8.47 & 0.09 & NT \\
50 & 19.6 & 7.99 & 0.17 & NT \\
100 & 19.5 & 7.93 & 0.31 & NT \\
200 & 19.6 & 7.40 & 0.53 & NT \\
300 & 19.3 & 7.34 & 0.45 & NT \\
$300 \mathrm{HO}$ & 19.5 & 7.20 & 0.50 & NT \\
\hline Grand Mean & 19.5 & 7.95 & 0.34 & NT \\
SD & 0.1 & 0.49 & 0.18 & NA \\
\hline
\end{tabular}

NT = non-toxic levels of total ammonia nitrogen (below chronlc and acute levels.

Note: Control water sample from $2 \mathrm{~B} 1$ ( $\mathrm{HGE}$ ) was accidentally pooled with the Block

1 (PPB) Control group, therefore only Iwo control samples were pooled for

determination of f GE control TAN.

Iten Number 3

Page 3 of 10 


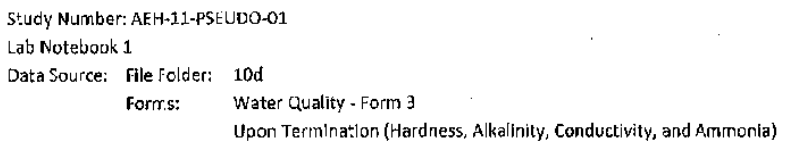

Test Article: $\quad$ MBI 401 (Pf-Cl. 145A [SDP]) Article Lot H: $\quad$ MBI-401-110308Al-BD-3

Exposure Dite: 12-May-11

Species Tested: FAM

Glochidla Lot \# 111600

Fatmucket (SDP) Total Ammonia Nitrogen

\begin{tabular}{|c|cccc|}
\hline $\begin{array}{c}\text { Treatment } \\
(\mathrm{mg} / \mathrm{L})\end{array}$ & $\begin{array}{c}\text { Temperature } \\
\left({ }^{\circ} \mathrm{c}\right)\end{array}$ & $\mathrm{pH}$ & $\begin{array}{c}\text { Total Ammonia } \\
\text { Nitrogen }\left(\mathrm{mg} \mathrm{NH} \mathbf{3}_{\mathbf{3}} \mathrm{N} / \mathrm{L}\right)\end{array}$ & $\begin{array}{c}\text { Ammonla } \\
\text { Toxicity }\end{array}$ \\
\hline \hline 0 & 20 & 8.45 & 0.10 & $\mathrm{NT}$ \\
50 & 20 & 8.11 & 0.25 & $\mathrm{NT}$ \\
100 & 19.8 & 7.61 & 0.24 & $\mathrm{NT}$ \\
200 & 19.9 & 7.48 & 0.44 & $\mathrm{NT}$ \\
300 & 19.7 & 7.25 & 0.63 & $\mathrm{NT}$ \\
$300 \mathrm{HD}$ & 19.7 & 7.17 & 0.55 & $\mathrm{NT}$ \\
\hline Grand Mear & 19.9 & 7.93 & 0.37 & $\mathrm{NT}$ \\
SD & 0.1 & 0.50 & 0.20 & $\mathrm{NA}$ \\
\hline
\end{tabular}

Item Number 3

Page 4 of 10 
Study Number: AEFl-11-FSEUDO-01

l.ab Notebook 1

Data Source: File Folder: $10 \mathrm{~d}$

Forms: Water Quality - Form 3

Upon Termination (Hardness, Alkalinity, Conductwily, Temperature, and pH)

LTRM Water Zuallty Laboratory Report
Test Art|cle: $\quad$ MBl 401 (Pf.CL 14j̈A [FOP])

Artic e Lot \#: 110607WB-FB-E

Exposure Date: $18-0 \mathrm{ct}-11$

Spec'es Tested: PPB

Glochidla Lot \# 115400

Plain Pocketbook (FDP) Total Ammonia Nitrogen

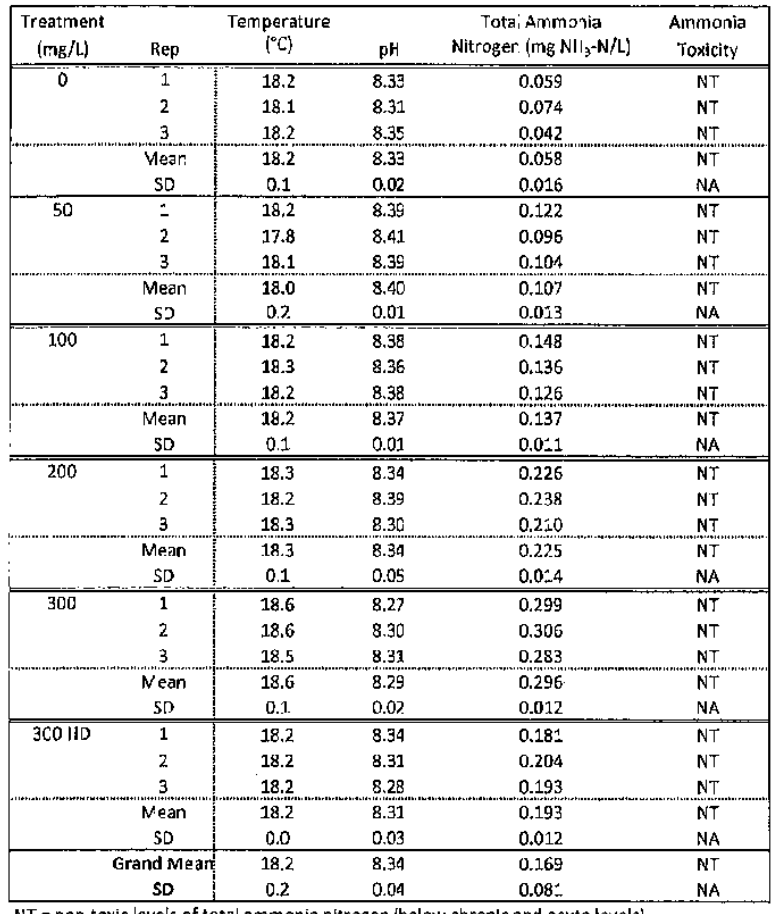

NT = non-toxic levels of total ammania rifrogen (below chronlc and acute levels].

them Number 3
Page 5 of 10 
Study Number; AEH-11-PSEUDO-01

Lat Notebook 1

Data Source: Flle Folder: 10

Forms: Water Quality - Form 3

Upon Termination (Hardness, Alke linity, Conductlylty, Temperature, and pH) LTRM Water Quallty Laboratory Report

\section{Washboard (FDP) Total Ammonia Nitrogen}

\begin{tabular}{|c|c|c|c|c|c|}
\hline $\begin{array}{l}\text { Treatment } \\
(\mathrm{mg} / \mathrm{L})\end{array}$ & Rep & $\begin{array}{l}\text { Temperature } \\
\left({ }^{\circ} \mathrm{C}\right)\end{array}$ & $\mathrm{pH}$ & $\begin{array}{c}\text { Total Ammonia } \\
\text { Nitrogen (Ing NH} \mathrm{N}_{3}-\mathrm{N} / \mathrm{L} \text { ) }\end{array}$ & $\begin{array}{l}\text { Amrronia } \\
\text { Toxicity }\end{array}$ \\
\hline \multirow[t]{3}{*}{0} & 1 & 18.7 & 8.41 & 0.027 & NT \\
\hline & 2 & 18.7 & 8.49 & 0.022 & VT \\
\hline & 3 & 18.6 & 8.48 & 0.030 & VT \\
\hline & Mear & 18.7 & 8.48 & 0.026 & VT \\
\hline & SD & 0.1 & 0.01 & 0.004 & $\mathrm{NA}$ \\
\hline \multirow[t]{3}{*}{50} & 1 & 18.5 & $8 . \overline{45}$ & 0.054 & NT \\
\hline & 2 & 18.1 & 8.47 & 0.058 & NT \\
\hline & 3 & 18.7 & 8.44 & 0.039 & NT \\
\hline & Mean & 18.4 & 8.45 & 0.050 & NT \\
\hline & SD & 0.3 & 0.02 & 0.010 & $\mathrm{NA}$ \\
\hline \multirow[t]{3}{*}{100} & 1 & 18.9 & 8.43 & 0.035 & $\overline{\mathrm{NT}}$ \\
\hline & 2 & 18.7 & 8.46 & 0.059 & $\mathrm{NI}$ \\
\hline & 3 & 18.6 & B.44 & 0.073 & $\mathrm{NT}$ \\
\hline & Mean & 18.7 & B.44 & 0.056 & $\mathrm{NT}$ \\
\hline & SD & 0.2 & 0.02 & 0,019 & $\mathrm{NA}$ \\
\hline \multirow[t]{3}{*}{200} & 1 & 19.1 & 8.41 & 0.053 & $\overline{\mathrm{NT}}$ \\
\hline & 2 & 18.6 & 8.40 & c.056 & NT \\
\hline & 3 & 19.1 & 8.42 & 0.033 & NT \\
\hline & Mean & 18.9 & 8.41 & 0.047 & NT \\
\hline & SD & 0.3 & 0.01 & 0.013 & NA \\
\hline \multirow[t]{3}{*}{300} & 1 & 18.0 & 8.42 & 0.111 & NT \\
\hline & 2 & 18.8 & 8.38 & 0.185 & NT \\
\hline & 3 & 19.2 & 8.35 & 0.074 & NT \\
\hline & Mean & 18.7 & 8.38 & 0.123 & NT \\
\hline & SD & 0.5 & 0.01 & 0.057 & NA \\
\hline \multirow[t]{3}{*}{$300 \mathrm{HD}$} & 1 & 18.6 & 8.42 & 0.096 & NT \\
\hline & 2 & 18.3 & 8.45 & 0.100 & $\mathrm{NT}$ \\
\hline & 3 & 18.6 & 8.40 & 0.063 & NT \\
\hline & Mean & 18.5 & 8.42 & 0.086 & NT \\
\hline & SD & 0.2 & 0.03 & 0.020 & NA \\
\hline & Grand Mearl & 18.7 & 8.43 & 0.065 & NT \\
\hline & SD & 0.3 & 0.04 & 0.039 & NA \\
\hline
\end{tabular}

$\mathrm{NT}=$ non-toxic levels of total ammanla nitrogen \{kelow chronic and acute levels\}.

1:em Number 3

Page 6 of 10
Test Ait cle; MBI 4C1 (PF-CL 145A [FOP])

Artcle Lot 4: $\quad 110607$ WB-FG-5

Exoosure Cate: 18 -Oct-11

Species Tested: WAS

Glochidia Lot \# 115500 
Stucy Number: AEH-11.PSEUDO-01

Lab Notebook 1

Data Source: Fle Pclder: 10

forms: Water Quality - form 3

Upun Terminattun (Hardness, Alkalinity, Conductivity, -emperature, and pH)

LTRM Water Quality Laboratory Repot
Test Article: $\quad$ MBi 40 : iPf-CL 145A [SDP]) Artkle Lot H: MBI-401 SDO 4655-12-Mix

Exposure Date: 18-Oct-11

Specles Tested: BLS

Glochidia Lot \# 120800

Black Sandshell (SDP) Total Ammonia Nitrogen

\begin{tabular}{|c|c|c|c|c|c|}
\hline $\begin{array}{l}\text { Treatment } \\
\text { (mg/L) }\end{array}$ & Rep & $\begin{array}{c}\text { Temperature } \\
\left({ }^{\circ} \mathrm{C}\right)\end{array}$ & $\mathrm{pH}$ & $\begin{array}{c}\text { Total AInmonia } \\
\left.\text { Nitrogen (mg NH} \mathrm{H}_{3}-\mathrm{N} / \mathrm{L}\right)\end{array}$ & $\begin{array}{l}\text { Ammonia } \\
\text { Toxlcity }\end{array}$ \\
\hline \multirow[t]{3}{*}{0} & $i$ & 20.1 & 8.36 & 0.082 & NT \\
\hline & 2 & 20.3 & 8.43 & 0.079 & NT \\
\hline & 3 & 20.5 & 8.45 & 0.080 & NT \\
\hline & Mear & 20.3 & 8.42 & 0.080 & NT \\
\hline & S) & 0.2 & 0.05 & 0.002 & $\mathrm{NA}$ \\
\hline \multirow[t]{3}{*}{50} & 1 & 20.4 & 8.30 & 0.215 & NT \\
\hline & 2 & 20.1 & 8.33 & 0.241 & $N T$ \\
\hline & 3 & 20.5 & 8.24 & 0.436 & NT \\
\hline & Mean & 20.3 & 8,20 & 0.237 & NT \\
\hline & so & 0.2 & 0.05 & 0.121 & NA \\
\hline \multirow[t]{3}{*}{100} & 1 & 20.3 & 8.15 & 0.724 & Chranle \\
\hline & 2 & 20.3 & 8.17 & 0.698 & Chronic \\
\hline & 3 & 21,0 & 8.23 & 0.719 & Chronle \\
\hline & Mean & 20.6 & 8.18 & 0.714 & Chronic \\
\hline & $S D$ & 0.4 & 0.04 & 0.014 & $N A$ \\
\hline \multirow[t]{3}{*}{200} & 1 & 20.0 & 8.24 & 0.916 & Chronic \\
\hline & 2 & 20.6 & 8.03 & 1.167 & Chronic \\
\hline & 3 & 20.5 & 8.12 & 1.078 & Chronic \\
\hline & Mean & 20.4 & 8.14 & 1.054 & Chronic \\
\hline & SD & 0.3 & 0.11 & 0,127 & $N A$ \\
\hline \multirow[t]{3}{*}{300} & 1 & 20.6 & 7.95 & 1.509 & Crronic \\
\hline & 2 & 20.6 & 8.13 & 1.187 & Crronic \\
\hline & 3 & 20.8 & 7.96 & 1,661 & Crronic \\
\hline & Mean & 20.7 & 8.02 & 1.452 & Chronic \\
\hline & $S D$ & 0.1 & 0.10 & 0.242 & NA \\
\hline \multirow[t]{3}{*}{$300 \mathrm{HD}$} & 1 & 20.3 & 8.28 & 0.245 & NT \\
\hline & 2 & 20.2 & 8.16 & 0.197 & NT \\
\hline & 3 & 18.4 & 8.46 & 0.247 & NT \\
\hline & Mean & 19.6 & 8.32 & $0.23 \mathrm{C}$ & NT \\
\hline & SD & 1.1 & 0.15 & 0.028 & NA \\
\hline & Grand Meart & 20.3 & 8.25 & 0.638 & Chronle \\
\hline & SD & 0.5 & 0.15 & 0.514 & NA \\
\hline
\end{tabular}

(n) 0.514

Item Number
Page 7 
Study Number: AEH-11-PSEUDO-01

Las Notebook 1

Data Source: File Folder: 10

Forms: Water Quality - Form 3

Upon Termination (Hardness, A kalinity, Conduct|vity, Temperature, and pH)

LTRM Wate:-Quality Laboratory Report
Test Article: $\quad M B \mid$ 401 (Pt-CL 145A [SDP] Article _ot H: M81-401 SDP 4655-12-MIX

Exposure Oate: $17 \cdot \operatorname{san}-12$

Species Tested: MUC

Glochldla Lot \# 120700

Mucket (SDP) Total Ammonia Nitrogen

\begin{tabular}{|c|c|c|c|c|c|}
\hline $\begin{array}{c}\text { Treatment } \\
(\mathrm{mg} / \mathrm{L})\end{array}$ & Rep & $\begin{array}{l}\text { Temperature } \\
\left({ }^{\circ} \mathrm{C}\right)\end{array}$ & $\mathrm{pH}$ & $\begin{array}{c}\text { Total Ammonia } \\
\text { Nitrogen }\left(\mathrm{mg} \mathrm{NH} \mathrm{H}_{3}-\mathrm{N} / \mathrm{L}\right)\end{array}$ & $\begin{array}{l}\text { Anmonta } \\
\text { Toxicity }\end{array}$ \\
\hline \multirow[t]{3}{*}{0} & 1 & 19.8 & 8.60 & 0.037 & NT \\
\hline & 2 & 19.8 & 8.57 & 0.052 & NT \\
\hline & 3. & 20.1 & 8.56 & 0.110 & NT \\
\hline & Mean & 19.9 & 8.58 & 0.066 & NT \\
\hline & SD & 0.2 & 0.02 & 0.039 & NA \\
\hline \multirow[t]{3}{*}{50} & 1 & 19.9 & 8.42 & 0.183 & NT \\
\hline & 2 & 20.1 & 8.36 & 0.533 & Chronic \\
\hline & 3 & 20.1 & 8.39 & 0.088 & NT \\
\hline & Mean & 20.0 & 8.39 & 0.268 & NT \\
\hline & SD & 0.1 & 0.03 & 0.234 & NA \\
\hline \multirow[t]{3}{*}{100} & 1 & 20.4 & 8.29 & 0.694 & Chronic \\
\hline & 2 & 20.2 & 8.25 & 0.430 & NT \\
\hline & 3 & 19.2 & 8.43 & 0.212 & $N T$ \\
\hline & Mean & $19.9^{\circ}$ & 8,33 & 0.445 & NT \\
\hline & SD & 0.6 & 0.09 & 0.241 & NA \\
\hline \multirow[t]{3}{*}{200} & 1 & 20.1 & 8.27 & 0.861 & Chronic \\
\hline & 2 & 19.9 & 8.28 & 0.777 & Chronic \\
\hline & 3 & 19.9 & 8.33 & 0,921 & Chronic \\
\hline & Mean & 20.0 & 8.29 & 0.853 & Chronic \\
\hline & SD & 0.1 & 0.03 & 0.072 & $\mathrm{NA}$ \\
\hline \multirow[t]{3}{*}{300} & 1 & 20.7 & 8.30 & 1.136 & Chronic \\
\hline & 2 & 19.8 & 8.21 & 0.916 & Chronic \\
\hline & 3 & 19.9 & 8.25 & 1,088 & Chronlc \\
\hline & Mean & 20.1 & 8.25 & 1,047 & Chronic \\
\hline & SD & 0.5 & 0.05 & 0,116 & NA \\
\hline \multirow[t]{3}{*}{$300 \mathrm{HD}$} & 1 & 19.9 & 8.19 & 0.537 & NT \\
\hline & 2 & 20.7 & 8.13 & 0.796 & Chronic \\
\hline & 3 & 19.7 & 8.37 & 0.409 & NT \\
\hline & Mean & 20.1 & 8.24 & 0.581 & Chronic \\
\hline & SD & 0.5 & 0.12 & 0.197 & $N A$ \\
\hline & Grand Mean & 20.5 & 8.36 & 0.543 & Chronic \\
\hline & SD & 0,4 & 0.13 & 0.371 & NA \\
\hline
\end{tabular}

NT = non-toxic levels of total ammon a nitrogen (below chronk and acute levels:.

Itern Number 3

Page 8 of 10 
Study Number: AEH-11-PSEUDO-01

Lab Notebook 1

Data Source: Fije Folder: $10 \mathrm{~d}$ Forns:

Water QLallty - Form 3

Upon Termination (I lardness, Alkalinity, Conduct|vily, Temperatura, and phl) LTRM Water Quallty Laboratory Report
Test Article: MB1 40: 'Pf-CL 145A [SDP]) Article Lot H: MBI-401 SOP 4655-12-MLX Exposure Date: 19-Jan-12 Species Tested: HIC Glochidia Lot \# 120900

Hickorynut (SDP) Total Ammonia Nitrogen

\begin{tabular}{|c|c|c|c|c|c|}
\hline $\begin{array}{l}\text { Treatment } \\
\text { (mg/l.) }\end{array}$ & Rep & $\begin{array}{l}\text { emperature } \\
\left({ }^{\circ} \mathrm{C}\right)\end{array}$ & $\mathrm{pH}$ & $\begin{array}{l}\text { Total Ammonia } \\
\text { Nitrogen }\left(m g ~ \mathrm{NH}_{3}-\mathrm{N} / \mathrm{L}\right)\end{array}$ & $\begin{array}{l}\text { Ammonia } \\
\text { Toxicity }\end{array}$ \\
\hline \multirow[t]{3}{*}{0} & 1 & 20.1 & 842 & 0.082 & NT \\
\hline & 2 & 20.0 & 8.47 & 0.084 & NT \\
\hline & 3 & 19.7 & 8.48 & 0.032 & NT \\
\hline & Vean & 19.9 & 8.46 & 0.066 & NT \\
\hline & SD & 0.2 & 0.03 & 0.029 & $\mathrm{NA}$ \\
\hline \multirow[t]{3}{*}{50} & 1 & 20.0 & 8.45 & 0.265 & NT \\
\hline & 2 & 20.1 & 8.29 & 0.266 & NT \\
\hline & 3 & 19.2 & 8.45 & 0.098 & NT \\
\hline & Mean & 19.8 & 8.40 & 0.210 & NT \\
\hline & SD & 0.5 & 0.09 & 0.097 & $\mathrm{NA}$ \\
\hline \multirow[t]{3}{*}{100} & 1 & 19.0 & 8.42 & 0.094 & NT \\
\hline & 2 & 19.9 & 8.35 & 0.533 & Chronic \\
\hline & 3 & 19.8 & 8.27 & 0.473 & NT \\
\hline & Mean & 19.6 & 8.35 & 0.367 & NT \\
\hline & SD & 0.5 & 0.08 & 0.238 & NA \\
\hline \multirow[t]{3}{*}{200} & 1 & 19.7 & 8.33 & $0 . \overline{213}$ & $\overline{N T}$ \\
\hline & 2 & 20.7 & 8.03 & 0.254 & NT \\
\hline & 3 & 20.4 & 8.13 & 0.263 & NT \\
\hline & Mean & 20.3 & 8.18 & 0.243 & NT \\
\hline & SD & 0.5 & 0.15 & 0.027 & $\mathrm{NA}$ \\
\hline \multirow[t]{3}{*}{300} & 1 & 20.6 & 8.05 & 0.231 & NT \\
\hline & 2 & 20.1 & 8.08 & 0.192 & NT \\
\hline & 3 & 20.3 & 7.98 & 0,482 & NT \\
\hline & Mean & 20.3 & 8.04 & 0.302 & NT \\
\hline & SO & 0.3 & 0.05 & 0.157 & NA \\
\hline \multirow[t]{3}{*}{$300 \mathrm{HD}$} & 1 & 20.3 & 8.05 & 0.885 & Chronic \\
\hline & 2 & 19.0 & 8.33 & 0.107 & $N T$ \\
\hline & 3 & 19.7 & 8.26 & $0 .<74$ & NT \\
\hline & Mean & 19.7 & 8.23 & 0.489 & NT \\
\hline & SD & 0.7 & 0.15 & 0.389 & NA \\
\hline
\end{tabular}

NT $=$ non-toxic levels of total ammonla nitrogen (below chronic and acute levels).

Inem Number $\frac{-3}{10}$ 
Study Yumber: AEH-11-PSELDOQ-01

lab Notebook 1

Data Source: File Folcer; 10

Forms: Water Quality - Forrs: 2 and 3

During Exposure (Dissolved Oxyger, pH, Temperatura)

Upon Termination (Hardness, Alkalfnity, Conductivity, and Ammonla)

LTRM Wate: Quality L.aboratory Report
Test Article: $M A B$ ' 401 (PF-CL 145A i5DP and FDP])

Article Lot H. MB -401.110308A1-BD-3,MBI-401 SDP 4555-12-MIX, 110607 WB-FB-E

Exposure Date: 12-May-11, 18-OCt-11, 17-Jan-12, 19-Jan-12

Specles Tested: PPE, HGE, FAM, WAS, BLS, MUC, HIC

Glochidda Lot \# : $11400,111500,111600,115400,1155 \mathrm{CO}, 120700,120800$,

$$
\therefore 20900
$$

Total Ammonia Nitrogen Summary

\begin{tabular}{|c|c|c|c|c|c|}
\hline $\begin{array}{c}\text { Treatment } \\
(\mathrm{mg} / \mathrm{L})\end{array}$ & $\begin{array}{c}\text { Species } \\
\text { (Fur'nulation) }\end{array}$ & $\begin{array}{l}\text { Mean Temperature } \\
\left({ }^{\circ} \mathrm{C}\right)\end{array}$ & $\begin{array}{c}\text { Mean } \\
\mathrm{pH}\end{array}$ & $\begin{array}{l}\text { Mean Total Ammonia } \\
\text { Nitrogen }\left(\mathrm{mg} \mathrm{NH} \mathrm{NH}_{\mathrm{g}}-\mathrm{N} / \mathrm{L}\right)\end{array}$ & $\begin{array}{l}\text { Ammonia } \\
\text { Toxicity }\end{array}$ \\
\hline \multirow[t]{10}{*}{0} & PFE (SDP) & 19.2 & (8.34 & 0.060 & $\begin{array}{l}\text { NT } \\
\end{array}$ \\
\hline & HGE (SOF) & 19.5 & 8.47 & 0.090 & NT \\
\hline & FAM (SDP) & 20.0 & 8.45 & 0.100 & NT \\
\hline & PFE (FDP) & 18.2 & 8.33 & 0.058 & NT \\
\hline & W/AS (FDP) & 18.7 & 8.48 & 0.026 & NT \\
\hline & BLS (SDP) & 20.3 & 8.42 & 0.080 & NT \\
\hline & MuC (SDP) & 19.9 & 8.58 & 0.066 & NT \\
\hline & $H I C(S D P)$ & 19.9 & 8.46 & 0.066 & NT \\
\hline & Mean & 19.5 & 8.45 & 0.068 & NT \\
\hline & SD & 0.7 & 0.08 & 0.023 & $\mathrm{NA}$ \\
\hline \multirow[t]{10}{*}{50} & $\overline{P P B}\{S D P\}$ & 19.2 & 8.25 & 0,080 & NT \\
\hline & HGE $\{S D P\}$ & 19.6 & 3.99 & 0.170 & NT \\
\hline & FAM (SOP) & 20,0 & 8.11 & 0.250 & NT \\
\hline & PPB (FEP) & 18.0 & 8.40 & 0.107 & NT \\
\hline & WAS (FDP) & 18.4 & 8.45 & 0.050 & NT \\
\hline & BIS (SDP) & 20.3 & 8.29 & 0.297 & NT \\
\hline & MUC (SDP) & 20.0 & 8.39 & 0.268 & NT \\
\hline & HIC (SDP) & 19.6 & 8.40 & 0.210 & NT \\
\hline & Mean & 19.4 & 8.31 & 0.179 & NT \\
\hline & SD & 0,8 & 0.16 & 0.092 & NA \\
\hline \multirow[t]{10}{*}{100} & PPB (SDP) & 19.0 & 8.03 & 0.130 & NT \\
\hline & HGE (SDP) & 19.5 & 7.93 & 0,310 & NT \\
\hline & FAM (SDP) & 19.8 & 7.61 & 0.240 & NT \\
\hline & PPB (FDF) & 18.2 & 8.37 & 0.137 & NT \\
\hline & WAS (FDP) & 18.7 & 8.44 & 0.056 & NT \\
\hline & B1.S [SDP) & 20.6 & 8.18 & 0.714 & Chronic \\
\hline & $\operatorname{MUC}\left(\mathrm{SO}^{\rho}\right)$ & 19.9 & 8.33 & 0.445 & NT \\
\hline & $H I C(S D P)$ & 19.6 & 8.35 & 0.367 & NT \\
\hline & Mean & 19.4 & 8.22 & 0.300 & $\mathrm{NI}$ \\
\hline & SD & 0.7 & 0,28 & 0.212 & NA \\
\hline \multirow[t]{10}{*}{200} & PPB (SWP) & 19.0 & 8.09 & 0.240 & NT \\
\hline & HGE (SDP) & 19.6 & 7.40 & 0.530 & NT \\
\hline & FAM (SDP) & 19,9 & $7.4 \mathrm{~B}$ & 0.440 & NT \\
\hline & $\mathrm{PPB}\{; \mathrm{FDP}\}$ & 18.3 & 8.34 & 0.225 & NT \\
\hline & WAS (FDP) & 18.9 & 8.41 & 0.047 & NT \\
\hline & BLS (SDF) & 20.4 & 8.14 & 1.054 & Chronic \\
\hline & MUC (SDP) & 20.0 & 8.29 & 0,853 & Chronic \\
\hline & $H \mid C$ (SDF) & 20,3 & 8.18 & 0.243 & NT \\
\hline & Mean & 19.5 & 8,15 & 0.454 & $N T$ \\
\hline & SD & 0.7 & 0.39 & 0.345 & NA \\
\hline \multirow[t]{10}{*}{300} & PPB (SDP) & 19.2 & 7.82 & 0.440 & NT \\
\hline & HGE (SDP) & 19.3 & 7.34 & 0.450 & NT \\
\hline & FAM (SDP) & 19.7 & 7,25 & 0.630 & NT \\
\hline & PPB (FDP) & 18.6. & 8.29 & 0.296 & NT \\
\hline & WAS (FDP) & 18.7 & 8.38 & 0.123 & $\mathrm{NT}$ \\
\hline & $3 . S$ (SDP) & 20.7 & 8.02 & 1.452 & Chronic \\
\hline & $M J C(S D P)$ & 20.1 & $8: 25$ & 1.047 & Chronic \\
\hline & $H(S O P)$ & 20.3 & 8.04 & 0.302 & NT \\
\hline & Mean & 19.6 & 8.07 & 0.593 & NT \\
\hline & SD & 0.8 & 0.43 & 0.445 & NA \\
\hline \multirow[t]{10}{*}{$300 \mathrm{HD}$} & PPB (SDP) & 19.1 & 8.07 & 0.510 & NT \\
\hline & HGE (SDP) & 19.5 & 7.20 & 0.500 & NT \\
\hline & FAM (SDP) & 29.7 & 7.17 & 0.550 & NT \\
\hline & PPB (FDP) & 18.2 & 8.31 & 0.193 & NT \\
\hline & WAS (FDP) & 18.5 & 8.42 & 0.086 & NT \\
\hline & BLS (SDP) & 19.6 & 8.32 & 0.230 & NT \\
\hline & MUC (SOP) & 20.1 & 8.24 & 0.581 & Chronic \\
\hline & $\mathrm{HIC}(\mathrm{SDP})$ & 19.7 & 8.23 & 0.489 & NT \\
\hline & Mean & 19.3 & 8.17 & 0.392 & NT \\
\hline & SD & 0.7 & 0.51 & 0.191 & NA \\
\hline
\end{tabular}

Item Number__

10 of 10 


\section{Appendix 7. Glochidia Viability}

\begin{tabular}{clcc}
\hline $\begin{array}{c}\text { Item } \\
\text { number }\end{array}$ & Item description & $\begin{array}{c}\text { Number } \\
\text { of } \\
\text { pages }\end{array}$ & $\begin{array}{c}\text { Report } \\
\text { page } \\
\text { number }\end{array}$ \\
\hline 1 & Initial (Pre exposure) Glochidia Viability - Data Summary & 2 & 354 \\
2 & SAS program for glochidia viability & 2 & 356 \\
3 & SAS log for glochidia viability & 4 & 358 \\
4 & SAS output for glochidia viability & 81 & 362 \\
5 & Glochidia Viability Assessment - 6 hour - Data Summary & 7 & 443 \\
6 & Glochidia Viability Assessment - 12hour - Data Summary & 7 & 450 \\
7 & Glochidia Viability Assessment - 24hour - Data Summary & 8 & 457 \\
8 & Glochidia Distribution Counts - Data Summary & 10 & 465 \\
\hline
\end{tabular}




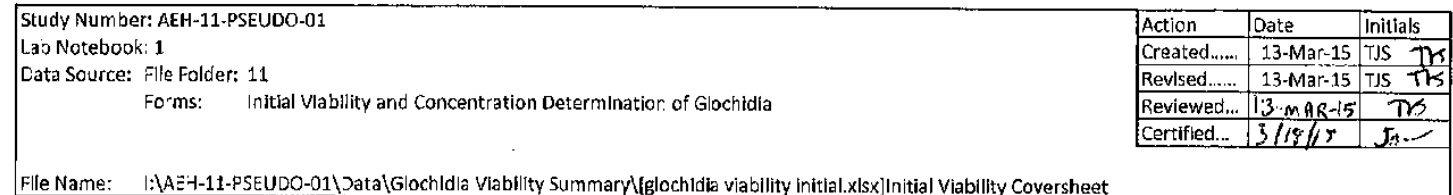

Initlal (Preexposure) Glochidia Vlability

Tast Article: Pscudomonas fluorescens ( $f f$-CL.145A) spray dried powder (SDP) and freeze dr ed powder (FDP)

Article Lot Hs: SDP $=$ MBt-401-110308AI-BD-3 and MBI-401 SDP 4655-12-Mix; FDP $=110607$ WB-FD-E

Exposure Dates: 12-May-11, 18-Oct-11, 17-Jan-12, and 19-Jan-12

Species Tested: PPG, FAM, HGE, WAS, BLS, MUC, and HIC

\section{Data Explanation:}

Species Abbreviations

PPB - plain pocketbook (Lampslis cardium )

FAM - fatmucket (lampsilts silizuoidea)

HGE - HIggIns eye (Lampsilis higginsii)

WAS - washboard (Megalonalas nervosa)

BLS - black sandshell (Ligumia recta)

MUC - mucket (Actinonaias ligamentina)

$\mathrm{HIC}$ - hickorynut (Obovaria olivaria)

Glochidla "closed before salt" and "open after salt" are considered non-viable.

\section{Data anomalies and deviations:}

Washboard used four donor mussels for glochldla; all other specics used three donor mussels.

Glochidia from black sandshell mussel $\$ 2$ was evalcated three times for viabillty; the combined value of all three samples is reported,

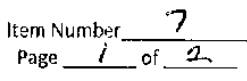


Study Number: A매-11-PSEUDO-01

Lab Notebook 1

Data Source: Fille Folder: 11

Forrs: Initial Viability and Concentration Determination of Gilochidia
Test Article: MBI 401 (PP-CL 145A [SDP] and [EDP]

Article Lot f: MB1-401-11C308Al-8D-3, MBI-401 SDP 4655-12-M1x, 1:0607WB-FD-E

Expusurs Dates: 12-May-11, 18-OCt-11, 17-Jan-12, and 19-Jan-12

5pecies Tested: PPB, FAM, HGE, WAS, BLS, MUC, ard HIC

Initial (Preexposure) Viability Summary

\begin{tabular}{|c|c|c|c|c|c|c|c|}
\hline Species & Formulation & Test Date & $\begin{array}{l}\text { Mussel } \\
\text { number }\end{array}$ & $\begin{array}{c}\text { Total } \\
\text { glochidfa } \\
\end{array}$ & $\begin{array}{c}\text { Glachidia closed } \\
\text { before salt } \\
\end{array}$ & $\begin{array}{c}\text { Glochidla open } \\
\text { after salt }\end{array}$ & $\begin{array}{l}\text { Percent } \\
\text { Viability } \\
\end{array}$ \\
\hline \multirow[t]{3}{*}{ PPB } & SDP & 12 -May:11 & 1 & 467 & 14 & 22 & 92.3 \\
\hline & & & 2 & 537 & 8 & 19 & 95.0 \\
\hline & & & ${ }^{3}$ & 927 & 10 & 45 & 94.1 \\
\hline & & & Grand Mean & & & & 93.8 \\
\hline & & & SD & & & & 1.4 \\
\hline \multirow[t]{3}{*}{ HGE } & SDP & 12-May-11 & 1 & 129 & 32 & 17 & 62.0 \\
\hline & & & 2 & 168 & 6 & 14 & 88.1 \\
\hline & & & $3^{3}$ & 357 & 13 & 14 & 92.4 \\
\hline & & & Grand Mean & & & & 80.8 \\
\hline & & & SD & . & & & 16.5 \\
\hline \multirow[t]{3}{*}{ FAM } & SDP & $12 \cdot$ Ma $y \cdot 11$ & 1 & 548 & 22 & 48 & 87.2 \\
\hline & & & 2 & 297 & 53 & 27 & 73.1 \\
\hline & & & $3^{3}$ & 456 & 34 & 55 & 80.5 \\
\hline & & & Grand Mean & & & & 80.3 \\
\hline & & & SD & & & & 7.1 \\
\hline \multirow[t]{3}{*}{ PPB } & FDP & $18-0 \mathrm{tat}-11$ & 1 & 212 & 4 & 12 & 92.5 \\
\hline & & & 2 & 222 & 26 & 13 & 82.4 \\
\hline & & & 3 & 150 & 15 & 16 & 79.3 \\
\hline & & & Grand Mean & & & & 84.7 \\
\hline & & & SD & & & & 6,9 \\
\hline \multirow[t]{4}{*}{ WAS } & FOP & 18 -0ct-11 & 1 & 124 & 1 & 1 & 98.4 \\
\hline & & & 2 & 155 & 1 & 0 & 99.4 \\
\hline & & & 3 & 51 & 4 & 1 & 90.2 \\
\hline & & & 4 & 104 & 0 & 0 & 100.0 \\
\hline & & & Grand Mean & & & & 96.5 \\
\hline & & & SD & & & & 5.5 \\
\hline \multirow[t]{3}{*}{ BLS } & $\operatorname{SDP}$ & $17-\tan -12$ & 1 & 312 & 10 & 22 & 89.7 \\
\hline & & & 2 & 1083 & 51 & 199 & 76.9 \\
\hline & & & 9 & 405 & 12 & 41 & 86.9 \\
\hline & & & Grand Mean & & & & 84.5 \\
\hline & & & SD & & & & 6.7 \\
\hline \multirow[t]{3}{*}{ MUC } & SDP & 17--an-12 & 1 & 161 & 6 & 8 & 91.3 \\
\hline & & & 2 & 153 & 6 & 3 & 94.1 \\
\hline & & & 3 & 287 & 3 & 18 & 927 \\
\hline & & & Grand Mean & & & & 92.7 \\
\hline & & & SD & & & & 1,4 \\
\hline \multirow[t]{3}{*}{ HIC } & SOP & $19-\operatorname{Jan}-12$ & 1 & 366 & 15 & 39 & 85.2 \\
\hline & & & 2 & 352 & 20 & 31 & 85.5 \\
\hline & & & 3 & 309 & 27 & 12 & 87.4 \\
\hline & & & $\begin{array}{c}\text { Grand Mean } \\
\text { SD }\end{array}$ & & & & $\begin{array}{l}86.0 \\
1.2\end{array}$ \\
\hline
\end{tabular}

Item Number
Page 2 of 2 
DM 'LOG; CleAR; OUTPUT; CleAR;'; * CLEAR LOG AND OUTPUT;

FOOTNOTE1 'Performed by J. Luoma; SAS version' \&SYSVER \&SYSTIME \&SYSDATE;

options $1 \mathrm{~s}=97 \mathrm{ps}=57$ formdlim=' -1 pageno = 1 nocenter nodate nosource2;

title1 $h=2$ 'Effects of Pseudomonas fluorescens (Pf-CL145A) to glochidia from seven unionid mussel sper title2 $h=2$ 'Statistical analysis of unadjusted glochidia viability following 24-h static exposure to title3 $h=2$ 'Analysis Completion Date:t8March2015 Analysis prepared by: JAL, SAS Version:'; title4 $h=2$ \&SYSVER \&SYSTIME \&SYSDATE;

$1 * * * * * * * * * * * * * * * * * * * * * * * * * * * * * * * * * * * * * * * * * * * * * * * * * * * * * * * * * * * * *$

* SAS ver 9.4 Analysis prepared by: JAL *

* Analysis completion date: 18March2015 5/2 *

$\star * * * * * * * * * * * * * * * * * * * * * * * * * * * * * * * * * * * * * * * * * * * * * * * * * * * * * * * * * * * * 1$

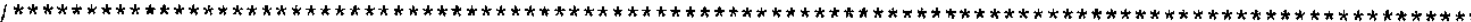

* Varlable Names:

* sps = three lettor code for mussel species

* BLS = Black sandshell (Ligumia recta)

* $\quad$ FAM = Fatmucket (Lampsilis siliquoidea

* WAS $=$ Washboard (Megalonaias nervosa)

* $\quad H G E=$ Higgins eye (Lampsilis higginsii)

* $\quad$ PPB = Plain pocketbook (Lapsilis cardium)

* HIC = Hickorynut (Obovaria olivaria)

* $\quad$ MUC $=$ Mucket (Actinonaias ligamentina)

* form = formulation (FDP [freeze dried powder] vs. SDP [spray dried powder])

* conc = concentration (i.n $\mathrm{mg} / \mathrm{L}$ )

* $\quad 0=\operatorname{control}(0 \mathrm{mg} / \mathrm{L})$

* $\quad 50=50 \mathrm{mg} / \mathrm{L}$ active ingredient

* $\quad 100=100 \mathrm{mg} / \mathrm{L}$ active ingredient

* $200=200 \mathrm{mg} / \mathrm{L}$ active ingredient

* $\quad 300=300 \mathrm{mg} / \mathrm{L}$ active ingredient

* $400=300 \mathrm{mg} / \mathrm{L}$ heat deative

* cham $=$ exposure chamber ID

* i.e., 3 A5 = test system (1, 2 or 3), Block ID (A or B), and Position in Block (1 - 6)

* time $=$ time post exposure assessment occurred

* tot $=$ total number of glochidia within sample

* $\mathrm{cbs}=$ number of glochidia closed prior to salt solution

* oas $=$ number of glochidia open after salt solution

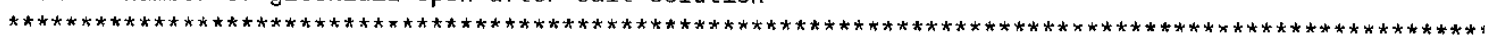

data glochidia; set Pseudo01.viability;

vi.a_glo = tot - cbs - oas;

petvia_glo $=$ via_glo/tot*100;

run;

proc sort data=glochidia; by sps form conc; run;

proc print data $=$ glochidia; run;

Title2 'Mean Unadjusted Viability of Glochidia by Species, Time, Form (SDP or FDP) and Treatment Grouk proc means data = glochidia mean std $c 1 m+f=8$;

by sps form;

class conc time;

var tot cbs oas via_glo pctvia_glo;

run;

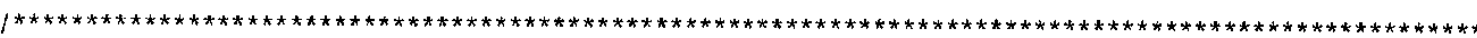


* The exposure concentrations were renamed to place an alpha character in front of all concentrations, * variable string to be first to set the variable character length (ie: $300 \mathrm{HD}$ vs 0 ). SAS assumes the

* therefore, the control was given the last alpha character and thereby other concentrations will be (

* $50 \mathrm{mg} / \mathrm{L}=\mathrm{a}$

* $100 \mathrm{Ng} / \mathrm{L}=\mathrm{b}$

*200 mg/L $=\mathrm{c}$

* $300 \mathrm{mg} / \mathrm{L}=\mathrm{d}$

* $300 \mathrm{mg} / \mathrm{L}$ HD (coded as 400 in dataset) $=e$

* $0 \operatorname{lng} / \mathrm{L}=\mathrm{f}$

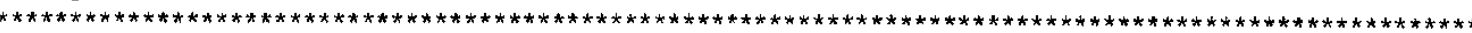

data glochidia2; set glochidia;

If conc $=$ ' $O$ ' then conca $=$ ' $f$ ';

If conc $=' 50$ ' then conca $=$ ' $\mathrm{a}$ ';

If conc $={ }^{\prime} 100^{\prime}$ then conca $={ }^{\prime} \mathrm{b} \mathrm{b}^{\prime}$;

If $\operatorname{conc}={ }^{\prime} 200$ ' then conca $=$ ' $\mathrm{c}$ '

If conc $=$ ' 300 ' then conca $=$ ' $d$ ';

If conc $=1400$ ' then conca $={ }^{\prime} e^{\prime}$;

run;

proc sort data=glochidia2; by form sps conca time; run;

proc glimmix data = glochidia2;

title2 'Overall Model: Glochidia Viability (unadjusted) - At 6, 12 and 24 h';

by form sps;

class conca time;

model vi.a_glo/tot $=$ conca $\mid$ time $/ d=$ bin link = logit noint s or;

lsmeans conca / pdiff cl ilink or;

lsmeans time / pdiff cl ilink or;

lsmeans conca*time / pdiff cl i.jink or;

random_residual_;

run;

quit; 


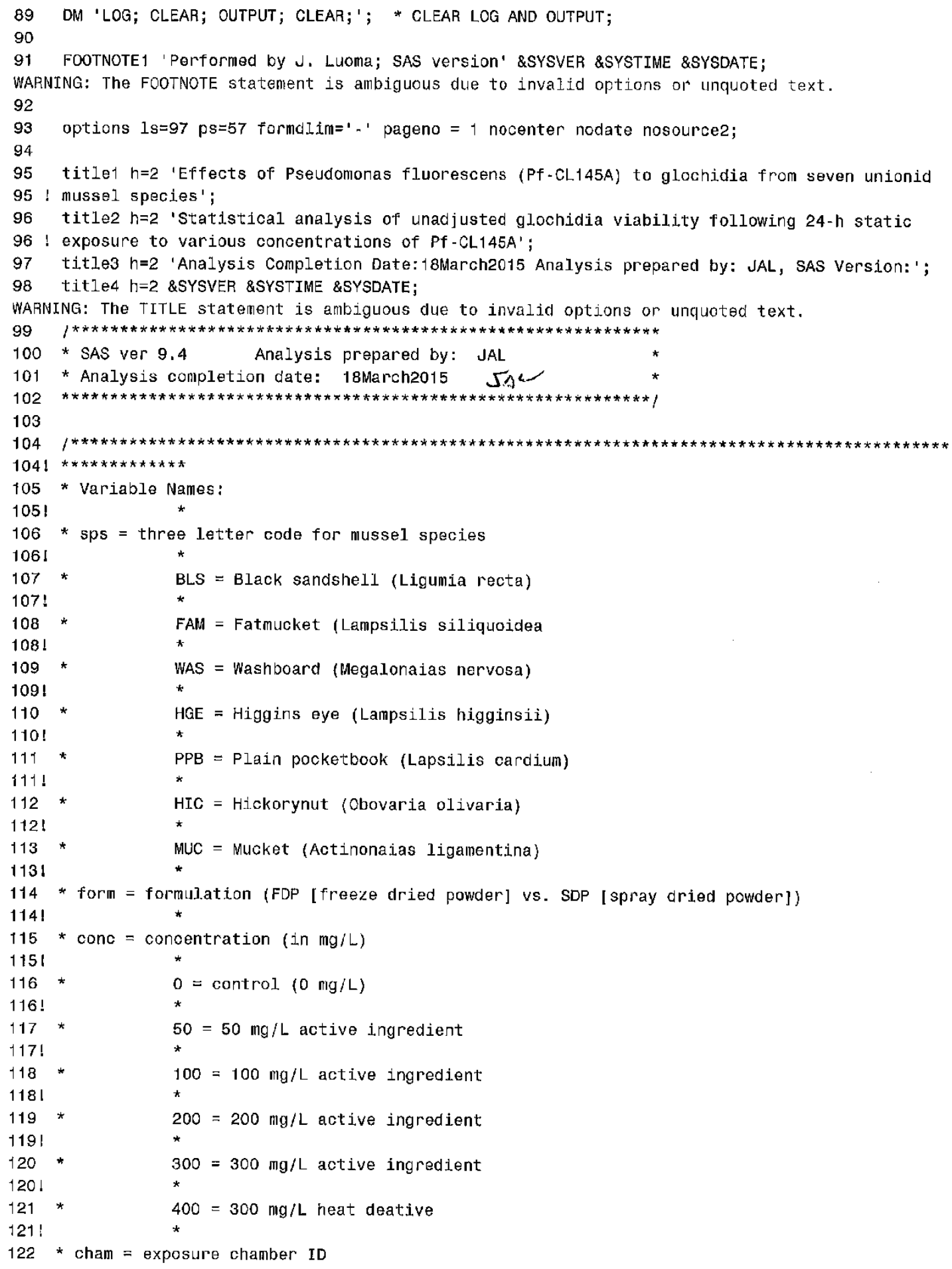




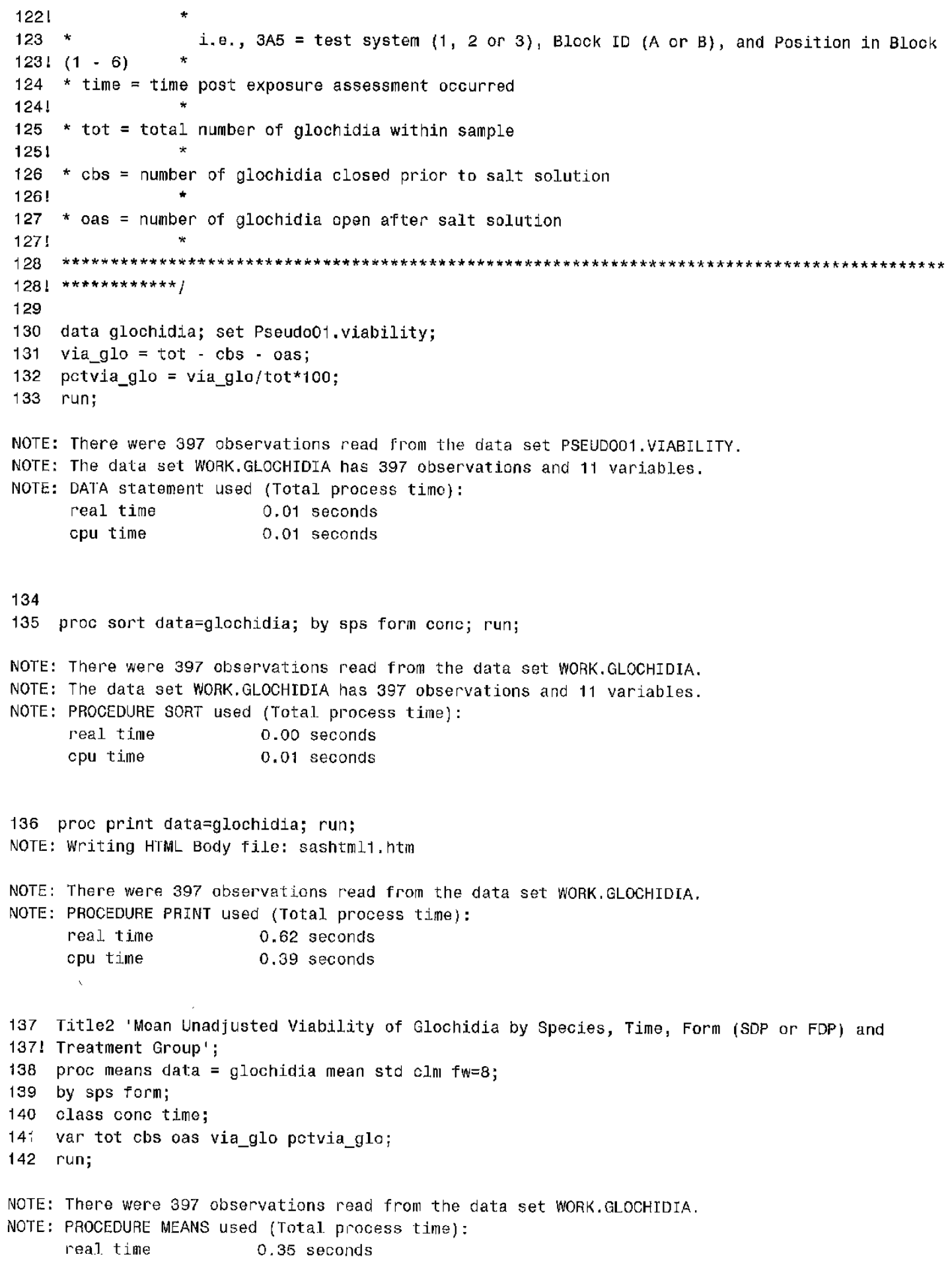




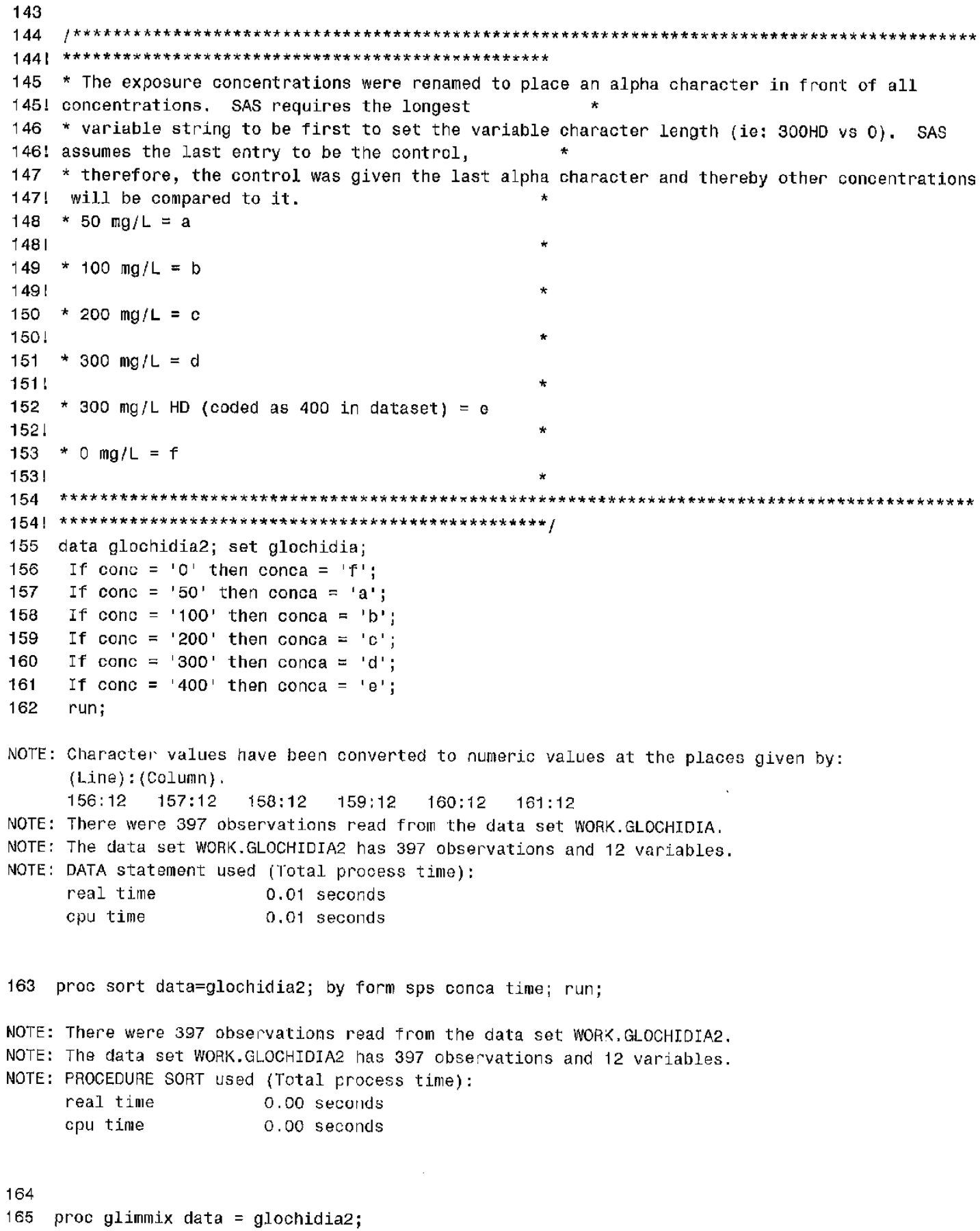




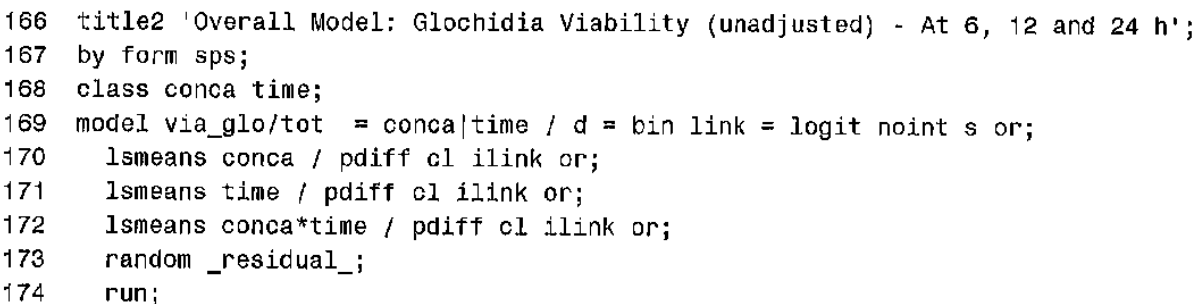

NOTE: The model does not contain an intercept, Columns of $X$ are scaled only and not centered. NOTE: Convergence criterion (ABSGCONV=0.00001) satisfied.

NOTE: The above message was for the following BY group: form=FDP sps=PPB

NOTE: The model does not contain an intercept. Columns of $X$ are scaled only and not centered.

NOTE: Convergence criterion (ABSGCONV $=0.00001$ ) satisfied.

NOTE: The above message was for the following BY group: form=FJP sps=WAS

NOTE: The model does not contain an intercept. Columns of $X$ are scaled only and not centered.

NOTE: Convergence criterion (ABSGCONV=0.00001) satisfied.

NOTE: The above message was for the following $B Y$ group: form=SDP sps=BLS

NOTE: The model does not contain an intercopt. Columns of $X$ are scaled only and not centered. NOTE: Convergence criterion (GCONV=1E-8) satisfied.

NOTE: The above message was for the following BY group: form $=$ SDP $\mathrm{sps}=\mathrm{FAM}$

NOTE: The model does not contain an intercept. Columns of $X$ are scaled only and not centered. NOTE: Convergence criterion (ABSGCONV=0.00001) satisfied.

NOTE: The above message was for the following BY group: form=SDP sps=HGE

NOTE: The model does not contain an intercept. Columns of $X$ are scaled only and rot centered. NOTE: Convergence criterion (GCONV $=1 E-8$ ) satisfied.

NOTE: The above message was for the following BY group: forml=SDP sps=HIC

NOTE: The model does not contain an intercept. Columns of $X$ are scaled only and not centered. NOTE: Convorgence oriterion (GCONV=1E-8) satisfied.

NOTE: The above message was for the following BY group: form $=$ SDP sps=MUC

NOTE: The model does not contain an intercept. Columns of $X$ are scaled only and not eentered. NOTE: Convorgence criterion (GCONV=1E-8) satisfied.

NOTE: The above message was for the following BY group: form $=S D P$ sps=PPB

NOTE: PROCEDURE GLIMAIX used (Total process time):

real time $\quad 1,42$ seconds

cpu time 1.29 seconds

175

176 quit; 


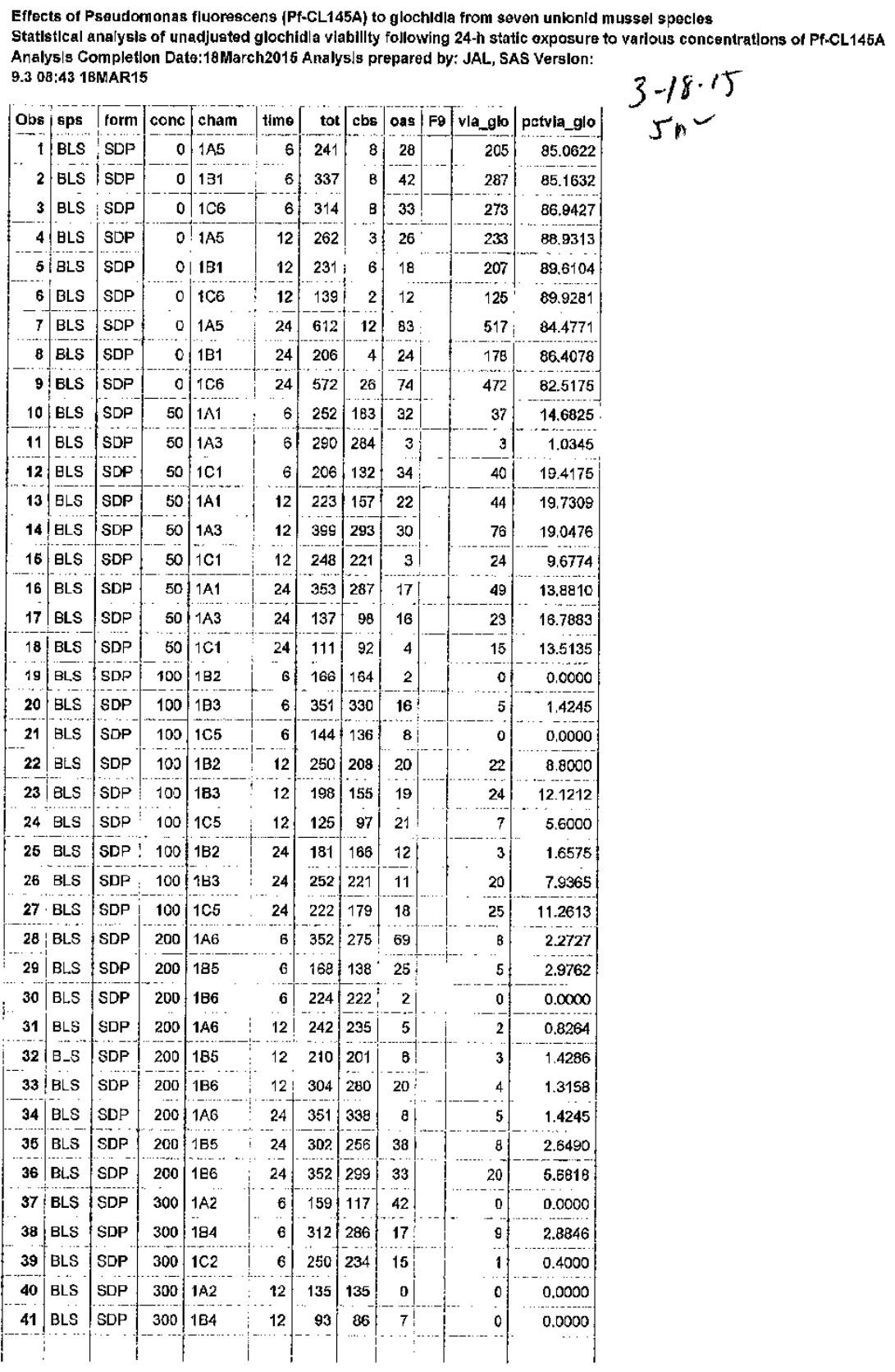

file://C:/Users/JLUOMA/AppData/I,ocal/Temp/1/SAS\%20Temporary\%20Files/_TD4836... 3/18/2015 


\begin{tabular}{|c|c|c|c|c|c|c|c|c|c|c|}
\hline 42 & BLS & $S D P$ & 300 & $1 \mathrm{C2}$ & 12 & 165 & 151 & 14 & 0 & 0.0000 \\
\hline 43 & BLS & SDP & 300 & $1 \mathrm{~A} 2$ & 24 & 284 & 270 & 14 & 0 & 0.0000 \\
\hline 44 & BLS & SDP & 300 & 184 & 24 & 255 & 247 & 5: & 3 & 1.1765 \\
\hline 45 & BLS & SOP & 300 & 102 & 24 & 383 & 373 & 6 & 4 & 1.0444 \\
\hline 46 & BLS & SDP & 400 & $1 \mathrm{~A} 4$ & 6 & 219 & 208 & 11 & 0 & 0.0000 \\
\hline 47 & BLS & SDP & 400 & $1 \mathrm{C3}$ & 6 & 254 & 213 & 41 & 0 & 0.0000 \\
\hline 48 & BLS & SDP & 400 & $1 \mathrm{CA}$ & 6 & 237 & 195 & 36 & 6 & 2.5316 \\
\hline 49 & BLS & SDP & 400 & $1 \mathrm{~A} 4$ & 12 & $281 \mid$ & 268 & 13 & 0 & 0.0000 \\
\hline 50 & BLS & SDP & 400 & $1 \mathrm{C3}$ & 12 & 157 & 147 & 8 & 2 & 1.2739 \\
\hline 51 & BLS & SDP & $4 \mathrm{CO}$ & $1 \mathrm{C} 4$ & 12 & 133 & 119 & 12 & 2 & 1.5038 \\
\hline 52 & ELS & SDP & 400 & $1 \mathrm{~A} 4$ & 24 & 485 & 471 & 11 & 3 & 0.6186 \\
\hline 53 & BLS & SDP & 400 & 1C3 & $24=$ & 381 & 367 & 8 & 6 & 1.5748 \\
\hline 54 & BLS & SDP & 400 & 104 & 24 & 302 & 295 & 7 & 0 & 0.0000 \\
\hline 55 & FAM & SDP & 0 & $3 \mathrm{~A} 2$ & 6 & 273 & 36 & 46 & 191 & 69.9634 \\
\hline 66 & FAM & SOP & 0 & $3 \mathrm{~B} 2$ & 6 & 210 & 29 & 34 & 147 & 70.0000 \\
\hline 57 & FAM & SDP & 0 & 3B3 & 6 & 265 & 32 & 29 & 204 & 76.9811 \\
\hline 68 & FAM & SDP & 0 & $3 A 2$ & 12 & 282 & 54 & 32 & 196 & 69.5035 \\
\hline 59 & FAM & SDP & 0 & 3B2 & 12 & 328 & 55 & 79 & 194 & 59.1463 \\
\hline 60 & FAM & SDP & 0 & 3B3 & 12 & 521 & 63 & 88 & 370 & 71.0173 \\
\hline 81 & FAM & SDP & 0 & $3 A_{2}$ & 24 & 307 & 48 & $55 !$ & 204 & 66.4495 \\
\hline 82 & FAM & SDP & 0 & 3B2 & 24 & 247 & 50 & 41 & 156 & 63.1579 \\
\hline 63 & FAM & SDP & 0 & $3 \mathrm{~B} 3$ & 24 & 244 & 19 & 33 & 192 & 78.6885 \\
\hline 64 & FAM & SDP & 50 & 3B5 & 6 & 456 & 93 & 98 & 265 & 58.1140 \\
\hline 65 & FAM & SDP & 50 & $3 \mathrm{C5}$ & 6 & 276 & 57 & 71 & 148 & 53.6232 \\
\hline 66 & ! FAM & SDP & 50 & $3 \mathrm{C} 6$ & 6 & 239 & 42 & 571 & 140 & 58.5774 \\
\hline 67 & FAM & SDP & 50 & 385 & 12 & 430 & 146 & 36 & 248 & 57.6744 \\
\hline 68 & FAM & SDP & 50 & $3 \mathrm{C} 5$ & 12 & 368 & 109 & 53 & 208 & 55.9783 \\
\hline 89 & FAM & SDP & 50 & $3 \mathrm{C6}$ & 12 & 207 & 33 & 42 & 132 & 63.7681 \\
\hline 70 & FAM & SDP & 50 & $3 \mathrm{B5}$ & 24 & 327 & 38 & 86 & 203 & 62.0795 \\
\hline 71 & |FAM & SDP & 50 & $3 c 5$ & 24 & 334 & 64 & 76 & 194 & 58.0838 \\
\hline 72 & FAM & SDP & 50 & $3 C 6$ & 24 & 278 & 70 & 68 & 140 & 50.3597 \\
\hline 73 & FAM & SDP & 100 & $3 A 1$ & 6 & 237 & 126 & 37 & 74 & 31.2236 \\
\hline 74 & FAM & SDP & 100 & $3 A 3$ & 6 & 303 & 238 & 25 & 40 & 13.2013 \\
\hline 75 & FAM & SDP & 100 & 3A5 & $6_{i}$ & 351 & 119 & 83 & 149 & 42.4501 \\
\hline 76 & FAM & SDP & 100 & $3 A 1$ & 12 & 409 & 183 & 48 & 178 & 43.5208 \\
\hline 77 & FAM & SDP & 100 & $3 A 3$ & 12 & 221 & 64 & 53 & 104 & 47.0588 \\
\hline 78 & FAM & SDP & 100 & $3 A 5$ & 12 & 159 & 68 & 27 & 64 & 40.2516 \\
\hline 79 & FAU & SDP & 100 & $3 A 1$ & 24 & 112 & 41. & 20 & 51 & 45.5357 \\
\hline 80 & | FAM & SDP & 100 & $3 A 3$ & 24 & 208 & 60 & 54 & 94 & 45.1923 \\
\hline 81 & FAN. & SDP & 100 & $3 A 5$ & 24 & 313 & 146 & 60 & 107 & 34.1853 \\
\hline 82 & FAM & SDP & 200 & 384 & 6 & 380 & 308 & 47 & 25 & 6.5789 \\
\hline 83 & FAM & SDP & 200 & $3 \mathrm{~B} 6$ & 6 & 526 & 407 & 53 & 66 & 12.5475 \\
\hline 84 & FAM & SDP & 200 & $3 C 4$ & 6 & 201 & 59 & $64 !$ & 78 & 38.8060 \\
\hline $85 !$ & IFAM & SOP & 200 & 3B4 & 12 & 198 & 127 & $40 !$ & 31 & 15.6566 \\
\hline 86 & FAM & SDP & 200 & 386 & 12 & 134 & 69 & 19 & 46 & 34.3284 \\
\hline 87 & FAM & SDP & 2001 & $3 \mathrm{C} 4$ & 12 & 214 & 172 & 23 & 19 & 8.8785 \\
\hline
\end{tabular}

file://C:/Users/JLUOMA/AppData/L,ocal/Temp/1/SAS\%20Temporary\%20Files/_TD4836... 3/18/2015 


\begin{tabular}{|c|c|c|c|c|c|c|c|c|c|c|}
\hline & FAM & SOP & 200 & 384 & 24 & 271 & 190 & 43 & 38 & 14.0221 \\
\hline B9 & FAM & SDP & 200 & 3B6 & 24 & 205 & \begin{tabular}{|l|}
147 \\
\end{tabular} & 28 & 30 & 14.6341 \\
\hline 90 & FAM & SDP & 200 & $3 C 4$ & 24 & 271 & 193 & 61 & 17 & 6.2731 \\
\hline 91. & FAM & SDP & 300 & 381 & 6 & 482 & 422 & 56 & 4 & 0.8299 \\
\hline 92 & FAM & SDP & 300 & $3 \mathrm{C1}$ & 6 & 501 & 224 & 121 & 156 & 31.1377 \\
\hline 93. & FAM & SDP & 300 & $3 C_{3}$ & 8 & 242 & 204 & 29 ! & $\mathbf{g}$ & 3.7190 \\
\hline 94 & FAM & SDP & 300 & 381 & 12 & 298 & 233 & 55 & 10 & 3.3557 \\
\hline 95 & FAM & SDP & 300 & $3 \mathrm{C1}$ & 12 & 240 & 195 & 29 & 16 & 6.6667 \\
\hline 96 & FAM & SDP & 300 & $3 \mathrm{C} 3$ & 12 & 206 & 173 & 26 & 7 & 3.3981 \\
\hline 97 & FAM & SDP & 300 & $3 \mathrm{~B} 1$ & 24 & 217 & 170 & 35 & $12 !$ & 5.5300 \\
\hline 98 & FAM & SDP & $3 c c$ & $3 \mathrm{C} 1$ & 24 & 176 & 145 & 23 & 8 & 4.5455 \\
\hline 99 & FAM & SDP & 300 & 303 & 24 & 215 & 130 & 68 ! & $17 !$ & 7.9070 \\
\hline 100 & FAM & SDP & 400 & $3 A 4$ & 6 & 216 & 155 & 56 & 5 & 2.3148 \\
\hline 101 & FAM & SOP & 400 & 3AB & 6 & 212 & 164 & 38 & 10 & 4.7170 \\
\hline 102 & FAM & SDP & 400 & $3 \mathrm{C} 2$ & 6 & 205 & $|166|$ & 39 & 0 & 0.0000 \\
\hline 103 & FAM & SDP & 400 & $3 A 4$ & 12 & 310 & 252 & 52 & 6 & 1.9355 \\
\hline 104 & FAM & SDP & 400 & $3 A 6$ & 12 & 298 & 229 & 54 & 15 & 5.0336 \\
\hline 105 & FAM & SDF & 400 & $3 \mathrm{C} 2$ & 12 & 187 & 131 & $56 !$ & 0 & 0.0000 \\
\hline 106 & FAM & SDP & 400 & $3 \mathrm{~A} 4$ & 24 & 281 & 250 & 29 & 2 & 0.7117 \\
\hline 107 & FA.M & SDP & 400 & $3 A 6$ & 24 & 86 & 69 & 15 & 2 & 2.3256 \\
\hline 108 & FAM & SDP & 400 & $3 \mathrm{C} 2$ & 24 & 336 & 293 & 36 & 7 & 2.0833 \\
\hline 109 & HGE & SDP & 0 & $2 \mathrm{~B} 1$ & 6 & 258 & 18 & 31 & 209 & 81.0078 \\
\hline $1+0$ & HGE & SDP & 0 & $2 \mathrm{C3}$ & 6) & 248 & 18 & 76 & 154 & 62.0968 \\
\hline 111 : & HGE & SDP & 0 & $2 \mathrm{C} 6$ & 6 & 168 & 10 & 34 & 124 & 73.8095 \\
\hline 112 & HGE & SDP & 0 & $2 \mathrm{~B} 1$ & 12 & 254 & 29 & 57 & 168 & 66.1417 \\
\hline 113 & HGE & SDP & 0 & $2 \mathrm{C} 3$ & 12 & 481 & $48 \mid 1$ & 124 & 309 & 64.2412 \\
\hline 114 & HGE & SDP & 0 & $2 \mathrm{CO}$ & 12 & 210 & 13 & 38 & 159 & 75.7143 \\
\hline 115 & भGE & SDP & 0 & $2 \mathrm{C} 3$ & 24 & 318 & 28 & 66 & 224 & 70,4403 \\
\hline $116^{\circ}$ & HGE & SDP! & 0 & 2C3-R2 & 24 & 377 & $46 \mid 1$ & 108 & 223 & 59.1512 \\
\hline 117 & HGE & SDP & a & $2 \mathrm{C6}$ & 24 & 557 & 61 & 70 & 426 & 76.4811 \\
\hline 118 & $\mathrm{HGE}$ & SDP & 01 & 2C6-R2 & 24 & 408 & 44 & 73 & 291 & 71.3235 \\
\hline 119 & HGE & SDP & 50 & $2 A 1$ & 6 & 224 & 11 & 39 & 174 & 77.6786 \\
\hline $120 !$ & HGE & SDP & 50 & $2 \mathrm{~A} 2$ & 6 & 616 & 26 & 88 & 502 & 81.4935 \\
\hline 121 & HGE & SDP & 50 & $2 A 4$ & B & 204. & 24 & 29 & 151 & 74.0196 \\
\hline 122 & HGE & SDP & 50 & $2 A 1$ & 12 & 225 & 38 & 48 & 139 & 61.7778 \\
\hline 123 & HGE & SDP & 50 & $2 \mathrm{~A} 2$ & 12 & 230 & 21 & 49 & 160 & 69.5652 \\
\hline 124 & HGE & SDP & 50 & $2 \mathrm{~A} 4$ & 12 & 339 & 36 & 50 & 253 & 74.6313 \\
\hline 125 & HGE & SDP & 50 & $2 A 1$ & 24 & 506 & $49^{i}$ & 67 & 390 & 77.0751 \\
\hline 126 & HGE & SDP & 50 & $2 A 2$ & 24 & 514 & 55 & 68 & 391 & 76.0700 \\
\hline 127 & HGE & SDP & 50 & $2 A 4$ & 24 & 464 & $34 ! 1$ & 102 & 328 & 70.6897 \\
\hline 128 & HGE & SDP & 100 & $2 A 3$ & 6 & 261 & 47 & 51 & 163 & 62.4521 \\
\hline 129 & HGE & SDP & 100 & $2 \mathrm{C} 4$ & 6 & 396 & \begin{tabular}{l|l}
40 & 1 \\
\end{tabular} & 101 & 255 & 64.3939 \\
\hline 130 & HGE & SDP & 100 & $2 \mathrm{C5}$ & 6 & 414 & 103 & 51 & 260 & 62.8019 \\
\hline 131 & HGE & SDP & 100 & $2 A 3$ & 12 & 235 & 50 & $3 \mathrm{~B}$ & 147 & 62.5532 \\
\hline 132 & HGE & SDP & 100 & $2 C 4$ & 12 & 273 & 35 & $60:$ & 178 & 65.2015 \\
\hline 133 & HGE & SOP & 100 & $2 \mathrm{C5}$ & 12 & 362 & 41 & 72 & 249 & 68.7845 \\
\hline
\end{tabular}

file://C:/Users/JLUOMA/AppData/I,ocal/Temp/1/SAS\%20Temporary\%20Files/_TD4836... 3/18/2015 


\begin{tabular}{|c|c|c|c|c|c|c|c|c|c|c|}
\hline & HGE $\mid$ & |SDP & 100 & $2 A 3$ & 24 & 629 & 95 & 87 & 447 & 71.0652 \\
\hline 135 & HGE & SDP & 100 & 204 & 24 & 423 & 87 & 69 & 267 & 63.1206 \\
\hline 136 & HGE & SDP & 100 & 205 & 24 & 413 & 105 & 51 & 257 & 62.2276 \\
\hline 137 & HGE & SDP & 200 & $2 A B$ & 6 & 492 & 120 & 62 & 310 & 63.0081 \\
\hline 138 & HGE & SDP & 200 & 2B2 & 3 & 357 & 73 & 93 & 191 & 53.5014 \\
\hline 139 & HGE & $\mathrm{SDP}$ & 200 & $2 B 5$ & 6 & 512 & 121 & 117 & 274 & 53.5156 \\
\hline 140 & HGE & SDP & 200 & $2 A B$ & 12 & 266 & 146 & 51 & 69 & 25.9398 \\
\hline 141 & HGE & SDP & 200 & $2 \mathrm{~B} 2$ & 12 & 344 & 65 & 73 & 206 & 59.8837 \\
\hline 142 & HGE & SDP & 200 & 285 & 12 & 274 & 81 & 48 & 145 & 52.9107 \\
\hline 143 & HGE & SDP & 200 & $2 A 6$ & 24 & 296 & 83 & 56 & 157 & 53.0405 \\
\hline 144 & HGE & SDP & 200 & 2B2 & 24 & $36 \mathrm{~S}$ & 135 & 54 & 180 & 48.7805 \\
\hline 146 & HGE & SDP & 200 & $2 \mathrm{~B} 5$ & 24 & 441 & 272 & 56 & 113 & 25.6236 \\
\hline 148 & H.GE & SDP & 300 & $2 \mathrm{B3}$ & 6 & 297 & 251 & 25 & 21 & 7.0707 \\
\hline 147 & HGE & SDP & 300 & $2 \mathrm{C} 1$ & 6 & 279 & 148 & 48 & 83 & 29.7491 \\
\hline 148 & HGE & SDP & 300 & $2 \mathrm{C} 2$ & 6 & 422 & 98 & 111 & 213. & 50.4739 \\
\hline 149 & HGE & $\mathrm{SDP}$ & 300 & 283 & 12 & 240 & 107 & 60 & 73 & 30.4167 . \\
\hline 160 & HGE & SDP & 300 & $2 \mathrm{Cl}$ & 12 & 267 & 67 & 95 & 105 & 39.3258 \\
\hline 151 & ' HGE & SDP & 300 & $2 \mathrm{C} 2$ & 12 & 215 & 52 & 63 & 100 & 46.5116 \\
\hline 152 & HGE & SDP ! & 300 & 2B3 & 24 & 171 & 109 & 54 & 8 & 4,6784 \\
\hline 153 & HGE & SDP & 300 & $2 \mathrm{C} 1$ & 24 & 354 & 287 & 34 & 33 & 9.3220 \\
\hline 154 & HGE & SDP & 300 & $2 \mathrm{C} 2$ & 24 & 140 & 38 & 28 & 74 & 52.8571 \\
\hline 155 & HOE & SDP & 400 & $2 \mathrm{~A} 5$ & 6 & 327 & 216 & 60 & 51 & 15.5963 \\
\hline 166 & HGE & SDP & 400 & 284 & 6 & 276 & 168 & 41 & 67 & 24.2754 \\
\hline 157 & HGE & SDP & 400 & $2 \mathrm{~B} 6$ & 6 & 139 & 102 & 37 & $0)$ & 0.0000 \\
\hline 158 & HGE & SDP & 400 & 2A5 & 12 & 229 & 161 ? & 44 & 24 & 10.4803 \\
\hline 159 & HGE & SDP & 400 & $2 \mathrm{~B} 4$ & 12 & 192 & 143 & 32 & 17 & 8.8542 \\
\hline 160 & HGE & SDP & 400 & 286 & 12 & 206 & 142 & 45 & 19 & 9.2233 \\
\hline 161 & HEE & SDP & 400 & $2 A 5$ & 24 & 137 & 89 & 42 & 6 & 4.3798 \\
\hline 162 & HGE & SDP & 400 & $2 \mathrm{E} 4$ & 24 & 216 & 88 & 74 & 54 & 25.0000 \\
\hline 163 & HGE & SDP & 400 & 286 & 24 & 330 & 229 & 60 & 41 & 12.4242 \\
\hline 164 & $\mathrm{H} \mid \mathrm{C}$ & SDP & 0 & $3 A 2$ & G & 258 & 65 & 21 & 172 & 66.6667 \\
\hline 166 & ! HIC & $\mathrm{SDP}^{-}$ & 0 & $3 \mathrm{C} 1$ & 6 & 156 & 8 & 12 & 136 & 87.1795 \\
\hline 166 & $\mathrm{HIC}$ & SDP & 0 & $3 c 4$ & 6 & 259 & 12 & 24 & 223 & 86.1004 \\
\hline 167 & HIC & SDP & 0 & $3 A 2$ & 12 & 257 & 6 & 22 & 229 & 89.1051 \\
\hline 168 & HIC & SDP & 0 & $3 \mathrm{C} 1$ & 12 & 216 & 19 & 26 & 171 & 79.1667 \\
\hline 169 & HIC & $S D P$ & 0 & $3 C 4$ & 12 & 144 & 5 & 8 & 131 & 90.9722 \\
\hline 170 & HIC & $S D P$ & 0 & $3 A 2$ & 24 & 92 & $B$ & 14 & 170 & 88.5417 \\
\hline 171 & HIC & SDP & 0 & $3 \mathrm{C} 1$ & 24 & 376 & 22 & 34 & 320 & 85.1064 \\
\hline 172 & HIC. & 'SDP & $0_{i}$ & $3 \mathrm{C} 4$ & 24 & 359 & 14 & 31 & 314 & 87.4652 \\
\hline 173 & $\mathrm{HIC}$ & SDP & 50 & 3A5 & 6. & 267 & 16 & 21 & 230 & 86.1423 \\
\hline 174 & \|\|$C$ & SDP & 50 & $3 \mathrm{~B} 3$ & 6 & 191 & 21 & 10 & 160 & 83.7696 \\
\hline 175 & HIC & SDP & 50 & 3B6 & 6 & 274 & 26 & 13 & 235 & 85.7664 \\
\hline 176 & {$[\mathrm{H} \mid \mathrm{C}$} & SDP & 50 & $3 A 5$ & 12 & 172 & 21 & 10 & 141 & 81.9767 \\
\hline 177 & $\mathrm{HIC}$ & SDP & 50 & 3B3 & 12 & 197 & 24 & 8 & 165 & 83.7563 \\
\hline 178 & HIC & SDP & 50 & 3B6 & 12 & 186 & 9 & 14 & 163 & 87.6344 \\
\hline 179 & IHIC & SDP & 50 & $3 A 5$ & 24 & 223 & 9 & 8 & 206 & 92.3767 \\
\hline
\end{tabular}

file://C:/Users/JI_UOMA/AppData/Local/Temp/1/SAS\%20Temporary\%20Files/_TD4836... 3/18/2015 


\begin{tabular}{|c|c|c|c|c|c|c|c|c|c|c|}
\hline & i HIC & SDP & 50 & 3B3 & 24 & 353 & 18 & 22 & 263 & 86.7987 \\
\hline 181 & $\mathrm{HIC}$ & SDP & 50 & 3B6 & 24 & 233 & 18 & 9 & 206 & $B B, 412 C$ \\
\hline 182 & HIC & SDP & 100 & 3B1 & 6 & 232 & 39 & 32 & 161 & 69.3966 \\
\hline 183 & HIC & SDP & 100 & $3 \mathrm{C} 2$ & 6 & 231 & 114 & 13 & 104 & 45.0216 \\
\hline 184 & HIC & SDP & 100 & $3 \mathrm{C} 6$ & 6 & 292 & 81 & 11 & 2001 & 68.4932 \\
\hline 185 & HIC & SDP & 100 & 381 & 12 & 214 & 36 & 6 & 172 & 80.3738 \\
\hline 186 & $\mathrm{HIC}$ & SDP & 100 & $3 C 2$ & 12 & 195 & 18 & 6 & 171 & 87,6923 \\
\hline 187 & HIC & SDP & 100 & $3 C 5$ & 12 & 216 & 75 & 4 & 137 & 63.4259 \\
\hline 188 & HIC & SOP & 100 & 3B1 & 24 & 404 & 36 & 21 & 347 & 85.8911 \\
\hline 189 & HIC & SDP & 100 & $3 \mathrm{C} 2$ & 24 & 278 & 59. & 17 & 202 & 72.6619 \\
\hline 190 & HIC & SDP & 100 & $3 \mathrm{C} 5$ & 24 & 257 & 51 & 10 & 196 & 76.2646 \\
\hline 181 & $\mathrm{HIC}$ & SDP & 200 & $3 A 3$ & 6 & 231 & 142 & 19 & 70 & 30.3030 \\
\hline 192 & HIC & SDP! & 200 & $3 A 4$ & 6 & 176 & 90 & 18 & 68 & 38.6364 \\
\hline 193 & $\mathrm{HIC}$ & SDP & 200 & $3 \mathrm{CC}$ & 6 & 285 & 117 & 17 & 151 & 52.9825 \\
\hline 194 & $\mathrm{HIC}$ & SDP & 200 & $3 A 3$ & 12 & 134 & 86 & 3 & 45 & 33.5821 \\
\hline 195 & $\mathrm{HIC}$ & SDP & 200 & $3 \mathrm{~A} 4$ & 12 & 186 & 84 & 8 & 94 & 50.5376 \\
\hline 196 & $\mathrm{HIC}$ & SDP & 200 & $3 \mathrm{C6}$ & 12 & 138 & 41 & 5 & 92 & 66.6667 \\
\hline 197 & $\mathrm{HIC}$ & SDP & 200 & $3 A 3$ & 24 & 349 & 114 & 10 & 225 & 64.4699 \\
\hline 198 & HIC & SDP & 200 & 3A4 & 24 & 203 & 78 & 12 & 113 & 55.6650 \\
\hline 199 & $\mathrm{HIC}$ & SDP & 200 & $3 \mathrm{C} 6$ & 24 & 197 & 66 . & $9 !$ & 122 & 61.9289 \\
\hline 200 & $\mathrm{HIC}$ & SDP & 300 & $3 A 6$ & 6 & 293 & 192 & 13 & 88 & 30.0341 \\
\hline 201 & HIC & SDP & 300 & 3B2 & 8 & 173 & 180 & 3 & 10 & 5.7803 \\
\hline 202 & $\mathrm{HIC}$ & SDP & 300 & $3 B 5$ & 6 & 266 & 226 & 10 & 30 & 11.2782 \\
\hline 203 & $\mathrm{H} \mid \mathrm{C}$ & SDP & 300 & $3 A 6$ & 12 & 204 & 123 & 8 & 73 & 35.7843 \\
\hline 204 & $\mathrm{HIC}$ & SDP & 300 & $3 \mathrm{~B} 2$ & 12 & 200 & 107 & 12 & 81 & 40.5000 \\
\hline 206 & $\mathrm{HIC}$ & SDP & 300 & $3 B 5$ & 12 & 206 & 98 & 23 & 85 & 41.2621 \\
\hline 206 & $\mathrm{HC}$ & SDP & 300 & $3 A 6$ & 24 & 191 & 138 & 9 & 44 & 23.0366 \\
\hline 207 & HIC & SDP & 300 & 382 & 24 & 140 & 98 & 4 & 38 & 27.1429 \\
\hline 208 & $\mathrm{HIC}$ & SDP & 300 & 385 & 24 & 231 & 156 & 7 & 68 & 29,4372 \\
\hline 209 & $\mathrm{H} \mid \mathrm{C}$ & SDP & 400 & BA1 & 6 & 362 & 262 & 25 & 75 & 20.7182 \\
\hline 210 & HIC & SDP & 400 & 384 & 6 & 317 & 274 & 16 & 27 & 8.5174 \\
\hline 211 & HIC & SDP & 400 & $3 \mathrm{C} 3$ & 6 & 246 & 209 & 71 & 30 & 12.1951 \\
\hline 212 & $\mathrm{HIC}$ & SOP & 400 & $3 A 1$ & 12 & 249 & 231 & 15 & 3 & 1.2048 \\
\hline 213 & $\mathrm{HIC}$ & SDP & 400 & $3 \mathrm{~B} 4$ & 12 & 338 & 313 & 22 & 3 & 0.8876 \\
\hline 214 ! & HIC & SDP & 400 & $3 \mathrm{C} 3$ & 12 & 251 & 244 & 3 & 4. & 1.5936 \\
\hline 215 & $\mathrm{HIC}$ & SDP & 400 & 3A1 & 24 & 290 & 287 & 2 & 1. & 0.3448 \\
\hline 216 & HIC & SDP & 400 & $3 \mathrm{BA}$ & 24 & 314 & 307 & 6 & 1) & 0.3185 \\
\hline 217 & HIC & SDP & 400 & 303 & 24 & 226 & 221 & 3 & 2 & 0.8850 \\
\hline 218 & MUC & SDP & 0 & $2 \mathrm{~B} 4$ & 6 & 98 & 15 & 17 & 166 & 83.8384 \\
\hline 219 & MUC & SDP & 0 & 285 & 6 & 132 & 2 & 12 & 118 & 89.3939 \\
\hline 220 & MUC & SDP & 0 & $2 \mathrm{C} 1$ & 6 & 257 & 7 & 12 & 238 & 92.6070 \\
\hline 221 & MUC & SDP & 0 & $2 B A$ & 12 & 178 & 2 & 11 & 165 & 92.6966 \\
\hline 222 & MUC & SDP & 0 & 285 & \begin{tabular}{|ll}
1 & 12 \\
\hdashline
\end{tabular} & 213 & 0 & 12 & 201 & 94.3662 \\
\hline 223 & MUC & SDP & 0 & $2 \mathrm{C} 1$ & 12 & 254 & 6 & 5. & 243 & $\begin{array}{r}95.6693 \\
\end{array}$ \\
\hline $\mathbf{2 2 4}$ & MUC & SDP & 0 & $2 \mathrm{~B} 4$ & 24 & $261 !$ & 7 & 19 & 235 & 90.0383 \\
\hline 225 & MUC & SDP & 0 & 2B5 & 24 & 361 & 1 & 20 & 340 & 94.1828 \\
\hline
\end{tabular}

file://C./Users/JI,UOMA/AppData/Local/Temp/1/SAS\%20'Temporary\%20Files/_TD4836... 3/18/2015 


\begin{tabular}{|c|c|c|c|c|c|c|c|c|c|c|}
\hline & $M L C$ & SDP & 0 & 201 & 24 & 251 & 8 & 8 & 235 & 93.6255 \\
\hline 227 & MUC & SOP & 50 & $2 A 4$ & 6 & 349 & 51 & 36 & 262 & 75.0716 \\
\hline 228 & $M \cup C$ & SDP & 50 & 286 & 6 & 128 & 43 & 3 & 82 & 64.0625 \\
\hline 229 & MUC & SDP & 50 & $2 \mathrm{C} 3$ & 6 & 295 & 59 & 12 & 224 & 75.9322 \\
\hline 230 & MUC & SDP & 50 & $2 \mathrm{A4}$ & 12 & 221 & 63 & 14 & 144 & 65.1584 \\
\hline 231 & MJC & SDP & 50 & 286 & 12. & 113 | & 53 & 4 & 56 & 49.5575 \\
\hline 232 & $M U C$ & SDP & 50 & $2 \mathrm{C3}$ & 12 & 193 & 17 & $12 j$ & 164 & 84.9741 \\
\hline 233 & MUC & SDP & 50 & $2 \mathrm{~A} 4$ & 24 & 328 & 49 & 14 & 265 & 80.7927 \\
\hline 234 & MUC & SDP & 50 & 236 & 24 & 146 & 48 & 8 & 89 & 60.9689 \\
\hline 236 & MUC & SDP & 50 & $2 \mathrm{C3}$ & 24 & 194 & 19 & 9 & 166 & 85.5670 \\
\hline 236 & MUC & SDP & 100 & 2A2 & 6. & 190 & 61 & 13 & 116 & 61.0526 \\
\hline 237 & MUC & SDP & 100 & $2 B 2$ & 6 & 154 & 26 & 7 & 121 & 78.5714 \\
\hline 238 & MUC & SDP & 100 & $2 \mathrm{C} 6$ & 5 & 466 & 78 & 29 & 359 & 77.0386 \\
\hline 239 & MUC & SDP & 100 & $2 A 2$ & 12. & 210 & 91. & 10 & 100 & 51.8048 \\
\hline 240 & MUC & SDP & 100 & $2 \mathrm{~B} 2$ & 12 & 138 & 46 & 14 & 78 & 56.5217 \\
\hline 241 & MUC & SDP & 100 & 206 & 12 & 215 & 26 & 7 & 182 & 84.6512 \\
\hline 242 & MUC & SDP & 100 & $2 \mathrm{~A} 2$ & 24 & 191 & 42 & 5 & 144 & 75.3927 \\
\hline 243 & MUC & SDP & 100 & 2B? & 24 & 200 & 87 & 11 & 111 & 53.1100 \\
\hline 244 & MUC & SDP & 100 & $2 \mathrm{Cz}$ & 24 & 213 & 71 & 10 & 132 & 61.9718 \\
\hline 246 & MUC & SDP & 200 & $2 A 5$ & 6 & 116 & 48 & 8 & 60 & 51.7241 \\
\hline 246 & MUC & SDP & 200 & 2A6 & 6 & 202 & 82 & 23 & 97 & 48.0198 \\
\hline 247 & MUC & SDP & 200 & $2 \mathrm{C} 2$ & 6 & 399 & 291 & 21 & 87 & 21.8045 \\
\hline 248 & MUC & SDP & 200 & $2 \mathrm{~A} 5$ & 12 & 175 & 83. & 17 & 75 & $\begin{array}{r}42.8571 \\
\end{array}$ \\
\hline 249 & MUC & SDP & 200 & $2 A 6$ & 12 & 179 & 92 & 11 & 76 & 42,4581 \\
\hline 260 & MUC & $S D P$ & 200 & $2 \mathrm{C2}$ & 12 & 362 & 227 & 9 & 126 & 34.8066 \\
\hline 251 & :vuc & SDP & 200 & $2 A 5$ & 24 & 251: & 170 & 16 & 65 & 25.8964 \\
\hline 252 & MUC & SDP & 200 & $2 A 6$ & 24 & 407 & 241 & 10 & 156 & 38.3292 \\
\hline 253 & MUC & SDP & 200 & $2 \mathrm{C} 2$ & 24 & 497 & 355 & 14 & 128 & $\begin{array}{r}25.7546 \\
\end{array}$ \\
\hline 254 & NUC & SDP! & 300 & $2 \mathrm{~B} 1$ & 6 & 265 . & 199 & 14 & 52 & 19.6226 \\
\hline 255 & MUC & SDP & 300 & $2 B 3$ & 6 & $251]$ & 232 & 6 & 13 & 5.1793 \\
\hline 256 & I MUC & SDP & 300 & $2 \mathrm{C} 4$ & 6 & 323 & 283 & 6 & 34 & 10.5263 \\
\hline 257 & MUC & SDP & 300 & 281 & 12 & 247 & 212 & 4 & 31 & 12.5506 \\
\hline 258 & MUC & SDP & 300 & 283 & 12 & 198 & 181 & 2 & 15 & 7.5758 \\
\hline 259 & MUC & SDP & 300 & $2 \mathrm{C} 4$ & 12 & 116 & $102 !$ & 2 & 12 & 10.3448 \\
\hline 260 & MUC & SUP & 300 & 2B1 & 24 & 192 & $171^{i}$ & 3 & 18 & 9.3750 \\
\hline 261 & Muc & SDP & 300 & $2 \mathrm{~B} 3$ & 24 & 297 & 281 & 9 & 7 & 2.3569 \\
\hline 262 & MUC & SDP & 300 & $2 \mathrm{C} 4$ & 2.4 & 451 & 417 & 6 & 28 & 6.2084 \\
\hline 263 & MUC & SDP & 400 & $2 \mathrm{A1}$ & 6 & 292 & 272 & 4 & 16 & 5.4795 \\
\hline 264 & MUC & SDP & 400 & $2 A 3$ & 6 & 355 & 342 & 8 & 5 & 1.4085 \\
\hline 265 & MUC & SDP & 400 & $2 \mathrm{C5}$ & 6 & 280 & 259 & 10 & 11 & 3.9286 \\
\hline 266 & $M \cup C$ & SDP & 400 & $2 \mathrm{~A} 1$ & 12 & 237 & 201 & 14 & 22 & 9.2827 \\
\hline 267 & MLC & SOP & 400 & $2 \mathrm{A3}$ & 12 & 127 & 100 & 11 & 16 & 12.5984 \\
\hline 268 & MUC & SDP & 400 & $2 C 5$ & 12 & 184 & 175 & 4 & 5 & 2.7174 \\
\hline 269 & MUC & SDP & 400 & $2 A 1$ & 24 & 292 & 278 & 6 & 8 & 2.7397 \\
\hline 270 & $M \cup C$ & SDP & 400 & $2 \mathrm{A3}$ & 24 & 308 & 276 & 4 & 28 & 9.0909 \\
\hline 271 & MUC & SDP & 400 & $2 \mathrm{CE}$ & 24 & 157 & 149 & $2 !$ & 6 & 3.8217 \\
\hline
\end{tabular}




\begin{tabular}{|c|c|c|c|c|c|c|c|c|c|c|}
\hline & PPB & FDP & 0 & 3AG & 6 & 214 & 17 & $16:$ & 181 & 84.5794 \\
\hline 273 & IPPB & FDP & 0 & 385 & 6 & 89 & 7 & 6 & 76 & 85.3933 \\
\hline 274 & PPB & I FDP & 0 & 336 & 6 & 125 & 18 & 11 & 96 & 76.8000 \\
\hline 275 & PPB & FDP & 0 & 3.46 & 12 & 33 & 3 & 20 & 110 & 82.7068 \\
\hline 276 & PPB & FDP & 0 & 385 & 12 & 314 & 8 & 61 & 245 & 78.0255 \\
\hline 277 & $P P B$ & FDP & 0. & $3 B 6$ & 12 & 182. & 5 & $23 \mid$ & 154 & 84.6154 \\
\hline 278 & PPB & FDP & 0 & $3 A 6$ & 24 & 573 & 21 & 74 & 478 & 83.4206 \\
\hline 279 & PPB & FDP & 0 & 3B5 & 24 & 279 & 7 & 47 & 225 & 80.6452 \\
\hline 280 & PPB & FDP & 0 & 3B6 & 24 & 278 & 17 & 34 & 227 & 81.6547 \\
\hline 281 & PPB & FDP & 50 & $3 A 4$ & 6 & 157 & 16 & 17 & 124 & 78.9809 \\
\hline 282 & PPB & FDP & $5 \mathrm{C}$ & $3 \mathrm{Cl}$ & 6 & 74 & 27 & 5: & 42 & 56.7568 \\
\hline 283 & PPB & FDP & 50 & $3 \mathrm{C} 2$ & 6 & 144 & 22 & 17 & 105 & 72.9167 \\
\hline 284 & PPB & FDP & 50 & $3 A 4$ & 12 & 280 & 24 & 62 & 204 & 70.3448 \\
\hline 285 & PPB & FDP & 50 & $3 \mathrm{C} 1$ & 12 & 160 & 8 & 29 & 123 & 76.8750 \\
\hline 286 & PPB & FDP & 50 & $3 \mathrm{C} 2$ & 12 & 606 & 34 & 70 & 502 & 82.8383 \\
\hline 287 & PPB & FDP & 50 & 3A4 & 24 & 164 & 39 & 21 & 104 & 63.4146 \\
\hline 288 & $\mathrm{PPB}$ & FDP & 50 & 301 & 24 & 368 & 33 & 45 & 290 & 78.8043 \\
\hline $289 !$ & PPB & FDP & 50 & $3 \mathrm{C} 2$ & 24 & 384 & 30 & 46 & 308 & 80.2083 \\
\hline 290 & PPB & FDP & 100 & 3B1 & 6 & 259 & 70 & 21 & 168 & 64.8649 \\
\hline 291 & PPB & FDP & 100 & 3B3 & 6 & 190 & 33 & 25 & 132. & 69.4737 \\
\hline 292 & PPB & $F D P$ & 100 & $3 \mathrm{C} 6$ & 6 & 161 & 47 & 13 & 101 & 62.7328 \\
\hline 283 & PPB & FDP & 100 & $3 \mathrm{~B} 1$ & 12 & 179 & 46 & 22 & 111 & 62.0112 \\
\hline 284 & PPB & FDP & 100 & 383 & 12. & 130 & 20 & 18 & 92 & 70.7692 \\
\hline 295 & PPB & FDP & 100 & 306 & 12 & 142 & 15 & 15 & 112 & 78.8732 \\
\hline 296 & PPB & FDP & 100 & 381 & 24 & 467 & 85 & 60 & 322 & 68.9507 \\
\hline 297 & $\mathrm{PPB}$ & FDP & 100 & $3 B 3$ & 24 & 405 & 93 & 55 & 257 & 63.4568 \\
\hline 298 & PF日 & FDP & 100 & $3 \mathrm{C6}$ & 24 & 288 & 38 & 46 & 204 & 70.8333 \\
\hline 299 & PPB & FDP & 200 & 3A2 & 6 & 256 & 86. & 52 & 118 & 46.0938 \\
\hline 300 & PPB & FDP & 200 & $3 \mathrm{cs}$ & 6 & 299 & 141 & 42 & 116 & 38.7960 \\
\hline 301 & PPB & FDP & 200 & $3 \mathrm{C} 5$ & 5 & 157 & 47 & 14 & 96 & 61.1465 \\
\hline 302 & PPB & FDP & 200 & $3 A 2$ & 12 & 249 & 161 & 38 & 50 & 20.0803 \\
\hline 303 & PPB & FDP & 200 & $3 c 3$ & 12 & 815 & 104 & 140 & 571 & 70.0613 \\
\hline 304 & PPB & FDP & 200 & $3 \mathrm{C5}$ & 12 & 200 & 151 & 20 & 29 & 14,5000 \\
\hline 305 ! & PPB & FDP & 200 & $3 A 2$ & 24 & 144 & 96 & 27 & 21 & 14.5833 \\
\hline 306 & PPB & FDP & 200 & 3C3 & 24 & 592 & 157 & 98 & 337 & 56.9257 \\
\hline 307 & PPB & FDP & 200 & 305 & 24 & 386 & $281 \mid$ & 39 & 66. & 17.0984 \\
\hline 308 & PPE & FDP & 300 & उA3 & $6^{\circ}$ & 259 & 63. & 64 & 132 & 50.9653 \\
\hline 309 & PPB & FDP & 300. & 384 & 6 & 272 & $149:$ & 58 & 65 & 23.8971 \\
\hline 310 & PPB & FDP & 300 & $3 \mathrm{C} 4$ & 6 & 655 & 218 & 128 & 309 & 47.1756 \\
\hline 311 & P & FDP & 300 & BA3 & 12 & 251 & 174 & 66 & 11 & 4.3825 \\
\hline 312 & PPB & FDP & 300 & 384 & 12 & 223 & 145 & 35 & 43 & 19.2825 \\
\hline 313 & PPE & FDP & 300 & $3 \mathrm{CA}$ & 12. & 348 & 288 & 46 & 13 & 3.7356 \\
\hline 314 & PPB & FDP & 300 & 3A3 & $24]$ & 199 & 132 & $45^{\circ}$ & 22 & 11.0553 \\
\hline 315 & PPB & FDP & 300 & $3 E 4$ & 24 & 714 & 442 & 171 & 101 & 14.1457 \\
\hline 316 & PPB & FDP & 300 & $3 C_{4}$ & 24 & $440]$ & 330 & 62 & 48 & 10.9091 \\
\hline 317 & PPB & FOP & 400 & $3 \mathrm{~A} 1$ & 6 & 200 & 144 & 45 & 11 & 5.5000 \\
\hline
\end{tabular}

file://C:/Users/JLUOMA/AppData/Local/Temp/1/SAS\%20Temporary\%20Files/_TD4836... 3/18/2015 


\begin{tabular}{|c|c|c|c|c|c|c|c|c|c|c|}
\hline & PPB & FDP & $4 C C$ & $3 A 5$ & 6 & 379 & 87 & 114 & 178 & 46.9657 \\
\hline 319 & PPB & FDP & 400 & $3 \mathrm{~B} 2$ & 3 & 418 & 321 & 60 & 37 & 8.8517 \\
\hline 320 & PPB & FDP & 400 & $3 \mathrm{~A} 1$ & 12 & 72 & 58 & 11 & 3 & 4.1667 \\
\hline 321 & PPB & FDP & 400 & $3 A 5$ & 12 & 48 & 38 & 7 & 3 & 6.2500 \\
\hline 322 & PPB & FDP & 400 & 3B2 & 12 & 242 & 211 & 21 & 10 & 4.1322 \\
\hline 323 & |PPB & FDP & 400 & $3 A 1$ & 24 & 370. & 277 & $78:$ & 15 & 4.0541 \\
\hline 324 & |PPB & FDP & 400 & $3 A 5$ & 24 & 253 & 207 & 38 & 8 & 3.1621 \\
\hline 326 & PPB & FDP & 400 & $3 B 2$ & 24 & 267 & 198 & 49 & 20 & 7.4906 \\
\hline $32 B$ & PPB & SDP & 0 & 1A3 & 6 & $16 \mathrm{C}$ & 2 & 12 & 146 & 91.2500 \\
\hline 327 & PPB & SDP & 0 & 1B1 & 6 & 312 & 3 & 18 & 291 & 93.2692 \\
\hline 328 & PPB & SDP & 0 & $1 \mathrm{C} 3$ & 6 & 426 & 7 & 25 & 394 & 92,4883 \\
\hline 329 & PPB & SDP & 0 & $1 \mathrm{~A} 3$ & 12 & 626 & 39 & 54 & 533 & 85.1438 \\
\hline 330 & | PPB & SDP & 0 & YB1 & 12 & 599 & 39 & 53 & 507 & 84.6411 \\
\hline 331 & PFB & SDP & 0 & 103 & 12 & 604 & 18 & 52 & 534 & 88.4106 \\
\hline 332 & PPB & SDP & 0 & $1 \mathrm{~A} 3$ & 24 & 429 & 12 & 26 & 391 & 91.1422 \\
\hline 333 & PPB & SDP & 0 & | $1 \mathrm{~B} 1$ & 24 & 514 & 15 & 22 & 477 & 92,8016 \\
\hline 334 & PPB & SDP & 0 & $1 \mathrm{c3}$ & 24 & 276 & 12 & 9 & 255 & 92,3913 \\
\hline 335 & PFB & SDP & 50 & $1 \mathrm{~A} 1$ & 6 & 189 & 16 & 10 & 163 & 86.2434 \\
\hline 336 & PFB & SDP & 50 & $1 \mathrm{~B} 5$ & 6 & 344 & 59 & $13 !$ & 272 & 79.0698 \\
\hline 337 & | PPB & SDP : & 50 & 101 & 6 & 447 & 129 & 12 & 306 & 68.4564 \\
\hline 338 & : PPB & SDP & 50 & $1 \mathrm{~A} 1$ & 12 & 265 & 24 & 14 & 227 & 85.6604 \\
\hline 339 & PPB & SDP & 60 & $1 \mathrm{~B} 5$ & 12 & 226 & 37 & 14 & 175 & 77.4336 \\
\hline 340 & PPB & SDP & 50 & 1C1 & 12 & 624 & 104 & 67 & 453 & 72.5962 \\
\hline 341 & PPB & SDP & 50 & $1 \mathrm{~A} 1$ & 24 & 459 & 55 & 28 & 376 & 81.8172 \\
\hline 342 & . PPB & SDP & 50 & :1B5 & 24 & 270 & 27 & 16 & 227 & 84.0741 \\
\hline 343 & PPB & SDP & 50 & 161 & 24 & 339 & 78 & 12 & 249 & 73.4513 \\
\hline 344 & |PPB & SDP & 100 & $1 \mathrm{~A} 2$ & 6 & 116 & 16 & 9 & 91 & 78.4483 \\
\hline 345 & PPB & SDP & 100 & $1 \mathrm{~A} 4$ & 6 & 535 & 70 & 42 & 424 & 79.1045 \\
\hline 346 & PPB & SDP & 100 & $1 \mathrm{~B} 4$ & 6 & 170 & 43 & 11 & 116 & 68.2353 \\
\hline 347 & PPB & SDP & 100 & $1 \mathrm{~A} 2$ & 12 & 353 & 321. & 32 & 0 & 0.0000 \\
\hline 348 & PPB & SOP & 100 & $1 A 4$ & 12 & 504 & 75 । & 40 & 389 & 77.1825 \\
\hline 349 & PPB & SDP & 100 & $1 \mathrm{~B} 4$ & 12 & 354 & $40:$ & 28 & 286 & 80.7910 \\
\hline 360 & p $P \theta_{0}$ & SDP & 100 & $1 \mathrm{~A} 2$ & 24 & 210 & $33^{i}$ & 16 & 161 & 76.6667 \\
\hline 351 & !PPB & SDP & 100 & $1 \mathrm{~A} 4$ & 24 & 310 & 34 & 125 & 151 & 48.7097 \\
\hline 352 & PPB & SDP & 100 & $1 \mathrm{~B} 4$ & 24 & 324 & 56 & 24 & 244 & 75.3086 \\
\hline 363 & PPB & $\operatorname{SDP}$ & 200 & 1B2 & 6 & 252 & 60 & 21 & 171 & 67.8571 \\
\hline 364 & PPB & SDP & 200 & $1 \mathrm{C} 4$ & 6 & 238 & 165 & 6 & 67 & 28.1513 \\
\hline 355 & НРB & SUH & 200 & $1 \mathrm{C6}$ & 6 & 145 & 47 & 10 & 88 & 60.6897 \\
\hline 356 & |PPB & SDP & 200 & 182 & 12 & 152 & 47 & $15:$ & 90 & 59.2105 \\
\hline 357 & PPB & SDP & 200 & $1 \mathrm{C} 4$ & 12 & 380 & 116 & 36 & 228 & 60.0000 \\
\hline 358 & PPB & SDP & 200 & $1 \mathrm{C6}$ & 12 & 177 & 129 & 11 & 37 & 20.9040 \\
\hline 359 & PPB & SOP & 200 & 182 & 24 & 187 & 50 & 13 & 124 & 66.3102 \\
\hline 360 & PPB & SDP & 200 & $1 \mathrm{CA}$ & 24 & 506 & 267 & 14 & 225 & 44.4664 \\
\hline 361 & PPB & SDP & 200 & 106 & 24 & 242 & 64 & 16 & 162 & 66.9421 \\
\hline 362 & PPB & SDP & 300 & $1 \mathrm{~A} 5$ & 6 & 114 & 75 & 14 & 25 & 21.9298 \\
\hline 363 & IPP3 & SDP & 300 & $1 \mathrm{C} 2$ & 6 & 512 & 464 & 31 & 17 & 3.3203 \\
\hline
\end{tabular}




\begin{tabular}{|c|c|c|c|c|c|c|c|c|c|c|}
\hline & PPB & SDP & 300 & $1 C 5$ & 6 & 268 & 234 & 22 . & 12 & 4.4776 \\
\hline 365 & PPB & SDP & 300 & $1 A .5$ & 12 & 1177 & |938 & 109 & 130 & 11.0450 \\
\hline 366 & PPB & SDP & 300 & $1 \subset 2$ & 12 & 305 & 284 & 13 & 8 & 2.8230 \\
\hline 367 & |PPB & SDP & 300 & $1 C 5$ & 12 & 460 & 403 & $30 !$ & 27 & 5.8696 \\
\hline 368 & PPB & SDP & 300 & 1A5 & 24 & 493 & 399 & 23 & 71 & 14.4016 \\
\hline 369 & PPB & SDP & 300 & 1C2 & 24 & 226 & 218 & 4 & 4 & 1.7699 \\
\hline 370 & PPB & SDP & 300 & 105 & 24 & 295 & 274 & 15 & 6 & 2.0339 \\
\hline 371 & |PPB & SDP & 400 & $1 A B$ & 6 & 147 & 108 & 30 & 9 & 6.1224 \\
\hline 372 & PPB & i SDP & 400 & 183 & 6 & 119 & 91 & 23: & 5 & 4.2017 \\
\hline 373 & PPB & SDP & 400 & 186 & 8 & 210 & 192 & 18 & 0 & 0.0000 \\
\hline $\begin{array}{lll} & 374\end{array}$ & PPB & SDP & 400 & IAG & 12 & 242 & 193 & 32 & 17 & 7.0248 \\
\hline 375 & |PpB & SDP & 400 & 183 & 12 & 225 & 171 & 37 & 17 & 7.5556 \\
\hline 376 & PPB & SDP & 400 & 186 & 12 & 322 & 280 & 33 & 9 & 2.7950 \\
\hline 377 & PPB & SDP & 400 & IAB & 24 & 204 & 147 & 40 & 17 & 8.3333 \\
\hline 378 & PPB & SDP & 400 & | 1B3 & 24 & 257 & 245 & 4 & 8 & 3.1128 \\
\hline 379 & PPB & SDP & 400 & 186 & 24 & 322 & 293 & 20 & 9 & 2.7950 \\
\hline 380 & IWAS & FDP & 0 & $1 \mathrm{~A} 5$ & 24 & 55 & $\theta$ & 11 & 35 & 63.6364 \\
\hline 381 & WAS & FDP & C & $1 \mathrm{B2}$ & 24 & 140 & 49 & $19 i$ & 72 & 51.4286 \\
\hline 382 & WAS & FDP & 0 & 184 & 24 & 73 & 32 & 11 & $3 c$ & 41.0959 \\
\hline 383 & WAS & FDP & 50 & $1 \mathrm{A1}$ & 24 & 55 & 16 & 14 & 25 & 45.4545 \\
\hline 384 & WAS & FDP & 50 & 1C1 & 24. & 65 & 19 & 9 & 37 & 56.9231 \\
\hline $3 B 5$ & WAS & FDP & 50 & $1 \mathrm{c} 2$ & 24 & 56 & 9. & 11 & 36 & 64.2857 \\
\hline 386 & WAS & FDP & 100 & $1 \mathrm{~A} 2$ & 24 & 67 & 23 & 23 & 21 & 31.3433 \\
\hline 387 & WAS & FDP & 100 & $1 \mathrm{~A} 6$ & 24 & 99 & 15 & 37 & 47 & 47.4747 \\
\hline 380 & WAS & FDP & 100 & 106 & 24 & 104 & 23 & 13 & 68 & 65.3846 \\
\hline 389 & WAS & FDP & 200 & B55 & 24 & 54 & 16 & 21 & 17 & 31.4815 \\
\hline 390 & WAS & |FDP & 200 & 103 & 24 & 60 & 12 & 19 & 29 & 48,3333 \\
\hline 391 & WAS & FDP & 200 & $1 \mathrm{C} 4$ & 24 & 64 & 14 & 6 & 44 & 68.7500 \\
\hline 392 ! & WAS & FDP & 300 & $1 \mathrm{~A} 4$ & 24 & 52 & 8 & 11 & 33 & 63.4615 \\
\hline 393 & WAS & FDP & 300 & 181 & 24 & 55 & 24 & 18 & 13 & 23.6364 \\
\hline 394 & WAS & FOP & 300 & $1 \mathrm{C5}$ & 24 & 69 & 8 & 38 & 23 & 39.3333 \\
\hline 395 & WAS & FDP & 400 & $1 \mathrm{~A} 3$ & 24 & 80 & 62 & 5 & 13 & 16.2500 \\
\hline 396 ! & WAS & FDP & 400 & 183 & 24 & 80 & 34 & 16 & 30 & 37.5000 \\
\hline 397 & WAS & FDP & 400 & $1 \mathrm{~B} 6$ & 24 & 83. & 37 & 24 & 22 & 26.5060 \\
\hline
\end{tabular}

Performed by J. Luoma; SAS version 9.3 08:43 18MAR15

file://C:/Users/JLUOMA/AppData/Local/Temp/1/SAS\%20Temporary\%20Files/ TD4836... 3/18/2015 


\begin{tabular}{|c|c|c|c|c|c|c|c|c|}
\hline \multicolumn{9}{|c|}{$\begin{array}{l}\text { Effects of Pseudomona } \\
\text { Mean Unadjusted V } \\
\text { The MEANS Procedure } \\
\text { sps=BLS fonm=SDP }\end{array}$} \\
\hline conc & $\operatorname{tim} \theta$ & N Obs & Varlable & Label & Mean & Std Dev & $\begin{array}{r}\text { Lower } 95 \% \\
\text { CL for Mean }\end{array}$ & $\begin{array}{l}\text { Upper } 95 \% \\
\text { CL for Mean }\end{array}$ \\
\hline 0 & 6 & & $\begin{array}{l}\text { tot } \\
\text { cbs } \\
\text { oas } \\
\text { vla_glo } \\
\text { pctvia_glo }\end{array}$ & $\begin{array}{l}\text { tot } \\
\text { cbs } \\
\text { oes }\end{array}$ & \begin{tabular}{|}
297.3 \\
8.0000 \\
34.3333 \\
255.0 \\
85.7227
\end{tabular} & $\begin{array}{r}50.1232 \\
0 \\
7.0946 \\
43.8634 \\
1.0577\end{array}$ & $\begin{array}{r}172.8 \\
16.7094 \\
146.0 \\
83.0952\end{array}$ & $\begin{array}{r}421.8 \\
51.9573 \\
364.0 \\
88.3503\end{array}$ \\
\hline & 12 & & \begin{tabular}{|l|} 
tot \\
cbs \\
oas \\
via_glo \\
pctvia_glo
\end{tabular} & $\begin{array}{l}\text { tot } \\
\text { cbs } \\
\text { oas }\end{array}$ & $\begin{array}{r}210.7 \\
3.6667 \\
18.6667 \\
188.3 \\
89.4899\end{array}$ & $\begin{array}{r}63.9713 \\
2.0817 \\
7.0238 \\
56.3678 \\
0.5092\end{array}$ & $\begin{array}{r}51.7530 \\
-1.5045 \\
1.2187 \\
48.3079 \\
88.2250\end{array}$ & $\begin{array}{r}369.6 \\
8.8378 \\
36.1147 \\
328.4 \\
90.7548\end{array}$ \\
\hline & 24 & 3 & $\begin{array}{l}\text { tot } \\
\text { cbs } \\
\text { oas } \\
\text { via_glo } \\
\text { pctvia_glo }\end{array}$ & $\begin{array}{l}\text { tot } \\
\text { cbs } \\
\text { oas }\end{array}$ & $\begin{array}{r}463.3 \\
14.0000 \\
60.3333 \\
389.0 \\
84, .6675\end{array}$ & $\begin{array}{r}223.8 \\
11.1355 \\
31.7857 \\
184.1 \\
1.9452 \\
\end{array}$ & $\begin{array}{r}-92.4995 \\
-13.6622 \\
-18.6288 \\
-68.3580 \\
79.6354\end{array}$ & $\begin{array}{r}1019.2 \\
41.6622 \\
139.3 \\
846.4 \\
89.2995\end{array}$ \\
\hline 50 & 6 & 3 & $\begin{array}{l}\text { lo: } \\
\text { cbs } \\
\text { oas } \\
\text { via_glo } \\
\text { pctvia_glo }\end{array}$ & $\begin{array}{l}\text { tot } \\
\text { cols } \\
\text { oas } \\
\text { ans }\end{array}$ & 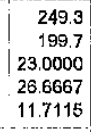 & $\begin{array}{r}42.0634 \\
77.3585 \\
17.3494 \\
20.5508 \\
9.5448\end{array}$ & $\begin{array}{r}144.8 \\
7.4976 \\
-20.0982 \\
-24.3842 \\
-11.9992\end{array}$ & $\begin{array}{r}353.8 \\
391.8 \\
66.0982 \\
77.7176 \\
35.4222\end{array}$ \\
\hline & 12 & 3 & \begin{tabular}{|l|} 
tot \\
cbs \\
cas \\
va__glo \\
pctvla_glo \\
\end{tabular} & $\begin{array}{l}\text { tot } \\
\text { cbs } \\
\text { oas }\end{array}$ & $\begin{array}{r}290.0 \\
223.7 \\
18.3333 \\
48.0000 \\
16.1520\end{array}$ & \begin{tabular}{|r|}
95.2208 \\
68.0392 \\
13.8684 \\
26.2298 \\
5.6175 \\
\end{tabular} & $\begin{array}{r}53.4584 \\
54.6479 \\
-16.1178 \\
-17.1583 \\
2.1972 \\
\hdashline .19\end{array}$ & $\begin{array}{r}526.5 \\
392.7 \\
52.7844 \\
113.2 \\
30.1067\end{array}$ \\
\hline & 24 & 3 & $\begin{array}{l}\text { tot } \\
\text { cbs } \\
\text { oas } \\
\text { via_glo } \\
\text { pctvla_g'o }\end{array}$ & $\begin{array}{l}\text { lot } \\
\text { cbs } \\
\text { oas }\end{array}$ & $\begin{array}{r}200.3 \\
159.0 \\
12.3333 \\
2.9 .0000 \\
14.7276\end{array}$ & $\begin{array}{r}132.9 \\
110.9 \\
7.2342 \\
17.7764 \\
1.7941 \\
\end{array}$ & $\begin{array}{r}-129.7 \\
-116.5 \\
-5.6374 \\
-15.1590 \\
10.2709\end{array}$ & $\begin{array}{r}530.4 \\
434.5 \\
30.3040 \\
73.1590 \\
19.1843\end{array}$ \\
\hline 100 & 6 & at & $\begin{array}{l}\text { tot } \\
\text { cbs } \\
\text { oas } \\
\text { via_glo } \\
\text { pctvia_glo }\end{array}$ & $\begin{array}{l}\text { tot } \\
\text { cbs } \\
\text { oas }\end{array}$ & $\begin{array}{r}220.3 \\
210.0 \\
8.6667 \\
1.6667 \\
0.4748\end{array}$ & $\begin{array}{r}113.7 \\
104.9 \\
7.0238 \\
2.8868 \\
0.8224\end{array}$ & $\begin{array}{r}-62.0983 \\
-50.4912 \\
-8.7813 \\
-5.5044 \\
-1.5682\end{array}$ & $\begin{array}{r}502.8 \\
470.5 \\
26.1147 \\
8.8378 \\
2.5179\end{array}$ \\
\hline & 12 & \begin{tabular}{r|r}
3 & $t$ \\
1 & 0 \\
$c$ &
\end{tabular} & $\begin{array}{l}\text { tot } \\
\text { cbs } \\
\text { oas } \\
\text { via_glo } \\
\text { potvia_glo }\end{array}$ & $\left\{\begin{array}{l}\text { tol } \\
\text { clos } \\
\text { oas }\end{array}\right.$ & $\begin{array}{r}191.0 \\
153.3 \\
20.0000 \\
17.6667 \\
8.8404 \\
\end{array}$ & $\begin{array}{r}62.7933 \\
55.5188 \\
1.0000 \\
9.2916 \\
3.2608\end{array}$ & $\begin{array}{r}35.0128 \\
15.4171 \\
17.5159 \\
-5.4149 \\
0.7401\end{array}$ & $\begin{array}{r}347.0 \\
291.2 \\
22.4841 \\
40.7482 \\
16.9407\end{array}$ \\
\hline & 24 & $\mid \begin{array}{l}1 \\
0 \\
0 \\
v \\
p\end{array}$ & $\begin{array}{l}\text { lot } \\
\text { cbs } \\
\text { oas } \\
\text { via_glo } \\
\text { pctvla_glo }\end{array}$ & $\left\{\begin{array}{l}\text { tot } \\
\text { cbs } \\
\text { oas }\end{array}\right.$ & $\begin{array}{r}218.3 \\
.88 .7 \\
3.6667 \\
16.0000 \\
6.9517\end{array}$ & $\begin{array}{r}35.6417 \\
28.7460 \\
3.7859 \\
11.5326 \\
4.8770\end{array}$ & $\begin{array}{r}129.8 \\
117.3 \\
4.2619 \\
-12.6485 \\
-5.1635\end{array}$ & $\begin{array}{r}306.8 \\
260.1 \\
23.0715 \\
44.6485 \\
19.0670\end{array}$ \\
\hline 200 & 6 & $\begin{array}{c}3 i t \\
0 \\
0 \\
v \\
p\end{array}$ & \begin{tabular}{|l|} 
tot \\
cbs \\
cas \\
via_glo \\
pctvia_glo
\end{tabular} & $\left\{\begin{array}{l}\text { lot } \\
\text { cbs } \\
\text { oas }\end{array}\right.$ & \begin{tabular}{|}
248.0 \\
211.7 \\
32.0000 \\
4.3333 \\
1.7496
\end{tabular} & $\begin{array}{r}94.3186 \\
69.0821 \\
34.0441 \\
4.0415 \\
1.5555\end{array}$ & $\begin{array}{r}13.6996 \\
40.0573 \\
-52.5702 \\
-5.7062 \\
-2.1145\end{array}$ & $\begin{array}{r}482.3 \\
383.3 \\
116.6 \\
14.3729 \\
5.6138 \\
\end{array}$ \\
\hline & 12 & $\begin{array}{r}3 t \\
0 \\
0 \\
v\end{array}$ & $\begin{array}{l}\text { tot } \\
\text { cbs } \\
\text { oas } \\
\text { vla_glo }\end{array}$ & $\begin{array}{l}\text { tot } \\
\text { cbs } \\
\text { oas }\end{array}$ & $\begin{array}{r}252.0 \\
238.7 \\
10.3333 \\
3.0000\end{array}$ & \begin{tabular}{|r|}
47.7912 \\
39.6274 \\
8.3865 \\
1.0000
\end{tabular} & $\begin{array}{r}133.3 \\
140.2 \\
-10.4999 \\
0.5159\end{array}$ & $\begin{array}{r}370.7 \\
337.1 \\
31.1665 \\
5.4841\end{array}$ \\
\hline
\end{tabular}

file://C:/Users/JLUOMA/AppData/Local/Temp/1/SAS\%20Temporary\%20Files/_TD4836... 3/18/2015 

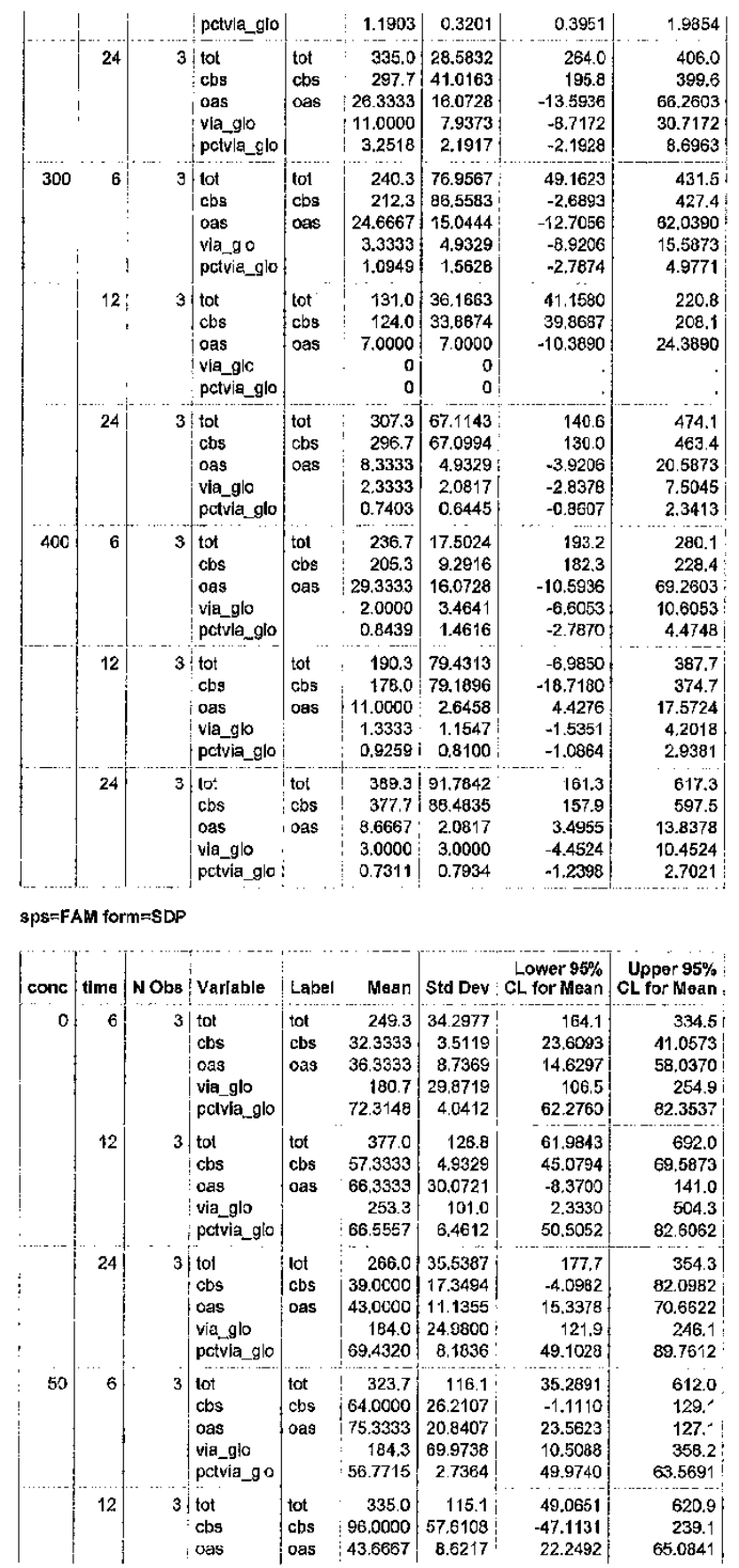

file://C:/Users/JUOMA/AppData/Local/Temp/1/SAS\%20Temporary\%20Files/_TD4836... 3/18/2015 


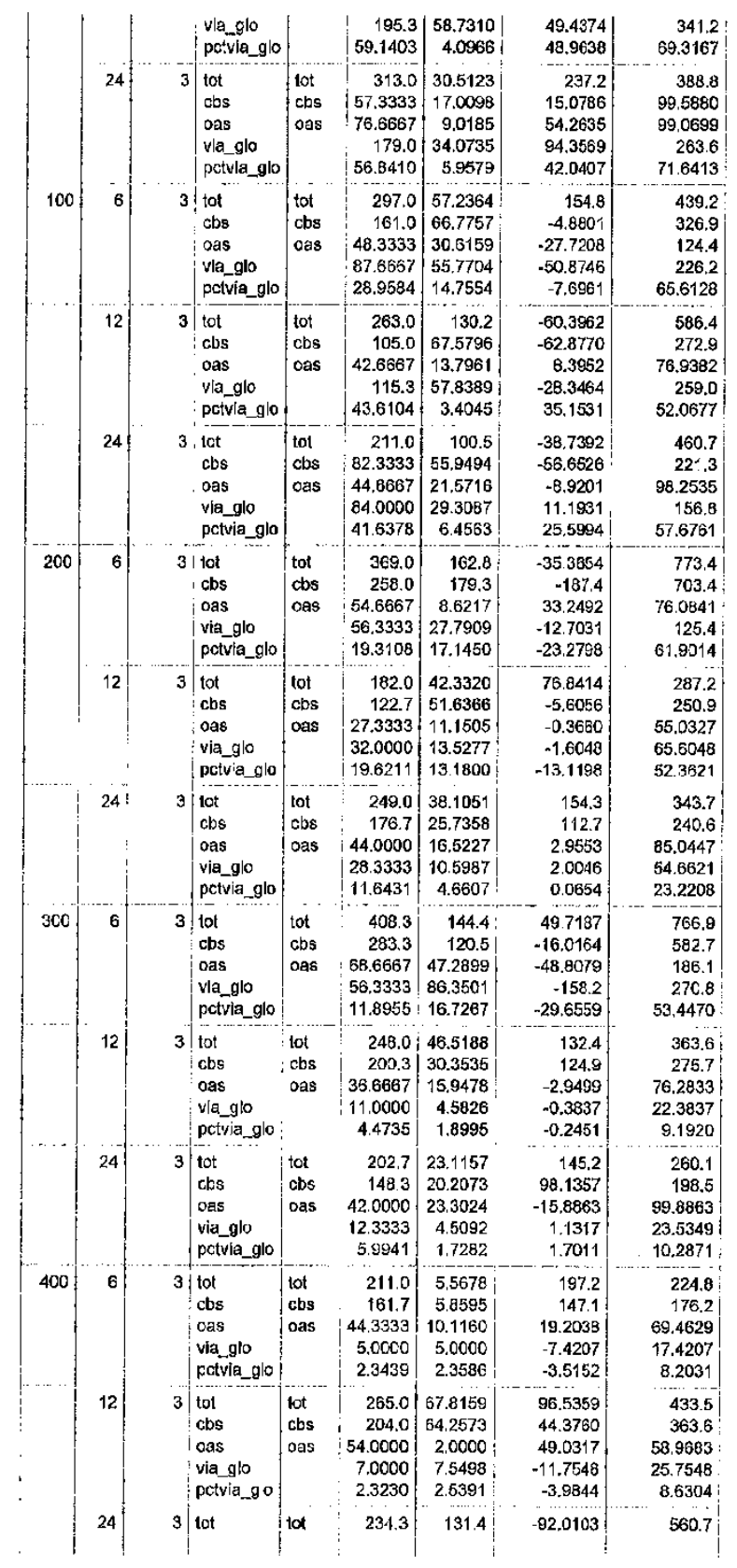

file://C:/Users/JLUOMA/AppData/Local/Temp/1/SAS\%20Temporary\%20Files/ TD4836... 3/18/2015 


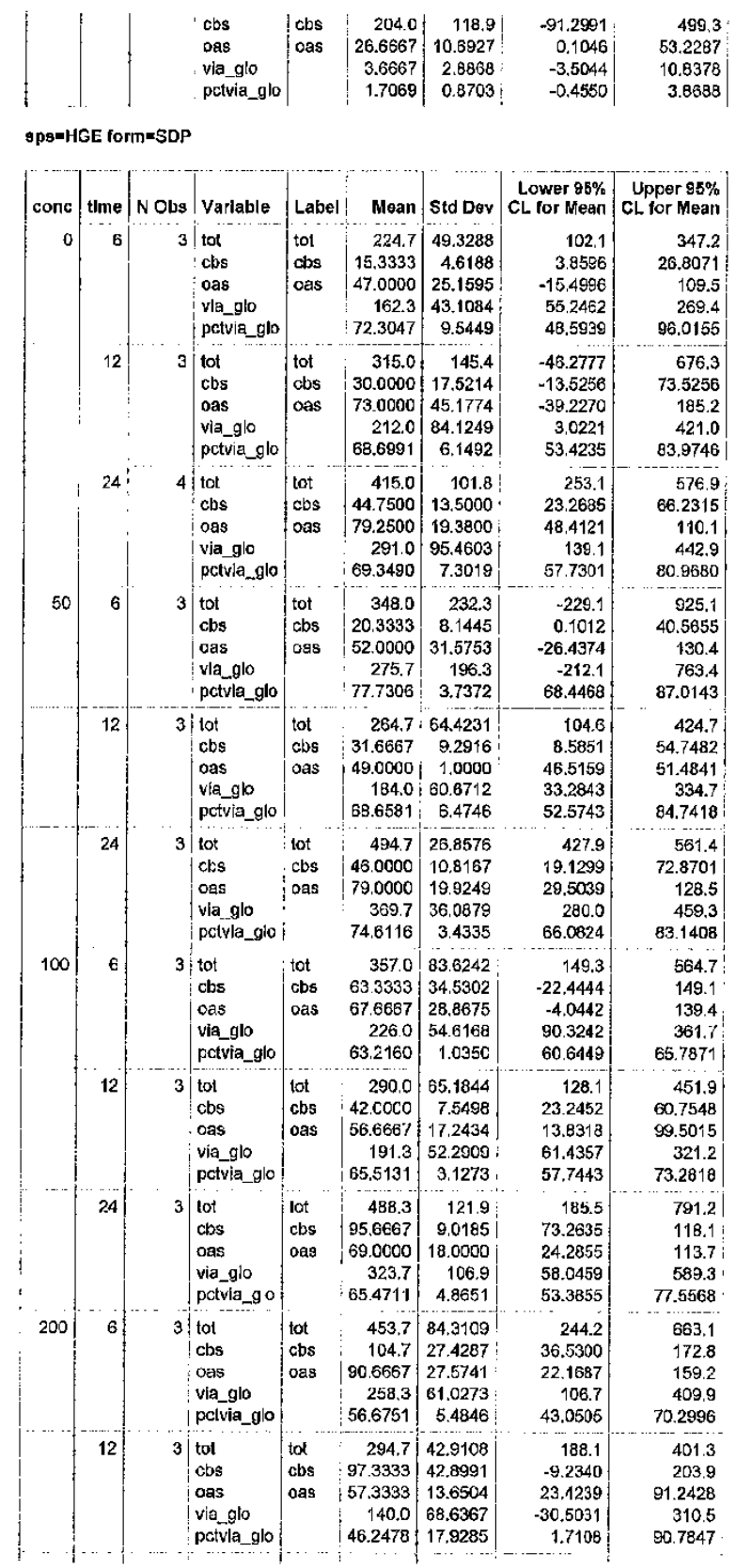

file://C:/Users/JLUOMA/AppData/L cocal/Temp/1/SAS\%20Temporary\%20Files/._TD4836... 3/18/2015 


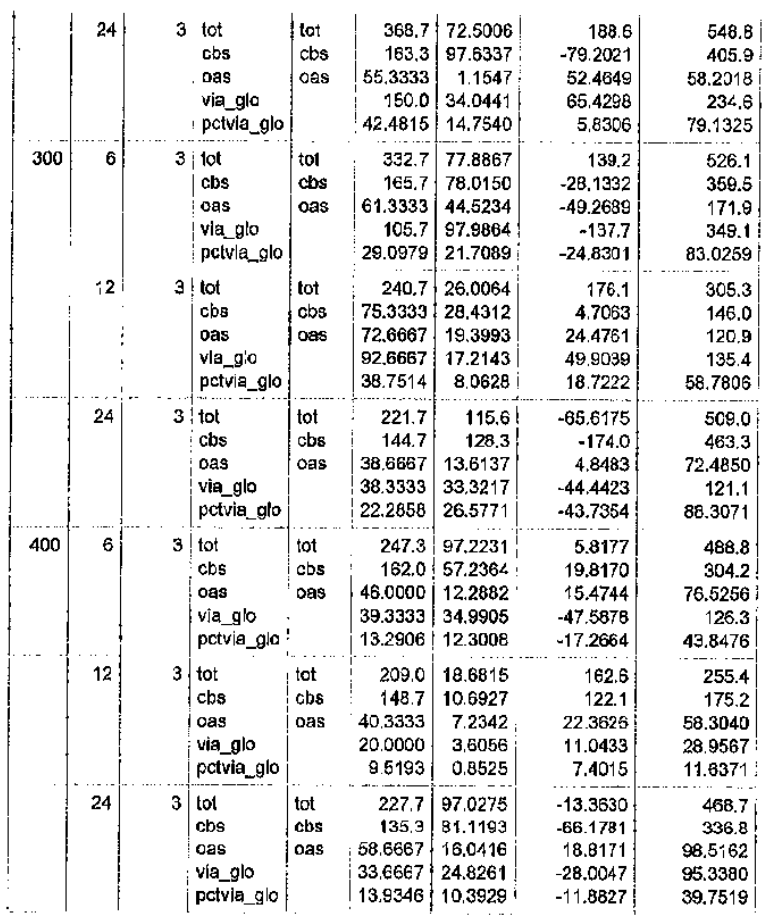

sps=HIC form=SDP

\begin{tabular}{|c|c|c|c|c|c|c|c|c|}
\hline cone & time & NObs & Variable & Labal & Mean & Std Dev & $\begin{array}{l}\text { Lower } 85 \% \\
\text { CL for Mean }\end{array}$ & $\begin{array}{r}\text { Upper } 95 \% \\
\text { CL for Mean }\end{array}$ \\
\hline 0 & $B$ & 3 & $\begin{array}{l}\text { tot } \\
\text { cbs } \\
\text { oas } \\
\text { vla_glo } \\
\text { pctvia_glo }\end{array}$ & $\begin{array}{l}\text { tot } \\
\text { cbs } \\
\text { oas }\end{array}$ & $\begin{array}{r}224.3 \\
28.3333 \\
19.0000 \\
177.0 \\
79.9822\end{array}$ & $\begin{array}{r}59.1805 \\
31.8172 \\
6.2450 \\
43.7150 \\
11.5442\end{array}$ & $\begin{array}{r}77.3208 \\
-50.7049 \\
3.4866 \\
68.4060 \\
51.3048\end{array}$ & $\begin{array}{r}371.3 \\
107.4 \\
34.5134 \\
285.6 \\
108.7\end{array}$ \\
\hline & 12 & 3 & $\begin{array}{l}\text { lot } \\
\text { cos } \\
\text { oas } \\
\text { v|a_glo } \\
\text { petvia_glo }\end{array}$ & \begin{tabular}{|l} 
tot \\
cbs \\
oas
\end{tabular} & $\begin{array}{r}205.7 \\
10.0000 \\
18.6667 \\
177.0 \\
86.4146\end{array}$ & $\begin{array}{r}57.2043 \\
7.8102 \\
9.4516 \\
49.2747 \\
6.3460\end{array}$ & $\begin{array}{r}63.5633 \\
-9.4017 \\
-4.812 .5 \\
54.5948 \\
70.6504\end{array}$ & $\begin{array}{r}347.8 \\
29.4017 \\
42.1458 \\
299.4 \\
102.2\end{array}$ \\
\hline & 24 & & $\begin{array}{l}\text { tot } \\
\text { cbs } \\
\text { oas } \\
\text { via_glo } \\
\text { pctvia glo }\end{array}$ & $\begin{array}{l}\text { tat } \\
\text { cbs } \\
\text { oas }\end{array}$ & $\begin{array}{r}309.0 \\
14.6667 \\
26.3333 \\
268.0 \\
87.0377\end{array}$ & \begin{tabular}{|r|}
101.7 \\
7.0238 \\
10.7858 \\
84.9235 \\
1.7571
\end{tabular} & $\begin{array}{l}56.4107 \\
-2.7813 \\
-0.4601 \\
57.0383 \\
82.6729\end{array}$ & \begin{tabular}{r|}
561.6 \\
32.1147 \\
53.1267 \\
479.0 \\
91.4026
\end{tabular} \\
\hline 50 & 6 & 3 & $\begin{array}{l}\text { i tot } \\
c b s \\
\text { oas } \\
\text { via_glo } \\
\text { petvia_glo }\end{array}$ & $\begin{array}{l}\text { tot } \\
\text { cbs } \\
\text { oas }\end{array}$ & $\begin{array}{r}244.0 \\
21.0000 \\
14.6667 \\
208.3 \\
85.2261\end{array}$ & $\begin{array}{r}46.0326 \\
5.0000 \\
5.8882 \\
41.9325 \\
1.2753\end{array}$ & $\begin{array}{r}129.6 \\
8.5793 \\
0.5413 \\
104.2 \\
82.0581\end{array}$ & $\begin{array}{r}358.4 \\
33.4207 \\
28.7921 \\
312.5 \\
88.3941\end{array}$ \\
\hline & 12 & 3 & $\begin{array}{l}\text { tct } \\
\text { clos } \\
\text { oas } \\
\text { via_glo }\end{array}$ & $\begin{array}{l}\text { tot } \\
\text { cbs } \\
\text { oas }\end{array}$ & $\begin{array}{r}185.0 \\
18.0000 \\
10.6667 \\
156.3\end{array}$ & $\begin{array}{r}12.5300 \\
7.9373 \\
3.0551 \\
13.3167\end{array}$ & $\begin{array}{r}153.9 \\
-1.7172 \\
3.0775 \\
123.3\end{array}$ & $\begin{array}{r}216.1 \\
37.7172 \\
18.2558 \\
189.4\end{array}$ \\
\hline
\end{tabular}

file://C:/Uscrs/JLUOMA/AppData/Local/Temp/1/SAS\%20Temporary\%20Files/_TD4836.. 3/18/2015 


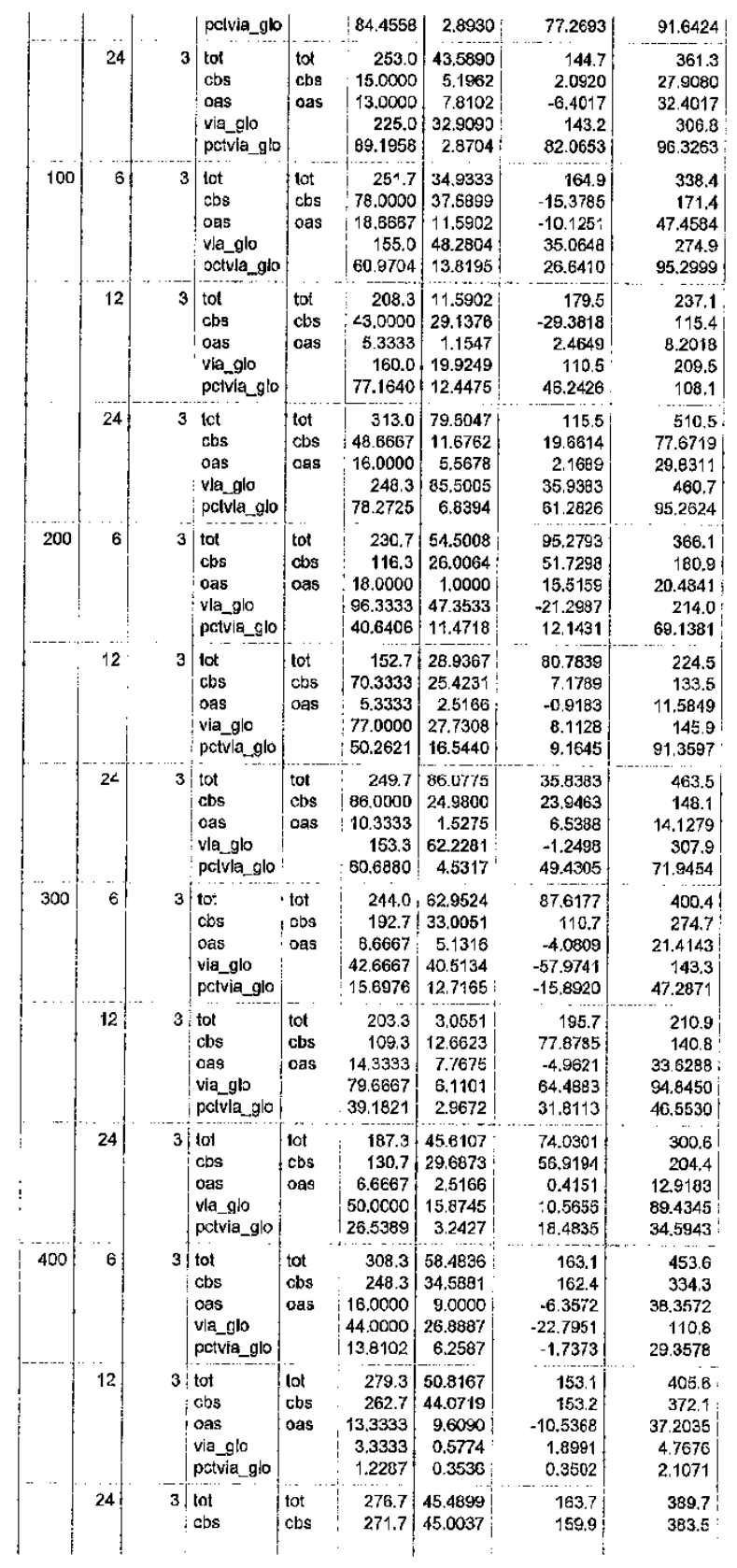

file://C:/Users/JLUOMA/AppData/Local/Temp/1/SAS\%20Temporary\%20Files/_TD4836... 3/18/2015 


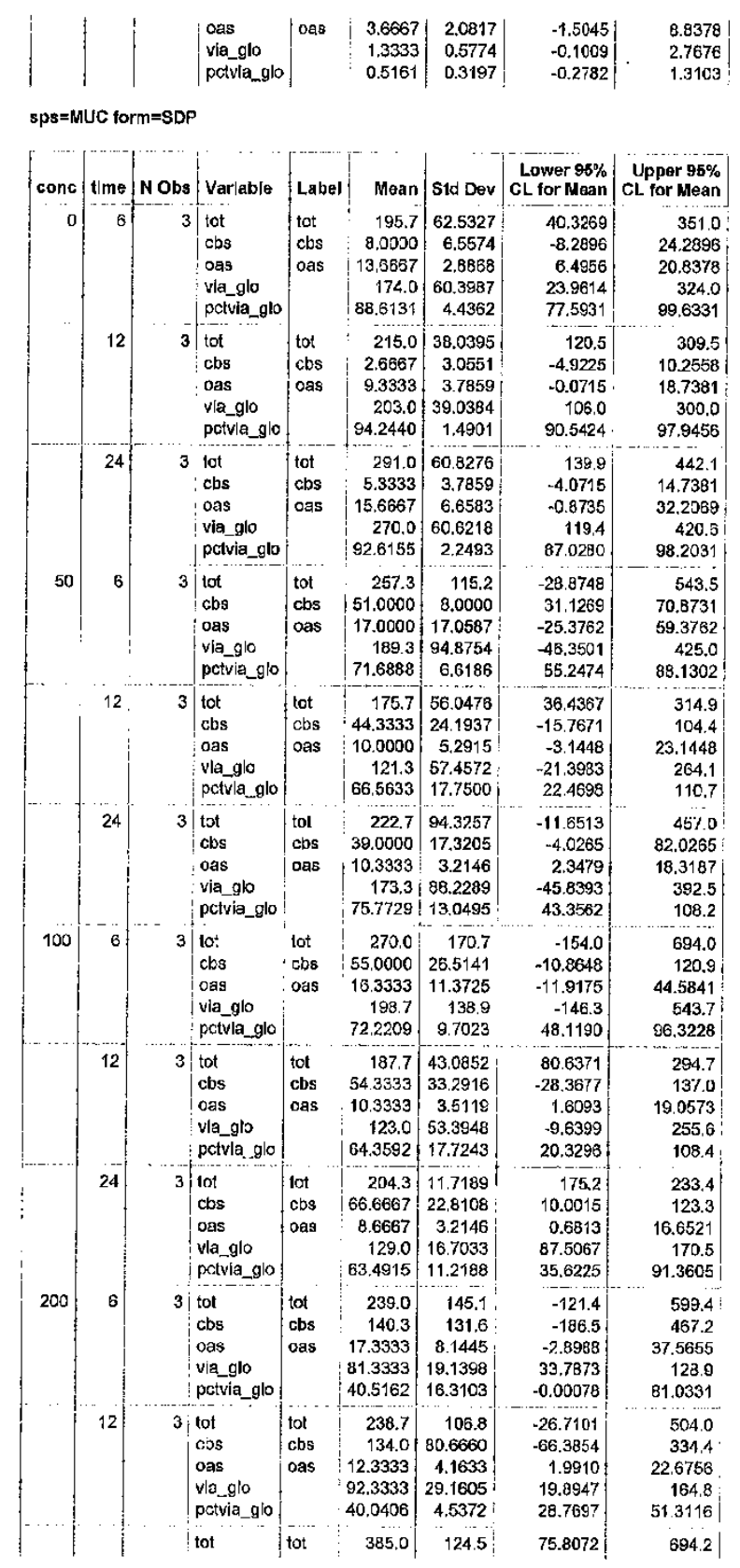

file://C:/Users/JLUOMA/AppData/Local/Temp/1/SAS\%20Temporary\%20Files/_TD4836... 3/18/2015 


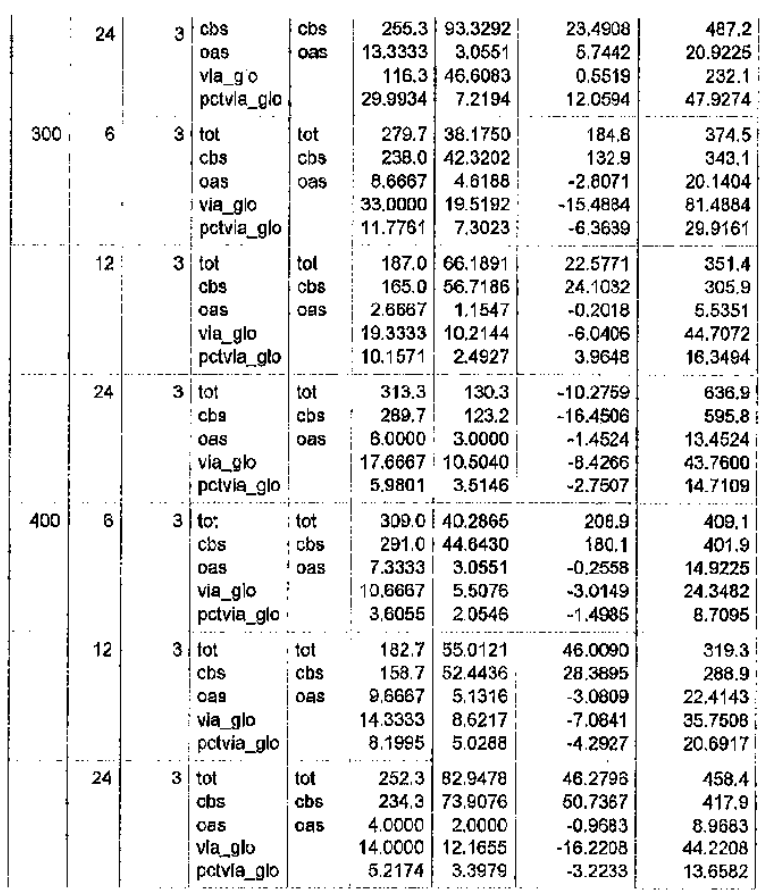

\begin{tabular}{|c|c|c|c|c|c|c|c|c|}
\hline conc & time & N Obs & Variable & Label & Mean & Std Dev | & $\begin{array}{l}\text { Lower } 95 \% \\
\text { CL for Mean }\end{array}$ & $\begin{array}{l}\text { Upper } 95 \% \\
\text { CL for Mean }\end{array}$ \\
\hline & 6 & 3 & \begin{tabular}{|l} 
tot \\
cbs \\
oas \\
via_glo \\
pctvia_go
\end{tabular} & $\begin{array}{l}\text { 1ot } \\
\text { cbs } \\
\text { oas }\end{array}$ & $\begin{array}{r}142.7 \\
14.0000 \\
11.0000 \\
117.7 \\
82.2576\end{array}$ & \begin{tabular}{|r|}
64.3454 \\
6.0828 \\
5.0000 \\
56.7524 \\
4.7439
\end{tabular} & $\begin{array}{r}-17.1762 \\
-1.1104 \\
-1.4207 \\
-20.8300 \\
70.4731\end{array}$ & $\begin{array}{r}302.5 \\
29.1104 \\
23.4207 \\
256.2 \\
94.0420\end{array}$ \\
\hline & 12 & 3 & $\begin{array}{l}\text { tot } \\
\text { cbs } \\
\text { ogs } \\
\text { via_glo } \\
\text { petvia_glo }\end{array}$ & $\left\{\begin{array}{l}\text { tot } \\
\text { cbs } \\
\text { oas }\end{array}\right.$ & $\begin{array}{r}209.7 \\
5.3333 \\
34.6667 \\
169.7 \\
81.7825\end{array}$ & $\begin{array}{r}83.6180 \\
2.5166 \\
22.8546 \\
68.8501 \\
3.3908\end{array}$ & \begin{tabular}{r|}
-22.8934 \\
-0.9183 \\
-22.1073 \\
-1.3864 \\
73.3594
\end{tabular} & $\begin{array}{r}442.2 \\
11.5849 \\
91.4407 \\
340.7 \\
90.2057\end{array}$ \\
\hline & 24 & 3 & \begin{tabular}{|l} 
tol \\
cbs \\
oas \\
vla_glo \\
petvla_glo
\end{tabular} & $\begin{array}{l}\text { tot } \\
\text { cbs } \\
\text { oas }\end{array}$ & $\begin{array}{r}376.7 \\
15.0000 \\
51.6667 \\
310.0 \\
81.9068\end{array}$ & $\begin{array}{r}170.0 \\
7.2111 \\
20.4042 \\
145.5 \\
1.4048\end{array}$ & $\begin{array}{r}-45.7122 \\
-2.9134 \\
0.9797 \\
-51.4314 \\
78.4171\end{array}$ & $\begin{array}{r}799.0 \\
32.9134 \\
102.4 \\
671.4 \\
85.3966\end{array}$ \\
\hline 50 & 6 & $\begin{array}{l}3 ! \\
!\end{array}$ & $\begin{array}{l}\text { tot } \\
\text { cbs } \\
\text { oas } \\
\text { vla_glo } \\
\text { pctvia_glo }\end{array}$ & $\begin{array}{l}\text { lot } \\
\text { cbs } \\
\text { oas }\end{array}$ & $\begin{array}{r}125.0 \\
21.6667 \\
13.0000 \\
90.3333 \\
69.5514\end{array}$ & \begin{tabular}{|r|}
44.6430 \\
5.5076 \\
6.9282 \\
42.9224 \\
11.4879
\end{tabular} & $\begin{array}{r}14.1006 \\
7.9851 \\
-4.2106 \\
-16.2918 \\
41.0139\end{array}$ & $\begin{array}{r}235.8 \\
35.3482 \\
30.2106 \\
197.0 \\
98.0889\end{array}$ \\
\hline & 12 & 3 & $\begin{array}{l}\text { tot } \\
\text { cos } \\
\text { oas } \\
\text { vla_glo } \\
\text { petvia_glo }\end{array}$ & $\begin{array}{l}\text { tot } \\
\text { cbs } \\
\text { oas }\end{array}$ & $\begin{array}{r}352.0 \\
22.0000 \\
53.6667 \\
276.3 \\
76.6850\end{array}$ & \begin{tabular}{r|r}
229.4 \\
13.1149 \\
21.7332 \\
199.6 \\
0.2489
\end{tabular} & $\begin{array}{r}-217.8 \\
-10.5792 \\
-0.3217 \\
-219.5 \\
61.1630\end{array}$ & $\begin{array}{r}921.8 \\
54.5792 \\
107.7 \\
772.1 \\
92.2 \mathrm{Cg1}\end{array}$ \\
\hline
\end{tabular}

file://C:/Users/JLUOMA/AppData/I,ocal/Temp/1/SAS\%20Temporary\%20Files/_TD4836... 3/18/2015 


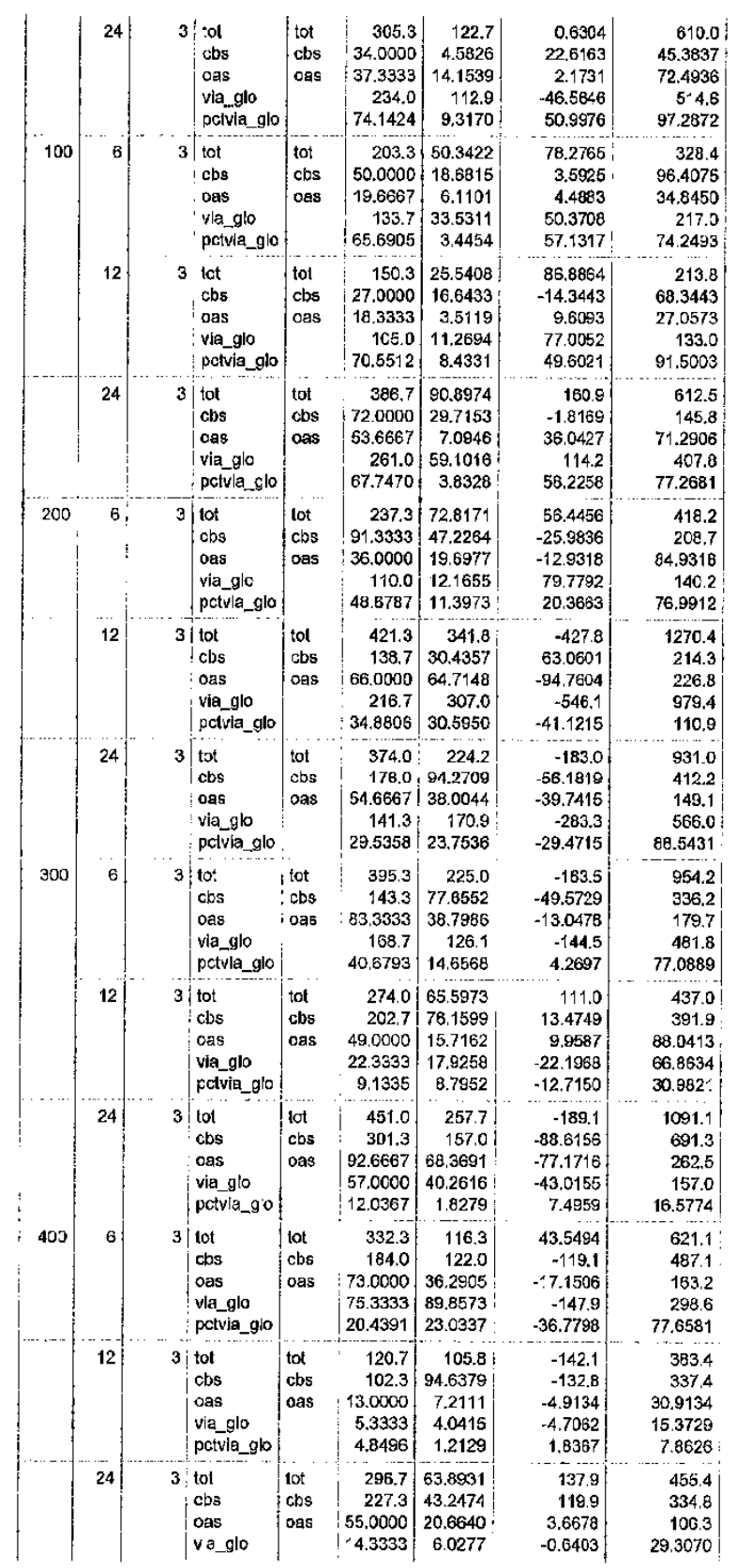

file://C:/Users/JLUOMA/AppData/L ocal/Temp/1/SAS\%20Temporary\%20Files/..TD4836... 3/18/2015 


\begin{tabular}{|c|c|c|c|c|c|c|c|c|}
\hline \multicolumn{5}{|c|}{$|+| p$} & 4.9022 & 2.2855 & -0.7754 & 10.5799 \\
\hline \multicolumn{9}{|c|}{ sps=PPB form=SDP } \\
\hline \multirow{3}{*}{ conc } & time & N Obs & Varlablo & Label & Mean & Std Dov & $\begin{array}{r}\text { Lower } 95 \% \\
\text { CL for Mean }\end{array}$ & $\begin{array}{l}\text { Upper 95\% } \\
\text { CL for Mean }\end{array}$ \\
\hline & 6 & 3 & $\begin{array}{l}\text { :ot } \\
\text { cbs } \\
\text { oas } \\
\text { vla_glo } \\
\text { petvla_glo }\end{array}$ & $\begin{array}{l}\text { tot } \\
\text { cbs } \\
\text { oas }\end{array}$ & $\begin{array}{r}299.3 \\
4.0000 \\
18.3333 \\
277.0 \\
92.3358\end{array}$ & $\begin{array}{r}133.5 \\
2.6458 \\
6.5064 \\
124.6 \\
1.0182\end{array}$ & $\begin{array}{r}-32.1789 \\
-2.5724 \\
2.1705 \\
-32.5020 \\
89.8065\end{array}$ & $\begin{array}{r}630.8 \\
10.5724 \\
34.4961 \\
586.5 \\
94.8652\end{array}$ \\
\hline & 12 & 3 & $\begin{array}{l}\text { tot } \\
\text { coss } \\
\text { oas } \\
\text { vla_glo } \\
\text { pctvia_glo }\end{array}$ & $\begin{array}{l}\text { lot } \\
\text { cbs } \\
\text { oes }\end{array}$ & $\begin{array}{r}609.7 \\
32.0000 \\
53.0000 \\
524.7 \\
86.0651\end{array}$ & $\begin{array}{r}14.3643 \\
12.1244 \\
1.0000 \\
15.3080 \\
2.0467\end{array}$ & $\begin{array}{r}574.0 \\
1.8814 \\
50.5159 \\
486.6 \\
80.9808\end{array}$ & $\begin{array}{r}645.3 \\
62.1186 \\
55.4841 \\
562.7 \\
91.1495\end{array}$ \\
\hline \multirow{3}{*}{50} & 24 & 3 & $\begin{array}{l}\text { ot } \\
\text { cbs } \\
\text { oas } \\
\text { via_glo } \\
\text { pctvla_glo }\end{array}$ & $\begin{array}{l}\text { tol } \\
\text { cbs } \\
\text { oas }\end{array}$ & $\begin{array}{r}4 C 6.3 \\
13.0000 \\
19.0000 \\
374.3 \\
92.1117\end{array}$ & $\begin{array}{r}120.6 \\
1.7321 \\
8.8882 \\
111.9 \\
0.8643\end{array}$ & $\begin{array}{r}106.7 \\
8.6973 \\
-3.0795 \\
96.2726 \\
89.9646\end{array}$ & $\begin{array}{r}705.9 \\
17.3027 \\
41.0795 \\
652.4 \\
94.2587\end{array}$ \\
\hline & 6 & 3 & $\begin{array}{l}\text { lot } \\
c b s \\
\text { oas } \\
\text { via_go } \\
\text { pctvia_glo }\end{array}$ & $\begin{array}{l}\text { tot } \\
\text { cos } \\
\text { oas }\end{array}$ & $\begin{array}{r}326.7 \\
68.0000 \\
11.6667 \\
247.0 \\
77.9232\end{array}$ & $\mid \begin{array}{r}129.9 \\
57.0351 \\
1.5275 \\
74,7081 \\
8.9488\end{array}$ & $\begin{array}{r}4.0506 \\
-73.6830 \\
7.8721 \\
61.4198 \\
55.6932\end{array}$ & $\begin{array}{r}648.3 \\
209.7 \\
15.4612 \\
432.6 \\
100.2\end{array}$ \\
\hline & 12 & 3 & $\begin{array}{l}\text { lot } \\
\text { cbs } \\
\text { oas } \\
\text { via_glo } \\
\text { petvia_glo }\end{array}$ & $\begin{array}{l}\text { tot } \\
\mathrm{cbs} \\
\text { oas }\end{array}$ & $\begin{array}{r}371.7 \\
55.0000 \\
31.6667 \\
285.0 \\
78.5634 \\
\end{array}$ & \begin{tabular}{|r|}
219.4 \\
42.9302 \\
30.5996 \\
147.8 \\
6.6050
\end{tabular} & $\begin{array}{r}-173.3 \\
-51.6445 \\
-44.3469 \\
-82.1485 \\
62.1557\end{array}$ & $\begin{array}{r}916.7 \\
161.6 \\
107.7 \\
652.1 \\
94.9711\end{array}$ \\
\hline \multirow{3}{*}{100} & 24 & 3 & $\begin{array}{l}\text { tot } \\
\text { cbs } \\
\text { oas } \\
\text { via_glo } \\
\text { pctvia_glo }\end{array}$ & $\begin{array}{l}\text { tot } \\
\text { cbs } \\
\text { oas }\end{array}$ & \begin{tabular}{|r|}
356.0 \\
53.3333 \\
18.6667 \\
284.0 \\
79.8142
\end{tabular} & \begin{tabular}{|r|}
95.6399 \\
25.5408 \\
6.3267 \\
80.4301 \\
5.6150
\end{tabular} & $\begin{array}{r}118.4 \\
-10.1136 \\
-2.0179 \\
84.2003 \\
65.8659\end{array}$ & $\begin{array}{r}593.6 \\
116.8 \\
39.3512 \\
483.8 \\
93.7625 \\
\end{array}$ \\
\hline & 6 & 3 & $\begin{array}{l}\text { tot } \\
\text { cbs } \\
\text { oas } \\
\text { via_glo } \\
\text { pctvla_glo }\end{array}$ & $\begin{array}{l}\text { tot } \\
\text { cbs } \\
\text { oas }\end{array}$ & $\begin{array}{r}274.0 \\
43.0000 \\
20.6667 \\
210.3 \\
752627\end{array}$ & $\begin{array}{r}228.5 \\
27.0000 \\
18.5023 \\
185.5 \\
6.0947\end{array}$ & $\begin{array}{r}-293.6 \\
-24.0717 \\
-25.2955 \\
-250.4 \\
60.1225\end{array}$ & $\begin{array}{r}841.6 \\
110.1 \\
66.6288 \\
671.0 \\
90.4028\end{array}$ \\
\hline & 12 & 3 & $\begin{array}{l}\text { tot } \\
\text { cbs } \\
\text { oas } \\
\text { via_glo } \\
\text { pctvia_glo }\end{array}$ & $\begin{array}{l}\text { tot } \\
\text { cbs } \\
\text { oas }\end{array}$ & $\begin{array}{r}403.7 \\
145.3 \\
33.3333 \\
225.0 \\
52.6578\end{array}$ & $\mid \begin{array}{r}86.8927 \\
153.1 \\
6.1101 \\
201.5 \\
45.6387\end{array}$ & $\begin{array}{r}187.8 \\
-235.1 \\
18.1550 \\
-275.7 \\
-60.7150\end{array}$ & $\begin{array}{r}619.5 \\
525.7 \\
48.5: 7 \\
725.7 \\
166.0\end{array}$ \\
\hline \multirow{3}{*}{200} & 24 & $\begin{array}{r}3 \\
! \\
!\end{array}$ & \begin{tabular}{|l|} 
tot \\
cbs \\
oas \\
ovia_glo \\
pctvia_glo
\end{tabular} & $\begin{array}{l}\text { fot } \\
\text { cbs } \\
\text { oas }\end{array}$ & \begin{tabular}{|r|}
281.3 \\
41.0000 \\
55.0000 \\
185.3 \\
66.8950
\end{tabular} & $\mid \begin{array}{l}62.1718 \\
13.0000 \\
60.7536 \\
51.0523 \\
15.7636\end{array}$ & $\begin{array}{r}126.9 \\
8.7062 \\
-95.9203 \\
58.5125 \\
27.7361\end{array}$ & \begin{tabular}{r|}
135.8 \\
73.2938 \\
205.9 \\
312.2 \\
106.1
\end{tabular} \\
\hline & 6 & 3 & $\begin{array}{l}\text { tol } \\
\text { cbs } \\
\text { oas } \\
\text { vla_glo } \\
\text { pctvla_glo }\end{array}$ & $\begin{array}{l}\text { tot } \\
\text { cbs } \\
\text { oas }\end{array}$ & $\begin{array}{r}211.7 \\
90.6667 \\
12.3333 \\
108.7 \\
52.2327\end{array}$ & \begin{tabular}{|r|}
58.1578 \\
64.7019 \\
7.7675 \\
54.9939 \\
21.1608
\end{tabular} & $\begin{array}{r}67.1946 \\
-70.0617 \\
-6.9621 \\
-27.9459 \\
-0.3337\end{array}$ & $\begin{array}{r}356.1 \\
251.4 \\
31.6288 \\
245.3 \\
104.8\end{array}$ \\
\hline & 12 & 3 & \begin{tabular}{|l} 
tot \\
cbs \\
oas \\
via_glo \\
pctvia_glo
\end{tabular} & $\begin{array}{l}\text { lot } \\
\text { cbs } \\
\text { oas }\end{array}$ & $\begin{array}{r}236.3 \\
97.3333 \\
20.6667 \\
-18.3 \\
46.7048\end{array}$ & $\begin{array}{r}125.0 \\
44.0719 \\
13.4288 \\
98.6019 \\
22.3477\end{array}$ & $\begin{array}{r}-74.2965 \\
-12.1474 \\
-12.6924 \\
-126.6 \\
-8.8059\end{array}$ & $\begin{array}{r}547.0 \\
206.8 \\
54.0257 \\
363.3 \\
102.2\end{array}$ \\
\hline & 24 & & $\begin{array}{l}\text { tot } \\
\text { cbs } \\
\text { oas }\end{array}$ & $\begin{array}{l}\text { tot } \\
\text { cbs } \\
\text { oas }\end{array}$ & $\begin{array}{r}311.7 \\
127.0 \\
14.3333\end{array}$ & $\begin{array}{r}170.5 \\
121.4 \\
1.5275\end{array}$ & $\begin{array}{r}-112.0 \\
-174.7 \\
10.5388\end{array}$ & $\begin{array}{r}735.3 \\
428.7 \\
18.1279\end{array}$ \\
\hline
\end{tabular}

file://C:/Users/JLUOMA/AppData/Local/I'Temp/1/SAS\%20Temporary\%20Files!_TD4836... 3/18/2015 


\begin{tabular}{|c|c|c|c|c|c|c|c|c|}
\hline & & & $\begin{array}{l}\text { vla_glo } \\
\text { pcivla_glo }\end{array}$ & & $\begin{array}{r}170.3 \\
59.2396\end{array}$ & \begin{tabular}{|l|}
51.0131 \\
12.7978
\end{tabular} & $\begin{array}{l}43,6098 \\
27.4480\end{array}$ & $\begin{array}{r}297.1 \\
91.0312\end{array}$ \\
\hline 300 & 6 & 3 & \begin{tabular}{|l|} 
tot \\
cbs \\
cas \\
vla_glo \\
pctvla_glo
\end{tabular} & $\begin{array}{l}\text { tol } \\
\text { ctos } \\
\text { oas }\end{array}$ & $\begin{array}{r}298.0 \\
257.7 \\
22.3333 \\
18.0000 \\
9.9092\end{array}$ & $\begin{array}{r}200.7 \\
195.6 \\
8.5049 \\
6.5574 \\
10.4262\end{array}$ & $\begin{array}{r}-200.5 \\
-228.2 \\
1.2060 \\
1.7104 \\
-15.8908\end{array}$ & $\begin{array}{r}796.5 \\
743.5 \\
43.4607 \\
34.2896 \\
35.8093\end{array}$ \\
\hline & 12 & 3 & $\begin{array}{l}\text { lot } \\
\text { cbs } \\
\text { oas } \\
\text { via_go } \\
\text { pctvia_glo }\end{array}$ & $\begin{array}{l}\text { tot } \\
\text { cbs } \\
\text { oas }\end{array}$ & $\begin{array}{r}647.3 \\
541.7 \\
50.6667 \\
55.0000 \\
6.5125\end{array}$ & $\begin{array}{r}465.2 \\
348.4 \\
51.2282 \\
65.6430 \\
4.2477 \\
\end{array}$ & $\begin{array}{r}-508.3 \\
-323.7 \\
-76.5914 \\
-108.1 \\
-4.0393\end{array}$ & $\begin{array}{r}1803.0 \\
1407.0 \\
177.9 \\
218.1 \\
17.0644\end{array}$ \\
\hline & 24 & 3 & $\begin{array}{l}\text { tot } \\
\text { clos } \\
\text { oas } \\
\text { via_gio } \\
\text { pctvia_glo }\end{array}$ & $\begin{array}{l}\text { tot } \\
\text { cbs } \\
\text { oss }\end{array}$ & $\begin{array}{r}338.0 \\
297.0 \\
14.0000 \\
27.0000 \\
6.0685\end{array}$ & \begin{tabular}{|r|}
138.6 \\
92.6661 \\
9.5394 \\
38.1182 \\
7.2179
\end{tabular} & $\begin{array}{r}-6.2929 \\
66.8047 \\
-9.6972 \\
-67.6910 \\
-11.8618\end{array}$ & $\begin{array}{r}682.3 \\
527.2 \\
37.6972 \\
121.7 \\
23.9888 \\
\end{array}$ \\
\hline 400 & 6 & & $\begin{array}{l}\text { tot } \\
\text { cbs } \\
\text { oas } \\
\text { vla_glo } \\
\text { pctvia_glo }\end{array}$ & \begin{tabular}{|l} 
tot \\
cbs \\
oas
\end{tabular} & \begin{tabular}{|r|}
158.7 \\
130.3 \\
23.6667 \\
4.6667 \\
3.4414
\end{tabular} & $\begin{array}{r}46.6093 \\
54.0771 \\
6.0277 \\
4.5092 \\
3.1312\end{array}$ & $\begin{array}{r}42.8852 \\
-4.0016 \\
8.6930 \\
-6.5349 \\
-4.3370\end{array}$ & $\begin{array}{r}274.4 \\
264.7 \\
38.6403 \\
15.8683 \\
11.2198\end{array}$ \\
\hline & 12 & 3 & $\begin{array}{l}\text { tot } \\
\text { cbs } \\
\text { oss } \\
\text { via_glo } \\
\text { pctvia_glo }\end{array}$ & $\begin{array}{l}\text { tot } \\
\text { cbs } \\
\text { oas }\end{array}$ & \begin{tabular}{|r|}
253.0 \\
214.7 \\
34.0000 \\
14,3333 \\
5.7918
\end{tabular} & $\begin{array}{r}51.7977 \\
67.6397 \\
2,6458 \\
4.6188 \\
2.6088 \\
\end{array}$ & $\begin{array}{r}134.3 \\
71.4818 \\
27.4275 \\
2.8595 \\
-0.6888\end{array}$ & $\begin{array}{r}391.7 \\
357.9 \\
40.5724 \\
25.8071 \\
12.2724\end{array}$ \\
\hline & 24 & 3 & $\begin{array}{l}\text { tot } \\
\text { cbs } \\
\text { oas } \\
\text { vla_glo } \\
\text { pctvla_glo }\end{array}$ & $\begin{array}{l}\text { tot } \\
\text { cbs } \\
\text { oas }\end{array}$ & $\begin{array}{r}261.0 \\
228.3 \\
21.3333 \\
11.3333 \\
4.7471\end{array}$ & $\begin{array}{r}59.1016 \\
74.4133 \\
18.0370 \\
4.9329 \\
3.1099\end{array}$ & $\begin{array}{r}114,2 \\
43.4805 \\
-23.4731 \\
-0.9208 \\
-2.9782\end{array}$ & \begin{tabular}{r|}
407.8 \\
413.2 \\
66.1397 \\
23.5873 \\
12.4724
\end{tabular} \\
\hline
\end{tabular}

\begin{tabular}{|c|c|c|c|c|c|c|c|c|}
\hline conc & time & N Obs & Variable & Label & Mean & Std Dov & $\begin{array}{l}\text { Lower } 95 \% \\
C\llcorner\text { for Mean }\end{array}$ & $\begin{array}{l}\text { Upper } 96 \% \\
\text { CL for Mean }\end{array}$ \\
\hline 0 & 24 & & $\begin{array}{l}\text { tot } \\
\text { cbs } \\
\text { oas } \\
\text { via_glo } \\
\text { pctivia_glo }\end{array}$ & $\begin{array}{l}\text { tot } \\
\text { cbs } \\
\text { oas }\end{array}$ & \begin{tabular}{|l|}
89.3333 \\
30.0000 \\
13.6667 \\
45.6667 \\
52.0536 \\
\end{tabular} & $\begin{array}{r}44.7921 \\
20.0749 \\
4.6188 \\
22.9420 \\
11.2832\end{array}$ & $\begin{array}{r}-21.9364 \\
-19.8687 \\
2.1929 \\
-11.3243 \\
24.0245\end{array}$ & $\begin{array}{r}200.6 \\
79.8687 \\
25.1404 \\
1027 \\
80.0827\end{array}$ \\
\hline 50 & 24 & 3 & $\begin{array}{l}\text { tot } \\
\text { cbs } \\
\text { oas } \\
\text { via_glo } \\
\text { petvia_glo }\end{array}$ & $\begin{array}{l}\text { tol } \\
\text { chs } \\
\text { oas }\end{array}$ & \begin{tabular}{|l|}
58.6667 \\
14.6667 \\
11.3333 \\
32.6667 \\
55.5544
\end{tabular} & $\begin{array}{l}5.5076 \\
5.1316 \\
2.5166 \\
6.6583 \\
9.4899\end{array}$ & $\begin{array}{r}44.9851 \\
1.9191 \\
5.0817 \\
16.1265 \\
31.9802\end{array}$ & $\begin{array}{l}72.3482 \\
27.4143 \\
17.5849 \\
49.2069 \\
79.1286\end{array}$ \\
\hline 100 & 24 & 3 & \begin{tabular}{|l|} 
lot \\
cbs \\
oas \\
via_glo \\
pctvia_glo
\end{tabular} & $\left\{\begin{array}{l}\text { tot } \\
\text { cbs } \\
\text { oas }\end{array}\right.$ & \begin{tabular}{|l|}
90.0000 \\
20.3333 \\
24.3333 \\
45.3333 \\
48.0675 \\
\end{tabular} & $\begin{array}{r}20.0749 \\
4.6188 \\
12.0554 \\
23.5443 \\
17.0284\end{array}$ & $\begin{array}{r}40.1313 \\
8.8596 \\
-5.6140 \\
-13.1539 \\
5.7666\end{array}$ & $\begin{array}{r}139.9 \\
31.8 \mathrm{C} 71 \\
54.2807 \\
103.8 ! \\
90.3685\end{array}$ \\
\hline 200 & 24 & 3 & $\begin{array}{l}\text { tol } \\
\text { cbs } \\
\text { oas } \\
\text { vla_glo } \\
\text { pclvia_glo }\end{array}$ & $\begin{array}{l}\text { tot } \\
\text { cbs } \\
\text { oas }\end{array}$ & \begin{tabular}{|l|}
59.3333 \\
14.0000 \\
15.3333 \\
30.0000 \\
49.5218 \\
\end{tabular} & \begin{tabular}{|r|}
5.0332 \\
2.0000 \\
8.1445 \\
13.5277 \\
18.6627 \\
\end{tabular} & $\begin{array}{r}46.8301 \\
9.0317 \\
-4.8968 \\
-3,6048 \\
3.1610\end{array}$ & $\begin{array}{l}71.8366 \\
18,9683 \\
35,5655 \\
63.6048 \\
95.8822\end{array}$ \\
\hline 300 & 24 & & $\begin{array}{l}\text { I tot } \\
\text { cbs } \\
\text { oas } \\
\text { via_gio } \\
\text { pctvia_glo }\end{array}$ & $\left\{\begin{array}{l}\text { tot } \\
\text { cbs } \\
\text { oas }\end{array}\right.$ & \begin{tabular}{|l|}
58.6667 \\
13.3333 \\
22.3333 \\
23.0000 \\
40.1437 \\
\end{tabular} & $\begin{array}{r}9.0738 \\
9.2376 \\
14.0119 \\
10.0000 \\
20.7677\end{array}$ & $\begin{array}{r}36.1262 \\
-9.6141 \\
-12.4742 \\
-1.8414 \\
-11.4461\end{array}$ & $\begin{array}{l}81.2072 . \\
36,2808 \\
57.1408 \\
47.8414 \\
91.7336\end{array}$ \\
\hline 400 & 24 & & $\begin{array}{l}\text { tot } \\
\text { cbs }\end{array}$ & $\begin{array}{l}\text { tot } \\
\text { cbs }\end{array}$ & \begin{tabular}{|}
81.0000 \\
44.3333
\end{tabular} & $\begin{array}{r}1.7321 \\
15.3731\end{array}$ & $\begin{array}{r}76.6973 \\
6.1443\end{array}$ & $\begin{array}{l}85.3027 \\
82.5223\end{array}$ \\
\hline
\end{tabular}

file://C:/Uscrs/JLUOMA/AppData/Local/Temp/1/SAS\%20Temporary\%20Files/_TD4836... 3/18/2015 


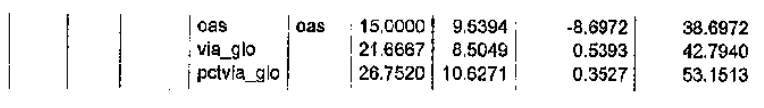

Performed by J. Luoma; SAS version $9.308: 43$ 18MAR15 


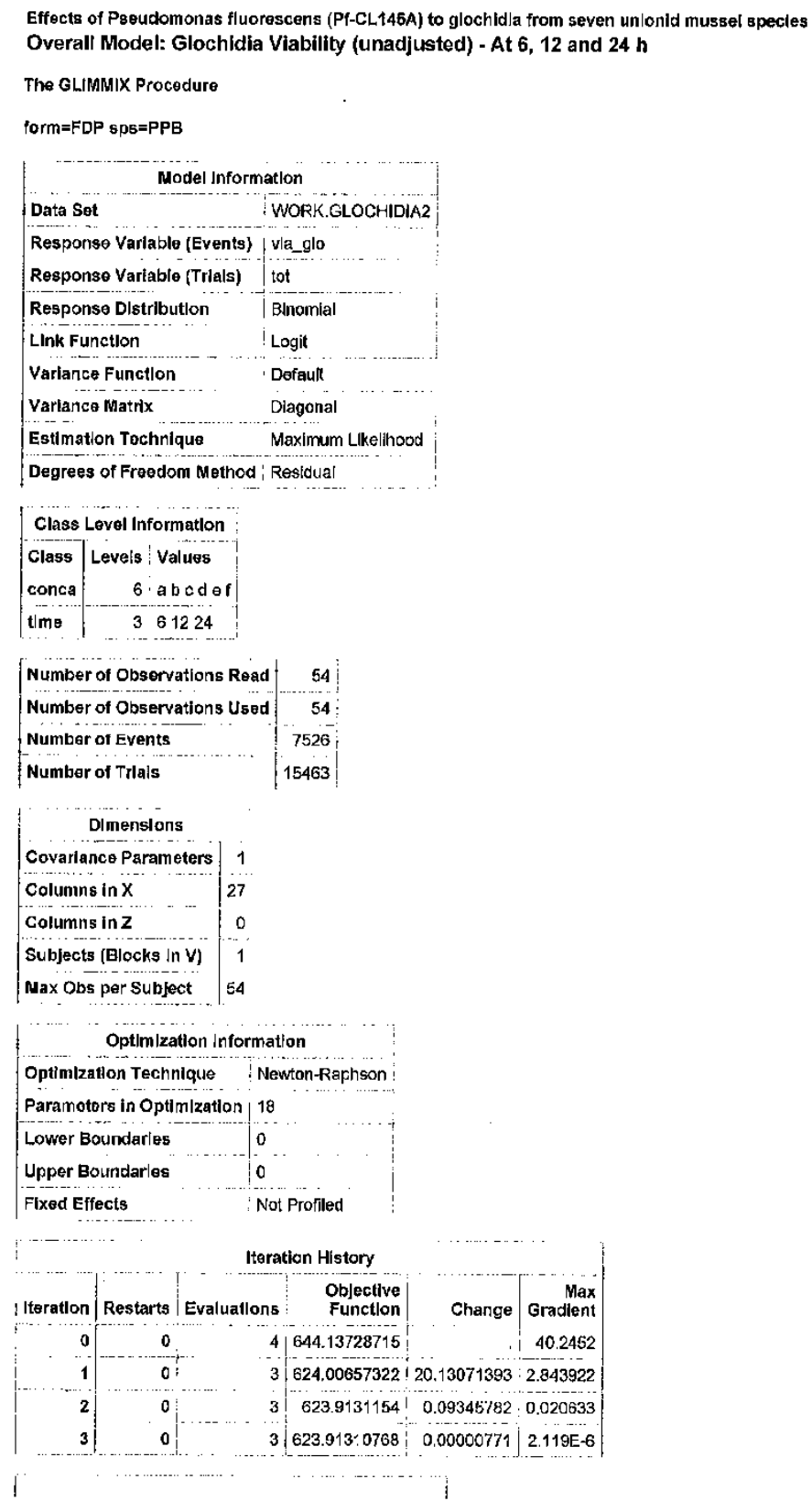

file://C:/Users/JLUOMA/AppData/Local/Temp/1/SAS\%20Temporary\%20Files/ TD4836... 3/18/2015 
Cenvergenca criterion (ABSGCONV=0.00001) satisfied.

\begin{tabular}{|l|r|}
\hline \multicolumn{1}{|c|}{ FIt Statistics } & \\
\hline -2 Log Likellhood & 1247.83 \\
\hline AIC (smaller is better) & 1283.83 \\
\hline ALCC (smaller is bottor) & 1303.37 \\
\hline BIC (smaller Is better) & 1319.63 \\
\hline CAIC (smaller Is better) & 1337.63 \\
HQIC (smaller Is better) & 1297.63 \\
\hline Pearson ChI-Square & 932.56 \\
\hline Pearson Chi-Square / DF & 25.90 \\
\hline
\end{tabular}

\begin{tabular}{|c|c|c|c|c|c|c|c|}
\hline \multirow[b]{2}{*}{ Effect } & \multicolumn{7}{|c|}{ Parameter Estimates } \\
\hline & conca & $\operatorname{tim} \theta$ & Estimate & Standard Error & DF & t Value & $\mathrm{Pr}>|\mathrm{t}|$ \\
\hline conca & a & & 1.1880 & 0.3974 & 36 & 2.99 & 0.0050 \\
\hline conca & b & & 0.7309 & 0.3191 & 36 & 2.29 & 0.0279 \\
\hline conca & c & & -0.4985 & 0.3134 & 36 & -1.59 & 0.1204 \\
\hline conca & d & & -1.9333 & 0.4164 & 36 & -4.64 & $<, 0001$ \\
\hline conca & 0 & & -2.9805 & 0.7956 & 36 & -3.75 & 0.0006 \\
\hline conca & $f$ & & 1.5369 & 0.3967 & 36 & 3.87 & 0.0004 \\
\hline time & & 6 & 0.01211 & 0.7590 & 36 & 0.02 & 0.9874 \\
\hline tIme & & 12 & -0.09191 & 0.6513 & 36 & -0.14 & 0.8886 \\
\hline $\operatorname{tim} \theta$ & & 24 & 0. & & 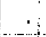 & & \\
\hline conca*tIme & $a$ & 6 & -0.2423 & 1.0386 & 36 & 0.23 & 0.8168 \\
\hline conca*time & $a$ & 12 & 0.1992 & 0.8529 & 36 & 0.23 & 0,8166 \\
\hline conca*time | & a & 24 & 0 & & $\cdot 1$ & & \\
\hline conca*tlme : & b & 6 & -0.09137 & 0.9309 & 36 & -0.10 & 0.9223 \\
\hline conea*time & $D$ & 12 & 0.2009 & 0.8937 & 36 & 0.22 & 0,8234 \\
\hline conca*tlmo & b & 24 & 0 & - & $\therefore$ & & \\
\hline concartine & c & 6 & 0.3400 & 0.9059 & 36 & 0.38 & 0.7096 \\
\hline conca*time & c & 12 & 0.6474 & 0.7774 & $36:$ & 0.83 & 0.4105 \\
\hline conca*time & c & 24. & 0 & & & & \\
\hline conca"time & d & 6 & 1.6256 & 0.9159 & 36 & 1.77 & 0.0844 \\
\hline conca*tme & $d$ & 12 & -0.3968 & 1.0092 & 36 & -0.39 & 0.6965 \\
\hline concattlme & d & 24 & 0 & & & & \\
\hline conca*time & $e$ & 6 & 1.7412 & 1.1651 & 36 & 1.49 & 0.1438 \\
\hline concattime & $\theta$ & 12 & $-0.00144 \mid$ & 1.6586 & 36 & -0.00 & 0.9993 \\
\hline concaktime & e & 24 & $0 !$ & & & & \\
\hline conca*tlme & $f$ & 6 & 0 & & & & \\
\hline conca*time & $f$ & 12 & 아 & & & & \\
\hline conca'tlme & $f$ & 24 & 0 & & & & \\
\hline Resldual & & & 25.9044 & & $\cdot !$ & & \\
\hline
\end{tabular}

\begin{tabular}{|c|c|c|c|c|}
\hline \multicolumn{5}{|c|}{ Type III Tests of Fixed Effects } \\
\hline Effect & Num DF & Den DF & F Value & $\mathrm{Pr}>\mathrm{F}$ \\
\hline conca & 5 & 36 & 20.90 & $<.0001$ \\
\hline time & 2 & 36 & 2.60 & 0.0880 \\
\hline
\end{tabular}

file://C:/Users/JLUOMA/AppData/Local/Temp/1/SAS\%20Temporary\%20Files/. TD4836... 3/18/2015 

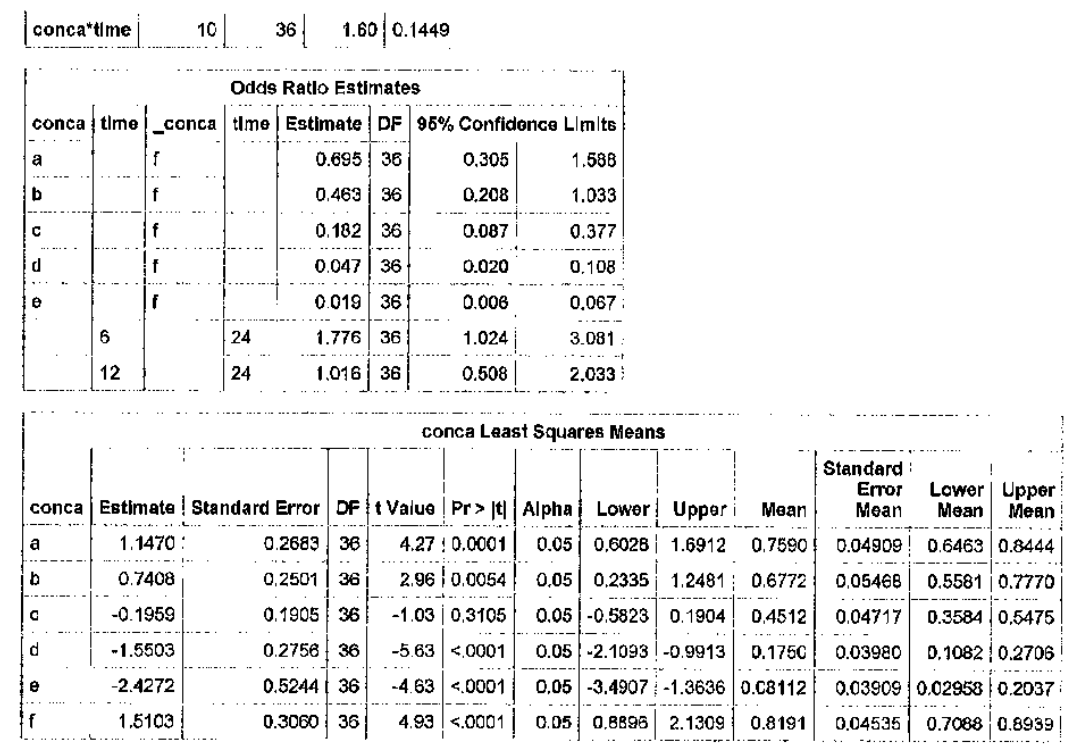

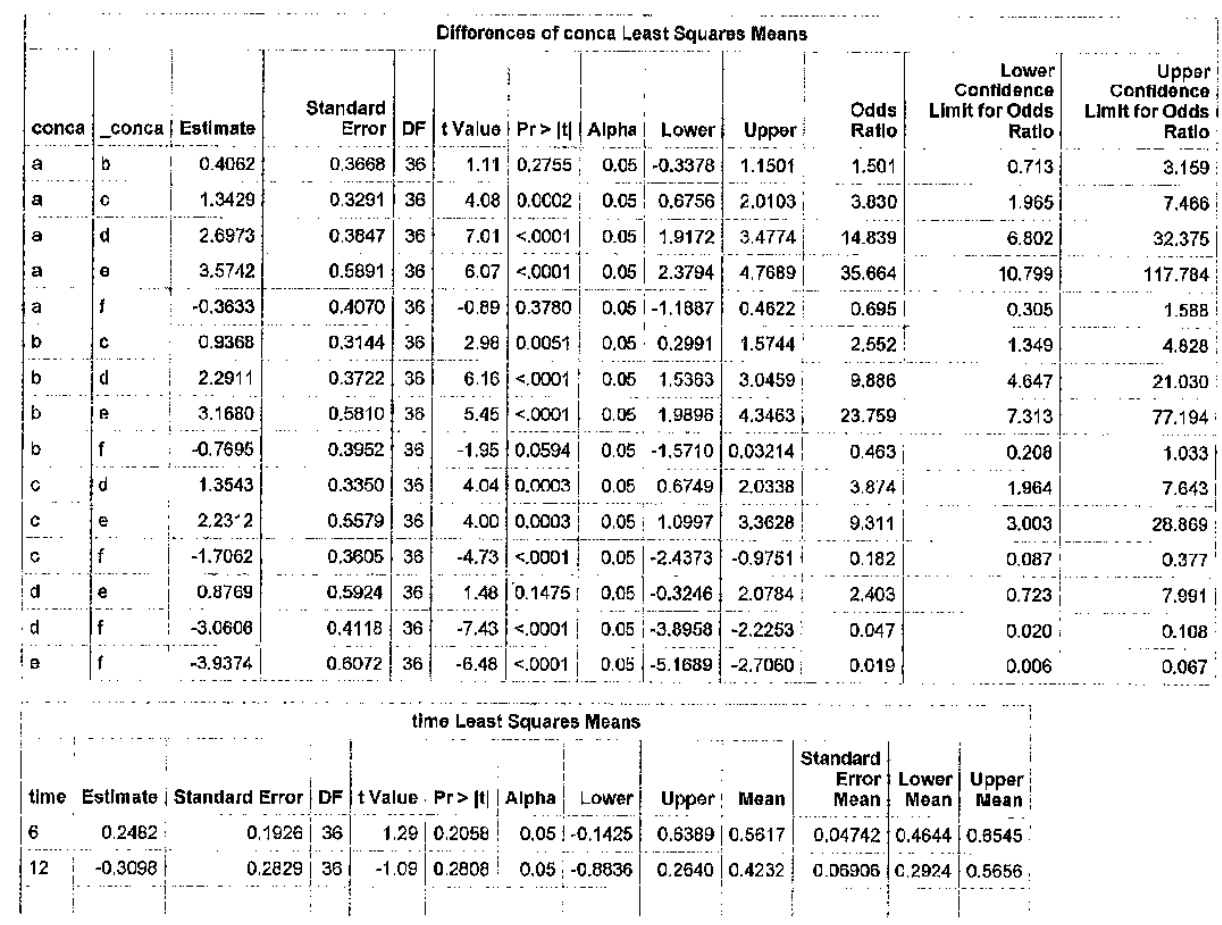


$|24|-0.3261|0.1916| 36|-1.70| 0.0974: 0.05|-0.7147| 0.06251|0.4192| 0.04665|0.3286| 0.5156 \mid$

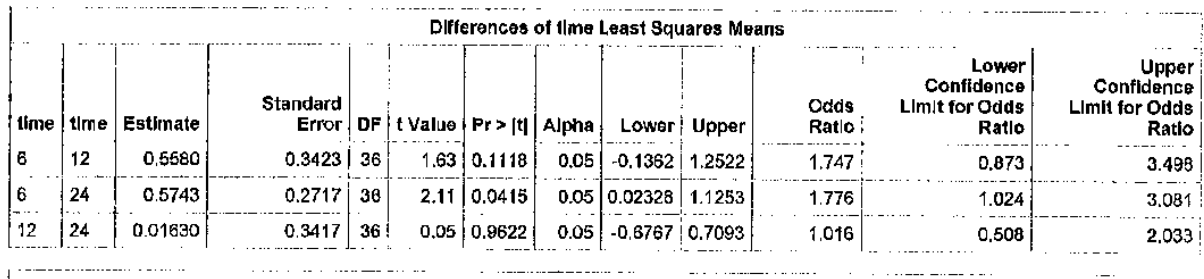

\begin{tabular}{|c|c|c|c|c|c|c|c|c|c|c|c|c|c|}
\hline conca & $\operatorname{tim} \theta$ & Estlmate & Standard Error & DF & t value & $\mathrm{Pr}>|\mathrm{t}|$ & Alpha & Lower & Upper & Mean & $\begin{array}{r}\text { Standard } \\
\text { Error } \\
\text { Mean }\end{array}$ & & $\begin{array}{l}\text { Upper } \\
\text { Mean }\end{array}$ \\
\hline & 6 & 0.9577 & 0.5871 & 36 & 1.63 & 0.1115 & 0.05 & -0.2329 & 2.1484 & 0.7227 & 0.1177 & 0.4420 & 0.8955 \\
\hline & 12 & 1.2953 & 0.3813 & 36 & 3.40 & 0.0017 & 0.05 & 0.5220 & 2.0685 & 0.7850 & 0.06434 & 0.6276 & 0.8878 \\
\hline & 24 & 1.1880 & 0,3974 & 36 & 2.99 & 0,0050 & 0.05 & 0.3819 & 1.9940 & 0.7664 & 0.07116 & 0.5943 & 0.8802 \\
\hline & 6 & 0.6516 & 0.4342 & 36 & 1.50 & $0.1<22$ & 0.05 & -0.2290 & 1.5323 & 0.6574 & 0.09780 & 0.4430 & 0,8223 \\
\hline & 12 & 0.8399 & 0.5222 & 36 & 1.61 & 0.1165 & 0.05 & -0.2192 & 1.8990 & 0.6984 & 0.1100 & 0.4454 & 0.8698 \\
\hline & 24 & 0.7309 & 0.3191 & 36 & 2.29 & 00279 & 0.05 & 0.08382 & 1.3780 & 0.6750 & 0.06999 & 0.5209 & 0.7987 \\
\hline & 6 & -0.1463 & 0.3825 & 36 & -0.38 & 0.7043 & 0.05 & $|-0.9221|$ & 0.6294 & 0.4635 & 0.09512 & 0.2845 & 0.6524 \\
\hline & 12 & 0.05698 & 0.2864 & 36 & 0.20 & 0,8434 & 0.05 & $\mid-0.5239$ & 0.6379 & 0.5142 & 0.07155 & 0.3719 & 0.6543 \\
\hline & 24 & -0.4985 & 0.3134 & 36 & -1.59 & 0.1264 & 0.05 & -1.1341 & 0.1371 & 0.3779 & 0.07367 & 0.2434 & 0.5342 \\
\hline & 6 & -0.2956 & 0.2988 & 36 & -0.99 & 0.3292 & 0.05 & -0.9016 & 0.3105 & 0.4266 & 0.07310 & 0.2887 & 0.5770 \\
\hline & 12 & $-2.422 \mathrm{C}$ & 0.6488 & 36 & -3.73 & 0.0007 & 0.05 & $|-3.7378|$ & -1.1062 & 0.08151 & 0.04867 & 0.02325 & 0.2186 \\
\hline & 24 & -1.9333 & 0.4164 & 36 & -4.64 & $<.0001$ & 0.05 & -2.7778 & -1.0888 & 0.1264 & 0.04598 & 0.05863 & 0.2518 \\
\hline & 6 & -1.2272 & 0.3850 & 36 & -3.19 & 0.0030 & 0.05 & $-2,0080$ & $-0.4464 \mid$ & 0.2267 & C. 06749 & 0.1184 & 0.3902 \\
\hline & 12 & $-3,0739$ & 1.3015 & 36 & -2.36 & 0.02 .37 & 0.05 & -5.7134 & -0.4343 & 0.04420 & 0.05498 & 0.003291 & 0.3931 \\
\hline & 24 & -2.9805 & 0.7956 & 36 & -3.75 & 0.0006 & 0.05 & -4.5941 & -1.3669 & 0.04831 & 0.03658 & 0.01001 & 0.2031 \\
\hline & 6 & 1.5490 & 0.6471 & 36 & 2.39 & 0.0220 & 0.05 & 0.2365 & 2.8614 & 0.8248 & 0.09353 & 0.5589 & 0,9459 \\
\hline & 12 & 1.4450 & 0.5165 & 36 & 2.80 & 0.0082 & 0.05 & 0.3975 & 2.4924 & 0.8092 & 0.07974 & 0.5981 & 0.9236 \\
\hline & 24 & 1.5369 & 0.3967 & 36 & 3.87 & 0.0004 & 0.05 & 0.7323 & 2.3414 & 0.8230 & 0.05779 & 0.6753 & 0.9123 \\
\hline
\end{tabular}

\begin{tabular}{|c|c|c|c|c|c|c|c|c|c|c|c|c|c|c|}
\hline & & & & & & & & :attlme & & & & & & \\
\hline conca & time & -conca & time & Estimate & $\begin{array}{r}\text { Standard : } \\
\text { Error }\end{array}$ & DF & I Valua & $\operatorname{Pr}>|t|$ & Alpha & Lower & Upper I & $\begin{array}{l}\text { Odds } \\
\text { Ratlo }\end{array}$ & $\begin{array}{r}\text { Lower } \\
\text { Confidence } \\
\text { Limlt for } \\
\text { Odds } \\
\text { Ratlo }\end{array}$ & $\begin{array}{r}\text { Upper } \\
\text { Confidence } \\
\text { Limit for } \\
\text { Odes } \\
\text { Ratlo }\end{array}$ \\
\hline a & 6 & a & 12 & -0.3375 & 0.7000 & 36 & -0.48 & 0.6326 & 0.05 & -1.7573 & 1.0822 & 0.714 & 0.173 & 2.951 \\
\hline a & 6 & a & 24 & -0.2302 & 0.7080 & 36 & $-0,32$ & 0.7473 & 0.05 & -1.6681 & 1.2076 & 0.794 & 0.189 & 3.345 \\
\hline a & 6 & b & 6 & 0.3061 & 0.7302 & 36 & 0.42 & 0.6776 & 0.05 & -1.1748 & 1.7870 & 1.358 & 0.309 & 5,972 \\
\hline a & 6 & $b$ & 12 & 0.1178 & 0.7857 & 36 & 0.15 & 0.8817 & 0.05 & -1.4757 & 1.7114 & 1.125 & 0.229 & 5.536 \\
\hline a & 6 & b & 24 & 0.2268 & 0.6682 & 36 & 0.34 & 0.7362 & 0.05 & -1283 & 1.5820 & 1.255 & 0.32 .4 & 4.865 \\
\hline a & 6 & c & 6 & 1.1041 & 0.7007 & 36 & $1.58:$ & 0.1239 & 0.05 & -0.3170 & 2.5251 & 3.016 & 0.728 & 12.493 \\
\hline a & 6 & c & 12 & 0.9008 & 0.6532 & 36 & 1.38 & 0.1764 & 0.05 & -0.4241 & 2.2256 & 2.461 & 0.654 & 9.258 \\
\hline a & 6 & c & 24 & 1.4562 & 0.6655 & 36 & 2.19 & 0.0352 & 0.05 & 0.1055 & 2,8059 & 4.290 & $1 .\{-2$ & 16.542 \\
\hline a & 6 & $d$ & 6 & 1.2533 & 0.6588 & 36 & 1.90 & 0.0651 & 0.05 & -0.08273 & 2.5893 & 3.502 & $0.92:$ & 13.320 \\
\hline a & 6 & d & 12 & 3.3798 & 0.8750 & 36) & $3.86 !$ & 0.0004 & 0.05 & 1.6052 & 5.1543 & 29.364 & 4.979 & 173,178 \\
\hline a & 6 & d & 24 & 2.8910 & 0.7198 & 36 & 4.02 & 0.0003 & $0.05 !$ & 1.4313 & 4.3508 & 18.012 & 4.194 & 77.540 \\
\hline
\end{tabular}

file://C:/Users/JLUOMA/AppData/Local/Temp/1/SAS\%20Temporary\%20Files/.. TD4836... 3/18/2015 


\begin{tabular}{|c|c|c|c|c|c|c|c|c|c|c|c|c|c|c|}
\hline a & 6 & e & $\theta$ & 2.1849 & 0.7021 & 36 & 3.11 & 0.0036 & 0.05 & 0.7610 & 3.6087 & 8.890 & 2.140 & 36.919 \\
\hline a & 6 & e & 12 & 4.0316 & 1.4278 & 36 & 2.82 & 0.0077 & 0.05 & 1.1359 & 6.9273 & 56.350 & 3.114 & $>999.989$ \\
\hline a & 6 & e & 24 & 3.9382 & 0.9888 & 36 & 3.98 & 0.0003 & 0.05 & 1.9329 & 5.9436 & 51.328 & 6.900 & 381.290 \\
\hline a & 6 & $f$ & 6 & -0.5913 & 0.8738 & 36 & -0.68 & 0.5029 & 0.05 & -2.3633 & 1.1808 & 0.554 & 0,094 & 3.257 \\
\hline a & 6 & if & 12 & -0.4872 & 0.7819 & 36 & -0.62 & 0.5371 & 0.05 & -2.0731 & 1.0988 & 0.614 & 0.126 & 3.000 \\
\hline a & 6 & 1 & 24 & -0.5791 & 0.7086 & 36 . & -0.82 & 0.4191 & 0.05 & -2.0161 & 0.8579 & 0.560 & 0.133 & 2.358 \\
\hline a & 12 & a & 24 & 0.1073 & 0.5507 & 36 & C.19 & 0.8466 & 0.05 & -1.0096 & 1.2243 & 1.113 & 0.364 & 3.402 \\
\hline a & 12 & b & 6 & 0.6438 & 0.5778 & 36 & 1.11 & 0.2727 & 0.05 & -0.5283 & 1.8156 & 1.903 & 0.590 & $\begin{array}{r}6.145 \\
\end{array}$ \\
\hline a & 12 & $b$ & 12 & 0.4554 & 0.6466 & 36 & 0.70 & 0.4858 & 0.05 & -0.8560 & $\begin{array}{l}1.7667 \\
\ldots . .\end{array}$ & 1.577 & 0.425 & $5.85^{-}$ \\
\hline a & 12. & b & 24 & 0.5644 & 0.4972 & 36 & 1.14 & 0.2638 & 0.05 & -0.4439 & 1.5727 & $1.758 !$ & 0.642 & 4.819 \\
\hline a & 12 & c & 6 & 1.4416 & 0.5401 & 36 & 2.67 & 0.0113 & 0.05 & D.3463 & 2.5369 & 4.227 & 1.414 & 12,641 \\
\hline a & 12 & c & 12 & 1.2383 & 0.4769 & 36 & 2,60 & 0.0135 & 0.05 & 0.2712 & 2.2054 & 3,450 & 1.311 & 9.074 \\
\hline $\mathrm{a}$ & 12 & c & 24 & 1.7938 & 0.4935 & 36 & 3.63 & 0.0009 & 0.05 & 0.7928 & 2.7947 & 6.012 & 2.210 & 16.357 \\
\hline a & 12 & d & 6 & 1.5908 & 0.4844 & 36 & 3.28 & 0.0023 & 0.05 & 0.6084 & 2.5733 & 4.908 & 1.837 & 13.108 \\
\hline a & 12 & d & 12 & 3.7173 & 0.7525 & 36 & 4.94 & $<.0001$ & 0.05 & 2.1911 & 5.2435 & 41.153 & 8.945 & 189.332 \\
\hline a & 2. & d & 24 & 3.2286 & 0.5646 & 36 & 5.72 & $<0001$ & 0.05 & 2.0835 & 4.3736 & 25.244 & 8.033 & 79.330 \\
\hline a & 12 & $\theta$ & 6 & 2.5224 & 0.5418 & 36 & 4.66 & $<.0001$ & 0.05 & 1.4235 & 3.6213 & 12.459 & 4.152 & $\begin{array}{r}37.387 \\
-\end{array}$ \\
\hline $\mathbf{a}$ & 12. & e. & 12 & 4.3691 & 1.3562 & 36 & 3.22 & 0.0027 & 0.05 & 1,6186 & 7.1196 & 78.974 & 5.046 & $>999.999$ \\
\hline a & 12 & $\theta$ & 24 & 4.2758 & $0.8823 i$ & 36 & 4.85 & $<.0001$ & 0.05 & 2.4865 & 6.0651 & 71.936 & 12.019 & 430.552 \\
\hline a & 12 & 1 & 6 & -0.2537 & 0.7511 & 36 & -0.34 & 0.7375 & 0.05 & -1.7770 & 1.2696 & 0.776 & 0.169 & $\begin{array}{l}3.559 \\
.\end{array}$ \\
\hline$a$ & 12 & $f$ & 12 & -0.1497 & 0.6420 & 36 & -0.23 & 0.8170 & 0.05 & -1.4617 & 1.1523 & 0.861 & 0.234 & 3.165 \\
\hline a & 12 & $f$ & 24 & -0.2416 & 0.5502 & 36. & $-0.44 !$ & 0.6632 & 0.05 & -1.3575 & 0.8743 & 0.785 & 0.257 & 2.397 \\
\hline a & 24 & b & 6 & 0.5363 & 0.5886 & 36 & 0.91 & 0.3683 & 0.05 & -0.6575 & 1.7301 & 1.710 & 0.518 & 5.641 \\
\hline a & 24 & b & 12 & 0.3480 & 0.6562 & 36 & 0.53 & 0.5991 & 0.05 & -0.9829 & 1.6790 & 1.416 & 0.374 & 5.360 \\
\hline a & 24 & b & 24 & 0.4571 & 05097 & 36 & 0.90 & 0.3758 & 0.05 & -0.5766 & 1.4907 & 1.579 & 0.562 & 4.440 \\
\hline a & 24 & $c$ & 6 & 1.3343 & 0.5516 & 36 & 2.42 & 0.0207 & 0.05 & 0.2156 & 2.4530 & 3.797 & 1.241 & 11.623 \\
\hline a & 24. & c & 12 & 1.1310 & 0.4890 & 36 & $2.31 i$ & 0.0268 & 0.05 & 0.1374 & 2.1245 & 3.099 & 1.147 & 8.369 \\
\hline a & 24 & $c$ & 24 & 1.6864 & 0.5061 & 38 & 3,33 & 0.0020 & 0.05 & 0,6600 & 2.7129 & 5.400 & 1.935 & 15.073 \\
\hline a & 24 & $d$ & 6 & 1.4835 & 0.4972 & 36 & 2.98 & 0.0051 & 0.05 & 0,4751 & 2.4919 & 4.408 & 1.608 & 12.085 \\
\hline a & 24 & d & 12. & 3.6100 & 0.7608 & 36 & 4.74 & $<.0001$ & 0.05 & 2.0669 & 5.15311 & 36.965 & 7.900 & 172,959 \\
\hline a & 24 & d & 24 & 3.1213 & 0.5756 & 36 & 5.42 & $<.0001$ & 0.05 & 1.9538 & 4.2887 & 22.675 & 7.056 & 72.871 \\
\hline$a$ & 24 & e & 6 & 2.4151 & 0.5533 & 36 & $4.36 \mid$ & $0.0001 \mid$ & 0.05 & 1.2929 & 3.5373 & 11.191 & 3.643 & 34.374 \\
\hline a & 24 & e & 12 & 4.2618 & 1.3608 & 36 & 3.13 & 0.0034 & 0.05 & 1.5019 & 7.0217 & 70.938 & 4.490 & $>999.999$ \\
\hline a & 24 & $e$ & 24 & 4.1685 & 0.8894 & 36 & 4.69 & $<, 0001$ & 0.05 & 2.3648 & 5.9722 & 64.616 & 10.641 & 392.353 \\
\hline a & 24 & f & 6 & -0.3610 & 0.7594 & 36 & -0.48 & 0.6374 & 0.05 & -1.9012 & 1.1792 & 0.697 & 0.149 & 3.252 \\
\hline a & 24 & $f$ & 12 & -0.2570 & 0.6517 & 36 & -0.39 & 0.6956 & $0.05^{\prime}$ & $-1,5787$ & 1.0647 & 0.773 & 0.206 & 2.900 \\
\hline a & 24 & $f$ & 24 & $\begin{array}{r}-0.3489 \\
-0.348\end{array}$ & 0.5615 & 36 & -0.62 & 0.5383 & 0.05 & -1.4878 & 0.7899 & 0.705 & 0.226 & 2.203 \\
\hline b & 6 & $b$ & 12 & -0.1883 & 0.6792 & 36 & -0.28 & 0.7832 & 0.05 & -1.5657 & 1.1891 & 0.828 & 0.209 & 3.284 \\
\hline b & 6 & b & 24 & -0.07926 & 0.5388 & 36 & -0.15 & 0.8839 & 0.05 & -1.172 .1 & 1.0135 & 0.924 & 0.310 & 2.755 \\
\hline b & 6 & c & 6 & 0.7980 & 0.5787 & 36 & 1.38 & 0.1764 & 0.05 & -0.3756 & 1.9715 & 2.221 & 0.687 & 7.182 \\
\hline b & 6 & c. & 12. & 0.5946 & 0.5202 & 36 & 1.14 . & 0.2605 & 0.05 & -0.4603 & 1.6496 & $1.8 * 2 !$ & 0.631 & 5.205 \\
\hline$b$ & 6 & c & 24 & 1.1501 & 0.5355 & 36 & 2.15 & 0.0385 & 0.05 & 0.06409 & 2.2361 & 3.159 & $1 . c \in 6$ & 0.357 \\
\hline b & 6 & $d$ & 6 & 0.9472 & 0.5271 & 36 & 80 & 0.0807 & 0.05 & -0.1218 & 2.0162 & 2.570 & 0.885 & 7.510 \\
\hline b & 6 & d & 12 & 3.0737 & 0.7807 & 36 & 3.94 & 0.0004 & 0.05 & 1.4903 & 4.6570 & 21.621 & 4.439 & 105.317 \\
\hline b & 6 & d & 24 & 2.5849 & 0.6016 & 36 & 4.30 & 0.0001 & 0.05 & 1.3648 & 3.8051 & 13.262 & 3.915 & 44.928 \\
\hline b & 6. & e & $\theta$ & 1.8788 & 0.5803 & 36 & 3.24 & 0.0026 & 0.05 & 0.7019 & 3.0557 & 6.546 & 2.017 & 21.236 \\
\hline
\end{tabular}

file://C:/Users/JLUOMA/AppData/L_ocal/Temp/1/SAS\%20Temporary\%20Files/_TD4836... 3/18/2015 


\begin{tabular}{|c|c|c|c|c|c|c|c|c|c|c|c|c|c|c|}
\hline & 6 & 0 & 12 & 3.7255 & 1.3720 & $38=$ & 2.72 & 0.0101 & 0.05 & 0.9429 & 6.5081 & 41.491 & 2.567 & 670.525 \\
\hline b & 6 & e & 24 & 3.6321 & 0.9064 & 36. & 4.01 & 0.0003 & 0.05 & 1.7039 & 5.4704 & 37.793 & 6.013 & 237.552 \\
\hline$b$ & 6 & $f$ & 6 & -0.8974 & 0.7793 & 36 & -1.15 & 0.2571 & 0.05 & -2.4779 & 0.6832 & 0.408 & 0.084 & 1.980 \\
\hline b & 6 & $f$ & 12 & -0.7933 & 0.6748 & 36 & -1.18 & 0.2474 & 0.05 & -2.1618 & $0.5752 !$ & 0.452 & 0.115 & 1.777 \\
\hline b & 6 & $f$ & 24 & -0.8852 & 0.5881 & 36 & -1.51 & 0.1410 & 0.05 & -2.0781 & 0.3076 & $0.4: 3$ & 0.125 & 1.360 \\
\hline b & 12 & b & 24 & 0.1090 & 0.6120 & 36 & 0.18 & 0.8595 & 0.05 & -1.1321 & 1.3502 & 1.115 & 0.322 & 3.858 \\
\hline b & 12 & c & 6 & 0.8862 & 0.6473 & 36 & 1.52 & 0.1353 & 0.05 & -0.3266 & 2.2991 & 2.681 & 0.721 & 9.965 \\
\hline b & 12 & c & 12 & 0.7829 & 0.5956 & 36 & 1.31 & 0.1970 & 0.05 & -0.4250 & 1.9909 & $2.188^{\circ}$ & 0.654 & 7.322 \\
\hline b & 12 & $c$ & 24 & 1,3384 & 0.6090 & 36 & 2.20 & 0.0345 & 0.05 & 0.1032 & 2.5736 & $3.813 i$ & 1.109 & 13.113 \\
\hline b & 12 & a & 6 & 1.1355 & 0.6017 & 36. & 1.89 & 0.0672 & 0.05 & -0.08476 & 2.3557 & 3.113 & 0.919 & 10.546 \\
\hline b & 12 & $d$ & 12 & 3.2619 & 0.8329 & 36 & 3.92 & 0.0004 & 0.05 & 1.5728 & 4.8511 & 26.100 & 4.820 & 141.324 \\
\hline b & 12 & $d$ & 24 & 2.7732 & 0.6679 & 36 & 4.15 & 0.0002 & 0.05 & 1.4180 & 4.1278 & 15.010 & 4,131 & 62.042 \\
\hline b & 12 & $\theta$ & 6 & 2.0671 & 0.6488 & 36 & 3.19 & 0.0030 & 0.05 & 0.7513 & 3.3829 & 7.902 & 2.120 & $\begin{array}{r}29.465 \\
-\end{array}$ \\
\hline b & 12 & 8 & 12 & 3.8138 & 1.4024 & 36 & 2.79 & 0.0084 & 0.05 & 1.0697 & 6.7579 & 50.087 & 2.914 & 860.813 \\
\hline b & 12 & $\theta$ & 24 & 3.8204 & 0.9517 & 36 & 4.01 & 0.0003 & 0.05 & 1.8903 & 5.7505 & 45.623 & 6.621 & 314.361 \\
\hline b & 12 & $f$ & 6 & -0.7091 & 0.8316 & 36 & -0.85 & 0.3995 & 0.05 & -2.3955 & 0.9774 & 0.492 & 0.091 & 2.658 \\
\hline b & 12 & $f$ & 12 & -0.6050 & 0.7345 & 36 & -0.82 & 0.4155 & 0.05 & -2.0946 & 0.8846 & 0.546 & 0.123 & 2.422 \\
\hline b & 12 & $f$ & 24 & -0.6969 & 0.6558 & 36 & -1.08 & 0.2950 & 0.05 & $-2,0270$ & 0.6331 & 0.498 & 0.132 & 1.883 \\
\hline b & 24 & $c$ & 6 & 0.8772 & 0.4981 & 36 & 1.76 & 0.0867 & 0.05 & -0.1330 & 1.8874 & 2.404 & 0.875 & 6.602 \\
\hline b & 24 & $c$ & 12 & 0.6739 & 0.4288 & 36 & 1.57 & 0.1248 & 0.05 & -0.1957 & 1.5435 & 1.962 & 0.822 & 4.681 \\
\hline b & 24 & 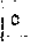 & 24 & 1.2294 & 0.4472 & 36 & 2.75 & 0.0093 & 0.05 & 0.3224 & 2.1364 & 3.419 & 1.380 & 8.469 \\
\hline b & 24 & $d$ & 6 & 1.0264 & 0.4371 & 36 & 2.35 & 0.0245 & 0.05 & 0.1399 & 1.9130 & 2.791 & 1.150 & 6.773 \\
\hline b. & 24 & d & 12 & 3.1529 & 0.7230 & 36 & 4.36 & 0.0001 & 0.05 & 1.6866 & 4.6192 & 23.404 & 5.401 & 101.417 \\
\hline b & 24 & d. & 24 & 2.6642 & $c .5246$ & 36 & 5.08 & $<, 0001$ & 0.05 & 1.6003 & 3.7281 & 14.356 & 4.954 & 41.601 \\
\hline b & 24 & e & 6 & 1.9580 & 0.5000 & 36 & 3.92 & 0.0004 & 0.05 & 0.9440 & 2.9721 & 7.085 & 2.570 & 18.533 \\
\hline b & 24 & e & 12 & 3.8047 & 1.3400 & 36 & 2.84 & 0.0074 & 0.05 & 1.0870 & 6.5224 & 44,913 & 2.965 & 680.243 \\
\hline b & 24 & e & 24 & 3.7114 & 0.8572 & 36 & 4.33 & $0.000 i$ & 0.05 & 1.9729 & 5.4499 & 40.911 & 7.191 & 232.732 \\
\hline b & 24 & 1 & 6 & -0.8181 & 0.7215 & 36 & -1.13 & 0.2643 & 0.05 & -2.2814 & 0.6452 & 0.441 & 0.102 & 1.806 \\
\hline b & 24 & 1 & 12 & -0.7141 & 0.6071 & 36 & -1.18 & 0.2472 & 0.05 & -1.9453 & 0.5172 & 0.490 & 0.143 & 1.677 \\
\hline b & 24 & $f$ & 24 & -0.8060 & 0.5091 & 36 & $-1.58 i$ & 0.1221 & 0.05 & -1.8385 & 0.2265 & 0.447 & 0.159 & 1.254 \\
\hline$c$ & 6 & $c$ & 12 & -0.2033 & 0.4779 & 36 & -0.43 & 0.6730 & 0.05 & -1.1725 & 0.7658 & 0.816 & 0.310 & 2.151 \\
\hline c & 6 & $\mathrm{c}$ & 24 & 0.3522 & 0.4945 & 36 & 0.71 & 0.4810 & 0.05 & -0.6507 & 1.3550 & 1.422 & 0.522 & 3.877 \\
\hline c & 6 & $d$ & 6 & 0.1492 & 0.4854 & 36 & 0.31 & 0.7603 & 0.05 & -0.8352 & 1.1336 & 161 & 0.434 & 3.107 \\
\hline c & 6 & $d$ & 12 & 2.2757 & 0.7532 & 36 & 3.02 & 0.0046 & 0.05 & 0.7482 & 3.8032 & 9.735 & 2.113 & 44.844 \\
\hline 0 & 6 & d & 24 & 1.7870 & 0.5654 & 36 & 3.16 & 0.0032 & 0.05 & 0,6402 & 2.9337 & 5,971 & 1.897 & 18.797 \\
\hline$c$ & 6 & e & 6 & 1.0008 & 0.5427 & 36 & 1.99 & $0.0540 !$ & 0.05 & $-0.01983 \mid$ & 2.1815 & 2.947 & 0.980 & 8.859 \\
\hline 5 & 6 & e & 12 & 2.9275 & 1.3565 & 36 & 2.16 & 0.0377 & 0.05 & 0.1763 & $5.6787 \mid$ & 18.6. 61 : & 1.193 & 292.573 \\
\hline c & $\theta$ & e & 24 & 2.8342 & 0.8828 & 36 & 3.21 & 0.0028 & 0.05 & 1.0438 & 4.6246 & 17.016 & 2.840 & 101.958 \\
\hline c & 6 & 1 & 6 & -1.6953 & 0.7517 & 36 & -2.26 & 0.0303 & 0.05 & -3.2199 & -0.1707 & 0.184 & 0.040 & 0.843 \\
\hline c & 6 & $f$ & 12 & -1.5913 & 0.6427 & 36 & -2.48 & 0.0181 & 0.05 & -2.8948 & -0.2878 & 0.204 & 0.055 & 0.750 \\
\hline c & 6 & $f$ & 24 & -1.6832 & 0.5511 & $36 \mid$ & -3.05 & 0.0042 & 0.05 & -2.8008 & -0.5656 & 0.186 & 0.061 & 0.568 \\
\hline e & 12 & $c$ & 24 & 0.5555 & 0.4246 & 36 & 1.31 & 0.1991 & 0.05 & -0.3058 & 1.4166 & 1.743 & 0.737 & 4.123 \\
\hline c & 12 & d & 6 & 0.3525 & 0.4139 & 36 & 0.85 & 0.4000 & 0.05 & -0.4869 & 1.1920 & 1.423 & 0.615 & 3.294 \\
\hline c & 12 & d & 12 & 2.4790 & 0.7092 & 36 & $3.50:$ & 0.0013 & 0.05 & 1.0406 & 3.9174 & 11.929 & 2.831 & 50.267 \\
\hline$c$ & 12 & d & 24 & 1.9903 & 0.5054 & 36 & 3.94 & 0.0004 & 0.05 & 0.9652 & 3.0153 & 7.318 & 2.625 & 20.395 \\
\hline c & 12. & e & 6 & 1.2841 & 0.4799 & 33 & 2.68 & 0.0111 & 0.05 & 0.3109 & $2.2573 !$ & 3.612 & 1.365 & 9.557 \\
\hline
\end{tabular}




\begin{tabular}{|c|c|c|c|c|c|c|c|c|c|c|c|c|c|c|}
\hline & 12 & e & 12 & 3.1308 & 1.3326 & 36 & 2.35 & |0.0244 & 0.05 & 0.4281 & 5.8336 & 22.893 & 1.534 & 341.569 \\
\hline c & 12 & e & 24 & 3.0375 & 0.8456 & 36 & 3.59 & 0.0010 & 0.05 & 1.3225 & 4.7525 & 20.853 & 3.753 & 115.868 \\
\hline c & 12 & $f$ & 6 & -1.4920 & 0.7077 & 36 & -2.11 & 0.0420 & 0.05 & -2.9273 & -0.05675 & 0.225 & 0.054 & 0.945 \\
\hline$c$ & 12 & $f$ & $: 2$ & -1.3880 & 0.5906 & 36 & -2.35 & 0.0244 & 0.05 & -2.5858 & -0.1902 & 0.250 & 0.075 & 0.827 \\
\hline c & 12 & f & 24 & $-1,4799$ & 0.4893 & 36 & -3.02 & 0.0046 & 0.05 & -2.4722 & -0.4875 & 0.228 & 0.034 & 0.614 \\
\hline c & 24 & d & 6 & -0.2029 & 0.4330 & 36 & -0.47 & 0.6421 & 0.05 & -1.0811 & 0,6753 & 0.816 & 0.339 & 1.965 \\
\hline c & 24 & $d$ & 12 & 1.9235 & 0.7205 & 36 & 2.67 & 0.0113 & 0.05 & 0.4623 & 3,3848 & 6.845 & 1.588 & 29.513 \\
\hline c & 24 & $d$ & 24 & 1.4348 & 0.5212 & 36 & 2.75 & 0.0092 & 0.05 & 0.3778 & 2,4918 & 4.199 & 1.459 & 12.083 \\
\hline c & 24 & :e & 6 & 0.7287 & 0.4964 & 36 & 1.47 & 0.1508 & 0.05 & -0.2781 & 1.7354 & 2.072 & 0.757 & 5.671 \\
\hline$c$ & 24 & [e & 12 & 2.5754 & 1.3387 & 36 & $1.92 i$ & 0.0623 & 0.05 & -0.1396 & 5.2904 & 13.136 & 0.870 & 198.415 \\
\hline$c$ & 24 & $e$ & 24 & 2.4820 & 0.8551 & 36 & 2.80 & 0.0063 & 0.05 & 0.7478 & 4.2163 & 11.965 & 2.112 & 67.780 \\
\hline c & 24 & $f$ & 6 & -2.0475 & 0.7190 & 36 & -2.85 & 0.0072 & 0.05 & -3.5057 & -0.5892 & 0.129 & 0.030 & 0.555 \\
\hline c & 24 & 1 & 12 & -1.9434 & 0.6041 & 36 & .3 .22 & 0.0027 & 0.05 & -3.1687 & -0.7182 & 0.143 & 0.042 & 0.488 \\
\hline $\mathrm{c}$ & 24 & $f$ & 24 & -2.0354 & 0.5056 & 36 & -4.03 & 0.0003 & 0.05 & -3.0607 & -1.0100 & 0.131 & 0.047 & 0.364 \\
\hline d & 6 & d & 12 & 2.1265 & c.7143 & 36 & 2.98 & 0.0052 & 0.05 & 0.6778 & 3.5751 & 8.385 & 1.970 & 35.700 \\
\hline d & 6 & d & 24 & 1.6377 & 0.5125 & 36 & 3.20 & 0.0029 & 0.05 & 0.5983 & 2.6772 & 5.144 & 1.819 & 14.545 \\
\hline d & 6 & e & 6 & 0.9316 & 0.4873 & 36 & 1.91 & 0.0639 & 0.05 & -0.05679 & 1.9200 & 2.539 & 0.945 & 6.821 \\
\hline d & 6 & e & 12 & 2.7783 & 1.3354 & 36 & 2.08 & 0.0447 & 0.05 & 0,07006 & 5.4865 & 16.092 & 1.073 & 241.7 \\
\hline d & 6 & e & 24 & 2,6849 & 0.8490 & 36 & $3.16 !$ & 0.0032 & 0.05 & 0.8613 & 4.4086 & 14,657 & 2.615 & 82.153 \\
\hline d & 6 & 1 & 6 & -1.8445 & 0.7128 & 36 & -2.59 & 0.0138 & 0.05 & -3.2901 & -0.3989 & 0.158 & 0.037 & 0.671 \\
\hline d & 6 & 1 & 12 & -1.7405 & 0.5967 & 36 & -2.92 & 0.0061 & 0.05 & -2.9507 & -0.5303 & 0.175 & 0.052 & 0.588 \\
\hline d & 6 & I & 24 & -1.8324 & 0.4967 & 36 & -3.69 & 0.0007 & 0.05 & -2.8397 & -0.8252 & 0.160 & 0.058 & 0.438 \\
\hline d & 12 & $d$ & 24 & -0.4887 & 0.7709 & 36 & -0.63 & 0.5301 & 0.05 & -2.0523 & 1.0748 & 0.613 & 0.128 & $2.929:$ \\
\hline$d$ & 12 & e & 6 & -1.1949 & 0.7544 & 36 & -1.58 & 0.1220 & 0.05 & -2.7249 & 0.3352 & 0.303 & 0.066 & 1.398 \\
\hline d & 12 & $\theta$ & 12 & 0.6518 & 1.4542 & 36 & 0.45 & 0.6567 & 0.05 & -2.2975 & 3.6012 & 1.919 & 0.101 & 36.641 \\
\hline$d$ & 12 & e & 24 & 0.5585 & 1.0266 & $36 !$ & 0.541 & 0.5898 & 0.05 & $-\{.5236$ & 2.8406 & 1.748 & 0.216 & 14.021 \\
\hline d & 12 & 1 & 6 & -3.9710 & 0.9164 & 36 & -4.33 & 0.0001 & 0.05 & -5.8295 & -2.1125 & 0.019 & 0.003 & 0.121 \\
\hline d & 12 & $f$ & 12 & -3.8670 & 0.8293 & 36 & -4.66 & $<.0001$ & 0.05 & -5.5488 & -2.1851 & 0.021 & 0.004 & 0.112 \\
\hline d & 2 & $f$ & 24 & -3.9589 & 0.7605 & 38 & -5.21 & $<.0001$ & 0.05 & -5.5012 & -2.4166 & 0.019 & 0.004 & 0.089 \\
\hline d & 24 & e & 6 & -0.7061 & 0.5671 & 36 & -1.25 & 0.2211 & 0.05 & -1.8563 & 0.4440 & 0.494 & 0.156 & 1.559 \\
\hline d & 24 & e & 12 & 1.1406 & 1.3665 & 36 & 0.83 & 0.4094 & 0.05 & -1.6308 & 3.9119 & 3.128 & 0.196 & 49.995 \\
\hline d & 24 & e & 24 & 1.0472 & 0.8980 & 36 & $11^{\wedge}$ & 0.2512 & 0.05 & -0.7740 & 2.8684 & 2.850 & 0.461 & 17.610 \\
\hline d & 24 & $f$ & 6 & -3.4823 & 0.7695 & 36 & -4.53 & $<.0001$ & 0.05 & -5.0430 & -1.9216 & 0.031 & 0.006 & 0.146 \\
\hline$d$ & 24 & $f$. & 12 & -3.3783 & 0.6634 & 36 & -5.09 & $<, 0001$ & 0.05 & 4.7238 & -2.0327 & 0.034 & 0.009 & 0.131 \\
\hline$d$ & 24 & if & 24 & -3.4702 & 0.5751 & 36 & -6.03 & $<0001$ & 0,05 & -4.6366 & -2.3037 & 0.031 & 0.010 & 0.100 \\
\hline e & 6 & e & 12 & 1.8467 & 1.3572 & 36 & 1.36 & 0.1821 & 0.05 & -0.9059 & 4.5993 & 6.339 & 0.404 & 98.416 \\
\hline e & 6 & $e$ & 24 & 1.7533 & 0.8839 & 36 & 1.98 & 0.0550 & 0.05 & -0.03923 & 3.5459 & 5.774 & 0.962 & 34.672 \\
\hline$\theta$ & 0 & $f$ & 6 & $-2.7761 \mid$ & 0.7530 & 36 & -3.69 & 0,0007 & 0.05 & -4.3033 & -1.2490 & 0.062 & 0.014 & 0.287 \\
\hline e & 6 & $f$ & 12 & -2.6721 & 0.6442 & 36 & -4.15 & $0.00 \mathrm{C} 2$ & 0.05 & -3.9786 & -1.3656 & 0.069 & 0.019 & 0.255 \\
\hline e & 6 & $f$ & 24 & -2.7640 & 0.5528 & 36 & -5.00 & $<.0001$ & 0.05 & -3.8852 & -1.6429 & 0.063 & 0.021 & 0.193 \\
\hline e & 12 & e & 24 & -0.09335 & 1.5254 & 36. & -0.06 & 0.9515 & 0.05 & -3.1870 & 3.0003 & 0.911 & 0.041 & 20.092 \\
\hline$e$ & 12 & $t$ & 6 & -4.6228 & 1.4535 & 36 & -3.18 & 0.0030 & 0.05 & -7.5707 & -1.6750 & $0.010^{\prime}$ & $<0.001$ & 0.187 \\
\hline e & 12 & $f$ & 12 & -4.5188 & 1.4002 & $36 \mid$ & -3.23 & 0.0027 & 0.05 & -7.3586 & -1.6790 & 0.011 & $<0.001$ & 0.187 \\
\hline e & 12 & $f$ & 24 & -4.6107 & 1.3606 & 36 & $-3,39$ & 0.0017 & 0.05 & -7.3702 & -1.8513 & 0.010 & $<0.001$ & 0.157 \\
\hline e & 24 & $f$ & 6 & -4.5295 & 1.0256 & 36 & -4.42 & $<, 0001$ & 0.05 & -6.6094 & -2.4495 & 0.011 & 0.001 & 0.086 \\
\hline e & 2.4 & $f$ & 12 & -4.4255 & 0.9486 & 36 & -467 & $<.0001$ & 0.05 & -6.3492 & -2.5017 & 0.012 & 0.002 & 0.082 \\
\hline
\end{tabular}




\begin{tabular}{|c|c|c|c|c|c|c|c|c|c|c|c|c|c|c|}
\hline & 24 & F & 24 & $-4,5174$ & 0.8890 & 36 & -5.08 & $<0001$ & 0.05 & $-6.3204\}$ & -2.7143 & 0.011 & 0.002 & 0.066 \\
\hline$f$ & 6 & f & 12 & 0.1040 & 0.8280 & 36 & 0.13 & 0.9007 & 0.05 & -1.5752 & 1.7832 & 1.110 & 0.207 & 5.949 \\
\hline$f$ & 6 & if & 24 & 0.01211 & 0.7590 & 36 & 0.02 & 0.9874 & 0.05 & -1.5273 & 1,5515 & 1.012 & 0.217 & 4.719 \\
\hline I & 12 & $f$ & 24 & -0.09191 & 0.6513 & 36 & -0.14 & 0.8886 & 0.05 & -1.4127 & 1.2289 & 0.912 & 0.243 & 3.417 \\
\hline
\end{tabular}

Performed by J. Luama; SAS versIon 9.3 08:43 18MAR15 


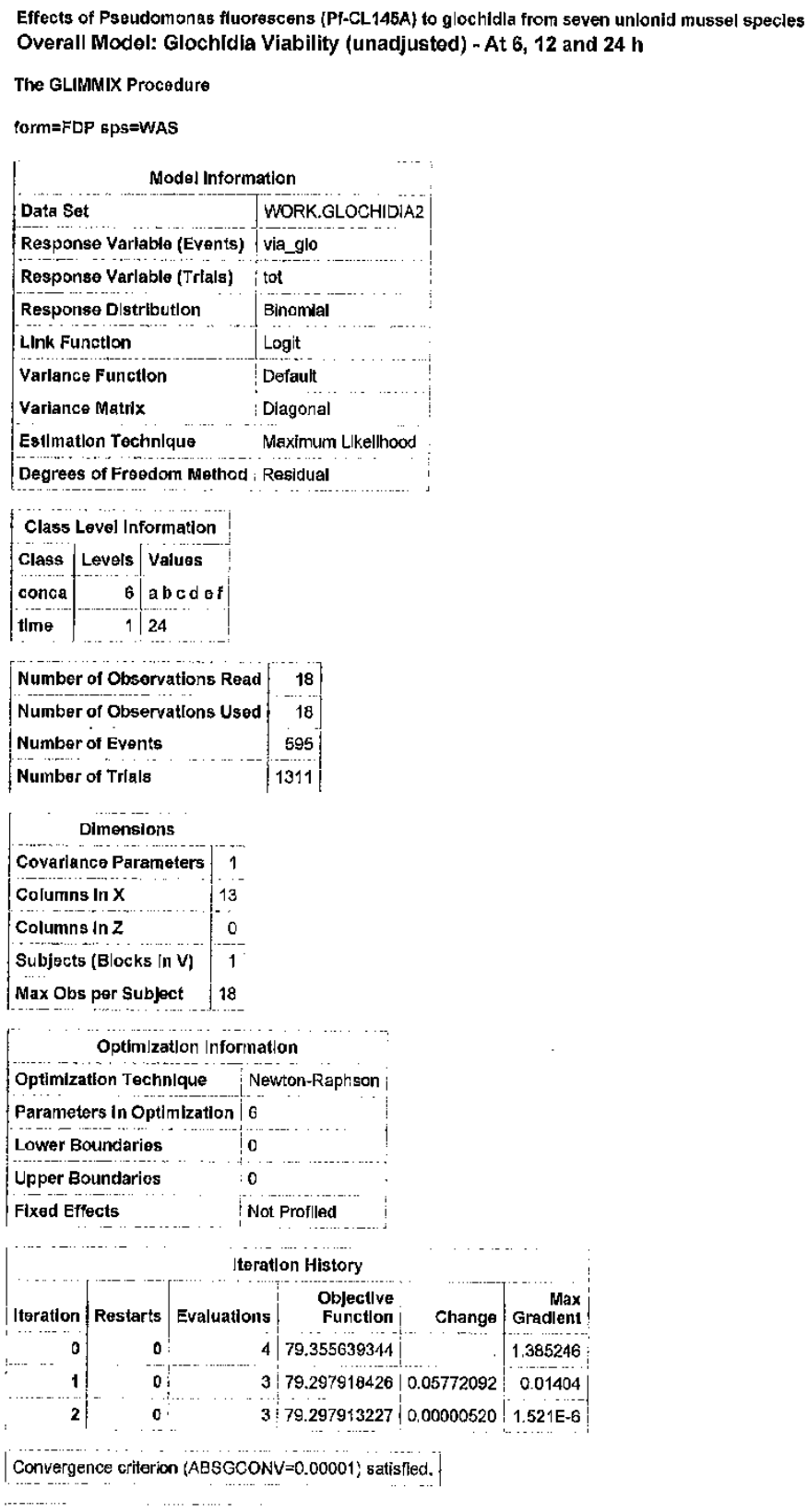

file://C:/Users/JLUOMA/AppData/Local/Temp/1/SAS\%20Temporary\%20Files/_TD4836... 3/18/2015 


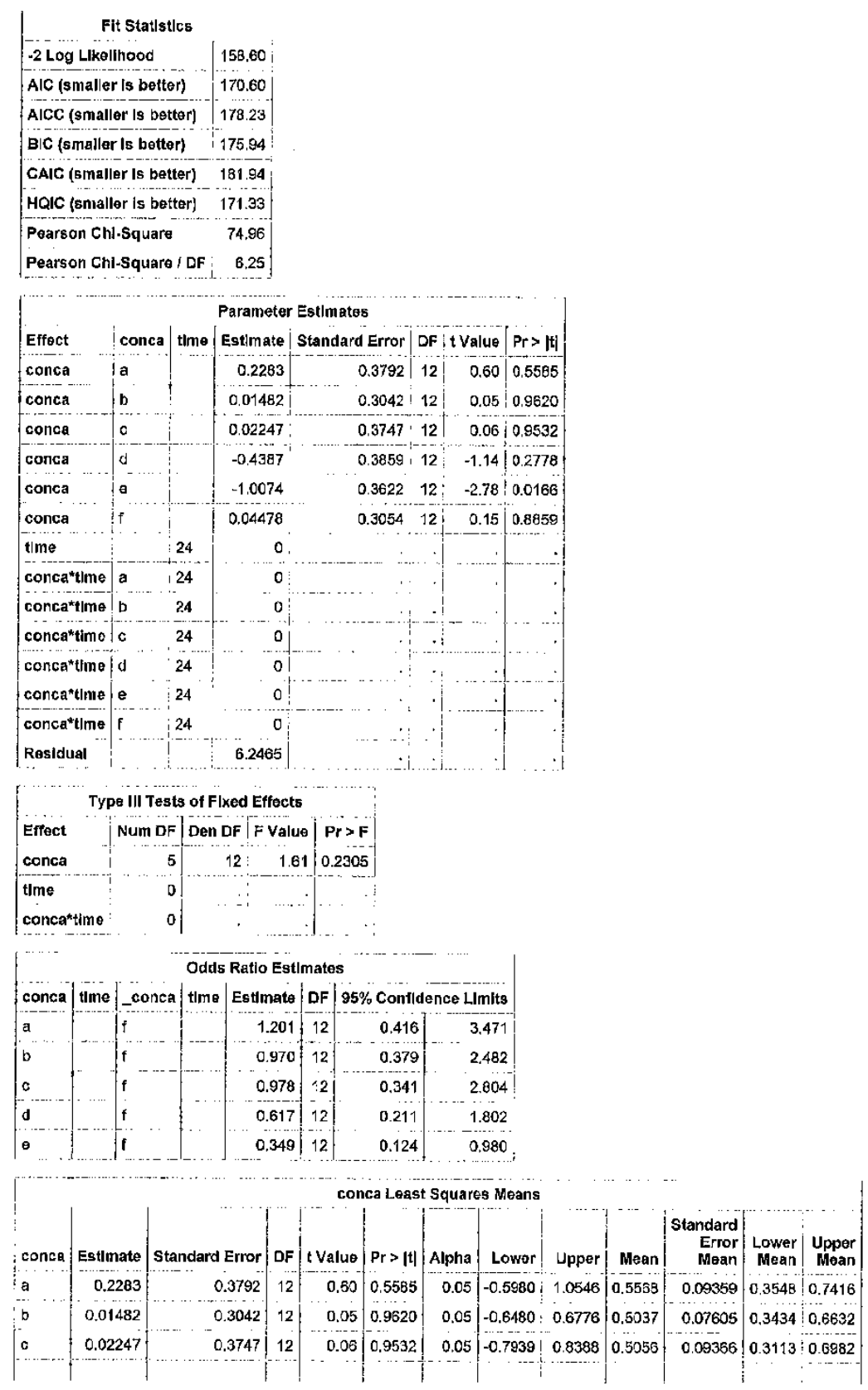

file://C:/Users/JLUOMA/AppData/Local/Temp/1/SAS\%20Temporary\%20Files/_TD4836... 3/18/2015 


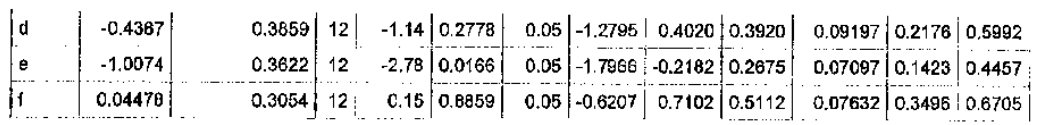

\begin{tabular}{|c|c|c|c|c|c|c|c|c|c|c|c|c|}
\hline \multirow[b]{2}{*}{ conca } & \multirow[b]{2}{*}{ conca } & \multirow[b]{2}{*}{ Estlmate } & \multirow[b]{2}{*}{$\begin{array}{c}\text { Standard } \\
\text { Error }\end{array}$} & \multicolumn{7}{|c|}{ Dlfferences of conca Least Squaros Moans } & \multirow[b]{2}{*}{$\begin{array}{r}\text { Lower } \\
\text { Conficence } \\
\text { Limit for Odds } \\
\text { Ratio }\end{array}$} & \multirow[b]{2}{*}{$\begin{array}{r}\text { Upper } \\
\text { Gonfidence } \\
\text { LImlt for Oodds } \\
\text { Ratlo }\end{array}$} \\
\hline & & & & DF & ivalue & $\begin{array}{l}\operatorname{Pr}>\mathbf{| t |} \\
\mid \mathbf{f}\end{array}$ & Alpha & Lower & Upper: & $\begin{array}{l}\text { Odds } \\
\text { Ratio }\end{array}$ & & \\
\hline a & $b$ & 0.2134 & 0.4862 & 12 & 0.44 & 0.6684 & 0.05 & -0.8458 & 1.2727 & 1.238 & 0.428 & 3.571 \\
\hline a & c & 0.2058 & 0.5331 & 12 & 0.39 & 0.7662 & $0.05 !$ & -0.9558 & 1.3673 & 1.228 & 0.385 & 3.925 \\
\hline a & d & 0.6670 & $0.541 \mathrm{C}$ & 12 & 1.23 & 0.2413 & 0.05 & -0.5119 & 1.8458 & 1.948 & 0.599 & 0.333 \\
\hline a & $\theta$ & 1.2357 & 0.5244 & 12 & 2.36 & 0.0363 & 0.05 & 0.09304 & 2.3783 & 3.441 & 1.098 & 10.786 \\
\hline a & f... & 0.1835 & 0.4869 & 12 & 0.38 & 0.7129 & 0.05 & -0.8775 & 1.2444 & 1.201 & 0.416 & 3.471 \\
\hline b & c & -0.00766 & 0.4826 & 12 & .0 .02 & 0.9876 & 0.05 & -1.0692 & 1.0439 ! & 0.992 & 0.347 & 2.840 \\
\hline b & d & 0.4535 & 0.4914 & 12 & 0.92 & 0.3742 & 0.05 & -0.6171 & 1.5242 & 1.574 & 0.540 & 4.591 \\
\hline b. & le & 1.0222 & 0.4730 & 12 & 2.16 & 0.0516 & 0.05 & -0.00839 & 2.0528 & 2.778 & 0.992 & 7.790 \\
\hline b & $i$ & -0.02997 & 0.4311 & 12 & -0.07 & 0.9457 & 0.05 & -0.9692 & 0.9093 & 0.970 & 0.379 & 2.482 \\
\hline c & d & 0.4612 & 0.5379 & 12 & 0.86 & 0.4080 & 0.05 & -0.7107 & 1.6331 & 1.586 & 0.491 & 5.120 \\
\hline c & e & 1.0299 & 0.5211 & 12 & 1.98 & 0.0716 & 0.05 & -0.1056 & 2.1653 & 2.801 & 0.900 & 8.717 \\
\hline$c$ & 1 & -0.02231 & 0.4834 & 12 & -0.05 & 0.9639 & 0.05 & -1.0755 & 1.0309 & 0.978 & 0.341 & 2.804 \\
\hline d & $\theta$ & 0.5687 & 0.5292 & 12 & 1.07 & 0.3037 & 0.05 & $\begin{array}{r}-0.5845 \\
-\end{array}$ & 1.7218 & 1.766 & 0.557 & 5.595 \\
\hline d & $f$ & -0.4835 & 0.4921 & 12 & -0.98 & $0.3453 \mid$ & 0.05 & -1.5558 & $0.5887 !$ & 0.617 & 0.211 & 1.802 \\
\hline e & 1 & -1.0522 & 0.4738 & 12 & -2.22 & 0.0464 & 0.05 & -2.0845 & -0.01989 & 0.349 & 0.124 & 0.980 \\
\hline
\end{tabular}

\begin{tabular}{|c|c|c|c|c|c|c|c|c|c|c|c|c|c|c|c|}
\hline & & & & & & ne Leas & st Squaros & 5 Means & & & & & & & \\
\hline time & Estlma & te I Stand & ard Er & or $\mid D F: t V$ & Value & $P r>14$ & Alpha & Lower & Upper & mean & $\begin{array}{r}\text { Standard } \\
\text { Error } \\
\text { Mean }\end{array}$ & $\begin{array}{l}\text { Lower } \\
\text { Mean }\end{array}$ & $\begin{array}{c}\text { Upper } \\
\text { mean }\end{array}$ & & \\
\hline 24 & $-0.18 \mathrm{c}$ & & 0.14 & $14 \mid 12 i$ & -1.31 & 0.2143 & 0.05 & -0.5038 & 0.1252 & 0.4528 & 0.03577 & 0.3766 & 0.5313 & & \\
\hline & & & & & & oncektin & me Least S & Squares & 5 Means & & & & & & \\
\hline conca & Itime & Estimate & Stan & dard Error & $\mathrm{DF}: \mathrm{t}$ & t Value & $|P r>| t|| A$ & Alpha & Lower & Uppor i & Mean & $\begin{array}{r}\text { tandard } \\
\text { Error } \\
\text { Mean }\end{array}$ & $\begin{array}{l}\text { Lower } \\
\text { Mean }\end{array}$ & $\begin{array}{l}\text { Upper } \\
\text { Mean }\end{array}$ & \\
\hline a & 24 & 0.2283 & & 0.3792 & 12 & 0.60 & 0.5585 & $0.05]-$ & -0.5980 & 1.0546 & 0.5568 & 0.09359 & 0.3548 & 0.7416 & \\
\hline b & 24 & 0.01482 & & 0.3042 & 12 & 0.05 & 0.9620 & $0.05 \mid \cdot 4$ & -0.6480 & $0.6776 \mid 0$ & 0.5037 & 0.07605 & 0.3434 & 0.6632 & \\
\hline$c$ & 24 & 0.02247 & & 0.3747 & 12 & 0.06 & $|0.9532|$ & $0.05:-0$ & -0.7939 & 0,838810 & 0.5058 & 0.09366 & 0.3113 & 0.6982 & \\
\hline d & 24 & -0.4387 & & 0.3859 & 12 & -1.14 & 0.2778 & $0.05 \mid-1$ & -1.2795 & $0.4020 \mid 0$ & 0.3920 & 0.09197 & 0.2176 & 0.5992 & \\
\hline e & 24 & -1.0074 & & 0.3622 & 12 & -2.78 & 0.0163 & $0.05 \mid-1$ & -1.7966 & -0.21820 & 0.2675 & 0.07097 & 0.1423 & 0.4457 & \\
\hline 1 & 24 & 0.04470 & & 0.3054 & $|12|$ & 0.15 & $\mid 0.8859$ & $0.05 \mid-0$ & -0.6207 & $0.7102 \mid 0$ & 0.5112 & 0.07632 & 0.3496 & 0.6705 & \\
\hline & & & & & & fference & es of cone & ca"tlme L & Least Squ & quares Mea & eans & & & & \\
\hline conca & time & -conca & time & Est|mate & $\begin{array}{l}\text { Standa } \\
\text { Err }\end{array}$ & $\begin{array}{l}\text { lard } i \\
\text { rror } \mid D r\end{array}$ & F t Value & $\begin{array}{l}\operatorname{Pr}> \\
|t|\end{array}$ & Alpha & Lower & Upper & $\begin{array}{l}\text { Odds } \\
\text { Ratio }\end{array}$ & Conf & $\begin{array}{r}\text { Lower } \\
\text { fidence } \\
\text { Imit for } \\
\text { Odds } \\
\text { Ratlo }\end{array}$ & $\begin{array}{r}\text { Upper } \\
\text { Confidence } \\
\text { LImit for } \\
\text { Odds } \\
\text { Ratlo }\end{array}$ \\
\hline a & 24 & fb & 24 & 0.2134 & 0.48 & $862 \mid 12$ & 0.44 & 0.6684 & 0.05 & -0.8458 & 1.2727 & 1.238 & & 0.429 & 3.571 \\
\hline a & 24 & lc & 24 & 0.2058 & 0.53 & $331 \mid 12$ & 0.39 & 0.7062 & 0.05 & -0.9558 & 1.3673 & 1.228 & & 0.385 & 3.925 \\
\hline a & 24 & d & 24 & 0.6670 & 0.54 & 41012 & 1.23 & 0.2413 & 0.05 & -0.5119 & 1.8458 & 1.948 & & 0.599 & 6.393 \\
\hline a & 24 & e & 24 & 1.2357 & 0.52 & $244 \mid 12$ & 2.36 & $|0.0363|$ & 0.05 & 0.09304 & 2.3783 & 3.441 & & 1.098 & 10.786 \\
\hline
\end{tabular}




\begin{tabular}{|c|c|c|c|c|c|c|c|c|c|c|c|c|c|c|}
\hline a & 24 & $f$ & 24 & 0.1835 & 0.4869 & 12 & 0.38 & | 0.7129 & 0.05 & -0.8775 & 1.2444 & .201 & 0.416 & 3.471 \\
\hline b & 24 & $c$ & 24 & -0.00766 & 0.4826 & 12 & -0.02 & $\mid 0.9876$ & $0.05^{1}$ & -1.0592 & 1.0439 & 0.992 & 0,347 & 2.840 \\
\hline b & 24 & d & 24 & 0.4535 & 0.4914 & 12 & 0.92 & $|0.3742|$ & 0.05 & -0.6171 & 1.5242 & 1.574 & 0.540 & 4.591 \\
\hline b & 24 & e & 24 & 1.0222 & 0.4730 & 12 & 2.16 & 0.0516 & 0.05 & -0.00839 & 2.0528 & 2.779 & 0.992 & 7.790 \\
\hline b & 24 & $f$ & 24 & -0.02997 & 0.4311 & 12 & -0.07 & 0.9457 & 0.05 & -0.9692 & 0.9093 & 0.970 & 0.379 & 2.482 \\
\hline c & 24 & d & 24 & 0.4612 & 0.5379 & 12 & 0.86 & $0.4080 \mid$ & 0.05 & -0.7107 & 1.6331 & 1.586 & 0.491 & 5.120 \\
\hline c & 24 & $\theta$ & 24 & 1.0299 & 0.5211 & 12 & 1.98 & 0.0718 & 0.05 & -c.1056 | & 2.1653 & 2.801 & 0.800 & 8.717 \\
\hline $\mathrm{c}$ & 24 & if & 24 & -0.02231 & 0.4834 & 12 & -0.05 & 0.9639 & 0.05 & -1.0755 & 1.0309 & 0.978 & 0.341 & 2.804 \\
\hline$d$ & 24 & $\theta$ & 24 & 0.5687 & 0.52 .92 & 12 & 1.07 & 0.3037 & 0.05 & -0.5845 & 1.7218 & 1.766 & 0.557 & 5.595 \\
\hline$d$ & 24 & $f$ & 24 & -0.4835 & 0.4921 & 12 & -0.98 & $0.3453 \mid$ & 0.05 & -1.5558 & 0.5887 & 0.617 & 0.211 & $1.802 !$ \\
\hline & 24 & $f$ & 24 & -1.0522 & 0.4738 & 12 & -2.22 & 0.0464 & 0.05 & -2.0845 & 0.01989 & 0.349 & 0.124 & 0.980 \\
\hline
\end{tabular}

Performed by J. Luoma; SAS version 9.3 08:43 18MAR15 


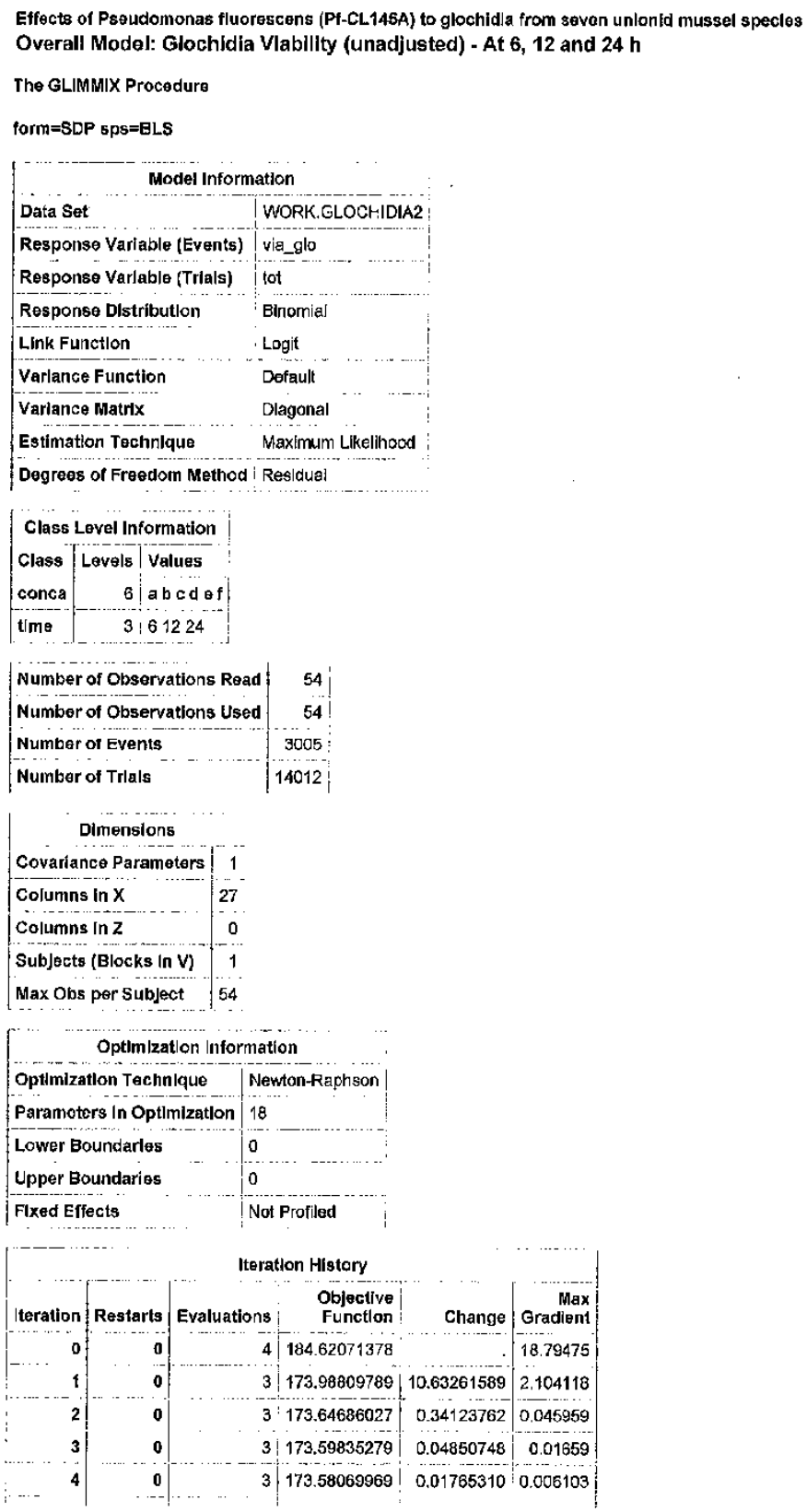

file://C:/Users/J.UOMA/AppData/Local/Temp/1/SAS\%20Temporary\%20Files/_TD4836... 3/18/2015 


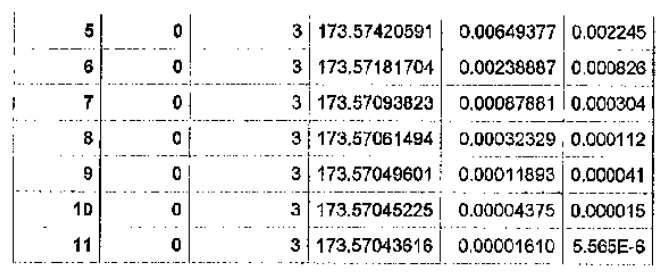

|Convergence criterion (ABSGCONV=0.00001) sattsfied.

\begin{tabular}{|l|r|}
\multicolumn{2}{|c}{ Fit Statlstlcs } \\
\hline-2 Log Likellhood & 347.14 \\
\hline AIC (s smaller is better) & 383.14 \\
\hline AICC (smaller is better) & 402.68 \\
\hline BIC (smaller Is better) & 418.94 \\
\hline CAIC (smailer Is better) & 436.94 \\
\hline HQIC (smaller Is better) & 396.95 \\
\hline Pearson Chi-Square & 137.50 \\
\hline Pearson Chi-Square I DF & 3.82 \\
\hline
\end{tabular}

\begin{tabular}{|c|c|c|c|c|c|c|c|}
\hline & & & Parameter & r Estimatos & & & \\
\hline Effect & conca & t tme & Estlmate & Standard Error I & DF & t Value & $\left|P_{\Gamma}>\right| t \mid$ \\
\hline conca & a & & -1.7763 & 0.2266 & 36 & -7.84 & $<, 0001$ \\
\hline conca & b & & -2.5373 & 0.2930 & $36:$ & -8.66 & $<, 0001$ \\
\hline conca & c & & $-3.3828 i$ & 0.3459 & 36 & -9.78 & $<<\mathrm{CCO}$ \\
\hline conca & d & & -4.8730 & 0.7415 & 36 & -6.57 & $\mid<.0 \mathrm{CO1}$ \\
\hline conca & e & & -4.8581 & 0.6540 & 36. & -7.43 & $<.0001$ \\
\hline conca & f & & 1.6550. & 0.1428 & 36 & 11.59 & $<.0001$ \\
\hline time & & 0 & 0.1407 & 0.2355 & $36 !$ & 0,60 & 0.5541 \\
\hline time & & 12 & 0.4771 & c.2901： & 36 & 1.64 & 0.1088 \\
\hline timo & & 24 & 0 & & . & & \\
\hline conca*tlme & a & 6 & -0.4866 & 0.4003 & 36 & -1.22 & 0.2321 \\
\hline Conca*time & a & 12 & -0.3185 & 0.4090 & 36 & -0.78 & 0.4412 \\
\hline conca*time & a & 24 & 0 & & & & \\
\hline Conca*time & b & 6 & -2.4801 & 0.9545 & 36 & -2.60 & 0.0135 \\
\hline conca*time ! & $b$ & 12 & -0.2233 & 0.4994 & 36 & -0.45 & 0.6575 \\
\hline conca*time & $b$ & 24 & 0 & & .1 & & \\
\hline conca*timo & c & $\theta$ & -0.7873 & $0.6886 !$ & ! $36 !$ & $-1,14$ & 0.2605 \\
\hline concantime & c & 12 & -1.5131 & 0.7958 & 36 & -1.90 & 0.0653 \\
\hline conca*time i & ic & 24 & 0 & & . & $\therefore$ & \\
\hline Concattime & $d$ & 6 & 0.4683 & 0.9963 & 36 & 0.47 & 0.6412 \\
\hline $\operatorname{conca^{*}t\operatorname {tim}\theta }$ & $d$ & 12 & -13.1562 & 638.55 & $36:$ & -0.02 & 0.9837 \\
\hline conca*time | & Id & 24 & 0 & . i & ! ! & & \\
\hline conca*tim | & e & 6 & -0.04760 & 1.0607 & 36 & -0.04 & 0,9645 \\
\hline conca*tlme & a & 12 & $-0.5731 !$ & 1.2139 & 36 & -0.47 & 0.6397 \\
\hline conca*tlme & a & 24 & 0 & &. & & \\
\hline concaktlme & $f$ & 6 & 0 & & & & \\
\hline
\end{tabular}

file://C:/Users/JI _UOMA/AppData/Local/Temp/1/SAS\%20Temporary\%20Files/ TD4836... 3/18/2015 


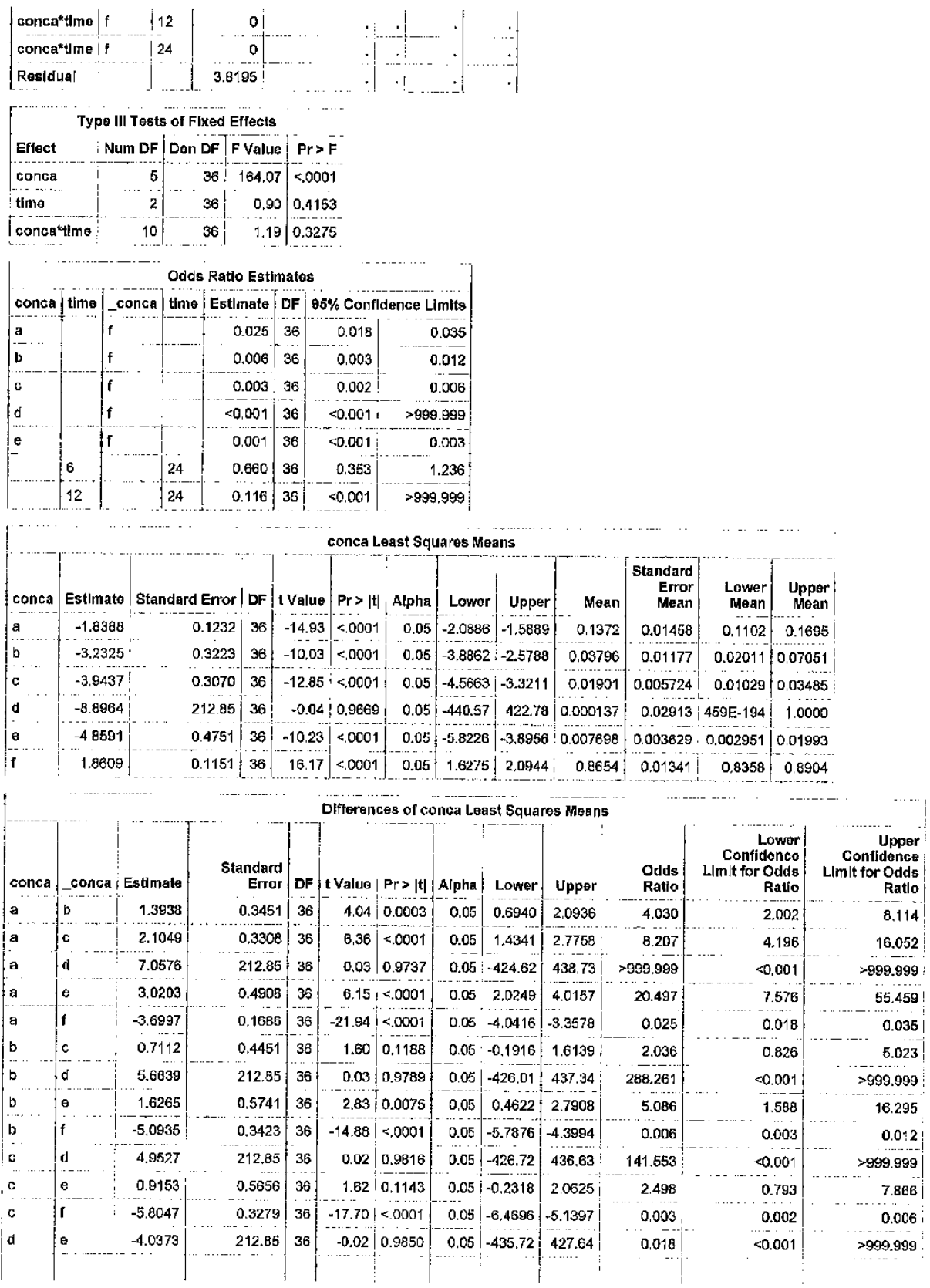




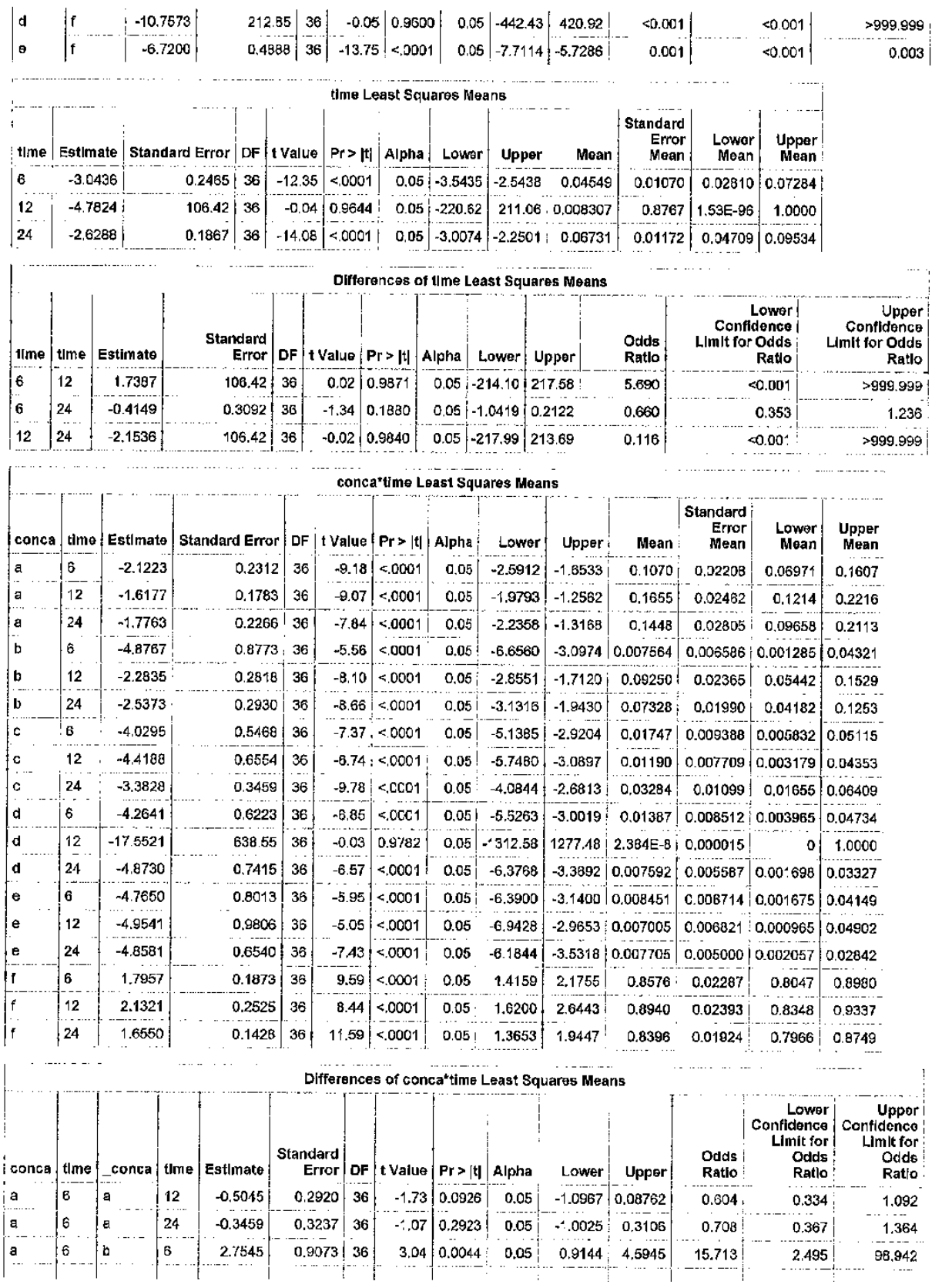

file://C:/Users/ILIJOMA/AppData/Local/Temp/1/SAS\%20Temporary\%20Files/ TD4836... 3/18/2015 


\begin{tabular}{|c|c|c|c|c|c|c|c|c|c|c|c|c|c|c|}
\hline a & 6 & b & 12 & 0.1613 & 0.3645 & 36 & 0.44 & 0.6608 & 0.05 & -0.5780 & 0.9005 & $1.175 !$ & 0,561 & 2,481 \\
\hline a & 6 & b & 24 & 0.4151 & 0.3733 & 36 & 1.11 & 0.2735 & 0.05 & -0.3419 & 1.1721 & 1.514 & c. 710 & 3.229 \\
\hline 日 & 6 & c & 6 & 1.9072 & 0.6937 & 36 & 3.21 & 0.0028 & 0.05 & 0.7031 & 3.1113 & 6.734 & 2.020 & 22,450 \\
\hline a & 6 & c & 12 & 2.2966 & C. 6950 & 36 & 3.30 & 0.0022 & 0.05 & 0.8871 & 3.7060 & 8.940 & 2.428 & 40.691 \\
\hline a & 6 & c & 24 & 1.2606 & 0.4161 & 36 & 3.03 & 0.0045 & 0.05 & 0.4167 & 2.1045 & 3.527 & 1.517 & 8.203 \\
\hline a & 6 & do & 6 & 2.1418 & 0.6639 & 36 & 3.23 & 0.0027 & 0.05 & 0.7953 & $3.4883 !$ & 8.515 & 2.215 & 32.730 \\
\hline a & 6 & d & 12 & 15.4298 & 638.55 & 36 & 0.02 & 0.9809 & 0.05 & -1279.60 & 1310.46 & $>999.999$ & $<0.001$ & $>999.999$ \\
\hline a & 6 & d & 24 & 2.7508 & 0.7767 & 36 & 3.54 & 0.0011 & 0.05 & 1.1755 & 4.3260 & 15.654 & 3.240 & 75.640 \\
\hline a & 6 & $\theta$ & 6 & 2.6428 & 0.8339 & 36 & 3.17 & 0.0031 & 0.05 & 0.9514 & 4.3341 & 14.052 & 2,589 & 76.254 \\
\hline a & 6 & e & 12 & 2.8318 & 1.0075 & 36 & 2.81 & 0.0079 & 0.05 & 0.7885 & 4.8751 & 16.976 & 2.200 & 130.989 \\
\hline a & 6 & $\theta$ & 24 & 2.7358 & 0.6936 & $36 !$ & 3.94 & 0.0004 & 0.05 & 1.3291 & 4.1426 & 15.422 & 3.777 & 62.966 \\
\hline a & 5 & f & 6 & -3.9180 & 0.2975 & 36. & $\mid-13.17$ & $<, 0001$ & 0.05 & -4.5214 & -3.3145 & 0.020 & 0.011 & 0.036 \\
\hline a & 6 & if & 12 & -4.2544 & $0.3424 \mid$ & 36; & -12.43 & $<.0001$ & 0.05 & -4.9488 & -3.5600 & 0.014 & 0.007 & 0.028 \\
\hline a & 6 & if & 24 & -3.7773 & 0.2718 & 36 & -13.90 & $<.0001$ & 0.05 & -4.3285 & i. -3.2261 & 0.023 & 0.013 & 0.040 \\
\hline a & 12 & $a$ & 24 & 0.1586 & 0.2883 & 36 & 0.55 & $\mid 0.5857$ & 0.05 & -0.4261 & 0.7433 & 1.172 & 0.653 & 2.103 \\
\hline a & 12 & b & 6 & 3.2590 & 0.8953 & 36 & 3.64 & 0.0008 & 0.05 & 1.4433 & 5.0747 & 26.023 & 4.235 & 159.919 \\
\hline a & 12 & $b$ & 12 & 0.6658 & 0.3335 & 36 & 2.00 & $0.0535 \mid$ & 0.05 & $\mid-0.01049$ & 1.3421 & 1.946 . & $0.990 !$ & 3.827 \\
\hline a & 12 & b & 24 & 0.9196 & 0.3430 & 36 & 2.68 & 0.0110 & 0.05 & 0.2240 & 1.6152 & $2.508 !$ & 1.259 & 5.029 \\
\hline a & 12 & c & 6 & 2.4117 & $0.5752 ;$ & 36 & 4.19 & $0.0002 \mid$ & 0.05 & 1.2452 & 3.5782 & 11.155 & $3.474 i$ & 35,810 \\
\hline a & 12 & o & 12 & 2.8011 & $0.6792:$ & 36 & 4.12 & 0.0002 & 0.05 & $1.4237 !$ & 4.1785 & 16.463 & 4.152 & 65.271 \\
\hline a & 12 & $\mathrm{c}$ & 24 & 1.7651 & 0.3892 & 36 & 4.54 & $<.0001$ & 0.05 & 0.9758 & 2.5544 & 5.842 & 2.653 & 12.863 \\
\hline a & 12 & $! d$ & 6 & 2.6464 & 0.6474 & 36 & 4.09 & 0 & 0.05 & 1.3334 & 3.9593 & 14.102 & 3.794 & 52.421 \\
\hline a & 12 & d & 12 & 15.9343 & 638.55 & 36 & 0.02 & 0.9802 & 0.05 & -1279.10 & 1310.96 & $>999.999$ & $<0.001$ & $>999.999$ \\
\hline a & 12 & $d$ & 24 & 3.2553 & 0.7626 & 36 & 4.27 & 0.0001 & 0.05 & 1.7086 & 4.8020 & 25.927 & 5.521 & 121.748 \\
\hline a & 12 & $e$ & 6 & 3.1473 & 08208 & 36 & 3.83 & 0.0005 & 0.05 & 1.4825 & 4.8120 & 23.273 & 4.404 & 122.982 \\
\hline a & 12 & e & 12 & 33363 & 0.9967 & 36 & 3.35 & 0.0019 & 0.05 . & 1.3150 & 5.3577 & 28.116 & 3.725 & 212.237 \\
\hline a & 12 & $\theta$ & 24 & 3.2404 & 0.6778 & 36 & 4.78 & $<.0001$ & 0.05 & 1.8656 & 4.6151 & 25.543 & $6.460^{\circ}$ & 100.995 \\
\hline a & 12 & i & 6 & -3.4134 & 0.2586 & 36 & -13.20 & $<.0001$ & 0.05 & -3.9378 & -2.8890 & 0.033 & 0.019 & 0.056 \\
\hline a & 12 & $f$ & 12 & -3.7499 & 0.3091 & 36 & -12.13 & $<, 0001$ & 0.05 & -4.3768 & -3.1230 & 0.024 & 0.013 & 0.044 \\
\hline a & 12 & f & 24 & -3.2728 & 0.2284 & 36 & -14.33 & $<, 0001$ & 0.05 & -3.7361 & -2.8095 & 0.038 & 0.024 & 0.060 \\
\hline a & 24 & b & 6 & 3.1004 & 0.9061 & $|36|$ & 3.42 & $|0.0016|$ & 0.05 & 1.2627 & 4.9381 & 22.207 & 3.535 & 139.505 \\
\hline a & 24 & b & 12 & 0.5072 & 0.3616 & 36 & 1.40 & $|0.1692|$ & 0.05 & $-0.2261 \mid$ & 1.2405 & 1.661 & 0.798 & 3.458 \\
\hline a & 24 & $b$ & 24 & 0.7610 & 0.3704 & 36 & 2.05 & 0.0472 & $0.05^{\prime}$ & 0.009803 & 1.5122 & 2.140 & 1.010 & 4.537 \\
\hline a & 24 & 6 & 6 & 2,2531 & 0.5919 & 36 & 3.81 & $|0.0005|$ & 0.05 & 1.0527 & 3.4536 & 9.518 & 2.865 & 31.614 \\
\hline s & 24 & c & 12 & $2.6425 \mid$ & 0.6934 & 36 & 3.81 & 0.0005 & 0.05 & 1.2362 & 4.0488 & 14.049 & 3.443 & 57.331 \\
\hline a & 24 & c & 24 & 1.6065 & 0.4135 & 36 & 3.88 & 0.0004 & 0.05 & 0.7679 & | 2.4452 & 4.985 & 2.155 & 11.533 \\
\hline a & 24 & d & 6 & 2.4878 & 0.6623 & 36 & 3.76 & $\mid 0.0003$ & 0.05. & 1.1446 & 3.8310 & 12.034 & 3.141 & 46.108 \\
\hline a & 24 & d & 12 & 15.7758 & 638.55 & 36 & 0.02 & 0.9804 & 0.05 & -1279.25 & 1310.81 & $=999.999$ & $<0.001$ & $>999.999$ \\
\hline a & 24 & d & 24 & 3.0967 & 0.7753 & 38 & 3.99 & 0,0003 & 0.05 & 1.5242 & $|4.6692|$ & 22.125 & 4.592 & 106.607 \\
\hline a & 24 & e & 6 & 2.9887 & 0.8327 & 36 & 3.59 & $\{0.0010\}$ & 0.05 & 1.3000 & 4.6774 & 19.860 & 3.669 & 107.494 \\
\hline a & 24 & $e$ & 12 & 3.1777 & 1.0064 & 36 & 3.16 & $|0.0032|$ & 0.05 & 1.1365 & 5.2189 & 23.993 & 3.116 & 184.734 \\
\hline 8 & 24 & e & 24 & 3.0818 & 0.6921 & 36 & 4.45 & $<0001$ & 0.05 & 1.6781 & 4.4854 & 21.797 & 5.355 & 88.715 \\
\hline a & 24 & $f$ & 6 & -3.5720 & 0.2939 & 36 & -12.15 & $<.0001$ & 0.05 & $-4.1681 \mid$ & -2.9759 & 0.028 & 0.015 & 0.051 \\
\hline$a$ & 24 & $f$ & 12 & -3.9084 & 0.3393 & 36 & -11.52 & $<.0001$ & 0.05 & -4.5965 & $\mid-3.2204$ & 0.020 & 0.010 & 0.040 \\
\hline a & 24 & $f$ & 24 & -3.4313 & 0.2678 & 36 & $-12,81$ & $<.0001$ & 0.05 & $-3,9745$ & -2.8881 & 0.032 & 0.019 & 0.056 \\
\hline b & 6 & b & 12 & $-2.5932 \mid$ & 0.9215 & 36 & -2.81 & 0.0079 & 0.05 & -4.4620 & -0.7243 & 0.075 & 0.012 & 0.485 \\
\hline
\end{tabular}




\begin{tabular}{|c|c|c|c|c|c|c|c|c|c|c|c|c|c|c|}
\hline & 6 & $b$ & 24 & -2.3394 & 0.9250 & 36 & -2.53 & 0.0160 & 0.05 & -4.2153 & -0.4635 & 0.096 & 0.015 & 0.629 \\
\hline b & 6 & c & 6 & -0.8473 & 1.0338 & 36 & -0.82 & 0.4179 & 0.05 & $-2,9439$ & 1.2494 & 0.429 & 0.053 & 3.488 \\
\hline b & 6 & c & 12 & -0.4579 & $\therefore 0951$ & 36 & -0.42 & 0.6783 & 0.05 & -2.6788 & 1.7631 & C.633 & 0.069 & 5.830 \\
\hline b & 6 & c & 24 & -1.4939 & 0.9431 & 36 & -1.58 & 0.1219 & 0.05 & -3.4065 & 0.4188 & 0.225 & 0.033 & 1.520 \\
\hline b & 6 & $d$ & 6 & -0.6126 & 1.0757 & 36 & -0.57 & 0.5725 & 0,05 & -2.7942 & 1.5689 & 0.542 & 0.051 & 4.801 \\
\hline b & 6 & d & 12 & 12.6754 & 638.55 & 36 & 0.02 & 0.9843 & 0.05 & |-1282.36 & $1307.71 \mid$ & $>999.999$ & $<0.001$ & $>999.999$ \\
\hline b & 6 & $d$ & 24 & -0.00371 & 1.1487 & 36 & -0.00 & $\mid 0.9974$ & 0.05 & -2.3334 & 2.3260 & 0.996 & 0.097 & 10.237 \\
\hline b & 6 & e & 6 & -0.1117 & 1.1882 & 36 & -0.09 & 0.9256 & 0.05 & -2.5214 & 2.2980 & 0.894 & 0.080 & 9.954 \\
\hline b & 6 & e & 12 & 0.07734 & 1.3158 & 36 & 0.06 & 0.9535 & 0.05 & $-2,5912$ & 2.7459 & 1.080 & 0.075 & 15.579 \\
\hline b & 6 & e & 24 & -0.01863 & 1.0943 & 36 & -0.02 & 0.9865 & 0.05 & $-2,2379$ & 2.2006 & 0.982 & 0.107 & 9.031 \\
\hline b & 6 & $f$ & 6 & -8.6724 & 0.8971 & 36 & -7.44 & $<.0001$ & 0.05 & -8.4918 & \begin{tabular}{|}
-4.8530 \\
\end{tabular} & $0.001 !$ & $<0.001$ & 0.008 \\
\hline b & 6 & $f$ & 12 & $-7,0089$ & C. 9130 & 36 & -7.68 & $<.0001$ & 0.05 & -8.8604 & -5.1573 & $<0.001$ & $<0.001$ & 0.006 \\
\hline$b$ & 6 & $f$ & 24 & -6.5317 & 0.8889 & 36 & -7.35 & $<.0001$ & 0.05 & -8.3345 & $|-4.7290|$ & 0.001 & $<0.001$ & 0.009 \\
\hline b & 12 & $b$ & 24 & 0.2538 & 0.4065 & 36 & 0.62 & 0.5364 & 0.05 & -0.5707 & $\begin{array}{r}1.0783 \\
-\quad .\end{array}$ & 1.289 & 0.565 & 2.940 \\
\hline b & 12 & c & 6 & 1.7459 & 0.6152 & 36 & 2.84 & 0.0074 & 0.05 & 0.4983 & 2.9936 & 5.731 & 1.646 & 19.957 \\
\hline b & 12 & $c$ & 12 & 2.1353 & 0.7134 & 36 & 2.99 & $|0.0050|$ & 0.05 & 0.6885 & 3.5821 & 8.460 & 1.991 & 35.949 \\
\hline b & 12 & $c$ & 24 & 1.0993 & 0.4462 & 36 & 2.46 & 0.0187 & 0.05 & 0.1944 & 2.0042 & 3.002 & 1.215 & 7.420 \\
\hline b & 12 & $d$ & 6 & 1.9806 & 0.6832 & 36 & 2.90 & 0.0063 & 0.05 & 0.5950 & 3.3661 & 7.247 & $1.813 !$ & 28.965 \\
\hline b & 12 & d & 12 & 15.2685 & 638,55 & 36 & 0.02 & 0.9811 & 0.05 & -1279.76 & 1310.30 & $>999.999$ & $<0.001$ & $>999.999$ \\
\hline b & 12 & d & 24 & 2.5895 & 0.7932 & 36 & 3.26 & 0.0024 & 0.05 & 0.9807 & 4.1982 & $13.323 i$ & 2.666 & 66.569 \\
\hline b & 12. & e & 6 & 2.4815 & 0.8494 & 36 & 2.92 & 0.0080 & 0.05 & 0.7589 & 4.2041 & 11.959 & 2.136 & 66,958 \\
\hline b & 12 & e & 12 & 2.6705 & 1.0203 & 36 & 2.62 & 0.0128 & 0.05 & 0.6013 & 4.7398 & 14.448 & 1.824 & 114.411 \\
\hline b & 12 & e & 24 & 2.5746 & 0.7121 & 36 & 3.62 ! & 0.0009 & 0.05 & $\begin{array}{r}1.1303 \\
-\end{array}$ & 4.0188 & 13.125 & 3.097 & 55.632 \\
\hline b & 12 & 1 & 6 & -4.0792 & 0.3383 & 36 & -12.06 & $<.0001$ & 0.05 & -4.7654 & -3.3930 & 0.017 & 0.009 & 0.034 \\
\hline b & 12 & $: f$ & 12 & -4.4157 & 03784 & 36 & $-11,67$ & $<, 0001$ & 0.05 & -5.1831 & -3.6483 & 0.012 & 0.006 & 0.026 \\
\hline$b$ & 12 & if & 24 & -3.9386 & 0.3159 & 36 & $\begin{array}{r}-12.47 \\
-\end{array}$ & $<.0001$ & 0.05 & -4.5793 & -3.2978 & 0.019 & 0.010 & 0.037 \\
\hline b & 24 & c & 6 & 1.4921 & 0.6204 & 36 & 2.41 & 0.0214 & 0.05 & 0.2339 & 2.7504 & 4.447 & 1.264 & 15.648 \\
\hline b & 24 & c & 12 & 1.8815 & 0.7179 & 36 & 2.62 & 0.0128 & 0.05 & 0.4256 & 3.3375 & 6.563 & 1.530 & 28.147 \\
\hline b & 24 & c & 24 & 0.8455 & 0,4534 & 36 & $1.87 \mid$ & 0.0703 & 0.05 & -0.07394 & 1.7650 & 2,329 & 0928 & 5.841 \\
\hline b & 24 & d & 6 & 1.7268 & 0.6879 & 36 & 2.51 & 0.0167 & 0.05 & 0.3317 & 3.1219 & $\begin{array}{r}5.622 \\
\end{array}$ & 1.393 & 22.688 \\
\hline b & 24 & d & 12 & 15.0148 & 638.55 & 36 & 0.02 & 0.9814 & 0.05 & -1280.02 & | 1310.04 & $>999.999$ & $<0.001$ & $>999.999$ \\
\hline b & 24 & $d$ & 24. & 2.3357 & 0.7973 & 36 & 2.93 & $0.0059 \mid$ & 0.05 & 0.7187 & 3.9527 & 0.337 & 2.052 & 52.074 \\
\hline b & 24 & e & 6 & 2.2277 & 0.8532 & 36 & 2.61 & 0.0131 & $0.05 !$ & 0.4974 & 3.9580 & 9.278 & 1,644 & 52.351 \\
\hline b & 24 & $\theta$ & 12 & 2.4167 & 1.0235 & 36 & 2,36 & $0.0237:$ & 0.05 & 0.3411 & 4.4924 & 11.209 & 1.406 & 89.336 \\
\hline$b$ & 24 & e & 24 & 2.3208 & 0.7166 & 36 & 3.24 & 0.0026 & $0.05^{i}$ & 0.8674 & 3.7741 & 10.183 & 2.381 & 43.560 \\
\hline b & 24 & $f$ & 6 & -4.3330 & 0.3478 & 36 & -12.46 & $<.0001$ & 0.05 & -5.0383 & -3.6277 & 0.013 & 0.006 & 0.027 \\
\hline b & 24 & I & 12 & -4.6695 & 0.3888 & 36 & -12.07 & $<.0001$ & 0.05 & -5.4540 & -3.8849 & 0.009 & 0.004 & 0.021 \\
\hline b & 24 & $f$ & 24 & -4.1923 & 0.3260 & 36 & -12.86 & $<.0001$ & 0.05 & -4.8535 & -3.5312 & 0.015 & 0.008 & 0.028 \\
\hline c & 6 & $c$ & 12 & 0.3894 & 0.8535 & 36 & 0.46 & 0.6510 & 0.05 & -1.3417 & 2.1204 & 1.476 & 0.261 & 8.335 \\
\hline c & 6 & $\mathrm{c}$ & 24 & -0.6466 & 0.6471 & 36 & -1.00 & 0.3243 & 0.05 & -1.9589 & 0.6657 & 0.524 & 0.141 & 1.946 \\
\hline c & 6 & $d$ & 6 & 0.2346 & 0.8285 & 36 & 0.28 & $0.7786:$ & 0.05 & -1.4456 & 1.9148 & 1.264 & 0.236 & $\begin{array}{l}6.786 \\
\cdots .-\end{array}$ \\
\hline c & 6 & $d$ & 12 & 13.5226 & 638.55 & 36 & 0.02 & 0.9832 & 0.05 & -1281.51 & 1308.55 & $>999.999$ & $<0.001$ & $>999.999$ \\
\hline c & 6 & $d$ & 24 & 0.8435 & 0.9213 & 36 & 0.92 & 0.3660 & 0.05 & $-1,0250$ & 2.7121 & 2.325 & 0.359 & 15.061 \\
\hline$c$ & 6 & e & 6 & 0.7356 & 0.9701 & 36 & 0.76 & 0.4532 & 0.05 & -1.2318 & 2.7029 & 2.087 & 0.292 & 14.924 \\
\hline$c$ & 8 & $\theta$ & 12 & 0.9246 & $\begin{array}{r}1.1228 \\
-\ldots\end{array}$ & 36 & 0.82 & 0.4156 & 0.05 & -1.3525 & 3.2017 & 2.521 & 0.259 & 24.574 \\
\hline$c$ & 6 & e & 24 & 0.8286 & 0.8525 & 36 & 0.97 & 0.3375 & 0.05 & -0.9003 & 2.5575 & 2.290 & 0.406 & 12.904 \\
\hline
\end{tabular}




\begin{tabular}{|c|c|c|c|c|c|c|c|c|c|c|c|c|c|c|}
\hline & 0 & $f$ & 6 & -5.8252 & 0.5780 & 36 & -10.08 & $<.0001$ & 0.05 & -6.9974 & -4.6529 & 0.003 & $<0.001$ & 0.010 \\
\hline & 6 & $f$ & 12 & -6.1616 & 0.6023 & 36 & -10.23 & $<.0001$ & 0.05 & -7.3832 & -4.9400 & 0.002 & $<0.001$ & 0,007 \\
\hline $\mathbf{c}$ & 6 & $f$ & 24 & -5.6845 & 0.5652 & 36 & -10.06 & $<, 0001$ & 0.05 & -6.8307 & -4.5382 & 0.003 & 0.001 & 0.011 \\
\hline 6 & 12 & c & 24 & -1.0360 & 0.7411 & 36 & -1.40 & $|0.1707|$ & 0.05 & -2.5389 & 0.4669 & 0.355 & 0.079 & 1.595 \\
\hline $\mathrm{c}$ & 12 & d & 6 & -0.1548 & 0.9038 & 36 & -0.17 & 0.8650 & 0.05 & -1.9877 & 1.6782 & $0.857 !$ & 0.137 & 5.356 \\
\hline C & 12 & d & 12 & 13.1332 & 638.55 & 36 & 0.02 & 0.8837 & 0.05 & -1281.90 & 1308.16 & $>999.999$ & $<0.001$ & $>999.999$ \\
\hline c & 12 & d & 24 & $\begin{array}{r}0.4542 \\
.\end{array}$ & 0.9896 & 36 & 0.46 & 0.6490 & 0.05 & -1.5528 & 2.4612 & 1.575 & 0.212 & 11.719 \\
\hline$c$ & 12 & e & 6 & 0.3462 & 1.0351 & 36 & 0.33 & 0.7400 & 0.05 & -1.7532 & 2.4455 & $1.414 i$ & 0.173 & 11.537 \\
\hline$c$ & 12 & e & 12 & 0.5352 & 1.1794 & 36 & 0.45 & $0.6527 \mid$ & 0.05 & -1.8568 & 2.9273 & 1.708 & 0.156 & 18.676 \\
\hline c & 12 & e & 24 & 0.4302 & 0.9258 & 36 & 0.47 & 0.6381 & 0.05 & -1.4384 & 2.3169 & 1.552 & 0.237 & 10.145 \\
\hline c & 12 & $\mathbf{f}$ & 6 & -6.2145 & 0.6816 & 36 & -8.12 & $<.0001$ & 0.05 & -7.5969 & -4.8322 & 0.002 & $<0.001$ & 0.008 \\
\hline c & 12 & $f$ & 12 & -6.5510 & 0.7023 & 36 & -9.33 & $<0001$ & 0.05 & $-7.9754 !$ & -5.1266 & 0.001 & $<0.001$ & 0.006 \\
\hline c & 12 & $f$ & 24 & -6.0739 & C.6707 & 36 & -5.06 & $<.000$ : & 0.05 & -7.4342 & -4.7135 & 0.002 & $<0.001$ & 0.009 \\
\hline c & 24 & d & 6 & 0.8812 & 0.7120 & 36 & 1.24 & 0.2239 & 0.05 & -0.5628 & 2,3253 & 2.414 & C.570 & 10.230 \\
\hline c & 24 & $d$ & 12 & 14.1692 & 638.55 & 36 & 0.02 & 0.9824 & 0.05 & -1280.86 & 1309.20 & $>999.999$ & $<0.001:$ & $>999.999$ \\
\hline c & 24 & d & 24 & 1.4902 & 0.8182 & 36 & 1.82 & 0.0769 & 0.05 & -0.1693 & 3.1496 & 4.438 & 0.844 & 23,327 \\
\hline c & 24 & e & 6 & 1,3822 & 0.8727 & 36 & 1.58 & 0.1220 & 0.05 & -0.3878 & $\begin{array}{l}3.1522 \\
-\ldots \ldots\end{array}$ & 3.984 & 0.679 & 23.337 \\
\hline c & 24. & e & 12 & 1.5712 & 1.0398 & 36 & 1.51 & 0.1395 & 0.05 & -0.5377 & 3.6801 & 4.812 & 0.584 & 39,651 \\
\hline$c$ & 24 & $\theta$ & 21 & 1.4752 & 0.7398 & 36 & 1.89 & 0.0538 & 0.05 & -0.02521 & 2.8757 & 4.372 & 0.975 & 19.603 \\
\hline c & 24 & if & 6 & -5.1785 & 0.3934 & $36 !$ & -13.16 & $<.0001$ & 0.05 & -5.9763 & -4.3808 & 0.006 & 0.003 & 0.013 \\
\hline c & 24 & $f$ & 12 & -5.5150 & 0.4283 & $36 !$ & -12.88 & $<.0001$ & 0.05 & -6.3836 & -4.6464 & 0.004 & 0.002 & 0.010 \\
\hline c & 24 & $f$ & 24 & -5.0379 & 0.3743 & 36 & -13.46 & $<.0001$ & 0.05 & -5.7969 & $\begin{array}{l}-4.2788 \\
-4\end{array}$ & 0.006 & 0.003 & 0.014 \\
\hline d & 6 & d & 12 & 13.2880 & 638.55 & 36 & 0.02 & 0.9835 & 0.05 & -1281.74 & 1308.32 & $>999,999$ & $<0.001$ & $>999.899$ \\
\hline d & 6 & d & 24 & 0.6089 & 0.9681 & 36 & 0.63 & 0.5333 & 0.05 & -1.3544 & 2.5722 & 1.838 & 0.258 & 13.095 \\
\hline d & 5. & $\theta$ & 6 & 0.5009 & 1.0146 & 36 & 0.49 & 0.6245 & 0.05 & -1.5567 & 2.5585 & 1.650 & 0.211 & 12.917 \\
\hline d & 6 & e & 12 & 0.6900 & 1.1614 & 36 & 0.59 & 0.5562 & 0.05 & -1.6655 & 3.0455 & 1.994 I & 0.189 & 21.020 \\
\hline d & 6 & $\mathrm{e}$ & 24 & 0.5940 & 0.9028 ! & 36 & 0.66 & 0.5147 & 0.05 & -1.2369 & 2.4249 & 1.811 & 0.290 & 11.301 \\
\hline d & 6 & ${ }^{f}$ & 6 & -6.0598 & 0.6499 & 36 & $\begin{array}{l}-9.32 \\
-\end{array}$ & $<.0001$ & 0.05 & -7.3779 & -4.7417 & 0.002 & $<0.001$ & 0.009 \\
\hline$d$ & 6 & $f$ & 2 & -6.3962 & 0.6716 & 36 & -9.52 & $<.0001$ & 0.05 & -7.7583 & -5.0341 & 0.002 & $<0.001$ & 0.007 \\
\hline d & 6 & I & 24 & -5.9191 & 0.6385 & 36 & -9.27 & $<.0001$ & 0.05 & $-7.2141 \mid$ & -4.6241 & 0.003 & $<0.001$ & 0.010 \\
\hline d & 12 & $\mathrm{~d}$ & 24 & -12.6791 & 638.65 & 36 & -0.02 & 0.9843 & 0.05 & $-1307.71 \mid$ & 1282.35 & $<0.001$ & $<0.001$ & $>999.999$ \\
\hline d & 12 & $e$ & 6 & $-12,7871$ & 638.55 & 36 & -0.02 & 0.9841 & 0.05 & -1307.82 & 1282.24 & $<0.001$ & $<0.001$ & $>999.999$ \\
\hline d & 12 & e & 12 & $-12,5980$ & 638.55 & 36 & -0.02 & 0.9844 & 0.05 & -1307.63 & 1282.43 & $<0.001$ & $-<0.001 !$ & $>999.999$ \\
\hline$d$ & 12 & $e$ & 24 & -12.6940 & 638.55 & 36 & -0.02 & 0.9842 & 0.05 & -1307.72 & 1282.34 & $<0.001$ & $<0.001$ & $>999.999$ \\
\hline d & 12 & 1 & 6 & -19.3478 & 638.55 & 36 & -0.03 & 0.9760 & 0.05 & $-1314,38$ & 1275.68 & $<0.001 !$ & $<0.001 \mid$ & $>899.999$ \\
\hline d & 12 & $f$ & 12 & -19.6842 & 638.55 & 36 & -0.03 & 0.9756 & $0.05 !$ & -1314.71 & 1275.35 & $<0.001$ & $<0.001$ & $>999.999$ \\
\hline$d$ & 12 & $f$ & 24 & -19.2071 & 638.55 & 36 & -0.03 & 0.9762 & $0.05 i$ & -1314.24 & 1275.82 & $<0.001$ & $<0.001$ & $>999.999$ \\
\hline d & 24 & e & 6 & -0.1080 & 1.0917 & 36 & -0.10 & 0.9217 & 0.05 & -23221 & 2.1061 & 0.898 & 0.098 & 8.216 \\
\hline d & 24 & e & 12 & 0.08105 & 1.2294 & 38 & 0.07 & 0.9478 & 0.05 ! & -2.4123 & 2.5744 & 1.084 & 0.090 & 13.123 \\
\hline$d$ & 24 & $e$ & 24 & -0.01493 & 0.9887 & 36 & -0.02 & 0.9880 & 0.05 & -2.0201 & 1.9902 & 0.985 & $0.133:$ & 7.317 \\
\hline d & 24 & 1 & 6 & -6.6687 & 0.7648 & 36 & -8.72 & $<, 0001$ & 0.05 & -8.2197 & -5.1177 & 0.001 & $<0.001$ & 0.006 \\
\hline d & 24 & 1 & 12 & -7.0051 & 0.7833 & 36 & -8.94 & $<.0001$ & 0.05 & -8.6938 & -5.4165 & $<0.001:$ & $<0.001$ & 0.004 \\
\hline d & 24 & $f$ & 24 & -6.5280 & 0.7551 & 36 & -8.64 & $<.0001$ & 0.05 & -8.0595 & -4.9066 & 0.001 & $<0.001$ & 0.007 \\
\hline$\theta$ & 6 & e & 12 & 0.1890 & 1.2663 & 36 & 0.15 & 0.8822 & 0.05 & $-2.3792 i$ & 2.7573 & 1.208 & 0.093 & 15.757 \\
\hline e & 6 & $\theta$ & 24 & 0.09307 & 1.0343 & 36 & 0.09 & 0.9288 & 0.05 & $-2 . \operatorname{CC} 45$ & 2.1906 & 1.098 & 0135 & 8.841 \\
\hline e & 6 & $f$ & $\theta$ & -6.5607 & 0.8228 & 36 & -7.97 & $<.0001$ & 0.05 & $-8.2295 i$ & -4.8919 & 0.001 & $<0.001$ & 0.008 \\
\hline
\end{tabular}




\begin{tabular}{|c|c|c|c|c|c|c|c|c|c|c|c|c|c|c|}
\hline & 6 & f & 12 & -6.8972 & 0.8401 & 36 & -8.21 & $<, 0001$ & 0.05 & -8.6010 & -5.1933 & 0.001 & $<0.001$ & 0.006 \\
\hline$\theta$ & 6 & 1 & 24 & -6.4200 & 0.8139 & 36 & -7.89 & $<, 0001$ & 0.05 & -8.0707 & -4.7694 & 0.002 & $<0.001$ & 0.008 \\
\hline$\theta$ & 12 & e & 24 & -0.09598 & 1.1787 & 36 & -0.08 & 0,9356 & 0.05 & -2.4864 & 2,2945 & 0.908 & 0.083 & 9.919 \\
\hline$\theta$ & 12 & $f$ & 6 & -6.7498 & 0.9983 & 36 & -6.76 & $<.0001$ & 0.05 & -8.7745 & -4.7250 & 0.001 & $<0.001$ & 0.008 \\
\hline e & 12 & 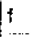 & 12 & -7.0862 & 1,0126 & 36 & -7.00 & $<.0001$ & 0.05 & -9.1399 & -5.0325 & $<0.001$ & $<0.00 \dagger$ & 0.007 \\
\hline e & 2 & $f$ & 24 & -6.6091 & 0.9910 & 36 & -6.67 & $<.0001$ & 0.05 & -8.6188 ! & -4.5993 & 0.001 & $<0.001$ & 0.010 \\
\hline e & 24 & $f$ & 6 & -6.6538 & 0.6803 & 36 & -9.78 & $<.0001$ & 0.05 & -8.0334 & -5.2742 & 0.001 & $<0.001$ & 0.005 \\
\hline e & 24 & $f$ & 12 & -6.9802 & 0.7010 & 38 & -9.97 & $<.0001$ & 0.05 & -8.4120 & -5.5685 & $<0.001$ & $<0.001$ & 0.004 \\
\hline e & 24 & f & 24 & -6.5131 & 0.6694 & 36 & -9.73 & $<.0001$ & 0.05 & -7.8707 & -5.1555 & 0.001 & $<0.001$ & $0.0 C 6$ \\
\hline$f$ & 6 & $f$ & 12 & -0.3364 & 0.3144 & 36 & $\therefore .07$ & 0.2917 & 0.05 & -0.9740 & 0.3011 & 0.714 & 0.378 & 1.351 \\
\hline it. & 6 & $\mathrm{f}$ & 24 & 0.1407 & 0.2355 & 36 & 0.60 & 0.5541 & 0.05 & -0.3370 & 0.6183 & 1.151 & 0.714 & 1.856 \\
\hline$f$ & 12 & $f$ & 24 & 0.4771 & 0.2901 & 36 & 1.64 & 0.1088 & 0.05 & -0.1113 & $1.0655 f$ & 1.611 & 0.895 & 2.902 \\
\hline
\end{tabular}

Performed by J. Luoma; SAS version 9.3 08:43 18MAR15 


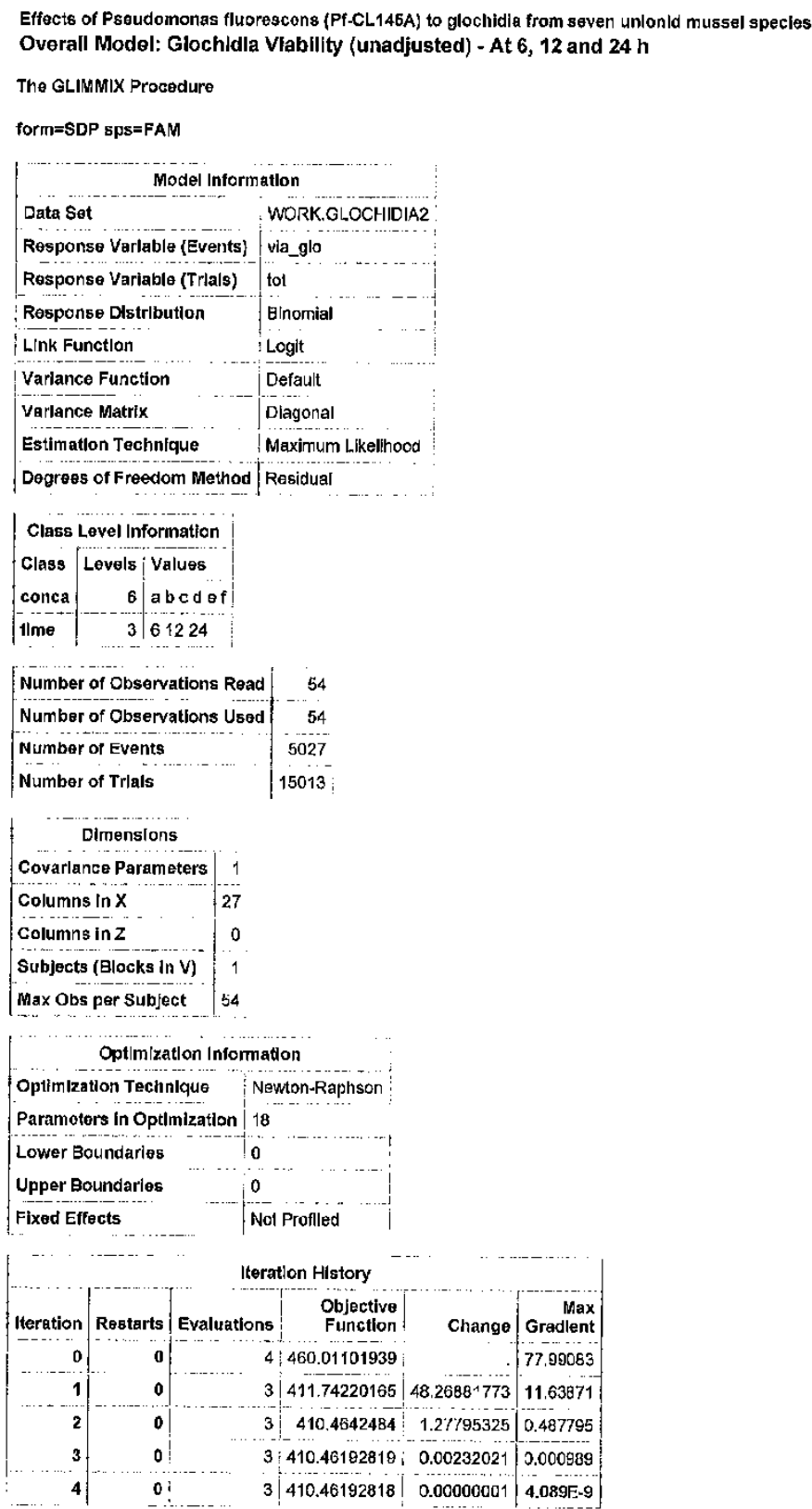

file:///C:/Users/J „UOMA/AppData/Local/Temp/1/SAS\%20Temporary\%20Files/_TD4836... 3/18/2015 


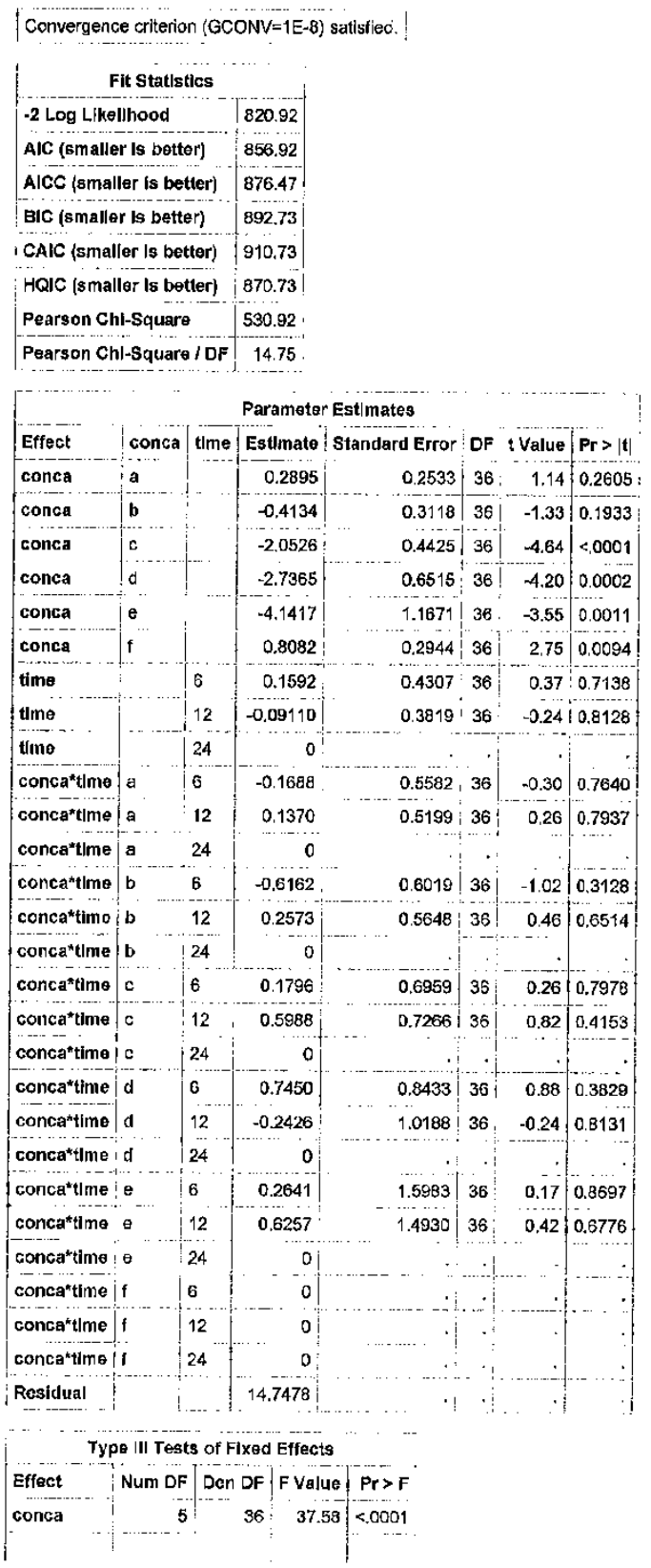

file://C:/Users/JUOMA/AppData/Local/Temp/1/SAS\%20Temporary\%20Files/_TD4836... 3/18/2015 


\begin{tabular}{|c|c|c|c|c|c|c|c|}
\hline \multirow{2}{*}{\multicolumn{2}{|c|}{$\begin{array}{l}\text { ' time } \\
\text { j conca*time }\end{array}$}} & 2 & & 0.25 & \multicolumn{3}{|c|}{0.7796} \\
\hline & & 10 & & 0.7 & \multicolumn{3}{|c|}{0.6883} \\
\hline \multicolumn{8}{|c|}{ Odds Ratlo Estimates } \\
\hline conca & time & _conca & time & Estimate & DF & $95 \%$ Confld & LImlts \\
\hline a & & $f$ & & 0.589 & 36 & 0.378 & 0.918 \\
\hline b & & if & & 0.262 & 36 & 0.162 & 0.421 \\
\hline c & & $f$ & & 0.074 & 36 & 0.042 & 0.132 \\
\hline$d$ & & $f$ & & 0.034 & 36 & 0.016 & 0.072 \\
\hline$\theta$ & & $f$ & & 0.010 & 36 & 0.003 & 0.033 \\
\hline & 6 & & 24 & 1.254 & 36 & 0.656 & 2.399 \\
\hline & 12 & & 24 & 1.148 & 36 & 0.594 & 2.220 \\
\hline
\end{tabular}

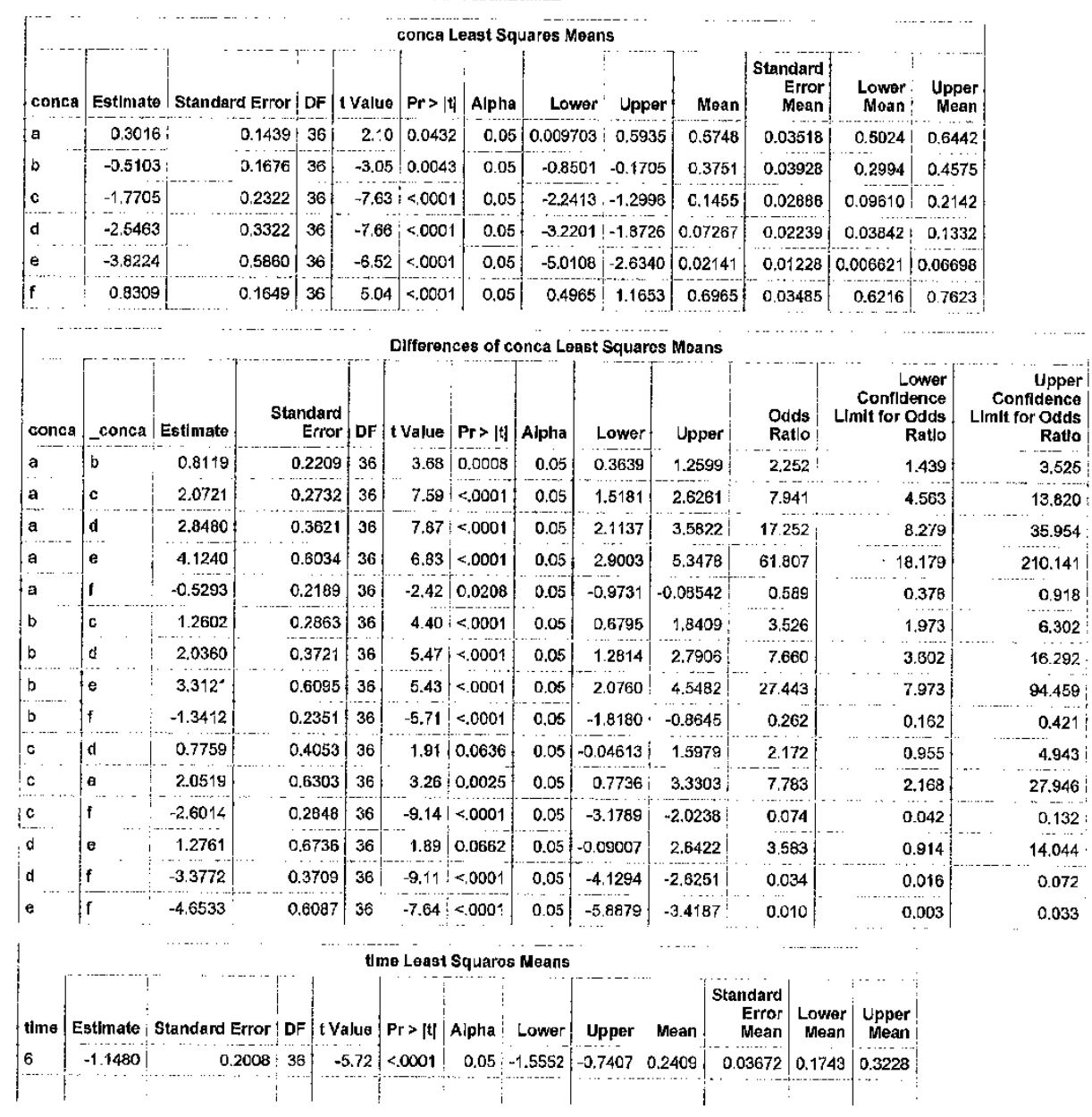

file://C:/Users/JLUOMA/AppData/Local/Temp/1/SAS\%20Temporary\%20Files/_TD4836... 3/18/2015 


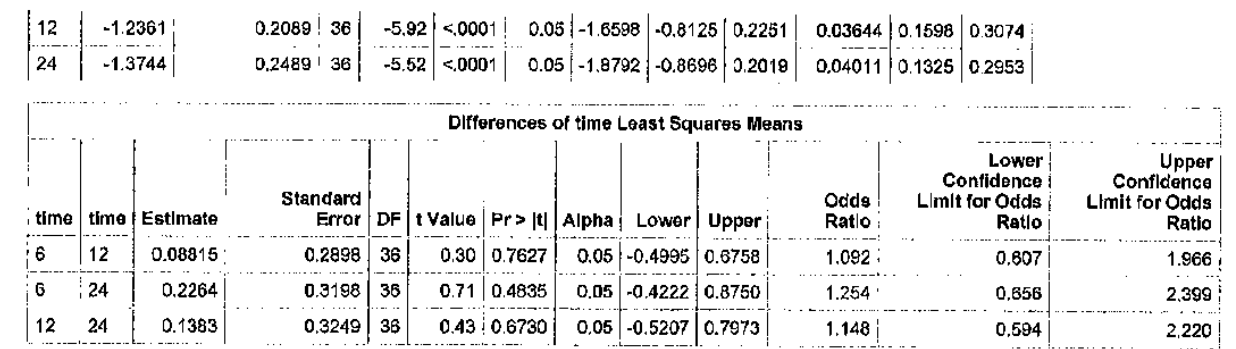

\begin{tabular}{|c|c|c|c|c|c|c|c|c|c|c|c|c|c|}
\hline conca & time & Estimate & Standard Error & $\mathrm{DF}$ & t Value & $|\operatorname{Pr}>| t \mid$ & Alpha & Lower & Upper & Moan & $\begin{array}{r}\text { Standard } \\
\text { Error } \\
\text { Mean }\end{array}$ & $\begin{array}{c}\text { Lower } \\
\text { Mean }\end{array}$ & $\begin{array}{l}\text { Upper } \\
\text { Mean }\end{array}$ \\
\hline a & 6 & 0.2799 & 0.2489 & 36 & 1.12 & 0.2683 & 0.05 & $|-0.2249|$ & 0.7847 & 0.5695 & 0.06102 & 0.4440 & 0.6867 \\
\hline a & 12 & 0.3364 & 0.2457 & 36 & 1.37 & |c.1806 & 0.05 & $\mid-0.1628$ & 0.8337 & 0,5831 & 0.05973 & 0.4594 & 0.6971 \\
\hline a & 24 & 0.2895 & 0.2533 & 36 & 1.14 & 0.2605 & 0.05 & -0.2241 & 0.8032 & 0.5719 & 0.06201 & 0.4442 & 0.6907 \\
\hline b & 6 & -0.8704 & 0.2821 & 36 & -3.09 & 0.0039 & 0.05 & -1.4424 & -0.2983 & 0.2952 & 0.05868 & 0.1912 & 0.4260 \\
\hline D & 12 & -0.2471 & 0.2755 & 36 & -0.90 & 0.3757 & 0.05 & -0.8059 & : 0.3117 & 0.4385 & 0.06784 & 0.3080 & 0.5773 \\
\hline b & 24 & -0.4134 & 0.3118 & 36 & -1.33 & 0.1933 & 0.05 & $|-1.0458|$ & 0.2190 & 0.3981 & 0.07472 & 0.2600 & 0.5545 \\
\hline c & 6 & -1.7139 & 0.3209 & 36 & -5.34 & $<.0001$ & 0.05 & $|-2,3647|$ & $\mid-1.0630$ & 0.1527 & 0.04151 & 0.08590 & 0.2567 \\
\hline c & 12 & -1.5449 & 0.4317 & 36 & -3.58 & 0.0010 & 0.05 & - -2.4205 & -0.6693 & 0.1758 & 0.06256 & 0.08162 & 0.3387 \\
\hline c & 24 & $-2,0526$ & 0.4425 & 36 & -4.64 & $<.0001$ & 0.05 & $\mid-2,9500$ & $|-1.1552|$ & 0.1138 & 0.04462 & 0.04974 & 0.2395 \\
\hline d & 6 & $-1,8323$ & 0.3182 & 36 & -5.76 & $<, 0001$ & 0.05 & -2.4776 & -1.1871 & 0.1380 & & 0.07744 & 02338 \\
\hline d & 12 & -3.0702 & 0.6838 & 36 & -4.49 & $<.000^{\circ}$ & 0.05 & -4.4571 & -1.6833 & 0.04435 & 0.02899 & 0.01146 & 0.1567 \\
\hline d & 24 & -2.7365 & 0.6515 & 36 & -4.20 & 0.0002 & 0.05 & -4.0577 & -1.4152 & 0.06086 & 0.03723 & 0.01699 & $\begin{array}{l}0.1954 \\
\end{array}$ \\
\hline$e$ & 6 & -3.7184 & 1.0035 & 36 & -3.71 & 0.0007 & 0.05 & -5.7537 & -1.6832 & 0.02370 & 0.02322 & 0.003161 & 0.1567 \\
\hline$e$ & 12 & -3.6070 & 0.8493 & 36 & -4.25 & 0.0001 & 0.05 & -5.3295 & -1.8846 & $|0.02642|$ & 0.02184 & 0.004823 & 0.1319 \\
\hline e & 24 & -4.1417 & 1.1671 & 36 & -3.55 & 0.0011 & 0.05 & -6.5086 & -1.7748 & $0.01565 \mid$ & 0.01798 & 0.001488 & 0.1449 \\
\hline & 6 & 0.9674 & 0.3143 & 36 & 3.08 & 0.0040 . & 0.05 & 0.3299 & 1.6049 & 0.7246 & 0.06273 & 0.5817 & 0.8327 \\
\hline & 12 & 0.7171 & 0.2432 & 36 & 295 & 0.0056 & 0.05 & 0.2233 & 1.2104 & 0.6720 & 0.05361 & 0.5557 & 0.7704 \\
\hline & 24 & 0.8082 & 0.2944 & 36 & 2.75 & 0.0094 & 0.05 & 0.2112 & 1.4053 & 0.6917 & 0.06278 & 0.5526 & 0.8030 \\
\hline
\end{tabular}

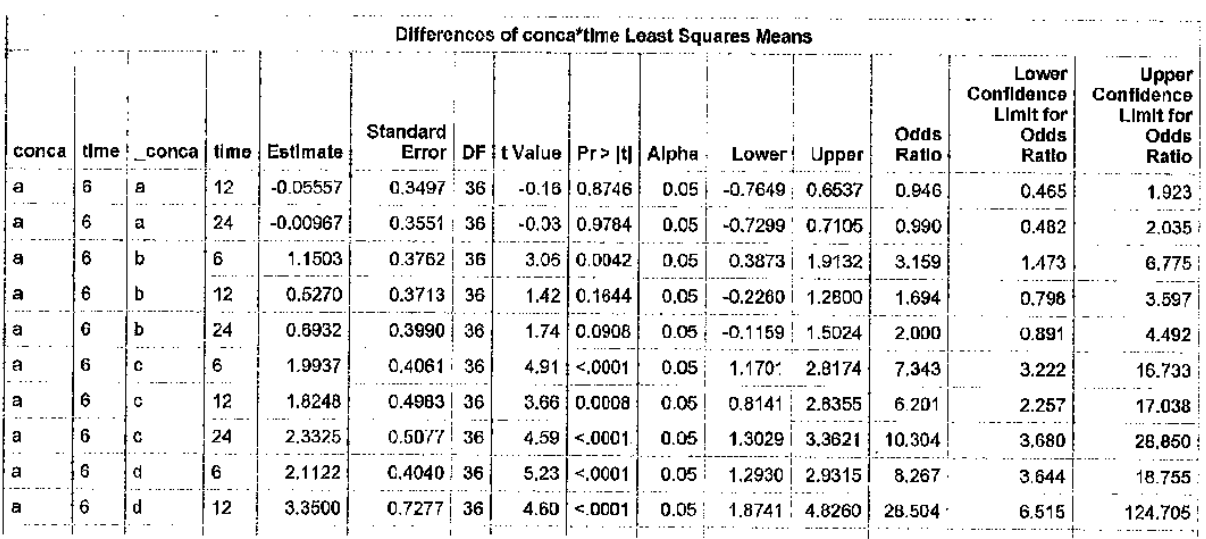

file://C:/Users/JL LUOMA/AppData/Local/Temp/1/SAS\%20Temporary\%20Files/ TD4836_.. 3/18/2015 


\begin{tabular}{|c|c|c|c|c|c|c|c|c|c|c|c|c|c|c|}
\hline a & 6 & d & 24 & 3.0163 & 0.6974 & 36 & 4.33 & 0.0001 & 0.05 & $1.6 \mathrm{C} 20$ & 4.4307 & 20.417 & 4.963 & 83.994 \\
\hline a & 6 & e & 6 & 3.9983 & 1.0339 & 36 & 3.87 & 0.0004 & 0.05 & 1.9014 & 6.0952 & 54,506 & 6.695 & 443.729 \\
\hline a & 6 & $e$ & 12 & 3.8869 & 0.8850 & 36 & 4.39 & $<.0001$ & 0.05 & 2.0920 & 5.6819 & 48.761 & 8.101 & 293.493 \\
\hline a & 6 & $\theta$ & 24 & 4.4216 & $1.1933:$ & 36 & 3.71 & 0.0007 & 0.05 & 2.0014 & 6.8417 & 83.227 & 7.400 & 936.079 \\
\hline a & 6 & $f$ & 6 & -0.6875 & 0.4009 & 36 & -1.71 & $|0.0950|$ & 0.05 & -1.5007 & 0.1256 & 0.503 & 0.223 & 1.134 \\
\hline a & 6 & $f$ & 12 & -0.4372 & 0.3480 & 36 & -1.26 & 0.2171 & 0.05 & -1.1430 & 0.2685 & 0.646 & 0.319 & 1.308 \\
\hline a & 6 & f & 24 & -0.5283 & 0.3855 & 36 & -1.37 & 0.1780 & 0.05 & -1.3102 & 0.2535 & 0.590 & 0.270 & 1.289 \\
\hline a & 12 & a & 24 & 0.04590 & 0.3529 & 36 & 0.13 & 0,8972 & 0.05 & -0.6697 & 0.7615 & 1.047 & 0.512 & 2.142 \\
\hline a & 12 & $b$ & 6 & 1.2058 & 0.3741 & 36 & 3.22 & 0.0027 & 0.05 & 0.4472 & 1.9045 & 3.340 & 1.564 & 7.131 \\
\hline$a$ & 12 & b & 12 & 0.5826 & 0.3692 & 36 & 1.58 & | 0.1233 & 0.05 & -0.1661 & 1.3313 & 1.791 & 0.847 & 3.786 \\
\hline a & 12 & $b$ & 24 & 0.7488 & 0.3970 & 36 & 1.89 & 0.0673 & 0.05 & $|-0.05630|$ & 1.5539 & 2.115 & 0.945 & 4.730 \\
\hline a & 12 & ic & 6 & 2.0493 & 0,4042 & 36 & 5.07 & $<.0001$ & 0.05 & 1.2295 & 2.8690 & 7.782 & 3.420 & 17.619 \\
\hline a & 12 & c & 12 & 1.8803 & 0.4968 & 36 & 3.79 & 0.0006 & 0.05 & 0.8729 & 2,8878 & 6.556 & 2.394 & 17.954 \\
\hline a & 12 & c & 24 & 2.3881 & 0.5061 ! & 36 & 4.72 & $<.0001$ & 0.05 & 1.3616 & 3.4145 & 10.892 & 3.903 & 30.402 \\
\hline a & 12 & $d$ & 6 & 2.1678 & 0.4020 & 36 & 5.39 & $<, 0001$ & 0.05 & 1.3525 & 2.9831 & 8.739 & 3.867 & 19.748 \\
\hline a & 12 & d & 12 & 3.4056 & 0.7266 & 36 & 4,69 & $<.0001$ & 0.05 & 1.9319 & 4.8793 & 30.133 & 6.933 & 131.541 \\
\hline a & 12 & d & 24 & 3.0719 & 0.6963 & 36 & 4.41 & $<.0001$ & 0.05 & 1.6598 & 4.4840 & 21.583 & 5.258 & 88.589 \\
\hline a & 12 & $\theta$ & 6 & 4.0539 & 1.0332 & 36 & 3.92 & 0.0004 & 0.05 & 1.9585 & 6.1492 & 57.621 & 7.089 & 468.357 \\
\hline a & 12 & $e$ & 12 & 3.9425 & 0.8841 & 38 & 4.46 & $<.0001$ & 0.05 & 2,1494 & 5.7356 & 51.547 & 8.580 & 309.701 \\
\hline a & 12 & e & 24 & 4.4771 & 1.1926 & 36 & 3.75 & 0.0006 & 0.05 & 2,0584 & 6.8959 & 87.983 & 7.833 & 988.238 \\
\hline a & 12 & $f$ & 6 & -0.6319 & 0.3990 & 36 & -1.58 & 0.1219 & 0.05 & -1.4411 & 0.1772 & 0.532 & 0.237 & 1.194 \\
\hline a & 12 & 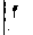 & 12 & -0.3817 & 0.3457 & 36 & -1.10 & 0.2769 & 0,05 & -1.0828 & 0.3195 & C.683 & 0.339 & 1.376 \\
\hline a & 12 & $f$ & 24 & -0.4728 & 0.3834 & 36 & -1.23 & 0.2256 & 0.05 & -1.2504 & 0.3049 & 0.623 & 0.2 .86 & 1.356 \\
\hline a & 24 & b & 6 & 1.1599 & 0.3791 & 36 & 3.06 & 0.0042 & 0.05 & 0.3911 & 1.9288 & 3.190 & 1.479 & 6.881 \\
\hline a & 24 & b & 12 & 0.5367 & 0.3743 & 36 & 1.43 & 0.1602 & 0.05 & -0.2223 & 1.2957 & 1.710 & 0.801 & 3.654 \\
\hline a & 24 & b & 24 & 0.7029 & 0.4017 & 36 & 1.75 & 0.0887 & 0.05 & -0.1118 & 1.5176 & 2.020 & 0.894 & 4.561 \\
\hline a & 24 & c & 6 & 2,0034 & 0.4088 & 36 & 4.90 & $<.0001$ & 0.05 & 1.1743 & 2.8325 & 7.414 & 3.236 & 16.988 \\
\hline a & 24 & c & 12 & 1.8344 & 0,5005 & 36 & 3.66 & 0.0008 & 0.05 & 0.8193 & 2.8496 & 6.262 & 2.269 & 17.281 \\
\hline $\mathbf{a}$ & 24 & c & 24 & 2.3422 & 0.5098 & 36 & 4.59 & $<.0001$ & 0.05 & 1.3082 & 3.3762 & 10.404 & 3.699 & 29.258 \\
\hline a & 24 & d & 6 & 2.1219 & 0.4067 & 36 & 5.22 & $<, 0001$ & 0.05 & $1.297^{\circ}$ & 2.9467 & 8.347 & 3.659 & 19.042 \\
\hline a & 24 & d & 12 & 3.3597 & 0.7292 & 36 & $\angle .61$ & $<<0001$ & 0.05 & 1.8807 & $4.838 \%$ & 28.781 & 6.558 & 126.303 \\
\hline a & 24 & d & 24 & 3.0260 & 0.6990 & 36 & 4.33 & 0.0001 & 0.05 & 1.6084 & 4.4436 & $20.6 \div 5$ & 4.995 & 85.061 \\
\hline a & 24 & $\theta$ & 6 & 4.0080 & 1.0350 & 36 & 3.87 & 0.0004 & 0.05 & 1.9089 & 6.1070 & 55.036 & 6.746 & 449.007 \\
\hline a & 24 & e & 12 & 3.8966 & 0.8863 & 36 & 4.40 & $<.0001$ & 0.05 & 2.0991 & 5.6940 & 49.235 & 8.159 & 297.092 \\
\hline$a$ & 24 & e & 24 & 4.4312 & 1,1942 & 36 & 3.71 & 0.0007 & 0.05 & 2.0092 & 6.8532 & 84.035 & 7.458 & 946.941 \\
\hline a & 24 & ff & 6 & -0.6778 & C.4037 & 36 & -1.68 & 0.1018 & 0.05 & $-1 .<965$ & 0.1408 & 0.508 & 0.224 & 1.151 \\
\hline a & 24 & $f$ & 12 & -0.4276 & $0.3511:$ & 36 & -1.22 & 0.2313 & 0.05 & -1.1397 & 0.2846 & 0.652 & 0.320 & 1.329 \\
\hline a & 24 & $f$ & 24 & -0.5187 & 0.3884 & 36 & -1.34 & 0.1901 & 0.05 & -1.3063 & 0.2689 & 0.595 & 0.271 & 1.309 \\
\hline b & 6 & b & 12 & -0.6233 & 0.3943 & 36 & -1.58 & 0.1227 & 0.05 & -1.4229 & 0.1764 & 0.536 & 0.241 & 1.193 \\
\hline & 6 & b & 24 & -0.4570 & $0.4205 !$ & 36 & -1.09 & 0.2843 & 0.05 & $-1,3098$ & 0.3957 & 0.633 & 0.270 & 1.485 \\
\hline & 6 & c & $B$ & 0.8435 & $0.4273:$ & 36 & 1.97 & 0.0561 & 0.05 & $-0,02305 \mid$ & 1.7100 & 2.324 & 0.977 & 5.529 \\
\hline 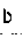 & 6 & c & 12 & 0.6745 & $0.5157 !$ & 36 & 1.31 & 0.1992 & 0.05 & -0.3714 & 1.7204 & 1.963 & 0.690 & 5.587 \\
\hline 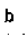 & 6 & c & 24 & 1.1822 & 0.5247 & 36 & 2.25 & 0.0304 & 0.05 & 0.1180 & 2.2464 & 3.262 & 1.125 & 9.464 \\
\hline & 6 & d & 6 & 0.9620 & 0.4252 & 36 & 2,26 & 0.0208 & 0.05 & $0.09962 !$ & 1.8243 & 2.617 & 1.105 & 6.198 \\
\hline 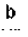 & 6 & d & 12 & 2.1998 & 0.7397 & 36 & 2.97 & 0.0052 & 0.05 & 0.6995 & 3.7000 & 9.023 & 2,013 & 40,448 \\
\hline & 6 & d & 24 & 1.8661 & 0.7099 & 36 & 2.63 & 0.0125 & 0.05 & 0.4263 & 3.3059 & 6.463 & 1.532 & 27.272 \\
\hline
\end{tabular}




\begin{tabular}{|c|c|c|c|c|c|c|c|c|c|c|c|c|c|c|}
\hline & 6 & e & 6 & 2.8481 & 1.0424 & 36 & 2.73 & $|0.0097|$ & 0.05 & 0.7340 & i 4.8622 & 17.254 & 2.083 & 142.901 \\
\hline b & 6 & e & 12 & 2.7367 & 0,8949 & 36 & 3.06 & 0.0042 & 0,05 & 0.92 .7 & 4.5517 & 15.435 & 2.513 & 94.789 \\
\hline b & 6 & e & 24 & 3.2713 & 1.2007 & 36 & 2.72 & 0.0099 & 0.05 & 0.8363 , & , 5.7064 & 26.346 . & 2.308 & 300.773 \\
\hline b & 6 & 1 & 6 & -1.8378 & 0.4223 & 36 & -4.35 & 0.0001 & 0.05 & $-2,6943$ & -0.9813 & 0.159 & 0.068 & 0.375 \\
\hline b & 6 & $f$ & 12 & $-1,5875$ & 0.3724 & 36 & -4.26 & 0.0001 & 0.05 & -2.3429 & $-0.8321 \mid$ & 0.204 & 0.096 & 0.435 \\
\hline b & 6 & f & 24 & -1.6786 & 0.4077 & 36 & -4.12 & 0.0002 & 0.05 & $-2,5055$ & $|-0.8517|$ & 0.187 & 0.082 & 0.427 \\
\hline$b$ & 12 & b & 24 & 0.1662 & 0.4161 & 36 & 0.40 & 0.6919 & 0.05 & -0.6777 & 1.0101 & 1.181 & 0508 & 2.746 \\
\hline b & 12 & c & 6 & 1.4667 & C.4230 & 36 & 3.47 & 0.0014 & 0.05 & 0.6089 & 2.3245 & 4.335 & 1.838 & 10.222 \\
\hline b & 12 & c & 12 & 1.2978 & 0.5122 & 36 & 2.53 & 0.0158 & 0.05 & 0.2591 & 2.3365 & 3.661 & 1.296 & 10.345 \\
\hline b & 12 & c & 24 & 1.8055 & 0.5212 & 36 & 3.46 & 0.0014 & 0.05 & 0.7483 & 2.8626 & 6.083 & 2.114 & 17.507 \\
\hline b & 12 & d & 6 & 1.5852 & 0.4209 & 36 & 3.77 & 0.0006 & 0.05 & 0.7316 & | 2.4388 & 4.880 & 2.078 & 11.459 \\
\hline b & 12 & d & 12 & 2.8230 & 0.7373 ! & 36 & 3.83 & 0.0005 & 0.05 & 1.3278 & 4.3183 & 16.828 & 3.773 & 75.069 \\
\hline b & 12 & d & 24 & 2.4893 & 0.7073 & 36 & 3.52 & 0.0012 & 0.05 & 1.0548 & 3.9239 & 12.053 & 2.871 & 50.597 \\
\hline b & 12 & $\theta$ & 6 & 3.4713 & 1.0407 & 36 & 3.34 & 0.0020 & 0.05 & 1.3608 & 5.5819 & 32.179 & 3.899 & 265.564 \\
\hline b & 12 & $\theta$ & 12 & 3.3599 & 0.8929 & 36 & 3.76 & 0.0006 & 0.05 & 1.5491 & 5.1708 & 28.787 & 4.707 & 176.051 \\
\hline b & 12 & e & 24 & 3.8946 & 1.1991 । & 36 & 3.25 & 0.0025 & 0.05 & 1.4626 & 6.3265 & 49.134 & 4.317 & 559.212 \\
\hline b & 12 & $f$ & 6 & -1.2145 & 0.4180 & 36 & $-2.91 !$ & 0.0662 & 0.05 & -2.0622 & -0.3668 & 0.297 & 0.127 & 0.693 \\
\hline b. & 12 & $f$ & 12 & -0.9642 & 0.3675 & 36 & $-2,62$ & 0.0127 & 0.05 & -1.7096 & $|-0,2189|$ & 0.381 & 0.181 & 0.803 \\
\hline b & 12 & $f$ & 24 & -1.0553 & 0.4032 & 36 & -2.62 & 0.0129 & 0.05 & -8731 & -0.2376 & 0.348 & 0.154 & 0.789 \\
\hline $\mathrm{b}$ & 24 & c & 6 & 1.3005 & 0.4475 & 36 & 2.91 & 0,0062 & 0.05 & 0.3930 & 2.2080 & 3.671 & 1.481 & 9.097 \\
\hline b & 24 & c & 12 & 1.1315 & 0.5326 & 36 & 2.12 & 0.0405 & $0.05 !$ & 0.05143 & | 2.2116 & 3.100 & $\{.053$ & 9.131 \\
\hline b & 24 & $c$ & 24 & 1.6392 & 0.5413 & 36 & 3.03 & 0.0045 & 0.05 & 0.5414 & 2.7371 & 5.151 & 1.718 & 15.442 \\
\hline b & 24 & d & 6 & 1.4190 & 0.4455 & 36 & 3.10 & 0.0030 & 0.05 & 0.5155 & 2.3225 & 4.133 & 1.574 & 10.201 \\
\hline b & 24 & $d$ & 12 & 2.6568 & 0.7516 & 36 & 3.53 & 0.0011 & 0.05 & 1.1325 & 4.1811 & 14.251 & 3.103 & 65.433 \\
\hline b & 24 & d & 24 & 2.3231 & 0.7223 & 36 & 3.22 & 0.0027 & 0.05 & 0.8583 & 3.7879 & 10.207 & 2.359 & 44.163 \\
\hline b & 21 & e & 6 & 3.3051 & 1.0508 & 36 & 3.15 & 0.0033 & 0.05 & 1.1738 & 5.4363 & 27.250 & 3.234 & 229.588 \\
\hline b & 24 & $e$ & 12 & 3.1937 & 0.9047 & 36 & 3.53 & 0.0012 & 0.05 , & 1,3588 & 5.0286 & 24.378 & 3.891 & 152.717 \\
\hline b & 24 & e & 24 & 3.7283 & 1.2080 & 36 & 3.09 . & 0.0039 & 0.05 & 1.2784 & 6.1783 & 41.609 & 3.591 & 482.148 \\
\hline b & 24 & $f$ & 6 & -1.3808 & 0.4428 & 36 & -3.12 & 0.0036 & 0.05 & -2.2787 & -0.4828 & 0.251 & 0.102 & 0.617 \\
\hline b & 24 & ! & 12 & -1.1305 & 0.3955 & 36 & -2.86 & 0.0070 & $0.05 i$ & -1.9325 & $-0.3285 \mid$ & 0.323 & 0.145 & 0.720 \\
\hline b & 24 & 1 & 24 & $-1,2216$ & 0.4288 & 36 & -2.85 & 0.0072 & 0.05 & -2.0913 & $|-0.3519|$ & 0.295 & C.124 & 0.703 \\
\hline c & 6 & c & 12 & -0.1690 & 0.5379 & 36 & -0.31 & 0.7553 & 0.05 & -1.2600 & 0.9220 & 0.845 & 0.284 & 2.514 \\
\hline c & 6 & c & 24 & 0.3388 & 0.5466 & 36 & 0.62 & 0.5393 & 0.05 & -0.7698 & 1.4473 & 1.403 & 0.463 & 4.252 \\
\hline c & 6 & d & 6 & 0.1185 & 0.4519 & 36 & 0.26 & 0.7947 & 0.05 & $-0.7980 i$ & 1.0350 & 1.126 & 0.450 & 2.815 \\
\hline c & 6 & d & 12 & 1.3563 & 0.7554 & 36 & 1.83 & 0.0810 & 0.05 & -0.1757 & 2.8883 & 3.882 & 0.839 & 17.964 \\
\hline c & 6 & $d$ & 24 & 1.0228 & 0.7262 & 36 & 1.41 & 0.1677 & 0.05 & -0.4502 & 2.4955 & 2.780 & 0.637 & 12.128 \\
\hline$c$ & 6 & e & 6 & 2.0046 & 1.0536 & 36 & 1.90 & 0.0651 & 0.05 & $-0 . \div 322$ & 4.1414 & 7.423 & 0.876 & 62.888 \\
\hline$c$ & 6 & e & 12 & 1.8932 & 0.8079 & 36 & 2.09 & 0.0442 & 0.05 & 0.05185 & 3.7345 & 6.641 & 1.053 & 41.869 \\
\hline$c$ & 6 & $\mathrm{e}$ & 24 & 2.4278 & 1,2104 & 36 & 2.01 & 0.0524 & 0.05 & -0.02692 & 4.8826 & 11.334 & 0.973 & 131.973 \\
\hline c & 6 & $f$ & 6 & -2.6812 & 0.4492 & 36 & -5.97 & $<.0001$ & 0.05 & -3.5923 & -1.7702 & 0.068 & 0.028 & 0.170 \\
\hline$c$ & 6 & 1 & 12 & -2.4310 & 0.4027 & 36 & -6.04 & $<.0001$ & 0.05 & -3.2476 & $-1,6143$ & 0.088 & 0.039 & 0.199 \\
\hline$c$ & 6 & $f$ & 24 & -2.5221 & 0.4355 & 36 & -5.79 & $<.0001$ & 0.05 & $-3,4053$ & -1.6388 & 0.080 & 0.033 & 0.194 \\
\hline o & 12 & c & 24 & 0.5077 & 0.6182 & 36 & C.82 & 0.4169 & 0.05 & -0.7461 & 1.7615 & $1.661 \mid$ & 0.474 & 5.821 \\
\hline$c$ & 12 & d & 6 & 0.2874 & 0.5363 & 36 & 0.54 & 0.5953 & 0.05 & -0.8002 & 1.3751 & 1.333 & 0.449 & $3.956 !$ \\
\hline c & 12 & d & 12 & 1,5253 & $0.8087 !$ & 36 & 1.89 & 0.0674 & 0.05 & -0.1148 & 3.1654 & 4.596 & 0.891 & 23.699 \\
\hline C & 12 & $d$ & 24 & 1.1916 & 0.7815 & 36 & 1.52 & $0.1361 \mid$ & 0.05 & -0.3935 & 2.7766 & 3.292 & 0.675 & 16.065 \\
\hline
\end{tabular}




\begin{tabular}{|c|c|c|c|c|c|c|c|c|c|c|c|c|c|c|}
\hline & 12 & e & 6 & 2.1735 & 1.0925 & 36 & 1.99 & 0.0543 & 0.05 & -0.04205 & 4.3891 & 8.788 & 0.959 & 80.570 \\
\hline c & 12 & e & 12 & 2.0622 & 0.9527 & 36 & 2.16 & 0.0371 & 0.05 & 0.1299 & 3.9944 & 7.863 & 1.139 & 54.294 \\
\hline c & 12 & e & 24 & 2.5968 & 1.2444 & 36 & 2.09 & 0.0440 & 0.05 & 0.07312 & 5.1205 & 13.421 & 1.076 & 167.412 \\
\hline c & 12 & 1 & 6 & $-2,5123$ & 0.5340 & 36 & -4.70 & $<.0001$ & 0.05 & -35954 & -1.4292 & 0.081 & 0.027 & 0.239 \\
\hline c & 12 & $f$ & 12 & -2.2620 & 0.4956 & 36 & -4.56 & $<.0001$ & 0.05 & -3.2670 & $|-1.2570|$ & 0.104 & 0.038 & 0.284 \\
\hline c & -2 & 1 & 24 & -2.3531 & 0.5226 & 36 & -4.50 & $<.0001$ & 0.05 & -3.4129 & -1.2933 & 0.095 & 0.033 & 0.274 \\
\hline$c$ & 24 & d & 6 & -0.2203 & 0.5450 & 36 & -0.40 & 0.6885 & 0.05 & -1.3256 & 0.8850 & $0.8 \mathrm{C} 2$ & 0.268 & 2.423 \\
\hline$\therefore$ & 24 & d & 12 & 1.0176 & 0.8145 & 36 & 1.25 & 0.2196 & 0.05 & -0.6344 & 2.6695 & 2.766 & 0.530 & 14.432 \\
\hline c & 24 & d & 24 & 0.6839 & 0.7875 & 36 & 0.87 & 0.3909 & 0.05 & -0.9133 & 2.2810 & 1.982 & 0.401 & 9.787 \\
\hline c & 24 & e & 6 & 1.6658 & 1.0967 & 36 & 1.52 & 0.1375 & 0.05 & -0.6585 & 3.8901 & 5.290 & 0.572 & 48,916 \\
\hline c & 24 & $\theta$ & 12 & 1.5544 & 0.9577 & 36 & 1.62 & 0.1133 & 0.05 & -0.3878 & 3.4967 & 4.732 & 0.679 & 33.005 \\
\hline c & 24 & e & 24 & 2.0891 & 1.2481 & 36 & 1.67 & 0.1028 & 0.05 & -0.4422 & |4.6204 & 8.077 & 0.643 & 101,533 \\
\hline c & 24 & $f$ & 6 & -3.0200 & 0.5428 & 36 & -5.56 & $<.0001$ & 0.05 & -4.1208 & -1.9192 & 0.049 & 0.016 & 0.147 \\
\hline$c$ & 24 & if & 12 & -2.7697 & 0.5049 & 36 & -5.49 & $<, 0001$ & 0.05 & -3.7937 & -1.7457 & 0.063 & 0.023 & 0.175 \\
\hline c & 24 & $f$ & 24 & $-2,8608$ & 0.5315 & , 36 & -5.38 & $<0001$ & 0.05 & -3.9387 & $\mid-1.7830$ & 0.057 & 0.019 & 0.168 \\
\hline$d$ & 6 & $d$ & 12 & 1.2378 & 0.7542 & 36 & 1.64 & 0.1095 & 0.05 & -0.2918 & 2.7675 & 3.448 & 0.747 & 15.919 \\
\hline$d$ & 6 & d & 24 & 0.9041 & 0.7250 & 36 & 1.25 & $0.22 \mathrm{CA}$ & 0.05 & -0.5663 & 2.3745 & 2.470 & 0.568 & 10.746 \\
\hline d & 6 & $\theta$ & 6 & 1.8861 & 1.0528 & , 36 & 1.79 & 0.0816 & 0.05 & -0.2490 & 4.0212 & 6.594 & 0.780 & 55.766 \\
\hline d & 6 & $\theta$ & 12 & 1.7747 & 0.9070 & 36 & 1.96 & 0.0582 & 0.05 & -0.06468 & 3.6141 & 5.899 & 0.937 & 37.118 \\
\hline d & 6 & $\theta$ & 24 & 2,3093 & 1.2097 & 36 & 1.91 & 0.0642 & 0.05 & -0.1439 & 4.7626 & 10.068 & 0.866 & 117.053 \\
\hline d & 6 & $f$ & 6 & -2.7997 & 0.4472 & 36 & -6.26 & $<<.0001$ & 0.05 & -3.7068 & -1.8927 & 0.061 & 0.025 & 0.151 \\
\hline d & 6 & f & 12 & -2.5495 & 0.4005 & 36 & -6.37 & $<, 0001$ & 0.05 & -3.3617 & -1.7372 & 0.078 & 0.035 & 0.176 \\
\hline d & 6 & $f$ & 24 & -2.6406 & 0.4335 & 36 & -6.09 & $<.0001$ & 0.05 & -3.5197 & -1.7614 & 0.071 & 0.030 & 0.172 \\
\hline d & 12 & d & 24 & -0.3337 & 0.9445 & 36 & -0.35 & 0.7259 & 0.05 & -2.2492 & 1.5818 & 0.716 & 0.105 & 4.864 \\
\hline d & 12 & e & 6 & 0.6483 & 1.2144 & 36 & 0.53 & 0.5967 & 0.05 & -1.8146 & 3.1111 & 1.912 & 0.163 & 22.446 \\
\hline d & 12 & e & 12 & 0.5369 & 1.0904 & 36 & 0.49 & 0.6254 & 0.05 & -1.6746 & 2.7483 & 1.711 & 0.187 & 15.616 \\
\hline$d$ & 12 & e & 24 & 1.0715 & 1.3527 & 36 & 0.79 & 0.4335 & 0.05 & -1.6718 & 3.8148 & 2.920 & 0.188 & 45.369 \\
\hline d & 12 & $f$ & 6 & -4.0376 & 0.7526 & 36 & -5.36 & $<.0001$ & 0.05 & -5.5640 & -2.5112 & 0.018 & 0.004 & 0.081 \\
\hline d & 12 & $f$ & 12 & -3.7873 & 0.7258 & 36 & -5.22 & $<.0001$ & 0,05 & | & -2.3153 & 0.023 & 0.005 & 0.099 \\
\hline d & 12 & $f$ & 24 & -3.8784 & 0.7445 & 36 & -5.21 & $<.0001$ & 0.05 & -5.3883 & $\therefore-2.3684$ & 0.021 & 0.005 & 0.094 \\
\hline d & 24 & e & 6 & 0.9820 & 1.1964 & 36 & 0.82 & 0.4172 & 0.05 & -1.4445 & 3,4005 & 2.670 & 0.236 & 30.219 \\
\hline d & 24 & 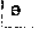 & 12 & 0.8706 & 1.0704 & 36 & 0.81 & 0.4214 & 0.05 & $-1,3003$ & 3.0414 & 2,388 & C. 272 & 20,935 \\
\hline d & 24 & e & 24 & 1.4052 & 1.3366 & 36 & 1.05 & 0.3001 & 0.05 & -1.3055 & 4.1159 & 4.076 & 0.271 & 61,309 \\
\hline d & 24 & $\stackrel{f}{\cdots}$ & 6 & -3.7039 & 0.7233 & 36 & -5.12 & $<0001$ & 0.05 & -5.1709 & -2.2369 & 0.025 & 0.006 & 0.107 \\
\hline d & 24 & $f$ & 12 & -3.4536 & 0.6954 & 36 & -4.97 & $<.0001$ & 0.05 & -4.8639 & $-2,0433$ & 0.032 & 0.008 & 0.130 \\
\hline d & 24 & 1 & 24 & -3.5447 & 0.7149 & \begin{tabular}{|l|}
36 \\
\end{tabular} & -4.96 & $<0001$ & 0.05 & -4.9946 & -2.0948 & 0.029 & 0.007 & 0.123 \\
\hline e & 6 & e & 12 & -0.1114 & 1.3147 & 36 & -0.08 & 0.9329 & 0.05 & -2.7777 & 2.5549 & 0.895 & 0.062 & 12.870 \\
\hline e & 6 & $\theta$ & 24 & 0.4233 & 1.5392 & 36 & 0.27 & 0.7849 & 0.05 & -2.6983 & 3.5449 & 1.527 & 0.067 & 34.635 \\
\hline e & 6 & $f$ & 6 & -4.6858 & 1.0516 & 36 & -4.46 & $<.0001$ & 0.05 & -6.8186 & -2.5531 & 0.009 & 0.001 & 0.078 \\
\hline 0 & 6 & $f$ & 12 & -4.4358 & 1.0326 & 36 & -4.30 & 0.0001 & 0.05 & -6.5297 , & -2.3414 & 0.012 & 0.001 & 0.096 \\
\hline e & 6 & 1 & 24 & -4.5267 & 1,0458 & 36 & -4.33 & 0.0001 & 0.05 & -6.6477 & -2.4057 & 0.011 & 0.001 & 0.090 \\
\hline 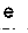 & 2 & $\theta$ & 24 & 0.5346 & 1.4434 & 36 & 0.37 & 0.7132 & 0.05 & -2.3927 & | 3.4620 & 1.707 & 0.091 & 31.879 \\
\hline 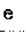 & 12 & $f$ & 6 & -4.5744 & 0.9056 & 36 & $\begin{array}{r}-5.05 \\
-5.05\end{array}$ & $<.0001$ & 0.05 & $-5, \Delta 111 \mid$ & | -2.7378 & 0.010 & 0.002 & 0.065 \\
\hline e & 12 & f & 12 & -4.3242 & 0.8835 & 36 & -4.89 & $<.0001$ & 0.05 & -6.1159 & -2.5324 & $0.013 !$ & 0.002 & 0.079 \\
\hline 6 & 12 & $f$ & 24 & -4.4153 & 0.8989 & 36 & -4.91 & $<.0001$ & 0.05 & -6.2383 & $-2,5922$ & 0.012 & 0.002 & 0.075 \\
\hline 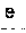 & 24 & $f$ & 6 & -5.1091 & 1.2086 & 36 & -4.23 & 0.0002 & 0.05 & -7.5603 & -2.6578 & 0.006 & $<0.001$ & 0.070 \\
\hline
\end{tabular}

file://C:/Users/JLUOMA/AppData/Local/Temp/1/SAS\%20Temporary\%20Iiles/ TD4836_.. 3/18/2015 


\begin{tabular}{|c|c|c|c|c|c|c|c|c|c|c|c|c|c|c|}
\hline & 24 & $f$ & 12 & -4.8588 & $1.1921 \mid$ & $|36|$ & -4.08 & 0.0002 & 0.05 & -7.2766 & $|-2.4411|$ & 0.008 & $<0.001$ & 0.087 \\
\hline$\theta$ & 24 & $f$ & 24 & -4.9199 & 1.2036 & 36 & $-4,11$ ! & 0.0002 & 0.05 & -7.3910 & -2.5089 & 0.007 & $<0.001$ & 0.081 \\
\hline f & 6 & $f$ & 12 & 0.2503 & 0.3974 & 36 & 0.63 & 0.5328 & 0.05 & -0.5558 & 1.0563 & 1.284 & 0.574 & 2.876 \\
\hline$f$ & 6 & f & 24 & 0.1592 & 0.4307 & 36 & $0.37 !$ & $\mid 0.7138$ & 0.05 & -0.7142 & 1.0326 & 1.173 & 0.490 & 2,808 \\
\hline & 12 & if & 24 & -0.09110 & 0.3819. & 36 & -0.24 & $|0.8128|$ & 0.05 & -0.8656 & | $0.8834 \mid$ & 0.913 & 0.421 & 1.981 \\
\hline
\end{tabular}

Performod by ل. Luoma; SAS version 9.3 08:43 18MAR15 


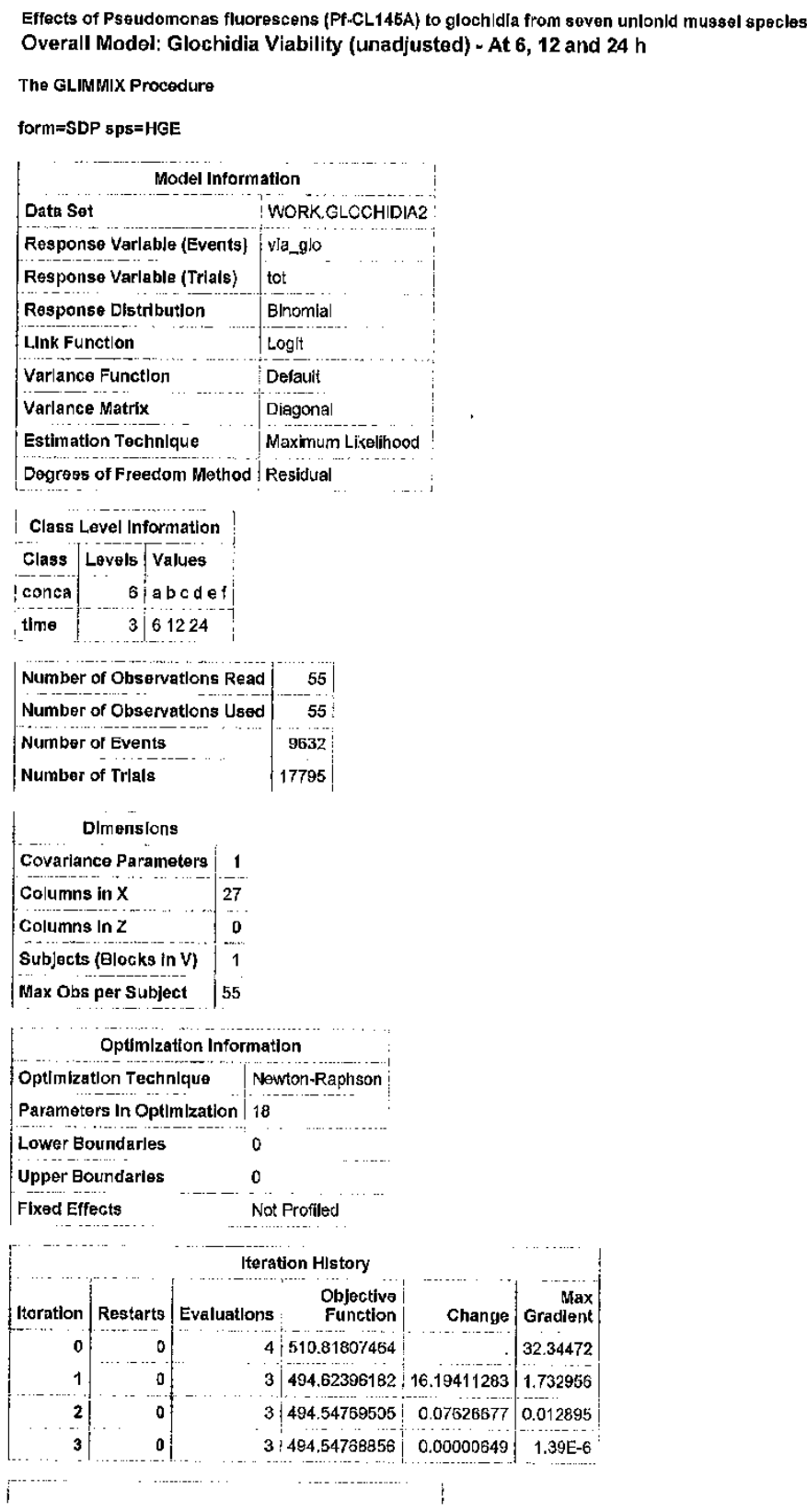

file://C:/Users/ILUOMA/AppData/Local/Temp/1/SAS\%20Temporary\%20Files/ TD4836_.. 3/18/2015 


Convergence criterion (ABSGCONV=0
\begin{tabular}{|c|c|} 
FIt Statistlcs \\
-2 Log Likelihood & 989.10 \\
\hline AIC (smailler Is better) & 1025.10 \\
\hline AICC (smaller is better) & 1044.10 \\
\hline EIC (smaller Is botter) & 1061.23 \\
\hline CAIC (smaller is better) & 1079.23 \\
HQIC (smaller Is better) & 1039.07 \\
\hline Pearson Chl-Square & 652.18 \\
Pearson Chi-Square I DF & 17.63 \\
\hline
\end{tabular}

\begin{tabular}{|c|c|c|c|c|c|c|c|}
\hline \multirow[b]{2}{*}{ Effect } & \multicolumn{6}{|c|}{ Parameter Estımates } & \multirow[b]{2}{*}{$\operatorname{Pr}>|1|$} \\
\hline & conca & t t/me & Estimato $\mathrm{St}$ & Standard Error & DF & t Value & \\
\hline conca & a & & 1.0843 & 0.2508 & 37 & 4.32. & 0.0001 \\
\hline conca & b & & 0.6758 & 0.2320 & $|37|$ & 2.91 & 0.0060 \\
\hline conca & c & & -0.3769 & 0.2570 & 37 & -1.47 & 0.1509 \\
\hline conca & d & & -1.5650 & 0.4305 & $37 \mid$ & -3.64 & 0.0008 \\
\hline conca & e & & -1.7513 & 0.4526 & 37 & -3.87 & 0.0004 \\
\hline conca & $f$ & & 0.8530 & 0.2251 & 37 & 3.79 & 0.0005 \\
\hline $\operatorname{tim} \theta$ & & 6 & 0.1041 & 0.4256 & 37 & 0.24 & 0.8081 \\
\hline tIme & & 12 & -0.1312 & 0.3680 & $37 \mid$ & -0.36 & 0.7235 \\
\hline time & & 24 & 0 & & & $\therefore$ & \\
\hline concantime & a & 6 & 0.1495 & 0.5887 & 37 & 0.25 & 0.8009 \\
\hline conca"time & a & 12 & -0.1285 & 0.5505 & 37 & -0.23 & $0.8: 67$ \\
\hline conca ${ }^{* t / m e}$ & $a$ & 24 & 01 & & $\therefore$ & & \\
\hline conca*time & b & 6 & -0.2346 & 0.5530 & | $37 \mid$ & -0.42 & 0.6739 \\
\hline conca'tlme & $b$ & 12 & 0.1177 & 0.5287 & $37 \mid$ & 0.22 & 0.8251 \\
\hline conca time & b & 24 & 0 & & 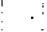 & & \\
\hline conca*time & c & 6 & 0.5523 & 0.5477 & 37 & 1.01 & 0.3198 \\
\hline conca*time & c & 12 & 0.4085 & 0.5305 & 37 & 0.77 & 0.4462 \\
\hline conca"t/me & ic & 24 & o & & - & $\therefore$ & \\
\hline conca"time & $d$ & 6 & 0.6962 & 0.6693 & 37 & 1.04 & 0,3050 \\
\hline conca*tlume & $d$ & 12 & $1.2280^{\prime}$ & 0.6510 & $|37|$ & 1.89 & 0.0671 \\
\hline conca*tlne & $d$ & 24 & 0 & & $\begin{array}{l}i \ldots . . . \\
i\end{array}$ & . & \\
\hline conca"tlme & e & 6 & -0.01823 & 0.7507 & 37 & -0.02 & 0.9808 \\
\hline concantim & $e$ & 12 & -0.3636 & 0.8155 & 37 & $-0,45$ & 0.6584 \\
\hline conca*time & e & 24 & 0 & & $\therefore$ & & \\
\hline conca $a^{*} \operatorname{lm} \theta$ & $r$ & 6 & $0 !$ & & & & \\
\hline conca*tume & f & 12 & 0 & & & & \\
\hline conca*time & i & 24 & 0 & & & & \\
\hline ResIdual & & & 17.6264 & & & & \\
\hline Typ & pe III Tes & sts of $F$ & FIxed Effects & & & & \\
\hline Effect & Num DF & F Den & In DF ' F value & $\mathrm{Pr}>\mathrm{F}$ & & & \\
\hline conca & & 5 & $\begin{array}{rr}37 & 27.49\end{array}$ & $\begin{array}{l}9 \\
-<.0001\end{array}$ & & & \\
\hline thme & & 2. & $37 \quad 1.34$ & \begin{tabular}{l|l}
4 & 0.2736
\end{tabular} & & & \\
\hline
\end{tabular}

file://C:/Uscrs/JLUOMA/AppData/Local/Temp/1/SAS\%20Temporary\%20Files/_TD4836... 3/18/2015 


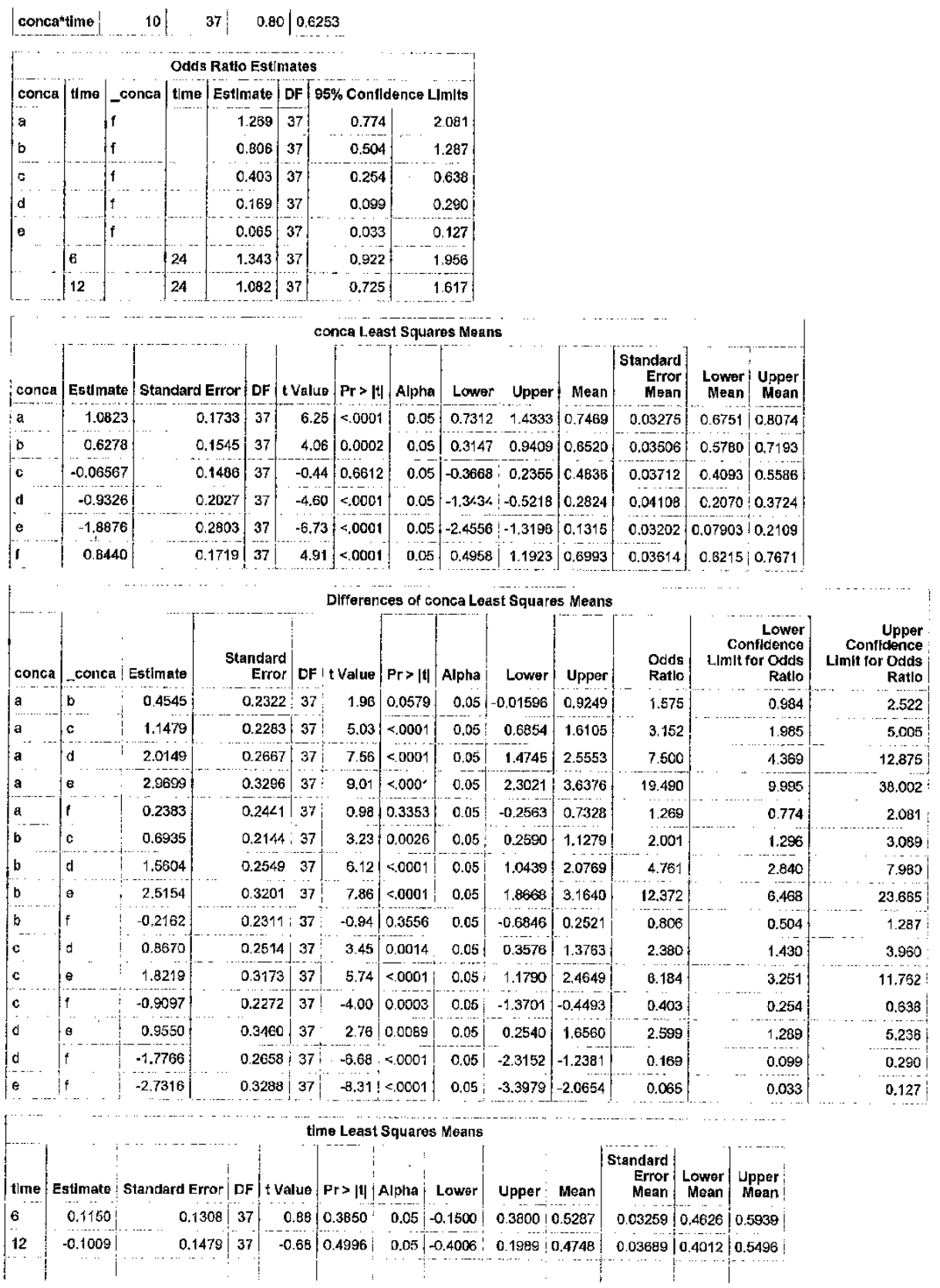


$|24|-0.1800|\quad 0.1316| 37|-1.37| 0.1796: 0.05|-0.4467| 0.08665: 0.4551|0.03264| 0.3901|0.5216|$

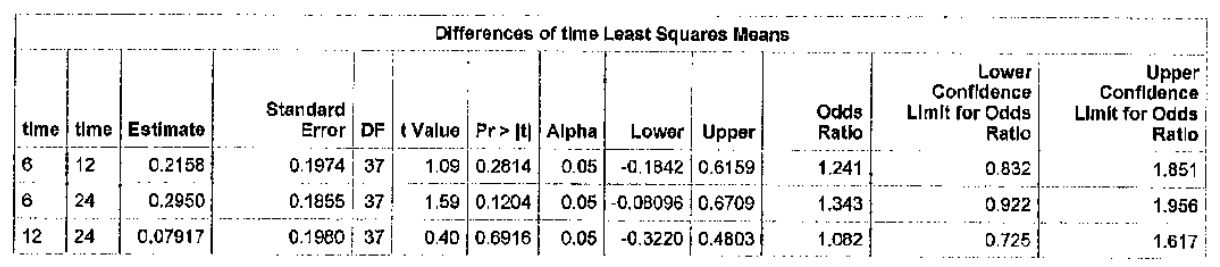

\begin{tabular}{|c|c|c|c|c|c|c|c|c|c|c|c|c|c|}
\hline conca & time & Estlmate & Standard Error & DF & t Value & $P r>\mid i t$ & Alpha & Lower & Upper: & Mean & $\begin{array}{c}\text { Standard } \\
\text { Error } \\
\text { Mean }\end{array}$ & $\begin{array}{c}\text { Lower } \\
\text { Mean }\end{array}$ & $\begin{array}{l}\text { Upper } \\
\text { Mean }\end{array}$ \\
\hline a & 6 & 1.3379 & 0.3202 & 37 & 4.18 & 0.0002 & $0.05:$ & 0.6891 & 1.9867 & 0.7921 & 0.05272 & 0.6658 & 0.8794 \\
\hline a & 12 & 0.8246 & 0.3237 & 37 & 2.55 & 0.0151 & 0.05 & 0.1688 & 1.4804 & 0.6952 & 0.06858 & 0.5421 & 0.8146 \\
\hline a & 24 & 1.0843 & 0.2508 & 37 & 4.32 & 0.0001 & 0.05 & 0.5761 & 1.5924 & 0.7473 & 0.04736 & 0.6402 & 0.8310 \\
\hline b & 6 & 0.5453 & 0.2662 & 37 & 2.05 & 0.0476 & 0.05 & 0.006019 & 1.0847 & 0.6331 & 0.06183 & 0.5015 & 0.7474 \\
\hline b & 12 & 0.5623 & 0.3004 & 37 & 2.20 & 0.0338 & 0.05 & 0.05355 & 1.2710 & 0.6598 & 0.06744 & 0.5134 & 0.7809 \\
\hline b & 24 & 0.6758 & 0.2320 & 37 & 2.91 & 0.0060 & 0.05 & 0.2057 & 1.1459 & 0.6628 & 0.05186 & 0.5512 & 0.7588 \\
\hline$c$ & 6 & 0.2795 & 0.2298 & 37 & 1.22 & 0.2316 & 0.05 & -0.1861 & 0.7452 & 0.5694 & 0.05635 & 0.4536 & 0.6781 \\
\hline c & 12 & -0.09963 & 0.2828 & 37 & -0.35 & 0.7266 & 0.05 & -0.6726 & 0.4733 & 0.4751 & 0.07052 & 0.3379 & 0.6162 \\
\hline c & 24 & -0.3769 & 0.2570 & 37 & -1.47 & 0.1509 & 0.05 & -0.8970 & 0.1438 & 0.4060 & $0.06202:$ & 0.2895 & 0.5358 \\
\hline d & 6 & -0.7647 & 0.2855 & 37 & -2.68 & 0.0110 & 0.05 & -1.3431 & -0.1863 & 0.3176 & 0,06187 & 0.2070 & 0.4536 \\
\hline d & 12 & -0.4682 & 0.3211 & 37 & -1.46 & 0.1532 & 0.05 & -1.1188 & $0.1824 i$ & 0.3850 & 0.07603 & 0.2462 & 0.5455 \\
\hline d & 24 & $-1,5650$ & 0.4305 & 37 & -3.64 & 0.0008 & 0.05 & -2.4372 & $\mid-0.6927$ & 0.1729 & 0.06157 & 0.08038 & 0.3334 \\
\hline e & 8 & -1.6655 & 0.4215 & 37 & -3.95 & 0.0003 & 0.05 & -2.5194 & -0.8115 & 0.1690 & 0.05636 & 0.07451 & 0.3076 \\
\hline e & 12 & -2.2460 & 0.5700 & 37 & -3.94 & 0.0003 & 0.05 & -3.4009 & $-1 . \operatorname{co1} 12$ & 0.09569 & 0.04932 & 0.03227 & 0.2514 \\
\hline e & 24 & -1.7513 & 0.4526 & 37 & -3.87 & 0.0004 & 0.05 & -2.6683 & -0.8344 & 0.1479 & 0.05703 & 0.06487 & 0.3027 \\
\hline$t$ & 6 & 0.9572 & 0.3612 & 37 & 2.65 & 0.0118 & 0.05 & 0.2253 & 1,6890 & 0.7226 & 0.07241 & 0.5551 & 0.8441 \\
\hline$f$ & 12 & 0.7219 & 0.2911 & 37 & 2.48 & 0.0178 & 0.05 & 0.1320 & 1.3117 & 0.6730 & 0.06407 & 0.5329 & 0.7878 \\
\hline$f$ & 24 & 0.8530 & 0.2251 & $\mid 37$ & 3.79 & 0.0005 & 0.05 & 0.3969 & 1.3092 & 0.7012 & 0.04717 & 0,5979 & 0.7874 \\
\hline
\end{tabular}

\begin{tabular}{|c|c|c|c|c|c|c|c|c|c|c|c|c|c|c|}
\hline \multirow[b]{2}{*}{ conca } & \multirow[b]{2}{*}{ time } & \multirow[b]{2}{*}{ - conca } & \multirow[b]{2}{*}{ | 1lme | } & \multirow[b]{2}{*}{ Estimate } & \multicolumn{7}{|c|}{ Differences of conca“time Least Squares Means } & \multirow[b]{2}{*}{$\begin{array}{l}\text { Odds } \\
\text { Ratio }\end{array}$} & \multirow[b]{2}{*}{$\begin{array}{r}\text { Lower } \\
\text { Confldence } \\
\text { Limlt for } \\
\text { Odds } \\
\text { Ratio }\end{array}$} & \multirow[b]{2}{*}{$\begin{array}{r}\text { Upper } \\
\text { Confidence } \\
\text { Limit for } \\
\text { Odds } \\
\text { Ratio }\end{array}$} \\
\hline & & & & & $\begin{array}{r}\text { Standard } \\
\text { Error }\end{array}$ & DF & t Value & $|\operatorname{Pr}>| t \mid$ & Alpha & Lower & Upper: & & & \\
\hline a & 6 & a & 12 & 0.5133 & C.4553. & 37 & 1.13 & 0.2669 & 0.05 & -0.4093 & $1.4358 \mathrm{i}$ & 1.671: & 0.664 & 4.203 \\
\hline a & 6 & a & 24 & 0.2536 & 0.4067 & 37 & 0.62 & 0.5368 & 0.05 & -0.5705 & 1.0778 & 1.289 & 0.565 & 2.938 \\
\hline a & 6 & b & 6 & 0.7926 & 0.4164 & 37 & 1.80 & 0.0648 & 0.05 & -0.05114 & 1.6363 & 2.209 & 0.950 & 5.136 \\
\hline a & 6 & b & 12 & 0.6756 & 0.4391 & 37 & 1.54 & 0.1324 & 0.05 & -0.2 .140 & 1.5653 & 1.965 & 0.807 & 4.784 \\
\hline a & 6 & b & 24 & 0,6621 & 0.3954 & 37 & 1.67 & 0.1025 & 0.05 & -0.1391 & $1.4634^{\prime}$ & 1.939 & 0.870 & 4.320 \\
\hline a & 6 & 0 & 6 & 1.0584 & 0.3942 & 37. & 2.69 & 0.0108 & 0.05 & 0.2597 & 1.8570 & 2.882 & 1.297 & 6.405 \\
\hline a & 6 & 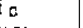 & 12 & 1.4375 & 0.4272 & $37 i$ & 3.37 & 0.0018 & 0.05 & 0.5720 & 2.3031 & 4.210: & 1.772 & 10.005 \\
\hline a & 6 & $\mathrm{c}$ & 24 & 1.7148 & 0.4106 & 37 & 4.18 & 0.0002 & 0.05 & 0.8829 & 2.5467 & 5.556 & $2.418:$ & 12.765 \\
\hline a & 6 & d & 6 & 2.1026 & 0.4290 & 37 & 4.90 & $<.0001$ & 0.05 & 1.2334 & 2.9718 & 8.187 & $3.433:$ & 19.527 \\
\hline a & 6 & d & 12 & 1.8061 & 0.4535 & 37 & 3.98 & 0.0003 & 0.05 & 0.8873 & 2.7249 & 6.087 & 2.428 & 15.256 \\
\hline a & 6 & d & 24 & 2.9029 & 0.5365 & 37 & 5.41 & $<, 0001$ & 0.05 & 1.8158 & 3.9900 & 18.227 & 6.146 & 54.055 \\
\hline
\end{tabular}




\begin{tabular}{|c|c|c|c|c|c|c|c|c|c|c|c|c|c|c|}
\hline a & 6 & e & 6 & 3.0034 & 0.5293 & 37 & 5.67 & $<.0001$ & 0.05 & 1.9309 & 4.0758 & 20.153 & 6.896 & 58.900 \\
\hline & 6 & $\theta$ & 12 & 3.5839 & 0.6538 & 37 & 5.48 & $<0.0001$ & 0.05 & 2.2593 & 4.9086 & 36.015 & 9.576 & 135.445 \\
\hline a & 6 & $\theta$ & 24 & 3.0893 & 0.5544 & 37 & 5.57 & $<, 0001$ & 0.05 & 1.9660 & $4.2126 !$ & ! 21.961 & 7.142 & 67.529 \\
\hline a & 6 & 1 & 6 & 0.3808 & 0.4827 & 37 & 0.79 & 0.4353 & 0.05 & -0.5973 & 1.3588 & 1.463 & 0.560 & 3.881 \\
\hline a & 6 & $f$ & 12 & 0.6161 & 0.4328 & 37 & 1.42 & 0.1630 & 0.05 & $-0,2608$ & 1.4929 & 1.852 & 0.770 & 4.450 \\
\hline a & 6 & f & 24 & 0.4849 & 0.3914 & 37 & 1.24 & 0.2233 & 0.05 & -0.3083 & 1.2780 & 1.624 & 0.735 & 3.589 \\
\hline a & 12 & $a$ & 24 & -0.2597 & 0.4095 & 37 & -0.63 & 0.5299 & 0.05 & -1.0893 & 0.5700 & 0.771 & 0.336 & 1.768 \\
\hline a & 12 & ib & 8 & 0.2793 & 0.4191 & 37 & 0.67 & 0.5093 & 0.05 & -0.5698 & 1.1284 & 1.322 & 0.566 & 3.091 \\
\hline a & 12 & b & 12 & 0.1623 | & 0.4416 & 37 & 0.37 & 0.7153 & 0.05 & -0.7325 & 1.0571 & 1.176 & 0.481 & 2,878 \\
\hline a & 12 & b & 24 & 0.1488 & 0.3982 & 37 & $0.37 !$ & 0.7108 & 0.05 & -0.6581 & 0.9557 & 1.160 & 0.518 & 2.601 \\
\hline $\mathbf{a}$ & 12 & c & 6 & 0.5451 & 0.3970 & 37 & 1.37 & 0.1780 & 0.05 & -0.2593 & 1.3494 & 1.725 & 0.772 & 3.855 \\
\hline a & 12 & c & 12 & 0.9242 & 0.4298 , & 37 & 2.15 & 0.0381 & 0.05 & 0.05339 & 1.7951 & 2.520 & 1.055 & 6.020 \\
\hline a & 12 & c & 24 & 1.2015 & 0.4133 & 37 & 2.91 । & 0.0061 & 0.05 & 0.3641 & 2.0389 & 3.325 & 1.439 & 7.682 \\
\hline a & 12 & d & 6 & 1.5893 & 0.4316 & 37 & 3.68 & 0.0007 & 0.05 & 0.7148 & 2.4637 & $4.900^{\prime}$ & 2.044 & 11.748 \\
\hline a & 12 & d & 12 & 1.2928 & 0.4559 & 37 & 2.84 & $|0.0074|$ & 0.05 & 0.3690 & 2.2166 & 3.643 & 1.446 & 9.176 \\
\hline a & 12 & $d$ & 24 & 2.3896 & 0.5386 & 37 & 4.44 & $<0001$ & 0.05 & 1.2983 & 3.4809 & 10.908 & 3.663 & 32.489 \\
\hline a & 12 & $\theta$ & 6 & $2.490 \dagger$ & 0.5314 & $37 \mid$ & 4.69 & $\mid<0001$ & 0.05 & 1.4133 & 3.5668 & 12.062 & 4.110 & 35.403 \\
\hline a & 12 & e & 12 & 3.0706 & 0.6555 & 37 & 4.68 & $<.0001$ & 0.05 & 1.7425 & 4.3987 & 21.555 & 5.712 & 81.346 \\
\hline a & 12 & e & 24 & 2.5760 & 0.5564 & 37 & 4.63 & $<.0001$ & 0.05 & 1.4486 & 3.7033 & 13.144 & 4.257 & 40.582 \\
\hline a & 12 & f & 6 & -0.1325 & 0.4850 & 37 & -0.27 & 0.7861 & 0.05 & -1.1152 & 0.8501 & $0.876 !$ & 0.328 & 2.340 \\
\hline a & 12 & 1 & 12 & 0.1028 & 0.4353 & 37 & 0.24 & $\mid 0.8147$ & 0.05 & -0.7793 & 0.9848 & 1.108 & 0.459 & 2.677 \\
\hline a & 12 & f & 24 & -0.02843 & 0.3943 & 37 & -0.07 & 0.9429 & 0.05 & -0.8273 & 0.7704 & 0.972 & 0.437 & 2.161 \\
\hline a & 24 & b & 6 & 0.5390 & 0.3657 & 37 & 1.47 & 0.1490 & $0.05^{\circ}$ & -0.2021 & 1.2800 & 1.714 & 0.817 & 3.596 \\
\hline a & 24. & $b$ & 12 & 0.4220 & 0.3913 & 37 & 1.08 & 0.2879 & 0.05 & -0.3709 & 1.2150 & 1.525 & $c .690$ & $3.37 \mathrm{C}$ \\
\hline a & 24 & b & 24 & 0.4085 & 0.3417 & 37 & 1.20 & 0.2394 & 0.05 & -0.2838 & 1.1008 & 1.505 & 0.753 & 3.006 \\
\hline a & 24 & c & 6 & 0.8047 & 0.3402 & $37 \mid$ & 2.37 & 0.0233 & 0.05 & 0.1155 & 1.4940 & 2.236 & 1.122 & $4,455 !$ \\
\hline a & 24 & $\mathrm{c}$ & 12 & 1.1839 & 0.3780 & 37 & 3.13 & 0.0034 & 0.05 & 0.4181. & 1.9497 & 3.267 & 1.519 & 7.027 \\
\hline a & 24 & $\mathrm{c}$ & 24 & 1.4612 & 0.3581 & 37 & 4.07 & 0.0002 & 0.05 & 0.7336 & 2.1888 & 4.311 & 2.083 & 8.924 \\
\hline a & 24 & d & 6 & 1.8489 & 0.3800 & 37 & 4.87 & $<.0001$ & 0.05 & 1.0790 & 2.8189 & 6.353 & 2.942 & 13.720 \\
\hline a & 24 & d & 12 & 1.5525 & 0.4074 & 37 & 3.81 & 0.0005 & 0.05 & 0.7270 & 2.3780 & 4.723 & 2.069 & 10.784 \\
\hline a & 24 & d & 24 & 2,6493 & 0.4982 & 37 & 5.32 & $<.0001$ & 0.05 & 6398 & 3.6588 & 14.144 & 5.154 & 38.813 \\
\hline a & 24 & e & 6 & 2.7498 & 0.4904 & 37 & 5.61 & ;<.0001 & 0.05 & 1.7560 & 3.7435 & 15.639 & 5.790 & 42.244 \\
\hline a & 24 & e & 12 & 3,3303 & 0.6227 & 37 & 5.35 & $<.0001$ & 0.05 & 2.0685 & 4.5920 & 27.947 & 7.914 & 98.693 \\
\hline$a$ & 24 & e & 24 & 2,8356 & 0.5174 & 37 & 5.48 & $<.0001$ & 0.05 & 1.7873 & 3.8840 & 17.041 & 5.973 & 48.618 \\
\hline a & 24 & if & 6 & 0.1271 & 0.4397 & 37 & 0.29 & 0.7741 & 0.05 & -0.7638 & 1.0181 & 1.136 & $0.466 \mid$ & $2.768:$ \\
\hline a & 24 & $f$ & 12 & 0.3624 & 0.3843 & 37 & $0.94 !$ & 0.3517 & 0.05 & -0.4152 & 1.1410 & 1.437 & 0.660 & 3.130 \\
\hline a & 24 & if & 24 & 0.2312 & 0.3370 & 37 & 0.69 & $0.4969 \mid$ & 0.05 & -0.4516 & 0.9141 & 1.260 & $0.637 \mid$ & 2,496 \\
\hline b & 6 & $b$ & 12 & -0.1169 & C.4011 & 37 & -0.29 & 0.7724 & 0.05 & -0.9302 & 0.6963 & 0.890 & $0.394 !$ & 2.006 \\
\hline b & 6 & $b$ & 24 & -0.1305 & 0.3531 & 37 & -0.37 ! & 0.7139 & 0.05 & -0.8459 & 0.5850 & 0.878 & 0.429 & 1.795 \\
\hline b & 6 & $\therefore$ & 6 & 0.2658 & 0.3517 & 37 & 0.76 & 0.4545 & 0.05 & -0.4468 & 0.9783 & 1.304 & 0.640 & 2.660 \\
\hline b & 6 & ? & 12 & 0.6450 & 0.3883 & 37 & 1.66 & 0.1052 & 0.05 & -0.1419 & 1.4318 & $1.906 !$ & 0.868 & 4.186 \\
\hline $\mathrm{b}$ & 6 & $\mathrm{c}$ & 24 & 0.9223 & 0.3700 & 37: & 2.49 & 0.0173 & 0.05 & C.1726 & 1.6719 & 2.515 & 1.188 & 5.322 \\
\hline b & 6 & d & 6 & 1.3100 & 0.3903 & 37 & 3.36 & 0.0018 & 0.05 & 0.5192 & 2.1008 & 3.706 & 1.681 & 8.173 \\
\hline b & 6 & d & 12 & 1.0135 & 0.4171 & 37 & 2.43 & 0.0201 & 0.05 & 0.1685 & 1.8586 & 2.755 & 1.183 & 6.415 \\
\hline b & 6 & d & 24 & 2.1103 & 0.5061 & 37 & 4.17 & 0.0002 & 0.05 & 1.0848 & 3.1358 & 8.251 & 2.959 & 23.008 \\
\hline b & 6 & e & 6 & 2.2108 & $0.4985 i$ & ; 37 & $4.44 \mid$ & $\mid<0001^{\circ}$ & 0.05 & 1.2008 & 3.2208 & 0.123 & 3.323 & 25.048 \\
\hline
\end{tabular}




\begin{tabular}{|c|c|c|c|c|c|c|c|c|c|c|c|c|c|c|}
\hline & 6 & e & 12 & 2.7914 & 0.6291 & 37 & 4.44 & $<.0001$ & 0.05 & 1.5168 & 4.0659 & 16.303 & 4.557 & 58.319 \\
\hline D & 6 & e & 24 & 2.2967 & 0.5250 & 37 & 4.37 & $<.0001$ & 0.05 & \begin{tabular}{l}
1.2329 \\
\hdashline .2
\end{tabular} & 3.3605 & 9.941 & 3.431 & 28.803 \\
\hline b & 6 & $f$ & 6 & -0.4118 & 0.4487 & 37 & -0.92 & 0.3646 & 0.05 & -1.3209 & 0.4973 & C.662 & 0.267 & 1.644 \\
\hline b & 6 & r & 12 & -0.1765 & 0.3945 & 37 & $-\mathrm{c} .45$ & 0.6571 & 0.05 & -0.9758 & 0.6228 & 0.838 & 0.377 & 1.864 \\
\hline b & 6 & $f$ & 24 & -0.3077 & C.3486 & 37 & -0.88 & 0.383 & 0.05 & -1.0141 & 0.3986 & 0.735 & $0.363 !$ & 1.490 \\
\hline b & 12 & $b$ & 24 & -0.01352 & 0,3796 & 37 & -0.04 & 0.9718 & 0.05 & -0.7826 & 0.7556 & 0.987 & 0.457 & 2.129 \\
\hline b. & 12 & c & 6 & 0.3827 & 0.3783 & 37 & 1.01 & $0.3182\}$ & 0.05 & -0.3837 & 1.1492 & 1.466 & 0.681 & 3.156 \\
\hline b & 12 & 6 & 12 & 0.7619 & 0.4126 & 37 & 1.85 & 0.0728 & 0.05 & -0.07404 & 1.5978 & 2.142 & 0.929 & 4.942 \\
\hline b & 12 & $c$ & 24 & 1.0392 & 0.3953 & 37 & 2.63 & 0.0124 & 0.05 & 0.2381 & 1.8402 & $2.827^{\prime}$ & 1.269 & 6.298 \\
\hline b & 12 & $d$ & 6 & 1.4269 & 0.4144 & 37 & 3.44 & 0.0014 & 0.05 & 0.5872 & 2.2666 & 4.166 & 1.799 & 9.647 \\
\hline b & 12 & d & 12 & 1.1305 & 0.4397 & 37 & $2.57 !$ & 0.0143 & 0.05 & 0.2395 & 2.0214 & 3.097 & 1.271 & 7.548 \\
\hline$b$ & 12 & d & 24 & 2.2273 & 0.5260 & 37 & $4.24 \mathrm{i}$ & 0,0001 & 0.05 & 1.1636 & 3.2909 & 9.274 & 3.201 & 26.867 \\
\hline b & 12 & e & 6 & 2.3277 & 0.5176 & 37 & 4.50 & $<0001$ & 0.05 & 1.2790 & 3.3764 & 10.255 & 3.593 & 29.266 \\
\hline b. & 12 & e & 12 & 2.9083 & 0.8443, & 37 & 4.51. & $1<.0001$ & 0.05 & 1.6028 & 4.2138 & 18.325 & 4.967 & 67.610 \\
\hline b & 12 & e & 24 & 2.4136 & 0.5432 & 37 & 4.44 & $<.0001$ & 0.05 & 1,3130 & 3.5142 & 11.174 & 3.717 & 33.590 \\
\hline b & 12 & 1 & 6 & -0.2949 & 0.4698 & 37 & -0.63 & 0.5341 & 0.05 & -1.2468 & 0.6570 & $0.745:$ & 0.287 & 1.929 \\
\hline$b$ & 12 & $f$ & 12 & -0.05959 & 0.4183 & 37 & $-0,14$ & 0.8875 & 0.05 & -0.9072 & 0.7881 & 0.942 & 0.404 & 2.199 \\
\hline b & 12 & 1 & 24 & -0.1908 & 0.3754 & 37 & -0.511 & 10.6144 & 0.05 & -0.9514 & 0.5699 & 0.826 & 0,386 & 1.768 \\
\hline $\mathrm{b}$ & 24 & c & 6 & 0.3962 & 0.3266 & 37 & 1.21 & 0.2327 & 0.05 & -0.2655 & 1.0580 & 1.486 & 0.767 & 2.881 \\
\hline b & 24 & c & 12 & 0.7754 & 0.3658 & 37 & 2.12 & 0.0408 & 0.05 & 0.03430 & 1.5165 & 2.172 & 1.035 & 4.566 \\
\hline b & 24 & c & 24 & 1.0527 & 0,3462 & 37 & $3 . \mathrm{CA}$ & 0.0043 & 0.05 & 0.3512 & 1.7542 & 2.865 & 1.421 & $5.779^{\circ}$ \\
\hline b & 24 & d & 6 & 1.4405 & 0.3679 & 37 & 3.82 & 0.0004 & 0.05 & 0.6951 & 2.1858 & 4.223 & 2.004 & 8.898 \\
\hline$b$ & 24 & d & 12. & 1.1440 & 0.3962 & 37 & 2.89 & 0.0064 & 0.05 & 0.3413 & 1.9467 & 3.139 & 1.407 & 7.005 \\
\hline b. & 24 & d & 24 & 2.2408 & 0.4890 & 37. & 4.58 & $\mid<, \infty 001$ & $0.05:$ & 1.2499 & 3.2317 & 9.401 & 3.490 & 25.322 \\
\hline b & 24 & e & 8 & 2.3413 & 0.4811 & 37 & 4.87 & $<.0001$ & 0.05 & 1.3665 & 3.3161 & 10.394 & 3.921 & 27.552 \\
\hline b & 24 & e & 12 & 2.9218 & 0.6154 & 37 & 4.75 & $<.0001$ & 0.05 & 1.6749 & 4.1687 & $18.575 !$ & 5.338 & 64.630 \\
\hline b & 24 & $\theta$ & 24 & 2.4271 & 0.5086 & 37 & 4.77 & $<.0001$ & 0.05 & 1.3967 & 3.4576 & 11,326 & 4.042 & 31.740 \\
\hline b & 24 & $f$ & 6 & -0.2814 & 0.4293 & 37 & -0.66 & 0.5162 & 0,05 & -1.1512 & 0.5885 & 0.755 & 0.316 & 1.801 \\
\hline b & 24 & $f$ & 12 & -0.04607 & 0.3723 & 37 & -0.12 & $\mid 0.9022$ & 0.05 & -0.8004 & 0.7087 & 0.955 & 0.449 & 2.030 \\
\hline b & 24 & f & 24 & -0.1773 & 0.3233 & 37 & $-0.55 !$ & 0.5868 & 0.05 & -0.8323 & 0.4778 & 0.838 & 0.435 & 1.613 \\
\hline$c$ & 6 & c & 12 & 0.3792 & 0.3644 & 37. & .04 & 0.3048 & 0.05 & -0.3591 & 1.1175 & 1.461 & 0.698 & $3.057:$ \\
\hline c & 6 & c & 24 & 0.6565 & 0.3448 & 37 & 1.90 & 0.0647 & $0.05=$ & -0.04210 & 1.3550 & 1.928 & 0.959 & 3.877 \\
\hline c & 6 & $d$ & 6 & 1.0442 & 0.3665 & 37. & 2.85 & 0.0071 & 0.05 & 0.3016 & 1.7868 & 2.841 & 1.352 & 5.970 \\
\hline c & 6 & d & 12 & 0.7477 & 0.3949 & 37 & 1.89 & 0.0661 & 0.05 & -0.05235 & 1.5478 & 2.112 & 0.949 & 4.701 \\
\hline c. & 6 . & d & 24 & 1.8445 & 0.4880 & 37 & 3.78 & 0.0006 & 0.05 & 0,8557 & 2.8333 & 6.325 & 2.353 & 17.002 \\
\hline c & 6 & e & 6 & 1.9450 & 0.4800 & 37. & 4.05 & 0.0003 & 0.05 & 0.9723 & 2.9177 & 6.994 & 2644 & 18.498 \\
\hline $\mathrm{c}$ & 6 & e & 12 & 2.5256 & 0.6146 & 37. & 4.11 & 0.0002 & 0.05 & 1.2803 & 3.7708 & 12.498 & 3.598 & 43.414 \\
\hline$c$. & 6 & a & 24 & 2.0309 & 0.5076 & 37 & 4.00 & 0.0003 & 0.05 & 1.0025 & 3.0593 | & 7.621 & 2.725 & 21.313 \\
\hline c & 6 & $f$ & 6 & -0.6776 & 0.4281 & 37 & -1.58 & 0.1220 & 0.05 & -1.5450 & 0.1898 & 0.508 & 0.213 & 1.209 \\
\hline c & 6 & $f$ & 12 & -0.4423 & 0.3709 & 37 & -1.19 & 0.2407 & 0.05 & -1.1939 & 0.3092 & 0.643 & 0.303 & 1.352 \\
\hline c & 6 & $f$ & 24 & -0.5735 & 0.3217 & 37 & -1.78 & 0.0829 & 0.05 & -1.2254 & 0.07836 & 0.564 & 0.294 & 1.082 \\
\hline c & 12 & c & 2.4 & 0.2773 & 0.3821 & 37 & 0.73 & 0.4726 & 0.05 & $-\mathrm{C.} 4869$ & 1.0515 & 1.320 & 0.608 & 2,862 \\
\hline $\mathrm{c}$ & 12 & d & 6 & 0.6650 & $0,4018 \mid$ & 37 & 1.66 & 0.1064 & 0.05 & -0.1491 & 1.4792 & $1.945:$ & 0.861 & 4,389 \\
\hline$c$ & 12 & id & 12 & 0.3686 & 0.4279 & $37^{\prime}$ & 0.86 & 0.3945 & 0,05 & -0.4983 & 1.2355 & 1.446 & 0.608 & 3.440 \\
\hline c & 12 & $d$ & 24 & 1.4654 & 0.5150 & $37:$ & 2.85 & 0.0072 & 0.05 & 0.4218 & $2.5089 !$ & 4.329 & 1.525 & 12.292 \\
\hline c & 12 & e & 6 & 1.5658 & 0.5075 & 37 & 3.09 & 0.0038 & 0.05 & 0.5375 & 2.5942 & $4.787^{i}$ & 1.712 & 13.386 \\
\hline
\end{tabular}




\begin{tabular}{|c|c|c|c|c|c|c|c|c|c|c|c|c|c|c|}
\hline & 12 & e & 12 & 2.1464 & 0.6363 & 37 & 3.37 & 0.0018 & 0.05 & 0.8572 & 3.4356 & 8.554 & 2.357 & 31.049 \\
\hline $\mathrm{c}$ & 12 & e & 24 & 1.6517 & 0.5336 & 37 & 3.10 & 0.0037 & 0.05 & 0.5705 & 2.7330 & 5.216 & 1.769 & 15,378 \\
\hline c & 12 & $f$ & 6 & -1.0568 & 0.4587 & 37 & $-2,30$ & 0.0269 & 0.05 & -1.9862 & -0.1274 & 0.348 & 0.137 & 0.880 \\
\hline c & 12 & $f$ & 12 & -0.8215 & 0.4058 & 37 & -2.02 & 0.0502 & 0.05 & -1.6438 & 0.000840 & 0.440 & 0.193 & 1.001 \\
\hline c & 12 & if & 24 & -0.9527 & 0.3614 & 37 & $-2.64:$ & 0.0122 & 0.05 & $-1,6850$ & -0.2203 & 0.386 & 0.185 & 0.802 \\
\hline c & 24 & d & 6 & 0.3877 & $0.3841 i$ & 37 & 1.01 & 0.3193 & 0.05 & -0.3905 & 1.1660 & 1.474 & 0.677 & 3.209 \\
\hline$c$ & 24 & d & 12 & 0.09129 & $0.4113^{\prime}$ & ' 37 & 0.22 & 0.8256 & 0.05 & -0.7420 & 0.9246 & 1.096 & 0.476 & 2.521 \\
\hline c & 24 & d & 24 & 1.1881 & 0.5014 & 37 & $2.37 \mathrm{i}$ & | 0.0231 & 0.05 & 0.1722 & 2.2039 & 3.281 & 1.188 & 9.060 \\
\hline c & 24 & e & 6 & 1.2886 & 0.4836 & 37 & 2.61 & 0.0130 & 0.05 & 0.2884 & 2.2887 & 3,628 & 1.334 & 8.862 \\
\hline c & 24 & e & 12 & 1.8691 & 0.6252 & 37 & 2.99 & 0.0049 & 0.05 & 0.6023 & 3.1359 & 6.482 & 1.826 & 23.010 \\
\hline c & 24 & e & 24 & 1.3744 & 0.5204 & 37 & 2.64 & 0.0120 & 0.05 & 0.3200 & 2.4289 & 3.953 & 1.377 & 11,347 \\
\hline c & 24 & f & 6 & -1.3341 & 0.4433 & 37 & -3.01 & 0.0047 & 0.05 & $-2,2322$ & -0.4359 & 0.263 & 0.107 & 0.647 \\
\hline G & 24 & $f$ & 12 & -1.0988 & 0.3883 & 37 & -2.83 & 0.0075 & 0.05 & -1.8856 & -0.3119 & 0.333 & 0.152 & 0.732 \\
\hline$c$ & 24 & $f$ & 24 & -1.2300 & 0.3416 & 37 & $-3,60$ & 0.0009 & 0.05 & -1.9222 & -0.5377 & 0.292 & 0.146 & 0.584 \\
\hline d & 6 & d & 12 & -0.2965 & 0.4296 & 37 & $-0,69$ & 0.4945 & 0.05 & -1.1670 & 0.5741 & 0.743 & 0.311 & 1.775 \\
\hline d & 6 & d & 24 & 0.8003 & 0.5165 & 37 & 1.55 & 0.1298 & 0.05 & -0.2463 & 1.8468 & 2.226 & 0.782 & $6.34 \mathrm{C}$ \\
\hline d & 6 & e & 6 & 0.9008 & 0.5090 & 37 & 1.77 & 0.0850 & 0.05 & -0.1306 & 1,9322 & 2.462 & 0.878 & 6.905 \\
\hline d & 6 & $e$ & 12 & 1.4814 & 0.6375 & 37 & 2.32 & $|0.0257|$ & 0.05 & 0.1898 & 2.7730 & 4.399 & 1.209 & 16.006 \\
\hline d & 6 & $\theta$ & 24 & 0.9867 & 0.5351 & 37 & 1.84 & 0.0732 & 0.05 & -0.09745 & 2.0708 & 2.682 & 0.907 & 7,931 \\
\hline d & 6 & f & 6 & -1.7218 & 0.4604 & 37 & -3.74 & 0.0006 & 0.05 & -2.6546 & .0 .7890 & 0.179 & 0.070 & 0,454 \\
\hline d & 6 & f & 12 & -1.4865 & 0.4077 & 37 & -3.65 & 0.0008 & 0.05 & -2.3127 & $-0,6604$ & 0.226 & 0.089 & 0.517 \\
\hline d & 6 & $f$ & 24 & -1.6177 & 0.3635 & 37 & -4.45 & $\mid<, 0001$ & 0.05 & -2.3543 & $-0.8 B 11$ & 0.198 & 0.095 & 0.414 \\
\hline d & 12 & d & 24 & 1.0968 & 0.5371 & 37 & 2.04 & 0.0483 & 0.05 & 0.008614 & 2.1850 & 2.995 & 1.009 & 8.890 \\
\hline d & 12 & $\theta$ & 6 & 1.1973 & 0.5298 & 37 & 2.26 & 0.0298 & 0.05 & 0.1237 & 2.2708 & 3.311 & 1.132 & 9.687 \\
\hline d & 12 & e & 12 & 1.7778 & 0.6542 & 37 & 2.72 & 0.0099 & 0.05 & 0.4523 & 3.1033 & 5.817 & 1.572 & 22.272 \\
\hline d & 12. & e & 24 & 1.2831 & 0.5549 & 37 & 2.31 & 0.0264 & 0.05 & 0.1588 & 2.4075 & 3.608 & 1.172 & 11.106 \\
\hline d & 12 & $f$ & 6 & -1.4254 & 0.4833 & 37 & -2.95 & 0.0055 & 0.05 & -2.4046 & -0.4461 & 0.240 & 0.090 & 0.640 \\
\hline d & 12 & $f$ & 12 & -1.1901 & 0.4334 & 37 & -2.75 & 0.0093 & 0.05 & -2.0683 & -0.3118 & 0.304 & 0.126 & 0.732 \\
\hline$d$ & 12 & $f$ & 24 & -1.3212 & 0,3922 & 37 & -3.37 & 0.0018 & 0.05 & -2.1158 & -0.5267 & 0.267 & $0.121 i$ & 0.591 \\
\hline d & 24 & e & 8 & 0.1005 & 0.6024 & $37 \mid$ & 0.17 & 0.8684 & 0.05 & -1.1202 & 1.3212 & 1.106 & 0.326 & 3.748 \\
\hline d & 24 & e & 12 & 0.6810 & 0.7143 & 37 & 0.95 & 0.3465 & 0.05 & -0.7662 & 2.1283 & 1.976 & 0.465 & 8.400 \\
\hline d & 24 & $e$ & 24 & 0.1864 & 0.6246 & 37 & 0.301 & 0.7671 & 0.05 & -1.0792 & 1.4519 & . .205 & 0.340 & 4.271 \\
\hline d & 24 & $f$ & 6 & -2.5221 & 0.5619 & 37 & -4.49 & $i<.0001$ & 0.05 & -3.6507 & -1.3835 & $0.080^{:}$ & 0.026 & 0.251 \\
\hline d & 24 & $f$ & 12 & -2.2868 & 0.5197 & 37 & -4.40 & $<.0001$ & 0.05 & -3.3398 & -1.2339 & 0.102 & 0.035 & 0.291 \\
\hline d & 24 & 1 & 24 & -2.4180 & 0.4858 & 37 & -4.98 & $<.0001$ & 0.05 & -3.4023 & -1.4337 & 0.089 & 0.033 & 0.238 \\
\hline$\theta$ & 6 & $\theta$ & 12 & 0.5805 & 0.7089 & 37 & 0.82 & 0.4180 & 0.05 & -0.8557 & 2.0160 & 1.787 & 0.425 & 7.515 \\
\hline$\theta$ & 6 & e & 24 & 0.08588 & 0.6184 & 37 & 0.14 & 0.8903 & 0.05 & -1.1671 & 1.3389 & 1.090 & 0.311 & 3.815 \\
\hline e & 6 & ! & 6 & -2.6226 & 0.5550 & 37 & -4.73 & $<.0001$ & 0.05 & -3.7473 & -1.4980 & 0.073 & 0.024 & 0.224 \\
\hline e & 6 & $f$ & 12 & -2.3873 & 0.5122 & 37 & -4.66 & $<.0001$ & 0.05 & $-3,4252$ & -1.3494 & 0.092 & 0.033 & 0.259 \\
\hline e & 6 & $f$ & 24 & -2.5185 & 0.4778 & 37 & -5.27 & $<.0001$ & 0.05 & -3.4866 & -1.5504 & 0.081 & 0.031 & 0.212 \\
\hline e & 12 & e & 24 & -0.4947 & 0.7278 & 37 & -0.68 & 0.5009 & 0.05 & -1.9693 & 0.9800 & 0.610 & 0.140, & 2.664 \\
\hline e & 12 & f & 6 & -3.2032 & 0.6748 & 37 & -4.75 & $i<.0001$ & 0.05 & -4.5704 & -1.8360 & 0.041 & 0.010 & 0.159 \\
\hline e & 12 & $f$ & 12 & -2.9679 & 0.6400 & 37 & -4.64 & $<, 000$ & 0.05 & -4.2647 & $-1.6711 \mid$ & 0.051 & 0.014 & 0.188 \\
\hline e & 12 & if & 24 & -3.0991 & 0.6128 & 37 & -5.06 & $<.0001$ & 0.05 & -4.3407 & -1.8574 & 0.045 & 0.013 & 0.156 \\
\hline$\theta$ & 24 & $f$ & 6 & -2.7085 & 0.5790 & 37 & -4.68 & $<.0001$ & 0.05 & -3.8817 & -1.5353 & 0.067 & 0.021 & 0.215 \\
\hline e & 24 & if & 12 & -2.4732 & 0.5381 & 37 & -4.60 & $<.0001$ & 0.05 & -3.5635 & -1.3829 & 0.084 & 0.028 & 0.251 \\
\hline
\end{tabular}




\begin{tabular}{|c|c|c|c|c|c|c|c|c|c|c|c|c|c|c|}
\hline & 24 & $f$ & 24 & -2.6044 & 0.5055 & 37 & -5.15 & $<<.0001:$ & 0.05 & -3.6285 & -1.5802 & 0.074 & 0.027 & 0.200 \\
\hline I & 6 & f & 12 & 0.2353 & 0.4639 & $|37|$ & 0.51 & 0.6150 & 0.05 & 0.7047 & 1.1753 & 1.266 & 0.494 & 3.239 \\
\hline 1 & 6 & if & 24 & 0.1041 & 0.4256 & 37 & 0.24 & 0.8081 & 0.05 & -0.7582 & 0.9665 & 1.110 & 0.468 & 2.629 \\
\hline & 12 & if & 24 & -0.1312 & 0.3680 & 37 & -0.36 & 0.7235 & 0.05 & -0.8769 & 0.6145 & $0.877 !$ & 0.416 & 1.849 \\
\hline
\end{tabular}

Performed by J. Luoma; SAS version 9.3 08:43 18MAR15 


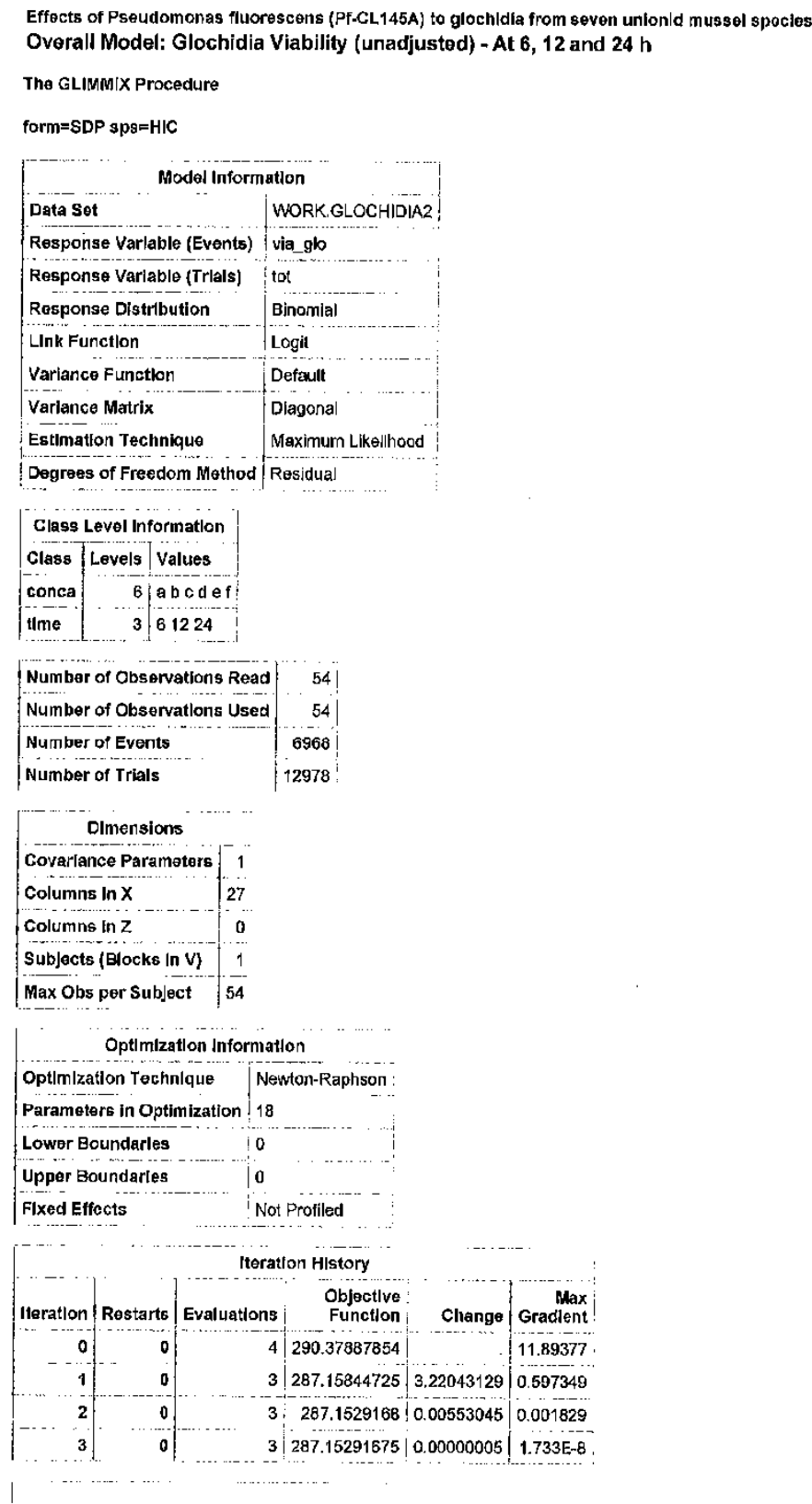

file://C:/Uscrs/JLUOMA/AppData/Local/Temp/1/SAS\%20Temporary\%20Files/. TD4836... 3/18/2015 
| Convergence criterion (GCONV=1E-8) satisied.

\begin{tabular}{|l|c|} 
FIt Stattstlcs & \\
-2 Log Llkellhood & 574.31 \\
\hline AIC (smaller is better) & 610.31 \\
\hline AICC (sinaller Is better) & 629.85 \\
\hline BIC (smaller is better) & 646.11 \\
\hline CAIC (smaller is better) & 664.11 \\
\hline HQIC (smaller Is better) & 624.11 \\
\hline Pearson Chl-Square & 298.77 \\
\hline Pearson ChI-Square I DF & 8.30 \\
\hline
\end{tabular}

Parameter Estimates

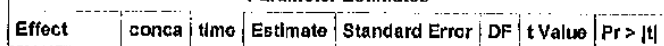

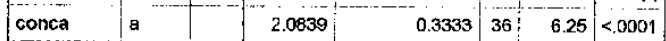

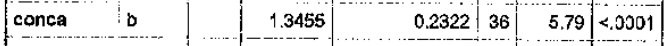

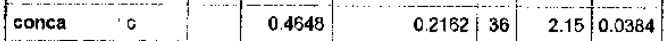

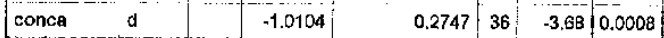

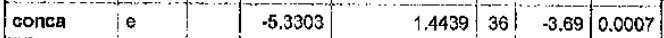

\begin{tabular}{|l|l|l|l|l|l|l|l|l|}
\hline conca & $f$ & 1.8774 & & 0.2789 & 36 & 6.73 & $<0001$
\end{tabular}

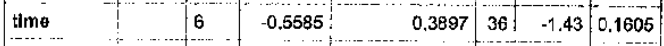

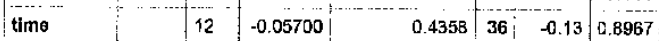

jtime

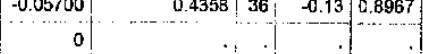

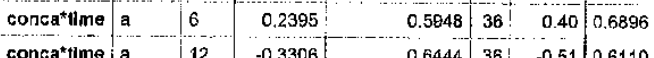

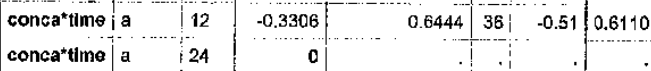

\begin{tabular}{l|l|l|l|l|l|l|l|}
\hline conca"time & b & 6 & -0.3149 & 0.5023 & 36 & -0.63 & 0.5346 \\
\hline
\end{tabular}

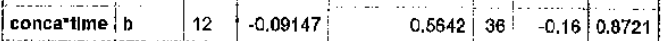

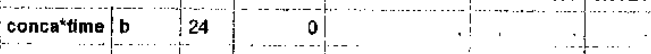

\begin{tabular}{l|l|l|ll|ll|l|} 
conca*tlme & c & 6 & -0.2388 & 0.4979 & 36 & -0.48 & 0.6344
\end{tabular}

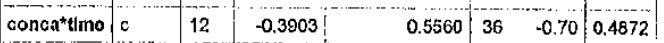

concantime $c$

\begin{tabular}{l|l|l|r|r|r|r|r|} 
conca'tlme & d & 6 & 0.01732 & 0.5531 & 36 & 0.03 & 0.9752
\end{tabular}

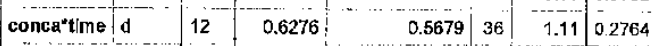

concantmojo

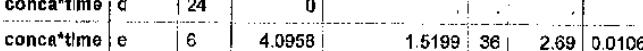

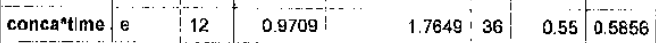

- 4

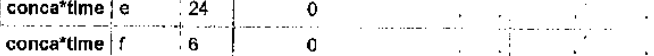

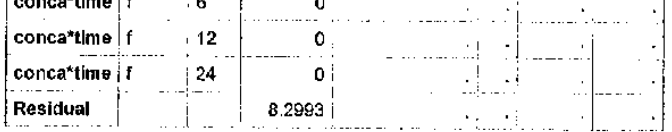

\begin{tabular}{|c|c|c|c|c|}
\hline \multicolumn{5}{|c|}{ Type III Tests of Flxed Effects } \\
\hline Effect & Num DF & Den DF & F Vaiue & $\mathrm{Pr}>\mathrm{F}$ \\
\hline conca & 5 & 36 & 55,04 & $<, \mathrm{CCO1}$ \\
\hline $\operatorname{tim} \theta$ & 2 & 36 & 0.04 & 0.9645 \\
\hline
\end{tabular}

file://C:/Uscrs/JLUOMA/AppData/Local/Temp/1/SAS\%20Temporary\%20Files/ TD4836,.. $3 / 18 / 2015$ 


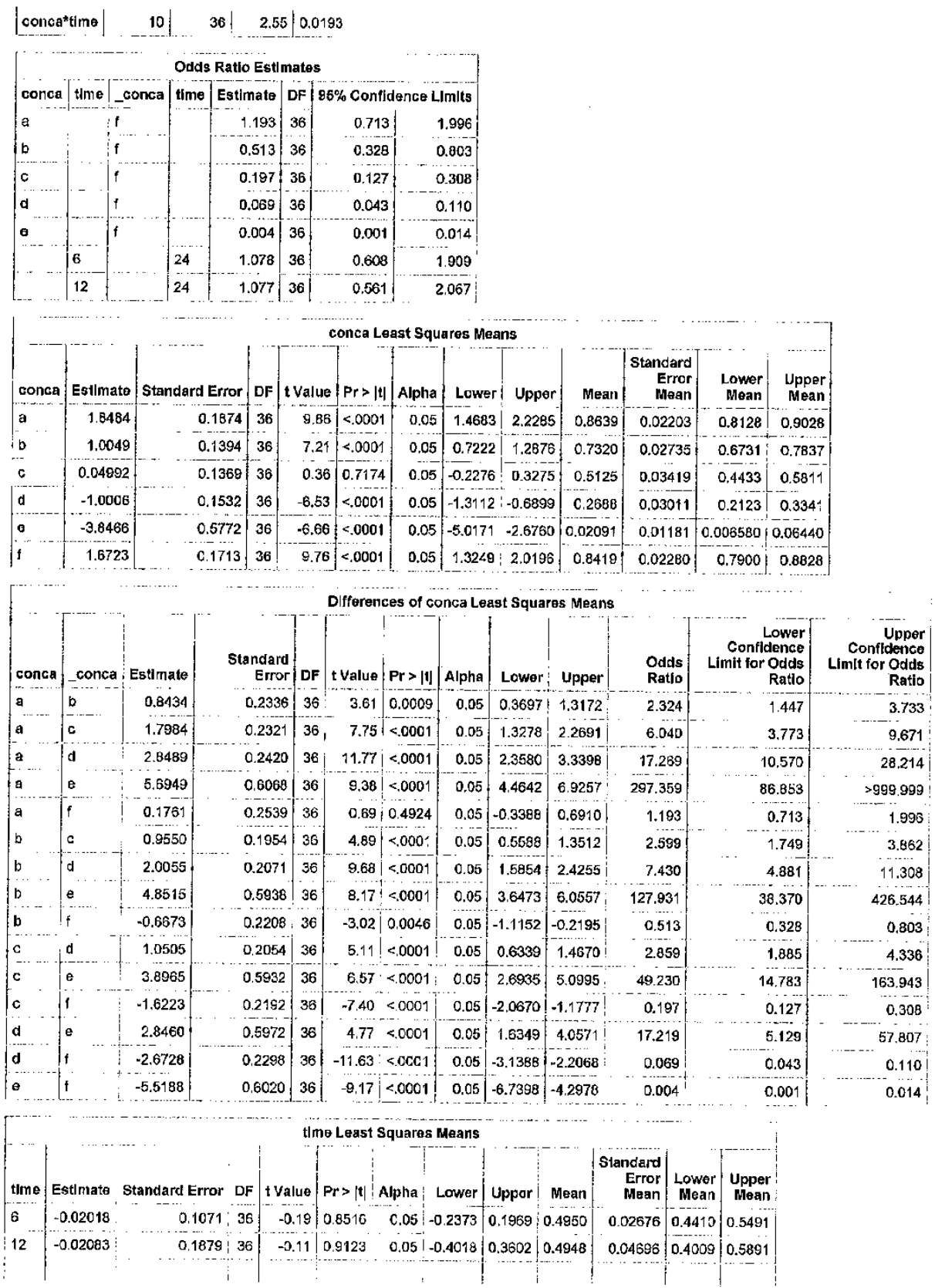

file://C:/Users/JLUOMA/AppDatz/Local/Temp/1/SAS\%20Temporary\%20Files/ TD4836... 3/18/2015 


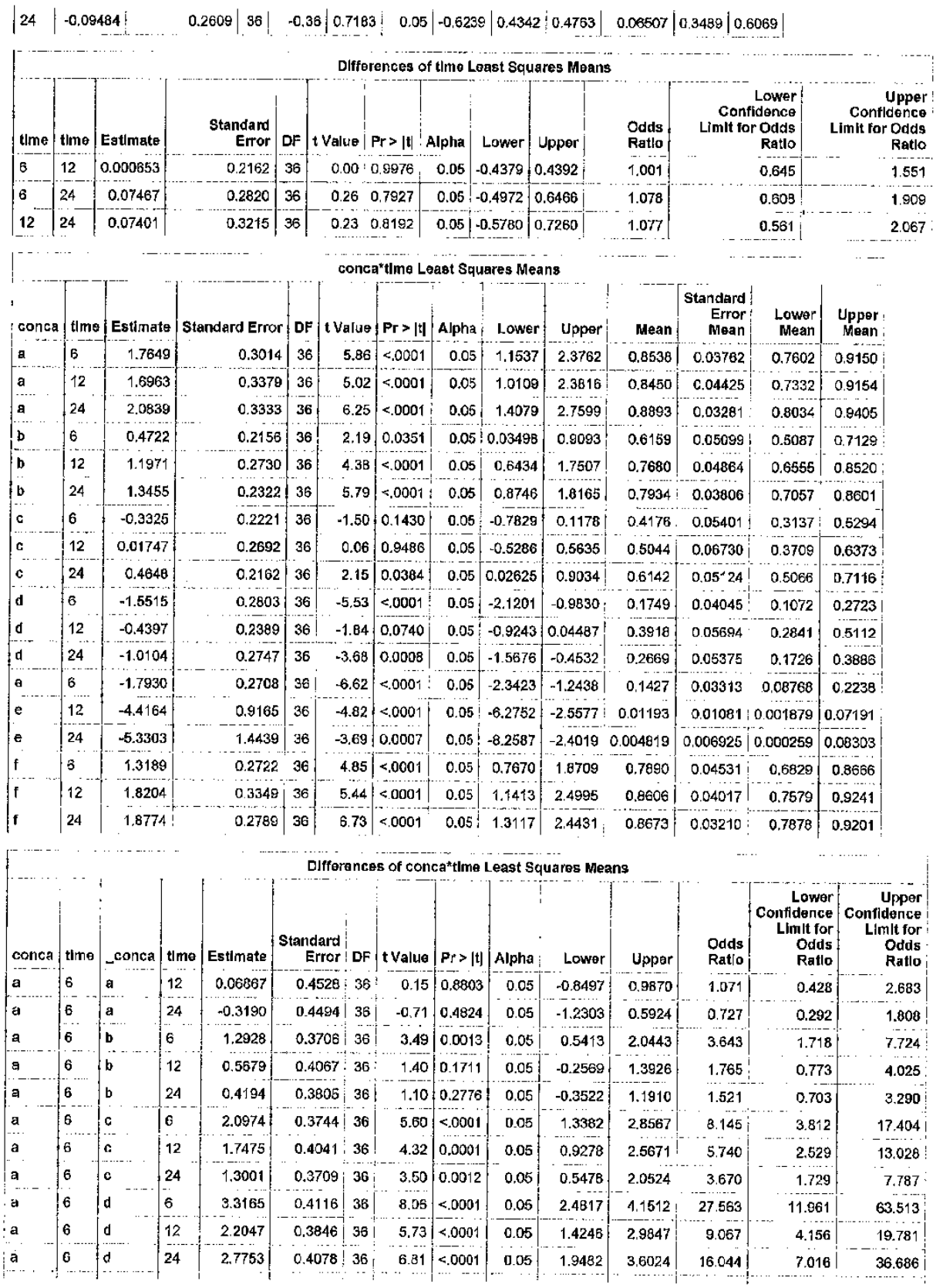




\begin{tabular}{|c|c|c|c|c|c|c|c|c|c|c|c|c|c|c|}
\hline a & 6 & e & 6 & 3.5579 & 0.4052 & 136 & 8.78 & $<, 0001$ & 0.05 & 2.7362 & 4.3797 & 35.091 & 15.428 & 79.815 \\
\hline $\mathbf{a}$ & 6 & e & 12 & 6.1814 & 0.9648 & 36 & 6.41 & $<.0001$ & 0.05 & 4.2247 & 8.1380 & 483.645 & 68.354 & $>999,999$ \\
\hline a & 6 & $\theta$ & 24 & 7.0952 & $\begin{array}{c}1.4750 \\
. .\end{array}$ & 36 & 4.81 & $<.0001$ & 0.05 & $\begin{array}{r}4.1037 \\
\end{array}$ & 10.0867 & $>999.999$ & 60.566 & $>999,999$ \\
\hline a & 6 & i & 6 & 0.4460 & 0.4061 & 36 & 1.10 & 0.2794 & 0.05 & -0.3776 & 1.2698 & 1.562 & 0.685 & 3.569 \\
\hline a & 6 & $f$ & 12 & -0.05549 & 0.4505 & 36 & -0.12 & 0,9027 & 0.05 & -0.9692 & 0.8582 & 0.946 & 0.379 & 2.359 \\
\hline a & 6 & $f$ & 24 & -0.1125 & 0.4107 & 36 & -0.27 & 0.7857 & 0.05 & -0.9453 & 0.7204 & 0.894 & 0.389 & 2.055 \\
\hline a & 12 & a & 24 & -0.3876 & 0.4747 & 36 & -0.82 & 0.4195 & 0.05 & -1.3503 & 0.5750 & 0.679 & 0.259 & 1.777 \\
\hline a & 12 & b. & 6 & $1.224^{4}$ & 0.4008 & 36 & 3.05 & 0.0042 & 0.05 & $c .4112$ & 2.0370 & 3.401 & 1.509 & 7,668 \\
\hline a & 12 & $b$ & 12 & 0.4992 & 0.4344 & 36 & 1.15 & 0.2581 & 0.05 & -0.3819 & 1.3803 & 1.647 & 0.683 & 3.976 \\
\hline a & 12 & b & 24 & 0.3507 & C. 4100 & 36 & C.86 & 0.3980 & 0.05 & -0.4808 & 1.1823 & 1.420 & 0.618 & 3.262 \\
\hline a & 12 & c & 6 & 2.0288 & 0.4044 & 36 & 5.02 & $<.0001$ & 0.05 & 1.2087 & 2.8489 & 7.605 & 3.349 & 17.268 \\
\hline a & 12 & $c$ & 12 & 1.6788 & 0.4321 & 36 & 3.89 & 0.0004 & 0.05 & 0.8025 & 2.5551 & 5.359 & 2.231 & 12.872 \\
\hline a & 12 & c & 24 & 1.2316 & 0.4012 & 36 & 3.07 & 0.0041 & 0.05 & 0.4178 & 2.0451 & 3.426 & 1.518 & 7.730 \\
\hline a & 12 & d & 6 & 3.2478 & 0.4391 & 36 & 7.40 & $<.0001$ & 0.05 & 2.3573 & 4.1383 & 25.734 & 10.563 & 62.694 \\
\hline a & 12 & d & 12 & 2.1360 & 0,4139 & 36 & 5.16 & $<.0001$ & 0.05 & 1.2966 & 2.9754 & 8.465 & 3.657 & 19.597 \\
\hline $\mathbf{a}$ & 12 & d & 24 & 2.7066 & 0.4355 & 36 & 6.21 & $<.0001$ & 0.05 & 1.8234 & 3.5899 & 14.979 & 6.193 & 36.231 \\
\hline a & 12 & e & 6 & -3.4893 & 0.4331 & 36 & 8.06 & $<.0001$ & 0.05 & 2.6110 & 4.3676 & $\begin{array}{r}32.762 \\
\hdashline\end{array}$ & 13.613 & 78.851 \\
\hline a & 12 & e & 12 & 6.1127 & 0.9768 & 36 & 6.26 & $<.0001$ & 0.05 & 4. $\because 316$ & 8.0937 & 451.549 & 62.279 & $>999.999$ \\
\hline a & 12 & e & 24 & 7.0266 & 1.4829 & 36 & 4.74 & $<, 0001$ & 0.05 & 4.0190 & 10.0341 & $>999.999$ & 56.648 & $>999.999$ \\
\hline a & 12 & 1 & 6 & 0.3773 & 0.4339 & 36 & 0.87 & 0.3903 & 0.05 & -0.5027 & 1.2573 & 1.458 & 0.605 & 3.516 \\
\hline a & 12 & $f$ & 12 & -0.1242 & 0.4757 & 36 & -0.26 & 0.7956 & 0.05 & -1.0890 & 0.8407 & 0.883 & 0.337 & 2.318 \\
\hline a & 12. & $f$ & 24 & -0.1812 & 0.4382 & 36 & $-0,41$ & 0.6817 & 0.05 & -1.0698 & 0.7075 & 0.834 & 0.343 & 2.028 \\
\hline a & 24. & b & 6 & 1.6117 & 0.3969 & 36 & 4.06 & 0.0003 & 0.05 & 0.8067 & 2.4168 & 5.012 & 2.241 & 11.210 \\
\hline a & 24 & b & 12 & 0.8868 & 0.4308 & 36 & 2.06 & 0.0468 & 0.05 & 0.01306 & 1.7600 & 2,427 & 1.013 & 5.810 \\
\hline$a$ & 24 & $b$ & 24 & 0.7384 & 0.4062 & | $36 !$ & 182 & 0.0774 & 0.05 & -0.08548 & 1.5622 & 2.093 & 0.918 & 4.769 \\
\hline a & 24 & $c$ & 6 & 2.4164 & 0.4005 & 36 & 6.03 & $<<0001$ & 0.05 & 1.6041 & 3.2287 & 11.206 & 4.974 & 25.246 \\
\hline a & 24 & $c$ & 12 & 2,0664 & 0.4285 & 36 & 4.82 & $<.0001$ & 0.05 & 1.1975 & 2.9354 & 7.897 & 3.312 & 18.829 \\
\hline a & 24 & $c$ & 24 & 1.6191 & 0.3973 & 36 & 4.08 & 0.0002 & 0.05 & 0,8133 & 2.4249 & 5.049 & 2.255 & 11,301 \\
\hline a & 24 & $d$ & 6 & 3.6354 & 0.4355 & | 36 & 8.35 & $<, 0001$ & 0.05 & 2.7522 & 4.5187 & 37.919 & 15.677 & 91.717 \\
\hline$a$ & 24 & $d$ & 12 & 2.5236 & 0.4101 & 36 & 6.15 & $<<0001$ & 0.05 & 1.6919 & 3.3554 & 12.474 & 5.430 & 28.656 \\
\hline a & 24 & d & 24 & 3.0943 & 0.4319 & 36 & 7.16 & $<.0001$ & 0.05 & 2.2183 & 3.9703 & 22.071 & 9.191 & 53.000 \\
\hline a & 24 & e & 6 & 3.8769 & 0.4295 & 36 & 9.03 & $<, 0001$ & 0.05 & 3.0059 & 4.7479 & 48.275 & 20.205 & 115.342 \\
\hline a & 24 & e & 12 & 6.5003 & 0.9752 & 36 & 6.67 & $<0001$ & 0.05 & 4.5225 & 8.4782 & 665.357 & 92.065 & $>999.999$ \\
\hline a & 24 & e & 24 & 7.4142 & 1.4819 & 36 & 5.00 & $<.0001$ & 0.05 & 4.4088 & 10.4196 & $>999.999$ & 82.172 & $>999.599$ \\
\hline a & 24 & 1 & 6 & 0.7650 & 0.4303 & $36 \mid$ & 1.78 & 0.0839 & 0.05 & -0.1078 & 1.6377 & 2.149 & 0.898 & 5.143 \\
\hline a & 24 & 1 & 12 & 0.2635 & 0.4725 & 36 & 0.56 & 0.5805 & 0.05 & -0.6947 & $1.2217 \mid$ & 1.301 & 0.499 & ${ }^{3.393}$ \\
\hline a & 24 & $f$ & 24 & 0.2065 & 0.4346 & 36 & 0.48 & 0.6376 & 0.05 & -0.6750 & 1.0879 & 1.229 & 0.509 & 2.968 \\
\hline b. & 6 & $b$ & 12 & -0.7219 & 0.3478 & 36 & -2.08 & 0.0443 & 0.05 & -1.4303 & -0.01944 & $0.484 !$ & 0.239 & 0.981 \\
\hline$b$ & 6 & $b$ & 24 & -0.8734 & 0.3168 & 30 & $-2,78$ & 0.0091 & 0.05 & -1.5159 & -0.2308 & 0.418 & 0.220 & 0.794 \\
\hline b & 6 & c & 6 & 0.8047 & 0.3095 & 36 & 2.60 & 0.0134 & 0.05 & 0.1770 & 1.4323 & $2.236 \mid$ & 1.194. & 4.188 \\
\hline$b$ & 6 & c & 12 & 0.4547 & 0.3449 & $36 !$ & 1.32 & 0.1957 & 0.05 & -0.2448 & 1.1542 & 1.576 & 0.783 & 3.171 \\
\hline b & 6 & c & 24 & 0.007357 & 0.3053 & 36 & 0.02 & $\mid 0.9809$ & 0.05 & -0.6119 & 0.6266 . & 1.007 & 0.542 & 1.871 \\
\hline b & 6 & $d$ & 6 & 2.0237 & 0.3536 & 36 & 5.72 & $<.0001$ & 0.05 & 1.3065 & 2,7409 . & 7.566 & 3.693 & $\begin{array}{ll}15.500 \\
.\end{array}$ \\
\hline b & 6. & d & 12 & 0.9119 & 0.3218 & 36 & 2.83 & 0.0075 & 0.05 & 0.2592 & 1.5646 & 2.489 & 1.296 & 4.781 \\
\hline b. & 6 & d & 24 & 1.4825 & 0.3492 & 36 & 4.25 & 0.0001 & 0.05 & 0.7743 & 2.1907 & 4.404 & $2.169:$ & 8.942 \\
\hline b & 6 & e & 6 & 2.2652 & 0.3461 & 36 & 6.54 & $<.0001$ & 0.05 & 1.5632 & 2.9672 & 9.633 & 4.774 & 19.437 \\
\hline
\end{tabular}




\begin{tabular}{|c|c|c|c|c|c|c|c|c|c|c|c|c|c|c|}
\hline & 6 & e & 12 & 4.8886 & 0.9415 & 36 & 5.19 & $<.0001$ & 0.05 & 2.9791 & 6.7980 & 132.766 & 19.671 & 896.078 \\
\hline b & 6 & e & 24 & 5.8025 & 1.4599 & 36 & 3.97 & 0.0003 & 0.05 & 2.8416 & 8.7633 & 331.112 & 17.144 & $>999.999$ \\
\hline b & 6 & f & 6 & $-0,8468$ & 0.3472 & 36 & -2.44 & 0.0198 & 0.05 & -1.5509 & -0.1426 & 0.429 & 0.212 & 0.867 \\
\hline b & 6 & $f$ & 12 & -1.3483 & 0.3982 & 38 & -3.39 & 0.0017 & 0.05 & -2.1559 & -0.5406 & 0.260 & 0.116 & 0.582 \\
\hline b & 6 & 1 & 24 & -1.4053 & 0.3625 & 36 & -3.99 & 0,0003 & 0.05 & -2.1202 & -0.6003 & 0.245 & 0.120 & 0.501 \\
\hline b & 12 & b & 24 & -0.1485 & 0.3584 & 36 & -0.41 & 0.6811 & 0.05 & -0.8753 & 0.5784 & 0.862 & 0417 & 1.783 \\
\hline b & 12 & c & 6 & 1.5296 & 0.3519 & 36 & 4.35 & 0.0001 & 0.05 & 0.8159 & 2.2433 & 4.616 & 2.261 & 9.424 \\
\hline$b$ & 12 & c & 12 & 1.1796 & 0.3834 & 36 & 3.08 & 0.0040 & 0.05 & 0.4020 & 1.9572 & 3.253 & 1.495 & 7.080 \\
\hline b & 12 & c & 24 & 0.7323 & 0.3483 & 36 & 2.10 & 0.0426 & 0.05 & 0.02595 & 1.4386 & 2.080 & 1.026 & 4.215 \\
\hline b & 12 & d & 6 & 2.7486 & 0.3913 & 36 & 7.02 & $<.0001$ & 0.05 & 1.9550 & 3.5422 & 15.621 & 7.064 & 34.541 \\
\hline b & 12 & d & 12 & 1.6368 & 0.3628 & 36 & 4.51 & $<<.0001$ & 0.05 & 0.9010 & 2.3726 & 5.139 & 2.462 & 10.725 \\
\hline b & 12 & $d$ & 24 & 2.2074 & 0.3873 & 36 & 5.70 & $<.0001$ & 0.05 & 1.4220 & 2.9929 & 9.092 & 4.145 & 19.944 \\
\hline b & 12 & $\theta$ & 6 & 2.9901 & 0.3845 & 36 & 7.78 & $<, 0001$ & 0.05 & 2.2102 & 3.7699 & 19.887 & 9.118 & 43.378 \\
\hline b & 12 & $\theta$ & 12 & 5.6135 & 0.9563 & 36 & 5.87 & $<.0001$ & 0.05 & 3.6741 & 7.5529 & 274.097 & 39.411 & $>999.999$ \\
\hline b & 12 & e & 24 & 6.5274 & 1.4695 & 36 & 4.44 & $<, 0001$ & 0.05 & 3.5471 & 9.5076 & 683.586 & 34.712 & $>999.999$ \\
\hline b & 12 & f & 5 & -0.1219 & 0.3855 & 36 & -0.32 & 0.7537 & 0.05 & -0.9037 & 0.6599 & 0.885 & 0.405 & 1.935 \\
\hline b & 12 & $f$ & 12 & -0.6234 & 0.4320 & 36 & -1.44 & 0.1577 & 0.05 & -1.4096 & 0.2529 & 0.536 & 0.223 & 1.288 \\
\hline b & 12 & $f$ & 24 & $-0,6804$ & 0.3903 & 36 & -1.74 & $\mid 0.0898$ & 0.05 & -1.4719 & 0.1112 & 0.506 & 0.229 & 1.118 \\
\hline b & 24 & c & 6 & 1.6780 & 0.3213 & 36. & 5.22 & $<.0001$ & 0.05 & 1.0264 & 2.3297 & 5.355 & 2.791 & 10.274 \\
\hline b & 24 & c & 12 & 1.3281 & 0.3555 & 36 & 3.74 & 0.0006 & 0.05 & 0.6070 & 2.0491 & 3.774 & 1.835 & 7.781 \\
\hline b & 24 & c & 24 & 0.8807 & 0.3173 & 36 & 2.78 & 0.0087 & 0.05 & 0.2372 & 1.5242 & 2.413 & 1.268 & 4.592 \\
\hline b & 24 & d & 6 & 2.8971 & 0.3640 & 36 & 7.96 & $<, 0001$ & 0.05 & 2.1588 & 3.6353 & 18.121 & 8.661 & 37.913 \\
\hline b & 24 & d & 12 & 1.7853 & 0.3332 & 36 & 5.36 & $<, 0001$ & 0.05 & 1.1095 & 2.4610 & 5.961 & 3.033 & 11.717 \\
\hline b & 24 & $d$ & 24 & 2,3559 & 0.3597 & 36 & 6.55 & $<, 0001$ & 0.05 & 1.6264 & 3.0854 & 10.548 & 5.085 & 21.877 \\
\hline$b$ & 24 & e & 6 & 3.1385 & $0.3667 !$ & 36 & 8.80 & $<<, 0001$ & 0.05 & 2.4151 & 3.8620 & 23.070 & 11.190 & 47.562 \\
\hline b & 24 & e & 12 & 5.7620 & 0.9454 & 36 & 6.09 & $<0001$ & 0.05 & 3,8445 & 7.6794 & 317.969 & 46.735 & $>999.999$ \\
\hline b & 24 & e & 24 & 6.6753 & 1.4625 & 36 & 4.56 & $<.0001$ & 0.05 & 3,7098 & 9.6418 & 793.003 & 40.847 & $>999.999$ \\
\hline b & 24 & $f$ & 6 & 0.02659 & 0.3578 & 36 & 0.07 & 0.9412 & 0.05 & -0.6990 & 0.7522 & 1.027 & 0.497 & 2.122 \\
\hline b & 24 & $f$ & 12 & -0.1749 & 0.4075 & 36 & -1.17 & 0.2515 & 0.05 & -1.3013 & 0.3515 & 0.622 & 0.272 & 1.421 \\
\hline b & 24 & 1 & 24 & -0.5319 & 0.3629 & 36 & -1.47 & 0.1514 & 0.05 & -1.2679 & 0.2042 & 0.587 & 0.281 & 1.226 \\
\hline$c$ & 6 & c & 12 & -0.3500 & 0.3490 & 36 & -1.00 & 0.3226 & 0.05 & -1.0578 & 0.3578 & 0.705 & 0.347 & 1.430 \\
\hline c & 6 & c & 24 & -0.7973 & 0.3100 & 38 & -2.57 & 0.0144 & 0.05 & -1.4259 & -0.1687 & 0.451 & 0.240 & 0.845 \\
\hline$c$ & 6 & d & 6 & 1.2190 & 0.3576 & 36 & 3.41 & 0.0016 & 0.05 & 0.4938 & 1.9443 & 3.384 & 1.638 & 6.989 \\
\hline c & 6 & d & 12 & 0.1072 & 0.3262 & $36 !$ & 0.33 & 0.7443 & 0.05 & -0.5543 & 0.7688 & 1.113 & 0.574 & 2.157 \\
\hline$c$ & 6 & d & 24 & 0.6779 & 0.3532 & 36 & 1.92 & 0.0629 & 0.05 & -0.03854 & 1.3943 & 1,970 & 0.962 & 4.032 \\
\hline$c$ & 6 & e & 6 & 1.4605 & 0.3502 & 36 & 4.17 & 0.0002 & 0.05 & 0.7502 & 2.1708 & 4.308 & 2.118 & 8.765 \\
\hline c & 6 & e & 12 & 4.0839 & 0.9430 & 36 & 4.33 & 0.0001 & 0.05 & 2.1714 & $5.9964 i$ & 59.378 & 8.771 & 401.988 \\
\hline $\mathrm{c}$ & 6 & $a$ & 24 & 4.9978 & 1.4509 & 36 & 3.42 & 0.0016 & 0.05 & 2.0350 & 7.9806 & 148.086 & 7.652 & $>999.999$ \\
\hline c & 6 & if & 6 & -1.6514 & 0.3513 & 36 & -4.70 & $<.0001$ & 0.05 & -2.3538 & -0.9391 & 0.192 & 0.094 & 0.391 \\
\hline C & 6 & I & 12 & -2.1529 & 0.4018 & 36 & -5.36 & $<, 0001$ & 0.05 & -2.9678 & $-1,3380$ & 0.116 & 0.051 & 0.262 \\
\hline 5 & 6 & $f$ & 24 & -2.2099 & 0.3565 & 36 & -6.20 & $<, 0001$ & 0.05 & -2.9330 & -1.4869 & 0.110 & 0.053 & 0.226 \\
\hline 6 & 12 & c & 24 & -0.4473 & 0.3453 & 36 & -1.30 & 0.2034 & 0.05 & -1.1477 & 0.2530 & 0.639 & 0.317 & 1.288 \\
\hline c & 12 & d & 6 & 1.5690 & 0.3887 & 36 & 4.04 & 0.0003 & 0.05 & 0.7807 & 2.3573 & 4.802 & 2.183 & 10.562 \\
\hline 6 & 12 & d & 12 & 0.4572 & 0.3600 & 36 & 1.27 & 0.2122 & 0.05 & -0.2729 & 1.1873 & 1.580 & 0.761 & 3.278 \\
\hline & 12 & d & 24 & 1.0279 & 0.3847 & 36 & 2.67 & 0.0113 & 0.05 & 0.2477 & 1.8080 & 2.795 & 1.281 & 6.058 \\
\hline & 12 & $\theta$ & 6 & 1.8105 & 0.3819 & 36 & 4.74 & $<.0001$ & 0.05 & 1.0360 & 2.5850 & 6.113 & 2.818 & 13.263 \\
\hline
\end{tabular}




\begin{tabular}{|c|c|c|c|c|c|c|c|c|c|c|c|c|c|c|}
\hline & 12 & e & 12 & 4.4339 & 0.9552 & 36 & 4.64 & $<.0001$ & 0.05 & 2.4966 & 6.3712 & 84.259 & 12.141 & 584.738 \\
\hline$c$ & 12 & ; & 24 & 5.3478 & 1.4688 & 36 & 3.64 & 0.0008 & 0.05 & 2.3689 & 8.3266 & 210.139 & 10.686 & $>999.999$ \\
\hline c & 12 & if & 6 & -1.3015 & 0.3828 & 36 & -3.40 & 0.0017 & 0.05 & -2.0779 & -0.5250 & 0.272 & 0.125 & 0.592 \\
\hline$c$ & 12 & 1 & 12 & -1.8029 & 0.4297 & 36 & -4.20 & 0.0002 & 0.05 & -2.6744 & -0.9315 & 0.165 & 0.069 & 0,394 \\
\hline c & 12 & $f$ & 24 & -1.8599 & 0.3877 & 36 & -4.80 & $<.0001$ & 0.05 & -2.6462 & -1.0737 & 0.156 & 0.071 & 0.342 \\
\hline$c$ & 24 & d & 6 & 2.0163 & 0.3540 & 36 & 5.70 & $<.0001$ & 0.05 & 1.2983 & 2.7344 & 7,511 & 3.663 & 15.400 \\
\hline c & 24 & d & 12 & 0.9045 & 0.3223 & 36 & 2.81 & 0.0030 & 0.05 & 0.2510 & 1.5581 & 2.471 & 1.285 & 4.750 \\
\hline c & 24 & $d$ & 24 & 1.4752 & 0.3496 & 30 & 4.22 & 0.0002 & 0.05 & 0.7661 & 2.1842 & 4.372 & 2.151 & 8.884 \\
\hline c & 24 & e & 6 & 2.2578 & 0.3466 & 36 & 6.52 & $<.0001$ & 0.05 & 1.5550 & 2,0307 & 9.562 & 4.735 & 19.311 \\
\hline c & 24 & $\theta$ & 12 & 4,8812 & 0.9417 & 36 & 5.18 & $<<.0001$ & 0.05 & 2.9715 & 6.7910 & 131.792 & 19.521 & 889.791 \\
\hline c & 24 & $\theta$ & 24 & 5.7951 & 1.4600 & 36 & 3.97 & 0.0003 & 0.05 & 2.8341 & 8.7561 & 328.685 & 17.015 & $>999,899$ \\
\hline c & 24 & if & 6 & -0.8541 & 0.3476 & 36 & $-2,46$ & 0.0190 & 0.05 & -1.5591 & -0.1491 & 0.426 & 0.210 & 0.861 \\
\hline c & 24 & $f$ & 12 & -1.3556 & 0.3986 & 36 & -3.40 & 0.0017 & 0.05 & -2.1640 & -0.5472 & 0.258 & 0.115 & 0.578 \\
\hline c & 24 & $f$ & 24 & -1.4126 & 0.3529 & 36 & -4.00 & 0.0003 & 0.05 & -2.1284 & -0.6968 & 0.244 & 0.119 & 0.498 \\
\hline d & 6 & d & 12 & -1.1118 & 0.3883 & 36 & -3.02 & 0.0046 & 0.05 & -1.8588 & -0.3648 & 0.329 & 0.156 & 0.694 \\
\hline d & 6 & d & 24 & -0.5412 & 0.3925 & 36 & -1.38 & 0.1765 & 0.05 & -1.3372 & 0.2549 & 0.582 & 0.263 & $1.2 \mathrm{EC}$ \\
\hline d & 6 & 0 & 6 & 0.2415 & 0.3898 & 36 & 0.62 & 0.5395 & 0.05 & -0.5490 & 1.0320 & 1.273 & 0.578 & 2.807 \\
\hline d & 6 & $\theta$ & 12 & 2.8649 & $c .9584$ & 36 & 2.99 & 0.0050 & 0.05 & 0.9212 & 4.8086 & 17.547 & 2.512 & 122,561 \\
\hline d & 6 & e & 24 & 3.7788 & 1.4709 & 36 & 2.57 & 0.0145 & 0.05 & C. 7957 & 6.7618 & 43.762 & 2.216 & 864.202 \\
\hline d & 6 & f & 5 & -2.8705 & 0.3907 & 36 & -7.35 & $<<.0001$ & 0.05 & -3.6629 & -2.0781 & 0.057 & 0.026 & 0.125 \\
\hline d & 6 & $f$ & 12 & -3.3720 & 0.4367 & 38 & -7.72 & $<.0001$ & 0.05 & -4.2576 & -2.4863 & 0.034 & 0.014 & 0.083 \\
\hline d & 6 & f & .24 & -3.4290 & 0.3954 & 36 & $-8,67$ & $<.0001$ & 0.05 & -4.2310 & -2.6270 & 0.032 & 0.015 & 0.072 \\
\hline d & 12 & $d$ & 2.4 & 0.5706 & 0.3641 & 36 & 1.57 & 0.1258 & 0.05 & -0.1678 & 1.3091 & 1.769 & 0.846 & 3.703 \\
\hline d & 12 & $\theta$ & 6 & 1.3533 & 0.3612 & 36. & 3.75 & 0.0006 & 0.05 & 0.6208 & 2.0857 & 3.870 & $\AA .860$ & 8.051 \\
\hline d & 12 & e & 12 & 3.9767 & 0.9471 & $36 !$ & 4.20 & 0,0002 & 0.05 & 2.0558 & 5.8975 & 53.340 & 7.813 & 364.143 \\
\hline d & 12 & $\theta$ & 24 & 4.8906 & 1.4635 & 36 & 3.34 & 0.0020 & 0.06 & 1.9224 & 7.8588 & 133.028 & 6.837 & $>999.999$ \\
\hline d & 12 & $f$ & 6 & -1.7587 & $0.3622:$ & 36 & -4.86 & $<<.0001$ & 0.05 & $-2,4932$ & -1.0242 & 0.172 & 0.083 & 0.359 \\
\hline d & 12 & f & 12. & -2.2602 & 0.4114 & 36 & -5.49 & $<<, 0001$ & 0.05 & -3.0945 & -1.4259 & 0.104 & 0.045 & 0.240 \\
\hline d & 12 & f & 24 & -2.3172 & 0.3673 & 36 & -0.31 & $<, 0001$ & 0.05 & -3.0620 & -1.5723 & 0.099 & 0.047 & $0.20 B$ \\
\hline d & 24 & e & 6 & 0.7826 & 0.3858 & 36 & 2.03 & 0.0499 & 0.05 & 0.000275 & 1.5650 & 2.187 & 1.000 & 4.783 \\
\hline d & 24 & $\theta$ & 12 & 3.4060 & 0.9568 & 36 & 3.56 & 0.0011 & 0.05 & 1.4656 & 5.3465 & 30.146 & 4.330 & 209.857 \\
\hline d & 24 & e & 24 & 4.3199 & 1.4698 & 36 & 2.94 & 0.0057 & 0.05 & 1.3390 & 7.3008 & 75.182 & 3.815 & $>999.999$ \\
\hline d & 24 & i & 6 & $-2,3293$ & 0.3867 & 36 & -6.02 & $1<0001$ & 0.05 & -3.1136 & -1.5450 & $c, 097$ & 0.044 & 0.213 \\
\hline d & 24 & $f$ & 12 & -2.8308 & 0.4331 & 36 & -6.54 & $i<.0001$ & 0.05 & -3.7092 & -1.9524 & 0.059 & 0.024 & 0.142 \\
\hline d & 24 & f & 24 & -2.8878 & 0.3915 & 36 & $-7,38$ & $\mid<.0001$ & 0.05 & -3.6818 & $-2,0938$ & 0.056 & C.025 & 0.123 \\
\hline$\theta$ & 6 & 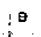 & 12 & 2.6234 & 0.9557 & 36 & 2.75 & 0.0094 & 0.05 & 0.6852 & 4.5616 & 13.783 & 1.984 & 95.734 \\
\hline$e$ & 6 & $\theta$ & 24 & 3.5373 & 1.4691 & 36 & 2.41 & 0.0213 & 0.05 & 0,5578 & 6.5167 & 34.373 & 1.747 & 676.353 \\
\hline$\theta$ & 6 & $f$ & 6 & -3.1120 & 0.3839 & 36 & -8.11 & $<, 0001$ & 0.05 & $-3.8 \mathrm{ECG}$ & -2.3333 & 0.045 & 0.020 & 0.097 \\
\hline e & 6 & $f$ & 12 & -3.6134 & 0.4307 & 36 & -8.39 & $<.0001$ & 0.05 & -4.4869 & -2.7400 & 0.027 & 0.011 & 0.065 \\
\hline e & 6 & $f$ & 24 & -3.6704 & 0.3888 & $36 !$ & -9.44 & $<.0001$ & $0.05:$ & -4.4589 & $-2,8820$ & 0.025 & 0.012 & 0.050 \\
\hline e & 12 & 10 & 24 & 0.9139 & 1.7102 & 36 & 0.53 & 0.5964 & 0.05 & -2.5546 & 4.3823 & 2.494 & 0.078 & 80.025 \\
\hline e & 12 & i & 6 & -5.7354 & 0.9560 & 36 & -6.00 & $<.0001$ & 0.05 & -7.6743 & -3.7964 & 0.003 & $<0.001$ & 0.022 \\
\hline e & 12 & f & 12 & -6.2368 & 0.9757 & 36 & -6.39 & $<.0001$ & 0.05 & -8.2157 & -4.2579 & 0.002 & $<0.001$ & 0.014 \\
\hline$\theta$ & 12 & 1 & 24 & -6.2938 & 0.9580 & 36 & -6.57 & $<.0001$ & 0.05 & -8.2367 & -4.3509 & 0.002 & $<0.001$ & 0.013 \\
\hline e & 24 & if & 6 & -6.6492 & 1.4693 & 36 & -4.53 & $<.0001$ & 0.05 & -9.6292 & -3.6683 & 0.001 & $<0.001$ & 0.025 \\
\hline 0 & 24 & $f$ & 12 & -7.1507 & 1.4822 & 38 & -4.82 & $<, 0001$ & 0.05 & -10.1568 & -4.1446 & $<0.001$ & $<0.001$ & 0.016 \\
\hline
\end{tabular}




\begin{tabular}{|c|c|c|c|c|c|c|c|c|c|c|c|c|c|c|}
\hline & 24 & $f$ & 24 & $-7,2077 \mid$ & 1.4708 & 36 & -4.90 & $<.0001$ & $0.05 !$ & -10.1902 & -4.2252 & $<0.001$ & $<0.001$ & 0.015 \\
\hline f & 6 & $f$ & 12 & -0.5015 & 0.4315 & 36 & -1.16 & 0.2528 & 0.05. & $-1,3766$ & 0.3737 & 0.606 & 0.252 & 1.453 \\
\hline$f$ & 6 & $f$ & 24 & -0.5585 & 0.3897 & 36 & -1.43 & 0.1605 & 0.05 & -1.3488 & 0.2319 & 0.572 & 0.260 & 1.261 \\
\hline & 12 & if & 24 & -0.05700 & 0.4358 ! & 36 & -0.13 & | $0.8967 \mid$ & 0.05 & -0.9409 & 0.8269 & 0.945 & 0.380 & 2.288 \\
\hline
\end{tabular}

Performed by J. Luome; SAS version 9.3 08:43 18MAR15 


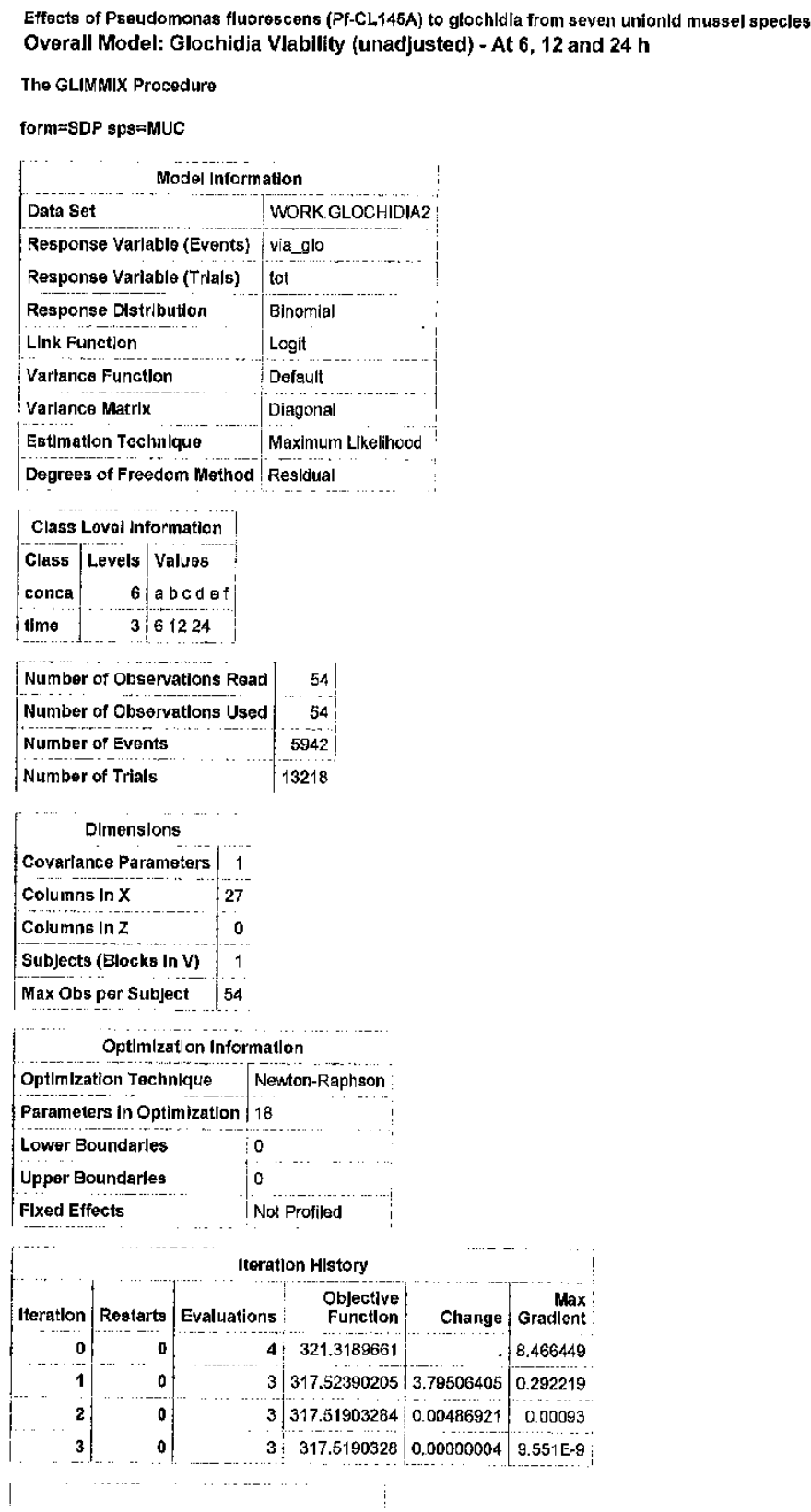

file://C:/Users/J_UOMA/AppData/Local/Temp/1/SAS\%20Temporary\%20Files/_TD4836... 3/18/2015 
| Convergence criterion (GCONV=1E-8) salisf ed, |

\begin{tabular}{|l|c|}
\hline FIt Statlstics & \\
\hline 2 Log Llkellhood & 635.04 \\
\hline AIC (smaller is better) & 671.04 \\
\hline AICC (smaller Is better) & 690.58 \\
\hline BIC (smallor is better) & 706.84 \\
\hline CAIC (smaller Is better) & 724.84 \\
\hline HQIC (smaller is botter) & 684.85 \\
\hline Poarson ChI-Square & 355.52 \\
\hline Pearson ChI-Square IDF & 9.88 \\
\hline
\end{tabular}

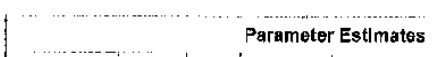

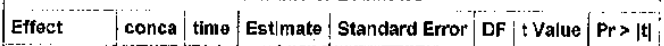

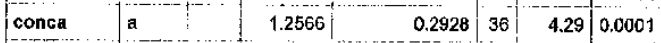

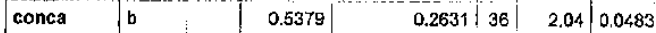

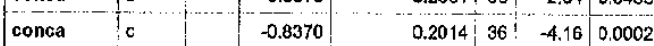

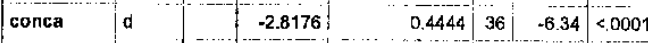
conca $|a|-|-2.8346|-20.4989|36|-5.68 \mid<, 0001$ \begin{tabular}{l|l|l|l|l|l|l|l} 
conca & $f$ & 2.5539 & $0.4110: 36$ & $6.21:<0001$
\end{tabular}

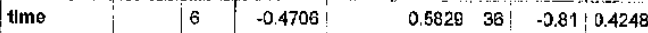
\begin{tabular}{l|l|l|l|l|l|l}
\hline time & 12 & 0.2744, & 0.6779 & 36 & 0.40 & 0.6880
\end{tabular}

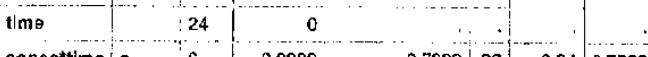

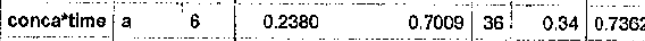

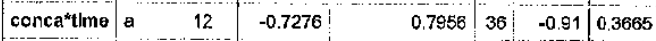
conca"time a 24

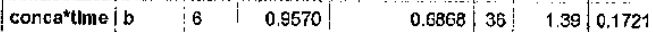
\begin{tabular}{l|llll|l|l|l|l|l}
\hline concatelme & $\mathrm{b}$ & 12 & -0.1694 & 0.7787 & 36 & $-0,22$ & 0.8291
\end{tabular} cancatimo b 24

\begin{tabular}{l|l|l|l|l|l|l|l|} 
conca*time & $c$ & 6 & 0.6457 & 0.6646 & 36 & 0.97 & 0.3378 \\
\hline
\end{tabular}

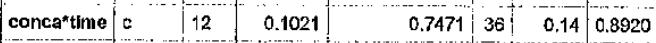
conca*tme $c \quad 24$

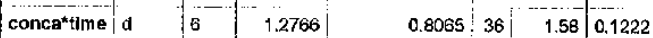

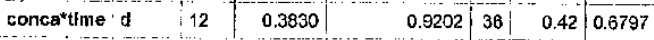
conca*tlme of

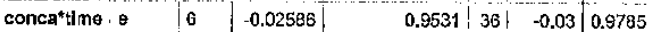

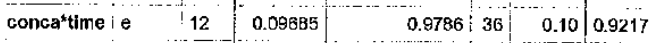
conca*time $e_{\text {e }}$ \begin{tabular}{l|l|l|l|l|l}
\hline concatime & $f$ & 6 & & 0 \\
\hline
\end{tabular} concaktime $\left.\right|_{f} \quad 12 \quad \mid-1$ concrtilme ir 24 Residual 9.8757 -

…............................

\begin{tabular}{|l|r|r|r|r|}
\multicolumn{3}{|c|}{ Type III Tests of Flxed Effects } \\
\hline Effect & Num DF & Den DF & F Value & Pr $>$ F \\
\hline conca & 5 & 36 & 72.78 & $<.0001$ \\
\hdashline tlme & 2 & 36 & 0.55 & 0.5814 \\
& & & &
\end{tabular}

file://C:/Users/JLUOMN/AppData/Local/T'Cmp/1/SAS\%20Temporary\%20Files/_TD4836... 3/18/2015 


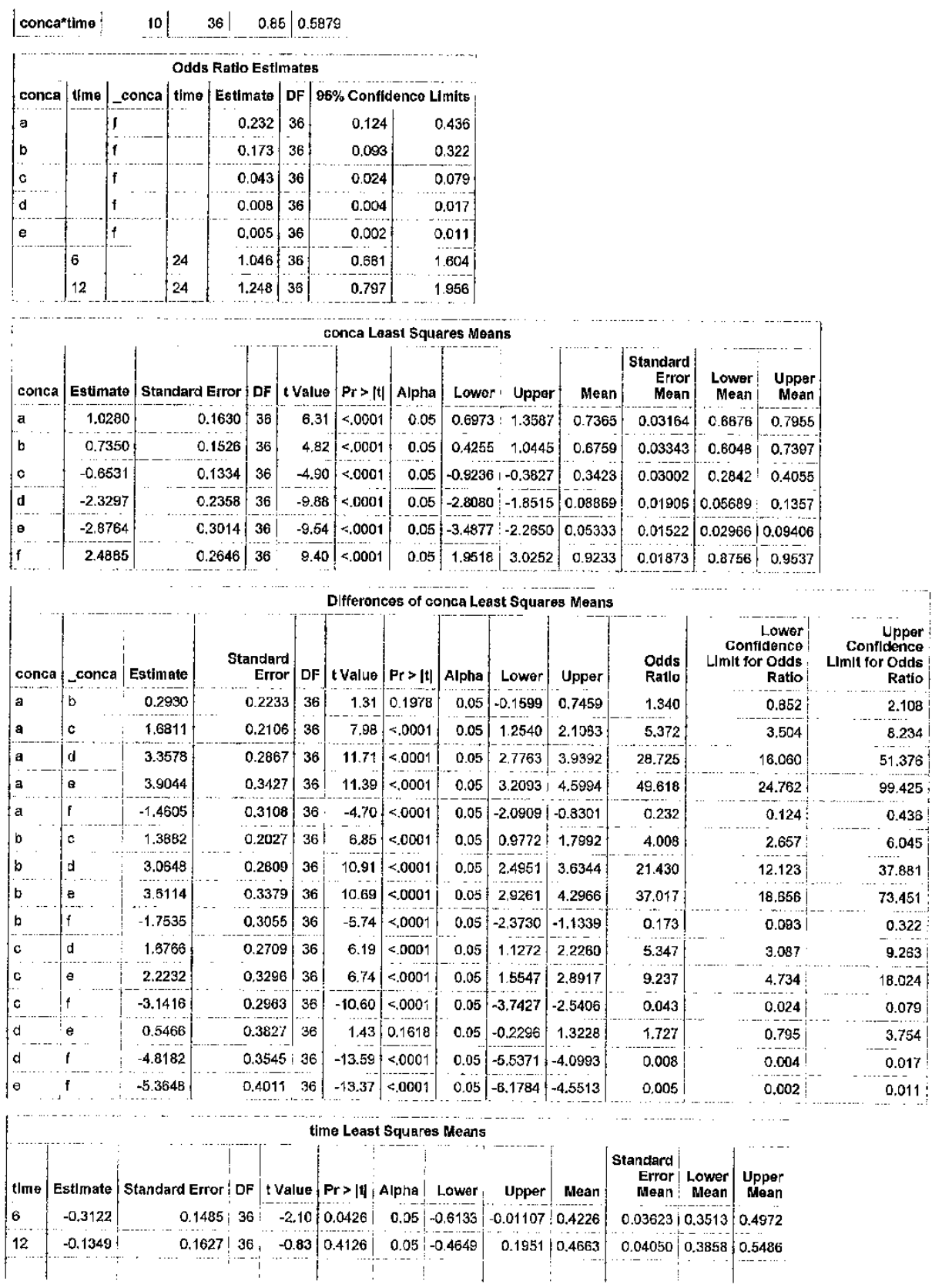

file://C:/Users/JLUOMAAppData/Local/Tomp/1/SAS\%20Temporary\%20Files/_TD4836... 3/18/2015 
$|24|-0.3568|\quad 0.1501| 36|-2.38| 0.0229|0.05|-0.6612|-0.05242| 0.4117|0.03635| 0.3405 \mid 0.4869$

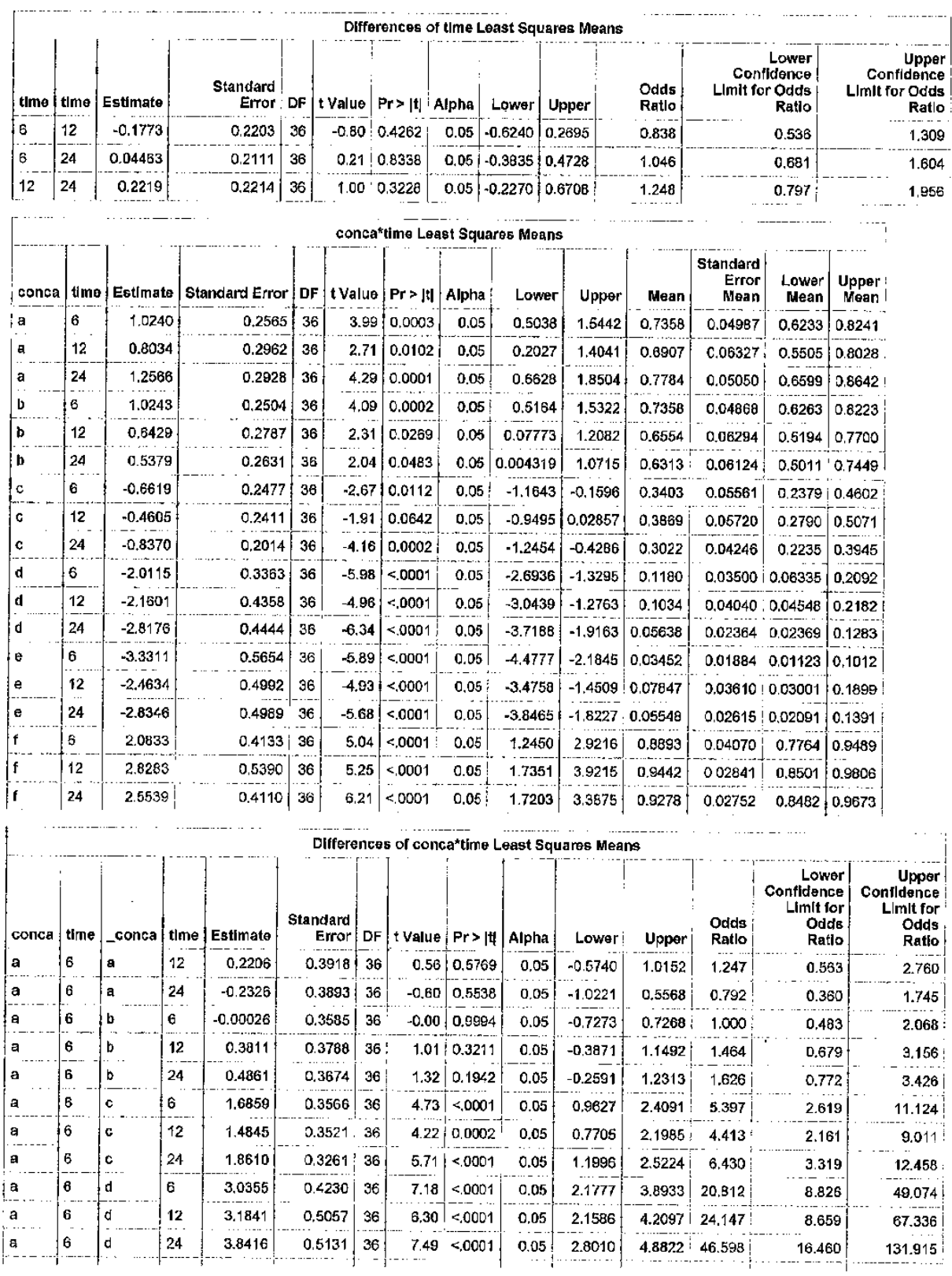




\begin{tabular}{|c|c|c|c|c|c|c|c|c|c|c|c|c|c|c|}
\hline a & 6 & e & 6 & 4.3551 & 0,6208 & 36 & 7.01 & $<.000^{\circ}$ & 0.05 & $3.096 \mathrm{C}$ & 5.6142 & 77.874 & 22.108 & 274.298 \\
\hline a & 6 & e & 12 & 3.4874 & 0.5613 & 36 & 6.21 & $<.0001$ & 0.05 & 2.3491 & 4.6257 & 32.690 & 10.476 & 102.070 \\
\hline a & 6 & e & 24 & 3.8586 & 0.5610 & 36 & 6.88 & $<, 0001 ;$ & 0.05 & 2.7208 & 4.9964 & 47,400 & 15.193 & 147.882 \\
\hline a & 6 & $f$ & 6 & -1.0593 & 0.4865 & 36 & -2.18 & $0.0361 \mid$ & 0.05 & -2.0459 & -0.07268 & 0.347 & 0.129 & 0.930 \\
\hline a & 6 & 1 & 12 & -1.8043 & 0.5969 & 36 & -3.02 & $0.00<6$ & 0.05 & -3.0149 & $-0,5036$ & 0.165 & $0.04 \theta$ & 0.552 \\
\hline a & 6 & 1 & 24 & -1.5299 & 0.4845 & 36 & -3.16 & 0.0032 & 0.05 & -2.5125 & -0.5473 & 0.217 & 0.081 & 0.579 \\
\hline a & 12 & a & 24 & -0.4532 & 0.4165 & 36 & -1.09 & 0.2837 & 0.05 & -1.2978 & 0.3914 & 0.636 & 0.273 & 1.478 \\
\hline a & 12 & b & 6 & -0.2209 & 0.3879 & 36 & -0.57 & 0.5726 & 0.05 & -1.0075 & 0.5658 & 0.802 & 0.365 & 1.761 \\
\hline a & 2 & $b$ & 12 & 0.1605 & 0.4067 & 36 & 0.39 & 0.6955 & 0.05 & -0.6643 & 0.9852 & 1.174 & 0.515 & 2.678 \\
\hline$a$ & 12 & $b$ & 24 & 0.2655 & 0.3061 & 36 & 0.67 & 0,5070 & 0.05 & -0.5379 & 1.0689 & 1.304 & 0.584 & 2.912 \\
\hline a & 12 & c & 6 & 1.4653 & 0.3861 & 36 & 3.80 & $0.0 \subset 05$ & 0.05 & 0.6823 & 2.2484 & 4.329 & 1.978 & 9.472 \\
\hline a & 12 & c & 12 & $\begin{array}{r}1.2639 \\
.\end{array}$ & 0.3819 & 36 & 3.31 & 0.0021 & 0.06 & 0.4893 & 2.0385 & $\begin{array}{r}3.539 \\
-\end{array}$ & 1.631 & 7.679 \\
\hline a & 12 & c & 24 & 1.6404 & 0,3581 & 36 & 4.58 & $<, 0001$ & 0.05 & 0.9141 & 2.3668 & 5.157 & 2.494 & 10,663 \\
\hline a & 12 & d & 6 & 2.8149 & 04481 & 36 & 6.28 & $<<0001$ & 0.05 & 1.9051 & 3.7238 & \begin{tabular}{|r}
16.692 \\
\end{tabular} & 6.727 & 41.421 \\
\hline a & 12 & 0 & 12 & 2.9636 & 0.5269 & 36 & 5.62 & $<.0001$ & 0.05 & 1.8950 & 4.0321 & 18.367 & 6.652 & 56.382 \\
\hline a & 12 & d & 24 & 3.6210 & 0.5340 & 36 & 6.78 & $<.0001$ & 0.05 & 2.5379 & 4.7040 & 37.373 & 12.653 & 110.389 \\
\hline a & 12 & $\theta$ & 6 & 4.1345 & 0.6383 & 36 & 6.48 & $<0001$ & 0.05 & 2.8401 & 5.4289 & 62.458 & 17.117 & 227.905 \\
\hline a & 12 & e & 12 & 3.2668 & 0.5805 & 36 & 5.63 & $<, 0001$ & 0.05 & 2.0895 & 4.4440 & | 26.226 & 8.081 & 85.115 \\
\hline a & 12 & $\theta$ & 24 & 3.6380 & 0.5802 & 36 & 6.27 & $<.0001$ & 0.05 & 2.4613 & 4.8148 & 38.016 & 11.720 & 123.319 \\
\hline a & 12 & $f$ & 6 & -1.2799 & 0.5085 & 38 & -2.52 & 0.0164 & $0.05:$ & -2.3112 & $=0.2486$ & 0.278 & 0.099 & 0.780 \\
\hline a & 12 & $f$ & 12 & -2.0249 & 0.6150 & 36 & -3.29 & 0.0022 & 0.05 & -3.2722 & -0.7776 & 0.132 & 0.038 & 0.460 \\
\hline a & 12 & if & 24 & $\begin{array}{r}-1.7505 \\
-1.750\end{array}$ & 0.5066 & 36 & -3.46 & 0.0014 & 0.05 & -2.7780 & -0.7230 & 0.174 & 0.062 & 0.485 \\
\hline a & 24 & b & 6 & 0.2324 & 0.3853 & 36 & 0.60 & 0.5602 & 0.05 & $-\mathrm{C.} 5490$ & 1.0137 & 1.262 & 0.578 & 2.756 \\
\hline a & 24 & $b$ & 12 & 0.6137 & 0.4042 & 36 & 1.52 & 0.1377 & 0.05 & -0.2061 & $\begin{array}{r}1.4335 \\
\ldots\end{array}$ & 1.847 & 0.814 & $\begin{array}{r}4.193 \\
. . .\end{array}$ \\
\hline a & 24 & b & 24 & 0.7187 & 0.3936 & 36 & 1.83 & 0.0762 & 0.05 & -0.07957 & 1.5170 & 2.052 & 0.924 & 4.569 \\
\hline a & 24 & c & 6 & 1.9185 & 0.3835 & 36 & 5.00 & $<.0001$ & 0.05 & 1.1408 & $2.6963 !$ & 6.811 & 3.129 & 14.825 \\
\hline a & 24 & c & 12 & 1.7171 & 0.3793 & 36 & 4.53 & $<<.0001$ & 0.05 & 0.9478 & 2.4864 & 5.568 & 2.580 & 12.017 \\
\hline a & 24 & $c$ & 24 & 2.0936 & 0.3553 & 36 & 5.89 & $<0001$ & 0.05 & $\begin{array}{r}1.3730 \\
\end{array}$ & 2.8143 & B.114: & 3.947 & 16.681 \\
\hline a & 24 & $d$ & 6 & 3.2681 & 0.4459 & 36 & 7.33 & $<<0001$ & 0.05 & 2.3636 & 4.1725 & 23.263 & 10.632 & $\begin{array}{r}64.875 \\
-\ldots\end{array}$ \\
\hline a & 24 & d & 12 & 3.4168 & 0.5250 & 36 & 6.51 & $<<0001$ & 0.05 & 2.3520 & 4.4815 & 30.471 & 10.507 & 88.368 \\
\hline a & 24 & d & 24 & 4.0742 & 0.5322 & 36 & 7.66 & $<0001$ & 0.05 & 2.9949 & 5.1534 & 58.802 & 19.984 & 173.023 \\
\hline a & 24 & e & 6 & 4.5877 & 0.6367 & 36 & 7.21 & $<.0001$ & 0.05 & 3.2964 & 5.8790 & 98.269 & 27.016 & 357.438 \\
\hline$a$ & 2.4 & le & 12 & $\begin{array}{r}3.7200 \\
\end{array}$ & 0.5787 & 36 & 6.43 & $<.0001$ & 0.05 & 2.5462 & 4.8937 & 41.263 & 12.759 & 133,449 \\
\hline a & 24 & $\theta$ & 24 & 4.0912 & 0.5785 & 36 & 7.07 & $<, 0001$ & 0.05 & 2.9180 & 5.2645 & 59.813 & 18.504 & 193.347 \\
\hline a & 24 & if & 6 & -0.8267 & 0.5065 & 36 & -1.63 & 0.1114 & 0.05 & -1.8540 & 0.2006 & 0.438 & 0.157 & 1.222 \\
\hline a & 24 & $f$ & 12 & -1.5717 & 0.6134 & 36 & -2.56 & 0.0147 & 0.05 & -2.8157 & $-0,3277$ & 0.208 & 0.060 & 0.721 \\
\hline$a$ & 24 & f. & 24 & -1.2973 & C.5046 & 36 & -2.57 & 0.0144 & 0.05 & -2.3208 & -0.2738 & $0.273:$ & 0.098 & 0.760 \\
\hline$b$ & 6 & $b$ & 12 & 0.3813 & 0.3717 & 36 & 1.02 & 0.3166 & 0.05 & -0.3786 & 1.1412 & 1.464 & 0.685 & 3.131 \\
\hline b & 6 & $b$ & 24 & 0.4864 & 0.3632 & 36 & $1.34 !$ & 0.1890 & 0.05 & -02503 & 1.2230 & 1.626 & 0.779 & $\begin{array}{r}3.397 \\
\ldots\end{array}$ \\
\hline b & 6 & c & 6 & 1.6862 & 0.3522 & 36 & 4.79 & $<<0001$ & 0.05 & 0.9718 & 2.4006 & 5,399 & 2.643 & 11.029 \\
\hline b & 6 & c & 12 & 1.4847 & 0.3477 & 36 & 4.27 & 0.000 & 0.05 & 0.7797 & 2.1898 & 4.414 & 2.181 & 8.934 \\
\hline b & 6 & c & 24 & 1.8613 & 0.3214 & $36 !$ & 5.79 & $\mid<0001$ & 0.05 & $1.2 \mathrm{Cg5}$ & 2.5130 & 6.432 & 3.352 & 12.342 \\
\hline b & 6 & $d$ & 6 & 3.0358 & 0.4193 & 36 & 7.24 & $<.0001$ & 0.05 & 2.1854 & 3.8862 & 20.818 & 8.894 & 48.725 \\
\hline b & 6 & d & 12 & 3.1844 & 0.5026 & 36 & 6.34 & $<<0001$ & 0.05 & 2.1651 & 4.2038 & 24.153 & 8.715 & 66.938 \\
\hline b & 6 & d & 24 & $\begin{array}{r}3.8418 \\
\end{array}$ & 0.5101 & 36 & 7.53 & $<<0001$ & 0.05 & 2.8073 & 4.8763 & 46.610 & 16.565 & 131.146 \\
\hline b & 6 & e & 6 & 4.3554 & 0.6184 & 36 & 7.04 & $<<.0001$ & 0.05 & 3.1013 & 5.6094 & 77.894 & 22.226 & 272.992 \\
\hline
\end{tabular}




\begin{tabular}{|c|c|c|c|c|c|c|c|c|c|c|c|c|c|c|}
\hline & 6 & $\theta$ & 12 & 3.4876 & 0.5585 & 36 & 6.241 & $1<, 0001$ & 0.05 & 2.3549 & 4.6203 & 32.708 & 10.537 & 101.529 \\
\hline b & 6 & $\theta$ & 24 & 3.8589 & 0.5583 & 36 & 6.91 & $<.0001$ & 0.05 & 2.7267 & 4.9911 & 47.412 & 15.282 & 147.098 \\
\hline b & 6 & $f$ & 6 & -1.0590 & $0.4833:$ & 36 & -2.19 & 0.0350 & 0.05 & $-2,0392$ & -0.07886 & $0.347 i$ & 0.130 & 0.924 \\
\hline b & 6 & $f$ & 12 & -1.8040 & 0.5944 & 36 & -3.04 & 0.0044 & 0.05 & -3.0094 & -0.5986 & 0.165 & 0.049 & 0.550 \\
\hline b & 6 & $f$ & 24 & -1.5296 & 0.4813 & 36 & -3.18 & 0,0030 & 0.05 & $-2,5059$ & -0.5535 & 0.217 & 0.082 & 0.575 \\
\hline b & 12 & b & 24 & 0.1050 & 0.3833 & 36 & 0.27 & 0.7856 & 0.05 & -0.5722 & 0.8823 & 1.111. & 0.511 & 2.417 \\
\hline b & 12 & c & 6 & 1.3049 & 0.3729 & 36 & 3.50 & 0.0013 & 0.05 & 0.5487 & 2.0611 & 3.687 & 1.731 & 7.854 \\
\hline b & 12 & c & 12 & 1.1034 & 0.3685 & 36 & 2.99 & 0.0050 & 0.05 & 0.3560 & 1.8508 & 3.014 & 1.428 & 6.365 \\
\hline b & 12 & c & 24 & 1.4800 & 0.3438 & 36 & 4.30 & 0.0001 & 0.05 & 0.7826 & 2.1773 & 4.393 & 2.187 & 8.822 \\
\hline b & 12 & d & 6 & $2.6545 i$ & 0.4368 & 36 & 6.08 & $<.0001$ & 0.05 & 1.7687 & 3.5403 & $14.217 !$ & 5.863 & 34.477 \\
\hline b & 12 & $d$ & 12 & 2.8031 & 0.5173 & 36. & 5.42 & $<, \infty 001$ & 0.05 & 1.7540 & $3.8522 !$ & 16.495 & 5.778 & 47.095 \\
\hline b & 12 & d & 24 & 3.4605 & 0.5245 & 36 & 6.60 & $<.0001$ & 0,05 & 2.3967 & 4.5243 & 31.833 & 10.987 & 92,231 \\
\hline b & 12 & e & 6 & 3.9740 & 0.6303 & 36 & 6.30 & $<.0001$ & 0.05 & 2.6957 & 5.2524 & 53.198 & 14.815 & 191.024 \\
\hline b & 12 & e & 12 & 3.1063 & 0.5717 & 36 & 5.43 & $<<.0001$ & 0.05 & $1.9467 f$ & 4.2658 & 22,338 & 7.006 & 71,225 \\
\hline b & 12 & e & 24 & 3.4776 & 0.5715 & 36 & 6.08 & $<<, 0001$ & 0.05 & 2.3185 & 4.6366 & 32.380 & 10.160 & 103.194 \\
\hline b & 12 & 1 & 6 & -1.4403 & 0.4985 & 36 & -2.89 & 0.0085 & 0.05 & -2.4514 & -0.4293 & 0.237 & 0.086 & 0.651 \\
\hline b & 12 & $f$ & 12 & -2.1854 & 0.6088 & 36 & -3.60 & 0.0009 & 0.05 & -3.4160 & -0.9547 & 0.112 & 0.033 & 0.385 \\
\hline b & 12 & 1 & 24 & -1.9110 & $0.4966 \mid$ & 36 & -3.85 & $|0.0005|$ & 0.05 & -2.9181 & -0.9038 & 0.148 & 0.054 & 0.405 \\
\hline$b$ & 24 & $\mathrm{c}$ & 6 & 1.1998 & 0.3613 & 36 & 3.32 & 0.0021 & 0.05 & 0.4670 & 1.9327 & 3.320 . & 1.595 & 6.908 \\
\hline b & 24 & $c$ & 12 & 0.9984 & 0.3569 & 36 & 2.80 & 0.0082 & 0.05 & 0.2746 & 1.7222 & 2.714 & 1,316 & 5.597 \\
\hline b & 24 & c & 24 & 1.3749 & 0.3313 & 36 & 4.15 & 0.0002 & 0.05 & 0.7030 & 2.0468 & 3.955 & 2.020 & 7.743 \\
\hline b & 24 & d & 6 & 2.5494 & 0.4270 & 36 & 5.97 & $<.0001$ & 0.05 & $1.6 \mathrm{a} 35$ & 3.4154 & 12.800 & 5.384 & 30.429 \\
\hline b & 24 & d & 12 & 2.6980 & 0.5090 & 36 & 5.30 & $<.0001$ & 0.05 & 1.6657 & 3.7304 & 14.851 & 5.289 & 41.696 \\
\hline b & 24 & a & 24 & 3.3554 & 0.5164 & 36 & 6.50 & $<.0001$ & 0.05 & 2.3081 & 4,4028 & 28.658 & 10.055 & 81.677 \\
\hline b & 24 & e & 6 & 3.8690 & 0.6236 & 36 & 6.20 & $<<.0001$ & 0.05 & 2.6043 & 5.1337 & 47.893 & 13.521 & 168.640 \\
\hline$b$ & 24 & e & 12 & 3.0012 & 0.5643 & 36 & 5.32 & $\mid<.0001$ & 0.05 & 1.8568 & 4.1457 & 20.111 & 6.403 & 63.162 \\
\hline b & 24 & $\theta$ & 24 & 3.3725 & C.5641 & 36 & 5.98 & $<.0001$ & 0.05 & 2.2285 & 4.5165 & 29.151 & 9.286 & 91.512 \\
\hline b & 24 & $f$ & 6 & -1.5454 & 0.4900 & 36 & -3.15 & 0.0032 & 0.05 & -2.5391 & -0.5517 & $0.2 \uparrow 3$ & 0.079 & 0.576 \\
\hline b & 24 & 1 & 12 & -2.2004 & 0.5998 & 36 & -3.82 & 0.0005 & 0.05 & -3.5069 & -1.0740 & 0.101 & 0.030 & 0.342 \\
\hline b & 24 & $f$ & 24 & -2.0160 & 0.4880 & 36 & -4.13 & 0.0002 & 0.05 & -3.0058 & $-1.0263 \mid$ & 0.133 & 0.050 & 0.358 \\
\hline $\mathrm{c}$ & 6 & c & 12 & -0.2014 & 0.3457 & 36 & -0.58 & 0.5637 & 0.05 & -0.9025 & 0.4896 & 0.818 & 0.406 & 1.648 \\
\hline c & 6 & c & 24 & 0.1751 & 0.3192 & $36:$ & 0.55 & 0.5868 & 0.05 & -0.4723 & 0.8225 & 1.191 & 0.624 & 2.276 \\
\hline$c$ & 6 & d & 6 & 1.3496 & 0.4177 & 36 & 3.23 & $\mid 0.0026$ & 0.05 & 0.5025 & $2.1967 \mid$ & 3.856 & 1.653 & 8.995 \\
\hline c & 6 & d & 12 & 1.4982 & 0.5013 & 36 & 2.99 & 0.0050 & 0.05 & 0.4816 & 2.5148 & 4.474 & 1.619 & 12.364 \\
\hline$c$ & 6 & d & 24 & 2.1556 & 0.5087 & 36 & 4.24 & 0.0002 & 0.05 & 1.1238 & 3.1874 & 8.633 & 3.077 & 24.225 \\
\hline$c$ & 6 & $\theta$ & 6 & 2.6692 & 0.6173 & 36 & 4.321 & $0.0001 \mid$ & 0.05 & 1.4173 & 3.9210 & 14.428 & 4.126 & 50.451 \\
\hline 6 & 6 & e & 12 & 1.8014 & $0.5573 !$ & 36 & 3.23 & 0.0026 & 0.05 & 0.6712 & 2.9317 & 6.058 & 1.957 & 18.759 \\
\hline c & 6 & e. & 24 & 2.1727 & 0.5570 & 36 & 3.90 & 0.0054 & 0.05 & 1.0429 & 3.3024 & 8.782 & 2.838 & 27.179 \\
\hline c & 6 & $f$ & 6 & -2.7452 & 04819 & 36 & $\boldsymbol{- 5 . 7 0 !}$ & $<<, 0001$ & 0.05 & -3.7225 & -1.7679 & 0.064 & 0.024 & 0.171 \\
\hline c & 6 & $f$ & 12 & -3.4902 & 0.5932 & 36 & $-5,88$ & $<<, 0001$ & 0.05 & -4.6933 & -2.2871 & 0.030 & 0.009 & 0.102 \\
\hline c & 6 & $f$ & 24 & -3.2158 & 0.4799 & 36 & -6.70 & $<, 0001$ & 0.05 & -4.1891 & -2.2425 & 0.040 & 0.015 & 0.106 \\
\hline c & 12 & $\mathrm{c}$ & 24 & 0.3765 & 0.3142 & 36 & 1.20 & 0.2385 & 0.05 & -0.2606 & 1.0137 & 1.457 & 0.771 & 2.756 \\
\hline c & 12 & $d$ & 6 & 1.5510 & 0.4138 & 36 & 3.75 & 0.0006 & 0.05 & 0.7118 & 2.3903 & 4.716 & 2.038 & 10.917 \\
\hline c & 12 & d & 12 & 1.6997 & 0.4980 & 36 & 3.41 & 0.0016 & 0.05 & 0.6896 & 2.7098 & 5.472 & 1.993 & 15.026 \\
\hline c & 12 & d & 24 & 2.3571 & 0.5056 & 36 & $4, \in \epsilon$ & $<<.0001$ & 0.05 & 1.3317 & $3.3824 \mid$ & 10.560 & 3.787 & 29.443 \\
\hline c & 12 & e & 6 & 2.8706 & 0.6147 & 36 & 4,67 & $<.0001$ & 0.05 & 1.6240 & 4.1172 & 17.648 & 5.074 & 61,386 \\
\hline
\end{tabular}




\begin{tabular}{|c|c|c|c|c|c|c|c|c|c|c|c|c|c|c|}
\hline & 12 & e & 12 & 2.0029 & 0.5544 & 36 & 3.61 & 0.0009 & 0.05 & 0.8785 & 3.1273 & 7.410 & 2.407 & 22.812 \\
\hline$c$ & 12 & e & 24 & 2.3741 & 0.5542 & 36 & 4.28 & 0.0001 & 0.05 & 1.2502 & 3.4980 & 10.742 & 3.491 & 33.050 \\
\hline 0 & 12 & 1 & 6 & -2.5438 & 0.4785 & 36 & $-5,32$ & $<, 0001$ & 0.05 & -3.5143 & -1.5732 & 0.079 & 0.030 & 0.207 \\
\hline c & 12 & 1 & 12 & -3.2888 & 0.5905 & 36 & -5.57 & $\mid<, 0001$ & 0.05 & -4.4864 & -2.0912 & 0.037 & 0.011 & 0.124 \\
\hline c & 12 & $f$ & 24 & -3.0144 & 0.4765 & 36 & -6.33 & $<<0001$ & 0.05 & $-3,9809$ & -2.0479 & 0.048 & 0.019 & 0.129 \\
\hline$c$ & 24 & d & 6 & 1.1745 & 0.3920 & 36 & 3.00 & $: 0.0049$ & 0.05 & 0.3795 & 1.9695 & 3.237 & 1.462 & 7.167 \\
\hline c & 24 & d & 12 & 1.3231 & 0.4801 & 36 & 2.76 & 0.0091 & 0.05 & 0.3495 & 2.2967 & 3.755 & 1.418 & 9.942 \\
\hline c & 24 & d & 24 & 1.9805 & 0.4879 & 36 & 4.06 & 0.0003 & 0.05 & 0.9911 & 2.9700 & 7.247 & 2.694 & 19.492 \\
\hline c & 24 & a & 6 & 2.4941 & 0.6002 & 36 & 4.16 & 0.0002 & 0.05 & 1.2769 & 3.7113 & 12.111 & 3.585 & 40.906 \\
\hline c & 24 & 0 & 12 & 1.6263 & 0,5383 & 36 & 3.02 & 0.0046 & 0.05 & 0.5348 & 2.7181 & 5.085 & 1.707 & 15.151 \\
\hline c & 24 & e & 24 & 1.9976 & 0.5380 & 36 & 371 & 0.0007 & 0.05 & 0.9054 & 3.0888 & 7.371 & 2.475 & 21.951 \\
\hline c & 24 & $f$ & 6 & -2.9203 & 0.4598 & 36 & -6.35 & $<<.0001$ & 0.05 & -3.8528 & -1.9878 & 0.054 & 0.021 & 0.137 \\
\hline c & 24 & f & 12 & -3.6653 & 0.5754 & 36 & -6.37 & $<.0001$ & 0.05 & -4.8323 & -2.4983 & 0.026 & 0.008 & 0.082 \\
\hline c & 24 & $f$ & 24 & -3.3909 & 0.4577 & 36 & -7.41 & $<.0001$ & 0.05 & -4.3192 & -2.4626 & 0.034 & 0.013 & 0.085 \\
\hline d & 6 & d & 12 & 0.1486 & 0.5505 & 36 & 0.27 & 0.7887 & 0.05 & -0.9678 & 1.2850 & 1.160 & 0.380 & 3.543 \\
\hline d & 6 & d & 24 & 0.8060 & 0.5573 & 36 & 1.45 & 0.1567 & 0.05 & -0.3242 & 1.9362 & 2.239 & 0.723 & 6.933 \\
\hline d & 6 & 0 & 6 & 1.3190 & 0.6578 & 36 & 2.011 & $|0.0524|$ & 0.05 & -0.01460 & 2.6537 & 3.742 & 0.986 & 14.207 \\
\hline d & 6 & e & 12 & 0.4518 & 0.6019 & 36 & 0.75 & 0.4578 & 0.05 & -0.7689 & 1.6726 & 1.571 & 0464 & 5.326 \\
\hline d & 6 & $\theta$ & 24 & 0.8231 & 0.6017 & 36 & 1.37 & 0.1798 & 0.05 & -0.3972 & 2.0434 & 2.278 & 0.672 & 7.717 \\
\hline d & 6 & $f$ & 6 & -4.0948 & 0.5329 & 36 & -7.68 & $\mid<\infty, \infty 001$ & 0.05 & --5.1755 & -3.0141 & 0.017 & 0.008 & 0.049 \\
\hline d & 6 & $f$ & 12 & -4.8398 & 0.6353 & 36 & -7.62 & $<, 0001$ & 0.05 & -6.1283 & -3.5513 & 0.008 & 0.002 & 0.029 \\
\hline d & 6 & $f$ & 24 & -4.5654 & 0.5311 & 38 & -8.60 & $<.0001$ & 0.05 & -5.6425 & -3.4883 & 0.010 & 0.004 & 0.031 \\
\hline d & 12. & d & 24 & 0.6574 & 0.6224 & 36 & 1.06 & 0.2979 & 0.06 & -0.6049 & 1.9197 & 1.930 & 0.546 & 6.819 \\
\hline d & 12 & e & 6 & 1.1709 & 0.7138 & 36 & 1.64 & 0.1090 & 0.05 & -0.2768 & 2.6187 & 3.225 & 0.758 & 13.717 \\
\hline d & 12 & e & 12 & 0.3032 & 0.6627 & 36 & 0.46 & 0.6500 & 0.05 & -1.0407 & 1.6472 & 1.354 & 0.353 & 5.192 \\
\hline d & 12 & e & 24 & 0.6745 & 0.6625 & 36 & 1.02 & $|0.3154|$ & 0.05 & -0.6691 & 2.0180 & 1.963 & 0.512 & 7.523 \\
\hline d & 12 & $p$ & 6 & -4.2434 & 0.6003 & 36 & -7.06 & $\mid<.0001$ & 0.05 & $-5,4616$ & -3.0253 & 0.014 & 0.004 & 0.049 \\
\hline d & 12 & $f$ & 12 & -4.9884 & 0.6931 & 36 & -7.20 & $<.0001$ & 0.05 & -6.3942 & -3.5827 & 0.007 & 0.002 & 0.028 \\
\hline d & 12 & $f$ & 24 & -4.7140 & 0.5990 & 36 & -7.87 & $<.0001$ & 0,05 & -5.9290 & -3.4991 & 0.009 & 0.003 & 0.030 \\
\hline d & 24 & e & 6 & 0.5135 & 0.7191 & 36 & 0.71 & 0.4798 & 0.05 & -0.9449 & 1.9720 & 1.671 & 0.389 & 7.185 \\
\hline$d$ & 24 & e & 12 & -0.3542 & 0.6683 & 36 & -0.53 & 0.5994 & 0.05 & -1.7097 & 1.0013 & 0.702 & 0.181 & 2.722 \\
\hline d & 24 & e & 24 & 0.01706 & 0.6681 & 36 & 0.03 & 0.9798 & 0.05 & -1.3380 & 1.3721 & 1.017 & 0.262 & 3.944 \\
\hline d & 24 & $f$ & 6 & -4.9008 & 0.6069 & 36 & -8.08 & $<.0001$ & 0.05 & -6.1317 & -3.6700 & 0.007 & 0.002 & 0.025 \\
\hline$d$ & 24 & if & 12 & -5.6459 & 0.6986 & 36 & -8.08 & $<.0001$ & 0.05 & -7.0626 & -4.22 .91 & 0.004 & $<0.001$ & 0.015 \\
\hline d & 24 & 1 & 24 & -5.3715 & 0.6053 & 36 & -8.87 & $<.0001$ & 0.05 & -6.5891 & -4.1438 & 0.005 & 0.001 & 0.016 \\
\hline e & 6 & e & 12 & -0.8677 & 0.7542 & 36 & $-1,15$ & 0.2676 & 0.05 & $-2,3974$ & 0.6619 & 0.420 & 0.091 & $\begin{array}{l}1.939 \\
0\end{array}$ \\
\hline e & 6 & e & 24 & -0.4965 & 0.7541 & 36 & $-\mathrm{C} .66$ & 0.5145 & 0.05 & $-2,0258$ & $1.032 B$ & 0.609 & 0.132 & 2.809 \\
\hline$\theta$ & 6 & $f$ & 6 & -5.4144 & 0.7004 & 36 & -7.73 & $<<0001$ & 0.05 & -6.8348 & -3.9940 & 0.004 & 0.001 & 0.018 \\
\hline e & 6 & $f$ & 12 & -6.1594 & 0.7811 & 36 & -7.89 & $<.000:$ & 0.05 & -7.7436 & -4.5751 & 0.002 & $<0.001$ & 0.010 \\
\hline e & 6 & I & 24 & -5.8850 & 0.6990 & 36. & -8.42 & $<.0001$ & 0.05 & $-7,3026$ & -4.4674 & 0.003 & $<0.001$ & 0.011 \\
\hline e & 12 & e & 24 & 0.3713 & 0.7058 & 36 & 0.53 & 0.6021 & 0.05 & -1.0602 & 1.8027 & 1.450 & 0.346 & 6.066 \\
\hline e & 12 & f & 6 & -4.5466 & 0.6481 & 36 & -7.01 & $<, 0001$ & 0.05 & -5.8611 & -3.2322 & 0.011 & 0.003 & 0.039 \\
\hline $\mathrm{e}$ & 12 & 1 & 12 & -5.2917 & 0.7347 & 36 & $-7,20$ & $<.0001$ & 0.05 & -6.7817 & -3.8016 & 0.005 & 0.001 & 0.022 \\
\hline$e$ & 12 & $f$ & 24 & -5.0173 & 0.6467 & 36 & -7.76 & $<.0001$ & 0.05 & -6.3287 & -3.7058 & 0.007 & 0.002 & 0.025 \\
\hline$\theta$ & 24 & $f$ & 6 & -4.9179 & 0.6479 & 36 & -7.59 & $<.0001$ & 0.05 & -6.2319 & $-3,6039$ & 0.007 & 0.002 & 0.027 \\
\hline$e$ & 24 & $f$ & 12 & -5.6629 & 0.7345 & 36 & -7.71 & $<.0001$ & 0.05 & -7.1525 & -4.1733 & 0.003 & $<0.001$ & 0.015 \\
\hline
\end{tabular}




\begin{tabular}{|c|c|c|c|c|c|c|c|c|c|c|c|c|c|c|}
\hline & 24 & $f$ & 24 & -5.3885 & 0.6464 & 36 & -8.34 & $<.0001$ & 0.05 & -6.6996 & $-4.0775 \mid$ & 0.005 & 0.001 & 0.017 \\
\hline f & 6 & 1 & 12 & -0.7450 & 0.6793 & 36 & -1.10 & 0.2800 & 0.05 & -2.1226 & 0.6326 & 0.475 & 0.120 & 1.882 \\
\hline$f$ & 6 & i & 24 & -0.4706 & 0.5829 & 36 : & -0.81 & 0.4248 & 0.05 & -1.6528 & 0.7116 & 0.625 & 0.192 & 2.037 \\
\hline$f$ & 12 & $f$ & 24 & 0.2744 & 0.8779 & 36 & 0.40 & 0.6880 & 0.05 & -1.1004 & 1.6492 & 1.316 & 0.333 & 5.203 \\
\hline
\end{tabular}

Performed by J. Luoma; SAS version 9.3 OB:43 18MAR15 


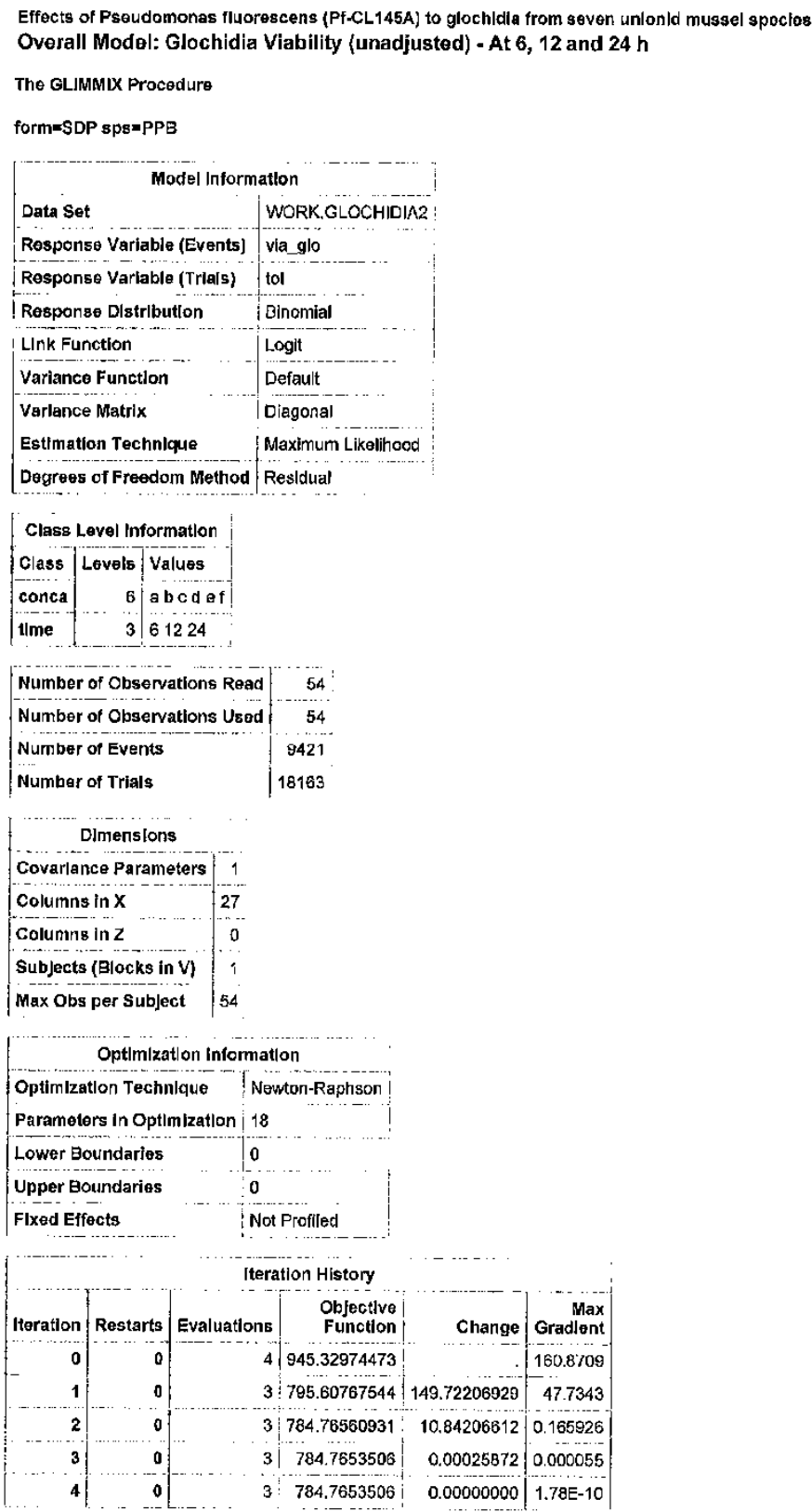

file://C:/Uscrs/JLUOMA/AppData/Local/Temp/1/SAS\%20Temporary\%20Files/ TD4836_.. 3/18/2015 


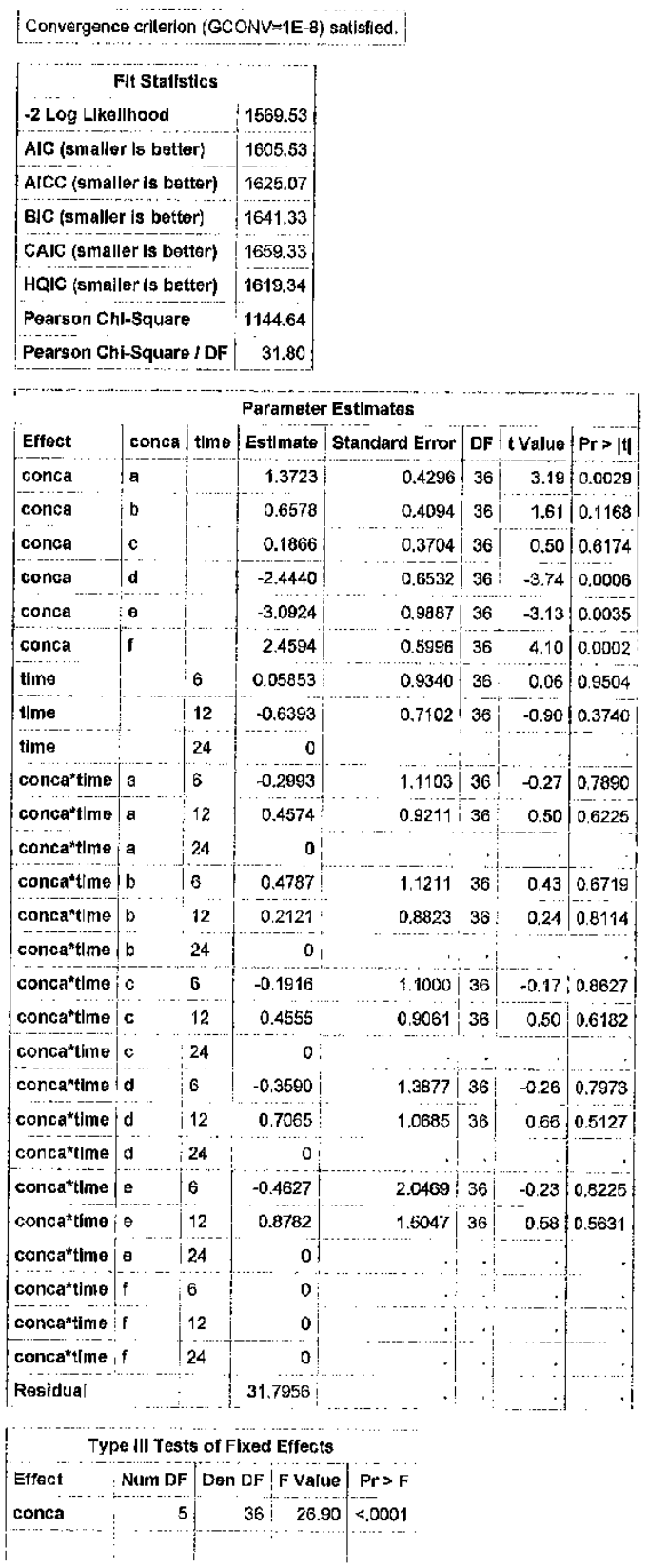

file://C:/Users/JLUOMA/AppData/Local/Temp/1/SAS\%20Temporary\%20Files/_TD4836... 3/18/2015 


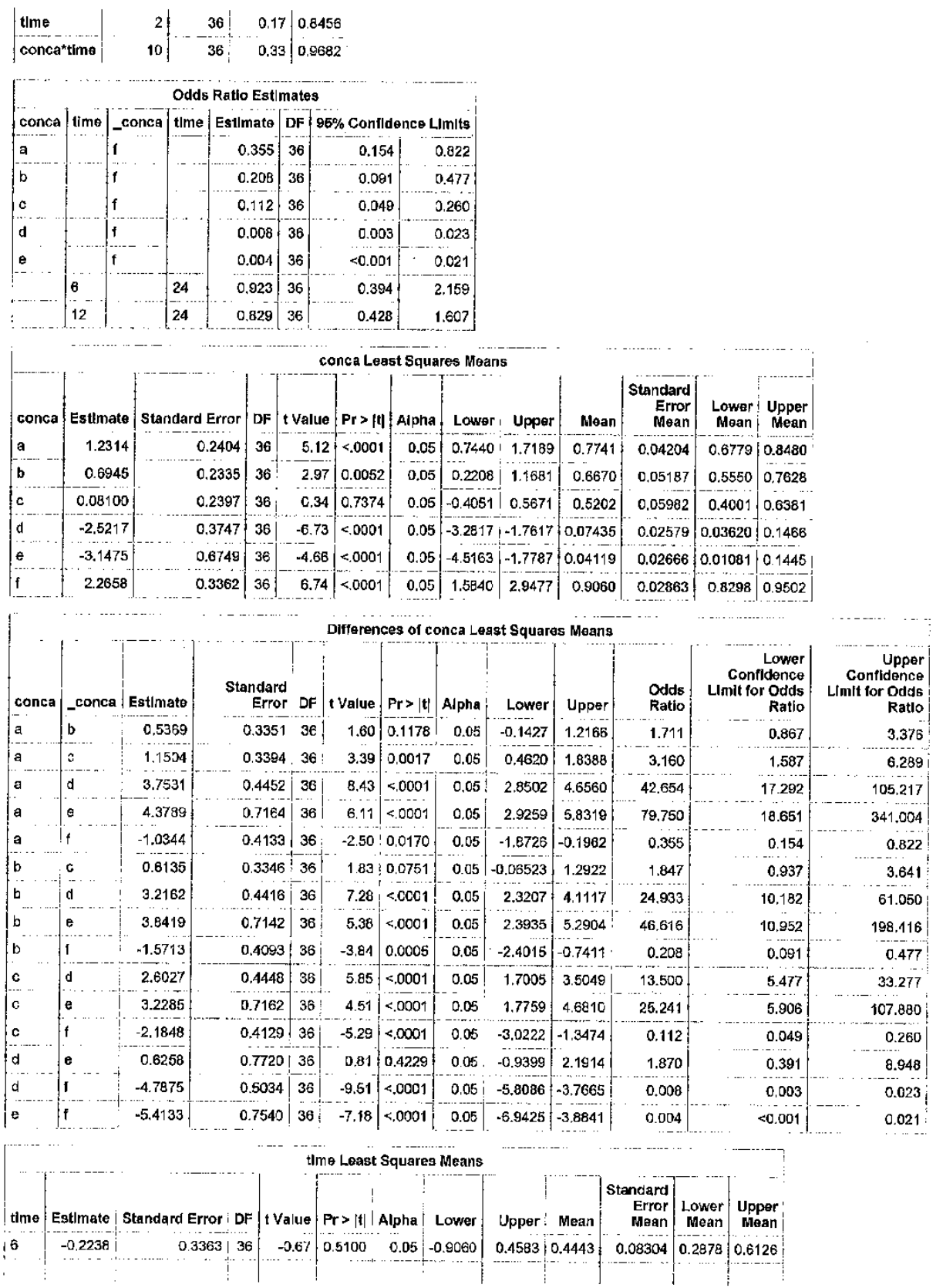

file://C:/Users/JLUOMA/AppData/Local/Temp/1/SAS\%20Temporary\%20Files/ TD4836... 3/18/2015 
\begin{tabular}{|l|l|l|l|l|l|l|l|l|l|l|l|l|l|}
12 & -0.3311 & 0.2097 & 36 & -1.58 & 0.1231 & 0.05 & -0.7563 & 0.09420 & 0.4180 & 0.05101 & 0.3194 & 0.5235 \\
\hline 24 & -0.1434 & & 0.2502 & 36 & -0.57 & 0.5702 & 0.05 & -0.6507 & 0.3610 & 0.4642 & 0.06222 & 0.3428 & 0.5900 \\
\hline
\end{tabular}

\begin{tabular}{|c|c|c|c|c|c|c|c|c|c|c|c|c|}
\hline \multirow[b]{2}{*}{ time } & \multirow[b]{2}{*}{ time } & \multirow[b]{2}{*}{ Estimate } & \multicolumn{9}{|c|}{ Differences of time Least Squares Means } & \multirow[b]{2}{*}{$\begin{array}{r}\text { Upper } \\
\text { Confidence } \\
\text { Limit for Odds } \\
\text { Ratlo }\end{array}$} \\
\hline & & & $\begin{array}{r}\text { Standard } \\
\text { Error }\end{array}$ & DF & t Value & $\operatorname{Pr}>|t|$ & Alpha & Lower & Upper & $\begin{array}{l}\text { Odds } \\
\text { Ratio }\end{array}$ & $\begin{array}{r}\text { Lower } \\
\text { Confldence } \\
\text { L/m|t for Odds } \\
\text { Ratlo }\end{array}$ & \\
\hline 6 & 12 & 0.1072 & 0.3964 & 36 & 0.27 & 0.7883 & 0.05 & $-0.6966 \mid$ & 0.9111 & 1.113 & 0.498 & 2,487 \\
\hline 6 & 24 & -0.08045 & 0.4192 & 36 & -0.19 & 0.8489 & 0.05 & $|-0.9306|$ & 0.7697 & 0.923 & 0.394 & 2.159 \\
\hline 12 & 24 & $-0,1877$ & 0.3264 & 36 & -0.57 & 0.5689 & 0.05 & -0.8497 & 0.4743 & 0.829 & 0.428 & 1.607 \\
\hline
\end{tabular}

\begin{tabular}{|c|c|c|c|c|c|c|c|c|c|c|c|c|c|}
\hline conca & tIme & Estlmate & Standard Error & DF & I Value & $P r>|t|$ & Alpha & Lowor ! & Upper & Mean & $\begin{array}{r}\text { Standard } \\
\text { Error } \\
\text { Mean }\end{array}$ & $\begin{array}{l}\text { Lower } \\
\text { Mean }\end{array}$ & $\begin{array}{l}\text { Upper } \\
\text { Mean }\end{array}$ \\
\hline $\mathrm{a}$ & 6 & 1.1315 & 0.4195 & 36 & 2.70 & 0.0106 & 0.05 & 0.2808 & 1.9822 & 0.7561 & 0.07735 & 0.5698 & 0.8789 \\
\hline a & 12 & 1.1904 & 0.3993 & 36 & 2.98 & 0.0051 & 0.05 & 0.3805 & 2.0003 & 0.7668 & 0.07141 & 0.5940 & 0.8808 \\
\hline a & 24 & 1.3723 & 0.4296 & 36 & 3.19 & 0.0029 & 0.05 & 0.5011 & 2.2435 & 0.7978 & C.06931 & 0.6227 & 0.9041 \\
\hline b & 6 & 1.1950 & 0.4657 & 36 & 2.57 & 0.0146 & 0.05 & 0.2506 & $2.1395 i$ & 0.7676 & 0.08306 & 0.5623 & 0.8947 \\
\hline b & 12 & 0.2306 & 0.3262 & 36 & 0.71 & 0.4842 & 0.05 & $|-0.4310|$ & 0.8922 & 0.5574 & 0.08048 & 0.3939 & 0.7093 \\
\hline b & 24 & 0.6578 & 0.4094 & 36 & 1.61 & $0 .: 168$ & 0.05 & $-0.1724 \mid$ & 1.4881 & 0.6588 & 0.09202 & 0.4570 & 0.8158 \\
\hline c & 6 & 0.05356 & 0.4477 & 36 & 0.12 & 0.9054 & 0.05 & -0.8544 & 0.9615 & 0.5134 & 0.1118 & 0.2985 । & 0.7234 \\
\hline c & 12 & 0.002821 & 0.4235 & 36 & 0.01 & 0.9947 & 0.05 & $-0.8562 \mid$ & 0.8618 & 0.5007 & 0.1059 & 0.2981 & 0.7030 \\
\hline c & 24 & 0.1866 & 0.3704 & 36 & 0.50 & 0.6174 & 0.05 & $|-0.5646|$ & 0.9379 & 0.5465 & 0.09180 & 0,3625 & 0.7187 \\
\hline d & 6 & -2.7444 & 0.7916 & 36 & -3.47 & 0.0014 & 0.05 & $-4,3499$ & $\left|-1 .{ }^{\wedge} 389\right|$ & 0.06040 & 0.04493 & 0.01274 & 0.2425 \\
\hline d & 12 & -2.3767 & 0.4589 & 36 & -5.18 & $<, 0001$ & 0.05 & -3.3074 & $-1.4460 \mid$ & 0.08496 & 0.03568 & 0.03532 & 0.1906 \\
\hline d & 24 & -2.4440 & 0.6532 & 36 & -3.74 & 0.0006 & 0.05 & 3.7686 & -1.1193 & 0.07988 & 0,04801 & 0.02256 & 0.2461 \\
\hline e & 6 & -3.4965 & 1.5297 & 36 & -2.29 & $\mid 0.0283$ & 0.05 & |-6.5989 | & -0.3942 & $0.02941 !$ & 0.04367 & 0.001360 & 0.4027 \\
\hline$\theta$ & 12 & -2.8535 & 0.8843 & 36 & $-3.23 j$ & $|0.0027|$ & 0.05 & -4.6170 & $|-1.0600|$ & 0.05450 & 0.04557 & 0.009499 & 0.2573 \\
\hline e & 24 & -3.0924 & 0.9887 & 36 & -3.13 & $0 . \cos 5$ & 0.05 & $-5.0978 \mid$ & $|-1.0871|$ & $\mid 0.04342$ & 0.04107 & $|0.006074|$ & 0.2522 \\
\hline $\mathrm{f}$ & 6 & 2,5179 & 0.7161 & 36 & 3.52 & 0.0012 & 0.05 & 1.0656 & 3.9703 & 0.9254 & $0.0494,4$ & 0.7438 & 0.9816 \\
\hline I & 12 & 1.8201 & 0.3806 & 36 & 4.78 & $<.0001$ & 0.05 & .0481 & 2.5921 & 0.8606 & 0.04567 & 0.7404 & 0.9304 \\
\hline f & 24 & 2.4594 & 0.5996 & 36 & 4.10 & 0.0002 & 0.05 & 1.2434 & 3.6755 & 0.9212 & 0.04350 & 0.7762 & 0.8753 \\
\hline
\end{tabular}

\begin{tabular}{|c|c|c|c|c|c|c|c|c|c|c|c|c|c|c|}
\hline \multirow[b]{2}{*}{ conca } & \multirow[b]{2}{*}{$\operatorname{tim} \theta$} & \multicolumn{13}{|c|}{ Differences of conca*time Least Squares Means } \\
\hline & & - conca & time & Estlmate & $\begin{array}{r}\text { Standard } \\
\text { Error }\end{array}$ & $\mathrm{DF}$ & t Value & $\mathrm{Pr}>|\mathrm{t}|$ & Alpha & Lower & Upper & $\begin{array}{l}\text { Odds } \\
\text { Ratio }\end{array}$ & $\begin{array}{r}\text { Lower } \\
\text { Confidence } \\
\text { LImit for } \\
\text { Odds } \\
\text { Ratlo }\end{array}$ & $\begin{array}{r}\text { Upper } \\
\text { Confldence } \\
\text { Llmit for" } \\
\text { Odds } \\
\text { Ratlo }\end{array}$ \\
\hline a & 6 & a & 12 & -0.05888 & 0.5792 & 36 & -0.10 & 0.9196 & 0.05 & -1.2335 & 1.1157 & C.943 & 0.231 & 3.052 \\
\hline a & 6 & a & 24 & -0.2408 & 0.6004 & 36 & $-0,40$ & 0.6908 & 0.05 & -1.4584 & 0.9769 & 0.786 & 0.233 & 2.656 \\
\hline a & 6 & b & 6 & -0.06350 & 0.6267 & 36 & -0.10 & 0.9199 & 0.05 & -1.3346 & 1.2076 & 0.938 & 0.263 & 3.345 \\
\hline a & 6 & b & 12 & 0.9010 & 0.5314 & 36 & 1.70 & 0.0986 & 0.05 & -0.1767 & 1.9787 & 2.462 & 0.838 & 7.233 \\
\hline a & 6 & b & 24 & 0.4737 & 0.5861 & 36 & 0.81 & 0.4243 & 0.05 & -0.7150 & 1.6624 & 1.606 & 0.489 & 5.272 \\
\hline a & 6 & $c$ & 6 & 1.0780 & 0.6135 & 36 & 1.76 & 0.0874 & 0.05 & -0.1662 & 2.3222 & 2.939 & 0.847 & 10.198 \\
\hline a & $\epsilon$ & c & 12 & 1.1287 & 0.5961 & 36 & 1.89 & 0.0663 & 0.05 & -0.08022 & 2.3377 & 3.092 & 0.923 & 10.357 \\
\hline a & 6 & c & 24 & 0.9449 & 0.5596 & 36 & 1,69 & 0.1000 & 0.05 & -0.1900 & 2.0798 & 2.573 & 0.827 & 8.003 \\
\hline a & 6 & d & 6 & 3.8760 & 0.8959 & 36 & 4.33 . & 0.0001 & 0.05 & 2.0590 & 5.6929 & 48.229 & 7.838 & 296.749 \\
\hline a & 6 & d & 12. & 3.5083 & 0.6217 & 36 & 5.54 & $<0001$ & 0.05 & $2.2474:$ & 4.7692 & 33.391 & 9.163 & 117.823 \\
\hline
\end{tabular}

file://C:/Users/JLUOMA/AppData/Local/Temp/1/SAS\%20Temporary\%20Files/ TD4836... 3/18/2015 


\begin{tabular}{|c|c|c|c|c|c|c|c|c|c|c|c|c|c|c|}
\hline $\mathbf{a}$ & 6 & d & 24 & 3.5755 & 0.7762 & 36 & 4.61 & $\mid<.0001$ & 0.05 & 2.0012 & 5.1498 & 35.712 & 7.398 & 172.397 \\
\hline & 6 & e & 6 & 4,6280 & 1,5862 & 36 & 2.92 & 0,0060 & 0.05 & 1.4112 & 7.8449 & 102.314 & 4.101 & $>999.999$ \\
\hline & 6 & 10 & 12 & 3.9851 & 0.9788 & 36 & 4.07 & 0.0002 & 0.05 & $2.00 \mathrm{co}$ & 5.9701 & 53.789 & 7.389 & 391.548 \\
\hline a & 6 & e & 24 & 4.2239 & 1.0740 & $36 \mid$ & 3.93 & 0.0004 & 0.05 & 2.0457 & 6.4022 & 68.300 & 7.734 & 603,151 \\
\hline a & 6 & $f$ & 6 & -1.3864 & 0.8299 & 36 & -1.67 & 0.1035 & 0.05 & -3.0696 & 0.2968 & 0.250 & 0.048 & $1.3<5$ \\
\hline a & 6 & $f$ & 12 & -0.6886 & 0.5664 & 36 & -1.22 & 0.2320 & $0.05:$ & -1.8373 & 0.4602 & 0.502 & 0.159 & 1.584 \\
\hline a & 6 & $f$ & 24 & -1.3279 & 0.7318 & 36 & -1.81 & 0.0779 & 0.05 & $-2,8119$ & 0.1562 & 0.265 & 0.060 & 1.169 \\
\hline a & 12 & $a$ & 24 & -0.1818 & 0.5865 & 36 & -0.31 & 0.7583 & 0.05 & -1.3714 & 1.0076 & 0,834 & 0.254 & 2.739 \\
\hline a & 12 & b & 6 & -0.00461 & 0.6135 & 36 & -0.01 & 0.9940 & 0.05 & $-1,2488$ & 1.2395 & 0.995 & 0.287 & 3.454 \\
\hline & 12 & b & 12 & 0.9598 & 0.5157 & 36 & 1.86 & 0.0709 & 0.05 & -0.08596 & 2.0056 & 2.611 & 0.918 & 7.431 \\
\hline & 12 & b & 24 & 0.5326 & 0.5719 & 36 & 0.93 & 0.3579 & 0.05 & -0.6272 & 1.6925 & 1.703 & 0.534 & 5.433 \\
\hline a & 12 & $c$ & 6 & 1.1369 & 0.5999 & 36 & 1.90 & 0.0861 & 0.05 & -0.07984 & 2.3536 & 3.117 & 0.923 & 10.523 \\
\hline $\mathbf{a}$ & 12 & c & 12 & 1.1876 & 0.5821 & 36 & 2.04 & 0.0487 & 0.05 & 0.007008 & 2.3682 & 3.279 & 1.007 & 10.678 \\
\hline a & 12 & $c$ & 24 & 1.0038 & 0.5447 & 36 & 1.84 & 0.0736 & 0.05 & -0.1009 & 2.1085 & 2.729 & 0.904 & 8.236 \\
\hline $\mathbf{a}$ & 12 & d & 8 & 3.9348 & 0.8868 & 36 & 4.44 & $<.0001$ & 0.05 & 2.1366 & 5.7330 & 51.154 & 8.471 & 308,906 \\
\hline a & 12 & d & 12 & 3.5672 & 0.6083 & 36 & 5.86 & $<.0001$ & 0.05 & 2.3334 & 4.8009 & 35.416 & 10.313 & 121,622 \\
\hline a & 12 & d & 24 & 3.6344 & 0.7656 & 36 & 4.75 & $<.0001$ & 0.05 & 2.0817 & 5.1870 & 37.878 & 8.018 & 178.935 \\
\hline a & 12 & e & 6 & 4.6869 & 1.5810 & 36 & 2.96 & 0.0053 & 0.05 & 1.4808 & 7.8933 & 108.510 & 4.396 & $>999,999$ \\
\hline a & 12 & $\theta$ & 12 & 4.0439 & 0.9703 & 36 & 4.17 & 0.0002 & 0.05 & 2.0750 & 8.0119 & 57.051 & 7.973 & 408.241 \\
\hline a & 12 & e & 24 & 4.2828 & 1.0663 & 36 & 4.02 & 0.0003 & 0.06 & 2.1201 & 6.4454 & 72.443 & 8,332 & 629.829 \\
\hline a & 12 & $f$ & 6 & $-1,3275$ & 0.8199 & 36 & -1.82 & 0.1142 & 0.05 & $-2,9904$ & 0.3354 & 0265 & 0.050 & 1.398 \\
\hline a & 12 & $f$ & 12 & -0.6297 & 0.5517 & 36 & -1.14 & 0.2612 & 0.05 & -1.7486 & 0.4892 & 0.533 & 0.174 & 1.631 \\
\hline a & 12 & $f$. & 24 & -1.2690 & 0.7204 & 36 & -1.76 & 0.0866 & 0.05 & -2.7301 & 0.1921 & 0.281 & 0.085 & 1.212 \\
\hline a & 24 & b & 6 & 0.1773 & 0.6335 & 36 & 0.28 & 0.7812 & 0.05 & -1.1070 & 1.4622 & 1.194 & 0.330 & 4.315 \\
\hline a & 24 & $b$ & 12 & 1.1417 & 0.5394 & 36 & 2.12 & 0.0413 & 0.05 & 0.04779 & 2.2357 & 3.132 & 1.049 & 9.353 \\
\hline a & 24 & b & 24 & 0.7145 & 0.5934 & 36 & 1.20 & 0.2364 & 0.05 & -0.4889 & 1.9179 & 2.043 & 0.613 & 6.807 \\
\hline a & 24 & ic & 6 & 1.3188 & 0.6204 & 36 & 2.13 & 0.0405 & 0.05 & 0.06043 & 2.5771 & 3.739 & 1.062 & 13.159 \\
\hline a & 24 & c & 12 & 1.3695 & 0.6032 & 36 & 2.27 & 0.0293 & 0.05 & 0.1461 & 2.5929 & 3.933 & 1.157 & 13.369 \\
\hline a & 24 & $c$ & 24 & 1.1857 & 0.5672 & $36:$ & 2.09 & 0.0437 & 0.05 & 0.03531 & 2.3360 & 3.273 & 1.036 & 10.340 \\
\hline a & 24 & d & 6 & 4.1167 & 0.9007 & $36:$ & 4.57. & $<.0001$ & 0.05 & 2.2901 & 5.9433 & 61.358 & 9.876 & 381.206 \\
\hline a & 24 & d & 12 & 3.7490 & 0.6286 & 36: & 5.96 & $<.0001$ & 0.05 & 2.4742 & 5.0239 & 42.480 & 11.872 & 151.993 \\
\hline a & 24 & d & 24 & 3.8163 & 0.7818 & 36 & 4.88 & $<.0001$ & 0.05 & 2.2308 & 5.4017 & 45.434 & 9.307 & 221.790 \\
\hline a & 24 & e & 6 & 4.8688 & 1.5889 & 36 & 3.06 & $0.0041 \mid$ & 0.05 & 1.6465 & 8.0912 & 130.167 & 5.189 & $>999.999$ \\
\hline a & 24 & $\mathrm{e}$ & 2 & 4.2258 & 0.9831 & 36 & 4.30 & 0.0001 & 0.05 & 2.2319 & 6.2197 & 68.432 & 9.318 & 502.575 \\
\hline a & 24 & $\theta$ & 24 & 4.4647 & $1.0780^{\circ}$ & 36 & 4.14 & 0,0002 & 0.05 & $2.2784 !$ & 6.6510 & 86.894 & 9.761 & 773.574 ! \\
\hline a & 24 & if & 6 & $\begin{array}{r}-1,1456 \\
\end{array}$ & 0.8351 & 36 & -1.37 & 0.1786 & 0.05 & -2.8392 & 0.5480 & 0.318 & 0.058 & 1.730 \\
\hline a & 24 & i. & 12 & -0.4478 & 0.5739 & 36 & -0.78 & 0.4404 & 0.05 & -1.6118 & 0.7162 & 0.639 & $\begin{array}{r}0.200 \\
\end{array}$ & 2.047 \\
\hline a & 24 & $i$ & 24 & -1.0871 & 0.7376 & 36 & -1.47 & 0.1492 & 0.05 & -2.5830 & 0.4088 & 0.337 & .0076 & 1.505 \\
\hline b & 6 & b & 12 & 0.9645 & 0.5686 & 36 & 1.70 & 0.0985 & 0.05 & -0.1887 & 2.1176 & 2.623 & 0.828 & 8.311 \\
\hline b & 6 & b & 24 & 0.5372 & 0.6200 & 36 & 0.87 & 0.3920 & 0.05 & -0.7203 & 1.7947 & 1.711 & 0.487 & $6.01 \mathrm{~B}$ \\
\hline b & 6 & c & 6 & 1.1415 & 0.6460 & 36 & $1.77 \mid$ & $\mid 0.0857$ & 0.05 & -0.1686 & 2.4516 & 3.131 & 0.845 & 11.607 \\
\hline b & 6 & c. & 12 & 1.1922 & 0.6295 & 36 & 1.89 & 0.0663 & 0.05 & -0.08443 & 2.4689 & 3.294 & 0.919 & 11.809 \\
\hline b & 6 & $c$ & 24 & 1.0084 & 0.5950 & 36 & 1.69 & 0.0988 & 0.05 & -0.1984 & 2.2152 & 2.741 & 0.820 & 9.163 \\
\hline b & 6 & $d$ & 6 & 3.9395 & 0.9184 & 36 & 4.29 & 0.0001 & 0.05 & 2.0768 & 5.8021 & 51.390 & 7.979 & 331.000 \\
\hline$b$ & 6 & d & 12 & $\begin{array}{r}3.5718 \\
-\end{array}$ & 0.6538 & 36 & 5.46 & $<, 0001$ & 0.05 & 2.2458 & 4.8977 & 35,579 & 9.448 & 133.986 \\
\hline b & 6 & d & 24 & 3.6390 & 0.8022 & 36 & 4.54 & $<.0001$ & 0.05 & 2.0121 & 5.2659 & 38.053 & 7.479 & 193.613 \\
\hline
\end{tabular}




\begin{tabular}{|c|c|c|c|c|c|c|c|c|c|c|c|c|c|c|}
\hline & 6 & e & 6 & 4.6915 & 1.5990 & 36 & 2.93 & 0.0058 & 0.05 & 1.4488 & 7.9345 & 109.021 & 4.257 & $>909.999$ \\
\hline b & 6 & e & 12 & 4.0486 & 0.9995 & 36 & 4.05 & 0.0003 & 0.05 & 2.0216 & 6.0755 & 57.315 & 7.550 & 435.088 \\
\hline b & 6 & e & 24 & 4.2874 & 1.0929 & 36 & 3.92 & 0.0004 & 0.05 & 2.0709 & 6.5040 & 72.778 & 7.932 & 687.377 \\
\hline b & 6 & 1 & 6 & $-1,3229$ & 0.8542 & 36 & -1.55 & 0.1302 & 0.05 & -3.0553 & 0.4095 & 0.268 & 0.047 & 1.506 \\
\hline b & 6 & $f$ & 12 & -0.6251 & 0.6015 & 36 & -1.04 & 0.3056 & 0.05 & -1.8449 & 0.5947 & 0.535 & 0.158 & 1.813 \\
\hline D & 6 & $f$ & 24 & -1.2644 & 0.7592 & 36 & $-1,67$ & 0.1045 & $0.05 !$ & -2.8041 & 0.2753 & 0.282 & 0.061 & 1.317 \\
\hline b & 12 & b & 24 & -0.4272 & 0.5235 & 36 & $\begin{array}{r}-0.82 \\
\ldots .\end{array}$ & 0.4198 & 0.05 & -1.4889 & 0.6344 & 0.652 & 0.226 & 1.886 \\
\hline b & 12 & c & 6 & 0.1770 & 0.5539 & 36 & 0.32 & 0.7511 & 0.05 & -0.9464 & 1.3005 & 1.194 & 0.388 & 3.671 \\
\hline b & 12 & c & 12 & 0.2278 & 0.5346 & 36 & 0.43 & 0.6726 & 0.05 & -0.8565 & 1.3120 & 1.256 & 0.425 & 3.714 \\
\hline b & 12 & $c$ & 24 & 0.04394 & 0.4936 & 36 & 0.09 & 0.9293 & 0.05 & -0.9571 & 1.0450 & 1.045 & 0.384 & 2.843 \\
\hline b & 12 & $d$ & 6 & 2.9750 & 0.8582 & 36 & 3.47 & 0.0014 & 0.05 & $\begin{array}{r}1.2385 \\
\end{array}$ & 4.7115 & 19,590 & 3.451 & 111.214 \\
\hline$b$ & 12 & d & 12 & 2.6073 & 0.5630 & 36 & 4.63 & $<.0001$ & 0.05 & 1.4654 & 3.7492 & 13.563 & 4.329 & 42,488 \\
\hline b & 12 & d & 24 & 2.6745 & 0.7301 & 36 & 3.66 & 0.0008 & 0.05 & .1938 & 4.1552 & 14.506 & 3.300 & 63.767 \\
\hline b & 12 & $\theta$ & 6 & $\begin{array}{r}3.7271 \\
\end{array}$ & \&5641 & 36 & 2.38 & 0.0226 & 0.05 & 0.5550 & 6.8992 & 41.558 & 1.742 & 991.478 \\
\hline b & 12 & 0 & 12 & 3.0841 & 0.9426 & 36 & 3.27 & 0.0024 & 0.05 & 1.1724 & 4.9958 & 21.848 & 3.230 & 147.786 \\
\hline b & 12 & e & 24 & 3.3230 & 1.0412 & 36 & 3.19 & 0.0029 & 0.05 & 1.2114 & 5.4346 & 27.742 & 3.358 & 229.190 \\
\hline b & 12 & $f$ & 6 & -2.2874 & 0.7869 & 36 & -2.91 & 0.0062 & 0.05 & -3.8833 & -0.6914 & 0.102 & 0.021 & 0.501 \\
\hline b & 12 & $f$ & 12 & -1.5895 & 0.5013 & 36 & -3.17 & 0.0031 & 0.05 & -2.6062 & -0.5728 & 0.204 & 0.074 & 0.564 \\
\hline b & 12 & f & 24 & -2.2288 & 0.6826 & 36 & -3.27 & 0.0024 & 0.05 & -3.6132 & -0.8445 & 0108 & 0.027 & 0.430 \\
\hline b & 24 & c & 6 & 0.6043 & 0.6066 & 36 & 1.00 & 0.3259 & 0.05 & -0.6261 & 1.8346 & 1.830 & 0.535 & B.263 \\
\hline b & 24 & c & 12 & 0.6550 & 0.5890 & 36 & 1.11 & 0.2735 & 0.05 & -0.5396 & 1.8496 & 1.925 & 0.583 & 6.357 \\
\hline b & 24 & c & 24 & 0.4712 & $0.552 \uparrow$ & 36 & 0.85 & 0.3991 & 0.05 & $-0,6485$ & 1.5909 & 1.602 & 0.523 & 4.908 \\
\hline b & 24 & d & 6 & 3.4022 & 0.8912 & 36 & 3.82 & 0.0005 & 0.05 & 1.5948 & 5.2097 & 30.031 & 4.927 & 183.034 \\
\hline$b$ & 24 & d & 12 & 3,0345 & 0.6150 & 36 & 4.93 & $<.0001$ & 0.05 & 1.7873 & 4.2817 & 20.791 & 5.974 & 72.367 \\
\hline b & 24 & $d$ & 24 & 3.1018 & 0.7708 & 36 & 4.02 & 0.0003 & 0.05 & 1.5384 & 4.6651 & 22.237 & 4.657 & 106.178 \\
\hline$b$ & 24 & $e$ & 6 & 4.1543 & 1.5835 & 36 & 2.62 & 0.0127 & 0.05 & 0.9428 & 7.3658 & 63.708 & 2.567 & $>999.999$ \\
\hline b & 24 & e & 12 & 3.5113 & 0.9745 & $36 i$ & 3.60 & 0.0009 & 0.05 & 1.5350 & 5.4877 & 33.493 & 4.641 & 241.701 \\
\hline b & 24 & e & 24 & 3.7502 & 1.0701 & 36 & 3.50 & 0.0012 & 0.05 & 1.5798 & 5.9205 & 42.529 & 4.854 & 372,611 \\
\hline b & 24 & $f$ & 6 & -1.8601 & 0.8249 & 36 & -2.26 & 0.0303 & 0.05 & -3.5330 & -0.1872 & 0.156 & 0.029 & 0.829 \\
\hline b & 24 & ! & 2 & -1.1623 & 0.5590 & | 36 & -2.08 & 0.0448 & 0.05 & -2.2960 & -0.02861 & 0.313 & 0.101 & 0.972 \\
\hline b & 24 & $f$ & 24 & -1.8016 & 0.7260 & 36 & -2.48 & 0.0179 & 0.05 & -3.2740 & -0.3292 & 0.165 & 0.038 & 0.720 \\
\hline c & 6 & $\therefore c$ & 12 & 0.05074 & $0.6163 i$ & 36 & 0.08 & 0.9348 & 0.05 & -1.1992 & 1.3006 & 1.052 & 0.301 & 3.672 \\
\hline c & 6 & $i^{c}$ & 24 & -0.1331 & 0.5811 & 36 & -0.23 & 0.8201 & 0.05 & -1.3115 & 1.0454 & 0.875 & 0.269 & 2,844 \\
\hline c & 6 & d & 6 & 2.7980 & 0.9094 & 36 & 3,08 & 0.0040 & 0.05 & 0.9535 & 4.6424 & 16.411 & 2.595 & 103.795 \\
\hline c & 6 & d & 12 & 2.4303 & 0.6411 & 36 & 3.79 & 0.0006 & 0.05 & 1.1301 & 3.7305 & 11.362 & 3.096 & 41.701 \\
\hline c & 6 & d & 24 & 2.4975 & 0.7919 & 36 & 3.15 & 0,0032 & 0.05 & 0,8915 & 4.1035 & 12.152 & 2.439 & 60.551 \\
\hline c & 6 & e & 6 & 3.5501 & 1.5939 & 36 & $2.23 !$ & 0.0323 & 0.05 & 0.3176 & 6.7825 & 34.816 & 1.374 & 882.313 \\
\hline$c$ & 6 & e & 12 & 2.9071 & 0.9912 & 36 & $2,93$. & 0.0058 & 0.05 & 0.8968 & 4.9173 & 18.303 & 2.452 & 136.638 \\
\hline$c$ & 6 & 0 & 24 & 3.1459 & $1 . C 854$ & 35 & 2.90 & 0.0063 & 0.05 & 0.9447 & 5.3472 & 23.241 & 2.572 & 210.016 \\
\hline c & 6 & f & 6 & -2.4644 & 0.8445 & 36 & -2.92 & 0.0060 & 0.05 & -4.1772 & -0.7516 & 0.085 & 0.015 & 0.472 \\
\hline$c$ & 6 & $f$ & 12 & $\begin{array}{l}-1.7666 \\
--\end{array}$ & 0.5876 & 36 & -3.01 & 0.0048 & 0.05 & $-2,9583$ & -0.5748 & 0.171 & 0.052 & 0.563 \\
\hline 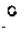 & 6 & $f$ & 24 & -2.4059 & 0.7483 & 36 & -3.22 & 0.0028 & 0.05 & -3.9235 & -0.8882 & 0.090 & 0.020 & 0.411 \\
\hline$c$ & 12 & $c$ & 24 & -0.1838 & 0.5627 & 36 & -0.33 & 0.7458 & 0.05 & $-1,3250$ & 0.9573 & 0.832 & 0.266 & 2.605 \\
\hline & 12 & d & 6 & 2.7472 & 0.8978 & 36 & 3.06 & 0.0042 & 0.05 & 0.9264 & 4.5681 & 15.599 & 2.525 & 96.357 \\
\hline & 12 & $d$ & 12 & 2.3796 & 0.6245 & 36 & 3.81 & 0.0005 & 0.05 & 1.1131 & 3.6461 & 10.800 & 3.044 & 38.324 \\
\hline & 12 & d & 24 & 2.4468 & 0.7785 & 36 & 3.14 & 0.0033 & 0.05 & 0.8680 & 4.0256 & 11.551 & 2.382 & 56.012 \\
\hline
\end{tabular}




\begin{tabular}{|c|c|c|c|c|c|c|c|c|c|c|c|c|c|c|}
\hline & 12 & e & 6 & 3.4893 & 1.5872 & 36 & 2.20 & 0.0340 & 0.05 & 0.2803 & 6.7184 & 33,093 & 1.323 & 827.487 \\
\hline c & 12 & e & 12 & 2.8563 & 0.9805 & 36 & 291 & 0.0061 & 0.05 & 0.8677 & 4.8450 & 17.398 & 2.382 & 127.097 \\
\hline c & 12 & e & 24 & 3.0952 & 1.0756 & 36 & 2.88 & 0,0067 & 0.05 & 0.9137 & 5.2767 & 22.092 & 2.494 & 195.722 \\
\hline c & 12 & 1 & 6 & -2.5151 & 0.8320 & 36 & -3.02 & 0.0046 & 0.05 & $-4.2 \mathrm{C} 25$ & $-0,8278$ & 0.081 & 0.015 & 0.437 \\
\hline c & 2 & $f$ & 12 & -1.8173 & 0.5694 & 36 & -3.19 & 0.0029 & 0.05 & -2.9722 & $-0,8624$ & 0.162 & 0.051 & 0.516 \\
\hline c & 12 & $f$ & 24 & -2.4566 & 0.7341 & 36 & -3.35 & 0.0019 & 0.05 & -3.9454 & $-0,9678$ & 0.086 & 0.019 & 0.380 \\
\hline$c$ & 24 & d & 6 & 2.8311 & 0.8740 & 36 & 3.35 & 0.0019 & 0.05 & 1.1585 & 4.7036 & 18.747 & 3.185 & 110,344 \\
\hline$c$ & 24 & d & 12 & 2.5634 & 0.5898 & 36 & 4.35 & 0.0001 & 0.05 & 1.3673 & 3.7594 & 12.980 & 3.925 & 42.824 \\
\hline c & 24 & d & 24 & 2.6306 & 0.7509 & 36 & 3.50 & 0.0012 & 0.05 & 1.1077 & 4.1535 & 13.882 & 3.027 & 63.654 \\
\hline c & 24 & e & 6 & 3.6834 & 1.5739 & 36 & 2.34 & 0.0249 & 0.05 & 0.4911 & 6.8752 & 39.771 & 1.634 & 967.923 \\
\hline c & 24 & $\theta$ & 12 & 3.0402 & 0.9588 & 36 & 3.17 & 0.0031 & 0.05 & 1.0957 & 4.9847 & 20.909 & 2.991 & 146.155 \\
\hline c & 24 & e & 24 & 3.2790 & 1.0558 & 36 & 3.11 & 0.0037 & 0.05 & 1.1376 & 5.4204 & 26.550 & 3.119 & 225,866 \\
\hline c & 24 & $f$ & 6 & -2.3313 & 0.8062 & 36 & -2.89 & 0.0065 & 0.05 & -3.9664 & -0.6962 & 0.097 & 0.019 & 0.498 \\
\hline c & 24 & $f$ & 12 & -1.6335 & 0.5311 & 36 & -3.08 & 0.0040 & 0.05 & -2.7107 & -0.5563 & 0.195 & 0.066 & 0.573 \\
\hline c & 24 & $f$ & 24 & -2.2728 & 0.7048 & 36 & -3.22 & 0.0027 & 0.05 & -3.7022 & -0.8434 & 0.103 & 0.025 & 0.430 \\
\hline d & 6 & d & 12 & -0.3677 & 0.9150 & 36 & -0.40 & 0.6902 & 0.05 & -2.2234 & 1.4881 & 0.692 & 0.108 & 4.428 \\
\hline$d$ & 6 & d & 24 & -0.3005 & 1.0263 & 36 & -0.29 & 0.7714 & 0.05 & -2.3819 & 1.7810 & 0.740 & 0.092 & 5.936 \\
\hline d & 6 & e & 6 & 0.7521 & 1.7224 & 36 & 0.44 & $|0.6650|$ & 0.05 & -2.7411 & 4.2452 & 2.121 & 0.065 & 69.772 \\
\hline d & 6 & e & 12 & 0.1091 & 1.1869 & 36 & 0.09 & 0.9273 & 0.05 & -2.2980 & 2,5162 & 1.115 & 0.100 & 12.382 \\
\hline d & 6 & $\theta$ & 24 & 0.3480 & 1.2666 & 36 & 0.27 & 0.7851 & 0.05 & -2.2208 & 2.9167 & 1.416 & 0.109 & 18.481 \\
\hline d & 6 & $f$ & 6 & -5.2624 & 1.0675 & 36 & -4.83 & $<.0001$ & 0.05 & -7.4273 & $-3,0974$ & 0.005 & $<0.001$ & 0.045 \\
\hline d & 6 & f & 12 & -4.5645 & 0.8784 & 36 & -5.20 & $<.0001$ & 0.05 & $-6,3460$ & -2.7831 & 0.010 & 0.002 & 0.062 \\
\hline d & 6 & f & 24 & -5.2038 & 0.9931 & 36 & -5.24 & $<<.0001$ & 0.05 & -7.2179 & -3.1898 & 0.005 & $<0.001$ & 0.041 \\
\hline d & 12 & d & 24 & 0.06722 & 0.7983 & 36 & 0.08 & 0.9334 & 0.05 & -1.5517 & 1.6862 & 1.070 & 0.212 & 5.399 \\
\hline d & 12 & e & 6 & 1.1198 & 1.5970 & 36 & 0.70 & 0.4877 & 0.05 & -2.1192 & 4.3587 & 3.064 & 0.120 & 78.157 \\
\hline d & 12 & e & 12 & 0.4768 & 0.9963 & 36 & 0.48 & 0.6352 & 0.05 & -1.5438 & 2.4974 & 1.611 & 0.214 & 12.151 \\
\hline d & 12 & e & 24 & 0.7156 & 1.0900 & 36 & 0.66 & 0.5157 & 0.05 & -1.4951 & 2.9264 & 2.045 & 0.224 & 18.660 \\
\hline d & 12 & f & 6 & -4.8947 & 0.8505 & 36 & -5.75 & $<.000$ & 0.05 & -6.6196 & -3.1697 & 0.007 & 0.001 & 0.042 \\
\hline d & 12 & 1 & 12 & -4.1968 & 0.5962 & 36 & -7.04 & $<.0001$ & 0.05 & -5.4060 & -2.9877 & 0.015 & 0.004 & 0.050 \\
\hline d & 12 & $f$ & 24 & -4.8361 & 0.7551 & 36 & -6.41 & $<.0001$ & 0.05 & -6.3675 & $-3,3048$ & 0.008 & 0.002 & 0.037 \\
\hline d & 24 & e & 6 & 1.0526 & 1.6633 & 36 & 0.63 & 0.5309 & 0.05 & -2.3208 & 4.4259 & 2.865 & 0.098 & 83.586 \\
\hline d & 24 & $e$ & 12 & 0.4096 & 1.0994 & 36 & 0.37 & 0.7117 & 0.05 & -1.8201 & 2.6392 & 1.506 & 0.162 & 14.003 \\
\hline d & 24 & e & 24 & 0.6484 & 1.1850 & 36 & 0.55 & 0.5876 & 0.05 & -1.7549 & 3.0517 & 1.913 & 0.173 & 21.152 \\
\hline d & 24 & I & 6 & -4.9619 & 0.9692 & 36 & -5.12 & $<.0001$ & 0.05 & 6.9276 & -2.9962 & 0.007 & $<0.001$ & 0.050 \\
\hline d & 24 & if & 12 & -4.2641 & 0.7560 & 36 & -5.64 & $<.0001$ & 0.05 & -5.7973 & -2.7309 & 0.014 & 0.003 & 0.065 \\
\hline d & 24 & if & 24 & -4.9034 & 0.8866 & 36 & -5.53 & $<0001$ & 0.05 & -6.7016 & -3.1052 & 0.007 & 0.001 & 0.045 \\
\hline e & 6 & $e$ & 12 & -0.6430 & 1.7669 & 36 & -0.36 & 0.7181 & 0.05 & -4.2265 & 2,9405 & 0.526 & 0.015 & 18.825 \\
\hline$\theta$ & 6 & e & 24 & -0.4041 & 1.8214 & 36 & -0.22 & 0.8257 & 0.05 & -4.0981 & 3.2899 & 0.668 & 0.017 & 26.839 \\
\hline $\boldsymbol{e}$ & 6 & $\mathrm{f}$ & $B$ & -6.0144 & 1.6890 & 36 & -3.56 & 0.0011 & 0.05 & -9.4399 & -2.5890 & 0.002 & $<0.001$ & 0.075 \\
\hline e & 6 & $f$ & 12 & -5.3186 & 1.5763 & 36 & -3.37 & 0.0018 & 0.05 & -8.5136 & -2.1197 & 0.005 & $<0.001$ & 0.120 \\
\hline e & 6 & $f$ & 24 & -5.9559 & 1.6430 & 36 & -3.63 & 0.0009 & 0.05 & -9.2881 & -2.6238 & 0.003 & $<0.001$ & 0.073 \\
\hline e & 12 & le & 24 & 0.2389 & 1.3265 & 36 & 0.18 & 0.8581 & 0.05 & -2.4515 & 2.9292 & 1.270 & 0.086 & 18.712 \\
\hline$\theta$ & 12 & 1 & 6 & -5.3715 & 1.1379 & 36 & -4.72 & $<0001$ & 0.05 & -7.6793 & $-3,0636$ & 0.005 & $<0.001$ & 0.047 \\
\hline 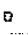 & 12 & 1 & 12 & -4.6736 & 0.9628 & 36 & -4.85 & $<, 0001$ & 0.05 & -6.6262 & -2.7210 & 0.009 & 0.001 & 0.066 \\
\hline$E$ & 12 & 1 & 24 & -53129 & 1.C684 & 36 & -4.97 & $<.0001$ & 0.05 & -74798 & -3.1460 & 0.005 & $<0,001$ & 0.043 \\
\hline e & 24 & 1 & 6 & -5.6103 & 1.2208 & 36 & -4.60 & $\mid<.0001$ & 0.05 & -8.0863 & -3.1343 & 0.004 & $<0.001$ & 0.044 \\
\hline
\end{tabular}




\begin{tabular}{|c|c|c|c|c|c|c|c|c|c|c|c|c|c|c|}
\hline & 24 & $f$ & $\mid 12$ & -4.9125 & 1.0595 & 36 & -4.64 & $<<.0001$ & 0.05 & -7.0612 & -2.7638 & 0.007 & $<0.001$ & 0.063 \\
\hline e & 24 & 1 & 24 & -5.5518 & 1.1563 & 36 & -4.80 & $<.0001$ & 0.05 & -7.8970 & -3.2066 & 0.004 & $<0.001$ & 0.040 \\
\hline 1 & 6 & 1 & 12 & 0.6978 & 0.8110 & 36 & 0.86 & 0.3952 & 0.05 ! & -0.9469 & 2.3426 & 2.009 & 0.388 & 10.408 \\
\hline f & 6 & $f$ & 24 & 0.05853 & 0.9340 & 36. & 0.06 & | $0.9504 \mid$ & 0.051 & -1.8357 & 1.9527 & 1.060 & 0.160 & 7.048 \\
\hline$f$ & 12 & $f$ & 24 & -0.6393 & 0.7102 & 38 & -0.90 & $0.3740 \mid$ & 0.05 & -2.0797 & 0.8011 & 0.528 & 0.125 & 2.228 \\
\hline
\end{tabular}

Performed by J. Luoma; SAS version 9.3 08:43 18MAR15

$$
3 / 18 / 15
$$




\begin{tabular}{|c|c|c|c|c|}
\hline Study Number & AEH-11-PSEUDO-01 & Action & Date & Initials. \\
\hline Lab Noteboak & 1 & Created...... & 7-Jun-12 & TMS Th \\
\hline File Folder & $12 \mathrm{~A}$ & Revised...... & 16-Mar-15 & JAL $\$$ SS \\
\hline \multirow[t]{2}{*}{ Raw Data Location } & \multirow[t]{2}{*}{$12 \mathrm{~A}$} & Reviewed... & $3-14 \cdot 15$ & $7 \% 5$ \\
\hline & & Cert'fied....... & $3-18-8)^{2}$ & $S_{4}=$ \\
\hline File Name: & \multicolumn{4}{|c|}{ I:AEH-11-PSEUDO-01LDatalGlochidia Viability Summary'(glochldia viability assessment 6h (adjusted).xlsx] Pocketbock SDP } \\
\hline
\end{tabular}

\section{Glochidia Viability Assessment - 6 hour}

Mussel species: Pocketbook Glochidła Lot number: 111400

Mean Inttial viability $=93.8 \%$

Test Chemical: Pseudomonas florescens Pf-CL 145A (SDP) Chemical lot t: MBI-401-110308AI-BD-3

Exposure Date: 12-May-11

Test System Locat'on: Block

Page 1 of 1

\begin{tabular}{|c|c|c|c|c|c|c|c|c|c|}
\hline Specles & $\begin{array}{c}\text { Test } \\
\text { Chamber }\end{array}$ & $\begin{array}{c}\text { Treatment } \\
\text { Group }\left(\mathrm{mg}_{\mathrm{g}} / \mathrm{L}\right)\end{array}$ & $\begin{array}{l}\text { Total Number } \\
\text { Glochlda } \\
\text { Counted }\end{array}$ & $\begin{array}{c}\text { Gochldia } \\
\text { Closed luefore } \\
\text { NaCil }\end{array}$ & $\begin{array}{c}\text { Glochidla Open } \\
\text { after NaCl }\end{array}$ & $\begin{array}{c}\text { Unadjusted } \\
\text { 6h vlablitity } \\
\text { (\%) }\end{array}$ & $\begin{array}{l}\text { Adjusted } 6 \mathrm{~h} \\
\text { viabillty (\%) }\end{array}$ & $\begin{array}{c}\text { Mean } \\
\text { adjusted } \\
\text { viability }(\%)\end{array}$ & $\begin{array}{l}\text { Standard } \\
\text { Devlation } \\
\text { (SD) }\end{array}$ \\
\hline Pocketbook & 181 & 0 & 312 & 3 & 18 & 93.3 & 99.4 & 98.4 & 1.1 \\
\hline Pocketbook & $1 \mathrm{C} 3$ & a & 426 & 7 & 25 & 92.5 & 98.6 & - & \\
\hline Pocketbook & $1 A 3$ & 0 & 160 & 2 & 12 & 91.3 & 97.3 & & \\
\hline Pocketbook & 185 & 50 & 341 & 59 & 13 & 79.1 & 84.3 & 83.1 & 9.5 \\
\hline Pocketbook & $1 \mathrm{~A} 1$ & 50 & 189 & 16 & 10 & 86.2 & 91.9 & & \\
\hline Pocketbook & $1 \mathrm{C} 1$ & 50 & 447 & 12.9 & 12 & 68.5 & 73.0 & & \\
\hline Pocketbook & 1B4 & 100 & 170 & 43 & 11 & 68.2 & 72.7 & 80.2 & 6.5 \\
\hline Pocketbook & $1 \mathrm{~A} 2$ & 100 & 116 & 16 & 9 & 784 & 83.5 & & \\
\hline Pocketbook & 1A4 & 100 & 536 & 70 & 42 & 79.1 & 84.3 & & \\
\hline Pocketbook & $16 \tilde{5}$ & 200 & 145 & 47 & 10 & 60.7 & 64.7 & 55.7 & 22.6 \\
\hline Pocketbook & 182 & 200 & 252 & 60 & 21 & 67.9 & 72.3 & & \\
\hline Pocketbook & $1 C 4$ & 200 & 2.38 & 165 & 6 & 23.2 & 30.0 & & \\
\hline Pocketbook & $1 \mathrm{~A} 5$ & 300 & 114 & 75 & 14 & 21.9 & 23.4 & 10.6 & 11.1 \\
\hline Pocketbook & $1 \mathrm{C} 2$ & 300 & 512 & 464 & 31 & 3.3 & 3.5 & & \\
\hline Pocketbook & 1C5 & 300 & 268 & 234 & 22 & 4.5 & 4.8 & & \\
\hline Pocketbook & $1 \mathrm{~A} G$ & $300 \mathrm{HD}$ & 147 & 108 & 30 & 6.1 & 6.5 & 3.7 & 3.3 \\
\hline Pocketbook & 183 & $300 \mathrm{HO}$ & 119 & 91 & 23 & 4.2 & 4.5 & & \\
\hline Pocketbook & 185 & ЗОоно & 210 & 192 & 18 & 0.0 & 0.0 & & \\
\hline
\end{tabular}

'If the number counted was glochldla cpen, the number of glochidia closed = total glochidia - open gloch|dla counted before $\mathrm{A}$ aCl.

Glochldla vlab' lity: (total \# glochicla - (glochidla closed before + open after NaCl))/total glochidla *100

2 Vlabllty adjus:ments were calcu ated by dividing the observed vlability by the mean Initlal viability for each spectes 


\begin{tabular}{|c|c|c|c|c|}
\hline Study Number & AEH-11-PSEUDO-01 & Actlon & Date & Inttials \\
\hline Lab Notebook & 1 & Created...... & 7.Jun-12 & TMSNA \\
\hline File Folder & $12 \mathrm{~A}$ & Revised...... & 16-Mat-15 & $J A L T S$ \\
\hline Raw data location & $12 \mathrm{~A}$ & Revlewed... & $3-17-15$ & 75 \\
\hline & & Certifled..... & $3 \cdot 18 \cdot 15$ & $5 n-$ \\
\hline
\end{tabular}

\section{Glochidia Viability Assessment - 6 hour}

Mussel species: Fatmucket Glochidia Lot number: 111600

Mean initial vability $=80.3 \%$

Test Chenical: Pseudomonas florescers Pf-CL 145A (SOP) Chem'cal lot \#: MBI-4C1-110308AI-BD-3

Exposure Date: 12-May-11

Test System Location: Block 3

Page 1 of 1

\begin{tabular}{|c|c|c|c|c|c|c|c|c|c|}
\hline Species & $\begin{array}{c}\text { Test } \\
\text { Chamber }\end{array}$ & $\begin{array}{l}\text { Treatment Group } \\
\qquad(\mathrm{mg} / \mathrm{L})\end{array}$ & $\begin{array}{l}\text { Total } \\
\text { Number } \\
\text { Glochida } \\
\text { Counted }\end{array}$ & $\begin{array}{l}\text { Glochidia } \\
\text { Closed } \\
\text { before } \mathrm{NaCl}^{1}\end{array}$ & $\begin{array}{l}\text { Glochidia } \\
\text { Open } \\
\text { after } \mathrm{NaCl}\end{array}$ & $\begin{array}{l}\text { Unadjusted } \\
\text { 6h viability } \\
\text { (\%) }\end{array}$ & $\begin{array}{c}\text { Adjusted } 6 \\
\text { h viability } \\
(\%)\end{array}$ & $\begin{array}{c}\text { Mean } \\
\text { adjusted } \\
\text { viability (\%) }\end{array}$ & $\begin{array}{l}\text { Standard } \\
\text { Deviation } \\
\text { (SD) }\end{array}$ \\
\hline Fatmucket & $3 \mathrm{~B} 2$ & 0 & 210 & 29 & 34 & 70.0 & 87.2 & 90,1 & 5.0 \\
\hline FatmLcket & 383 & 0 & 265 & 32 & 29 & 77.0 & 95.9 & & \\
\hline Fatmucket & $3 \mathrm{~A} 2$ & 0 & 273 & 36 & 46 & 70.0 & 87.1 & & \\
\hline Fatmucket & 385 & 50 & 456 & 93 & 98 & 58.1 & 72.4 & 70.7 & 3.4 \\
\hline Fatmucket & $3 \mathrm{C5}$ & 50 & 276 & 57 & 71 & 53.6 & 66.8 & & \\
\hline Fatmucket & $3 C 6$ & 50 & 239 & 42 & 57 & 38.6 & 72.9 & & \\
\hline Fatmucke: & $3 A 1$ & 100 & 237 & 126 & 37 & 31.2 & 38.9 & 36.1 & 18.4 \\
\hline Fatmucket & 3A3 & 100 & 303 & 238 & 25 & 13.2 & 16.4 & & \\
\hline Fatmucket & $3 A 5$ & 100 & 351 & 119 & 83 & 42.5 & 52.9 & & \\
\hline Fatmucket & $3 B 4$ & 200 & 380 & 308 & 47 & 6.6 & 8.2 & 24.0 & 21.4 \\
\hline Fatmucket & $3 B 6$ & 200 & 52.6 & 407 & 53 & 12.5 & 15.6 & & \\
\hline Fatrnucket & $3 C 4$ & 200 & 201 & 59 & 64 & 38.8 & 48.3 & & \\
\hline Fatmucket & $3 C 1$ & 300 & 501 & 224 & 121 & 31.1 & 38.8 & 14.8 & 20.8 \\
\hline Fatmucket & $3 C 3$ & 300 & 242 & 204 & 29 & 3.7 & 4.6 & & \\
\hline Fatmucket & 3B1 & 300 & 482 & 422 & 56 & 0.8 & 1.0 & & \\
\hline Fatmucket & $3 \mathrm{A4}$ & $300 \mathrm{HD}$ & 216 & 155 & 56 & 2.3 & 2.9 & 2.9 & 2.9 \\
\hline Fatmucket & $3 A 6$ & $300 \mathrm{HD}$ & 212 & 164 & 38 & 4.7 & 5.9 & & \\
\hline Fatmucket & $3 C 2$ & $300 \mathrm{HD}$ & 205 & 166 & 39 & 0.0 & 0.0 & & \\
\hline
\end{tabular}

1 If the number counted was glochldia open, the number of glochidia closed = total glochldla - open glochldia counted before NaCl.

Glochidia viability: (total \# glochidia - (glochidia closed before + open after NaCl)/total glochidia *100

${ }^{2} V$ iability adjustments were calculated by divlding the observed viability by the mear. initlal viability for each species 


\begin{tabular}{|c|c|c|c|c|}
\hline Study Number & AEH-11-PSEUDO-01 & Action & Date & Initlals \\
\hline Lab Notebook & 1 & Created...... & 7-Jun-1.2 & TMS $\pi$ \\
\hline File folder & $12 \mathrm{~A}$ & Revised...... & 16-Mar-15 & $\mathrm{JAL} 12$ \\
\hline \multirow[t]{2}{*}{ Raw data location } & $12 \mathrm{~A}$ & Revlewed... & $3-17-15$ & 725 \\
\hline & & Certif ed......... & $3.18(5-1)$ & $\sqrt{4}$ \\
\hline
\end{tabular}

Glochidia Viability Assessment - 6 hour

Mussel species: Higgins Eye Gloci cia Lot number: $\mathbf{1 1 1 5 0 0}$

Test Chemlcal: Pseudomonas florescens Pf-CL 145A (SDP) Chemlcal lot \#: MBl-401-110308A1-BD-3

Exposure Date: 12-May-11

Mear Initlal vlabj: $\mid$ ty $=80.8 \%$

Test System Location: Block 2

Page 1 of 1

\begin{tabular}{|c|c|c|c|c|c|c|c|c|c|}
\hline Specles & $\begin{array}{c}\text { Test } \\
\text { Chamber }\end{array}$ & $\begin{array}{c}\text { Treatment } \\
\text { Group } \\
\text { (mg/L) }\end{array}$ & $\begin{array}{l}\text { Total } \\
\text { Number } \\
\text { Glochida } \\
\text { Counted }\end{array}$ & $\begin{array}{l}\text { Glochldia } \\
\text { Closed } \\
\text { before NaCl }{ }^{1}\end{array}$ & $\begin{array}{l}\text { Glochidia } \\
\text { Open } \\
\text { after } \mathrm{NaCl}\end{array}$ & $\begin{array}{l}\text { Unadjusted } \\
6 \text { h viability } \\
\text { (\%) }\end{array}$ & $\begin{array}{l}\text { Adjusted 6h } \\
\text { Viabillty }(\%)^{2}\end{array}$ & $\begin{array}{c}\text { Mean } \\
\text { ad]usted } \\
\text { viability (\%) }\end{array}$ & $\begin{array}{c}\text { Standard } \\
\text { Devlatlon } \\
\text { (SD) }\end{array}$ \\
\hline Higgins Eye & $2 \mathrm{C} 3$ & 0 & 248 & 18 & 76 & 62.1 & 76.9 & 89.5 & 11.8 \\
\hline Higglns Eye & $2 \mathrm{C} 6$ & 0 & 168 & 10 & 34 & 73.8 & 91.3 & & \\
\hline Hlggins Eye & $2 B 1$ & 0 & 258 & 18 & 31 & 81.0 & 100.3 & & \\
\hline Hlggins Eye & $2 \mathrm{~A} 1$ & 50 & 224 & 11 & 39 & 77.7 & 96.1 & 96.2 & $4.6^{\circ}$ \\
\hline Higgins Eye & $2 A 2$ & 50 & 616 & 26 & 88 & 81,5 & 100.9 & & \\
\hline Higgins Eýe & $2 A 4$ & 50 & 204 & 24 & 29 & 74.0 & 91.6 & & \\
\hline Hlggins Eye & $2 A 3$ & 100 & 261 & 47 & 51 & 62.5 & 77.3 & 78.2 & 1.3 \\
\hline Higgins Eye & $2 \mathrm{C} 4$ & 100 & 396 & 40 & 101 & 64.4 & $\cdot 79.7$ & & \\
\hline Hlggins Eye & $2 \mathrm{C5}$ & 100 & 414 & 103 & 51 & 62.8 & 77.7 & & \\
\hline Hlge'ns Eye & $2 A G$ & 200 & 492 & 120 & 62 & 63.0 & 78.0 & 70.1 & 6.8 \\
\hline HigeIns Eye & $2 B 2$ & 200 & 357 & 73 & 93 & 53.5 & 66.2 & & \\
\hline Higgins Eye & 285 & 200 & 512 & 121 & 117 & 53.5 & 66.2 & & \\
\hline Higglns Eye & $2 \mathrm{C} 2$ & 300 & 422 & 98 & 111 & 50.5 & 62.5 & 36.0 & 26.9 \\
\hline Hlggirs Eye & $2 c 1$ & 300 & 279 & 148 & 48 & 29.7 & 36.8 & & \\
\hline Higgirs Eye & $2 \mathrm{B3}$ & 300 & 297 & 251 & 25 & 7.1 & 8.8 & & \\
\hline Higg'rs tye & $2 \mathrm{~A} 5$ & $300 \mathrm{HD}$ & 327 & 216 & 60 & 15.6 & 19.3 & 16.4 & 15.2 \\
\hline Higgins Eye & 234 & $300 \mathrm{HD}$ & 276 & 168 & 41 & 24.3 & 30.0 & & \\
\hline Higgins Eye & 236 & $300 \mathrm{HD}$ & 139 & 102 & 37 & 0.0 & 0.0 & & \\
\hline
\end{tabular}

${ }^{1}$ If the number counted was glochidia open, the number of glochid' a closed a total glochidia - open glochidia counted before NaCl.

${ }^{2}$ Viablity adjustments were claculated by dividing tine observed vlability by the mean initial vlability for each species 


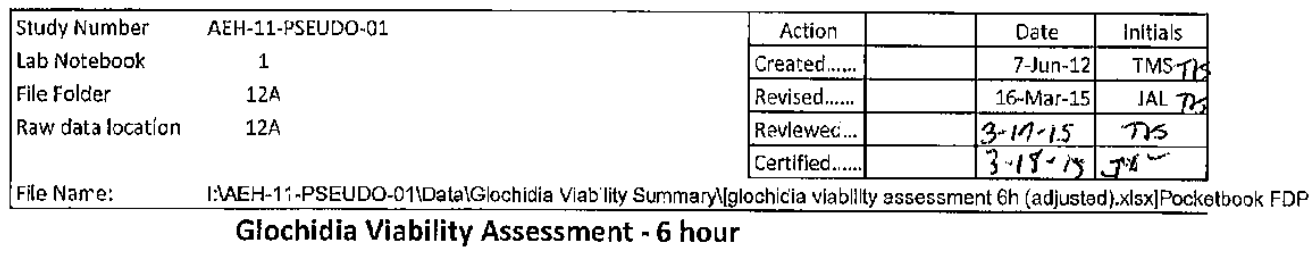

Mussel species: Pocketbock Glochiclla Lot number: 115400

Vean Inltlal vlab'llty $=84.7 \%$

Test Chemlcal: Pseudomonas florescens Pf-CL 14SA (FDP) Chemical lot number: 110607WB-FD-E

Exposure Date: 18 -Oct-11

Page 1 of 1

\begin{tabular}{|c|c|c|c|c|c|c|c|c|c|}
\hline Spacles & $\begin{array}{c}\text { Test } \\
\text { Chamber }\end{array}$ & $\begin{array}{l}\text { Treatment } \\
\text { Group } \\
\text { (mg/L) }\end{array}$ & $\begin{array}{c}\text { Total } \\
\text { Number } \\
\text { Glochida } \\
\text { Counted }\end{array}$ & $\begin{array}{c}\text { Glochidla } \\
\text { Closed } \\
\text { before NaCl' }\end{array}$ & $\begin{array}{l}\text { Glochidia } \\
\text { Open } \\
\text { after NaCl }\end{array}$ & $\begin{array}{c}\text { Unadjusted } \\
\text { 6h vability } \\
(\%)\end{array}$ & $\begin{array}{c}\text { Adjusted } 6 \mathrm{~h} \\
\text { Vlabillty } \\
\{\%\}^{2}\end{array}$ & $\begin{array}{c}\text { Mean } \\
\text { adjusted } \\
\text { viability }[\%]\end{array}$ & $\begin{array}{c}\text { Standard } \\
\text { Deviatlon } \\
\text { (SD) }\end{array}$ \\
\hline Pocketbook & $3 A 6$ & 0 & 214 & 17 & 16 & 84.6 & 99.9 & 97.1 & 5.6 \\
\hline Pocketbook & $3 \mathrm{~B} 5$ & 0 & 89 & 7 & 6 & 85.4 & 100.8 & & \\
\hline Pocketbook & 386 & 0 & 125 & 18 & 11 & 76.8 & 90.7 & & \\
\hline Pocketbook & $3 \mathrm{~A} 4$ & 50 & 157 & 16 & 17 & 79,0 & 93.2 & 82.2 & 13.6 \\
\hline Pocketbook & $3 C 1$ & 50 & 74 & 27 & 5 & 56.8 & 67.0 & & \\
\hline Pocketiook & $3 \mathrm{C} 2$ & 50 & 144 & 22 & 17 & 72.9 & 86.1 & & \\
\hline Pocketrook & $3 \mathrm{~B} 1$ & 100 & 259 & 70 & 21 & 64.9 & 76.6 & 77.6 & 4.1 \\
\hline Pocketbook & $3 \mathrm{~B} 3$ & 100 & 190 & 33 & 25 & 69.5 & 82.0 & & \\
\hline Pocketbook & $3 \mathrm{C6}$ & 100 & 161 & 47 & 13 & 62.7 & 74.1 & & \\
\hline Pocketbook & $3 A 2$ & 200 & 256 & 86 & 52 & 46.1 & 54.4 & 57.5 & 13.5 \\
\hline Pocketbook & $3 \mathrm{C3}$ & 200 & 299 & 141 & 42 & 38.8 & 45.8 & & \\
\hline Pocketboo's & $3 C 5$ & 200 & 157 & 47 & 14 & 61.1 & 72.2 & & \\
\hline Pocketbook & $3 A 3$ & 300 & 259 & 63 & 64 & 51.0 & $60 . \bar{z}$ & 48.0 & 17.3 \\
\hline Pocketbook & $3 \mathrm{~B} 4$ & 300 & 272 & 149 & 58 & 23.9 & 28.2 & & \\
\hline Pocketbook & $3 \mathrm{CA}$ & 300 & 655 & 218 & 128 & 47.2 & 55.7 & & \\
\hline Pocketbook & $3 A 1$ & 300HD & 200 & 144 & 45 & 5.5 & 6.5 & 24.1 & 27.2 \\
\hline Pocketbook & $3 A 5$ & $300 \mathrm{HD}$ & 379 & 87 & 114 & 47.0 & 55.4 & & \\
\hline Pocketbook & $3 B 2$ & $300 \mathrm{HD}$ & 418 & 321 & 60 & 8.9 & 10.5 & & \\
\hline
\end{tabular}

The number counted was $\vec{g}$ ochidia open, the number of glochidla closed = total glochidia - open glochicia counted beforc Naci.

Glochldia viability: (total \# glcchidia - (glochidla closed before + open after Nacl))/total glochidia *100

${ }^{2}$ Viabllity adjustments were calculated by dlvidling the observed viabillty by the mean Initlal viability for each species 


\begin{tabular}{|c|c|c|c|c|}
\hline Study Number & AEH-11-PSEUDO-01 & Actlon & Date & Iritlals \\
\hline Lab Notebook & 1 & Created...... & 7-Jun-12 & TMS Th \\
\hline File Folder & $12 \mathrm{~A}$ & Revised...... & 16-Mar-15 & JAL TS \\
\hline \multirow[t]{2}{*}{ Raw Data Location } & $12 \mathrm{~A}$ & Revlewed... & $3-17-15$ & $7 \mathrm{~s}$ \\
\hline & & Cert|fled....... & $5 \cdot 18-15$ & $\sqrt{4} \div$ \\
\hline
\end{tabular}

Glochidia Viability Assessment - 6 hour

Mussel species: Black Sandshell Glochidia Lot number: 120800

Mean initial vataility $=84.5 \%$

Test Chemical: Pseudomonas forescens Pf-CL 145A (SOP) Chemical lot H: MBi-401. SI)P 4655-12-Mix

Exposure Date: $17-\mathrm{jan}-12$

Test System Locatlon: Block 1

Page 1 of 1

\begin{tabular}{|c|c|c|c|c|c|c|c|c|c|}
\hline Mussel Species & $\begin{array}{c}\text { Test } \\
\text { Chamber }\end{array}$ & $\begin{array}{c}\text { Treatment } \\
\text { Group } \\
(\mathrm{mg} / \mathrm{L})\end{array}$ & $\begin{array}{c}\text { Total } \\
\text { Number } \\
\text { Glochlda } \\
\text { Counted }\end{array}$ & $\begin{array}{c}\text { Glochidia } \\
\text { Closed } \\
\text { before } \\
\mathrm{NaCl}^{1}\end{array}$ & $\begin{array}{c}\text { Glochidla } \\
\text { Open } \\
\text { after } \\
\mathrm{NaCl} \\
\end{array}$ & $\begin{array}{c}\text { Unadjusted } \\
\text { 6h vlability } \\
\text { (\%) }\end{array}$ & $\begin{array}{l}\text { Adjusted 6h } \\
\text { Vabllity }(\%)^{2}\end{array}$ & $\begin{array}{c}\text { Mean } \\
\text { adjusted } \\
\text { viablity (\%) }\end{array}$ & $\begin{array}{c}\text { Standard } \\
\text { Devlation } \\
\text { (SD) }\end{array}$ \\
\hline Black Sandshell & 1A5 & 0 & 241 & 8 & 28 & 85.1 & 100.7 & 101.4 & 1.3 \\
\hline Black Sandshell & 131 & 0 & 337 & 8 & 42 & 85.2 & 100.8 & & \\
\hline Black Sandshell & $1 \mathrm{CS}$ & 0 & 314 & 8 & 33 & 86.9 & 102.3 & & \\
\hline Black Sandshell & $1 \mathrm{~A} 1$ & 50 & 252 & 183 & 32 & 14.7 & 17.4 & 13.9 & 11.3 \\
\hline Black Sandshell & $1 \mathrm{~A} 3$ & 50 & 290 & 284 & 3 & 1.0 & 1.2 & & \\
\hline Black Sandshell & $1 \mathrm{Cl}$ & 50 & 206 & 132 & 34 & 19.4 & 23.0 & & \\
\hline Black Sandstell & $1 \mathrm{~B} 2$ & 100 & 166 & 164 & 2 & 0.3 & 0.0 & 0.6 & 1.0 \\
\hline Black Sandstell & 183 & 100 & 351 & 330 & 16 & 1.4 & 1.7 & & \\
\hline Elack Sandstell & $1 \mathrm{C5}$ & 100 & 244 & 136 & 8 & 0.0 & 0.0 & & \\
\hline Black Sandshell & IAG & 200 & 352 & 275 & 69 & 2.3 & 2.7 & 2.1 & 1.8 \\
\hline Elack Sandshelf & $1 \mathrm{~B} \overline{5}$ & 200 & 168 & 138 & 25 & 3.0 & 3.5 & & \\
\hline Black Santishell & 186 & 200 & 224 & 222 & 2 & 0.0 & 0.0 & & \\
\hline Hlack Sandshell & $1 \mathrm{~A} 2$ & 300 & 159 & 117 & 42 & 0.0 & 0.0 & 1.3 & 1.8 \\
\hline Black Sandshell & $1 \mathrm{~B} 4$ & 300 & 312 & 286 & 17 & 2.9 & 3.4 & & \\
\hline Black Sandshell & $1 C 2$ & 300 & 250 & 234 & 15 & 0.4 & 0.5 & & \\
\hline Black Sandishell & $1 \mathrm{~A} 4$ & $300 \mathrm{HD}$ & 219 & 208 & 11 & 0.0 & 0.0 & 1.0 & 1.7 \\
\hline Black Sandshell & $1 \mathrm{C} 3$ & $300 \mathrm{HD}$ & 254 & 213 & 41 & 0.0 & 0.0 & & \\
\hline Black Sandshell & $1 \mathrm{C} 4$ & $300 \mathrm{HD}$ & 237 & 195 & 36 & 2.5 & 3.0 & & \\
\hline
\end{tabular}

'If the number counted was glochidia open, the number of glochidia closed = total glochidia - open glochidia counted before $\mathrm{NaCl}$.

Glochitdla viability; (total \# glochidia - (glochidia closed before + open after NaCl)//otal glochldla *100

${ }^{2}$ Viability adjustments were calculated by div|ding the observed vlability by the mean Initial vlability for each species 


\begin{tabular}{|c|c|c|c|c|}
\hline Study Number & AEH-11-PSEUDO-01 & Action & Date & Initials \\
\hline Lab Nuleboor & 1 & Created...... & 7-Jun-12 & TMS Dg \\
\hline File Folder & $12 \mathrm{~A}$ & Revised..... & 16-Mar-15 & JAL Th \\
\hline \multirow[t]{2}{*}{ Raw Data Location } & $12 \mathrm{~A}$ & Reviewed... & $3-1 \div-15$ & ThS \\
\hline & & Certified...... & $3-19+15$ & 50 \\
\hline
\end{tabular}
Glochidia Viability Assessment - 6 hour

Mussel specles: Mucket Glochldia Lot number: $120700 \quad$ Mean init'al viablity $-92.7 \%$

Test Chemical; Fseudomonas florescens Pf-CL 145A (SDP) Chemical lot H: MBl-401 SDP 4G55-12-Mix

Fxposure Date: 17-Jan-12

Test System Location: Block 2

Page 1 of 1

\begin{tabular}{|c|c|c|c|c|c|c|c|c|c|}
\hline Species & $\begin{array}{c}\text { Test } \\
\text { Chamber }\end{array}$ & $\begin{array}{c}\text { Treatment } \\
\text { Group } \\
(\mathrm{mg} / \mathrm{h})\end{array}$ & $\begin{array}{c}\text { Total } \\
\text { Number } \\
\text { Glochida } \\
\text { Counted }\end{array}$ & $\begin{array}{c}\text { Glochidia } \\
\text { Closed } \\
\text { before } \\
\mathrm{NaCl}^{1}\end{array}$ & $\begin{array}{l}\text { Glochidia } \\
\text { Open } \\
\text { after } \mathrm{NaCl}\end{array}$ & $\begin{array}{c}\text { Unadjusted } \\
\text { 6h viability } \\
(\%)\end{array}$ & $\begin{array}{c}\text { Adjusted 6h } \\
\text { Viability } \\
(\%)^{2}\end{array}$ & $\begin{array}{c}\text { Mean } \\
\text { adjusted } \\
\text { vlability (\%) }\end{array}$ & $\begin{array}{c}\text { Standard } \\
\text { Deviation } \\
\text { (SD) }\end{array}$ \\
\hline Mucket & 2834 & 0 & 198 & 15 & 17 & 83.8 & 90.4 & 95.6 & 4.8 \\
\hline Mucket & $2 B 5$ & 0 & 132 & 2 & 12 & 89.4 & 96.4 & & \\
\hline Mucket & $2 C 1$ & 0 & 257 & 7 & 12 & 92.6 & 99.9 & & \\
\hline Mucket & $2 \mathrm{~A} 4$ & 50 & 349 & 51 & 36 & 75.1 & 81.0 & $\pi / 3$ & 7.1 \\
\hline Mucket & $2 B 6$ & 50 & 128 & 43 & 3 & 64.1 & 69.1 & & \\
\hline Mucket & $2 \mathrm{CC}$ & 50 & 295 & 59 & 12 & 75.9 & 81.9 & & \\
\hline Mucket & $2 \mathrm{~A} 2$ & 100 & 190 & 61 & 13 & 61.1 & 65.9 & 77.9 & 10.5 \\
\hline Mucket & $2 \dot{\mathrm{B}} 2$ & 100 & 154 & 26 & 7 & 78.6 & 84.8 & & \\
\hline Mucket & $2 \mathrm{C6}$ & 100 & 466 & 78 & 29 & 77.0 & 83.1 & & \\
\hline Mucket & $2 A 5$ & 200 & 116 & 48 & 8 & 51.7 & 55.8 & 43.7 & 17.6 \\
\hline Mucket & $2 A G$ & 200 & 202 & 82 & 23 & 48.0 & 51.8 & & \\
\hline Mucket & $2 \mathrm{C} 2$ & 200 & 399 & 291 & 21 & 21.8 & 23.5 & & \\
\hline Mucket & 281 & 300 & 265 & 199 & 14 & 19.6 & 21.2 & 12.7 & 7.9 \\
\hline Mucket & $2 \mathrm{~B} 3$ & 300 & 251 & 232 & 6 & 5.2 & 5.6 & & \\
\hline Mucket & 264 & 300 & 323 & 283 & 6 & 10.5 & 11.4 & & \\
\hline Mucket & $2 \mathrm{~A} 1$ & $300 \mathrm{HD}$ & 292 & 272 & 4 & 5.5 & 5.9 & 3.9 & 2.2 \\
\hline Mucket & $2 A 3$ & $300 \mathrm{HD}$ & 355 & 342 & 8 & 1.4 & 1.5 & & \\
\hline Mucket & $2 \mathrm{CS}$ & $300 \mathrm{HD}$ & 280 & 259 & 10 & 3.9 & 4.2 & & \\
\hline
\end{tabular}

If the number counted was glochidia open, the number of glochldla closed = total glochldia - open glochldia counted before $\mathrm{NaCl}$ Glochidia viability: (total \# glochidia - (glochidia closed before + open after NaC.))/total glochidia *100

${ }^{2}$ Vlabllity adjustments were calculated by divlding the observed viability by the mean initial viability for each species 


\begin{tabular}{|c|c|c|c|c|}
\hline Study Number & AEH-11-PSEUCDO-01 & Action & Date & Ini:lals \\
\hline Lab Notebook & 1 & Created..... & 7-Jun-12 & TNS TH \\
\hline File Folder & $12 A$ & Revised...... & 17-Mar 15 & TIS THS \\
\hline \multirow[t]{2}{*}{ Raw Data Location } & $12 \mathrm{~A}$ & Reviewed... & $\frac{3-17-15}{1}$ & T.5 \\
\hline & & Certified...... & $3 \cdot 15 \cdot 15$ & $5 x-$ \\
\hline
\end{tabular}

Mussel species: Hickorymut Glochtd a Lot number: 120900 Mean initial vlabillty $=86.0 \%$

Test Chemical: Pseudomanas florescens Pf-CL 1ASA (SDP) Chemical lot number: MB|-401 SDP 4655-12-Mi|X

Exposure Date: 19-Jan-12

Page 1 of 1

Test System Location: Block 3

\begin{tabular}{|c|c|c|c|c|c|c|c|c|c|}
\hline Species & $\begin{array}{c}\text { Test } \\
\text { Chamber }\end{array}$ & $\begin{array}{c}\text { Treatment } \\
\text { Group } \\
(\mathrm{mg} / \mathrm{L}) \\
\end{array}$ & $\begin{array}{c}\text { Total } \\
\text { Number } \\
\text { Glochlda } \\
\text { Counted }\end{array}$ & $\begin{array}{c}\text { Glochidia } \\
\text { Closed } \\
\text { before } \\
\mathrm{NaCl}^{1}\end{array}$ & $\begin{array}{l}\text { Glodidia } \\
\text { Open } \\
\text { after } \mathrm{NaCl}\end{array}$ & $\begin{array}{l}\text { Unad]usted } \\
\text { 6h vlability } \\
\text { (\%) }\end{array}$ & $\begin{array}{l}\text { Adlusted } 6 \mathbf{h} \\
\text { Viabllity }(\%)^{2}\end{array}$ & $\begin{array}{c}\text { Mean } \\
\text { adjusted } \\
\text { viability }(\%)\end{array}$ & $\begin{array}{c}\text { Standard } \\
\text { Devation } \\
\text { (SO) }\end{array}$ \\
\hline Hickorynut & $3 A_{2}$ & 0 & 258 & 65 & 21 & 66.7 & 77.5 & 93.0 & 13,4 \\
\hline Hilckorynut & $3 \mathrm{Cl}$ & 0 & 156 & 8 & 12 & 87.2 & 101.4 & & \\
\hline Hickorynut & $3 \mathrm{C4}$ & 0 & 259 & 12 & 24 & 86.1 & 100.1 & & \\
\hline Hickorynut & 3AS & 50 & 267 & 16 & 21 & 86.1 & 100.2 & 99.1 & 1.5 \\
\hline Hickorynut & 383 & 50 & 191 & 21 & 10 & 83.8 & 97.4 & & \\
\hline Hickorynu: & 3B5 & 50 & 274 & 26 & 13 & 85.8 & 99.7 & & \\
\hline HIckorynu: & 381 & 100 & 232 & 39 & 32 & 69.4 & 80.7 & 70.9 & 16.1 \\
\hline Hlckorynut: & $3 \mathrm{C} 2$ & 100 & 231 & 114 & 13 & 45.0 & 57.4 & & \\
\hline Hlckorynut & $3 \mathrm{C5}$ & 100 & 292 & 81 & 11 & 68.5 & 79.6 & & \\
\hline Hickorynut & 3A3 & 200 & 231 & 142 & 19 & 30.3 & 35.2 & 47.3 & 13.3 \\
\hline Hllckorynut & $3 \mathrm{~A} 4$ & 200 & 176 & 90 & 18 & 38.6 & 44.9 & & \\
\hline HIckoryn $t$ & 366 & 200 & 285 & $11 \%$ & 17 & 53.0 & 61,6 & & \\
\hline HIckorynst & 3A5 & 300 & 293 & 192 & 13 & 30.0 & 34,9 & 18.3 & 14.8 \\
\hline HICkorynst & 382 & 300 & 173 & 160 & 3 & 5.8 & 6.7 & & \\
\hline Hlekorynut & 385 & 300 & 266 & 226 & 10 & 11.3 & 13.1 & & \\
\hline H|ckorynut & $3 A 1$ & $300 \mathrm{HD}$ & 362 & 262 & 25 & 20.7 & 24.1 & 16.1 & 7.3 \\
\hline H|ckoryาut & 38.4 & $300 \mathrm{HD}$ & 317 & 274 & 16 & 8.5 & 3.9 & & \\
\hline Hickorynut & $3 \mathrm{C3}$ & $300 \mathrm{HD}$ & 246 & 209 & 7 & 12.2 & 14.2 & & \\
\hline
\end{tabular}

If the number counted was glochidia open, the number of glochidla closed = total glochidia - open glochidia counted before $\mathrm{NaCl}$.

Glochidia viability: (total \# glochldia - (glochldia closed before + open after NaCl))/total glochidia *100

${ }^{2}$ Viability adjustments were calculated by dividing the observed viabiity by the mean initial viability for each species 


\begin{tabular}{|c|c|c|c|c|}
\hline Study Number & AEH-11-PSEUDO-01 & Action & Date & Initiais \\
\hline Lab Notebook & 1 & Created...... & 7-Jun-12 & TMS TF \\
\hline File Folder & $12 \mathrm{~A}$ & Revised...... & 16-Mar-15 & IAL.TIS \\
\hline \multirow[t]{2}{*}{ Raw Data Location } & \multirow[t]{2}{*}{$12 \mathrm{~A}$} & Reviewed... & $17 \cdot m A R \cdot 15$ & 76 \\
\hline & & Certlfied....... & $3 / 15015$ & $\sqrt{7}-$ \\
\hline file Name: & \multicolumn{4}{|c|}{ 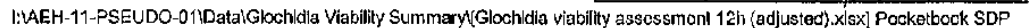 } \\
\hline
\end{tabular}

\section{Glochidia Viability Assessment - 12 hour}

Mussel species: Pocketbook Glochldia Lot number: 111400

Mean Inttlal viabllity $=93.8 \%$

Test Chemical: Pseudomonas florescens PF-C. 145A (SDP) Chemlcal lot *: MBI-401-110308A|-8D-3

Exposure Date: 12-May-11

Test System Locatlon: Block 1

Page 1 of 1

\begin{tabular}{|c|c|c|c|c|c|c|c|c|c|}
\hline Species & $\begin{array}{c}\text { Test } \\
\text { Chamber }\end{array}$ & $\begin{array}{c}\text { Trestment } \\
\text { Group (mg/t) }\end{array}$ & $\begin{array}{c}\text { Total Number } \\
\text { Glochilda } \\
\text { Counted }\end{array}$ & $\begin{array}{l}\text { Closed before } \\
\text { NaCl }^{1}\end{array}$ & $\begin{array}{l}\text { Glochidla Open } \\
\text { after Nad }\end{array}$ & $\begin{array}{l}\text { Unadjusted } \\
\text { 12h vlabllity } \\
\text { (\%) }\end{array}$ & $\begin{array}{l}\text { Adjusted 12h } \\
\text { vability (\%) }\end{array}$ & $\begin{array}{c}\text { Mean } \\
\text { adjusted } \\
\text { v[abnity }\{\%\}\end{array}$ & $\begin{array}{c}\text { Standard } \\
\text { Devlation } \\
\text { [SD] }\end{array}$ \\
\hline Pocketbosis & 181 & 0 & 599 & 39 & 53 & 84.6 & 90.2 & 91.8 & 2,2 \\
\hline Pocketbook & $1 \mathrm{C3}$ & 0 & 604 & 18 & 52 & 88.4 & 94.3 & & \\
\hline Pocketbook & $1 \mathrm{AB}$ & 0 & 626 & 39 & 54 & 85.1 & 90.8 & & \\
\hline Pocketbook & 185 & 50 & 226 & 37 & 14 & 77.4 & 82.6 & 83.8 & 7.0 \\
\hline Pocketbook & $1 \mathrm{~A} 1$ & 50 & 265 & 24 & 14 & 85.7 & 91.3 & & \\
\hline Pocketbook & $1 \mathrm{Cl}$ & 50 & 624 & 104 & 67 & 72.6 & 77.4 & & \\
\hline Pocketbook & 184 & 100 & 354 & 40 & 28 & 80.8 & 86.1 & 56.1 & 48.7 \\
\hline Pocketbook & $1 \mathrm{~A} 2$ & 100 & 353 & 321 & 32 & 0.0 & 0.0 & & \\
\hline Pocketbook & $1 \mathrm{~A} 4$ & 100 & 504 & 75 & 40 & 77.2 & 82.3 & & \\
\hline Pocketbook & $1 \mathrm{CE}$ & 200 & 177 & 129 & 11 & 20.9 & 22.3 & 49.8 & 23.8 \\
\hline Pocketbook & $1 B 2$ & 200 & 152 & 47 & 15 & 59.2 & 63.1 & & \\
\hline Pocketbook & $1 \mathrm{CH}$ & 200 & 380 & 116 & 36 & 60.0 & 64.0 & & \\
\hline Pocketbook & $1 \mathrm{~A} .5$ & 300 & 1177 & 938 & 109 & 11.0 & -1.8 & 6.9 & 4.5 \\
\hline Pocketbook & $1 \mathrm{C} 2$ & 300 & 305 & 284 & 13 & 2.6 & 2.8 & & \\
\hline Pocketbook & $1 \mathrm{CS}$ & 300 & 460 & 403 & 30 & 5.9 & 6.3 & & \\
\hline Pocketbook & 1A6 & $300 \mathrm{HD}$ & 242 & 193 & 32 & 7.0 & 7.5 & 6.2 & 28 \\
\hline Pocketbook. & $1 B 3$ & $300 \mathrm{HD}$ & 225 & 172 & 37 & 7.6 & 8.1 & & \\
\hline Pocketbook & 186 & $300 \mathrm{HD}$ & 322 & 280 & 33 & 2,8 & 3,0 & & \\
\hline
\end{tabular}

'If tlie number counted was glochldia open, the number of glochldla closed $*$ :otal glochidia - open gloceldila cour ted before $\mathrm{NaC}$."

Glochldia vjablity: (total \# glochildia - (glochidia closed before + open after NaEl))//otal glochidia *100

${ }^{2}$ V|ab||ity adjustments were calculated by dividing the observed viablilty by the mean initial viability for each species 


\begin{tabular}{|c|c|c|c|c|}
\hline Study Number & AEH-11-PSEUDO-01 & Action & Qate & Initials \\
\hline Lab Notebook & 1 & Created...... & $7-J u n-12$ & TMS ins \\
\hline File Folder & $12 \mathrm{~A}$ & Revlsed...... & 17-Mar-15 & TJS TS \\
\hline \multirow[t]{2}{*}{ Raw data location } & $12 \mathrm{~A}$ & Reviewed... & $17, m n R+15$ & $T x$ \\
\hline & & Certifled...... & $3 / 1565$ & $\sqrt{11}=$ \\
\hline
\end{tabular}

\section{Glochidia Viability Assessment - 12 hour}

Mussel species: Fatmucket Gloci cia Lot number; 111600

Mean inital viability $=80.3 \%$

Test Chemical: Pseudomonas fiorescens Pf-CL 145A (SOP) Chemlcal lot f: MBI-401-110308Al-BD-3

Exposure Date: 12-May-11

Test System Locatlon: Block 3

Page 1 of 1

\begin{tabular}{|c|c|c|c|c|c|c|c|c|c|}
\hline Spacies & $\begin{array}{c}\text { Test } \\
\text { Chamber }\end{array}$ & $\begin{array}{c}\text { Treatment Group } \\
(\mathrm{mg} / \mathrm{L})\end{array}$ & $\begin{array}{c}\text { Total } \\
\text { Number } \\
\text { Glochlda } \\
\text { Counted }\end{array}$ & $\begin{array}{c}\text { Glochldia } \\
\text { Closed } \\
\text { before } \mathrm{NaCl}^{1}\end{array}$ & $\begin{array}{c}\text { Glochldia } \\
\text { Open } \\
\text { after } \mathrm{NaCl}\end{array}$ & $\begin{array}{c}\text { Unadjusted } \\
12 \text { h viability } \\
(\%)\end{array}$ & $\begin{array}{c}\text { Adjusted } \\
12 \mathrm{~h} \\
\text { viablity } \\
(\%) \\
\end{array}$ & $\begin{array}{c}\text { Mean } \\
\text { adjusted } \\
\text { viability (\%) }\end{array}$ & $\begin{array}{l}\text { Standard } \\
\text { Deviatlon } \\
\text { (SD) }\end{array}$ \\
\hline Fatmucket & $3 \mathrm{~B} 2$ & 0 & 328 & 55 & 79 & 59.1 & $73 . \%$ & 82.9 & 8.0 \\
\hline Fatmucket & $3 B 3$ & 0 & 521 & 63 & 88 & 71.0 & 88.4 & & \\
\hline Fatmucket & $3 A 2$ & 0 & 282 & 51 & 32 & 69.5 & 86.6 & & \\
\hline Fatmucket & $3 B 5$ & 50 & 430 & 146 & 36 & 57.7 & 71.8 & 73.6 & 5.1 \\
\hline Fatmucket & $3 \mathrm{CS}$ & 50 & 368 & 109 & 53 & 56.0 & 69.7 & & \\
\hline Fatmucket & $3 \mathrm{CG}$ & 50 & 207 & 33 & 42 & 63.8 & 79.4 & & \\
\hline Fatmucket & $3 \mathrm{~A} 1$ & 100 & 409 & 183 & 48 & 43.5 & 54,2 & 54,3 & 4.2 \\
\hline Fatmucket & $3 A 3$ & 100 & 221 & 64 & 53 & 47.1 & 58.6 & & \\
\hline Fatmucket & $3 A 5$ & 100 & 159 & 68 & 27 & 40.3 & 50.1 & & \\
\hline Fatmucket & $3 B 4$ & 200 & 198 & 127 & 40 & 15.7 & 19.5 & 24.4 & 16.4 \\
\hline Fatmucket & $3 \mathrm{~B} 6$ & 200 & 134 & 69 & 19 & 34.3 & 42.8 & & \\
\hline Fatmucket & $3 C_{4}$ & 200 & 214 & $1 / 2$ & 23 & 8.9 & 11.1 & & \\
\hline Fatmucket & $3 \mathrm{Cl}$ & 300 & 240 & 195 & 29 & 6.7 & 8.3 & 5.6 & 2.4 \\
\hline Fatmucket & $3 C 3$ & 300 & 206 & 173 & 26 & 3.4 & 4.2 & & \\
\hline Fatmucket & 3B1 & 300 & 298 & 233 & 55 & 3.4 & 4.2 & & \\
\hline Fatniucket & $3 A 4$ & $300 \mathrm{HD}$ & 310 & 252 & 52 & 1.9 & 2.4 & 2.9 & 3,2 \\
\hline Fatmucket & $3 A 6$ & $300 \mathrm{HD}$ & 298 & 229 & 54 & 5.0 & 6.3 & & \\
\hline Fatmucket & $3 C_{2}$ & $300 \mathrm{HD}$ & 187 & 131 & 56 & 0.0 & 0,0 & & \\
\hline
\end{tabular}

1 If the number counted was glochidia open, the number of glochldla closed = total glochldla - open glochldia counted before $\mathrm{NaCl}$. Glochldia viability: (total \# glochidia - (glochidia closed before + open after NaCl)\}/total glochidla *1CO

${ }^{2}$ Viability adjustments were calculated by dividing the observed vlablity by the mean initlal vlabillty for each species 


\begin{tabular}{|c|c|c|c|c|}
\hline Study Number & AEH-11-PSEUDO-01 & Action & Date & nitials \\
\hline Lab Notebook & 1 & Created...... & 7.Jun-12 & TMSTAS \\
\hline File Folder & $12 \mathrm{~A}$ & Revlscd...... & 16-Mar-15 & JAL NSS \\
\hline \multirow[t]{2}{*}{ Raw data location } & \multirow[t]{2}{*}{$12 \mathrm{~A}$} & Revlewed... & $17 M A R-15$ & $\pi S$ \\
\hline & & Certifled...... & $13 / 18 / 14$ & Jiw \\
\hline
\end{tabular}

Glochidia Vlability Assessment - 12 hour

Mussel species: Higgins Eve Glochidia Lot number: 111500

Test Chernical: Pseudomonas florescens Pf-CL 145A (SDP) Chemical lot H: MBl-401-110308AI-BD-3

Exposure Jate: $12-\mathrm{May}-11$

Mean inltial viability $=80.8 \%$

Test System Locatlon: Block 2

Page 1 of 1

\begin{tabular}{|c|c|c|c|c|c|c|c|c|c|}
\hline Species & $\begin{array}{c}\text { Test } \\
\text { Chamber }\end{array}$ & $\begin{array}{c}\text { Treatment } \\
\text { Group } \\
(\mathrm{mg} / \mathrm{L})\end{array}$ & $\begin{array}{c}\text { Total } \\
\text { Number } \\
\text { Glochida } \\
\text { Counted }\end{array}$ & $\begin{array}{c}\text { Glochidla } \\
\text { Closed } \\
\text { before }\left.\mathrm{NaCl}\right|^{2}\end{array}$ & $\begin{array}{l}\text { Glochidia } \\
\text { Open } \\
\text { after } \mathrm{NaCl}\end{array}$ & $\begin{array}{c}\text { Unadjusted } \\
\text { 12h vlabllity } \\
(\%)\end{array}$ & $\begin{array}{l}\text { Adjusted } 12 \mathrm{~h} \\
\text { Viability }(\%)^{2}\end{array}$ & $\begin{array}{c}\text { Mean } \\
\text { adjusted } \\
\text { viabllity }(\%)\end{array}$ & $\begin{array}{c}\text { Standard } \\
\text { Devlatlon } \\
\text { (5D) }\end{array}$ \\
\hline Hlggirs Eye & $2 \mathrm{C3}$ & 0 & 481 & 48 & 124 & 64,2 & 79.5 & 85.0 & 7.6 \\
\hline Higgirs Eye & $2 \mathrm{C} 6$ & 0 & 210 & 13 & 38 & 75.7 & 93.7 & & \\
\hline Hlegirs Eye & $2 B 1$ & 0 & 254 & 29 & 57 & 66.1 & 81.9 & & \\
\hline Hleg|ris Eye & $2 \mathrm{~A} 1$ & 50 & 225 & 38 & 48 & 61.8 & 76.5 & 85.0 & 8.0 \\
\hline HiggIns Eye & $2 A 2$ & 50 & 230 & 21 & 49 & 69.6 & 86.1 & & \\
\hline Hlggins Eye & $2 A 4$ & so & 339 & 36 & 50 & 74.6 & 92.4 & & \\
\hline Hlggins Eye & $2 A 3$ & 100 & 235 & 50 & 38 & 62.6 & 77.4 & 81.1 & 3.9 \\
\hline Hrgeins Eye & $2 \mathrm{CA}$ & 100 & 273 & 35 & 60 & 65.2 & 80.7 & & \\
\hline Higglns Eye & $2 C 5$ & 100 & 362 & 41 & 72 & 68.8 & 85.1 & & \\
\hline HIggins Eye & $2 A 6$ & 200 & 266 & 146 & 51 & 25.9 & 32.1 & 57.2 & 22.2 \\
\hline HiggIns Eye & 282 & 200 & 344 & 65 & 73 & 59.9 & 74.1 & & \\
\hline Higgins Eye & 285 & 200 & 274 & 81 & 48 & 52.9 & 65.5 & & \\
\hline Higgins Eye & $2 \mathrm{C} 2$ & 300 & 215 & 52 & 63 & 46.5 & 57.6 & 48.0 & 10.0 \\
\hline Higgins Eye & $2 \mathrm{Cl}$ & 300 & 267 & 67 & 95 & 39.3 & 48.7 & & \\
\hline Higgins Eye & $2 \mathrm{~B} 3$ & 300 & 240 & 107 & 60 & 30.4 & 37.6 & & \\
\hline Higgins Eye & $2 A 5$ & $300 \mathrm{HO}$ & 229 & 161 & 44 & 10.5 & 13.0 & 11.8 & 1.1 \\
\hline HIgg|ns Eye & $2 \mathrm{~B} 4$ & $30 \mathrm{HD}$ & 192 & 143 & 32 & 8.9 & 11.0 & & \\
\hline Higgins Eye & $2 \mathrm{~B} 6$ & $300 \mathrm{HD}$ & 206 & 142 & 45 & 9.2 & 11,4 & & \\
\hline
\end{tabular}

${ }_{1}^{1}$ If the number counted was glochidia open, the number of glochicia closed $=$ total glochldla - open glochidia counted before $\mathrm{NaCl}$

${ }^{2}$ Vlabllity adjustments were claculated by dividing the observed viability by the mean initial viability for each spec!es 


\begin{tabular}{|c|c|c|c|c|}
\hline Study Number & AEH-11-PSEUDO-01 & Action & Date & nitials \\
\hline Lab Notebook & 1 & Created...... & 7-Jun-:2 & TMS Ds \\
\hline Fle Folder & $12 \mathrm{~A}$ & Revised...... & 16-Mar- $\$ 5$ & JALTSS \\
\hline \multirow[t]{2}{*}{ Raw data location } & $12 \mathrm{~A}$ & Reviewed... & In-MAR.J5 & $\pi b$ \\
\hline & & Certified...... & $3 / / v / 15$ & 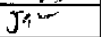 \\
\hline
\end{tabular}

File Name: I:AAEH-11-PSEUDO-011Da_alGlochidia Viakility Summaryl[Glochidia viajility assessment 12h (adjusted).xlsx]Pockatbook FDP Glochidia Viability Assessment - 12 hour

Mussel species: Pocketbook Glochidla Lot number: 115400

Mean Initial vability $=84.7 \%$

Test Chemical: Pseudomonas florescens Pf-CL. 245A (FDP] Chemical lot number: 110607 WB-FD- $z$

Exposure Date: 18 -Oct-11

Page 1 of 1 .

Test System Location: Block 3

\begin{tabular}{|c|c|c|c|c|c|c|c|c|c|}
\hline Specles & $\begin{array}{c}\text { Test } \\
\text { Chamber }\end{array}$ & $\begin{array}{l}\text { Treatment } \\
\text { Group } \\
\text { (mg/L) }\end{array}$ & $\begin{array}{l}\text { Total } \\
\text { Number } \\
\text { Glochlda } \\
\text { Counted }\end{array}$ & $\begin{array}{l}\text { Glochidia } \\
\text { Closed } \\
\text { before } \mathrm{NaCl}^{1}\end{array}$ & $\begin{array}{l}\text { Glochidia } \\
\text { Open } \\
\text { after } \mathrm{NaCl}\end{array}$ & $\begin{array}{c}\text { Unadjusted } \\
12 \mathrm{~h} \text { vlability } \\
\text { (\%) }\end{array}$ & $\begin{array}{c}\text { Adjusted } \\
\text { 12h } \\
\text { Viabllity } \\
(\%)^{2}\end{array}$ & $\begin{array}{c}\text { Mean } \\
\text { adjusted } \\
\text { vlabillty (\%) }\end{array}$ & $\begin{array}{l}\text { Standard } \\
\text { Deviatlon } \\
\text { (SD) }\end{array}$ \\
\hline Pocketbook & उA6 & 0 & 133 & 3 & 20 & 82.7 & 97.6 & 96.6 & 4.0 \\
\hline Pocketbook & $3 B 5$ & 0 & 314 & 8. & 61 & 78.0 & 92.1 & & \\
\hline Pocketbook & $3 B 6$ & 0 & 182 & 5 & 23 & 84.6 & 99.9 & & \\
\hline Pocketbook & $3 A 4$ & 50 & 290 & 24 & 62 & 70.3 & 83.1 & 90.5 & 7.4 \\
\hline Pocketbook & $3 \mathrm{C} 1$ & 50 & 160 & 8 & 29 & 76.9 & 90.8 & & \\
\hline Pocketbook & $3 \mathrm{C} 2$ & 50 & 606 & 34 & $\%$ & 82.8 & 97.8 & & \\
\hline Pocketbook & 381 & 100 & 179 & 46 & 22 & 62,0 & 73.2 & 83.3 & 10.0 \\
\hline Pocketbook & 383 & 100 & 130 & 20 & 18 & 70.8 & 83.6 & & \\
\hline Pocketbook & $3 C 6$ & 100 & 142 & 15 & 15 & 78.9 & 93.1 & & \\
\hline Pocketbook & $3 A 2$ & 200 & 249 & 161 & 38 & 20.1 & 23.7 & 41.2 & 36.1 \\
\hline Pocketbonk & $3 c 3$ & 200 & 815 & 104 & 140 & 70.1 & 82.7 & & \\
\hline Pocke:book & $3 C 5$ & 200 & 200 & 151 & 20 & 14.5 & 17.1 & & \\
\hline Pocke:book & $3 A 3$ & 300 & 251 & 174 & 66 & 4.4 & 5.2 & 10.8 & 10.4 \\
\hline Pocketbook & 384 & 300 & 223 & 145 & 35 & 19.3 & 22.8 & & \\
\hline Pocke:book & $3 C 4$ & 300 & 348 & 289 & 46 & 3.7 & 4.4 & & \\
\hline Pocketbook & $3 A 1$ & $300 \mathrm{HD}$ & 72 & 58 & 11 & 4.2 & 4.9 & 5.7 & 1.4 \\
\hline Pocke:book & 345 & $30 \mathrm{HD}$ & 48 & 38 & 7 & 6.3 & 7.4 & & \\
\hline Pocketbook & 382 & $300 \mathrm{HD}$ & 242 & 211 & 21 & 4.1 & 4.9 & & \\
\hline
\end{tabular}

If the number counted was glochidia open, the rumber of glochidia closed = to:a glochidla - open glochidia counted before $\mathrm{NaCl}$

Glochtdla viabillty: (total \# gloch dia - (glochidia closed before + open after NaCl))/total glochidia *100

${ }^{2}$ Viability adjustments were calculated by dividing the observed viability by the mean inl tial viability for each species 


\begin{tabular}{|c|c|c|c|c|}
\hline \multirow{2}{*}{$\begin{array}{l}\text { Study Number } \\
\text { Lab Notebook }\end{array}$} & \multirow{2}{*}{$\begin{array}{l}\text { AEH-11-PSEUDO-01 } \\
1\end{array}$} & \multirow{2}{*}{\begin{tabular}{|c|} 
Action \\
Created......
\end{tabular}} & Date & \multirow{2}{*}{$\frac{\text { Instials }}{\text { TMSThS }}$} \\
\hline & & & 7-Jun-12 & \\
\hline File Folder & $12 \mathrm{~A}$ & Revised...... & 16-Mar-15 & JAL DS \\
\hline \multirow[t]{2}{*}{ Raw Data Location } & \multirow[t]{2}{*}{$12 \mathrm{~A}$} & Revlewed... & $M-M A R-15$ & ThS \\
\hline & & Certifled....... & $36 \times 1 / 5$ & $50-$ \\
\hline Flle Name: & \multicolumn{4}{|c|}{ d] usted) xlsx]Black Sandshell } \\
\hline
\end{tabular}
Glochidia Viability Assessment - 12 hour

Mussel specles: Black Sandshell Glochldia Lot number: 120800 Mean initial viabiltty $=84.5 \%$ Test Chemlca: Pseudomonas florescens Pf-CL 145A (SDP) Chemical lot \#: MB|-401 SDP 4655-12-MIX Exposure Date: $17 . J a n-12$

Page 1 of 1 Test Systent Loca:ion: Block 1

\begin{tabular}{|c|c|c|c|c|c|c|c|c|c|}
\hline Mussel Species & $\begin{array}{c}\text { Test } \\
\text { Chamber }\end{array}$ & $\begin{array}{l}\text { Treatment } \\
\text { Group } \\
\text { (mg/L) }\end{array}$ & $\begin{array}{c}\text { Total } \\
\text { Number } \\
\text { Glochida } \\
\text { Counted }\end{array}$ & $\begin{array}{c}\text { Glochidia } \\
\text { Closed } \\
\text { before } \\
\mathrm{NaCl}^{1}\end{array}$ & $\begin{array}{l}\text { Glochidia } \\
\text { Open } \\
\text { after } \\
\mathrm{NaCl}\end{array}$ & $\begin{array}{l}\text { Unadjusted } \\
12 \mathrm{~h} \text { viabillty } \\
\text { (\%) }\end{array}$ & $\begin{array}{l}\text { Adjusted } 12 \mathrm{~h} \\
\text { Vlability }(\%)^{2}\end{array}$ & $\begin{array}{c}\text { Mean } \\
\text { adjusted } \\
\text { vjabllity (\%) }\end{array}$ & $\begin{array}{c}\text { Standard } \\
\text { Deviation } \\
\text { (SD) }\end{array}$ \\
\hline Black Sandshell & $1 \wedge 5$ & 0 & 262 & 3 & 26 & 88.9 & 105.2 & 105.9 & 0.6 \\
\hline Black Sandshell & 181 & 0 & 231 & 6 & 18 & 89.6 & 106.0 & & \\
\hline Black Sandshell & $1 C 6$ & 0 & 139 & 2 & 12 & 89.9 & 106.4 & & \\
\hline Black Sandshell & $1 \mathrm{~A} 1$ & 50 & 223 & 157 & 22 & $19: 7$ & 23.4 & 19.1 & 6.6 \\
\hline Black Sandshell & $1 \mathrm{AB}$ & 50 & 399 & 293 & 30 & 19.0 & 22.5 & & \\
\hline Black Sandshel & $1 \mathrm{C} 1$ & 50 & 248 & 22.1 & 3 & 9.7 & 11.5 & & \\
\hline Black Sandshell & 182 & 100 & 250 & 208 & 20 & 8.8 & 10.4 & 10.5 & 3.9 \\
\hline Black Sandshell & 183 & 100 & 198 & 155 & 19 & 12.1 & 14.3 & & \\
\hline Black Sandshell & $1 C 5$. & 100 & 125 & 97 & 21 & 5.6 & 6.6 & & \\
\hline Black Sandshell & $1 A G$ & 200 & 242 & 235 & 5 & 0.8 & 1.0 & 1.4 & 0.4 \\
\hline Black Sandshell & 185 & 200 & 210 & 201 & 6 & 1,4 & 1.7 & & \\
\hline Black Sandshell & 186 & 200 & 304 & 280 & 20 & 1.3 & 1.6 & & \\
\hline Black Sandshell & 1A2 & 300 & 135 & 135 & 0 & 0.0 & 0.0 & 0.0 & 0.0 \\
\hline Black Sandshell & $1 B A$ & 300 & 93 & 86 & 7 & 0.0 & 0.0 & & \\
\hline Black Sandshell & $1 \mathrm{C} 2$ & 300 & 165 & 151 & 14 & 0,0 & 0.0 & & \\
\hline Black Sandshell & IA4 & $30 \mathrm{HO}$ & 281 & 268 & 13 & 0.0 & 0.0 & 1.1 & 1.0 \\
\hline Black Sandshell & $1 \mathrm{C3}$ & $300 \mathrm{HO}$ & 157 & 147 & 8 & 1.3 & 1.5 & & \\
\hline Black Sandshell & $1 \mathrm{CA}$ & $300 \mathrm{HO}$ & 133 & 119 & 12 & 1.5 & 1.8 & & \\
\hline
\end{tabular}




\begin{tabular}{|c|c|c|c|c|}
\hline Study Number & AEH-11-PSEUDO-01 & Action & Date & |nitials \\
\hline Lab Notebook & 1 & Created...... & 7-Jun-12 & TMS ThS \\
\hline File Folder & $12 \mathrm{~A}$ & Revised...... & 16-Mar-15 & JALTBS \\
\hline \multirow[t]{2}{*}{ Raw Data Location } & \multirow[t]{2}{*}{$12 A$} & Reviewed... & $11 m A R+15$ & ThS \\
\hline & & Certified....... & $3 / 18 / 15$ & $\sqrt{6}$ \\
\hline File Name; & \multicolumn{4}{|c|}{ I:AEH-11-PSEUDO-011DatalGlochldla Vlability Surnmaryl[Glochidia viabllily essessment 12:1 (adjusted),xlsx]Mccket } \\
\hline
\end{tabular}

Glochidia Viability Assessment - 12 hour

Mussel species: Mucket Glochidia Lot number: 120700

Mean initial viability $=92.7 \%$

Test Chemical: Pseudomonas florescens Pf-CL 145A (SDP) Chemical lot H: MB|-401 SDP 4655-12-Mix

Fxposure Date: 17-Jan-12

Test System Location: Block 2

Page 1 of 1.

\begin{tabular}{|c|c|c|c|c|c|c|c|c|c|}
\hline Specles & $\begin{array}{c}\text { Test } \\
\text { Chamber }\end{array}$ & $\begin{array}{l}\text { Treatment } \\
\text { Group } \\
\text { (mg/L) }\end{array}$ & $\begin{array}{c}\text { Total } \\
\text { Number } \\
\text { Glochida } \\
\text { Counted }\end{array}$ & $\begin{array}{c}\text { Glochidia } \\
\text { Closed } \\
\text { before } \\
\mathrm{NaCl}^{1}\end{array}$ & $\begin{array}{l}\text { Glochidla } \\
\text { Open } \\
\text { after } \mathrm{NaCl}\end{array}$ & $\begin{array}{c}\text { Unadjusted } \\
12 \mathrm{~h} \text { viability } \\
\text { (\%) }\end{array}$ & $\begin{array}{c}\text { Adjusted } \\
12 \mathrm{~h} \text { Vlability } \\
{[\%]^{2}}\end{array}$ & $\begin{array}{c}\text { Mean } \\
\text { adjusted } \\
\text { viabiltty (\%) }\end{array}$ & $\begin{array}{c}\text { Standard } \\
\text { Deviation } \\
\text { (SD) }\end{array}$ \\
\hline Mucket & $2 B 4$ & 0 & 178 & 2 & 11 & 92.7 & 100.0 & 101.7 & 1.6 \\
\hline Mucket & $2 B 5$ & 0 & 2.13 & 0 & 12 & 94.4 & 101.8 & & \\
\hline Mucket & $2 \mathrm{C1}$ & 0 & 254 & 6 & 5 & 95.7 & 103.2 & & \\
\hline Mucket & $2 \mathrm{~A} 4$ & 50 & 221 & 63 & 14 & 65.2 & 70.3 & 71.8 & 19.1 \\
\hline Mucket & $2 B 6$ & 50 & 113 & 53 & 4 & 49.6 & 53.5 & & \\
\hline Mucket & $2 \mathrm{C3}$ & so & 193 & 17 & 12 & 85.0 & 91.7 & & \\
\hline Mucket & $2 \mathrm{~A} 2$ & 100 & 210 & 91 & 10 & 51,9 & 56.0 & 69.4 & 19.1 \\
\hline Mucket & $2 B 2$ & 100 & 138 & 46 & 14 & 56.5 & 61.0 & & \\
\hline Mucket & 256 & 100 & 215 & 26 & 7 & 84.7 & 91.3 & & \\
\hline Mucket & $2 \mathrm{AS}$ & 200 & 175 & 83 & 17 & 42.9 & 46.2 & 43.2 & 4.9 \\
\hline Mucket & $2 A 6$ & 200 & 179 & 92 & 11 & 42.5 & 45.8 & & \\
\hline Mucket & $2 \mathrm{C} 2$ & 200 & 362 & 227 & 9 & 34.8 & 37.5 & & \\
\hline Mucket & 231 & 300 & 247 & 212 & 4 & 12.6 & 13.5 & 11.0 & 2.7 \\
\hline Mucket & 233 & 300 & 198 & 181 & 2 & 7.6 & 8.2 & & \\
\hline Mucket & $2 \mathrm{Ca}$ & 300 & 116 & 102 & 2 & 10.3 & 11.2 & & \\
\hline Mucket & $2 \mathrm{~A} 1$ & $300 \mathrm{HD}$ & 237 & 201 & 14 & 9.3 & 10.0 & 8.8 & 5.4 \\
\hline Mucket & $2 \mathrm{~A} 3$ & $300 \mathrm{HD}$ & 127 & 100 & 11 & 12.6 & 13.6 & & \\
\hline Mucket & $2 \mathrm{C5}$ & $300 \mathrm{HD}$ & 184 & 175 & 4 & 2.7 & 2.9 & & \\
\hline
\end{tabular}

${ }^{1}$ If the number counted was glochidia upen, the number of glochidia closed = total glochidia - open glochidia counted before Nac:.

Glochidia vlabiltty: (total \# glochidla - (glochldla closed before + open after Naci))/total glochtdia *100

${ }^{2}$ Viabi!|ty adjustments were calculated by divlding the observed viabllity by the mean initial viability for each spesles 


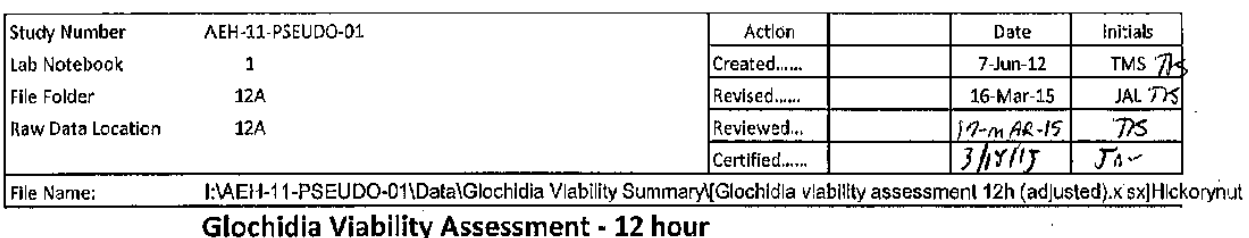
Glochidia Viability Assessment - 12 hour

Mussel specles: Hickorynut Glochldla Lot number: 120903

Mean Initial viability $=86.0 \%$

Test Chemical: Pseudornonas florescens Pf-CL 145A (SDP) Chernical lot number: MB|-401 SDP 4555-12-MIx

Exposure Date: 19-Jan-12

Page 1 of 1

\begin{tabular}{|c|c|c|c|c|c|c|c|c|c|}
\hline Species & $\begin{array}{c}\text { Test } \\
\text { Chamber }\end{array}$ & $\begin{array}{l}\text { Treatment } \\
\text { Group } \\
\text { (mg/) }\end{array}$ & $\begin{array}{c}\text { Total } \\
\text { Number } \\
\text { Glochida } \\
\text { Counted }\end{array}$ & $\begin{array}{c}\text { Glochidia } \\
\text { Closed } \\
\text { before } \\
\mathrm{NaCl}^{1}\end{array}$ & $\begin{array}{l}\text { Glochidla } \\
\text { Open } \\
\text { after } \mathrm{NaCl}\end{array}$ & $\begin{array}{c}\text { Unadjusted } \\
12 \mathrm{~h} \text { viability } \\
\text { (\%) }\end{array}$ & $\begin{array}{l}\text { Adjusted } 12 \mathrm{~h} \\
\text { Viabillty }(\%)^{2}\end{array}$ & $\begin{array}{c}\text { Mean } \\
\text { adjusted } \\
\text { viability }(\%)\end{array}$ & $\begin{array}{c}\text { Standard } \\
\text { Devlation } \\
\text { (SD) }\end{array}$ \\
\hline Hlickorynut & 3A2 & 0 & 257 & 6 & 22 & 89.1 & 103.6 & 100.5 & 7.4 \\
\hline Hickorynut & $3 \mathrm{Cl}$ & 0 & 216 & 19 & 26 & 79.2 & 92.1 & & \\
\hline HIckorynut & $3 c 4$ & 0 & 144 & 5 & 8 & 91.0 & 105.8 & & \\
\hline Hickorynut & $3 A 5$ & 50 & 172 & 21 & 10 & 82.0 & 95.3 & 98.2 & 3.4 \\
\hline HIckorynut & 383 & 50 & 197 & 24 & 8 & 83.8 & 97.4 & & \\
\hline Hlckorynut & $3 \mathrm{~B} 6$ & 50 & 186 & 9 & 14 & 87.6 & 101.9 & & \\
\hline Hlckorynut & 981 & 100 & 214 & 36 & 6 & 80.4 & 93.5 & 89.7 & 14.5 \\
\hline Hlckorynut & $3 \mathrm{C} 2$ & 100 & 195 & 18 & 6 & 87.7 & 102.0 & & \\
\hline HIckorynut & $3 \mathrm{CS}$ & 100 & 216 & 75 & 4 & 63.4 & 73.8 & & \\
\hline Hlckorynut & JAS & 200 & 134 & 86 & 3 & 33.6 & 39.0 & 58.4 & 19.2 \\
\hline Hlckorynut & 3A4 & 200 & 186 & 84 & 8 & 50.5 & 58.8 & & \\
\hline Hlekorynut & $3 C 6$ & 200 & 138 & 41 & 5 & 66.7 & 77.5 & & \\
\hline Hlekorynut & $3 A 6$ & 300 & 204 & 123 & 8 & 35.8 & 41.6 & 45.6 & 3.5 \\
\hline Hlckorynut & $3 B 2$ & 300 & 200 & 107 & 12 & 40.5 & 47.1 & & \\
\hline Hickorynut & 3BS & 300 & 206 & 98 & 23 & 41.3 & 48.0 & & \\
\hline Hlckorynut & gA1 & $300 \mathrm{HD}$ & 249 & 231 & 15 & 1.2 & 1.4 & 1,4 & 0.4 \\
\hline Hlckorynut & 384 & $300 \mathrm{HD}$ & 338 & 313 & 22 & 0.9 & 1.0 & & \\
\hline HIckorynut & 363 & $300 \mathrm{HD}$ & 251 & 244 & 3 & 1.6 & 1.9 & & \\
\hline
\end{tabular}

If the number counted was glochidla open, the number of glochidla closed $=$ total glochidia - open glochidia courted before NaCl.

Glochidia viability: (total \# glcchidia - (Elochldia closed before + open after NaCl)]/total glochidia *100

${ }^{2}$ Vlability acjustments were calculated by dividing the abserved viability by the mean initial viability for each species 


\begin{tabular}{|c|c|c|c|c|}
\hline Study Number & AEH-11-PSEUDO-01 & Action & Date & Inbtlals \\
\hline Lab Notebook & 1 & Created...... & 7-Jun-12 & TMSTHS \\
\hline File Folder & $12 \mathrm{~A}$ & Revised...... & 16-Mar-15 & IAL TS \\
\hline \multirow[t]{2}{*}{ Raw data locatio } & \multirow[t]{2}{*}{$12 \mathrm{~A}$} & Reviewed... & M.MAR-15 & 75 \\
\hline & & Certlfled...... & $3 / 15 / 15$ & $57-$ \\
\hline
\end{tabular}

Glochidla Viability Assessment - 24 hour

Mussel species: Higgins Eye Glochidla Lot number: 111500 Test Chemlca : Pseudomonas florescens Pf-CL 145A (SDP) Chemical lot il: MBI-401-1103כ8A-BD-3

Exposure Date: 12-Mar-11

Maan Initial viability $=80.8 \%$

Test System Lozation: Block 2

Page 1 of 1

\begin{tabular}{|c|c|c|c|c|c|c|c|c|c|}
\hline Specles & $\begin{array}{c}\text { Test } \\
\text { Chamber }\end{array}$ & $\begin{array}{l}\text { Treatinent } \\
\text { Group } \\
\text { (mg/L) }\end{array}$ & $\begin{array}{c}\text { Total } \\
\text { Number } \\
\text { Glochlda } \\
\text { Counted }\end{array}$ & $\begin{array}{c}\text { Glochldla } \\
\text { Closed } \\
\text { before } \mathrm{NaCl}^{1}\end{array}$ & $\begin{array}{l}\text { Glochldla } \\
\text { Open } \\
\text { after } \mathrm{NaCl}\end{array}$ & $\begin{array}{c}\text { Unadjusted } \\
24 h \text { vlability } \\
\text { (\%) }\end{array}$ & $\begin{array}{l}\text { Adjusted 24h } \\
\text { Vlabllity (\%) }\end{array}$ & $\begin{array}{c}\text { Mean } \\
\text { adjusted } \\
\text { vlabillty }(\%)\end{array}$ & $\begin{array}{l}\text { Standard } \\
\text { Dewatlon } \\
\text { (SD) }\end{array}$ \\
\hline Higgins Eye & $2 \mathrm{C3}$ & 0 & 318 & 28 & 66 & 70.4 & 87.2 & 85,8 & 9.0 \\
\hline Higgins Eye & $2 \mathrm{C} 3-\mathrm{R}^{\mathrm{d}}$ & 0 & 377 & 46 & 108 & 59.2 & 73.2 & & \\
\hline Helggins Eye & $2 \mathrm{CG}$ & 0 & 557 & 61 & 70 & 76.5 & $\$ 4.7$ & & \\
\hline Heggins Eye & $2 \mathrm{C} 6-\mathrm{R}^{4}{ }^{4}$ & 0 & 408 & 44 & 73 & 71.3 & 88.3 & & . \\
\hline Higgins Eye & $2 B 1$ & 0 & lost $t^{3}$ & - & . & - & . & & \\
\hline Higgins Eye & $2 \mathrm{~A} 1$ & 50 & 506 & & 67 & 77.1 & 95.4 & 92.3 & 4.2 \\
\hline Hlgeins Eye & $2 \mathrm{~A} 2$ & 50 & 514 & 55 & 68 & 76.1 & 94.1 & & \\
\hline Higglns Eye & 2A4 & 50 & 464 & 34 & 102 & 70.7 & 87.5 & & \\
\hline Hlggins Eye & $2 \mathrm{~A} 3$ & 100 & 629 & 95 & 87 & 71.1 & 88.0 & 81.0 & 6.0 \\
\hline HiggIns Eye & $2 \mathrm{CA}$ & 100 & 423 & 87 & 69 & 63.1 & 78.1 & & \\
\hline Hilggins Eye & $2 C 5$ & 200 & 413 & 105 & 51 & 62.2 & 77.0 & & \\
\hline HiggIns Eye & $2 A G$ & 200 & 296 & 83 & 56 & 53.0 & 65.6 & 52.6 & 18.3 \\
\hline Hlgglns Eve & $2 \mathrm{~B} 2$ & 200 & 369 & 135 & 54 & 48.8 & $6 \mathrm{CC} .4$ & & \\
\hline Higgins Eve & $2 B 5$ & 200 & 441 & 272 & 56 & 25.6 & 31.7 & & \\
\hline Hlggins Eye & $2 \mathrm{C} 2$ & $300^{\circ}$ & 140 & 38 & 28 & 52.9 & 65.4 & 27.6 & 32.9 \\
\hline Hlggins Eye & $2 \mathrm{Cl}$ & 300 & 354 & 287 & 34 & 9.3 & 11,5 & & \\
\hline Hlggins Eye & $2 \mathrm{~B} 3$ & 300 & 171 & 109 & 54 & 4.7 & 5.8 & & \\
\hline Higgins Eye & $2 \mathrm{~A} 5$ & $300 \mathrm{HD}$ & 137 & 89 & 42 & 4.4 & 5.4 & 17.2 & 12.9 \\
\hline Higgins Eye & $2 B 4$ & $300 \mathrm{HO}$ & $2: 6$ & 88 & 74 & 25.0 & 30.9 & & \\
\hline Higgins Eve & $2 \mathrm{~B} 6$ & $300 \mathrm{HO}$ & 330 & 229 & 60 & 12.4 & 15.4 & & \\
\hline
\end{tabular}

${ }^{1}$ If the number counted was gloch:dia open, the number of glochidia closed = total glochidia - open glochldia counted before $\mathrm{NaCl}$.

'Vlability adjustments were calculated by dividing the obse'ved viability by the mean Inlt|al vlabllity for each species

${ }^{3}$ Sample lost, the 281 chamber was accidentally pooled with control charnbers from Block 1 before the $24 \mathrm{~h}$ glochidla sample collected.

"Two replicate sarrples were collected from the control samples $2 \mathrm{C} 3$ and $2 \mathrm{C} 6$ stnce sample $2 \mathrm{B1}$ was lost.

Glochídia viabil ty: (total \# gloclidia - (glochidia closed before + open after NaC)|)/total glochidia *100 


\begin{tabular}{|c|c|c|c|c|}
\hline Study Number & AEH-21-PSEUDO-01 & Action & Date & Initials \\
\hline Lab Notebook & 1 & Created...... & 7-Jun-12 & TMSTKS \\
\hline File folder & $12 \mathrm{~A}$ & Revised....... & 16-Mar-15 & JAL.TKS \\
\hline \multirow[t]{2}{*}{ Raw data location } & \multirow[t]{2}{*}{$12 \mathrm{~A}$} & Revlewed... & $17-M A R=15$ & Ths \\
\hline & & Certified....... & $3 / 19 / 15$ & $5=$ \\
\hline
\end{tabular}

\section{Glochidia Viability Assessment - 24 hour}

Mussel species: Fatmucket Glochidia Lot number: 111600

Mean inltial vlablitity $=80.3 \%$

Test Chemical: Pseuctomonas fiorescens Pf-CL 145A (SUP) Chemical lot \#: MBI-401-110308Al-BC-3

Exposure Date: 12-May-11

Test System Locatlon: Block 3

Page 1 of 1

\begin{tabular}{|c|c|c|c|c|c|c|c|c|c|}
\hline Specles & $\begin{array}{c}\text { Test } \\
\text { Chamber }\end{array}$ & $\begin{array}{l}\text { Treatment Group } \\
(\mathrm{mg} / \mathrm{l})\end{array}$ & $\begin{array}{l}\text { Total } \\
\text { Number } \\
\text { Glochida } \\
\text { Counted }\end{array}$ & $\begin{array}{c}\text { Glochidia } \\
\text { Closed } \\
\text { before } \mathrm{NaCl}^{1}\end{array}$ & $\begin{array}{c}\text { Glochldia } \\
\text { Open } \\
\text { after } \mathrm{NaCl}\end{array}$ & $\begin{array}{c}\text { Unadjusted } \\
24 h \text { viability } \\
\text { (\%) }\end{array}$ & $\begin{array}{l}\text { Adjusted } \\
24 h \\
\text { Vability } \\
(\%)^{2}\end{array}$ & $\begin{array}{c}\text { Mean } \\
\text { adjusted } \\
\text { viablity }(\%)\end{array}$ & $\begin{array}{c}\text { Standard } \\
\text { Deviation } \\
\text { (SD) }\end{array}$ \\
\hline Fatmucket & $3 \mathrm{~B} 2$ & 0 & 247 & 50 & 41 & 63.2 & 78.7 & 86.5 & 10.2 \\
\hline Fatmucket & 383 & 0 & 244 & 19 & 33 & 78.7 & 98.0 & & \\
\hline Fatmucket & $3 A 2$ & 0 & 307 & 48 & 55 & 66.4 & 82,8 & & \\
\hline Fatmucket & 385 & 50 & 327 & 38 & 86 & 62.1 & 77.3 & 70.8 & 7.4 \\
\hline Fatmucket & $3 C 5$ & 50 & 334 & 64 & 76 & 58.1 & 72.3 & & \\
\hline Fatmucket & 306 & 50 & 278 & 70 & 68 & 50.4 & 62.7 & & \\
\hline Fatmucket & JA1 & 100 & 112 & 41 & 20 & 45.5 & 56.7 & 51.9 & 8.0 \\
\hline Fatmucket & $3 A 3$ & 100 & 208 & 60 & 54 & 45.2 & 56.3 & & \\
\hline Fatmucket & $3 A 5$ & 100 & 313 & 146 & 60 & 34.2 & 42.6 & & \\
\hline Fatmucket & 384 & 200 & 271 & 190 & 13 & 14.0 & 17.5 & 14.5 & 5.8 \\
\hline Fatmucket & 386 & 200 & 205 & 147 & 28 & 14.6 & 18.2 & & \\
\hline Fatmucket & $3 \mathrm{CA}$ & 200 & 271 & 103 & 61 & 6.3 & 7.8 & & \\
\hline Fatmucket & $3 \mathrm{C} 1$ & 300 & 176 & 145 & 23 & 4.5 & 5.7 & 7.5 & 2.2 \\
\hline Fatmucket & $3 C_{3}$ & 300 & 215 & 130 & 68 & 7.9 & 9,8 & & \\
\hline Fatmucket & 381 & 300 & 217 & 170 & 35 & 5.5 & 6,9 & & \\
\hline Fatmucket & $3 A A$ & $300 \mathrm{HJ}$ & 281 & 250 & 29 & 0.7 & 0.9 & 2.1 & 1.1 \\
\hline Fatnucket & $3 A 6$ & $300 \mathrm{HO}$ & 86 & 69 & 15 & 2.3 & 2.9 & & \\
\hline Fatmucket & $3 \mathrm{C} 2$ & 300110 & 336 & 293 & 36 & 2.1 & 2,6 & & \\
\hline
\end{tabular}

1 If the number counted was glochidia open, the number of glochicia closed = total glochidia - open glochldila counted before $\mathrm{NaCl}$.

Glochidia vability: (total \# glochidia - (glochidla closed before + open after NaCl) / total glochidia *1CO

${ }^{2}$ Viabllity adjustrents were calculated by dividing the observed viablity by the mean initial viability for each species 


\begin{tabular}{|c|c|c|c|c|}
\hline \multirow{2}{*}{$\begin{array}{l}\text { Study Number } \\
\text { Lab Notebook }\end{array}$} & \multirow{2}{*}{$\begin{array}{c}\text { AEH-11-PSEUOO-01 } \\
1\end{array}$} & \multirow{2}{*}{\begin{tabular}{|c|} 
Action \\
Created....... \\
\end{tabular}} & \multirow{2}{*}{\begin{tabular}{|l|} 
Date \\
7 -Jun-12 \\
\end{tabular}} & \multirow{2}{*}{$\frac{\text { Initials }}{\text { TMSTS }}$} \\
\hline & & & & \\
\hline File Folder & $12 \mathrm{~A}$ & Revised...... & 16-Mar-15 & JAL TS \\
\hline \multirow[t]{2}{*}{ Raw Data Location } & $12 \mathrm{~A}$ & Revlewed... & 11-MAR-15 & Ths \\
\hline & & Certified...... & $3 / 15 / 5$ & $J_{1}-$ \\
\hline
\end{tabular}

\section{Glochidia Viability Assessment - 24 hour}

Mussel species: Pocketbook Glochidla Lot number; 111400

Mean initlai viability $=93.8 \%$

Test Chemlcal: Pseudornonas flurescens Pf-CL 145A (SDP) Chemical lot H: MB|-401-110308A|-BD-3

Exposure Date: 12-May-11

Test System Location: Block 1

Page 1 of 1

\begin{tabular}{|c|c|c|c|c|c|c|c|c|c|}
\hline Specles & $\begin{array}{c}\text { Test } \\
\text { Chamber }\end{array}$ & $\begin{array}{c}\text { Treatment } \\
\text { Group }(\mathrm{mg} / \mathrm{L})\end{array}$ & $\begin{array}{l}\text { Total Number } \\
\text { Glochlda } \\
\text { Counted }\end{array}$ & $\begin{array}{c}\text { Glochldla } \\
\text { Closed } \\
\text { before } \mathrm{NaG}^{1}\end{array}$ & $\begin{array}{c}\text { Glochidla } \\
\text { Open } \\
\text { after } \mathrm{NaCl}\end{array}$ & $\begin{array}{l}\text { Unadjusted } \\
\text { 24h viabillty } \\
\text { (\%) }\end{array}$ & $\begin{array}{l}\text { Adjusted 24h } \\
\text { Viability }\{\%\}^{2}\end{array}$ & $\begin{array}{c}\text { Mean } \\
\text { acqusted } \\
\text { vlability (\%) }\end{array}$ & $\begin{array}{c}\text { Standard } \\
\text { Deviation } \\
\text { (SD) }\end{array}$ \\
\hline Pocketbook & 181 & 0 & 514 & 15 & 22 & 92,8 & 98.9 & 98.2 & 1.0 \\
\hline Pocketbook & $1 \mathrm{CJ}$ & 0 & 276 & 1.2 & 9 & 92.4 & 98.5 & & \\
\hline Pocketlook & $1 \mathrm{AB}$ & 0 & 367 & 9 & 24 & 91.0 & 97.0 & & \\
\hline Pocketbook & 185 & 50 & 270 & 27 & 16 & 84.1 & 89.6 & 85.1 & 6.0 \\
\hline Pocketbook & IA] & 50 & 459 & 55 & 28 & 81.9 & 87.3 & & \\
\hline Pocketbonk & $1 \mathrm{C1}$ & 50 & 339 & 78 & 12 & 73.5 & 78.3 & & \\
\hline Pockethook & 184 & 100 & 324 & 56 & 24 & 75.3 & 80.3 & 71.3 & 16.8 \\
\hline Pocketbook & $1 A 2$ & 100 & 210 & 33 & 16 & 76.7 & 81.7 & & \\
\hline Pocketbook & $1 \mathrm{A4}$ & 100 & 310 & 34 & 125 & 48.7 & 51.9 & & \\
\hline Pocketbook & 16.6 & 2.00 & 242 & 64 & 16 & 66.9 & 71.4 & 63.2 & 13.6 \\
\hline Pocketbook & 182 & 200 & 187 & 30 & 13 & 66.3 & 70.7 & & \\
\hline Pocketbook & 164 & 200 & 505 & 267 & 14 & 44.5 & 47.4 & & \\
\hline Pocketbook & $1 \mathrm{~A} 5$ & 300 & 493 & 399 & 23 & 14.4 & 25.4 & 6.5 & 7.7 \\
\hline Pocketbook & $1 \mathrm{C} 2$ & 300 & 226 & 218 & 4 & 1.8 & 1.9 & & \\
\hline Pocketbook & $1 \mathrm{C5}$ & 300 & 295 & 274 & 15 & 2.0 & 2.2 & & \\
\hline Pocketbook & $1 A 6$ & $300 \mathrm{HD}$ & 204 & 147 & 40 & 8.3 & 8.9 & 5.1 & 3.3 \\
\hline Pocketbook & 183 & $300 \mathrm{HO}$ & 257 & 245 & 4 & 3.1 & 3.3 & & \\
\hline Pocketbook & 186 & $300 \mathrm{HD}$ & 322 & 293 & 20 & 2.8 & 3.0 & & \\
\hline
\end{tabular}




\begin{tabular}{|c|c|c|c|c|}
\hline Study Number & AEH-11-PSEUDO-01 & Action & Date & Initials \\
\hline Lab Notebook & 1 & Created...... & 7-Jun-12 & TMSTBS \\
\hline File Folder & $12 \mathrm{~A}$ & Revlsed...... & 16-Mar-1.5 & JAL TSS \\
\hline Raw data location & $12 \mathrm{~A}$ & Reviewed... & 11-MAR-15 & Trs \\
\hline & & Certified...... & $3 / 18 / \pi$ & $j \pi \omega$ \\
\hline
\end{tabular}

File Name: $\quad$ I:AEH-11-PSEUDO-01LDatalGlochidia Viability SummamiGlochidia viability assessment 24h (adjusted).xlsx]Pocketbook FDP Glochidia Viability Assessment - 24 hour

Mussel species: Pocketbook Glocidia Lot number: 115400 Mean initial viability $=84.7 \%$

Test Chemical: Pseudornonas florescens Pf-CL 145A (FDP) Chernical lot number; 110607WB-FD-E Exposure Date: 18-Oct-11

Test System Location: Block 3

Page 1 of 1

\begin{tabular}{|c|c|c|c|c|c|c|c|c|c|}
\hline Species & $\begin{array}{c}\text { Test } \\
\text { Chamber }\end{array}$ & $\begin{array}{l}\text { Treatment } \\
\text { Group } \\
\text { (mg/L) }\end{array}$ & $\begin{array}{l}\text { Total } \\
\text { Number } \\
\text { Glochida } \\
\text { Counted }\end{array}$ & $\begin{array}{c}\text { Glochldia } \\
\text { Closed } \\
\text { before } \mathrm{NaCl}^{1}\end{array}$ & $\begin{array}{l}\text { Glochidla } \\
\text { Open } \\
\text { after } \mathrm{NaCl}\end{array}$ & $\begin{array}{l}\text { Unadjusted } \\
24 h \text { viability } \\
\text { (\%) }\end{array}$ & $\begin{array}{l}\text { Adjusted } \\
24 h \\
\text { Viability } \\
(\%)^{2}\end{array}$ & $\begin{array}{c}\text { Mean } \\
\text { adjusted } \\
\text { viability }(\%)\end{array}$ & $\begin{array}{l}\text { Standard } \\
\text { Deviatlon } \\
\text { (SD) }\end{array}$ \\
\hline Pocketbook & $3 A 6$ & 0 & 573 & 21 & 74 & 83.4 & 98.5 & 96.7 & 1.7 \\
\hline Pocketbook & 3B5 & 0 & 279 & 7 & 47 & 80.6 & 95.2 & & \\
\hline Pocketbook & $3 \mathrm{~B} 6$ & 0 & 278 & 17 & 34 & 81.7 & 96.4 & & \\
\hline Pocketbook & $3 \mathrm{~A} 4$ & 50 & 164 & 39 & 21 & 63.4 & 74.9 & 87.5 & 11.0 \\
\hline Pocketbook & $3 \mathrm{C} 1$ & 50 & 368 & 33 & 45 & 78.8 & 93.0 & & \\
\hline Pocketbook & $3 C 2$ & 50 & 384 & 30 & 46 & 80.2 & 94.7 & & \\
\hline Pocketbook & $3 \mathrm{~B} 1$ & 100 & 467 & 85 & 60 & 69.0 & 81.4 & 80.0 & 4.5 \\
\hline Pocketbook & $3 \mathrm{~B} 3$ & 100 & 405 & 93 & 55 & 63.5 & 74.9 & & \\
\hline Pocketbook & $3 \mathrm{C6}$ & 100 & 288 & 38 & 46 & 70.8 & 83.6 & & \\
\hline Pocketbook & $3 A 2$ & 200 & 144 & 96 & 27 & 14.6 & 17,2 & 34.9 & 28.0 \\
\hline Pocketboo' & $3 \mathrm{C} 3$ & 200 & 592 & 157 & 98 & 56.9 & 67.2 & & \\
\hline Pocketboo< & $3 \mathrm{C} 5$ & 200 & 386 & $28:$ & 39 & $1 \% 1$ & 20.2 & & \\
\hline Pocketboor & $3 A 3$ & 300 & 199 & 132 & 45 & 11.1 & 13.1 & 14.2 & 2.2 \\
\hline Pocketbook & 3B4 & 300 & 714 & 442 & 271 & 14.1 & 16.7 & & \\
\hline Pocketbook & $3 \mathrm{CA}$ & 300 & 440 & 330 & 62 & 10.9 & 12.9 & & \\
\hline Pocketbook & $3 \mathrm{~A} 1$ & $300 k 1 D$ & 370 & 277 & 78 & 4.1 & 4.8 & 5.8 & 2,7 \\
\hline Pocketbook & $3 A 5$ & $300 \mathrm{HD}$ & 253 & 207 & 38 & 3.2 & 3.7 & & \\
\hline Pucketbook & 3B2 & $300 \mathrm{HD}$ & 267 & 1.98 & 49 & 7.5 & 8.8 & & \\
\hline
\end{tabular}




\begin{tabular}{|c|c|c|c|c|}
\hline Study Number & AEH-11-PSEUDO-01 & Action & Date & Initials \\
\hline Lab Notebook & 1 & Created...... & 30-May-12 & TJSTTS \\
\hline File Folder & $12 \mathrm{C}$ & Revised...... & 16-Mar-15 & $\mathrm{JAL} / \mathrm{F}$ \\
\hline Raw Data location & $12 \mathrm{C}$ & Certified...... & $3 / 18 / 15$ & $\mathrm{~J}^{n_{1}}$ \\
\hline File Name: & \multicolumn{4}{|c|}{ 1:IAEH-11-PSEUDO-01IDatalGlochicia Viability Summaryl[giochidia viabllity assessment 24h.xlsx]Washboard } \\
\hline
\end{tabular}

Glochidia Viability Assessment - 24 hour

Mussel species: Washboard Glochidia Lot number: 115500

Mean initial viability $=96.5 \%$

Test Chemlcal: Pseudomonas florescens Pf-CL 145A (FDP) Chemical lot number: 110607WB-FD-E

Exposure Date: $18-0 c t-11$

Test System Location: Block 1

Page 1 of 1

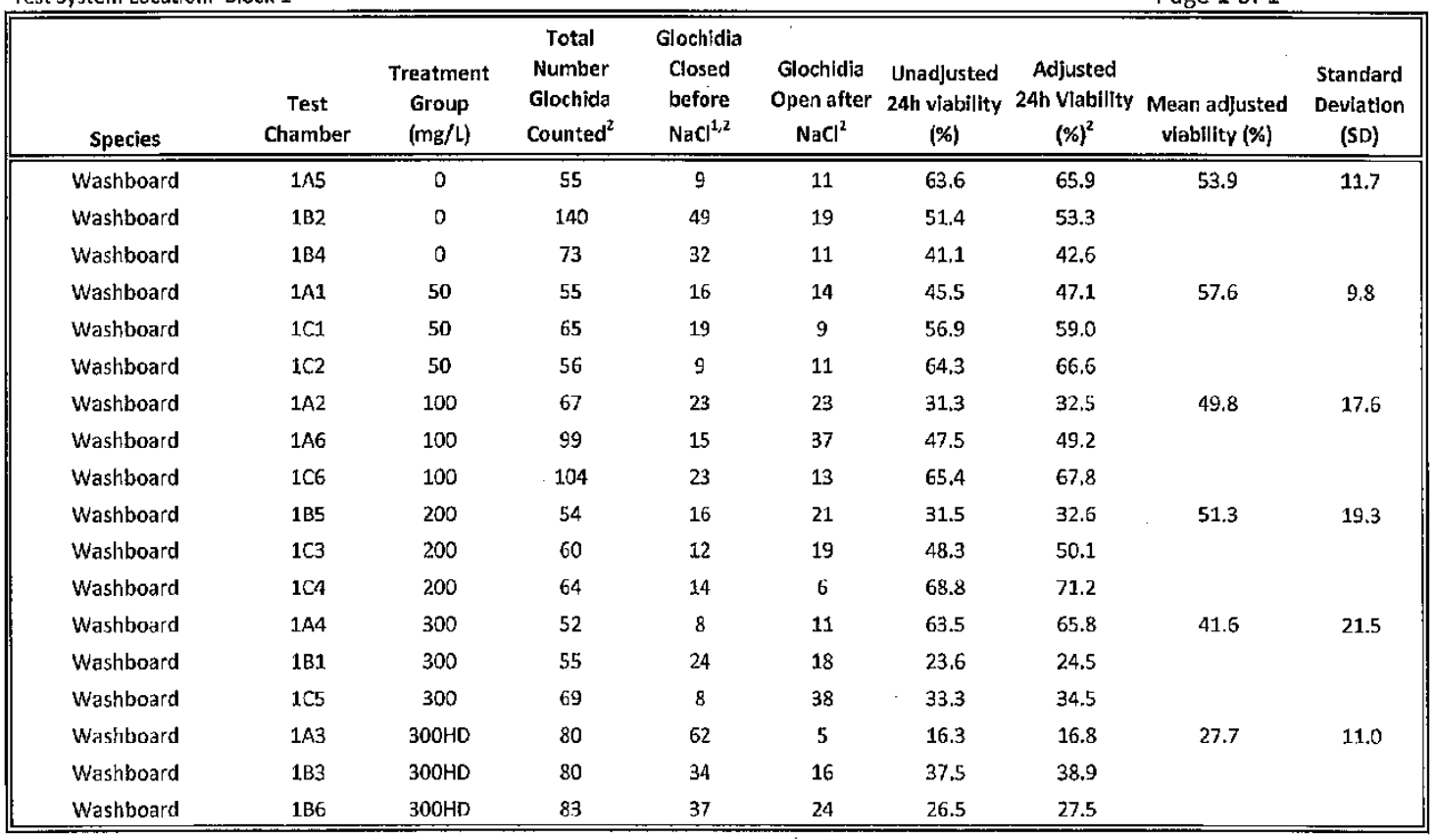

1 If the number counted was glochidia open, the number of glochidia closed = total glochidia - open glochidia counted before $\mathrm{NaCl}$.

2 Washboard samples were manual processed with a dissection scope, chambers with multiple replicates were pooled for one value. Glochidia viability: (total \# glochldia - (glochidia closed before + open after $\mathrm{NaCl}$ )/total glochidia *100

${ }^{3}$ Viability adjustments were calculated by dividing the observed viability by the mean initial viability for each species 


\begin{tabular}{|c|c|c|c|c|}
\hline Study Number & AEH-11-PSEUDO-01 & Action & Date & Initials \\
\hline L.ab Notebook & 1 & Created...... & 7-Jun-12 & TMST73 \\
\hline File Folder & $12 \mathrm{~A}$ & Revised....... & 16-Mar-15 & JAL. THS \\
\hline \multirow[t]{2}{*}{ Raw Data Location } & \multirow[t]{2}{*}{$12 \mathrm{~A}$} & Revlewed... & $17-M A R-15$ & Ths \\
\hline & & Certi ${ }^{2}$ led...... & $3 / 18 / 15$ & $5 \div 6$ \\
\hline Flle Name: & \multicolumn{4}{|c|}{ I:AEH-11-PSEUDO-01:DatalGiochldla Viability Sumrnary\}[Glochldia vlabillty assessmen! 24h (adjusted).xlsx\}Black Sandshell } \\
\hline
\end{tabular}

Glochidia Viability Assessment - 24 hour

Mussel species: Black Sandshell Glochidla Lot r.umber: 120800

Mean init|al viability $=81.5 \%$

Test Chemlcal: Pseudiomonas florescens Pf-CL 145A [SDP) Chem|cal lot \#: MBP-401 SOP 4655-12-Mix

Exposure Date: 17-Jan-12

Test System Lacation: Block 1

Page 1 of 1

\begin{tabular}{|c|c|c|c|c|c|c|c|c|c|}
\hline Mussel Specles & $\begin{array}{c}\text { Test } \\
\text { Chamber }\end{array}$ & $\begin{array}{c}\text { Treatment } \\
\text { Group } \\
\text { (mg/L) }\end{array}$ & $\begin{array}{l}\text { Total } \\
\text { Number } \\
\text { Glochlda } \\
\text { Counted }\end{array}$ & $\begin{array}{c}\text { Glochldia } \\
\text { Closed } \\
\text { before } \\
\mathrm{NaCl}^{1}\end{array}$ & $\begin{array}{l}\text { Glochldla } \\
\text { Open } \\
\text { after } \\
\mathrm{NaCl}\end{array}$ & $\begin{array}{l}\text { Unadjusted } \\
\text { 24h viability } \\
\text { (\%) }\end{array}$ & $\begin{array}{l}\text { Adjusted 24h } \\
\text { Viabllity }(\%)^{2}\end{array}$ & $\begin{array}{c}\text { Mean } \\
\text { adjusted } \\
\text { viabllity }(\%)\end{array}$ & $\begin{array}{l}\text { Standard } \\
\text { Devlation } \\
\text { (SD) }\end{array}$ \\
\hline Black Sandshell & IAS & 0 & 612 & 12 & 83 & 84.5 & 100.0 & 100.0 & 2.3 \\
\hline Black Sandshell & $1 \mathrm{B1}$ & 0 & 206 & 4 & 24 & 86.4 & 102.3 & & \\
\hline Black Sandishell & $1 \mathrm{C} 6$ & 0 & 572 & 26 & 74 & 82.5 & 97.7 & & \\
\hline Black Sandshell & 1A1 & 50 & 353 & 287 & 17 & 13.9 & 16.4 & 17.4 & 2,1 \\
\hline Black Sandshell & $1 \mathrm{~A} 3$ & 50 & 137 & 98 & 16 & 16.8 & 19.9 & & \\
\hline Black Sandshell & $1 C 1$ & 50 & 111 & 92 & 4 & 13.5 & 16.0 & & \\
\hline Black Sandshell & $1 \mathrm{B2}$ & 100 & 181 & 166 & 12 & 1.7 & 2.0 & 8.2 & 5.8 \\
\hline Black Sandshell & 183 & 100 & 252 & 221 & 11 & 7.9 & 9.4 & & \\
\hline Elack Sandshell & $1 C 5$ & 100 & 222 & 179 & 18 & 11,3 & 13.3 & & \\
\hline Black Sandshell & $1 \mathrm{~A} \sigma$ & 200 & 351 & 338 & 8 & 1.4 & 1.7 & 3.8 & 2.6 \\
\hline Black Sandshell & 185 & 200 & 302 & 255 & 38 & 2.5 & 3.1 & & \\
\hline Black Sandshell & $1 \mathrm{~B} 6$ & 200 & 352 & 299 & 33 & 5.7 & 6.7 & & \\
\hline Black Sandshell & $1 \mathrm{AZ}$ & 300 & 284 & 270 & 14 & 0.0 & 0.0 & 0.9 & 0.8 \\
\hline Biack Sandshelf & 184 & 300 & 255 & 247 & 5 & 1,2 & 1,4 & & \\
\hline Black Sandshell & $1 \mathrm{C} 2$ & 300 & 383 & 373 & 6 & 1.0 & 1.2 & & \\
\hline Black Sandshell & $1 \wedge 4$ & $30 \mathrm{HD}$ & 485 & 471 & 11 & 0.6 & 0.7 & 0.9 & 0.9 \\
\hline Black Sand'shell & $1 C 3$ & $300 \mathrm{HD}$ & 381 & 367 & 8 & 1.6 & 1.9 & & \\
\hline Bdack Sandshell & $1 \mathrm{C} 4$ & $300 \mathrm{HD}$ & 302 & 295 & 7 & 0.0 & 0.0 & & \\
\hline
\end{tabular}

1 If the number counted was g.ochidia open, the number of glochidia closed = total glochidla - open glochidia counted before NaCl.

Glochidia viabllity: (total \# glochldia - (glochidia closed before + oper after NaCl))/total glochidia "100

${ }^{2}$ Vlability adjustments were calculated by divlding the observed viability by the mean Initial viabi' ity for each species 


\begin{tabular}{|c|c|c|c|c|}
\hline Study Number & AEH-11-PSEUDOיD1 & Action & Date & Initiais \\
\hline Lab Notebook & 1 & Created...... & 7-jun-12 & TMSTB \\
\hline File Folder & $12 \mathrm{~A}$ & Revised..... & 16-Mar-15 & JAL THS \\
\hline \multirow[t]{2}{*}{ Raw Data Location } & \multirow[t]{2}{*}{$12 \mathrm{~A}$} & Reviewed... & A.MAR-15 & $7 n s$ \\
\hline & & Certifled....... & $3 / 18 / 15$ & $\Pi^{\prime}$ \\
\hline Flle Name: & \multicolumn{4}{|c|}{ I:AEH-11-PSEUDO-011DatalGlochidla Vlability Summaryl[Glochidla vlablity assessment 24h (acfusted).xlsx]Mucket } \\
\hline
\end{tabular}

Glochidia Viability Assessment - 24 hour

Mussel specles; Mucket Glochlóia Lot number: 120700

Mean In tial vlabllity $=92.7 \%$

Test Chemlcal: Pseudomonas florescens Pf-CL 145A (SDP) Chemical lot \#: MBI-401 SDP 4655- «2-Mix

Exposure Date: 1 ił Jan-12

Test System Location: Block 2

\begin{tabular}{|c|c|c|c|c|c|c|c|c|c|}
\hline Specles & $\begin{array}{c}\text { Test } \\
\text { Chamber }\end{array}$ & $\begin{array}{c}\text { Treatment } \\
\text { Group } \\
(\mathrm{mg} / \mathrm{L})\end{array}$ & $\begin{array}{c}\text { Total } \\
\text { Number } \\
\text { Glochida } \\
\text { Counted }\end{array}$ & $\begin{array}{c}\text { Glochidia } \\
\text { Closed } \\
\text { before } \\
\mathrm{NaCl}^{1} \\
\end{array}$ & $\begin{array}{l}\text { Glochldia } \\
\text { Open } \\
\text { after } \mathrm{NaCl}\end{array}$ & $\begin{array}{l}\text { Unadjusted } \\
24 \text { h viability } \\
\text { (\%) }\end{array}$ & $\begin{array}{c}\text { Adjusted } \\
\text { 24h Vlability } \\
(\%)^{2}\end{array}$ & $\begin{array}{c}\text { Mean } \\
\text { ad)usted } \\
\text { viabillity (\%) }\end{array}$ & $\begin{array}{l}\text { Standard } \\
\text { Devlation } \\
\text { (SD) }\end{array}$ \\
\hline Mucket & $2 B 4$ & 0 & 261 & 7 & 19 & 90.0 & 97.1 & 99.9 & 2.4 \\
\hline Mucket & $2 B 5$ & 0 & 361 & 1 & 20 & 94.2 & 101.6 & & \\
\hline Mucket & $2 \mathrm{Cl}$ & 0 & 251 & 8 & 8 & 93.6 & 101.0 & & \\
\hline Mucket & $2 A 4$ & 50 & 328 & 49 & 14 & 80.8 & 87.2 & 81.7 & 14.1 \\
\hline Mucket & 286 & 50 & 146 & 49 & 8 & 61.0 & 65.8 & & \\
\hline Mucket & $2 \mathrm{C3}$ & 50 & 194 & 19 & 9 & 85.6 & 92.3 & & \\
\hline Mucket & $2 \mathrm{AZ}$ & 100 & 191 & 42 & 5 & 75.4 & 81.3 & 68.5 & 12.1 \\
\hline Mucket & 282 & 100 & 209 & 87 & 11 & 53.1 & 57.3 & & \\
\hline Mucket & $2 \mathrm{Co}$ & 100 & 213 & 71 & 10 & 62.0 & 66.9 & & \\
\hline Mucket & $2 \mathrm{~A} 5$ & 200 & 251 & 170 & 16 & 25.9 & 27.9 & 32.4 & 7.8 \\
\hline Mucket & $2 A 6$ & 200 & 407 & 241 & 10 & 38.3 & 41.3 & & \\
\hline Mucket & $2 \mathrm{C} 2$ & 200 & 497 & 355 & 14 & 25.8 & 27.8 & & \\
\hline Mucket & 2B1 & 300 & 192 & 171 & 3 & 9.4 & 10.1 & 6.5 & 3.8 \\
\hline Mucket & $2 \mathrm{~B} 3$ & 300 & 297 & 281 & 9 & 2.4 & 2.5 & & \\
\hline Mucket & $2 \mathrm{C4}$ & 300 & 451 & 417 & 6 & 6.2 & 6.7 & & \\
\hline Mucket & $2 \mathrm{AI}$ & $300 \mathrm{HO}$ & 292. & 278 & 6 & 2.7 & 3.0 & 5.6 & 3.7 \\
\hline Miccket & $2 \mathrm{~A} 3$ & 300110 & 308 & 276 & 4 & 9.1 & 9.8 & & \\
\hline Nucket & $2 \mathrm{C5}$ & $300 \mathrm{HD}$ & 157 & 149 & 2 & 3.8 & 4.1 & & \\
\hline
\end{tabular}

If the number counted was glochilda open, the number of glochidla closed = total glochidia - cFen glochidia counted before $\mathrm{NaCl}$

Glochidia vlablity: (total \# glochldia - (glochidia closed before + open after NaCl))/total glochidla *100

${ }^{2}$ Viability adjustments were calculated by dividing the observed vaability by the mean initial viability for each specles 


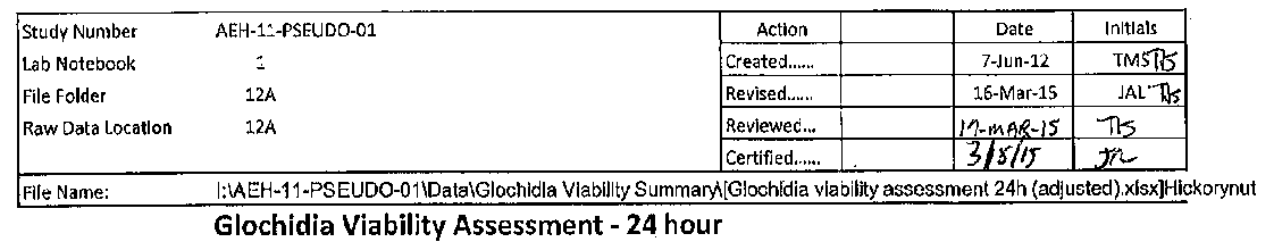

Mussel speclas: Hlckorynut Glochldla jot number: 120900 Mean initial vlabl $\mid$ ty $=86.0 \%$

Test Chemlcal: Pseudomonas florescens Pf-CL 145A [SDP) Cheinlcal lot number: MBI-4O1 SDP 4655-12-Mix

Exposure Date: 19-Jan-12.2

Page 1 of 1

Test System Locatlon: Block 3

\begin{tabular}{|c|c|c|c|c|c|c|c|c|c|}
\hline Species & $\begin{array}{c}\text { Test } \\
\text { Chamber }\end{array}$ & $\begin{array}{c}\text { Treatment } \\
\text { Group } \\
\text { (mg/L) }\end{array}$ & $\begin{array}{c}\text { Total } \\
\text { Number } \\
\text { Glachlda } \\
\text { Counted }\end{array}$ & $\begin{array}{c}\text { Glochldla } \\
\text { Closed } \\
\text { before } \\
\mathrm{NaCl}^{1} \\
\end{array}$ & $\begin{array}{c}\text { Glochldla } \\
\text { Open } \\
\text { after } \mathrm{NaCl}\end{array}$ & $\begin{array}{c}\text { Unadiusted } \\
\text { 24h viability } \\
\text { (\%) }\end{array}$ & $\begin{array}{c}\text { Adjusted } \\
24 \mathrm{~h} \text { Viabillty } \\
(\%)^{2}\end{array}$ & $\begin{array}{c}\text { Mean } \\
\text { adjusted } \\
\text { viability (\%) }\end{array}$ & $\begin{array}{l}\text { Standard } \\
\text { Deviatlon } \\
\text { (SO) }\end{array}$ \\
\hline HIckorynut & $3 A^{2}$ & 0 & 192 & 8 & 14 & 88.5 & 103.0 & 101.2 & 2.0 \\
\hline Hlckorynut & $3 C 1$ & 0 & 376 & 22 & 34 & 85.1 & 99.0 & & \\
\hline HIckorynut & $3 \mathrm{CA}$ & 0 & 359 & 14 & 31 & 87.5 & 101.7 & & \\
\hline HIekorynut & $3 A 5$ & 50 & 223 & 9 & 8 & 92,4 & 107.4 & 103.7 & 3.3 \\
\hline Hlckorynut & 3B3 & 50 & 303 & 18 & 22 & 86.8 & 100.9 & & \\
\hline Hickorynut & 386 & 50 & 233 & 18 & 9 & 88.4 & 102.8 & & \\
\hline Hkkorynut & 3в1 & 100 & 404 & 36 & 21 & 83.9 & 99.9 & 91.0 & 8.0 \\
\hline Hickorynut & $3 C 2$ & 100 & 278 & 59 & 17 & 72.7 & 84.5 & & \\
\hline Hickorynut & $9 \mathrm{Cs}$ & 100 & 257 & 51 & $10^{\circ}$ & 76.3 & 88.7 & & \\
\hline Hikkorynut & $3 \mathrm{AB}$ & 200 & 349 & 114 & 10 & 64.5 & 75.0 & 70.6 & 5.3 \\
\hline Hlckorynut & $3 A 4$ & 200 & 203 & 78 & 12 & 55.7 & 64.7 & & \\
\hline Hickorynut & $3 \mathrm{C} 6$ & 200 & 197 & 66 & 9 & 61.9 & 72.0 & & \\
\hline Hlckarynut & JA6 & 300 & 191 & 138 & 9 & 23.0 & 26.8 & 30.9 & 3.8 \\
\hline Hickorynut & $3 B 2$ & 300 & 140 & 98 & 4 & 27.1 & 31.6 & & \\
\hline Hlckorynut & $3 B 5$ & 300 & 231 & 156 & 7 & 29,4 & 34.2 & & \\
\hline Hickorynut & SA1 & $300 \mathrm{HD}$ & 290 & 287 & 2 & 0.3 & 0.4 & 0.6 & 0.4 \\
\hline Hlckorynut & 384 & $300 \mathrm{HD}$ & 314 & 307 & 5 & 0.3 & 0.4 & & \\
\hline Hickorynut & $3 C 3$ & $300 \mathrm{HD}$ & 226 & 221 & 3 & 0.9 & 1.0 & & \\
\hline
\end{tabular}




\begin{tabular}{|c|c|c|c|c|}
\hline \multirow{2}{*}{\multicolumn{2}{|c|}{$\begin{array}{l}\text { S:udy Number: AEH-11-PSEUDO-01 } \\
\text { lab Notebook: } 1\end{array}$}} & Action & Date & Initials \\
\hline & & Created...... & 17-Mar-15 & TIS TPS \\
\hline \multirow[t]{3}{*}{ Data Source: } & Flle Folder: $12 \mathrm{~d}$ & Revised....... & 18-Mar-15 & IJS THS \\
\hline & Forms: Glochidia Photomicrograph Counting Record & Reviewed... & 18.MAR-1S & T25 \\
\hline & & Certified... & $1.2 / 12 / 15$ & $\sqrt[5 n]{n}$ \\
\hline
\end{tabular}

\section{Glochidia Distribution Counts}

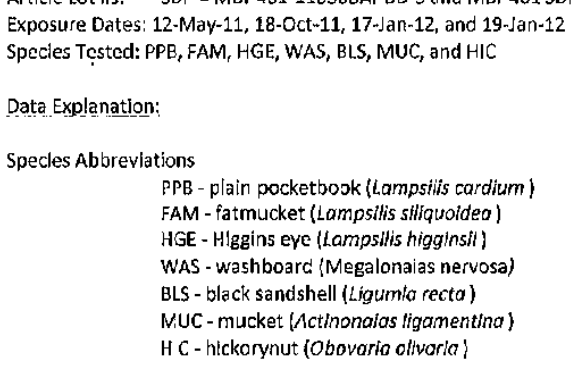

Throughour distribution of glochidia to test chambers, $n=16100 \mu \mathrm{L}$ samples of glochldia were collected, photographed, and enumerated (from photomicrographs) to estlmate the number of glochidia distributed to test chambers.

These enumerations were used to calculate the mean number of glochidia distributed to each chamber $[5 \times 100 \mu \mathrm{L}$ mean $=$ estimated glochidla per $500 \mu \mathrm{L}$ test chamber).

Data anomalles and deviations:

Hlckorynut samples contained large numbers of glochldia, and therefcre, some samples were divided and multiple photographs were captured for enumeration. 
Stucy Number: AEH-11-PSEUDO-01

Lab Notebook 1

Da:a Source: Flle =olcer: $12 \mathrm{~d}$

Forms: Glochidia Photomicrograph Counting Record
Test Article: NBI 401 (PF-CL 145A [SDP])

Article Lot \#: MBI-401-110308AI-BD-3

Exposure Dates: 12-May-11

Species Tested: PPB

Plain pocketbook (SDP) Distribution Glochidia Count

\begin{tabular}{|c|c|c|c|c|c|}
\hline Species & Formulation & Test date & $\begin{array}{l}\text { Distribution } \\
\text { round }\end{array}$ & $\begin{array}{c}\text { Rep } \\
\text { number }\end{array}$ & $\begin{array}{c}\text { Total glochidia } \\
\text { per } 100 \mu \mathrm{L}\end{array}$ \\
\hline \multirow[t]{16}{*}{ PPB } & SDP & 12-May-11 & 1 & 1 & 689 \\
\hline & & & & 2 & 715 \\
\hline & & & & 3 & 450 \\
\hline & & & 2 & 1 & 577 \\
\hline & & & & 2 & 1136 \\
\hline & & & & 3 & 1056 \\
\hline & & & 3 & 1 & 679 \\
\hline & & & . & 2 & 544 \\
\hline & & & & 3 & 667 \\
\hline & & & 4 & 1 & 731 \\
\hline & & & & 2 & 787 \\
\hline & & & & 3 & 520 \\
\hline & & & 5 & 1 & 249 \\
\hline & & & & 2 & 659 \\
\hline & & & & 3 & 770 \\
\hline & & & & 4 & 506 \\
\hline & & & & Mean (per $100 \mu L)$ & 671 \\
\hline & & & & SD & 215 \\
\hline & Glochidia & Distrlbution & Estimate & Mean $($ per $500 \mu \mathrm{L})$ & 3355 \\
\hline
\end{tabular}

Item Number 9

Page 2 of 10 
Study Number: AEH-11-PSEUDO-01

Lab Notebook 1

Data Source: File Folder: 12d

Forms: Glochldia Photomicrograpt. Counting Record
Test Article: MB1 401 (Pf-CL 145A [SDP]) Article Lot \#: MBI-401-110308A-BD-3 Exposure Dates: 12-May-11

Species Tested: FAM

Fatmucket (SDP) Distribution Glochidia Count

\begin{tabular}{|c|c|c|c|c|c|}
\hline Species & Formulation & Test date & $\begin{array}{c}\text { Distrlbution } \\
\text { round }\end{array}$ & $\begin{array}{c}\text { Rep } \\
\text { rumber }\end{array}$ & $\begin{array}{c}\text { Total glochldia } \\
\text { per } 100 \mu L\end{array}$ \\
\hline \multirow[t]{16}{*}{ FAM } & SDP & 12-May-11 & 1 & 1 & 677 \\
\hline & & & & 2 & 1211 \\
\hline & & & & 3 & 630 \\
\hline & & & 2 & 1 & 552 \\
\hline & & & & 2 & 635 \\
\hline & & & & 3 & 413 \\
\hline & & & 3 & 1 & 399 \\
\hline & & & & 2 & 329 \\
\hline & & & & 3 & 508 \\
\hline & & & 4 & 1 & 481 \\
\hline & & & & 2 & 381 \\
\hline & & & & 3 & 350 \\
\hline & & & 5 & 1 & 450 \\
\hline & & & & 2 & 436 \\
\hline & & & & 3 & 423 \\
\hline & & & & 4 & 771 \\
\hline & & & & Mean (per $100 \mu \mathrm{L})$ & 540 \\
\hline & & & & SD & 219 \\
\hline & Glochidl & DIstrlbution & Estlmate & Mean (per $500 \mu \mathrm{L})$ & 2702 \\
\hline
\end{tabular}

item Number $\quad 9$

Page $\frac{3}{3}$ of 10 
Stucy Number: AEH-11-PSEUDO-01

Lab Notebook 1

Da:a Source: File Folder: $12 d$

Forms: Glachidia Photomicrograph Counting Record
Test Article: $\mathrm{MIBI} 401$ (Pf-CL 1ASA [SOP])

Artlcle Lot H: MBI-401-110308AT-BD-3

Exposure Dates: 12-May-11

Species Tested: HGE

\section{Higgins eye (SDP) Distribution Glochidia Count}

\begin{tabular}{|c|c|c|c|c|c|}
\hline Species & Formulation & Test dațe & $\begin{array}{c}\text { Distribution } \\
\text { round }\end{array}$ & $\begin{array}{c}\text { Rep } \\
\text { number }\end{array}$ & $\begin{array}{c}\text { Totai glochldla } \\
\text { per } 100 \mu \mathrm{L}\end{array}$ \\
\hline \multirow[t]{16}{*}{ HGE } & SDP & 12-May-11 & 1 & 1 & 1107 \\
\hline & & & & 2 & 823 \\
\hline & & & & 3 & 636 \\
\hline & & & 2 & 1 & 843 \\
\hline & & & & 2 & 871 \\
\hline & & & & 3 & 812 \\
\hline & & & 3 & 1 & 746 \\
\hline & & & & 2 & 640 \\
\hline & & & & 3 & 549 \\
\hline & & & 4 & 1 & 802 \\
\hline & & & & 2 & 593 \\
\hline & & & & 3 & 357 \\
\hline & & & 5 & 1 & 724 \\
\hline & . & & & 2 & 684 \\
\hline & & & & 3 & 481 \\
\hline & & & & 4 & 298 \\
\hline & & & & Mean (per $100 \mu \mathrm{L}$ ) & 685 \\
\hline & & & & So & 204 \\
\hline & Glochid & Distributiol & Est/mate & Mean (per $500 \mu$ 니 & 3427 \\
\hline
\end{tabular}

Item Number $\frac{9}{4}$ 
Study Number: AEH-11-PSEUDO-C1

Lab Notebook 1

Data Source: File Folder: $12 d$

Forms: Glochldla Photomlcrograph Counting Record lest Article: $\mathrm{YBI} 401$ \{Pf-CL 145A [FDP] Artlcle Lot H: 110607WB-FD-E

Exposure Dates: 18-Oct-11

Species Tested: PPB

Plain pocketbook (FDP) Distribution Glochidia Count

\begin{tabular}{|c|c|c|c|c|c|}
\hline Species & Formulation & Test date & $\begin{array}{l}\text { Distribution } \\
\text { round }\end{array}$ & $\begin{array}{c}\text { Rep } \\
\text { number }\end{array}$ & $\begin{array}{c}\text { Total giachidia } \\
\text { per } 100 \mu \mathrm{L} \text {. }\end{array}$ \\
\hline \multirow[t]{16}{*}{ PPB } & FDP & 18-Oct-11 & 1 & 1 & 762 \\
\hline & & & & 2 & 1075 \\
\hline & & & & 3 & 501 \\
\hline & & & & 4 & 620 \\
\hline & & & 2 & 1 & 986 \\
\hline & & & & 2 & 1023 \\
\hline & & & & 3 & 493 \\
\hline & & & 3 & 1 & 1152 \\
\hline & & & & 2 & 968 \\
\hline & & & & 3 & 1221 \\
\hline & & & 4 & 1 & 1278 \\
\hline & & & & 2 & 1033 \\
\hline & & & & 3 & 971 \\
\hline & & & 5 & 1 & 718 \\
\hline & & & & 2 & 1126 \\
\hline & & & & 3 & 800 \\
\hline & & & & Nean (per $100 \mu \mathrm{L}$ ) & 920 \\
\hline & & & & SD & 245 \\
\hline & Glochid & Distribution & Estimate & Mean (per $500 \mu$ L) & 4602 \\
\hline
\end{tabular}

tem Number $\frac{9}{\text { of } 10}$ 
Study Number: AEH-11-PSEUDO-01

Lab Notebook 1

Data Source: File Folder: $12 \mathrm{~d}$

Forms: Glochidia Photomicrogrash Counting Record

Test Article: MB| 401 (Pf-CL. 145A [FDP)) Article lot \#: 110607 WB-FD-E

Exposure Dates: 18-Oct-11

Specles Tested: WAS

Washboard (FDP) Distribution Glochidia Count

\begin{tabular}{|c|c|c|c|c|c|}
\hline Species & Formulation & Test date & $\begin{array}{c}\text { Distribution } \\
\text { round }\end{array}$ & $\begin{array}{c}\text { Rep } \\
\text { number }\end{array}$ & $\begin{array}{c}\text { Total glochidia } \\
\text { per } 100 \mu \mathrm{t}\end{array}$ \\
\hline \multirow[t]{16}{*}{ WAS } & F.DP & 18-0ct-11 & 1 & 1 & 115 \\
\hline & & & & 2 & 7 \\
\hline & & & & 3 & 139 \\
\hline & & & & 4 & 110 \\
\hline & & & ?. & 1 & 215 \\
\hline & . & & & 2 & 149 \\
\hline & & & & 3 & 255 \\
\hline & & & 3 & 1 & 204 \\
\hline & & & & 2 & 179 \\
\hline & & & & 3 & 288 \\
\hline & & & 4 & 1 & 201 \\
\hline & & & & 2 & 303 \\
\hline & & & & 3 & 171 \\
\hline & & & 5 & 1 & 131 \\
\hline & & & & 2 & 152 \\
\hline & & & & 3 & 80 \\
\hline & & & & Mean (per $100 \mu \mathrm{L}$ ) & 169 \\
\hline & & & & SD & 75 \\
\hline & Glochidi & Distributio & Estimate & Mean (per $500 \mu \mathrm{L}$ ) & 843 \\
\hline
\end{tabular}

tem Number 9

Page 6 of 10 
Study Number: AE'-1-11-PSEUDO-01

Lab Notebook 1

Data Source: F'le Fclder: $12 d$

Forms: Glochidia Photomicrograph Counting Record

Test Art|cle: MBI 401 (Pf-CL 145A [SDㅁ]) Article Lot \#: MBI-401 SDP 4655-12-Mix Exposure Dates: 17-Jan-12

Specles Tested: BLS

Black sandshell (SDP) Distribution Glochidia Count

\begin{tabular}{|c|c|c|c|c|c|}
\hline Species & Formulation & Test date & $\begin{array}{c}\text { Distributton } \\
\text { round }\end{array}$ & $\begin{array}{c}\text { Rep } \\
\text { number }\end{array}$ & $\begin{array}{c}\text { Total glochidia } \\
\text { per } 100 \mu \mathrm{l}\end{array}$ \\
\hline \multirow[t]{19}{*}{ PPB } & SDP & 17-Jan-12 & 1 & 1 & 322 \\
\hline & & & & 2 & 456 \\
\hline & & & & 3 & 382 \\
\hline & & & 2 & 1 & 468 \\
\hline & & & & 2 & 546 \\
\hline & & & & 3 & 430 \\
\hline & & & 3 & 1 & 309 \\
\hline & & & & 2 & 367 \\
\hline & & & & 3 & 235 \\
\hline & & & 4 & 1 & 453 \\
\hline & & & & 2 & 348 \\
\hline & & & & 3 & 514 \\
\hline & & & 5 & 1 & 604 \\
\hline & & & & 2 & 415 \\
\hline & & & & 3 & 324 \\
\hline & & & & 4 & 295 \\
\hline & & & & Mean (per $100 \mu \mathrm{t})$ & 404 \\
\hline & & & & SD & 100 \\
\hline & Glochld| & Distributlon & Estimate & Mean $($ per $500 \mu \mathrm{L})$ & 2021 \\
\hline
\end{tabular}

Item Number $\frac{9}{\text { Page. }-1}$ 
Study Number: AEH-11-PSEUDO-01

Lab Notebook 1

Data Source: File Folder: $12 d$

Forms: Glochidla Photomicrograps Counting Record

Test Article: MB| 401 (Pf-CL 145A 'SDP) Artlcie Lot H: MB|-401 SDP 4655-12-Mix Exposure Dates: 17-ian-12

Species Tested: MUC

Mucket (SDP) Distribution Glochidia Count

\begin{tabular}{|c|c|c|c|c|c|}
\hline Specles & Formulation & Test date & $\begin{array}{l}\text { Distribution } \\
\text { round }\end{array}$ & $\begin{array}{c}\text { Rep } \\
\text { number }\end{array}$ & $\begin{array}{c}\text { Total glochidia } \\
\text { per } 100 \mu \mathrm{L}\end{array}$ \\
\hline \multirow[t]{16}{*}{ MUC } & SDP & $17-\operatorname{Jan}-12$ & 1 & 1 & 97 \\
\hline & & & & 2 & 576 \\
\hline & & & & 3 & 727 \\
\hline & & & 2 & 1 & 521 \\
\hline & & & & 2 & 754 \\
\hline & & & & 3 & 218 \\
\hline & & & 3 & 1 & 531 \\
\hline & & & & 2 & 350 \\
\hline & & & & 3 & 780 \\
\hline & & & 4 & 1 & 618 \\
\hline & & & & 2 & 470 \\
\hline & & & & 3 & 285 \\
\hline & & & 5 & 1 & 309 \\
\hline & & & & 2 & 198 \\
\hline & & & & 3 & 278 \\
\hline & & & & 4 & 329 \\
\hline & & & & Mean (per $100 \mu \mathrm{l}$ ) & 440 \\
\hline & & & & SD & 212 \\
\hline & Glochldi: & |stributio & mate & Mean (per $500 \mu \mathrm{L})$ & 2200 \\
\hline
\end{tabular}

Iten Number $\frac{9}{\text { of } 10}$ 
StLdy Number: AEH-11-PSEUDO-01 Lab Notebook 1

Data Source: File Folder: 12

Forrาs: Glochidia Photomicrograph Counting Record
Test Article: $M B ! 401$ [Pf-CL 145A [SDP]) Article Lot H: MBI-401 SDP 4655-12-Mix Exposure Dates: $19 \cdot$ Jan-12

Species Tested: HIC

Hickorynut (SDP) Distribution Glochidia Count

\begin{tabular}{|c|c|c|c|c|c|}
\hline Species & Formulation & Test date & $\begin{array}{c}\text { Distribution } \\
\text { round }\end{array}$ & $\begin{array}{c}\text { Rep } \\
\text { nunber }\end{array}$ & $\begin{array}{l}\text { Total glochidla } \\
\text { per } 100 \mu \mathrm{L}\end{array}$ \\
\hline \multirow[t]{19}{*}{$\mathrm{HIC}$} & SDP & 19-Jan-12 & 1 & 1 & 779 \\
\hline & & & & 2 & 995 \\
\hline & & & & 3 & 2555 \\
\hline & & & 2 & 1 & 593 \\
\hline & & & & 2 & 1105 \\
\hline & & & & 3 & 521 \\
\hline & & & 3 & 1 & 359 \\
\hline & & & & 2 & 853 \\
\hline & & & & 3 & 708 \\
\hline & & & 4 & 1 & 637 \\
\hline & & & & 2 & 748 \\
\hline & & & & 3 & 1067 \\
\hline & & & 5 & 1 & 630 \\
\hline & & & & 2 & 545 \\
\hline & & & & 3 & 701 \\
\hline & & & & 4 & 603 \\
\hline & & & & Mean (per $100 \mu \mathrm{L})$ & 837 \\
\hline & & & & SD & 501 \\
\hline & Glochidl & Distributlor & Estimate & Mean (per $500 \mu \mathrm{l}$ ) & 4187 \\
\hline
\end{tabular}

Item Number $\frac{9}{9}$ of 10 
StLCy Number: AEH-11-PSEUDO-01

Lab Notebook 1

Data Source: File Folder: $12 d$

Forms: Glochidia Protomicrograph Counting Record
Test Article: M81 401 (Pf-CL 145A [SDP] and [FDP])

Article Lot t: MB|-401-110308Al-BD-3, MB|-401 SDP 4655-12-Mix, 110607WB--ED-E

Exposure Oates: 12-May-11, 18-Oct-11, 17-Jan-12, and 19-Jan-12

Species Tested: PPB, FAM, HGE, WAS, BIS, MUC, and HIC

Distribution Glochidia Count Summary

\begin{tabular}{|c|c|c|c|c|}
\hline Species & Formulatior & Test dale & $\begin{array}{c}\text { Mean glochidla } \\
\text { per sample }(100 \mu \mathrm{L})\end{array}$ & 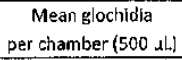 \\
\hline PPB & SOP & 12-MaV-11 & 671 & 3355 \\
\hline FAM & SDP & $1 \geqslant$ May-11 & 540 & 2702 \\
\hline HGE & SOP & 12-May-11 & 685 & 3427 \\
\hline PPB & FDP & $18-0 \mathrm{ct}-11$ & 920 & 4602 \\
\hline WAS & FDP & $18-0 \mathrm{ct}-11$ & 169 & $B 43$ \\
\hline BLS & SOP & 17.Jan-12 & 404 & 2021 \\
\hline MUC & SOP & 17-Jan-12 & 440 & 2200 \\
\hline$H K$ & SOP & 19-Jan-12 & 837 & 4187 \\
\hline
\end{tabular}

Itcm Number 9 


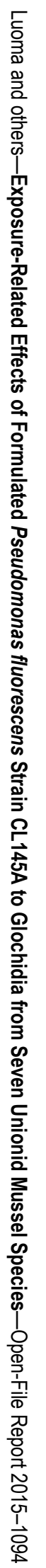

ISSN 2331-1258 (online) http://dx.doi.org/10.3133/ofr20151094 